\title{
Recent CMV Research
}

\author{
Edited by \\ Anamaris M. Colberg-Poley
}

Printed Edition of the Special Issue Published in Viruses 
Anamaris M. Colberg-Poley (Ed.)

\section{Recent CMV Research}


This book is a reprint of the special issue that appeared in the online open access journal Viruses (ISSN 1999-4915) in 2013

(http://www.mdpi.com/journal/viruses/special_issues/recent_cmv_research).

\section{Guest Editor}

Anamaris M. Colberg-Poley

Professor of Integrative Systems Biology and of Pediatrics

George Washington University School of Medicine and Health Sciences

Senior Investigator,

Research Center for Genetic Medicine,

Children's National Health System

Washington, DC 20010, USA

\section{Editorial Office}

MDPI AG

Klybeckstrasse 64

Basel, Switzerland

Publisher

Shu-Kun Lin

Managing Editor

Delia Costache

\section{Edition 2014}

MDPI • Basel • Beijing • Wuhan

ISBN 978-3-906980-53-9 (Hbk)

ISBN 978-3-906980-54-6 (PDF)

(C) 2014 by the authors; licensee MDPI, Basel, Switzerland. All articles in this volume are Open Access distributed under the Creative Commons License (CC BY), which allows users to download, copy and build upon published articles even for commercial purposes, as long as the author and publisher are properly credited, which ensures maximum dissemination and a wider impact of our publications. However, the dissemination and distribution of physical copies of this book as a whole is restricted to MDPI, Basel, Switzerland. 


\title{
Table of Contents
}

\author{
Anamaris M. Colberg-Poley
}

Preface Guest Editor.

Kayla Dufrene, Roberta L. DeBiasi and Anamaris M. Colberg-Poley

Introduction: Preface of the Special Issue: "Recent CMV Research"

Reprinted from: Viruses 2014, 6(1), 336-339

www.mdpi.com/1999-4915/6/1/336

\section{Pathogenesis and its Control}

\section{John H. Sinclair and Matthew B. Reeves}

Human Cytomegalovirus Manipulation of Latently Infected Cells

Reprinted from: Viruses 2013, 5(11), 2803-2824

www.mdpi.com/1999-4915/5/11/2803.

Martin Zydek, Matthew Petitt, June Fang-Hoover, Barbara Adler,

Lawrence M. Kauvar, Lenore Pereira and Takako Tabata

HCMV Infection of Human Trophoblast Progenitor Cells of the Placenta Is Neutralized by a Human Monoclonal Antibody to Glycoprotein B and Not by Antibodies to the Pentamer Complex

Reprinted from: Viruses 2014, 6(3), 1346-1364

www.mdpi.com/1999-4915/6/3/1346.

Sonia M. Restrepo-Gualteros, Lina E. Jaramillo-Barberi, Monica Gonzalez Santos,

Carlos E. Rodriguez-Martinez, Geovanny F. Perez, Maria J. Gutierrez and

Gustavo Nino

Characterization of Cytomegalovirus Lung Infection in Non-HIV Infected Children

Reprinted from: Viruses 2014, 6(5), 2038-2051

www.mdpi.com/1999-4915/6/5/2038

Jesse D. Deere and Peter A. Barry

Using the Nonhuman Primate Model of HCMV to Guide Vaccine Development

Reprinted from: Viruses 2014, 6(4), 1483-1501

www.mdpi.com/1999-4915/6/4/1483

\section{Immune and DNA Damage Responses Linked to CMV}

Patrick J. Hanley and Catherine M. Bollard

Controlling Cytomegalovirus: Helping the Immune System Take the Lead

Reprinted from: Viruses 2014, 6(6), 2242-2258

www.mdpi.com/1999-4915/6/6/2242 
Emily V. Stevenson, Donna Collins-McMillen, Jung Heon Kim, Stephen J. Cieply, Gretchen L. Bentz and Andrew D. Yurochko

HCMV Reprogramming of Infected Monocyte Survival and Differentiation: A Goldilocks

Phenomenon

Reprinted from: Viruses 2014, 6(2), 782-807

www.mdpi.com/1999-4915/6/2/782......

Annette Fink, Angeliqué Renzaho, Matthias J. Reddehase and

Niels A. W. Lemmermann

The p36 Isoform of Murine Cytomegalovirus m152 Protein Suffices for Mediating Innate and Adaptive Immune Evasion

Reprinted from: Viruses 2013, 5(12), 3171-3191

www.mdpi.com/1999-4915/5/12/3171

Annette Fink, Julia K. Büttner, Doris Thomas, Rafaela Holtappels,

Matthias J. Reddehase and Niels A. W. Lemmermann

Noncanonical Expression of a Murine Cytomegalovirus Early Protein CD8 T-Cell Epitope as an Immediate Early Epitope Based on Transcription from an Upstream Gene

Reprinted from: Viruses 2014, 6(2), 808-831

www.mdpi.com/1999-4915/6/2/808

\section{Bindu Raghavan, Charles H. Cook and Joanne Trgovcich}

The Carboxy Terminal Region of the Human Cytomegalovirus Immediate Early 1 (IE1)

Protein Disrupts Type II Inteferon Signaling

Reprinted from: Viruses 2014, 6(4), 1502-1524

www.mdpi.com/1999-4915/6/4/1502

\section{Xiaofei E and Timothy F. Kowalik}

The DNA Damage Response Induced by Infection with Human Cytomegalovirus and Other Viruses

Reprinted from: Viruses 2014, 6(5), 2155-2185

www.mdpi.com/1999-4915/6/5/2155

\section{Amit S. Kulkarni and Elizabeth A. Fortunato}

Modulation of Homology-Directed Repair in T98G Glioblastoma Cells Due to Interactions between Wildtype p53, Rad51 and HCMV IE1-72

Reprinted from: Viruses 2014, 6(3), 968-985

www.mdpi.com/1999-4915/6/3/968 


\section{The CMV Life Cycle}

Laura Graf, Rike Webel, Sabrina Wagner, Stuart T. Hamilton, William D. Rawlinson, Heinrich Sticht and Manfred Marschall

The Cyclin-Dependent Kinase Ortholog pUL97 of Human Cytomegalovirus Interacts with Cyclins

Reprinted from: Viruses 2013, 5(12), 3213-3230

www.mdpi.com/1999-4915/5/12/3213

\section{Rico Rana and Bonita J. Biegalke}

Human Cytomegalovirus UL34 Early and Late Proteins Are Essential for Viral Replication

Reprinted from: Viruses 2014, 6(2), 476-488.

www.mdpi.com/1999-4915/6/2/476

\section{Ina Niemann, Anna Reichel and Thomas Stamminger}

Intracellular Trafficking of the Human Cytomegalovirus-Encoded 7-trans-Membrane Protein Homologs pUS27 and pUL78 during Viral Infection: A Comparative Analysis

Reprinted from: Viruses 2014, 6(2), 661-682.

www.mdpi.com/1999-4915/6/2/661

Rebecca Marie Smith, Srivenkat Kosuri and Julie Anne Kerry

Role of Human Cytomegalovirus Tegument Proteins in Virion Assembly

Reprinted from: Viruses 2014, 6(2), 582-605.

www.mdpi.com/1999-4915/6/2/582

Vanessa M. Noriega, Thomas J. Gardner, Veronika Redmann, Gerold Bongers,

Sergio A. Lira and Domenico Tortorella

Human Cytomegalovirus US28 Facilitates Cell-to-Cell Viral Dissemination

Reprinted from: Viruses 2014, 6(3), 1202-1218

www.mdpi.com/1999-4915/6/3/1202

\section{The Use of Novel Technologies}

Sabine Reyda, Nicole Büscher, Stefan Tenzer and Bodo Plachter

Proteomic Analyses of Human Cytomegalovirus Strain AD169 Derivatives Reveal Highly

Conserved Patterns of Viral and Cellular Proteins in Infected Fibroblasts

Reprinted from: Viruses 2014, 6(1), 172-188.

www.mdpi.com/1999-4915/6/1/172

Mark R. Schleiss, Shane McAllister, Anibal G. Armién, Nelmary Hernandez-Alvarado, Claudia Fernández-Alarcón, Jason C. Zabeli, Thiruvarangan Ramaraj,

John A. Crow and Michael A. McVoy

Molecular and Biological Characterization of a New Isolate of Guinea Pig Cytomegalovirus

Reprinted from: Viruses 2014, 6(2), 448-475

www.mdpi.com/1999-4915/6/2/448 
Josephine S. Gnanandarajah, Peter A. Gillis, Nelmary Hernandez-Alvarado, LeeAnn Higgins, Todd W. Markowski, Heungsup Sung, Sheila Lumley and Mark R. Schleiss

Identification by Mass Spectrometry and Immune Response Analysis of Guinea Pig Cytomegalovirus (GPCMV) Pentameric Complex Proteins GP129, 131 and 133

Reprinted from: Viruses 2014, 6(2), 727-751. 391

www.mdpi.com/1999-4915/6/2/727

\section{Francisco Puerta Martínez and Qiyi Tang}

Identification of Cellular Proteins that Interact with Human Cytomegalovirus Immediate-

Early Protein 1 by Protein Array Assay

Reprinted from: Viruses 2014, 6(1), 89-105

www.mdpi.com/1999-4915/6/1/89

Endrit Elbasani, Ildar Gabaev, Lars Steinbrück, Martin Messerle and Eva Maria Borst

Analysis of Essential Viral Gene Functions after Highly Efficient Adenofection of Cells with Cloned Human Cytomegalovirus Genomes

Reprinted from: Viruses 2014, 6(1), 354-370.... 435

www.mdpi.com/1999-4915/6/1/354

Shivaprasad Bhuvanendran, Kyle Salka, Kristin Rainey, Sen Chandra Sreetama, Elizabeth Williams, Margretha Leeker, Vidhya Prasad, Jonathan Boyd, George H. Patterson, Jyoti K. Jaiswal and Anamaris M. Colberg-Poley

Superresolution Imaging of Human Cytomegalovirus vMIA Localization in Sub-

Mitochondrial Compartments

Reprinted from: Viruses 2014, 6(4), 1612-1636 453

www.mdpi.com/1999-4915/6/4/1612

\section{Steven Sijmons, Marc Van Ranst and Piet Maes}

Genomic and Functional Characteristics of Human Cytomegalovirus Revealed by NextGeneration Sequencing

Reprinted from: Viruses 2014, 6(3), 1049-1072 479 www.mdpi.com/1999-4915/6/3/1049

Zhu Yang, Gia-Phong Vu, Hua Qian, Yuan-Chuan Chen, Yu Wang, Michael Reeves, Ke Zen and Fenyong Liu

Engineered RNase P Ribozymes Effectively Inhibit Human Cytomegalovirus Gene

Expression and Replication

Reprinted from: Viruses 2014, 6(6), 2376-2391 503

www.mdpi.com/1999-4915/6/6/2376 


\section{Preface}

In developed countries, human cytomegalovirus (CMV) is the major infectious cause of congenital birth defects including microcephaly, mental retardation, sensorineural hearing loss, and intrauterine growth restriction. Nonetheless, because of the potential for teratogenicity and toxic effects, no prenatal therapeutic treatment is currently approved by the Food and Drug Administration for congenital CMV infection. Therefore there is great interest in understanding CMV growth and blocking or altogether preventing CMV infection.

When I received the email from the Viruses Editor-in-Chief, Eric Freed, about editing a Special Issue of Viruses on Recent CMV Research, I debated with myself and my husband (a Pediatric Infectious Disease physician) about the need for another dedicated CMV issue. There were several recent books, chapters and special issues on CMV including the comprehensive "Cytomegaloviruses From Molecular Pathogenesis to Intervention" edited by Matthias Reddehase with the assistance of Niels Lemmerman (Caister Academic Press, 2013) as well as a recent supplement (Supplement 4) of Clinical Infectious Diseases (2013, volume 57) on Prenatal Therapy of Congenital Cytomegalovirus Infection. Nonetheless, having participated in the $4^{\text {th }}$ Congenital CMV Conference in San Francisco and then being interviewed by Kayla Dufrene, a young woman who had suffered congenital CMV infection convinced me of the vital importance of continued efforts to understand the CMV life cycle and for developing new approaches to preventing and treating CMV diseases. With this in mind, I accepted serving as Guest Editor for the Viruses Special Issue on Recent CMV Research. Following my husband's advice, I asked Kayla if we could use her autobiography, which she wrote following our meeting for her writing class at Gallaudet University, as the Introduction for our Special Issue. She accepted and it is the first paper in this book (Dufrene et al., 2014). Her autobiography is the backdrop for this issue and should serve as inspiration for many CMV investigators. Tellingly, Kayla has also been invited to speak about her life's story at the 2014 Cytomegalovirus Public Health and Policy Conference in Utah. She provides an unparalleled perspective for the CMV field.

I am very pleased with this Viruses Special Issue. Of particular interest to families and caregivers affected by CMV diseases are several papers: addressing prevention of CMV infection of trophoblast cells (Zydek et al., 2014), CMV latency (Sinclair and Reeves, 2013), as well as of CMV lung infections in non-HIV infected children (Restrepo-Gualteros et al., 2014). Our ability to enhance immune responses for controlling CMV infection (Hanley and Bollard, 2014) and new strategies for CMV vaccine development guided by non-human primate studies (Deere and Barry, 2014) are discussed in two excellent reviews. Several articles address the CMV manipulation of the immune system, both innate and adaptive immune responses (Stevenson et al., 2014, Fink et al, 2013, 2014, Raghavan et al., 2014) and of DNA damage responses (E and Kowalik, 2014; Kulkarni and Fortunato, 2014).

The CMV life cycle itself is the subject of several papers, including demonstration that the UL97 protein interacts with cell cyclins (Graf et al., 2014), the UL34 early and late proteins 
are required for infection (Rana and Biegalke, 2014), the intracellular trafficking of CMV proteins (Niemann et al., 2014), the roles of CMV tegument proteins in virion assembly (Smith et al., 2014), and the effects of US28 protein on cell-to-cell spread of CMV (Noriega et al., 2014).

Development of new drugs for CMV depends heavily on the generation of novel understanding of CMV lifecycle and the mechanisms it uses in its progression. With this in mind, many studies in this book has made significant contributions of state-of-the-art technologies to the field including the use of mass spectrometry (Reyda et al., 2014; Gnanandarajah et al., 2014), protein arrays (Puerta Martínez and Tang, 2014), adenofection for efficient transfection of CMV genomes (Elbasani et al., 2014), superresolution microscopy (Bhuvanendran et al., 2014), next generation sequencing (Sijmons et al., 2014) and targeted ribozymes (Yang et al., 2014) to enhance our understanding of CMV infection.

Finally, I want to thank all the colleagues who contributed papers to this Special Issue, which has impressed the publishers and the readers alike. In fact the publisher selected two of the papers from this special issue (Zydek et al., 2014; Deere and Barry, 2014) to highlight in the MDPI Magazine. It is my hope that this special issue provides a valuable resource for the families and investigators in the CMV field.

Anamaris M. Colberg-Poley

Guest Editor 


Reprinted from Viruses. Cite as: Dufrene, K.; DeBiasi, R.L.; Colberg-Poley, A.M. Preface of the Special Issue: "Recent CMV Research". Viruses 2014, 6, 336-339.

Preface

\title{
Preface of the Special Issue: "Recent CMV Research"
}

\author{
Kayla Dufrene ${ }^{1}$, Roberta L. DeBiasi ${ }^{2,3}$ and Anamaris M. Colberg-Poley ${ }^{2,4,5, *}$ \\ 1 Gallaudet University, 800 Florida Avenue NE, Washington, DC 20002, USA; \\ E-Mail: kayla.dufrene@gallaudet.edu
}

2 Departments of Pediatrics, Children's National Medical Center, 111 Michigan Avenue, NW Washington, DC 20010, USA; E-Mail: rdebiasi@childrensnational.org

3 Microbiology, Immunology and Tropical Medicine, George Washington University, Washington, DC 20037, USA

4 Integrative Systems Biology, Research Center for Genetic Medicine, Children's National Medical Center, 111 Michigan Avenue, NW Washington, DC 20010, USA

5 Biochemistry and Molecular Medicine, School of Medicine and Health Sciences, George Washington University, Washington, DC 20037, USA

* Author to whom correspondence should be addressed;

E-Mail: acolberg-poley@childrensnational.org; Tel.: +1-202-476-3984; Fax: +1-202-476-6014.

Received: 9 December 2013 / Accepted: 14 January 2014 / Published: 22 January 2014

\section{Foreword}

This Viruses Special Issue on Recent Cytomegalovirus (CMV) Research is dedicated to the patients who have suffered CMV infection and to their parents, families and caregivers. We are including as a Preface to this issue the insights of a young college student, Kayla Dufrene, who suffered congenital CMV infection and contacted me and Dr. Roberta DeBiasi, to interview us to learn more about CMV. As I was just returning to the DC area from the 4th Congenital CMV Conference in San Francisco, I was particularly receptive to her request. When we met Kayla, we were both impressed with her personal strength and ability to cope with her disabilities and needed medical treatments. Despite it all, Kayla has an exceptionally positive outlook on life, feeling even lucky. She has not only coped, but has transcended her difficulties. I am proud to say that she was on the Dean's List (Figure 1) at Gallaudet University. Ultimately, her hope lies in our fields' efforts to develop a vaccine to prevent CMV disease in other children.

Her autobiography (in her own words) is our Preface. For those of us who work on the virus and anyone interested in the consequences of CMV disease, it is a touching and inspiring read (Figure 2). 
Anamaris Colberg-Poley, Guest Editor, Viruses, Special Issue, Recent CMV Research and Roberta L. DeBiasi, Professor of Pediatrics, GWU, Acting Chief, Division of Pediatric Infectious Diseases, Children's National Medical Center.

Figure 1. Kayla finding out that she made the Dean's List at Gallaudet University.

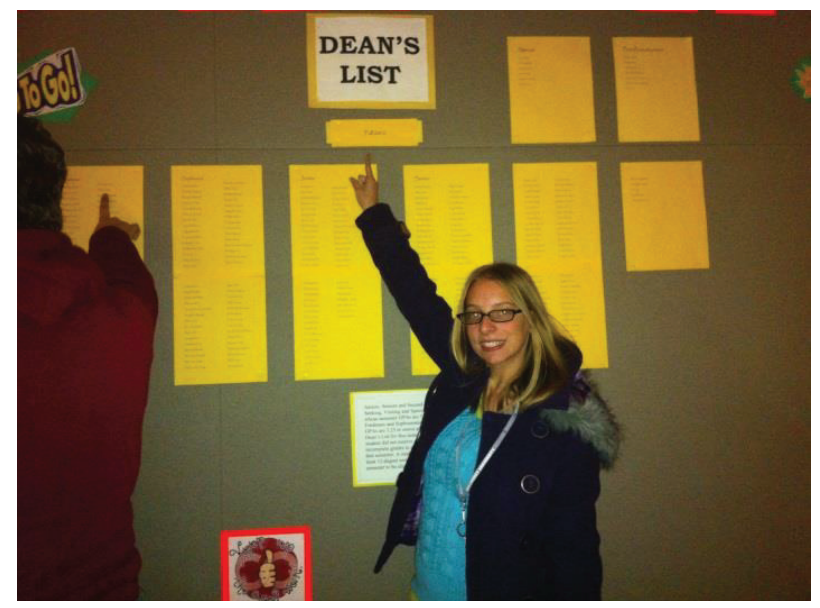

\section{Preface: Cytomegalovirus - Patient Monograph — Kayla Dufrene}

Prior to writing a research paper for a college assignment, I never felt the need to learn more about Cytomegalovirus (CMV). I didn't know a lot about CMV, I just grew up hearing my Mom tell doctors that it's what I was born with. It's the reason why I have hearing loss, bad eyesight, and muscle problems in my legs, and also the cyst in my brain. It's also why I have had to endure two eye surgeries and surgery on both my hips. When I was born, I was very sick. Besides having CMV I had an enlarged liver (when the liver swells beyond its normal size) and yellow jaundice (yellowing of the skin). I was very tiny and had to stay in the hospital for a month. The doctors told my birth parents I would either die or not have a good quality of life. The decision was then made to put me up for adoption.

Figure 2. Kayla enjoying the cherry blossoms.

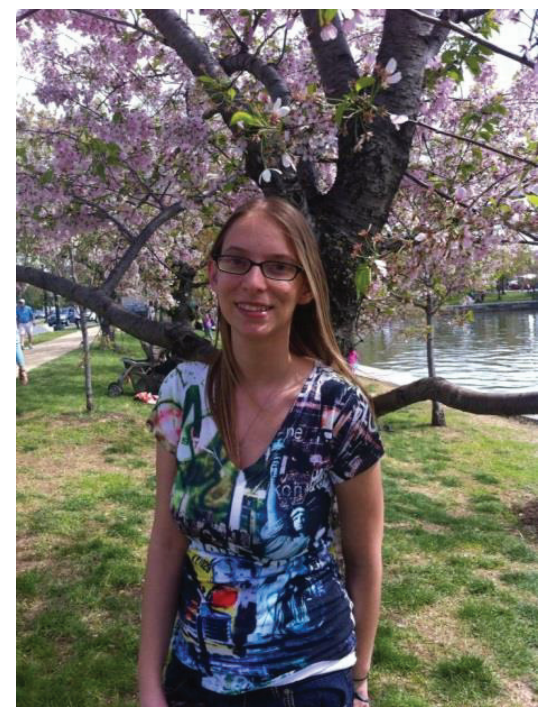


It turns out I definitely DID want to know more about CMV. What are the symptoms? Is there a cure? Is this a genetic disease? Is there genetic testing? How does it affect my body? How does someone get CMV? Is it an STD? How is it diagnosed? Can I get it later in life? Is there a vaccine for infants? Is there a test for CMV? Is it contagious? Who can get CMV? How many people are diagnosed with cytomegalovirus? How has it affected me? Do I still have it in my system? How does my case compare to others? How will it affect me later in life? Could it affect my sex partner? Could I really have died? Did it affect my birth mom? Is it a genetic disease and something I have to worry about in the future?

After reading texts and online references on Gallaudet's library webpage, I had a foundation for my search and I was ready to start building from there. I developed a desire for more knowledge and answers to a more of my questions. I learned that CMV is a more popular subject than I thought. When I read about the impact it can have on the family, it made me understand a little more why my birth parents put me up for adoption. They couldn't have known the extent that I would be affected yet here I am, 19 years later, a student at Gallaudet University. I was very excited to continue my search and learn more about the disease that has made me the person who I am today.

My reading taught me that development of a CMV vaccine was a national top priority and I wanted to learn more about the efforts that doctors and researchers are going through to make a vaccine a reality. I know firsthand the effects of CMV: Knowing a vaccine could have prevented a lot of what I had to go through and could prevent newborns in the future from being affected by CMV I believe is worthwhile. CMV is very common and the effects it can have on a child and the family can be very hard. I know these from my own experiences of being picked on in school for wearing a hearing aid and being "the girl who walked a little funny". I was excited to read about clinical trials that are testing potential vaccines for CMV. One of the articles explained a clinical trial to develop a vaccine to prevent CMV among mothers and infants. The possibility of a vaccine is very real. CMV has affected my life from the surgeries to the teasing in school and the thought that a vaccine could have prevented all of that is mind-boggling. My life could have turned out differently.

My reading sparked my interest in interviewing someone knowledgeable about CMV research and disease. The deadline for having an interviewee was quickly approaching. I thought long and hard about where I could find someone whom I could interview. I then thought that since CMV is found in babies, maybe I should ask a pediatrician. I found out that the Children's National Medical Center was just a few metro stops from Gallaudet and I was lucky to identify both a scientist (Dr. Colberg-Poley) and a doctor (Dr. Roberta DeBiasi) who focus their careers in this very area. They were both more than happy to meet with me. On the day of the interview, I was really nervous and wanted to make sure I had everything I needed prepared. I explained to them that I was writing a paper on a topic that had to relate to me so I chose CMV. I told them how I was born with CMV and of how it was the reason I had two eye surgeries when I was a kid, a hip surgery on both hips, and why I wear glasses and a hearing aid. They were thrilled to have me there, eager to share their knowledge about CMV, and just as excited to meet and speak with me as I was excited to meet with them.

Dr. DeBiasi shared that in her entire career, she had never met someone as an adult who had been diagnosed with CMV as a baby. She said it was a good experience for her and an honor to 
meet me. I shared many of my frustrations, such as the fact that no one figured out I was deaf until third grade, and I was able to ask if I born deaf or if it was detected late. I learned that even though the virus is latent in my body (just like anyone else who is infected with CMV at any time in their life); it is not something I have to worry about when I have a sexual relationship or children. I learned that it is not a genetic illness, and not something that I am going to pass on to my children genetically. I also learned that it is hard for doctors to accept that there are diseases they can diagnose, but for which they can't do anything about, and they want to help change that.

The one big thing that I took away was that I'm going to be okay and I do not have to stress about my future like wondering if CMV was going to affect my future children. I also realized that, when my birth mom put me up for adoption, she couldn't have known what the future held for me. I'm glad that she's okay. My adoptive mom did a very brave thing of adopting a baby that she knew was sick and never bat an eye and, over the last 19 years, I have not once heard her complain. Hearing Dr. Colberg-Poley and Dr. DeBiasi describe how there are even worse possible outcomes for people with CMV makes me feel extremely lucky. Something so small created a lot of problems for me growing up but it has made me a stronger person and more understanding of other people who have other problems. If it wasn't for cytomegalovirus I wouldn't have hearing problems and I wouldn't be at Gallaudet University and I never would have had the chance to find out all of this information about CMV.

Doing the interviews was the most beneficial thing to me because I have never had anyone to answer my questions. I was not sure where my search would lead me and what I would find out. I have always lived with the fear of not knowing how Cytomegalovirus would affect my future. I have always had questions like: What are the symptoms? Is there a cure? Is this a genetic disease? Do I still have it in my system? How does my case compare to others? How will it affect me later in life? Could it affect my sex partner? But I never had any answers. Now I have all the answers to all the questions I have ever asked. The search was hard and stressful but I'm glad I had the opportunity to finally learn about CMV. I have now made contact with people of whom I can ask questions that may arise later. During the interviews I learned things that I could not get through any article or any book and I got real answers. It's amazing that something as small as a tiny virus could have such a huge impact and effect on my life.

After my reading and interviews, I left feeling much more knowledgeable about CMV. Dr. Colberg-Poley and DeBiasi answered all of my questions and made me leave with a new-found confidence. I'm going to be okay in the future and so will my kids. CMV affected me as a baby and growing up it caused me a lot of problems, but it's because of having Cytomegalovirus that I've had the experiences I've had and why I'm at Gallaudet University. When I started my research, I was not sure where it would lead me or what I would find. I have learned a lot about the virus and a lot about myself and just because the past was tough, it doesn't mean the future has to be. 


\title{
CMV Pathogenesis and its Control
}

Reprinted from Viruses. Cite as: Sinclair, J.H.; Reeves, M.B. Human Cytomegalovirus Manipulation of Latently Infected Cells. Viruses 2013, 5, 2803-2824.

Review

\section{Human Cytomegalovirus Manipulation of Latently Infected Cells}

\section{John H. Sinclair ${ }^{1}$ and Matthew B. Reeves ${ }^{2, *}$}

1 Department of Medicine, University of Cambridge, Addenbrooke's Hospital, Hills Road, Cambridge, CB2 0QQ, UK; E-Mail: js152@cam.ac.uk

2 Institute of Immunity and Transplantation, Division of Infection and Immunity, University College London, Royal Free Campus, Rowland Hill Street, London, NW3 2PF, UK

* Author to whom correspondence should be addressed; E-Mail: matthew.reeves@ucl.ac.uk; Tel.: +44-(0)207-794-0500 (ext. 33109).

Received: 17 October 2013; in revised form: 11 November 2013 / Accepted: 13 November 2013 / Published: 21 November 2013

\begin{abstract}
Primary infection with human cytomegalovirus (HCMV) results in the establishment of a lifelong infection of the host which is aided by the ability of HCMV to undergo a latent infection. One site of HCMV latency in vivo is in haematopoietic progenitor cells, resident in the bone marrow, with genome carriage and reactivation being restricted to the cells of the myeloid lineage. Until recently, HCMV latency has been considered to be relatively quiescent with the virus being maintained essentially as a "silent partner" until conditions are met that trigger reactivation. However, advances in techniques to study global changes in gene expression have begun to show that HCMV latency is a highly active process which involves expression of specific latency-associated viral gene products which orchestrate major changes in the latently infected cell. These changes are argued to help maintain latent infection and to modulate the cellular environment to the benefit of latent virus. In this review, we will discuss these new findings and how they impact not only on our understanding of the biology of HCMV latency but also how they could provide tantalising glimpses into mechanisms that could become targets for the clearance of latent HCMV.
\end{abstract}

Keywords: cytomegalovirus; latency; immune evasion; apoptosis; gene expression; cellular signalling 


\section{Introduction}

Human Cytomegalovirus (HCMV) remains a major cause of disease in a number of patient populations who have compromised immune systems, as well as providing an increasing threat to critically ill immuno-competent patients [1-4]. These pathologies associated with opportunistic HCMV infections can be, in part, associated with a key characteristic of the virus: the ability to establish lifelong latent infection of the human host and, crucially, reactivate [2,5]. A wealth of studies from a number of laboratories using naturally latently infected cells has led to an informed consensus that the cells of the myeloid lineage represent at least one important site of HCMV latency, persistence, and reactivation (reviewed in [6]). Thus, at a cellular level, there is a clear and intimate link between myeloid differentiation and natural HCMV reactivation [7-14]. Furthermore, the use of experimental infection of non-permissive primary cells and cell lines in vitro are generating snapshots of the complex regulation of HCMV gene expression at a molecular level [15-25]. However, these studies have focussed predominantly on the regulation of major immediate early (MIE) gene expression because the critical switch to a reactivating phenotype is dependent on the triggering of MIE gene expression from quiescence.

In many cases, the species specificity of HCMV has driven these analyses to be performed in experimental cell culture models and, ultimately, on tissue derived from healthy HCMV seropositive individuals which has then been analysed ex vivo. As a result, the mechanisms that control HCMV latency and persistence in vivo, at an organism level, have relied on the extrapolation of studies performed in vitro or using animal model surrogates such as murine CMV [26]; guinea pig CMV [27] and, more recently, non-human primate CMV strains [28]. Consequently, the inability to perform analogous studies in humans has likely contributed to the perception that HCMV latency is essentially a relatively quiescent infection. However, as techniques for studying HCMV at a molecular level have become increasingly powerful, it is now emerging that latent HCMV infection profoundly modulates the latently infected cell and the surrounding cellular environment. These effects act in concert to maintain latent carriage and this depends on, at least in part, the expression of a subset of virally encoded gene products.

In this short review, we will examine our current knowledge of HCMV latency with particular emphasis on recent data which suggest that HCMV imparts a distinctive signature on latently infected cells. These latency-associated changes underpin the successful persistence of this virus in vivo and, importantly, could direct novel therapeutic strategies to target latency and reactivation of this important human pathogen.

\section{Background-HCMV Latency and Reactivation}

Following primary infection, HCMV establishes a latent infection of the CD34+ haematopoietic cell population in the bone marrow [29,30]. The prevailing view is that, ultimately, the major immediate early promoter (MIEP) is profoundly suppressed in these cells [6] and that this is achieved through cellular transcriptional repressors directing histone-modifying enzymes to impart repressive post-translational modifications of MIEP-associated histones [6]. During latency, the chromatin structure of the MIEP bears all the hallmarks of transcriptional repression: tri-methylation of histone H3 (lysine 9 and 27) and recruitment of heterochromatin protein-1 (HP-1) coupled with a 
concomitant absence of histone acetylation on histone H4 [11,16,17,25]. Consequently, HCMV MIE gene expression, and lytic gene expression in general, is profoundly repressed in CD34+ progenitor cells. This chromatin phenotype is maintained in the monocyte cells derived from these progenitors [11,31] and it is only upon cellular differentiation that robust IE gene expression is observed $[7,8,11,12,32]$. The detection of IE gene expression in dendritic cells (DCs) is consistent with the histone modifications present at the MIEP in these terminally differentiated myeloid cells [11,31]. For instance, HP-1 is no longer associated with the MIEP-likely due to extensive de-methylation of histones at lysine residue 9 (methylation at this residue being important for HP-1 binding to chromatin [33]) and, in these cells, the MIEP is associated with predominantly acetylated histones. Thus, the presence of repressive or activatory chromatin marks around the MIEP correlates with the expression of viral major IE RNA and the latency/reactivation phenotype of the virus [11,31]. Importantly, and consistent with molecular analyses, infectious HCMV progeny cannot be recovered from myeloid progenitor cells i.e., CD34+ cells or granulocyte-macrophage progenitors (GMPs) unless they are co-cultured under conditions that promote cellular differentiation or activation [9,11,34]. Analogous models of histone-mediated regulation of viral lytic gene expression also underpin studies of herpes simplex virus and Epstein-Barr virus and thus represent a common unifying theme in the biology of herpesvirus latency and reactivation $[35,36]$.

The molecular model of HCMV latency in the myeloid lineage, derived from analyses of natural latency, has been reviewed extensively elsewhere [6,37,38] and has helped provide an initial understanding of the underlying mechanism for the differentiation-dependent reactivation of HCMV. It is worth noting, however, that other studies using experimental infection models of latency and reactivation have essentially recapitulated the key observations made with natural models of latent infection and this gives confidence that wider studies involving experimentally latent models will have in vivo relevance.

\subsection{The Transcriptional Landscape of Latent HCMV}

HCMV encodes anywhere between 170 and 751 ORFs all of which are believed to be expressed at some stage during lytic infection [39,40]. Furthermore, the virus also encodes a number of microRNAs (miRNAs) which, during lytic infection, have been shown to target and regulate both cell and viral gene expression [41-43]. In contrast, the transcriptional landscape in latency is less clear. The earliest studies identified a number of transcripts arising from the MIE region of HCMV but no function was assigned to them $[44,45]$. Furthermore, deletion of the putative ORFs encoded by these latency-associated transcripts appeared to have little effect on HCMV latency in vitro [46]. As such, it was speculated that HCMV could exist in latency in a relatively quiescent state and that the normal transit and differentiation of latently infected CD34+ cells into the periphery was sufficient to trigger HCMV reactivation. Indeed, transcriptional quiescence during latency would provide the ideal mechanism for evasion of the robust immune responses known to be present in HCMV seropositive individuals [47]. However, a number of aspects of the known biology of HCMV are at odds with the view that HCMV is maintained in a totally quiescent state. For instance, if virus is carried long-term in the myeloid lineage, how is the latent genome maintained in cells which will, at least at some stage of their lifespan, proliferate? Although no latent origin of replication has been definitively identified for HCMV, it has been suggested that a mutation in the MIE region had a 
carriage defect during latency in GMPs [48] and more recent work has suggested UL84 may act to maintain viral sequences [25]. Furthermore, an overt characteristic of HCMV latency is the carriage of the viral genome in the cells of the myeloid lineage and, particularly, the monocyte lineage [49-51] but not lymphocyte or polymorphonuclear cells [50] despite the fact that latent infection is seeded in a pluripotent progenitor cell type [29,30]. Potentially, this could be explained in alternative ways: the virus actively promotes myelopoiesis of infected CD34+ cells or, HCMV may preferentially promote the survival of myeloid committed progenitors or, finally, HCMV cannot combat anti-viral mechanisms in cells committed to the lymphoid lineage. Arguably, all these scenarios suggest an active process involving viral latency-associated functions during latent infection.

A number of studies over the last 10 years or so have applied increasingly sensitive techniques to determine whether viral gene expression occurs during latent infection. Two independent microarray analyses identified a number of transcripts expressed during experimental latency [34,52] and, importantly, some have been subsequently confirmed during natural latency; including UL138, UL81-82ast (LUNA), as well as a splice variant of UL111A, which encodes a viral interleukin 10 (vIL-10) termed LAcmvIL-10 [24,53-55]. These, and subsequent studies, have also shown that the initial infection of undifferentiated myeloid cells with HCMV to establish experimental latency results in a burst of temporally dysregulated viral transcription from a number of gene loci, including MIE gene expression, at very early times post infection [25,32,34]. However, it remains unclear what this means in the context of latent infection. It is tempting to speculate that this gene expression is important for preparing the cell for latency-akin to that proposed for the establishment of EBV latency [56]. However, there is no evidence, as yet, that cells which initially express lytic antigens go on to establish long-term latency. It is possible that the extremely high MOIs used to establish latent infections in vitro results in a sub-population of lytically or abortively infected cells which are, ultimately, unviable and die, leaving the true latent population.

Regardless, what is generally accepted is that HCMV has a very distinct transcriptional profile during latent infection, quite different from lytic infection. The expression of a number of viral genes has now been described during latency and these are summarised in Table 1. For the remainder of this review, we will focus on emerging stories regarding the manipulation of latently infected cells by HCMV and how, in some instances, viral gene products may contribute to this.

Table 1. Gene products and functions during latency and lytic infection.

\begin{tabular}{cccc}
\hline Gene Product & Latent Function & Lytic Function & References \\
\hline CLTs & Unknown & $\begin{array}{c}\text { Regulation of anti-viral 2'5' OAS } \\
\text { expression (ORF94) }\end{array}$ & {$[44-46,57]$} \\
\hline UL138 & $\begin{array}{c}\text { Regulation of TNFRI (up) and MRP1 } \\
\text { (down), repression of the MIEP(?) }\end{array}$ & $\begin{array}{c}\text { Regulation of TNFRI (up) and } \\
\text { MRP1 (down), virus maturation } \\
(133-138 \text { locus) }\end{array}$ & {$[53,58-61]$} \\
\hline UL81-82ast & Promotes UL138 gene expression. & Unknown & {$[24,55,62]$} \\
\hline LAvIL-10 & Down-regulation of MHC class II & Unknown - cmvIL-10 expressed & {$[54,63]$} \\
& expression, immune evasion & during lytic infection & \\
\hline Lnc4.9 & Binds Polycomb repressor complex 2, & Unknown & {$[25]$} \\
& Silencing of the MIEP & & \\
\hline
\end{tabular}


Table 1. Cont.

\begin{tabular}{cccc}
\hline Gene Product & Latent Function & Lytic Function & References \\
\hline UL84 & Genome maintenance & $\begin{array}{c}\text { DNA replication, UTPase activity, } \\
\text { transcriptional regulation }\end{array}$ & {$[25,64-67]$} \\
& Unknown & GPCR, induces cell signalling and & {$[68-74]$} \\
US28 & cell migration, agonist of the MIEP & \\
& Unknown & TNF superfamily member, hijacks & {$[75-78]$} \\
& & NF-kB signalling, immune evasion? & \\
\hline UL144 & & &
\end{tabular}

\section{Mechanisms Targeted during HCMV Latency}

\subsection{Viral Evasion of Cell Death}

Pro-death signals in response to infection represent a very significant obstacle for many pathogens. Consequently, key players in the cellular apoptotic response become important targets for the virus - and HCMV is no exception. HCMV encodes an impressive armoury of anti-apoptotic functions that it expresses throughout lytic infection and which all contribute to efficient virus infection [79-84]. However, there is no evidence that any of these already-described anti-apoptotic viral genes associated with lytic infection are also expressed during latency. Clearly, if the virus was to be carried truly silently during latency then, arguably, there would be little requirement for any increased protection from cell death. However, it is becoming increasingly evident that HCMV does actively modulate multiple functions of the latently infected cell and that these, in effect, stress the cell to the point that viral functions are needed to protect the latently infected cell from such stress-induced pro-death signals.

In the context of infection, be it latent or lytic, the initiation of cell death can arise at the earliest point of infection: at entry [85]. Pathogen recognition receptors (PRRs) can detect pathogen-associated molecular patterns (PAMPs), triggering cell death - and this is an important part of an intrinsic immune response [85]. Clearly, during lytic infection, the rapid expression of virally encoded anti-apoptotic proteins could quickly provide protection against such extrinsic death response signals [80]. However, during HCMV infection of cells destined to become latently infected with the associated suppression of the lytic transcription programme, it appears that virus binding, in itself, activates cell survival signals [86,87]. This occurs in both CD34+ cells and CD14+ cells, albeit with the employment of different signalling pathways in the two cell types as well as cell-specific differences in the duration of the survival response. Nevertheless, the up-regulation of an important cellular anti-apoptotic protein, MCL-1 [88], appeared to be important for protection in both cell types $[86,87]$. Thus, although the exact mechanisms of protection varied in these different cell types, the outcome was the same.

The transitory nature of the ERK-MAPK dependent survival signal observed in CD34+ cells [86] argues that is likely to be important for overcoming the initial death signals triggered by cellular recognition of virus shortly after binding and/or entry. Consequently, it could be argued that long-term anti-death signals may not be required by a virus which is truly silenced in latency. However, recent work suggests that long-term anti-death signals may be important during latent infection with HCMV (Figure 1). For instance, experimental latent infection of granulocyte-macrophage 
progenitors has been shown to result in long-term up-regulation of PEA-15 RNA [89]. As PEA-15 is an anti-apoptotic factor that blocks both TNFR1 and Fas-L triggered apoptosis [90], clearly its up-regulation could be part of a protective response mediated by latent infection. Consistent with this, latently infected CD34+ cells are protected from FAS-L induced cell death [91]. Furthermore, given that it has been shown that the UL138 gene product up-regulates TNFR1 expression during lytic [59,60] and latent infection [61], potentially sensitising latently infected cells to TNFR1 mediated apoptosis, the concomitant up-regulation of PEA-15 would be a sensible pro-survival strategy. Other preliminary data from the Sinclair laboratory has also shown that a number of other cellular proteins with potent anti-apoptotic function are up-regulated in latently infected CD34+ cells (J.S. unpublished data) and this includes the PEA-15 protein, further supporting a model by which induction of PEA-15 during latent infection, at least in part, protects latently infected cells from pro-death signals. Furthermore, it is likely that these effects are driven by secreted products in the latency-associated secretome [92], since inhibition of latency-induced cellular IL-10 was sufficient to block this survival effect [91].

Figure 1. Protection of latently infected cells from cell death. CD34+ cells latently infected with HCMV down-regulate the expression of mir92a. A key target of miRNA is the GATA-2 transcription factor which, consequently, is up-regulated. This promotes increased transcription of cellular (IL-10) and viral (LUNA) genes. LUNA expression promotes UL138 gene expression-a gene product shown to up-regulate cell surface levels of TNFRI, a potentially pro-apoptotic signalling factor. However, HCMV also up-regulates a number of anti-apoptotic factors including PEA-15. This occurs, in part, via the expression of IL-10 and potentially could provide a mechanism to protect cells from extrinsic cell death signalling.

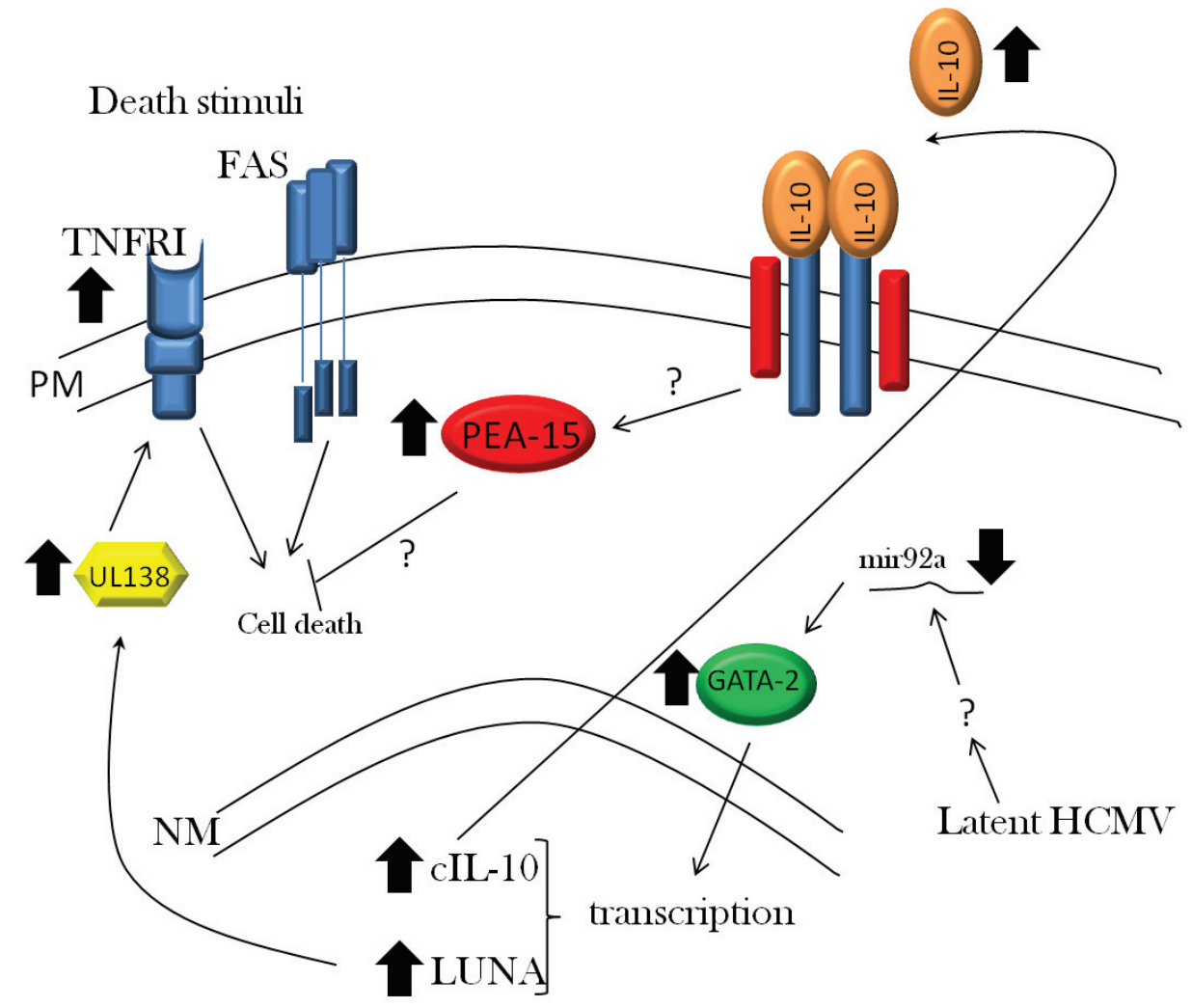




\subsection{Viral Evasion of the Immune Response during Latent Infection}

HCMV infection is known to generate a robust $\mathrm{T}$ cell response in vivo with between 0.5 and $10 \%$ of all cytotoxic T lympohcytes (CTLs) recognising HCMV antigens [47]. The CTL response to HCMV is dominated by two abundant viral antigens-pp65 and IE72 [47], although CTLs which recognise most, if not all, lytic antigens have been detected [93]. Pertinent to this review is that significant $\mathrm{T}$ cell responses against antigens also expressed during latency are present in healthy HCMV carriers [94,95] and thus, in theory, a latently infected cell should be visible to these $\mathrm{T}$ cells. However, recent work suggests that latent infection results in a number of mechanisms which act in concert to disrupt these $\mathrm{T}$ cell responses, thereby preventing clearance of latently infected cells by the adaptive arm of the host immune response (Figure 2).

A recent analysis of experimentally latently infected CD34+ cells detected a unique cell secretome signature associated with latency [92]. Intriguingly, this secretome was observed to promote the migration of Th1 CD4+ T cells to the latently infected cell. However, the anti-viral effector functions of these recruited cytotoxic $\mathrm{T}$ cells was countered by the concomitant latency-associated expression of two key cellular cytokines, transforming growth factor-beta (TGF- $\beta$ ) and interleukin-10 (cIL-10). Both TGF- $\beta$ and cIL-10 have profound immune-modulatory capacity [96] and, consistent with this, blocked the CD4 $\mathrm{T}$ effector functions [92]. Although the exact mechanisms that resulted in up-regulated expression of TGF- $\beta$ and cIL-10 during latency are unclear, elevated cIL-10 production was observed to be, at least partly, dependent on the up-regulation of the cellular GATA-2 transcription factor resulting from a concomitant down-regulation of the cellular microRNA mir92a [91]. Furthermore, other recent work has illustrated that a proportion of the CD4+ T cell response directed against latent antigens consists of $\mathrm{T}$ regulatory (Treg) cells [95]. This study in healthy donors showed that, whilst cytotoxic CD4 $+\mathrm{T}$ cell responses against latent antigens were detectable, they were dominated by IL-10 expressing Treg cells. Consequently, the recruitment of Treg cells to a latently infected cell (74) would augment the effects of the immune-suppressive secretome around the latently infected cells dampening down CTL effector cell function [92]. Given the extremely low frequency of latently infected cells in a healthy seropositive individual [97], it is likely that the microenvironment around a latently infected cell would have little overall impact on the normal immune homeostasis of the bone marrow but may be locally sufficient to ensure latently infected cells evade elimination by the immune system.

Induction of cIL-10 by latent virus clearly appears to be of real import for latent carriage and this view is, perhaps, reinforced by the fact that HCMV also encodes an IL-10 homolog, known as cmvIL-10, which is expressed solely during lytic infection, as well as an alternatively spliced form (LAcmvIL-10) expressed during both latent infection and lytic infection [98,99]. Interestingly, lytic infection-associated cmvIL-10 has retained many of the immune-suppressive functions associated with its cellular counterpart [100-102] and, indeed, signals via the human IL-10 receptor [98,103]. Consistent with a role for cmvIL-10-mediated immune evasion are studies in rhesus CMV that have demonstrated a role in viral dissemination [104] — presumably via a temporary dampening of the immune response. It is tempting to speculate that failure to evade the immune response during a primary infection could profoundly impact on the set point of latency but, unfortunately, this has not been possible to analyse. 
Figure 2. Evasion of the immune response to HCMV. The up-regulation of IL-10 in latently infected CD34+ cells is concomitant with TGF-b up-regulation via an unknown mechanism. However, the expression of two potent immune-suppressive cytokines inhibits the effector functions of CD4 Th1 cells recruited to a latently infected cell. Furthermore, both cellular IL-10 and viral IL-10 (LAcmvIL-10) act in concert to promote the down-regulation of HLA-DR MHC class II molecules on the surface of latently infected cells. Although the mechanism used by LAcmvIl-10 is not yet understood but is known not to occur via binding to the cellular IL-10 receptor.

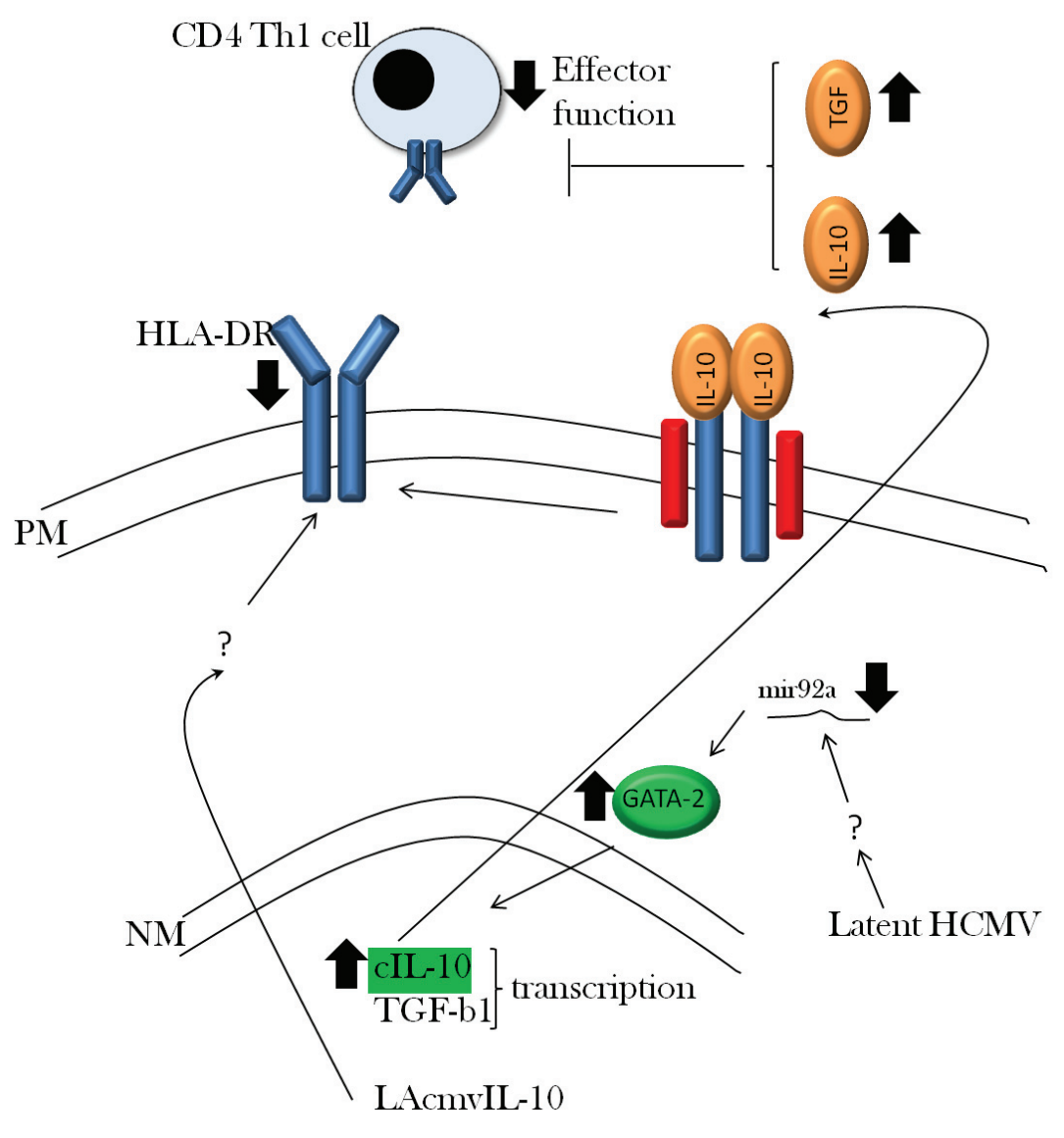

As stated above, the cmvIL-10 gene encodes a number of biological properties that could impinge on HCMV latency and reactivation. Multiple studies have shown that cmvIL-10 promotes MHC class I and II down-regulation [102], prevents DC maturation and function [105,106] and promotes the polarisation of macrophages to an M2c phenotype [107] — which is considered to be a relatively inactive macrophage phenotype compared with the classic inflammatory M1 phenotype. As such, all these functions would be consistent with a role in immune evasion. However, the alternatively spliced LAcmvIL-10, though also detected during lytic infection, is the isoform expressed during latent infection [54] but does not exhibit many of the properties of cmvIL-10 [63] — presumably, in part, due to its inability to bind the cIL-10 receptor [63]. Crucially, however, both latently infected GMPs and monocytes have been shown to exhibit a dramatic decrease in cell surface expression of MHC class II [32,108] — a function associated with LAcmvIL-10 [63]. Importantly the deletion of the UL111A locus from the virus (and thus LAcmvIL-10) has illustrated that latently cells become sensitive to CD4+ recognition and killing [109] as well as impacting on the 
normal differentiation of myeloid progenitor cells to a DC phenotype [110]. Thus despite a loss of many of the functions associated with cmvIL-10, the LAcmvIL-10 isoform has retained biological properties that could contribute to successful persistence during latency in vivo.

\subsection{Viral Regulation of Immediate Early Gene Expression}

As already discussed, the regulation of HCMV MIE gene expression during latency involves the action of higher order chromatin structure. As such, it has been hypothesised that the assembly and modification of histones at the MIEP is an intrinsic response dictated by the cellular environment. Indeed, at low MOIs during lytic infection, there appears to be pre-immediate early gene expression event where the MIEP is associated with methylated histones [111]. This may well represent an anti-viral response to foreign DNA that is mediated by ND10 bodies and their components and is overcome by the action of incoming viral pp71 tegument protein and, subsequently, newly expressed IE72 which has been reviewed extensively elsewhere [112-114]. In contrast to lytic infection, the intrinsic repression of the MIEP is not overcome in non-productive myeloid cells. One study has proposed that unknown mechanisms that exclude pp71 from the nucleus in CD34+ cells contributes to this [23], although the high levels of transcriptional repressors present in these cells is also likely to be important; consistent with this, the transfected MIEP is intrinsically less active in undifferentiated myeloid cells [115]. Indeed, a number of transcriptional repressors of the MIEP have been identified (such as YY1 and ERF) and these are believed to recruit histone methyltransferases $[116,117]$ to the MIEP in undifferentiated myeloid cells and this is important for generating the signature repressive chromatin phenotype associated with the MIEP of latent HCMV [11].

However, more recent work suggests that HCMV gene products themselves may be actively helping to manage MIE regulation during HCMV latency. Although the prevailing view of the MIEP during latency in CD34+ cells is a promoter predominantly associated with repressive chromatin marks (i.e., histone methylation and HP-1 binding), chromatin and its post-translational modification is highly dynamic. Studies analysing the chromatin state of well-characterised silenced cellular genes, in e.g., stem cells, suggest that all cellular promoters bear at least some hallmarks of transcription [118]. Histone methylation at lysine 4 (a marker of a recently transcribed promoter) has been identified at "silent promoters" and, consistent with this, small RNA fragments were identified which would correspond to aborted transcription events [118]. Thus the notion of "chromatin breathing," even at repressed promoters, is not uncommon. Given the potent activity of the MIEP, there is a strong argument that the MIEP is unlikely to be completely transcriptionally repressed, even in the most undifferentiated myeloid cell, and that this will call for additional mechanisms to eliminate any residual low level, uncontrolled MIE expression (Figure 3). 
Figure 3. HCMV mediated repression of IE gene expression. Latent infection of $\mathrm{CD} 34+$ cells is characterised by a repression of the major immediate early promoter (MIEP). Classically, the MIEP has been shown to be repressed by multiple cellular transcriptional repressors known to interact with components of the histone modifying enzyme families. However, these events may be augmented by the activity of further viral mechanisms. The expression of LUNA during latency has been shown to be important for UL138 expression - a protein postulated to repress the MIEP. Furthermore, the expression of the long non-coding 4.9kb RNA (lnc4.9 RNA) during latency has been suggested to promote the recruitment of polycomb repressor complex 2 (PRC2) to the MIEP via direct binding of the RNA. Recruitment of PRC2 would promote a chromatin structure inhibitory for MIE transcription. Finally, the expression of a viral miRNA, mir112.1, has been hypothesised to be important for silencing translation from MIE transcript UL123 during latency.

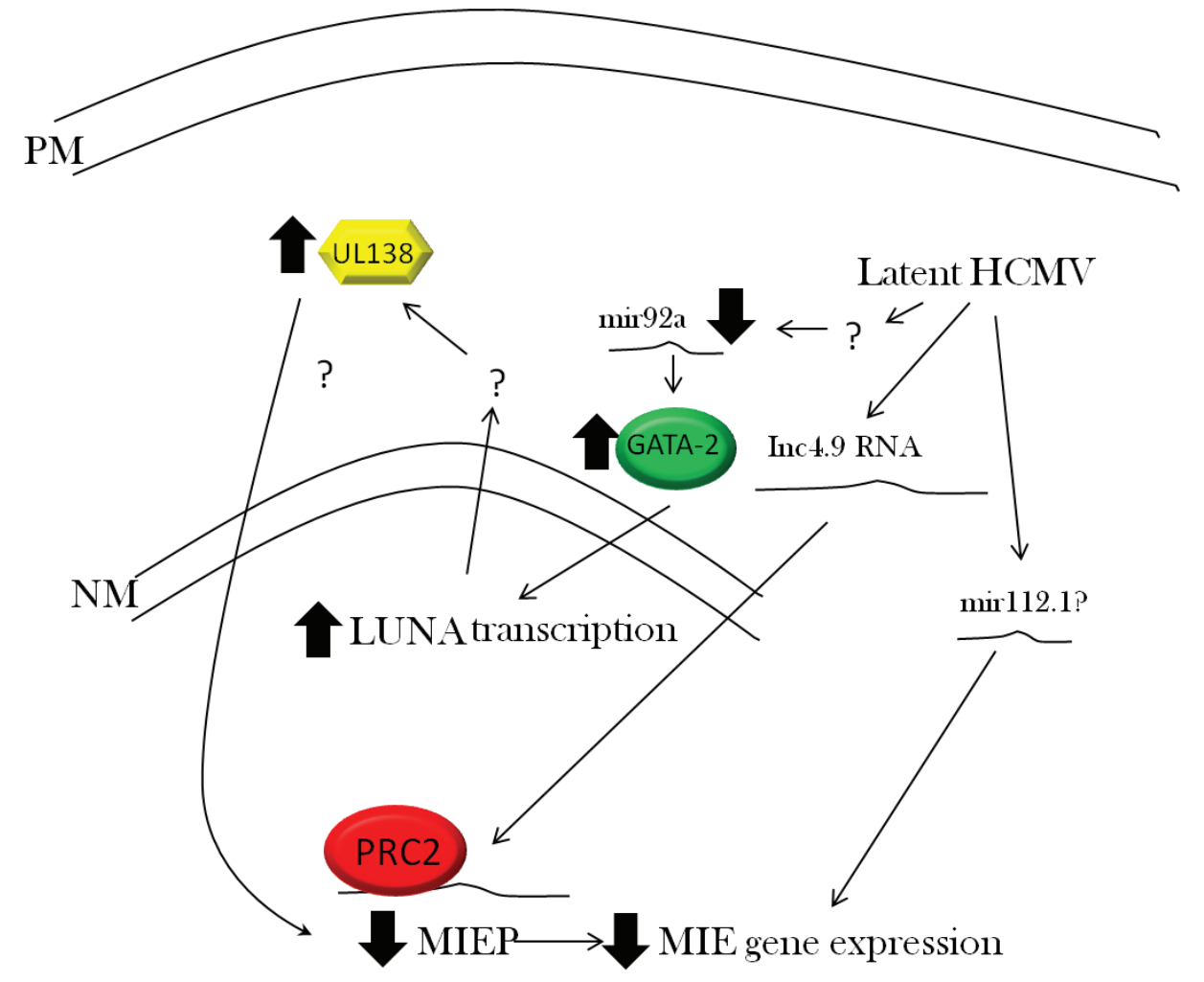

During lytic infection, HCMV expresses a virally encoded microRNA (mirUL-112-1) that specifically target IE72 encoding UL123 transcripts [119,120] and inhibits IE72 translation [120]. Deletion of mirUL-112-1 has no overt phenotype in infected fibroblasts, likely due to the substantial levels of IE72 transcript accompanying lytic infection [120]. However, it has been postulated that low levels of IE72 RNA may be targeted efficiently and, hence, miRUL112-1 may have more of a role during latency [119]—where untimely IE72 expression could be problematic but where the less abundant levels of IE72 RNA could be more effectively controlled by a miRNA-mediated mechanisms. In effect, the microRNA acts as a safety net to ensure that the functional impact of any sporadic activity of the MIEP, and any resultant IE transcripts, are minimised during latent infection. 
A more recent study [25] that used a deep sequencing approach to re-visit latent gene expression in experimental as well as naturally latent tissue samples, identified the expression of a number of viral transcripts including a $4.9 \mathrm{~kb}$ long non-coding RNA (lnc4.9). Interestingly, this transcript was observed to associate with the polycomb repressor complex 2 (PRC2) — with direct analogy to the KSHV PAN RNA species that also bind this complex [121]. Indeed, the PRC2 complex has also been shown to regulate HSV latency, although this is thought to occur independently of direct binding to the LAT RNA [122]. Furthermore, the binding of components of PRC2 and also the lnc4.9 RNA was observed in experimentally latently infected cells [25]. The net result of such interactions would be to augment the silencing of the viral MIEP linked with histone tri-methylation at lysine 27 on histone H3. The overall contribution of the lnc4.9 RNA to HCMV latency remains to be determined; however, analysis of whether virus mutants that fail to express lnc 4.9 are defective in their establishment and maintenance of latency could help determine whether this interaction is as an essential component of the mechanisms required to maintain HCMV latency.

Finally, other recent work has also suggested that the MIEP may repressed by a virally encoded factor during latency [23]. Treatment with histone deacetylase inhibitors (HDACi) is insufficient to promote the reactivation of IE gene expression in CD34+ cells latently infected with clinical strains of HCMV [23]. Conversely, however, CD34+ cells latently infected with laboratory isolates are responsive to HDACi. Consequently, this has suggested that a viral factor present in clinical isolates is involved in chromatin-mediated suppression of MIEP activity during experimental latency [23]. The likely candidate is UL138, which is expressed only in clinical isolates [39] and is known to be expressed during latent infection [34,53]. However, published studies suggest that the importance of UL138 for latency is not due to any direct effect on MIEP activity as a transcriptional repressor [58], thereby remaining in line with the predominant localisation of UL138 protein to the Golgi apparatus during lytic infection and transfection [58]. One caveat to this, though, is that the localisation of the UL138 protein has not been extensively analysed during latent infection and hence, at this stage, a role for UL138 in the repression of the viral MIEP during latency awaits further analyses.

\subsection{Viral Regulation of Latent Gene Expression}

In addition to the regulation of MIE gene expression, there is emerging evidence that HCMV also expresses functions to ensure efficient latent gene expression during latent infection (Figure 3). This, in itself, argues that latent viral gene products are likely to have important functions during latency and, importantly, that these could act as potential therapeutic targets for latent infection.

The modulation of the cellular miRNAome during latent infection could provide potent fine-tuning mechanisms to optimise both viral and cellular gene expression [91]. As discussed earlier, the down-regulation of cellular hsa-miR-92a by HCMV is important for the increased cIL-10 production that results in downstream effects on viability and immune modulation [91,92]. However, the down-regulation of hsa-miR-92a also results in an increase in the levels of the GATA-2 transcription factor [91]. The GATA family of proteins are considered key regulators of haematopoiesis and myeloid cell production $[123,124]$ and, thus, the targeting of this transcription factor in the knowledge that HCMV persists in the myeloid lineage appears more than coincidental. However, a more direct effect of GATA-2 regulation is observed on latent gene expression. A 
number of promoters of latently expressed genes contain consensus sequences for GATA-2 binding sites $[77,91,125,126]$ and two of these, LUNA and UL144, have been directly demonstrated to be GATA-2 responsive [77,126]. Recent work has shown that the down-regulation of the hsa-mir92a observed in HCMV infected CD34+ cells results in increased GATA-2 levels during latency, subsequently leading to increased levels of GATA-2-dependent latent gene expression [91].

Although we are far from completely elucidating the function of viral gene products during latency, observations, to date, strongly argue that latent infection with HCMV results in a latency-associated transcription profile of viral gene expression, resulting in an orchestrated change in the cell to support latent carriage. The regulation of latent viral gene expression, as well as the role of latent viral functions and their effects on cellular gene expression, are clearly inextricably linked. For instance, recent work from the St Jeor laboratory has shown that the expression of LUNA during latent infection is also important for latency-associated UL138 gene expression [62]. Consequently, a pathway of interactions appears to occur during latency which is exemplified by latency, thus resulting in the targeting of cellular hsa-miR92a; this, in turn, up-regulates cellular GATA-2 expression [91], leading to a downstream impact on latency-associated LUNA gene expression [77,91] and ultimately ensuring the expression of UL138 [62], which has been proposed to be a key determinant of latency $[23,53]$.

Clearly, this simplified example of a linear pathway of viral and cellular interactions is likely to give way to far more complex networks of host-virus interactions as we begin to understand the multi-functional role of viral proteins, non-coding RNAs, and miRNAs during HCMV latency and their impact on the latent cell.

\section{Concluding Remarks}

The advances in molecular techniques for performing large-scale analyses at the cell level are allowing ever more detailed analyses of aspects of HCMV biology which, previously, were all but impossible due the limitations of sensitivity and the availability of tractable primary cell models. These approaches have already begun to illustrate the complexity of HCMV latency and to provide an intriguing view of the concerted efforts HCMV employs to maintain the latent state.

It is evident that the reductionist approach of these types of studies, as well as the difficulty in further examining in vitro findings in vivo, warrants necessary caution to prevent overinterpretation. Accordingly, a key development in the future of HCMV studies of latency and reactivation will be the tractability and applicability of the humanised mouse model to studies of HCMV [127]. Caveats with this system also remain; although the humanised mouse can be used to assess HCMV reactivation in the myeloid lineage in vivo, this is still occurring in the background of mouse tissue that does not support extensive HCMV replication [128]. Consequently, such analyses are, arguably, restricted to the very initial events of HCMV reactivation occurring within a specific niche of human cells. Furthermore, the extent to which the human haematopoietic system develops from engrafted human CD34+ cells in the mouse (for instance, murine and human cytokines do not crosstalk unequivocally) is unclear and, more generally, the extent to which mouse models of disease truly reflect the human condition is an area of ongoing debate [129,130]. Nevertheless, the humanised mouse model could provide the potential to examine a number of predictions regarding 
HCMV latency derived from in vitro studies, as well as certain aspects of the development of the immune response to latent HCMV.

These cautionary notes aside, the identification of viral gene functions expressed during experimental latency (many of which, importantly, can be validated in naturally latent cells ex vivo) is beginning to provide a tantalising glimpse into the once-perceived "black box" of latency. As we begin to understand the functions of these latency-associated gene products, and assess their precise role in HCMV latency and reactivation, they are also likely to become potential targets for therapeutics. These approaches could range from the targeting of factors important for HCMV reactivation (our own unpublished work suggests that the LUNA gene product may encode a function that could be a future therapeutic target) or for the direct targeting of latently infected cells using chemotherapeutic or immunotherapeutic means [61].

Anti-viral strategies for HCMV have, to date, relied on targeting of replicating virus during lytic infection. However, understanding the complex interplay between the virus and the host during latency will give important insights into how to explore potential therapeutic options that target latent virus in what was previously considered to be in an "untargetable state."

\section{Acknowledgments}

We would like to thank members past and present of the Sinclair laboratory and the numerous colleagues in the field whose work has contributed to this review. We also apologise to those colleagues whose work has not been cited due to space limitations. Finally, we gratefully acknowledge funding from the UK Medical Research Council (J.H.S. G:0701279 and M.B.R. G:0900466) which supports the current research in our laboratories and also the support of NIHR UK Biomedical Research Centre (J.H.S.).

\section{Conflicts of Interest}

The authors declare no conflict of interest.

\section{References}

1. Grilli, E.; Galati, V.; Bordi, L.; Taglietti, F.; Petrosillo, N. Cytomegalovirus pneumonia in immunocompetent host: Case report and literature review. J. Clin. Virol. 2012, 55, 356-359.

2. Limaye, A.P.; Kirby, K.A.; Rubenfeld, G.D.; Leisenring, W.M.; Bulger, E.M.; Neff, M.J.; Gibran, N.S.; Huang, M.L.; Santo Hayes, T.K.; Corey, L.; et al. Cytomegalovirus reactivation in critically ill immunocompetent patients. JAMA 2008, 300, 413-422.

3. Legendre, C.; Pascual, M. Improving outcomes for solid-organ transplant recipients at risk from cytomegalovirus infection: Late-onset disease and indirect consequences. Clin. Infect. Dis. 2008, 46, 732-740.

4. Ljungman, P.; Hakki, M.; Boeckh, M. Cytomegalovirus in hematopoietic stem cell transplant recipients. Hematol. Oncol. Clin. N. Am. 2011, 25, 151-169.

5. Crough, T.; Khanna, R. Immunobiology of human cytomegalovirus: from bench to bedside. Clin. Microbiol. Rev. 2009, 22, 76-98. 
6. Sinclair, J.; Sissons, P. Latency and reactivation of human cytomegalovirus. J. Gen. Virol. 2006, 87, 1763-1779.

7. Taylor-Wiedeman, J.; Sissons, P.; Sinclair, J. Induction of endogenous human cytomegalovirus gene expression after differentiation of monocytes from healthy carriers. $J$. Virol. 1994, 68, 1597-1604.

8. Soderberg-Naucler, C.; Fish, K.N.; Nelson, J.A. Reactivation of latent human cytomegalovirus by allogeneic stimulation of blood cells from healthy donors. Cell 1997, 91, 119-126.

9. Hahn, G.; Jores, R.; Mocarski, E.S. Cytomegalovirus remains latent in a common precursor of dendritic and myeloid cells. Proc. Natl. Acad. Sci. USA 1998, 95, 3937-3942.

10. Zhuravskaya, T.; Maciejewski, J.P.; Netski, D.M.; Bruening, E.; Mackintosh, F.R.; St Jeor, S. Spread of human cytomegalovirus (HCMV) after infection of human hematopoietic progenitor cells: Model of HCMV latency. Blood 1997, 90, 2482-2491.

11. Reeves, M.B.; MacAry, P.A.; Lehner, P.J.; Sissons, J.G.; Sinclair, J.H. Latency, chromatin remodeling, and reactivation of human cytomegalovirus in the dendritic cells of healthy carriers. Proc. Natl. Acad. Sci. USA 2005, 102, 4140-4145.

12. Reeves, M.B.; Compton, T. Inhibition of inflammatory interleukin-6 activity via extracellular signal-regulated kinase-mitogen-activated protein kinase signaling antagonizes human cytomegalovirus reactivation from dendritic cells. J. Virol. 2011, 85, 12750-12758.

13. Huang, M.M.; Kew, V.G.; Jestice, K.; Wills, M.R.; Reeves, M.B. Efficient human cytomegalovirus reactivation is maturation dependent in the Langerhans dendritic cell lineage and can be studied using a CD14+ experimental latency model. J. Virol. 2012, 86, 8507-8515.

14. Minton, E.J.; Tysoe, C.; Sinclair, J.H.; Sissons, J.G. Human cytomegalovirus infection of the monocyte/macrophage lineage in bone marrow. J. Virol. 1994, 68, 4017-4021.

15. Abraham, C.G.; Kulesza, C.A. Polycomb repressive complex 2 silences human cytomegalovirus transcription in quiescent infection models. J. Virol. 2013, doi:10.1128/JVI.02420-13.

16. Murphy, J.C.; Fischle, W.; Verdin, E.; Sinclair, J.H. Control of cytomegalovirus lytic gene expression by histone acetylation. EMBO J. 2002, 21, 1112-1120.

17. Reeves, M.B.; Lehner, P.J.; Sissons, J.G.; Sinclair, J.H. An in vitro model for the regulation of human cytomegalovirus latency and reactivation in dendritic cells by chromatin remodelling. J. Gen. Virol. 2005, 86, 2949-2954.

18. Ioudinkova, E.; Arcangeletti, M.C.; Rynditch, A.; De Conto, F.; Motta, F.; Covan, S.; Pinardi, F.; Razin, S.V.; Chezzi, C. Control of human cytomegalovirus gene expression by differential histone modifications during lytic and latent infection of a monocytic cell line. Gene 2006, $384,120-128$.

19. Meier, J.L. Reactivation of the human cytomegalovirus major immediate-early regulatory region and viral replication in embryonal NTera2 cells: Role of trichostatin A, retinoic acid, and deletion of the 21-base-pair repeats and modulator. J. Virol. 2001, 75, 1581-1593.

20. Keller, M.J.; Wu, A.W.; Andrews, J.I.; McGonagill, P.W.; Tibesar, E.E.; Meier, J.L. Reversal of human cytomegalovirus major immediate-early enhancer/promoter silencing in quiescently infected cells via the cyclic AMP signaling pathway. J. Virol. 2007, 81, 6669-6681. 
21. Yuan, J.; Liu, X.; Wu, A.W.; McGonagill, P.W.; Keller, M.J.; Galle, C.S.; Meier, J.L. Breaking human cytomegalovirus major immediate-early gene silence by vasoactive intestinal peptide stimulation of the protein kinase A-CREB-TORC2 signaling cascade in human pluripotent embryonal NTera2 cells. J. Virol. 2009, 83, 6391-6403.

22. O'Connor, C.M.; Murphy, E.A. A myeloid progenitor cell line capable of supporting human cytomegalovirus latency and reactivation, resulting in infectious progeny. J. Virol. 2012, 86, 9854-9865.

23. Saffert, R.T.; Penkert, R.R.; Kalejta, R.F. Cellular and viral control over the initial events of human cytomegalovirus experimental latency in CD34+ cells. J. Virol. 2010, 84, 5594-5604.

24. Reeves, M.B.; Sinclair, J.H. Analysis of latent viral gene expression in natural and experimental latency models of human cytomegalovirus and its correlation with histone modifications at a latent promoter. J. Gen. Virol. 2010, 91, 599-604.

25. Rossetto, C.C.; Tarrant-Elorza, M.; Pari, G.S. Cis and trans acting factors involved in human cytomegalovirus experimental and natural latent infection of CD14 (+) monocytes and CD34 (+) cells. PLoS Pathog. 2013, 9, e1003366.

26. Reddehase, M.J.; Simon, C.O.; Seckert, C.K.; Lemmermann, N.; Grzimek, N.K. Murine model of cytomegalovirus latency and reactivation. Curr. Top. Microbiol. Immunol. 2008, 325, 315-331.

27. Schleiss, M.R. Nonprimate models of congenital cytomegalovirus (CMV) infection: gaining insight into pathogenesis and prevention of disease in newborns. ILAR J. 2006, 47, 65-72.

28. Yue, Y.; Barry, P.A. Rhesus cytomegalovirus a nonhuman primate model for the study of human cytomegalovirus. Adv. Virus Res. 2008, 72, 207-226.

29. Sindre, H.; Tjoonnfjord, G.E.; Rollag, H.; Ranneberg-Nilsen, T.; Veiby, O.P.; Beck, S.; Degre, M.; Hestdal, K. Human cytomegalovirus suppression of and latency in early hematopoietic progenitor cells. Blood 1996, 88, 4526-4533.

30. Mendelson, M.; Monard, S.; Sissons, P.; Sinclair, J. Detection of endogenous human cytomegalovirus in CD34+ bone marrow progenitors. J. Gen. Virol. 1996, 77, 3099-3102.

31. Reeves, M.B.; Sinclair, J.H. Circulating dendritic cells isolated from healthy seropositive donors are sites of human cytomegalovirus reactivation in vivo. J. Virol. 2013, 87, 10660-10667.

32. Hargett, D.; Shenk, T.E. Experimental human cytomegalovirus latency in CD14+ monocytes. Proc. Natl. Acad. Sci. USA 2010, 107, 20039-20044.

33. Bannister, A.J.; Zegerman, P.; Partridge, J.F.; Miska, E.A.; Thomas, J.O.; Allshire, R.C.; Kouzarides, T. Selective recognition of methylated lysine 9 on histone H3 by the HP1 chromo domain. Nature 2001, 410, 120-124.

34. Goodrum, F.D.; Jordan, C.T.; High, K.; Shenk, T. Human cytomegalovirus gene expression during infection of primary hematopoietic progenitor cells: A model for latency. Proc. Natl. Acad. Sci. USA 2002, 99, 16255-16260.

35. Knipe, D.M.; Cliffe, A. Chromatin control of herpes simplex virus lytic and latent infection. Nat. Rev. Microbiol. 2008, 6, 211-221.

36. Tempera, I.; Lieberman, P.M. Chromatin organization of gammaherpesvirus latent genomes. Biochim. Biophys. Acta 2010, 1799, 236-245. 
37. Reeves, M.B. Chromatin-mediated regulation of cytomegalovirus gene expression. Virus. Res. 2011, 157, 134-143.

38. Sinclair, J. Chromatin structure regulates human cytomegalovirus gene expression during latency, reactivation and lytic infection. Biochim. Biophys. Acta 2010, 1799, 286-295.

39. Gatherer, D.; Seirafian, S.; Cunningham, C.; Holton, M.; Dargan, D.J.; Baluchova, K.; Hector, R.D.; Galbraith, J.; Herzyk, P.; Wilkinson, G.W.; et al. High-resolution human cytomegalovirus transcriptome. Proc. Natl. Acad. Sci. USA 2011, 108, 19755-19760.

40. Murphy, E.; Yu, D.; Grimwood, J.; Schmutz, J.; Dickson, M.; Jarvis, M.A.; Hahn, G.; Nelson, J.A.; Myers, R.M.; Shenk, T.E. Coding potential of laboratory and clinical strains of human cytomegalovirus. Proc. Natl. Acad. Sci. USA 2003, 100, 14976-14981.

41. Pfeffer, S.; Sewer, A.; Lagos-Quintana, M.; Sheridan, R.; Sander, C.; Grasser, F.A.; van Dyk, L.F.; Ho, C.K.; Shuman, S.; Chien, M.; et al. Identification of microRNAs of the herpesvirus family. Nat. Methods 2005, 2, 269-276.

42. Grey, F.; Antoniewicz, A.; Allen, E.; Saugstad, J.; McShea, A.; Carrington, J.C.; Nelson, J. Identification and characterization of human cytomegalovirus-encoded microRNAs. J. Virol. 2005, 79, 12095-12099.

43. Dunn, W.; Trang, P.; Zhong, Q.; Yang, E.; van Belle, C.; Liu, F. Human cytomegalovirus expresses novel microRNAs during productive viral infection. Cell. Microbiol. 2005, 7, 1684-1695.

44. Kondo, K.; Kaneshima, H.; Mocarski, E.S. Human cytomegalovirus latent infection of granulocyte-macrophage progenitors. Proc. Natl. Acad. Sci. USA 1994, 91, 11879-11883.

45. Kondo, K.; Xu, J.; Mocarski, E.S. Human cytomegalovirus latent gene expression in granulocyte-macrophage progenitors in culture and in seropositive individuals. Proc. Natl. Acad. Sci. USA 1996, 93, 11137-11142.

46. White, K.L.; Slobedman, B.; Mocarski, E.S. Human cytomegalovirus latency-associated protein pORF94 is dispensable for productive and latent infection. J. Virol. 2000, 74, 9333-9337.

47. Jackson, S.E.; Mason, G.M.; Wills, M.R. Human cytomegalovirus immunity and immune evasion. Virus. Res. 2011, 157, 151-160.

48. Mocarski, E.S.; Hahn, G.; White, K.L.; Xu, J.; Slobedman, B.; Hertel, L.; Aguirre, S.A.; Noda, S. Myeloid Cell Recruitment and Function in Pathogenesis and Latency. In Cytomegaloviruses: Molecular Biology and Immunology; Reddehase, M.J., Ed.; Caister Academic Press: Poole, UK, 2006; Volume 1, pp. 463-482.

49. Taylor-Wiedeman, J.; Sissons, J.G.; Borysiewicz, L.K.; Sinclair, J.H. Monocytes are a major site of persistence of human cytomegalovirus in peripheral blood mononuclear cells. J. Gen. Virol. 1991, 72, 2059-2064.

50. Taylor-Wiedeman, J.; Hayhurst, G.P.; Sissons, J.G.; Sinclair, J.H. Polymorphonuclear cells are not sites of persistence of human cytomegalovirus in healthy individuals. J. Gen. Virol. 1993, 74, 265-268.

51. Bevan, I.S.; Daw, R.A.; Day, P.J.; Ala, F.A.; Walker, M.R. Polymerase chain reaction for detection of human cytomegalovirus infection in a blood donor population. Br. J. Haematol. 1991, 78, 94-99. 
52. Cheung, A.K.; Abendroth, A.; Cunningham, A.L.; Slobedman, B. Viral gene expression during the establishment of human cytomegalovirus latent infection in myeloid progenitor cells. Blood 2006, 108, 3691-3699.

53. Goodrum, F.; Reeves, M.; Sinclair, J.; High, K.; Shenk, T. Human cytomegalovirus sequences expressed in latently infected individuals promote a latent infection in vitro. Blood 2007, 110, 937-945.

54. Jenkins, C.; Abendroth, A.; Slobedman, B. A novel viral transcript with homology to human interleukin-10 is expressed during latent human cytomegalovirus infection. J. Virol. 2004, 78, 1440-1447.

55. Bego, M.; Maciejewski, J.; Khaiboullina, S.; Pari, G.; St Jeor, S. Characterization of an antisense transcript spanning the UL81-82 locus of human cytomegalovirus. J. Virol. 2005, 79, 11022-11034.

56. Kalla, M.; Hammerschmidt, W. Human B cells on their route to latent infection-early but transient expression of lytic genes of Epstein-Barr virus. Eur. J. Cell. Biol. 2012, 91, 65-69.

57. Tan, J.C.; Avdic, S.; Cao, J.Z.; Mocarski, E.S.; White, K.L.; Abendroth, A.; Slobedman, B. Inhibition of 2',5'-oligoadenylate synthetase expression and function by the human cytomegalovirus ORF94 gene product. J. Virol. 2011, 85, 5696-5700.

58. Petrucelli, A.; Rak, M.; Grainger, L.; Goodrum, F. Characterization of a novel Golgi apparatus-localized latency determinant encoded by human cytomegalovirus. J. Virol. 2009, $83,5615-5629$.

59. Le, V.T.; Trilling, M.; Hengel, H. The cytomegaloviral protein pUL138 acts as potentiator of tumor necrosis factor (TNF) receptor 1 surface density to enhance ULb'-encoded modulation of TNF-alpha signaling. J. Virol. 2011, 85, 13260-13270.

60. Montag, C.; Wagner, J.A.; Gruska, I.; Vetter, B.; Wiebusch, L.; Hagemeier, C. The latency-associated UL138 gene product of human cytomegalovirus sensitizes cells to tumor necrosis factor alpha (TNF-alpha) signaling by upregulating TNF-alpha receptor 1 cell surface expression. J. Virol. 2011, 85, 11409-11421.

61. Weekes, M.P.; Tan, S.Y.; Poole, E.; Talbot, S.; Antrobus, R.; Smith, D.L.; Montag, C.; Gygi, S.P.; Sinclair, J.H.; Lehner, P.J. Latency-associated degradation of the MRP1 drug transporter during latent human cytomegalovirus infection. Science 2013, 340, 199-202.

62. Keyes, L.R.; Hargett, D.; Soland, M.; Bego, M.G.; Rossetto, C.C.; Almeida-Porada, G.; St Jeor, S. HCMV protein LUNA is required for viral reactivation from latently infected primary CD14(+) cells. PLoS One 2013, 7, e52827.

63. Jenkins, C.; Garcia, W.; Godwin, M.J.; Spencer, J.V.; Stern, J.L.; Abendroth, A.; Slobedman, B. Immunomodulatory properties of a viral homolog of human interleukin-10 expressed by human cytomegalovirus during the latent phase of infection. J. Virol. 2008, 82, 3736-3750.

64. Pari, G.S.; Anders, D.G. Eleven loci encoding trans-acting factors are required for transient complementation of human cytomegalovirus oriLyt-dependent DNA replication. J. Virol. 1993, 67, 6979-6988.

65. Sarisky, R.T.; Hayward, G.S. Evidence that the UL84 gene product of human cytomegalovirus is essential for promoting oriLyt-dependent DNA replication and formation of replication compartments in cotransfection assays. J. Virol. 1996, 70, 7398-7413. 
66. Spector, D.J.; Tevethia, M.J. Protein-protein interactions between human cytomegalovirus IE2-580aa and pUL84 in lytically infected cells. J. Virol. 1994, 68, 7549-7553.

67. Colletti, K.S.; Xu, Y.; Yamboliev, I.; Pari, G.S. Human cytomegalovirus UL84 is a phosphoprotein that exhibits UTPase activity and is a putative member of the $\mathrm{DExD} / \mathrm{H}$ box family of proteins. J. Biol. Chem. 2005, 280, 11955-11960.

68. Boomker, J.M.; The, T.H.; de Leij, L.F.; Harmsen, M.C. The human cytomegalovirus-encoded receptor US28 increases the activity of the major immediate-early promoter/enhancer. Virus. Res. 2006, 118, 196-200.

69. Beisser, P.S.; Laurent, L.; Virelizier, J.L.; Michelson, S. Human cytomegalovirus chemokine receptor gene US28 is transcribed in latently infected THP-1 monocytes. J. Virol. 2001, 75, 5949-5957.

70. Billstrom, M.A.; Johnson, G.L.; Avdi, N.J.; Worthen, G.S. Intracellular signaling by the chemokine receptor US28 during human cytomegalovirus infection. J. Virol. 1998, 72, 5535-5544.

71. Casarosa, P.; Bakker, R.A.; Verzijl, D.; Navis, M.; Timmerman, H.; Leurs, R.; Smit, M.J. Constitutive signaling of the human cytomegalovirus-encoded chemokine receptor US28. J. Biol. Chem. 2001, 276, 1133-1137.

72. Gao, J.L.; Murphy, P.M. Human cytomegalovirus open reading frame US28 encodes a functional beta chemokine receptor. J. Biol. Chem. 1994, 269, 28539-28542.

73. Miller, W.E.; Houtz, D.A.; Nelson, C.D.; Kolattukudy, P.E.; Lefkowitz, R.J. G-protein-coupled receptor (GPCR) kinase phosphorylation and beta-arrestin recruitment regulate the constitutive signaling activity of the human cytomegalovirus US28 GPCR. J. Biol. Chem. 2003, 278, 21663-21671.

74. Streblow, D.N.; Soderberg-Naucler, C.; Vieira, J.; Smith, P.; Wakabayashi, E.; Ruchti, F.; Mattison, K.; Altschuler, Y.; Nelson, J.A. The human cytomegalovirus chemokine receptor US28 mediates vascular smooth muscle cell migration. Cell 1999, 99, 511-520.

75. Poole, E.; King, C.A.; Sinclair, J.H.; Alcami, A. The UL144 gene product of human cytomegalovirus activates NFkappaB via a TRAF6-dependent mechanism. EMBO J. 2006, $25,4390-4399$.

76. Poole, E.; Atkins, E.; Nakayama, T.; Yoshie, O.; Groves, I.; Alcami, A.; Sinclair, J. NF-kappaB-mediated activation of the chemokine CCL22 by the product of the human cytomegalovirus gene UL144 escapes regulation by viral IE86. J. Virol. 2008, 82, 4250-4256.

77. Poole, E.; Walther, A.; Raven, K.; Benedict, C.A.; Mason, G.M.; Sinclair, J. The myeloid transcription factor GATA-2 regulates the viral UL144 gene during human cytomegalovirus latency in an isolate-specific manner. J. Virol. 2013, 87, 4261-4271.

78. Benedict, C.A.; Butrovich, K.D.; Lurain, N.S.; Corbeil, J.; Rooney, I.; Schneider, P.; Tschopp, J.; Ware, C.F. Cutting edge: A novel viral TNF receptor superfamily member in virulent strains of human cytomegalovirus. J. Immunol. 1999, 162, 6967-6970.

79. Goldmacher, V.S.; Bartle, L.M.; Skaletskaya, A.; Dionne, C.A.; Kedersha, N.L.; Vater, C.A.; Han, J.W.; Lutz, R.J.; Watanabe, S.; Cahir McFarland, E.D.; et al. A cytomegalovirus-encoded mitochondria-localized inhibitor of apoptosis structurally unrelated to Bcl-2. Proc. Natl. Acad. Sci. USA 1999, 96, 12536-12541. 
80. McCormick, A.L. Control of apoptosis by human cytomegalovirus. Curr. Top. Microbiol. Immunol. 2008, 325, 281-295.

81. Reeves, M.B.; Davies, A.A.; McSharry, B.P.; Wilkinson, G.W.; Sinclair, J.H. Complex I binding by a virally encoded RNA regulates mitochondria-induced cell death. Science 2007, $316,1345-1348$.

82. Skaletskaya, A.; Bartle, L.M.; Chittenden, T.; McCormick, A.L.; Mocarski, E.S.; Goldmacher, V.S. A cytomegalovirus-encoded inhibitor of apoptosis that suppresses caspase-8 activation. Proc. Natl. Acad. Sci. USA 2001, 98, 7829-7834.

83. Moorman, N.J.; Cristea, I.M.; Terhune, S.S.; Rout, M.P.; Chait, B.T.; Shenk, T. Human cytomegalovirus protein UL38 inhibits host cell stress responses by antagonizing the tuberous sclerosis protein complex. Cell. Host. Microbe. 2008, 3, 253-262.

84. Terhune, S.; Torigoi, E.; Moorman, N.; Silva, M.; Qian, Z.; Shenk, T.; Yu, D. Human cytomegalovirus UL38 protein blocks apoptosis. J. Virol. 2007, 81, 3109-3123.

85. Everett, H.; McFadden, G. Apoptosis: An innate immune response to virus infection. Trends Microbiol. 1999, 7, 160-165.

86. Reeves, M.B.; Breidenstein, A.; Compton, T. Human cytomegalovirus activation of ERK and myeloid cell leukemia-1 protein correlates with survival of latently infected cells. Proc. Natl. Acad. Sci. USA 2012, 109, 588-593.

87. Chan, G.; Nogalski, M.T.; Bentz, G.L.; Smith, M.S.; Parmater, A.; Yurochko, A.D. PI3K-dependent upregulation of Mcl-1 by human cytomegalovirus is mediated by epidermal growth factor receptor and inhibits apoptosis in short-lived monocytes. J. Immunol. 2010, 184, 3213-3222.

88. Perciavalle, R.M.; Opferman, J.T. Delving deeper: MCL-1's contributions to normal and cancer biology. Trends Cell. Biol. 2013, 23, 22-29.

89. Slobedman, B.; Stern, J.L.; Cunningham, A.L.; Abendroth, A.; Abate, D.A.; Mocarski, E.S. Impact of human cytomegalovirus latent infection on myeloid progenitor cell gene expression. J. Virol. 2004, 78, 4054-4062.

90. Condorelli, G.; Vigliotta, G.; Cafieri, A.; Trencia, A.; Andalo, P.; Oriente, F.; Miele, C.; Caruso, M.; Formisano, P.; Beguinot, F. PED/PEA-15: An anti-apoptotic molecule that regulates FAS/TNFR1-induced apoptosis. Oncogene 1999, 18, 4409-4415.

91. Poole, E.; McGregor Dallas, S.R.; Colston, J.; Joseph, R.S.; Sinclair, J. Virally induced changes in cellular microRNAs maintain latency of human cytomegalovirus in CD34(+) progenitors. J. Gen. Virol. 2011, 92, 1539-1549.

92. Mason, G.M.; Poole, E.; Sissons, J.G.; Wills, M.R.; Sinclair, J.H. Human cytomegalovirus latency alters the cellular secretome, inducing cluster of differentiation (CD)4+ T-cell migration and suppression of effector function. Proc. Natl. Acad. Sci. USA 2012, 109, 14538-14543.

93. Sylwester, A.W.; Mitchell, B.L.; Edgar, J.B.; Taormina, C.; Pelte, C.; Ruchti, F.; Sleath, P.R.; Grabstein, K.H.; Hosken, N.A.; Kern, F.; et al. Broadly targeted human cytomegalovirus-specific CD4+ and CD8+ T cells dominate the memory compartments of exposed subjects. J. Exp. Med. 2005, 202, 673-685.

94. Tey, S.K.; Goodrum, F.; Khanna, R. CD8+ T-cell recognition of human cytomegalovirus latency-associated determinant pUL138. J. Gen. Virol. 2010, 91, 2040-2048. 
95. Mason, G.; Jackson, S.E.; Okecha, G.; Poole, E.; Sissons, J.G.P.; Sinclair, J.; Wills, M.R. Human cytomegalovirus latency-associated proteins elicit immune-suppressive IL-10 producing CD4+ T cells. PLoS Pathog. 2013, 10, e1003635.

96. Opal, S.M.; DePalo, V.A. Anti-inflammatory cytokines. Chest 2000, 117, 1162-1172.

97. Slobedman, B.; Mocarski, E.S. Quantitative analysis of latent human cytomegalovirus. J. Virol. 1999, 73, 4806-4812.

98. Kotenko, S.V.; Saccani, S.; Izotova, L.S.; Mirochnitchenko, O.V.; Pestka, S. Human cytomegalovirus harbors its own unique IL-10 homolog (cmvIL-10). Proc. Natl. Acad. Sci. USA 2000, 97, 1695-1700.

99. Lockridge, K.M.; Zhou, S.S.; Kravitz, R.H.; Johnson, J.L.; Sawai, E.T.; Blewett, E.L.; Barry, P.A. Primate cytomegaloviruses encode and express an IL-10-like protein. Virology 2000, 268, 272-280.

100. Nachtwey, J.; Spencer, J.V. HCMV IL-10 suppresses cytokine expression in monocytes through inhibition of nuclear factor-kappaB. Viral. Immunol. 2008, 21, 477-482.

101. Spencer, J.V. The cytomegalovirus homolog of interleukin-10 requires phosphatidylinositol 3-kinase activity for inhibition of cytokine synthesis in monocytes. J. Virol. 2007, 81, 2083-2086.

102. Spencer, J.V.; Lockridge, K.M.; Barry, P.A.; Lin, G.; Tsang, M.; Penfold, M.E.; Schall, T.J. Potent immunosuppressive activities of cytomegalovirus-encoded interleukin-10. J. Virol. 2002, 76, 1285-1292.

103. Jones, B.C.; Logsdon, N.J.; Josephson, K.; Cook, J.; Barry, P.A.; Walter, M.R. Crystal structure of human cytomegalovirus IL-10 bound to soluble human IL-10R1. Proc. Natl. Acad. Sci. USA 2002, 99, 9404-9409.

104. Chang, W.L.; Barry, P.A. Attenuation of innate immunity by cytomegalovirus IL-10 establishes a long-term deficit of adaptive antiviral immunity. Proc. Natl. Acad. Sci. USA 2011, 107, 22647-22652.

105. Chang, W.L.; Baumgarth, N.; Yu, D.; Barry, P.A. Human cytomegalovirus-encoded interleukin-10 homolog inhibits maturation of dendritic cells and alters their functionality. J. Virol. 2004, 78, 8720-8731.

106. Raftery, M.J.; Wieland, D.; Gronewald, S.; Kraus, A.A.; Giese, T.; Schonrich, G. Shaping phenotype, function, and survival of dendritic cells by cytomegalovirus-encoded IL-10. J. Immunol. 2004, 173, 3383-3391.

107. Avdic, S.; Cao, J.Z.; McSharry, B.P.; Clancy, L.E.; Brown, R.; Steain, M.; Gottlieb, D.J.; Abendroth, A.; Slobedman, B. Human cytomegalovirus interleukin-10 polarizes monocytes toward a deactivated M2c phenotype to repress host immune responses. J. Virol. 2013, 87, $10273-10282$.

108. Slobedman, B.; Mocarski, E.S.; Arvin, A.M.; Mellins, E.D.; Abendroth, A. Latent cytomegalovirus down-regulates major histocompatibility complex class II expression on myeloid progenitors. Blood 2002, 100, 2867-2873. 
109. Cheung, A.K.; Gottlieb, D.J.; Plachter, B.; Pepperl-Klindworth, S.; Avdic, S.; Cunningham, A.L.; Abendroth, A.; Slobedman, B. The role of the human cytomegalovirus UL111A gene in down-regulating $\mathrm{CD} 4+\mathrm{T}$-cell recognition of latently infected cells: Implications for virus elimination during latency. Blood 2009, 114, 4128-4137.

110. Avdic, S.; Cao, J.Z.; Cheung, A.K.; Abendroth, A.; Slobedman, B. Viral interleukin-10 expressed by human cytomegalovirus during the latent phase of infection modulates latently infected myeloid cell differentiation. J. Virol. 2011, 85, 7465-7471.

111. Groves, I.J.; Reeves, M.B.; Sinclair, J.H. Lytic infection of permissive cells with human cytomegalovirus is regulated by an intrinsic "pre-immediate-early" repression of viral gene expression mediated by histone post-translational modification. J. Gen. Virol. 2009, 90, 2364-2374.

112. Maul, G.G. Initiation of cytomegalovirus infection at ND10. Curr. Top. Microbiol. Immunol. 2008, 325, 117-132.

113. Kalejta, R.F. Functions of human cytomegalovirus tegument proteins prior to immediate early gene expression. Curr. Top. Microbiol. Immunol. 2008, 325, 101-115.

114. Stamminger, T. Interactions of human cytomegalovirus proteins with the nuclear transport machinery. Curr. Top. Microbiol. Immunol. 2008, 325, 167-185.

115. Sinclair, J.; Sissons, P. Latent and persistent infections of monocytes and macrophages. Intervirology 1996, 39, 293-301.

116. Wright, E.; Bain, M.; Teague, L.; Murphy, J.; Sinclair, J. Ets-2 repressor factor recruits histone deacetylase to silence human cytomegalovirus immediate-early gene expression in non-permissive cells. J. Gen. Virol. 2005, 86, 535-544.

117. Liu, R.; Baillie, J.; Sissons, J.G.; Sinclair, J.H. The transcription factor YY1 binds to negative regulatory elements in the human cytomegalovirus major immediate early enhancer/promoter and mediates repression in non-permissive cells. Nucleic. Acids. Res. 1994, 22, 2453-2459.

118. Guenther, M.G.; Levine, S.S.; Boyer, L.A.; Jaenisch, R.; Young, R.A. A chromatin landmark and transcription initiation at most promoters in human cells. Cell 2007, 130, 77-88.

119. Murphy, E.; Vanicek, J.; Robins, H.; Shenk, T.; Levine, A.J. Suppression of immediate-early viral gene expression by herpesvirus-coded microRNAs: implications for latency. Proc. Natl. Acad. Sci. USA 2008, 105, 5453-5458.

120. Grey, F.; Meyers, H.; White, E.A.; Spector, D.H.; Nelson, J. A human cytomegalovirus-encoded microRNA regulates expression of multiple viral genes involved in replication. PLoS Pathog. 2007, 3, e163.

121. Rossetto, C.C.; Tarrant-Elorza, M.; Verma, S.; Purushothaman, P.; Pari, G.S. Regulation of viral and cellular gene expression by Kaposi's sarcoma-associated herpesvirus polyadenylated nuclear RNA. J. Virol. 2013, 87, 5540-5553.

122. Cliffe, A.R.; Coen, D.M.; Knipe, D.M. Kinetics of facultative heterochromatin and polycomb group protein association with the herpes simplex viral genome during establishment of latent infection. mBio 2013, 4, doi:10.1128/mBio.00590-12.

123. Tsai, F.Y.; Keller, G.; Kuo, F.C.; Weiss, M.; Chen, J.; Rosenblatt, M.; Alt, F.W.; Orkin, S.H. An early haematopoietic defect in mice lacking the transcription factor GATA-2. Nature 1994, 371, 221-226. 
124. Ling, K.W.; Ottersbach, K.; van Hamburg, J.P.; Oziemlak, A.; Tsai, F.Y.; Orkin, S.H.; Ploemacher, R.; Hendriks, R.W.; Dzierzak, E. GATA-2 plays two functionally distinct roles during the ontogeny of hematopoietic stem cells. J. Exp. Med. 2004, 200, 871-882.

125. Reeves, M.; Sinclair, J. Regulation of human cytomegalovirus transcription in latency: Beyond the major immediate-early promoter. Viruses 2013, 5, 1395-1413.

126. Reeves, M.; Woodhall, D.; Compton, T.; Sinclair, J. Human cytomegalovirus IE72 protein interacts with the transcriptional repressor hDaxx to regulate LUNA gene expression during lytic infection. J. Virol. 2010, 84, 7185-7194.

127. Smith, M.S.; Goldman, D.C.; Bailey, A.S.; Pfaffle, D.L.; Kreklywich, C.N.; Spencer, D.B.; Othieno, F.A.; Streblow, D.N.; Garcia, J.V.; Fleming, W.H.; et al. Granulocyte-colony stimulating factor reactivates human cytomegalovirus in a latently infected humanized mouse model. Cell Host Microbe 2010, 8, 284-291.

128. Jurak, I.; Brune, W. Induction of apoptosis limits cytomegalovirus cross-species infection. EMBO J. 2006, 25, 2634-2642.

129. Mestas, J.; Hughes, C.C., Of mice and not men: Differences between mouse and human immunology. J. Immunol. 2004, 172, 2731-2738.

130. Seok, J.; Warren, H.S.; Cuenca, A.G.; Mindrinos, M.N.; Baker, H.V.; Xu, W.; Richards, D.R.; McDonald-Smith, G.P.; Gao, H.; Hennessy, L.; et al. Genomic responses in mouse models poorly mimic human inflammatory diseases. Proc. Natl. Acad. Sci. USA 2013, 110, 3507-3512. 
Reprinted from Viruses. Cite as: Zydek, M.; Petitt, M.; Fang-Hoover, J.; Adler, B.; Kauvar, L.M.; Pereira, L.; Tabata, T. HCMV Infection of Human Trophoblast Progenitor Cells of the Placenta Is Neutralized by a Human Monoclonal Antibody to Glycoprotein B and Not by Antibodies to the Pentamer Complex. Viruses 2014, 6, 1346-1364.

Article

\section{HCMV Infection of Human Trophoblast Progenitor Cells of the Placenta Is Neutralized by a Human Monoclonal Antibody to Glycoprotein B and Not by Antibodies to the Pentamer Complex}

Martin Zydek ${ }^{1}$, Matthew Petitt ${ }^{1}$, June Fang-Hoover ${ }^{1}$, Barbara Adler ${ }^{2}$, Lawrence M. Kauvar $^{3}$, Lenore Pereira ${ }^{1, *}$ and Takako Tabata ${ }^{1}$

1 Department of Cell and Tissue Biology, University of California San Francisco, 513 Parnassus Avenue, San Francisco, CA 94143, USA; E-Mails: martin.zydek@ucsf.edu (M.Z.); matthew.petitt@ucsf.edu (M.P.); june.fang-hoover@ucsf.edu (J.F.-H.); takako.tabata@ucsf.edu (T.T.)

2 Division of Virology, Max von Pettenkofer-Institute, Ludwig-Maximilians-University Munich, Pettenkoferstr.9A, D-80336 Munich, Germany; E-Mail: adler_b@mvp.uni-muenchen.de

3 Trellis Bioscience, LLC, 2-B Corporate Drive, South San Francisco, CA 94080, USA; E-Mail: 1kauvar@trellisbio.com

* Author to whom correspondence should be addressed; E-Mail: lenore.pereira@ucsf.edu; Tel.: +1-415-476-8248; Fax: +1-415-476-3983.

Received: 22 January 2014; in revised form: 27 February 2014 / Accepted: 27 February 2014 / Published: 19 March 2014

Abstract: Human cytomegalovirus (HCMV) is the major viral cause of congenital
infection and birth defects. Primary maternal infection often results in virus
transmission, and symptomatic babies can have permanent neurological deficiencies
and deafness. Congenital infection can also lead to intrauterine growth restriction, a
defect in placental transport. HCMV replicates in primary cytotrophoblasts (CTBs), the
specialized cells of the placenta, and inhibits differentiation/invasion. Human
trophoblast progenitor cells (TBPCs) give rise to the mature cell types of the chorionic
villi, CTBs and multi-nucleated syncytiotrophoblasts (STBs). Here we report that
TBPCs are fully permissive for pathogenic and attenuated HCMV strains. Studies with
a mutant virus lacking a functional pentamer complex (gH/gL/pUL128-131A) showed
that virion entry into TBPCs is independent of the pentamer. In addition, infection is
blocked by a potent human neutralizing monoclonal antibody (mAb), TRL345, reactive 
with glycoprotein B (gB), but not mAbs to the pentamer proteins pUL130/pUL131A. Functional studies revealed that neutralization of infection preserved the capacity of TBPCs to differentiate and assemble into trophospheres composed of CTBs and STBs in vitro. Our results indicate that $\mathrm{mAbs}$ to $\mathrm{gB}$ protect trophoblast progenitors of the placenta and could be included in antibody treatments developed to suppress congenital infection and prevent disease.

Keywords: HCMV; congenital; trophoblast; progenitors; neutralizing; placenta; development; neutralization; pentamer; hyperimmune globulin

\section{Introduction}

Human cytomegalovirus (HCMV) is the major viral cause of congenital infections and affects $1 \%-3 \%$ of live births in the U.S. [1]. Virus transmission to the fetus occurs in $40 \%-50 \%$ of cases of primary infection and can result in birth defects, including severe neurological disorders, impaired hearing, vision loss and intrauterine growth restriction (IUGR), a defect that results from compromised placental function [2-4]. In contrast, due to immunity from maternal antibodies, recurrent infection is associated with a reduced viral transmission rate $(0.1 \%$ to $2 \%)$ [5]. Although recent studies indicate that the frequency of hearing loss is similar in pregnancies complicated by primary and recurrent infection, the severity is greater in cases of primary infection [6,7].

Currently, there are no approved therapeutics for congenital HCMV infection in cases of primary maternal infection due to concerns over the toxicity and teratogenicity of available antiviral drugs (reviewed in [8,9]). A recently developed therapeutic approach is the administration of hyperimmune globulin (HIG), a pooled immunoglobulin preparation from donors with high-avidity anti-HCMV antibodies. In initial (non-randomized) clinical studies, treatment of pregnant women with HIG within 4 to 6 weeks after seroconversion was found to improve fetal outcome [10-13]. Analysis of placentas revealed that viral replication was reduced and compensatory development enabled growth of chorionic villi increasing the placental surface perfused by maternal blood $[12,14]$. However, reported studies were small and the results should be confirmed by double-blinded clinical trials [15]. To date, administration of HIG has been approved by the Food and Drug Administration only for transplant recipients [16-18]. As promising novel therapeutics, human monoclonal antibodies (mAbs) specific for epitopes on HCMV proteins have been developed using technological advancement of human B-cell cloning from highly seropositive donors [19-23].

Chorionic villi, the functional units of the placenta, are composed of a stromal core that contains blood vessels continuous with the fetal vasculature. The core is surrounded by a basement membrane populated by villus cytotrophoblasts (vCTBs), covered by a multinucleated syncytiotrophoblast (STB) layer. At the tips of the villi, vCTBs differentiate and switch their adhesion phenotype to that of invasive cytotrophoblasts (iCTBs), which penetrate the decidua and remodel uterine arteries, providing maternal blood to the placenta $[24,25]$. STBs, which are bathed in maternal blood, mediate transport of nutrients and oxygen to the fetal bloodstream. In contrast to CTBs, whose 
proliferative potential is limited [26,27], trophoblast progenitor cells (TBPCs), resident in the chorion, proliferate and differentiate into the mature trophoblast populations [28].

Previous studies from our lab suggested that during the course of transmission, HCMV first replicates in uterine blood vessels, then spreads to differentiating iCTBs, where infection downregulates cell-matrix adhesion molecules and impairs cell differentiation/invasion [29-33]. Studies of congenital infection in placentas from early gestation and at term indicated a central role for high-avidity, neutralizing antibodies in suppression of infection [34-36]. We recently reported that some cases of IUGR have underlying primary or recurrent maternal infection, which impaired placental development [4]. In a case of IUGR associated with preterm delivery, chorionic villi contained unusual clusters of extravillous, cytokeratin 7 (CK7)-positive CTBs, suggesting arrested differentiation. In cases of symptomatic congenital infection, infected cell proteins were detected in TBPCs in the chorion, suggesting that virus replication could interfere with TBPC self-renewal and differentiation [37].

HCMV encodes several glycoprotein complexes in the virion envelope that function in attachment and penetration of host cells. A highly studied envelope protein important for virion entry into all cell types is $\mathrm{gB}[38,39]$. $\mathrm{gB}$ facilitates attachment to the cell surface by binding to heparin sulfate glycosaminoglycans [40,41], cellular receptors [42,43] and integrins [44,45] and, in cooperation with $\mathrm{gH} / \mathrm{gL}$ complexes, mediates membrane fusion [46]. In contrast to the fibroblast-adapted (attenuated) strains AD169 and Towne, clinical strains, such as VR1814 and TB40/E, are pathogenic and express the pentamer glycoprotein complex $\mathrm{gH} / \mathrm{gL} / \mathrm{pUL} 128-131 \mathrm{~A}$, which is required for virus entry into epithelial and endothelial cells $[47,48]$ but not fibroblasts $[49,50]$. AD169 and Towne have acquired mutations in the UL128-131A locus, lack a functional pentamer and are impaired in infection of epithelial and endothelial cells [51,52].

Recently, TBPCs were isolated from the chorions of human placentas, and lines of continuously self-renewing cells were established [27]. These TBPCs express the trophoblast marker CK7, factors for stem cell self-renewal, including HMGA2 (high-mobility group AT-hook 2), and proteins required for trophoblast fate specification, GATA-3, GATA-4, Eomes (Eomesodermin) and GCM-1 (glial cell missing homolog 1). When cultured under differentiation conditions, TBPCs aggregate and form spheres (trophospheres) that upregulate HLA-G, a marker of differentiating iCTBs, and increase expression of human placental lactogen and human chorionic gonadotropin (normally secreted from STBs) [27], in accord with differentiation in utero.

Here, we have identified glycoproteins involved in entry of HCMV virions into TBPCs established from first and second trimester placentas and measured the extent to which neutralizing $\mathrm{mAbs}$ to $\mathrm{gB}$ and the pentameric complex block infection. We found that TBPCs are susceptible to infection with both the pathogenic VR1814 and the attenuated AD169 strain and that both strains undergo lytic viral replication in TBPCs. In addition, we found that virion entry into TBPCs was independent of the pentamer complex, as shown by infection with a UL131A-deficient mutant $[53,54]$ and confirmed by failure of anti-pentamer mAbs to block VR1814 infection. Importantly, virion entry was found to depend on $\mathrm{gB}$ function. Consequently, neutralization of infection with an anti-gB mAb (TRL345) was found to preserve the ability of TBPCs to differentiate and form trophospheres. 


\section{Results and Discussion}

\subsection{TBPCs from First and Second Trimesters Are Permissive for Pathogenic and Attenuated HCMV Strains}

TBPC expression of markers for trophoblasts (CK7), stem cell self-renewal (HMGA2) and trophoblast fate acquisition (GATA-4 and GATA-3) was confirmed by immunostaining. Over 90\% of TBPCs in culture expressed these markers (Figure 1).

Figure 1. TBPCs express markers of trophoblasts and pluripotency. TBPCs from 7.3 weeks (left panel) and 15.6 weeks (right panel) of gestation were immunostained for CK7 (A,E), HMGA2 (B,F), GATA-4 (C,G) or GATA-3 (D,H). Nuclei were counterstained with DAPI. Scale bar $=100 \mu \mathrm{m}$.

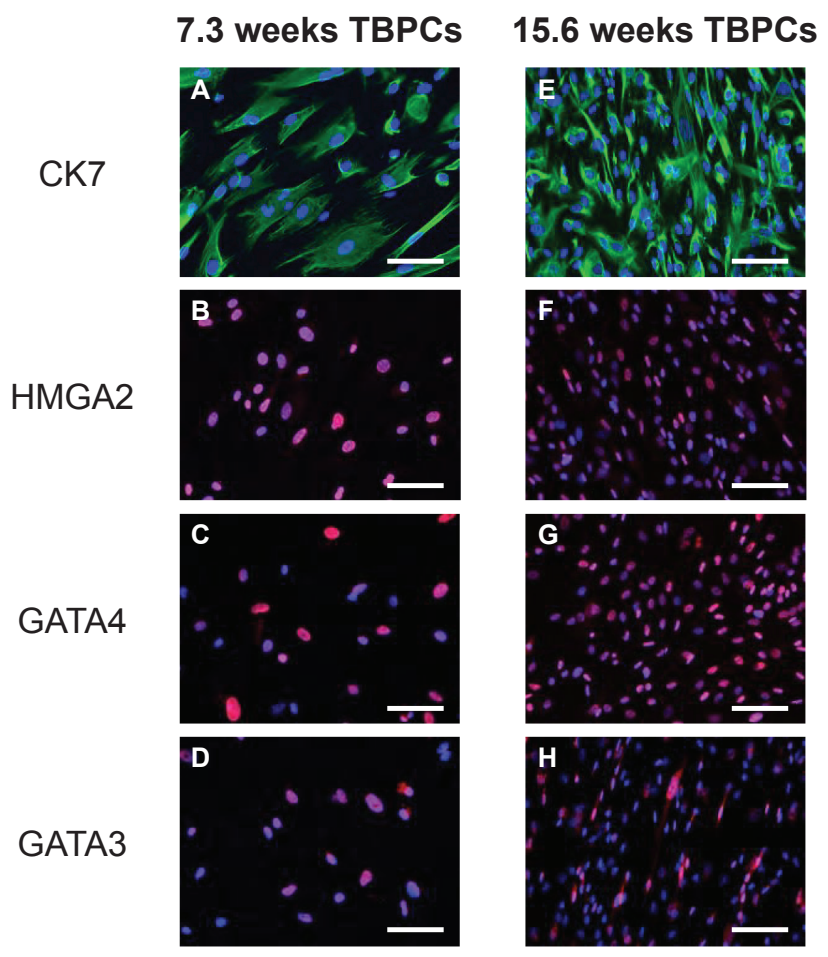

To determine whether TBPCs isolated from placentas at 7.3 and 15.6 weeks of gestation were susceptible to infection, suspensions of VR1814 and AD169 were adsorbed to TBPCs and the cells immunostained at the indicated time points for expression of immediate-early protein IE1, early proteins pUL112/pUL113 and late protein pp28. We found that viral proteins were expressed at comparable levels in VR1814- and AD169-infected cells (Figure 2), indicating that TBPCs were susceptible to lytic HCMV infection. In 15.6 weeks TBPCs, nearly all cells expressed IE1 in nuclei at 1 day post infection (dpi), and pUL112/pUL113 were detected at 2 dpi. pp28 was present in most cells at 4 dpi (Figure 2A) suggesting viral DNA replication [55]. In 7.3 weeks TBPCs, fewer cells expressed viral proteins. Approximately $70 \%$ expressed IE1 at 1 dpi and $85 \%$ at 4 dpi (Figure 2B). pUL112/pUL113 and pp28 were expressed in nearly all cells by 7 dpi, suggesting delayed viral gene expression. Nonetheless, almost all TBPCs from both gestational ages expressed late infected-cell proteins within a week. Altogether, our results indicate that first and second trimester 
TBPCs are permissive for pathogenic and attenuated virus strains. Because results for first and second trimester TBPCs were similar, only TBPCs from 15.6 weeks of gestation were used for subsequent experiments.

Figure 2. VR1814 and AD169 replicate in TBPCs. Cells from 15.6 weeks (A) or 7.3 weeks (B) of gestation were infected (MOI 2) and immunostained for HCMV IE1, pUL112/pUL113 or pp28 at the indicated time points (green). Mock-infected controls were immunostained for CK7 (red). Nuclei were counterstained with DAPI (blue). Scale bar $=100 \mu \mathrm{m}$.

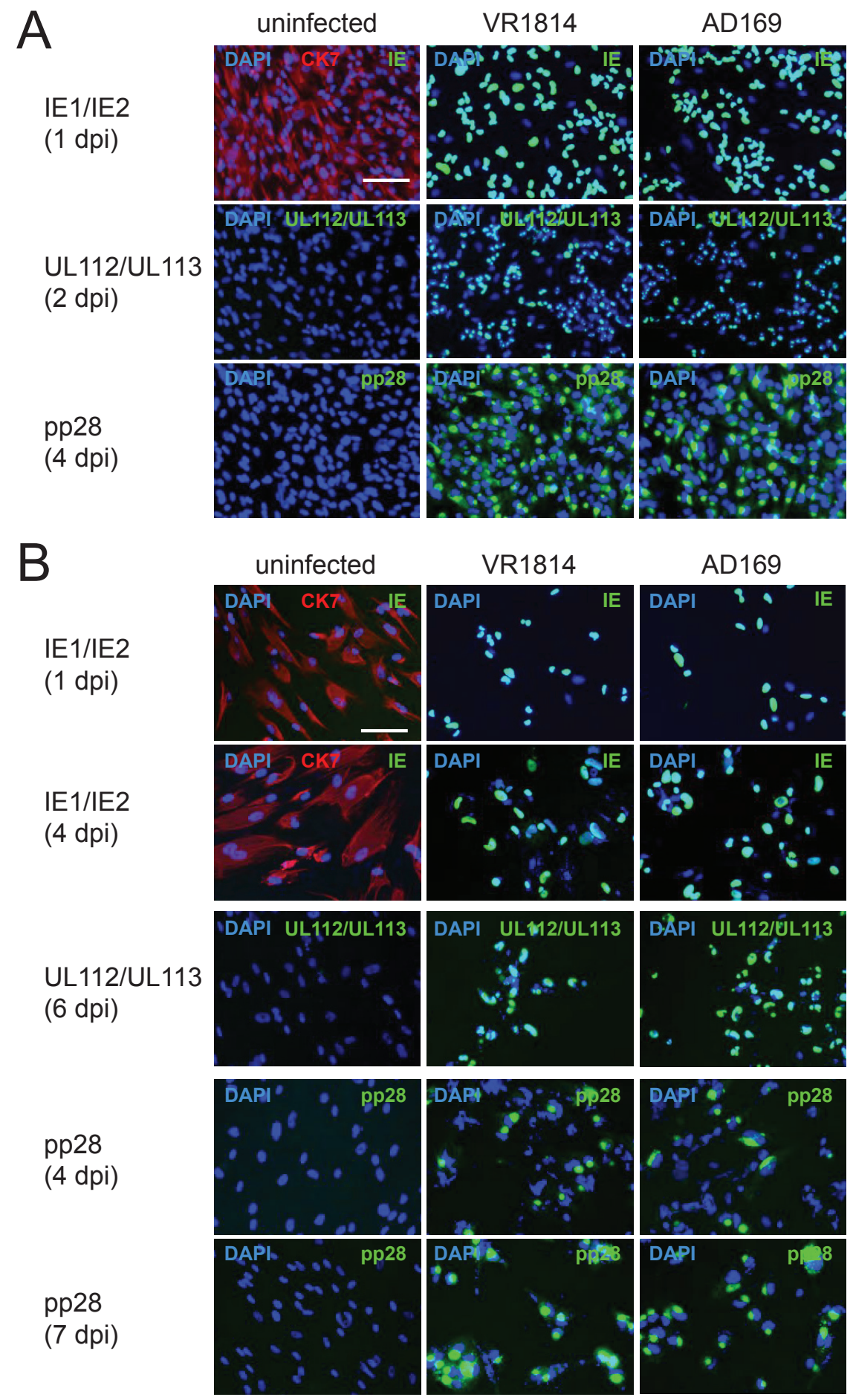




\subsection{HCMV-Infected TBPCs Produce Infectious Progeny Virions}

To determine if TBPCs were fully permissive and produced progeny virions, the cells were infected with either VR1814 or AD169, conditioned medium (CM) was collected at $48 \mathrm{~h}$ intervals, and viral progeny were quantified by titration on human foreskin fibroblasts (HFFs). For comparison of virus yield, HFFs were infected and analyzed in parallel. We found that relatively high levels of progeny virions were released from infected TBPCs (total $\sim 10^{6}$ PFU per $2 \mathrm{~cm}^{2}$ plate on peak day), about 10- to 15-fold lower than the amount released from HFFs (Figure 3A). Consistent with the comparable levels of IE, early and late protein expression (Figure 2A), no significant differences were found between the levels of VR1814 and AD169 progeny released. The results indicate that, following virion entry into TBPCs, both pathogenic and attenuated HCMV strains complete the lytic infectious cycle.

Next, we determined whether viral progeny released from TBPCs retain tropism. CM harvested from VR1814- and AD169-infected TBPCs (8 dpi) were adsorbed to human retinal pigment epithelial cells (ARPE-19) or HFFs. Immunostaining for IE proteins showed that AD169 progeny failed to infect ARPE-19 cells (Figure 3B) whereas VR1814 progeny from infected TBPCs retained the ability to infect ARPE-19 and HFFs. These results suggest that progeny virions released from TBPCs in the infected chorion retain tropism and hence could spread to other placental cells - vCTBs, iCTBs, stromal fibroblasts in the villous core and endothelial cells in blood vessels - thereby promoting viral dissemination in utero.

Figure 3. (A) Infected TBPCs release progeny virions. CM from VR1814- and AD169-infected TBPCs and HFFs (MOI 0.1) were collected at the indicated time points and titrated on HFFs. (B) Progeny virions from TBPCs retain tropism. CM from VR1814- and AD169-infected TBPCs (8 dpi) was added to HFFs and ARPE-19 cells, and IE1 was immunostained (1 dpi). Nuclei were counterstained with DAPI. Scale bar $=200 \mu \mathrm{m}$.

A

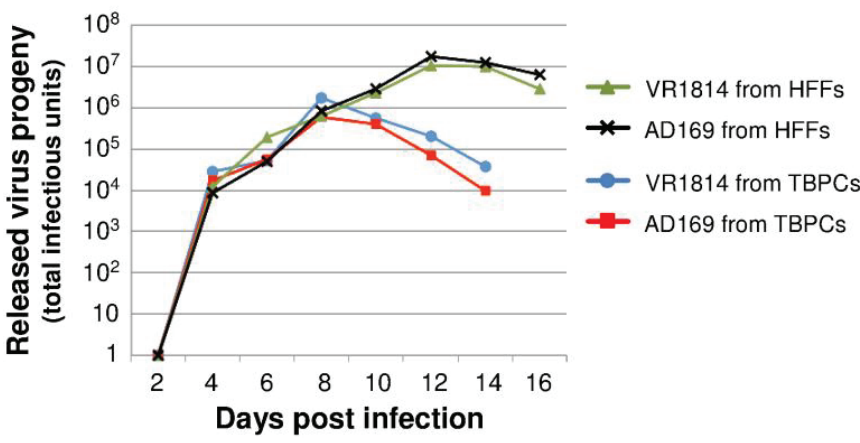

B
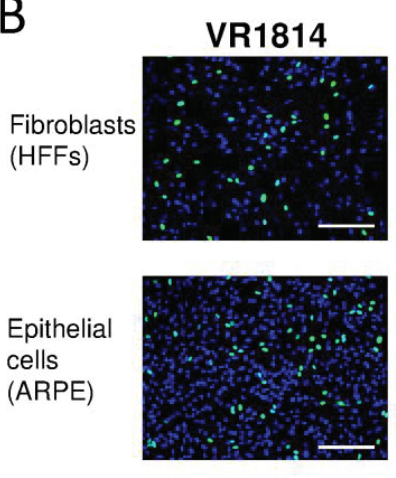

AD169
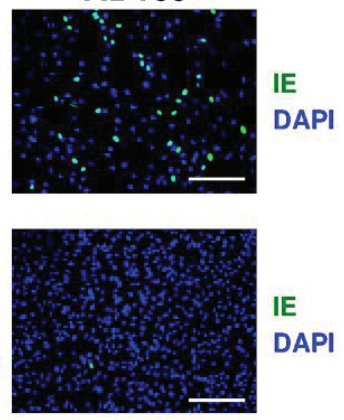

\subsection{Viral Entry into TBPCs Is Independent of the Pentamer Complex}

The finding that TBPCs can be infected with AD169 suggested that the pentamer complex is not required for virion entry into these cells. To test this hypothesis, we performed experiments with the TB40/E parent virus and its UL131A-deficient mutant (AUL131A) [53]. The mutant has an early stop codon in the UL131A ORF, does not express a functional pentamer, and fails to enter 
epithelial and endothelial cells [53]. We confirmed the phenotype of parental TB40/E and $\Delta$ UL131A by infecting ARPE-19 cells and HFFs. As expected HFFs supported infection with both viruses as determined by IE protein expression at 2 dpi (Figure 4G-L), whereas ARPE-19 cells were only permissive for the parental virus TB40/E (Figure 4C,D), but not $\triangle$ UL131A (Figure 4E,F). To test TBPCs, we incubated the cells with TB40/E and $\triangle$ UL131A and found that in both cases $>90 \%$ of cells expressed IE proteins at 2 dpi (Figure 4M-S). The results confirmed that the pentamer complex is dispensable for virion entry into TBPCs. These data also suggest that virion entry into TBPCs follows the pathway described for fibroblasts, which involves gB-mediated fusion at the plasma membrane at neutral $\mathrm{pH}[56]$, as opposed to the entry pathway into epithelial and endothelial cells, where virion uptake entails endocytosis $[57,58]$.

Figure 4. HCMV pentamer complex is dispensable for virion entry into TBPCs. ARPE-19 cells $(\mathbf{A}-\mathbf{F})$, HFFs $(\mathbf{G}-\mathbf{L})$ and TBPCs $(\mathbf{M}-\mathbf{S})$ were mock infected or infected (MOI 2) with parental TB40/E and UL131A-deficient mutant ( $\triangle$ UL131A) and immunostained for IE1 proteins (2 dpi). Nuclei were counterstained with DAPI (blue). Scale bar $=100 \mu \mathrm{m}$.

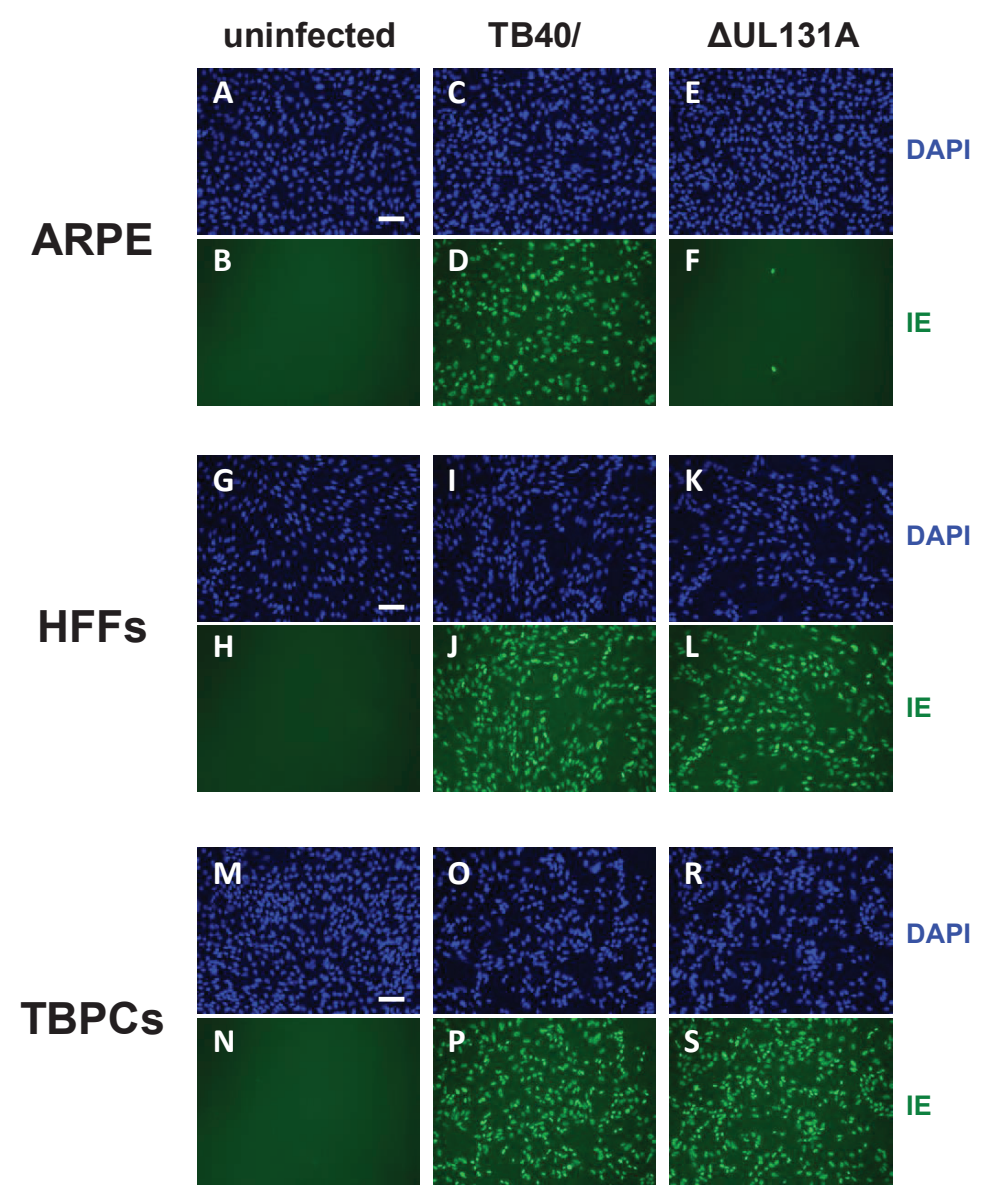

\subsection{TBPC Infection Is Blocked by mAbs to gB But Not mAbs to the Pentamer Complex}

Unlike HIG preparations, human mAbs to viral glycoproteins are epitope specific and can have especially high affinity. Among the important HCMV proteins that have been targeted by human $\mathrm{mAbs}$ are $\mathrm{gB}[23]$ and components of the pentamer complex [19]. The finding that a viral mutant 
lacking a functional pentamer infects TBPCs at levels comparable to those of the parent, which expresses a functional pentamer, suggested that the pentamer is dispensable for virion entry. Consequently, we expected that mAbs that prevent HCMV entry into a broad range of cells (e.g., gB-specific mAbs) would block TBPC infection, whereas mAbs to the pentamer would not.

To test this hypothesis, we carried out neutralization assays with human mAbs to the pentamer component pUL130/pUL131A (mAbs 5A2 and 1F11) and a human mAb to gB (TRL345). These mAbs were selected by B-cell cloning and reported to have potent HCMV neutralizing titer in epithelial cells (anti-pUL130/pUL131A and anti-gB) and fibroblasts (anti-gB) [19,23]. First, we verified that the mAbs and the HIG preparation blocked VR1814 infection of ARPE-19 cells in a dose-dependent manner (Figure 5). The control mAb (Synagis) lacked neutralizing activity (Figure 5A). Next, we found that mAbs to the pentamer (mAbs 5A2 and 1F11) failed to block VR1814 infection of TBPCs (Figure 5B), whereas anti-gB mAb TRL345 exhibited a dose-dependent neutralizing activity, almost completely blocking VR1814 infection (99\% reduction) at $10 \mu \mathrm{g} / \mathrm{mL}$ (Figure 5B). HIG showed some neutralizing activity in TBPCs at high concentrations ( $\sim 2 \%$ mean reduction at $10 \mu \mathrm{g} / \mathrm{mL}, \sim 81 \%$ at $100 \mu \mathrm{g} / \mathrm{mL}$ ), but the efficiency was highly variable. It is difficult to compare HIG with HCMV neutralizing mAbs because the former is a polyclonal antibody mixture prepared from seropositive donors and contains mostly antibodies without antiviral activity. In contrast, each $\mathrm{mAb}$ recognizes a specific functional epitope on a single viral protein. Nonetheless, anti-gB mAb TRL345 blocked infection of TBPCs at $1 \mu \mathrm{g} / \mathrm{mL}$ to a greater extent than $\mathrm{HIG}$ at $100 \mu \mathrm{g} / \mathrm{mL}$. These results indicate that a mAb to gB efficiently neutralizes HCMV infection of TBPCs as compared with HIG.

\subsection{Neutralization of VR1814 by Anti-gB mAb TRL345 Restores TBPC Differentiation}

When cultured under differentiation conditions, TBPCs form trophospheres and express markers of differentiated iCTBs and STBs [27], a process that mimics early differentiation in utero. In contrast, infected TBPCs fail to differentiate and form trophospheres [37]. We therefore examined whether TRL345-mediated virus neutralization could preserve the ability of TBPCs to differentiate and form trophospheres. For these experiments, TBPCs were infected with VR1814 or a virus-antibody mixture containing $20 \mu \mathrm{g} / \mathrm{mL}$ anti-gB mAb (TRL345), $20 \mu \mathrm{g} / \mathrm{mL}$ anti-pentamer $\mathrm{mAb}(5 \mathrm{~A} 2)$ or $20 \mu \mathrm{g} / \mathrm{mL}$ negative control mAb (Synagis), or they were left uninfected. At $3 \mathrm{dpi}$, culture conditions were changed to induce differentiation (see Experimental Section). At 4 dpi, differentiating TBPCs (uninfected) formed trophospheres with diameters of approximately 200-250 $\mu \mathrm{m}$ (Figure 6A,F). In contrast, infected TBPCs generally failed to form spheres, and the few spheres that assembled had diameters of approximately $50 \mu \mathrm{m}$ or less (Figure 6B,G). TBPCs incubated with anti-gB mAb-treated VR1814 retained the capacity to differentiate and formed large trophospheres (Figure 6D,I). In contrast, TBPCs incubated with VR1814 treated with mAbs to the pentamer or the negative control mAb failed to form large trophospheres (Figure 6C,E,H,J), consistent with the failure of pentamer-specific mAbs to block infection (Section 2.4). Thus, neutralizing anti-gB mAb TRL345 precludes HCMV infection of TBPCs and rescues differentiation. 
Figure 5. Infection of TBPCs is blocked by a $\mathrm{mAb}$ to $\mathrm{gB}$ but not $\mathrm{mAbs}$ to the pentamer complex. (A) VR1814 (MOI 0.01) was pre-incubated with medium alone, a serial $\log _{10}$ dilution of the indicated mAb $(0.01-10 \mu \mathrm{g} / \mathrm{mL})$ or HIG $(0.1-100 \mu \mathrm{g} / \mathrm{mL})$. Virus-antibody mixtures were then applied to ARPE-19 cells. At 2 dpi, IE1 was immunostained and numbers of infected cells were counted. Data were normalized to the no Ab control. Error bars indicate standard deviation. (B) TBPCs were incubated with virus-antibody mixtures and analyzed as described for panel $\mathbf{A}$.
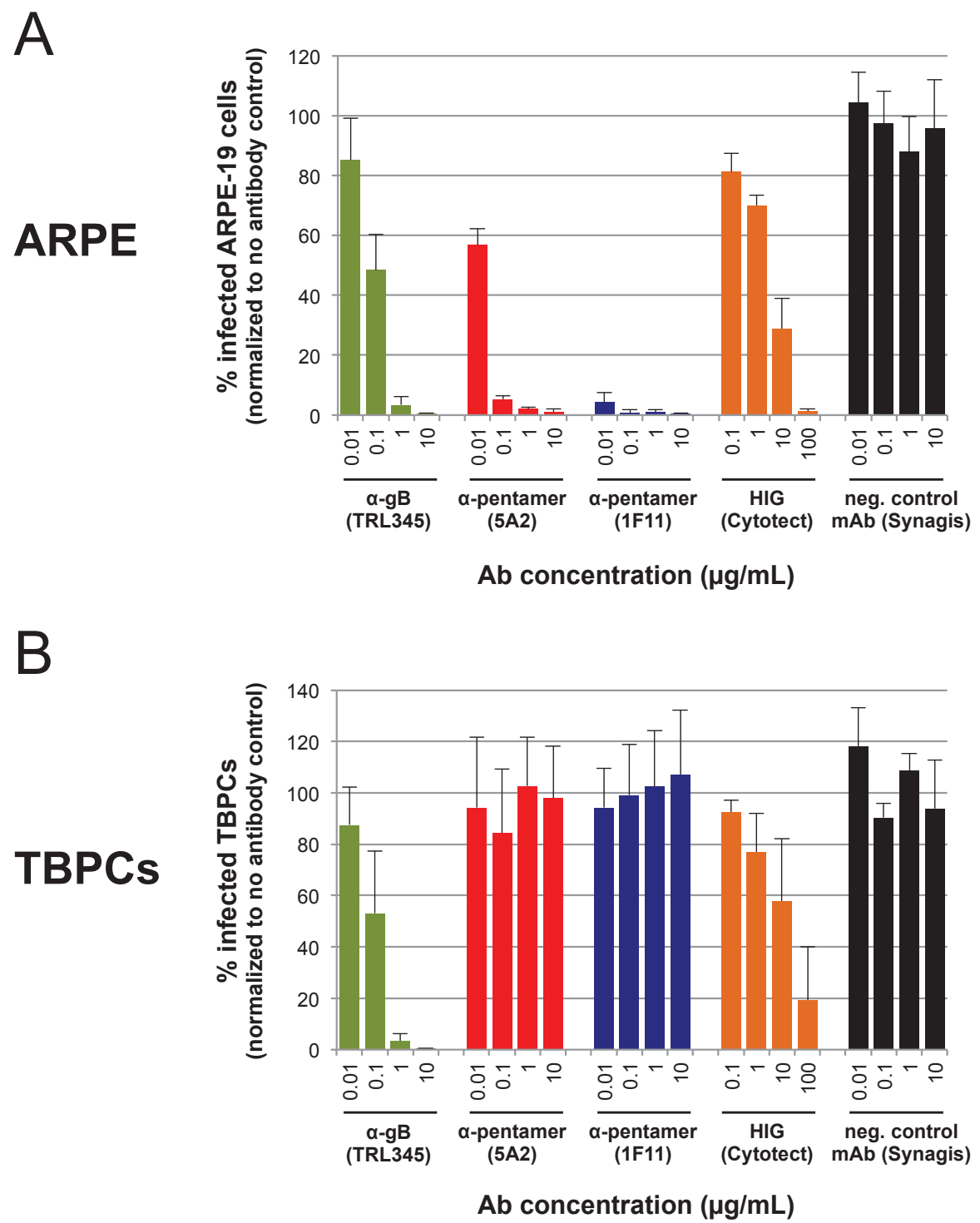

\subsection{Discussion}

The studies presented here show that HCMV undergoes lytic replication in TBPCs and that viral entry into these cells depends on the functions of $\mathrm{gB}$ but not those of the pentamer complex. VR1814-infected TBPCs release significant levels of progeny virions that retain epithelial cell tropism. These findings suggest infection of TBPCs could inhibit villous growth and differentiation and promote viral spread among various cell types in the placenta. Infection could decrease the population of TBPCs (Figures 2-4) and impair differentiation (Figure 6), thereby reducing the mature trophoblast populations, CTBs and STBs. 
Figure 6. Neutralizing anti-gB mAb TRL345 precludes VR1814 infection and rescues TBPC differentiation. VR1814 was pretreated with $20 \mu \mathrm{g} / \mathrm{mL}$ of the indicated $\mathrm{mAbs}$, and the mixtures were adsorbed to TBPCs. At 3 dpi, the cells were reseeded on matrigel and cultured in differentiation medium for $24 \mathrm{~h}$ followed by light microscopic analysis. Lower magnification photographs (upper panel), higher magnification photographs (lower panel). Scale bars $=200 \mu \mathrm{m}$.

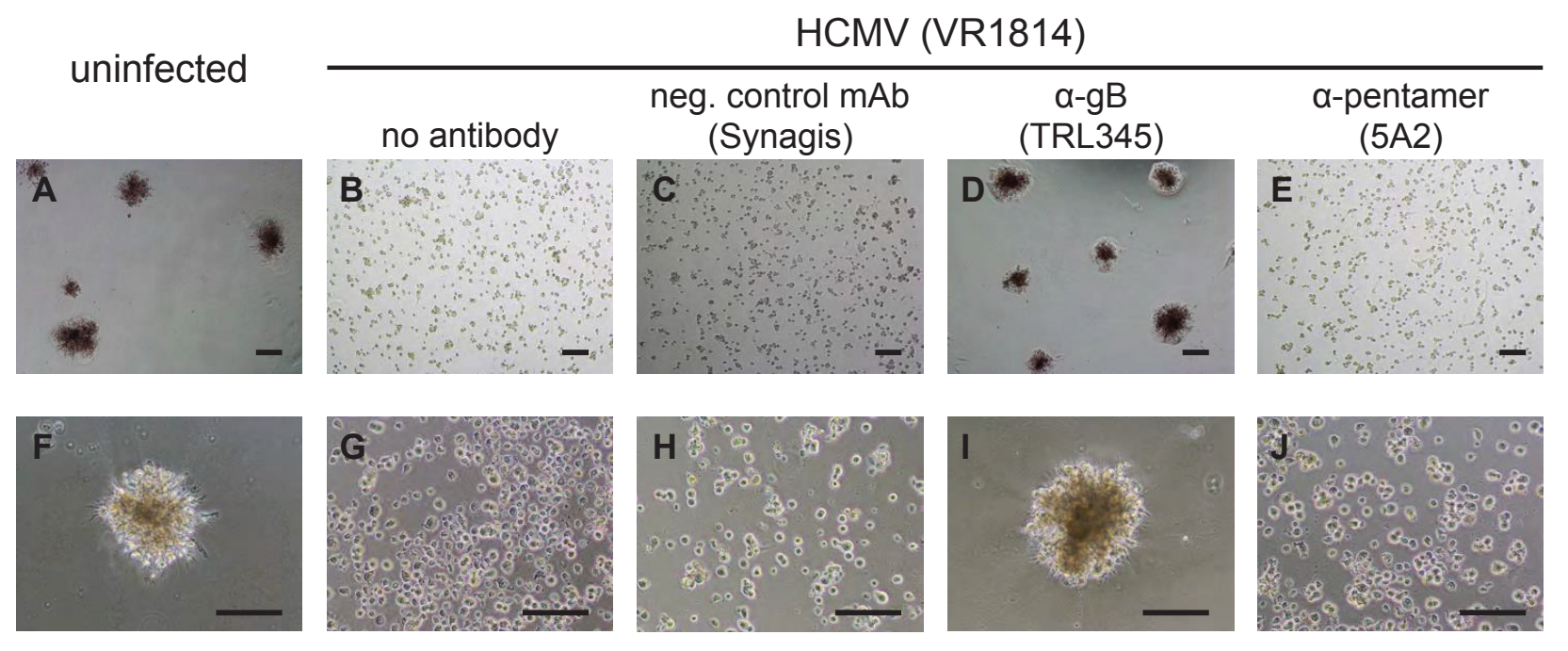

These findings could also explain the observation that CTBs failed to differentiate into STBs in cases of IUGR with congenital HCMV infection, suggesting infection and paracrine effects could subvert placental development [4]. Passive immunization with HIG enables compensatory growth of chorionic villi, increasing STB surface area and exchange [14], suggesting that neutralizing antibodies reduce virus replication and improve outcome. HIG was reported to prevent virus transmission to the placenta and fetus when administrated soon after maternal seroconversion [10-13]. In accord with these findings, HIG reduces viral spread in the decidua in an ex vivo tissue model [59]. Animal models that simulate congenital infection are rare because of the unique anatomy and biology of the hematogenous human placenta. However, the guinea pig has been used to measure efficacy of guinea pig CMV (gpCMV) antibodies in reducing transplacental infection [60]. When pregnant guinea pigs were infected and passively immunized with gpCMV neutralizing antiserum, fetal survival increased significantly, and placental inflammation and IUGR were reduced [60]. Likewise, a gpCMV gB subunit vaccine elicited protective neutralizing antibodies in dams, resulting in lower rates of fetal infection and reduced pup mortality [61].

HCMV entry into fibroblasts requires $\mathrm{gB}$ and $\mathrm{gH} / \mathrm{gL}[46,49,50]$, whereas entry into epithelial and endothelial cells requires $\mathrm{gB}$ and the pentamer complex $\mathrm{gH} / \mathrm{gL} / \mathrm{pUL} 128-131 \mathrm{~A}[47-51,62]$. Consequently, anti-gB antibodies neutralize virus entry into all cell types, whereas antibodies to the pentamer components pUL128, pUL130 and pUL131A selectively block infection of epithelial and endothelial cells $[19,63]$. Interestingly, antibodies to the pentamer complex are the major active component of HIG [64], and delayed development of these antibodies correlates with transplacental transmission to the fetus [65]. Our results suggest that anti-pentamer mAbs may reduce but not prevent virus spread in the developing placenta, as they fail to protect TBPCs, and other cell types, including stromal fibroblasts from the villus core [29] and uterine smooth muscle cells (data not 
shown). Importantly, anti-gB mAbs, but not anti-pentamer mAbs, preserve the ability of TBPCs to differentiate. Moreover, we found that anti-gB mAb TRL345 had a higher efficiency and consistency as compared to HIG (Figure 5A,B). This high potency of mAb TRL345 is a consequence of the targeted, highly conserved AD-2 (Site I) epitope on gB [66], which the antibody binds to with high affinity [23]. While this neutralizing epitope is essential for $\mathrm{gB}$ function, it is poorly immunogenic, which explains why antibodies to this epitope are rare in human blood and consequently not present in HIG preparations $[19,23,66]$. HCMV infection inhibits TBPC self-renewal, proliferation and differentiation, which are required for proper villous development [37]. High affinity, potent human mAbs to $\mathrm{gB}$, which functions in virion entry into a broad range of cell types, should be considered as a potential biotherapy for congenital HCMV infection, alone or in combination with pentamer complex targeting mAbs that more efficiently block infection of epithelial and endothelial cells. Additional advantages include a well-defined composition that reduces lot-dependent effects and increases consistency, suggesting mAbs could have considerable efficacy in clinical trials.

\section{Experimental Section}

\subsection{Cells}

TBPCs (from 7.3 and 15.6 weeks of gestation) were established as reported [27]. Cells were grown on gelatin-coated plates in DMEM/F12 supplemented with $10 \mathrm{ng} / \mathrm{mL}$ basic FGF (R\&D Systems, Minneapolis, MN, USA), 10\% FCS, $10 \mu \mathrm{M}$ SB431542 (Tocris Bioscience, Minneapolis, MN, USA), 100 units $/ \mathrm{mL}$ penicillin, $100 \mu \mathrm{g} / \mathrm{mL}$ streptomycin and $0.25 \mu \mathrm{g} / \mathrm{mL}$ fungizone. To exclude the presence of placental fibroblasts in the TBPC cultures, cells were immunostained for cytokeratin 7 (CK7), GATA-3 and GATA-4 as described in Section 3.3. These proteins are expressed in TBPCs but not in placental fibroblasts. ARPE-19 cells and HFFs were cultured in DMEM supplemented with FCS and penicillin/streptomycin as described previously $[67,68]$.

\subsection{Viruses and Infections}

VR1814 (an endothelial and epithelial cell-tropic pathogenic strain of HCMV) [69] was propagated in HUVEC [70], followed by a single passage in HFFs to obtain high titer stocks. AD169 (attenuated laboratory strain), TB40/E-derived parental virus (vBAC4-luc) and $\triangle$ UL131A mutant (vBAC4-luc/UL131Astop) were propagated in HFFs [53]. For infection (MOI is indicated in figure legends), virus was diluted in DMEM, $1 \% \mathrm{FCS}, 100$ units $/ \mathrm{mL}$ penicillin, $100 \mu \mathrm{g} / \mathrm{mL}$ streptomycin and adsorbed for $2 \mathrm{~h}$. Virus suspensions were aspirated, cells washed with PBS and provided with fresh medium.

\subsection{Immunofluorescence}

Cells were fixed with $4 \%$ paraformaldehyde, permeabilized with $0.5 \%$ Triton X-100 and blocked with normal serum. Cells were incubated with primary antibody, followed by fluorescein isothiocyanate (FITC) or rhodamine red-X (RRX) labeled secondary antibodies (Jackson ImmunoResearch Laboratories, West Grove, PA, USA). For detection of TBPC markers, antibodies to cytokeratin 7 (CK7, monoclonal rat IgG, clone 7D3 [71]), GATA-3 (polyclonal goat 
IgG, R\&D Systems, Minneapolis, MN, USA), GATA-4 (polyclonal goat IgG, R\&D Systems) and HMGA2 (polyclonal rabbit IgG, Abcam, Cambridge, MA, USA) were used. Murine monoclonal antibodies used to detect viral proteins were CH433 (anti-IE1, immediate-early protein), CH19 (anti-pp28, true-late protein) [72] and M23 (anti-pUL112/pUL113, early proteins) [73]. Nuclei were counterstained with DAPI (Sigma-Aldrich, St. Louis, MO, USA). Analyses were performed with a Nikon Eclipse TS100 inverted fluorescence microscope equipped with a Nikon DS-F12 camera controlled by Nikon NIS Elements 4.0 imaging software [74].

\subsection{Detection and Quantification of Viral Progeny}

To quantify viral progeny, TBPCs or HFFs were infected with VR1814 or AD169 (MOI 0.1). Conditioned media (CM) was collected every $48 \mathrm{~h}$. Subsequently, virus titers in CM were determined with an immunofluorescence-based infectivity assay in HFFs at 24 hpi [70]. For quantification, at least six representative pictures were taken and IE1-positive cells counted using Fiji Image J software [75,76].

\subsection{Virus Neutralization Assay}

HCMV neutralizing assays were performed as described before [36]. Briefly, TBPCs or ARPE-19 cells were seeded in 24-well plates. Diluted mAbs (final concentration $0.01 \mu \mathrm{g} / \mathrm{mL}, 0.1 \mu \mathrm{g} / \mathrm{mL}$, $1 \mu \mathrm{g} / \mathrm{mL}$ or $10 \mu \mathrm{g} / \mathrm{mL}$ ) or HIG (Cytotect, Biotest, Boca Raton, FL, USA, final concentration $0.1 \mu \mathrm{g} / \mathrm{mL}, 1 \mu \mathrm{g} / \mathrm{mL}, 10 \mu \mathrm{g} / \mathrm{mL}$ or $100 \mu \mathrm{g} / \mathrm{mL}$ ) were incubated with VR1814 (MOI 0.01, in $300 \mu \mathrm{L}$ total volume) for $1 \mathrm{~h}$ at $37^{\circ} \mathrm{C}$ with moderate agitation. Virus-antibody mixtures were adsorbed to cells for $2 \mathrm{~h}$, followed by a wash step with PBS. The cells were then supplemented with fresh growth medium. At 2 dpi cells were fixed, permeabilized and immunostained for IE1 and analyzed by immunofluorescence microscopy (Section 3.3). IE1-positive cells were quantified as described in Section 3.4. Alternatively, viral titers were adjusted to give a total of 600 to 800 IE1-positive cells/well in 24 well plates. To target gB, human mAb TRL345 (Trellis Bioscience, LLC, South San Francisco, NC, USA [23]) was used. To target the pentamer, human mAb 1F11 or 5A2 (cloned from a published sequence [19] by Trellis Bioscience) directed to pUL130/pUL131A was used. Synagis [77], reactive with respiratory syncytial virus, was used as negative control mAb.

\subsection{Trophosphere Formation}

TBPCs were mock infected or infected with VR1814 or a mixture of virus and mAb as described above. The corresponding $\mathrm{mAb}$ used for pretreatment was present throughout the experiment $(20 \mu \mathrm{g} / \mathrm{mL})$. At $3 \mathrm{dpi}$, TBPCs were dissociated and reseeded in matrigel-coated plates $\left(100,000\right.$ cells per $2 \mathrm{~cm}^{2}$ ) in differentiation medium (Knockout Serum Replacement Medium, Gibco, Grand Island, NE, USA) supplemented with 10\% FCS, 100 units $/ \mathrm{mL}$ penicillin, $100 \mu \mathrm{g} / \mathrm{mL}$ streptomycin, $0.25 \mu \mathrm{g} / \mathrm{mL}$ fungizone, $10 \mathrm{ng} / \mathrm{mL}$ FGF4 (Sigma-Aldrich, St. Louis, MO, USA) and $40 \mathrm{ng} / \mathrm{mL}$ EGF (Invitrogen, Grand Island, NE, USA) [27]. Formation of trophospheres was analyzed by microscopy at 4 dpi. 


\section{Conclusions}

Human TBPCs in the chorion differentiate into CTBs and STBs, the two major cell types of chorionic villi required for placental development. The HCMV pathogenic strain VR1814 and the attenuated strain AD169 replicate fully in TBPCs, and the infectious progeny released retain tropism. Infection is blocked by a human mAb TRL345 to $\mathrm{gB}$ but not mAbs to the pentamer complex. Infected TBPCs are functionally impaired and fail to develop into large trophospheres comprised of cells that express the properties of differentiated CTBs and STBs. Altogether, our results suggest that congenital HCMV infection could be suppressed with a broadly effective neutralizing $\mathrm{mAb}$ to $\mathrm{gB}$ to prevent infection of TBPCs and, together with mAbs that protect specialized placental cells, could reduce virus transmission at the uterine-placental interface.

\section{Acknowledgments}

This work was supported by grants from the National Institutes of Health RO1AI046657, R56AI073752, RO1AI21420, R56AI21739 (L.P.), R21HD061890 (T.T.) and R44AI102396 (L.K.). Martin Zydek was supported by a postdoctoral fellowship of the Deutsche Forschungsgemeinschaft DFG (ZY110/1-1). Barbara Adler was supported by the Deutsche Forschungsgemeinschaft DFG (AD131/3-1 and AD131/3-2). We thank Laura Scrivano for constructing the UL131A-deficient mutant and Susan Fisher, Olga Genbacev and Nicholas Larocque of the Eli and Edythe Broad Center of Regenerative Medicine, University of California San Francisco, for excellent scientific and technical advice. We are grateful to our deceased colleague William Usinger for his contributions.

\section{Author Contributions}

M.Z., T.T. and L.P. designed research; M.Z. and J.F.-H. performed research; B.A. and L.M.K. contributed new reagents and scientific advice; M.Z., M.P. and T.T. analyzed data; M.Z., M.P., T.T. and L.P. wrote the paper.

\section{Conflicts of Interest}

TRL345 is a mAb from Trellis Bioscience in which Larry Kauvar has an equity interest.

\section{References and Notes}

1. Britt, W.J. Congenital cytomegalovirus infection. In Sexually Transmitted Diseases and Adverse Outcomes of Pregnancy; Hitchcock, P.J., MacKay, H.T., Wasserheit, J.N., Eds.; ASM Press: Washington, DC, USA, 1999; pp. 269-281.

2. Demmler, G.J. Congenital cytomegalovirus infection and disease. Adv. Pediatr. Infect. Dis. 1996, 11, 135-162.

3. Rivera, L.B.; Boppana, S.B.; Fowler, K.B.; Britt, W.J.; Stagno, S.; Pass, R.F. Predictors of hearing loss in children with symptomatic congenital cytomegalovirus infection. Pediatrics 2002, 110, 762-767. 
4. Pereira, L.; Petitt, M.; Fong, A.; Tsuge, M.; Tabata, T.; Fang-Hoover, J.; Maidji, E.; Zydek, M.; Zhou, Y.; Inoue, N.; et al. Intrauterine growth restriction caused by underlying congenital cytomegalovirus infection. J. Infect. Dis. 2014, doi:10.1093/infdis/jiu019.

5. Fowler, K.B.; Stagno, S.; Pass, R.F. Maternal immunity and prevention of congenital cytomegalovirus infection. JAMA 2003, 289, 1008-1011.

6. Yamamoto, A.Y.; Mussi-Pinhata, M.M.; Isaac Mde, L.; Amaral, F.R.; Carvalheiro, C.G.; Aragon, D.C.; Manfredi, A.K.; Boppana, S.B.; Britt, W.J. Congenital cytomegalovirus infection as a cause of sensorineural hearing loss in a highly immune population. Pediatr. Infect. Dis. J. 2011, 30, 1043-1046.

7. Ross, S.A.; Fowler, K.B.; Ashrith, G.; Stagno, S.; Britt, W.J.; Pass, R.F.; Boppana, S.B. Hearing loss in children with congenital cytomegalovirus infection born to mothers with preexisting immunity. J. Pediatr. 2006, 148, 332-336.

8. Mercorelli, B.; Sinigalia, E.; Loregian, A.; Palu, G. Human cytomegalovirus DNA replication: Antiviral targets and drugs. Rev. Med. Virol. 2008, 18, 177-210.

9. Benoist, G.; Leruez-Ville, M.; Magny, J.F.; Jacquemard, F.; Salomon, L.J.; Ville, Y. Management of pregnancies with confirmed cytomegalovirus fetal infection. Fetal. Diagn. Ther. 2013, 33, 203-214.

10. Nigro, G.; Adler, S.P.; Parruti, G.; Anceschi, M.M.; Coclite, E.; Pezone, I.; Di Renzo, G.C. Immunoglobulin therapy of fetal cytomegalovirus infection occurring in the first half of pregnancy - A case-control study of the outcome in children. J. Infect. Dis. 2012, 205, 215-227.

11. Buxmann, H.; Stackelberg, O.M.; Schlosser, R.L.; Enders, G.; Gonser, M.; Meyer-Wittkopf, M.; Hamprecht, K.; Enders, M. Use of cytomegalovirus hyperimmunoglobulin for prevention of congenital cytomegalovirus disease: A retrospective analysis. J. Perinat. Med. 2012, 40, 439-446.

12. Nigro, G.; Adler, S.P.; La Torre, R.; Best, A.M. Passive immunization during pregnancy for congenital cytomegalovirus infection. New Engl. J. Med. 2005, 353, 1350-1362.

13. Nigro, G.; Torre, R.L.; Pentimalli, H.; Taverna, P.; Lituania, M.; de Tejada, B.M.; Adler, S.P. Regression of fetal cerebral abnormalities by primary cytomegalovirus infection following hyperimmunoglobulin therapy. Prenat. Diagn. 2008, 28, 512-517.

14. Maidji, E.; Nigro, G.; Tabata, T.; McDonagh, S.; Nozawa, N.; Shiboski, S.; Muci, S.; Anceschi, M.M.; Aziz, N.; Adler, S.P.; Pereira, L. Antibody treatment promotes compensation for human cytomegalovirus-induced pathogenesis and a hypoxia-like condition in placentas with congenital infection. Am. J. Pathol. 2010, 177, 1298-1310.

15. Adler, S.P.; Nigro, G. Findings and conclusions from $\mathrm{cmv}$ hyperimmune globulin treatment trials. J. Clin. Virol. 2009, 46, S54-S57.

16. Nightingale, S.L. From the food and drug administration. JAMA 1990, 264, 2863.

17. Snydman, D.R.; Werner, B.G.; Heinze-Lacey, B.; Berardi, V.P.; Tilney, N.L.; Kirkman, R.L.; Milford, E.L.; Cho, S.I.; Bush, H.L., Jr.; Levey, A.S.; et al. Use of cytomegalovirus immune globulin to prevent cytomegalovirus disease in renal-transplant recipients. New Engl. J. Med. 1987, 317, 1049-1054.

18. Ballow, M. Mechanisms of action of intravenous immune serum globulin therapy. Pediatr. Infect. Dis. J. 1994, 13, 806-811. 
19. Macagno, A.; Bernasconi, N.L.; Vanzetta, F.; Dander, E.; Sarasini, A.; Revello, M.G.; Gerna, G.; Sallusto, F.; Lanzavecchia, A. Isolation of human monoclonal antibodies that potently neutralize human cytomegalovirus infection by targeting different epitopes on the gh/gl/ul128-131a complex. J. Virol. 2010, 84, 1005-1013.

20. Collarini, E.J.; Lee, F.E.; Foord, O.; Park, M.; Sperinde, G.; Wu, H.; Harriman, W.D.; Carroll, S.F.; Ellsworth, S.L.; Anderson, L.J.; et al. Potent high-affinity antibodies for treatment and prophylaxis of respiratory syncytial virus derived from $b$ cells of infected patients. J. Immunol. 2009, 183, 6338-6345.

21. Harriman, W.D.; Collarini, E.J.; Sperinde, G.V.; Strandh, M.; Fatholahi, M.M.; Dutta, A.; Lee, Y.; Mettler, S.E.; Keyt, B.A.; Ellsworth, S.L.; et al. Antibody discovery via multiplexed single cell characterization. J. Immunol. Methods 2009, 341, 135-145.

22. Wrammert, J.; Smith, K.; Miller, J.; Langley, W.A.; Kokko, K.; Larsen, C.; Zheng, N.Y.; Mays, I.; Garman, L.; Helms, C.; et al. Rapid cloning of high-affinity human monoclonal antibodies against influenza virus. Nature 2008, 453, 667-671.

23. McCutcheon, K.M.; Gray, J.; Chen, N.Y.; Liu, K.; Park, M.; Ellsworth, S.; Tripp, R.A.; Mark Tompkins, S.; Johnson, S.K.; et al. Multiplexed screening of natural humoral immunity identifies antibodies at fine specificity for complex and dynamic viral targets. MAbs 2014, 6, $460-473$.

24. Damsky, C.H.; Fisher, S.J. Trophoblast pseudo-vasculogenesis: Faking it with endothelial adhesion receptors. Curr. Opin. Cell Biol. 1998, 10, 660-666.

25. Zhou, Y.; Fisher, S.J.; Janatpour, M.; Genbacev, O.; Dejana, E.; Wheelock, M.; Damsky, C.H. Human cytotrophoblasts adopt a vascular phenotype as they differentiate. A strategy for successful endovascular invasion? J. Clin. Investig. 1997, 99, 2139-2151.

26. Hemberger, M.; Udayashankar, R.; Tesar, P.; Moore, H.; Burton, G.J. Elf5-enforced transcriptional networks define an epigenetically regulated trophoblast stem cell compartment in the human placenta. Hum. Mol. Genet. 2010, 19, 2456-2467.

27. Genbacev, O.; Donne, M.; Kapidzic, M.; Gormley, M.; Lamb, J.; Gilmore, J.; Larocque, N.; Goldfien, G.; Zdravkovic, T.; McMaster, M.T.; Fisher, S.J. Establishment of human trophoblast progenitor cell lines from the chorion. Stem Cells 2011, 29, 1427-1436.

28. Genbacev, O.; Lamb, J.D.; Prakobphol, A.; Donne, M.; McMaster, M.T.; Fisher, S.J. Human trophoblast progenitors: Where do they reside? Semin. Reprod. Med. 2013, 31, 56-61.

29. Fisher, S.; Genbacev, O.; Maidji, E.; Pereira, L. Human cytomegalovirus infection of placental cytotrophoblasts in vitro and in utero: Implications for transmission and pathogenesis. J. Virol. 2000, 74, 6808-6820.

30. Maidji, E.; Genbacev, O.; Chang, H.T.; Pereira, L. Developmental regulation of human cytomegalovirus receptors in cytotrophoblasts correlates with distinct replication sites in the placenta. J. Virol. 2007, 81, 4701-4712.

31. Pereira, L.; Maidji, E.; McDonagh, S.; Genbacev, O.; Fisher, S. Human cytomegalovirus transmission from the uterus to the placenta correlates with the presence of pathogenic bacteria and maternal immunity. J. Virol. 2003, 77, 13301-13314.

32. Pereira, L.; Maidji, E.; McDonagh, S.; Tabata, T. Insights into viral transmission at the uterine-placental interface. Trends Microbiol. 2005, 13, 164-174. 
33. Tabata, T.; McDonagh, S.; Kawakatsu, H.; Pereira, L. Cytotrophoblasts infected with a pathogenic human cytomegalovirus strain dysregulate cell-matrix and cell-cell adhesion molecules: A quantitative analysis. Placenta 2007, 28, 527-537.

34. McDonagh, S.; Maidji, E.; Chang, H.T.; Pereira, L. Patterns of human cytomegalovirus infection in term placentas: A preliminary analysis. J. Clin. Virol. 2006, 35, 210-215.

35. Maidji, E.; McDonagh, S.; Genbacev, O.; Tabata, T.; Pereira, L. Maternal antibodies enhance or prevent cytomegalovirus infection in the placenta by neonatal fc receptor-mediated transcytosis. Am. J. Pathol. 2006, 168, 1210-1226.

36. Nozawa, N.; Fang-Hoover, J.; Tabata, T.; Maidji, E.; Pereira, L. Cytomegalovirus-specific, high-avidity igg with neutralizing activity in maternal circulation enriched in the fetal bloodstream. J. Clin. Virol. 2009, 46, S58-S63.

37. Tabata, T.; Petitt, M.; Zydek, M.; Fang-Hoover, J.; Larocque, N.; Tsuge, M.; Gormley, M.; Genbacev, O.; Pereira, L. Human cytomegalovirus infection of trophoblast progenitor cells inhibits early steps of differentiation. To be submitted for publication, 2014.

38. Isaacson, M.K.; Compton, T. Human cytomegalovirus glycoprotein b is required for virus entry and cell-to-cell spread but not for virion attachment, assembly, or egress. J. Virol. 2009, 83, 3891-3903.

39. Wille, P.T.; Wisner, T.W.; Ryckman, B.; Johnson, D.C. Human cytomegalovirus (hemv) glycoprotein gb promotes virus entry in trans acting as the viral fusion protein rather than as a receptor-binding protein. mBio 2013, 4, e00332-00313.

40. Compton, T.; Nowlin, D.M.; Cooper, N.R. Initiation of human cytomegalovirus infection requires initial interaction with cell surface heparan sulfate. Virology 1993, 193, 834-841.

41. Carlson, C.; Britt, W.J.; Compton, T. Expression, purification, and characterization of a soluble form of human cytomegalovirus glycoprotein b. Virology 1997, 239, 198-205.

42. Wang, X.; Huong, S.M.; Chiu, M.L.; Raab-Traub, N.; Huang, E.S. Epidermal growth factor receptor is a cellular receptor for human cytomegalovirus. Nature 2003, 424, 456-461.

43. Soroceanu, L.; Akhavan, A.; Cobbs, C.S. Platelet-derived growth factor-alpha receptor activation is required for human cytomegalovirus infection. Nature 2008, 455, 391-395.

44. Feire, A.L.; Roy, R.M.; Manley, K.; Compton, T. The glycoprotein b disintegrin-like domain binds beta 1 integrin to mediate cytomegalovirus entry. J. Virol. 2010, 84, 10026-10037.

45. Feire, A.L.; Koss, H.; Compton, T. Cellular integrins function as entry receptors for human cytomegalovirus via a highly conserved disintegrin-like domain. Proc. Natl. Acad. Sci. USA 2004, 101, 15470-15475.

46. Vanarsdall, A.L.; Ryckman, B.J.; Chase, M.C.; Johnson, D.C. Human cytomegalovirus glycoproteins $\mathrm{gb}$ and $\mathrm{gh} / \mathrm{gl}$ mediate epithelial cell-cell fusion when expressed either in cis or in trans. J. Virol. 2008, 82, 11837-11850.

47. Hahn, G.; Revello, M.G.; Patrone, M.; Percivalle, E.; Campanini, G.; Sarasini, A.; Wagner, M.; Gallina, A.; Milanesi, G.; Koszinowski, U.; et al. Human cytomegalovirus ul131-128 genes are indispensable for virus growth in endothelial cells and virus transfer to leukocytes. J. Virol. 2004, 78, 10023-10033. 
48. Ryckman, B.J.; Chase, M.C.; Johnson, D.C. Hcmv gh/g1/ul128-131 interferes with virus entry into epithelial cells: Evidence for cell type-specific receptors. Proc. Natl. Acad. Sci. USA 2008, 105, 14118-14123.

49. Sinzger, C.; Digel, M.; Jahn, G. Cytomegalovirus cell tropism. Curr. Top. Microbiol. Immunol. 2008, 325, 63-83.

50. Vanarsdall, A.L.; Johnson, D.C. Human cytomegalovirus entry into cells. Curr. Opin. Virol. 2012, 2, 37-42.

51. Wang, D.; Shenk, T. Human cytomegalovirus ul131 open reading frame is required for epithelial cell tropism. J. Virol. 2005, 79, 10330-10338.

52. Dolan, A.; Cunningham, C.; Hector, R.D.; Hassan-Walker, A.F.; Lee, L.; Addison, C.; Dargan, D.J.; McGeoch, D.J.; Gatherer, D.; Emery, V.C.; et al. Genetic content of wild-type human cytomegalovirus. J. Gen. Virol. 2004, 85, 1301-1312.

53. Scrivano, L.; Sinzger, C.; Nitschko, H.; Koszinowski, U.H.; Adler, B. Hcmv spread and cell tropism are determined by distinct virus populations. PLoS Pathog. 2011, 7, e1001256.

54. Adler, B.; Scrivano, L.; Ruzcics, Z.; Rupp, B.; Sinzger, C.; Koszinowski, U. Role of human cytomegalovirus ul131a in cell type-specific virus entry and release. J. Gen. Virol. 2006, 87, 2451-2460.

55. Stinski, M.F. Sequence of protein synthesis in cells infected by human cytomegalovirus: Early and late virus-induced polypeptides. J. Virol. 1978, 26, 686-701.

56. Compton, T.; Nepomuceno, R.R.; Nowlin, D.M. Human cytomegalovirus penetrates host cells by ph-independent fusion at the cell surface. Virology 1992, 191, 387-395.

57. Ryckman, B.J.; Jarvis, M.A.; Drummond, D.D.; Nelson, J.A.; Johnson, D.C. Human cytomegalovirus entry into epithelial and endothelial cells depends on genes ul128 to ul150 and occurs by endocytosis and low-ph fusion. J. Virol. 2006, 80, 710-722.

58. Sinzger, C. Entry route of homv into endothelial cells. J. Clin. Virol. 2008, 41, 174-179.

59. Weisblum, Y.; Panet, A.; Zakay-Rones, Z.; Haimov-Kochman, R.; Goldman-Wohl, D.; Ariel, I.; Falk, H.; Natanson-Yaron, S.; Goldberg, M.D.; Gilad, R.; et al. Modeling of human cytomegalovirus maternal-fetal transmission in a novel decidual organ culture. J. Virol. 2011, $85,13204-13213$.

60. Bratcher, D.F.; Bourne, N.; Bravo, F.J.; Schleiss, M.R.; Slaoui, M.; Myers, M.G.; Bernstein, D.I. Effect of passive antibody on congenital cytomegalovirus infection in guinea pigs. J. Infect. Dis. 1995, 172, 944-950.

61. Schleiss, M.R.; Bourne, N.; Stroup, G.; Bravo, F.J.; Jensen, N.J.; Bernstein, D.I. Protection against congenital cytomegalovirus infection and disease in guinea pigs, conferred by a purified recombinant glycoprotein b vaccine. J. Infect. Dis. 2004, 189, 1374-1381.

62. Straschewski, S.; Patrone, M.; Walther, P.; Gallina, A.; Mertens, T.; Frascaroli, G. Protein pul128 of human cytomegalovirus is necessary for monocyte infection and blocking of migration. J. Virol. 2011, 85, 5150-5158.

63. Saccoccio, F.M.; Sauer, A.L.; Cui, X.; Armstrong, A.E.; Habib el, S.E.; Johnson, D.C.; Ryckman, B.J.; Klingelhutz, A.J.; Adler, S.P.; McVoy, M.A. Peptides from cytomegalovirus ul130 and ul131 proteins induce high titer antibodies that block viral entry into mucosal epithelial cells. Vaccine 2011, 29, 2705-2711. 
64. Fouts, A.E.; Chan, P.; Stephan, J.P.; Vandlen, R.; Feierbach, B. Antibodies against the

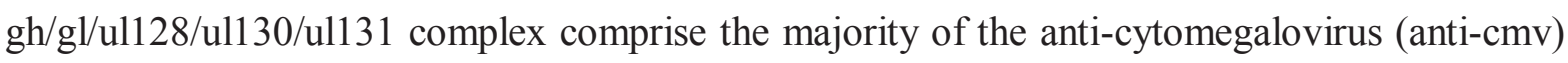
neutralizing antibody response in cmv hyperimmune globulin. J. Virol. 2012, 86, 7444-7447.

65. Lilleri, D.; Kabanova, A.; Revello, M.G.; Percivalle, E.; Sarasini, A.; Genini, E.; Sallusto, F.; Lanzavecchia, A.; Corti, D.; Gerna, G. Fetal human cytomegalovirus transmission correlates with delayed maternal antibodies to gh/gl/pul128-130-131 complex during primary infection. PLoS One 2013, 8, e59863.

66. Ohlin, M.; Sundqvist, V.A.; Mach, M.; Wahren, B.; Borrebaeck, C.A. Fine specificity of the human immune response to the major neutralization epitopes expressed on cytomegalovirus gp58/116 (gb), as determined with human monoclonal antibodies. J. Virol. 1993, 67, 703-710.

67. Tugizov, S.; Maidji, E.; Xiao, J.; Pereira, L. An acidic cluster in the cytosolic domain of human cytomegalovirus glycoprotein $\mathrm{b}$ is a signal for endocytosis from the plasma membrane. J. Virol. 1999, 73, 8677-8688.

68. Navarro, D.; Paz, P.; Tugizov, S.; Topp, K.; La Vail, J.; Pereira, L. Glycoprotein b of human cytomegalovirus promotes virion penetration into cells, transmission of infection from cell to cell, and fusion of infected cells. Virology 1993, 197, 143-158.

69. Grazia Revello, M.; Baldanti, F.; Percivalle, E.; Sarasini, A.; De-Giuli, L.; Genini, E.; Lilleri, D.; Labo, N.; Gerna, G. In vitro selection of human cytomegalovirus variants unable to transfer virus and virus products from infected cells to polymorphonuclear leukocytes and to grow in endothelial cells. J. Gen. Virol. 2001, 82, 1429-1438.

70. Maidji, E.; Percivalle, E.; Gerna, G.; Fisher, S.; Pereira, L. Transmission of human cytomegalovirus from infected uterine microvascular endothelial cells to differentiating/invasive placental cytotrophoblasts. Virology 2002, 304, 53-69.

71. Damsky, C.H.; Fitzgerald, M.L.; Fisher, S.J. Distribution patterns of extracellular matrix components and adhesion receptors are intricately modulated during first trimester cytotrophoblast differentiation along the invasive pathway, in vivo. J. Clin. Investig. 1992, 89, 210-222.

72. Pereira, L.; Hoffman, M.; Gallo, D.; Cremer, N. Monoclonal antibodies to human cytomegalovirus: Three surface membrane proteins with unique immunological and electrophoretic properties specify cross-reactive determinants. Infect. Immun. 1982, 36, 924-932.

73. Iwayama, S.; Yamamoto, T.; Furuya, T.; Kobayashi, R.; Ikuta, K.; Hirai, K. Intracellular localization and DNA-binding activity of a class of viral early phosphoproteins in human fibroblasts infected with human cytomegalovirus (towne strain). J. Gen. Virol. 1994, 75, 3309-3318.

74. Nis-elements, 4.0; Laboratory Imaging, Ltd.: Prague, Czech Republic, 2011.

75. Schneider, C.A.; Rasband, W.S.; Eliceiri, K.W. Nih image to imagej: 25 years of image analysis. Nat. Methods 2012, 9, 671-675.

76. Fiji (fiji is just imagej), version 1.0; University of Wisconsin at Madison: Madison, WI, USA, 2011.

77. Null, D.; Bimle, C.; Weisman, L.; Johnson, K.; Steichen, J.; Gratton, T.; Singh, S.; Wang, E.; Asztalos, E.; Loeffler, A.M.; et al. Palivizumab, a humanized respiratory syncytial virus monoclonal antibody, reduces hospitalization from respiratory syncytial virus infection in high-risk infants. Pediatrics 1998, 102, 531-537. 
Reprinted from Viruses. Cite as: Restrepo-Gualteros, S.M.; Jaramillo-Barberi, L.E.; Gonzalez-Santos, M.; Rodriguez-Martinez, C.E.; Perez, G.F.; Gutierrez, M.J.; Nino, G. Characterization of Cytomegalovirus Lung Infection in Non-HIV Infected Children. Viruses 2014, 6, 2038-2051.

Article

\section{Characterization of Cytomegalovirus Lung Infection in Non-HIV Infected Children}

Sonia M. Restrepo-Gualteros ${ }^{\text {1,2 }}$, Lina E. Jaramillo-Barberi ${ }^{3,4}$, Monica Gonzalez-Santos ${ }^{\mathbf{5}}$, Carlos E. Rodriguez-Martinez ${ }^{5,6,7}$, Geovanny F. Perez ${ }^{8}$, Maria J. Gutierrez ${ }^{9}$ and Gustavo Nino ${ }^{8,10, *}$

1 Division of Pediatric Pulmonology, Fundacion Hospital La Misericordia, Bogota 111411, Colombia; E-Mail: sm.restrepo@uniandes.edu.co

2 Department of Pediatrics, School of Medicine, Universidad de los Andes, Fundacion Santa Fe de Bogota, Bogota 110111, Colombia

3 Department of Pathology, School of Medicine, Universidad Nacional de Colombia, Bogota 111321, Colombia; E-Mail: millito59@hotmail.com

4 Department of Pathology, Fundación Hospital La Misericordia, Bogota 111411, Colombia

5 Department of Pediatrics, School of Medicine, Universidad Nacional de Colombia, Bogota 111321, Colombia; E-Mails: mikg376@gmail.com (M.G.-S.); carlos2671@gmail.com (C.E.R.-M.)

6 Department of Pediatric Pulmonology and Pediatric Critical Care Medicine, School of Medicine, Universidad El Bosque, Bogota 110111, Colombia

7 Research Unit, Military Hospital of Colombia, Bogota 111321, Colombia

8 Division of Pulmonary and Sleep Medicine, Children's National Medical Center, Washington, DC 20010, USA; E-Mail: gperez@childrensnational.org

9 Division of Pediatric Rheumatology, Allergy and Immunology, Pennsylvania State University College of Medicine, Hershey, PA 17033, USA; E-Mail: mgutierrez@hmc.psu.edu

10 Department of Integrative Systems Biology, Center for Genetic Medicine Research, Children's National Medical Center, George Washington University, Washington, DC 20010, USA

* Author to whom correspondence should be addressed; E-Mail: gnino@childrensnational.org; Tel.: +1-202-476-3194; Fax: +1-202-476-5864.

Received: 21 January 2014; in revised form: 29 March 2014 / Accepted: 3 April 2014 / Published: 7 May 2014

Abstract: Cytomegalovirus (CMV) is a prevalent pathogen in the immunocompromised host and invasive pneumonia is a feared complication of the virus in this population. In this pediatric case series we characterized CMV lung infection in 15 non-HIV infected 
children (median age 3 years; IQR 0.2-4.9 years), using current molecular and imaging diagnostic modalities, in combination with respiratory signs and symptoms. The most prominent clinical and laboratory findings included cough (100\%), hypoxemia (100\%), diffuse adventitious breath sounds (100\%) and increased respiratory effort (93\%). All patients had abnormal lung images characterized by ground glass opacity/consolidation in $80 \%$ of cases. CMV was detected in the lung either by CMV PCR in bronchoalveolar lavage ( $82 \%$ detection rate) or histology/immunohistochemistry in lung biopsy $(100 \%$ detection rate). CMV caused respiratory failure in $47 \%$ of children infected and the overall mortality rate was $13.3 \%$. Conclusion: CMV pneumonia is a potential lethal disease in non-HIV infected children that requires a high-index of suspicion. Common clinical and radiological patterns such as hypoxemia, diffuse adventitious lung sounds and ground-glass pulmonary opacities may allow early identification of CMV lung infection in the pediatric population, which may lead to prompt initiation of antiviral therapy and better clinical outcomes.

Keywords: CMV; lung; pneumonia; children; ground glass

\section{Introduction}

Cytomegalovirus (CMV) is one of the most important causes of opportunistic lung infection in the pediatric population [1]. Risk factors include human immunodeficiency virus (HIV) infection and immunocompromised status after bone marrow/stem cell transplantation (BMT/SCT) and solid organ transplantation [2]. Despite being a life threatening condition, if diagnosis is made in a timely manner, CMV pneumonia can potentially be cured with appropriate antiviral therapy (i.e., ganciclovir) [3]. Unfortunately, the clinical diagnosis of CMV lung infection is challenging in children and often requires a high-index of suspicion, especially in non-HIV infected cases and patients not severely immunocompromised [4].

Most of the literature about CMV lung infection in children focuses on the epidemiology of this condition in the pediatric immunocompromised population [1]. There are few reports that address the specific clinical and pulmonary imaging findings that may suggest this difficult diagnosis. Smith et al. in 1977 described the clinical respiratory findings and radiological appearance of CMV pulmonary infection in children using plain X-ray films [5]. This seminal work enhanced the awareness of the atypical course of CMV infection in the lungs, often described as "CMV pneumonitis" [5], which includes non-specific pulmonary findings such as interstitial patterns with increased bronchopulmonary markings and bronchiolar disease (diffuse air trapping) [5]. Interestingly, there has been a dramatic change in both the epidemiology of CMV infection and diagnostic techniques available for its diagnosis in children over the last 30 years [6]. Now-a-days we have an increasing population of non-HIV infected infants and children who have received different modalities of immunomodulators (i.e., steroids) for various conditions including BMT/SCT, solid-organ transplant and systemic autoimmune/inflammatory disorders that put them at risk for CMV lung infection [7]. In addition, better molecular diagnostics (i.e., CMV qualitative real-time 
PCR) as well as great progress in performing pediatric bronchoscopy and detailed lung imaging (i.e., high-resolution computerized tomography, HRCT) have improved our ability to detect CMV pulmonary infection, re-defining the concept of CMV pneumonia, which is now known to occur in children that are not severely immunocompromised [8].

The goal of this article is to characterize CMV lung infection in non-HIV infected children using current molecular and imaging diagnostic modalities, in combination with traditional respiratory signs and symptoms. To this end, we present a case series of 15 children with CMV lung infection focusing on their clinical presentation, radiological patterns, bronchoscopic findings and the molecular approaches used. Our results highlight that the diagnosis of CMV pneumonia in children is challenging but the common clinical and radiological patterns such as hypoxemia, diffuse adventitious lung sounds and ground-glass pulmonary opacities, can provide critical clues to allow early identification of CMV lung infection in the pediatric population.

\section{Experimental}

\subsection{Patients}

Fifteen cases of CMV pulmonary infection in non-HIV pediatric patients were studied. Neonates, infants and children (0-14 years) of both genders were included. All patients were seen at La Misericordia Children's Hospital in Bogota, Colombia, which is the largest University-based Pediatric Hospital in the country. Cases were collected between January 2010 and November 2013. Children with HIV infection were not included. No other medical conditions were considered as exclusion criteria. CMV lung infection cases were defined as positive pulmonary detection of CMV by polymerase chain reaction (PCR) or immunofluorescence (lung biopsy) and clinical evidence of lung involvement on exam and from radiographic images of the chest.

\subsection{Clinical Assessment}

Clinical variables were obtained by electronic medical record review (EMR) and included symptoms involving the respiratory system, such as cough and signs/symptoms indicative of increased breathing effort including tachypnea, retractions, nasal flaring and/or dyspnea. Other respiratory variables included abnormal breath sounds (rales, wheezing, rhonchus, etc.), pulsoximetry values and the need/duration for mechanical ventilation. General symptoms like fever and weight loss were also recorded. Duration of disease, time to diagnosis and length of hospitalization were included as well.

\subsection{Diagnostic Studies}

Clinical specimens used for the diagnosis of CMV lung infection included serum and bronchoalveolar lavage (BAL). CMV detection was performed using real time PCR methodology (Light Cycler CMV Quantitative Kit, Roche Diagnostics, Indianapolis, IN, USA), which is routinely conducted in our institution. CMV viral culture is not available and not routinely performed in our hospital. BAL was obtained via fiberoptic bronchoscopy from the right middle lobe, lingula or affected site according to ERS guidelines [9]. Neutrophilia in BAL was defined as 
$>3 \%$ based on prior studies and bronchoscopy guidelines for children [9]. In five patients, open lung biopsy was also performed and the lung tissue was submitted for histopathological analysis to identify typical CMV features (i.e., inclusion bodies) and for immunohistochemistry studies using CMV-specific antibodies. Chest radiographs and high resolution CT scans (HRCT) were performed in all patients using pediatric protocols and general anesthesia if appropriate. Radiological findings were analyzed using standardized Fleischner Society [10] terminology by pediatric radiologists and independently by at least two pediatric pulmonologists. Other tests performed included CMV serology (serum IgM by enzyme-linked immunosorbent assay) and follow-up studies for CMV infection including serum CMV PCR/viral load measurements as well as ongoing monitoring of hemogram and liver function testing for drug toxicity.

\section{Results}

The baseline characteristics of the study subjects are presented in Table 1. The median age of the 15 children with CMV lung infection was 3 years (IQR $0.2-4.9$ years.), which most likely reflects the population treated by the authors and not necessarily the group that is more likely to be affected by CMV. The majority were immunocompromised (13/15, 87\%) according to immune cell counts, immunoglobulin levels or concomitant use of immunosuppressant agents (steroids). Specifically, children on systemic steroids $(11 / 15,73 \%)$ had low values for age of either IgG (6/11, $54 \%$ ), absolute lymphocyte counts $(8 / 11,73 \%)$, absolute neutrophil counts $(1 / 11,9 \%)$, or total CD4 counts $(2 / 11,18 \%)$. Other important risk factors included malnutrition, hypogammaglobulinemia, hematologic malignancy and bone marrow/solid organ transplantation (Table 1). All transplant patients included had prior record of negative serology for CMV (donor and recipient).

Table 1. Baseline characteristics for pediatric subjects.

\begin{tabular}{cc}
\hline $\mathbf{N}$ & $\mathbf{1 5}$ \\
\hline Male, n (\%) & $8(53)$ \\
\hline Age (year), median (IQR) & $3(0.25-4.9)$ \\
\hline Comorbidity & $13 / 15(87)$ \\
Immunocompromised, n (\%) & $11(73)$ \\
Systemic steroid use, n (\%) & $8(53)$ \\
Hypogammaglobulinemia & $6(40)$ \\
Malnutrition, n (\%) & $3(20)$ \\
Acute leukemia, n (\%) & $2(13)$ \\
Autoimmune disease, n (\%) & $2(13)$ \\
Allogeneic bone marrow/stem cell transplant, n (\%) & $1(6)$ \\
Renal transplant, n (\%)
\end{tabular}

$I Q R$, interquartile range.

\subsection{Clinical Characterization of CMV Lung Infection}

Clinical presentation of CMV lung infection was variable among pediatric subjects (Table 2). The median time of symptom onset was 14 days (IQR 3-20 days) with a median time to diagnosis of 26 days (IQR 11-37 days). All subjects presented with cough and 93\% had increased respiratory effort/dyspnea as reported by the parent and/or patient. Other non-specific symptoms included fever and weight loss, which were present in $10(67 \%)$ and $8(53 \%)$ subjects respectively. The two 
most prominent abnormal laboratory findings were anemia (53\%) and abnormal liver function (47\%), which has been previously reported as a hallmark of acute CMV infection [11]. Other common hematologic abnormalities included thrombocytopenia (40\%), leukocytosis (40\%) and leukopenia (27\%).

The clinical respiratory assessment was abnormal in all children with CMV pulmonary infection (Table 3). Physical examination findings included diffuse abnormal breath sounds (100\% of patients) including wheezing $(80 \%)$, rales $(73 \%)$ and rhonchus (67\%). All subjects showed hypoxemia at presentation and $47 \%$ required mechanical ventilation for respiratory failure. The median duration of mechanical ventilation was eight days (IQR 7-26 days). Overall mortality rate was $13 \%(n=2)$. One subject died due to severe CMV infection and one due to massive central nervous system bleeding, secondary to severe thrombocytopenia associated with systemic lupus erythematosus.

Table 2. Clinical presentation of Cytomegalovirus (CMV) lung infection in children.

\begin{tabular}{cc}
\hline Duration & \\
\hline Onset of symptoms (days), median (IQR) & $14(3-20)$ \\
Time to diagnosis (days), median (IQR) & $26(11-37)$ \\
\hline Presenting symptoms & $15(100)$ \\
Cough, n (\%) & $14(93)$ \\
Increased breathing effort, n (\%) & $10(67)$ \\
Fever, n (\%) & $8(53)$ \\
\hline Weight loss, n (\%) & $8(53)$ \\
\hline Laboratory findings & $6(40)$ \\
Anemia, n (\%) & $6(40)$ \\
Thrombocytopenia, n (\%) & $4(27)$ \\
Leukocytosis, n (\%) & $7(47)$ \\
Leukocytopenia, n (\%) &
\end{tabular}
$I Q R$, interquartile range.

Table 3. Respiratory Manifestations of CMV lung infection in children.

\begin{tabular}{cc}
\hline Clinical variables & \\
\hline Abnormal lung sounds, n (\%) & $15(100)$ \\
Wheezing, n (\%) & $12(80)$ \\
Rales, n (\%) & $11(73)$ \\
Rhonchus, n (\%) & $10(67)$ \\
Hypoxemia, n (\%) & $15(100)$ \\
Mechanical ventilation (MV), n (\%) & $7(47)$ \\
Days of MV, median (IQR) & $8(7-26)$ \\
Death, n (\%) & $2(13)$ \\
\hline Radiological variables & $15(100)$ \\
\hline Abnormal lung images, n (\%) & $12(80)$ \\
Ground-glass opacity, n (\%) & $12(80)$ \\
Consolidation, n (\%) & $6(40)$ \\
Atelectasis, n (\%) & $5(33)$ \\
Air trapping, n (\%) & $3(20)$ \\
Nodular pattern, n (\%) & $1(6)$ \\
Reticular pattern, n (\%) & $1(6)$ \\
Tree-in-bud pattern, n (\%)
\end{tabular}




\subsection{Radiological Findings in CMV Lung Infection}

All patients had abnormal lung images. The most prominent radiological findings on plain chest radiographs and chest CT were consolidation and ground-glass opacity (80\%) (Figures 1-5), indicating partial filling of alveolar air spaces, interstitial thickening and/or partial collapse of the alveoli during CMV pulmonary infection [10]. The result of these alveolar lesions is lung haziness in chest-X-ray (CXR) and ground-glass opacity on the CT scan $[10,12]$. In our pediatric case series, ground-glass opacity and consolidations were diffuse (Figures 1-5) and co-existed in eight patients (53\%). In cases where CXR showed minimal abnormalities, the use of high-resolution CT scan (HRCT) enhanced the ability to see ground-glass opacity and consolidations (Figure 4). Atelectasis was present in $40 \%$ of cases and air trapping in 33\%. Other radiological lung patterns were rare and included nodular (20\%), reticular (6\%) and tree-in-bud pattern $(6 \%)$ (Table 3$)$.

Figure 1. Cytomegalovirus (CMV) lung infection after steroid therapy. Three year old girl treated with systemic corticosteroid for autoimmune lymphoproliferative syndrome (ALPS). Chest-X-ray (CXR) shows diffuse haziness more prominent in the left base (A), which correspond to ground-glass images in CT scan (B). H\&E staining revealed monocyte infiltration with cytomegalic changes in lung biopsy (black squares in panel C). Immunohistochemical detection of CMV (brown staining in D) showed typical CMV inclusion bodies (squares). Slides shown with 100× magnification.

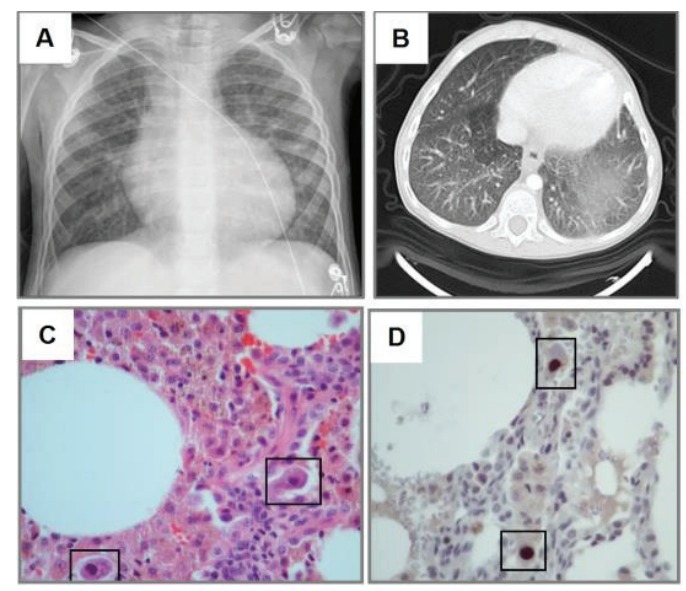

Figure 2. Ground-glass pattern in CMV lung infection after stem cell transplant (SCT). Four year old boy with acute lymphoid leukemia presenting with hypoxemia and bilateral rales after allogeneic SCT. Diffuse haziness is seen in CXR (A), and CT scan (B). There are interspersed airway lumen marks known as "dark bronchus" signs indicating ground-glass pattern (arrows in $\mathbf{B}$ ).
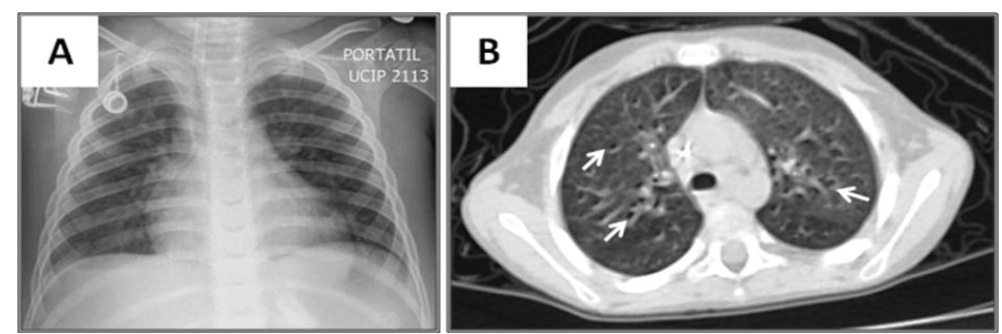
Figure 3. Ground-glass/Consolidation pattern in neonatal CMV lung infection. One month old term baby with lung haziness in CXR (A). CT scan revealed diffuse ground-glass and consolidations in both lung bases (B). Cytomegalovirus polymerase chain reaction) (CMV PCR) was $(+)$ in serum but $(-)$ in bronchoalveolar lavage. Lung biopsy revealed dense monocyte infiltration with cytomegalic changes (black squares in panel C). Immunohistochemical detection of CMV in the lung (brown staining in D) revealed characteristic inclusion bodies (arrows). Slides shown with 40× magnification.

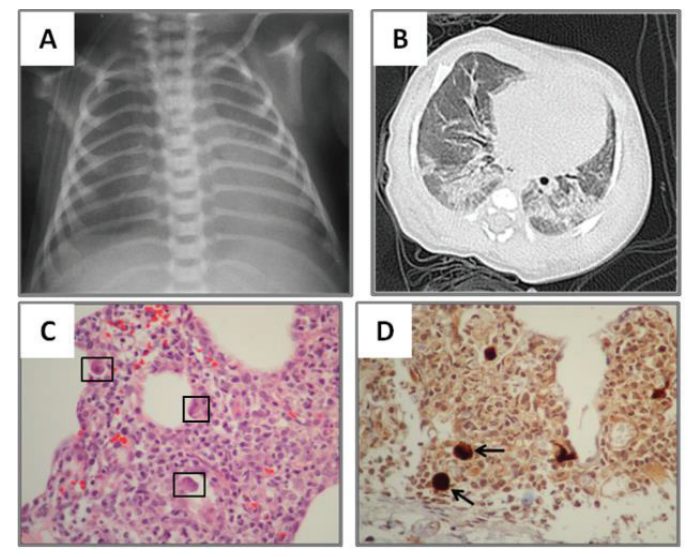

Figure 4. Ground-glass pattern in CMV lung infection detected with high resolution CT scan (HRCT). Four year old boy with hypoxemia and diffuse rales/wheezing after bone marrow transplant. Plain CXR revealed non-specific mild increased lung markings. HRCT identified subtle ground-glass pattern in both bases. CMV diagnosis was confirmed by $(+)$ PCR in bronchoalveolar lavage.

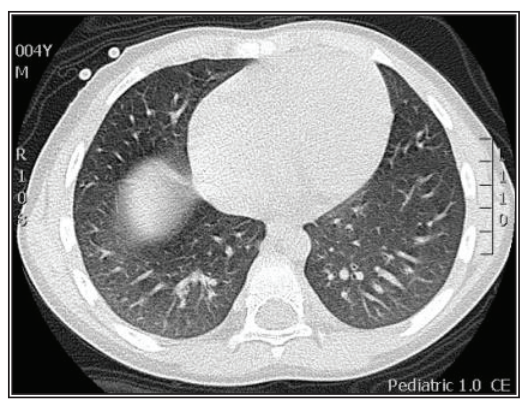

Figure 5. Consolidation pattern in CMV lung infection. Fourteen year old boy with history of renal transplant. A retrocardial consolidation is seen in CT scan along with scattered opacities with lower density resembling ground glass pattern (arrows). Bronchoalveolar lavage of the left lower lobe revealed a (+) PCR for CMV.

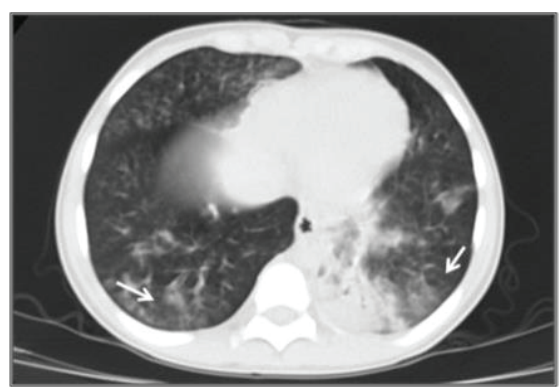




\subsection{Diagnostic and Treatment Approaches of CMV Lung Infection in Children}

The diagnostic approaches included a combination of CMV IgM serology, CMV serum PCR/viral load, bronchoscopy with bronchoalveolar lavage (BAL) with quantitative CMV PCR and lung biopsy (Table 4). The most common diagnostic modalities used were CMV serum $\mathrm{PCR} /$ viral load (14/15), which yielded a positive result in $50 \%$ of cases with a median CMV viral load of 268 copies/ $\mu$ L (IQR 20-20,000 copies/ $\mu \mathrm{L}$ ), and BAL (12/15) which gave positive CMV PCR in $82 \%$ of cases (Table 4). Most children with CMV lung infection had neutrophilia in BAL cellularity (92\%) (Table 4). Lung biopsy was performed in five subjects and CMV was confirmed by histopathology and immunohistochemical detection (Figures 1, 3 and 6). A number of patients (14/15) were treated with IV ganciclovir during 14 to 21 days at dose of ( $5 \mathrm{mg} / \mathrm{kg}$ every $12 \mathrm{~h}$ ), which is the standard CMV treatment in our institution. Patients were followed for an average period of 2-3 weeks after therapy. One patient was not treated because of full resolution of CMV infection before starting antiviral therapy. Four subjects also received oral valganciclovir treatment after IV ganciclovir. Antiviral therapy was effective in 13/14 patients (one patient died due to severe CMV infection) and no significant side-effects were reported.

Table 4. Diagnostic and treatment approaches of CMV lung infection in children.

\begin{tabular}{cc}
\hline Intervention & \\
\hline Bronchoalveolar lavage (BAL), $\mathbf{n}(\%)$ & $\mathbf{1 2}(\mathbf{8 0})$ \\
\hline CMV PCR (+) in BAL, $\mathbf{n}(\%)$ & $9 / 11(82)$ \\
Neutrophilia in BAL, $(\%)$ & $11 / 12(92)$ \\
\hline Lung biopsy, $\mathbf{n}(\mathbf{\%})$ & $\mathbf{5 ( 3 3 )}$ \\
\hline Biopsy confirmed CMV, $\mathbf{n}(\%)$ & $5 / 5(100)$ \\
\hline CMV serum PCR viral load, $\mathbf{n}(\%)$ & $\mathbf{1 4 / 1 5 ( 9 3 )}$ \\
\hline CMV PCR viral load (+) in serum & $7 / 14(50)$ \\
CMV copies/ $\mu \mathrm{L}$, median (IQR) & $268(20-20,000)$ \\
\hline CMV serology, $\mathbf{n}(\%)$ & $\mathbf{5 / 1 5 ( 3 3 )}$ \\
\hline CMV IgM (+) in serum & $4 / 5(80)$ \\
\hline CMV treatment, $\mathbf{n}(\%)$ & $\mathbf{1 4 / 1 5 ( 9 3 )}$ \\
\hline Ganciclovir, n (\%) & $14 / 14(100)$ \\
Valganciclovir, n (\%) & $4 / 14$ \\
\hline$I Q R$, interquartile range; $P C R$, polymerase chain reaction.
\end{tabular}

Figure 6. Cytopathic changes in CMV pulmonary infection after steroid therapy. Thirteen year old boy treated with systemic corticosteroid for bronchiolitis obliterans and severe asthma presenting with acute hypoxemia and diffuse rales/wheezing. Lung biopsy revealed diffuse cytopathic changes in pneumocytes infected by CMV (squares in $\mathbf{A}$ and magnified image in $\mathbf{B}$ ) including cellular enlargement and abnormal nucleus. Figures show H\&E staining with magnification $40 \times(A)$ and $100 \times(B)$.
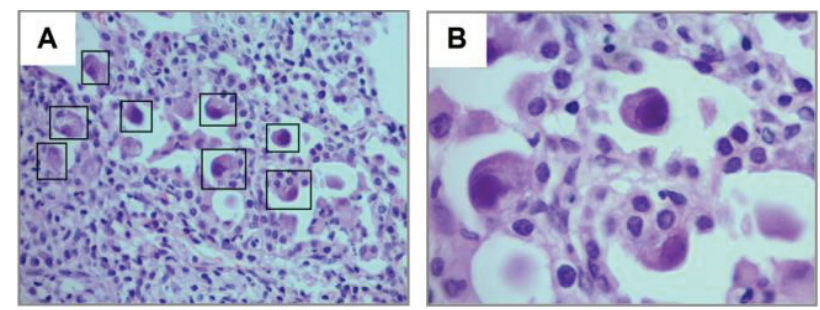


\section{Discussion}

CMV is a herpes virus that can produce life-threatening pulmonary infections in immunocompromised hosts [4]. Although effective antiviral therapy for CMV is available [11,13], timely diagnosis remains a major challenge for this condition, particularly in the pediatric population where CMV often presents with atypical patterns of lung infection [5,14]. The diagnosis of CMV pneumonia can be even more difficult in situations when there is no high-index of suspicion, for instance, in patients that do not have an underlying immunodeficiency syndrome (i.e., HIV-infected patients). Indeed, a detailed clinical characterization of CMV lung infection in this age group is critical to select patients that may warrant invasive procedures to obtain lung samples (i.e., pediatric bronchoscopy or open lung biopsy) and may benefit from prompt CMV therapy before confirmatory testing is available. Given the paucity of data about the clinical features of CMV pulmonary infection in children, this paper aims to fill this gap in the literature detailing the clinical and radiological features of this condition in a case series $(n=15)$ of non-HIV infected pediatric patients with pulmonary CMV infection. Our data illustrates that despite significant variability in the clinical presentation, there are specific features consistently present in pediatric CMV pulmonary infection, including hypoxemia, diffuse adventitious sounds and ground-glass consolidation pattern in lung CT scan, which together may offer crucial clues in the diagnosis of this condition in children.

As previously reported in the adult literature, we found that the majority of children that developed CMV pneumonia were immunocompromised $(87 \%)$ and $73 \%$ of patients were on systemic steroid therapy for different conditions including asthma, systemic lupus erythematous, leukemia treatment, renal transplant and allogeneic bone marrow/stem cell transplant. In addition, $8 / 15$ of the subjects (53\%) had hypogammaglobulinemia, which was mostly secondary to steroid treatment and/or malnutrition in our case series. These risk factors have been previously reported in the literature [15], and reflect the need to have proper humoral and cell-mediated immunity to clear CMV infection in the lungs $[3,16,17]$. During the study period we did not identify children with HIV and pulmonary CMV in our institution. This may reflect the population treated by the authors and not the overall trend of CMV infections in pediatric HIV. In this regard, it is important to emphasize that children with HIV may have different patterns of CMV lung disease. For instance, HIV itself is often associated with non-malignant lymphocytic infiltrative disorders, including nonspecific interstitial pneumonitis and lymphocytic interstitial pneumonitis (LIP) [18], which could potentially change the radiological appearance and the clinical manifestations of opportunistic lung infections like CMV. In addition, HIV patients might have more extra-pulmonary manifestations of CMV (retinitis and hepatitis) as well as other opportunistic lung pathogens such as Pneumocystis Jirovecci [19] that may lead to more severe disease and worse prognosis.

In terms of the clinical presentation and respiratory compromise, the onset of symptoms had a mean time of 14 days however there was considerable variation ranging from three days in a patient that required early mechanical ventilatory support, and up to three weeks in one child with nonspecific symptoms, a feature that has previously labeled CMV as an unpredictable respiratory infection [5]. Overall there was also great heterogeneity in the severity of the respiratory compromise. While one patient had self-limited clinical course that did not required therapy with ganciclovir, 
another had severe CMV infection and died despite antiviral therapy. In contrast, there was a consistent homogenous pattern of initial respiratory symptoms/signs that included a combination of cough, hypoxemia, increased breathing effort (i.e., retractions or dyspnea) and diffuse abnormalities in lung auscultation, which were present in virtually all cases (Table 3). The diffuse adventitious lung sounds identified were described as either wheezing, rales and/or rhonchus, which suggests variable degrees of involvement of lung parenchyma and conductive airways during CMV infection, compatible with what is generally described in CMV lung pathology [20]. In association with respiratory symptoms, about half of the patients had constitutional manifestations (fever or weight loss) and accompanying laboratory abnormalities that included anemia (53\%), thrombocytopenia (40\%), leukocytosis (40\%), leukopenia (27\%) and elevated liver enzymes (47\%). These abnormal laboratory findings are in overall agreement with prior reports of CMV lung infection in children [11,21].

One of the most important findings of our study was the current difficulty/delay in establishing the diagnosis of pediatric CMV lung infection. There was a median time to diagnosis of 26 days (IQR 11-37 days), which could be explained by the low clinical suspicion in the initial management of these non-HIV infected neonates and children. Indeed, relatively prompt diagnosis was obtained in those individuals with BMT, SMC and bone marrow transplantation (Figures 2, 4 and 5), which are well-known risk factors for CMV and therefore bronchoscopy/BAL was performed early. On the other hand individuals without underlying immunodeficiency (i.e., steroid-dependent asthma; Figure 6) or malnutrition were subject to longer time to diagnosis. Another factor that contributed to CMV diagnosis delay was the turnover time for the molecular studies. The CMV molecular diagnostic approach was performed using several modalities, including CMV viral load in peripheral blood, CMV qualitative PCR amplification in bronchoalveolar lavage (BAL) fluid and IgG serology for CMV. Prior reports have been found in which measurements of CMV viral DNA done in samples of BAL or sputum are better to make a diagnosis of CMV pneumonia, compared with the gold standard demonstration of cytomegalic inclusions in lung tissue [22]. Honda, J., et al. studied $363 \mathrm{CMV}$ adult patients with 882 samples of sputum, BAL, peripheral blood and urine, showing a positive predictive value (PPV) and negative predictive value (NPV) of $100 \%$ and $98.8 \%$ for BAL samples, $95.5 \%$ and $99.7 \%$ for sputum samples; and a sensitivity $90.9 \%$ and specificity $100 \%$ for BAL and $95.5 \%$ and $99.7 \%$ for sputum [22]. In our pediatric case series, CMV PCR in BAL was obtained in $11 / 15$ and was positive in $82 \%$ of the cases $(9 / 11)$; which may reflect the technical difficulties of obtaining a proper BAL specimen in the pediatric population, particularly in neonates [9]. Interestingly, most children with CMV lung infection had neutrophilia instead of lymphocytosis in BAL cellularity, which has been described in the adult literature [7,23]. The later may be attributed to bacterial superinfection, immunosuppression (affecting lymphocyte function and proliferation) or differences in the airway immune response to CMV in the pediatric population. In addition to bronchoscopy, four patients also underwent lung biopsy, two of these had PCR negative for CMV in BAL and the other two had a PCR positive in BAL but biopsy was performed due to additional concerns about other pulmonary infectious/inflammatory processes (i.e., persistent hypoxemia and alveolar hemorrhage). There was one patient who did not undergo a flexible bronchoscopy with BAL before open lung biopsy. In all five cases, lung biopsy confirmed the diagnosis of CMV, demonstrating cytopathic changes (Figure 6) and positive immunohistochemistry (Figures 1 and 3). Additional pathological findings in lung biopsies were 
diffuse monocytic infiltration (Figures 1,3 and 6) alveolar hemorrhage and pulmonary vascular disease in a patient with history of hemosiderosis and pulmonary hypertension. Because not all patients had the same CMV testing done (i.e., viral load) we could not do correlations of laboratory values with clinical parameters or the severity of CMV lung infection.

The initial imaging diagnostic approach consisted of chest radiography and later chest CT to further characterize the parenchymal involvement and to help in the decision making for further testing such as flexible bronchoscopy and/or lung biopsy. One hundred percent of patients had abnormalities on radiological exams; the most frequent findings were consolidation, usually compromising the dependent lung regions (bi-basal consolidation; Figure 3) and ground-glass opacities caused by the partial displacement of air due to filling of alveolar spaces, interstitial thickening and/or partial collapse of alveoli leading to enhanced small airway lumen marks, which is a radiological sign known as "dark bronchus" [10] (Figure 2). The ground glass/consolidation pattern has been previously identified in adults with CMV infection [24,25]. These radiological findings underlie the clinical presentation of CMV lung infection in our pediatric series that included increased respiratory effort, abnormal breath sounds and hypoxemia secondary to diminished alveolo-capillar gas diffusion and abnormal ventilator (V)/perfusion (Q) match in the lungs $[25,26]$. Importantly, CT scan was superior to CXR in detailing the radiological pattern of CMV lung infection in our pediatric case series. This is in agreement with Smith et al. who previously reported children with CMV pulmonary disease having minimal non-specific abnormalities in CXR [5]. In this context, it is noteworthy to mention that newer CT scan lung modalities (high-resolution) are now being proposed to diagnose different pediatric pulmonary conditions and thus avoid open lung biopsies [27]. For instance, some types of children's interstitial lung disease (chILD) such as neuroendocrine cell hyperplasia in infancy (NEHI), have specific patterns of disease in CT scan that may be sufficient to make a diagnosis when combined with a specific set of clinical features [27]. Based on our case series, we propose a group of clinical criteria (Table 5) based on risk factors (immunosuppression), symptoms/signs and lung imaging that when present must raise concern for CMV pulmonary infection in children and should prompt further confirmatory investigation (i.e., bronchoscopy) or empiric therapy depending on the clinical situation.

Table 5. Key features of CMV lung infection in non-HIV infected children.

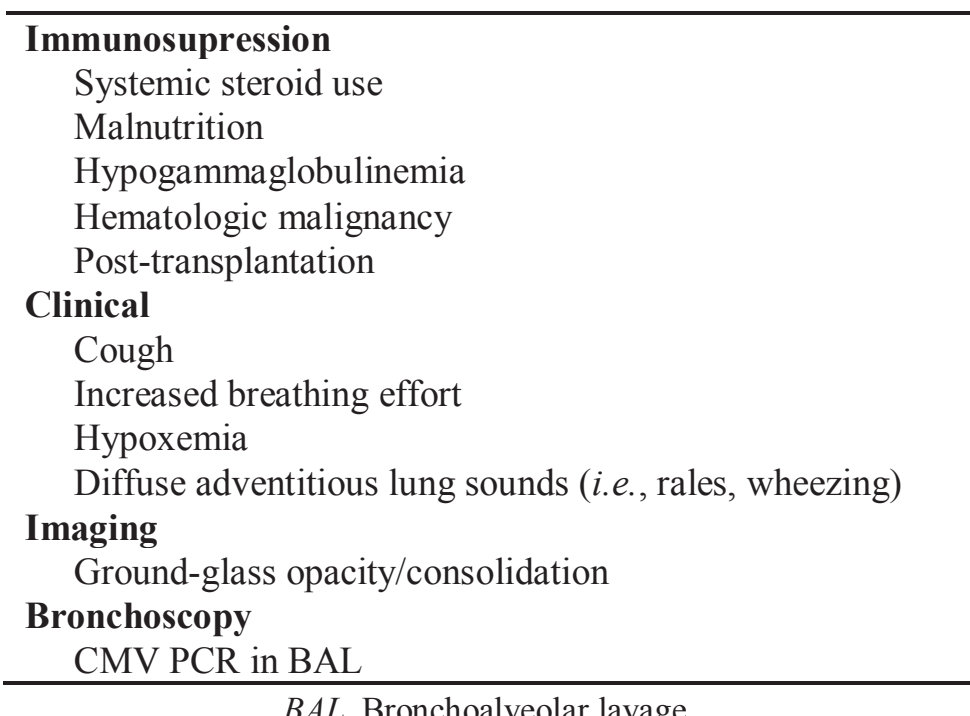

$B A L$, Bronchoalveolar lavage. 


\section{Conclusions}

In summary, CMV infection of the lung is a prevalent condition in the immunocompromised host with a variable clinical presentation. Clinical clues that should prompt suspicion of CMV pneumonia in this population are the presence of respiratory symptoms and abnormal lung exam. The initial diagnostic approach should include a chest- $X$ ray which can shows signs consistent with alveolar air displacement, however these changes can be subtle and easily missed on interpretation, thus making a chest $\mathrm{CT}$ a better radiological tool to evaluation the lung involvement in the immunocompromised host. The best test to confirm the diagnosis is a CMV PCR performed in BAL, bronchoscopy. The prompt identification of CMV lung infection in children without underlying immunodeficiency syndrome (i.e., non-HIV infected) requires a high-index of suspicion based on common respiratory sign/symptoms/images (Table 5) and is crucial to institute early antiviral therapy, which can significantly impact the clinical outcome of neonates and children with this potentially life-threatening infection.

\section{Acknowledgments}

We thank German Camacho-Moreno (Pediatric Infectious Disease, La Misericordia Children's Hospital, Bogota, Colombia) and Paulina Ojeda (Pathology, Santa Clara Hospital, Bogota, Colombia) for their careful review and valuable and insightful suggestions.

This work was supported in part by NIH Grants HL090020 and HD001399 (GN).

\section{Author Contributions}

Sonia M. Restrepo-Gualteros and Gustavo Nino defined the research theme. Sonia M. Restrepo-Gualteros, Lina E. Jaramillo-Barberi, Monica Gonzalez-Santos and Gustavo Nino collected and analyzed the data. Sonia M. Restrepo-Gualteros, Maria J. Gutierrez, Geovanny F. Perez, Carlos E. Rodriguez-Martinez and Gustavo Nino discussed analyses, interpretation and presentation of results and wrote the paper. All authors have contributed to, reviewed and approved the manuscript.

\section{Conflicts of Interest}

The authors declare no conflict of interest.

\section{References and Notes}

1. Manicklal, S.; Emery, V.C.; Lazzarotto, T.; Boppana, S.B.; Gupta, R.K. The "silent" global burden of congenital citomegalovirus. Clin. Microbiol. Rev. 2013, 26, 86-102.

2. Russel, M.; Palmer, A.; Michaels, M. Citomegalovirus infection in pediatric inmunocompromised hosts. Infect. Disord. Drug Targets 2011, 11, 437-448.

3. Gandhi, M.; Khanna, R. Human cytomegalovirus: Clinical aspects, immune regulation, and emerging treatments. Lancet Infect. Dis. 2004, 4, 725-738.

4. Radigan, K.; Wunderink, R. Epidemic viral pneumonia and other emerging pathogens. Clin. Chest Med. 2011, 32, 451-467. 
5. Smith, S.D.; Cho, C.T.; Brahmacupta, N.; Lenahan, M.F. Pulmonary involvement with cytomegalovirus infections in children. Arch. Dis. Child. 1977, 52, 441-446.

6. Zhou, W.; Lin, F.; Teng, L.; Li, H.; Hou, J.; Tong, R.; Zheng, C.; Lou, Y.; Tan, W. Prevalence of herpes and respiratory viruses in induced sputum among hospitalized children with non typical bacterial community-acquired pneumonia. PLoS One 2013, 8, e79477.

7. Jouneau, S.; Poineuf, J.S.; Minjolle, S.; Tattevin, P.; Uhel, F.; Kerjouan, M.; le Hô, H.; Desrues, B. Wich patients should be tested for viruses on bronchoalveolar lavage fluid? Eur. J. Clin. Microbiol. Infect. Dis. 2013, 32, 671-677.

8. Cunha, B.A. Cytomegalovirus pneumonia: Community-acquired pneumonia in immunocompetent hosts. Infect. Dis. Clin. North Am. 2010, 24, 147-158.

9. De Blic, J.; Midulla, F.; Barbato, A.; Clement, A.; Dab, I.; Eber, E.; Green, C.; Grigg, J.; Kotecha, S.; Kurland, G.; et al. Bronchoalveolar lavage in children. ERS Task Force on bronchoalveolar lavage in children. European Respiratory Society. Eur. Respir. J. 2000, 15, 217-231.

10. Hansel, D.M.; Bankier, A.A.; MacMahon, H.; McLoud, T.C.; Müller, N.L.; Remy, J. Fleischner society: Glossary of terms for thoracic imaging. Radiology 2008, 246, 697-722.

11. Doan, T.; Phung, T.T.; Pham, H.V.; Pham, S.H.; Nguyen, L.T. Effect of ganciclovir for the treatment of severe cytomegalovirus-associated pneumonia in children without a specific immunocompromised state. BMC Infect. Dis. 2013, 13, e424.

12. Jogeesvaran, K.H.; Owens, C. Chronic diseases of lung parenchyma in children: The role of imaging. Pediatr. Radiol. 2010, 40, 850-858.

13. Alarcon, A.A.; Baquero-Artigao, F.; Grupo de estudio de la infección por citomegalovirus de la Sociedad Española de Infectología Pediátrica. Review and guidelines on the prevention, diagnosis and treatment of post-natal cytomegalovirus infection. An. Pediatr. 2011, 74, doi:10.1016/j.anpedi.2010.05.024.

14. Escribano, A.; Chilet, M.; Clari, M.Á.; Lucas, R.; Costa, E.; Bravo, D.; Muñoz-Cobo, B.; Borrás, R.; Navarro, D. Frequent detection of cytomegalovirus (CMV) DNA in the lower respiratory tract in $\mathrm{CMV}$-seropositive pediatric patients with underlying chronic bronchopulmonary diseases lacking canonical immunosuppression. J. Med. Virol. 2013, 85, 888-892.

15. Khan, N. The immunological burden of human cytomegalovirus infection. Arch. Immunol. Ther. Exp. 2007, 55, 299-308.

16. Lidehäll, A.K.; Engman, M.L.; Sund, F.; Malm, G.; Lewensohn-Fuchs, I.; Ewald, U.; Tötterman, T.H.; Karltorp, E.; Korsgren, O.; Eriksson, B.M. Cytomegalovirus-specific CD4 and CD8 T cell responses in infants and children. Scand. J. Immunol. 2013, 77, 135-143.

17. La Rosa, C.; Diamond, D.J. The immune response to human CMV. Future Virol. 2012, 7, 279-293.

18. Corrin, B.; Nicholson, A.G. Pathology of the Lungs, 2nd ed.; Elsevier: London, UK, 2006; Chapter 5, pp. 159-160.

19. Griffiths, M.H.; Miller, R.F.; Semple, S.J. Interstitial pneumonitis in patients infected with the human immunodeficiency virus. Thorax 1995, 50, 1141-1146. 
20. Jeena, P.M.; Coovadia, H.M.; Chrystal, V. Pneumocystis carinii and cytomegalovirus infections in severely ill, HIV-infected African infants. Ann. Trop. Paediatr. 1996, 16, 361-368.

21. Avila-Agüero, M.L.; Paris, M.M.; Alfaro, W.; Avila-Agüero, C.R.; Faingezicht, I. Ganciclovir therapy in cytomegalovirus (CMV) infection in immunocompetent pediatric patients. Int. J. Infect. Dis. 2003, 7, 278-281.

22. Honda, J.; Yonemitsu, J.; Kitajima, H.; Yosida, N.; Fumirori, T.; Oizumi, K. Clinical utility of capillary polymerase chain reaction for diagnosis of Cyto-megalovirus pneumonia. Scand. J. Infect. Dis. 2001, 33, 702-705.

23. Ljungman, P. Cytomegalovirus pneumonia: Presentation, diagnosis, and treatment. Semin. Respir. Infect. 1995, 10, 209-215.

24. Moon, J.H.; Kim, E.A.; Lee, K.S.; Kim, T.S.; Jung, K.J.; Song, J.H. Cytomegalovirus pneumonia: High-resolution CT findings in ten non-AIDS immunocompromised patients. Korean J. Radiol. 2000, 1, 73-78.

25. West, B.W. Respiratory Physiology the Essentials: Ventilation-Perfusion Relationships, 9th ed.; Lippincott Williams \& Wilkins: Baltimore, MD, USA, 2012; pp. 56-76.

26. Agrawal, A.; Agrawal, A.; Bansal, V.; Pandit, M. A systematic approach to interpretation of heterogeneous lung attenuation on computed tomography of the chest. Lung India 2013, 30, 327-334.

27. Popler, J.; Gower, W.A.; Mogayzel, P.J., Jr.; Nogee, L.M.; Langston, C.; Wilson, A.C.; Hay, T.C.; Deterding, R.R. Familial neuroendocrine cell hyperplasia of infancy. Pediatr. Pulmonol. 2010, $45,749-755$. 
Reprinted from Viruses. Cite as: Deere, J.D.; Barry, P.A. Using the Nonhuman Primate Model of HCMV to Guide Vaccine Development. Viruses 2014, 6, 1483-1501.

Review

\title{
Using the Nonhuman Primate Model of HCMV to Guide Vaccine Development
}

\author{
Jesse D. Deere ${ }^{1}$ and Peter A. Barry ${ }^{2, *}$
}

1 Center for Comparative Medicine, University of California, Davis, Davis, CA 95616, USA; E-Mail: jddeere@ucdavis.edu

2 Center for Comparative Medicine, Department of Pathology and Laboratory Medicine, California National Primate Research Center, University of California, Davis, Davis, CA 95616, USA

* Author to whom correspondence should be addressed; E-Mail: pabarry@ucdavis.edu; Tel.: +1-530-752-6912.

Received: 7 February 2014; in revised form: 11 March 2014 / Accepted: 12 March 2014 /

Published: 27 March 2014

\begin{abstract}
The natural history of human cytomegalovirus (HCMV) is inextricably associated with mucosal surfaces. The vast preponderance of primary infections occur following mucosal exposure to infectious virions, and the high seroprevalence of HCMV throughout the world is due to long-term excretion of HCMV in bodily fluids from multiple mucosal sites. Accumulating evidence presents a model where the earliest virus-host interactions following infection dictate the long-term pattern of infection, alter innate immune responses that skew adaptive responses to enable persistence within an immune host, and are essential for reinfection of a host with prior immunity. HCMV has evolved a complex repertoire of viral functions fine-tuned to manipulate the immune environment both locally at the sites of infection and systemically within an infected host. Collectively, viral immune modulation represents a significant impediment for an HCMV vaccine. As HCMV can disseminate beyond mucosal surfaces to reinfect immune hosts, it may not matter whether prior immunity results from prior infection or immunization. A better understanding of the earliest virus-hosts interactions at mucosal surfaces may identify elements of the viral proteome that are especially susceptible to vaccine-mediated disruption and prevent challenge virus from disseminating to distal sites, particularly the maternal-fetal interface.
\end{abstract}

Keywords: cytomegalovirus; nonhuman primate; RhCMV; immune modulation; persistence; vaccine; reinfection 


\section{Introduction}

Herpesviridae are a family of ubiquitous pathogens with ancient origins, dating back 400 million years, and they have co-evolved with their radiating host species since the progenitor herpes virus species arose [1]. The extent of co-evolution is such that herpes simplex virus (HSV-1) sequences have been used to track human global migration out of Africa [2]. If evolutionary longevity and the percentage of infected hosts measure the success of a pathogen, this family is very successful. While the classification of Herpesviridae is based on virion structure, all herpes viruses share the phenotype of establishing a lifelong persistence within the infected and, usually, immune-competent host. Mechanisms of persistence remain an active area of research into herpes virus natural histories. While large gaps in our knowledge of persistence remain to be filled, it is becoming clear that there are common themes amongst all herpes viruses layered onto virus-specific functions that modulate host cell signaling, trafficking, activation, antigen presentation, and lifespan. Based on our current understanding, some viral immune modulation mechanisms can be sub-grouped by family (alpha-, beta-, or gamma-herpesvirinae) or can be species-specific. One unifying feature of all herpes viruses is the central role of epithelial cells in the infectious cycle in mediating the initial portal of entry during infection of an individual in addition to serving as the exit site for secretion into bodily fluid to maintain the virus within the larger population of susceptible hosts. In between these points of the HCMV infectious cycle, herpes viruses disseminate to family-specific cell types of persistence for the alpha-, beta-, or gammaherpesvirinae, including neurons, $\mathrm{CD} 34^{+}$and other cell types, and B cells, respectively. It is highly likely that the raison d'être for sites of persistence is to provide a privileged site for viral genomes, resistant to immune-mediated clearance, that can occasionally or subliminally reactivate to produce infectious virions that can traffic back to mucosal epithelial cells and be secreted in bodily fluids. Analysis of the central role of mucosal epithelial cells in the life cycle of human cytomegalovirus (HCMV) illustrates the complexities and the challenges confronting development of HCMV vaccines designed to prevent primary infection and, hopefully, reinfection of those with preexisting HCMV immunity.

\section{HCMV Natural History}

\subsection{HCMV Shedding in Bodily Fluids}

HCMV is the prototypical betaherpesvirus with a complex natural history, a large and incompletely defined genetic coding capacity, and a seemingly contradictory virus-host relationship. HCMV is ubiquitous throughout the world, and seroprevalence rates range from $\sim 50 \%$ in the U.S. to close to $100 \%$ in some developing countries [3]. Primary HCMV infection in immune-competent individuals is generally associated with either transient and mild clinical outcomes or inapparent seroconversion. Based on the reported rate of congenital infection $(\sim 0.7 \%)$, primary infections result almost exclusively following mucosal exposure to horizontally transmitted virions in bodily fluids. Despite mucosal epithelia serving as the entry point for the preponderance of HCMV infections, there is virtually nothing known about the virological and immunological cascade of events within the mucosa and submucosa. 
Like all herpes viruses, HCMV establishes a lifelong persistence characterized by the presence of cells harboring latent viral genomes that periodically reactivate to produce infectious virions. The frequency of shedding varies dependent in part on age, health, the particular bodily fluid, and physiologic status. In children under the age of five years infected congenitally or within the first years of birth, the frequency of HCMV in saliva and/or urine has been reported as high as $80 \%$ with rates declining as the children age (12\%-23\%) [4,5]. In healthy non-human immunodeficiency virus (HIV)-infected adults, cross-section surveys of HCMV excretion in cervical swabs, saliva, and/or urine indicate rates of detection between 7\%-24\%, with higher rates observed in the context of HIV coinfection (non-HAART treated) or sexually transmitted diseases [4,6-9]. Longitudinal studies of seroimmune healthy women demonstrate that $83 \%$ exhibited at least one positive urine sample with HCMV [8]. Rates of HCMV reactivation during pregnancy lead to the conclusion that HCMV is exquisitely suited for shedding in breast milk. Three studies have observed frequencies of HCMV detection in breast milk from $89 \%-100 \%$ [10-12]. While it is well established that HCMV can establish near complete latency in certain cell types (e.g., CD34 ${ }^{+}$myeloid progenitor cells), characterized by an extremely limited pattern of HCMV transcripts [13-18], long-term shedding in mucosal fluids is the norm during persistent infection. Even more pronounced than primary infection, persistent HCMV shedding is not associated with disease in those infected individuals with functional immune systems. As with the large gaps in our knowledge of initial virus-host interactions at mucosal surfaces during primary infection, the mechanistic basis for the frequency and magnitude of mucosal shedding are virtually unexplored for HCMV.

\subsection{HCMV Immune Responses}

The general absence of HCMV clinical sequelae associated with primary infection and the almost complete absence during persistent infection emphasize the protective nature of host antiviral immune responses. Primary HCMV infection stimulates robust immune responses, including broadly neutralizing antibodies to multiple envelope glycoproteins [19-21] and especially prominent antiviral $\mathrm{T}$ cell responses [22,23]. The protective efficacy of these immune responses is emphasized by the fact that declines in functional immunity, either by iatrogenic immunosuppression or immune deficiency, is associated with significantly increased risks of HCMV morbidity and mortality. Similarly, congenital HCMV infection is a leading infectious cause of hearing loss and developmental disorders in infants in the U.S. [21,24]. Primary HCMV infection in the mother puts the infant at greater risk of symptomatic disease [19]. Likewise, the immunological immaturity of fetuses portends increased risk of developmental deficits following congenital HCMV infection (reviewed in [25]). Due to the severe diseases that HCMV can cause in people with limited functional immunity, an HCMV vaccine has been a public health priority for the past several decades [24,26-30]. An overriding theme of HCMV vaccine development has been to recapitulate through vaccination the dominant immune responses generated during primary infection.

A potential limitation of this approach is that peripheral immune responses (e.g., neutralizing antibodies, antigen-specific $\mathrm{T}$ cells) constitute the vast majority of immune responses analyzed in infected individuals. While informative, limitation of immune responses to the periphery may result in failure to identify critical tissue-specific immune responses. In particular, mucosal immune responses in situ, including innate responses, to HCMV are notably understudied relative to 
peripheral $\mathrm{B}$ and $\mathrm{T}$ cell responses. A limited number of studies have detected very low but detectable binding and/or neutralizing antibody responses to HCMV in saliva following either natural infection and, importantly, immunization with live attenuated virus or recombinant $\mathrm{gB}$ subunit vaccination [31-33]. Saliva antibody responses, which are not detectable in all those with serum antibodies to $\mathrm{HCMV}$, include IgG, IgA, and sIgA. Neutralizing antibodies have differentially been associated with the $\operatorname{IgA}$ and $\operatorname{IgG}$ fractions.

Three features of these studies that may be relevant to vaccine design include the following. (1) Vaccination can lead to detectable increases in saliva antibodies [33]; (2) Saliva IgG titers are a fraction of serum antibody IgG titers but generally correlate with peripheral IgG [31,33]; (3) There is no apparent association between cessation of HCMV shedding in saliva of young HCMV-infected children and development of detectable neutralizing antibodies in saliva [32]. As mucosal surfaces represent the first line of defense against HCMV infection, it stands to reason that a more thorough understanding of the earliest host-pathogen interactions at mucosal surfaces is critical for the future development of vaccine strategies designed to disrupt the natural pattern of infection, persistence, and shedding. Enhancement of innate immune responses at the site of infection might tip the balance in favor of the host and allow for viral clearance or reduced viral shedding from mucosal surfaces later in the replication cycle.

\subsection{HCMV Persistence}

The preceding discussion about the mostly protective immune responses resulting from primary infection presents something of a conundrum about the quality of the antiviral immune responses. Like all herpes viruses, HCMV persists for the lifetime of immune-competent hosts, and the longitudinal shedding of virus in bodily fluids demonstrates that viral gene expression occurs in the presence of the very same immune responses that protect against viral sequelae. Simply put, HCMV stimulates immune responses that effectively limit infection and mostly protect the host from disease, yet are insufficient to clear persistent reservoirs of infected cells. The chronicity of antiviral immunity following primary infection strongly implies chronic viral gene expression at a level sufficient to maintain antibody and $\mathrm{T}$ cell responses [23]. There is no solid evidence that lifelong HCMV infection and gene expression result in overt HCMV pathology, which leads to the conclusion that HCMV has evolved a lifecycle to maximize its potential to excrete infectious virions that can disseminate to susceptible hosts. Beyond the potential for spreading virus horizontally and vertically, there are numerous reports in the last several years suggesting that chronic HCMV infection may be associated with increased risk of some age-related diseases. It should be stressed, however, that the linkages are statistical associations without conclusive proof of HCMV causality [24,34]. It may turn out that, as human lifespan increases well beyond historic levels, chronic HCMV infection may have adverse effects in the context of an aging immune system.

\subsection{HCMV Reinfection}

Another aspect of the restricted immune protection conferred by primary infection is the failure of preexisting immunity to fully protect from reinfection with a different strain of HCMV $[21,35,36]$. Multiple studies have documented that HCMV can reinfect individuals with prior HCMV 
immunity [8,21,37-43]. Congenital infection rates of $1 \%$ have been reported in populations with $100 \%$ seroprevalence of HCMV. The demonstration that seropositive women who give birth to a congenitally infected infant acquired new antigenic reactivity to HCMV antigens between pregnancies is strong evidence that prior immunity is incompletely protective against reinfection with antigenic HCMV variants. This is particularly remarkable because, in healthy long-term HCMV carriers, $\sim 10 \%$ of memory $\mathrm{T}$ cells are HCMV-specific, and neutralizing antibodies are generated against multiple viral glycoproteins including $\mathrm{gB}$ [23,44-48]. In another study, 30\% of seropositive women that were longitudinally evaluated developed novel HCMV antibody specificities, equivalent to an annual rate of HCMV reinfection of 10\% [49]. Studies of reinfection indicate a viral mechanism to surmount preexisting immune memory to initiate a new infection, leading to the clinical ramification that infection of an immune host is probably independent of whether prior immunity is from prior infection or prior vaccination. The reinfection studies also suggest that the true disease burden might be underestimated, particularly so since the reinfection studies to date are based on looking at an extremely limited number of epitope variants.

\subsection{HCMV Immune Modulation}

The ability of HCMV to persist in an immune-competent host and reinfect immune individuals is undoubtedly related to the extraordinary devotion of HCMV coding content to viral proteins that modulate host immune cell function. Based on the number of open reading frames that can be deleted without impairing replication in fibroblasts, it is likely that $\sim 50 \%$ of viral ORF are involved in an extensive repertoire of immune modulating functions [50,51]. Given the protracted evolution of herpes viruses, in general, and HCMV, in particular, it is highly likely that evolution of viral functions that modulate innate and adaptive immunity define the mechanistic basis for the phenotypic features that define HCMV as the virus that it is. Consistent with this interpretation, it is, perhaps, noteworthy that there have been no descriptions of immune escape variants arising during subclinical persistent infections, or fulminant outcomes associated with immunosuppression or immunodeficiency. In contrast, drug-resistant variants readily arise after initiation of antiHCMV chemotherapies [52], which, based on recent estimates of sequence variation occurring during HCMV infection, should come as no surprise [53]. One interpretation of these discordant responses to immune-mediated and antiviral-mediated selective pressures might be the result of the protracted evolution of herpes viruses in hosts possessing innate and adaptive immune systems. HCMV evolved the broad repertoire of immunomodulatory functions that presumably confers a selective advantage over genetic drift as a means to persist within a host and disseminate throughout the population. In contrast, anti-HCMV chemotherapies are very recent treatment modalities, and therefore, there are no apparent HCMV-encoded functions that can directly counteract the different anti-HCMV drugs in clinical use.

\subsection{Future Directions for HCMV Vaccine Development}

One of the earliest calls for development of an HCMV vaccine stemmed from the clinical recognition of the grave infectious threat that intrauterine HCMV represents to fetal growth and development [27]. Based on his 15-year retrospective analysis of congenital HCMV cases, 
Hanshaw concluded that, "... any thoughtful program aimed at prevention or treatment deserves consideration". Multiple lines of reasoning, focusing on (1) lifelong viral gene expression and shedding; (2) the magnitude of host cell immune responses required to prevent sequelae; and (3) reinfection of immune hosts, lead to the argument that HCMV is unlike any other virus for which vaccines have been clinically approved. Accordingly, "any thoughtful program aimed at prevention" of HCMV infection is likely to require adjuncts to the current paradigm of preventing attachment of HCMV virions to susceptible cells by gB-mediated neutralization. There are numerous challenges confronting HCMV vaccine development including a complex natural history, incompletely defined correlates of immune protection, and financial and logistical challenges in sufficiently powered clinical trials. Recent studies using the rhesus macaque model of HCMV demonstrate that infection of rhesus macaques with rhesus CMV (RhCMV) offers novel insight into vaccine strategies that have clinical relevance for development of HCMV vaccines. The remainder of this review will focus on the mechanisms of RhCMV infection, transmission, persistence, and reinfection and how the results of these studies inform new HCMV vaccine modalities.

\section{RhCMV Natural History}

\subsection{Endemic RhCMV Infectious Cycle in Rhesus Macaques (Macaca mulatta)}

Development of the RhCMV model of HCMV persistence and pathogenesis has benefitted by characterization of RhCMV natural history in large breeding cohorts to establish normative parameters of endemic infections to guide experimental studies. One unifying element of population studies is the central role of mucosal surfaces in the infectious cycle of RhCMV. Studies have demonstrated that the kinetics of RhCMV dissemination throughout mixed cohorts of uninfected and infected animals is a function of the persistent shedding of virus in bodily fluids of infected animals and the repeated mucosal exposure of uninfected animals to virus. RhCMV is endemic in breeding cohorts of rhesus macaques, as well as in native populations of wild macaques, and almost $100 \%$ of naive animals seroconvert by one year of age, well before the onset of sexual maturity around four years of age [54-57]. There are no known clinical sequelae associated with primary RhCMV infection in immune competent animals, which stands in contrast to the severe pathogenic outcomes following SIV immunodeficiency [58-60] or iatrogenic immunosuppression [56]. Seroconversion of previously naïve animals is often, but not always, contemporaneous with the detection by TaqMan real-time PCR of low quantities of RhCMV genomes in plasma $[61,62]$. The quantities of viral DNA detected in plasma are lower than those detected in saliva later in infection, consistent with hematogenous spread of progeny virions from local portals of entry to distal sites throughout the body. Like HCMV, RhCMV can be detected in multiple cell types throughout the body [58,60], and the virus efficiently seeds those anatomic sites where virus can be shed.

Recapitulating the virus-host relationship for HCMV, RhCMV persists, and chronic RhCMV gene expression is the norm during the life of the infected animal, despite the presence of neutralizing antibody and $\mathrm{T}$ cell responses [63-65]. Key sites of persistence include the salivary glands and genitourinary tract. Infected animals can persistently and asymptomatically shed virus in bodily fluids (e.g., saliva and urine), often in high quantities, for years after primary 
infection [66-69]. Multiple genetic variants have been detected in fluids of naturally infected animals [69-71], although there are no reports of deep sequencing to quantify the extent of sequence diversity. Because of persistent shedding in bodily fluids and the social dynamics of large macaque cohorts, RhCMV rapidly spreads through the cohort. In fact, RhCMV has an exceedingly high force of infection in naïve hosts. Two studies of mixed cohorts of infected and uninfected macaques demonstrate that the doubling rate of seroconversion of uninfected animals is five to nine weeks $[54,61]$.

There have been no reports to date of vertical transmission of RhCMV, possibly due to the universal seroprevalence of RhCMV in breeding age female macaques [56]. Consequently, the high seroprevalence of RhCMV in large cohort is the direct consequence of repeated mucosal exposure of uninfected animals to horizontally transmitted virions in bodily fluids of infected animals. As such, this aspect of RhCMV natural history reflects the challenges that confront HCMV vaccine trials, namely repeated close contact of vaccinated/uninfected women to those shedding potentially antigenically variant HCMV in bodily fluids. One implication from the studies of RhCMV in mixed populations is that there are at least two strategies to minimize the risk of primary infection: (1) vaccinate those at-risk for primary infection; and (2) vaccinate those who may become the source of horizontally transmitted virus to minimize the potential for shedding of virus in bodily fluids (such as young children). While detailed molecular and virological investigations of RhCMV infection following natural exposure have not been reported, experimental data regarding cellular tropism and the virally encoded genes that are essential in this process provide insights into parts of the viral proteome that may be especially susceptible to vaccine-mediated disruption.

\subsection{Early Virus-Host Interactions during Experimental Infection}

Early virus-host interactions within the mucosa following natural exposure to RhCMV have not yet been explored. However, several studies using experimental inoculation via subcutaneous (SC) delivery of virus have revealed a salient aspect about early virus-host interactions relevant to HCMV vaccine design. In particular, the acute virus-host interactions following SC inoculation determine the long-term patterns of RhCMV infection.

Several strains of RhCMV have been described in the literature, and strain-specific differences depend on the extent of fibroblast adaptation. RhCMV strain 68-1 is the prototypical strain that was isolated after co-culture of urine from a rhesus macaque on human fibroblasts $[66,67]$. Sequence analysis of the 68-1 genome after serial passage in human and/or rhesus fibroblasts revealed that the $\mathrm{UL} / \mathrm{b}$ ' region of the genome had undergone rearrangements and loss of coding capacity, in particular RhUL128, RhUL130, and three CXC chemokine-like ORF [69,72]. Based on precedence with the HCMV gH-based pentamer $[73,74]$, the loss of RhUL128 and RhUL130 results in severe attenuation of RhCMV tropism for epithelial and some endothelial cells in culture $[75,76]$. RhCMV strains UCD52 and UCD59 were also isolated initially on human and macaque fibroblasts, and subsequently passed exclusively on primary monkey kidney epithelial cells [61,77]. Sequence analyses of both UCD52 and UCD59 demonstrated that the UL/b' regions of both strains retain the full coding capacity of wild-type RhCMV (i.e., never passed in culture) [69], and both retain epithelial cell tropism in vitro. Of particular note for vaccine strategies, strain-specific differences in the $\mathrm{UL} / \mathrm{b}$ ' coding content result in distinct phenotypes of acute infection in vivo following SC inoculation. 
Skin biopsies of the SC inoculation site obtained seven days after infection with either UCD52 or UCD59 are noted for prominent neutrophilic infiltration with extensive infection of endothelial cells (CD31-positive), fibroblasts (vimentin-positive), and macrophages (CD68-positive) [60]. In contrast, biopsies from animals infected seven days prior with 68-1 were noted for a predominant mononuclear cell infiltrate, and infected cells were predominantly fibroblasts and a reduced infection frequency in macrophages was observed (compared to UCD52/UCD59). Notably, no endothelial cells infected with 68-1 were detected (infection of epithelial cells was not evaluated in this study). This study confirmed that the expanded tropism conferred by the $\mathrm{gH}$-anchored pentamer (in UCD52 and UCD59) observed in vitro is also observed in vivo. In addition, investigation of the earliest virus-hosts interactions highlights how RhCMV acutely alters local innate host responses, presumably due to the presence (UCD52/UCD59) or absence (68-1) of viral CXC chemokine-like ORF. As will be discussed below (Sections 3.3 and 3.4), differences in UL/b' coding capacity also result in distinct long-term parameters of RhCMV infection.

\subsection{Rapid Emergence of RhCMV Variants with Complete UL/b' Coding Capacity during Experimental Infection}

The strong positive selective pressures conferred by a full complement of UL/b' coding capacity was recently described in a serendipitous finding with another annotated strain of RhCMV [78]. RhCMV strain 180.92 was isolated from a simian immunodeficiency virus (SIV)-infected monkey and serially passed on human and monkey fibroblasts without plaque purification [79]. Sequence analysis of overlapping cosmid clones generated from cells infected with the 180.92 stock revealed a large rearrangement in UL/b' that resulted in the deletion of most of UL/b'-encoded ORF, compared to 68-1 and wild-type RhCMV, but left intact the RhUL128-131 coding regions. RhCMV 180.92 exhibits greater epithelial/endothelial cell tropism than 68-1, consistent with retention and expression of the complete $\mathrm{gH}$-anchored pentamer in 180.92, although the ability to infect epithelial/endothelial cells is reduced compared to a repaired version of 68-1 (BRh68-1.2) in which the RhUL128 and RhUL130 genes were engineered back into 68-1 [75]. Reduced growth of 180.92 in epithelial/endothelial cells compared to BRh68-1.2, is likely due to other UL/b' ORF distinct from RhUL128-131. Stocks of RhCMV 180.92 were prepared from bulk infected cell cultures, and plaque purification was never done during serial passage. PCR analysis of the 180.92 stock subsequently revealed the presence of a minor sequence variant comprising $<15 \%$ of the total RhCMV genomes within the 180.92 stock. PCR amplification and sequencing of molecular clones determined that the minor sequence variant was, in fact, a full-length RhCMV genome, presumably representing the remnant of the original unrearranged genome prior to fibroblast adaptation. Reexamination of tissues from these animals and other animals unexpectedly revealed that there was a rapid emergence of the full-length variant despite it being a minor constituent in the virus stock [78].

Six RhCMV uninfected macaques, including one that was SIV-infected, were inoculated intravenously with the RhCMV 180.92 stock, and longitudinal plasma, saliva, and urine samples were collected and analyzed for RhCMV. Using primer sets that distinguished the truncated and full-length genomic variants, it became apparent that the full-length variant rapidly became the dominant genomic form ( $>95 \%)$ in blood by three weeks post inoculation. Analysis of RhCMV in 
the tissues from the SIV/RhCMV coinfected animal similarly demonstrated high-level genome copy numbers of the full-length in tissues. In contrast, the truncated genomic variant exhibited significantly lower genome copy numbers in plasma, tissues, urine, and saliva. These data demonstrate that the truncated 180.92 variant is severely attenuated in vivo, despite the presence of the RhUL128-131 ORF. The results emphasize that additional UL/b' ORF are essential for optimal replication and dissemination of RhCMV in vivo, and accordingly, these particular viral proteins represent potential vaccine targets to minimize hematogenous spread to tissues throughout the body.

\subsection{Long-term Parameters of RhCMV Infection during Experimental Infection}

The preceding rationale about vaccine-mediated targeting those UL/b' ORF outside of RhUL128-131 can also be applied to RhUL128-131, based on differences in long-term infections of variants containing or lacking RhUL128-131. In addition to an apparent defect in cell tropism during acute infection (described above), 68-1 is significantly attenuated for shedding in bodily fluids [60,61]. Uninfected animals inoculated SC with UCD52 and UCD59 are noted for persistent detection of RhCMV in the saliva and urine of most animals ( $75 \%$ ) beginning six to eight weeks after inoculation [61]. The prolonged detection of RhCMV following experimental inoculation reflects the pattern of RhCMV shedding in animals naturally exposed to RhCMV [66-68]. In marked contrast, 68-1 DNA is either below the limit of PCR detection, or infrequently detected at low copy numbers in either bodily fluid [80-82]. All of these studies used PCR detection of RhCMV DNA as a surrogate for virus isolation to assess virus shedding. As a measure of the biological relevance of PCR detection, animals inoculated with UCD52 or UCD59 can transmit virus to uninfected cage-mates, whereas horizontal transmission of 68-1 has not been observed [61]. This finding is particularly relevant for vaccine development strategies aimed at reducing viral shedding as a means to control horizontal infection. Together, these observations suggest impaired dissemination and shedding of 68-1 in vivo and demonstrate the essential nature of mucosal tissues in CMV infection and the requirement for broad cellular tropism. It should be noted that RhCMV 68-1 is not replication impaired in vivo due to the absence of a functional gH-anchored pentamer and the CXC-chemokine-like ORF. The pattern of acute infection with 68-1 (described in Section 3.2) [60] demonstrates that 68-1 can utilize, presumably, gB-mediated entry into permissive cells, and 68-1 can disseminate throughout the body [83]. Moreover, studies using RhCMV 68-1 as a vaccine vector to express SIV antigens have observed some excretion of the engineered 68-1 vectors in urine of inoculated animals [22,84].

Although the mechanisms involved in mucosal transmission are poorly understood, disruption of the natural pattern of mucosal shedding through vaccination, by either reducing the incidence of shedding in infected people or enhancing mucosal immune responses to prevent infection or reinfection, is a novel strategy that is supported by recent observations in macaques. A better understanding of the early interactions between RhCMV and its host, and the mucosal immune responses involved in RhCMV infection, are essential for this vaccine development strategy. 


\subsection{RhCMV Immune Modulation}

It is an accepted precept of vaccinology that the quality and quantity of adaptive immune responses are dependent on the quality and quantity of innate responses [85]. Accordingly, there is increasing effort in designing adjuvants that direct adaptive immunity in a direction that increase protective efficacy. Acute differences in innate immune responses at sites of inoculation following SC exposure to either UCD52/UCD59 or 68-1 (described above) [60] imply that RhCMV (and by extension HCMV) similarly exerts an adjuvant-like effect on the local innate effector cells to direct adaptive immune responses towards a situation that favors viral persistence at the expense of immune-mediated clearance of RhCMV-infected cells. The RhCMV functions that presumably mediated the relative differences in the nature of the inflammatory response (polymorphonuclear cells for UCD52/UCD59 and mononuclear cells for 68-1) is due to the presence of the CXC-like ORF in UCD52/UCD59, although no functional activity for these ORF has been reported [69]. The concept that a critical step in early RhCMV-host interactions involves rapid manipulation of innate responses is bolstered by studies investigating the role of the virally encoded interleukin-10 like protein of RhCMV.

Primate CMVs, with the exception of chimpanzee CMV, encode a viral IL-10 gene that is an evolutionary remnant of a transduced cellular IL-10 (cIL-10) gene by a progenitor primate CMV at some point after the split of rodents and primates [56,86,87]. Despite extensive genetic drift of the HCMV and RhCMV viral IL-10 proteins (cmvIL-10 and rhcmvIL-10, respectively) from the cIL-10 proteins of their respective hosts, these viral orthologs retain high affinity for the cognate cIL-10 receptor [50] and nearly identical immunosuppressive properties in vitro to those exhibited by cIL-10 [56,87]. The conservation of functionality of cmvIL-10 and rhcmvIL-10 to that of cIL-10 leads to the proposition that expression of an IL-10-like protein in the context of viral infection suppresses acute immune responses to viral infection.

The role of rhcmvIL-10 in primary infection was analyzed in macaques following SC inoculation with $68-1$ or a $68-1$ rhcmvIL-10 knockout virus (68-1 $\Delta \mathrm{IL}-10)$ [81]. Tissue biopsies taken from animals seven days after inoculation with 68-1 had markedly reduced cellularity at the inoculation site, but greater numbers of tissue macrophages, than 68-1 $\Delta \mathrm{IL}-10$ inoculated animals [81]. In addition, there were fewer myeloid dendritic cells and decreased priming of naïve $\mathrm{CD} 4^{+} \mathrm{T}$ cells in the draining lymph nodes of 68-1 infected animals versus $68-1 \Delta \mathrm{IL}-10$ infected animals. Animals inoculated with 68-1 also showed impaired RhCMV-specific IgG responses and lower IgG avidity, compared with $68-1 \Delta \mathrm{IL}-10$ infected animals. In sum, the functional absence of rhcmvIL-10 in 68-1 $\Delta \mathrm{IL}-10$ resulted in increased innate immune recognition of viral antigens and increased adaptive immune responses. These results lead to a model whereby a critical step in RhCMV infection is the immediate manipulation of the local immune environment mediated, in part, by rhcmvIL-10. Based on this premise, secreted proteins, such as rhcmvIL-10, should represent reasonable vaccine targets to induce, particularly, antibodies that neutralize their function.

A recent survey of RhCMV-infected macaques revealed that one consequence of the extensive genetic drift of rhcmvIL-10 from the cIL-10 protein of its host is that what was once a self-protein soon after transduction of the cIL-10 gene has become highly immunogenic in the context of viral infection [88]. All RhCMV-infected animals develop high avidity binding antibodies to rhcmvIL-10, 
and almost $100 \%$ of the animals also exhibit some level of antibodies that neutralize rhomvIL-10 function in bioassays. Importantly, the rhcmvIL-10-specific neutralizing antibody responses generated in these macaques did not cross-react with cIL-10. The cmvIL-10 protein is also immunogenic in HCMV-infected humans [89]. Together, these data provide proof-of-concept for targeting viral IL-10, an immune modulator, as a vaccine strategy to interrupt viral persistence.

To accomplish antigenic stimulation without triggering the cIL-10 receptor, a non-functional rhcmvIL-10 has been designed, cloned into an expression cassette, expressed, and purified [90]. Immunization of naïve macaques with a DNA prime/protein boost immunization strategy stimulated antibody responses that neutralized wild-type rhcmvIL-10 function without cross-neutralization of rhesus cIL-10 [90]. SC challenge of vaccinated animals with UCD59 resulted in significantly lower frequencies and loads of RhCMV DNA in plasma, saliva, and urine, compared to mock-vaccinated control animals [91]. These data are unprecedented for CMV vaccine strategies in that targeting a single viral immune modulator (i.e., rhcmvIL-10) elicited immune-mediated protection against viral challenge without inducing antibodies that neutralized RhCMV infection of cells. Given the large devotion of the HCMV coding capacity to immune modulating proteins (discussed in Section 2.5), the results targeting rhcmvIL-10 offer a compelling rationale to explore additional viral antigens involved in immune modulation.

\subsection{RhCMV Reinfection}

A considerable challenge to reducing congenital infection is the ability of HCMV to reinfect women with pre-conceptional immunity and subsequently cross the maternal-fetal interface (discussed in Section 2.4). The challenge arises because the mechanism for reinfection is unknown and, consequently, there are no prevention strategies short of absolutely minimizing exposure to virus shedding contacts. Fortunately, a study of RhCMV sheds light on viral mechanisms to overcome prior immunity.

Evidence for RhCMV infection of immune competent hosts is suggestive from natural history studies but incontrovertible from experimental studies. As described above (Section 3.1), multiple genetic variants of RhCMV have been amplified by PCR from RhCMV-immune animals. While these reports are consistent with reinfection, given the repeated exposure to infectious virus in bodily fluids of infected cohorts, it cannot be ruled out that the animal, from which saliva samples were analyzed, was not infected with more than one variant during primary exposure to RhCMV. In contrast, experimental inoculation of immune animals with genetically tagged variants of RhCMV is exceedingly efficient. Engineered variants of RhCMV 68-1 that express the SIV proteins (gag, retanef) have been used to reinfect RhCMV immune animals [84]. Animals inoculated with the RhCMV vector expressing the SIV protein uniformly develop SIV protein-specific $\mathrm{T}$ cells responses, and the RhCMV/SIV vector can be recovered in the urine of reinfected animals. Reinfection can be observed following inoculation with as little as 100 PFU, highlighting the ability of RhCMV to overcome existing antiviral immune responses [22].

Based on this, Hansen et al. reasoned that an essential step in reinfection is overcoming "an initial immunological checkpoint", and they addressed whether the RHCMV orthologs of the HCMV proteins disrupting MHC-I antigen presentation mediate RhCMV reinfection (US2, 3, 6, and 11) [22]. Engineered deletion of the RhUS2-11 genes did not impair the ability of the deletant 
variant to replicate in cultured fibroblasts, compared to the unmodified parental vector. RhCMVAUS2-11 expressing SIV gag was also able to establish infection in RhCMV-uninfected macaques, as demonstrated by recovery of RhCMV $\Delta$ US2-11 in the urine of infected animals and development of RhCMV-specific and gag-specific T cell responses. Notably, RhCMV $\Delta$ US2-11 was incapable of reinfecting RhCMV-immune animals following SC inoculation with $10^{7} \mathrm{PFU}$. These results demonstrate that manipulation of the host immunity is essential for reinfection, and, together with studies of acute RhCMV infection, illustrate the role RhCMV immune modulating proteins play in the infectious cycle of RhCMV in vivo.

\section{Conclusions}

The results of the Phase II gB vaccine trial [29] represents an important step towards meeting the clarion call of Hanshaw to develop a vaccine that protects against the devastating consequences of intrauterine HCMV infection [27]. This clinical trial now represents the "gold standard" against which subsequent HCMV vaccine trials will be compared, and yet, it is not certain at this time whether the level of observed protection compels a Phase III trial. Key issues about how best to move forward include (1) optimization of $\mathrm{gB}$ immunization to induce greater protective neutralizing responses; (2) inclusion of additional envelope glycoproteins to induce broader neutralizing responses against other virologically relevant cell types (i.e., gH pentamer-mediated infection of epithelial and endothelial cells); and/or (3) targeting other parts of the HCMV proteome not involved in attachment/entry of susceptible cells. The vaccine potential for broadening neutralizing antibody responses beyond $\mathrm{gB}$ has been demonstrated recently by restoring the $\mathrm{gH}$ pentameric complex in the AD169 vaccine strain of HCMV [92,93]. While still in an early developmental stage, this strategy may help to address the difficult problem of the broad cellular tropism of HCMV. HCMV persistence in immune competent hosts and, in particular, the ability of HCMV to reinfect immune individuals leads to the proposition that HCMV is unlike most every other virus for which protective vaccines have been developed. Varicella zoster virus (VZV), an alphaherpesvirus and the only herpes virus for which there is a commercially available vaccine, persists in the infected host in a truly latent state, and recurrent disease is the result of reactivation of this latent virus after host immune responses have waned or become impaired. The attenuated VZV vaccine strain, Oka, was generated via serial passage in cell culture of a clinical VZV isolate. Similar strategies for HCMV have failed to generate a successful vaccine candidate (e.g., Towne) [94,95]. In addition, prior immunity to HCMV does not protect from reinfection despite robust adaptive immune responses. The ability to overcome extant neutralizing antibody responses and extraordinarily large HCMV-specific T cell responses emphasize the unprecedented role of HCMV-encoded immune modulating functions in HCMV natural history. Given the expense involved in conducting sufficiently powered clinical Phase II and Phase III trials, there is an essential need for rigorous pre-clinical studies that can inform which vaccine modalities should advance to human testing.

Recent studies of RhCMV point to multiple ORF that can be targeted to change the course of acute infection, dissemination via the blood to tissues throughout the body, and the ability to seed distal sites where progeny virions are shed into bodily fluids. These antigens include: gB, pp65, the gH-pentamer complex, CXC chemokines, rhcmvIL-10, and other ORF in UL/b' besides RhUL128-131. However, multiple RhCMV vaccine studies using a variety of antigen delivery 
modalities have demonstrated partial protection, at best, and the results are consistent with the complexities of HCMV vaccine design. It should be stressed that all RhCMV vaccine studies, and to our knowledge all animal vaccine models of HCMV vaccines, use direct parenteral introduction of challenge virus. This bypasses the potential for mucosal immunity in the vaccinee to control primary viral challenge, and subsequent studies in animal models should work to recapitulate the repeated mucosal exposures to HCMV that vaccinees will encounter.

\section{Acknowledgments}

This manuscript was made possible by grant support from NIH to PAB (AI063356, AI097629, AI049342) and the California National Primate Research Center (OD011107), and the Margaret Deterding Infectious Disease Research Support Fund.

\section{Author Contributions}

J.D.D. and P.A.B. contributed equally to this review.

\section{Conflicts of Interest}

The authors declare no conflict of interest.

\section{References and Notes}

1. McGeoch, D.J.; Gatherer, D. Integrating reptilian herpesviruses into the family herpesviridae. J. Virol. 2005, 79, 725-731.

2. Kolb, A.W.; Ane, C.; Brandt, C.R. Using HSV-1 Genome Phylogenetics to Track Past Human Migrations. PLoS One 2013, 8, e76267.

3. Cannon, M.J.; Schmid, D.S.; Hyde, T.B. Review of cytomegalovirus seroprevalence and demographic characteristics associated with infection. Rev. Med. Virol. 2010, 20, 202-213.

4. Cannon, M.J.; Hyde, T.B.; Schmid, D.S. Review of cytomegalovirus shedding in bodily fluids and relevance to congenital cytomegalovirus infection. Rev. Med. Virol. 2011, 21, 240-255.

5. Noyola, D.E.; Valdez-Lopez, B.H.; Hernandez-Salinas, A.E.; Santos-Diaz, M.A.; Noyola-Frias, M.A.; Reyes-Macias, J.F.; Martinez-Martinez, L.G. Cytomegalovirus excretion in children attending day-care centers. Arch. Med. Res. 2005, 36, 590-593.

6. Berntsson, M.; Dubicanac, L.; Tunback, P.; Ellstrom, A.; Lowhagen, G.B.; Bergstrom, T. Frequent detection of cytomegalovirus and Epstein-Barr virus in cervical secretions from healthy young women. Acta Obstet. Gynecol. Scand. 2013, 92, 706-710.

7. De Franca, T.R.; de Albuquerque Tavares Carvalho, A.; Gomes, V.B.; Gueiros, L.A.; Porter, S.R.; Leao, J.C. Salivary shedding of Epstein-Barr virus and cytomegalovirus in people infected or not by human immunodeficiency virus 1. Clin. Oral Investig. 2012, 16, 659-664.

8. Arora, N.; Novak, Z.; Fowler, K.B.; Boppana, S.B.; Ross, S.A. Cytomegalovirus viruria and DNAemia in healthy seropositive women. J. Infect. Dis. 2010, 202, 1800-1803. 
9. Gautheret-Dejean, A.; Aubin, J.T.; Poirel, L.; Huraux, J.M.; Nicolas, J.C.; Rozenbaum, W.; Agut, H. Detection of human Betaherpesvirinae in saliva and urine from immunocompromised and immunocompetent subjects. J. Clin. Microbiol. 1997, 35, 1600-1603.

10. Hayashi, S.; Kimura, H.; Oshiro, M.; Kato, Y.; Yasuda, A.; Suzuki, C.; Watanabe, Y.; Morishima, T.; Hayakawa, M. Transmission of cytomegalovirus via breast milk in extremely premature infants. J. Perinatol. 2011, 31, 440-445.

11. Hamprecht, K.; Maschmann, J.; Vochem, M.; Dietz, K.; Speer, C.P.; Jahn, G. Epidemiology of transmission of cytomegalovirus from mother to preterm infant by breastfeeding. Lancet 2001, 357, 513-518.

12. Jim, W.T.; Shu, C.H.; Chiu, N.C.; Chang, J.H.; Hung, H.Y.; Peng, C.C.; Kao, H.A.; Wei, T.Y.; Chiang, C.L.; Huang, F.Y. High cytomegalovirus load and prolonged virus excretion in breast milk increase risk for viral acquisition by very low birth weight infants. Pediatr. Infect. Dis. J. 2009, 28, 891-894.

13. Maciejewski, J.P.; Bruening, E.E.; Donahue, R.E.; Mocarski, E.S.; Young, N.S.; St Jeor, S.C. Infection of hematopoietic progenitor cells by human cytomegalovirus. Blood 1992, 80, 170-178.

14. Mendelson, M.; Monard, S.; Sissons, P.; Sinclair, J. Detection of endogenous human cytomegalovirus in CD34+ bone marrow progenitors. J. Gen. Virol. 1996, 77, 3099-3102.

15. Kondo, K.; Kaneshima, H.; Mocarski, E.S. Human cytomegalovirus latent infection of granulocyte-macrophage progenitors. Proc. Natl. Acad. Sci. USA 1994, 91, 11879-11883.

16. Kondo, K.; Xu, J.; Mocarski, E.S. Human cytomegalovirus latent gene expression in granulocyte-macrophage progenitors in culture and in seropositive individuals. Proc. Natl. Acad. Sci. USA 1996, 93, 11137-11142.

17. Goodrum, F.; Reeves, M.; Sinclair, J.; High, K.; Shenk, T. Human cytomegalovirus sequences expressed in latently infected individuals promote a latent infection in vitro. Blood 2007, 110, 937-945.

18. Cheung, A.K.; Abendroth, A.; Cunningham, A.L.; Slobedman, B. Viral gene expression during the establishment of human cytomegalovirus latent infection in myeloid progenitor cells. Blood 2006, 108, 3691-3699.

19. Fowler, K.B.; Stagno, S.; Pass, R.F.; Britt, W.J.; Boll, T.J.; Alford, C.A. The outcome of congenital cytomegalovirus infection in relation to maternal antibody status. N. Engl. J. Med. 1992, 326, 663-667.

20. Rasmussen, L.; Matkin, C.; Spaete, R.; Pachl, C.; Merigan, T.C. Antibody response to human cytomegalovirus glycoproteins $\mathrm{gB}$ and $\mathrm{gH}$ after natural infection in humans. J. Infect. Dis. 1991, 164, 835-842.

21. Boppana, S.B.; Rivera, L.B.; Fowler, K.B.; Mach, M.; Britt, W.J., Intrauterine transmission of cytomegalovirus to infants of women with preconceptional immunity. N. Engl. J. Med. 2001, 344, 1366-1371.

22. Hansen, S.G.; Powers, C.J.; Richards, R.; Ventura, A.B.; Ford, J.C.; Siess, D.; Axthelm, M.K.; Nelson, J.A.; Jarvis, M.A.; Picker, L.J.; et al. Evasion of CD8+ T cells is critical for superinfection by cytomegalovirus. Science 2010, 328, 102-106. 
23. Sylwester, A.W.; Mitchell, B.L.; Edgar, J.B.; Taormina, C.; Pelte, C.; Ruchti, F.; Sleath, P.R.; Grabstein, K.H.; Hosken, N.A.; Kern, F.; et al. Broadly targeted human cytomegalovirus-specific CD4+ and CD8+ T cells dominate the memory compartments of exposed subjects. J. Exp. Med. 2005, 202, 673-685.

24. Griffiths, P.D. Burden of disease associated with human cytomegalovirus and prospects for elimination by universal immunisation. Lancet Infect. Dis. 2012, 12, 790-798.

25. Crough, T.; Khanna, R. Immunobiology of human cytomegalovirus: From bench to bedside. Clin. Microbiol. Rev. 2009, 22, 76-98.

26. Elek, S.D.; Stern, H. Development of a vaccine against mental retardation caused by cytomegalovirus infection in utero. Lancet 1974, 1, 1-5.

27. Hanshaw, J.B. Congenital cytomegalovirus infection: A fifteen year perspective. J. Infect. Dis. 1971, 123, 555-561.

28. Plotkin, S.A. Vaccines for varicella-zoster virus and cytomegalovirus: Recent progress. Science 1994, 265, 1383-1385.

29. Pass, R.F.; Zhang, C.; Evans, A.; Simpson, T.; Andrews, W.; Huang, M.L.; Corey, L.; Hill, J.; Davis, E.; Flanigan, C.; et al. Vaccine prevention of maternal cytomegalovirus infection. $N$. Engl. J. Med. 2009, 360, 1191-1199.

30. Stratton, K.R.; Durch, J.S.; Lawrence, R.S. Vaccines for the 21st Century: A Tool for Decision Making; National Academy Press: Washington, DC, USA, 2000; p. Appendix 4:165-172.

31. Saccoccio, F.M.; Gallagher, M.K.; Adler, S.P.; McVoy, M.A. Neutralizing activity of saliva against cytomegalovirus. Clin. Vaccine Immunol. 2011, 18, 1536-1542.

32. Tamura, T.; Chiba, S.; Chiba, Y.; Nakao, T. Virus excretion and neutralizing antibody response in saliva in human cytomegalovirus infection. Infect. Immun. 1980, 29, 842-845.

33. Wang, J.B.; Adler, S.P.; Hempfling, S.; Burke, R.L.; Duliege, A.M.; Starr, S.E.; Plotkin, S.A. Mucosal antibodies to human cytomegalovirus glycoprotein B occur following both natural infection and immunization with human cytomegalovirus vaccines. J. Infect. Dis. 1996, 174, 387-392.

34. Simanek, A.M.; Dowd, J.B.; Pawelec, G.; Melzer, D.; Dutta, A.; Aiello, A.E. Seropositivity to cytomegalovirus, inflammation, all-cause and cardiovascular disease-related mortality in the United States. PLoS One 2011, 6, e16103.

35. Bale, J.F., Jr.; Petheram, S.J.; Souza, I.E.; Murph, J.R. Cytomegalovirus reinfection in young children. J. Pediatr. 1996, 128, 347-352.

36. Chandler, S.H.; Handsfield, H.H.; McDougall, J.K. Isolation of multiple strains of cytomegalovirus from women attending a clinic for sexually transmitted disease. J. Infect. Dis. 1987, 155, 655-660.

37. Sohn, Y.M.; Park, K.I.; Lee, C.; Han, D.G.; Lee, W.Y. Congenital cytomegalovirus infection in Korean population with very high prevalence of maternal immunity. J. Korean Med. Sci. 1992, 7, 47-51.

38. Gaytant, M.A.; Rours, G.I.; Steegers, E.A.; Galama, J.M.; Semmekrot, B.A. Congenital cytomegalovirus infection after recurrent infection: Case reports and review of the literature. Eur. J. Pediatr. 2003, 162, 248-253. 
39. Gaytant, M.A.; Steegers, E.A.; Semmekrot, B.A.; Merkus, H.M.; Galama, J.M. Congenital cytomegalovirus infection: Review of the epidemiology and outcome. Obstet. Gynecol. Surv. 2002, 57, 245-256.

40. Gandhoke, I.; Aggarwal, R.; Lal, S.; Khare, S. Congenital CMV infection in symptomatic infants in Delhi and surrounding areas. Indian J. Pediatr. 2006, 73, 1095-1097.

41. Yamamoto, A.Y.; Mussi-Pinhata, M.M.; Boppana, S.B.; Novak, Z.; Wagatsuma, V.M.; Oliveira Pde, F.; Duarte, G.; Britt, W.J. Human cytomegalovirus reinfection is associated with intrauterine transmission in a highly cytomegalovirus-immune maternal population. Am. J. Obstet. Gynecol. 2010, 202, 297.e1-297.e8.

42. Ross, S.A.; Fowler, K.B.; Ashrith, G.; Stagno, S.; Britt, W.J.; Pass, R.F.; Boppana, S.B. Hearing loss in children with congenital cytomegalovirus infection born to mothers with preexisting immunity. J. Pediatr. 2006, 148, 332-336.

43. Wang, C.; Zhang, X.; Bialek, S.; Cannon, M.J. Attribution of congenital cytomegalovirus infection to primary versus non-primary maternal infection. Clin. Infect. Dis. 2011, 52, e11-e13.

44. Britt, W.J.; Mach, M. Human cytomegalovirus glycoproteins. Intervirology 1996, 39, 401-412.

45. Revello, M.G.; Gerna, G. Human cytomegalovirus tropism for endothelial/epithelial cells: Scientific background and clinical implications. Rev. Med. Virol. 2010, 20, 136-155.

46. Cui, X.; Meza, B.P.; Adler, S.P.; McVoy, M.A. Cytomegalovirus vaccines fail to induce epithelial entry neutralizing antibodies comparable to natural infection. Vaccine 2008, 26, 5760-5766.

47. Macagno, A.; Bernasconi, N.L.; Vanzetta, F.; Dander, E.; Sarasini, A.; Revello, M.G.; Gerna, G.; Sallusto, F.; Lanzavecchia, A. Isolation of human monoclonal antibodies that potently neutralize human cytomegalovirus infection by targeting different epitopes on the gH/gL/UL128-131 A complex. J. Virol. 2010, 84, 1005-1013.

48. Britt, W.J. Neutralizing antibodies detect a disulfide-linked glycoprotein complex within the envelope of human cytomegalovirus. Virology 1984, 135, 369-378.

49. Ross, S.A.; Arora, N.; Novak, Z.; Fowler, K.B.; Britt, W.J.; Boppana, S.B. Cytomegalovirus reinfections in healthy seroimmune women. J. Infect. Dis. 2010, 201, 386-389.

50. Dunn, W.; Chou, C.; Li, H.; Hai, R.; Patterson, D.; Stolc, V.; Zhu, H.; Liu, F. Functional profiling of a human cytomegalovirus genome. Proc. Natl. Acad. Sci. USA 2003, 100, 14223-14228.

51. Yu, D.; Silva, M.C.; Shenk, T. Functional map of human cytomegalovirus AD169 defined by global mutational analysis. Proc. Natl. Acad. Sci. USA 2003, 100, 12396-12401.

52. Hakki, M.; Chou, S. The biology of cytomegalovirus drug resistance. Curr. Opin. Infect. Dis. 2011, 24, 605-611.

53. Renzette, N.; Gibson, L.; Bhattacharjee, B.; Fisher, D.; Schleiss, M.R.; Jensen, J.D.; Kowalik, T.F. Rapid intrahost evolution of human cytomegalovirus is shaped by demography and positive selection. PLoS Genet. 2013, 9, e1003735.

54. Vogel, P.; Weigler, B.J.; Kerr, H.; Hendrickx, A.; Barry, P.A. Seroepidemiologic studies of cytomegalovirus infection in a breeding population of rhesus macaques. Lab. Anim. Sci. 1994, $44,25-30$. 
55. Andrade, M.R.; Yee, J.; Barry, P.; Spinner, A.; Roberts, J.A.; Cabello, P.H.; Leite, J.P.; Lerche, N.W. Prevalence of antibodies to selected viruses in a long-term closed breeding colony of rhesus macaques (Macaca mulatta) in Brazil. Am. J. Primatol. 2003, 59, 123-128.

56. Früh, K.; Malouli , D.; Oxford, K.; Barry, P. Non-Human-Primate Models of Cytomegalovirus Infection, Prevention, and Therapy. In CYTOMEGALOVIRUSES: From Molecular Pathogenesis to Therapy; Reddehase, M., Ed.; Caister Academic Press/Horizon: Norfolk, UK, 2013; Volume 2.

57. Jones-Engel, L.; Engel, G.A.; Heidrich, J.; Chalise, M.; Poudel, N.; Viscidi, R.; Barry, P.A.; Allan, J.S.; Grant, R.; Kyes, R. Temple monkeys and health implications of commensalism, Kathmandu, Nepal. Emerg. Infect. Dis. 2006, 12, 900-906.

58. Baskin, G.B. Disseminated cytomegalovirus infection in immunodeficient rhesus monkeys. Am. J. Pathol. 1987, 129, 345-352.

59. Kaur, A.; Kassis, N.; Hale, C.L.; Simon, M.; Elliott, M.; Gomez-Yafal, A.; Lifson, J.D.; Desrosiers, R.C.; Wang, F.; Barry, P.; et al. Direct relationship between suppression of virus-specific immunity and emergence of cytomegalovirus disease in simian AIDS. J. Virol. 2003, 77, 5749-5758.

60. Assaf, B.T.; Mansfield, K.G.; Westmoreland, S.V.; Kaur, A.; Oxford, K.L.; Diamond, D.J.; Barry, P.A. Patterns of acute rhesus cytomegalovirus (RhCMV) infection predict long-term RhCMV infection. J. Virol. 2012, 86, 6354-6357.

61. Oxford, K.L.; Strelow, L.; Yue, Y.; Chang, W.L.; Schmidt, K.A.; Diamond, D.J.; Barry, P.A. Open reading frames carried on UL/b' are implicated in shedding and horizontal transmission of rhesus cytomegalovirus in rhesus monkeys. J. Virol. 2011, 85, 5105-5114.

62. Wussow, F.; Yue, Y.; Martinez, J.; Deere, J.D.; Longmate, J.; Herrmann, A.; Barry, P.A.; Diamond, D.J. A Vaccine Based on the Rhesus Cytomegalovirus UL128 Complex Induces Broadly Neutralizing Antibodies in Rhesus Macaques. J. Virol. 2013, 87, 1322-1332.

63. Yue, Y.; Zhou, S.S.; Barry, P.A. Antibody responses to rhesus cytomegalovirus glycoprotein B in naturally infected rhesus macaques. J. Gen. Virol. 2003, 84, 3371-3379.

64. Yue, Y.; Kaur, A.; Zhou, S.S.; Barry, P.A. Characterization and immunological analysis of the rhesus cytomegalovirus homologue (Rh112) of the human cytomegalovirus UL83 lower matrix phosphoprotein (pp65). J. Gen. Virol. 2006, 87, 777-787.

65. Pitcher, C.J.; Hagen, S.I.; Walker, J.M.; Lum, R.; Mitchell, B.L.; Maino, V.C.; Axthelm, M.K.; Picker, L.J. Development and homeostasis of $\mathrm{T}$ cell memory in rhesus macaque. J. Immunol. 2002, 168, 29-43.

66. Asher, D.M.; Gibbs, C.J., Jr.; Lang, D.J. Rhesus monkey cytomegaloviruses: Persistent asymptomatic viruses. Bacteriol. Proc. 1969, 69, 191.

67. Asher, D.M.; Gibbs, C.J., Jr.; Lang, D.J.; Gajdusek, D.C.; Chanock, R.M. Persistent shedding of cytomegalovirus in the urine of healthy Rhesus monkeys. Proc. Soc. Exp. Biol. Med. 1974, 145, 794-801.

68. Huff, J.L.; Eberle, R.; Capitanio, J.; Zhou, S.S.; Barry, P.A. Differential detection of B virus and rhesus cytomegalovirus in rhesus macaques. J. Gen. Virol. 2003, 84, 83-92.

69. Oxford, K.L.; Eberhardt, M.K.; Yang, K.W.; Strelow, L.; Kelly, S.; Zhou, S.S.; Barry, P.A. Protein coding content of the UL)b' region of wild-type rhesus cytomegalovirus. Virology 2008, 373, 181-188. 
70. Alcendor, D.J.; Barry, P.A.; Pratt-Lowe, E.; Luciw, P.A. Analysis of the rhesus cytomegalovirus immediate-early gene promoter. Virology 1993, 194, 815-821.

71. Barry, P.A.; Alcendor, D.J.; Power, M.D.; Kerr, H.; Luciw, P.A. Nucleotide sequence and molecular analysis of the rhesus cytomegalovirus immediate-early gene and the UL121-117 open reading frames. Virology 1996, 215, 61-72.

72. Hansen, S.G.; Strelow, L.I.; Franchi, D.C.; Anders, D.G.; Wong, S.W. Complete sequence and genomic analysis of rhesus cytomegalovirus. J. Virol. 2003, 77, 6620-6636.

73. Wang, D.; Shenk, T. Human cytomegalovirus virion protein complex required for epithelial and endothelial cell tropism. Proc. Natl. Acad. Sci. USA 2005, 102, 18153-18158.

74. Ryckman, B.J.; Rainish, B.L.; Chase, M.C.; Borton, J.A.; Nelson, J.A.; Jarvis, M.A.; Johnson, D.C. Characterization of the human cytomegalovirus gH/gL/UL128-131 complex that mediates entry into epithelial and endothelial cells. J. Virol. 2008, 82, 60-70.

75. Lilja, A.E.; Shenk, T. Efficient Replication of Rhesus Cytomegalovirus Variants in Multiple Rhesus and Human Cell Types. Proc. Natl. Acad. Sci. USA 2008, 105, 19950-19955.

76. Carlson, J.R.; Chang, W.L.; Zhou, S.S.; Tarantal, A.F.; Barry, P.A. Rhesus brain microvascular endothelial cells are permissive for rhesus cytomegalovirus infection. J. Gen. Virol. 2005, 86, 545-549.

77. Abel, K.; Martinez, J.; Yue, Y.; Lacey, S.F.; Wang, Z.; Strelow, L.; Dasgupta, A.; Li, Z.; Schmidt, K.A.; Oxford, K.L.; et al. Vaccine-induced control of viral shedding following rhesus cytomegalovirus challenge in rhesus macaques. J. Virol. 2011, 85, 2878-2890.

78. Assaf, B.T.; Mansfield, K.G.; Westmoreland, S.V.; Strelow, L.; Barry, P.A.; Kaur, A. Limited Dissemination and Shedding of the UL128-Complex-Intact, UL/b'-Defective Rhesus Cytomegalovirus Strain 180.92. J. Virol., submitted.

79. Rivailler, P.; Kaur, A.; Johnson, R.P.; Wang, F. Genomic sequence of rhesus cytomegalovirus 180.92: Insights into the coding potential of rhesus cytomegalovirus. J. Virol. 2006, 80, 4179-4182.

80. Yue, Y.; Kaur, A.; Eberhardt, M.K.; Kassis, N.; Zhou, S.S.; Tarantal, A.F.; Barry, P.A. Immunogenicity and protective efficacy of DNA vaccines expressing rhesus cytomegalovirus glycoprotein B, phosphoprotein 65-2, and viral interleukin-10 in rhesus macaques. J. Virol. 2007, 81, 1095-1109.

81. Chang, W.L.; Barry, P.A. Attenuation of innate immunity by cytomegalovirus IL-10 establishes a long-term deficit of adaptive antiviral immunity. Proc. Natl. Acad. Sci. USA 2010, 107, 22647-22652.

82. Abel, K.; Strelow, L.; Yue, Y.; Eberhardt, M.K.; Schmidt, K.A.; Barry, P.A. A heterologous DNA prime/protein boost immunization strategy for rhesus cytomegalovirus. Vaccine 2008, 26, 6013-6025.

83. Lockridge, K.M.; Sequar, G.; Zhou, S.S.; Yue, Y.; Mandell, C.P.; Barry, P.A. Pathogenesis of experimental rhesus cytomegalovirus infection. J. Virol. 1999, 73, 9576-9583.

84. Hansen, S.G.; Vieville, C.; Whizin, N.; Coyne-Johnson, L.; Siess, D.C.; Drummond, D.D.; Legasse, A.W.; Axthelm, M.K.; Oswald, K.; Trubey, C.M.; et al. Effector memory T cell responses are associated with protection of rhesus monkeys from mucosal simian immunodeficiency virus challenge. Nat. Med. 2009, 15, 293-299. 
85. Pulendran, B.; Ahmed, R. Immunological mechanisms of vaccination. Nat. Immunol. 2011, 12, 509-517.

86. Lockridge, K.M.; Zhou, S.S.; Kravitz, R.H.; Johnson, J.L.; Sawai, E.T.; Blewett, E.L.; Barry, P.A. Primate cytomegaloviruses encode and express an IL-10-like protein. Virology 2000, 268, 272-280.

87. Slobedman, B.; Barry, P.A.; Spencer, J.V.; Avdic, S.; Abendroth, A. Virus-encoded homologs of cellular interleukin-10 and their control of host immune function. J. Virol. 2009, 83, 9618-9629.

88. Eberhardt, M.K.; Chang, W.L.; Logsdon, N.J.; Yue, Y.; Walter, M.R.; Barry, P.A. Host immune responses to a viral immune modulating protein: Immunogenicity of viral interleukin-10 in rhesus cytomegalovirus-infected rhesus macaques. PLoS One 2012, 7, e37931.

89. De Lemos Rieper, C.; Galle, P.; Pedersen, B.K.; Hansen, M.B. Characterization of specific antibodies against cytomegalovirus (CMV)-encoded interleukin 10 produced by $28 \%$ of CMV-seropositive blood donors. J. Gen. Virol. 2011, 92, 1508-1518.

90. Logsdon, N.J.; Eberhardt, M.K.; Allen, C.E.; Barry, P.A.; Walter, M.R. Design and analysis of rhesus cytomegalovirus IL-10 mutants as a model for novel vaccines against human cytomegalovirus. PLoS One 2011, 6, e28127.

91. Eberhardt, M.; Deshpande, A.; Chang, W.-L.; Barthold, S.; Walter, M.; Barry, P. Vaccination Against a Virally-Encoded Cytokine Significantly Restricts Viral Challenge. J. Virol. 2013, 87, 11323-11331.

92. Fu, T.M.; Wang, D.; Freed, D.C.; Tang, A.; Li, F.; He, X.; Cole, S.; Dubey, S.; Finnefrock, A.C.; ter Meulen, J.; Shiver, J.W.; Casimiro, D.R. Restoration of viral epithelial tropism improves immunogenicity in rabbits and rhesus macaques for a whole virion vaccine of human cytomegalovirus. Vaccine 2012, 30, 7469-7474.

93. Freed, D.C.; Tang, Q.; Tang, A.; Li, F.; He, X.; Huang, Z.; Meng, W.; Xia, L.; Finnefrock, A.C.; Durr, E.; et al. Pentameric complex of viral glycoprotein $\mathrm{H}$ is the primary target for potent neutralization by a human cytomegalovirus vaccine. Proc. Natl. Acad. Sci. USA 2013, 110, E4997-E5005.

94. Plotkin, S.A.; Furukawa, T.; Zygraich, N.; Huygelen, C. Candidate cytomegalovirus strain for human vaccination. Infect. Immun. 1975, 12, 521-527.

95. Plotkin, S.A.; Starr, S.E.; Friedman, H.M.; Gonczol, E.; Brayman, K. Vaccines for the prevention of human cytomegalovirus infection. Rev. Infect. Dis. 1990, 12, S827-S838. 



\title{
Immune and DNA Damage Responses Linked to CMV
}

Reprinted from Viruses. Cite as: Hanley, P.J.; Bollard, C.M. Controlling Cytomegalovirus: Helping the Immune System Take the Lead. Viruses 2014, 6, 2242-2258.

Review

\section{Controlling Cytomegalovirus: Helping the Immune System Take the Lead}

\section{Patrick J. Hanley * and Catherine M. Bollard}

Program for Cell Enhancement and Technologies for Immunotherapy, Sheikh Zayed Institute for Pediatric Surgical Innovation, and Center for Cancer and Immunology Research, Children's National Health System, Washington, DC 20010, USA; E-Mail: CBollard@childrensnational.org

* Author to whom correspondence should be addressed; E-Mail: PHanley@childrensnational.org; Tel.: +1-202-476-6368; Fax: +1-202-476-5808.

Received: 4 February 2014; in revised form: 9 May 2014 / Accepted: 13 May 2014 /

Published: 27 May 2014

\begin{abstract}
Cytomegalovirus, of the Herpesviridae family, has evolved alongside humans for thousands of years with an intricate balance of latency, immune evasion, and transmission. While upwards of $70 \%$ of humans have evidence of CMV infection, the majority of healthy people show little to no clinical symptoms of primary infection and CMV disease is rarely observed during persistent infection in immunocompetent hosts. Despite the fact that the majority of infected individuals are asymptomatic, immunologically, CMV hijacks the immune system by infecting and remaining latent in antigen-presenting cells that occasionally reactivate subclinically and present antigen to $\mathrm{T}$ cells, eventually causing the inflation of $\mathrm{CMV}$-specific $\mathrm{T}$ cells until they can compromise up to $10 \%$ of the entire $\mathrm{T}$ cell repertoire. Because of this impact on the immune system, as well as its importance in fields such as stem cell and organ transplant, the relationship between CMV and the immune response has been studied in depth. Here we provide a review of many of these studies and insights into how CMV-specific T cells are currently being used therapeutically.
\end{abstract}

Keywords: Cytomegalovirus (CMV); Adoptive immunotherapy; $\mathrm{T}$ cell; immunotherapy; cellular therapy; transplant 


\section{Biology of Cytomegalovirus}

The virus family Herpesviridae consists of three subfamilies of viruses, alpha, beta, and gamma. Betaherpesviruses contain the four major components of this family: the core, the capsid, the tegument, and the envelope and establish latency in cells of the myeloid lineage and CD34+ cells [1-3]. The tegument contains the majority of virion-associated proteins [4,5]. Tegument proteins have two reported functions, though the proteins that facilitate them are not mutually exclusive [6]. The first function is the disassembly of the virion during entry and assembly of the virion during egress [7]. The second function is inhibiting the host immune response to infection, though as discussed later, they may also promote the response as well [6]. Many of these proteins are associated with immune evasion, and are therefore packaged within the virion and delivered to the host soon after uncoating of the virus. The most abundant tegument protein is the lower matrix phosphoprotein of $65 \mathrm{kDa}$ (pp65), or Unique Long (UL)83 [5]. One important function of pp65 is immune evasion. Other tegument proteins devoted to immune evasion include the upper matrix protein pp71, UL36, UL38, and IRS1/TRS1 [8,9].

Immediate early (IE) proteins are translated within $2 \mathrm{~h}$ of infection and do not require the de novo synthesis of viral proteins for their translation [10]. These IE proteins then control subsequently gene expression and virus replication. As such, suppression of IE proteins is thought to contribute to CMV latency whereas the expression of IE genes is associated with reactivation [11]. The laboratories of both Hahn and Fietze have shown that proinflammatory cytokines such as GM-CSF and TNF- $\alpha$ can induce the differentiation of monocytes into macrophages or dendritic cells, which is thought to activate the IE1 promoter and stimulate reactivation [12-14]. However, how cytokines cause HCMV reactivation is still mostly unknown [12,14]. Because IE proteins are expressed first after reactivation, $\mathrm{T}$ cells targeting these proteins are of paramount importance, as highlighted in the field of transplantation where $\mathrm{T}$ cells IE proteins are important for protection after solid organ transplant [15].

\section{CMV Infection in the Immune Compromised Host}

CMV has long been one of the most problematic pathogens after stem cell transplantation (SCT) and organ transplant [16-19]. While effective antiviral drugs, viral monitoring, and donor/recipient matching have lowered the likelihood of disease after SCT, the mortality rate in patients who develop CMV-associated pneumonia remains strikingly high (around 80\%-90\%) [17,18]. Additionally, the recipient's CMV-seropositivity remains an independent risk factor for morbidity and mortality after SCT. In the case of SCT, the highest risk of CMV reactivation is when the recipient is seropositive and the stem cell donor is seronegative [20,21]. This is because the recipient has latent (or active) CMV that can no longer be controlled by the recipient's immune system after it is depleted with conditioning regimens and the stem cell donor graft does not contain protective CMV-specific memory T cells. In contrast, the risk of CMV-related complications, including death, after solid organ transplant (SOT) is greatest when the organ donor is CMV-seropositive and the recipient is $\mathrm{CMV}$-seronegative, though the severity tends to vary based on the organ being transplanted [22-25]. With the advent of CMV prophylaxis, an unexpected complication has 
emerged with an increased incidence of late-onset CMV disease; after day 100, late CMV disease may be as high as 17\% in CMV-seropositive recipients undergoing SCT [26].

\section{Innate Immunity to CMV}

An in-depth review of the immune response to CMV can be found here [9]. Some of the best evidence for the role of the innate immune system in mice is in experiments using beige mice that have known defects in Natural Killer (NK) cell-mediated cytotoxicity and are highly susceptible to murine Cytomegalovirus (MCMV). However, protection against MCMV can be restored by transferring NK cells from normal mice [27]. Despite the elegant studies suggesting the importance of NK cells in controlling MCMV, similar studies in humans are lacking for HCMV [28]. However, Biron et al. have reported in the New England Journal of Medicine NK cell-deficient individuals who are susceptible to herpes virus infections, including HCMV. In vitro experiments using IL-2 activated human NK cells have also demonstrated that NK cells can inhibit CMV replication in CMV-infected fibroblasts by inducing IFN-beta release from infected fibroblasts [29]. Activated NK cells also released IFN-gamma which can impede viral replication [9]. Boehme et al. have shown that HCMV glycoproteins B and H also activate Toll Like Receptor (TLR) 2 on fibroblast, resulting in $\mathrm{NF \kappa B}$ activation and subsequent inflammatory cytokine secretion, suggesting that NK cells are not the only innate cell responsible for protection from CMV [30]. Indeed, monocytes, macrophages and dendritic cells are cells permissible for viral reactivation and once infected release inflammatory cytokines, in addition to presenting antigen to T cells [31].

\section{Humoral Immunity to CMV}

The importance of an antibody response to CMV is demonstrated in guinea pig models where antibodies protect the animals from reaching a lethal infective dose, but do not prevent infection, suggesting a role for the humoral immune response in limiting the severity of the disease by controlling CMV viral load [32]. After a primary infection in humans, antibodies against a number of proteins from HCMV are detectable in the serum. These antibodies recognize an array of proteins from different parts of the virus, including pp65 and pp150 from the tegument, the glycoproteins $\mathrm{gB}$ and $\mathrm{gH}$ from the envelope, as well as proteins involved in transcription such as IE-1 [33]. Most CMV-seropositive humans have antibodies directed against gB, with over $50 \%$ of all neutralizing antibodies recognizing an epitope of $\mathrm{gB}[33,34]$. The importance of humoral immunity is also demonstrated in congenital CMV infection where pregnant women who develop primary CMV infection carry a $40 \%$ chance of HCMV transmission to the fetus $[35,36]$. In cases where the mother is able to provide transplacental IgG antibodies, the severity is less [37]. Moreover, identifying a successful vaccine that elicits functional neutralizing antibodies to CMV and can prevent congenital CMV has become a priority [40]. Relevant articles discussing the role of the humoral immune response can be found here [39-41].

\section{Cellular Immunity to CMV}

CMV infection commands an overwhelming response from all facets of the immune system. As discussed above, the humoral response and innate response to CMV are indeed significant and 
contribute to controlling the infection, but the cellular immune response is necessary to control latency and impede viral replication in latently infected individuals (Figure 1) [42]. The most compelling evidence for the immunogenicity of CMV involves the cellular arm of the immune system where up to $10 \%$ of all circulating CD8+ $\mathrm{T}$ cells can be directed towards CMV - a staggering number given the plethora of pathogens we encounter in our lifetime [43]. With the extraordinary percentage of $\mathrm{T}$ cells targeting $\mathrm{CMV}$, it has been postulated that over time, immune surveillance is less effective in CMV+ individuals and, although controversial, CMV-seronegative individuals have been reported to live longer than their CMV-seropositive counterparts [44]. Recent reports on the "aging" of the immune response to CMV have begun to shed light on the mechanism behind this observation $[45,46]$.

Figure 1. Cellular and humoral immunity to Cytomegalovirus.

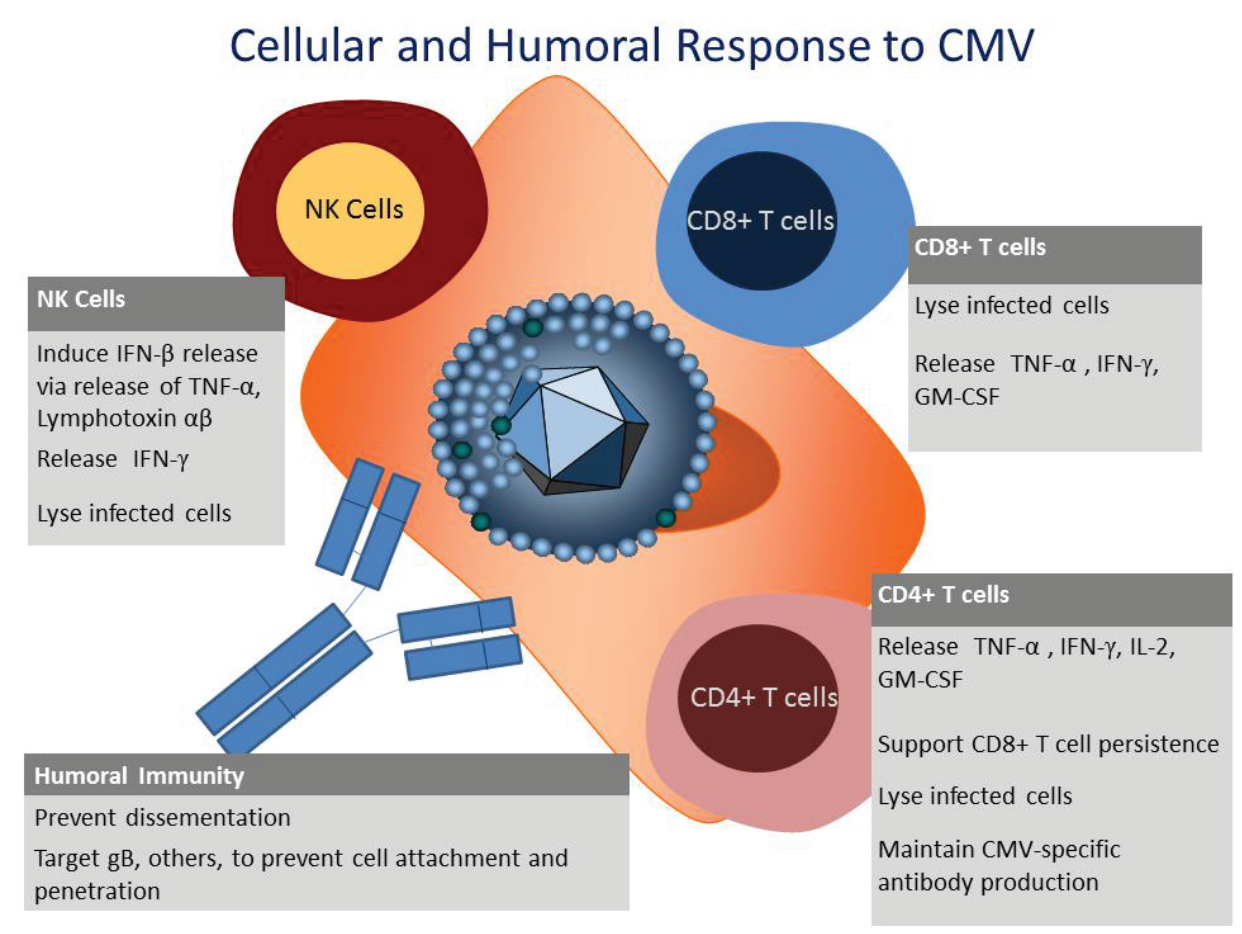

\section{CD8+ T Cell Response to CMV}

The most studied, understood, and manipulated facet of the immune response to CMV is the cellular response, in particular the protective role of CD8 $+\mathrm{T}$ cells. The presence of CMV-specific $\mathrm{T}$ cells was first reported in a SCT study noting that the majority of patients without CMV-specific $\mathrm{T}$ cells had overwhelming CMV disease [47]. This led to studies evaluating the ex vivo expansion of CMV specific T cells from CMV-seropositive individuals [48] and the extensive evaluation of the CMV-specific $\mathrm{T}$ cell response in healthy individuals versus immune compromised populations. The importance of CD8 $+\mathrm{T}$ cells has been reported in solid organ transplant as well [49]. In renal transplant recipients, the presence of $\mathrm{CD} 8+\mathrm{T}$ cells coincides with protection from CMV [50-52] and in both heart and lung transplant recipients, the presence of IE-1-specific $\mathrm{T}$ cells correlated with protection from CMV disease [15]. 
The protectiveness of CD8+ T cells was first shown in a study by Riddell et al. where T cells were expanded ex vivo using CMV-infected fibroblasts [48]. Expanded T cells were then infused into patients after SCT. Riddell et al. reported that prior to the infusion of the T cells, the CMV-specific immunity was undetectable in these patients. However, as early as $48 \mathrm{~h}$ after infusion there was a detectable CMV-specific response and after three weeks the CMV-specific $\mathrm{T}$ cells response was as great or greater than CMV-specific immune responses detected in immunocompetent donors $[48,53]$.

The cell mediated immune response targets proteins in all three of these stages of the viral replication cycle: immediate early, early, and late. By targeting immediate-early and early genes, $\mathrm{T}$ cells can protect against reactivation from latent viruses [54]. Although targeting late proteins would typically delay the cellular response to CMV since these proteins are not expressed until 24-h after infection, many of the late proteins are structural proteins such as the tegument protein pp65 which is already present within the virion and can be immediately presented to $\mathrm{T}$ cells by infected APCs.

In spite of the plethora of CMV antigens targeted by the CD8+ T cell response, a hierarchy of antigenic frequency exists, though it varies on the method of detection. Collectively, pp65 and the immediate-early 1 (IE-1) are two of the most targeted antigens of CMV by CD8+ T cells $[55,56]$.

\section{CD4+ T Cell Response to CMV}

As is the case with CD8+ T cells, approximately $9 \%$ of all circulating CD4+ T cells recognize $\mathrm{CMV}$, an astounding number given the immune repertoire devoted to protection from one pathogen [56]. These $\mathrm{T}$ cells recognize at least 125 open reading frames of CMV or $59 \%$ of all open reading frames. Antigen recognition by CD4+ T cells is similar to CD8+ T cells in that $\mathrm{T}$ cells recognizing pp65 are some of the most abundant but $\mathrm{T}$ cells recognizing other antigens such as $\mathrm{gB}$, UL86, pp28, IE-2, UL36, UL48, pp10, UL113, and IRS-1 are also highly abundant [56].

The importance of CD4 $+\mathrm{T}$ cells is apparent in a model of CD4-T cell-depleted mice where mice infected with MCMV had an increased incidence of MCMV reactivation [57]. It has also been reported that $\mathrm{CD} 4+\mathrm{T}$ cells contribute to controlling MCMV in mice depleted of CD8 $+\mathrm{T}$ cells, but viral clearance is significantly delayed in most tissues and is never cleared from the salivary glands where a persistent infection develops [58].

The significance of CD4+ T cells is better understood and supported in HCMV. Lung transplant recipients with low frequencies of CMV-specific CD4+ T cells have difficulty clearing CMV [49]. In a parallel to the finding in mice, children with CMV who have few CD4+ T cells have prolonged shedding of CMV in the salivary glands, and dysfunctional CD4+ $\mathrm{T}$ cells have been reported during primary infection $[59,60]$. Studies from SCT recipients suggest that CD4+ T cells are linked with protection from disease and are necessary for the recovery of donor-derived CD8+ T cells [61]. What is perhaps more interesting is that the persistence of adoptively transferred $\mathrm{T}$ cells has been reported to depend on the presence of CD4+ T helper cells [53].

In the first study of adoptively transferred CMV-specific $\mathrm{T}$ cells (predominantly CD8+), no patients developed CMV viremia but only patients who had detectable CD4+ $\mathrm{T}$ cell responses showed persistence of the transferred CD8 + T cells [53]. In other words, the highly successful trials mentioned above that utilized CD8 $+\mathrm{T}$ cells specific for various antigens of CMV required not only the transferred CD8+ T cells, but endogenous CD4+ cells as well. 
A compelling argument for the role of CD4+ T cells in immunity to HCMV derives from a study conducted by Einsele et al. in which CD4+ T cells (without CD8+ cells) were transferred to antiviral-resistant SCT recipients with CD4+ T cell deficiencies. Remarkably, all patients exhibited rapid antiviral activity and the cells then persisted at levels similar to immunocompetent healthy donors. Einsele et al. observed that the presence of CD4 $+\mathrm{T}$ cells allowed endogenous CD8+ $\mathrm{CMV}$-specific $\mathrm{T}$ cells to expand, in contrast to transferring CD8+ T cells that eventually decline without CD4+ T cell help [61].

\section{CMV Evasion from the Immune System}

Through thousands of years of evolution, CMV and humans have reached a balance whereby CMV is able to transmit virus from host-to-host yet not cause significant pathology to immunocompetent individuals. One way $\mathrm{CMV}$ is able to persist is by employing numerous immune evasion genes that are expressed in both the unique short (US) and unique long (UL) region of the genome (Table 1) [62]. As with all herpes viruses, CMV interferes with MHC class I presentation to $\mathrm{CD} 8+\mathrm{T}$ cells in a number of ways. Interestingly, CMV also appears to stealthily modulate the immune response to itself by using decoys and choosing which epitopes and antigens it allows the immune system to target. More specifically, phosphorylation of IE-1 by pp65 blocks the processing of IE-1 in the proteosome [63]. This evasiveness is likely a reason why IE-1 was not identified earlier as an important immunogen of CMV.

Table 1. CMV genes involved in immune evasion.

\begin{tabular}{|c|c|c|}
\hline Mechanism of evasion & CMV Gene product & Effect on immune system \\
\hline MHC Class I down-regulation [64] & $\begin{array}{l}\text { US2, US3, US6, } \\
\text { US11 }\end{array}$ & $\begin{array}{c}\text { Decreased presentation of CMV } \\
\text { antigens to CD8+ T cells }\end{array}$ \\
\hline CMV-IE-1 sequestration [63] & UL83 (pp65) & $\begin{array}{l}\text { T cells cannot target first genes } \\
\text { expressed upon reactivation }\end{array}$ \\
\hline MHC Class II down-regulation [65-67] & IE/E product & $\begin{array}{c}\text { Decreased presentation of CMV } \\
\text { antigens to CD4+ T cells }\end{array}$ \\
\hline MHC Class I homolog [68] & UL18 & Inhibition of NK cell lysis \\
\hline $\begin{array}{l}\text { Inhibitory receptors, downregulation of } \\
\text { ligands }[69,70]\end{array}$ & UL40, UL16, UL142 & Evasion of NK cells \\
\hline Chemokine receptor [71] & US28 & Immune homing interference \\
\hline IL-10 homolog [1] & UL111a & Immune suppression \\
\hline Inhibitors of apoptosis $[72,73]$ & UL36, UL37 & $\begin{array}{c}\text { Decrease in phagocytosis of } \\
\text { infected cells by APCs }\end{array}$ \\
\hline $\begin{array}{l}\text { Downregulation of MICB } \\
\text { expression }[74,75]\end{array}$ & $\begin{array}{l}\text { MicroRNAs } \\
\text { (miR-UL112) }\end{array}$ & $\begin{array}{c}\text { Decreased recognition by NK cells } \\
\text { and T cells via NKG2D }\end{array}$ \\
\hline
\end{tabular}

Another way CMV disrupts antigen presentation is by disrupting the transporter associated with antigen processing (TAP). US6 binds with high affinity to the ER-lumenal side of the transporter, effectively altering the affinity of TAP for ATP. Overall, at least four US genes are involved in down regulation expression of MHC Class I: US2, US3, US6, and US11 [64,76]. 
Dendritic cells play an important role in the primary immune response to CMV as they orchestrate the priming of naïve $\mathrm{T}$ cells in the lymph nodes. Not surprisingly, CMV targets dendritic cells and halts their maturation, forcing them into a state of functional paralysis and preventing them from presenting CMV antigens to T cells [62,77]. What's more unexpected is that DCs infected with MCMV are not just unresponsive to MCMV, but MCMV-infected DCs do not secrete IL-12 or IL-2 even after treatment with the potent stimulus lipopolysaccharide (LPS) [77]. It has been suggested that CMV not only evades the immune system, but it also suppresses it. Secondary CMV-associated diseases exist and are a result of CMV modulation of the immune response in ways mentioned above. Additional immunomodulatory genes expressed by CMV and their function are listed in Table 1, but for a more comprehensive review of immune evasion by CMV see the reviews cited here [78-80].

\section{Immunotherapy: Adoptive Transfer of CMV-Specific T Cells after Transplant}

As discussed above, some of the most insightful data about the role of $\mathrm{T}$ cells in protection from CMV came from adoptively transferring $\mathrm{T}$ cells to patients who received stem cell transplants. Since the first method of generation CMV-specific T cells that was utilized almost 20 years ago by Riddell et al. using CMV-infected fibroblasts to expand $\mathrm{T}$ cells, numerous other methods have been developed to offer protection after SCT [81]. The most common and perhaps most simple is pulsing antigen presenting cells - typically dendritic cells - with overlapping peptides spanning the entire pp65 antigen [82]. Although efficacious, this technique requires the lengthy and difficult generation of DCs, a non-trivial amount of donor-derived blood, and requires highly trained technicians and expensive equipment and manufacturing facilities.

In 2006, Leen, Bollard, and Rooney et al. generated T cells specific for three viruses (CMV, $\mathrm{EBV}$, and adenovirus) by using a recombinant adenovirus that expressed pp65, hereafter named Ad5f35pp65 [83]. This approach utilized monocytes and EBV transformed LCL transduced with the Ad5f35pp65 vector as APCs, thus obviating the need for high volumes of donor blood and targeted 3 viruses in a single culture. While this strategy was effective in vivo and less labor intensive, the expansion of virus-specific $\mathrm{T}$ cells is still a lengthy process that takes upwards of one month, not including the months it takes to generate EBV-LCL [83].

To circumvent the need for multiple expansions and a lengthy expansion process, Peggs et al. recently described a method where they pulsed leukocytes with overlapping peptides for pp65. Activated $\mathrm{T}$ cells are then selected based upon their secretion of IFN-gamma and then frozen for infusion. Including QA/QC testing, total time to infusion was less than two weeks and the entire selection process took $<24 \mathrm{~h}$. However, the risk of such a rapid manufacturing strategy is GVHD since alloreactive $\mathrm{T}$ cells may be still present in the infused product. Indeed, in the Peggs study, most patients were protected from CMV at a dose of only $1 \times 10^{4} \mathrm{CD} 3+$ cells $/ \mathrm{kg}$ but 8 of 18 patients developed acute GvHD, three of which were grade II or higher. Six patients also experienced chronic GvHD [84].

Memory $\mathrm{T}$ cells are critical in non- $\mathrm{T}$ cell depleted grafts because the virus-specific memory $\mathrm{T}$ cells present in the grafts confer protection against viral infections and reactivation. For this reason, CMV-reactivation is highest when the transplant recipient is CMV-seropositive and the transplant donor is CMV-seronegative, as is often the case in cord blood transplantation and CMV-seronegative 
donors [85]. Memory $\mathrm{T}$ cells are also a valuable resource when expanding virus-specific $\mathrm{T}$ cells ex vivo from seropositive donors as these $\mathrm{T}$ cells can be expanded by simply culturing virus-specific memory $\mathrm{T}$ cells with antigen-presenting cells loaded with the antigen of interest. When transferred to transplant recipients after transplant, these cells have been protective against $\mathrm{CMV}, \mathrm{EBV}$, and adenovirus without severe adverse events (Table 2) $[83,86]$. The challenge, however, has been the ex vivo generation of antigen-specific $\mathrm{T}$ cells from antigen-inexperienced sources of $\mathrm{T}$ cells such as cord blood. Instead of expanding the pre-existing memory $\mathrm{T}$ cell population, naïve $\mathrm{T}$ cells need to be primed in vitro to respond to the antigen of interest [19].

Table 2. Studies of adoptively transferred CMV-specific T cells.

\begin{tabular}{cc}
\hline Group & Method of Expansion/Selection \\
\hline Riddell, 1992, 1995 [48,53] & Expansion using CMV-infected fibroblasts \\
\hline Einsele, 2002 [61] & Expansion with CMV lysate \\
\hline Cobbold, 2005 [87] & Tetramer Selection using magnetic beads \\
\hline Leen, 2006 [83] & $\begin{array}{c}\text { Antigen-presenting cells (Dendritic cells, EBV-LCL) transduced with } \\
\text { an adenoviral vector encoding CMVpp65 }\end{array}$ \\
\hline Micklethwaite, 2008 [86] & Antigen-presenting cells (Dendritic cells) transduced with an \\
adenoviral vector encoding CMVpp65
\end{tabular}

Because of these challenges, only a few reports document the generation of single antigen-specific $\mathrm{T}$ cells from naive donors [90-93]. In an attempt to target 3 viruses simultaneously, our group reported the ability to generate CMV, EBV, and adenovirus-specific CTL from the $20 \%$ fraction of a cord blood unit by using dendritic cells transduced with an Ad5/f35-CMV-pp65 vector as well as the cytokines IL-7, IL-12, and IL-15 [94]. Responding T cells were shown to be derived from the naïve $\mathrm{T}$ cell population and responded to typical and atypical, novel CMV-pp65 epitopes. A clinical trial using CB-derived multi-virus specific $\mathrm{T}$ cells for the prevention and treatment of viral infection after CBT is open and has started to accrue patients. (Clinical Trial \#: NCT01017705) [81,88]. Recently, we and other groups have also reported the ability to generate CMV-specific T cells from CMV-seronegative donors; $[95,96]$ the clinical efficacy of these T cells will be tested in a Phase 1 clinical study (Clinical Trial \#: NCT01945814).

Another option for recipients of $\mathrm{CB}$ and $\mathrm{CMV}$-seronegative donors is the use of third party, CMV-specific $\mathrm{T}$ cells. Thirdy party virus specific $\mathrm{T}$ cells have been evaluated clinically in several trials with promising results [97-101]. Leen et al. recently published a multi-institutional study of best-matched, MULTI virus-specific T cells and reported that responses with third party CTL were similar to those from donor-derived CTL in their previous studies $[83,100]$. This study highlights the importance of epitope recognition when selecting the optimal third party $\mathrm{T}$ cell lines. 


\section{Summary}

The human body has done an exquisite job over thousands of years to prevent or impede the manifestations of CMV and as a result, the majority of the population can live with CMV without knowing they have it. However, when the balance shifts towards CMV reactivation, usually as a result of treatment modalities or in some cases other infections, options are available to treat CMV infection, and the use of immunotherapy is rapidly becoming one of the favored options. The use of third-party, epitope-targeted CMV-specific T cells provides a unique platform similar to other pharmacotherapies in that they are rapidly available, are short lived, are effective, and are not associated with significant toxicities. However, for chronically suppressed patients, such as those undergoing SOT, the use of autologous CMV-specific $\mathrm{T}$ cells might be the ideal solution as long-term protection is necessary. Overall, improvements to $\mathrm{T}$ cell manufacturing technologies will provide a new and widely used treatment for CMV infection after transplant.

\section{Acknowledgments}

This work was supported by a post-doctoral fellowship, PF-13-046-01-LIB from the American Cancer Society awarded to PJH, and CPRIT RO1 RP100469 and NCI PO1 CA148600-02 awards to $\mathrm{CMB}$.

\section{Author Contributions}

P.J.H. and C.M.B. both contributed to writing the manuscript.

\section{Conflicts of Interest}

The authors declare no conflict of interest.

\section{References and Notes}

1. Cheung, A.K.; Gottlieb, D.J.; Plachter, B.; Pepperl-Klindworth, S.; Avdic, S.; Cunningham, A.L.; Abendroth, A.; Slobedman, B. The role of the human cytomegalovirus UL111A gene in down-regulating CD4+ T-cell recognition of latently infected cells: Implications for virus elimination during latency. Blood 2009, 114, 4128-4137.

2. Goodrum, F.D.; Jordan, C.T.; High, K.; Shenk, T. Human cytomegalovirus gene expression during infection of primary hematopoietic progenitor cells: A model for latency. Proc. Natl. Acad. Sci. USA 2002, 99, 16255-16260.

3. Reeves, M.B.; Sinclair, J.H. Analysis of latent viral gene expression in natural and experimental latency models of human cytomegalovirus and its correlation with histone modifications at a latent promoter. J. Gen. Virol. 2010, 91, 599-604.

4. Varnum, S.M.; Streblow, D.N. Monroe, M.E.; Smith, P.; Auberry, K.J.; Pasa-Tolic, L.; Wang, D.; Camp, D.G., Jr.; Rodland, K.; Wiley, S.; et al. Identification of proteins in human cytomegalovirus (HCMV) particles: The HCMV proteome. J. Virol. 2004, 78, 10960-10966.

5. Kalejta, R.F. Tegument proteins of human cytomegalovirus. Microbiol. Mol. Biol. Rev. 2008, 72, 249-265. 
6. Human Herpesviruses: Biology, Therapy, and Immunoprophylaxis; Arvin, A., Campadelli-Fiume, G., Mocarski, E., Moore, P.S., Roizman, B., Whitley, R., Yamanishi, K., Eds.; Cambridge University Press: Cambridge, UK, 2007.

7. Roby, C.; Gibson, W. Characterization of phosphoproteins and protein kinase activity of virions, noninfectious enveloped particles, and dense bodies of human cytomegalovirus. J. Virol. 1986, 59, 714-727.

8. Gandhi, M.K.; Khanna, R. Human cytomegalovirus: Clinical aspects, immune regulation, and emerging treatments. Lancet Infect. Dis. 2004, 4, 725-738.

9. Loewendorf, A.; Benedict, C.A. Modulation of host innate and adaptive immune defenses by cytomegalovirus: Timing is everything. J. Intern. Med. 2010, 267, 483-501.

10. Sissons, J.G.; Bain, M.; Wills, M.R. Latency and reactivation of human cytomegalovirus. J. Infect. 2002, 44, 73-77.

11. Paulus, C.; Nevels, M. The human cytomegalovirus major immediate-early proteins as antagonists of intrinsic and innate antiviral host responses. Viruses 2009, 1, 760-779.

12. Hahn, G.; Jores, R.; Mocarski, E.S. Cytomegalovirus remains latent in a common precursor of dendritic and myeloid cells. Proc. Natl. Acad. Sci. USA 1998, 95, 3937-3942.

13. Gerna, G.; Percivalle, E.; Lilleri, D.; Lozza, L.; Fornara, C.; Hahn, G.; Baldanti, F.; Revello, M.G. Dendritic-cell infection by human cytomegalovirus is restricted to strains carrying functional UL131-128 genes and mediates efficient viral antigen presentation to CD8+ T cells. J. Gen. Virol. 2005, 86, 275-284.

14. Fietze, E.; Prosch, S.; Reinke, P.; Stein, J.; Docke, W.D.; Staffa, G.; Löning, S.; Devaux, S.; Emmrich, F.; von Baehr, R. Cytomegalovirus infection in transplant recipients. The role of tumor necrosis factor. Transplantation 1994, 58, 675-680.

15. Bunde, T.; Kirchner, A.; Hoffmeister, B.; Habedank, D.; Hetzer, R.; Cherepnev, G.; Proesch, S.; Reinke, P.; Volk, H.D.; Lehmkuhl, H.; et al. Protection from cytomegalovirus after transplantation is correlated with immediate early 1 -specific CD8 T cells. J. Exp. Med. 2005, 201, 1031-1036.

16. Kennedy-Nasser, A.A.; Bollard, C.M.; Myers, G.D.; Leung, K.S.; Gottschalk, S.; Zhang, Y.; Liu, H.; Heslop, H.E.; Brenner, M.K.; Krance, R.A. Comparable outcome of alternative donor and matched sibling donor hematopoietic stem cell transplant for children with acute lymphoblastic leukemia in first or second remission using alemtuzumab in a myeloablative conditioning regimen. Biol. Blood Marrow Transplant. 2008, 14, 1245-1252.

17. Mori, T.; Kato, J. Cytomegalovirus infection/disease after hematopoietic stem cell transplantation. Int. J. Hematol. 2010, 91, 588-595.

18. Boeckh, M.; Nichols, W.G. The impact of cytomegalovirus serostatus of donor and recipient before hematopoietic stem cell transplantation in the era of antiviral prophylaxis and preemptive therapy. Blood 2004, 103, 2003-2008.

19. Hanley, P.J.; Cruz, C.R.; Shpall, E.J.; Bollard, C.M. Improving clinical outcomes using adoptively transferred immune cells from umbilical cord blood. Cytotherapy 2010, 12, 713-720. 
20. Ugarte-Torres, A.; Hoegh-Petersen, M.; Liu, Y.; Zhou, F.; Williamson, T.S.; Quinlan, D.; Sy, S.; Roa, L.; Khan, F.; Fonseca, K.; et al. Donor serostatus has an impact on cytomegalovirus-specific immunity, cytomegaloviral disease incidence, and survival in seropositive hematopoietic cell transplant recipients. Biol. Blood Marrow Transplant. 2011, 17, 574-585.

21. Jaskula, E.; Bochenska, J.; Kocwin, E.; Tarnowska, A.; Lange, A. CMV Serostatus of Donor-Recipient Pairs Influences the Risk of CMV Infection/Reactivation in HSCT Patients. Bone Marrow Res. 2012, 2012, 375075.

22. Falagas, M.E.; Paya, C.; Ruthazer, R.; Badley, A.; Patel, R.; Wiesner, R.; Griffith, J.; Freeman, R.; Rohrer, R.; Werner, B.G.; et al. Significance of cytomegalovirus for long-term survival after orthotopic liver transplantation: A prospective derivation and validation cohort analysis. Transplantation 1998, 66, 1020-1028.

23. Paya, C.V. Prevention of cytomegalovirus disease in recipients of solid-organ transplants. Clin. Infect. Dis. 2001, 32, 596-603.

24. Cope, A.V.; Sabin, C.; Burroughs, A.; Rolles, K.; Griffiths, P.D.; Emery, V.C. Interrelationships among quantity of human cytomegalovirus (HCMV) DNA in blood, donor-recipient serostatus, and administration of methylprednisolone as risk factors for HCMV disease following liver transplantation. J. Infect. Dis. 1997, 176, 1484-1490.

25. Sia, I.G.; Wilson, J.A.; Groettum, C.M.; Espy, M.J.; Smith, T.F.; Paya, C.V. Cytomegalovirus (CMV) DNA load predicts relapsing CMV infection after solid organ transplantation. J. Infect. Dis. 2000, 181, 717-720.

26. Boeckh, M. Current antiviral strategies for controlling cytomegalovirus in hematopoietic stem cell transplant recipients: Prevention and therapy. Transpl. Infect. Dis. 1999, 1, 165-178.

27. Shellam, G.R.; Allan, J.E.; Papadimitriou, J.M.; Bancroft, G.J. Increased susceptibility to cytomegalovirus infection in beige mutant mice. Proc. Natl. Acad. Sci. USA 1981, 78, 5104-5108.

28. Biron, C.A.; Byron, K.S.; Sullivan, J.L. Severe herpesvirus infections in an adolescent without natural killer cells. N. Engl. J. Med. 1989, 320, 1731-1735.

29. Iversen, A.C.; Norris, P.S.; Ware, C.F.; Benedict, C.A.; Human, N.K. Cells inhibit cytomegalovirus replication through a noncytolytic mechanism involving lymphotoxin-dependent induction of IFN-beta. J. Immunol. 2005, 175, 7568-7574.

30. Boehme, K.W.; Guerrero, M.; Compton, T. Human cytomegalovirus envelope glycoproteins $\mathrm{B}$ and $\mathrm{H}$ are necessary for TLR2 activation in permissive cells. J. Immunol. 2006, 177, 7094-7102.

31. Rossini, G.; Cerboni, C.; Santoni, A.; Landini, M.P.; Landolfo, S.; Gatti, D.; Gribaudo, G.; Varani, S. Interplay between human cytomegalovirus and intrinsic/innate host responses: A complex bidirectional relationship. Mediat. Inflamm. 2012, 2012, 607276.

32. Bratcher, D.F.; Bourne, N.; Bravo, F.J.; Schleiss, M.R.; Slaoui, M.; Myers, M.G.; Bernstein, D.I. Effect of passive antibody on congenital cytomegalovirus infection in guinea pigs. J. Infect. Dis. 1995, 172, 944-950.

33. Britt, W.J.; Vugler, L.; Butfiloski, E.J.; Stephens, E.B. Cell surface expression of human cytomegalovirus (HCMV) gp55-116 (gB): Use of HCMV-recombinant vaccinia virus-infected cells in analysis of the human neutralizing antibody response. J. Virol. 1990, 64, 1079-1085. 
34. Gonczol, E.; deTaisne, C.; Hirka, G.; Berencsi, K.; Lin, W.C.; Paoletti, E.; Plotkin, S. High expression of human cytomegalovirus (HCMV)-gB protein in cells infected with a vaccinia-gB recombinant: The importance of the gB protein in HCMV immunity. Vaccine 1991, 9, 631-637.

35. Stagno, S.; Pass, R.F.; Cloud, G.; Britt, W.J.; Henderson, R.E.; Walton, P.D.; Veren, D.A.; Page, F.; Alford, C.A. Primary cytomegalovirus infection in pregnancy. Incidence, transmission to fetus, and clinical outcome. J. Am. Med. Assoc. (JAMA) 1986, 256, 1904-1908.

36. Revello, M.G.; Gerna, G. Diagnosis and management of human cytomegalovirus infection in the mother, fetus, and newborn infant. Clin. Microbiol. Rev. 2002, 15, 680-715.

37. Schleiss, M.R. Cytomegalovirus in the neonate: Immune correlates of infection and protection. Clin. Dev. Immunol. 2013, 2013, 501801.

38. Krause, P.R.; Bialek, S.R.; Boppana, S.B.; Griffiths, P.D.; Laughlin, C.A.; Ljungman, P.; Ljungman, P.; Mocarski, E.S.; Pass, R.F.; Read, J.S.; et al. Priorities for CMV vaccine development. Vaccine 2013, 32, 4-10.

39. Alonso Arias, R.; Moro-Garcia, M.A.; Echeverria, A.; Solano-Jaurrieta, J.J.; Suarez-Garcia, F.M.; Lopez-Larrea, C. Intensity of the humoral response to cytomegalovirus is associated with the phenotypic and functional status of the immune system. J. Virol. 2013, 87, 4486-4495.

40. Zhu, J.; Shearer, G.M.; Marincola, F.M.; Norman, J.E.; Rott, D.; Zou, J.P.; Epstein, S.E. Discordant cellular and humoral immune responses to cytomegalovirus infection in healthy blood donors: Existence of a Th1-type dominant response. Int. Immunol. 2001, 13, 785-790.

41. Landini, M.P.; Lazzarotto, T.; Xu, J.; Geballe, A.P.; Mocarski, E.S. Humoral immune response to proteins of human cytomegalovirus latency-associated transcripts. Biol. Blood Marrow Transplant. 2000, 6, 100-108.

42. Moss, P.; Rickinson, A. Cellular immunotherapy for viral infection after HSC transplantation. Nat. Rev. Immunol. 2005, 5, 9-20.

43. Crough, T.; Khanna, R. Immunobiology of human cytomegalovirus: From bench to bedside. Clin. Microbiol. Rev. 2009, 22, 76-98.

44. Olsson, J.; Wikby, A.; Johansson, B.; Lofgren, S.; Nilsson, B.O.; Ferguson, F.G. Age-related change in peripheral blood T-lymphocyte subpopulations and cytomegalovirus infection in the very old: The Swedish longitudinal OCTO immune study. Mech. Ageing Dev. 2000, 121, $187-201$.

45. Spielmann, G.; Bollard, C.M.; Bigley, A.B.; Hanley, P.J.; Blaney, J.W.; Lavoy, E.C.; Pircher, H.; Simpson, R.J. The effects of age and latent cytomegalovirus infection on the redeployment of $\mathrm{CD} 8+\mathrm{T}$ cell subsets in response to acute exercise in humans. Brain Behav. Immunity 2013, doi:10.1016/j.bbi.2013.05.003.

46. Simpson, R.J.; Lowder, T.W.; Spielmann, G.; Bigley, A.B.; LaVoy, E.C.; Kunz, H. Exercise and the aging immune system. Ageing Res. Rev. 2012, 11, 404-420.

47. Reusser, P.; Riddell, S.R.; Meyers, J.D.; Greenberg, P.D. Cytotoxic T-lymphocyte response to cytomegalovirus after human allogeneic bone marrow transplantation: Pattern of recovery and correlation with cytomegalovirus infection and disease. Blood 1991, 78, 1373-1380.

48. Riddell, S.R.; Watanabe, K.S.; Goodrich, J.M.; Li, C.R.; Agha, M.E.; Greenberg, P.D. Restoration of viral immunity in immunodeficient humans by the adoptive transfer of $\mathrm{T}$ cell clones. Science 1992, 257, 238-241. 
49. Sester, U.; Gartner, B.C.; Wilkens, H.; Schwaab, B.; Wossner, R.; Kindermann, I.; Girndt, M.; Meyerhans, A.; Mueller-Lantzsch, N.; Schäfers, H.J. Differences in CMV-specific T-cell levels and long-term susceptibility to CMV infection after kidney, heart and lung transplantation. Am. J. Transplant. 2005, 5, 1483-1489.

50. Radha, R.; Jordan, S.; Puliyanda, D.; Bunnapradist, S.; Petrosyan, A.; Amet, N.; Toyoda, M. Cellular immune responses to cytomegalovirus in renal transplant recipients. Am. J. Transplant. 2005, 5, 110-117.

51. Reusser, P.; Cathomas, G.; Attenhofer, R.; Tamm, M.; Thiel, G. Cytomegalovirus (CMV)-specific $\mathrm{T}$ cell immunity after renal transplantation mediates protection from CMV disease by limiting the systemic virus load. J. Infect. Dis. 1999, 180, 247-253.

52. Sester, M.; Sester, U.; Gartner, B.C.; Girndt, M.; Meyerhans, A.; Kohler, H. Dominance of virus-specific CD8 T cells in human primary cytomegalovirus infection. J. Am. Soc. Nephrol. (JASN) 2002, 13, 2577-2584.

53. Walter, E.A.; Greenberg, P.D.; Gilbert, M.J.; Finch, R.J.; Watanabe, K.S.; Thomas, E.D.; Riddell, S.R. Reconstitution of cellular immunity against cytomegalovirus in recipients of allogeneic bone marrow by transfer of T-cell clones from the donor. N. Engl. J. Med. 1995, 333, 1038-1044.

54. Hanley, P.J.; Shaffer, D.R.; Cruz, C.R.; Ku, S.; Tzou, B.; Liu, H.; Demmler-Harrison, G.; Heslop, H.E.; Rooney, C.M.; Gottschalk, S.; et al. Expansion of T cells targeting multiple antigens of cytomegalovirus, Epstein-Barr virus and adenovirus to provide broad antiviral specificity after stem cell transplantation. Cytotherapy 2011, 13, 976-986.

55. Wills, M.R.; Carmichael, A.J.; Mynard, K.; Jin, X.; Weekes, M.P.; Plachter, B.; Sissons, J.G. The human cytotoxic T-lymphocyte (CTL) response to cytomegalovirus is dominated by structural protein pp65: Frequency, specificity, and T-cell receptor usage of pp65-specific CTL. J. Virol. 1996, 70, 7569-7579.

56. Sylwester, A.W.; Mitchell, B.L.; Edgar, J.B.; Taormina, C.; Pelte, C.; Ruchti, F.; Sleath, P.R.; Grabstein, K.H.; Hosken, N.A.; Kern, F.; et al. Broadly targeted human cytomegalovirus-specific CD4+ and CD8+ T cells dominate the memory compartments of exposed subjects. J. Exp. Med. 2005, 202, 673-685.

57. Polic, B.; Hengel, H.; Krmpotic, A.; Trgovcich, J.; Pavic, I.; Luccaronin, P.; Jonjić, S.; Koszinowski, U.H. Hierarchical and redundant lymphocyte subset control precludes cytomegalovirus replication during latent infection. J. Exp. Med. 1998, 188, 1047-1054.

58. Jonjic, S.; Pavic, I.; Lucin, P.; Rukavina, D.; Koszinowski, U.H. Efficacious control of cytomegalovirus infection after long-term depletion of CD8+ T lymphocytes. J. Virol. 1990, 64, 5457-5464.

59. Tu, W.; Chen, S.; Sharp, M.; Dekker, C.; Manganello, A.M.; Tongson, E.C.; Maecker, H.T.; Holmes, T.H.; Wang, Z.; Kemble, G.; et al. Persistent and selective deficiency of CD4+ T cell immunity to cytomegalovirus in immunocompetent young children. J. Immunol. 2004, 172, 3260-3267.

60. Antoine, P.; Olislagers, V.; Huygens, A.; Lecomte, S.; Liesnard, C.; Donner, C.; Marchant, A. Functional exhaustion of CD4+ $\mathrm{T}$ lymphocytes during primary cytomegalovirus infection. J. Immunol. 2012, 189, 2665-2672. 
61. Einsele, H.; Roosnek, E.; Rufer, N.; Sinzger, C.; Riegler, S.; Loffler, J.; Grigoleit, U.; Moris, A.; Rammensee, H.G.; Kanzm L.; et al. Infusion of cytomegalovirus (CMV)-specific T cells for the treatment of CMV infection not responding to antiviral chemotherapy. Blood 2002, 99, 3916-3922.

62. Lehner, P.J.; Wilkinson, G.W. Cytomegalovirus: From evasion to suppression? Nat. Immunol. 2001, 2, 993-994.

63. Gilbert, M.J.; Riddell, S.R.; Plachter, B.; Greenberg, P.D. Cytomegalovirus selectively blocks antigen processing and presentation of its immediate-early gene product. Nature 1996, 383, 720-722.

64. Park, B.; Oh, H.; Lee, S.; Song, Y.; Shin, J.; Sung, Y.C.; Hwang, S.Y.; Ahn, K. The MHC class I homolog of human cytomegalovirus is resistant to down-regulation mediated by the unique short region protein (US)2, US3, US6, and US11 gene products. J. Immunol. 2002, $168,3464-3469$.

65. Miller, D.M.; Rahill, B.M.; Boss, J.M.; Lairmore, M.D.; Durbin, J.E.; Waldman, J.W.; Sedmak, D.D. Human cytomegalovirus inhibits major histocompatibility complex class II expression by disruption of the Jak/Stat pathway. J. Exp. Med. 1998, 187, 675-683.

66. Le Roy, E.; Muhlethaler-Mottet, A.; Davrinche, C.; Mach, B.; Davignon, J.L. Escape of human cytomegalovirus from HLA-DR-restricted CD4(+) T-cell response is mediated by repression of gamma interferon-induced class II transactivator expression. J. Virol. 1999, 73, 6582-6589.

67. Tomazin, R.; Boname, J.; Hegde, N.R.; Lewinsohn, D.M.; Altschuler, Y.; Jones, T.R.; Cresswell, P.; Nelson, J.A.; Riddell, S.R.; Johnson, D.C. Cytomegalovirus US2 destroys two components of the MHC class II pathway, preventing recognition by CD4+ T cells. Nat. Med. 1999, 5, 1039-1043.

68. Reyburn, H.T.; Mandelboim, O.; Vales-Gomez, M.; Davis, D.M.; Pazmany, L.; Strominger, J.L. The class I MHC homologue of human cytomegalovirus inhibits attack by natural killer cells. Nature 1997, 386, 514-517.

69. Wang, E.C.; McSharry, B.; Retiere, C.; Tomasec, P.; Williams, S.; Borysiewicz, L.K.; Braud, V.M.; Wilkinson, G.W. UL40-mediated NK evasion during productive infection with human cytomegalovirus. Proc. Natl. Acad. Sci. USA 2002, 99, 7570-7575.

70. Dunn, C.; Chalupny, N.J.; Sutherland, C.L.; Dosch, S.; Sivakumar, P.V.; Johnson, D.C.; Cosman, D. Human cytomegalovirus glycoprotein UL16 causes intracellular sequestration of NKG2D ligands, protecting against natural killer cell cytotoxicity. J. Exp. Med. 2003, 197, 1427-1439.

71. Casarosa, P.; Bakker, R.A.; Verzijl, D.; Navis, M.; Timmerman, H.; Leurs, R.; Martine, J.S. Constitutive signaling of the human cytomegalovirus-encoded chemokine receptor US28. J. Biol. Chem. 2001, 276, 1133-1137.

72. Skaletskaya, A.; Bartle, L.M.; Chittenden, T.; McCormick, A.L.; Mocarski, E.S.; Goldmacher, V.S. A cytomegalovirus-encoded inhibitor of apoptosis that suppresses caspase-8 activation. Proc. Natl. Acad. Sci. USA 2001, 98, 7829-7834. 
73. Zhang, A.; Hildreth, R.L.; Colberg-Poley, A.M. Human cytomegalovirus inhibits apoptosis by proteasome-mediated degradation of Bax at endoplasmic reticulum-mitochondrion contacts. J. Virol. 2013, 87, 5657-5668.

74. Stern-Ginossar, N.; Elefant, N.; Zimmermann, A.; Wolf, D.G.; Saleh, N.; Biton, M.; Horwitz, E.; Prokocimer, Z.; Prichard, M.; Hahn, G.; et al. Host immune system gene targeting by a viral miRNA. Science 2007, 317, 376-381.

75. Stern-Ginossar, N.; Gur, C.; Biton, M.; Horwitz, E.; Elboim, M.; Stanietsky, N.; Mandelboim, M.; Mandelboim, O. Human microRNAs regulate stress-induced immune responses mediated by the receptor NKG2D. Nat. Immunol. 2008, 9, 1065-1073.

76. Mocarski, E.S., Jr. Immunomodulation by cytomegaloviruses: Manipulative strategies beyond evasion. Trends Microbiol. 2002, 10, 332-339.

77. Andrews, D.M.; Andoniou, C.E.; Granucci, F.; Ricciardi-Castagnoli, P.; Degli-Esposti, M.A. Infection of dendritic cells by murine cytomegalovirus induces functional paralysis. Nat. Immunol. 2001, 2, 1077-1084.

78. Noriega, V.; Redmann, V.; Gardner, T.; Tortorella, D. Diverse immune evasion strategies by human cytomegalovirus. Immunol. Res. 2012, 54, 140-151.

79. Miller-Kittrell, M.; Sparer, T.E. Feeling manipulated: Cytomegalovirus immune manipulation. Virol. J. 2009, 6, doi:10.1186/1743-422X-6-4.

80. Jackson, S.E.; Mason, G.M.; Wills, M.R. Human cytomegalovirus immunity and immune evasion. Virus Res. 2011, 157, 151-160.

81. Saglio, F.; Hanley, P.J.; Bollard, C.M. The time is now: Moving toward virus-specific T cells after allogeneic hematopoietic stem cell transplantation as the standard of care. Cytotherapy 2014, 16, 149-159.

82. Gerdemann, U.; Katari, U.L.; Papadopoulou, A.; Keirnan, J.M.; Craddock, J.A.; Liu, H.; Martinez, C.A.; Kennedy-Nasser, A.; Leung, K.S.; Gottschalk, S.M.; et al. Safety and clinical efficacy of rapidly-generated trivirus-directed $\mathrm{T}$ cells as treatment for adenovirus, EBV, and CMV infections after allogeneic hematopoietic stem cell transplant. Mol. Ther. 2013, 21, 2113-2121.

83. Leen, A.M.; Myers, G.D.; Sili, U.; Huls, M.H.; Weiss, H.; Leung, K.S.; Carrum, G.; Krance, R.A.; Chang, C.C.; Molldrem, J.J.; et al. Monoculture-derived T lymphocytes specific for multiple viruses expand and produce clinically relevant effects in immunocompromised individuals. Nat. Med. 2006, 12, 1160-1166.

84. Peggs, K.S.; Thomson, K.; Samuel, E.; Dyer, G.; Armoogum, J.; Chakraverty, R.; Pang, K.; Mackinnon, S.; Lowdell, M.W. Directly selected cytomegalovirus-reactive donor T cells confer rapid and safe systemic reconstitution of virus-specific immunity following stem cell transplantation. Clin. Infect. Dis. 2011, 52, 49-57.

85. Kroger, N.; Zabelina, T.; Kruger, W.; Renges, H.; Stute, N.; Schrum, J.; Kabisch, H.; Schafhausen, P.; Jaburg, N.; Löliger, C.; et al. Patient cytomegalovirus seropositivity with or without reactivation is the most important prognostic factor for survival and treatment-related mortality in stem cell transplantation from unrelated donors using pretransplant in vivo T-cell depletion with anti-thymocyte globulin. Br. J. Haematol. 2001, 113, 1060-1071. 
86. Micklethwaite, K.P.; Clancy, L.; Sandher, U.; Hansen, A.M.; Blyth, E.; Antonenas, V.; Sartor, M.M.; Bradstock, K.F.; Gottlieb, D.J. Prophylactic infusion of cytomegalovirus-specific cytotoxic T lymphocytes stimulated with Ad5f35pp65 gene-modified dendritic cells after allogeneic hemopoietic stem cell transplantation. Blood 2008, 112, 3974-3981.

87. Cobbold, M.; Khan, N.; Pourgheysari, B.; Tauro, S.; McDonald, D.; Osman, H.; Assenmacher, M.; Billingham, L.; Colin. Adoptive transfer of cytomegalovirus-specific CTL to stem cell transplant patients after selection by HLA-peptide tetramers. J. Exp. Med. 2005, 202, 379-386.

88. Hanley, P.J.; Martinez, C.; Leung, K.; Savoldo, B.; Dotti, G.; Brenner, M.K.; Rooney, C.; Heslop, H.; Krance, R.; Shpall, E.J.; et al. Improving Immune Reconstitution after cord blood transplantation using ex vivo expanded virus-specific $\mathrm{T}$ cells: A phase I clinical study. ASH Annu. Meet. Abstr. 2013, 15, S21.

89. Blyth, E.; Clancy, L.; Simms, R.; Ma, C.K.; Burgess, J.; Deo, S.; Byth, K.; Dubosq, M.C.; Shaw, P.J.; Micklethwaite, K.P.; et al. Donor-derived CMV-specific T cells reduce the requirement for $\mathrm{CMV}$-directed pharmacotherapy after allogeneic stem cell transplantation. Blood 2013, 121, 3745-3758.

90. Sun, Q.; Burton, R.L.; Pollok, K.E.; Emanuel, D.J.; Lucas, K.G. CD4(+) Epstein-Barr virus-specific cytotoxic T-lymphocytes from human umbilical cord blood. Cell. Immunol. 1999, 195, 81-88.

91. Savoldo, B.; Cubbage, M.L.; Durett, A.G.; Goss, J.; Huls, M.H.; Liu, Z.; Teresita, L.; Gee, A.P.; Ling, P.D.; Brenner, M.K.; et al. Generation of EBV-specific CD4+ cytotoxic T cells from virus naive individuals. J. Immunol. 2002, 168, 909-918.

92. Park, K.D.; Marti, L.; Kurtzberg, J.; Szabolcs, P. In vitro priming and expansion of cytomegalovirus-specific Th1 and Tc1 T cells from naive cord blood lymphocytes. Blood 2006, 108, 1770-1773.

93. Hanley, P.J.; Lam, S.; Shpall, E.J.; Bollard, C.M. Expanding cytotoxic T lymphocytes from umbilical cord blood that target cytomegalovirus, Epstein-Barr virus, and adenovirus. J. Vis. Exp. 2012, e3627, doi:10.3791/3627.

94. Hanley, P.J.; Cruz, C.R.; Savoldo, B.; Leen, A.M.; Stanojevic, M.; Khalil, M.; Decker, W.; Molldrem, J.J.; Liu, H.; Gee, A.P.; et al. Functionally active virus-specific T cells that target CMV, adenovirus, and EBV can be expanded from naive T-cell populations in cord blood and will target a range of viral epitopes. Blood 2009, 114, 1958-1967.

95. Jedema, I.; van de Meent, M.; Pots, J.; Kester, M.G.; van der Beek, M.T.; Falkenburg, J.H. Successful generation of primary virus-specific and anti-tumor T-cell responses from the naive donor T-cell repertoire is determined by the balance between antigen-specific precursor T cells and regulatory T cells. Haematologica 2011, 96, 1204-1212.

96. Hanley, P.J.; Cruz, R.Y.; Melenhorst, J.; Scheinberg, J.; Blaney, J.; Savoldo, B.; Dotti, G.; Heslop, H.; Rooney, C.M.; Shpall, E.J.; et al. Naive T-cell-derived CTL recognize atypical epitopes of CMVpp65 with higher avidity than CMV-seropositive donor-derived CTL-A basis for treatment of post-transplant viral infection by adoptive transfer of T-cells from virus-naive donors. Cytotherapy 2013, 15, S9. 
97. Barker, J.N.; Doubrovina, E.; Sauter, C.; Jaroscak, J.J.; Perales, M.A.; Doubrovin, M.; Prockop, S.E.; Koehne, G.; O’Reilly, R.J. Successful treatment of EBV-associated posttransplantation lymphoma after cord blood transplantation using third-party EBV-specific cytotoxic T lymphocytes. Blood 2010, 116, 5045-5049.

98. Doubrovina, E.; Oflaz-Sozmen, B.; Prockop, S.E.; Kernan, N.A.; Abramson, S.; Teruya-Feldstein, J.; Hedvat, C.; Chou, J.F.; Heller, G.; Barker, J.N.; et al. Adoptive immunotherapy with unselected or EBV-specific $\mathrm{T}$ cells for biopsy-proven EBV+ lymphomas after allogeneic hematopoietic cell transplantation. Blood 2012, 119, 2644-2656.

99. Uhlin, M.; Okas, M.; Gertow, J.; Uzunel, M.; Brismar, T.B.; Mattsson, J. A novel haplo-identical adoptive CTL therapy as a treatment for EBV-associated lymphoma after stem cell transplantation. Cancer Immunol. Immunother. (CII) 2010, 59, 473-437.

100. Leen, A.M.; Bollard, C.M.; Mendizabal, A.M.; Shpall, E.J.; Szabolcs, P.; Antin, J.H.; Kapoor, N.; Pai, S.Y.; Rowley, S.D.; Kebriaei, P.; et al. Multicenter study of banked third-party virus-specific $\mathrm{T}$ cells to treat severe viral infections after hematopoietic stem cell transplantation. Blood 2013, 121, 5113-5123.

101. Qasim, W.; Derniame, S.; Gilmour, K.; Chiesa, R.; Weber, M.; Adams, S.; Rao, K.; Amrolia, P.; Goulden, N. Third-party virus-specific $\mathrm{T}$ cells eradicate adenoviraemia but trigger bystander graft-versus-host disease. Br. J. Haematol. 2011, 154, 150-153. 

Reprinted from Viruses. Cite as: Stevenson, E.V.; Collins-McMillen, D.; Kim, J.H.; Cieply, S.J.; Bentz, G.L.; Yurochko, A.D. HCMV Reprogramming of Infected Monocyte Survival and Differentiation: A Goldilocks Phenomenon. Viruses 2014, 6, 782-807.

Review

\title{
HCMV Reprogramming of Infected Monocyte Survival and Differentiation: A Goldilocks Phenomenon
}

\author{
Emily V. Stevenson ${ }^{1,2}$, Donna Collins-McMillen ${ }^{1,2}$, Jung Heon Kim ${ }^{1,2}$, Stephen J. Cieply ${ }^{1,2}$, \\ Gretchen L. Bentz ${ }^{1, \dagger}$ and Andrew D. Yurochko ${ }^{1,2,3, *}$
}

1 Department of Microbiology and Immunology, Louisiana State University Health Sciences Center-Shreveport, Shreveport, LA 71130, USA; E-Mails: esteve@1suhsc.edu (E.V.S.); dcol12@1suhsc.edu (D.C.-M.); jkim4@1suhsc.edu (J.H.K.); sciepl@1suhsc.edu (S.J.C.); Bentz_GL@Mercer.edu (G.L.B.)

2 Center for Molecular and Tumor Virology, Louisiana State University Health Sciences Center-Shreveport, Shreveport, LA 71130, USA

3 Feist-Weiller Cancer Center, Louisiana State University Health Sciences Center-Shreveport, Shreveport, LA 71130, USA

$\dagger$ Present address: Division of Basic Medical Sciences, Macon Campus, Mercer University School of Medicine, 1550 College Street, Macon, GA 31207, USA.

* Author to whom correspondence should be addressed; E-Mail: ayuroc@1suhsc.edu; Tel.: +1-318-675-8332.

Received: 23 December 2013; in revised form: 4 February 2014 / Accepted: 4 February 2014 / Published: 13 February 2014

\begin{abstract}
The wide range of disease pathologies seen in multiple organ sites associated with human cytomegalovirus (HCMV) infection results from the systemic hematogenous dissemination of the virus, which is mediated predominately by infected monocytes. In addition to their role in viral spread, infected monocytes are also known to play a key role in viral latency and life-long persistence. However, in order to utilize infected monocytes for viral spread and persistence, HCMV must overcome a number of monocyte biological hurdles, including their naturally short lifespan and their inability to support viral gene expression and replication. Our laboratory has shown that HCMV is able to manipulate the biology of infected monocytes in order to overcome these biological hurdles by inducing the survival and differentiation of infected monocytes into long-lived macrophages capable of supporting viral gene expression and replication. In this current review, we describe the unique aspects of how HCMV promotes monocyte survival and differentiation by inducing a "finely-tuned" macrophage cell
\end{abstract}


type following infection. Specifically, we describe the induction of a uniquely polarized macrophage subset from infected monocytes, which we argue is the ideal cellular environment for the initiation of viral gene expression and replication and, ultimately, viral spread and persistence within the infected host.

Keywords: HCMV; monocyte; macrophage; polarization; survival; signaling; receptor-ligand; viral persistence; viral dissemination

\section{Introduction}

Human cytomegalovirus (HCMV), a ubiquitous betaherpesvirus that infects $60 \%-90 \%$ of the population worldwide, establishes a lifelong infection of the host [1]. The disease manifestations associated with HCMV infection are dependent upon the immune status of the host. In healthy individuals, primary infection with HCMV is typically asymptomatic, although mononucleosis-like symptoms have been reported [2,3]. Long-term infection with HCMV, however, is associated with chronic inflammation, which links HCMV infection to the development of cardiovascular diseases and some types of cancers in otherwise immunocompetent individuals [3-5]. In contrast, HCMV infection causes severe morbidity and mortality in immunocompromised individuals, including AIDS patients, transplant recipients, and developing fetuses [3,6]. In these susceptible populations lacking normal immune control of the virus, HCMV infection can lead to a severe disease state that is characterized by multi-organ system involvement [7].

The ability of HCMV to establish persistent infection within the host and the wide range of associated disease pathologies in multiple organ sites are a result of the systemic dissemination of the virus following primary infection. The dissemination strategy of HCMV involves a hematogenous step that is mediated predominately by infected peripheral blood monocytes. There are multiple lines of evidence supporting a specific role for monocytes in the hematogenous dissemination of HCMV. An HCMV cell-associated viremia is known to occur early after infection $[8,9]$ and HCMV viral DNA is found predominately in peripheral blood monocytes and polymorphonuclear leukocytes (PMNs), rather than in lymphocytes [10-12]. Both monocytes and PMNs have been shown to play a role in the transfer of HCMV to blood vessel endothelial cells, suggesting a role for both cell types in the spread of HCMV [11]. Neither cell type is able to support viral replication [11]; however, viral gene expression and replication have been identified in monocyte-derived macrophages [13-16]. Furthermore, monocytes are the most prevalent infiltrating cell type found within HCMV-infected tissues [11,17], and a critical role for monocytes in the dissemination strategy of HCMV has been clearly defined in an animal model using the related murine CMV [18]. Based on these studies and others (reviewed in [19,20]), our laboratory focused on primary HCMV infection and the specific role monocytes play in viral dissemination [20,21]. We argue that monocytes serve as "Trojan Horses" to carry HCMV to multiple organ sites in the absence of de novo viral gene expression, as monocytes are initially non-permissive for viral gene expression and replication and only become permissive upon their differentiation into macrophages [20-27]. 
Monocytes/myeloid cells are not only key to viral spread following primary infection, but are central to the entire viral persistence strategy, as myeloid progenitors have been shown to be critical for the establishment of viral latency within the bone marrow [8,10,28-30]. Furthermore, reactivation of the virus occurs in these latently infected myeloid precursor cells that then leave the bone marrow as differentiated monocytes - thus also serving as a source of life-long periodic viral shedding [31-33]. Therefore, cells of the monocytic lineage play a defined and crucial role in the overall success of the virus in establishing a productive and persistent infection within the host and also in the transfer of HCMV to new hosts. Our recent studies have focused on determining how $\mathrm{HCMV}$ is able to manipulate the biology of newly infected monocytes during primary infection in order to promote the viral dissemination required for productive and persistent infection.

\section{HCMV Rapidly Changes the Monocyte Cellular Environment Following Primary Infection to Establish a Pro-Viral Inflammatory Phenotype that Mediates Viral Dissemination}

Due to the immune surveillance function of peripheral blood monocytes, this cell type can readily migrate through the blood vessel endothelium and infiltrate into peripheral organs, making infected monocytes ideal candidates for widespread delivery of the virus to nearly all organ tissues. Monocytes possess, however, a short lifespan of approximately 1-3 days within the bloodstream [34,35]. As previously mentioned, newly infected monocytes do not support de novo viral lytic gene expression or replication [36], thus new viral gene products are not synthesized that can alter the cellular environment to favor the success of the virus within the cell, as seen in other cell types infected by HCMV [37-41]. Despite this lack of new viral gene expression, our laboratory has observed rapid signaling and activation-induced changes in infected monocytes following viral binding that result in the production of a pro-inflammatory monocyte phenotype that favors viral spread [21-23,27,42-44]. Furthermore, we have shown that these phenotypic changes are mediated by the signaling that results from viral engagement of the epidermal growth factor receptor (EGFR) and the $\beta_{1}$ and $\beta_{3}$ integrins on the surface of monocytes $[20,24-26,45,46]$. Based on our recent studies $[20,25,26,45,46]$ and the work of others [47-49], we have gained a better understanding of the early binding events that occur during HCMV infection of monocytes and how these events translate into the activation of cellular signaling pathways and functional changes in infected monocytes that promote the viral dissemination and persistence strategy [20,21].

During primary infection of monocytes, initial viral tethering likely occurs through engagement of glycoprotein $\mathrm{M}(\mathrm{gM}) / \mathrm{gN}$ to heparin sulfate proteoglycans [50]. This initial viral tethering event is then followed by the binding of the $\mathrm{gH} / \mathrm{gL} / \mathrm{UL} 128-131$ complex to specific proteinaceous receptors, the $\beta_{1}$ and $\beta_{3}$ integrins [26,46] and the binding of other viral glycoprotein(s), such as gB to EGFR on the surface of monocytes [45]. These receptor-binding events then trigger the activation of signaling cascades downstream of EGFR and integrins. Our data suggest that there are both distinct and overlapping signaling events that mediate viral entry into monocytes and that initiate specific monocyte biological changes $[20,25,26,45,46]$. For instance, the binding to and activation of both EGFR and integrins are required for viral entry into monocytes $[26,45,46]$. Moreover, the activation of both EGFR and integrins by HCMV is required for increased monocyte cellular motility, which is essential for the overall dissemination strategy of HCMV [26,45]. However, although both EGFR and integrin signaling are required for HCMV-induced enhanced 
monocyte motility, our data indicate that HCMV activation of EGFR alone promotes the upregulation of an actin cytoskeletal regulator, N-WASP, which we showed to be required for enhanced monocyte motility [45]; while activation of integrins alone promotes the activation and upregulation of the actin cytoskeletal regulator, paxillin, which we show also induces monocyte motility and plays a key role in the viral entry process [26]. These data suggest that EGFR and integrin signaling events overlap to promote HCMV-induced monocyte motility through actin cytoskeletal rearrangement, although the specific signaling cascades downstream of these receptors regulate distinct cellular molecules. We also note that the requirement of both EGFR and integrin signaling for actin cytoskeletal rearrangement in the process of viral entry into monocytes links the receptor-mediated activation of cellular motility to the viral entry process, as the cytoskeletal events regulating enhanced motility also regulate viral entry [26,45,46]. The EGFR- and integrin-mediated enhanced motility of infected monocytes, when paired with a virus-induced increase in monocyte adhesion molecules and in monocyte transendothelial migration, likely allows the infected monocyte to exit the bloodstream and to enter peripheral organ tissues [22,23]. Furthermore, HCMV-induced activation of EGFR alone results in the prolonged survival of infected monocytes by altering the normal cellular apoptotic machinery through the prolonged expression of Mcl-1, providing the virus with a longer-lived cellular host [22,25]. The enhanced survival of infected monocytes is also potentiated by HCMV-induced differentiation of infected monocytes to naturally long-lived tissue macrophages within the organ tissues [21,24]. Following the differentiation of infected monocytes to macrophages, viral replication can then commence $[21,51,52]$, resulting in progeny virions capable of infecting the necessary surrounding cell types required to promote viral shedding to other hosts $[13,21]$ or capable of infecting myeloid progenitor cells within the bone marrow to establish the latent infection required for lifelong viral persistence within the host $[19,28,29,32]$.

These earlier studies highlight the ability of HCMV to alter the biological function of infected monocytes to promote changes that are critical for the outcome of the viral life cycle and for the viral persistence strategy. Furthermore, our studies demonstrate that the HCMV-induced changes in infected monocytes are initiated by cellular surface receptor binding-induced signaling in the cell, in the absence of de novo viral gene expression. In fact, newer studies have uncovered that a high degree of regulatory control is utilized by HCMV during infection of monocytes through the utilization of these two separate cellular receptors, EGFR and integrins, to promote a "finely-tuned" monocyte/macrophage cell type that is required for viral spread, replication, and persistence.

\section{Precise Regulation of the Monocyte/Macrophage Apoptotic Program Is Required for HCMV-Induced Monocyte-to-Macrophage Differentiation}

We have shown that HCMV effectively initiates molecular changes that reprogram infected monocytes to establish a pro-viral phenotype that mediates viral dissemination. We suggest that for the virus to successfully have evolved to infect monocytes and utilize them as "Trojan Horses" for the spread of HCMV to target organs, the virus had to overcome at least two significant biological obstacles. First, HCMV had to adapt to the naturally short lifespan of monocytes of around 48 to 72 hours in circulation [34,35], a scenario that is particularly problematic for HCMV, with its slow DNA replication cycle of several days to weeks in vivo [53]. Second, the virus had to adapt to the 
differentiation barrier, because as stated previously, monocytes are not initially permissive for de novo viral gene expression and replication [14,52,53], while their differentiated counterparts, macrophages, are permissive [21]. We have evidence that HCMV navigates these biological hurdles by extending the lifespan of infected monocytes and directing their differentiation into long-lived macrophages, which are capable of supporting viral replication and the production of progeny virions $[21,24,25,44]$. In this manner, it appears that HCMV has evolved a mechanism to molecularly bridge the survival-differentiation hurdle in order to generate a long-lived cell type that serves as a reservoir for viral persistence in host organ tissue.

In order to promote the extended survival of HCMV-infected monocytes, HCMV must escape both the intrinsic programming that causes monocytes to die within 48-72 hours of entering circulation [34,35] and the cellular antiviral pro-apoptotic response [54]. Because monocytes are not productive for viral gene expression and replication, viral immediate early proteins do not participate in the rescue of infected monocytes from various apoptotic mechanisms. Accordingly, the virus appears to have evolved a mechanism whereby it relies on cellular Bcl-2 family anti-apoptotic proteins in order to navigate critical cellular checkpoints and to escape apoptosis. The Bcl-2 family of proteins consists of multiple mitochondrial membrane permeabilization regulators that function to either promote or inhibit apoptosis [55]. Anti-apoptotic Bcl-2 members (Mcl-1, Bcl-2, and Bcl-xL) have been reported to mediate survival of myeloid cells under some conditions [56-61]. Our laboratory recently reported that Mcl-1 functions to mediate the early survival of infected monocytes [25]. Monocytes have a biologically limited lifespan of about 48-72 hours in the circulation, during which they must make a cell fate decision to either undergo monocyte-to-macrophage differentiation [24,25] or to fulfill their natural "default" biological programming and undergo apoptosis. Under homeostatic conditions, monocytes exhibit high levels of Mcl-1 upon entry into circulation and upon isolation in our system, followed by a significant reduction in Mcl-1 protein levels over their 48-72 hour lifespan [25]. The decline of pro-survival Mcl-1 between 48 and 72 hours post infection is consistent with the in vivo lifespan of these cells and appears to constitute a potential molecular viability gate, which monocytes must negotiate in order to escape their intrinsic apoptotic fate [25]. Furthermore, this expression pattern suggests that, under homeostatic conditions, Mcl-1 may function as a molecular clock to ensure a controlled population of unstimulated short-lived monocytes [25]. These data are consistent with what is known about Mcl-1 and its anti-apoptotic function in myeloid leukemia cells [62-64]. The Mcl-1 gene was originally identified as being over-expressed in a human myeloid leukemia cell line during monocyte-to-macrophage differentiation [62]. Mcl-1 has since been identified as a member of the Bcl-2 gene family that, under homeostatic conditions, serves to regulate two important myeloid cell processes: survival and differentiation [63,65]. Because of its pro-survival function in cells, over-expression of Mcl-1 enhances the survival of cancerous cells and contributes to drug resistance in a variety of cancers [66-69]. Our data suggest that HCMV usurps the pro-survival function of Mcl-1 to promote the enhanced survival of infected monocytes. In contrast to uninfected monocytes, Mcl-1 levels remain stable through the first 24 hours following HCMV infection; after 24 hours, Mcl-1 levels in infected monocytes decline until expression finally reaches the same levels as those observed in mock-infected cells at 72 hours post infection [25]. By extending the expression of Mcl-1 in HCMV-infected monocytes when compared to their 
mock-infected counterparts, HCMV infection allows these cells to navigate the cell viability checkpoint at 48 hours post infection. Thus, HCMV appears to upregulate Mcl-1 expression in infected monocytes to overcome the intrinsic apoptotic clock that controls the monocyte population under homeostatic conditions, in order to extend the lifespan of the infected cell [25]. In support, siRNA-mediated knock down of Mcl-1 levels leads to a reversal of the apoptotic resistance observed in HCMV-infected monocytes, confirming that Mcl-1 is responsible for the enhanced survival of monocytes during the initial 24-48 hours following infection [25].

Molecularly, survival and differentiation are distinct processes in monocytes that are, nonetheless, intimately linked. Prolonged survival of monocytes is necessary to afford the time required for differentiation to occur; however, an excess of pro-survival signals can block differentiation [70]. Thus, the survival and differentiation programs in monocytes appear to represent a "Goldilocks" phenomenon whereby only precise expression levels of the cellular products involved in both survival and differentiation will result in effective monocyte-to-macrophage differentiation. In fact, myeloid cell differentiation has been shown to require low level expression of multiple "pro-apoptotic" players, particularly the caspases [70]. For example, caspase-3 and caspase-8 play a significant role in monocyte-to-macrophage differentiation induced by stimulation with macrophage colony-stimulating factor (M-CSF): both caspases are required for differentiation to occur [70]. However, the expression of caspase-3 and caspase- 8 is lower during differentiation than during apoptosis [70], suggesting a threshold level of caspase expression exists that is necessary for differentiation, while remaining low enough to avoid triggering apoptosis. These studies implicate that the process of monocyte-to-macrophage differentiation may represent, molecularly, an incomplete apoptosis-like program.

In support, recent work from our laboratory has shown that HCMV controls monocyte-to-macrophage differentiation through the partial activation of caspase-3 [24]. Furthermore, we demonstrated that, prior to the 48-hour cell fate checkpoint, Mcl-1 acts to inhibit the cleavage and activation of caspase-3, which in turn promotes monocyte survival [24]. However, once infected monocytes survive to and through the 48-hour viability gate, the loss of Mcl-1 allows a basal activation of caspase-3 that is required for monocyte-to-macrophage differentiation [24]. Thus, by differentially regulating Mcl-1 expression levels, HCMV walks a fine line between survival and death; between differentiation and non-differentiation, to create the long-lived macrophage that is central to its viral survival/persistence strategy.

Our more recent studies investigating the prolonged survival of HCMV-infected monocytes have further illustrated the involvement of a possible incomplete apoptosis-like program in HCMV-induced monocyte-to-macrophage differentiation. During our analysis of a panel of assays to identify apoptotic cell death, we identified that despite the well-characterized enhanced survival of HCMV-infected monocytes [24,25], HCMV-infected cells did not exhibit a decrease in the expression of several apoptosis-associated markers as would be expected, but rather showed an increase in some quantifiable phenomena associated with apoptosis (Figure 1). Flow cytometric analysis of Annexin V and propidium iodide (PI) staining in HCMV-infected versus mock-infected monocytes at 72 hours post infection revealed a greater than two fold increase in the percentage of Annexin V positive, PI negative cells in HCMV-infected monocytes (Figure 1A). Cells staining as Annexin $\mathrm{V}$ positive, PI negative are prototypically considered to be in the early stages of apoptosis, 
because Annexin V binds to phosphatidyl serine (PS), an inner membrane component that flips to the outer membrane of cells during apoptosis [71]. However, the presence of PS on the outer membrane of cells is also a natural occurrence during monocyte-to-macrophage differentiation, and PS on the outer membrane is required for the phagocytic function of macrophages [72]. With an already documented increase in cell viability during HCMV infection of monocytes [45] and in the absence of an increase in PI staining, which would represent true cell death; the increase in the number of cells expressing PS (by Annexin V staining) in HCMV-infected monocytes likely represents the larger number of cells undergoing monocyte-to-macrophage differentiation that we have previously documented [21,24]. Furthermore, quantification of fluorescent cells in images captured by an IncuCyte ZOOM ${ }^{\mathrm{TM}}$ analyzer of HCMV-infected versus mock-infected monocytes that were treated with CellPlayerTM 96 -well Caspase-3/7 reagent (which fluorescently labels cells containing activated or cleaved caspase-3/7) over a timecourse of 72 hours indicated an increase in the number of cells containing cleaved caspase-3/7 in HCMV-infected cells compared to mock-infected cells (Figure 1B). We previously showed that HCMV-infected monocytes had increased levels of the full-length $32 \mathrm{kDa}$ and partially cleaved $20-\mathrm{kDa}$ forms of caspase-3, while showing similar levels of the fully-cleaved $17 \mathrm{kDa}$ form of caspase-3, when compared to mock-infected monocytes by three days post infection [24]. Additionally, we have observed an increase in caspase-7 expression and cleavage in infected monocytes 3-5 days post infection [73]. Although the activation of caspase-3/7 is typically indicative of an apoptotic process in cells, our previous data showed that caspase-3 activity was required for HCMV-induced monocyte-to-macrophage differentiation [24]; however, we have not yet addressed a potential role for caspase-7 in the differentiation of infected monocytes to macrophages. With a defined role for caspase-3 activation in myeloid cell differentiation $[24,64]$ and the recently documented role for caspase-7 in the differentiation of dental hard tissue producing cells, such as odontoblasts and ameloblasts [74], we suggest that the increase in the number of cells expressing activated caspase-3 and -7 in HCMV-infected monocytes, again reflects a greater number of cells undergoing monocyte-to-macrophage differentiation, rather than apoptosis. To verify that HCMV-infected monocytes exhibited lower amounts of apoptosis than their uninfected counterparts despite the measurable increase in PS expression and caspase-3/-7 activation, we performed a DNA laddering assay to detect the presence of fragmented DNA (a hallmark of complete apoptosis) at 72 hours (data not shown) and 120 hours post infection (a time that we could identify any cellular apoptosis that may potentially be initiated at 72 hours) (Figure 1C). We observed a small amount of DNA laddering in HCMV-infected monocytes (Figure 1C, Lane 3), indicating that some HCMV-infected cells had undergone apoptosis. However, we identified that uninfected monocytes (Figure 1C, Lane 2) had an increased amount of DNA laddering when compared to HCMV-infected monocytes (Figure 1C, Lane 3), indicating the presence of increased apoptosis in uninfected monocytes. Therefore, it appears that although multiple biological changes occur during HCMV infection of monocytes that have been linked to the initiation of an apoptotic cascade, full apoptosis does not occur in the majority of HCMV-infected monocytes. We argue that the process of macrophage differentiation likely requires the specific regulation of a partial apoptotic-like cascade (involving multiple steps including the activation of caspases and the surface expression of PS) that drives the myeloid differentiation process to a 
"point of no return" for the macrophage, but that does not result in a full apoptotic response that would result in cell death.

Figure 1. Human cytomegalovirus (HCMV) induces the upregulation of apoptosis-associated markers in the absence of increased apoptosis. Primary human peripheral blood monocytes were isolated as previously described [22,44]. Monocytes were mock- or HCMV-infected (Towne/E p.41 MOI 5) for 72 hours at $37{ }^{\circ} \mathrm{C}$ in $5 \%$ $\mathrm{CO}_{2}$, and kept either in suspension (A and $\mathbf{C}$ ) or adherent in a 96 well plate (B) in $1 \%$ human serum RPMI 1640 (Cellgro). (A) At 72 hours post infection, monocytes were harvested, stained with Annexin V-FITC and PI, and analyzed by flow cytometry. The average percentage of Annexin V positive, PI negative cells in each sample from three separate donors is represented. Student's paired t-test was used to determine statistical significance $(* p<0.05)$. (B) At the time of infection, triplicate samples were treated with $5 \mu \mathrm{M}$ CellPlayerTM 96-Well Caspase-3/-7 reagent. Samples were placed in an IncuCyte ${ }^{\mathrm{TM}}$ Zoom live imaging system at $37^{\circ} \mathrm{C}$ and $5 \% \mathrm{CO}_{2}$. Phase contrast and green fluorescence images of each sample at identical areas were acquired every six hours for 72 hours. The number of green objects detected per image was normalized to the number of cells detected by phase contrast per respective image. The resulting green object count for each sample is represented. (C) At 120 hours post infection, fragmented DNA was harvested from samples using a Calbiochem ${ }^{\circledR}$ Suicide Track $^{\mathrm{TM}}$ DNA Ladder Isolation kit. Isolated DNA was run on a $1.5 \%$ agarose/TAE gel at 50 constant volts. The gel was then stained with ethidium bromide and apoptotic DNA was visualized by UV illumination. All samples were run on the same gel under the same conditions; however, a lane containing an irrelevant treatment option was removed for the final figure. Lane 1 contains the $1 \mathrm{~kb}$ DNA standard ladder, Lane 2 contains DNA from mock-infected monocytes, and Lane 3 contains DNA from HCMV-infected monocytes.

A.

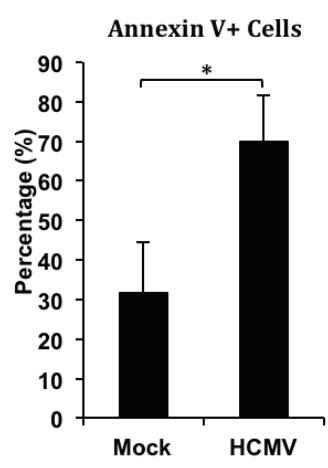

C.

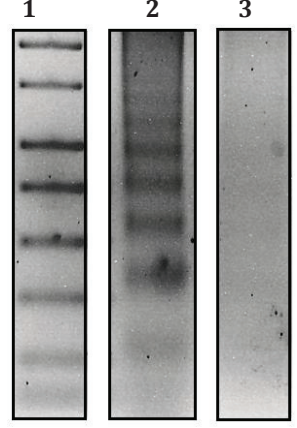

B.

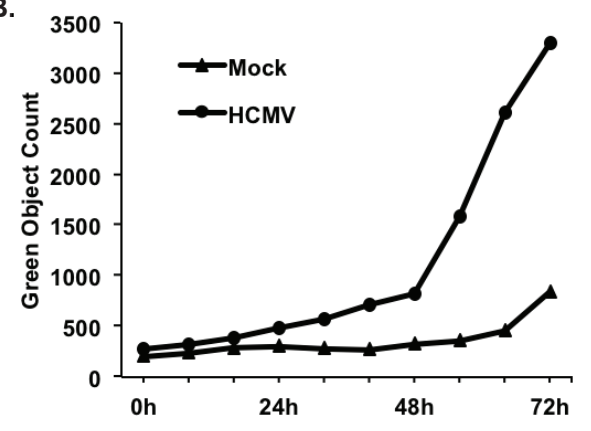




\section{HCMV-Induced Monocyte-to-Macrophage Differentiation Is Required for Viral Replication and for Long-Term Persistent Infection}

Infection of monocytes by HCMV was initially thought to represent a non-productive and abortive infection for the virus because monocytes do not support viral replication $[8,12,36]$. However, as previously discussed, HCMV-infected monocytes are clearly implicated in the overall dissemination of HCMV within the infected host [8-12,17,18]. The circulating surveillance function of monocytes within the bloodstream makes this highly motile cell type ideal for delivery of the virus to multiple organ tissues, but in the absence of viral replication, infection of monocytes by HCMV would appear to serve no productive function for the overall viral persistence strategy. On the other hand, viral replication has been shown to occur in monocyte-derived macrophages [13-16,75] and in macrophages from infected individuals [13,76]. However, differentiated macrophages are not found within the bloodstream and can, therefore, not play a predominant role in the hematogenous dissemination strategy of HCMV during primary infection. Our laboratory demonstrated that HCMV is able to promote the transendothelial migration of infected monocytes to facilitate their exit out of the bloodstream and entry into the surrounding tissues [21,22]. Studies of a mouse model of CMV infection support the extravasation of infected monocytes out of the bloodstream and their movement into organ tissues [77]. In this model, a primarily mononuclear cell-associated CMV viremia was identified in the infected mice; that was then followed by a dissemination of the virus to the liver and spleen [77]. Furthermore, we showed that primary infection of monocytes by HCMV drives the differentiation of infected monocytes into macrophages that can then support viral replication and the release of infectious virions $[21,24,78,79]$. Although infected monocytes appear to be driven towards differentiation into macrophages within hours following infection [78,79], the complete productive monocyte-to-macrophage differentiation process appears to take up to two weeks post infection [21]. HCMV-infected monocytes exhibit gene expression changes that are associated with macrophage differentiation as early as four hours post infection [78,79] and have an upregulation of macrophage markers, such as CD68, as early as 24 hours post infection [21]. HCMV-infected monocytes gain an increase in macrophage-associated phagocytosis function by three days post infection [21]. The infected monocyte/macrophage begins to express the macrophage differentiation marker, HLA-DR, in low levels by four days post infection, with increasing expression levels that peak around two weeks post infection [21]. The HCMV immediate early (IE) gene products, IE1 and IE2, and the late gene product, $\mathrm{gH}$, can then be detected within these fully differentiated macrophages between 2-3 weeks post infection, which coincides with the release of infectious virus into the macrophage supernatant $[21,46]$. Together, these data demonstrate that primary infected peripheral blood monocytes not only serve as viral carriers for hematogenous dissemination, but that they also serve as sources of viral persistence through their production of progeny virions.

The release of newly produced infectious virions by HCMV-infected monocytes that have undergone differentiation into tissue macrophages would allow the virus to establish a long-term infection within peripheral organ tissue. It is likely that this viral persistence in macrophages links HCMV infection to the overt organ pathology observed in immunocompromised hosts. Newly released infectious virions within various host organs would then be able to infect additional 
recruited myeloid cells and nearby epithelial cells at sites of bodily fluid excretion in order to facilitate chronic viral shedding and passage to new hosts [20,22]. In support, following the initiation of viral gene expression around two weeks post infection, we detect the release of infectious HCMV into the cellular supernatant at around three weeks post infection, which we showed continues through five weeks post infection [21]. Furthermore, we now have data indicating that HCMV-induced differentiation of infected monocytes results in a long-lived macrophage phenotype that becomes a long-term persistent source for the production of infectious HCMV (Table 1). In fact, HCMV-infected monocyte-derived macrophages secreted infectious virus into the cellular supernatant for at least 16 weeks post infection, albeit at very low levels (Table 1). Therefore, when paired with our previous data indicating that HCMV drives the motility and transendothelial migration [21,26,45] and the prolonged survival [25] and differentiation of infected monocytes $[21,22,24]$, these data suggest that primary infected peripheral blood monocytes serve a critical role in the overall long-term persistence strategy of HCMV. Through the induction of these highly specific biological changes in infected monocytes, HCMV promotes the establishment of a long-lived macrophage phenotype that supports the chronic production of infectious virus, likely contributing to continuous shedding of the virus for months after initial infection. Clinical data support that chronic shedding of the virus following primary infection is a common occurrence, particularly among children in daycare settings, who have been shown to shed the virus for up to 23 months [80]. Furthermore, because monocytes/macrophages are the primary cell type involved in carrying reactivated HCMV, the replication of reactivated HCMV within these cells may contribute to the observed chronic shedding of HCMV in the breast milk of postpartum mothers [81]. Therefore, the replication of HCMV within differentiated monocytes/macrophages likely serves as a critical component for the long-term production and shedding of HCMV in both primary and reactivated infections.

Table 1. Long-term release of HCMV from infected monocytes/macrophages. Monocytes were mock- or HCMV-infected (Towne/E MOI 5) and then cultured for 16 weeks. At the indicated times post infection, cell supernatants were collected and added to fibroblasts. The presence or absence of detectable IE1 expression was determined by immunohistochemistry staining using an anti-IE1 antibody (Millipore MAB810), Histostain-Plus Kit (Invitrogen cat. 859043), and AEC Substrate Kit (Invitrogen cat. 00-2007) at 24 hours post infection, as an indication for productive virus release by the long-term infected cells. "-" represents the absence of released virus and the numbers represent the calculated plaque forming units per 500 macrophages (averaged from two donors).

\begin{tabular}{cccccc}
\hline Treatment & $\mathbf{2}$ weeks & $\mathbf{4}$ weeks & $\mathbf{8}$ weeks & $\mathbf{1 2}$ weeks & $\mathbf{1 6}$ weeks \\
\hline Mock & - & - & - & - & - \\
HCMV & - & 20 & 26 & 30 & 10 \\
\hline
\end{tabular}




\section{HCMV Reprograms the Polarization of Infected Monocytes towards a Unique Macrophage Phenotype in Order to Promote Viral Replication and Persistence}

Recent advances in macrophage biology research have uncovered that the differentiation of monocytes into macrophages can result in a wide spectrum of resulting phenotypes that have a variety of biological functions [82-85]. These macrophage phenotypes have been categorized broadly into "M1", or classically-activated, pro-inflammatory macrophages, and "M2", or alternatively-activated, anti-inflammatory macrophages, although the phenotypes classified within these categories are broadly pleotropic [82-85]. Differentiation towards an M1 macrophage phenotype typically occurs following activation by interferon- $\gamma$ (IFN- $\gamma$ ), granulocyte-macrophage colony-stimulating factor (GM-CSF), tumor necrosis factor (TNF), or lipopolysaccharide (LPS), resulting in a host defense or tumor resistance phenotype [83-85]. M1 macrophages are characterized by their secretion of large amounts of proinflammatory cytokines, including interleukin (IL)-6, IL-12, IL-23, and TNF $[84,86,87]$. On the other hand, differentiation towards an M2 macrophage phenotype typically occurs following activation by IL-4, IL-10, IL-13, or M-CSF, resulting in a regulatory, anti-parasitic, wound healing, or tumor promotion phenotype [83-85]. M2 macrophages are characterized by the secretion of very low levels of inflammatory cytokines and the production of high amounts of IL-10 [84,86,87]. Moreover, M2 macrophages have been further classified into three subcategories (M2a, M2b, and M2c), which differ by stimulating cytokines, upregulated macrophage markers, cytokine production, and distinct biological function [84]. For example, M2a macrophages are stimulated by IL-4 and IL-13, express scavenger receptors on their surfaces, and function primarily in anti-parasitic activity or allergic inflammation; M2b macrophages are stimulated by Toll-like receptors or IL-1 receptors, express CD86, secrete TNF, IL-1, and IL-6, and are involved in the regulation of immune responses; and M2c macrophages are stimulated by IL-10, secrete TGF- $\beta$, and are involved in tissue remodeling [84]. Therefore, the specific activating ligand involved in the stimulation of monocytes can greatly influence the overall differentiation and polarization of monocytes to macrophages.

Regardless of stimuli, the process of monocyte-to-macrophage differentiation results in a series of cellular phenotypes that have some degree of shared functional and/or cytokine expression traits along with distinct characteristics. Thus, due to the wide range of potential differentiation-inducing ligands, the overall resulting macrophage cell type under various stimuli represents a diversity of potential cell types. Our laboratory was among the first to demonstrate that a virally-induced monocyte-to-macrophage differentiation process occurs and that it is likely required for viral replication and long-term viral persistence $[21,24,78,79]$. Because macrophages can be polarized to function generally as either pro-inflammatory/anti-microbial or anti-inflammatory/pro-tumorigenic, the specific type of macrophage polarization that results during HCMV infection of monocytes would have significant implications for viral dissemination and persistence. Our laboratory's data indicate that HCMV induces the pro-inflammatory activation of infected monocytes, which we argue is critical for viral spread within the host $[21,23,45]$. However, the induction of a pro-inflammatory or M1 phenotype typically results in an anti-microbial functional that is characteristic of a host immune response $[83,84,88]$, which would likely be detrimental to the viral life cycle. Nonetheless, because of the plasticity of macrophage differentiation along with our data that the stimulation of 
macrophage differentiation by LPS results in a macrophage phenotype that is distinct from HCMV-induced macrophage differentiation [21], we suggest that HCMV utilizes this plasticity to regulate the polarization of infected monocytes/macrophages towards a distinct phenotype that ensures successful viral survival and persistence. One mechanism to test this notion of a distinct cell type is global transcriptome microarray analyses. We used this global transcriptome approach paired with an analysis of secreted chemokine analysis to decipher the distinct polarization of HCMV-infected monocytes/macrophages [78,79]. These studies identified that HCMV-infected monocytes exhibit a unique reprogramming of their differentiation and polarization in which both M1- and M2-associated genes are expressed and chemokines are secreted [78,79]. Specifically, we identified that subsets of M1-associated gene transcripts (65\%) and M2-associated gene transcripts (4\%) were upregulated during HCMV infection of monocytes, and subsets of M1-associated chemokines (44\%) and M2-associated chemokines (33\%) were more abundant in infected monocytes. These data implicate that HCMV-infected monocytes/macrophages fall somewhere along the macrophage polarization continuum to resemble a more "M1-like" pro-inflammatory phenotype $[78,79]$. We propose that HCMV specifically modulates the polarization of infected monocytes/macrophages to induce an activated, mostly M1-like phenotype in order to promote the pro-inflammatory changes (such as adhesion, motility, and survival) that are required for effective viral dissemination while dampening a potential anti-viral immune response by promoting the upregulation of key M2-associated genes (such as IL-10 and CCL18).

To expand our microarray analyses, we have focused on the identification of specific gene expression changes that occur during monocyte-to-macrophage differentiation and polarization under various stimuli. These studies were designed to allow us to determine specific genes that are key to the overall differentiation of monocytes to macrophages, regardless of stimuli, and moreover, to identify the specific subsets of gene expression changes that are shared among HCMV-infected and M1 or M2 polarized macrophages. We hoped from these studies to gain a better understanding of the similarities and differences of $\mathrm{HCMV}$-induced macrophage polarization versus traditional M1 or M2 polarization. To address this question, monocytes were mock- or HCMV-infected, or treated with LPS (to induce M1 polarization) or M-CSF (to induce M2 polarization), for two weeks. At two weeks, RNA was harvested for microarray analysis. These new microarray analyses have uncovered the differences in the overall gene expression profile and the changes that occur during the differentiation of HCMV-infected versus LPS-treated (M1) versus M-CSF-treated (M2) macrophages, when compared to mock-infected monocytes/macrophages (Figure 2A,B). Our data indicate that the general ligand-induced monocyte-to-macrophage differentiation process includes a subset of gene expression changes that is present regardless of stimulating ligand or polarization (upregulation of 2,455 genes; downregulation of 450 genes) (Figure 2A,B). That is, there is a core set of likely "pan" macrophage genes induced and/or reduced. However, the differentiation of monocytes towards an M1 or M2 macrophage phenotype also resulted in a large pool of distinct gene expression profiles. That is, we observed a large number of gene expression changes that appear unique to the specific macrophage polarization phenotype (upregulation of 739 M-CSF-specific genes and 588 LPS-specific genes; downregulation of 961 M-CSF-specific genes and 685 LPS-specific genes) (Figure 2A,B). Furthermore, these analyses revealed that HCMV-induced monocyte-to-macrophage differentiation results in subsets of gene expression 
changes that are shared with either M1 or M2 macrophages, with more genes shared with the M1 macrophage polarization phenotype (upregulation of 936 LPS-shared genes; downregulation of 634 LPS-shared genes) than the M2 macrophage phenotype (upregulation of $422 \mathrm{M}$-CSF-shared genes; downregulation of 305 M-CSF-shared genes) (Figure 2A,B). We also observed a distinct subset of gene expression changes in infected monocytes/macrophages that were not observed in either M1 or M2 polarized macrophages (upregulation of 1,371 HCMV-specific genes; downregulation of 1,263 HCMV-specific genes) (Figure 2A,B), suggesting that the HCMV-induced differentiation program of infected monocytes truly represents a unique differentiation phenotype.

Figure 2. HCMV infection of monocytes induces a unique subset of gene expression changes when compared to monocytes polarized towards an M1 or M2 phenotype. Monocytes were mock- or HCMV-infected (Towne/E p.41 MOI 5), or treated with LPS $(100 \mathrm{ng} / \mathrm{mL})$ or M-CSF $(100 \mathrm{ng} / \mathrm{mL})$ for two weeks at $37{ }^{\circ} \mathrm{C}$ and $5 \% \mathrm{CO}_{2}$ under adherent conditions. At two weeks, RNA was harvested from each sample. Harvested RNA was used to perform microarray analysis on Affymetrix U133 Plus 2.0 genechips. Probe sets to represent individual genes were selected by using JetSet [89]. The genes that were upregulated (A) or downregulated (B) ( $\geq 2$ fold change when compared to mock-infected monocytes) were compared between treatments and the number of unique or shared genes among samples is represented.

A.

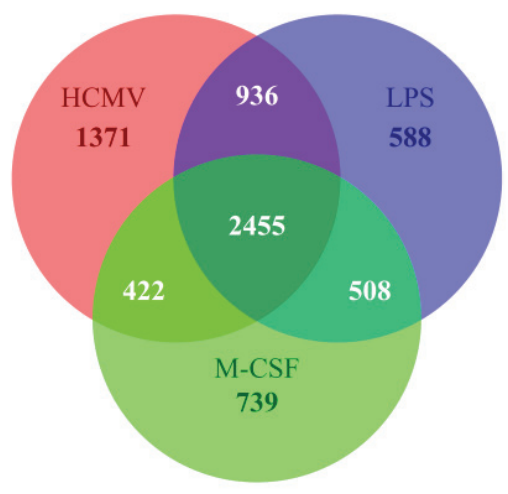

B.

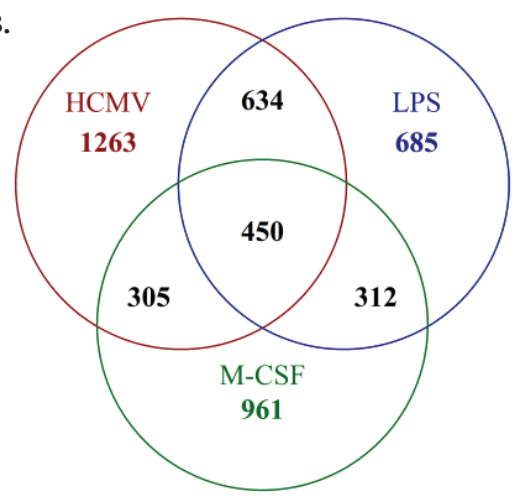

In support of our gene array analyses, flow cytometric analysis revealed an increase in the expression of both M1- and M2-associated macrophage markers in HCMV-infected monocytes when compared to mock-infected monocytes at five days post infection (Figure 3). Of note-although uninfected monocytes are programmed to die between 48-72 hours in circulation, a certain percentage of cells $(\sim 40 \%)$ receive enough stimuli in our in vitro culture system to survive and undergo a small degree of differentiation. However, when compared to the percentage of cells found in HCMV-infected monocytes ( $>80 \%$ survival), it is clear that infection serves as both a survival and a differentiation stimuli. Analysis of co-staining with CD71 (a general macrophage marker), CD86 (a cell surface marker that is typically expressed on M1 macrophages), and CD163 (a cell surface marker that is typically expressed on M2 macrophages) revealed that HCMV infection of monocytes induces the upregulation of specific macrophage markers, resulting in a larger number of cells within the doublet staining macrophage subsets. In addition, the macrophage marker expression profile of HCMV-infected monocytes was distinct from that seen with M-CSF- or GM-CSF-treated monocytes, suggesting that various stimulating ligands induce distinct macrophage phenotype 
populations (data not shown). Furthermore, analyses of the expression of select M1- and M2-associated genes in monocytes/macrophages at 24 and 72 hours following treatment with M-CSF, GM-CSF or LPS or infection with HCMV supported the finding that HCMV infection of monocytes/macrophages results in a distinct polarization profile that shares some characteristics with M1 and/or M2 macrophages (data not shown). However, further studies involving additional donors are required to effectively decipher gene profile changes that occur during macrophage polarization between 24 and 72 hours. Taken together, these data demonstrate that HCMV-infection of monocytes/macrophages drives their differentiation/polarization towards a macrophage phenotype that shares some characteristics with M1-polarized macrophages and M2-polarized macrophages, but that, overall, represents a distinct polarization phenotype; that is, the specific HCMV-induced polarization phenotype does not fall into the category of strictly "M1" or "M2", but exists somewhere along the M1/M2 continuum, skewed towards a more M1-like phenotype.

Figure 3. HCMV infection of monocytes induces a macrophage polarization phenotype that is distinct from M1 or M2 polarization. Monocytes were mock- or HCMV-infected (Towne/E p.41 MOI 5) at $37{ }^{\circ} \mathrm{C}$ and $5 \% \mathrm{CO}_{2}$ for five days. Monocytes/macrophages were washed with PBS, blocked in 5\% fetal bovine serum and stained with Alexa fluor-conjugated antibodies specific for CD14 (a myeloid specific marker), CD71 (a macrophage specific marker), CD86 (an M1 macrophage associated marker), or CD163 (an M2 macrophage associated marker). Cells were then analyzed by flow cytometry and gated by forward and side scatter and for CD14 positive cells. Scatter quadrant plots were generated indicating the number of cells expressing the various staining profiles.
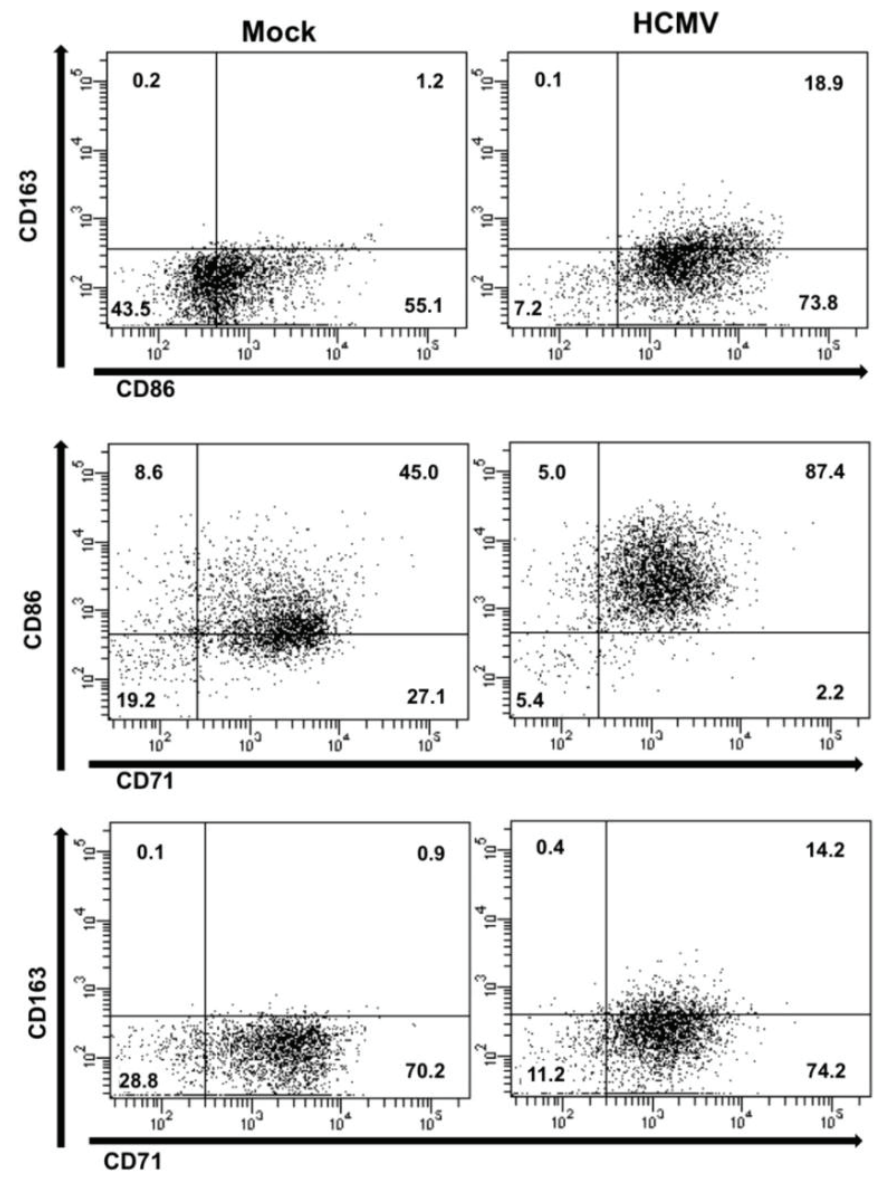
Overall, we propose that this unique macrophage polarization phenotype uncovered by our studies reflects the manipulation of the differentiation and polarization program of infected monocytes by HCMV to promote a macrophage phenotype that is required for the overall persistence strategy of the virus. We predict that the early pro-inflammatory phenotype of HCMV-infected monocytes/macrophages regulates the motility and migration of the infected cells in order to promote their exit out of the bloodstream, and it helps to trigger the pro-survival signals necessary to overcome the cell's intrinsic apoptotic program (inflammatory activation is known to induce prolonged monocyte survival [90]). However, because some inflammatory cytokines have also been linked to apoptosis in Mycobacterium-infected monocytes [90], the expression of some M2-associated anti-inflammatory genes in HCMV-infected monocytes likely helps to dampen the cell's intrinsic apoptotic host response to infection. Through the specific regulation of the differentiation and polarization of infected monocytes/macrophages, we argue that HCMV promotes the specific cellular environment that is required for viral replication and overall persistence. This notion is supported by studies involving the infection of already differentiated M1 or M2 macrophages by HCMV, which indicate that although both macrophage phenotypes are susceptible to HCMV infection, HCMV is able to infect a higher percentage of M2 macrophages than M1 macrophages, indicating that M2 macrophages are more susceptible to HCMV infection [91,92]. These studies also indicated that HCMV induced the secretion of pro-inflammatory cytokines and chemokines in both M1 and M2 macrophages following infection, but that infected M2 macrophages released a higher amount of infectious virus [93]. These data suggest that HCMV not only induces the polarization of differentiating monocytes, but also alters the polarization profile of already differentiated macrophages by inducing the expression of pro-inflammatory molecules. Furthermore, these data suggest that the expression of specific M2-associated molecules confers some type of advantage for the virus during infection, indicating that the specific macrophage polarization phenotype in infected cells may determine the overall outcome of HCMV replication and gene expression. In support, it was shown that the specific phenotype of differentiated macrophages resulting from infected monocytes strictly affected HCMV replication and the production of infectious virus from infected monocytes/macrophages, as the absence of IFN- $\gamma$ and IL-2 nearly abolished viral replication [52]. Moreover, IFN- $\gamma$ and TNF- $\alpha$ were shown to be required for the differentiation of HCMV-permissive monocyte-derived macrophages, and the presence of these cytokines during the differentiation process conferred HCMV resistance to these antiviral cytokines [93]. Taken together, these studies illustrate the requirement of a very distinct macrophage phenotype, expressing specific proinflammatory (M1-associated) cytokines and/or cellular factors (such as IFN- $\gamma$ and TNF- $\alpha$ ) and certain anti-inflammatory (M2-associated) cytokines and/or cellular factors (such as IL-10), for the establishment of the ideal tissue environment for effective HCMV replication within monocyte-derived macrophages. Therefore, we propose that the "fine-tuning" of the infected macrophage polarization phenotype likely produces a cellular environment that expresses all of the cytokines and cellular factors that are required for the hematogenous spread of HCMV and for the initiation of HCMV replication in macrophages, while avoiding certain detrimental outcomes like a robust antiviral immune response. Specifically, we predict that the expression of M1-associated cytokines likely promotes the required biological changes for enhanced motility, transendothelial migration, and survival to promote viral spread and 
replication and to help provide resistance to any later production of these pro-inflammatory cytokines, while the expression of M2-associated cytokines promotes an environment that is better-suited for viral replication and that helps to dampen immune detection of the virus.

\section{Discussion}

The hematogenous dissemination strategy of HCMV allows the virus to spread throughout the entire body of the host and to gain access to nearly every organ tissue. This systemic spread is critical for the establishment of viral latency within the bone marrow and the life-long persistent infection that is associated with HCMV infection $[19,28,29]$. Because peripheral blood monocytes play an immune surveillance role within the host and can, therefore, easily transition out of the bloodstream to enter into peripheral organ tissues, monocytes represent ideal candidates to serve as viral carriers for the spread of HCMV during infection. Furthermore, the clinical disease manifestations associated with HCMV infection are thought to result from the infiltration of infected monocytes during the hematogenous spread of the virus [94]. However, because peripheral blood monocytes have a short lifespan [34,35] and do not support de novo viral gene expression or replication until differentiation into macrophages [36], HCMV must first overcome these biological hurdles in order to utilize monocytes for viral spread.

Our laboratory has demonstrated that the binding of HCMV glycoproteins to EGFR and the $\beta_{1}$ and $\beta_{3}$ integrins on the surface of monocytes triggers the activation of multiple signaling cascades that mediate the pro-inflammatory activation of infected monocytes $[26,45]$. We propose that this pro-inflammatory monocyte activation is required for the induction of multiple monocyte biological changes that are critical for viral spread and persistence, including an enhanced motility and transendothelial migration, which help to facilitate the extravasation of infected monocytes out of the bloodstream and into the surrounding tissues [21-23,45]. However, due to the natural short lifespan of these cells, in the absence of a viral-induced pro-survival signal, infected monocytes would likely represent an "end-point-cellular-host" for the virus. Our data have shown that HCMV promotes the extended survival of infected monocytes through the prolonged expression of the cellular anti-apoptotic molecule, Mcl-1 early after infection, which promotes the survival of infected monocytes through a 48-hour viability checkpoint, a time at which uninfected monocytes appear to be programmed to die by apoptosis [25] (Figure 4). Although the prolonged survival of infected monocytes is critical for providing a longer-lived cellular host for the virus, in order for HCMV replication to occur, differentiation of infected monocytes into long-lived macrophages is also required [8,13-15,95] (Table 1). Further complicating this scenario, cellular differentiation can by blocked by an abundance of pro-survival signaling [70], suggesting that the survival and differentiation programs in infected monocytes must be intimately linked and tightly co-regulated during HCMV infection in order for viral replication to occur. That is, the virus must regulate the precise expression of pro-survival signals in order to navigate through critical cell fate decision checkpoints to promote survival, while simultaneously ensuring that differentiation also appropriately commences in the presence of these pro-survival signals. Based on our studies, we have developed a model describing this link between HCMV-induced monocyte survival and differentiation. Early after infection, following the extravasation of infected monocytes into the surrounding tissues, the primary hurdle for HCMV is the "ticking clock" of monocyte pre-programmed cellular death around 
a 48-hour viability gate (Figure 4). Therefore, HCMV regulates the prolonged expression of Mcl-1 to drive the survival of the infected cell through this 48 -hour viability gate (Figure 4). Following the successful navigation through this cell survival "crisis point", the primary hurdle for HCMV becomes the inability to initiate viral gene expression and replication, thereby necessitating a switch from a primarily cell survival program to a primarily cellular differentiation program (Figure 4). During the cellular differentiation phase of infection, Mcl-1 expression levels are depleted to allow for differentiation, while a low level increase in other pro-survival signals (including Bcl-2 expression) is observed, which we believe allows the infected cell to maintain survival throughout the differentiation process without directly interfering with the differentiation process (Figure 4).

In an effort to identify the molecular mechanisms for how HCMV effectively regulates the distinct processes of survival and differentiation during infection, we initiated studies to molecularly define the players involved in driving the monocyte-to-macrophage differentiation process that may also be linked to alterations in the cell survival program. We have shown that Mcl-1 expression early after infection serves to inhibit the apoptosis of infected cells through the inhibition of caspase-3 cleavage/activation, prior to the 48-hour viability gate (Figure 4) [24]. Furthermore, we showed that once Mcl-1 levels are depleted after the 48-hour viability checkpoint, HCMV utilizes the partial activation of caspase-3, a typically pro-apoptotic molecule that has also been shown to regulate macrophage differentiation [64], in order to drive differentiation of infected monocytes (Figure 4) [24]. Our new studies confirm an increase in the activation of caspases during HCMV infection of monocytes (Figure 1B) and indicate that HCMV infection also induces the expression of PS on the surface of infected monocytes (Figure 1A). Although these findings are typically associated with enhanced cellular apoptosis, we nonetheless observe a prolonged survival [25] and a lower amount of DNA laddering in infected monocytes (Figure 1C), indicating that HCMV promotes resistance to apoptosis in infected monocytes. Moreover, both the activation of caspases [24,64,70,74] and the expression of PS [96] have been shown to occur during the differentiation of monocytes to macrophages. Therefore, we argue that following the successful navigation of infected monocytes through the critical 48-hour cell fate decision checkpoint (which is mediated by the increased expression of cell survival signals, including Mcl-1 [25]), HCMV then initiates the expression of a series of "pro-apoptotic" signals in precise quantities that help to drive the differentiation of infected monocytes to macrophages, but that does not fully activate the apoptotic response (Figure 4). Furthermore, the expression of PS has recently been found to have an anti-inflammatory effect by inducing the expression of anti-inflammatory cytokines that can counteract the effects of pro-inflammatory cytokines within inflamed tissues [97-100]. These data suggest that the surface expression of PS in infected monocytes may not only indicate the differentiation of infected monocytes into macrophages, but may also aid in the distinct polarization of infected monocytes/macrophages by enhancing the expression of anti-inflammatory, or M2-associated cytokines to counteract any potential negative or anti-viral effects of pro-inflammatory, or M1-associated cytokines that are expressed during infection. 
Figure 4. HCMV regulates the distinct processes of survival and differentiation during infection through the precise expression of specific pro- and anti-apoptotic molecules. Our data have shown that HCMV infection induces the enhanced expression of Mcl-1 in infected monocytes in order to mediate their prolonged survival through a 48-hour viability checkpoint, a time in which uninfected monocytes are programmed to die [24,25]. Mcl-1 levels gradually decrease through 48 hours after infection, before becoming undetectable. Following 48 hours, HCMV induces the enhanced expression of Bcl-2 and phosphatidyl serine (PS) membrane expression and an increase in the partial activation of caspase- 3 in infected monocytes. The expression of low levels of Bcl-2 helps to support the survival of infected monocytes after the 48-hour viability gate, while precise regulation of the membrane expression of PS and the partial activation of caspase-3 help to drive the differentiation of infected monocytes towards replication-permissive macrophages. Overall, through the tight regulation of pro- and anti-apoptotic molecules, HCMV is able to overcome critical biological hurdles, such as the short lifespan of monocytes and the limit of de novo viral gene expression and/or replication in monocytes.

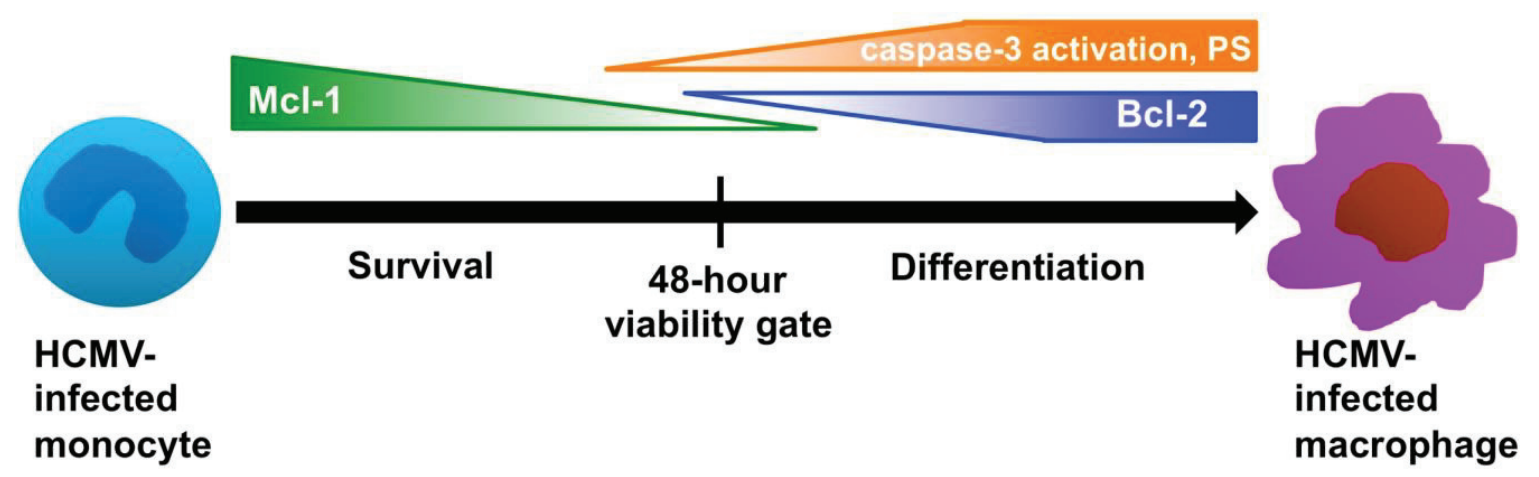

\section{Conclusions}

Although the precise mechanisms for how HCMV directs the polarization of infected monocytes/macrophages remain undetermined, our data clearly indicate that HCMV serves as a unique ligand that induces the differentiation and polarization of infected monocytes/macrophages towards a distinct phenotype that shares common characteristics with both M1 and M2 polarized macrophages [78,79] (Figures 2 and 3). In this manner, HCMV appears to specifically regulate the polarization of infected monocytes/macrophages in order to achieve an effective balance between pro-inflammatory and anti-inflammatory signals, which may establish the cellular environment that is conducive for dissemination and persistence of HCMV (Figure 5). Furthermore, we suggest that the specific polarization of HCMV-infected monocytes into distinct macrophages allows the virus to reprogram its cellular environment to achieve effective viral replication within a now naturally long-lived tissue macrophage (Figure 5). Combined with our polarization data, our identification of the ability of infected monocytes/macrophages to serve as long-term sources of productive viral release (Table 1) suggests that HCMV specifically manipulates monocyte cell biology to utilize this cell type not only as a vessel for successful dissemination, but to also promote long-term persistence within infected tissues. Furthermore, these studies strengthen previous studies indicating that infected 
macrophages do, in fact, serve as a critical cellular component in the production of HCMV within tissues, tying infected monocytes/macrophages to viral spread, persistence, and pathogenesis within organs $[13,101]$. Overall, it seems that in order to successfully reach the point of viral replication, HCMV must orchestrate the precise expression of multiple pro-survival and pro-apoptosis signals, as well as pro-inflammatory and anti-inflammatory mediators, to achieve optimal "Goldilocks" expression levels of these critical cellular signals in infected monocytes/macrophages. Furthermore, as evidenced by the partial activation of caspase- 3 and the extracellular membrane-associated expression of PS on infected monocytes/macrophages, HCMV has likely evolved to utilize multiple molecular mechanisms that link the survival and differentiation/polarization programs of infected cells.

Figure 5. HCMV polarizes infected monocytes/macrophages towards a distinct phenotype possessing aspects of both M1 and M2 macrophages in order to promote viral spread and replication. Our current data indicate that HCMV induces the simultaneous expression of M1-associated molecules (IL-6, TNF- $\alpha$, CD86) and M2-associated molecules (IL-10 and CD163), suggesting that HCMV-infected macrophages fall somewhere along the macrophage polarization continuum between an M1 and M2 phenotype (with a skewing towards a more M1-like phenotype) with an expression of more M1-associated genes and chemokines than M2-associated genes and chemokines [78,79]. We propose that through tight regulation of the expression of specific M1 (pro-inflammatory) and M2 (anti-inflammatory) macrophage markers and chemokines, HCMV is able to utilize key pro-viral aspects of both polarization phenotypes, while simultaneously avoiding potential detrimental anti-viral effects. Specifically, HCMV likely utilizes M1-associated macrophage markers and chemokines to promote the pro-inflammatory activation of infected monocytes/macrophages to ensure proper cellular motility, migration, and monocyte recruitment, while utilizing M2-associated macrophage markers and chemokines to dampen the tissue-damaging effects of the pro-inflammatory response and any potential anti-viral responses. Overall, through the precise regulation of macrophage polarization, HCMV likely induces an ideal cellular environment that can support effective long-term viral replication, which ensures the overall success of the viral spread and persistence strategy.

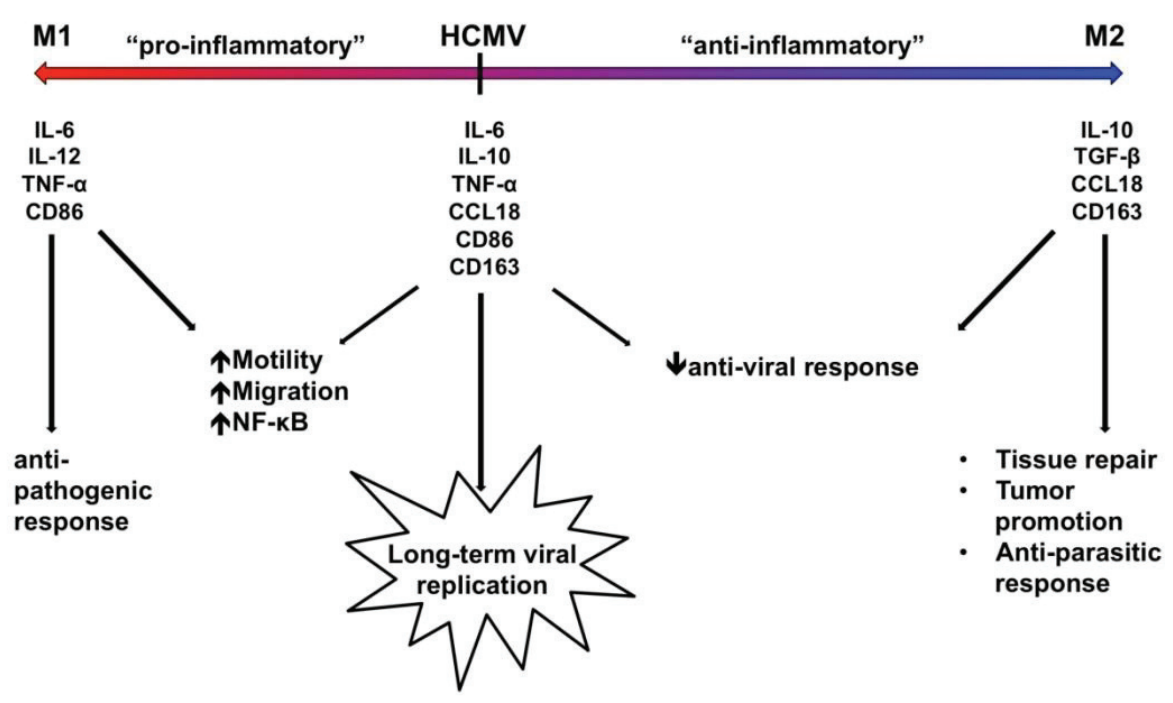




\section{Acknowledgments}

This work was supported by a Malcolm Feist Cardiovascular Research Pre-Doctoral Fellowship and American Heart Association Pre-Doctoral Fellowship (12PRE11840019) and U.S. National Institutes of Health grants (A156077, HD051998, and P20-RR018724).

\section{Author Contributions}

E.V.S., D.C.-M., S.J.C., G.L.B., and A.D.Y. designed experimental research; E.V.S., D.C.-M., S.J.C., and G.L.B. performed experimental research; E.V.S., D.C.-M., J.H.K., and A.D.Y. wrote the paper.

\section{Conflicts of Interest}

The authors declare no conflict of interest.

\section{References and Notes}

1. Mocarski, E.S.; Courcelle, C.T. Cytomegaloviruses and their replication. In Fields Virology, 4th ed.; Knipe, D.M., Howley, P.M., Eds.; Lippincott Williams \& Wilkins: Philadelphia, PA, USA, 2001; Volume 2, pp. 2629-2673.

2. Horwitz, C.A.; Henle, W.; Henle, G. Diagnostic aspects of the cytomegalovirus mononucleosis syndrome in previously healthy persons. Postgrad. Med. 1979, 66, 153-158.

3. Nogalski, M.T.; Collins-McMillen, D.K.; Yurochko, A.D. Overview of human cytomegalovirus pathogenesis. In Human Cytomegoloviruses; Yurochko, A.D., Miller, W.E., Eds.; Methods in Molecular Biology; Human Press: New York, NY, USA, 2014; in press.

4. Soderberg-Naucler, C. HCMV microinfections in inflammatory diseases and cancer. J. Clin. Virol. 2008, 41, 218-223.

5. Soderberg-Naucler, C. Does cytomegalovirus play a causative role in the development of various inflammatory diseases and cancer? J. Intern. Med. 2006, 259, 219-246.

6. Crough, T.; Khanna, R. Immunobiology of human cytomegalovirus: From bench to bedside. Clin. Microbiol. Rev. 2009, 22, 76-98.

7. Drew, W.L. Nonpulmonary manifestations of cytomegalovirus infection in immunocompromised patients. Clin. Microbiol. Rev. 1992, 5, 204-210.

8. Sinclair, J.; Sissons, P. Latent and persistent infections of monocytes and macrophages. Intervirology 1996, 39, 293-301.

9. Sinzger, C.; Jahn, G. Human cytomegalovirus cell tropism and pathogenesis. Intervirology 1996, 39, 302-319.

10. Taylor-Wiedeman, J.; Sissons, J.G.; Borysiewicz, L.K.; Sinclair, J.H. Monocytes are a major site of persistence of human cytomegalovirus in peripheral blood mononuclear cells. J. Gen. Virol. 1991, 72, 2059-2064.

11. Gerna, G.; Baldanti, F.; Revello, M.G. Pathogenesis of human cytomegalovirus infection and cellular targets. Hum. Immunol. 2004, 65, 381-386. 
12. Gerna, G.; Zipeto, D.; Percivalle, E.; Parea, M.; Revello, M.G.; Maccario, R.; Peri, G.; Milanesi, G. Human cytomegalovirus infection of the major leukocyte subpopulations and evidence for initial viral replication in polymorphonuclear leukocytes from viremic patients. J. Infect. Dis. 1992, 166, 1236-1244.

13. Sinzger, C.; Plachter, B.; Grefte, A.; The, T.H.; Jahn, G. Tissue macrophages are infected by human cytomegalovirus in vivo. J. Infect. Dis. 1996, 173, 240-245.

14. Ibanez, C.E.; Schrier, R.; Ghazal, P.; Wiley, C.; Nelson, J.A. Human cytomegalovirus productively infects primary differentiated macrophages. J. Virol. 1991, 65, 6581-6588.

15. Taylor-Wiedeman, J.; Sissons, P.; Sinclair, J. Induction of endogenous human cytomegalovirus gene expression after differentiation of monocytes from healthy carriers. J. Virol. 1994, 68, 1597-1604.

16. Sinclair, J.H.; Baillie, J.; Bryant, L.A.; Taylor-Wiedeman, J.A.; Sissons, J.G. Repression of human cytomegalovirus major immediate early gene expression in a monocytic cell line. J. Gen. Virol. 1992, 73, 433-435.

17. Booss, J.; Dann, P.R.; Griffith, B.P.; Kim, J.H. Host defense response to cytomegalovirus in the central nervous system. Predominance of the monocyte. Am. J. Pathol. 1989, 134, 71-78.

18. Stoddart, C.A.; Cardin, R.D.; Boname, J.M.; Manning, W.C.; Abenes, G.B.; Mocarski, E.S. Peripheral blood mononuclear phagocytes mediate dissemination of murine cytomegalovirus. J. Virol. 1994, 68, 6243-6253.

19. Jarvis, M.A.; Nelson, J.A. Mechanisms of human cytomegalovirus persistence and latency. Front. Biosci. 2002, 7, d1575-1582.

20. Chan, G.; Nogalski, M.T.; Stevenson, E.V.; Yurochko, A.D. Human cytomegalovirus induction of a unique signalsome during viral entry into monocytes mediates distinct functional changes: A strategy for viral dissemination. J. Leukoc. Biol. 2012, 92, 743-752.

21. Smith, M.S.; Bentz, G.L.; Alexander, J.S.; Yurochko, A.D. Human cytomegalovirus induces monocyte differentiation and migration as a strategy for dissemination and persistence. J. Virol. 2004, 78, 4444-4453.

22. Smith, M.S.; Bentz, G.L.; Smith, P.M.; Bivins, E.R.; Yurochko, A.D. HCMV activates $\mathrm{PI}(3) \mathrm{K}$ in monocytes and promotes monocyte motility and transendothelial migration in a PI(3)K-dependent manner. J. Leukoc. Biol. 2004, 76, 65-76.

23. Smith, M.S.; Bivins-Smith, E.R.; Tilley, A.M.; Bentz, G.L.; Chan, G.; Minard, J.;

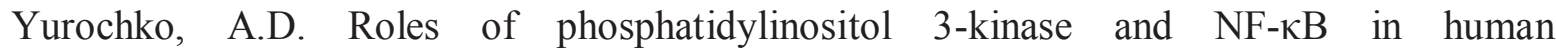
cytomegalovirus-mediated monocyte diapedesis and adhesion: Strategy for viral persistence. J. Virol. 2007, 81, 7683-7694.

24. Chan, G.; Nogalski, M.T.; Yurochko, A.D. Human cytomegalovirus stimulates monocyte-to-macrophage differentiation via the temporal regulation of caspase 3. J. Virol. 2012, 86, 10714-10723.

25. Chan, G.; Nogalski, M.T.; Bentz, G.L.; Smith, M.S.; Parmater, A.; Yurochko, A.D. PI3K-dependent upregulation of Mcl-1 by human cytomegalovirus is mediated by epidermal growth factor receptor and inhibits apoptosis in short-lived monocytes. J. Immunol. 2010, $184,3213-3222$. 
26. Nogalski, M.T.; Chan, G.; Stevenson, E.V.; Gray, S.; Yurochko, A.D. Human cytomegalovirus-regulated paxillin in monocytes links cellular pathogenic motility to the process of viral entry. J. Virol. 2011, 85, 1360-1369.

27. Yurochko, A.D. Human cytomegalovirus modulation of signal transduction. Curr. Top. Microbiol. Immunol. 2008, 325, 205-220.

28. Streblow, D.N.; Nelson, J.A. Models of HCMV latency and reactivation. Trends Microbiol. 2003, 11, 293-295.

29. Bego, M.G.; St Jeor, S. Human cytomegalovirus infection of cells of hematopoietic origin: HCMV-induced immunosuppression, immune evasion, and latency. Exp. Hematol. 2006, 34, $555-570$.

30. Goodrum, F.D.; Jordan, C.T.; High, K.; Shenk, T. Human cytomegalovirus gene expression during infection of primary hematopoietic progenitor cells: A model for latency. Proc. Natl. Acad. Sci. USA 2002, 99, 16255-16260.

31. Keyes, L.R.; Hargett, D.; Soland, M.; Bego, M.G.; Rossetto, C.C.; Almeida-Porada, G.; St Jeor, S. HCMV protein luna is required for viral reactivation from latently infected primary cd14(+) cells. PLoS One 2012, 7, e52827.

32. O'Connor, C.M.; Murphy, E.A. A myeloid progenitor cell line capable of supporting human cytomegalovirus latency and reactivation, resulting in infectious progeny. J. Virol. 2012, 86, 9854-9865.

33. Huang, M.M.; Kew, V.G.; Jestice, K.; Wills, M.R.; Reeves, M.B. Efficient human cytomegalovirus reactivation is maturation dependent in the langerhans dendritic cell lineage and can be studied using a CD14+ experimental latency model. J. Virol. 2012, 86, 8507-8515.

34. Hume, D.A.; Ross, I.L.; Himes, S.R.; Sasmono, R.T.; Wells, C.A.; Ravasi, T. The mononuclear phagocyte system revisited. J. Leukoc. Biol. 2002, 72, 621-627.

35. Whitelaw, D.M. Observations on human monocyte kinetics after pulse labeling. Cell Tissue Kinet. 1972, 5, 311-317.

36. Maciejewski, J.P.; Bruening, E.E.; Donahue, R.E.; Sellers, S.E.; Carter, C.; Young, N.S.; St Jeor, S. Infection of mononucleated phagocytes with human cytomegalovirus. Virology 1993, 195, 327-336.

37. Terhune, S.; Torigoi, E.; Moorman, N.; Silva, M.; Qian, Z.; Shenk, T.; Yu, D. Human cytomegalovirus UL38 protein blocks apoptosis. J. Virol. 2007, 81, 3109-3123.

38. Chang, W.L.; Baumgarth, N.; Yu, D.; Barry, P.A. Human cytomegalovirus-encoded interleukin-10 homolog inhibits maturation of dendritic cells and alters their functionality. J. Virol. 2004, 78, 8720-8731.

39. Bronzini, M.; Luganini, A.; Dell'Oste, V.; de Andrea, M.; Landolfo, S.; Gribaudo, G. The US16 gene of human cytomegalovirus is required for efficient viral infection of endothelial and epithelial cells. J. Virol. 2012, 86, 6875-6888.

40. Castillo, J.P.; Kowalik, T.F. Human cytomegalovirus immediate early proteins and cell growth control. Gene 2002, 290, 19-34.

41. Engel, P.; Angulo, A. Viral immunomodulatory proteins: Usurping host genes as a survival strategy. Adv. Exp. Med. Biol. 2012, 738, 256-276. 
42. DeMeritt, I.B.; Milford, L.E.; Yurochko, A.D. Activation of the NF-кB pathway in human cytomegalovirus-infected cells is necessary for efficient transactivation of the major immediate-early promoter. J. Virol. 2004, 78, 4498-4507.

43. DeMeritt, I.B.; Podduturi, J.P.; Tilley, A.M.; Nogalski, M.T.; Yurochko, A.D. Prolonged activation of NF- $\kappa \mathrm{B}$ by human cytomegalovirus promotes efficient viral replication and late gene expression. Virology 2006, 346, 15-31.

44. Yurochko, A.D.; Huang, E.S. Human cytomegalovirus binding to human monocytes induces immunoregulatory gene expression. J. Immunol. 1999, 162, 4806-4816.

45. Chan, G.; Nogalski, M.T.; Yurochko, A.D. Activation of EGFR on monocytes is required for human cytomegalovirus entry and mediates cellular motility. Proc. Natl. Acad. Sci. USA 2009, 106, 22369-22374.

46. Nogalski, M.T.; Chan, G.C.; Stevenson, E.V.; Collins-McMillen, D.K.; Yurochko, A.D. The HCMV gH/gL/UL128-131 complex triggers the specific cellular activation required for efficient viral internalization into target monocytes. PLoS Pathog. 2013, 9, e1003463.

47. Straschewski, S.; Patrone, M.; Walther, P.; Gallina, A.; Mertens, T.; Frascaroli, G. Protein puUL128 of human cytomegalovirus is necessary for monocyte infection and blocking of migration. J. Virol. 2011, 85, 5150-5158.

48. Viswanathan, K.; Smith, M.S.; Malouli, D.; Mansouri, M.; Nelson, J.A.; Fruh, K. Bst2/tetherin enhances entry of human cytomegalovirus. PLoS Pathog. 2011, 7, e1002332.

49. Zheng, Q.; Tao, R.; Gao, H.; Xu, J.; Shang, S.; Zhao, N. HCMV-encoded UL128 enhances TNF-alpha and IL-6 expression and promotes pbmc proliferation through the MAPK/ERK pathway in vitro. Viral Immunol. 2012, 25, 98-105.

50. Compton, T. Receptors and immune sensors: The complex entry path of human cytomegalovirus. Trends Cell Biol. 2004, 14, 5-8.

51. Soderberg-Naucler, C.; Streblow, D.N.; Fish, K.N.; Allan-Yorke, J.; Smith, P.P.; Nelson, J.A. Reactivation of latent human cytomegalovirus in $\mathrm{CD} 14(+)$ monocytes is differentiation dependent. J. Virol. 2001, 75, 7543-7554.

52. Soderberg-Naucler, C.; Fish, K.N.; Nelson, J.A. Growth of human cytomegalovirus in primary macrophages. Methods 1998, 16, 126-138.

53. Mocarski, E.S.; Shenk, T.; Pass, R.F. Cytomegaloviruses; Lippincott Williams \& Wilkins: Philadelphia, PA, USA, 2007; Volume 2.

54. Barber, G.N. Host defense, viruses and apoptosis. Cell Death Differ. 2001, 8, 113-126.

55. Adams, J.M.; Cory, S. The Bcl-2 protein family: Arbiters of cell survival. Science 1998, 281, $1322-1326$.

56. Zhang, J.; Li, Y.; Yu, M.; Chen, B.; Shen, B. Lineage-dependent NF-кB activation contributes to the resistance of human macrophages to apoptosis. Hematol. J. 2003, 4, 277-284.

57. Perlman, H.; Georganas, C.; Pagliari, L.J.; Koch, A.E.; Haines, K., 3rd; Pope, R.M. Bcl-2 expression in synovial fibroblasts is essential for maintaining mitochondrial homeostasis and cell viability. J. Immunol. 2000, 164, 5227-5235.

58. Lin, E.Y.; Orlofsky, A.; Wang, H.G.; Reed, J.C.; Prystowsky, M.B. A1, a Bcl-2 family member, prolongs cell survival and permits myeloid differentiation. Blood 1996, 87, 983-992. 
59. Lagasse, E.; Weissman, I.L. Enforced expression of Bcl-2 in monocytes rescues macrophages and partially reverses osteopetrosis in op/op mice. Cell 1997, 89, 1021-1031.

60. Liu, H.; Perlman, H.; Pagliari, L.J.; Pope, R.M. Constitutively activated Akt-1 is vital for the survival of human monocyte-differentiated macrophages. Role of Mcl-1, independent of nuclear factor (NF)-кB, Bad, or caspase activation. J. Exp. Med. 2001, 194, 113-126.

61. Sanz, C.; Benito, A.; Silva, M.; Albella, B.; Richard, C.; Segovia, J.C.; Insunza, A.; Bueren, J.A.; Fernandez-Luna, J.L. The expression of $\mathrm{Bcl}-\mathrm{x}$ is downregulated during differentiation of human hematopoietic progenitor cells along the granulocyte but not the monocyte/macrophage lineage. Blood 1997, 89, 3199-3204.

62. Kozopas, K.M.; Yang, T.; Buchan, H.L.; Zhou, P.; Craig, R.W. MCL1, a gene expressed in programmed myeloid cell differentiation, has sequence similarity to BCL2. Proc. Natl. Acad. Sci. USA 1993, 90, 3516-3520.

63. Zhou, P.; Qian, L.; Kozopas, K.M.; Craig, R.W. Mcl-1, a Bcl-2 family member, delays the death of hematopoietic cells under a variety of apoptosis-inducing conditions. Blood 1997, $89,630-643$.

64. Sordet, O.; Rebe, C.; Plenchette, S.; Zermati, Y.; Hermine, O.; Vainchenker, W.; Garrido, C.; Solary, E.; Dubrez-Daloz, L. Specific involvement of caspases in the differentiation of monocytes into macrophages. Blood 2002, 100, 4446-4453.

65. Yang, T.; Buchan, H.L.; Townsend, K.J.; Craig, R.W. Mcl-1, a member of the Bcl-2 family, is induced rapidly in response to signals for cell differentiation or death, but not to signals for cell proliferation. J. Cell. Physiol. 1996, 166, 523-536.

66. Thallinger, C.; Wolschek, M.F.; Wacheck, V.; Maierhofer, H.; Gunsberg, P.; Polterauer, P.; Pehamberger, H.; Monia, B.P.; Selzer, E.; Wolff, K.; Jansen, B. Mcl-1 antisense therapy chemosensitizes human melanoma in a SCID mouse xenotransplantation model. J. Investig. Dermatol. 2003, 120, 1081-1086.

67. Wirth, T.; Kuhnel, F.; Fleischmann-Mundt, B.; Woller, N.; Djojosubroto, M.; Rudolph, K.L.; Manns, M.; Zender, L.; Kubicka, S. Telomerase-dependent virotherapy overcomes resistance of hepatocellular carcinomas against chemotherapy and tumor necrosis factor-related apoptosis-inducing ligand by elimination of Mcl-1. Cancer Res. 2005, 65, 7393-7402.

68. Kobayashi, S.; Werneburg, N.W.; Bronk, S.F.; Kaufmann, S.H.; Gores, G.J. Interleukin-6 contributes to Mcl-1 up-regulation and TRAIL resistance via an Akt-signaling pathway in cholangiocarcinoma cells. Gastroenterology 2005, 128, 2054-2065.

69. Song, L.; Coppola, D.; Livingston, S.; Cress, D.; Haura, E.B. Mcl-1 regulates survival and sensitivity to diverse apoptotic stimuli in human non-small cell lung cancer cells. Cancer Biol. Ther. 2005, 4, 267-276.

70. Nicholson, D.W.; Thornberry, N.A. Caspases: Killer proteases. Trends Biochem. Sci. 1997, 22, 299-306.

71. Hamon, Y.; Broccardo, C.; Chambenoit, O.; Luciani, M.F.; Toti, F.; Chaslin, S.; Freyssinet, J.M.; Devaux, P.F.; McNeish, J.; Marguet, D.; Chimini, G. Abc1 promotes engulfment of apoptotic cells and transbilayer redistribution of phosphatidylserine. Nat. Cell Biol. 2000, 2, 399-406. 
72. Callahan, M.K.; Williamson, P.; Schlegel, R.A. Surface expression of phosphatidylserine on macrophages is required for phagocytosis of apoptotic thymocytes. Cell Death Differ. 2000, 7, 645-653.

73. Collins-McMillen, D.K. Department of Microbiology and Immunology, Louisiana State University, Shreveport, LA, USA. Unpublished work, 2014.

74. Matalova, E.; Lesot, H.; Svandova, E.; Vanden Berghe, T.; Sharpe, P.T.; Healy, C.; Vandenabeele, P.; Tucker, A.S. Caspase-7 participates in differentiation of cells forming dental hard tissues. Dev. Growth Differ. 2013, 55, 615-621.

75. Fish, K.N.; Depto, A.S.; Moses, A.V.; Britt, W.; Nelson, J.A. Growth kinetics of human cytomegalovirus are altered in monocyte-derived macrophages. J. Virol. 1995, 69, 3737-3743.

76. Bissinger, A.L.; Sinzger, C.; Kaiserling, E.; Jahn, G. Human cytomegalovirus as a direct pathogen: Correlation of multiorgan involvement and cell distribution with clinical and pathological findings in a case of congenital inclusion disease. J. Med. Virol. 2002, 67, 200-206.

77. Collins, T.M.; Quirk, M.R.; Jordan, M.C. Biphasic viremia and viral gene expression in leukocytes during acute cytomegalovirus infection of mice. J. Virol. 1994, 68, 6305-6311.

78. Chan, G.; Bivins-Smith, E.R.; Smith, M.S.; Smith, P.M.; Yurochko, A.D. Transcriptome analysis reveals human cytomegalovirus reprograms monocyte differentiation toward an M1 macrophage. J. Immunol. 2008, 181, 698-711.

79. Chan, G.; Bivins-Smith, E.R.; Smith, M.S.; Yurochko, A.D. NF-кB and phosphatidylinositol 3-kinase activity mediates the HCMV-induced atypical M1/M2 polarization of monocytes. Virus Res. 2009, 144, 329-333.

80. Adler, S.P. Molecular epidemiology of cytomegalovirus: A study of factors affecting transmission among children at three day-care centers. Ped. Infect. Dis. J 1991, 10, 584-590.

81. Stagno, S.; Reynolds, D.W.; Pass, R.F.; Alford, C.A. Breast milk and the risk of cytomegalovirus infection. N. Engl. J. Med. 1980, 302, 1073-1076.

82. Tugal, D.; Liao, X.; Jain, M.K. Transcriptional control of macrophage polarization. Arterioscler. Thromb. Vasc. Biol. 2013, 33, 1135-1144.

83. Lawrence, T.; Natoli, G. Transcriptional regulation of macrophage polarization: Enabling diversity with identity. Nat. Rev. Immunol. 2011, 11, 750-761.

84. Mantovani, A.; Sica, A.; Sozzani, S.; Allavena, P.; Vecchi, A.; Locati, M. The chemokine system in diverse forms of macrophage activation and polarization. Trends Immunol. 2004, 25, 677-686.

85. Murray, P.J.; Wynn, T.A. Protective and pathogenic functions of macrophage subsets. Nat. Rev. Immunol. 2011, 11, 723-737.

86. Sica, A.; Mantovani, A. Macrophage plasticity and polarization: In vivo veritas. J. Clin. Investig. 2012, 122, 787-795.

87. Mantovani, A.; Biswas, S.K.; Galdiero, M.R.; Sica, A.; Locati, M. Macrophage plasticity and polarization in tissue repair and remodelling. J. Pathol. 2013, 229, 176-185.

88. Sica, A.; Invernizzi, P.; Mantovani, A. Macrophage plasticity and polarization in liver homeostasis and pathology. Hepatology 2013, doi:10.1002/hep.26754.

89. Li, Q.; Birkbak, N.J.; Gyorffy, B.; Szallasi, Z.; Eklund, A.C. Jetset: Selecting the optimal microarray probe set to represent a gene. BMC Bioinf. 2011, 12, 474. 
90. Parihar, A.; Eubank, T.D.; Doseff, A.I. Monocytes and macrophages regulate immunity through dynamic networks of survival and cell death. J. Innate Immunol. 2010, 2, 204-215.

91. Romo, N.; Magri, G.; Muntasell, A.; Heredia, G.; Baia, D.; Angulo, A.; Guma, M.; Lopez-Botet, M. Natural killer cell-mediated response to human cytomegalovirus-infected macrophages is modulated by their functional polarization. J. Leukoc. Biol. 2011, 90, 717-726.

92. Bayer, C.; Varani, S.; Wang, L.; Walther, P.; Zhou, S.; Straschewski, S.; Bachem, M.; Soderberg-Naucler, C.; Mertens, T.; Frascaroli, G. Human cytomegalovirus infection of M1 and M2 macrophages triggers inflammation and autologous T-cell proliferation. J. Virol. 2013, 87, 67-79.

93. Soderberg-Naucler, C.; Fish, K.N.; Nelson, J.A. Interferon-gamma and tumor necrosis factor-alpha specifically induce formation of cytomegalovirus-permissive monocyte-derived macrophages that are refractory to the antiviral activity of these cytokines. J. Clin. Investig. 1997, 100, 3154-3163.

94. Britt, W. Manifestations of human cytomegalovirus infection: Proposed mechanisms of acute and chronic disease. Curr. Top. Microbiol. Immunol. 2008, 325, 417-470.

95. Fish, K.N.; Stenglein, S.G.; Ibanez, C.; Nelson, J.A. Cytomegalovirus persistence in macrophages and endothelial cells. Scand J. Infect. Dis. Suppl. 1995, 99, 34-40.

96. Callahan, M.K.; Halleck, M.S.; Krahling, S.; Henderson, A.J.; Williamson, P.; Schlegel, R.A. Phosphatidylserine expression and phagocytosis of apoptotic thymocytes during differentiation of monocytic cells. J. Leukoc. Biol. 2003, 74, 846-856.

97. Ma, H.M.; Wu, Z.; Nakanishi, H. Phosphatidylserine-containing liposomes suppress inflammatory bone loss by ameliorating the cytokine imbalance provoked by infiltrated macrophages. Lab. Investig. 2011, 91, 921-931.

98. Harel-Adar, T.; Ben Mordechai, T.; Amsalem, Y.; Feinberg, M.S.; Leor, J.; Cohen, S. Modulation of cardiac macrophages by phosphatidylserine-presenting liposomes improves infarct repair. Proc. Natl. Acad. Sci. USA 2011, 108, 1827-1832.

99. Wu, Z.; Nakanishi, H. Phosphatidylserine-containing liposomes: Potential pharmacological interventions against inflammatory and immune diseases through the production of prostaglandin E(2) after uptake by myeloid derived phagocytes. Arch. Immunol. Ther. Exp. 2011, 59, 195-201.

100. Yeom, M.; Hahm, D.H.; Sur, B.J.; Han, J.J.; Lee, H.J.; Yang, H.I.; Kim, K.S. Phosphatidylserine inhibits inflammatory responses in interleukin-1 $\beta$-stimulated fibroblast-like synoviocytes and alleviates carrageenan-induced arthritis in rat. Nutr. Res. 2013, 33, 242-250.

101. Sinzger, C.; Grefte, A.; Plachter, B.; Gouw, A.S.; The, T.H.; Jahn, G. Fibroblasts, epithelial cells, endothelial cells and smooth muscle cells are major targets of human cytomegalovirus infection in lung and gastrointestinal tissues. J. Gen. Virol. 1995, 76, 741-750. 
Reprinted from Viruses. Cite as: Fink, A.; Renzaho, A.; Reddehase, M.J.; Lemmermann, N.A.W. The p36 Isoform of Murine Cytomegalovirus m152 Protein Suffices for Mediating Innate and Adaptive Immune Evasion. Viruses 2013, 5, 3171-3191.

Article

\title{
The p36 Isoform of Murine Cytomegalovirus m152 Protein Suffices for Mediating Innate and Adaptive Immune Evasion
}

\author{
Annette Fink, Angeliqué Renzaho, Matthias J. Reddehase * and Niels A. W. Lemmermann * \\ Institute for Virology, University Medical Center of the Johannes Gutenberg-University Mainz, \\ Obere Zahlbacher Str. 67, Mainz D-55131, Germany; E-Mails: finka@uni-mainz.de (A.F.); \\ renzaho@uni-mainz.de (A.R.) \\ * Authors to whom correspondence should be addressed; \\ E-Mails: matthias.reddehase@uni-mainz.de (M.J.R.); lemmermann@uni-mainz.de (N.A.W.L.); \\ Tel.: +49-6131-19-9230 (M.J.R.); Tel.: +49-6131-19-9184 (N.A.W.L.); \\ Fax: +49-6131-19-9038 (M.J.R. \& N.A.W.L.).
}

Received: 4 November 2013; in revised form: 6 December 2013 / Accepted: 10 December 2013 / Published: 16 December 2013

\begin{abstract}
The MHC-class I (MHC-I)-like viral (MHC-Iv) $\mathrm{m} 152$ gene product of murine cytomegalovirus (mCMV) was the first immune evasion molecule described for a member of the $\beta$-subfamily of herpesviruses as a paradigm for analogous functions of human cytomegalovirus proteins. Notably, by interacting with classical MHC-I molecules and with MHC-I-like RAE1 family ligands of the activatory natural killer (NK) cell receptor NKG2D, it inhibits presentation of antigenic peptides to CD8 T cells and the NKG2D-dependent activation of NK cells, respectively, thus simultaneously interfering with adaptive and innate immune recognition of infected cells. Although the $m 152$ gene product exists in differentially glycosylated isoforms whose individual contributions to immune evasion are unknown, it has entered the scientific literature as m152/gp40, based on the quantitatively most prominent isoform but with no functional justification. By construction of a recombinant mCMV in which all three $\mathrm{N}$-glycosylation sites are mutated (N61Q, N208Q, and N241Q), we show here that $N$-linked glycosylation is not essential for functional interaction of the m152 immune evasion protein with either MHC-I or RAE1. These data add an important functional detail to recent structural analysis of the m152/RAE1 $\gamma$ complex that has revealed $N$-glycosylations at positions Asn61 and Asn208 of m152 distant from the m152/RAE1 $\gamma$ interface.
\end{abstract}


Keywords: antigen presentation; BAC mutagenesis; CD8 T cells; cytomegalovirus; viral immune evasion; natural killer (NK) cells; $N$-linked glycosylation

\section{Introduction}

Murine cytomegalovirus (mCMV) encodes a set of MHC class I (MHC-I)-like molecules (MHC-Iv) from the "mCMV-private" m145 gene family that are involved in evasion of natural killer (NK) cell recognition of infected cells by interfering with cell surface expression of MHC-I-like cellular ligands of the activatory NK cell receptor NKG2D. Specifically, m145 interferes with MULT1 [1], m152 with RAE1 family members [2], and m155 with H60 [3,4] (for reviews, see [5-9]).

Besides representing the first confirmed MHC-Iv type immune evasion molecule of a CMV [10-12], m152 is special in that it targets not only RAE1 family ligands of NKG2D for subverting innate immune recognition of infected cells but also classical MHC-I allomorphs for inhibiting the recognition of infected cells by virus epitope-specific CD8 T cells, hence subverting also adaptive immunity ([13-15]; for reviews see [16-18]).

Mechanistically, regarding its interference with the classical MHC-I pathway of antigen-presentation, $\mathrm{m} 152$ is thought to interact transiently with nascent peptide MHC-I complexes (pMHC-I) in the ER and disconnects them from the constitutive vesicular flow to the cell surface by retaining them in the ER-Golgi Intermediate Compartment (ERGIC)/cis-Golgi [11,12], which classifies m152 as the prototype of a "retainer"-type immune evasion molecule (for reviews, see [16,19]). Accordingly, the frequent statement that MHC-I cell surface expression is "downmodulated" by m152 may be somewhat misleading. More precisely, the function of this immune evasion molecule is to interfere with trafficking of newly generated pMHC-I from the ER to the cell surface, while loss of virus-specific as well as overall cell surface pMHC-I rather results from cell-surface MHC-I turnover in absence of resupply [20]. Transient interaction between pMHC-I and the luminal portion of $\mathrm{m} 152$, which is a type-I transmembrane protein, proved to be sufficient for catalyzing durable pMHC-I retention, while dissociated $\mathrm{m} 152$ passes the Golgi apparatus and eventually becomes degraded in the lysosome [21]. Regarding m152's interference with cell surface expression of NKG2D ligands of the RAE1 family, the association with m152 varies between different RAE1 isoforms, with the greatest affinity observed for RAE1 $\gamma$ [22]. RAE1 $\delta$ appears to be special in that its nascent form is effectively retained by $\mathrm{m} 152$, whereas loss of the mature, surface-resident form is prevented by absence of a PLWY motif [23]. Based on the high affinity of m152's interaction with RAE1 $\gamma$, Wang and colleagues [24] succeeded in resolving the X-ray crystal structure of the m152/RAE1 $\gamma$ complex, and they defined intermolecular contacts showing that m152 interacts in a pincer-like manner with two sites on the $\alpha 1$ and $\alpha 2$ helices of RAE1 $\gamma$.

In infected cells, m152 is found in differentially glycosylated isoforms, of which a $40 \mathrm{kDa}$ molecular species is most prominent [12]. This has led to equate m152 with gp40 in its immunoevasive functions, both in innate and adaptive immune recognition of infected cells, although the isoform(s) actually interacting with and catalyzing retention of classical MHC-I and RAE1 molecules as well as a possible contribution of carbohydrate moieties to the retention 
function have never been established. The crystal structure of the m152/RAE1 $\gamma$ complex indeed revealed electron density for two single $N$-acetyl glucosamine residues at Asn61 and Asn208 [24], which shows that the $N$-glycosylation does at least not interfere with the physical association between $\mathrm{m} 152$ and RAE1 $\gamma$. Whether or not it is actually needed for the immunoevasive function is the question that we have pursued here.

\section{Results and Discussion}

\subsection{Impaired Immune Evasion Function Coincides with Quantitative Underrepresentation of Glycosylation Isoform gp48 of 152}

Our original interest in the role of glycosylation isoforms of $\mathrm{m} 152$ was based on the incidental observation of inconsistent reduction in overall cell surface display of classical MHC-I molecules in two mCMV mutants deleted for immune evasion gene $m 06$ [25] but supposed to be identical in the expression of the two remaining mCMV-encoded class-I trafficking regulators $\mathrm{m} 152$ and $\mathrm{m} 04$, namely mutants mCMV- $\Delta \mathrm{m} 06^{\mathrm{L}} \quad[18,26]$ and $\mathrm{mCMV}-\Delta \mathrm{m} 06^{\mathrm{W}}$ [27]. Identical expression of m04/gp34 [28] by these two mutants has been documented previously [18], so that suspicion focused on a possibly aberrant expression of m152.

As shown in Figure 1, left column, infected cultures of mouse embryo fibroblasts (MEF) consist of two cell populations with clearcut distinction between "uninfected" cells characterized by missing expression of the ER-resident viral early (E) phase glycoprotein m164/gp36.5 [29] and high cell surface expression of classical MHC-I, and infected cells characterized by expression of the infection marker m164/gp36.5 and levels of MHC-I cell surface expression that vary depending upon the expression of immune evasion genes [18,26,30]. As a side aspect for clarity, it should be noted that at an MOI of 4 all cells present in infected cell cultures likely have virus attached, but apparently not all cells are permissive for the viral gene expression program. Notably, these cells also fail to express a fluorescent reporter, such as GFP, even under the control of the human CMV IE promoter-enhancer in a respective recombinant virus mCMV-GFP [31] (Appendix Figure A1), which suggests immediate silencing of incoming viral genomes.

After infection with virus mCMV- $\Delta \mathrm{m} 06 \mathrm{~m} 152$, infected $\mathrm{m} 164^{\text {pos }}$ cells (arrow-marked) showed an intermediate level of cell surface MHC-I, which was strongly reduced by expression of m152 in cells infected with $\mathrm{mCMV}-\Delta \mathrm{m} 06^{\mathrm{L}}$. Surprisingly, although $\mathrm{mCMV}-\Delta \mathrm{m} 06^{\mathrm{W}}$, which is the prototypic m06 deletion mutant used in several publications by a number of investigators, was expected to show the same phenotype, immune evasion was found to be alleviated [30], corresponding to an only moderate reduction of cell surface MHC-I expression (Figure 1, left column). Notably, these findings for the three viruses were paralleled by cell surface levels of RAE1 in the respective $\mathrm{m} 164^{\text {pos }}$ cell populations (Figure 1, right column), which clearly points to $\mathrm{m} 152$ expression as being the key to this phenomenon, since RAE1, unlike classical MHC-I, is targeted by m152 selectively. 
Figure 1. Inconsistent effects of m152 expression in cells infected with $\Delta \mathrm{m} 06$ mutants. Left column panels: Cytofluorometric analysis of cell surface MHC-I ( $\left.\mathrm{H}-2 \mathrm{~K}^{\mathrm{d}}\right)$ expression (abscissa; FL-2, PE fluorescence intensity) and expression of the ER-resident, E-phase infection marker m164/gp36.5 (ordinate; FL-1, Alexa Fluor488 fluorescence intensity) in $\mathrm{BALB} / \mathrm{c}$ MEF infected with the indicated immune evasion gene deletion mutants of mCMV. Data are displayed as density plots (color-coded with red and blue representing highest and lowest density, respectively). The arrows point to the population of actually infected MEF present within the infected cell cultures beyond to cells that are non-permissive for productive cycle infection and express high levels of MHC-I not downmodulated by immune evasion gene expression. Right column panels: Cell surface expression of pan-RAE1 (abscissa; FL-4, APC fluorescence intensity) by the gated population of infected MEF. To serve as a reference for an easier comparison, the dashed line marks the peak RAE1 expression in absence of immune evasion molecules m06 and m152.
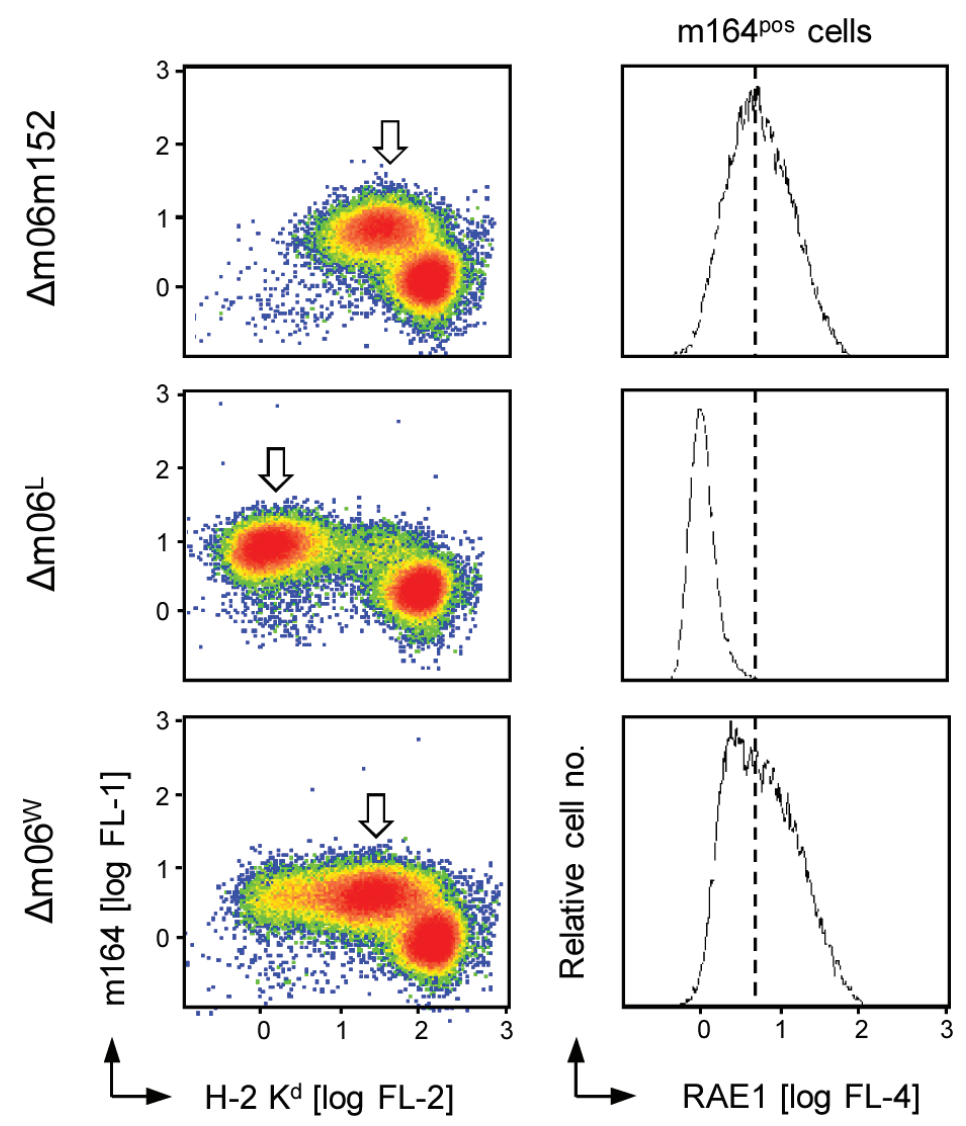

We therefore studied the expression of m152 by these two m06 deletion mutants in comparison to mCMV-WT.BAC. Generally, as shown by us previously in Western blot analyses, the amount of the $40 \mathrm{kDa}$ molecular species of the m152 protein is strongly reduced in cells infected with mCMV- $\Delta \mathrm{m} 06^{\mathrm{W}}$ compared to cells infected with WT virus or mCMV $-\Delta \mathrm{m} 06^{\mathrm{L}}$ [18]. That the protein is not completely absent has been shown by Western blot in the original report on $m C M V-\Delta m 06^{\mathrm{W}}$ [27], and we could confirm this by sensitive immunofluorescence analysis of infected cells as well as by immunohistological detection of m152 protein [18] in tissue sections of mice infected with mCMV- $\Delta \mathrm{m} 06^{\mathrm{W}}$ [32]. 
Here we have modified the approach of detecting m152 proteins in that they were first enriched by m152-specific immunoprecipitation before their analysis by Western blot (Figure 2). For WT virus and mutant virus $\mathrm{mCMV}-\Delta \mathrm{m} 06^{\mathrm{L}}$, this more sensitive analysis revealed $\mathrm{m} 152$ isoforms of $48 \mathrm{kDa}$ and $40 \mathrm{kDa}$, whereas the $48 \mathrm{kDa}$ molecular species proved to be severely underrepresented selectively in cells infected with virus $\mathrm{mCMV}-\Delta \mathrm{m} 06^{\mathrm{W}}$ (Figure $2 \mathrm{~A}$ ). In accordance with previous work by Ziegler and colleagues describing the more abundant $40 \mathrm{kDa}$ molecular species [21], both isoforms turned out to be resistant against treatment with endoglycosidase $\mathrm{H}$ (Endo $\mathrm{H}$ ), while removal of all $\mathrm{N}$-linked carbohydrates using PNGase $\mathrm{F}$ identified them as differentially $N$-glycosylated isoforms gp48 and gp40 that, according to their Endo $\mathrm{H}$ resistance, must have passed the Golgi apparatus, and led to a deglycosylated (more precisely: $N$-linked carbohydrate-deprived) isoform p36 (Figure 2B). As indicated by increasing abundance of gp48 corresponding to decreasing abundance of gp40 over time in absence of further protein synthesis, gp40 is apparently a precursor of the higher-glycosylated isoform gp48. It should be noted that the reason for the difference between the two $\Delta$ m06 mutants is still unknown, as sequencing did not reveal any mutation in the coding region, the $3^{\prime}$ and $5^{\prime}$ untranslated regions (UTRs), and the promoter region of transcription unit $\mathrm{m} 152$ of the two $\Delta \mathrm{m} 06$ viruses (data not shown).

Based on these findings we surmised the gp48 glycosylation isoform of m152 might be the functional isoform of m152 in immune evasion.

\subsection{Mapping of N-glycosylation Sites in 152 and Generation of a Recombinant Virus Expressing only Isoform p36 of the m152 Gene Product}

There exist three potential $\mathrm{N}$-glycosylation sites in the amino acid sequence of the m152 protein, namely at amino acid positions Asn61, Asn208, and Asn241. Transfection of COS7 cells with expression plasmids carrying single mutations N61Q, N208Q, and N241Q revealed a predicted ca. $2 \mathrm{kDa}$ molecular mass shift from $40 \mathrm{kDa}$ to $38 \mathrm{kDa}$ only after mutations N61Q or N208Q (Figure 3), indicating that the site Asn241 is not used in presence of either of the other two sites, at least not in COS7 cells. Interestingly, as mentioned above, the crystal structure of the m152/RAE1 $\gamma$ complex, for which the ectodomain of His6-tagged m152 was expressed in Drosophila S2 cells, in fact revealed usage of both Asn61 and Asn208, but not of Asn241 [24]. In accordance with these structural data, double mutation N61/208Q revealed a molecular species of $36 \mathrm{kDa}$ consistent with p36 found after de- $N$-glycosylation with PNGase F. In line with non-usage of Asn241, mutation of all three sites also revealed p36 as the most prominent band. A minor signal at lower apparent molecular mass turned out to be visible only upon transfection (see below) and, therefore, we did not pursue its identity any further.

For analyzing any functional impact of N-glycosylation on the immune evasion function of m152, we constructed and characterized mutant virus mCMV-m152 $\Delta 3 \mathrm{Glyc}$. Fidelity of triple mutagenesis N61Q, N208Q, and N241Q is verified by sequencing (Figure 4A), and Western blot analysis revealed a single molecular species of $36 \mathrm{kDa}$, that is the p36 isoform of $\mathrm{m} 152$ (Figure 4B). Note that a lower molecular mass species, whose existence was suggested by transfection with the triple-N/Q expression plasmid (see above), is not seen in cells infected with the corresponding virus mCMV-m152 $\triangle 3$ Glyc. 
Figure 2. Protein expression and glycosylation analysis of molecular species of m152 after infection of $\mathrm{BALB} / \mathrm{c}$ MEF with $\mathrm{mCMV}-\Delta \mathrm{m} 06^{\mathrm{W}}$ or $-\Delta \mathrm{m} 06^{\mathrm{L}}$ compared to mCMV-WT. BAC (WT). MEF were infected with the indicated viruses and, from $6 \mathrm{~h}$ p.i. onward, further translation was blocked with cycloheximide ( $\mathrm{CHX})$ for the indicated times to reveal shifts to higher glycosylated isoforms over time. To increase the sensitivity of detection by enrichment of m152 isoforms prior to Western blot analysis, $430 \mu \mathrm{g}$ of total cell protein lysates were subjected to immunoprecipitation (IP) by using magnetic Dynabeads incubated with monoclonal antibody 152.01. (A) Molecular identification of m152 isoforms. Solubilized IP-precipitates were subjected to SDS-PAGE (12.5\%) and molecular species of m152 were detected by Western blot using monoclonal antibody M3D10; (B) Glycosylation analysis. Prior to SDS-PAGE and Western blotting, bead-bound IP-precipitates were mock treated (upper panel) or were treated with either Endo H (centre panel) or PNGase F (lower panel) for 60 min n.i., not infected; no precip., IP procedure performed without a precipitating antibody.
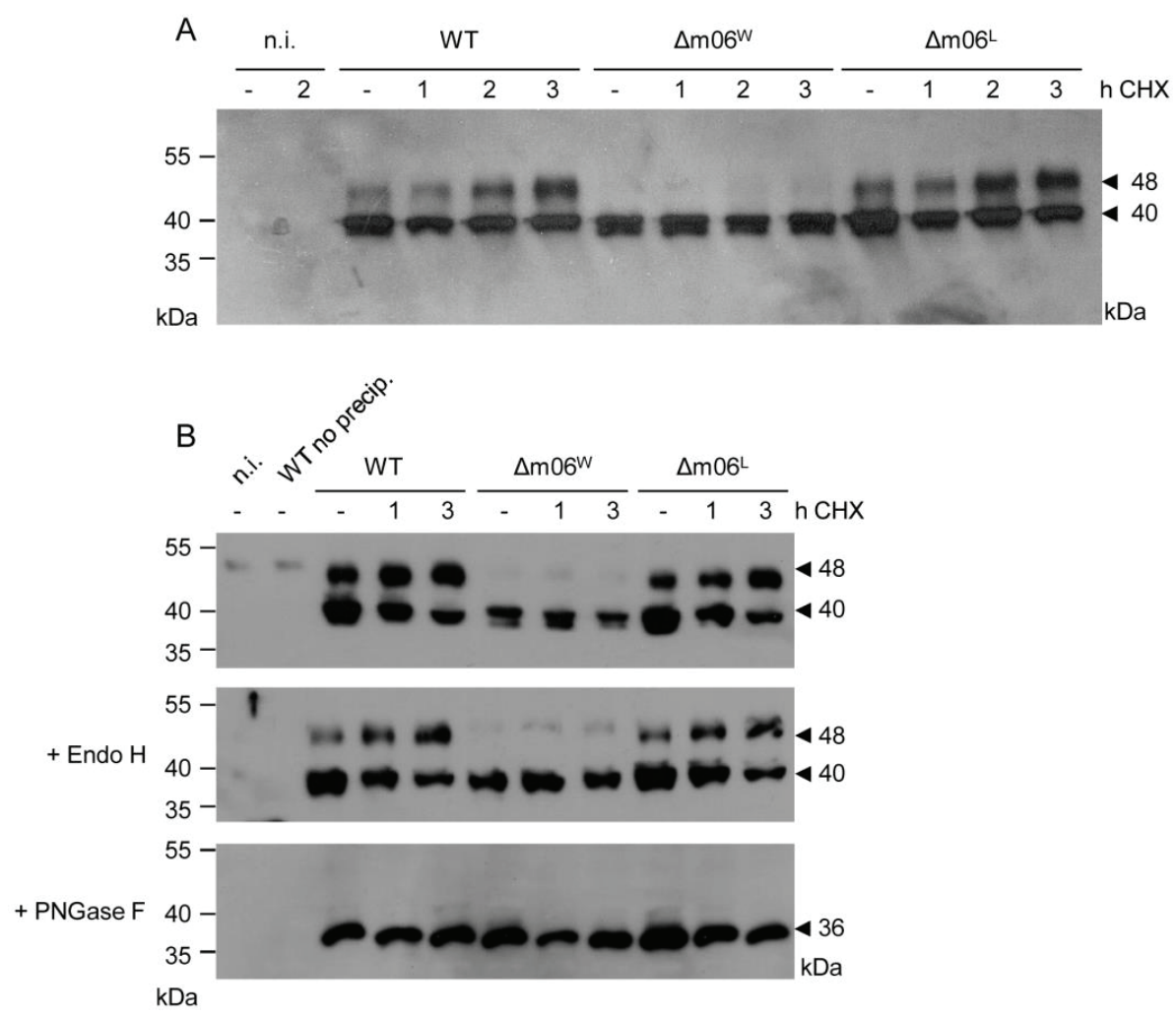

Log-linear growth curves in organs of immunocompromised mice revealed identical, exponential growth of mCMV-WT.BAC and mutant virus mCMV-m152 $\triangle 3 \mathrm{Glyc}$ in the spleen but suggest some growth attenuation of the mutant virus in lungs and liver as indicated by a somewhat prolonged virus doubling time in these organs (Appendix Figure A2). Although some residual innate immunity from NK cells remains in BALB/c mice immunocompromised by a 6-7 Gy dose of $\gamma$-irradiation [33,34], a link of growth attenuation of mCMV-m152 $\Delta 3$ Glyc to a potentially impaired innate immune evasion of NK cell control is considered less likely, as one would not expect this to apply to liver and lungs but not to the spleen where NK cells are, in principle, operative as well. Furthermore, the log-linear growth curves of virus mCMV- $\Delta \mathrm{m} 152$ that activates NK cells through 
its failure to prevent RAE1 cell surface expression did not reveal an attenuation in liver and lungs (unpublished data), which excludes attenuation of mCMV-m152 $\triangle 3$ Glyc due to NK cell control; hence, the reason for the slight growth attenuation of mCMV-m152 $\Delta 3 \mathrm{Glyc}$ in liver and lungs remains to be revealed but is most likely unrelated to $\mathrm{m} 152$ and its glycosylation status.

Figure 3. Mutational analysis of $N$-glycosylation sites in $\mathrm{m} 152$. Western blot analysis of $\mathrm{m} 152$ isoforms (not enriched by a preceding IP) expressed in COS7 cells transfected with expression plasmids carrying single or combined N/Q mutations at potential $N$-glycosylation sites. In essence, COS7 cells were transfected with $4 \mu \mathrm{g}$ of the indicated expression vectors. After $48 \mathrm{~h}$, total protein extraction was performed and $30 \mu \mathrm{g}$ of the protein lysates were subjected to SDS-PAGE (12.5\%) followed by Western blot analysis using monoclonal antibody M3D10 for detection.

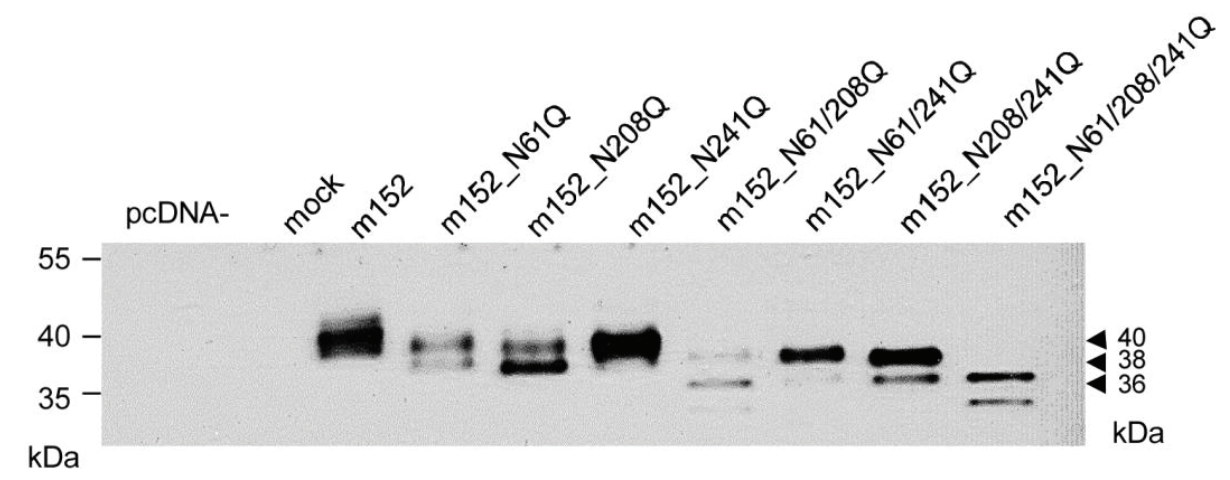

\subsection{The $36 \mathrm{kDa}$ Isoform of $\mathrm{m} 152$ Expressed by Virus $m C M V$-m15243Glyc is Sufficient to Inhibit Antigen Presentation to CD8 T Cells}

One function of m152 is to retain peptide-loaded classical MHC-I molecules in the ERGIC, thereby reducing the number of pMHC-I complexes displayed at the cell surface for recognition by CD8 T cells. The presentation of pMHC-I complexes can be evaluated by quantitating the number of virus epitope-specific but polyclonal CD8 T cells sensitized to secrete IFN- $\gamma$ upon contact with infected cells. As shown in Figure 5 for memory CD8 T cells derived from latently infected BALB/c and C57BL/6 mice representing haplotypes $\mathrm{H}-2^{\mathrm{d}}$ and $\mathrm{H}-2^{\mathrm{b}}$, respectively, recognition of WT virus-infected MEF was low compared to cells infected with the immune evasion gene deletion mutant mCMV- $\Delta \mathrm{m} 152$. Importantly, in this respect, the m152 N-glycosylation-deprived mutant virus mCMV-m152 $\Delta 3$ Glyc behaved like WT virus, with no noticeable difference in the degree of immune evasion. Thus, N-glycosylation of m152 is not critically involved in the immune evasion function of $\mathrm{m} 152$ in antigenic peptide presentation by classical MHC-I molecules of two different MHC haplotypes.

\subsection{The $36 \mathrm{kDa}$ Isoform of $m 152$ Expressed by Virus $m C M V$-m15243Glyc is Sufficient to Inhibit Activation of NK Cells through RAE1/NKG2D Interaction}

Although it is proposed that the identified crystal structure of the complex formed between m152 and the MHC-I-like NKG2D ligand RAE1 is a paradigm for MHC/MHC interactions, including the interaction of the MHC-I-like m152 molecule with classical MHC-I [24], $N$-glycosylation 
might nonetheless modulate m152/MHC-I and m152/RAE1 interactions differently and possibly with different functional consequences. We therefore also studied the impact of $N$-glycosylation of m152 on RAE1 cell surface expression by comparing cells infected with mCMV-m152 3 Glyc with cells infected with WT virus, known to downmodulate cell surface RAE1, and mutant virus mCMV- $\Delta$ m152, known to leave RAE1 untouched (Figure 6). Again, analysis was restricted to infected (arrow-marked) cells characterized by expression of the infection marker m164/gp36.5 and low cell surface expression of classical MHC-I that, in the case of these three viruses (and different to the viruses used in Figure 1), was mediated primarily by immune evasion protein m06, which is known to be the main regulator of overall cell surface MHC-I expression [18,26,27]. Importantly, $\mathrm{m} 152 \Delta 3 \mathrm{Glyc}$, that is the $\mathrm{m} 152$ isoform $\mathrm{p} 36$, proved to be fully competent in downmodulating cell surface RAE1.

Figure 4. Verification of $\mathrm{N} / \mathrm{Q}$ mutations in the m152 coding sequence of recombinant BAC plasmid and virus m152 $\triangle 3$ Glyc. (A) Chromatograms of BAC plasmid sequencing. Upper panels: WT.BAC (WT) sequence regions of interest with Asn (N) at aa positions 61, 208 and 241 (positions defined by protein start). Indicated are nucleotide positions $n$ of the WT.Smith genome [35]. Lower panels: m152 $\Delta 3$ Glyc.BAC sequence regions of interest with Gln (Q) at the respective aa positions. Signals from nucleotides A, T, G, and $\mathrm{C}$ are shown in green, red, black, and blue, respectively; (B) Western blot analysis of 152 protein expression. BALB/c MEF were infected with mCMV-WT.BAC (WT) or mCMV-m152 $\Delta 3 \mathrm{Glyc}$. At the indicated times p.i., proteins were extracted and $30 \mu \mathrm{g}$ of whole protein lysates were subjected to SDS-PAGE (12.5\%) followed by Western blot analysis using monoclonal antibody M3D10 for detection of m152 isoforms.

A

WT

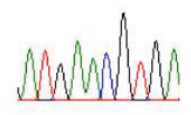

ATG $\frac{Q}{220}$ CAGGA

m152_ $\Delta 3 G l y c$

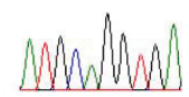

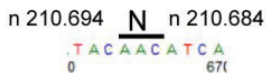

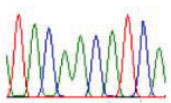

$\frac{Q}{T A C C A G A T C A}{ }_{660}$

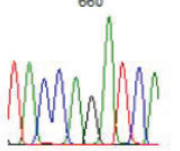

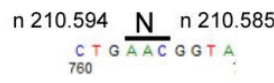
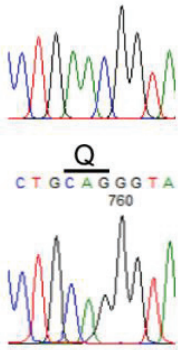

$\mathrm{B}$

WT

m152 33 Glyc

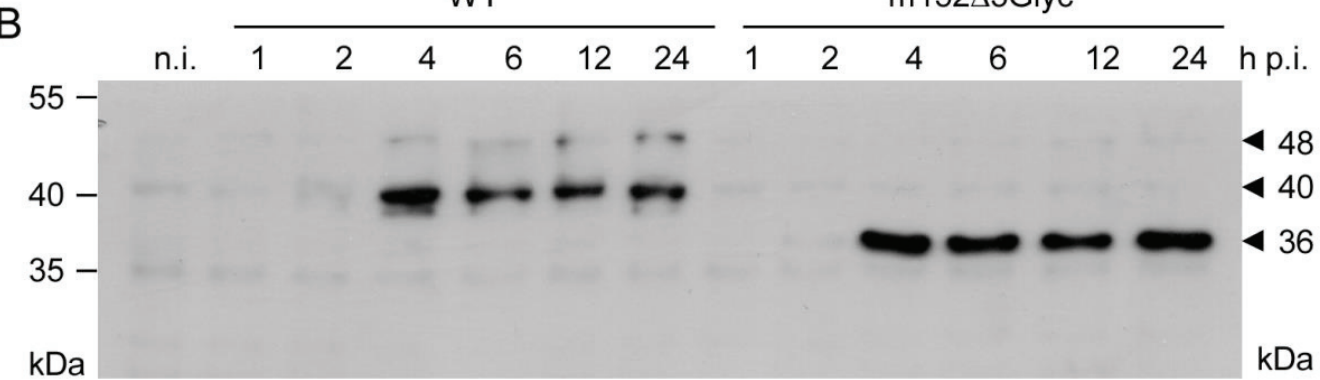


Figure 5. Inhibition of antigen presentation to CD $8 \mathrm{~T}$ cells by $\mathrm{m} 152$ independent of its $N$-glycosylation status. Polyclonal memory CD8 T cells derived from the spleens of latently infected BALB/c (left panel) and C57BL/6 (right panel) mice at seven months after intraplantar infection with mCMV-WT.BAC were used as effector cells in an IFN- $\gamma$ ELISpot assay for sensing the presentation of antigenic peptides on BALB/c and $\mathrm{C} 57 \mathrm{BL} / 6 \mathrm{MEF}$, respectively, infected with the indicated viruses either expressing all isoforms of m152 (WT), lacking m152 ( $\Delta \mathrm{m} 152)$, or expressing just the unglycosylated p36 isoform (m152 $33 \mathrm{Glyc})$. n.i., uninfected MEF. Bars represent numbers of responding CD8 T cells, error bars represent $95 \%$ confidence intervals.
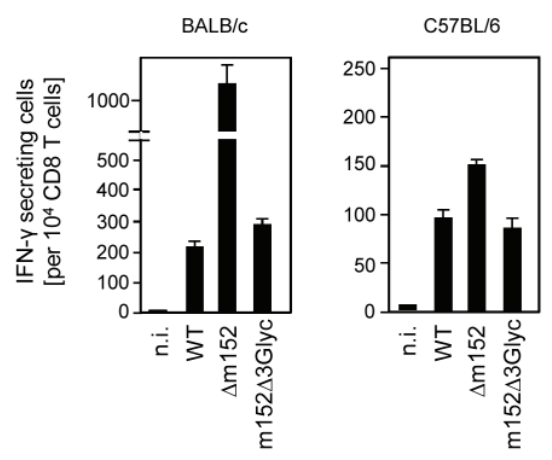

Figure 6. Inhibition of RAE1 cell surface expression by $\mathrm{m} 152$ independent of its $\mathrm{N}$-glycosylation status. Cytofluorometric analysis of pan-RAE1 cell surface expression (right column panels: abscissa; FL-4, APC fluorescence intensity) on BALB/c MEF infected with the indicated viruses either expressing all isoforms of m152 (WT), lacking $\mathrm{m} 152(\Delta \mathrm{m} 152)$, or expressing just the unglycosylated p36 isoform (m152 $\Delta 3 \mathrm{Glyc})$. To serve as a reference for an easier comparison, the dashed line marks the peak RAE1 expression in absence of immune evasion molecule $\mathrm{m} 152$. The analysis was restricted to actually infected cells (left column panels: arrow-marked population) by electronic gating on cells expressing E-phase infection marker m164/gp36.5 (ordinate; FL-1, Alexa Fluor488 fluorescence intensity) and low levels of MHC-I (H-2 K $\mathrm{K}^{\mathrm{d}}$, abscissa; FL-2, PE fluorescence intensity). For data display, see legend to Figure 1.

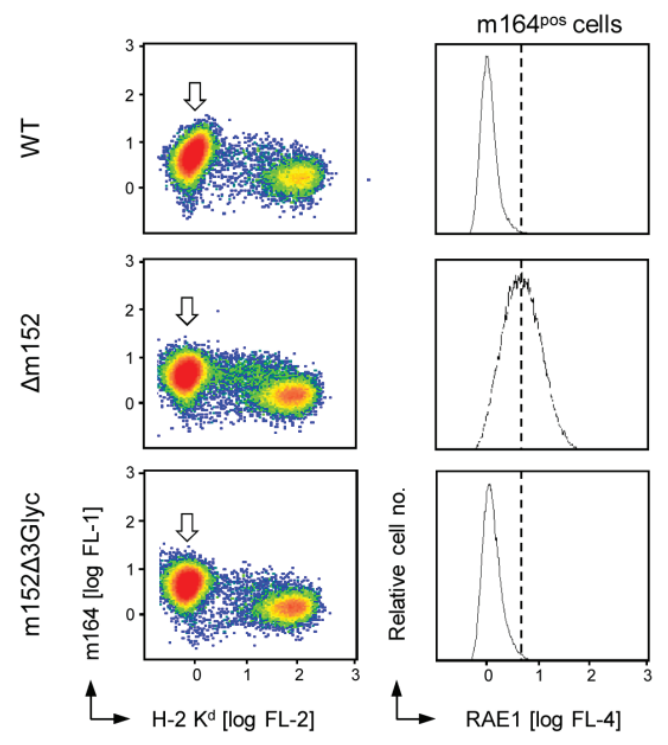


The effects on RAE1 cell surface expression were functionally reflected in vivo by the antiviral activity of NK cells in spleen and lungs in an established 3-day NK cell assay (for a review, see [6]) (Figure 7A). Specifically, NK cell control of virus replication, which is effective after infection with the $\mathrm{m} 152$ deletion virus $\mathrm{mCMV}-\Delta \mathrm{m} 152$, was significantly alleviated in BALB/c mice infected with either the WT virus $(p<0.0001)$ or the mutant virus mCMV-m152 $\Delta 3$ Glyc $(p<0.0001)$. The somewhat lower replication of mCMV-m152 33 Glyc compared to WT virus in the lungs likely relates to its slight growth attenuation already seen in Appendix Figure A2 for the lungs of immunocompromised mice (see the discussion above). This definitively does not indicate a reduced NKG2D ligation-specific NK cell evasion of virus mCMV-m152 $\Delta 3$ Glyc since, in contrast to $\mathrm{mCMV}-\Delta \mathrm{m} 152$, its replication in the lungs was not increased by pan-NK cell depletion or by antagonistic anti-NKG2D antibody blocking the activatory RAE1/NKG2D interaction (Figure 7B).

In conclusion, N-glycosylation of m152 does not appear to be critical—and is definitively not essential-for m152/RAE1 interaction that prevents NKG2D-dependent activation of NK cells.

\section{Experimental}

\subsection{Cells, Viruses, and Mice}

Primary BALB/c or C57BL/6 mouse embryo fibroblasts (MEF) were cultivated in modified Eagle's medium (MEM) supplemented with 10\% fetal calf serum (FCS) and antibiotics. For transfection experiments, COS7 cells were cultivated and seeded $24 \mathrm{~h}$ prior to transfection in DMEM medium supplemented with $10 \%$ FCS and antibiotics.

Virus derived from BAC plasmid pSM3fr [36] was used as "wild-type" virus, mCMV-WT.BAC (WT). Recombinant viruses $\mathrm{mCMV}-\Delta \mathrm{m} 06^{\mathrm{W}}$ and $\mathrm{mCMV}-\Delta \mathrm{m} 06^{\mathrm{L}}$ are described in references [26] and [27], respectively. Virus mCMV-GFP has been described by Angulo and colleagues [31].

$\mathrm{BALB} / \mathrm{c}$ mice were bred and maintained under SPF conditions at the "Central Laboratory Animal Facility (CLAF)" of the University Medical Center Mainz. All experimental procedures were performed in compliance with the 'International Guiding Principles for Biomedical Research Involving Animals' guidelines. The experiments were approved according to German federal law under permission number AZ 1.5 177-07-04/051-61.

\subsection{Infection Conditions and Virus Growth Kinetics in Immunocompromised Mice}

For in vitro assays, 3rd-passage MEF were infected with the indicated viruses at a multiplicity of infection (MOI) of 4 by using the method of centrifugal enhancement of infectivity $([37,38]$ and references therein).

For the in vivo NK cell assay (see below), 8-10 week-old BALB/c mice were infected intravenously (i.v.) with $2 \times 10^{5} \mathrm{PFU}$ (non-enhanced) of mCMV-WT.BAC or the indicated BAC-derived recombinant viruses, all diluted in $50 \mu \mathrm{L}$ of PBS.

Priming of mice for the generation of memory CD8 $\mathrm{T}$ cells was performed by intraplantar infection with $1 \times 10^{5} \mathrm{PFU}$ (non-enhanced) of mCMV-WT.BAC.

Log-linear in vivo virus growth curves were determined by intraplantar infection (see above) of $\mathrm{BALB} / \mathrm{c}$ mice immunocompromised by hematoablative treatment with a single 6.5 Gy dose of 
total-body $\gamma$-irradiation and subsequent monitoring of organ infection on days $2,4,6,8$, and 10 . Infection of lungs and spleen was quantitated by determining virus titers (see above) in the respective organ homogenates. Infection of the liver was quantitated by immunohistochemistry (IHC) and counting of infected liver cells, which are predominantly hepatocytes, in representative $10 \mathrm{~mm}^{2}$ areas of tissue sections, based on detection of the intranuclear immediate-early (IE) protein IE1-pp89/76. Virus doubling times (vDT) and their 95\% confidence intervals (CI) were calculated from the slopes of regression lines determined by linear regression analysis with the statistics software Mathematica [39]. All methods were described in greater detail previously [38].

Figure 7. NK-cell evasion by $\mathrm{m} 152$ independent of its $\mathrm{N}$-glycosylation status. (A) Modulation of the in vivo NK cell control of virus replication by 152 expression in spleen (left panel) and lungs (right panel) of mice infected with viruses expressing all isoforms of m152 (WT, black-filled circles), lacking m152 ( $\Delta \mathrm{m} 152$, open circles), or expressing just the p36 isoform (m152 $33 \mathrm{Glyc}$, gray-filled circles); (B) Replication of mCMV-m152 $\triangle 3$ Glyc in the lungs is not influenced by NK cells. Left panel: Growth attenuation of virus mCMV- $\Delta \mathrm{m} 152$ dependent upon activation of NK cells through ligation of the activatory receptor NKG2D. Right panel: NK cell-independent growth of mCMV-m152 33 Glyc. The day-3 NK cell assay was performed with BALB/c mice left undepleted (control), BALB/c mice depleted of pan-NK cells with anti-asialo GM-1 antibodies administered $24 \mathrm{~h}$ before infection, or BALB/c mice in which ligation of NKG2D on NK cells was blocked by antagonistic anti-NKG2D antibodies administered $6 \mathrm{~h}$ before infection. Throughout, symbols represent virus titers (determined as plaque-forming units, PFU, by virus plaque assay performed under conditions of centrifugal enhancement of infectivity) for individual BALB/c mice infected i.v. three days before with $2 \times 10^{5}$ PFU of the indicated viruses. Median values are marked by short horizontal bars. The dotted lines indicate the detection limit. The statistical significance of differences was tested based on log-transformed ordinate values by the unpaired, two-tailed Student's t test with Welch's correction not assuming equal variance. Differences are considered non-significant for $p>0.05$, significant for $p<0.01$, and highly significant for $p<0.001$.

A

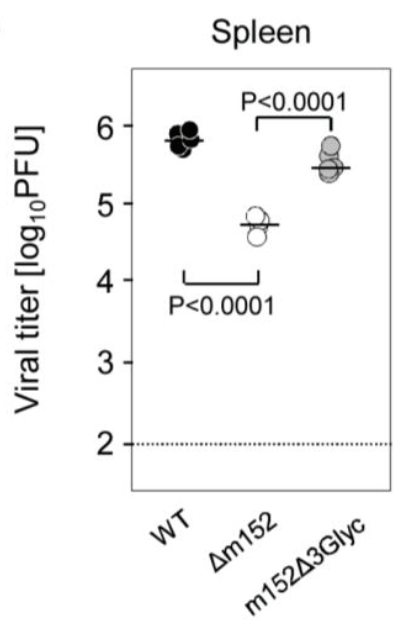

B

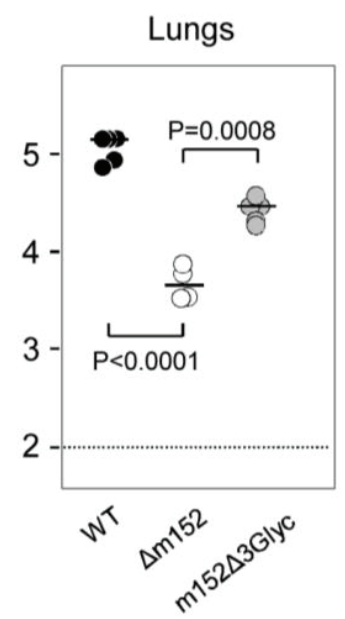

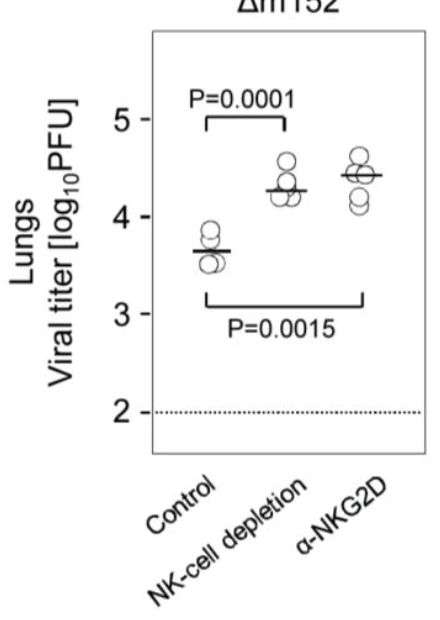

m152 $\Delta 3$ Glyc

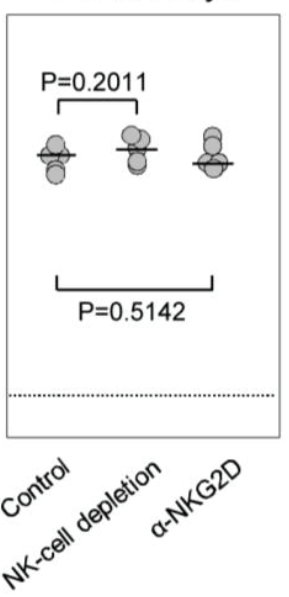




\subsection{In Vivo NK Cell Assays}

On Day 3 after i.v. infection (see Section 3.2), virus titers in homogenates of spleen and lungs were determined under conditions of centrifugal enhancement of infectivity. The involvement of NK cells was tested by NK-cell depletion with polyclonal rabbit antibody directed against mouse asialo GM1 (20 $\mu \mathrm{L}$ in $500 \mu \mathrm{L}$ of PBS i.v., WAKO Chemicals, Richmond, VA, USA) administered on day-1, ca. $24 \mathrm{~h}$ before infection. For the specific blockade of NK-cell activation through RAE1/NKG2D ligation, hamster monoclonal antibody (clone C7) directed against mouse NKG2D [40] was administered i.v. (300 $\mu \mathrm{g}$ in $500 \mu \mathrm{L}$ of PBS) $6 \mathrm{~h}$ before infection.

\subsection{ELISpot Analysis}

An IFN- $\gamma$-based enzyme-linked immunospot (ELISpot) assay was performed to quantify responding memory CD8 T cells after stimulation with infected MEF. Polyclonal memory CD8 T cells were immunomagnetically purified from spleen cell populations of latently infected mice at 7 months after primary intraplantar infection with mCMV-WT.BAC. The assay has been described in detail previously ([41] and references therein). In brief, graded numbers of CD8 T cells were incubated in triplicate cultures for $18 \mathrm{~h}$ with $1 \times 10^{5} \mathrm{MEF}$ infected with an MOI of 4 (centrifugal infection) for $90 \mathrm{~min}$ prior to the cocultivation during which infection proceeds. Frequencies of IFN- $\gamma$-secreting cells and the corresponding 95\% confidence limits were calculated from the spot counts by intercept-free linear regression analysis with the statistics software Mathematica [39].

\subsection{Cytofluorometric Analysis}

At 16 h p.i. (MOI 4, with centrifugal enhancement), BALB/c MEF were stained for cell surface MHC-I with PE-conjugated monoclonal antibody mouse anti-mouse H-2 K ${ }^{\mathrm{d}}$ (FL-2, clone SF1-1.1; BD Pharmingen, catalog no. 553566, Heidelberg, Germany), for cell surface RAE1 with APC-labeled monoclonal antibody rat anti-mouse pan-RAE1 (FL-4, clone 186107; R\&D Systems, catalog no. FAB17582A; Wiesbaden, Germany), and for intracellular expression of m164/gp36.5 indirectly with a rabbit anti-m164 antiserum [30] and Alexa Fluor488-conjugated goat-anti-rabbit IgG (FL-1, Life technologies, catalog no. A11008; Darmstadt, Germany). GFP fluorescence was measured in fluorescence channel 1 (FL-1). Analysis was performed with a Beckman Coulter FC500 cytofluorometer and CXP software [42].

\subsection{Construction of Expression Plasmids}

To generate expression plasmids, PCR was performed using mCMV-WT.BAC genomic DNA with oligonucleotides m152-HpaI_for/rev (Appendix Table A1) amplifying the full length ORFm152 with the following protocol parameters: $5 \mathrm{~min}$ at $95{ }^{\circ} \mathrm{C} ; 18$ cycles of $30 \mathrm{~s}$ at $94{ }^{\circ} \mathrm{C}, 90 \mathrm{~s}$ at $62{ }^{\circ} \mathrm{C}$, and $2 \mathrm{~min}$ at $68{ }^{\circ} \mathrm{C}$; 12 cycles of $30 \mathrm{~s}$ at $94{ }^{\circ} \mathrm{C}, 2 \mathrm{~min}$ at $45{ }^{\circ} \mathrm{C}$ and $2 \mathrm{~min}$ at $68{ }^{\circ} \mathrm{C}$; followed by final extension for $10 \mathrm{~min}$ at $68{ }^{\circ} \mathrm{C}$. The PCR product was subcloned into vector pcDNA3.1 within the EcoR I restriction site. To insert nucleotide exchanges CAT-GCG at nt positions 211.131-211.129, 210.690-210.688, and 210.591-210.589, the construct was subjected to site-directed mutagenesis using the Quick Change II Site-Directed Mutagenesis Kit (Agilent, 
catalog No. 200524, Böblingen, Germany) with the oligonucleotide pairs m152_N61Q_for/_rev, m152_N208Q_for/_rev, and m152_N241Q_for/rev (Appendix Table 1), respectively. The mutagenesis resulted in constructs pcDNA-m152N61Q, pcDNA-m152N208Q, and pcDNAm152N241Q. Plasmid pcDNA-m152N61Q was subjected to a second round of mutagenesis using the respective oligonucleotides resulting in the constructs pcDNA-m152N61/208Q and pcDNA-m152N61/241Q. Plasmid pcDNA-m152N208Q was used as template for the construction of pcDNA-m152N208/241Q, and, in a third round, mutagenesis of pcDNA-m152N61/208Q resulted in the generation of pcDNA-m152N61/208/241Q (m152 $\Delta 3$ Glyc). The successful replacement was confirmed by sequencing (GATC; Konstanz, Germany).

\subsection{Generation of Recombinant Viruses}

To generate recombinant virus mCMV-m152 $\triangle 3 \mathrm{Glyc}$, BAC mutagenesis was performed. In a first step, mCMV-WT.BAC DNA was used as template for PCR with the oligonucleotides m152_BAC-for/rev (Appendix Table A1) to amplify ORFm152 with the following protocol parameters: $5 \mathrm{~min}$ at $95{ }^{\circ} \mathrm{C} ; 35$ cycles of $15 \mathrm{~s}$ at $94{ }^{\circ} \mathrm{C}, 1 \mathrm{~min}$ at $55^{\circ} \mathrm{C}$ and $7 \mathrm{~min} 40 \mathrm{~s}$ at $68{ }^{\circ} \mathrm{C}$; followed by final extension for $10 \mathrm{~min}$ at $72{ }^{\circ} \mathrm{C}$. After Sac I/Xma I restriction, the product was subcloned into pBluescript, resulting in pB-m152_flank. The m152_208/241 fragment from pcDNA-m152N208/241Q (see above) was inserted into pB-m152_flank by Nhe I/Sph I subcloning. The thus modified m152 sequence was inserted into the shuttle vector pST76K_SR by Sac I/Xma I restriction, resulting in pST76K-m152_N208/241Q_flank. This construct was used for allelic exchange of ORFm152 in BAC plasmid pSM3fr as described [43], resulting in BAC-m152_N208/241.

For replacement of the third glycosylation site, BAC-m152_N208/241 DNA was introduced into E. coli GS1783 and recombination was performed by Red-mediated markerless DNA recombination as described by Tischer and colleagues [44]. In brief, oligonucleotides pEPKan-S_m152N61Q_for/rev (Appendix Table A1) were used for a PCR with plasmid pEP-kanS as a template. The resulting products were transformed into GS1783 cells carrying BAC-m152_N208/241Q. After Redrecombination, arabinose-induced $I-S c e I$ expression, and a second round of Red-recombination, m152_N61/208/241Q (m152 33 Glyc) BAC DNA was purified and successful mutagenesis was confirmed by sequencing (GATC; Konstanz, Germany).

The generation of recombinant BAC plasmid pSM3fr_ $\triangle \mathrm{m} 152$ was performed in E. coli strain SW105 as described by Warming and colleagues [45] by PCR-based deletion of ORFm152 between the nucleotides 211,378 und 210,245 [35]. For this, a kanR cassette flanked by homologous viral sequences was amplified from plasmid pKD46 using oligonucleotides m152_del_for/rev (Appendix Table A1) and inserted into the viral genome by ET-recombination. The kanR cassette was subsequently excised by FLP recombinase [45].

Virus reconstitution and purification of a high titer virus stock were performed as described [38].

\subsection{Protein Extraction and Analysis}

MEF were seeded in $10 \mathrm{~cm}$ cell culture dishes and infected with an MOI of 4 under conditions of centrifugal enhancement of infectivity. At defined times p.i., cells were washed with ice-cold PBS and scraped-off. After centrifugation ( $5 \mathrm{~min}, 3,000 \mathrm{rpm}, 4^{\circ} \mathrm{C}$ ), the cells in the pellet were 
lysed for $15 \mathrm{~min}$ on ice in $200 \mu \mathrm{L}$ Lysis Buffer/dish $(0.2 \mathrm{M} \mathrm{NaCl}, 1.5 \mathrm{mM} \mathrm{MgCl}, 4 \mathrm{mM}$ EDTA, 4 mM EGTA, 1\% Triton-X100, 20 mM HEPES; with complete proteinase inhibitor (diluted 1:25; Roche, catalog No.11697498001) and 1mM DTT added shortly before use). After centrifugation (10 min, 14,000 rpm, $4{ }^{\circ} \mathrm{C}$ ), supernatants were collected and the amount of protein was determined by BCA-Assay (Thermo Scientific, catalog No. 23225, Dreieich, Germany). $30 \mu \mathrm{g}$ of total protein was separated on an SDS-PAGE followed by Western blot analysis. Isoforms of m152 were detected with monoclonal antibody M3D10 (1:250) [21].

\subsection{Transfection}

$5 \times 10^{5}$ COS7 cells per $10-\mathrm{cm}$ dish were seeded and $4 \mu \mathrm{g}$ DNA was transfected with Polyfect (Qiagen, catalog No. 301105, Hilden, Germany) following the manufacturer's instructions. $48 \mathrm{~h}$ later, cells were harvested and total protein was extracted. $30 \mu \mathrm{g}$ of protein lysates were separated on a $12.5 \%$ SDS-PAGE followed by Western Blot analysis.

\subsection{Immunoprecipitation}

MEF were infected at an MOI of 4 (centrifugal enhancement) and translation was blocked by cycloheximide (CHX) $(100 \mu \mathrm{g} / \mathrm{mL})$ from $6 \mathrm{~h}$ p.i. onward. At the indicated times after addition of CHX, protein extraction was performed and $430 \mu \mathrm{g}$ of protein lysates were incubated overnight with anti-m152 monoclonal antibody (clone 152.01; 1:100)-pretreated Dynabeads (Life technologies, catalog no. 11201D, Darmstadt, Germany). Unbound proteins were removed by repetitive washing with PBS. Precipitated m152 protein isoforms were identified by SDS-PAGE separation and Western blot analysis.

\subsection{Deglycosylation with Endo H and PNGase F}

Immunoprecipitated, bead-bound $\mathrm{m} 152$ isoforms were mock treated or incubated with either 750U EndoH (NEB, catalog no. P0702S, Frankfurt, Germany) or 1000U PNGase F (NEB, catalog No. P0704S, Frankfurt, Germany) for $60 \mathrm{~min}$ at $37^{\circ} \mathrm{C}$, followed by SDS-PAGE separation and Western blot analysis.

\section{Conclusions}

The recently established crystal structure of the m152/RAE1 $\gamma$ complex has revealed two single $\mathrm{N}$-acetyl glucosamine residues at Asn61 and Asn208 of m152 [24]. As these two positions are distant from the identified m152/RAE $1 \gamma$ interface, Wang and colleagues proposed that these $N$-glycosylations are unlikely to affect that interaction. Here we show functional data confirming this view experimentally. In a transfection system with single mutations of the three potential $N$-glycosylation sites of m152, namely Asn61, Asn208, and Asn241, evidence of usage of the respective site was provided only for Asn61 and Asn208, precisely the two sites actually found to be $N$-glycosylated in the crystallized m152/RAE1 $\gamma$ complexes. Lack of m152 N-glycosylation in an N61Q-N208Q-(N241Q) triple mutated virus mCMV-m152 3 Glyc did not prevent downmodulation of RAE1 from the surface of infected cells, indicating that $N$-glycosylation at Asn61 and Asn208 is 
not needed for m152/RAE1 $\gamma$ complex formation nor does it alter the complex in a mode relevant for its function in NK cell evasion. Interestingly, the same also applied to the interaction between m152 and classical MHC-I in the inhibition of pMHC-I cell surface trafficking for antigen presentation to CD8 $\mathrm{T}$ cells. This supports the title notion by Wang and colleagues that the structural basis of m152 interaction with RAE1 $\gamma$ may reveal a paradigm for MHC/MHC interaction in immune evasion [24].

In addition, these data have shown that absence of the higher glycosylated isoform gp48 of m152 has no functional consequence in immune evasion and thus cannot explain the impaired immune evasion capacity of virus mutant $\mathrm{mCMV}-\Delta \mathrm{m} 06^{\mathrm{W}}$ compared to $\mathrm{mCMV}-\Delta \mathrm{m} 06^{\mathrm{L}}$. Since the Asn61/208 dually $N$-glycosylated isoform gp40 is Endo H-resistant, it must have reached the Golgi apparatus, whereas pMHC-I complexes and RAE1 stick in the ERGIC/cis-Golgi. Unless we assume retrograde trafficking of gp40 back into the ER, for which there exists no evidence and for which there is no rationale as glycosylation is not needed for function, the Endo H-resistant isoform gp40 is unlikely the molecule that mediates immune evasion by interacting with and catalyzing the retention of pMHC-I and RAE1. Since $N$-glycosylation takes place in the ER, it remains open to question if nascent p36 interacts with pMHC-I and RAE-1 before or after the glycosylations at Asn61 and Asn208. In either case, our data have revealed that unglycosylated p36 can interact and that the two $\mathrm{N}$-glycosylations are not required for the innate and adaptive immune evasion functions of 152 .

\section{Acknowledgments}

The authors thank S. Jonjic (University of Rijeka, Croatia) and E. Kremmer (Helmholtz Zentrum München, Munich, Germany) for generously supplying us with monoclonal antibodies, and Kirsten Freitag for help with cytofluorometric analyses.

This work was supported by the Deutsche Forschungsgemeinschaft, SFB490 individual projects E2 (A.R. and M.J.R.) and E4 (A.F. and M.J.R.), and the Clinical Research Group KFO183 (A.F., N.A.W.L., and M.J.R.). N.A.W.L. received intramural funding in the young investigator program MAIFOR of the University Medical Center of the Johannes Gutenberg-University Mainz.

\section{Conflicts of Interest}

The authors declare no conflict of interest.

\section{References and Notes}

1. Krmpotic, A.; Hasan, M.; Loewendorf, A.; Saulig, T.; Halenius, A.; Lenac, T.; Polic, B.; Bubic, I.; Kriegeskorte, A.; Pernjak-Pugel, E.; et al. NK cell activation through the NKG2D ligand MULT-1 is selectively prevented by the glycoprotein encoded by mouse cytomegalovirus gene m145. J. Exp. Med. 2005, 201, 211-220.

2. Lodoen, M.; Ogasawara, K.; Hamerman, J.; Arase, H.; Houchins, J.; Mocarski, E.; Lanier, L. NKG2D-mediated natural killer cell protection against cytomegalovirus is impaired by viral gp40 modulation of retinoic acid early inducible 1 gene molecules. J. Exp. Med. 2003, 197, $1245-1253$. 
3. Lodoen, M.; Abenes, G.; Umamoto, S.; Houchins, J.; Liu, F.; Lanier, L. The cytomegalovirus m155 gene product subverts natural killer cell antiviral protection by disruption of H60-NKG2D interactions. J. Exp. Med. 2004, 200, 1075-1081.

4. Hasan, M.; Krmpotic, A.; Ruzsics, Z.; Bubic, I.; Lenac, T.; Halenius, A.; Loewendorf, A.; Messerle, M.; Hengel, H.; Jonjic, S.; et al. Selective down-regulation of the NKG2D ligand H60 by mouse cytomegalovirus m155 glycoprotein. J. Virol. 2005, 79, 2920-2930.

5. Jonjić, S.; Babić, M.; Polić, B.; Krmpotić, A. Immune evasion of natural killer cells by viruses. Curr. Opin. Immunol. 2008, 20, 30-38.

6. Lenac, T.; Arapović, J.; Traven, L.; Krmpotić, A.; Jonjić, S. Murine cytomegalovirus regulation of NKG2D ligands. Med. Microbiol. Immunol. 2008, 197, 159-166.

7. Lisnić, V.J.; Krmpotić, A.; Jonjić, S. Modulation of natural killer cell activity by viruses. Curr. Opin. Microbiol. 2010, 13, 530-539.

8. Slavuljica, I.; Krmpotić, A.; Jonjić, S. Manipulation of NKG2D ligands by cytomegaloviruses: Impact on innate and adaptive immune response. Front. Immunol. 2011, 2, doi:10.3389/fimmu.2011.00085.

9. Vidal, S.; Krmpotić, A.; Pyzik, M.; Jonjić, S. Innate immunity to cytomegalovirus in the murine model. In Cytomegaloviruses: From Molecular Pathogenesis to Intervention; Reddehase, M.J., Ed.; Caister Academic Press: Wymondham, Norfolk, UK, 2013; pp. 191-213.

10. Thäle, R.; Szepan, U.; Hengel, H.; Geginat, G.; Lucin, P.; Koszinowski, U.H. Identification of the mouse cytomegalovirus genomic region affecting major histocompatibility complex class I molecule transport. J. Virol. 1995, 69, 6098-6105.

11. Del Val, M.; Hengel, H.; Häcker, H.; Hartlaub, U.; Ruppert, T.; Lucin, P.; Koszinowski, U. Cytomegalovirus prevents antigen presentation by blocking the transport of peptide-loaded major histocompatibility complex class I molecules into the medial-Golgi compartment. J. Exp. Med. 1992, 176, 729-738.

12. Ziegler, H.; Thäle, R.; Lucin, P.; Muranyi, W.; Flohr, T.; Hengel, H.; Farrell, H.; Rawlinson, W.; Koszinowski, U. A mouse cytomegalovirus glycoprotein retains MHC Class I complexes in the ERGIC/ cis-Golgi compartments. Immunity 1997, 6, 57-66.

13. Krmpotic, A.; Messerle, M.; Crnkovic-Mertens, I.; Polic, B.; Jonjic, S.; Koszinowski, U. The immunoevasive function encoded by the mouse cytomegalovirus gene m152 protects the virus against T cell control in vivo. J. Exp. Med. 1999, 190, 1285-1296.

14. Krmpotić, A.; Busch, D.H.; Bubić, I.; Gebhardt, F.; Hengel, H.; Hasan, M.; Scalzo, A.A.; Koszinowski, U.H.; Jonjić, S. MCMV glycoprotein gp40 confers virus resistance to CD8 ${ }^{+} \mathrm{T}$ cells and NK cells in vivo. Nat. Immunol. 2002, 3, 529-535.

15. Holtappels, R.; Podlech, J.; Pahl-Seibert, M.; Jülch, M.; Thomas, D.; Simon, C.O.; Wagner, M.; Reddehase, M.J. Cytomegalovirus misleads its host by priming of CD8 T cells specific for an epitope not presented in infected tissues. J. Exp. Med. 2004, 199, 131-136.

16. Reddehase, M.J. Antigens and immunoevasins: Opponents in cytomegalovirus immune surveillance. Nat. Rev. Immunol. 2002, 2, 831-844.

17. Lemmermann, N.A.; Böhm, V.; Holtappels, R.; Reddehase, M.J. In vivo impact of cytomegalovirus evasion of CD8 T-cell immunity: Facts and thoughts based on murine models. Virus Res. 2011, 157, 161-174. 
18. Lemmermann, N.A.; Fink, A.; Podlech, J.; Ebert, S.; Wilhelmi, V.; Böhm, V.; Holtappels, R.; Reddehase, M.J. Murine cytomegalovirus immune evasion proteins operative in the MHC class I pathway of antigen processing and presentation: State of knowledge, revisions, and questions. Med. Microbiol. Immunol. 2012, 201, 497-512.

19. Hansen, T.H.; Bouvier, M. MHC class I antigen presentation: Learning from viral evasion strategies. Nat. Rev. Immunol. 2009, 9, 503-513.

20. Lemmermann, N.A.; Gergely, K.; Böhm, V.; Deegen, P.; Däubner, T.; Reddehase, M.J. Immune evasion proteins of murine cytomegalovirus preferentially affect cell surface display of recently generated peptide presentation complexes. J. Virol. 2010, 84, 1221-1236.

21. Ziegler, H.; Muranyi, W.; Burgert, H.; Kremmer, E.; Koszinowski, U. The luminal part of the murine cytomegalovirus glycoprotein gp40 catalyzes the retention of MHC class I molecules. EMBO J. 2000, 19, 870-881.

22. Zhi, L.; Mans, J.; Paskow, M.J.; Brown, P.H.; Schuck, P.; Jonjić, S.; Natarajan, K.; Margulies, D.H. Direct interaction of the mouse cytomegalovirus m152/gp40 immunoevasin with RAE-1 isoforms. Biochemistry 2010, 49, 2443-2453.

23. Arapovic, J.; Lenac, T.; Antulov, R.; Polic, B.; Ruzsics, Z.; Carayannopoulos, L.N.; Koszinowski, U.H.; Krmpotic, A.; Jonjic, S. Differential susceptibility of RAE-1 isoforms to mouse cytomegalovirus. J. Virol. 2009, 83, 8198-8207.

24. Wang, R.; Natarajan, K.; Revilleza, M.; Boyd, L.; Zhi, L.; Zhao, H.; Robinson, H.; Margulies, D. Structural basis of mouse cytomegalovirus m152/gp40 interaction with RAE1 $\gamma$ reveals a paradigm for MHC/MHC interaction in immune evasion. Proc. Natl. Acad. Sci. USA 2012, 109, 3578-3587.

25. Reusch, U.; Muranyi, W.; Lucin, P.; Burgert, H.G.; Hengel, H.; Koszinowski, U.H. A cytomegalovirus glycoprotein re-routes MHC class I complexes to lysosomes for degradation. EMBO J. 1999, 18, 1081-1091.

26. Fink, A.; Lemmermann, N.A.; Gillert-Marien, D.; Thomas, D.; Freitag, K.; Böhm, V.; Wilhelmi, V.; Reifenberg, K.; Reddehase, M.J.; Holtappels, R. Antigen presentation under the influence of "immune evasion" proteins and its modulation by interferon-gamma: Implications for immunotherapy of cytomegalovirus infection with antiviral CD8 T cells. Med. Microbiol. Immunol. 2012, 201, 513-525.

27. Wagner, M.; Gutermann, A.; Podlech, J.; Reddehase, M.J.; Koszinowski, U.H. Major histocompatibility complex class I allele-specific cooperative and competitive interactions between immune evasion proteins of cytomegalovirus. J. Exp. Med. 2002, 196, 805-816.

28. Kleijnen, M.; Huppa, J.; Lucin, P.; Mukherjee, S.; Farrell, H.; Campbell, A.; Koszinowski, U.H.; Hill, A.; Ploegh, H. A mouse cytomegalovirus glycoprotein, gp34, forms a complex with folded class I MHC molecules in the ER which is not retained but is transported to the cell surface. EMBO J. 1997, 16, 685-694

29. Däubner, T.; Fink, A.; Seitz, A.; Tenzer, S.; Müller, J.; Strand, D.; Seckert, C.K.; Janssen, C.; Renzaho, A.; Grzimek, N.K.; et al. A novel transmembrane domain mediating retention of a highly motile herpesvirus glycoprotein in the endoplasmic reticulum. J. Gen. Virol. 2010, 91, $1524-1534$. 
30. Holtappels, R.; Gillert-Marien, D.; Thomas, D.; Podlech, J.; Deegen, P.; Herter, S.; Oehrlein-Karpi, S.; Strand, D.; Wagner, M.; Reddehase, M.J. Cytomegalovirus encodes a positive regulator of antigen presentation. J. Virol. 2006, 80, 7613-7624.

31. Angulo, A.; Ghazal, P.; Messerle, M. The major immediate-early gene ie 3 of mouse cytomegalovirus is essential for viral growth. J. Virol. 2000, 74, 11129-11136.

32. Podlech, J. University Medical Center Mainz, Mainz, Germany. Personal communication, 2013.

33. Erlach, K.C.; Böhm, V.; Knabe, M.; Deegen, P.; Reddehase, M.J.; Podlech, J. Activation of hepatic natural killer cells and control of liver-adapted lymphoma in the murine model of cytomegalovirus infection. Med. Microbiol. Immunol. 2008, 197, 167-178.

34. Slavuljica, I.; Busche, A.; Babić, M.; Mitrović, M.; Gašparović, I.; Cekinović, D.; Markova Car, E.; Pernjak Pugel, E.; Ciković, A.; Lisnić, V.J.; et al. Recombinant mouse cytomegalovirus expressing a ligand for the NKG2D receptor is attenuated and has improved vaccine properties. J. Clin. Investig. 2010, 120, 4532-4545.

35. Rawlinson, W.; Farrell, H.; Barrell, B. Analysis of the complete DNA sequence of murine cytomegalovirus. J. Virol. 1996, 70, 8833-8849.

36. Wagner, M.; Jonjic, S.; Koszinowski, U.; Messerle, M. Systematic excision of vector sequences from the BAC-cloned herpesvirus genome during virus reconstitution. J. Virol. 1999, 73, 7056-7060.

37. Kurz, S.; Steffens, H.; Mayer, A.; Harris, J.; Reddehase, M. Latency versus persistence or intermittent recurrences: Evidence for a latent state of murine cytomegalovirus in the lungs. J. Virol. 1997, 71, 2980-2987.

38. Lemmermann, N.A.; Podlech, J.; Seckert, C.; Kropp, K.; Grzimek, N.K.; Reddehase, M.J.; Holtappels, R. CD8 T-cell immunotherapy of cytomegalovirus disease in the murine model. In Methods in Microbiology; Kabelitz, D., Kaufmann, S., Eds.; Academic Press: London, UK, 2010; pp. 369-420.

39. Wolfram Mathematica, version 9, Wolfram Research, Champaign, IL, USA, 2012.

40. Ho, E.L.; Carayannopoulos, L.N.; Poursine-Laurent, J.; Kinder, J.; Plougastel, B.; Smith, H.R.; Yokoyama, W.M. Costimulation of multiple NK cell activation receptors by NKG2D. J. Immunol. 2002, 169, 3667-3675.

41. Böhm, V.; Simon, C.; Podlech, P.; Seckert, C.; Gendig, D.; Deegen, P.; Gillert-Marien, D.; Lemmermann, N.A.; Holtappels, R.; Reddehase, M.J. The immune evasion paradox: Immunoevasins of murine cytomegalovirus enhance priming of CD8 $\mathrm{T}$ cells by preventing negative feedback regulation. J. Virol. 2008, 82, 11637-11650.

42. CXP Acquisition, version 2.2, Beckman Coulter, Indianapolis, IN, USA, 2006.

43. Borst, E.; Posfai, G.; Pogoda, M.; Messerle, M. Mutagenesis of herpesvirus BACs by allele replacement. In Methods Mol. Biol., Bacterial Artificial Chromosomes; Zhao, S., Stodolsky, M., Eds.; Humana Press: Totowa, NJ, USA, 2004; pp. 269-280.

44. Tischer, B.; von Einem, J.; Kaufer, B.; Osterrieder, N. Two-step Red-mediated recombination for versatile high-efficiency markerless DNA manipulation in Escherichia coli. Biotechniques 2006, 40, 191-197.

45. Warming, S.; Costantino, N.; Court, D.; Jenkins, N.; Copeland, G. Simple and highly efficient BAC recombineering using galK selection. Nucleic Acids Res. 2005, 33, e36. 


\section{Appendices}

Figure A1. Reporter virus revealing cells non-permissive for the viral replicative cycle within infected mouse embryo fibroblast cell cultures. Cytofluorometric analysis of cell surface MHC-I (H-2 K $\mathrm{K}^{\mathrm{d}}$ ) expression (abscissa; FL-2, PE fluorescence intensity) and expression of the fluorescent reporter protein GFP (ordinate; FL-1) in BALB/c MEF infected with reporter virus mCMV-GFP. Data are displayed as a density plot (color-coded with red and blue representing highest and lowest density, respectively). The red arrows point to three prominent cell populations. Population 1: Cells not expressing the GFP reporter under control of the human CMV promoter-enhancer but expressing a high level of cell surface MHC-I, which indicates lack of immune evasion gene expression. Population 2: Infected cells expressing high levels of reporter protein GFP but low levels of MHC-1 due to the action of the immune evasion proteins. Population 3: Putatively infected cells, in which GFP expression became silenced and in which MHC-I expression is low due to a preceding action of the immune evasion proteins.

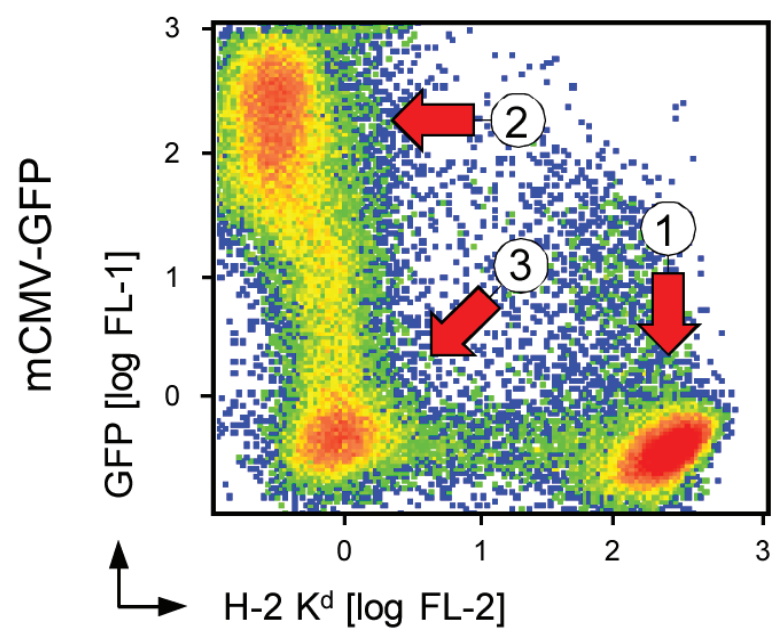


Figure A2. Comparative growth kinetics of mCMV-WT.BAC and mCMV-m152 $\triangle 3 \mathrm{Glyc}$ in immunocompromised mice. Prior to intraplantar infection with $1 \times 10^{5} \mathrm{PFU}$ of the viruses under comparison, $\mathrm{BALB} / \mathrm{c}$ mice were immunocompromised by hematoablative total-body $\gamma$-irradiation with a single dose of $6.5 \mathrm{~Gy}$. At the indicated times p.i., virus multiplication was quantitated in host organs. (A) Virus titers (plaque forming units, $\mathrm{PFU}$ ) in spleen (upper two panels) and lungs (lower two panels), determined by virus plaque assay under conditions of centrifugal enhancement of infectivity; (B) Infection of the liver quantitated by counting the number of infected liver cells, which are mostly hepatocytes, stained by IHC specific for intranuclear IE1 protein. Symbols represent individual mice. Median values are marked by short horizontal bars. Virus doubling times in hours (vDT, with 95\% confidence intervals in parentheses) were calculated from the slopes of log-linear regression lines determined by using the statistics software Mathematica [39]. Note that slopes, unlike the absolute ordinate values, reflect virus growth within the respective tissues/organs, independent of possible differences in the spread to these organs and not influenced by unintentional systematic error or statistical variance in inoculum virus doses.

A
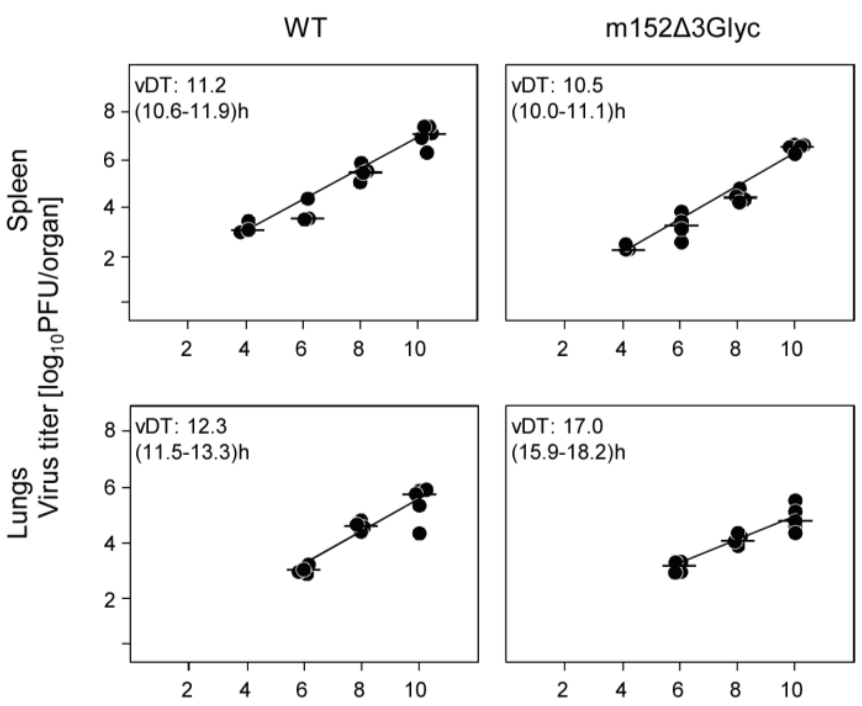

B WT

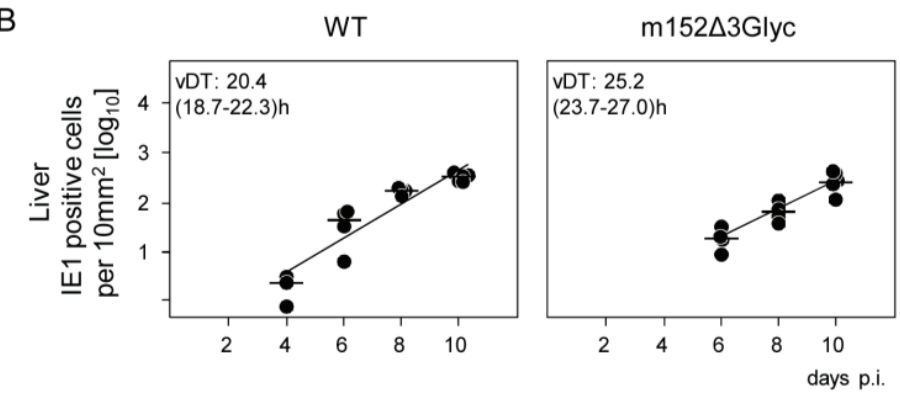


Table A1. Oligonucleotides used in this study; nucleotides inserting aa substitutions are highlighted in bold face.

\begin{tabular}{cc}
\hline Name & Sequence \\
\hline m152-HpaI_for & 5'-GGAGTTAACCATATAAAAGCTGTCCCCCATGCCATTCGAT \\
CAGACGCGGGCTACTCCCGAAAGAGTAAC-3' \\
m152-HpaI-rev & 5'-GGAGTTAACTGACTAATAAGTTATCTTTATTGTACAAGTGT \\
m152_N_83_Q_for & TGTGTGTTATCCCTGAGCCCATTTTCAG-3' \\
m152_N_83_Q_rev & 5'-CATTTTCCTTGGATGCAGGTGAGCGAGCTGG-3' \\
m152_N_230_Q_for & 5'-CCAGCTCGCTCACCTGCATCCAAGGAAAATG-3' \\
m152_N_230_Q_rev & 5'-GGTTCCGTTGGCGTACCAGATCAGTCTCGCGAACGG-3' \\
m152_N_230_Q_for & 5'-CCGTTCGCGAGACTGATCTGGTACGCCAACGGAACC-3' \\
m152_N_263_Q_rev & 5'-GGTTCCGTTGGCGTACCAGATCAGTCTCGCGAACGG-3' \\
m152_BAC_for & 5'-CGCGAAGTCGGTACTACCCTGCAGGTCTCTGAGATCGAGC-3' \\
m152_BAC_rev & 5'-GCGAGTTCGTCTCGAAG-3' \\
pEPKan-S_ & 5'-TAGACCGCCGACAATCAG-3' \\
m152N83Q_for & GCAGGTGAGCGAGCTGGCGCAGCAACCAATTAACCAATTCTGATTAG-3' \\
pEPKan-S_ & 5'-ACGAAGAACGCACTCTCCTGCGCCAGCTCGCTCACCTGCATCCAAGGAA \\
m152N83Q_rev & AATGTTTCACCAGGATGACGACGATAAGTAGGG-3' \\
m152_del_fwd & 5'-TGTCACCGCTCCACGTTTCACCGTCGGTCTCCCGATCGCTAG \\
m152_del_rev & CCTGTACACAGGAACACTTAACGGCTGA-3' \\
& 5'-GAGCACCCGACGATCTGACATTGTCCAGTGTGCCGGTCGCA \\
& CGAACATCAAGGACGACGACGACAAGTAA-3' \\
\hline
\end{tabular}



Reprinted from Viruses. Cite as: Fink, A.; Büttner, J.K.; Thomas, D.; Holtappels, R.; Reddehase, M.J.; Lemmermann, N.A.W. Noncanonical Expression of a Murine Cytomegalovirus Early Protein CD8 T-Cell Epitope as an Immediate Early Epitope Based on Transcription from an Upstream Gene. Viruses 2014, 6, 808-831.

Article

\title{
Noncanonical Expression of a Murine Cytomegalovirus Early Protein CD8 T-Cell Epitope as an Immediate Early Epitope Based on Transcription from an Upstream Gene
}

\author{
Annette Fink, Julia K. Büttner, Doris Thomas, Rafaela Holtappels, Matthias J. Reddehase * \\ and Niels A. W. Lemmermann * \\ Institute for Virology and Research Center for Immunology (FZI), University Medical Center of \\ the Johannes Gutenberg-University Mainz, Obere Zahlbacher Str. 67, Mainz D-55131, Germany; \\ E-Mails: finka@uni-mainz.de (A.F.); julia_buettner@uni-mainz.de (J.K.B.); \\ thomdo00@uni-mainz.de (D.T.); R.Holtappels@uni-mainz.de (R.H.) \\ * Authors to whom correspondence should be addressed; \\ E-Mails: matthias.reddehase@uni-mainz.de (M.J.R.); lemmermann@uni-mainz.de (N.A.W.L.); \\ Tel.: +49-6131-17-9230 (M.J.R); +49-6131-17-9184 (N.A.W.L); \\ Fax: +49-6131-17-9038 (M.J.R \& N.A.W.L.).
}

Received: 23 December 2013; in revised form: 17 January 2014 / Accepted: 26 January 2014 / Published: 14 February 2014

\begin{abstract}
Viral CD8 T-cell epitopes, represented by viral peptides bound to major histocompatibility complex class-I (MHC-I) glycoproteins, are often identified by "reverse immunology", a strategy not requiring biochemical and structural knowledge of the actual viral protein from which they are derived by antigen processing. Instead, bioinformatic algorithms predicting the probability of $C$-terminal cleavage in the proteasome, as well as binding affinity to the presenting MHC-I molecules, are applied to amino acid sequences deduced from predicted open reading frames (ORFs) based on the genomic sequence. If the protein corresponding to an antigenic ORF is known, it is usually inferred that the kinetic class of the protein also defines the phase in the viral replicative cycle during which the respective antigenic peptide is presented for recognition by $\mathrm{CD} 8 \mathrm{~T}$ cells. We have previously identified a nonapeptide from the predicted ORFm164 of murine cytomegalovirus that is presented by the MHC-I allomorph $\mathrm{H}-2 \mathrm{D}^{\mathrm{d}}$ and that is immunodominant in BALB/c (H-2 ${ }^{\mathrm{d}}$ haplotype) mice. Surprisingly, although the ORFm164 protein gp36.5 is expressed as an Early (E) phase protein, the m164 epitope is presented already during the Immediate Early (IE) phase,
\end{abstract}


based on the expression of an upstream mRNA starting within ORFm167 and encompassing ORFm164.

Keywords: antigenic peptides; antigen presentation; CD8 T cell epitope; gene expression; immediate-early protein; murine CMV ORF m164; open reading frame; RACE mapping; reverse immunology; transcription start site; translation start site

\section{Introduction}

In listings of antigenic peptides identified in viral open reading frames (ORFs) by "reverse immunology" [1-3], annotated viral proteins are usually co-listed under the implicit inference that the antigenic peptides are derived by antigen processing when the respective proteins are synthesized, and, thus, belong to the same kinetic class in the cascade regulation of viral gene expression. Although such a "canonical" expression and presentation of an antigenic peptide as a peptide-MHC (pMHC) complex almost certainly applies, because, as far as we are aware of, no viral protein or even self protein is exempt from becoming degraded in one of the antigen processing pathways, the kinetic class assignment of an antigenic peptide and its corresponding protein can nonetheless differ due to "noncanonical" entry into a processing pathway. One mechanism of noncanonical antigen deployment and pre-immediate early presentation is delivery of virion structural proteins during the viral entry process [4-6], whereas canonical expression and presentation would occur not before the late (L) phase, close to the time of virion assembly and release and, thus, possibly too late for antiviral immune protection. A second virion entry-associated mechanism could be the delivery of viral RNA enclosed in virions [7], leading to pre-immediate early gene expression independent from the canonical kinetic class of the respective viral RNA. Such mechanisms can be of functional relevance for cell surface presentation of antigenic peptides prior to the expression of viral immune evasion proteins that would otherwise interfere [8-10]. Here, we report on an example for a third mechanism of noncanonical antigenic peptide presentation.

We have previously identified an antigenic peptide in ORFm164 of murine cytomegalovirus (mCMV) by employing the strategy of "reverse immunology" [11]. CD8 T cells derived from infected BALB/c (haplotype H-2 ${ }^{\mathrm{d}}$ ) mice were found to respond to MHC $\left(\mathrm{H}-2 \mathrm{D}^{\mathrm{d}}\right)$ gene-transfected $\mathrm{L}$ cells (fibroblasts of the unrelated haplotype $\mathrm{H}-2^{\mathrm{k}}$ ) exogenously loaded with high performance liquid chromatography (HPLC)-separated naturally processed peptides derived from mCMVinfected BALB/c mouse embryo fibroblasts (MEF). This was the first indication of the existence of mCMV peptide(s) presented by MHC-I ${ }^{\mathrm{d}}$, as the only antigenic mCMV peptide known at that time was the Immediate Early (IE) peptide IE1 presented by MHC-I L ${ }^{\mathrm{d}}$ [12]. Algorithms predicting H-2 D binding nonapeptides were applied to the viral genome-wide coding sequence and predicted a panel of high-scoring peptides of which a peptide with the amino acid sequence AGPPRYSRI encoded by ORFm164 was functionally verified by the response of CD8 T cells. Along with the IE1 epitope and with more recently identified epitopes from ORFs M105 and $m 145$ [13], the m164 epitope proved to be immunodominant with respect to the frequency of CD8 T cells responding to it during the immune response to acute infection. In addition to the IE1 epitope, which is the prototype of an epitope 
that induces expansion of the memory CD8 T-cell pool at extralymphoid sites of latent mCMV infection [14], a phenomenon known today as "memory inflation" ([15], reviewed in [16-18]), the m164 epitope turned out to be the second inducer of memory inflation in the H-2 ${ }^{\mathrm{d}}$ haplotype, a property that it shares with three epitopes in the H-2 $2^{\mathrm{b}}$ haplotype [19] and that is thought to indicate episodes of limited viral gene expression during latency (for recent reviews, see [20,21]). As the ORFm164 protein, a non-essential ER-resident type-I glycoprotein of $36.5 \mathrm{kDa}$, is expressed as an Early (E) phase protein [22], it was assumed that the m164 peptide is presented not until the E phase. Interestingly, cytolytic $\mathrm{T}$ lymphocytes (CD8 $\left.{ }^{+} \mathrm{CTL}\right)$ of an $\mathrm{m} 164$-specific $\mathrm{CTL}$ line (m164-CTLL) recognized infected fibroblasts in the E phase despite all immune evasion molecules being expressed, a finding that was explained by high numbers of pMHC-I (m164 peptide-D ${ }^{\mathrm{d}}$ ) complexes exhausting the inhibitory capacity of the immune evasion proteins, thus allowing the escape of some of the pMHC-I complexes to the cell surface sufficient for recognition [23]. Importantly, in an experimental approach of CMV immunotherapy (for reviews, see [24,25]), m164-CTLL proved to protect against multiple organ CMV disease upon adoptive cell transfer into infected, immunocompromised recipient mice [11].

Here, we report the astounding finding that this "E phase peptide", as well as a transgenic peptide replacing it in a recombinant $\mathrm{MCMV}$, are recognized by CTLL of the respective cognate specificities on infected cells metabolically arrested in the IE phase, thus representing a case of "noncanonical presentation". This presentation is not explained by virion entry-dependent delivery of antigenic virion protein or epitope-encoding virion-associated RNA, but is based on IE phase expression of an unrelated mRNA originating in ORFm167 and encompassing the epitope-encoding sequence in ORFm164. These data call for caution in assigning epitope presentation to a kinetic phase based just on the kinetic class to which the corresponding viral protein belongs.

\section{Results and Discussion}

\subsection{Antigenic Sequences Encoded by the Predicted ORFm164 Are Presented on Infected Cells Metabolically Arrested in the IE Phase as Well as in the E Phase}

MEFs were infected under established conditions of IE phase or E phase arrest (Figure 1A). For IE phase arrest, infection was performed in the presence of the reversible protein synthesis inhibitor cycloheximide $(\mathrm{CH})$ that becomes replaced after $3 \mathrm{~h}$ with the irreversible transcription inhibitor actinomycin D (ActD). This allows for selective and enhanced transcription from IE genes followed by IE protein synthesis, and prevents transactivation of E gene transcription. E phase arrest is achieved by $16 \mathrm{~h}$ of infection in the continuous presence of the DNA synthesis inhibitor phosphonoacetic acid (PAA) preventing, by definition, the transition from the $\mathrm{E}$ phase to the $\mathrm{L}$ phase. The mutation strategy for manipulating antigenicity and immunogenicity of ORFm164 (reviewed in [26]) is sketched in Figure 1B. In a first approach, MEF were infected either with BAC-cloned wildtype (WT) virus mCMV-WT.BAC coding for the authentic ORFm164 epitope AGPPRYSRI or with a mutant virus mCMV-m164Ala, in which the $C$-terminal amino acid isoleucine of the antigenic sequence is genetically replaced with alanine. This strategy prevents $C$-terminal proteasomal cleavage of the antigenic peptide in the first place. In addition, it reduces binding affinity to the presenting MHC-I molecule $\mathrm{D}^{\mathrm{d}}$ in case that inefficient, residual proteasomal cleavage might still generate 
some AGPPRYSRA peptide. We have previously introduced $C$-terminal residue mutagenesis as an ideal control for testing epitope specificity of the CD8 T cell response [13,27-30], leaving other parameters of infection bona fide untouched. In a second approach, referred to as "orthotopic peptide swap", the authentic antigenic peptide AGPPRYSRI was replaced at precisely the same site with the ovalbumin-derived model peptide sequence SIINFEKL in recombinant virus mCMV-SIINFEKL [29]. This peptide swap is associated with a switch in the presenting MHC-I molecule, namely from $\mathrm{H}-2 \mathrm{D}^{\mathrm{d}}$ to $\mathrm{K}^{\mathrm{b}}$. For serving as an epitope specificity control, recombinant virus mCMV-SIINFEKA was generated based on the rationale already explained above [29].

Figure 1. Presentation of intrinsic and transgenic m164 peptides in the IE and E phase. (A) Scheme of the experimental protocol for selective arrest of viral gene expression in infected fibroblasts by metabolic inhibitors. For arrest in the IE phase, MEF are infected under conditions of centrifugal enhancement, resulting in an MOI of 4 , in the presence of cycloheximide $(\mathrm{CH}, 100 \mu \mathrm{g} / \mathrm{mL})$ that becomes replaced at $3 \mathrm{~h}$ post-infection (p.i.) with actinomycin D (ActD, $5 \mu \mathrm{g} / \mathrm{mL})$. For arrest in the E phase, MEF are infected likewise for $16 \mathrm{~h}$ in the continuous presence of PAA $(250 \mu \mathrm{g} / \mathrm{mL})$. At $5 \mathrm{~h}$ and $16 \mathrm{~h}$ p.i., respectively, these cells are used in an IFN- $\gamma$-based ELISpot assay as stimulator cells for CTL from CTL lines (CTLL) of defined antigenic peptide specificities; (B) Scheme of the mutagenesis rationale for modification of $\mathrm{m} 164$ protein antigenicity and immunogenicity in recombinant viruses. Shown is an overview map of the mCMV genomic region encompassing ORFs m163-m165. ORFs are symbolized by boxes, with the blue box representing ORFm164 encoding protein gp36.5 that comprises the intrinsic antigenic peptide AGPPRYSRI, which is presented by the MHC-I molecule $\mathrm{H}-2 \mathrm{D}^{\mathrm{d}}$. Hatched boxes indicate overlaps with neighboring ORFs. Amino acid sequences of the intrinsic and the replacing transgenic CD8 T-cell epitope, which is presented by $\mathrm{H}-2 \mathrm{~K}^{\mathrm{b}}$, are shown in blue and green one-letter code, respectively. The $C$-terminal residue mutation to Ala is highlighted by red capital letter. Epitope-flanking residues are shown in smaller font; (C) Frequencies of cells of the indicated CTLL that respond in an IFN- $\gamma$-based ELISpot assay to sensitization by MEF of the presenting MHC haplotype infected with the indicated viruses under the indicated conditions of gene expression phase arrest. (Left two panels) Bars represent most probable numbers determined by intercept-free linear regression analysis based on triplicate assay cultures and graded numbers of CTL seeded; error bars indicate the $95 \%$ confidence intervals. n.i., no infection (uninfected MEF); ActD, MEF infected for $5 \mathrm{~h}$ in the presence of ActD preventing transcription from the very beginning; UV-IE, IE conditions applied to cells mock-infected with virus inactivated by UV-light $\left(254 \mathrm{~nm}, c a .4500 \mathrm{~J} / \mathrm{m}^{2}\right)$. IE and E, MEF infection under conditions of IE and E phase arrest, respectively, as explained above. (Right panel) Bars represent median values of absolute spot counts from triplicate assay cultures for CTL seeded in graded numbers as indicated. Variance bars show the range.

A

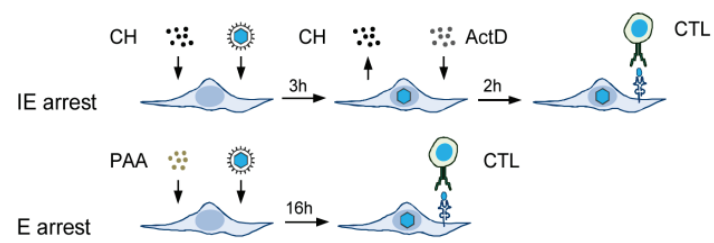


Figure 1. Cont.

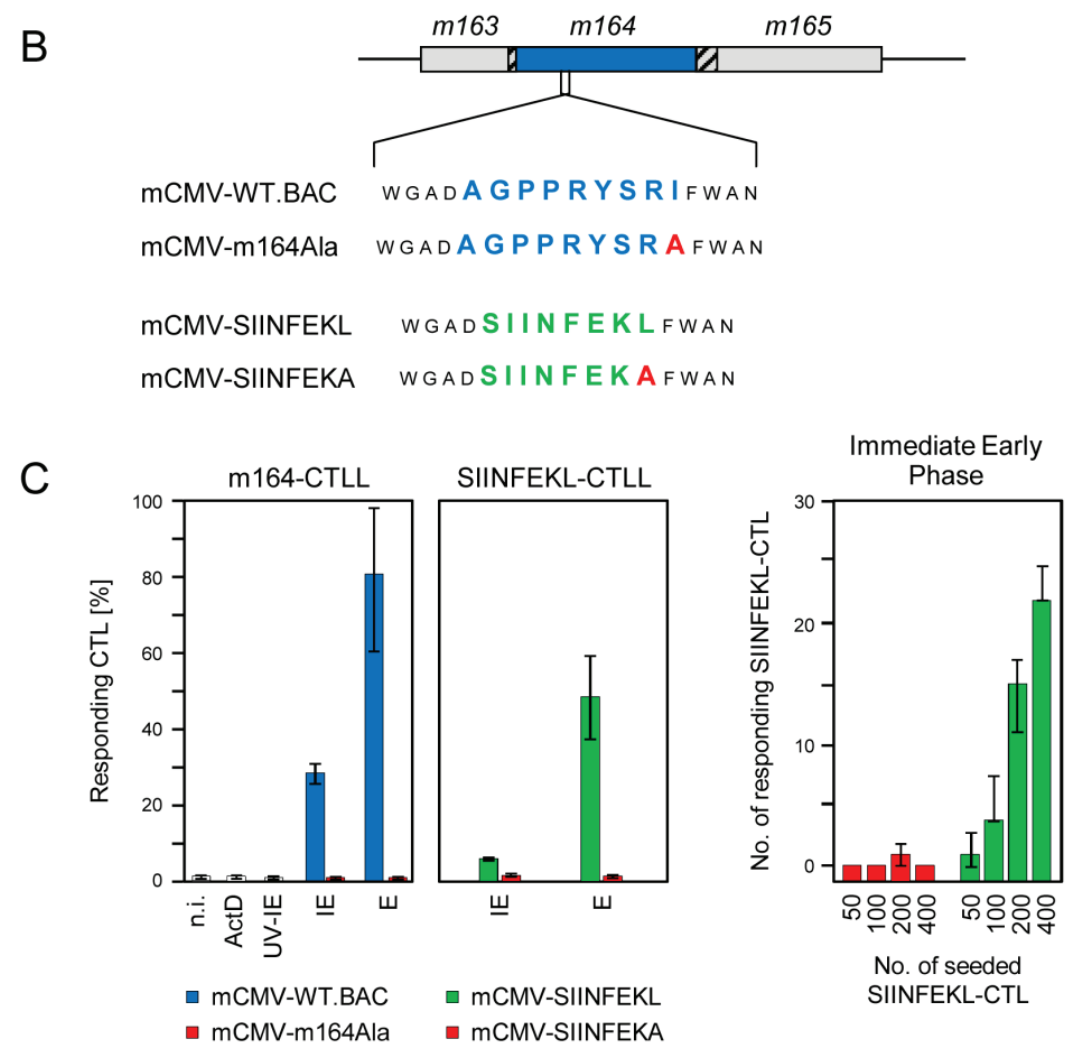

In the experiment to the first approach (Figure 1C, far left panel) m164-CTLL recognized WT virus-infected target cells in the E phase, as it was to be expected. Surprisingly, however, a significant proportion of the m164-CTL, supposedly those with the highest functional avidity [25,31], also recognized target cells arrested in the IE phase. Recognition in both the IE and the E phase was abolished after infection with the epitope deletion virus mCMV-m164Ala, thereby excluding the theoretical possibility that inhibitor treatment of infected target cells might have induced $\mathrm{T}$ cell receptor (TCR)-independent, non-epitope specific signaling that activates CTLL. Known noncanonical modes of epitope presentation were also ruled out: (i) infection in the presence of inhibitor ActD prevented presentation of the m164 epitope. This revealed the requirement for de novo transcription and thus excluded a noticeable contribution of virion-associated RNA, and (ii) UV-inactivated virus did not lead to the presentation of the m164 epitope, which was not unexpected in light of the fact that no ORFm164-encoded protein was detected in the virion proteome [32]. These findings were corroborated by the experiment to the second approach (Figure 1C, center and far right panels). Although the response of SIINFEKL-specific CTL to the $\mathrm{K}^{\mathrm{b}}$-presented epitope was lower compared to recognition of the authentic m164 peptide by m164-specific CTL, which is not unusual as processing rates and MHC-I binding affinity differ between different epitopes, the key message of presentation in both the IE and $\mathrm{E}$ phase was reproduced, and epitope-specificity was confirmed for both phases by missing recognition of cells infected with mCMV-SIINFEKA.

In conclusion, antigenic peptides naturally present or experimentally placed in the sequence of the E phase protein $\mathrm{m} 164 / \mathrm{gp} 36.5$ are noncanonically expressed and presented in the IE phase, an obvious paradox that needed to be resolved. 


\subsection{Detection of Distinct $m R N A$ Species in the IE and $E$ Phases by $m 164$}

\section{Sequence-Specific Hybridization}

As virion proteins and virion-associated RNA, apparently, did not account for presentation of m164 peptide in the IE phase (recall Figure 1C, left panel), and as recognition by CD8 T cells was clearly epitope-specific (recall Figure 1C, all panels), there must exist an IE mRNA encoding a protein that encompasses the antigenic sequence. Northern blot analysis (Figure 2), using a DIG-labeled m164-specific single-strand RNA-probe for hybridization with total RNA, revealed only an E phase RNA of slightly more than $2 \mathrm{~kb}$ in size present $3 \mathrm{~h}$ after infection in the absence of protein synthesis inhibition by $\mathrm{CH}$ but absent in the presence of $\mathrm{CH}$. When sensitivity was increased by using poly $(\mathrm{A})^{+}$RNA for hybridization with the riboprobe, this mRNA species was far more prominent but remained absent in cells infected in the presence of $\mathrm{CH}$, thus identifying it as a true $\mathrm{E}$ phase transcript. The nature of a low-abundance $\mathrm{CH}$-sensitive $\mathrm{E}$ phase transcript of $c a .4 \mathrm{~kb}$, which is present only in infected cells, was not pursued further as our interest was focused on the existence of an IE RNA. Conversely, however, this sensitive analysis indeed revealed an mRNA species of $>5 \mathrm{~kb}$ that became visible only after infection in the presence of $\mathrm{CH}$, thus identifying it as an IE phase transcript. The blot did not reveal splice products that would be expected to be more stable and, thus, more prominent than a long splice precursor. In conclusion, m164 sequence is indeed present in distinct mRNA species of $c a .2 \mathrm{~kb}$ and $>5 \mathrm{~kb}$ expressed in the $\mathrm{E}$ and the IE phase, respectively.

Figure 2. Identification of m164 transcripts by Northern blot analysis. Total cellular RNA or poly $(\mathrm{A})^{+}$RNA were isolated at $3 \mathrm{~h}$ p.i. from MEF infected in the presence $(+)$ or absence $(-)$ of $\mathrm{CH}(100 \mu \mathrm{g} / \mathrm{mL})$ and were analyzed by Northern blotting using a DIG-labeled m164-specific RNA probe. The arrow highlights a specific band of $>5 \mathrm{~kb}$ representing an IE mRNA.

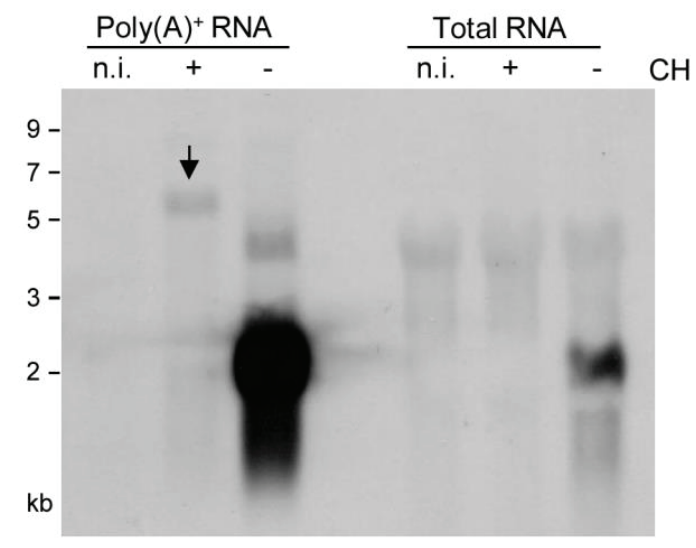

\subsection{Mapping of the m164 E Phase Start Sites of Transcription and Translation}

It was obvious to predict that the $c a .2 \mathrm{~kb}$ mRNA that is abundantly expressed in the E phase codes for the previously identified $\mathrm{E}$ phase protein m164/gp36.5 [22], which is considerably smaller than the gp48.6 (includes $2 \mathrm{kDa}$ from the identified single $N$-glycosylation [22]) predicted for a protein starting at the first of three AUG (Met1, Met27, and Met91) translation start sites in 
the predicted ORFm $164[33,34]$. In fact, relative to Met1, the $N$-terminus of the mature protein was definitively identified by mass spectrometric sequence analysis to be Ser108. We, therefore, originally speculated that the translation start site might be the third AUG (Met91). Accordingly, we proposed a 17-aa signal peptide of which existence was bioinformatically supported by the SignalP 3.0 algorithm [35] predicting signal peptide peptidase cleavage between Ala107 and Ser108 [22].

With the initial aim to verify this assumption, mutational analysis was performed by transfection of COS7 cells with a complete set of expression plasmids comprising all possible combinations of ATG to GCG mutations of the three proposed start codons (Figure 3A). This analysis revealed that all three AUG start codons can be used, at least upon transfection of COS7 cells, when usage is enforced by absence of competing start codons. Unexpectedly, however, the different-lengths primary translation products were all co- or posttranslationally processed to yield the $36.5 \mathrm{kDa}$ mature protein (Figure 3B), which also showed the typical ER and nuclear rim/outer nuclear membrane localization (Figure 3C) described for protein m164/gp36.5 previously [22]. Translation was prevented only by mutation of all three ATG in expression plasmid $\triangle \mathrm{ATG}$ (Figure $3 \mathrm{~B}$ ), thus showing that no start codon other than AUG is used.

Figure 3. Mapping of AUG start codon usage by comprehensive mutational analysis through cell transfection with a complete set of expression plasmids. (A) Overview scheme of combinatorial ATG to GCG mutations for the three ATG codons present in the predicted ORFm164; (B) Western blot analysis of gene products of mutated $m 164$ ORFs. COS7 cells were transfected with $4 \mu \mathrm{g}$ of expression plasmids carrying single or combined ATG to GCG mutations at potential start sites. After $48 \mathrm{~h}$, total protein was extracted and $30 \mu \mathrm{g}$ of the protein lysates were subjected to sodium dodecyl sulfate polyacrylamide gel electrophoresis (SDS-PAGE) (12.5\%) followed by Western blot analysis for detection of m164 protein species by using polyclonal antibody directed against a $C$-terminal peptide; (C) m164/gp36.5 localization in the ER, independent of usage of the 1st, 2nd, or 3rd AUG as start codon. COS7 cells were seeded on glass coverslips and were transfected with $0.4 \mu \mathrm{g}$ of pcDNA_m164_ATG_1/2/3,_1, 22, or _3. At $24 \mathrm{~h}$ after transfection, cells were fixed and protein m164/gp36.5 was detected with the polyclonal antibody. DNA was stained by Hoechst-dye.

A

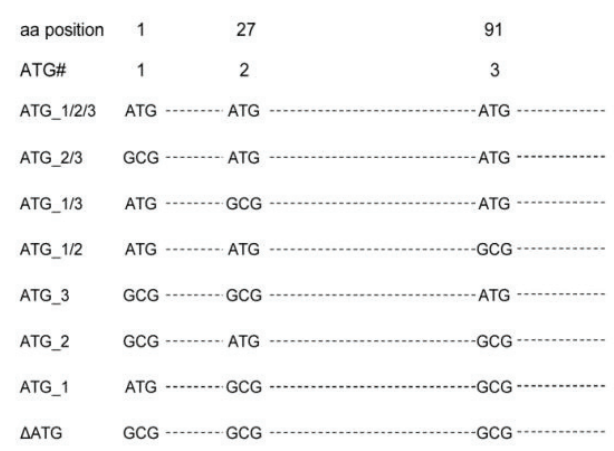

B

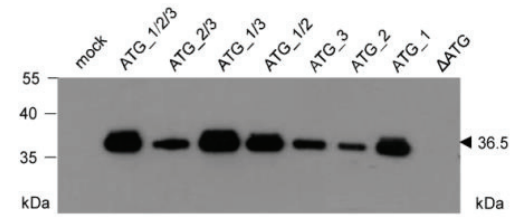

C

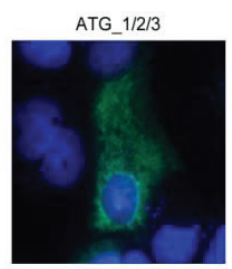

ATG 2

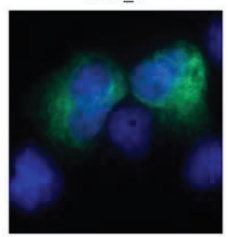

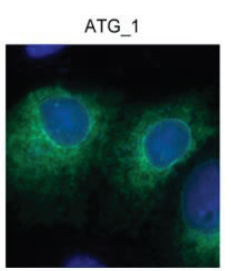

ATG 3

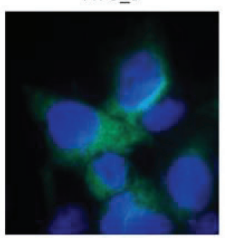


This analysis, however, did not reveal the start AUG that is actually used when any of the three can be chosen, although increased signal strengths with expression plasmids containing ATG_1, alone or in combinations, indicated preferential usage of the first AUG (Figure 3B). Translation start at the first AUG is also strongly suggested by ribosome profiling, showing reads beginning at position -12 relative to the AUG, which is the correct distance from a start site [36,37]. To finally settle this question under the more real conditions of infection, we constructed recombinant virus mCMV-m164_ $\triangle \mathrm{ATG}$ _1, with only the first start codon mutated, to infect MEF. As revealed by Western blot analysis, this mutation abolished expression of gp36.5 (Figure 4A).

Figure 4. (A) Verification of the 1 st AUG as start codon. MEF were infected with mCMV-WT.BAC or mCMV-m164__ATG_1, and at $6 \mathrm{~h}$ p.i. proteins were extracted and $30 \mu \mathrm{g}$ of whole protein lysates were subjected to SDS-PAGE (12.5\%) followed by Western blot analysis detecting protein $\mathrm{m} 164 / \mathrm{gp} 36.5$; (B) Verification of the E phase mRNA encoding the ORFm164 product. The in vitro transcribed RNAs (see Appendix Figure A1) were used as templates for in vitro translation. Shown is the autoradiograph of $\left[{ }^{35} \mathrm{~S}\right]$-labeled translation products separated by SDS-PAGE. Lane 1: proteins translated from the RACEm164 in vitro transcript. Lane 2: proteins translated from the ORFm164 in vitro transcript. Lane 3: BMV (Brome Mosaic Virus) RNA control supplied in the Ambion MEGAscript SP6 kit (Life Technologies, Catalog No. AM1330, Darmstadt, Germany); (C) Map of the m164 mRNA and its localization as revealed by 5'- and 3'-RACE shown in Appendix Figure A1.

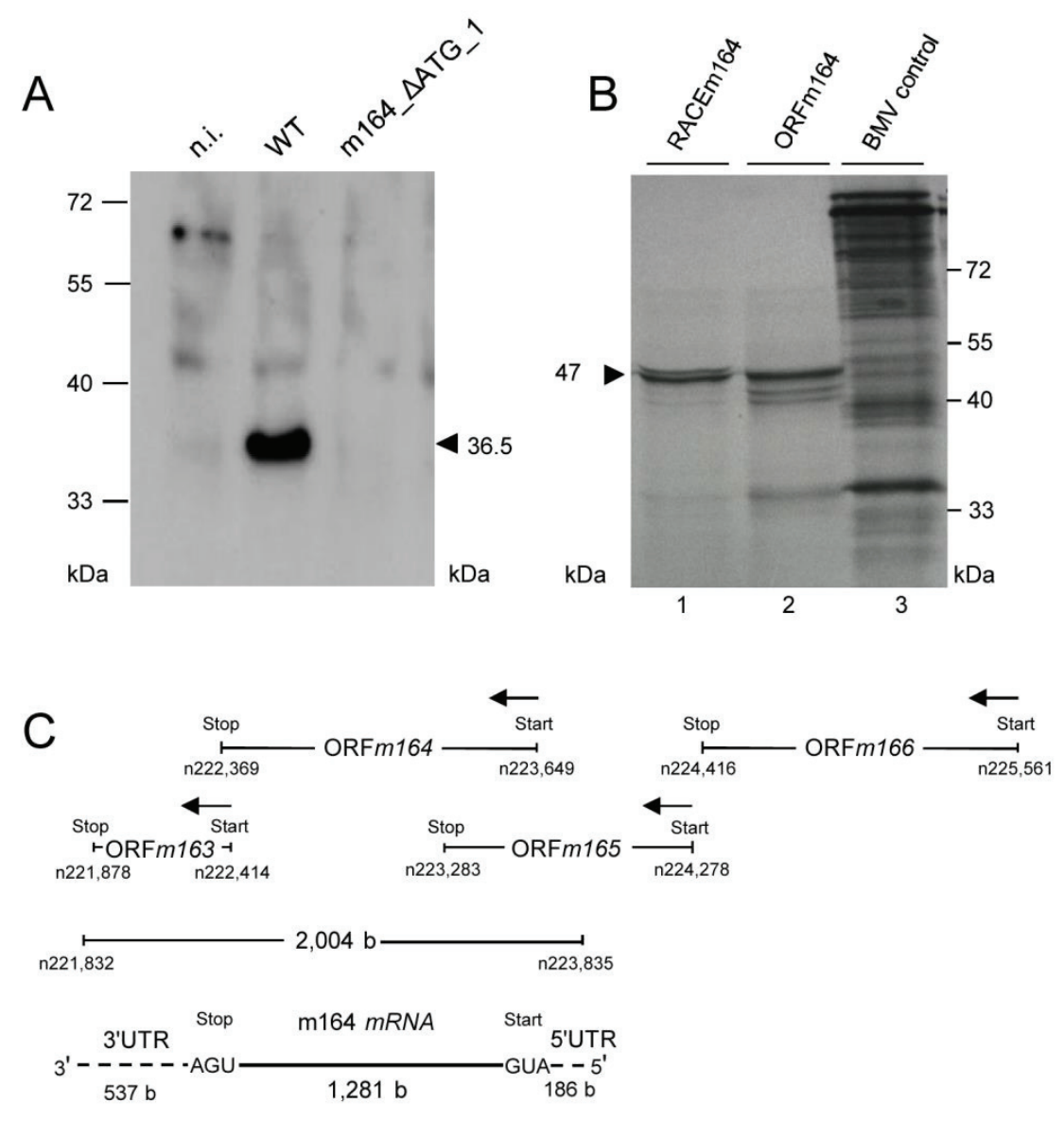


Having, thus confirmed ORFm164, the slightly more than $2 \mathrm{~kb}$ length of the predominant $\mathrm{E}$ phase mRNA exceeded the calculated $1281 \mathrm{~b}$ of the ORF, which is difficult to explain just by the poly $(\mathrm{A})^{+}$tail and rather suggests the existence of UTRs. To address this issue, rapid amplification of cDNA ends (RACE) analysis was performed and in fact identified 3' and 5' UTRs of 537 bp and $186 \mathrm{bp}$, respectively (Appendix Figure A1). This result defined a length of the mRNA of 2,004 bp (polyadenylated tail not included), which is compatible with the length revealed by Northern blot analysis of poly(A) ${ }^{+}$E phase m164 transcripts (recall Figure 2). Finally, in vitro transcribed ORF RNA as well as RACE RNA (Appendix Figure A1C) were translated in vitro and both resulted in a dominant product of $c a .47 \mathrm{kDa}$ (Figure 4B) in accordance with the calculated molecular mass of the unprocessed $46.6 \mathrm{kDa}$ primary translation product starting at Met1 (see above). Thus, in conclusion, the $c a .2 \mathrm{~kb}$ E phase poly $(\mathrm{A})^{+} \mathrm{RNA}$ is, in fact, the one that codes for the $\mathrm{E}$ phase protein m164/gp36.5. To sum these results up, Figure 4C shows a revised map of m164 and its neighboring genes with which it largely overlaps.

\subsection{Confirmation of an Upstream IE Phase RNA Encompassing the Complete m164 E Phase RNA Sequence}

What, finally, is the $>5 \mathrm{~kb}$ IE phase poly $(\mathrm{A})^{+}$RNA detected with the m164 sequence-specific riboprobe in the Northern blot (recall Figure 2)? RACE mapping of the complete m164 E phase transcript allowed us to design RT-PCRs with one primer placed at the 3 ' terminus and a second primer placed either to the left (RT-PCR I) or to the right (RT-PCR II) of the $5^{\prime}$ terminus, that is inside or outside of the E phase transcript, respectively (for the strategy, see Figure 5A).

With total RNA from infected cells, the inside 5'-end primer resulted in the expected amplificate of 2,001 bp in RT-PCR I, corresponding to the RACE-mapped E phase mRNA. Notably, in RTPCR II, the outside 5'-end primer also gave an amplificate, thus indicating the existence of an RNA that initiates upstream of the $5^{\prime}$ end and includes the complete m164 E phase transcript sequence (Figure 5B). As RT-PCR I detects both transcripts, the signal was only in part sensitive to $\mathrm{CH}$ treatment of the infected cells, with the remaining signal coming from IE RNA. In contrast, an enhanced signal seen by RT-PCR II after $\mathrm{CH}$ treatment shows that it results from IE RNA. Upstream "walking" of the 5'-end primer (Appendix Figure A2) located the start site of this IE mRNA between nucleotides n226,549 and n226,903 within ORFm167 (for a summarizing map, see Figure 6), resulting in a length of $>5 \mathrm{~kb}$ compatible with the length seen for the IE phase poly $(\mathrm{A})^{+}$ RNA detected by the Northern blot analysis (recall Figure 2). As this RNA ends before the start of the predicted ORFm167, it cannot be the regular m167 transcript, whereas ORFm166 [38] is completely included. Notably, in accordance with IE gene expression upstream of $m 164$, by using an mCMV ORF microarray, the group of T.E. Shenk has defined a cluster of IE genes within restriction fragment Hind IIIE, namely genes m166-m169 [39-41].

As the full-length m166 mRNA with a putative 5' UTR has not yet been mapped, it might overlap with ORFm167 so that the transcription start site of m166 mRNA remains a candidate. Alternatively, the long m164 epitope sequence-encompassing IE phase mRNA might represent a new transcript using an alternative start site within ORFm167. Theoretically, as no in-frame translation start sites are present upstream of ORFm164, the IE mRNA might represent a polycistronic transcript using the ORFm164 start site, either directly or with an upstream internal 


\section{4}

ribosomal entry site (IRES). Bioinformatical analysis [42] indeed revealed a putative group 4 IRES [43] 1,044 bp upstream of the confirmed m164 AUG. Though IRES sequences are rarely described for herpesviral transcripts, it remains to be tested if this IRES is functional.

Figure 5. Verification of the m164 IE phase mRNA by RT-PCR. (A) Schematic map of the RACE-defined m164 mRNA with its 3'- and 5'-UTRs. RACE analysis (see Appendix Figure A1) predicted an mRNA of 2004 b. Positions of oligonucleotides binding inside or outside of the RACE product are indicated. Calculated sizes of the respective RT-PCR products are shown in lines I and II; (B) MEF were infected with mCMV-WT.Smith in presence $(+)$ or absence $(-)$ of ActD $(5 \mu \mathrm{g} / \mathrm{mL})$ or $\mathrm{CH}$ $(100 \mu \mathrm{g} / \mathrm{mL})$ as indicated. At $1.5 \mathrm{~h}$ or $3 \mathrm{~h}$ p.i. total RNA was isolated and subjected to RT-PCRs using oligonucleotides for product I or II (see Appendix Table A1). Amplificates were analyzed on a 1\% agarose gel. M, marker; n.i. not infected; bp, basepairs. Controls with no RT step were negative throughout (not shown).

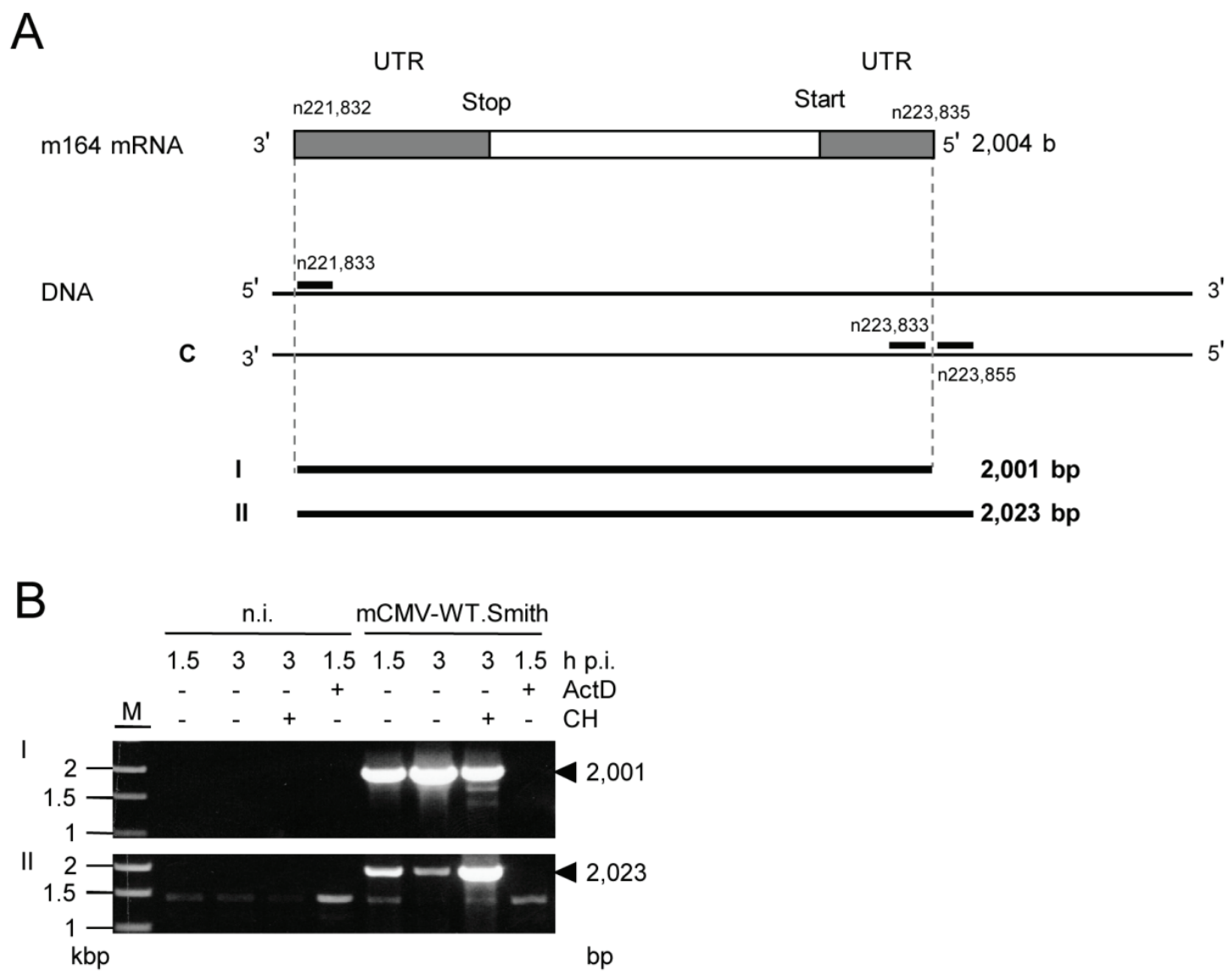

As Western blot analysis with antibodies directed against a $C$-terminal peptide of ORFm164 did neither detect m164/gp36.5 nor larger or smaller isoforms expressed in the IE phase ([22], and data not shown), translation from the long IE mRNA and consequent m164 epitope presentation in the IE phase may result from a smaller isoform or even oligopeptide lacking the $C$-terminus.

In any case, this study has revealed the existence of an IE phase mRNA that starts upstream of the E phase m164 transcript and that spans the complete m164 sequence, including the sequence that encodes the CD8 T-cell epitope of which presentation was, so far, assigned only to the E phase. 
Figure 6. Provisional map of the $>5 \mathrm{~kb}$ m164 IE mRNA and its localization within the mCMV genome. Positions of oligonucleotides binding inside or outside of the IE transcript are shown. Unmapped sequences are indicated as hatched boxes. A predicted IRES is indicated.

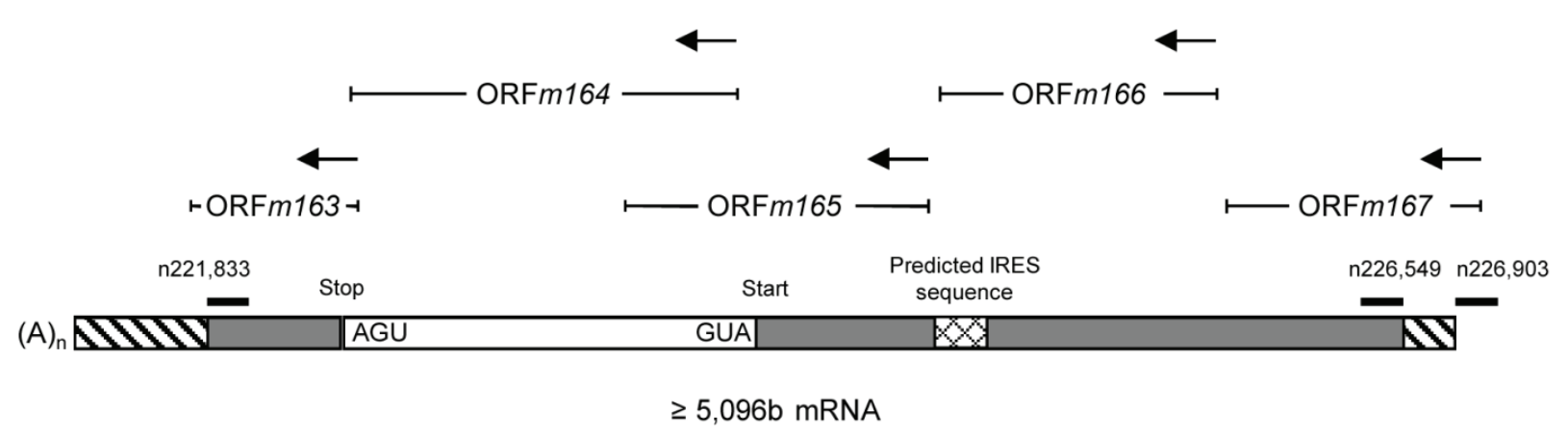

\section{Experimental Section}

\subsection{Cells, Viruses, and Mice}

Primary BALB/c or C57BL/6 mouse embryo fibroblasts (MEF) were prepared as described previously [44] and cultivated in modified Eagle's medium (MEM) supplemented with $10 \%$ fetal calf serum (FCS) and antibiotics. For transfection experiments, COS7 cells were cultivated and seeded $24 \mathrm{~h}$ prior to transfection in DMEM medium supplemented with $10 \%$ FCS and antibiotics.

The m164 peptide (AGPPRYSRI)-specific, polyclonal cytolytic T-lymphocyte (CTL) line (m164-CTLL), and the SIINFEKL-specific CTLL were generated and tested for functional avidity as described previously $[44,45]$.

High virus titer stocks of mCMV Smith strain (mCMV-WT.Smith; ATCC VR-194/1981 re-accessioned as VR-1399), mCMV.WT.BAC [46], mCMV-m164Ala [13], mCMV-SIINFEKL [29], and mCMV-SIINFEKA [29] were prepared from infected MEF according to standard procedures $[44,45]$. Unless stated otherwise, infection was regularly performed with mCMV-WT.Smith.

$\mathrm{BALB} / \mathrm{c}$ and C57BL/6 mice were bred and maintained under SPF conditions at the "Central Laboratory Animal Facility (CLAF)" of the University Medical Center Mainz. All experimental procedures were performed in compliance with the "International Guiding Principles for Biomedical Research Involving Animals" guidelines [47]. The experiments were approved according to German federal law under permission number AZ 23 177-07/G 09-1-004.

\subsection{Infection Conditions}

Confluent MEF were infected in the second or third cell culture passage under conditions of centrifugal enhancement of infectivity with $0.2 \mathrm{PFU} /$ cell of the indicated viruses, resulting in an effective multiplicity of infection (MOI) of $\sim 4$ PFU per cell. For viral transcriptional arrest in the IE-phase, cells were infected in presence of cycloheximide $(\mathrm{CH} ; 100 \mu \mathrm{g} / \mathrm{mL}$; Sigma-Aldrich, Catalog No. C7698, Steinheim, Germany) and, 3 h later, CH was replaced with Actinomycin D (ActD; $5 \mu \mathrm{g} / \mathrm{mL}$; Sigma-Aldrich, Catalog No. A9415, Steinheim, Germany). For E phase arrest, 
cells were infected in the presence of phosphonoacetic acid (PAA; $250 \mu \mathrm{g} / \mathrm{mL}$, Catalog No. 284270, Steinheim, Germany) [44].

\subsection{Construction of Expression Plasmids}

To amplify the full-length sequence of ORFm164, PCR was performed using mCMV-WT.BAC genomic DNA as template with oligonucleotides m164_rev_BamHI and m164_full_Hind III (Appendix Table A1) with the following protocol parameters: $5 \mathrm{~min}$ at $95{ }^{\circ} \mathrm{C} ; 35$ cycles of $10 \mathrm{~s}$ at $94{ }^{\circ} \mathrm{C}, 30 \mathrm{~s}$ at $60{ }^{\circ} \mathrm{C}$, and $40 \mathrm{~s}$ at $72{ }^{\circ} \mathrm{C}$ followed by final primer extension for $10 \mathrm{~min}$ at $72{ }^{\circ} \mathrm{C}$. The PCR product was subcloned into vector pcDNA3.1 after BamH I/Hind III restriction. To insert nucleotide exchanges ATG to GCG at nt positions 223, 649-223, 647; 223, 571-223, 569 and 223, 379-223, 377 the construct was subjected to site-directed mutagenesis using the Quick Change II Site-Directed Mutagenesis Kit (Agilent, Catalog No. 200524, Böblingen, Germany) with the oligonucleotide pairs m164_1stATG_for/rev, m164_2ndATG_for/rev, and m164_3rdATG_for/rev (Appendix Table A1), respectively. The mutagenesis resulted in constructs pcDNA-m164_ATG_2/3, pcDNA-m164_ATG_1/3, and pcDNA-m164_ATG_1/2. Plasmid pcDNA-m164_ATG_2/3 was subjected to a second round of mutagenesis using the respective oligonucleotides resulting in the constructs pcDNA-m164_ATG_3 and pcDNA-m164_ATG_2. Plasmid pcDNA-m164_ATG_1/3 was used as template for construction of pcDNA-m164_ATG_1. A third round of mutagenesis using pcDNA-m164_ATG_3 as a template resulted in pcDNA-m164__ATG. The successful replacement was confirmed by sequencing (GATC; Konstanz, Germany).

\subsection{Construction of Recombinant $m C M V$}

To generate recombinant virus mCMV-m164_AATG_1, BAC mutagenesis was performed by Red-mediated markerless DNA recombination as described by Tischer and colleagues [47]. In brief, oligonucleotides pEPKan-S_m164_1stATG_for and pEPKan-S_rev (see Appendix Table A1) were used for a PCR with plasmid pEP-kanS [48] as a template. The resulting products were subjected to a second round of PCR using oligonucleotides pEPKan-S_m164_1stATG_rev and m164_1stATG_short. The resulting product was transformed into GS1783 cells carrying WT.BAC. After Red-recombination, arabinose-induced I-SceI expression, and a second round of Red-recombination, m164_SATG_1 BAC DNA was purified, and successful replacement of the ATG was confirmed by sequencing (GATC; Konstanz, Germany).

\subsection{Protein Extraction and Analysis}

MEF were seeded on 10-cm diameter cell culture dishes and infected with an MOI of 4 under conditions of centrifugal enhancement of infectivity. At defined times p.i., cells were washed with ice-cold PBS and scraped-off. After centrifugation ( $5 \mathrm{~min}, 3,000 \mathrm{rpm}, 4{ }^{\circ} \mathrm{C}$ ), the pelleted cells were lysed for $15 \mathrm{~min}$ on ice in $200 \mu \mathrm{L}$ Lysis Buffer/dish composed of $0.2 \mathrm{M} \mathrm{NaCl}, 1.5 \mathrm{mM} \mathrm{MgCl}$, 4 mM EDTA, 4 mM EGTA, 1\% Triton X-100, 20 mM HEPES; with complete proteinase inhibitor (diluted 1:25; Roche, Catalog No. 11697498001, Mannheim, Germany) and 1 mM DTT added shortly before use. After centrifugation (10 $\left.\mathrm{min}, 14,000 \mathrm{rpm}, 4{ }^{\circ} \mathrm{C}\right)$, supernatants were collected and the amount of protein was determined by BCA-Assay (Thermo Scientific, Catalog No. 23225, 
Dreieich, Germany). An amount of $30 \mu \mathrm{g}$ of total protein was separated on an SDS-PAGE followed by Western blot analysis. Detection of m164 proteins was performed with polyclonal affinity-purified rabbit antibodies directed against a $C$-terminal peptide (1:500) [49] and an HRP-conjugated anti-rabbit antibody as secondary Ab (DakoCytomation, Catalog No. P045001, Hamburg, Germany).

\subsection{Transfection}

A total of $5 \times 10^{5} \mathrm{COS} 7$ cells per $10-\mathrm{cm}$ diameter dish were seeded and $4 \mu \mathrm{g}$ DNA was transfected with Polyfect (Qiagen, Catalog No. 301105, Hilden, Germany) according to the manufacturer's instructions. Forty-eight hours later, cells were harvested and total protein was extracted as described above. An amount of $30 \mu \mathrm{g}$ of protein lysates were separated on a $12.5 \%$ SDS-PAGE followed by m164 protein-specific Western blot analysis.

\subsection{Immunofluorescence Analysis}

COS7 cells seeded on glass coverslips and transfected with $0.4 \mu \mathrm{g}$ DNA were fixed with $4 \%$ (wt/vol) paraformaldehyde in PBS supplemented with 4\% (wt/vol) sucrose. After incubation for $1 \mathrm{~h}$ in blocking buffer (PBS with $0.3 \%$ (vol/vol) Triton X-100 and 15\% (vol/vol) FCS), intracellular m164 protein was detected by polyclonal affinity-purified rabbit antibodies (1:100) [49] and Alexa Fluor 546-conjugated goat anti-rabbit antibody (Life Technologies, Catalog No. A11010, Darmstadt, Germany) as secondary antibody. Cell nuclei were stained by $5 \mathrm{~min}$ incubation with the DNA-binding blue fluorescent dye Hoechst 33342 (1:5,000; Life Technologies, Catalog No. H3570, Darmstadt, Germany).

\subsection{Northern Blot using RNA Probes}

MEF were treated $15 \mathrm{~min}$ before infection either with $100 \mu \mathrm{g} / \mathrm{mL} \mathrm{CH}$ or $5 \mu \mathrm{g} / \mathrm{mL} \mathrm{ActD}$, or were left untreated. Cells were infected under conditions of centrifugal enhancement. Highly purified polyadenylated RNA was isolated from the cell lysate at $1.5 \mathrm{~h}$ (untreated and ActD treated cells) or $3 \mathrm{~h}$ (untreated and $\mathrm{CH}$ treated cells) p.i. by using oligo(dT)-coated superparamagnetic 50-nm diameter microbeads ( $\mu$ MACS mRNA isolation kit; Miltenyi Biotec Systems, Catalog No. 130-075-201, Bergisch-Gladbach, Germany). To generate two complementary hybridization probes, a 431 bp DNA fragment of the m164 sequence (nt 222,625-nt 223,055) was amplified by PCR using the primers mRNAint_for and mRNAint_rev and the plasmid pSM3fr [46] as template. The resulting PCR product was subcloned into pDrive cloning vector (Qiagen, Catalog No. 223122, Hilden, Germany) resulting in pDrive-m164probe. After linearization of the plasmid with BamHI for transcription with T7, the MAXIscript Kit SP6/T7 (Life technologies, Catalog No. AM1320, Darmstadt, Germany) was used to synthesize single strand RNA probes labeled by random-priming with the DIG RNA Labeling Mix (Roche, Catalog No. 1277073, Mannheim, Germany) in the presence of DIG-UTP:dTTP (1:3). Amounts of $4 \mu \mathrm{g}$ of poly(A) ${ }^{+}$RNA or $2 \mu \mathrm{g}$ of total RNA per lane were separated in $1.2 \%$ Agarose $/ 37 \%$ formaldehyde gels and transferred onto positively charged nylon membranes. An amount of $700 \mathrm{ng}$ of the DIG-labeled RNA-probe (T7) was diluted in $70 \mathrm{~mL}$ DIG Easy Hyb buffer prior to hybridization, which was performed as described above. 


\subsection{Mapping of $m R N A$ by $5^{\prime} / 3^{\prime} R A C E$}

To map the full-length m164 transcript by 5'/3' RACE (rapid amplification of cDNA ends) [50], the 2nd generation kit (Roche, Catalog No. 03353621, Mannheim, Germany) was used for the analysis of poly $(\mathrm{A})^{+} \mathrm{RNA}$ isolated in the $\mathrm{E}$ phase of the viral replicative cycle at $6 \mathrm{~h}$ post-infection (p.i.).

In brief, poly $(\mathrm{A})^{+}$RNA was enriched from total RNA by the Oligotex mRNA Mini Kit (Qiagen, Catalog No. 72022; Hilden, Germany) according to the manufacturer's instructions. 5' RACE analysis started with an RT-step transcribing m164 mRNA partially into cDNA by using the Flank_1 primer (see Appendix Table A1), followed by digestion of residual poly(A) ${ }^{+}$RNA with RNase $H$. The transcription start site of gene m164 was identified by tailing the cDNA at its 3 ' end using terminal deoxynucleotidyl transferase with substrate dATP. The $3^{\prime}$ region of the cDNA [corresponding to the $5^{\prime}$ end of the poly $(\mathrm{A})^{+}$RNA] was then amplified using a standard PCR protocol with the provided oligo-dT anchor primer and the antisense primer Nested_Flank_2 (see Appendix Table A1) designed to bind internally within the predicted ORFm164.

3' RACE analysis started with the synthesis of cDNA using the oligo-dT anchor primer, followed by digestion of residual poly $(\mathrm{A})^{+}$RNA with RNase $\mathrm{H}$. The 5 ' region of the cDNA (corresponding to the $3^{\prime}$ end of the poly $(\mathrm{A})^{+}$RNA) was then amplified by using the provided PCR anchor primer and the ORFm164 sequence-specific internal primer Nested_Flank_3 (see Appendix Table A1). Reaction products were analyzed by $1.5 \%$ agarose gel electrophoresis and were sequenced.

\subsection{Primer Walking}

Fifteen minutes prior to infection, MEF were either treated with $100 \mu \mathrm{g} / \mathrm{mL} \mathrm{CH}$ or $5 \mu \mathrm{g} / \mathrm{mL}$ $\mathrm{ActD}$, or were left untreated. Infection was performed under conditions of centrifugal enhancement. Highly purified polyadenylated RNA was isolated from the cell lysate at $1.5 \mathrm{~h}$ (untreated and ActD treated cells) or at $3 \mathrm{~h}$ (untreated and $\mathrm{CH}$ treated cells) p.i. by using oligo(dT)-coated superparamagnetic $50 \mathrm{~nm}$-diameter microbeads ( $\mu$ MACS mRNA isolation kit; Miltenyi Biotec Systems, Catalog No. 130-075-201, Bergisch-Gladbach, Germany). For reverse transcription reactions, the OneStep RT-PCR Kit (Qiagen, Catalog No. 210210, Hilden, Germany) was used according to the manufacturer's protocol. Reactions were carried out with an automated thermal cycler (GeneAmp PCR System 9700; Life technologies, Darmstadt, Germany), using the primer pairs shown in Appendix Table A1. The time-temperature profiles were as follows: reverse transcription for $30 \mathrm{~min}$ at $50{ }^{\circ} \mathrm{C}$, initial PCR activation step for $15 \mathrm{~min}$ at $95{ }^{\circ} \mathrm{C}, 35$ cycles of denaturation for $30 \mathrm{~s}$ at $94{ }^{\circ} \mathrm{C}$, and annealing for $30 \mathrm{~s}$ with annealing temperatures and elongation conditions specified in Appendix Table A2. Final extension was performed for $10 \mathrm{~min}$ at $68{ }^{\circ} \mathrm{C}$ or $72{ }^{\circ} \mathrm{C}$, dependent on the respective elongation temperature. Amplification products were visualized by standard procedures of $1 \%(\mathrm{wt} / \mathrm{vol})$ agarose gel electrophoresis with ethidium bromide.

\subsection{In Vitro Transcription and Translation}

To introduce the SP6 promoter sequence [51] in front of the RACEm164 and the ORFm164 sequence, PCRs were performed with primer pairs SP6_m164RACE_for and SP6_m164RACE_rev or SP6_m164ORF_for and SP6_m164ORF_rev (see Appendix Table A1), respectively. An amount of 
$1 \mu \mathrm{g}$ of purified PCR product was used as template for in vitro transcription according to the manufacturer's protocol for "Transcription Reaction Assembly" of the Ambion MEGAscript SP6 kit (Life technologies, Catalog No. AM1330, Darmstadt, Germany). After $3.5 \mathrm{~h}$ of incubation at $37{ }^{\circ} \mathrm{C}$ for transcription, DNA template was removed by digestion with DNase, and the RNA was cleaned with RNeasy Mini Elute Cleaning kit (Qiagen, Catalog No. 74134, Hilden, Germany) and analyzed on a $1 \%$ agarose/formaldehyde gel.

In vitro translation of the in vitro transcripts was performed with the Promega wheat germ extract (Promega, Catalog No. L4380, Mannheim, Germany). The reaction conditions were $90 \mathrm{~min}$ at $25{ }^{\circ} \mathrm{C}$ with $80 \mathrm{ng}$ of template RNA. The resulting protein was labeled with Redivue L- $\left[{ }^{35} \mathrm{~S}\right]$ methionine (Amersham Biosciences, Catalog No. AG1094, Little Chalfont, UK) and analyzed by $12.5 \%$ SDS-PAGE followed by autoradiography.

\subsection{ELISpot Assay}

Presentation of the m164- and SIINFEKL-epitope was determined after endogenous antigen processing in infected MEF (BALB/c, haplotype $\mathrm{H}-2^{\mathrm{d}}$ and $\mathrm{C} 57 \mathrm{BL} / 6$, haplotype $\mathrm{H}-2^{\mathrm{b}}$, respectively) by using cognate CTLL as responder cells in a standard IFN- $\gamma$-based ELISpot assay. For selective arrest of the viral gene expression in the IE or E phase, MEFs were treated with inhibitors as described previously [44]. The assay was performed as described ([52,53] and references therein) and frequencies of IFN- $\gamma$-secreting cells and the corresponding 95\% confidence intervals were calculated by intercept-free linear regression analysis using the software Mathematica [54].

\section{Conclusions and Outlook}

These data have revealed a paradigmatic example of a CD8 T-cell epitope that is encoded by two overlapping mRNAs expressed in different phases of the coordinately regulated gene expression program in the productive viral cycle $[55,56]$. As overlapping genes are a more general feature of CMV genomes [33,57], more such cases might be found in the future and lead to a revision of the assignment of antigenic peptides to a certain kinetic class in epitope listings. This is of relevance for the order in which epitopes are expressed relative to immune evasion proteins or for discussing viral gene expression during CMV latency. In the specific case of the memory inflation-inducing m164 peptide of mCMV it was assumed that the expansion of the m164 epitope-specific memory CD8 T-cell pool indicates E gene expression during latency [11,20,21]. With the new knowledge presented here, future experiments will have to address the question of whether the m164 peptide that is supposed to drive m164-specific memory inflation during latency indeed results from the E phase transcript or, alternatively, results from the here identified IE phase transcript. Sporadic expression of IE genes from the major IE locus during viral latency has been documented [27,58-60]. Thus, "noncanonical" expression and presentation of the m164 epitope from the long IE phase transcript could explain m164-specific memory inflation during viral latency in absence of reactivation of the coordinately regulated viral transcriptional program. 


\section{Acknowledgments}

The authors appreciated the contributions of Torsten Däubner and Christof Seckert at earlier stages of this project, and thank Dennis Strand and the Confocal Laser Scanning Microscopy Core Facility of the Forschungszentrum Immunologie (FZI) at the University Medical Center of the Johannes Gutenberg-University Mainz for advice and assistance with image collection.

This work was supported by the Deutsche Forschungsgemeinschaft, SFB490, individual projects E3 (R.H. and D.T.) and E4 (A.F. and M.J.R.), and the Clinical Research Group KFO183 (A.F., N.A.W.L., and M.J.R.). N.A.W.L. and R.H. received intramural funding in the young investigator program MAIFOR and in program IFF-I, respectively, of the University Medical Center of the Johannes Gutenberg-University Mainz, Mainz, Germany. The Forschungszentrum Immunologie (FZI) funded J.K.B.

\section{Author Contributions}

Conceived and designed the experiments and analyzed the data: Annette Fink, Julia K. Büttner, Rafaela Holtappels, Matthias J. Reddehase and Niels A. W. Lemmermann. Performed the experiments: Annette Fink, Julia K. Büttner, Doris Thomas. Wrote the paper: Annette Fink, Julia K. Büttner, Matthias J. Reddehase and Niels A. W. Lemmermann.

\section{Conflicts of Interest}

The authors declare no conflict of interest.

\section{References and Notes}

1. Falk, K.; Rötzschke, O.; Stevanović, S.; Jung, G.; Rammensee, H.G. Allele-specific motifs revealed by sequencing of self-peptides eluted from MHC molecules. Nature 1991, 351, 290-296.

2. Rammensee, H.; Bachmann, J.; Emmerich, N.P.; Bachor, O.A.; Stevanović, S. SYFPEITHI: Database for MHC ligands and peptide motifs. Immunogenetics 1999, 50, 213-219.

3. Tenzer, S.; Peters, B.; Bulik, S.; Schoor, O.; Lemmel, C.; Schatz, M.M.; Kloetzel, P.M.; Rammensee, H.G.; Schild, H.; Holzhütter, H.G. Modeling the MHC class I pathway by combining predictions of proteasomal cleavage, TAP transport and MHC class I binding. Cell Mol. Life Sci. 2005, 62, 1025-1037.

4. Reddehase, M.J.; Keil, G.M.; Koszinowski, U.H. The cytolytic T lymphocyte response to the murine cytomegalovirus. II. Detection of virus replication stage-specific antigens by separate populations of in vivo active cytolytic T lymphocyte precursors. Eur. J. Immunol. 1984, 14, 56-61.

5. McLaughlin-Taylor, E.; Pande, H.; Forman, S.J.; Tanamachi, B.; Li, C.R.; Zaia, J.A.; Greenberg, P.D.; Riddell, S.R. Identification of the major late human cytomegalovirus matrix protein pp65 as a target antigen for $\mathrm{CD} 8^{+}$virus-specific cytotoxic T lymphocytes. J. Med. Virol. 1994, 43, 103-110. 
6. Frankenberg, N.; Lischka, P.; Pepperl-Klindworth, S.; Stamminger, T.; Plachter, B. Nucleocytoplasmic shuttling and CRM1-dependent MHC class I peptide presentation of human cytomegalovirus pp65. Med. Microbiol. Immunol. 2012, 201, 567-579.

7. Bresnahan, W.A.; Shenk, T. A subset of viral transcripts packaged within human cytomegalovirus particles. Science 2000, 288, 2373-2376.

8. Reddehase, M.J. Antigens and immunoevasins: Opponents in cytomegalovirus immune surveillance. Nat. Rev. Immunol. 2002, 2, 831-844.

9. Powers, C.; DeFilippis, V.; Malouli, D.; Früh, K. Cytomegalovirus immune evasion. Curr. Top. Microbiol. Immunol. 2008, 325, 333-359.

10. Hansen, T.H.; Bouvier, M. MHC class I antigen presentation: Learning from viral evasion strategies. Nat. Rev. Immunol. 2009, 9, 503-513.

11. Holtappels, R.; Thomas, D.; Podlech, J.; Reddehase, M.J. Two antigenic peptides from genes $\mathrm{m} 123$ and $\mathrm{m} 164$ of murine cytomegalovirus quantitatively dominate CD8 T-cell memory in the H-2d haplotype. J. Virol. 2002, 76, 151-164.

12. Reddehase, M.J.; Rothbard, J.B.; Koszinowski, U.H. A pentapeptide as minimal antigenic determinant for MHC class I-restricted T lymphocytes. Nature 1989, 337, 651-653.

13. Holtappels, R.; Simon, C.O.; Munks, M.W.; Thomas, D.; Deegen, P.; Kühnapfel, B.; Däubner, T.; Emde, S.; Podlech, J.; Grzimek, N.K.; et al. Subdominant CD8 T-cell epitopes account for protection against cytomegalovirus independent of immunodomination. J. Virol. 2008, 82, 5781-5796.

14. Holtappels, R.; Pahl-Seibert, M.F.; Thomas, D.; Reddehase, M.J. Enrichment of immediate-early 1 (m123/pp89) peptide-specific CD8 T cells in a pulmonary CD62L(lo) memory-effector cell pool during latent murine cytomegalovirus infection of the lungs. J. Virol. 2000, 74, 11495-11503.

15. Karrer, U.; Sierro, S.; Wagner, M.; Oxenius, A.; Hengel, H.; Koszinowski, U.H.; Phillips, R.E.; Klenerman, P. Memory inflation: Continuous accumulation of antiviral CD8 ${ }^{+} \mathrm{T}$ cells over time. J. Immunol. 2003, 170, 2022-2029.

16. Klenerman, P.; Dunbar, P.R. CMV and the art of memory maintenance. Immunity 2008, 29, $520-522$.

17. Snyder, C.M. Buffered memory: A hypothesis for the maintenance of functional, virus-specific $\mathrm{CD}^{+} \mathrm{T}$ cells during cytomegalovirus infection. Immunol. Res. 2011, 51, 195-204.

18. O’Hara, G.A.; Welten, S.P.; Klenerman, P.; Arens, R. Memory T cell inflation: Understanding cause and effect. Trends Immunol. 2012, 33, 84-90.

19. Munks, M.W.; Cho, K.S.; Pinto, A.K.; Sierro, S.; Klenerman, P.; Hill, A.B. Four distinct patterns of memory CD8 $\mathrm{T}$ cell responses to chronic murine cytomegalovirus infection. J. Immunol. 2006, 177, 450-458.

20. Seckert, C.K.; Griessl, M.; Büttner, J.K.; Scheller, S.; Simon, C.O.; Kropp, K.A.; Renzaho, A.; Kühnapfel, B.; Grzimek, N.K.; Reddehase, M.J. Viral latency drives "memory inflation": A unifying hypothesis linking two hallmarks of cytomegalovirus infection. Med. Microbiol. Immunol. 2012, 201, 551-566. 
21. Seckert, C.K.; Grieß1, M.; Büttner, J.K.; Freitag, K.; Lemmermann, N.A.W.; Hummel, M.A.; Liu, X.-F.; Abecassis, M.I.; Angulo, A.; Messerle, M.; et al. Immune surveillance of cytomegalovirus latency and reactivation in murine models: Link to "memory inflation". In Cytomegaloviruses: From Molecular Pathogenesis to Intervention; Reddehase, M.J., Ed.; Caister Academic Press: Wymondham, Norfolk, UK, 2013; pp. 374-416.

22. Däubner, T.; Fink, A.; Seitz, A.; Tenzer, S.; Müller, J.; Strand, D.; Seckert, C.K.; Janssen, C.; Renzaho, A.; Grzimek, N.K.; et al. A novel transmembrane domain mediating retention of a highly motile herpesvirus glycoprotein in the endoplasmic reticulum. J. Gen. Virol. 2010, 91, $1524-1534$.

23. Holtappels, R.; Grzimek, N.K.; Simon, C.O.; Thomas, D.; Dreis, D.; Reddehase, M.J. Processing and presentation of murine cytomegalovirus pORFm164-derived peptide in fibroblasts in the face of all viral immunosubversive early gene functions. J. Virol. 2002, 76, 6044-6053.

24. Holtappels, R.; Böhm, V.; Podlech, J.; Reddehase, M.J. CD8 T-cell-based immunotherapy of cytomegalovirus infection: "Proof of concept" provided by the murine model. Med. Microbiol. Immunol. 2008, 197, 125-134.

25. Ebert, S.; Podlech, J.; Gillert-Marien, D.; Gergely, K.M.; Büttner, J.K.; Fink, A.; Freitag, K.; Thomas, D.; Reddehase, M.J.; Holtappels, R. Parameters determining the efficacy of adoptive CD8 T-cell therapy of cytomegalovirus infection. Med. Microbiol. Immunol. 2012, 201, $527-539$.

26. Lemmermann, N.A.; Kropp, K.A.; Seckert, C.K.; Grzimek, N.K.; Reddehase, M.J. Reverse genetics modification of cytomegalovirus antigenicity and immunogenicity by CD8 T-cell epitope deletion and insertion. J. Biomed. Biotechnol. 2011, 2011, doi:10.1155/2011/812742.

27. Simon, C.O.; Holtappels, R.; Tervo, H.-M.; Böhm, V.; Däubner, T.; Oehrlein-Karpi, S.A.; Kühnapfel, B.; Renzaho, A.; Strand, D.; Podlech, J.; et al. CD8 T cells control cytomegalovirus latency by epitope-specific sensing of transcriptional reactivation. J. Virol. 2006, 80, 10436-10456.

28. Böhm, V.; Podlech, J.; Thomas, D.; Deegen, P.; Pahl-Seibert, M.; Lemmermann, N.A.; Grzimek, N.K.; Oehrlein-Karpi, S.A.; Reddehase, M.J.; Holtappels, R. Epitope-specific in vivo protection against cytomegalovirus disease by CD8 $\mathrm{T}$ cells in the murine model of preemptive immunotherapy. Med. Microbiol. Immunol. 2008, 197, 135-144.

29. Lemmermann, N.A.; Gergely, K.M.; Böhm, V.; Deegen, P.; Däubner, T.; Reddehase, M.J. Immune evasion proteins of murine cytomegalovirus preferentially affect cell surface display of recently generated peptide presentation complexes. J. Virol. 2010, 84, 1221-1236.

30. Ebert, S.; Lemmermann, N.A.; Thomas, D.; Renzaho, A.; Reddehase, M.J.; Holtappels, R. Immune control in the absence of immunodominant epitopes: Implications for immunotherapy of cytomegalovirus infection with antiviral CD8 T cells. Med. Microbiol. Immunol. 2012, 201, $541-550$.

31. Nauerth, M.; Weißbrich, B.; Knall, R.; Franz, T.; Dössinger, G.; Bet, J.; Paszkiewicz, P.J.; Pfeifer, L.; Bunse, M.; Uckert, W.; et al. TCR-ligand koff rate correlates with the protective capacity of antigen-specific $\mathrm{CD}^{+} \mathrm{T}$ cells for adoptive transfer. Sci. Transl. Med. 2013, 5, doi:10.1126/scitranslmed.3005958. 
32. Caposio, P.; Streblow, D.; Nelson, J.A. Cytomegalovirus proteomics. In Cytomegaloviruses: From Molecular Pathogenesis to Intervention; Reddehase, M.J., Ed.; Caister Academic Press: Wymondham, Norfolk, UK, 2013; Volume I, pp. 86-108.

33. Redwood, A.J.; Shellam, G.R.; Smith, L.M. Molecular evolution of murine cytomegalovirus genomes. In Cytomegaloviruses: From Molecular Pathogenesis to Intervention; Reddehase, M.J., Ed.; Caister Academic Press: Wymondham, Norfolk, UK, 2013; Volume I, pp. 23-37.

34. Rawlinson, W.; Farrell, H.; Barrell, B. Analysis of the complete DNA sequence of murine cytomegalovirus. J. Virol. 1996, 70, 8833-8849.

35. Bendtsen, J.D.; Nielsen, H.; von Heijne, G.; Brunak, S. Improved prediction of signal peptides: SignalP 3.0. J. Mol. Biol. 2004, 340, 783-795.

36. Dölken, L. Department of Medicine, University of Cambridge, Cambridge, UK. Personal communication, 2013.

37. Erhard, F. Institut für Informatik, Ludwigs-Maximilians-Universität München, München, Germany. Personal communication, 2013.

38. Zhu, J.; Chen, J.; Hai, R.; Tong, T.; Xiao, J.; Zhan, X.; Lu, S.; Liu, F. In vitro and in vivo characterization of a murine cytomegalovirus with a mutation at open reading frame m166. J. Virol. 2003, 77, 2882-2891.

39. Mercer, J.A.; Marks, J.R.; Spector, D.H. Molecular cloning and restriction endonuclease mapping of the murine cytomegalovirus genome (Smith Strain). Virology 1983, 129, 94-106.

40. Wing, B.A.; Browne, E.P.; Shenk, T.E. Modulation of cellular gene expression by MCMV evaluated by DNA microarray analysis. In Proceedings of the 26th International Herpesvirus Workshop, Regensburg, Germany, 14-17 May 2001; Abstract Number 1.08.

41. Shenk, T.E. Department of Molecular Biology, Princeton University, Princeton, NJ, USA. Personal communication, 2007.

42. Hong, J.-J.; Wu, T.-Y.; Chang, T.-Y.; Chen, C.-Y. Viral IRES prediction system-A web server for prediction of the IRES secondary structure in silico. PLoS One 2013, 8, e79288.

43. Baird, S.D.; Turcotte, M.; Robert, G.; Korneluk, R.G.; Holcik, M. Searching for IRES. RNA 2006, 12, 1755-1785.

44. Podlech, J.; Holtappels, R.; Grzimek, N.K.; Reddehase, M.J. Animal models: Murine cytomegalovirus. In Methods in Microbiology, Immunology of Infection, 2nd ed.; Kaufmann, S., Kabelitz, D., Eds.; Academic Press: London, UK, 2002; pp. 493-525.

45. Lemmermann, N.A.; Podlech, J.; Seckert, C.K.; Kropp, K.A.; Grzimek, N.K.; Reddehase, M.J.; Holtappels, R. CD8 T-cell immunotherapy of cytomegalovirus disease in the murine model. In Methods in Microbiology; Kabelitz, D., Kaufmann, S., Eds.; Academic Press: Oxford. UK, 2010; Volume 37, pp. 369-420.

46. Wagner, M.; Jonjić, S.; Koszinowski, U.H.; Messerle, M. Systematic excision of vector sequences from the BAC-cloned herpesvirus genome during virus reconstitution. J. Virol. 1999, 73, 7056-7060.

47. CIOMS and ICLAS release the new International Guiding Principles for Biomedical Research Involving Animals. Available online: http://www.cioms.ch/images/stories/CIOMS/IGP2012.pdf (accessed on 1 December 2013). 
48. Tischer, B.K.; von Einem, J.; Kaufer, B.; Osterrieder, N. Two-step Red-mediated recombination for versatile high-efficiency markerless DNA manipulation in Escherichia coli. Biotechniques 2006, 40, 191-197.

49. Holtappels, R.; Gillert-Marien, D.; Thomas, D.; Podlech, J.; Deegen, P.; Herter, S.; Oehrlein-Karpi, S.A.; Strand, D.; Wagner, M.; Reddehase, M.J. Cytomegalovirus encodes a positive regulator of antigen presentation. J. Virol. 2006, 80, 7613-7624.

50. Frohman, M.A. On beyond classic RACE (rapid amplification of cDNA ends). PCR Methods Appl. 1994, 4, 40-58.

51. Krieg, P.A.; Melton, D.A. Functional messenger RNAs are produced by SP6 in vitro transcription of cloned cDNAs. Nucleic Acids Res. 1984, 12, 7057-7070.

52. Pahl-Seibert, M.F.; Juelch, M.; Podlech, J.; Thomas, D.; Deegen, P.; Reddehase, M.J.; Holtappels, R. Highly protective in vivo function of cytomegalovirus IE1 epitope-specific memory CD8 $829 \mathrm{~T}$ cells purified by T-cell receptor-based cell sorting. J. Virol. 2005, 79, $5400-5413$.

53. Böhm, V.; Simon, C.O.; Podlech, J.; Seckert, C.K.; Gendig, D.; Deegen, P.; Gillert-Marien, D.; Lemmermann, N.A.; Holtappels, R.; Reddehase, M.J. The immune evasion paradox: Immunoevasins of murine cytomegalovirus enhance priming of CD8 T cells by preventing negative feedback regulation. J. Virol. 2008, 82, 11637-11650.

54. Wolfram Mathematica, version 9; Wolfram Research: Champaign, IL, USA, 2012.

55. Honess, R.W.; Roizman, B. Regulation of herpesvirus macromolecular synthesis. I. Cascade regulation of the synthesis of three groups of viral proteins. J. Virol. 1974, 14, 8-19.

56. Fortunato, E.A.; Spector, D.H. Regulation of human cytomegalovirus gene expression. Adv. Virus Res. 1999, 54, 61-128.

57. Davison, A.J.; Holton, M.; Dolan, A.; Dargan, D.J.; Gatherer, D.; Hayward,G.S. Comparative genomics of primate cytomegaloviruses. In Cytomegaloviruses: From Molecular Pathogenesis to Intervention; Reddehase, M.J., Ed.; Caister Academic Press: Wymondham, Norfolk, UK, 2013; Volume I, pp. 1-22.

58. Kurz, S.K.; Rapp, M.; Steffens, H.P.; Grzimek, N.K.; Schmalz, S.; Reddehase, M.J. Focal transcriptional activity of murine cytomegalovirus during latency in the lungs. J. Virol. 1999, 73, 482-494.

59. Grzimek, N.K.; Dreis, D.; Schmalz, S.; Reddehase, M.J. Random, asynchronous, and asymmetric transcriptional activity of enhancer-flanking major immediate-early genes ie1/3 and ie2 during murine cytomegalovirus latency in the lungs. J. Virol. 2001, 75, 2692-2705.

60. Simon, C.O.; Seckert, C.K.; Dreis, D.; Reddehase, M.J.; Grzimek, N.K. Role for tumor necrosis factor alpha in murine cytomegalovirus transcriptional reactivation in latently infected lungs. J. Virol. 2005, 79, 326-340.

61. Ebeling, A.; Keil, G.M.; Knust, E.; Koszinowski, U.H. Molecular cloning and physical mapping of murine cytomegalovirus DNA. J. Virol. 1983, 47, 421-433. 


\section{Appendices}

Figure A1. Mapping of the E phase m164 transcript with its $5^{\prime}$ and $3^{\prime}$ untranslated regions (UTRs). (A) Hind III restriction map [61] of the mCMV-WT.Smith genome (GenBank Accession No. MCU68299), and map position of the predicted ORFm164 [34] located within the Hind $\mathrm{E}$ fragment on the $\mathrm{C}$ (complementary) strand. It overlaps with neighboring ORFs $m 163$ and $m 165$ by 45 bp and 366 bp, respectively; (B) Schematic illustration of the strategy of 5' and 3' RACE for identifying UTRs. For 5' RACE, poly $(\mathrm{A})^{+}$mRNA is reverse transcribed using an internal primer Flank_1. An oligo-dA sequence is appended to the $3^{\prime}$ end of the resulting m164 cDNA fragment. PCR performed with an oligo-dT anchor primer and an internal primer Nested Flank_2 will reveal the expected $965 \mathrm{bp}$ of predicted ORF sequence (up to the complementary start codon CAT) plus an additional unknown number of $5^{\prime}$ bp (5' X; dashed line). For $3^{\prime}$ RACE, poly $(A)^{+}$RNA is reverse transcribed by using an oligo-dT anchor primer. The resulting cDNA is amplified by using the PCR anchor primer and an internal primer Nested Flank_3 to reveal the expected $984 \mathrm{bp}$ of predicted ORF sequence (up to complementary stop codon TCA) plus an additional unknown number of $3^{\prime}$ bp (3' Y; dashed line); (C) Left two panels: Ethidium bromide-stained 1\% agarose gels identifying 1,151 bp 5' and 1,521 bp 3' RACE products, allowing the calculation of 5' $\mathrm{X}$ and $3^{\prime} \mathrm{Y}$, respectively. Sequencing revealed 2,004 bp of non-overlapping sequence representing a 2,004 base-long mRNA, referred to as RACEm164 RNA. Right panel: in vitro transcripts. Ethidium bromide-stained agarose/formaldehyde gel showing the in vitro transcription products ( $1 \mu \mathrm{g}$ RNA per lane) obtained with DNA templates representing either the full-length mRNA [except the poly(A) tail] as determined by RACE analysis (RACEm164) or the sequence of only the predicted ORF (ORFm164), both 5' extended with the 20-bp SP6 promoter sequence ATTTAGGTGACACTATAGAA. CUU, additional codon derived from the SP6 promoter sequence.

A

mCMV-WT.Smith genome

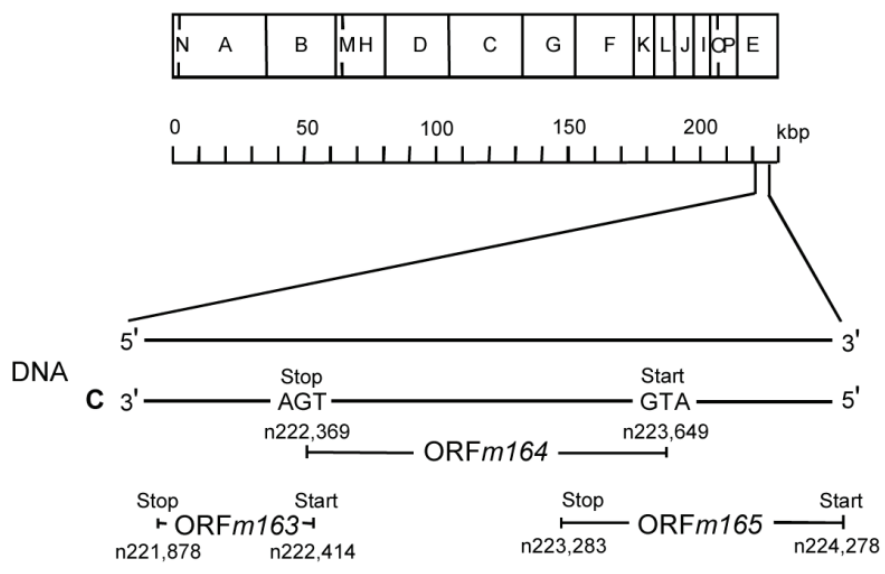


Figure A1. Cont.

B

$$
\text { DNA }
$$
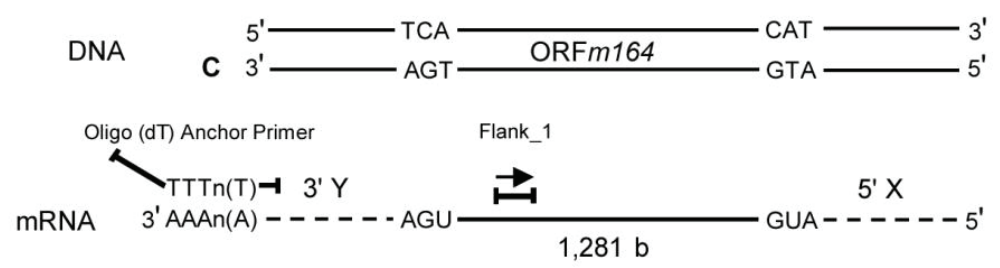

3' RACE:

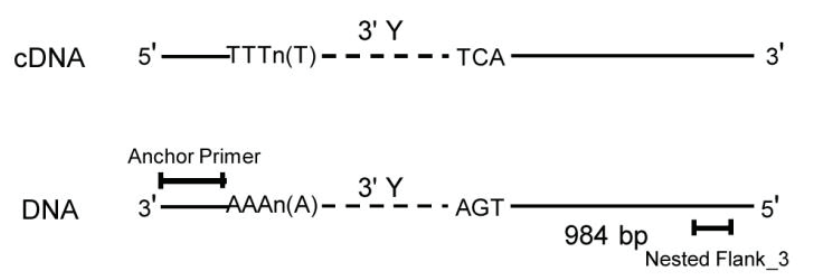

\section{5' RACE:}

Nested Flank_2

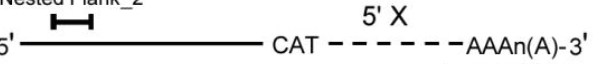
-TTTn(T)

Nested Flank_2 Oligo (dT) Anchor Primer $5^{\prime}$

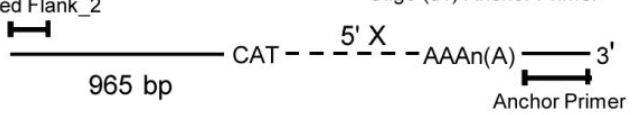

C

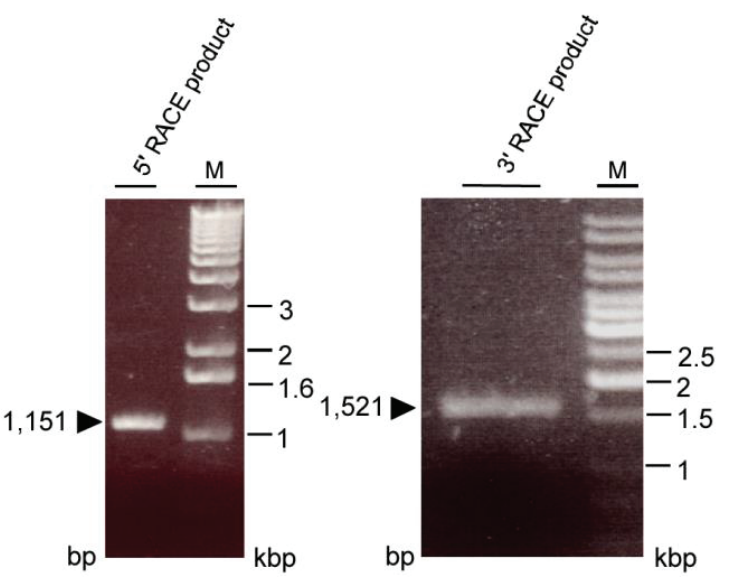

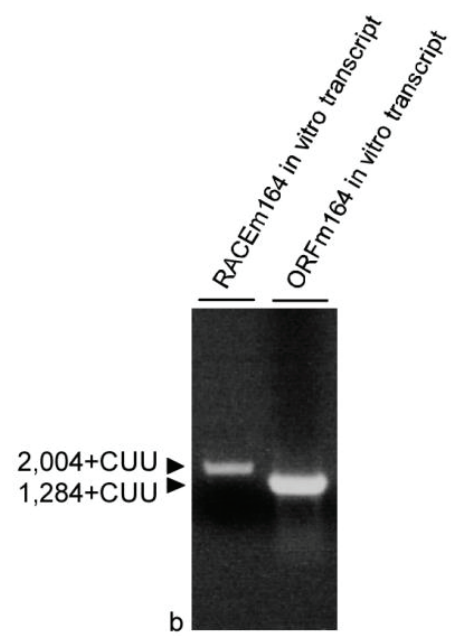


Figure A2. RT-PCR primer walking for searching the 5' end of the long IE m164 mRNA (A) Map indicating relative positions of ORFs $m 164$ to m166. I-IV show predicted lengths of RT-PCR products and the positions of the oligonucleotides on the mCMV genome (map not drawn to scale); (B) MEF were infected with mCMV-WT.Smith in presence $(+)$ or absence $(-)$ of ActD $(5 \mu \mathrm{g} / \mathrm{mL})$ or $\mathrm{CH}(100 \mu \mathrm{g} / \mathrm{mL})$. At $1.5 \mathrm{~h} \mathrm{p}$.i. (for ActD) or $3 \mathrm{~h}$ p.i. (for $\mathrm{CH}$ ), total RNA was isolated and subjected to RT-PCR. Products were analyzed on a $1 \%$ agarose gel stained with ethidium bromide. M, marker; n.i., not infected; (C) Left panel: map indicating relative positions of oligonucleotides and RT-PCR products (map not drawn to scale); right panel: MEF were infected with mCMV-WT.Smith in absence (-) or presence of $\mathrm{CH}$ or ActD, and total RNA was isolated at $3 \mathrm{~h}$ p.i. (for $\mathrm{CH}$ ) or $1.5 \mathrm{~h}$ p.i. (for Act.D) and subjected to RT-PCR using oligonucleotides for products I-VIII (see Appendix Table A1). DNA isolated from infected cells not treated with inhibitors was used as a positive control.

A

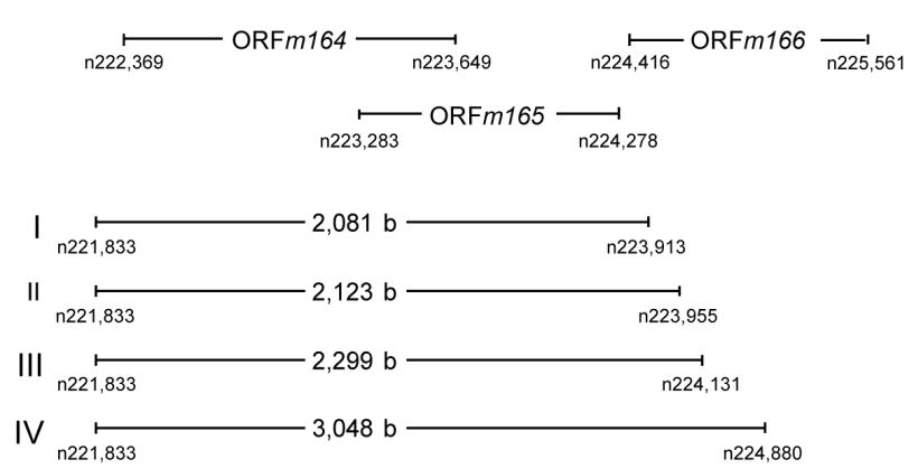

B

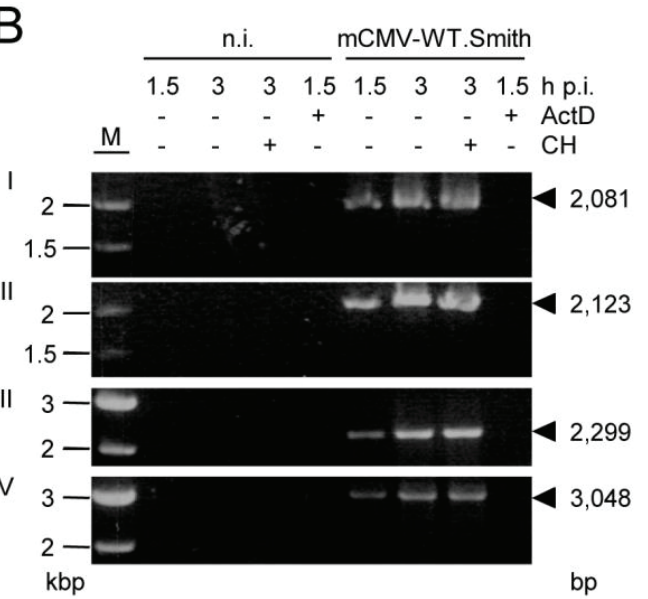

C
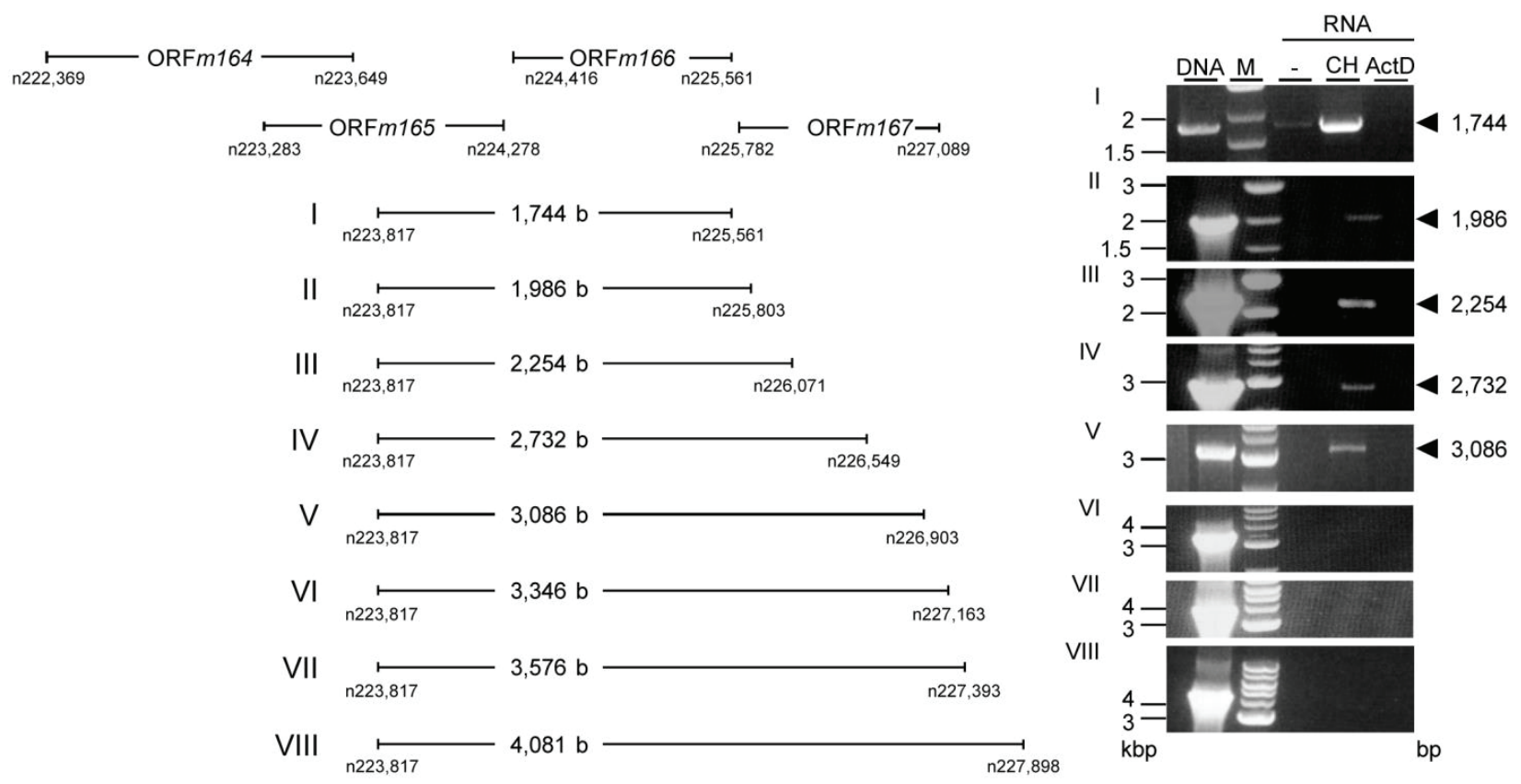
Table A1. Oligonucleotides used in this study; nucleotides inserting ATG to GCG substitutions are highlighted in bold face red letters.

\begin{tabular}{|c|c|}
\hline Name & Sequence \\
\hline pEPKan-S_m164_1stATG_for & $\begin{array}{c}\text { 5'-CCTGCCAGATCTTGGGGCCTCGGGTCGCCACACACGTGGG } \\
\text { AAGGCGGCGTTTCTCCGCGGCTGTCGGCAACCAATTAAC } \\
\text { CAATTCTGATTAG-3' }\end{array}$ \\
\hline pEPKan-S_m164_1stATG_rev & $\begin{array}{c}\text { 5'-GCTGCGCGTCTCCCCCTCCGACAGCCGCGGAGAAACGCCGCC } \\
\text { TTCCCACGTGTGTGGCGAGGATGACGACGATAAGTAGGG-3' }\end{array}$ \\
\hline m164_1stATG_short & 5'-CCTGCCAGATCTTGGGGCCTCGGG-3' \\
\hline pEPKan-S_rev & 5'-AGGATGACGACGATAAGTAGGG-3' \\
\hline mRNAint_for & 5'-GGCTTCTCGGGCCTCGTCGTGTGC-3' \\
\hline mRNAint_rev & 5'-GCCACGTGGACGGGTCGACATTCG-3' \\
\hline m164mRNA_rev & 5'-ACGCACATCACCAGTG-3' \\
\hline mRNAin_for & 5'-GTTTAGTCGGGAGCGTG-3' \\
\hline m164mRNAout_for & 5'-GTGAGGTGGTGGATAGATAC-3' \\
\hline m164mRNAout50_for & 5'-GTGTGAGCAGTATCTGGG-3' \\
\hline m164mRNAout100_for & 5'-GAGACGGCTAGCGAATC-3' \\
\hline m164mRNAout300_for & 5'-GCGGACTACATCTCGATC-3' \\
\hline m164mRNAout1000_for & 5'-GAGTGGAGCGAGAACAAC-3' \\
\hline m164mRNAin_rev & 5'-CACGCTCCCGACTAAAC-3' \\
\hline m166_ORF_end_for & 5'-ATGGCGCTTCCAGCAATTCC-3' \\
\hline m166_ORF_end250_for & 5'-CAGGTTCCGCAACAGTC-3' \\
\hline m166_ORF_end500_for & 5'-ATCGGATGGTGCTTCAG-3' \\
\hline m166_ORF_end1000_for & 5'-AAGCCTCCGAGGAAGAC-3' \\
\hline m166.5_ORF_end_for & 5'-GGCGGTAGAACAGTTGC-3' \\
\hline m166.5_ORF_end250_for & 5'-CCTTTGAGTGTGGAAAGTTTG-3' \\
\hline m166.5_ORF_end500_for & 5'-TTACCCGCGTCACATGG-3' \\
\hline m166.5_ORF_end1000_for & 5'-CCGTCCTTAGGTTTCGTTTC-3' \\
\hline Flank_1 & 5'-CACGAAGGCGGCGAGGAAAC-3' \\
\hline Nested Flank_2 & 5'-GACGACGCACGATACGCAGAC-3' \\
\hline Nested Flank_3 & 5'-CTCATCTACGCGGCGGCTTCAG-3' \\
\hline m164_rev_BamHI & 5'-ATATGGATCCGGAGAAGAGTCAGAATCATGACGAACG-3' \\
\hline m164_full_HindIII & 5'-ATATAAGCTTCTCCTGCCAGATCTTG-3' \\
\hline m164_3rdATG_rev & 5'-GCGTAGATGAGGGCGAGCGCCCCGGCCTTGAAGAGTCG-3' \\
\hline m164_3rdATG_for & 5'-CGACTCTTCAAGGCCGGGGCGCTCGCCCTCATCTACGC-3' \\
\hline m164_2ndATG_rev & 5'-GCAACGCGTCGTCGGCACGCCGGCAACTCGGGTTCCCCG-3' \\
\hline m164_2ndATG_for & 5'-CGGGGAACCCGAGTTGCCGGCGTGCCGACGACGCGTTGC-3' \\
\hline m164_1stATG_rev & $\begin{array}{c}\text { 5'-CCGACAGCCGCGGAGAAACGCCGCCTTCCCACG } \\
\text { TGTGTG-3'G }\end{array}$ \\
\hline m164_1stATG_for & $\begin{array}{l}\text { 5'-CCACACACGTGGGAAGGCGGCGTTTCTCCGCG } \\
\text { GCTGTCG-3' }\end{array}$ \\
\hline
\end{tabular}


Table A2. PCR cycling conditions used in this study.

\begin{tabular}{|c|c|c|c|c|c|}
\hline Product & Primer rev & Primer for & Annealing & Elongation & Size [bp] \\
\hline \multicolumn{6}{|c|}{ Figure 4} \\
\hline $\mathrm{I}$ & m164mRNA_rev & m164mRNAin_for & $51.7^{\circ} \mathrm{C}$ & $1 \min 30 \mathrm{~s} ; 72^{\circ} \mathrm{C}$ & 2,001 \\
\hline II & m164mRNA_rev & m164mRNAout_for & $51.7^{\circ} \mathrm{C}$ & $90 \mathrm{~s} ; 72{ }^{\circ} \mathrm{C}$ & 2,023 \\
\hline \multicolumn{6}{|c|}{ Figure A2A } \\
\hline $\mathrm{I}$ & m164mRNA_rev & m164mRNAout50_for & $51.7^{\circ} \mathrm{C}$ & $2 \min 10 \mathrm{~s} ; 68^{\circ} \mathrm{C}$ & 2,081 \\
\hline II & m164mRNA_rev & m164mRNAout100_for & $51.7^{\circ} \mathrm{C}$ & $2 \min 10 \mathrm{~s} ; 68^{\circ} \mathrm{C}$ & 2,123 \\
\hline III & m164mRNA_rev & m164mRNAout300_for & $51.7^{\circ} \mathrm{C}$ & $2 \min 18 \mathrm{~s} ; 68^{\circ} \mathrm{C}$ & 2,299 \\
\hline IV & m164mRNA_rev & m164mRNAout1000_for & $51.7^{\circ} \mathrm{C}$ & $3 \mathrm{~min} ; 68^{\circ} \mathrm{C}$ & 3,048 \\
\hline \multicolumn{6}{|c|}{ Figure A2C } \\
\hline I & m164mRNAin_rev & m166_ORF_end_for & $55^{\circ} \mathrm{C}$ & $1 \min 30 \mathrm{~s} ; 72^{\circ} \mathrm{C}$ & 1,744 \\
\hline II & m164mRNAin_rev & m166_ORF_end250_for & $55^{\circ} \mathrm{C}$ & $1 \min 30 \mathrm{~s} ; 72^{\circ} \mathrm{C}$ & 1,986 \\
\hline III & m164mRNAin_rev & m166_ORF_end500_for & $55^{\circ} \mathrm{C}$ & $2 \min 18 \mathrm{~s} ; 68^{\circ} \mathrm{C}$ & 2,254 \\
\hline IV & m164mRNAin_rev & m166_ORF_end1000_for & $55^{\circ} \mathrm{C}$ & $3 \mathrm{~min} ; 68^{\circ} \mathrm{C}$ & 2,732 \\
\hline $\mathrm{V}$ & m164mRNAin_rev & m166.5_ORF_end_for & $55^{\circ} \mathrm{C}$ & $3 \mathrm{~min} ; 68^{\circ} \mathrm{C}$ & 3,086 \\
\hline VI & m164mRNAin_rev & m166.5_ORF_end250_for & $55^{\circ} \mathrm{C}$ & $3 \min 20 \mathrm{~s} ; 68^{\circ} \mathrm{C}$ & 3,346 \\
\hline VII & m164mRNAin_rev & m166.5_ORF_end500_for & $55^{\circ} \mathrm{C}$ & $3 \min 30 \mathrm{~s} ; 68^{\circ} \mathrm{C}$ & 3,576 \\
\hline VIII & m164mRNAin_rev & m166.5_ORF_end1000_for & $55^{\circ} \mathrm{C}$ & $4 \mathrm{~min} ; 68^{\circ} \mathrm{C}$ & 4,081 \\
\hline
\end{tabular}


Reprinted from Viruses. Cite as: Raghavan, B.; Cook, C.H.; Trgovcich, J. The Carboxy Terminal Region of the Human Cytomegalovirus Immediate Early 1 (IE1) Protein Disrupts Type II Inteferon Signaling. Viruses 2014, 6, 1502-1524.

Article

\title{
The Carboxy Terminal Region of the Human Cytomegalovirus Immediate Early 1 (IE1) Protein Disrupts Type II Inteferon Signaling
}

\author{
Bindu Raghavan ${ }^{\dagger}$, Charles H. Cook and Joanne Trgovcich * \\ Department of Surgery, The Ohio State University, 473 West 12th Avenue, Columbus, OH 43210, \\ USA; E-Mail: bindu.raghavan@monsanto.com (B.R.); Charles.Cook@osumc.edu (C.H.C.) \\ $\dagger$ Current Address: Monsanto Company, 800 North Lindbergh Blvd, St. Louis, MO 63167, USA. \\ * Author to whom correspondence should be addressed; E-Mail: joanne.trgovcich@osumc.edu; \\ Tel.: +1-614-688-3353.
}

Received: 31 December 2013; in revised form: 7 March 2014 / Accepted: 7 March 2014 /

Published: 2 April 2014

\begin{abstract}
Interferons (IFNs) activate the first lines of defense against viruses, and promote innate and adaptive immune responses to viruses. We report that the immediate early 1 (IE1) protein of human cytomegalovirus (HCMV) disrupts signaling by IFN $\gamma$. The carboxyl-terminal region of IE1 is required for this function. We found no defect in the initial events in IFN $\gamma$ signaling or in nuclear accumulation of signal transducer and activator of transcription 1 (STAT1) in IE1-expressing cells. Moreover, we did not observe an association between disruption of IFN $\gamma$ signaling and nuclear domain 10 (ND10) disruption. However, there is reduced binding of STAT1 homodimers to target gamma activated sequence (GAS) elements in the presence of IE1. Co-immunoprecipitation studies failed to support a direct interaction between IE1 and STAT1, although these studies revealed that the C-terminal region of IE1 was required for interaction with STAT2. Together, these results indicate that IE1 disrupts IFN $\gamma$ signaling by interfering with signaling events in the nucleus through a novel mechanism.
\end{abstract}

Keywords: human cytomegalovirus; interferons; interferon $\gamma$; immediate early 1; IE1; STAT1; STAT2; ND10; antiviral defense 


\section{Introduction}

After primary infection, human cytomegalovirus (HCMV) persists for the lifetime of the host, avoiding elimination by the host immune system [1]. In congenitally infected infants and immunosuppressed persons, HCMV can cause serious diseases [2]. Aspects of both HCMV disease and persistence in human populations are suspected to be linked to the multiple mechanisms this virus has evolved to modulate human immune responses.

Interferons direct intrinsic cellular defenses and early immune responses against HCMV and other viruses. IFNs are classified into three categories: type I (IFNs- $\alpha, \beta, \omega, \varepsilon, \kappa)$, type II (IFN $\gamma$ ) and type III (IFN $\lambda$ ), though type I and II interferons are the best studied with respect to their antiviral functions [3]. IFNs have intrigued virologists for over 50 years, not only for providing insight into viral biology and the molecular combat that occurs in infected cells, but also for the potential promise of exploiting the IFN system for therapeutic control and prevention of viral diseases. IFNs function to limit virus replication and pathogenesis through stimulation of intrinsic cellular defense mechanisms that contribute to innate immunity [4,5], and by promoting and regulating adaptive immune responses [6,7].

The antiviral functions of IFNs are initiated by binding with their receptors [4,8-10]. The type I receptor is composed of two proteins, IFN Alpha Receptor 1 (IFNAR1) and IFN Alpha Receptor 2 (IFNAR2). The cytoplasmic tails of the receptors associate with protein tyrosine kinases, Janus Kinase 1 (JAK1) and Tyrosine Kinase 2 (TYK2). Binding of Type I IFN to its cognate receptor leads to phosphorylation of JAK1 and TYK2 and tyrosine residues on the cytoplasmic tails of the receptors. Phosphorylation of the receptors enables docking of Signal Transducer and Activator of Transcription 1 and 2 (STAT1 and STAT2) via Src Homology 2 (SH2) domains and tyrosine phosphorylation and heterodimerization of the STATs. The STAT1-STAT2 heterodimers forms complexes with interferon regulatory factor (IRF) 9 that translocates to the nucleus and binds Interferon Stimulated Response Elements (ISRE) in the promoters of type I IFN induced genes [8,9]. Type II IFNs bind to a different receptor composed of Interferon Gamma Receptor 1 and 2 (IFNGR1 and IFNGR2) proteins, associated with the tyrosine kinases JAK1 and JAK2. IFN $\gamma$ signaling results in formation of phosphorylated STAT1 homodimers that translocate to the nucleus and bind Gamma Activated Sequence (GAS) elements in the promoters of type II IFN inducible genes [3,9,10].

Interferons can stimulate the expression of over 300 cellular genes known as Interferon Stimulated Genes (ISGs) [11]. The proteins encoded by ISGs have antiviral and immunomodulatory activity. These gene products interfere with the viral life cycle by degrading viral nucleic acids, inhibiting viral protein synthesis, interference with viral protein trafficking, or inducing apoptosis leading to death of virally infected cells [10]. In addition to their direct antiviral role, IFNs can control viral pathogenesis by enhancing adaptive immune responses, enhancing antigen presentation to cells of the immune system, and recruiting immune cells to sites of infection [6,7]. In particular, type II IFN is important in stimulating the activity of NK cells, macrophages, $\mathrm{T}$ lymphocytes and dendritic cells (DCs) [7].

The cytomegaloviruses employ multiple mechanisms to disrupt IFN induction [12-14] and IFN signaling [15-20]. HCMV targets the type I pathway by disrupting STAT1 and STAT2. Paulus and colleagues demonstrated that HCMV immediate early 1 (IE1/IE72) encoded by the UL123 gene 
forms a physical complex with STAT1 and STAT2 thereby blocking signaling after nuclear translocation and before DNA binding [21]. Binding of IE1 to STAT2 requires the short acidic and serine/proline-rich low-complexity motifs in the carboxy-terminal region of IE1 [22]. Huh and colleagues demonstrated that disruption of IFN $\beta$ activity related to binding the acidic domain of immediate early 1 (IE1) to STAT2 in a sumoylation-dependent manner [23]. Le et al. further reported that STAT2 is targeted for proteasome-mediated degradation at early to late times of infection that was dependent on expression of an early gene [24].

The type II IFN signaling pathway has been studied in both MCMV- and HCMV-infected cells. A blockade in IFN $\gamma$-mediated regulation of several genes is observed in murine cytomegalovirus (MCMV) infected macrophages [25], and the MCMV M27 protein has been shown to disrupt IFN $\gamma$ signaling through a novel, STAT2-dependent mechanism [26]. Initial reports that HCMV also targets type II IFN signaling derived from the observation that IFN $\gamma$-induced CIITA induction was disrupted downstream of STAT1 nuclear translocation as early as 6 hours after HCMV infection [27]. This appeared to be due to impaired binding of STAT1 to GAS elements at very early times in HCMV infected cells [28]. Subsequently, it was reported that IFN $\gamma$ signaling in HCMV infected cells is also disrupted through degradation of JAK1 [29]. Furthermore, Baron and Davignon described impaired STAT1 tyrosine phosphorylation in response to IFN $\gamma$ in the 12 to 24 hour time period after infection with HCMV [30]. This was found to be linked to activation of the SH2 domain-containing phosphatase 2 (SHP2) acting on phosphorylated STAT1. Remarkably, Knoblach and colleagues described an activation of a type II interferon-like host response in cells induced to express IE1 [31]. The activation ISGs by IE1 in this report was attributed to activation of STAT1 and was independent of IFN $\gamma$. Altogether there is a lack of clarity on how HCMV influences type II IFN signaling and the viral gene products involved.

Here we report that expression of the HCMV UL123 gene that codes for IE1/IE72 also interferes with IFN $\gamma$ signaling in human primary fibroblasts. IE1 is a promiscuous transactivator of viral and cellular genes [32] and, as discussed above, is known to antagonize type I interferon signaling $[21,23,24]$. Our findings suggest that IE1 can disrupt signaling by both type I and Type II interferons. Furthermore we have determined that the carboxyl-terminal region of IE1 that includes the acidic domain is required for this function. We found no defect in the initial events in IFN $\gamma$ signaling in IE1-overexpressing cells, nor did we observe an association between disruption of IFN $\gamma$ signaling and ND10 disruption. Moreover, IE1 does not interfere with nuclear accumulation of STAT1. However there is reduced binding of STAT1 homodimers to target GAS elements in the presence of IE1. This activity does not appear to require a direct interaction of IE1 and STAT1 and suggests that IE1 disrupts IFN $\gamma$ signaling in the nucleus and through a novel mechanism.

\section{Results and Discussion}

\subsection{The HCMV IE1 Gene Disrupts Signaling by Type II Interferon}

To identify the HCMV genes involved in disruption of IFN signaling a cDNA library of the HCMV laboratory strain AD169 was constructed [33]. The human fibrosarcoma cell line 2C4 was used in a preliminary screen for HCMV cDNA clones that have a role in regulating IFN signaling. $2 \mathrm{C} 4$ is a fibrosarcoma cell line engineered to express the T-cell antigen CD2 under the control of 
the promoter element of the Interferon Induced Transmembrane protein 1 (IFITM1) gene (a.k.a. 9-27, IFI17, CD225) which respond to both type I and II IFNs by increasing cell surface expression of CD2 [34]. 2C4 cells transfected with any HCMV cDNA clone involved in disruption of IFN signaling would thus be expected to exhibit reduced accumulation of cell surface of CD2. Using this system we observed that transfection of cDNA clone pIE622, harboring the full length cDNA sequence for the HCMV UL123 gene, was associated with diminished CD2 cell surface levels relative to empty vector-transfected cells after exposure to IFN $\beta$, and to a lesser extent, IFN $\gamma$ (data not shown). This suggested a role for IE1 in disruption of both type I and type II IFN signaling. The UL123 gene codes for the HCMV major transcriptional transactivator protein, IE1. In addition to its role as a promiscuous transactivator of viral and cellular genes, IE1 is known to have multiple functions including disruption of ND10 nuclear bodies [35], antagonism of histone deaceytlase (HDAC3) [36], anti-apoptotic function [37], chromatin tethering [38], and interference with signaling by type I interferons [21].

To confirm a role for IE1 in disruption of type II IFN signaling, we examined the effect of IE1 in a more physiologically relevant human fibroblast cell line. Basal expression of MHC II Transcriptional Activator (CIITA) is very low in fibroblasts but becomes highly induced after IFN $\gamma$ treatment. CIITA transcript levels were quantified by Real-Time RT-PCR in MRC5 fibroblasts after nucleofection with pIE622 expressing the full length IE1. In MRC5 cells, nucleofection resulted in greater than $90 \%$ transfection efficiency (data not shown). CIITA mRNA levels were compared in IFN $\gamma$ treated cells and untreated cells. In untreated cells, expression of IE1 was associated with a slight induction of CIITA or IRF1 ( 0.9 to 3 fold). In IFN $\gamma$ treated cells, we found that CIITA induction in IE1 expressing cells was only $39 \%$ of that observed in cells nucleofected with empty vector (Figure 1A). We also examined the IFN $\gamma$-induced expression of a second gene, IRF1, in IE1 expressing fibroblasts. We found that the increase in expression of IRF1 in response to IFN $\gamma$ is reduced by $52 \%$ in IE1 expressing cells as compared to cells nucleofected with empty vector (Figure 1B) relative to untreated cells. These findings confirm that IE1 is able to impair, but not completely block IFN $\gamma$-induced upregulation of CIITA and IRF1 transcript levels in human fibroblasts.

\subsection{Mapping of the IE1 Protein Region Involved in Disruption of IFN-Induced Gene Expression}

The HCMV IE1 protein is a multifunctional protein with various functions attributed to different domains of the protein (Figure 2A). We generated a series of FLAG epitope-tagged truncation mutants to identify the protein region required for attenuation of IFN $\gamma$-induced gene expression as described in Materials and Methods. We generated five different FLAG-tagged truncation mutants plus a full length FLAG-tagged version of IE1 (Figure 2A). These six different clones were nucleofected into MRC5 cells, followed by treatment with IFN $\gamma$ for 6 hours. RNA extracted from these cells was analyzed for levels of CIITA transcript induction by Real-Time RT-PCR. We found that there was a reduction in the IFN $\gamma$-induced expression of CIITA in cells expressing full length and truncated versions of IE1, with the exception of the $\mathrm{C}$-terminal truncation missing residues 345-491 ( $\triangle \mathrm{AD})$ that includes the acidic domain and chromatin tethering domain (Figure $2 \mathrm{~B})$. We consistently observed higher levels of CIITA induction in cells expressing the $\Delta 345-491$ protein relative to our vector alone transfections. Although the reason for this is not clear, it may be that the 
IE1 protein without the $\mathrm{C}$-terminal domain provides a signal that amplifies IFN $\gamma$-induced CIITA gene expression. This may also relate to the report linking inducible IE1 expression to STAT1-dependent interferon-like gene induction [31]. One possibility is that IE1 may interact with cellular proteins that stimulate a type II interferon response, but this activity is masked by the dominant inhibitory role of the C-terminal 147 residues in our studies. These contradictory data may also reflect the different transfection systems or viral strains used in these two studies.

Figure 1. Diminished interferon-induced gene expression in human fibroblasts expressing IE1. MRC-5 fibroblasts were nucleofected with plasmid pIE622 harboring the UL123 gene specifying IE1, or the pFIN2 empty vector. At 24 hours post-nucleofection, the cells were exposed to $100 \mathrm{U} / \mathrm{mL}$ of IFN $\gamma$ or were left untreated. At 6 hours after treatment, total RNA was isolated and subjected to Real Time RT-PCR analysis as described in Materials and Methods. The fold increase in CIITA (A) $(p$ value $=0.053)$ or IRF1 (B) ( $p$ value 0.017) transcript levels in IFN treated cells relative to untreated cells was determined by the $\Delta \Delta \mathrm{Ct}$ method. Data shown are the average of three independent experiments.

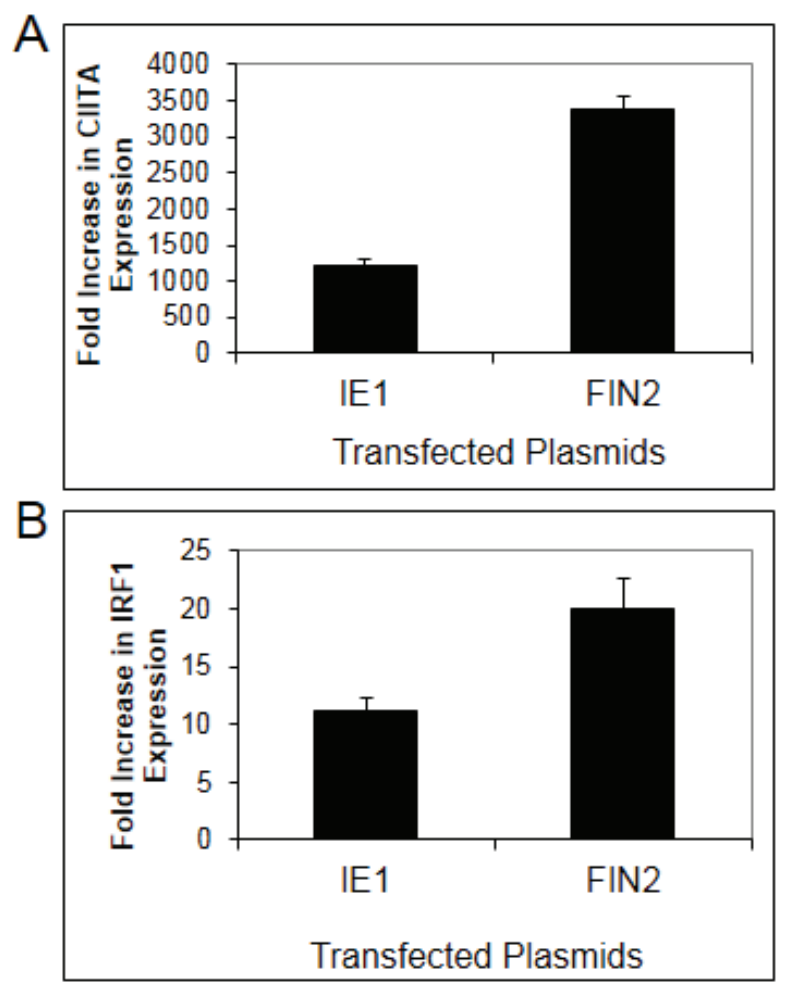

Figure 2. The carboxy-terminal region of IE1 is required for disruption of IFN $\gamma$ signaling. (A) Schematic of the IE1 protein and truncation mutants generated in this study. Top line: a schematic of the IE1 protein indicating the amino acid positions comprising the coding exons (below) and the positions of the known functional domains (above). The coding exons are shown in white boxes. The functional domains of exon 4 are shown in shaded boxes and include the leucine zipper (L), the zinc finger (ZF) and the acidic domain (AD). A schematic of the five different IE1 N-terminal truncation mutants generated in this study are shown below. The sixth mutant is a 
C-terminal truncation mutant. The designation of the plasmids harboring the truncation mutants is based on the deletion of relevant exons or functional domains shown on the right, and the deleted amino acid residues are shown in parentheses. The gene sequences were inserted in frame to sequences specifying a FLAG epitope (depicted as a flag). (B) Interferon Stimulated Gene (ISG) induction in fibroblasts expressing truncated IE1 proteins. The indicated plasmids were nucleoporated into MRC5 cells and 24 hours after nucleofection the cells were treated with $100 \mathrm{U} / \mathrm{mL} \mathrm{IFN} \gamma$. At 6 hours after treatment total RNA was isolated. Real Time RT-PCR analysis was carried out for CIITA and 18S rRNA (endogenous control). The fold increase in CIITA transcript levels in IFN treated cells relative to untreated cells was determined by the $\Delta \Delta \mathrm{Ct}$ method. Shown is the average of two independent experiments. A film image of immunoblot analysis is shown below. In replicate cultures of the first experiment, cells were treated as above and solubilized. Equal amounts of protein from each lysate were subjected to electrophoresis in a denaturing polyacrylamide gel. Proteins were transferred to nitrocellulose sheets and reacted with the indicated antibodies. Anti-FLAG antibody was used to detect the presence of FLAG tagged IE1 proteins and antibody to GAPDH was used to evaluate protein loading.

A
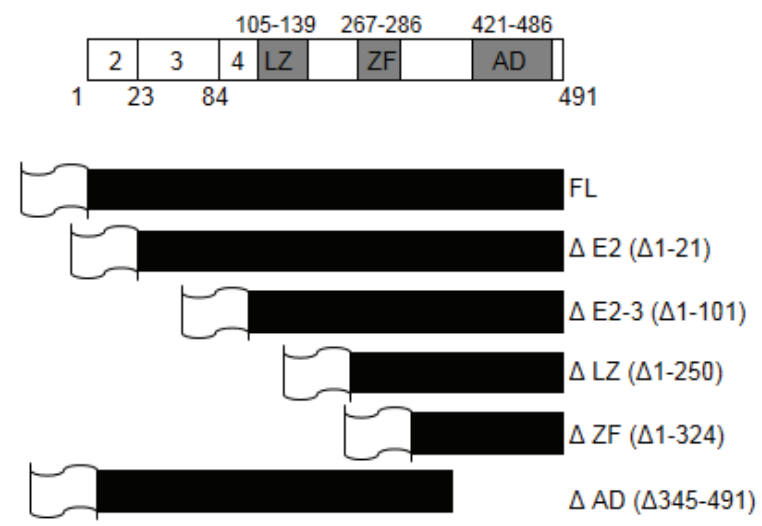

$\mathrm{B}$
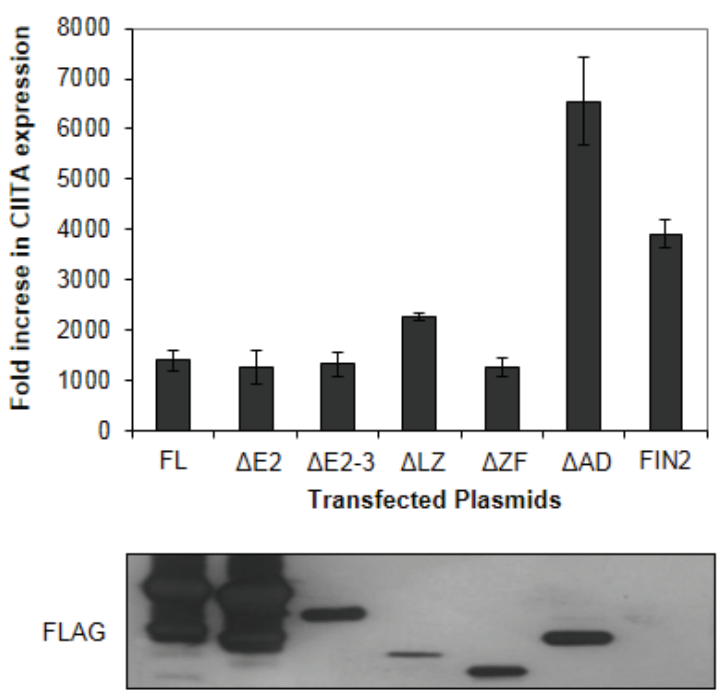

GAPDH

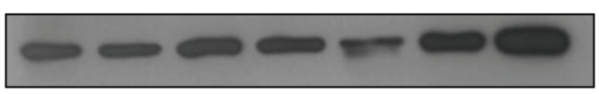


Promyelocytic leukemia protein (PML) is involved in transcriptional repression and is a master organizer of nuclear domain (ND)10 structures. Since IE1 has been reported to be involved in the disruption of ND10 structures in the nucleus [35,39-43] and because PML is known to mediate antiviral activities of IFNs in HSV-infected cells [44] and to confer intrinsic immunity against CMV $[45,46]$, we set out to test the hypothesis that the function of IE1 in disrupting IFN $\gamma$-induced gene expression is linked to its role in disrupting ND10 structures by immunofluorescence microscopy. We found that in fibroblasts expressing the full length IE1 and the $\Delta 345-491$ C-terminal deletion mutant of IE1, PML was dispersed throughout the nucleus, indicating disruption of ND10s (Figure 3). As expected, PML staining was in the form of punctate dots in the nucleus in the absence of IE1. Therefore, the C-terminal deletion mutant retains the ability to disrupt ND10s. We conclude that IE1-mediated disruption of the IFN $\gamma$ signaling pathway is not strictly linked to IE1-mediated dispersal of ND10s.

Figure 3. Disruption of ND10 structures in cells expressing IE1 and the C-terminal truncation mutant. Immunofluorescence images of IE1 and Promyelocytic leukemia protein (PML) in fibroblast cells. MRC5 cells were nucleofected with the indicated constructs. At 24 hours after nucleofection cells were fixed in methanol and reacted with mouse monoclonal anti-IE1 antibody (Mab810) followed by anti-mouse alexafluor 488 as secondary antibody. The cells were then exposed to rhodamine conjugated anti PML antibody (PGM3). IE1 reactivity is visualized in green (left column), PML reactivity is visualized in red (middle column) and merged images are shown in the right column.

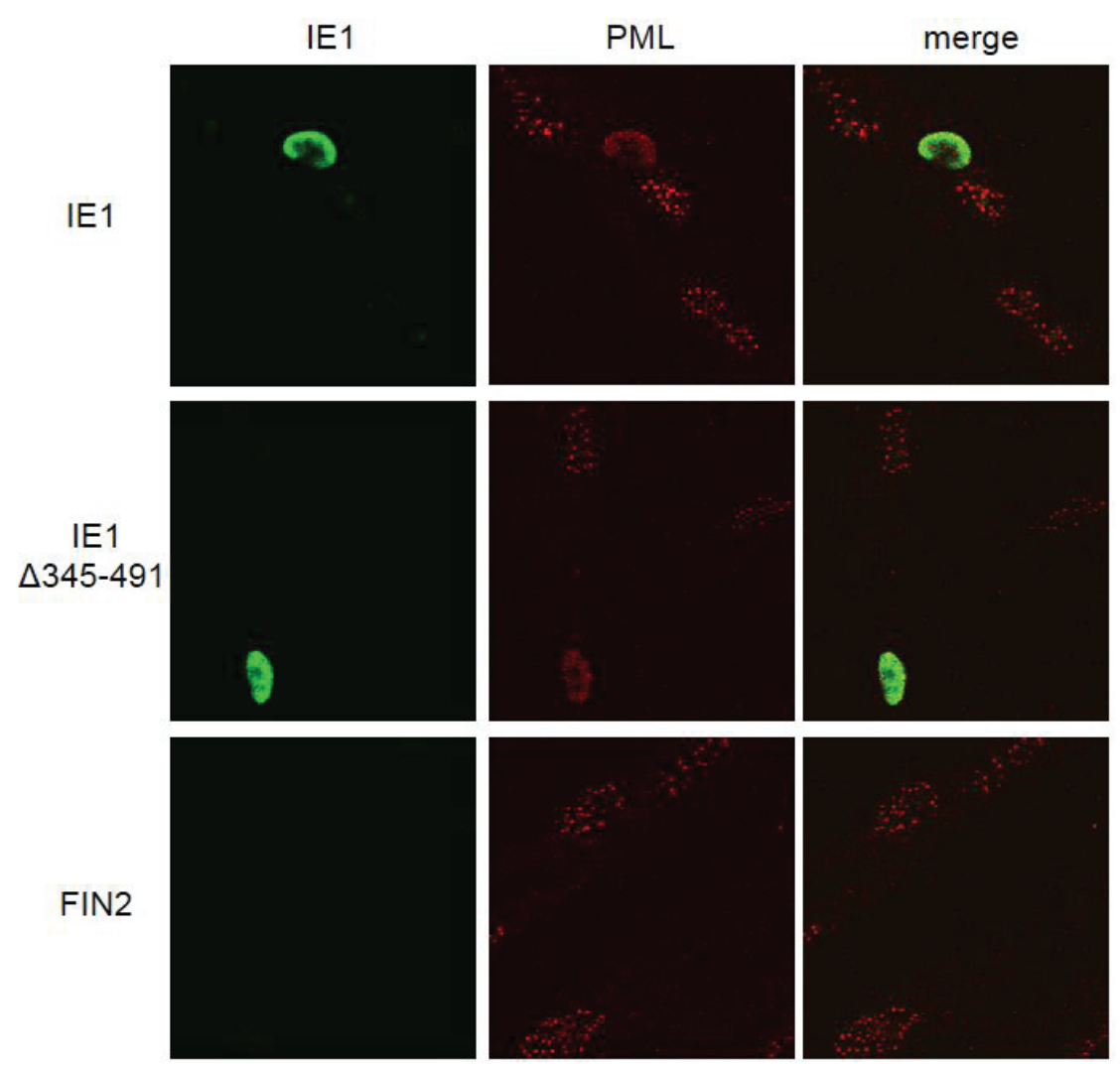


Earlier there have been conflicting reports, one suggesting that the acidic domain is important for PML targeting and disruption of ND10 structures [41] and the other suggesting that the acidic domain is not involved in this function of IE1 [39]. Yet another study revealed an intermediate role for the acidic domain IE1 wherein it was required to target ND10 structures but could not disrupt them [47]. Our results indicate that a C-terminal truncation mutant that includes the entire acidic domain is able to disrupt ND10s similar to the full length IE1 protein in MRC5 cells. Our results are in agreement the findings of Wilkinson et al. [39]. Although these results to do not preclude a role for PML in IE1-mediated IFN signal disruption, they do demonstrate that this function of IE1 does not involve disruption of ND10 structures.

\subsection{IE1 Does Not Perturb Initial Events in IFNy-Mediated Signal Transduction}

We next examined the state and abundance of proteins involved in IFN $\gamma$ signaling in IE1-expressing cells. We found no difference in the levels of total JAK1, JAK2 and STAT1 in IE1-expressing cells as compared to cells expressing the $\Delta 345-491$ mutant or cells nucleofected with empty vector (Figure 4). Activation of STAT1 by IFN $\gamma$ was not affected by IE1 since similar levels of tyrosine 701-phosphorylated STAT1 were observed after 30 minutes of IFN $\gamma$ treatment in cells nucleofected with full length IE1, the $\Delta 345-491$ mutant $(\triangle \mathrm{AD})$, or empty vector (Figure 4). Similarly, no differences in accumulation of serine 727-phosphorylated STAT1 were observed (data not shown). We also found that translocation of STAT1 to the nucleus was not affected by IE1 when examined by immunofluorscence microscopy (Figure 5A). Consistent with the microscopy analysis, the accumulation of STAT1 protein in the nucleus of IE1 expressing cells was similar to that of cells expressing the $\Delta 345-491$ deletion mutant $(\triangle \mathrm{AD})$ and cells transfected with empty vector (Figure 5B). Thus, IE1 does not act by limiting the abundance of key IFN $\gamma$ signal transduction molecules, nor does it disrupt STAT1 phosphorylation and nuclear translocation.

\subsection{Type II Interferon-Induced Binding of STAT1 to GAS Elements Is Reduced in the Presence of IE1}

We next determined whether STAT1 molecules in the nucleus were competent to bind the GAS element derived from the promoter of the IRF1 gene by EMSA. The full length IE1, the $\Delta 345-491$ mutant $(\triangle \mathrm{AD})$, and empty vector (FIN2) were nucleofected into MRC5 cells. These cells were either treated with IFN $\gamma$ for 30 minutes or left untreated. Nuclear extracts from these cells were incubated with a radiolabeled 22mer IRF1 GAS element probe. The amount of probe shifted in nuclear extracts from IE1-expressing cells is diminished relative to extracts derived from empty vector- or $\Delta 345-491$-nucleofected cells (Figure 6A, compare lane 6 to lanes 5 and 7). These bands could be supershifted using a STAT1 antibody indicating that the shift was caused by a complex containing STAT1 (lanes 11-13). This demonstrates that there is reduced binding of STAT1 to GAS elements in the presence of IE1. Studies were also conducted with nuclear extracts from HCMV-infected cells at 12 hours after infection. We could detect only a minor STAT1-shifted probe band in HCMV infected cells compared to uninfected cells (Figure 6B, lane 2), which may be related to the much higher levels of IE1 in infected cells relative to cells nucleofected with full length IE1 (data not shown). Together, these data indicate that expression of HCMV IE1 is sufficient to impair STAT1 binding to GAS elements, and that this activity requires the C-terminal region of 
IE1 including residues 344 to 491 . These data also suggest that the impairment of functional STAT1 dimer binding to GAS elements at 12 hours after infection is a result of IE1 expression.

Figure 4. IE1 does not alter the state or abundance of key signaling molecules of the IFN $\gamma$ pathway. Film images of electrophoretically separated cell lysates reacted with antibodies to IFN $\gamma$ pathway signaling components. MRC5 cells were nucleofected with plasmid harboring full length IE1 (FL), the $\Delta 345-491 \mathrm{C}$-terminal truncation IE1 ( $\triangle \mathrm{AD}$ ) or empty vector (FIN2). At 24 hours after nucleofection, cells were exposed to IFN $\gamma$ for 30 minutes or left untreated. Cells were solublized and equal amounts of protein from each lysate were subjected to electrophoresis in a denaturing polyacrylamide gel. Proteins were transferred to nitrocellulose sheets and reacted with the indicated antibodies. Anti-FLAG antibody was used to detect the presence of FLAG tagged IE1 proteins and antibody to GAPDH was used to evaluate protein loading.

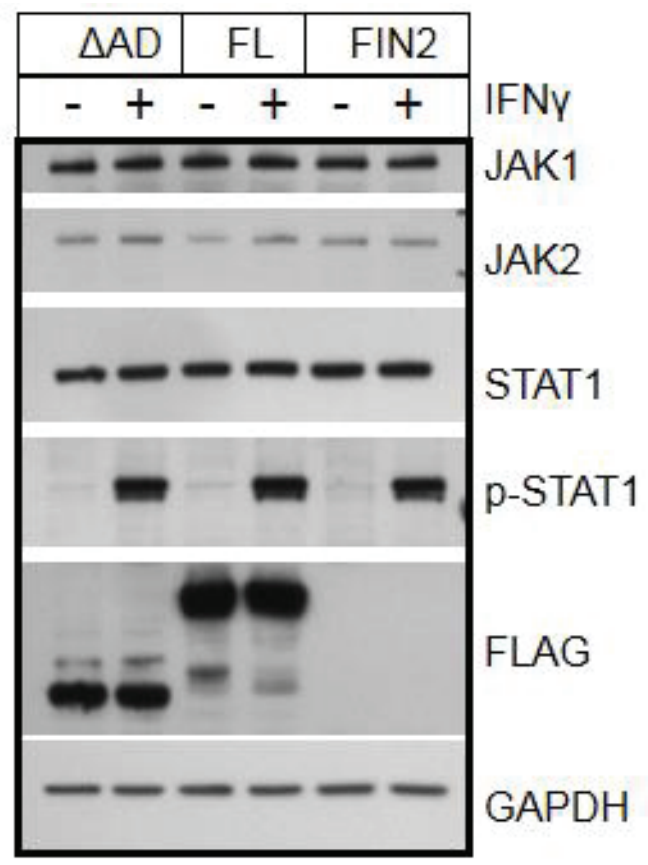

IE1 has been reported to antagonize HDAC activity [36]. Although HDAC activity is typically associated with transcriptional repression, it is associated with transcriptional activation of ISGs [48]. It is tempting to speculate that antagonism of HDAC activity by IE1 is linked to disruption of interferon signaling. Further implicating a role for chromatin organization is that the C-terminal region of IE1 includes the region previously shown to be required for chromatin tethering [38]. However, this possibility is difficult to reconcile with the observation that IE1 disrupts STAT1-binding to a GAS element probe used in our EMSA assays, which is not dependent on chromatin structure. We therefore propose that a novel function of IE1 impairs STAT1 binding to target GAS elements. 
Figure 5. Nuclear translocation of STAT1 is not altered by IE1. (A) Immunofluorescence images of IE1 and STAT1 in cells with and without IFN $\gamma$ treatment. MRC5 cells were nucleofected with the full length IE1 (FL) or empty vector (FIN2). After 24 hours cells were exposed to IFN $\gamma$ for 30 minutes or left untreated. Cells were fixed in methanol and reacted with mouse monoclonal anti-IE1 antibody (Mab810) and rabbit polyclonal anti-STAT1 antibody followed by anti-mouse alexafluor 543 and anti-rabbit alexafluor 488 as secondary antibodies. STAT1 reactivity is visualized in green (left column), IE1 reactivity is visualized in red (middle column) and merged images are shown in the right column. (B) Film images of electrophoretically separated cell lysates reacted with antibodies to STAT1 and GAPDH (loading control). MRC5 cells were nucleofected with plasmid harboring full length IE1 (FL), the $\Delta 345-491$ C-terminal truncation IE1 $(\triangle \mathrm{AD})$ or empty vector (FIN2). At 72 hours after nucleofection, cells were exposed to IFN $\gamma$ for 30 minutes or left untreated. Cells were harvested and nuclear and cytoplasmic fractions were separated. Equal amounts of protein from the nuclear and cytoplasmic lysates were subjected to electrophoresis in a denaturing polyacrylamide gel. Proteins were transferred to nitrocellulose sheets and reacted with the indicated antibodies.
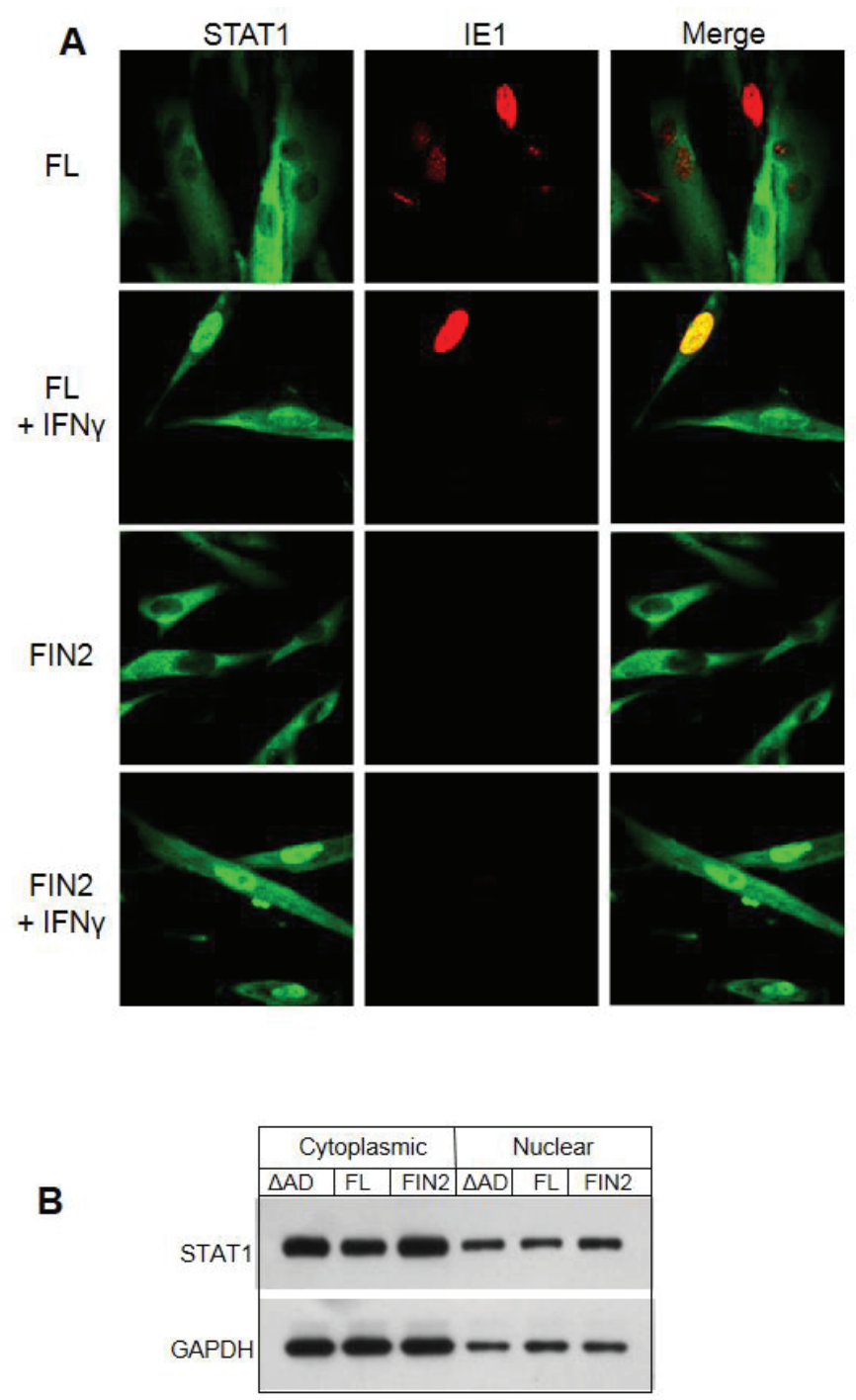
Figure 6. Reduced binding of STAT1 to GAS elements in the presence of IE1. Film image of an Electrophoretic Mobility Shift Assay shows a reduction in the amount of IRF1 GAS element probe that is shifted upon mixture with nuclear extracts from MRC5 cells ectopically expressing IE1 (A) and HCMV infected cells (B). In A, MRC5 cells were nucleofected with plasmid harboring full length IE1 (FL), the $\Delta 345-491$ C-terminal truncation IE1 $(\triangle \mathrm{AD})$ or empty vector (FIN2). At 24 hours after nucleofection, cells were exposed to $100 \mathrm{U} / \mathrm{mL}$ of IFN $\gamma$ or left untreated. At 30 minutes after treatment, cells were solubilized and nuclear extracts were isolated as described in Materials and Methods. Nuclear extracts were mixed with the GAS element of the IRF1 gene with or without addition of unlabeled probe and with or without anti-STAT1 antibody. Resulting complexes were resolved by electrophoresis on a $4.5 \%$ native polyacrylamide gel. In a second experiment (B), MRC5 cells were exposed to $1 \mathrm{PFU}$ per cell of the AD169 strain of HCMV or left uninfected. At 12 hours after infection, cells were solubilized and processed as described above. The black arrow indicates the shifted bands and the grey arrow indicates the supershifted bands.

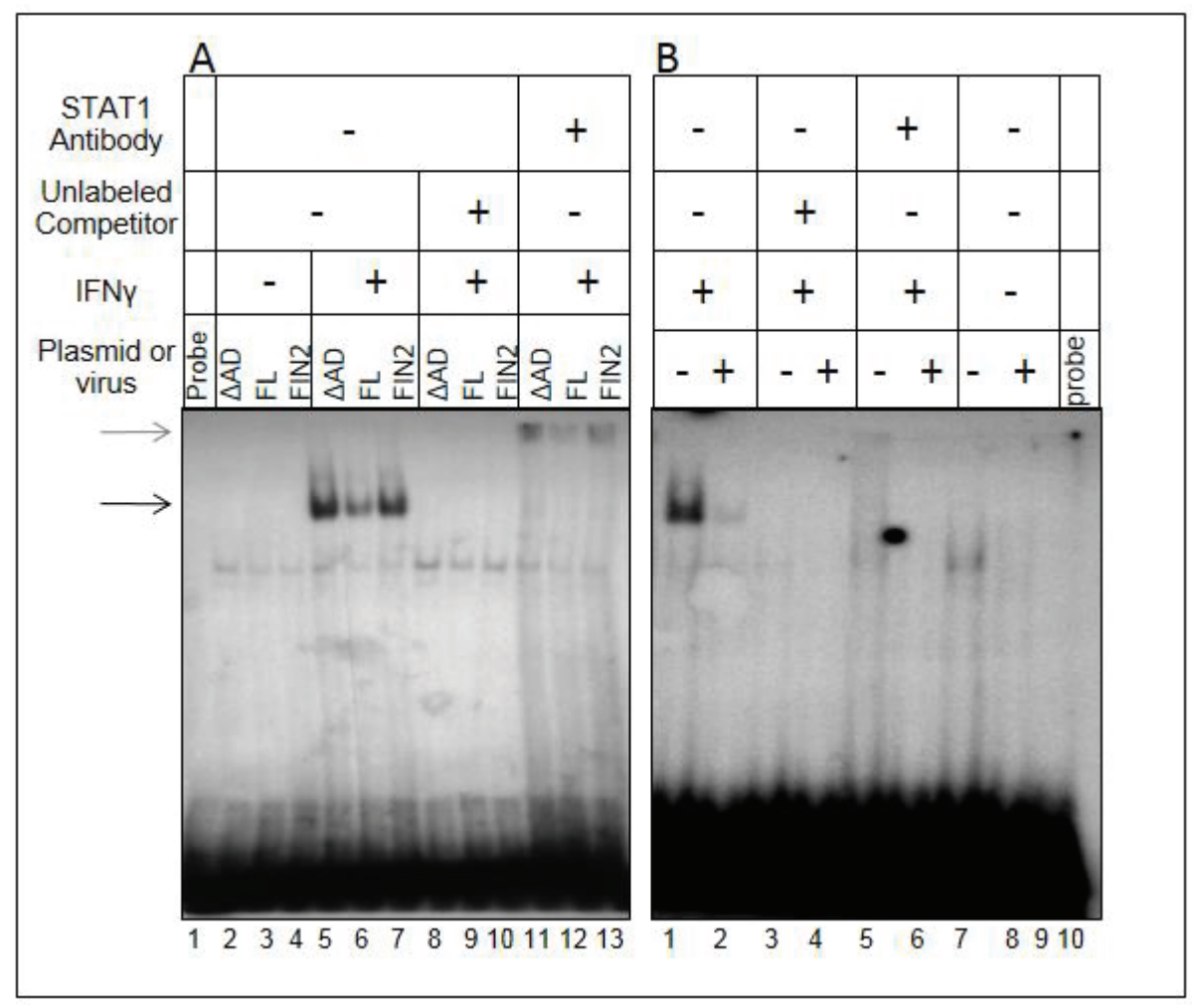

\subsection{STAT1 Fails to Co-Immunoprecipitate with IE1}

In the EMSA studies, the size of the shifted band in the presence or absence of STAT1 antibody is the same whether or not IE1 is present. This argues against a scenario in which IE1 acts by directly binding to STAT1-bound GAS elements. In order to test this directly, we carried out co-immunoprecipitation studies. MRC-5 fibroblast cells were exposed to 3 PFU per cell of the AD169 strain of HCMV. At 12 hours after infection, cells were treated with interferon for 30 minutes. Cells were solubilized and IE1 was isolated using an antibody that recognizes an 
epitope in exon 2. Under these conditions, we found no evidence of an interaction between STAT1 and IE1 (Figure 7A). Similarly, STAT1 failed to specifically co-immunoprecipitate with either the full length or the $\triangle 345-491$ mutant of IE1 in nucleofection studies (Figure 7B, left panel). We did observe a small amount of STAT1 isolated from cells expressing both the full length IE1 and the C-terminal mutant upon longer exposure (right panel). However, in several replicate experiments, similar amounts of STAT1 were also observed in immunoprecipitations performed using lysates of empty-vector-transfected cells (data not shown). We therefore conclude that the small amount of STAT1 isolated upon isolation of IE1 and the $\Delta 345-491$ mutant IE1 represents nonspecific binding. These studies suggest that IE1does not require a sustained interaction with STAT1 to disrupt IFN $\gamma$ signaling.

A blockade in IFN $\gamma$-mediated regulation of several genes is observed in murine cytomegalovirus (MCMV) infected macrophages [25], and the MCMV M27 protein has been shown to disrupt IFN $\gamma$ signaling through a novel, STAT2-dependent mechanism [26]. Therefore, we examined whether STAT2 co-immunoprecipitates with IE1 in IFN $\gamma$ treated human fibroblasts. As expected based previously published studies [21-24], we did observe an interaction of STAT2 with the full length IE1 in total lysates of nucleofected cells and HCMV infected cells. Similar to previously published results, this interaction maps to the C-terminal region of IE1 and the $\Delta 345-491$ mutant does not appear to interact with STAT2 under these conditions (Figure 7B). In addition, very little or no phosphorylated STAT2 accumulated in nuclei of MRC5 human fibroblasts in response to IFN $\gamma$ treatment, though phosphorylated STAT2 was observed in nuclei after IFN $\beta$ treatment (Figure 8). Our findings raise the possibility that IFN $\gamma$ signaling is different in murine and human fibroblasts. Specifically, it seems that murine, but not human fibroblasts, utilize phosphorylated STAT2 to transduce and amplify signals by IFN $\gamma$. Together, these studies suggest that IE functions via a novel mechanism in the nucleus to interfere with the type II IFN signal transduction pathway.

An important question raised by our studies is whether IE1 employs the same strategy, or entirely different strategies to disrupt type I and type II interferon signaling. Relevant are the following observations: (i) co-immunoprecipation studies using total lysates indicate an interaction between the HCMV IE1 acidic domain and STAT2 in human cells consistent with other studies ([21-24] and this report), and IE1 and STAT2 colocalize in ND10 structures and metaphase chromosomes [23]; (ii) in human fibroblasts, phophorylated STAT2 does not accumulate in the nucleus upon IFN $\gamma$ treatment ([24] and this report); (iii) there are conflicting reports of an interaction between IE1 of the Towne strain and STAT1 [21,23] and we were unable to find compelling evidence for a specific interaction between IE1 of strain AD169 and STAT1; and (iv) nevertheless, IE1 expression interferes with binding of STAT1 molecules to GAS elements. Based on these observations, it is tempting to conclude that an interaction between IE1 and STAT2 is necessary and sufficient to interfere with type I IFN signaling. However, such an interaction does not readily account for the activity of IE1 in attenuating binding of STAT1 molecules to GAS elements in response to type II interferon. On the other hand, the ability of IE1 to disrupt STAT1 binding to target DNA elements through an indirect mechanism could impede signals transduced by both type I and type II interferons. We suggest further studies are needed to resolve the role of IE1 in these signaling pathways. 
Figure 7. IE1 does not interact with STAT1 by co-immunoprecipitation. Film images of electrophoretically separated cell lysates and proteins isolated by immunoprecipation. (A) MRC5 cells were exposed to HCMV AD169 at 3 PFU per cell or left untreated. At 12 hours after infection cells were treated with $100 \mathrm{U} / \mathrm{mL}$ of IFN $\gamma$ for 30 minutes. The cells were harvested and solubilized. $350 \mu \mathrm{g}$ of total protein from each sample was reacted with anti-IE1 antibody (mouse monoclonal Mab810). The isolated proteins along with the $10 \mu \mathrm{g}$ total lysates were resolved by denaturing PAGE and transferred to a nitrocellulose membrane. The membrane was reacted with antibodies for STAT1, IE1 and STAT2 and the antibody reactive bands were visualized by chemiluminescence. The left panel shows films exposed for 10 seconds and the right panel shows films exposed for 5 minutes. The arrow indicates the position of the isolated, full length IE1. (B) MRC5 cells were nucleofected with full length IE1 (FL), the $\Delta 345-491$ C-terminal truncation IE1 $(\triangle \mathrm{AD})$ or empty vector (FIN2). 48 hours after nucleofection cells were exposed to $100 \mathrm{U} / \mathrm{mL}$ of IFN $\gamma$ for 30 minutes. The preparation of cell lysates, immunoprecipitations, PAGE and western hybridization and detection were carried out as above. The left panel shows films exposed for 10 seconds and the right panel shows films exposed for 5 minutes. The short and long arrows indicate the position of the $\Delta 345-491$ IE1 in the total lysate and immunoprecipitates respectively.
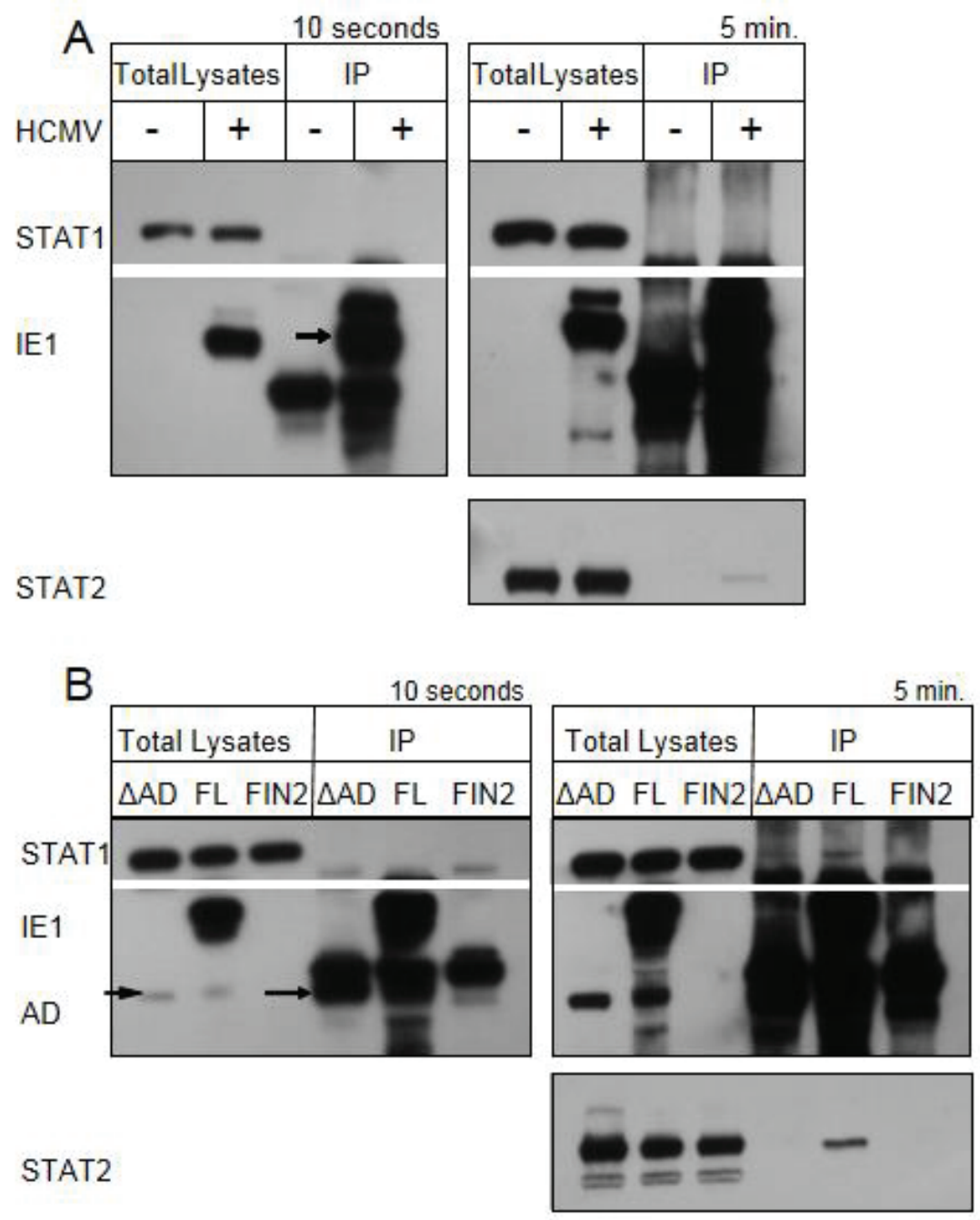
Many DNA viruses encode proteins that target the JAK- STAT signaling pathway including adenovirus E1A [49,50], human papilloma virus (HPV) E7 protein [51], hepatitis B virus (HBV) terminal protein [52], and polyoma virus large T antigen [53]. Among the herpesviruses, the EBV immediate early protein BZLF1 has been shown to interfere with STAT1 phosphorylation and nuclear translocation in response to IFN $\gamma$, as well as IFN $\gamma$ receptor expression [54]. Similarly, herpes simplex virus (HSV) was shown to interfere with type I IFN signaling by decreasing the levels of several signaling molecules, including JAK1 and STAT2, which was in part mediated by the virion host shutoff protein encoded by UL41 [55,56], by inducing suppressor of cytokine signaling-3 (SOCS3) [57], and by the activity of the ICP27 protein in inhibiting STAT1 phosphorylation and nuclear translocation [58]. HSV also targets the IFN $\gamma$ pathway by modifying the IFNGR1 through the activity of the US3 and UL13 protein kinases, and indirectly by the activities of virion host shutoff protein [59]. With the partial exception of HSV virion host shutoff protein, all of these viruses target early events in the signaling pathway, especially STAT1 phosphorylation and nuclear translocation, to attenuate IFN mediated signaling. Our findings indicate that HCMV does not target these proximal events in IFN signaling but rather it reduces STAT1 binding to target promoter elements in the nucleus. Elucidating the mechanism and consequences of this novel immune evasion strategy may provide important insights into the pathogenesis of HCMV infections and the regulation of interferon-induced gene expression.

Figure 8. Phosphorylated STAT2 does not accumulate in the nucleus of IFN $\gamma$-treated MRC-5 cells. Film images of electrophoretically separated nuclear lysates reacted with antibodies to p-STAT2 and Lamin (loading control). MRC5 cells were nucleofected with full length IE1 (FL), the $\triangle 345-491$ C-terminal truncation IE1 ( $\triangle \mathrm{AD})$ or empty vector (FIN2). At 72 hours after nucleofection, cells were exposed to IFN $\gamma$ or IFN $\beta$ for 30 minutes or left untreated. Cells were harvested and nuclear and cytoplasmic fractions were separated. Equal amounts of protein from the nuclear lysates were subjected to electrophoresis in a denaturing polyacrylamide gel. Proteins were transferred to nitrocellulose sheets and reacted with the indicated antibodies.

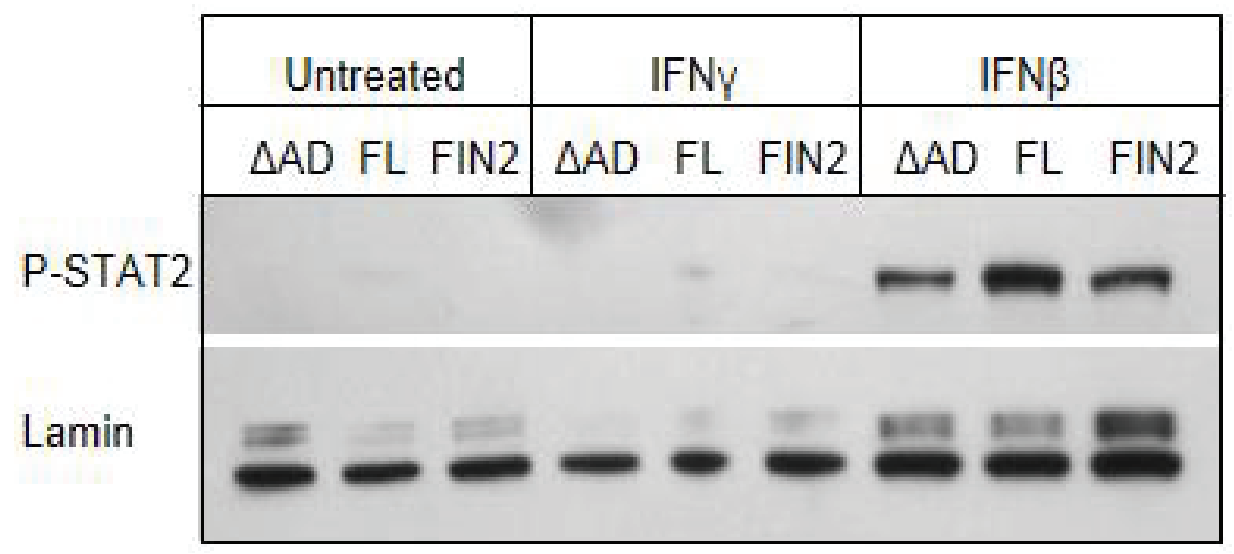




\section{Experimental Section}

\subsection{Cells and Viruses}

Human embryonic lung fibroblasts (MRC5) were obtained from American Type Culture Collection (ATCC, Manassas, VA, USA) and maintained in ATCC-recommended complete media. Human fibrosarcoma cell line 2C4 was a kind gift from George Stark (Cleveland Clinic Lerner Research Institute, Cleveland, OH, USA) [34]. 2C4 cells were maintained in DMEM supplemented with 10\% FBS, Glutamine and Sodium Pyruvate. HCMV strain AD169 was obtained from ATCC and propagated in MRC5 cells. Viral titers were determined by standard plaque assay [60].

Plasmids and vectors: Construction of the AD169 cDNA library is described elsewhere [33]. cDNA clones containing different truncated versions of the IE1 gene were selected from the cDNA library. The following clones were used in this study and harbor viral gene sequences with the indicated genomic coordinates: pie639 (173157-171814), pie535 (172658-171814), pie836 (172436-171814). The sequences specifying the FLAG epitope tag were inserted at the 5 ' end of each truncation mutant by replacing the Nde I-Eco R1 fragment of each clone with the Nde I-Eco R1 fragment from the pCMV3TAG vector (Stratagene, La Jolla, CA, USA) that includes both the CMVIE1 promoter sequence and the sequences specifying the FLAG tag. The resultant plasmids specified 5' FLAG tag sequences in frame with different truncated versions of IE1. For the C-terminal deletion mutant, a Bgl II fragment from cDNA clone pie622 (a full length IE1 cDNA clone), was inserted into the pCMV3TAG vector containing a FLAG tag at the 5' end in the same coding frame. The genomic coordinates for the C-terminal deletion mutant are (173705-173626 + 173511-173327 + 173156-172377). To generate a full length UL123 gene sequence in frame with FLAG sequences, the IE1 cDNA was PCR amplified from pie622 using the following primers: 'AGAGTGACTCACCAAGCTTGACACGATG and CGAGGCTGATCAGCTTAATTAACC. The PCR products were digested with Hind III and Pac I and inserted into HindIII- and PacI-digested pCMV3TAG vector. To generate a UL123 construct missing exon 2 in frame with FLAG sequences, pie622 was used as template to PCR amplify the appropriate gene regions using the following primers: CCTTCCTCCAAGCTTCCACGGCC and CGAGGCTGATCAGCTTAATTAACC which begins at genomic coordinate 173,645. The PCR products were digested with Hind III and Pac I and ligated into HindIII and PacI digested pCMV3TAG vector.

\subsection{Transfection, Nucleofection and Infection}

40,000 2C4 cells were plated per well of a 12-well plate. Within 24 hours of plating cells were transfected with the cDNA clones specifically using the Fugene HD transfection reagent (Roche, Indianapolis, IN, USA) according to manufacturer's recommendations ( $1 \mu \mathrm{g}$ plasmid DNA/well with $3 \mu \mathrm{L}$ of transfection reagent) [61]. Typically, this procedure resulted in $40 \%-50 \%$ transfection efficiency (data not shown). Transfections were optimized to reduce non-specific cellular interferon responses [61]. For nucleofections, $1.5 \times 10^{6}$ MRC5 cells were resuspended in $100 \mu \mathrm{L}$ of basic nucleofector solution for primary fibroblasts with $3 \mu \mathrm{g}$ of plasmid DNA and nucleofected using U23 program in the nucleofector device (Amaxa, Cologne, Germany). After nucleofection cells were immediately distributed equally in three wells of a six-well cluster. Nucleofection and 
transfection efficiencies were determined by immunofluorescence microscopy analysis of IE1 expression and/or immunofluorescence microscopy analysis of GFP expression in parallel transfections using plasmid pEGFP-N3 (Clontech, Mountainview, CA, USA). Nucleofection resulted in greater than $90 \%$ transfection efficiency (data not shown). For infections, confluent MRC5 cells in $75 \mathrm{~cm}^{2}$ tissue culture flasks were exposed to one or three plaque forming unites (PFU) per cell of HCMV strain AD169 and harvested 24 hours post infection. Wherever indicated, cells were treated with $100 \mathrm{U} / \mathrm{mL}$ of human recombinant IFN $\beta$ or IFN $\gamma$ (Chemicon (now EMD Millipore), Billerica, MA, USA). All interferon treatments were performed 24 hours after transfection or nucleofection.

\subsection{Immunoblotting and Immunoprecipitation}

Infected or nucleofected cells were rinsed with versene (PBS + 1 mM EDTA) to dislodge cells. No trypsin was used in these studies. Harvested cells were pelleted by centrifugation at $1500 \mathrm{rpm}$ for 5 minutes and resuspended in lysis buffer $(25 \mathrm{mM}$ Tris, $150 \mathrm{mM} \mathrm{NaCl}, 1 \% \mathrm{NP} 40,10 \mathrm{mM} \mathrm{NaF}$, $0.1 \mathrm{mM} \mathrm{NaVO}_{4}, 1 \mathrm{mM}$ EDTA and 1\% (vol/vol) protease inhibitor cocktail (Sigma, St. Louis, MO, USA). The cells were then sonicated in a Misonix (Farmingdale, NY, USA) Cuphorn Sonicator for 100 seconds ( $5 \mathrm{~s}$ on, $5 \mathrm{~s}$ off) at setting 5 . Sonicated cells were centrifuged at 13,000 rpm for 5 minutes to pellet insoluble material. Protein was quantified using Bradford dye (Bio-Rad, Hercules, CA, USA) according to manufacturer recommendations. For immunoprecipitation, cell lysates were precleared by incubating with protein A agarose beads for 30 minutes at $4{ }^{\circ} \mathrm{C}$. The precleared lysates were incubated with primary antibody at $4{ }^{\circ} \mathrm{C}$ overnight followed by incubation with Protein A beads for 2 hours at $4{ }^{\circ} \mathrm{C}$. The isolated complexes were rinsed four times in lysis buffer and eluted by boiling in $2 \times$ Laemmli buffer with $5 \% \beta$-mercaptoethanol. Proteins were separated by SDS-PAGE, transferred to a nitocellulose membrane (Whatman (now GE Healthcare Bio-Sciences), Pittshburgh, PA, USA), reacted with primary antibodies overnight, exposed to HRP- conjugated secondary antibody, and detected by ECL (Amersham (now GE Healthcare Bio-Sciences), Pittshburgh, PA, USA). Antibodies to the following were used: JAK1 (Upstate (now EMD Millipore), Billerica, MA), JAK2 (Santacruz Biotechnology, Dallas, TX, USA), STAT1 (Santacruz), STAT2 (Cell Signaling, Danvers, MA, USA), phosphotyrosine STAT 1 (tyr 701, Cell Signaling), phosphoserine STAT1 (ser 727, Cell Signaling), FLAG (Sigma), Glyceraldehyde 3-phosphate dehydrogenase (GAPDH) (Chemicon), phosphoSTAT2 (Upstate), IE1 exon 2 antibody (Chemicon), IE1 exon 4 antibody p63-27 (gift of Dr. William Britt, University of Alabama at Birmingham, Birmingham, AL, USA).

\subsection{Flow Cytometry}

2C4 cells were rinsed with versene and nonezymatically dislodged from wells at 24 hours after IFN treatment. Cells were resuspended in flow buffer (PBS with 1\% FBS) and reacted with FITC-conjugated anti-CD2 antibody (Dako, Carpinteria, CA, USA) according to manufacturers recommendations. Cells were rinsed with flow buffer and CD2 fluorescence was measured in a Facscalibur Flow Cytometer (Becton Dickenson, Franklin Lakes, NJ, USA). The data were analyzed with the aid of CELLQuest 3.0 software (Becton Dickinson) [62]. 


\subsection{Real Time RT-PCR}

Total RNA from MRC-5 cells was isolated using Trizol (Invitrogen (now Life Technologies), Grand Island, NY, USA) according to the manufacturer's protocol. Next, $1 \mu \mathrm{g}$ of total RNA from each sample was used to perform first strand cDNA synthesis using Superscript reverse transcriptase (RT) (Invitrogen) as per manufacturer's recommendations. The cDNA was diluted 1:4 and $8 \mu \mathrm{L}$ was used per real-time polymerase chain reaction (PCR) of $25 \mu \mathrm{L}$ total volume. For reactions using SYBR green, the primers used for CIITA and GAPDH (endogenous control) are described elsewhere [63]. For reactions using $\mathrm{Taqman}^{\mathrm{TM}}$ the following primer and probe sets were used: CIITA-Hs00172106_mL, IRF1-Hs00233698_mL and 18SrRNA-4310893E (Applied Biosystems (now Life Technologies), Grand Island, NY, USA). All Real-Time reactions were set up in triplicate in 96 well format and analyzed in an ABI prism 7900HT Real-Time instrument. Fold change in CIITA or IRF1 expression in IFN treated samples compared to untreated samples was calculated using the $2^{-\Delta \Delta \mathrm{Ct}}$ method after normalizing to the endogenous control (either GAPDH or 18SrRNA) [64].

\subsection{Microscopy}

Nucleofected or transfected cells were plated on $12 \mathrm{~mm}$ coverslips in 24 well plates. Wherever indicated cells were exposed to $100 \mathrm{U} / \mathrm{mL}$ IFN for 30 minutes. Cells were rinsed with PBS and fixed in methanol for 1 hour on ice. The cells were then air dried and stored at $4{ }^{\circ} \mathrm{C}$. Cells were rehydrated in PBS for 5 minutes and incubated in blocking buffer (PBS, 10\% human serum, 1\% BSA) for $1 \mathrm{hr}$. This was followed by incubation with primary antibody in blocking buffer overnight at $4{ }^{\circ} \mathrm{C}$. Cells were rinsed three times for 20 minutes with PBS. This was followed by exposure to fluorescently labeled secondary antibody for 1 hour at $4{ }^{\circ} \mathrm{C}$. Excess secondary antibody was rinsed off with three rinses with PBS. The coverslips were allowed to air dry and then were mounted on glass slides using the Prolong antifade kit (Molecular Probes (now Life Technologies), Grand Island, NY, USA). Microscopy was performed using a Zeiss LSM 510 instrument. Each fluorescent dye was scanned separately to avoid any spectral overlap. Images were prepared using Zeiss LSM 5 Software, Version 3.5 [65]. The following primary antibodies were used: anti-IE1 exon 2 antibody (Chemicon), anti-IE1 exon 4 antibody p63-27 (gift of Dr. William Britt), anti-PML (Santacruz), anti-STAT1 (Santa Cruz).

\subsection{Electrophoretic Mobility Shift Assay (EMSA)}

MRC5 cells were plated at a density of $1 \times 10^{6}$ cells per $75 \mathrm{~cm}^{2}$ flask post nucleofection. Cells were exposed to $100 \mathrm{U} / \mathrm{mL}$ IFN $\gamma$ for 30 minutes or left untreated. Cells were rinsed with PBS, dislodged from the plates, and nuclear extracts were prepared using Nuclear extract kit (Activ Motif, Carlsbad, CA, USA). A 22mer Interferon Response Factor 1 (IRF1) GAS element probe (GATCGATTTCCCCGAAATCATG) was radiolabeled with $\gamma[\mathrm{P}]^{32}$ ATP using T4 polynucleotide kinase enzyme (Invitrogen) according to the manufacturer's recommendations. $4 \mu \mathrm{g}$ of nuclear extract was incubated with $1 \times$ binding buffer, $0.5 \mu \mathrm{g}$ poly dI:C, $0.1 \mu \mathrm{g}$ poly L-lysine and $2 \mathrm{ng}$ of labeled probe for 15 minutes at room temperature. For competition assays, a 200 -fold excess of 
unlabeled probe was added to the reaction mix. For supershift studies, $5 \mu \mathrm{g}$ of rabbit polyclonal anti STAT1 antibody (Santacruz) was added to the reaction and incubated for 15 minutes at room temperature prior to adding the labeled probe. The reactions were resolved on a $4.5 \%$ native polyacrylamide gel in $0.5 \times \mathrm{TBE}$ buffer and visualized by autoradiography.

\section{Conclusions}

The major findings of this report are as follows: (i) IE1, the immediate early transcriptional transactivator of HCMV, was identified as a candidate protein that disrupts IFN signaling by screening a HCMV cDNA library using the $2 \mathrm{C} 4$ reporter cell line and primary human fibroblasts; (ii) Using a series of truncation mutants we assigned this function of IE1 to the C-terminal 147 residues of the IE1 protein; (iii) IE1-mediated disruption of IFN $\gamma$ signaling appears to be independent of IE1-mediated ND10 disruption; (iv) IE1 does not inhibit the proximal events of type II IFN signaling; and (v) Although IE1 does not appear to directly interact with STAT1, expression of IE1 interferes with STAT1 binding to target DNA elements. Together our data indicate that a novel mechanism is employed by IE1 to interfere with the IFN $\gamma$ signal transduction pathway. In earlier studies, Miller et al. [28] have reported a reduction in STAT1 homodimer binding to GAS elements starting at 12 hours of infection and continuing up to 72 hours of infection. Furthermore, LeRoy et al. reported a defect in CIITA induction by IFN $\gamma$ that occurs downstream of STAT1 nuclear translocation in infected cells [27]. Our results suggest that these phenomena are mediated by IE1. Although only cells of the immune system including NK cells and $\mathrm{T}$ cells produce IFN $\gamma$, most or all nucleated cells in the body can express IFN $\gamma$ receptors and thus elaborate antiviral responses when stimulated by IFN $\gamma$. It is known that during a primary infection $\mathrm{CD}^{+} \mathrm{T}$ cells play an important role in controlling the spread of the virus. During this time it may be advantageous for the virus to be able to dampen signaling induced by IFN $\gamma$, which is produced abundantly by $\mathrm{CD} 8^{+} \mathrm{T}$ cells. This could not only counteract IFN $\gamma$-mediated antiviral responses, but also interfere with IFN $\gamma$-induced expression of MHC class I and MHC class II molecules, assembly of the immunoproteasome [66] and the presentation of viral antigens. Therefore, viral disruption of IFN $\gamma$-mediated signaling may promote virus replication by attenuating both intrinsic cellular defense mechanisms and adaptive immune responses.

\section{Acknowledgments}

This work was supported by AI51411-03 (JT) and GM066115 (CHC) from the National Institute of Health and from Support from the Ohio State University College of Medicine. We thank George Stark for the 2C4 cells, William Britt for providing us with antibodies, and Mark Kotur and Guojuan Zhang for technical assistance.

\section{Author Contributions}

Experiments were designed by JT and BR and conducted by BR. The manuscript was written by JT, BR and CHC. 


\section{Conflicts of Interest}

The authors declare no conflict of interest.

\section{References and Notes}

1. Vancikova, Z.; Dvorak, P. Cytomegalovirus infection in immunocompetent and immunocompromised individuals-A review. Curr. Drug Targets Immune Endocr. Metabol. Disord. 2001, 1, 179-187.

2. Britt, W.J.; Alford, C. A Cytomegalovirus. In Fields Virology; Fields, B., Knipe, D.M., Howley, P.M., Eds.; Lippincott-Raven: Philadelphia, PA, USA, 1996; Volume 2, pp. 2493-2523.

3. Borden, E.C.; Sen, G.C.; Uze, G.; Silverman, R.H.; Ransohoff, R.M.; Foster, G.R.; Stark, G.R. Interferons at age 50: Past, current and future impact on biomedicine. Nat. Rev. Drug Discov. 2007, 6, 975-990.

4. Samuel, C.E. Antiviral actions of interferons. Clin. Microbiol. Rev. 2001, 14, 778-809, table of contents.

5. Goodbourn, S.; Didcock, L.; Randall, R.E. Interferons: Cell signalling, immune modulation, antiviral response and virus countermeasures. J. Gen. Virol. 2000, 81, 2341-2364.

6. Le Bon, A.; Tough, D.F. Type I interferon as a stimulus for cross-priming. Cytokine Growth Factor Rev. 2008, 19, 33-40.

7. Stetson, D.B.; Medzhitov, R. Type I interferons in host defense. Immunity 2006, 25, 373-381.

8. Platanias, L.C. Mechanisms of type-I- and type-II-interferon-mediated signalling. Nat. Rev. Immunol. 2005, 5, 375-386.

9. Schindler, C.; Levy, D.E.; Decker, T. JAK-STAT signaling: From interferons to cytokines. J. Biol. Chem. 2007, 282, 20059-20063.

10. Stark, G.R.; Kerr, I.M.; Williams, B.R.; Silverman, R.H.; Schreiber, R.D. How cells respond to interferons. Annu. Rev. Biochem. 1998, 67, 227-264.

11. Der, S.D.; Zhou, A.; Williams, B.R.; Silverman, R.H. Identification of genes differentially regulated by interferon alpha, beta, or gamma using oligonucleotide arrays. Proc. Natl. Acad. Sci. USA 1998, 95, 15623-15628.

12. Taylor, R.T.; Bresnahan, W.A. Human cytomegalovirus immediate-early 2 protein IE86 blocks virus-induced chemokine expression. J. Virol. 2006, 80, 920-928.

13. Taylor, R.T.; Bresnahan, W.A. Human cytomegalovirus immediate-early 2 gene expression blocks virus-induced beta interferon production. J. Virol. 2005, 79, 3873-3877.

14. Taylor, R.T.; Bresnahan, W.A. Human cytomegalovirus IE86 attenuates virus- and tumor necrosis factor alpha-induced NFkappaB-dependent gene expression. J. Virol. 2006, 80, 10763-10771.

15. Amsler, L.; Verweij, M.C.; Defilippis, V.R. The Tiers and Dimensions of Evasion of the Type I Interferon Response by Human Cytomegalovirus. J. Mol. Biol. 2013, 425, 4857-4871.

16. DeFilippis, V.R. Induction and evasion of the type I interferon response by cytomegaloviruses. Adv. Exp. Med. Biol. 2007, 598, 309-324.

17. Marshall, E.E.; Geballe, A.P. Multifaceted evasion of the interferon response by cytomegalovirus. J. Interferon. Cytokine Res. 2009, 29, 609-619. 
18. Powers, C.; DeFilippis, V.; Malouli, D.; Fruh, K. Cytomegalovirus immune evasion. Curr. Top. Microbiol. Immunol. 2008, 325, 333-359.

19. Vandevenne, P.; Sadzot-Delvaux, C.; Piette, J. Innate immune response and viral interference strategies developed by human herpesviruses. Biochem. Pharmacol. 2010, 80, 1955-1972.

20. Loenen, W.A.; Bruggeman, C.A.; Wiertz, E.J. Immune evasion by human cytomegalovirus: lessons in immunology and cell biology. Semin. Immunol. 2001, 13, 41-49.

21. Paulus, C.; Krauss, S.; Nevels, M. A human cytomegalovirus antagonist of type I IFN-dependent signal transducer and activator of transcription signaling. Proc. Natl. Acad. Sci. USA 2006, 103, 3840-3845.

22. Krauss, S.; Kaps, J.; Czech, N.; Paulus, C.; Nevels, M. Physical requirements and functional consequences of complex formation between the cytomegalovirus IE1 protein and human STAT2. J. Virol. 2009, 83, 12854-12870.

23. Huh, Y.H.; Kim, Y.E.; Kim, E.T.; Park, J.J.; Song, M.J.; Zhu, H.; Hayward, G.S.; Ahn, J.H. Binding STAT2 by the acidic domain of human cytomegalovirus IE1 promotes viral growth and is negatively regulated by SUMO. J. Virol. 2008, 82, 10444-10454.

24. Le, V.T.; Trilling, M.; Wilborn, M.; Hengel, H.; Zimmermann, A. Human cytomegalovirus interferes with signal transducer and activator of transcription (STAT) 2 protein stability and tyrosine phosphorylation. J. Gen. Virol. 2008, 89, 2416-2426.

25. Popkin, D.L.; Watson, M.A.; Karaskov, E.; Dunn, G.P.; Bremner, R.; Virgin, H.W.T. Murine cytomegalovirus paralyzes macrophages by blocking IFN gamma-induced promoter assembly. Proc. Natl. Acad. Sci. USA 2003, 100, 14309-14314.

26. Zimmermann, A.; Trilling, M.; Wagner, M.; Wilborn, M.; Bubic, I.; Jonjic, S.; Koszinowski, U.; Hengel, H. A cytomegaloviral protein reveals a dual role for STAT2 in IFN-\{gamma signaling and antiviral responses. J. Exp. Med. 2005, 201, 1543-1553.

27. Le Roy, E.; Muhlethaler-Mottet, A.; Davrinche, C.; Mach, B.; Davignon, J.L. Escape of human cytomegalovirus from HLA-DR-restricted CD4(+) T-cell response is mediated by repression of gamma interferon-induced class II transactivator expression. J. Virol. 1999, 73, $6582-6589$.

28. Miller, D.M.; Zhang, Y.; Rahill, B.M.; Kazor, K.; Rofagha, S.; Eckel, J.J.; Sedmak, D.D. Human cytomegalovirus blocks interferon-gamma stimulated up-regulation of major histocompatibility complex class I expression and the class I antigen processing machinery. Transplantation 2000, 69, 687-690.

29. Miller, D.M.; Rahill, B.M.; Boss, J.M.; Lairmore, M.D.; Durbin, J.E.; Waldman, J.W.; Sedmak, D.D. Human cytomegalovirus inhibits major histocompatibility complex class II expression by disruption of the Jak/Stat pathway. J. Exp. Med. 1998, 187, 675-683.

30. Baron, M.; Davignon, J.L. Inhibition of IFN-gamma-induced STAT1 tyrosine phosphorylation by human CMV is mediated by SHP2. J. Immunol. 2008, 181, 5530-5536.

31. Knoblach, T.; Grandel, B.; Seiler, J.; Nevels, M.; Paulus, C. Human cytomegalovirus IE1 protein elicits a type II interferon-like host cell response that depends on activated STAT1 but not interferon-gamma. PLoS Pathog. 2011, 7, e1002016.

32. Stenberg, R.M. The human cytomegalovirus major immediate-early gene. Intervirology 1996, 39, 343-349. 
33. Zhang, G.; Raghavan, B.; Kotur, M.; Cheatham, J.; Sedmak, D.; Cook, C.; Waldman, J.; Trgovcich, J. Antisense transcription in the human cytomegalovirus transcriptome. J. Virol. 2007, 81, 11267-11281.

34. Watling, D.; Guschin, D.; Muller, M.; Silvennoinen, O.; Witthuhn, B.A.; Quelle, F.W.; Rogers, N.C.; Schindler, C.; Stark, G.R.; Ihle, J.N.; et al. Complementation by the protein tyrosine kinase JAK2 of a mutant cell line defective in the interferon-gamma signal transduction pathway. Nature 1993, 366, 166-170.

35. Korioth, F.; Maul, G.G.; Plachter, B.; Stamminger, T.; Frey, J. The nuclear domain 10 (ND10) is disrupted by the human cytomegalovirus gene product IE1. Exp. Cell Res. 1996, 229, 155-158.

36. Nevels, M.; Paulus, C.; Shenk, T. Human cytomegalovirus immediate-early 1 protein facilitates viral replication by antagonizing histone deacetylation. Proc. Natl. Acad. Sci. USA 2004, 101, 17234-17239.

37. McCormick, A.L. Control of apoptosis by human cytomegalovirus. Curr. Top. Microbiol. Immunol. 2008, 325, 281-295.

38. Reinhardt, J.; Smith, G.B.; Himmelheber, C.T.; Azizkhan-Clifford, J.; Mocarski, E.S. The carboxyl-terminal region of human cytomegalovirus IE1491aa contains an acidic domain that plays a regulatory role and a chromatin-tethering domain that is dispensable during viral replication. J. Virol. 2005, 79, 225-233.

39. Wilkinson, G.W.; Kelly, C.; Sinclair, J.H.; Rickards, C. Disruption of PML-associated nuclear bodies mediated by the human cytomegalovirus major immediate early gene product. J. Gen. Virol. 1998, 79, 1233-1245.

40. Ahn, J.H.; Hayward, G.S. The major immediate-early proteins IE1 and IE2 of human cytomegalovirus colocalize with and disrupt PML-associated nuclear bodies at very early times in infected permissive cells. J. Virol. 1997, 71, 4599-4613.

41. Ahn, J.H.; Brignole, E.J., 3rd.; Hayward, G.S. Disruption of PML subnuclear domains by the acidic IE1 protein of human cytomegalovirus is mediated through interaction with PML and may modulate a RING finger-dependent cryptic transactivator function of PML. Mol. Cell Biol. 1998, 18, 4899-4913.

42. Ahn, J.H.; Hayward, G.S. Disruption of PML-associated nuclear bodies by IE1 correlates with efficient early stages of viral gene expression and DNA replication in human cytomegalovirus infection. Virology 2000, 274, 39-55.

43. Xu, Y.; Ahn, J.H.; Cheng, M.; apRhys, C.M.; Chiou, C.J.; Zong, J.; Matunis, M.J.; Hayward, G.S. Proteasome-independent disruption of PML oncogenic domains (PODs), but not covalent modification by SUMO-1, is required for human cytomegalovirus immediate-early protein IE1 to inhibit PML-mediated transcriptional repression. J. Virol. 2001, 75, 10683-10695.

44. Chee, A.V.; Lopez, P.; Pandolfi, P.P.; Roizman, B. Promyelocytic leukemia protein mediates interferon-based anti-herpes simplex virus 1 effects. J. Virol. 2003, 77, 7101-7105.

45. Tavalai, N.; Papior, P.; Rechter, S.; Leis, M.; Stamminger, T. Evidence for a role of the cellular ND10 protein PML in mediating intrinsic immunity against human cytomegalovirus infections. J. Virol. 2006, 80, 8006-8018. 
46. Tavalai, N.; Papior, P.; Rechter, S.; Stamminger, T. Nuclear domain 10 components promyelocytic leukemia protein and hDaxx independently contribute to an intrinsic antiviral defense against human cytomegalovirus infection. J. Virol. 2008, 82, 126-137.

47. Lee, H.R.; Kim, D.J.; Lee, J.M.; Choi, C.Y.; Ahn, B.Y.; Hayward, G.S.; Ahn, J.H. Ability of the human cytomegalovirus IE1 protein to modulate sumoylation of PML correlates with its functional activities in transcriptional regulation and infectivity in cultured fibroblast cells. J. Virol. 2004, 78, 6527-6542.

48. Nusinzon, I.; Horvath, C.M. Interferon-stimulated transcription and innate antiviral immunity require deacetylase activity and histone deacetylase 1. Proc. Natl. Acad. Sci. USA 2003, 100, $14742-14747$.

49. Leonard, G.T.; Sen, G.C. Effects of adenovirus E1A protein on interferon-signaling. Virology 1996, 224, 25-33.

50. Leonard, G.T.; Sen, G.C. Restoration of interferon responses of adenovirus E1A-expressing HT1080 cell lines by overexpression of p48 protein. J. Virol. 1997, 71, 5095-5101.

51. Barnard, P.; McMillan, N.A. The human papillomavirus E7 oncoprotein abrogates signaling mediated by interferon-alpha. Virology 1999, 259, 305-313.

52. Wu, M.; Xu, Y.; Lin, S.; Zhang, X.; Xiang, L.; Yuan, Z. Hepatitis B virus polymerase inhibits the interferon-inducible MyD88 promoter by blocking nuclear translocation of Stat1. J. Gen. Virol. 2007, 88, 3260-3269.

53. Weihua, X.; Ramanujam, S.; Lindner, D.J.; Kudaravalli, R.D.; Freund, R.; Kalvakolanu, D.V. The polyoma virus $\mathrm{T}$ antigen interferes with interferon-inducible gene expression. Proc. Natl. Acad. Sci. USA 1998, 95, 1085-1090.

54. Morrison, T.E.; Mauser, A.; Wong, A.; Ting, J.P.; Kenney, S.C. Inhibition of IFN-gamma signaling by an Epstein-Barr virus immediate-early protein. Immunity 2001, 15, 787-799.

55. Chee, A.V.; Roizman, B. Herpes simplex virus 1 gene products occlude the interferon signaling pathway at multiple sites. J. Virol. 2004, 78, 4185-4196.

56. Yokota, S.; Yokosawa, N.; Kubota, T.; Suzutani, T.; Yoshida, I.; Miura, S.; Jimbow, K.; Fujii, $\mathrm{N}$. Herpes simplex virus type 1 suppresses the interferon signaling pathway by inhibiting phosphorylation of STATs and janus kinases during an early infection stage. Virology 2001, 286, 119-124.

57. Yokota, S.; Yokosawa, N.; Okabayashi, T.; Suzutani, T.; Miura, S.; Jimbow, K.; Fujii, N. Induction of suppressor of cytokine signaling-3 by herpes simplex virus type 1 contributes to inhibition of the interferon signaling pathway. J. Virol. 2004, 78, 6282-6286.

58. Johnson, K.E.; Song, B.; Knipe, D.M. Role for herpes simplex virus 1 ICP27 in the inhibition of type I interferon signaling. Virology 2008, 374, 487-494.

59. Liang, L.; Roizman, B. Expression of gamma interferon-dependent genes is blocked independently by virion host shutoff RNase and by US3 protein kinase. J. Virol. 2008, 82, $4688-4696$.

60. Wentworth, B.B.; French, L. Plaque assay of cytomegalovirus strains of human origin. Proc. Soc. Exp. Biol. Med. 1970, 135, 253-258. 
61. Raghavan, B.Z.G.; Kotur, M.; Cheatham, J.; Trgovcich, J. Superiority of Fugene HD transfection reagent in minimizing non-specific cellular interferon responses. Roche Biochemica 2006, 3, 20-23.

62. CELLQuest Software Reference Manual; Becton Dickinson: San Jose, CA, USA, 1998.

63. Beresford, G.W.; Boss, J.M. CIITA coordinates multiple histone acetylation modifications at the HLA-DRA promoter. Nat. Immunol. 2001, 2, 652-657.

64. Livak, K.J.; Schmittgen, T.D. Analysis of relative gene expression data using real-time quantitative PCR and the 2(-Delta Delta C(T)) Method. Methods 2001, 25, 402-408.

65. LSM 5 Software, Version 3.5; Carl Zeiss, Inc.: Jena, Germany, 2006.

66. Khan, S.; Zimmermann, A.; Basler, M.; Groettrup, M.; Hengel, H. A cytomegalovirus inhibitor of gamma interferon signaling controls immunoproteasome induction. J. Virol. 2004, $78,1831-1842$. 

Reprinted from Viruses. Cite as: E, X.; Kowalik, T.F. The DNA Damage Response Induced by Infection with Human Cytomegalovirus and Other Viruses. Viruses 2014, 6, 2155-2185.

Review

\title{
The DNA Damage Response Induced by Infection with Human Cytomegalovirus and Other Viruses
}

\author{
Xiaofei $\mathrm{E}^{1,2}$ and Timothy F. Kowalik ${ }^{1,2, *}$
}

1 Department of Microbiology and Physiological Systems, University of Massachusetts Medical School, 368 Plantation St, Worcester, MA 01605, USA; E-Mail: xiaofei.e@umassmed.edu

2 Program in Immunology and Microbiology, University of Massachusetts Medical School, 368 Plantation St, Worcester, MA 01605, USA

* Author to whom correspondence should be addressed; E-Mail: timothy.kowalik@umassmed.edu; Tel.: +1-508-856-6035; Fax: +1-508-856-5920.

Received: 31 January 2014; in revised form: 2 May 2014 / Accepted: 8 May 2014 /

Published: 23 May 2014

\begin{abstract}
Viruses use different strategies to overcome the host defense system. Recent studies have shown that viruses can induce DNA damage response (DDR). Many of these viruses use DDR signaling to benefit their replication, while other viruses block or inactivate DDR signaling. This review focuses on the effects of DDR and DNA repair on human cytomegalovirus (HCMV) replication. Here, we review the DDR induced by HCMV infection and its similarities and differences to DDR induced by other viruses. As DDR signaling pathways are critical for the replication of many viruses, blocking these pathways may represent novel therapeutic opportunities for the treatment of certain infectious diseases. Lastly, future perspectives in the field are discussed.
\end{abstract}

Keywords: HCMV; cell cycle; DNA damage response; DDR; ATM

\section{Introduction}

Human cytomegalovirus (HCMV) is a $\beta$-herpesvirus and is genetically the most complex viral pathogen of humans. Though HCMV infection rarely causes symptomatic disease in immunocompetent individuals, it can establish lifelong latency/persistence following primary infection and can be reactivated under some conditions. In general, it is the causative agent of a variety of disorders in immunocompromised and immunosuppressed individuals. HCMV-associated pneumonitis and retinitis are among the most prevalent complications following primary infection 
or reactivation of latent HCMV reservoirs. HCMV infections are serious threats to the health of HIV-positive individuals and transplant patients. Primary or reactivated HCMV infections can place pregnancies at risk as the virus can be transmitted to fetuses. In the United States, about 1\% of newborns are congenitally infected with HCMV. Most congenitally infected infants and children do not present with health problems, but $22 \%-38 \%$ of infected infants are born with symptoms, including microcephaly and mental retardation [1-3]. HCMV is also the leading cause of nonfamilial hearing loss. A strong association between HCMV and glioblastomas has been established [4], but its direct role in tumorigenesis is, at this juncture, unclear.

Many environmental factors and physiological processes can damage DNA. The presence of abnormal DNA structures can induce many types of DNA signaling pathways. Mammalian viruses use different strategies to antagonize host defense systems, including altering host DNA damage response (DDR) to facilitate their replication. This review concentrates on the effects of DDR on HCMV replication and draws comparison to other viruses that induce DDR.

\section{HCMV}

HCMV virions are structurally complex. It is an enveloped DNA virus that contains a dsDNA genome of 235 kilobase pairs, which is the largest genome of any human virus [5]. The genome encodes approximately 200 open reading frames [6,7] although a recent study suggests that many additional, small open reading frames are also transcribed and translated [8]. Recent study shows that HCMV genomes exists as complex mixtures of variants in patients, which may add another layer of genetic complexity to viral infections [9]. The viral genome is encased within a capsid and surrounded by a protein layer called the tegument $[10,11]$. This structure contains proteins that are delivered to cells upon infection and can act before the onset of viral immediate early (IE) gene expression to help initiate a productive infection. As examples of tegument proteins with such activity, pp71, promotes the degradation of hypophosphorylated forms of $\mathrm{pRB}, \mathrm{p} 107$, and $\mathrm{p} 130$, thus stimulating activities associated with cell cycle progression, whereas tegument delivered pUL69 arrests the cell cycle in a late $\mathrm{G}_{1} / \mathrm{S}$-like state [12].

Productive HCMV replication and gene expression has been subdivided into three kinetic classes: immediate early (IE), early (E), and late (L) $[13,14]$. IE genes are the first to be expressed and do not require de novo protein synthesis for their expression. The IE proteins have many functions which collectively prepare the host cell and viral genome for E gene expression and viral DNA replication. In general terms, E gene products are associated with promoting viral DNA replication. The replication of viral DNA is closely associated with expression of L genes. IE and E gene products also regulate late gene expression [15]. The 72-kDa IE1 protein and 86-kDa IE2 proteins are the first and, for IE1 at least, among the most abundantly expressed proteins during HCMV infection. Both proteins have long been recognized as transcriptional regulators, but they also interact with numerous cellular proteins including RB family members $[16,17]$. They are produced from differentially spliced transcripts under the control of strong promoter-enhancer element known as the major immediate early promoter (MIEP).

HCMV early genes require prior de novo synthesis of viral IE and cellular proteins for their transcription. The earliest of the early gene transcripts appear and accumulate to peak levels by 8 hours postinfection (e.g., UL112-113), while temporally later early transcripts can be detected 
just prior to the onset of viral DNA replication (e.g.,TRL4) and accumulate to peak levels when viral DNA replication is allowed to proceed [18]. Most of the viral early genes function in one of two ways. Some of the early genes are directly involve in viral DNA synthesis, cleavage and packaging of the viral genome, and contribute to assembly of the virus particles. Some other genes function to produce cellular and extracellular environments that are suitable for viral gene expression and replication, either by modulating factors involved in the regulation of cellular DNA synthesis or by altering the host's immune response to the virus. Some examples of the early genes are the UL112-113 nuclear phosphoproteins and HCMV viral DNA replication proteins, including DNA polymerase processivity factor (UL44) and single-stranded DNA binding protein (SSB) (UL57), which are localized in nuclear replication compartments [19].

Following viral DNA replication, delayed early and late viral genes are expressed which, in general, encode the structural components of the virion. UL55 (gB), UL75 (gH) and UL99 (pp28) as well as components of the capsid are products of late genes. While much study has been carried on the regulation of HCMV IE and E gene expression, little is known about the specific mechanisms of regulating late gene expression.

A key biological property of HCMV is to maintain a lifelong relationship with its host by way of latent or persistent infections. During latency, only a subset of viral genes is expressed. The mechanisms governing the establishment and maintenance of latency and reactivation of HCMV from latency are complex and now coming into focus. HCMV resides latently in hematopoietic cells of the bone marrow. Several in vitro systems have been developed as models for HCMV latency. Nelson and colleagues [20,21] have used allogenic stimulation to study HCMV reactivation in monocytes that harbor viral genomes. CD4+ and CD8 $+\mathrm{T}$ lymphocytes, cytokines, IFN- $\gamma$, and tumor necrosis factor- $\alpha$ can facilitate viral reactivation [21,22]. Mocarski and colleagues [23,24] have studied HCMV latency in granulocyte-macrophage progenitors expressing CD33 and dendritic cell markers. They have identified several HCMV transcripts expressed during latency following in vivo or in vitro infection. Goodrum and colleagues have investigated a primary CD34 $(+)$ hematopoietic progenitor cell system as an experimental model to study HCMV latency and reactivation [25]. Using an HCMV gene array, they examined HCMV gene expression in these cells. CD34+ cells exhibit distinct patterns of viral gene expression from that observed during productive or nonproductive infections. Furthermore, pUL138 was identified as an HCMV protein that promotes an infection with the hallmarks of latency [26,27]. Sinclair and colleagues analyzed the secretome of cells carrying latent HCMV and have identified changes in several secreted cellular proteins known to be involved in regulation of the immune response and chemoattraction [28]. Their results identified a strategy by which sites of latent HCMV can firstly recruit CD4+ T cells and then inhibit their antiviral effector functions. All told, much more needs to be learned in order to develop a clear understanding of HCMV latency.

In sum, HCMV infection strategies and viral replication are complex and reflective of the large genome, numbers of proteins (and miRNAs), and broad cell tropism. 


\section{Cell Cycle Checkpoints}

Cell cycle checkpoints are regulatory steps in pathways that govern the order and timing of cell cycle transitions to ensure completion of one cellular event prior to commencement of another. Checkpoints also offer the opportunity to repair damaged DNA. Most eukaryotic cells proceed through an ordered cell cycle, $\mathrm{G} 1 \rightarrow \mathrm{S} \rightarrow \mathrm{G} 2 \rightarrow \mathrm{M}$ phase, during which the chromosomes and other cell material double in number with each of the daughter cells receiving one copy of the doubled genetic material. The cell cycle is completed when each daughter cell has its own intact outer membrane. Regulation of the cell cycle is the key for the normal development of multi cellular organisms.

In the cell cycle, the G1 phase represents an organizing state prior to DNA replication and where decisions regarding cell cycle progress are made. Factors that influence progression through G1 include cell size, metabolic state, cell signaling, and perhaps a need to repair damaged DNA. Inconsistency among these states or excessive DNA damage can lead cells to exit the cell cycle and undergo senescence or apoptosis. S phase is where DNA synthesis takes place resulting in the duplication of the cellular genome. In the G2 phase, the cell prepares for the process of mitosis, and the associated cell division to form two daughter cells. This stage provides another opportunity for recognition and repair of damaged DNA. Thus, under normal conditions, the progression of DNA replication and mitosis is signaled by the intracellular checkpoints primarily at the G1 and G2, respectively.

\section{DNA Damage Response (DDR)}

Many external and internal effectors, such as ionizing radiation and reactive oxygen species, can directly damage DNA. The presence of abnormal DNA structures, including single-stranded break, double-strand breaks, modification of incorporated nucleotides or aberrant replication fork structures, as well as alterations in higher-order chromatin structure, can induce one or more DDR. For a more complete review of DNA damage and responses, please refer to these publications [29-33].

Activation of the DDR plays a key role in avoiding or reducing errors. Although cells use different signaling pathways (Figure 1) to deal with different environment stresses, there are common elements. Signaling networks in response to DNA damage consists of sensors, transducers, and effectors. Sensors detect damaged DNA and signal to transducers. Transducers amplify and transfer the signal to effectors. Effectors then execute the cellular response to initiate cell cycle checkpoint activation, DNA repair or apoptosis. Cellular responses to DNA damage are crucial for maintaining genome integrity. Defects in the DDR system are also associated with several inherited human disorders [34-36] and cancers [37,38]. The DDR system tends to be more error prone than genomic replication and any remaining damage or incorrectly repaired damage may play a role in the development of pathologies such as birth defects, cancer or aging.

DNA lesions trigger the activation of various kinases, which play important roles in DDR (Figure 1). The phosphatidylinositol-3-kinase-like family, including ataxia-telangiectasia mutated (ATM), ataxia-telangiectasia Rad3-related (ATR) and DNA-dependent protein kinase catalytic subunit (DNA-PKcs) play central roles in DNA damage checkpoints. ATM is defective in ataxia-telangiectasia mutated disease $(\mathrm{A}-\mathrm{T})$, which is characterized by cancer susceptibility, 
radiosensitivity and neurological defects [39]. ATM is the primary mediator of the response to DNA double strand breaks (DSBs); ATM has been traditionally considered a nuclear protein that functions in response to genotoxic damage though ATM also participates in the oxidative stress response and cytoplasmic signaling [40-44]. ATR activation is generally associated with single-stranded DNA breaks and stalled DNA replication forks. DNA-Pkcs is an important enzyme involved in the non-homologous-end-joining pathway of double strand break repair [45]. The phosphorylation of these proteins plays a crucial role in the activation of various effector proteins. A large-scale proteomic study on ATM and ATR substrates identified more than 700 proteins that are phosphorylated in response to DNA damage [46].

Figure 1. DNA damage-induced cell cycle checkpoint network. Schematic representation of ATM, ATR and DNA-PK signaling pathways. DNA-PK responds to DNA double-strand breaks and regulates nonhomologous end joining (NHEJ). The DNA ends are captured by the KU heterodimer. Ku regulatory proteins recruit DNA-PK to double-strand breaks; two DNA-PKcs molecules in concert tether DNA ends together and recruit the DNA Ligase IV-XRCC4 complex to rejoin broken DNA ends. ATM responds to DNA double-strand breaks; phosphorylates H2AX and NBS1, which localize to sites of DNA damage, where upon the MRN complexes form. ATM activation regulates cell-cycle checkpoints through the phosphorylation of $\mathrm{CHK} 2$ and p53. ATR is activated in response to single-stranded DNA (ssDNA). Activation of ATR requires TopBP1. ATR is recruited to RPA-coated ssDNA by its binding partner ATRIP. ATR regulates the cell-cycle through activation of CHK1. Both ATM and ATR are required to activate the p38MAPK/MK2 effector kinase complex downstream of TAO kinases in response to DNA damage. The three effector kinases, CHK1, CHK2, and MK2 are directly responsible for inhibitory phosphorylation on members of the Cdc25 family. Arrows indicate the flow of the respective DDR pathways.

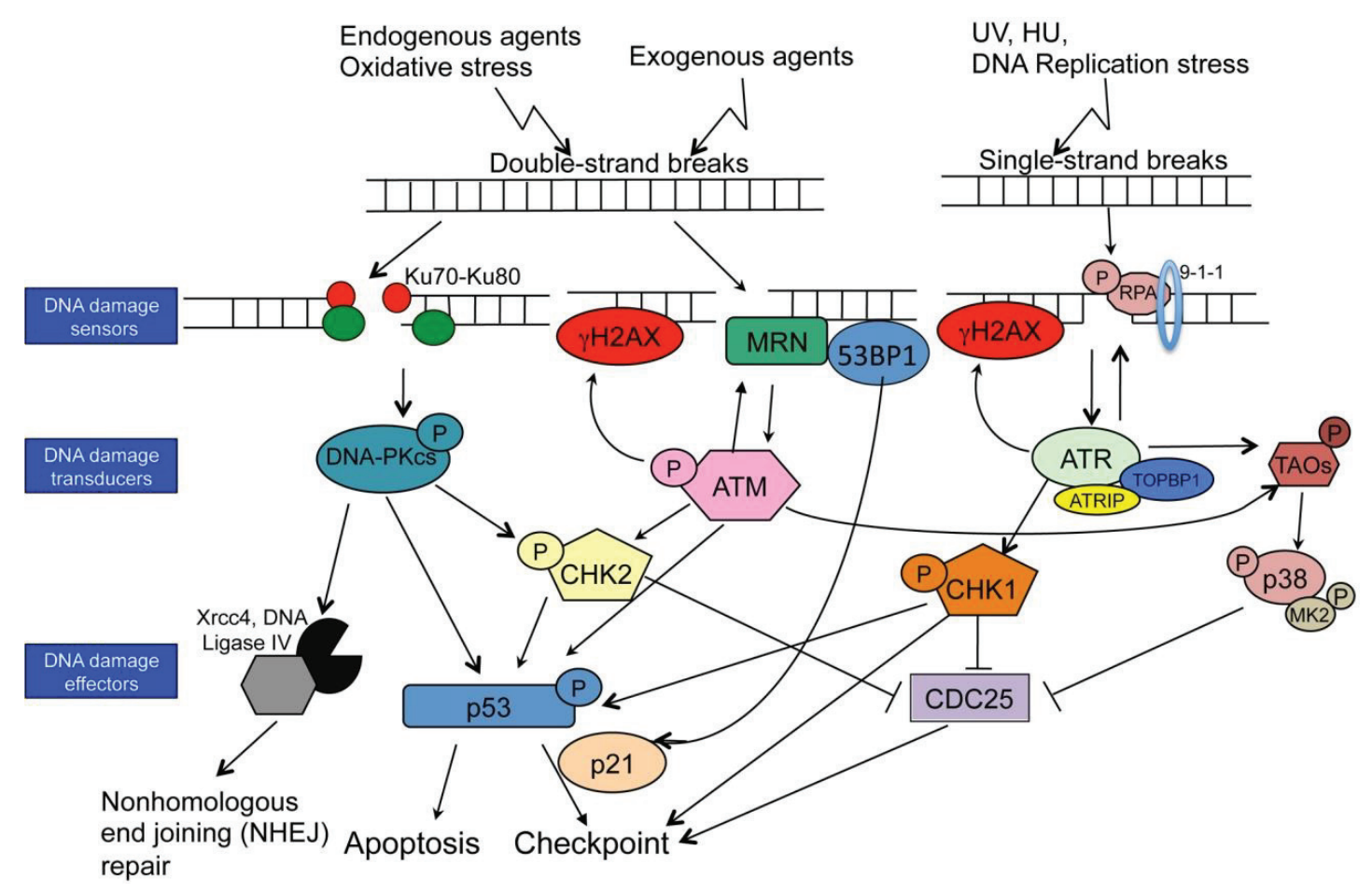




\section{The pRB-E2F Complex}

The RB protein family consists of three members - pRB, p107, and p130 — which maintain cells in a quiescent state as well as regulate the transition from $\mathrm{G} 0 / \mathrm{G} 1$ - to S-phase by modulating the activity of the E2F family of transcription factors. E2F transcription factors are the major downstream targets of the RB family of proteins, and are necessary for the expression of many genes that are required for cell cycle progression [47-50]. RB family members exert their growth regulatory functions partly by inhibiting the transcriptional activity of E2F [51-55]. Conversely, disruption of the $R B$ gene by deletion or mutation, or inactivation of $\mathrm{pRB}$ by phosphorylation or interaction with viral oncoproteins, cause the release of free or now depressed and transcriptionally active E2F1-3 resulting in cell cycle progression [56-58]. Sustained inactivation of pRB often results in apoptosis or deregulated proliferation.

The E2F family, including E2F1-8, can be divided into two subgroups based on their primary function, activator E2Fs: E2F1, 2 and E2F3a, and suppressor E2Fs: E2F3b, E2F4-8. E2Fs 1-3a are required for the transactivation of target genes involved in the $\mathrm{G}_{1} / \mathrm{S}$ transition. E2F3 encodes two proteins, E2F3a and E2F3b that differ in expression pattern and function. E2F3a is a transcriptional activator mainly expressed during S phase, while E2F3b acts as a transcriptional repressor, which is constantly expressed during cell cycle. In contrast, E2F4 and E2F5 possess predominantly repressive activity. E2F4 and E2F5 bind to p107 and p130 with high affinity, and recent studies have demonstrated that they also interact with pRB [59-63]. E2F6 and E2F7 are also considered to be transcriptional repressors [64-69]. E2F6-8 are distinct from the other E2Fs in that they do not bind to pocket proteins. E2F6 is known to interact and form complexes with the Polycomb Group $(\mathrm{PcG})$ proteins. It is not yet clear what the identity is of the interacting partners of these E2Fs. Deregulation of the pRB-E2F interaction results in hyperproliferation, lack of differentiation, genomic instability and can lead to cancer.

\section{The Link between Cell Cycle and DDR}

\subsection{CHK1 and CHK2 Kinases Control the Cell Cycle in Response to DNA Damage}

DNA damage poses a continuous threat to genomic integrity in mammalian cells. In order to prevent the propagation of damaged DNA through the cell cycle, cells have evolved DDR that coordinate cell cycle progression and checkpoints with the repair of DNA lesions. Mammalian cells initiate cell cycle arrest at different phases of the cell cycle in response to various forms of genotoxic stress to allow time for DNA repair. Cell cycle arrest and apoptosis are two of the downstream consequences of DDR [70]. CHK1 and CHK2 are checkpoint kinases that are activated by ATM or ATR and phosphorylate cell-cycle components to cause the arrest of the cell cycle [71,72] (Figure 1). Although CHK1 and CHK2 have overlapping roles, CHK1 kinase is restricted to $\mathrm{S}$ and $\mathrm{G} 2$ where its activity is amplified in the presence of different types of DNA damage [73]. CHK2 is expressed throughout the cell cycle and is also activated in the presence of DNA damage [74]. Usually, CHK1 or CHK2 phosphorylated p53 and CDC25 propagate signals to arrest cells or to undergo apoptosis depending on cell type and extent of DNA damage. However, DNA lesions sometimes do not induce cell cycle checkpoint responses, such as DNA damage 
during G2 phase of the green alga, Scenedesmus quadricauda [75]; or the level of DNA damage is low enough that the cell can deal with the lesions in the absence of a checkpoint response.

\subsection{A Novel Cell Cycle Checkpoint Kinase Pathway, MK2, that also Induced Cell Cycle Arrest}

Over the last decade, a number of publications point to a crucial role for the p38MAPK/MAPKAP-K2 (MK2) complex as an integral part of the DDR network [76,77]. There are four p38MAPK isoforms denoted $\alpha, \beta, \gamma$ and $\delta$ [78]. p38 $\alpha$ and p38 $\beta$ have been shown to be activated by DNA damage-specific agents, such as cisplatin, doxorubicin, and temozolomiode [76,77,79-81]. p38 $\alpha$ forms a nuclear complex with its downstream substrate MK2 and that upon activation of p38MAPK in this complex, p38MAPK phosphorylates and activates MK2. p38MAPK/MK2 complex is a third checkpoint effect or module that operates parallel to CHK1 and is activated downstream of ATM and ATR [76,77,82] (Figure 1). More detailed information on this DNA damage checkpoint signaling pathways can be found elsewhere [82-84].

\section{Many Viruses can Induce DNA Damage Responses and Modulate Cell Cycle Progression}

DDR can be activated not only by external sources of DNA damage, but also by intracellular conditions, such as oncogene overexpression, loss of tumor suppressors, and viral infections. Recent studies demonstrate that infections by DNA viruses or viruses with a DNA genome stage during infection induce host DDR (Table 1) [85-89]. Somewhat unexpectedly, some viruses with RNA genomes can also induce DDR. The mechanisms responsible for DDR induction by RNA viruses are less clear and possibly indirect.

The manipulation of the cell cycle by viruses is closely related to activation of the DDR and is usually associated with DNA double-strand break signaling pathways. As examples, oncoproteins of DNA tumor viruses, such as adenovirus E1A, simian virus $40 \mathrm{~T}$ antigen, and papillomavirus E7, each interact with the RB family of tumor suppressors, leading to E2F-mediated cell cycle stimulation, apoptosis induction, and cellular transformation. These proteins utilize a conserved LXCXE motif, which is also found in cellular proteins, to target the RB family. The subsequent induction of cell cycle checkpoints and activation of ATM/ATR/DNAPKcs pathways have been reported to accompany infection by a number of different viruses. Given that DDR usually results in cell cycle checkpoints and apoptosis, infection-associated DDR were initially considered to be antiviral, as in the case of adenovirus serotype 5 (Ad5) [90]. However, for many other viruses that induce a host DDR, the response appears to modulate the cell cycle at a precise point that favors virus replication [91-93]. Thus, manipulation of the cell cycle and the associated DDR may be a commonly employed strategy of viruses to create a favorable cellular environment for replication.

Several mammalian viruses evolved mechanisms to manipulate DDR pathways for their own benefit by exploiting or actively inhibiting different parts of the pathways [91]. For example, simian virus type 40 (SV40), herpes simplex virus 1(HSV-1), HCMV, and Epstein-Barr virus (EBV) all activate ATM and downstream signaling during infection, which is accompanied by recruitment of ATM and other repair proteins to sites of viral DNA replication [94-97] (Table 1). 
Table 1. A list of viruses that both induce and require host DNA damage responses (DDR) for productive infections.

\begin{tabular}{|c|c|c|c|c|c|}
\hline $\begin{array}{l}\text { Virus that Induce DNA } \\
\text { damage response (DDR) }\end{array}$ & Abbreviation & Virus type & DDR factors activated & $\begin{array}{l}\text { DDR factors required } \\
\text { for virus replication }\end{array}$ & References \\
\hline Human cytomegalovirus & HCMV & $\begin{array}{l}\text { dsDNA, } \\
\beta \text {-herpesvirus }\end{array}$ & $\begin{array}{l}\text { ATM, CHK2, p53, H2AX } \\
\text { NBS1, CHK1 }\end{array}$ & ATM, p53, H2AX & {$[94,98]$} \\
\hline $\begin{array}{c}\text { Herpes simplex virus type } \\
1 \\
\end{array}$ & HSV-1 & $\begin{array}{l}\text { dsDNA, } \\
\alpha \text {-herpesvirus }\end{array}$ & ATM, CHK2, 53BP1, NBS1 & ATM, Mre11 & [95] \\
\hline Epstein-Barr virus & EBV & $\begin{array}{l}\text { dsDNA, } \\
\gamma \text {-herpesvirus }\end{array}$ & $\begin{array}{l}\text { ATM, CHK2, Nbs1, H2AX, } \\
\text { p53, CHK1 }\end{array}$ & $\mathrm{XPC}$ & {$[96,99]$} \\
\hline $\begin{array}{c}\text { Murine gammaherpesvirus } \\
68 \\
\end{array}$ & $\gamma \mathrm{HV} 68$ & $\begin{array}{l}\text { dsDNA, } \\
\gamma \text {-herpesvirus }\end{array}$ & ATM, H2AX, p53, CHK1 & ATM, H2AX & {$[100,101]$} \\
\hline Simian virus type 40 & SV40 & $\begin{array}{l}\text { dsDNA, } \\
\text { polyomavirus }\end{array}$ & ATM, CHK1, CHK2, p53 & ATM, Rad51, FancD2 & {$[97,102,103]$} \\
\hline Human papillomavirus & HPV & $\begin{array}{l}\text { dsDNA, } \\
\text { papillomavirus }\end{array}$ & $\begin{array}{l}\text { ATM, CHK2, H2AX, } \\
\text { NBS1, CHK1, BRCA1 }\end{array}$ & ATM, CHK2 & [104-108] \\
\hline Human parvovirus B19 & B19V & $\begin{array}{l}\text { ssDNA, } \\
\text { parvovirus }\end{array}$ & $\begin{array}{l}\text { ATM, CHK2, ATR, } \\
\text { DNA-PKcs, CHK1, } \\
\text { Ku70/Ku80, H2AX, RPA-32 }\end{array}$ & $\begin{array}{c}\text { ATR, CHK1, } \\
\text { DNA-PKcs, Ku70/ku80 }\end{array}$ & [109-111] \\
\hline Adeno-associated virus & AAV & $\begin{array}{l}\text { ssDNA, } \\
\text { parvovirus }\end{array}$ & $\begin{array}{l}\text { ATM, CHK2, DNA-PKcs, } \\
\text { SMC1, } \\
\text { H2AX, CHK1, RPA32 }\end{array}$ & DNA-Pkcs & {$[112,113]$} \\
\hline $\begin{array}{c}\text { Human T-cell } \\
\text { lymphotrophic virus type } \\
1\end{array}$ & HTLV1 & $\begin{array}{l}\mathrm{ssRNA} / \mathrm{dsDN} \\
\text { A, retrovirus }\end{array}$ & $\begin{array}{l}\text { ATM, CHK2, H2AX, } \\
\text { NBS1, DNA-PKcs }\end{array}$ & N/A & [114-119] \\
\hline $\begin{array}{l}\text { Human immunodeficiency } \\
\text { virus type } 1 \\
\end{array}$ & HIV-1 & $\begin{array}{l}\text { ssRNA/dsDN } \\
\text { A, lentivirus } \\
\end{array}$ & $\begin{array}{l}\text { ATM, H2AX, p53, NBS1, } \\
\text { ATR, CHK1, P38MAPK }\end{array}$ & ATM & [120-122] \\
\hline Rift Valley fever virus & RVFV & $\begin{array}{l}\text { ssRNA, } \\
\text { arbovirus }\end{array}$ & ATM, CHK2, H2AX, p53 & ATM, CHK2, p53 & {$[123,124]$} \\
\hline Hepatitis $\mathrm{C}$ virus & $\mathrm{HCV}$ & $\begin{array}{l}\text { ssRNA, } \\
\text { flavivirus }\end{array}$ & ATM, CHK2, H2AX, CHK1 & ATM, CHK2 & {$[125,126]$} \\
\hline
\end{tabular}

HSV-1, an alphaherpesvirus, has a complex relationship with the DDR, in that it activates many components of the ATM-dependent signaling pathway, such as phosphorylation of CHK2, 53BP1, and NBS1, while inhibiting the DNA-PKcs and ATR kinases [95,127-129]. MRE11-RAD40-NBS1 (MRN) complex formation (Figure 1) and activated ATM promote HSV-1 replication [95]. HSV-1 codes for an immediate early transcription factor, ICP0 that promotes cell cycle arrest by inducing the tumor suppressor p53 and its downstream target proteins (p21, GADD45, and MDM2).

Epstein-Barr virus (EBV) is a gammaherpesvirus that induces the phosphorylation of ATM, NBS1, H2AX, CHK2, and p53 during lytic infection [99]. Phosphorylated ATM, NBS1 and Mre1 1 proteins are recruited to EBV replication compartments. XPC, a sensor of DDR that functions in nucleotide excision repair, is required for EBV replication [96].

The murine gammaherpesvirus 68 ( $\gamma \mathrm{HV} 68$ ) latency-associated, anti-interferon M2 protein induces ATM activation and histone acetylation [100] presumably to limit the induction of a virus-induced DNA damage signaling cascade. However, $\gamma \mathrm{HV} 68$ protein kinase orf36 activates the DDR and 
facilitates lytic replication in primary macrophages. H2AX, an orf36 substrate, can enhance MHV68 replication [101].

The large T antigen encoded by the SV40 polyomavirus, deregulates multiple DNA damage signaling and repair pathways [97]. ATM mediated phosphorylation of SV40 large T antigen is detected at the onset of viral replication, and is required for optimal viral DNA synthesis [102]. Inhibition of ATM activity decreases SV40 DNA accumulation [102,103], and delays the assembly of viral replication compartments and recruitment of cellular DNA repair proteins to these sites.

Human papillomavirus (HPV) proteins induce a DDR characterized by the activation of the ATM kinase substrates CHK2, NBS1, and BRCA1 [104-108]. ATM kinase activity is required for HPV genome amplification in differentiating cells but not for episome maintenance in undifferentiated cells [106]. HPV does not induce degradation of MRN components but instead keeps them at high levels throughout differentiation [106].

As a single stranded DNA virus, human parvovirus B19 (B19V) induces a broad range of DDR by triggering activation of all PI-3-like kinases associated with DNA repair pathways during infection [109-111]. Phosphorylated ATM, ATR, and DNA-PKcs, and their downstream targets (CHK2, CHK1, and Ku70/Ku80 complex, respectively) are all localized within B19V replication compartments. However, B19 virus apparently only uses ATR-CHK1 signaling to promote its replication [111].

Relatedly, adeno-associated viruses (AAV), another member of Parvoviridae, do not have an absolute requirement for ATM kinase activity. DNA-PK is the primary mediator of damage signaling in response to AAV replication [113]. Immunofluorescence revealed that some activated damage proteins are found in a pan-nuclear pattern (phosphorylated ATM, SMC1, and H2AX), while others such as DNA-PK components (DNA-PKcs, Ku70, and Ku86) and RPA32 accumulate at AAV replication compartments. DNA-PK enhances recombinant AAV (rAAV) replication through the interaction of $\mathrm{Ku}$ proteins and AAV-ITRs [112].

ATM protein can also enhance the replication of retroviruses and lentiviruses [120,121], such as human immunodeficiency virus type 1 (HIV-1) where ATM can enhance HIV replication by stimulating Rev function [120,122]. Similar observations have been made during human T-cell lymphotropic virus (HTLV1) infections [114-119].

In most cases, ATM signaling has been demonstrated to be beneficial for DNA virus replication. It has also been suggested that RNA viruses activate DDR functions that can be beneficial. A study shows the induction of DNA damage signaling upon infection with Rift Valley Fever Virus (RVFV), an RNA virus, that results in cell cycle arrest and increased viral replication [124]. ATM and a number of its substrates, CHK2, H2AX, and p53, were phosphorylated following RVFV infection. The use of ATM and CHK2 inhibitors or p53-null cells demonstrates that they are required for RVFV replication [123,124]. Another recently identified example of an RNA virus that activates DDR is HCV, which replicates better in the presence of ATM and CHK2, and expresses viral proteins that bind ATM and sensitize cells to DNA damage $[125,126]$. Other studies observe that HCV NS2 protein inhibits DNA damage signaling by sequestering p53 in the cytoplasm while the viral core protein interacts with NBS1 protein, leading to inhibition of MRN complex formation thereby blocking ATM activation and signaling in response to DSBs [130,131]. 
Clearly the association between RNA virus infection and DDR is complex, with additional study needed to better understand the molecular underpinnings and biology of this relationship.

In contrast, some viruses do not use DDR signaling for replication [91]. Specially, serotype-specific inactivation of the cellular DDR during adenovirus infection has been found [132]. For example, human adenovirus serotype $5(\mathrm{Ad} 5)$ encoded E4 proteins inactivate the MRN complex early in infection, either via E1b55K/E4orf6-mediated degradation of MRN [90,133] or E4orf3-mediated mislocalization of MRN into nuclear tracks [134,135] and cytoplasmic aggresomes [136,137]. In addition to preventing ATM and ATR-mediated damage signaling, inactivation of MRN promotes Ad5 DNA replication [134,138,139]. Likewise, Kaposi's sarcoma-associated herpesvirus (KSHV) viral interferon regulatory factor 1 ( $v I R F 1)$ compromises an ATM/p53-mediated DNA damage checkpoint by targeting both upstream ATM kinase and downstream p53 tumor suppressor [140]. Whether this activity is essential for productive or latent infection awaits further study.

In summary, though there are some viruses that do not use DDR for their replication (i.e., Ad5), there are numerous viruses (Table 1) that induce DDR pathways and require ATM or other PI-3-like kinases for productive infection. For more detailed information of DDR and viruses in general, see the following reviews [85-89].

\section{HCMV Modulates the Cell Cycle and Checkpoints}

HCMV infection can alter the cell cycle status (Figure 2) and induce a DDR (Figure 3). Most cells infected with HCMV are driven into a unique G1/S-like state that provides enzymes and metabolites necessary for viral DNA replication [141-143]. At the same time, the virus directly suppresses competitive cellular DNA synthesis [144-147]. This unusual G1/S-like state is dependent upon several viral proteins including at least the tegument proteins pUL69 [148], pp71 [149], and pUL97 [150], pUL35 [151], the immediate early proteins, IE1 [152] and IE2 protein [153], and polymerase accessory protein pUL44 [154], while several other viral proteins prevent apoptosis signaling that would normally result from deregulating the cell cycle (Figure 2).

The p53 checkpoint protein functions primarily as a transcriptional activator with target genes including the CDK inhibitor, p21, as well as Mdm2, a negative regulator of $\mathrm{p} 53$. The levels of p53 and its phosphorylation are increased during HCMV infection [94,98,141]. Phosphorylation-mediated activation of p53 induces an arrest of the cell cycle, which is mainly due to the induction of $\mathrm{p} 21$, sustained arrest leading to apoptosis. During HCMV infection, phosphorylation of p53 following DDR signaling prevents its interaction with MDM2, thereby stabilizing p53 by preventing its ubiquitin-mediated degradation [155], and contributing to the G1/S-like cell cycle arrest through the induction of p21 expression [152]. HCMV infection also activates several factors that normally induce cell cycle progression, including E2Fs [156]. In summary, the interaction between HCMV and cell cycle regulatory mechanisms is complex, with some viral factors eliciting cell cycle arrest and others promoting cell cycle progression. Thus, HCMV infection-induces a cell cycle arrest that leaves cells in a unique state that favors its replication, a theme that appears to be common to herpesviruses during productive infections [92]. 
Figure 2. The relationship between cell cycle and the DDR induced by HCMV. Lines depicted in green represent activities that promote cell cycle progression or prevent the cells from undergoing apoptosis. Lines depicted in red represent activities that can negatively affect cell cycle progress within the cell.

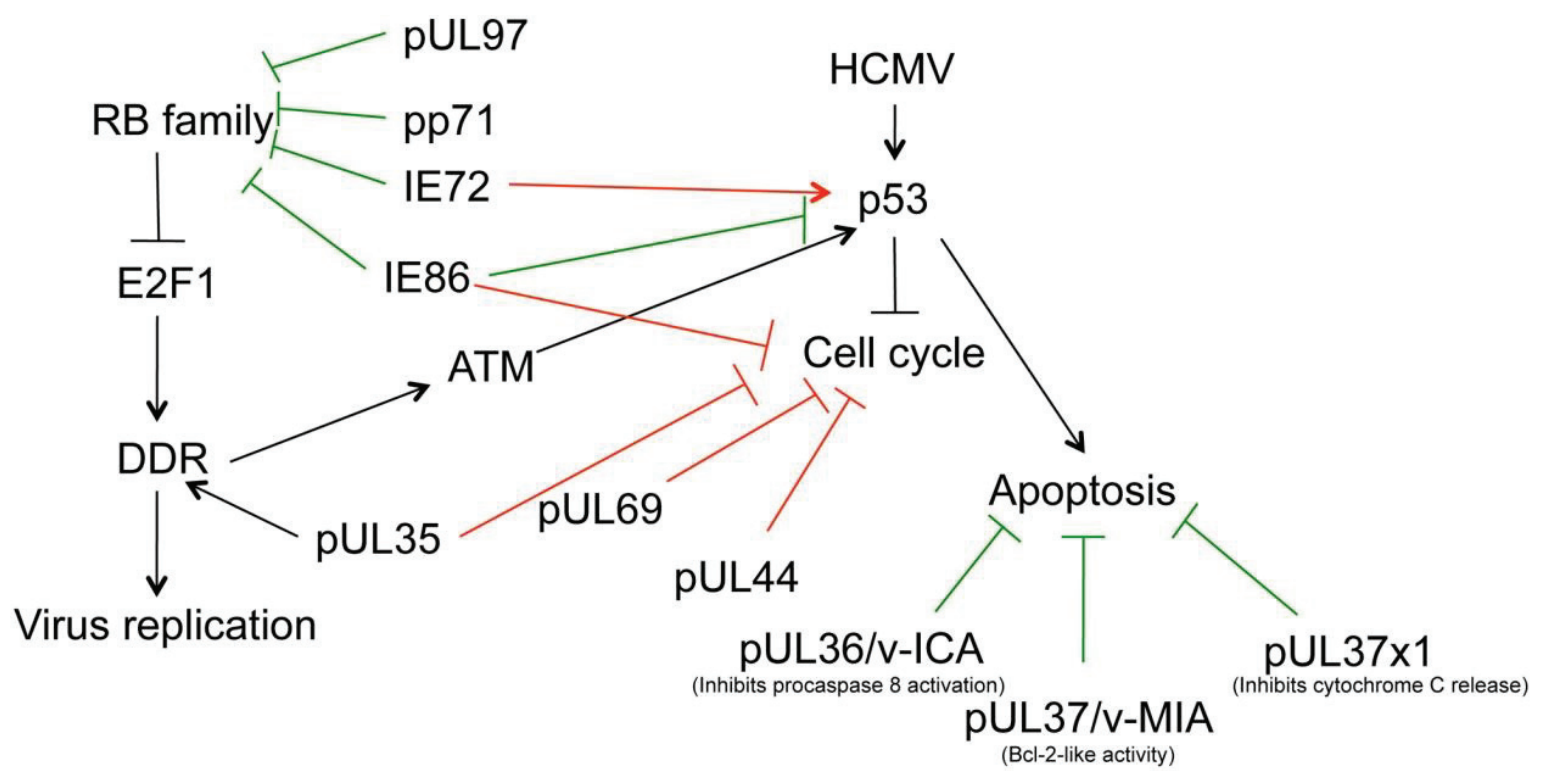

Figure 3. Model of the host DNA damage response induced by HCMV infection.

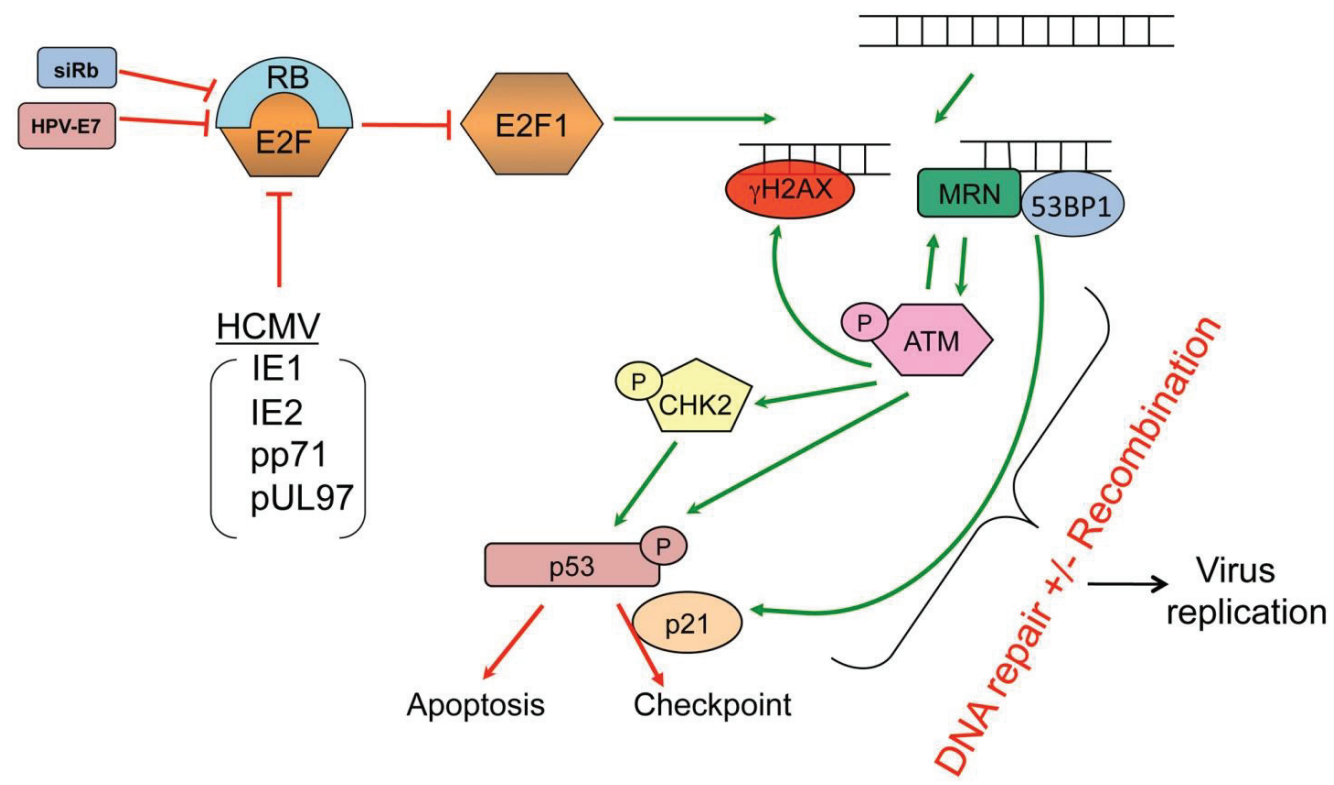

\section{HCMV, Deregulation of the Cell Cycle, and DNA Damage Signaling}

HCMV infection induces a DDR that includes activation of ATM, H2AX, NBS1, CHK2, CHK1, and p53 [94,98,157]. How does HCMV induce DDR signaling? HCMV encode four proteins, IE1, IE2, pp71 and pUL97 that can either bind to or phosphorylate RB family members (Figure 2) $[17,149,150,158-163]$. Binding by these viral proteins to or phosphorylation of $\mathrm{pRB}$ causes the release of E2F proteins. Of these, the now de-repressed E2F1 can induce DSBs [164]. The resultant ATM activation and its downstream phosphorylation targets, including $\mathrm{H} 2 \mathrm{AX}$ and 
p53, contribute to HCMV replication [94]. These DDR phenotypes are similar to productive infection with another herpesvirus, HSV-1 $[85,89,95,128]$. In addition, HCMV pUL35 can active DDR, causing $\gamma \mathrm{H} 2 \mathrm{AX}$ and 53BP1 foci formation and induce a cell cycle arrest [151] which likely supports viral replication [151].

HCMV, in particular, encodes several proteins that both modulate cell cycle controls and the host DDR to promote viral replication. The following subsections focus on how some of these viral proteins, in particular, IE1, IE2, pp71, pUL97, pUL69, mediate these activities.

\subsection{IE1 Can Inactivate 107 and 130}

UL123, encoding IE1 (sometimes referred to IE1-72 or IE72), is the first transcribed HCMV gene. As a predominantly nuclear protein, IE1 is a promiscuous transactivator that also interacts and modulates the function of p107, a member of the RB protein family [17,165]. IE1 has been suggested to exhibit kinase activity [156,163]. In vitro kinase assays suggest that both p107 and p130 can be phosphorylated by IE1 and that phosphorylation of these two RB proteins is sufficient to disrupt their interaction with E2F4 [163]. These findings imply that IE1 specifically targets at least two RB proteins for inactivation, which causes the derepression of E2F-responsive promoters. Even with RB family member inactivation and derepression of E2Fs, IE1 expression is unable to induce S-phase entry in primary fibroblasts due to a p53-dependent arrest [152]. This ability of p53 to block the IE1-mediated induction of S-phase is dependent on the p21 CDK inhibitor [157].

IE1 also disrupts PML bodies [166-168] (also known as promyelocytic oncogenic domains (PODs) or nuclear domains-10 (ND-10)). PML is involved in cellular growth regulation, transcription, DNA replication and repair, and posttranscriptional regulation of gene expression [169]. PML body integrity is also a component of the host intrinsic antiviral defense and the displacement of PML protein from PML bodies by IE1 contributes for sustained viral gene expression [170,171].

\subsection{IE2 Binds $p R B$ and $p 53$}

Studies have shown that the protein encoded by the largest transcript of UL122, IE2 (also known as IE2-86 or IE86) specifically interacts with pRB [16,160,172,173]. Expression of IE2 is sufficient to alleviate pRB repression of E2F-responsive promoters $[16,47,160]$. IE2 can also interact with p53 [174-176]. However, expression of IE2 in a human cell line blocked cell cycle progression in G1-phase similar to the phenotype observed when permissive cells are infected with HCMV [153]. Moreover, a study using DNA microarrays to analyze the effects of IE2 protein on cellular gene expression reveals that the $86 \mathrm{kDa}$ form of IE2 induces the expression of numerous factors associated with cell cycle regulation and bioenzymatic machinery necessary for DNA replication. For example, IE2 expression results in an increase in the mRNA levels of B-myb, cyclin E, cdk-2, E2F1, ribonucleotide reductase subunit-1 and -2, thymidine synthetase, MCM3, and MCM7, among other factors associated with S phase [177]. This study shows that most of the genes induced by IE2 are E2F targets. What is striking is that even though IE2 inactivates pRB, derepresses E2F activity, and can inhibit cell cycle arrest functions of $\mathrm{p} 53$, this viral protein does not induce $\mathrm{S}$ phase as measured by cellular DNA replication. This observation is in stark contrast to pRB and p53 targeting proteins encoded by other viruses such as SV40 lg T or the combination of 
adenovirus E1A and E1B-55K proteins, which are potent inducers of cell cycle progression and $\mathrm{S}$ phase. A clearer understanding is needed as to why IE2 does not induce $\mathrm{S}$ phase.

\section{3. pp 71 Binds to $p R B, p 107$ and $p 130$}

The pp71 phosphoprotein, expressed from the UL82 ORF, is a tegument protein that localizes to the nucleus immediately after virion entry during productive infections $[178,179]$. Functionally, pp71 is a transcription factor that is packaged within the viral tegument and is essential for the adequate accumulation of IE1 and IE2 by transactivating the major IE promoter and accelerating the infection cycle of HCMV [180]. Studies examining the relationship between pp71 and the cell cycle reveal that pp71 contains a sequence (LACSD) that is similar to the RB-binding motif (LxCxE) present in viral oncoproteins encoded by the small DNA tumor viruses. This motif is required for the induction of DNA synthesis in quiescent cells and for degradation of the RB family by viral oncoproteins $[149,162]$. pp71 binds to all three RB family members and promotes the G1/S cell cycle state during infection [149].

\section{4. pUL97 Phosphorylates RB Protein}

pUL97, another tegument protein, is a multifunctional viral protein kinase which is required at multiple steps during viral replication. Deletion of the $\mathrm{p} U L 97$ region from the viral genome or pharmacological inhibition of pUL97 kinase activity drastically reduces viral replication. A number of cellular and viral interacting proteins and substrates of pUL97 have been described, including viral pUL69 [181], pUL44 [182], autophosphorylated pUL97 [183,184], and, given its cdk-like activity, cellular RB family proteins are also phosphorylated by pUL97 [150]. In addition, pUL97 is able to phosphorylate nuclear lamins, which contributes to the HCMV-induced reorganization of the nuclear lamina [185].

\section{5. pUL69 Modulates CDK Function}

As mentioned earlier, IE1, IE2 and pUL97 can interact or phosphorylate RB family members to inactivate protein function, which can, under certain circumstances, result in stimulation of cell cycle progression. However, during HCMV infection, cell cycle progression is blocked at multiple points, including the $\mathrm{G}_{1}$-to-S-phase transition. pUL69, a phosphorylated virion tegument protein [186], is reported to be a factor responsible for this cell cycle block [148]. Host CDKs and pUL97 phosphorylate pUL69 and modulate its nuclear localization and activity [181]. Although the mechanism by which pUL69 induces an accumulation of cells in G1 is not clear, these findings indicate that HCMV tegument proteins (pUL69, pp71, and pUL97) can have an immediate effect on the cell cycle. It seems that HCMV has developed a strategy to inhibit cellular DNA replication and, at the same time, alter the host environment in a manner that ensures the replication of viral DNA.

\subsection{E2F1-mediated DNA Damage Response}

In the normal condition, RB family members bind to E2F family members to limit cell cycle progression. During HCMV infection, at least four viral proteins (IE1, IE2, pp71 and pUL97) 
inactivate $\mathrm{RB}$ family proteins resulting in the release of E2F proteins. These derepressed E2F proteins then alter the expression of $\mathrm{S}$ phase genes that contribute activities and substrates that support viral DNA replication. In addition, deregulated E2F1 induces DSBs and stimulates a robust host DDR [94]. The mechanism responsible for this phenomenon is unclear but it is specific to E2F1 as other RB-targeted E2Fs, namely E2F2 or E2F3, do not induce double strand DNA breaks (DSBs) or DDR [164]. This relationship between E2F1 and DSBs appears relevant to HCMV, as it is the only activator E2F that contributes significantly to viral replication [94]. Moreover, ATM, the signal transducing kinase of many DDR, functions downstream of E2F1 deregulation and is also required for HCMV replication [94].

\section{Which DDR Factors Contribute to HCMV Replication?}

ATM has been implicated as a target of several DNA viruses (Table 1), which activate or inhibit the ATM signaling pathway [85]. Depletion or inhibition of ATM by RNA interference or by pharmacological compounds, respectively, demonstrate that ATM is a key kinase in DDR and also the most common DDR factor contributing to virus replication [94]. ATM and at least some downstream targets, like H2AX [94], p53 [187] contribute to HCMV replication. In addition, at least one DNA repair factor, DDB2 [188], influences HCMV replication.

\section{Difference and Similarities in the DDR Induced by HCMV and Other Viruses}

\subsection{HCMV Is Similar to Other Viruses that Use DDR (ATM Signaling) for Replication}

As listed in Table 1, many viruses can activate ATM, and most of them can use ATM signaling to promote viral replication [94,95,97,99-104,106,108-110,113,114,116,120,122,124,125]. HCMV infection as well as IE1 or IE2 transduction can activate ATM [94,98,157]. Using caffeine, a PI3 kinase-like inhibitor, KU 55933, an ATM-specific inhibitor [189], AT cells derived from patients with ataxia telangiectasia, or siRNAs to deplete ATM, results in reduced or blocked HCMV replication [94]. Here, HCMV is similar to many other viruses with DNA stages in their replication strategy, such as HSV-1, HIV, MHV-68, SV-40, and HPV, in its requirement for ATM signaling for replication.

\subsection{HCMV is Different from Other Viruses Not Using ATM or that Block DDR for Replication}

HCMV is different from Ad5 and KSHV or B19V in its requirement for ATM signaling for efficient replication. Ad5 has evolved mechanisms to inhibit DNA damage signaling and repair during infection by degrading and mislocalizing components of the Mre11-Rad50-NBS1 (MRN) DNA damage recognition complex [135]. In addition, the Ad5 E3 ligase complex (comprised of E1B-55K and E4 adenoviral proteins) is able to target a number of cellular DNA repair proteins for proteasomal degradation including the RecQ helicase, bloom helicase (BLM) [190]. KSHV vIRF1 protein compromises an ATM/p53-mediated DDR by targeting both upstream ATM kinase activity and also downstream p53 tumor suppressor function by facilitating its proteasome-mediated degradation [140]. However, a recent study shows that during early de novo infection of primary endothelial cells, KSHV induces DDR signaling and ATM kinase activation as measured by H2AX 
phosphorylation, which are essential for KSHV's latent gene expression and establishment of latency [191]. Taken together, it seems that KSHV is able to both inhibit, and induce the cellular DDR dependent upon its replication strategy. Infection with B19V induces a broad range of DNA damage responses by activating three upstream kinases: ATM, ATR, and DNA-PKcs. Disruption of either the ATR or DNA-PKcs, but not ATM, signaling pathways significantly reduced the efficiency of B19V replication without affecting the resultant cell cycle arrest [111]. Thus, it appears that B19V uses ATR and DNAPKcs, but not ATM to facilitate its replication. Likewise, DNA-PKcs contribute to the replication of adeno-associated virus (AAV), another parvovirus [112]. Interestingly, AAV and Ad5 coinfections activate a broad DDR that is different from that seen during Ad5 or AAV infection alone [113].

\section{HCMV Infection Results in the Relocalization of DDR Proteins to Virus Replication Compartments (RCs)}

Viral RCs are sites of viral DNA replication and maturation. RCs begin as multiple, discrete structures early in infection, then move and coalesce into a single, large structure that can be referred to as a "mature" RC [94,192]. HCMV DNA replication happens in the RCs, similar to HSV-1 $[95,193,194]$. During HCMV infection, many DDR proteins are relocalized to the RCs including $\gamma \mathrm{H} 2 \mathrm{AX}, \mathrm{p} 53, \mathrm{pATM}, \mathrm{MRE11}, \mathrm{CHK} 2, \mathrm{NBS} 1$, Rad50, ATRIP, and CHK1 [94,98]. Although the mechanism(s) responsible for this relocation is unclear, these DDR proteins might interact with the viral DNA replication machinery in RCs to regulate viral DNA replication, gene expression, or recruit DNA repair proteins to repair damage to viral DNA.

\section{Does DNA Damage Exist in Cellular or Viral DNA During Infection?}

Many DNA viruses and retroviruses can tether or integrate their genome into the host DNA during infection $[195,196]$. It has been suggested that viral genetic material can be recognized by the host as damage DNA and stimulate cellular DNA repair mechanisms [197]. Given these observations, questions arise regarding whether host DNA is damaged during infections and whether DNA damage exists in viral DNA.

Host chromosome breaks have been observed in HCMV-infected cells. Infection of cells during S-phase results in two specific breaks on chromosome 1 at positions 1q42 and 1q21 [198]. HCMV infection-associated damage to chromosome 1 appears not to be cell-type-or-strain specific. Cells infected with HSV also show an increased incidence of chromosome breaks [199,200]. Chromatid gaps and breaks were found to accumulate in region 3 of the $\mathrm{X}$ chromosome and in region 7 of chromosome 1 [201]. Adenovirus type 12 E1B protein can induce damage specifically at 17q21-22, lp36, 1q21, and lq42-43 and at random sites in cellular chromosomes [202]. For HCMV, it is not clear if infection causes extensive host DNA damage or whether the two DNA break is sufficient to initiate the observed host DDR.

Some studies suggest that viral adsorption and penetration is required for inducing chromosomal breaks whereas viral protein expression is not required for induction of damage [198]. Alternately, a viral or cellular protein component of the incoming virion may be responsible for the induced damage. Our laboratory has observed DDR at very early times of infection, even before de novo 
viral protein expression. Input tegument proteins have been suggested to be partly responsible for DDR soon after viral entry, perhaps by inactivating pRB and derepressing E2F1 [199].

Another possible source of the DDR is that incoming, virion-delivered viral DNA or the nascent viral DNA generated during replication, is damaged or contains mutations. Evidence for these possibilities exists for HSV [194,200-204]. One can imagine that the oxidative state of virally infected cells, in particular HCMV infected cells [205] may result in oxidation of nucleotides such as guanines in viral DNA. It is also possible that, even though herpesviruses encode replicase complexes with proof reading activity, the high levels of (viral) DNA replication during infection may result in an accumulation of mutations that stimulate the relocalization of DDR proteins to viral RCs.

Activation of DDR does not necessarily require DNA lesions. Prolonged binding of DNA repair factors to chromatin can elicit DNA damage response in an ATM- and DNA-PK dependent manner in the absence of DNA lesions [206]. The herpesviral genomes are synthesized in a rolling circle manner to produce head-to-tail concatemers that are subsequently cleaved into unit-length genomes [207] and either the replication complexes or the cleaved DNA may be recognized as damaged DNA and trigger a DDR. In addition, viral infections confront cells with large amounts of exogenous genetic material that might be broadly recognized as abnormal [197] or the physical interaction of DNA repair factors with chromatin can be sufficient to activate the DDR signaling cascade [206]. Another possibility is that infected cells recognize viral replication as a genotoxic stress and elicit a DDR. In the case of HCMV infection, the inactivation of RB family members by IE1, IE2, pp71, and pUL97 and subsequent deregulation of E2F1 appears to result in DSBs in human fibroblasts [94]. Thus, it is possible that a trigger of the virus-induced DDR is not necessarily the recognition of linear viral DNA as double-strand breaks or actual damage to DNA, but rather, it is the recruitment of DNA damage repair factors to RCs or, very likely, a combination of these possibilities. Clearly, much work is needed to understand the interplay of viral infection, the presence of viral DNA and proteins, the remodeling of host cells function and the resultant DDR.

\section{Future Perspectives}

Although it is well accepted that activation of host DDR is a common theme of infections with DNA viruses, many of which require DDR signaling to replicate, more detailed study is still needed to better understand the "hows" and "whys" of this relationship. Most small DNA viruses capable of infecting nondividing cells induce $S$ phase in order to activate the host DNA replication machinery to provide the nucleotide triphosphates and host replication machinery necessary for viral DNA replication [93]. However, many large DNA viruses, such as herpesviruses, code for not only their own viral replicase enzymes, but also factors involved in deoxynucleotide synthesis and do not require a canonical S phase to support viral replication [92]. It has been thought that the host DNA polymerases do not play a role in herpesviral DNA replication. However, our unpublished studies suggest that host DNA polymerases may contribute to HCMV replication with the implication that theses enzymes may contribute activities that are different from the viral polymerase [208]. It should be interesting to explore the roles of host DNA polymerases in the replication of HCMV and other viruses. 
Components of the ATM-pathway are activated and recruited to sites of viral DNA synthesis including HCMV infected cells. Although ATR- and ATM-mediated pathways are related and both can be activated by similar genotoxic events, HSV-1 distinguishes between these two pathways, inactivating ATR pathway and potentially using ATM pathway. In contrast, it has been noted that the steady-state levels of ATR increase in HCMV infected cells at 48 hpi accompanied by a shift in mobility [98]. The ATR pathway also responds to DSBs, generally more slowly than ATM [209]. Determining the contribution of ATR signaling in relation to ATM signaling during HCMV infection should provide important insight into the specifics of DDR signaling as it pertains to HCMV infection and viral DNA replication,

HSV and HCMV infections, and the adenovirus type 12 E1B protein induce chromosome breaks in specific chromosome regions. The particular nonrandom distribution of chromosome aberrations in the HSV and HCMV infected cells raise a question as to whether virion genome deposition or early replication localized to particular chromosome regions are responsible for these effects.

The mechanism by which E2F1 stimulates host DDR is not well understood. Inactivation of RB and the subsequent deregulation of E2F1, but not the related family members, E2F2 or E2F3, leads to an accumulation of DSBs in human fibroblasts as observed by the $\gamma \mathrm{H} 2 \mathrm{AX}$ immunostaining and neutral comet assays [164]. The mechanism by which E2F1 stimulates host DDR needs to be further studied during infection by HCMV and other viruses.

Ubiquitination and sumolyation is one of the most common mechanisms by which viruses target cellular proteins. Viruses can encode their own ubiquitin ligases, such as the ICP0 protein of HSV-1. ICP0 interacts with PML isoform I and induces its SUMO-independent degradation [210]. Other viral proteins, such as Ad-E1B55K/E4orf6, KSHV-LANA, HPV-E6, and HIV-Vpr, can recruit and redirect cellular ubiquitin ligase complexes to target proteins. A recent study shows that a DNA repair factor, DDB2, can contribute to HCMV replication [188]. DDB2 is a component of a Cul4A-Ub ligase complex. The DDB1-CUL4A ${ }^{\mathrm{DDB} 2}$ complex is a cullin-RING (i.e., E3) Ub-ligase that targets histone $\mathrm{H} 2 \mathrm{~A}$ at UV-damaged DNA sites. Whether the ubiquitination ability of this complex is involved in HCMV replication needs further investigation.

One wonders if the intimate relationship between the host DDR and HCMV infection can be leveraged to treat HCMV-associated diseases. For example, there are no licensed treatments for pregnant women who undergo primary infection with HCMV during pregnancy, which places the fetus at risk for symptomatic congenital infection. This is at least partly due to the fact that drugs that are effective against HCMV infection have serious teratogenic side effects, which make them inappropriate for use during pregnancy. HCMV is also a common complication during tissue transplantation. Here, ganciclovir is commonly used to treat HCMV disease. However, ganciclovir induces leukocytopenia, an unwanted side effect in patients already receiving immunosuppressants. Perhaps, ATM inhibitors or more appropriately, drugs that target ATM responsive factors can be used alone or as adjuvant therapies to prevent congenital CMV infections or other diseases associated with infection. Continued study of infection-induced DDR pathways is essential for a better understanding of host-pathogen interactions and the development of host-targeted therapeutics. 


\section{Acknowledgments}

We thank the members of the Kowalik laboratory for comments on the manuscript. We apologize to the many groups whose primary research papers were not cited due to unintentional omissions on our part. This publication was supported by grants from the National Institutes of Health (R01AI076189; R01HD061959). The contents of this publication are solely the responsibility of the authors and do not necessarily represent the official views of the NIH.

\section{Authors Contributions}

Both authors wrote the manuscript and generated the figures.

\section{Conflicts of Interest}

The authors declare no conflicts of interest.

\section{References and Notes}

1. Vancikova, Z.; Dvorak, P. Cytomegalovirus infection in immunocompetent and immunocompromised individuals-A review. Curr. Drug Targets Immune Endocr. Metabol. Disord. 2001, 1, 179-187.

2. Cheeran, M.C.; Lokensgard, J.R.; Schleiss, M.R. Neuropathogenesis of congenital cytomegalovirus infection: disease mechanisms and prospects for intervention. Clin. Microbiol. Rev. 2009, 22, 99-126. Table of Contents.

3. Griffiths, P.D.; Walter, S. Cytomegalovirus. Curr. Opin. Infect. Dis. 2005, 18, 241-245.

4. Dziurzynski, K.; Chang, S.M.; Heimberger, A.B.; Kalejta, R.F.; McGregor Dallas, S.R.; Smit, M.; Soroceanu, L.; Cobbs, C.S. Consensus on the role of human cytomegalovirus in glioblastoma. Neuro Oncol. 2012, 14, 246-255.

5. Dolan, A.; Cunningham, C.; Hector, R.D.; Hassan-Walker, A.F.; Lee, L.; Addison, C.; Dargan, D.J.; McGeoch, D.J.; Gatherer, D.; Emery, V.C.; et al. Genetic content of wild-type human cytomegalovirus. J. Gen. Virol. 2004, 85, 1301-1312.

6. Chee, M.S.; Bankier, A.T.; Beck, S.; Bohni, R.; Brown, C.M.; Cerny, R.; Horsnell, T.; Hutchison, C.A., 3rd; Kouzarides, T.; Martignetti, J.A.; et al. Analysis of the protein-coding content of the sequence of human cytomegalovirus strain AD169. Curr. Top. Microbiol. Immunol. 1990, 154, 125-169.

7. Rigoutsos, I.; Novotny, J.; Huynh, T.; Chin-Bow, S.T.; Parida, L.; Platt, D.; Coleman, D.; Shenk, T. In silico pattern-based analysis of the human cytomegalovirus genome. J. Virol. 2003, 77, 4326-4344.

8. Stern-Ginossar, N.; Weisburd, B.; Michalski, A.; Le, V.T.; Hein, M.Y.; Huang, S.X.; Ma, M.; Shen, B.; Qian, S.B.; Hengel, H.; et al. Decoding human cytomegalovirus. Science 2012, 338, 1088-1093.

9. Renzette, N.; Bhattacharjee, B.; Jensen, J.D.; Gibson, L.; Kowalik, T.F. Extensive genome-wide variability of human cytomegalovirus in congenitally infected infants. PLoS Pathog. 2011, 7, e1001344. 
10. Varnum, S.M.; Streblow, D.N.; Monroe, M.E.; Smith, P.; Auberry, K.J.; Pasa-Tolic, L.; Wang, D.; Camp, D.G., 2nd; Rodland, K.; Wiley, S.; et al. Identification of proteins in human cytomegalovirus (HCMV) particles: The HCMV proteome. J. Virol. 2004, 78, 10960-10966.

11. Baldick, C.J., Jr.; Shenk, T. Proteins associated with purified human cytomegalovirus particles. J. Virol. 1996, 70, 6097-6105.

12. Hayashi, M.L.; Blankenship, C.; Shenk, T. Human cytomegalovirus UL69 protein is required for efficient accumulation of infected cells in the G1 phase of the cell cycle. Proc. Natl. Acad. Sci. USA 2000, 97, 2692-2696.

13. DeMarchi, J.M.; Schmidt, C.A.; Kaplan, A.S. Patterns of transcription of human cytomegalovirus in permissively infected cells. J. Virol. 1980, 35, 277-286.

14. Wathen, M.W.; Stinski, M.F. Temporal patterns of human cytomegalovirus transcription: Mapping the viral RNAs synthesized at immediate early, early, and late times after infection. J. Virol. 1982, 41, 462-477.

15. Stamminger, T.; Fleckenstein, B. Immediate-early transcription regulation of human cytomegalovirus. Curr. Top. Microbiol. Immunol. 1990, 154, 3-19.

16. Fortunato, E.A.; Sommer, M.H.; Yoder, K.; Spector, D.H. Identification of domains within the human cytomegalovirus major immediate-early 86-kilodalton protein and the retinoblastoma protein required for physical and functional interaction with each other. J. Virol. 1997, 71, 8176-8185.

17. Poma, E.E.; Kowalik, T.F.; Zhu, L.; Sinclair, J.H.; Huang, E.S. The human cytomegalovirus IE1-72 protein interacts with the cellular p107 protein and relieves p107-mediated transcriptional repression of an E2F-responsive promoter. J. Virol. 1996, 70, 7867-7877.

18. Fortunato, E.A.; Spector, D.H. Regulation of human cytomegalovirus gene expression. Adv. Virus Res. 1999, 54, 61-128.

19. Penfold, M.E.; Mocarski, E.S. Formation of cytomegalovirus DNA replication compartments defined by localization of viral proteins and DNA synthesis. Virology 1997, 239, 46-61.

20. Soderberg-Naucler, C.; Fish, K.N.; Nelson, J.A. Reactivation of latent human cytomegalovirus by allogeneic stimulation of blood cells from healthy donors. Cell 1997, 91, 119-126.

21. Soderberg-Naucler, C.; Streblow, D.N.; Fish, K.N.; Allan-Yorke, J.; Smith, P.P.; Nelson, J.A. Reactivation of latent human cytomegalovirus in $\mathrm{CD} 14(+)$ monocytes is differentiation dependent. J. Virol. 2001, 75, 7543-7554.

22. Soderberg-Naucler, C.; Fish, K.N.; Nelson, J.A. Interferon-gamma and tumor necrosis factor-alpha specifically induce formation of cytomegalovirus-permissive monocyte-derived macrophages that are refractory to the antiviral activity of these cytokines. J. Clin. Investig. 1997, 100, 3154-3163.

23. Kondo, K.; Kaneshima, H.; Mocarski, E.S. Human cytomegalovirus latent infection of granulocyte-macrophage progenitors. Proc. Natl. Acad. Sci. USA 1994, 91, 11879-11883.

24. Hahn, G.; Jores, R.; Mocarski, E.S. Cytomegalovirus remains latent in a common precursor of dendritic and myeloid cells. Proc. Natl. Acad. Sci. USA 1998, 95, 3937-3942. 
25. Goodrum, F.D.; Jordan, C.T.; High, K.; Shenk, T. Human cytomegalovirus gene expression during infection of primary hematopoietic progenitor cells: A model for latency. Proc. Natl. Acad. Sci. USA 2002, 99, 16255-16260.

26. Petrucelli, A.; Rak, M.; Grainger, L.; Goodrum, F. Characterization of a novel Golgi apparatus-localized latency determinant encoded by human cytomegalovirus. J. Virol. 2009, $83,5615-5629$.

27. Goodrum, F.; Reeves, M.; Sinclair, J.; High, K.; Shenk, T. Human cytomegalovirus sequences expressed in latently infected individuals promote a latent infection in vitro. Blood 2007, 110, 937-945.

28. Mason, G.M.; Poole, E.; Sissons, J.G.; Wills, M.R.; Sinclair, J.H. Human cytomegalovirus latency alters the cellular secretome, inducing cluster of differentiation (CD)4+ T-cell migration and suppression of effector function. Proc. Natl. Acad. Sci. USA 2012, 109, 14538-14543.

29. Jackson, S.P.; Bartek, J. The DNA-damage response in human biology and disease. Nature 2009, 461, 1071-1078.

30. Halazonetis, T.D.; Gorgoulis, V.G.; Bartek, J. An oncogene-induced DNA damage model for cancer development. Science 2008, 319, 1352-1355.

31. Nyberg, K.A.; Michelson, R.J.; Putnam, C.W.; Weinert, T.A. Toward maintaining the genome: DNA damage and replication checkpoints. Annu. Rev. Genet. 2002, 36, 617-656.

32. Elledge, S.J. Cell cycle checkpoints: Preventing an identity crisis. Science 1996, 274, 1664-1672.

33. Sancar, A.; Lindsey-Boltz, L.A.; Unsal-Kacmaz, K.; Linn, S. Molecular mechanisms of mammalian DNA repair and the DNA damage checkpoints. Annu. Rev. Biochem. 2004, 73, $39-85$.

34. O’Driscoll, M.; Gennery, A.R.; Seidel, J.; Concannon, P.; Jeggo, P.A. An overview of three new disorders associated with genetic instability: LIG4 syndrome, RS-SCID and ATR-Seckel syndrome. DNA Repair (Amst) 2004, 3, 1227-1235.

35. Shiloh, Y. ATM and related protein kinases: Safeguarding genome integrity. Nat. Rev. Cancer 2003, 3, 155-168.

36. Taylor, A.M.; Groom, A.; Byrd, P.J. Ataxia-telangiectasia-like disorder (ATLD)-its clinical presentation and molecular basis. DNA Repair (Amst) 2004, 3, 1219-1225.

37. Kastan, M.B.; Bartek, J. Cell-cycle checkpoints and cancer. Nature 2004, 432, 316-323.

38. Khanna, K.K.; Jackson, S.P. DNA double-strand breaks: Signaling, repair and the cancer connection. Nat. Genet. 2001, 27, 247-254.

39. Shiloh, Y.; Kastan, M.B. ATM: Genome stability, neuronal development, and cancer cross paths. Adv. Cancer Res. 2001, 83, 209-254.

40. Alexander, A.; Cai, S.L.; Kim, J.; Nanez, A.; Sahin, M.; MacLean, K.H.; Inoki, K.; Guan, K.L.; Shen, J.; Person, M.D.; et al. ATM signals to TSC2 in the cytoplasm to regulate mTORC1 in response to ROS. Proc. Natl. Acad. Sci. USA 2010, 107, 4153-4158.

41. Guo, Z.; Kozlov, S.; Lavin, M.F.; Person, M.D.; Paull, T.T. ATM activation by oxidative stress. Science 2010, 330, 517-521. 
42. Hinz, M.; Stilmann, M.; Arslan, S.C.; Khanna, K.K.; Dittmar, G.; Scheidereit, C. A cytoplasmic ATM-TRAF6-cIAP1 module links nuclear DNA damage signaling to ubiquitin-mediated NF-kappaB activation. Mol. Cell 2010, 40, 63-74.

43. Li, B.; Wang, X.; Rasheed, N.; Hu, Y.; Boast, S.; Ishii, T.; Nakayama, K.; Nakayama, K.I.; Goff, S.P. Distinct roles of c-Abl and Atm in oxidative stress response are mediated by protein kinase C delta. Genes Dev. 2004, 18, 1824-1837.

44. Abraham, R.T. Cell cycle checkpoint signaling through the ATM and ATR kinases. Genes Dev. 2001, 15, 2177-2196.

45. Burma, S.; Chen, B.P.; Chen, D.J. Role of non-homologous end joining (NHEJ) in maintaining genomic integrity. DNA Repair (Amst) 2006, 5, 1042-1048.

46. Matsuoka, S.; Ballif, B.A.; Smogorzewska, A.; McDonald, E.R., 3rd; Hurov, K.E.; Luo, J.; Bakalarski, C.E.; Zhao, Z.; Solimini, N.; Lerenthal, Y.; et al. ATM and ATR substrate analysis reveals extensive protein networks responsive to DNA damage. Science 2007, 316, $1160-1166$.

47. Nevins, J.R. E2F: A link between the Rb tumor suppressor protein and viral oncoproteins. Science 1992, 258, 424-429.

48. Dyson, N. pRB, p107 and the regulation of the E2F transcription factor. J. Cell. Sci. Suppl. 1994, 18, 81-87.

49. La Thangue, N.B. DP and E2F proteins: Components of a heterodimeric transcription factor implicated in cell cycle control. Curr. Opin. Cell Biol. 1994, 6, 443-450.

50. La Thangue, N.B. E2F and the molecular mechanisms of early cell-cycle control. Biochem. Soc. Trans. 1996, 24, 54-59.

51. Chellappan, S.P.; Hiebert, S.; Mudryj, M.; Horowitz, J.M.; Nevins, J.R. The E2F transcription factor is a cellular target for the RB protein. Cell 1991, 65, 1053-1061.

52. Helin, K.; Lees, J.A.; Vidal, M.; Dyson, N.; Harlow, E.; Fattaey, A. A cDNA encoding a pRB-binding protein with properties of the transcription factor E2F. Cell 1992, 70, 337-350.

53. Kaelin, W.G., Jr.; Krek, W.; Sellers, W.R.; DeCaprio, J.A.; Ajchenbaum, F.; Fuchs, C.S.; Chittenden, T.; Li, Y.; Farnham, P.J.; Blanar, M.A.; et al. Expression cloning of a cDNA encoding a retinoblastoma-binding protein with E2F-like properties. Cell 1992, 70, 351-364.

54. Qin, X.Q.; Livingston, D.M.; Ewen, M.; Sellers, W.R.; Arany, Z.; Kaelin, W.G., Jr. The transcription factor E2F-1 is a downstream target of RB action. Mol. Cell. Biol. 1995, 15, $742-755$.

55. Ikeda, M.A.; Jakoi, L.; Nevins, J.R. A unique role for the Rb protein in controlling E2F accumulation during cell growth and differentiation. Proc. Natl. Acad. Sci. USA 1996, 93, 3215-3220.

56. Chellappan, S.; Kraus, V.B.; Kroger, B.; Munger, K.; Howley, P.M.; Phelps, W.C.; Nevins, J.R. Adenovirus E1A, simian virus 40 tumor antigen, and human papillomavirus E7 protein share the capacity to disrupt the interaction between transcription factor E2F and the retinoblastoma gene product. Proc. Natl. Acad. Sci. USA 1992, 89, 4549-4553.

57. Nevins, J.R. Disruption of cell-cycle control by viral oncoproteins. Biochem. Soc. Trans. 1993, 21, 935-938. 
58. Nevins, J.R. Cell cycle targets of the DNA tumor viruses. Curr. Opin. Genet. Dev. 1994, 4, 130-134.

59. Moberg, K.; Starz, M.A.; Lees, J.A. E2F-4 switches from p130 to p107 and pRB in response to cell cycle reentry. Mol. Cell. Biol. 1996, 16, 1436-1449.

60. Ginsberg, D.; Vairo, G.; Chittenden, T.; Xiao, Z.X.; Xu, G.; Wydner, K.L.; DeCaprio, J.A.; Lawrence, J.B.; Livingston, D.M. E2F-4, a new member of the E2F transcription factor family, interacts with p107. Genes Dev. 1994, 8, 2665-2679.

61. Hijmans, E.M.; Voorhoeve, P.M.; Beijersbergen, R.L.; van’t Veer, L.J.; Bernards, R. E2F-5, a new E2F family member that interacts with p130 in vivo. Mol. Cell. Biol. 1995, 15, 3082-3089.

62. Vairo, G.; Livingston, D.M.; Ginsberg, D. Functional interaction between E2F-4 and p130: Evidence for distinct mechanisms underlying growth suppression by different retinoblastoma protein family members. Genes Dev. 1995, 9, 869-881.

63. Beijersbergen, R.L.; Kerkhoven, R.M.; Zhu, L.; Carlee, L.; Voorhoeve, P.M.; Bernards, R. E2F-4, a new member of the E2F gene family, has oncogenic activity and associates with p107 in vivo. Genes Dev. 1994, 8, 2680-2690.

64. Morkel, M.; Wenkel, J.; Bannister, A.J.; Kouzarides, T.; Hagemeier, C. An E2F-like repressor of transcription. Nature 1997, 390, 567-568.

65. Cartwright, P.; Muller, H.; Wagener, C.; Holm, K.; Helin, K. E2F-6: A novel member of the E2F family is an inhibitor of E2F-dependent transcription. Oncogene 1998, 17, 611-623.

66. Gaubatz, S.; Wood, J.G.; Livingston, D.M. Unusual proliferation arrest and transcriptional control properties of a newly discovered E2F family member, E2F-6. Proc. Natl. Acad. Sci. USA 1998, 95, 9190-9195.

67. Trimarchi, J.M.; Fairchild, B.; Verona, R.; Moberg, K.; Andon, N.; Lees, J.A. E2F-6, a member of the E2F family that can behave as a transcriptional repressor. Proc. Natl. Acad. Sci. USA 1998, 95, 2850-2855.

68. de Bruin, A.; Maiti, B.; Jakoi, L.; Timmers, C.; Buerki, R.; Leone, G. Identification and characterization of E2F7, a novel mammalian E2F family member capable of blocking cellular proliferation. J. Biol. Chem. 2003, 278, 42041-42049.

69. Di Stefano, L.; Jensen, M.R.; Helin, K. E2F7, a novel E2F featuring DP-independent repression of a subset of E2F-regulated genes. EMBO J. 2003, 22, 6289-6298.

70. Roos, W.P.; Kaina, B. DNA damage-induced cell death by apoptosis. Trends Mol. Med. 2006, 12, 440-450.

71. Zhou, B.B.; Bartek, J. Targeting the checkpoint kinases: Chemosensitization versus chemoprotection. Nat. Rev. Cancer 2004, 4, 216-225.

72. Bartek, J.; Lukas, J. Chk1 and Chk2 kinases in checkpoint control and cancer. Cancer Cell 2003, 3, 421-429.

73. Zhao, H.; Watkins, J.L.; Piwnica-Worms, H. Disruption of the checkpoint kinase 1/cell division cycle 25A pathway abrogates ionizing radiation-induced S and G2 checkpoints. Proc. Natl. Acad. Sci. USA 2002, 99, 14795-14800. 
74. Lukas, C.; Bartkova, J.; Latella, L.; Falck, J.; Mailand, N.; Schroeder, T.; Sehested, M.; Lukas, J.; Bartek, J. DNA damage-activated kinase Chk2 is independent of proliferation or differentiation yet correlates with tissue biology. Cancer Res. 2001, 61, 4990-4993.

75. Hlavova, M.; Cizkova, M.; Vitova, M.; Bisova, K.; Zachleder, V. DNA damage during G2 phase does not affect cell cycle progression of the green alga Scenedesmus quadricauda. PLoS One 2011, 6, e19626.

76. Manke, I.A.; Nguyen, A.; Lim, D.; Stewart, M.Q.; Elia, A.E.; Yaffe, M.B. MAPKAP kinase-2 is a cell cycle checkpoint kinase that regulates the $\mathrm{G} 2 / \mathrm{M}$ transition and $\mathrm{S}$ phase progression in response to UV irradiation. Mol. Cell 2005, 17, 37-48.

77. Reinhardt, H.C.; Aslanian, A.S.; Lees, J.A.; Yaffe, M.B. p53-deficient cells rely on ATM- and ATR-mediated checkpoint signaling through the p38MAPK/MK2 pathway for survival after DNA damage. Cancer Cell 2007, 11, 175-189.

78. Kyriakis, J.M.; Avruch, J. Mammalian mitogen-activated protein kinase signal transduction pathways activated by stress and inflammation. Physiol. Rev. 2001, 81, 807-869.

79. Raman, M.; Earnest, S.; Zhang, K.; Zhao, Y.; Cobb, M.H. TAO kinases mediate activation of p38 in response to DNA damage. EMBO J. 2007, 26, 2005-2014.

80. Hirose, Y.; Katayama, M.; Stokoe, D.; Haas-Kogan, D.A.; Berger, M.S.; Pieper, R.O. The p38 mitogen-activated protein kinase pathway links the DNA mismatch repair system to the G2 checkpoint and to resistance to chemotherapeutic DNA-methylating agents. Mol. Cell. Biol. 2003, 23, 8306-8315.

81. Mikhailov, A.; Shinohara, M.; Rieder, C.L. Topoisomerase II and histone deacetylase inhibitors delay the G2/M transition by triggering the p38 MAPK checkpoint pathway. $J$. Cell Biol. 2004, 166, 517-526.

82. Reinhardt, H.C.; Yaffe, M.B. Kinases that control the cell cycle in response to DNA damage: Chk1, Chk2, and MK2. Curr. Opin. Cell Biol. 2009, 21, 245-255.

83. Bouwman, P.; Jonkers, J. The effects of deregulated DNA damage signalling on cancer chemotherapy response and resistance. Nat. Rev. Cancer 2012, 12, 587-598.

84. Lazzaro, F.; Giannattasio, M.; Puddu, F.; Granata, M.; Pellicioli, A.; Plevani, P.; Muzi-Falconi, M. Checkpoint mechanisms at the intersection between DNA damage and repair. DNA Repair (Amst) 2009, 8, 1055-1067.

85. Lilley, C.E.; Schwartz, R.A.; Weitzman, M.D. Using or abusing: Viruses and the cellular DNA damage response. Trends Microbiol. 2007, 15, 119-126.

86. McFadden, K.; Luftig, M.A. Interplay between DNA tumor viruses and the host DNA damage response. Curr. Top. Microbiol. Immunol. 2013, 371, 229-257.

87. Nikitin, P.A.; Luftig, M.A. At a crossroads: Human DNA tumor viruses and the host DNA damage response. Future Virol. 2011, 6, 813-830.

88. Turnell, A.S.; Grand, R.J. DNA viruses and the cellular DNA-damage response. J. Gen. Virol. 2012, 93, 2076-2097.

89. Weitzman, M.D.; Lilley, C.E.; Chaurushiya, M.S. Genomes in conflict: Maintaining genome integrity during virus infection. Annu. Rev. Microbiol. 2010, 64, 61-81.

90. Stracker, T.H.; Carson, C.T.; Weitzman, M.D. Adenovirus oncoproteins inactivate the Mre11-Rad50-NBS1 DNA repair complex. Nature 2002, 418, 348-352. 
91. Chaurushiya, M.S.; Weitzman, M.D. Viral manipulation of DNA repair and cell cycle checkpoints. DNA Repair (Amst) 2009, 8, 1166-1176.

92. Flemington, E.K. Herpesvirus lytic replication and the cell cycle: Arresting new developments. J. Virol. 2001, 75, 4475-4481.

93. Nascimento, R.; Costa, H.; Parkhouse, R.M. Virus manipulation of cell cycle. Protoplasma 2012, 249, 519-528.

94. E, X.; Pickering, M.T.; Debatis, M.; Castillo, J.; Lagadinos, A.; Wang, S.; Lu, S.; Kowalik, T.F. An E2F1-mediated DNA damage response contributes to the replication of human cytomegalovirus. PLoS Pathog. 2011, 7, e1001342.

95. Lilley, C.E.; Carson, C.T.; Muotri, A.R.; Gage, F.H.; Weitzman, M.D. DNA repair proteins affect the lifecycle of herpes simplex virus 1. Proc. Natl. Acad. Sci. USA 2005, 102, 5844-5849.

96. Lu, C.C.; Chen, Y.C.; Wang, J.T.; Yang, P.W.; Chen, M.R. Xeroderma pigmentosum C is involved in Epstein Barr virus DNA replication. J. Gen. Virol. 2007, 88, 3234-3243.

97. Boichuk, S.; Hu, L.; Hein, J.; Gjoerup, O.V. Multiple DNA damage signaling and repair pathways deregulated by simian virus 40 large T antigen. J. Virol. 2010, 84, 8007-8020.

98. Luo, M.H.; Rosenke, K.; Czornak, K.; Fortunato, E.A. Human cytomegalovirus disrupts both ataxia telangiectasia mutated protein (ATM)- and ATM-Rad3-related kinase-mediated DNA damage responses during lytic infection. J. Virol. 2007, 81, 1934-1950.

99. Kudoh, A.; Fujita, M.; Zhang, L.; Shirata, N.; Daikoku, T.; Sugaya, Y.; Isomura, H.; Nishiyama, Y.; Tsurumi, T. Epstein-Barr virus lytic replication elicits ATM checkpoint signal transduction while providing an S-phase-like cellular environment. J. Biol. Chem. 2005, 280, 8156-8163.

100. Liang, X.; Pickering, M.T.; Cho, N.H.; Chang, H.; Volkert, M.R.; Kowalik, T.F.; Jung, J.U. Deregulation of DNA damage signal transduction by herpesvirus latency-associated M2. J. Virol. 2006, 80, 5862-5874.

101. Tarakanova, V.L.; Leung-Pineda, V.; Hwang, S.; Yang, C.W.; Matatall, K.; Basson, M.; Sun, R.; Piwnica-Worms, H.; Sleckman, B.P.; Virgin, H.W. Gamma-herpesvirus kinase actively initiates a DNA damage response by inducing phosphorylation of $\mathrm{H} 2 \mathrm{AX}$ to foster viral replication. Cell Host Microbe 2007, 1, 275-286.

102. Shi, Y.; Dodson, G.E.; Shaikh, S.; Rundell, K.; Tibbetts, R.S. Ataxia-telangiectasia-mutated (ATM) is a T-antigen kinase that controls SV40 viral replication in vivo. J. Biol. Chem. 2005, 280, 40195-40200.

103. Zhao, X.; Madden-Fuentes, R.J.; Lou, B.X.; Pipas, J.M.; Gerhardt, J.; Rigell, C.J.; Fanning, E. Ataxia telangiectasia-mutated damage-signaling kinase- and proteasome-dependent destruction of Mre11-Rad50-Nbs1 subunits in Simian virus 40-infected primate cells. J. Virol. 2008, 82, 5316-5328.

104. Fradet-Turcotte, A.; Bergeron-Labrecque, F.; Moody, C.A.; Lehoux, M.; Laimins, L.A.; Archambault, J. Nuclear accumulation of the papillomavirus E1 helicase blocks S-phase progression and triggers an ATM-dependent DNA damage response. J. Virol. 2011, 85, 8996-9012. 
105. Kadaja, M.; Isok-Paas, H.; Laos, T.; Ustav, E.; Ustav, M. Mechanism of genomic instability in cells infected with the high-risk human papillomaviruses. PLoS Pathog. 2009, 5, e1000397.

106. Moody, C.A.; Laimins, L.A. Human papillomaviruses activate the ATM DNA damage pathway for viral genome amplification upon differentiation. PLoS Pathog. 2009, 5, e1000605.

107. Reinson, T.; Toots, M.; Kadaja, M.; Pipitch, R.; Allik, M.; Ustav, E.; Ustav, M. Engagement of the ATR-dependent DNA damage response at the human papillomavirus 18 replication centers during the initial amplification. J. Virol. 2013, 87, 951-964.

108. Sakakibara, N.; Mitra, R.; McBride, A.A. The papillomavirus E1 helicase activates a cellular DNA damage response in viral replication foci. J. Virol. 2011, 85, 8981-8995.

109. Lou, S.; Luo, Y.; Cheng, F.; Huang, Q.; Shen, W.; Kleiboeker, S.; Tisdale, J.F.; Liu, Z.; Qiu, J. Human parvovirus B19 DNA replication induces a DNA damage response that is dispensable for cell cycle arrest at phase G2/M. J. Virol. 2012, 86, 10748-10758.

110. Luo, Y.; Kleiboeker, S.; Deng, X.; Qiu, J. Human parvovirus B19 infection causes cell cycle arrest of human erythroid progenitors at late $\mathrm{S}$ phase that favors viral DNA replication. J. Virol. 2013, 87, 12766-12775.

111. Luo, Y.; Lou, S.; Deng, X.; Liu, Z.; Li, Y.; Kleiboeker, S.; Qiu, J. Parvovirus B19 infection of human primary erythroid progenitor cells triggers ATR-Chk1 signaling, which promotes B19 virus replication. J. Virol. 2011, 85, 8046-8055.

112. Choi, Y.K.; Nash, K.; Byrne, B.J.; Muzyczka, N.; Song, S. The effect of DNA-dependent protein kinase on adeno-associated virus replication. PLoS One 2010, 5, e15073.

113. Schwartz, R.A.; Carson, C.T.; Schuberth, C.; Weitzman, M.D. Adeno-associated virus replication induces a DNA damage response coordinated by DNA-dependent protein kinase. J. Virol. 2009, 83, 6269-6278.

114. Baydoun, H.H.; Bai, X.T.; Shelton, S.; Nicot, C. HTLV-I tax increases genetic instability by inducing DNA double strand breaks during DNA replication and switching repair to NHEJ. PLoS One 2012, 7, e42226.

115. Belgnaoui, S.M.; Fryrear, K.A.; Nyalwidhe, J.O.; Guo, X.; Semmes, O.J. The viral oncoprotein tax sequesters DNA damage response factors by tethering MDC1 to chromatin. J. Biol. Chem. 2010, 285, 32897-32905.

116. Chandhasin, C.; Ducu, R.I.; Berkovich, E.; Kastan, M.B.; Marriott, S.J. Human T-cell leukemia virus type 1 tax attenuates the ATM-mediated cellular DNA damage response. J. Virol. 2008, 82, 6952-6961.

117. Chlichlia, K.; Khazaie, K. HTLV-1 Tax: Linking transformation, DNA damage and apoptotic T-cell death. Chem. Biol. Interact. 2010, 188, 359-365.

118. Durkin, S.S.; Guo, X.; Fryrear, K.A.; Mihaylova, V.T.; Gupta, S.K.; Belgnaoui, S.M.; Haoudi, A.; Kupfer, G.M.; Semmes, O.J. HTLV-1 Tax oncoprotein subverts the cellular DNA damage response via binding to DNA-dependent protein kinase. J. Biol. Chem. 2008, 283, 36311-36320.

119. Kinjo, T.; Ham-Terhune, J.; Peloponese, J.M., Jr.; Jeang, K.T. Induction of reactive oxygen species by human T-cell leukemia virus type 1 tax correlates with DNA damage and expression of cellular senescence marker. J. Virol. 2010, 84, 5431-5437. 
120. Ariumi, Y.; Trono, D. Ataxia-telangiectasia-mutated (ATM) protein can enhance human immunodeficiency virus type 1 replication by stimulating Rev function. J. Virol. 2006, 80, 2445-2452.

121. Lau, A.; Swinbank, K.M.; Ahmed, P.S.; Taylor, D.L.; Jackson, S.P.; Smith, G.C.; O’Connor, M.J. Suppression of HIV-1 infection by a small molecule inhibitor of the ATM kinase. Nat. Cell Biol. 2005, 7, 493-500.

122. Perfettini, J.L.; Nardacci, R.; Bourouba, M.; Subra, F.; Gros, L.; Seror, C.; Manic, G.; Rosselli, F.; Amendola, A.; Masdehors, P.; et al. Critical involvement of the ATM-dependent DNA damage response in the apoptotic demise of HIV-1-elicited syncytia. PLoS One 2008, 3, e2458.

123. Austin, D.; Baer, A.; Lundberg, L.; Shafagati, N.; Schoonmaker, A.; Narayanan, A.; Popova, T.; Panthier, J.J.; Kashanchi, F.; Bailey, C.; et al. p53 Activation following Rift Valley fever virus infection contributes to cell death and viral production. PLoS One 2012, 7, e36327.

124. Baer, A.; Austin, D.; Narayanan, A.; Popova, T.; Kainulainen, M.; Bailey, C.; Kashanchi, F.; Weber, F.; Kehn-Hall, K. Induction of DNA Damage Signaling upon Rift Valley Fever Virus Infection Results in Cell Cycle Arrest and Increased Viral Replication. J. Biol. Chem. 2012, 287, 7399-7410.

125. Ariumi, Y.; Kuroki, M.; Dansako, H.; Abe, K.; Ikeda, M.; Wakita, T.; Kato, N. The DNA damage sensors ataxia-telangiectasia mutated kinase and checkpoint kinase 2 are required for hepatitis C virus RNA replication. J. Virol. 2008, 82, 9639-9646.

126. Wen, C.; He, X.; Ma, H.; Hou, N.; Wei, C.; Song, T.; Zhang, Y.; Sun, L.; Ma, Q.; Zhong, H. Hepatitis $\mathrm{C}$ virus infection downregulates the ligands of the activating receptor NKG2D. Cell. Mol. Immunol. 2008, 5, 475-478.

127. Parkinson, J.; Lees-Miller, S.P.; Everett, R.D. Herpes simplex virus type 1 immediate-early protein vmw110 induces the proteasome-dependent degradation of the catalytic subunit of DNA-dependent protein kinase. J. Virol. 1999, 73, 650-657.

128. Wilkinson, D.E.; Weller, S.K. Recruitment of cellular recombination and repair proteins to sites of herpes simplex virus type 1 DNA replication is dependent on the composition of viral proteins within prereplicative sites and correlates with the induction of the DNA damage response. J. Virol. 2004, 78, 4783-4796.

129. Wilkinson, D.E.; Weller, S.K. Herpes simplex virus type I disrupts the ATR-dependent DNA-damage response during lytic infection. J. Cell Sci. 2006, 119, 2695-2703.

130. Bittar, C.; Shrivastava, S.; Bhanja Chowdhury, J.; Rahal, P.; Ray, R.B. Hepatitis C virus NS2 protein inhibits DNA damage pathway by sequestering p53 to the cytoplasm. PLoS One 2013, 8, e62581.

131. Machida, K.; McNamara, G.; Cheng, K.T.; Huang, J.; Wang, C.H.; Comai, L.; Ou, J.H.; Lai, M.M. Hepatitis C virus inhibits DNA damage repair through reactive oxygen and nitrogen species and by interfering with the ATM-NBS1/Mre11/Rad50 DNA repair pathway in monocytes and hepatocytes. J. Immunol. 2010, 185, 6985-6998.

132. Forrester, N.A.; Sedgwick, G.G.; Thomas, A.; Blackford, A.N.; Speiseder, T.; Dobner, T.; Byrd, P.J.; Stewart, G.S.; Turnell, A.S.; Grand, R.J. Serotype-specific inactivation of the cellular DNA damage response during adenovirus infection. J. Virol. 2011, 85, 2201-2211. 
133. Karen, K.A.; Hoey, P.J.; Young, C.S.; Hearing, P. Temporal regulation of the Mre11-Rad50-Nbs1 complex during adenovirus infection. J. Virol. 2009, 83, 4565-4573.

134. Evans, J.D.; Hearing, P. Relocalization of the Mre11-Rad50-Nbs 1 complex by the adenovirus E4 ORF3 protein is required for viral replication. J. Virol. 2005, 79, 6207-6215.

135. Carson, C.T.; Orazio, N.I.; Lee, D.V.; Suh, J.; Bekker-Jensen, S.; Araujo, F.D.; Lakdawala, S.S.; Lilley, C.E.; Bartek, J.; Lukas, J.; et al. Mislocalization of the MRN complex prevents ATR signaling during adenovirus infection. EMBO J. 2009, 28, 652-662.

136. Liu, Y.; Shevchenko, A.; Berk, A.J. Adenovirus exploits the cellular aggresome response to accelerate inactivation of the MRN complex. J. Virol. 2005, 79, 14004-14016.

137. Araujo, F.D.; Stracker, T.H.; Carson, C.T.; Lee, D.V.; Weitzman, M.D. Adenovirus type 5 E4orf3 protein targets the Mre11 complex to cytoplasmic aggresomes. J. Virol. 2005, 79, $11382-11391$.

138. Mathew, S.S.; Bridge, E. The cellular Mre11 protein interferes with adenovirus E4 mutant DNA replication. Virology 2007, 365, 346-355.

139. Lakdawala, S.S.; Schwartz, R.A.; Ferenchak, K.; Carson, C.T.; McSharry, B.P.; Wilkinson, G.W.; Weitzman, M.D. Differential requirements of the C terminus of Nbs1 in suppressing adenovirus DNA replication and promoting concatemer formation. J. Virol. 2008, 82, 8362-8372.

140. Shin, Y.C.; Nakamura, H.; Liang, X.; Feng, P.; Chang, H.; Kowalik, T.F.; Jung, J.U. Inhibition of the ATM/p53 signal transduction pathway by Kaposi's sarcoma-associated herpesvirus interferon regulatory factor 1. J. Virol. 2006, 80, 2257-2266.

141. Fortunato, E.A.; Spector, D.H. p53 and RPA are sequestered in viral replication centers in the nuclei of cells infected with human cytomegalovirus. J. Virol. 1998, 72, 2033-2039.

142. Fortunato, E.A.; McElroy, A.K.; Sanchez, I.; Spector, D.H. Exploitation of cellular signaling and regulatory pathways by human cytomegalovirus. Trends Microbiol. 2000, 8, 111-119.

143. Kalejta, R.F.; Shenk, T. Manipulation of the cell cycle by human cytomegalovirus. Front. Biosci. 2002, 7, d295-d306.

144. Biswas, N.; Sanchez, V.; Spector, D.H. Human cytomegalovirus infection leads to accumulation of geminin and inhibition of the licensing of cellular DNA replication. J. Virol. 2003, 77, 2369-2376.

145. Wiebusch, L.; Uecker, R.; Hagemeier, C. Human cytomegalovirus prevents replication licensing by inhibiting MCM loading onto chromatin. EMBO Rep. 2003, 4, 42-46.

146. Bresnahan, W.A.; Boldogh, I.; Thompson, E.A.; Albrecht, T. Human cytomegalovirus inhibits cellular DNA synthesis and arrests productively infected cells in late G1. Virology 1996, 224, 150-160.

147. Dittmer, D.; Mocarski, E.S. Human cytomegalovirus infection inhibits G1/S transition. J. Virol. 1997, 71, 1629-1634.

148. Lu, M.; Shenk, T. Human cytomegalovirus UL69 protein induces cells to accumulate in G1 phase of the cell cycle. J. Virol. 1999, 73, 676-683.

149. Kalejta, R.F.; Bechtel, J.T.; Shenk, T. Human cytomegalovirus pp71 stimulates cell cycle progression by inducing the proteasome-dependent degradation of the retinoblastoma family of tumor suppressors. Mol. Cell. Biol. 2003, 23, 1885-1895. 
150. Hume, A.J.; Finkel, J.S.; Kamil, J.P.; Coen, D.M.; Culbertson, M.R.; Kalejta, R.F. Phosphorylation of retinoblastoma protein by viral protein with cyclin-dependent kinase function. Science 2008, 320, 797-799.

151. Salsman, J.; Jagannathan, M.; Paladino, P.; Chan, P.K.; Dellaire, G.; Raught, B.; Frappier, L. Proteomic profiling of the human cytomegalovirus UL35 gene products reveals a role for UL35 in the DNA repair response. J. Virol. 2012, 86, 806-820.

152. Castillo, J.P.; Yurochko, A.D.; Kowalik, T.F. Role of human cytomegalovirus immediate-early proteins in cell growth control. J. Virol. 2000, 74, 8028-8037.

153. Wiebusch, L.; Hagemeier, C. Human cytomegalovirus 86-kilodalton IE2 protein blocks cell cycle progression in G(1). J. Virol. 1999, 73, 9274-9283.

154. Kwon, Y.; Kim, M.N.; Young Choi, E.; Heon Kim, J.; Hwang, E.S.; Cha, C.Y. Inhibition of p53 transcriptional activity by human cytomegalovirus UL44. Microbiol. Immunol. 2012, 56, 324-331.

155. Pietenpol, J.A.; Stewart, Z.A. Cell cycle checkpoint signaling: Cell cycle arrest versus apoptosis. Toxicology 2002, 181-182, 475-481.

156. Margolis, M.J.; Pajovic, S.; Wong, E.L.; Wade, M.; Jupp, R.; Nelson, J.A.; Azizkhan, J.C. Interaction of the 72-kilodalton human cytomegalovirus IE1 gene product with E2F1 coincides with E2F-dependent activation of dihydrofolate reductase transcription. J. Virol. 1995, 69, 7759-7767.

157. Castillo, J.P.; Frame, F.M.; Rogoff, H.A.; Pickering, M.T.; Yurochko, A.D.; Kowalik, T.F. Human cytomegalovirus IE1-72 activates ataxia telangiectasia mutated kinase and a p53/p21-mediated growth arrest response. J. Virol. 2005, 79, 11467-11475.

158. Prichard, M.N.; Sztul, E.; Daily, S.L.; Perry, A.L.; Frederick, S.L.; Gill, R.B.; Hartline, C.B.; Streblow, D.N.; Varnum, S.M.; Smith, R.D.; et al. Human cytomegalovirus UL97 kinase activity is required for the hyperphosphorylation of retinoblastoma protein and inhibits the formation of nuclear aggresomes. J. Virol. 2008, 82, 5054-5067.

159. Zhang, Z.; Huong, S.M.; Wang, X.; Huang, D.Y.; Huang, E.S. Interactions between human cytomegalovirus IE1-72 and cellular p107: Functional domains and mechanisms of up-regulation of cyclin E/cdk2 kinase activity. J. Virol. 2003, 77, 12660-12670.

160. Hagemeier, C.; Caswell, R.; Hayhurst, G.; Sinclair, J.; Kouzarides, T. Functional interaction between the HCMV IE2 transactivator and the retinoblastoma protein. EMBO J. 1994, 13, 2897-2903.

161. Kalejta, R.F. Human cytomegalovirus pp71: A new viral tool to probe the mechanisms of cell cycle progression and oncogenesis controlled by the retinoblastoma family of tumor suppressors. J. Cell. Biochem. 2004, 93, 37-45.

162. Kalejta, R.F.; Shenk, T. Proteasome-dependent, ubiquitin-independent degradation of the Rb family of tumor suppressors by the human cytomegalovirus pp71 protein. Proc. Natl. Acad. Sci. USA 2003, 100, 3263-3268.

163. Pajovic, S.; Wong, E.L.; Black, A.R.; Azizkhan, J.C. Identification of a viral kinase that phosphorylates specific E2Fs and pocket proteins. Mol. Cell. Biol. 1997, 17, 6459-6464.

164. Pickering, M.T.; Kowalik, T.F. Rb inactivation leads to E2F1-mediated DNA double-strand break accumulation. Oncogene 2006, 25, 746-755. 
165. Johnson, R.A.; Yurochko, A.D.; Poma, E.E.; Zhu, L.; Huang, E.S. Domain mapping of the human cytomegalovirus IE1-72 and cellular p107 protein-protein interaction and the possible functional consequences. J. Gen. Virol. 1999, 80, 1293-1303.

166. Ahn, J.H.; Hayward, G.S. The major immediate-early proteins IE1 and IE2 of human cytomegalovirus colocalize with and disrupt PML-associated nuclear bodies at very early times in infected permissive cells. J. Virol. 1997, 71, 4599-4613.

167. Wilkinson, G.W.; Kelly, C.; Sinclair, J.H.; Rickards, C. Disruption of PML-associated nuclear bodies mediated by the human cytomegalovirus major immediate early gene product. J. Gen. Virol. 1998, 79, 1233-1245.

168. Xiaofei, E.; Stadler, B.M.; Debatis, M.; Wang, S.; Lu, S.; Kowalik, T.F. RNA interference-mediated targeting of human cytomegalovirus immediate-early or early gene products inhibits viral replication with differential effects on cellular functions. J. Virol. 2012, $86,5660-5673$.

169. Borden, K.L. Pondering the promyelocytic leukemia protein (PML) puzzle: Possible functions for PML nuclear bodies. Mol. Cell. Biol. 2002, 22, 5259-5269.

170. Saffert, R.T.; Kalejta, R.F. Promyelocytic leukemia-nuclear body proteins: Herpesvirus enemies, accomplices, or both? Future Virol. 2008, 3, 265-277.

171. Saffert, R.T.; Kalejta, R.F. Inactivating a cellular intrinsic immune defense mediated by Daxx is the mechanism through which the human cytomegalovirus pp71 protein stimulates viral immediate-early gene expression. J. Virol. 2006, 80, 3863-3871.

172. Sommer, M.H.; Scully, A.L.; Spector, D.H. Transactivation by the human cytomegalovirus IE2 86-kilodalton protein requires a domain that binds to both the TATA box-binding protein and the retinoblastoma protein. J. Virol. 1994, 68, 6223-6231.

173. Choi, K.S.; Kim, S.J.; Kim, S. The retinoblastoma gene product negatively regulates transcriptional activation mediated by the human cytomegalovirus IE2 protein. Virology 1995, 208, 450-456.

174. Speir, E.; Modali, R.; Huang, E.S.; Leon, M.B.; Shawl, F.; Finkel, T.; Epstein, S.E. Potential role of human cytomegalovirus and p53 interaction in coronary restenosis. Science 1994, 265, 391-394.

175. Tsai, H.L.; Kou, G.H.; Chen, S.C.; Wu, C.W.; Lin, Y.S. Human cytomegalovirus immediate-early protein IE2 tethers a transcriptional repression domain to p53. J. Biol. Chem. 1996, 271, 3534-3540.

176. Bonin, L.R.; McDougall, J.K. Human cytomegalovirus IE2 86-kilodalton protein binds p53 but does not abrogate G1 checkpoint function. J. Virol. 1997, 71, 5861-5870.

177. Song, Y.J.; Stinski, M.F. Effect of the human cytomegalovirus IE86 protein on expression of E2F-responsive genes: A DNA microarray analysis. Proc. Natl. Acad. Sci. USA 2002, 99, 2836-2841.

178. Ruger, B.; Klages, S.; Walla, B.; Albrecht, J.; Fleckenstein, B.; Tomlinson, P.; Barrell, B. Primary structure and transcription of the genes coding for the two virion phosphoproteins pp65 and pp71 of human cytomegalovirus. J. Virol. 1987, 61, 446-453. 
179. Hensel, G.M.; Meyer, H.H.; Buchmann, I.; Pommerehne, D.; Schmolke, S.; Plachter, B.; Radsak, K.; Kern, H.F. Intracellular localization and expression of the human cytomegalovirus matrix phosphoprotein pp71 (ppUL82): Evidence for its translocation into the nucleus. J. Gen. Virol. 1996, 77, 3087-3097.

180. Baldick, C.J., Jr.; Marchini, A.; Patterson, C.E.; Shenk, T. Human cytomegalovirus tegument protein pp71 (ppUL82) enhances the infectivity of viral DNA and accelerates the infectious cycle. J. Virol. 1997, 71, 4400-4408.

181. Rechter, S.; Scott, G.M.; Eickhoff, J.; Zielke, K.; Auerochs, S.; Muller, R.; Stamminger, T.; Rawlinson, W.D.; Marschall, M. Cyclin-dependent Kinases Phosphorylate the Cytomegalovirus RNA Export Protein pUL69 and Modulate Its Nuclear Localization and Activity. J. Biol. Chem. 2009, 284, 8605-8613.

182. Marschall, M.; Freitag, M.; Suchy, P.; Romaker, D.; Kupfer, R.; Hanke, M.; Stamminger, T. The protein kinase pUL97 of human cytomegalovirus interacts with and phosphorylates the DNA polymerase processivity factor pUL44. Virology 2003, 311, 60-71.

183. Schregel, V.; Auerochs, S.; Jochmann, R.; Maurer, K.; Stamminger, T.; Marschall, M. Mapping of a self-interaction domain of the cytomegalovirus protein kinase pUL97. J. Gen. Virol. 2007, 88, 395-404.

184. Michel, D.; Kramer, S.; Hohn, S.; Schaarschmidt, P.; Wunderlich, K.; Mertens, T. Amino acids of conserved kinase motifs of cytomegalovirus protein UL97 are essential for autophosphorylation. J. Virol. 1999, 73, 8898-8901.

185. Marschall, M.; Marzi, A.; aus dem Siepen, P.; Jochmann, R.; Kalmer, M.; Auerochs, S.; Lischka, P.; Leis, M.; Stamminger, T. Cellular p32 recruits cytomegalovirus kinase pUL97 to redistribute the nuclear lamina. J. Biol. Chem. 2005, 280, 33357-33367.

186. Winkler, M.; Stamminger, T. A specific subform of the human cytomegalovirus transactivator protein pUL69 is contained within the tegument of virus particles. J. Virol. 1996, 70, 8984-8987.

187. Casavant, N.C.; Luo, M.H.; Rosenke, K.; Winegardner, T.; Zurawska, A.; Fortunato, E.A. Potential role for p53 in the permissive life cycle of human cytomegalovirus. J. Virol. 2006, 80, 8390-8401.

188. E, X.; Savidis, G.; Chin, C.R.; Wang, S.; Lu, S.; Brass, A.L.; Kowalik, T.F. A Novel DDB2-ATM Feedback Loop Regulates Human Cytomegalovirus Replication. J. Virol. 2014, $88,2279-2290$.

189. Hickson, I.; Zhao, Y.; Richardson, C.J.; Green, S.J.; Martin, N.M.; Orr, A.I.; Reaper, P.M.; Jackson, S.P.; Curtin, N.J.; Smith, G.C. Identification and characterization of a novel and specific inhibitor of the ataxia-telangiectasia mutated kinase ATM. Cancer Res. 2004, 64, 9152-9159.

190. Orazio, N.I.; Naeger, C.M.; Karlseder, J.; Weitzman, M.D. The adenovirus E1b55K/E4orf6 complex induces degradation of the Bloom helicase during infection. J. Virol. 2011, 85, $1887-1892$. 
191. Singh, V.V.; Dutta, D.; Ansari, M.A.; Dutta, S.; Chandran, B. Kaposi’s Sarcoma-Associated Herpesvirus Induces the ATM and H2AX DNA Damage Response Early during De Novo Infection of Primary Endothelial Cells, Which Play Roles in Latency Establishment. J. Virol. 2014, 88, 2821-2834.

192. Chang, L.; Godinez, W.J.; Kim, I.H.; Tektonidis, M.; de Lanerolle, P.; Eils, R.; Rohr, K.; Knipe, D.M. Herpesviral replication compartments move and coalesce at nuclear speckles to enhance export of viral late mRNA. Proc. Natl. Acad. Sci. USA 2011, 108, E136-E144.

193. Taylor, T.J.; Knipe, D.M. Proteomics of herpes simplex virus replication compartments: Association of cellular DNA replication, repair, recombination, and chromatin remodeling proteins with ICP8. J. Virol. 2004, 78, 5856-5866.

194. Wilkinson, D.E.; Weller, S.K. The role of DNA recombination in herpes simplex virus DNA replication. IUBMB Life 2003, 55, 451-458.

195. Kaufer, B.B.; Jarosinski, K.W.; Osterrieder, N. Herpesvirus telomeric repeats facilitate genomic integration into host telomeres and mobilization of viral DNA during reactivation. J. Exp. Med. 2011, 208, 605-615.

196. Li, M.; Mizuuchi, M.; Burke, T.R., Jr.; Craigie, R. Retroviral DNA integration: Reaction pathway and critical intermediates. EMBO J. 2006, 25, 1295-1304.

197. Weitzman, M.D.; Carson, C.T.; Schwartz, R.A.; Lilley, C.E. Interactions of viruses with the cellular DNA repair machinery. DNA Repair (Amst) 2004, 3, 1165-1173.

198. Fortunato, E.A.; Dell'Aquila, M.L.; Spector, D.H. Specific chromosome 1 breaks induced by human cytomegalovirus. Proc. Natl. Acad. Sci. USA 2000, 97, 853-858.

199. Kwak, S.; E, X.; Kowalik, T.F. An HCMV virion-associated tegument protein initiates activation of the host DNA damage response to infection. University of Massachusetts Medical School, Worcester, MA, USA, 2014, to be submitted for publication.

200. Millhouse, S.; Su, Y.H.; Zhang, X.; Wang, X.; Song, B.P.; Zhu, L.; Oppenheim, E.; Fraser, N.W.; Block, T.M. Evidence that herpes simplex virus DNA derived from quiescently infected cells in vitro, and latently infected cells in vivo, is physically damaged. J. Neurovirol. 2010, 16, 384-398.

201. Stich, H.F.; Hsu, T.C.; Rapp, F. Viruses and Mammalian Chromosomes. I. Localization of Chromosome Aberrations after Infection with Herpes Simplex Virus. Virology 1964, 22, 439-445.

202. Peat, D.S.; Stanley, M.A. Chromosome damage induced by herpes simplex virus type 1 in early infection. J. Gen. Virol. 1986, 67, 2273-2277.

203. Schramayr, S.; Caporossi, D.; Mak, I.; Jelinek, T.; Bacchetti, S. Chromosomal damage induced by human adenovirus type 12 requires expression of the E1B 55-kilodalton viral protein. J. Virol. 1990, 64, 2090-2095.

204. RW, H.Y.; Oakes, J.E.; Kudler, L. In vitro repair of the preexisting nicks and gaps in herpes simplex virus DNA. Virology 1977, 76, 286-294.

205. Tilton, C.; Clippinger, A.J.; Maguire, T.; Alwine, J.C. Human cytomegalovirus induces multiple means to combat reactive oxygen species. J. Virol. 2011, 85, 12585-12593.

206. Soutoglou, E.; Misteli, T. Activation of the cellular DNA damage response in the absence of DNA lesions. Science 2008, 320, 1507-1510. 
207. McVoy, M.A.; Adler, S.P. Human cytomegalovirus DNA replicates after early circularization by concatemer formation, and inversion occurs within the concatemer. J. Virol. 1994, 68, $1040-1051$.

208. E, X.; Kowalik, T.F. A role for host DNA polymerases in the replication of herpesviral DNA. University of Massachusetts Medical School, Worcester, MA, USA, 2014, to be submitted for publication.

209. Zhou, B.B.; Elledge, S.J. The DNA damage response: Putting checkpoints in perspective. Nature 2000, 408, 433-439.

210. Cuchet-Lourenco, D.; Vanni, E.; Glass, M.; Orr, A.; Everett, R.D. Herpes simplex virus 1 ubiquitin ligase ICP0 interacts with PML isoform I and induces its SUMO-independent degradation. J. Virol. 2012, 86, 11209-11222. 
Reprinted from Viruses. Cite as: Kulkarni, A.S.; Fortunato, E.A. Modulation of Homology-Directed Repair in T98G Glioblastoma Cells Due to Interactions between Wildtype p53, Rad51 and HCMV IE1-72. Viruses 2014, 6, 968-985.

Article

\title{
Modulation of Homology-Directed Repair in T98G Glioblastoma Cells Due to Interactions between Wildtype p53, Rad51 and HCMV IE1-72
}

\author{
Amit S. Kulkarni ${ }^{1}$ and Elizabeth A. Fortunato ${ }^{2, *}$ \\ 1 Tumorvirologie (F010), Deutsches Krebsforschungszentrum, Im Neuenheimer Feld 242, 69120, \\ Heidelberg, Germany; E-Mail: amitkulkar@gmail.com \\ 2 Department of Biological Sciences and the Center for Reproductive Biology, University of \\ Idaho, 875 Perimeter Drive, Mailstop 3051, Moscow, ID 83844, USA \\ * Author to whom correspondence should be addressed; E-Mail: 1fort@uidaho.edu; \\ Tel.: +1-208-885-6966; Fax: +1-208-885-7905.
}

Received: 24 January 2014; in revised form: 15 February 2014 / Accepted: 17 February 2014 / Published: 26 February 2014

\begin{abstract}
Human cytomegalovirus (HCMV) is a ubiquitous pathogen capable of causing life threatening consequences in neonates and immune-compromised individuals. HCMV inflicts site-specific double strand breaks (DSBs) in the cellular genome. DNA damage infliction raises the corollary question of virus modulation of DNA repair. We recently reported HDR was stimulated in wt human foreskin fibroblasts (HFFs) during fully permissive infection or expression of the HCMV protein IE1-72 (IE72). These studies have been extended into semi-permissive T98G glioblastoma cells. T98Gs encode a mutant p53, which may contribute to their high baseline rate of HDR. We fully expected HCMV infection to increase HDR in T98Gs, similar to its effects in HFFs. Surprisingly in T98Gs HCMV infection, or sole expression of IE72, decreased HDR by two-fold. Transient expression of wt p53 in T98Gs also reduced HDR by two-fold. Dual transient expression of wt p53 and IE72 restored high baseline HDR levels. GST pulldown experiments revealed that both IE72 and wt p53 bound the important HDR protein, Rad51. We conclude that the expression of certain HCMV proteins can modulate HDR in an infected cell, dependent upon p53 status. We propose a model of the protein interactions explaining this behavior.
\end{abstract}

Keywords: human cytomegalovirus; homology directed repair; p53; IE72; Rad51 


\section{Introduction}

Human cytomegalovirus (HCMV) is a member of the $\beta$-herpesvirus family and is endemic in the human population. HCMV can cause congenital birth defects, primarily of neurological origin [1-3]. It also causes severe systemic disease following reactivation in immunosuppressed individuals [4]. The virus' role in oncomodulation of cancers has also recently been the subject of intensive investigation (for review see [5,6]).

One of the direct effects of HCMV infection in human foreskin fibroblasts (HFFs), is the induction of two site-specific breaks at chromosome 1q42 and 1q23.3 in the host cellular DNA [7,8]. The physiological significance of HCMV-induced specific breaks or whether they can be repaired is unknown. However, the proximity of the 1q23.3 breakpoint to two hearing impairment (HI) loci, DFNA49 and DFNA7, and in the vicinity of the myelin protein zero (MPZ) gene [9], prompts speculation on this break's correlation with the hearing loss observed in congenitally infected infants.

Mammalian cells are exposed to both intrinsic (i.e., reactive oxygen species) and extrinsic (i.e., genotoxic chemicals) agents capable of inflicting cellular DNA damage. DNA damage triggers a cascade of complex protein signaling pathways. This combination of pathways constitutes the DNA damage response (DDR). Depending on the type and extent of DNA damage different repair pathways are activated. In the case of double-stranded breaks (DSBs), two repair pathways are principally activated (as reviewed in [10,11]). One is the error free homology-directed repair (HDR) pathway, which commonly involves strand invasion of a sister chromatid. The other pathway utilizes the more error-prone non-homologous end-joining (NHEJ) or single-strand annealing mechanisms.

We recently reported interactions between HCMV and HDR in permissive HFFs [12]. HCMV infection caused a two-fold increase in HDR of an I-SceI induced site-specific DNA DSB, from a baseline level of $4 \%$ to a stimulated level of $8 \%$. Expression of the HCMV IE72 protein on its own produced comparable results. Here the same DSB repair assay has found that HCMV regulates HDR in the semi-permissive T98G cells in a surprising and counterintuitive manner, hinging on the p53 status of these cells. A model to explain the interaction of HCMV IE72 with p53 and the HDR machinery is proposed.

\section{Results}

\subsection{Introduction of the pDRGFP Substrate into T98G Cells to Assess HDR}

Our earlier study assessed whether HCMV infection affected HDR of an integrated substrate at I-SceI-induced DSBs in fully permissive HFFs. This work found that HDR, as measured by an increase in $\mathrm{GFP}^{+}$cells, was stimulated in HFFs during HCMV infection and, further, that expression of the HCMV protein IE72 on its own also stimulated HDR [12]. The assay utilized a non-functional GFP reporter substrate susceptible to DSBs upon introduction of I-SceI [13,14] (see Figure 1A for diagram of the substrate). If the DSB was successfully repaired by HDR, functional GFP was reconstituted. HDR was scored by microscopic analysis for cellular $\mathrm{GFP}^{+}$. These fibroblast experiments have now been extended into the semi-permissive cell line T98G. Three different stable clones of T98Gs with an integrated pDRGFP substrate were generated. The results 
obtained using all clones were consistent, therefore here we report only representative studies carried out in Clone 10.

Clone 10 was synchronized in $\mathrm{G}_{0}$ and then infected with an Adenovirus (Ad) expressing the I-SceI endonuclease (NGUS24i), or an Ad control virus (dL70-3) [15,16] at various MOIs (5, 10, 25, 50, 100, and 200) or were mock-infected. Cells were analyzed for efficiency of I-SceI endonuclease production after NGUS24i infection. The I-SceI endonuclease in this virus is tagged with hemagglutinin (HA), permitting detection of protein expression using an anti-HA Ab by IF. $100 \%$ of the cells infected with NGUS24i displayed HA-positivity and no staining was observed in mock-infected cells (Figure 1B) or dL70-3-infected cells (data not shown).

NGUS24i infection produced $\mathrm{GFP}^{+}$cells. This indicated I-SceI-induced site-specific DNA breaks had been successfully repaired by HDR. In our hands NGUS24i infection effects were greatest using an MOI of 200 and continuous incubation for $72 \mathrm{~h}$ prior to harvest. Clone 10 was also examined for spontaneous HDR events. Figure 1B displays representative experiments depicting the absence of any $\mathrm{GFP}^{+}$cells in mock-infected Clone 10. These results demonstrated that the Ad-based enzyme delivery system in conjunction with the integrated pDRGFP substrate worked as well in the T98G cells as had been previously observed in the HFFs [12].

Figure 1. Efficient expression of I-SceI from an Adenoviral vector based system in T98Gs with an integrated pDRGFP substrate. (A) Schematic diagram of reporter plasmid pDRGFP as described in [12]. (B) Representative IF staining of Ad-infected Clone 10. Cells were seeded onto glass coverslips and infected with Ad expressing I-SceI (NGUS24i). Coverslips were harvested at $72 \mathrm{~h}$ post infection. $\mathrm{GFP}^{+}$cells were scored as HDR competent. Cells were stained with HA-specific Ab to detect HA-I-SceI expressed from NGUS24i. Scale bar $=5 \mu \mathrm{m}$ for all panels and all figures.

A.

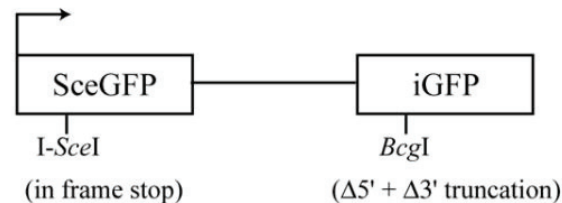

B.

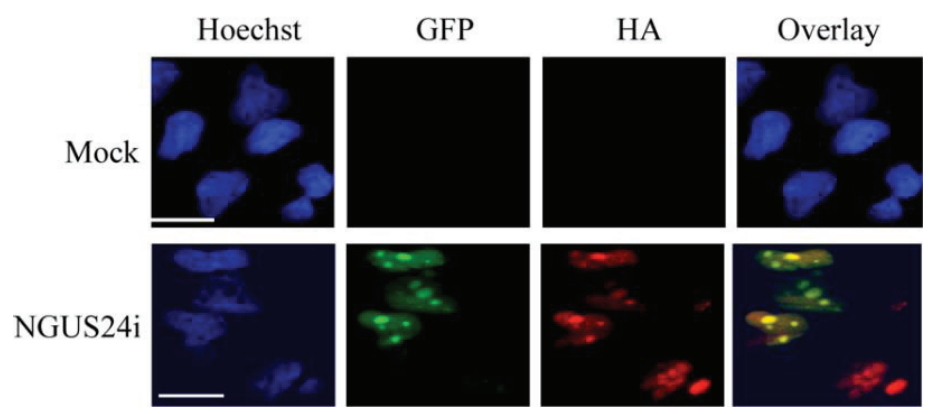

2.2. HDR at I-SceI Induced DSBs Was Downregulated in HCMV-Infected T98G Cells

Previously we showed that in a semi-permissive infection of T98G cells, although all cells take up virus, only a modest percentage $(\sim 30 \%-40 \%)$ of cells express IE72 antigen (Ag) in contrast to 
$100 \% \mathrm{Ag}^{+}$cells in a fully permissive infection [17]. Clone 10 was $\mathrm{G}_{\mathrm{o}}$ synchronized, released from synchronization and then mock-infected or HCMV-infected at an MOI of 10 for $48 \mathrm{~h}$. These cells were subsequently superinfected with NGUS24i or dL70-3 and harvested at $72 \mathrm{~h}$ post Ad infection. Cells were stained for the expression of HCMV IE72 by IF to monitor initiation and progression of HCMV infection. Approximately $30 \%$ of cells were IE72 ${ }^{+}$at $120 \mathrm{~h}$ post infection (hpi), which agreed with previous experiments [17]. Cells were also scored for $\mathrm{GFP}^{+}$. Representative IF images for these infections are shown in Figure 2A.

Figure 2. HCMV infection decreased HDR in T98Gs in the presence of stably integrated pDRGFP substrate. Cells were seeded onto glass coverslips and infected with HCMV or Ad individually or dually with HCMV and Ad in succession. Coverslips were harvested at $72 \mathrm{~h}$ post Ad superinfection with either I-SceI expressing (NGUS24i) or control (dL70-3) virus. $\mathrm{GFP}^{+}$cells were scored as HDR competent. Clone 10 was stained by IF for the presence of IE72. (A) Representative IF staining for $\mathrm{GFP}^{+}+\mathrm{IE}^{+}$ cells in Clone 10. (B) Percentage of $\mathrm{GFP}^{+}$cells in Clone 10 after infection. (C) Representative protein profile for steady state levels of HA-tagged I-SceI in Clone 10 infected with NGUS24i alone or dually infected with HCMV and NGUS24i. Actin was used as a loading control. For IF studies at least 300 cells were scored for each type of infection. Values on top of the bar indicate fold decrease in percent of $\mathrm{GFP}^{+}$cells in dually infected versus NGUS24i alone experiments. Error bars represent one SD.

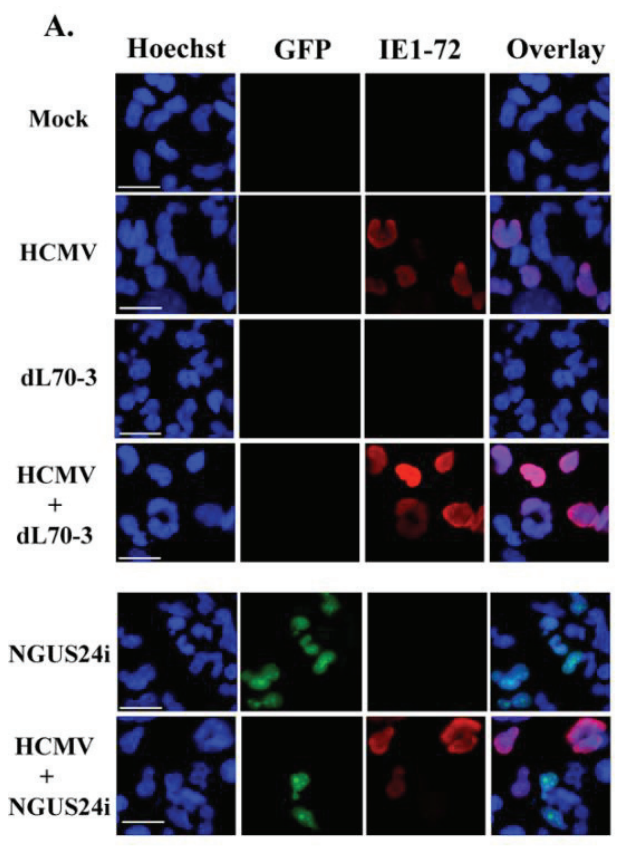

B.

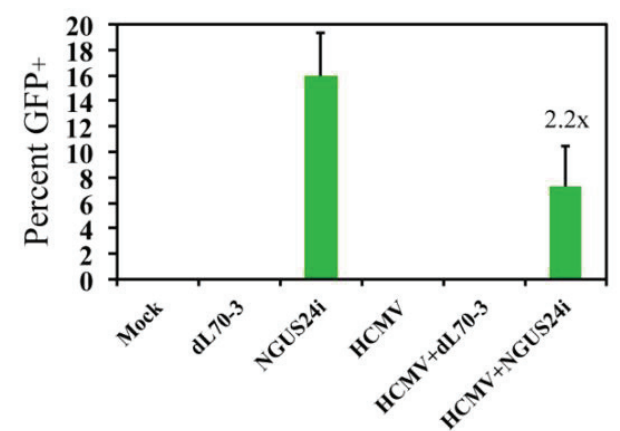

C.

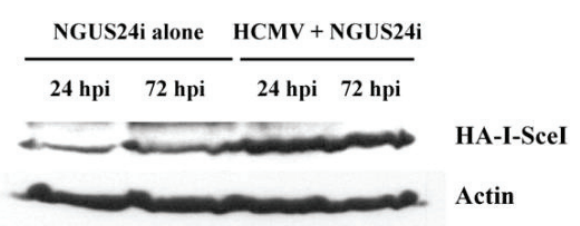

T98Gs displayed a very high baseline rate of HDR after DSB induction with I-SceI. Ad NGUS24i infection resulted in $\sim 16 \% \mathrm{GFP}^{+}$cells (as compared to $4 \%$ in HFFs) [12]. No $\mathrm{GFP}^{+}$cells were observed in either dL70-3-infected or mock-infected cells. An average of $\sim 7 \% \mathrm{GFP}^{+}$cells were detected in HCMV-infected cells subsequently superinfected with NGUS24i to induce DSBs, an $\sim 2$ fold decrease (Figure 2B). Although not quite reaching a degree of statistical significance, this decrease was consistently reproducible (fold changes of 2.7 and 1.9 in two experiments). 
Downregulation of HDR due to HCMV infection was not associated with changes in steady state levels of I-SceI expression. Immunoblot analysis found no decrease in I-SceI expression in HCMV-infected T98Gs compared to mock-infected T98Gs (Figure 2C). Actually, somewhat the reverse was found with slightly higher levels of I-SceI observed in lysates derived from an equivalent number of virus-infected cells. Thus lower expression of the I-SceI enzyme was not the cause of the somewhat paradoxical decrease in HDR.

\subsection{Transient Expression of IE72 Alone Was Sufficient to Downregulate HDR in T98Gs}

A number of studies have suggested that IE72 plays a vital role in fine tuning the host cell, including activating early gene expression needed for viral replication ([18] and as reviewed in [19]). We had previously shown that expression of IE72 alone was sufficient to enhance HDR in HFFs [12]. Having found that full HCMV infection reduced HDR in T98Gs, we repeated the IE72 expression assays in these cells. Parallel experiments to those above were performed in Clone 10 nucleofected with vector-alone (pSG5) or an IE72-expressing construct (pSGIE72). These cells were then Ad infected. Cells were subsequently scored for IE72 and GFP positivity. Approximately $90 \%$ cells were $\mathrm{IE}^{+}{ }^{+}$(data not shown).

No $\mathrm{GFP}^{+}$cells were observed in vector alone (pSG5), pSG5 + dL70-3-infected (control Ad), IE72 alone or IE72 + dL70-3-infected cells. In close agreement with the previous results an average of $\sim 16 \% \mathrm{GFP}^{+}$cells were detected in cells nucleofected with pSG5 and NGUS24i-infected (I-SceI expressing Ad). In cells nucleofected with the IE72 construct and NGUS24i-infected, an average of $9 \% \mathrm{GFP}^{+}$cells were scored, a 1.8 fold decrease (Figure 3A). This decrease was approximately equivalent to the reduction in $\mathrm{GFP}^{+}$of $\mathrm{HCMV}$-infected cells. Again, although this decrease was not statistically significant, it was consistently reproducible (fold changes of 1.9 and 1.5 in two experiments). Therefore, as was seen in HFFs, transient expression of IE72 alone largely recapitulated the HCMV-infection results, however, with the same perplexing downregulation of HDR in T98Gs.

\subsection{Expression of wt p53 in T98Gs Caused the Same Decrease in HDR as IE72}

T98Gs have a mutagenic profile and harbor a mutation in $\mathrm{p} 53$ at $\mathrm{R} 273 \mathrm{H}$, in the DNA binding domain [20]. It is widely accepted that wt p53 suppresses HDR (as reviewed in [21]). Was the mutation in p53 responsible for the elevated levels of $\operatorname{HDR}(\sim 16 \%$, Figure $2 \mathrm{~B})$ in uninfected T98Gs compared to HFFs $(\sim 4 \%$, [12])? If so, introduction of wt p53 would be expected to decrease HDR levels in uninfected T98G cells and, similarly, p53 DNA binding domain mutants would not be expected to alter HDR levels. Experiments parallel to those discussed for IE72 were performed in Clone 10. It should be noted that although all the constructs used in this study were GFP-tagged, the wt p53 and the mutant p53 genes were expressed from the weak wt p53 promoter and, as we have previously observed [22], no detectable GFP expression above background was observed in control populations of these cells (data not shown). Clone 10 was nucleofected with vector-alone (pCDNA3-T7) or constructs [22] expressing wt p53, a non-acetylatable p53 mutant (K382R), p53 DNA binding domain mutants (R273H, R175H, R248W, G154V), or a p53 N-terminal multi-site phosphorylation mutant and subsequently Ad-infected. Cells were scored for $\mathrm{GFP}^{+}$(Figure $3 \mathrm{~B}$ ). 
Figure 3. Nucleofection with IE72 or wt p53 alone decreased HDR, whereas co-expression did not alter high level HDR in T98Gs. Cells were nucleofected with the indicated plasmids and then infected with either I-SceI expressing (NGUS24i) or control (dL70-3) Ad. Coverslips were harvested at 40 h post Ad infection. GFP ${ }^{+}$cells were scored as HDR competent. (A) Percentage of $\mathrm{GFP}^{+}$in Clone 10 after nucleofection of IE72 or pSG5 control. (B) Percentage of $\mathrm{GFP}^{+}$in Clone 10 nucleofected with either wt p53 or p53 mutants (K382R, N-term, R273H, R175H, R248W, G154V). (C) Percentage of $\mathrm{GFP}^{+}$in Clone 10 nucleofected with wt p53 and/or IE72. At least 300 cells were scored for each type of infection. Error bars represent one SD. Values on top of the bars indicate fold decrease (or increase for the dual IE72/wt p53 nucleofection) in percent of $\mathrm{GFP}^{+}$cells. *, ** and *** indicate increasing levels of statistical significance (see text for details). Error bars represent one SD.

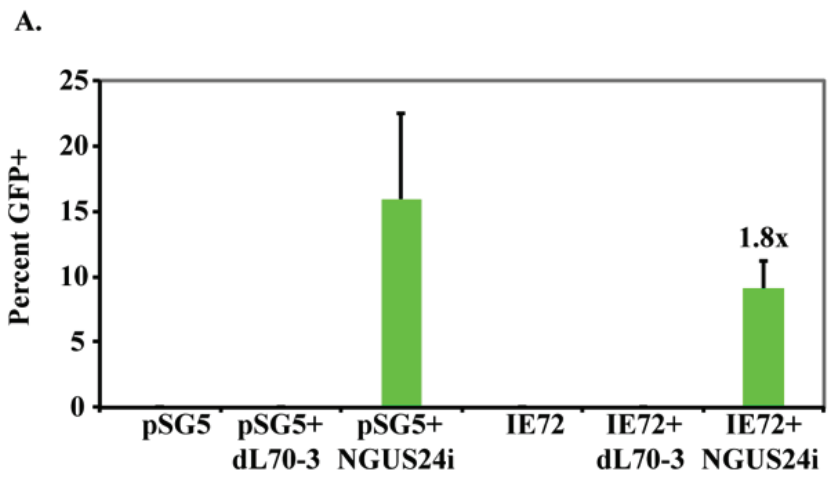

B.

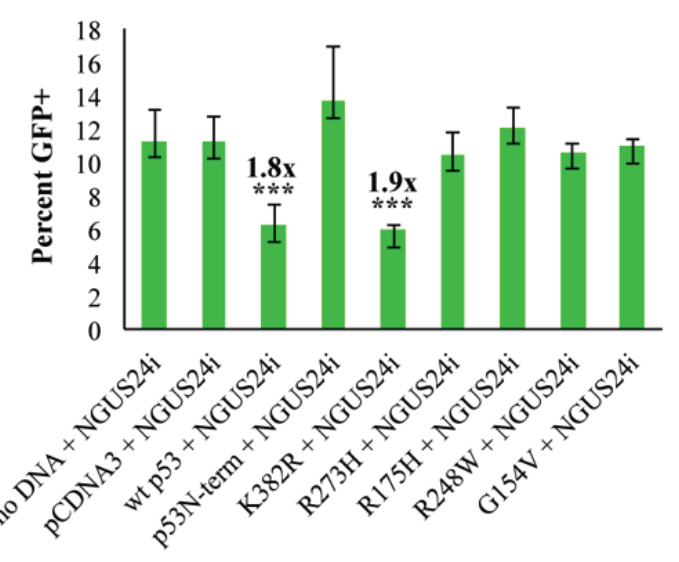

C.

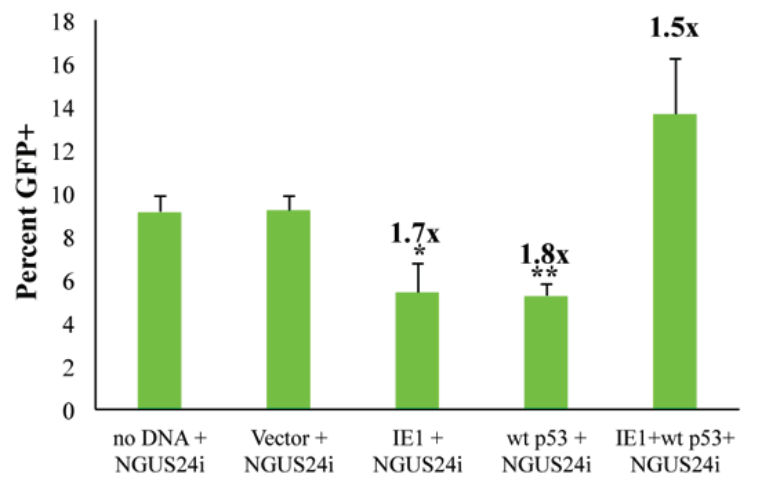


Immediately evident was that introduction of even low levels of wt p53 into T98Gs resulted in accelerated cell death, with very few cells surviving until $72 \mathrm{~h}$ post Ad infection. The infection protocol was modified to overcome this technical problem. Ad infection MOIs were reduced from 200 to 5. An MOI of 5 still delivered I-SceI enzyme to the entire population, as detected by $\mathrm{HA} \mathrm{Ab}$ staining (data not shown), however the lower MOI reduced baseline DSB-induced HDR events to a more modest, but still prevalent, $\sim 11 \%$. More importantly, the reduced MOI allowed sufficiently high cell survival for a long enough duration to obtain meaningful population counts. Eight hours post-nucleofection, cells were Ad infected and $\mathrm{GFP}^{+}$was scored at $40 \mathrm{~h}$ post $\mathrm{Ad}$ infection. Following this modified protocol, control experiments using any of the constructs followed by infection with the dL70-3 control virus showed no $\mathrm{GFP}^{+}$cells (data not shown). Nucleofection with no DNA, or introduction of vector alone (pCDNA3) followed by I-SceI-expressing NGUS24i-infection produced an average $\sim 11 \% \mathrm{GFP}^{+}$cells (Figure $3 \mathrm{~B}$ ). Cells nucleofected with wt p53 or the p53 K382R mutant produced an average of $\sim 6 \% \mathrm{GFP}^{+}$, an $\sim 2$ fold decrease. This decrease was consistently reproducible and highly statistically significant for both constructs ( $p$ value $<0.0001$ and 0.0004 , respectively). The introduction of the p53 DNA binding domain mutants $(\mathrm{R} 273 \mathrm{H}, \mathrm{R} 175 \mathrm{H}, \mathrm{R} 248 \mathrm{~W}, \mathrm{G} 154 \mathrm{~V})$, or the p53 N-terminal multi-site phosphorylation mutant yielded no change in the percent of $\mathrm{GFP}^{+}$from the vector alone. Thus, introduction of wt p53 into the T98G cells suppressed the high level HDR allowed by the endogenous mutant p53 [20].

\subsection{Co-Expression of IE72 and wt p53 Negated Their Individual Effects on HDR}

Surprisingly, in the T98G microenvironment, expression of either IE72 or wt p53 reduced HDR, therefore we tested co-introduction of both proteins. Using the modified protocol described above, once again control experiments using dL70-3 virus produced no $\mathrm{GFP}^{+}$cells (data not shown). We observed that nucleofection with no DNA, or introduction of backbone vector (pCDNA3) followed by I-SceI-expressing NGUS24i produced an average $\sim 9 \% \mathrm{GFP}^{+}$cells. Introduction of either IE72 or wt p53 alone reduced $\mathrm{GFP}^{+}$to $\sim 5 \%$, an $\sim 2$ fold decrease as expected, both of which were statistically significant ( $p=0.01$ and $p=0.0014$, respectively). Much to our surprise, dual introduction of IE72 and wt p53 produced an average of $13.6 \% \mathrm{GFP}^{+}$cells, an $\sim 1.4$ fold increase from the baseline rate of $\sim 9 \%$, an increase that was only marginally statistically significant $(p=0.043)$ (fold changes of 1.3, 1.7, 1.5 in three experiments) (Figure 3C). This suggested that in T98G cells interaction between wt p53 and IE72 negated their individual effects on the HDR machinery.

\subsection{In Vitro Binding Assays Found Both wt p53 and IE72 Bound Rad51}

T98G cells harbor a mutant p53 (R273H mutation) [20]. The mutation abolishes specific DNA binding. The p53 transient expression experiments established that an intact DNA binding domain and phosphorylatable N-terminus were required to decrease HDR in these cells. Previous studies have determined that recombination is controlled, at least in part, by p53 binding to the strand invasion protein Rad51, which modulates Rad51's function (as reviewed in [21]). An intact DNA binding domain in the p53 protein is required for this interaction [23-25]. Further, T98G cells mount a DSB response [20] and express high levels of the DSB repair proteins, including 
Rad51 [20,26]. Previous work had established that p53 could bind both Rad51 and IE72 [27], and that interaction between $\mathrm{p} 53$ and IE72 could negate p53's normal DNA binding ability through its core region [27]. This information suggested that the effects noted in the above transient expression and co-expression experiments might be the result of IE72 binding either wt p53 or Rad51. In vitro mixing experiments of radiolabeled Rad51 and pGEX72, GST wt p53 or pGEX-KG (control GST alone) were performed. As can be seen in Figure 4, both p53 and IE72 were capable of binding Rad51, although IE72 appeared to be slightly less avid for the protein. While not excluding other possible explanations, this binding, in combination with the results from the expression experiments, have prompted us to propose a protein interaction model capable of explaining the observed behaviors (see Figure 5 below). This model may have significant bearing on questions entirely unrelated to HCMV-infection in the semi-permissive cell type T98G.

Figure 4. GST pulldown experiments revealed IE72 and wt p53 bound to Rad51. GST proteins were incubated with radiolabeled Rad51 for $1 \mathrm{~h}$ as described in experimental details. Input lanes represent $1 / 10$ th the total starting reaction. Negative controls are incubations with in vitro translation mixture containing an equivalent amount of radioactivity.

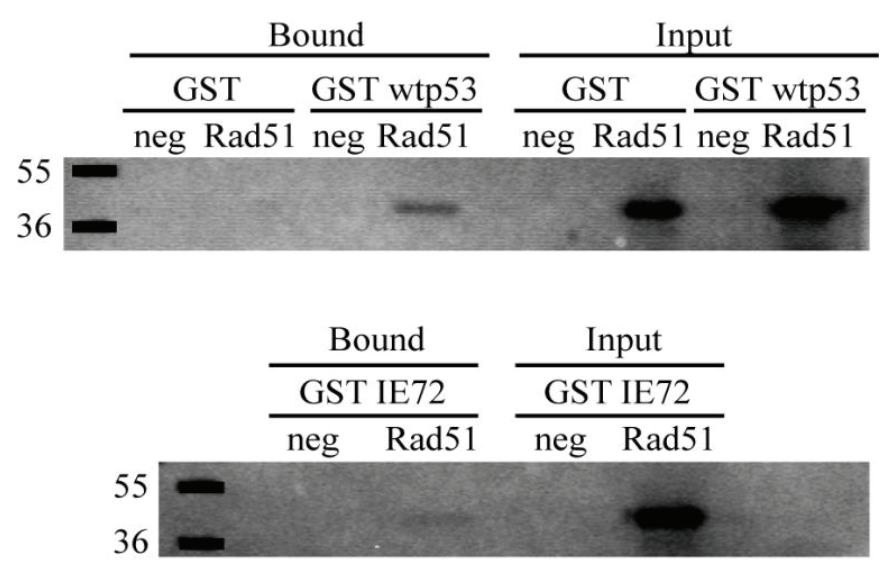

\section{Experimental Section}

\subsection{Cells and Virus Growth}

T98G glioblastoma cells and clones expressing pDRGFP were propagated in Earle's minimal essential media (MEM) supplemented with 10\% heat-inactivated fetal bovine serum (FBS), L-glutamine $(2 \mathrm{mM})$, penicillin $(200 \mathrm{U} / \mathrm{mL})$, streptomycin $(200 \mu \mathrm{g} / \mathrm{mL})$, and amphotericin B $(1.5 \mu \mathrm{g} / \mathrm{mL})$. Cells were grown in incubators maintained at $37{ }^{\circ} \mathrm{C}$ and $5 \% \mathrm{CO}_{2}$. The Towne strain of HCMV was obtained from the ATCC (\# VR 977), propagated under standard procedures and used at a multiplicity of infection (MOI) of 10 in all experiments. The recombinant Ad strains dL70-3, used as a control virus, and NGUS24i, encoding the I-SceI enzyme, (both kind gifts of Frank Graham and Philip Ng, McMaster University, Hamilton, ON, Canada $[15,16]$ ) were grown and titrated in replication-permissive human 293A cells. The MOI of Ad viruses used was established separately depending on experimental procedure (see above for particular details). 


\subsection{Transfections}

Lipofectamine reagent (Invitrogen/Life Technologies, Grand Island, NY, USA) was used according to manufacturer's instructions to introduce pDRGFP [13,14] into T98Gs. Following transfection, cells were grown in non-selective medium for $48 \mathrm{~h}$. Subsequently, puromycin $(1 \mu \mathrm{g} / \mathrm{mL})$ was added to the media to select for integrated pDRGFP. Cloning rings were used to isolate puromycin-resistant colonies. Colonies were separated and subjected to another cycle of puromycin selection prior to freezing and storage in liquid nitrogen. All colonies were tested to ensure the absence of $\mathrm{GFP}^{+}$without DSB induction. Three stable clones of T98GpDRGFP were tested and produced comparable results in the assays described above. T98GpDRGFP clone 10 (Clone 10) was used for all experiments reported here.

\subsection{Nucleofections}

Nucleofection of T98Gs was optimized using a protocol provided by Amaxa Biosystems (program O-016). Each nucleofection used $\sim 5 \times 10^{5}$ cells. IE72 transient expression studies used either $6 \mu \mathrm{g}$ of control vector pSG5 (Stratagene/Agilent Technologies, Santa Clara, CA, USA) or $6 \mu \mathrm{g}$ of pSGIE72 [28]. p53 transient expression experiments used the following constructs in the listed quantities: $6 \mu \mathrm{g}$ of vector pCDNA3-T7 alone (with the CMV promoter removed, as a control) or $6 \mu \mathrm{g}$ of p53 derivative vectors containing (1) a wt p53 promoter-wt p53-green fluorescent protein (GFP) cassette (wtp53pCDNA3-T7) or (2) an equivalent vector cassette containing one of the following p53 mutations: K382R, R273H, R175H, R248W, G154V or an N-terminal multi-site phosphorylation mutant [29]. All of these have been previously described [22]. After nucleofection, cells were immediately transferred to plates containing coverslips and pre-warmed medium.

\subsection{Molecular Cloning}

The pCDNA3-T7 vector was modified by insertion into its polylinker of a BamHI-NotI fragment containing DsRed2 (from pDsRed2-N1vector; Clontech Laboratories, Mountain View, CA, USA) to produce pCDNA3-DsRed. The wt p53 promoter-wt p53 coding sequence was cleaved from pCLNCX-p53pro-p53GFP [22] at the KpnI and AgeI sites. Finally, this fragment was inserted upstream of DsRed2 in the polylinker of pCDNA3-DsRed. This ultimately produced the pCDNA3-p53pro-p53DsRed construct. Dual transient expression studies with wt p53 and IE72 used $3 \mu \mathrm{g}$ each of pCDNA3-p53pro-p53DsRed and pSGIE72. Alternatively, one of these constructs $(3 \mu \mathrm{g})$ was transfected along with the corresponding control backbone of the other construct $(3 \mu \mathrm{g})$.

\subsection{DSB Repair Assay}

The DSB repair assay was carried out as described previously [12]. The results reported were carried out in mock- or HCMV-infected Clone 10. 


\subsection{Virus Infection}

Clone 10 was synchronized in $G_{o}$ by serum starvation for $3 \mathrm{~d}$. Cells were then washed with PBS, trypsinized and re-plated at a density of 5 or $10 \times 10^{5}$ cells/60-mm dish containing glass coverslips. After allowing $2 \mathrm{~h}$ for attachment, the cells were mock- or HCMV-infected. Twenty-four hours later the virus or mock inoculums were removed (unless otherwise noted). After an additional $24 \mathrm{~h}$, cells were washed once with PBS and subsequently superinfected for 30 minutes in pre-warmed media. Super-infection was with control Ad dL70-3 or with I-SceI-expressing Ad NGUS24i [12]. Cells were then re-fed with complete media. Cells were fixed and permeabilized $72 \mathrm{~h}$ later unless otherwise noted. Coverslips were scored for $\mathrm{GFP}^{+}$cells and $\mathrm{IE}^{+} 2^{+}$cells.

\subsection{Antibodies (Ab)}

Primary mouse monoclonal antibodies $(\mathrm{mAbs})$ used were: anti-pan actin $\left(\mathrm{IgG}_{1}\right)$ (Neomarkers/Thermo Scientific, Fremont, CA, USA); anti-hemagglutinin (HA)(12CA5) $\left(\operatorname{IgG}_{2 b}\right)\left(\right.$ Abcam, Cambridge, MA, USA); anti-IE1 ( $\operatorname{IgG}_{2 \mathrm{a}}$ ) (a kind gift from Bill Britt, University of Alabama, Birmingham, AL, USA); and anti-IE1 and -IE2 (Ch16.0; $\mathrm{IgG}_{1}$ ) (Virusys Corporation, Taneytown, MD, USA). Secondary Abs used were as follows: for immunoblot detection, horseradish peroxidase (HRP)-linked sheep anti-mouse (GE Healthcare Life Sciences, Pittsburgh, PA, USA), and for IF analysis, tetramethyl rhodamine isothiocyanate (TRITC)-conjugated anti-mouse $\operatorname{IgG}_{1}$, $\mathrm{IgG}_{2 \mathrm{a}}$ and $\mathrm{IgG}_{2 \mathrm{~b}}$ (Jackson ImmunoResearch Laboratories, West Grove, PA, USA).

\subsection{Immunofluorescence (IF)}

Cells were seeded into dishes containing glass coverslips. Coverslips were collected and processed at indicated times pi, as described previously [30]. 300-500 cells per coverslip were counted and scored for $\mathrm{GFP}^{+}$and either $\mathrm{HA}^{+}$or $\mathrm{IE}^{+} 2^{+}$cells in each experiment. All experimental results represent the average of at least two independent experiments. Error bars represent $+/-$ one standard deviation (SD). Statistical analysis was performed using unpaired, two-tailed student t-tests.

\subsection{Immunoblotting}

Virus- and mock-infected cells were harvested at 48, 72, and 96 hpi and lysates were processed as previously described $[22,31]$.

\subsection{GST Binding Studies}

Overnight bacterial cultures (100 $\mathrm{mL}$ in $\mathrm{LB}+100 \mu \mathrm{g} / \mathrm{mL}$ ampicillin) containing the GST fusion plasmids pGEX-human p53 (1-393) (Addgene plasmid \#24860; [32]), pGEX72 [28] or pGEX-KG [33] were diluted 1:10 (to a final volume of $1 \mathrm{~L} \mathrm{LB} / \mathrm{amp}$ ) and incubated an additional $1 \mathrm{~h}$ at $37{ }^{\circ} \mathrm{C}$ with shaking. Cultures were chilled to room temperature (RT), then $1 \mathrm{~mL} 0.5 \mathrm{M}$ IPTG was added. After an additional incubation at RT for $2 \mathrm{~h}$, bacteria were pelleted at $5000 \mathrm{rpm}$ for $10 \mathrm{~min}$ and cells were resuspended in $50 \mathrm{~mL} \mathrm{NETN} \mathrm{+} \mathrm{protease} \mathrm{inhibitors} \mathrm{(PIs)}(20 \mathrm{mM}$ Tris $\mathrm{pH} 8,100 \mathrm{mM} \mathrm{NaCl}, 1 \mathrm{mM}$ EDTA, $0.5 \% \mathrm{NP} 40,2 \mu \mathrm{g} / \mathrm{mL}$ aprotinin, $2 \mu \mathrm{g} / \mathrm{mL}$ leupeptin, $100 \mathrm{mM}$ DTT). This suspension was then frozen at $-80{ }^{\circ} \mathrm{C}$ in $5 \mathrm{~mL}$ aliquots for future use. 
Assays were performed using one of the $5 \mathrm{~mL}$ aliquots thawed on ice. Fifty $\mu \mathrm{L}$ of a $10 \mathrm{mg} / \mathrm{mL}$ lysozyme solution was added and the cell suspension was incubated on ice for $30 \mathrm{~min}$. The suspension was sonically disrupted to lyse the cells. Debris was pelleted at 10,000 rpm for 5 min and the supernatant transferred to a new tube. Sixty $\mu \mathrm{L}$ of a $50 \%$ glutathione agarose bead slurry (vol/vol in NETN) was added and rocked at $4{ }^{\circ} \mathrm{C}$ for $1 \mathrm{~h}$. Beads were then washed in $500 \mu \mathrm{L}$ $\mathrm{NETN}+$ PIs three times prior to final re-suspension in $500 \mu \mathrm{L} \mathrm{NETN}+$ PIs. Fifty $\mu \mathrm{L}$ of this bead suspension ( $25 \mu \mathrm{L}$ bead equivalents) were incubated with $20 \mu \mathrm{L}$ Laemmli reducing sample buffer (2\% SDS, 10\% glycerol, $100 \mathrm{mM}$ DTT, $60 \mathrm{mM}$ Tris $\mathrm{pH}$ 6.8, Bromophenol blue dye, aprotinin and leupeptin $(2 \mu \mathrm{g} / \mathrm{mL}$ each) $)$, and then boiled for $5 \mathrm{~min}$. The resulting supernatant was loaded onto a $12 \%$ SDS-PAGE gel. The gel was stained with Coomassie dye to visualize the GST fusion proteins and to determine equivalent protein amounts for use in the following binding reactions. Roughly equivalent amounts of protein, as estimated from the Coomassie staining, were used in the reactions (approximately $30 \mu \mathrm{L}$ beads of pGEX72 and pGEX-p53 and $7.5 \mu \mathrm{L}$ of pGEX-KG were used in the reactions shown in Figure 4).

Pet24d-Rad51 [34] was in vitro translated using the Promega quick-coupled TNT reaction kit per manufacturer's instructions. In each binding assay, five $\mu \mathrm{L}$ of translated Rad51 (or just TNT reaction mixture) was incubated while rocking for $1 \mathrm{~h}$ at $4{ }^{\circ} \mathrm{C}$ with the GST fusion proteins in a 500 $\mu \mathrm{L}$ reaction of NETN + PIs. After incubation, $40 \mu \mathrm{L}$ of a $50 \%$ Protein A Sepharose slurry (vol/vol in NETN) was added to each tube (to increase the bead volume). Ten percent of the total volume $(54 \mu \mathrm{L})$ was removed to serve as the input fraction (no washes of these beads followed). The beads in the remaining sample were pelleted and then washed four times with $500 \mu \mathrm{L}$ of buffer (the first two washes in NETN, the last two in RIPA ( $150 \mathrm{mM} \mathrm{NaCl}, 1 \%$ Nonidet P-40, $0.5 \%$ deoxycholate, $0.1 \%$ SDS, $50 \mathrm{mM}$ Tris (pH 8), $5 \mathrm{mM}$ EDTA). After the final spin, $15 \mu \mathrm{L}$ of urea sample buffer was added to the beads and the samples were boiled for $5 \mathrm{~min}$. The supernatants were run on a $12 \%$ SDS-PAGE gel. Gels were dried and exposed to Kodak X-omat film.

\section{Conclusions}

A cell's ability to repair insult to its DNA is a process that is essential to its survival. The concept that virus infection may alter this capability has begun to be investigated. In theory a virus might benefit from manipulation of the repair processes, while simultaneously (and likely corollary) degrading the integrity of a host's genome. Existing studies have examined a variety of different repair pathways in the context of expression of a single viral protein and have assessed that protein's capability to influence the repair of exogenously introduced damage in the cellular DNA [35-46]. The effects on repair during a complete infection have also been examined [12,47-56]. A handful of these studies have looked specifically at $\operatorname{HDR}[12,35,37,45,49]$. The very large majority of these studies found viral protein expression (or full infection) decreased cellular repair capabilities.

Two cellular proteins, p53 and Rad51, are exceedingly important in the context of HDR. It is widely accepted that wt p53 regulates HDR, primarily through interactions with Rad51 (as reviewed in [21]). In vitro reactions have found p53 binds Rad51 and, by means of this interaction, inhibits strand exchange and branch migration of recombination intermediates [57]. An intact core DNA binding domain of the p53 protein is required for direct binding between the p53 and $\operatorname{Rad} 51$ proteins (and inhibition of the latter's activities) [23-25]. It has also been shown that following 
induction of damage wt p53 inhibits transactivation of the Rad51 promoter, thereby acting as a repressor of Rad51 transcription and protein expression [23]. It has also been shown that a phosphorylatable N-terminus is important for regulation and binding of $\operatorname{Rad} 51$ [58]. Many tumors (and cell lines derived from them) have so called "hotspot" mutations in p53, including the T98G cells used in this study [20]. Reintroduction of wt p53 into these tumor cells reduced HDR via decreasing Rad51 activity [59].

Multiple viruses have been shown to interact with the Rad51 protein. Epstein-Barr Virus (EBV), a herpesvirus, and SV40 have both been found to require the presence of Rad51 within their viral replication centers. Knockdown of Rad51 in infected cells dramatically decreased viral replication [60,61]. The opposite was true for HIV; stimulation of Rad51 activity inhibited HIV replication, primarily due to an inhibitory effect of Rad51 on the virus' Integrase enzyme [62].

The context of the T98G cellular environment is important for consideration of HDR in these cells. Under normal stress T98Gs can complete HDR and have normal levels of Rad51 [20]. Baseline levels of HDR in T98G cells after insult are quite high, perhaps in part due to the large proportion of these rapidly dividing cells being in the S/G2 phases of the cell cycle, during which HDR is most common [25,59]. However, the higher rate of HDR may also be attributable to the presence of the mutation in the p53 DNA binding domain (R273H) within these cells [20], which prevents p53's normal binding to, and regulation of, Rad51.

The model in Figure 5 is proposed to explain the dichotomy of results between the T98G results reported here and our earlier HFF study. In wt cells p53 normally inhibits Rad51 activity. In the model, the prediction is that the mutant p53-containing T98G cells, where p53 cannot bind Rad51 [23-25], would have high baseline recombination rates, as seen. The model further predicts that, introduction on their own (indicated by the arrow in the figure) of either wt p53 or IE72 (which we have shown can bind Rad51 in vitro) into these cells would decrease HDR by their binding to and inhibiting Rad51. Co-expression of both wt p53 and IE72 did not inhibit Rad51. We propose in these experiments wt p53 and IE72 bound to one another, inhibiting normal p53 activity [27]. This seems a reasonable assumption, given previous reports that IE72 binds directly to p53 and inhibits core domain binding ability [27]. If the introduced wt p53 and IE72 were interacting and p53's normal activity was inhibited, Rad51 would again be free to cause the high recombination rates observed.

Also in our model, in wt cells p53 normally inhibits Rad51, establishing baseline HDR activity levels. HCMV-infection of wt fibroblast cells causes p53 to become tightly associated with the viral replication centers [63]. We believe in an infected cell this viral manipulation of p53 prevents the normal binding of $\mathrm{p} 53$ and Rad51. The disruption of this normal condition in turn frees Rad51 to increase recombination in both the viral and cellular DNA. Experiments in wt cells found that expression of the single HCMV protein IE72 was sufficient to increase HDR levels [12]. The proposed model attributes the increase in HDR in wt cells to IE72 binding to wt p53, thereby inhibiting the ability of wt $\mathrm{p} 53$ to bind and regulate Rad51.

The proposed model offers a mechanistic explanation for our previously reported results in wt HFFs [12]. The focus of the earlier HFF study was the recognition of viral manipulation of a cellular repair mechanism to its own ends in the context of a fully permissive infection. The experiments performed in $\mathrm{T} 98 \mathrm{G}$ cells were expected to corroborate that data. Fortuitously, the 
particular microenvironment of the mutant glioblastoma cells allowed elucidation of what we believe to be the protein interactions responsible.

The results of this study may have implications for the initiation of glioblastomas. It has been reported that in excess of $90 \%$ of glioblastomas contain HCMV genetic material [64,65]. A latently HCMV-infected neural progenitor/stem cell which reactivated could, and most likely would, express IE72. We have found that expression of IE72 in a wt cell was capable of increasing unscheduled recombination, a hallmark of genetic instability and perhaps the first step toward hyperproliferation and gliomagenesis. The common perception of HCMV as a relatively harmless pathogen belie not only its capacity to cause serious birth defects, inflict direct DNA damage and downregulate repair of the cellular genome, but perhaps its capability to initiate an often lethal and devastating cancer. We have experiments underway to determine if the protein interactions found in this study are capable of intitiating oncogenic effects in tissue culture experiments.

Figure 5. A model for the interactions between wt p53, Rad51 and IE72 in HFFs and T98Gs. Interactions between these three proteins and the ramifications to HDR depend completely on the cellular microenvironment and the presence or absence of wt p53. See text for description.

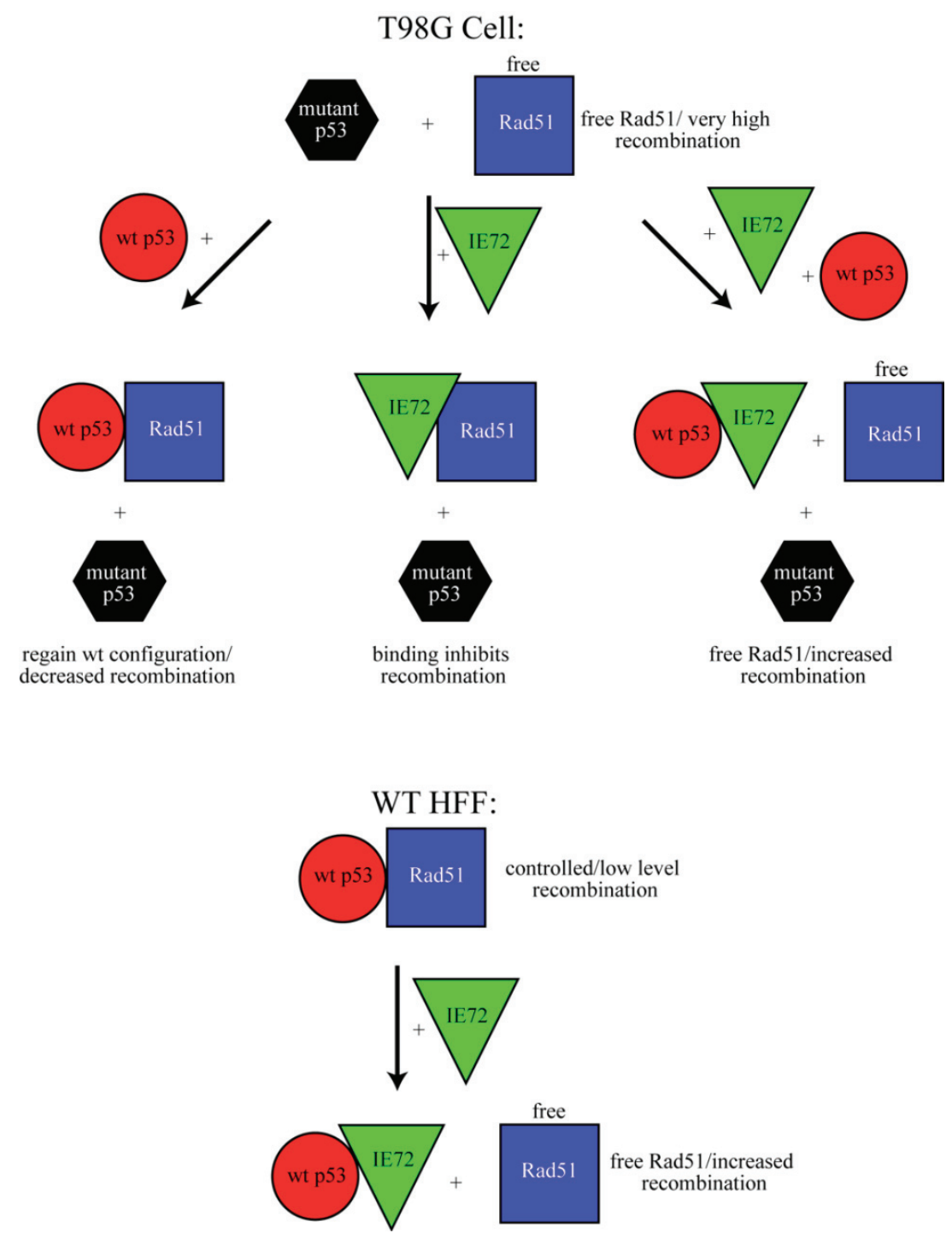




\section{Acknowledgments}

This work was supported by NIH grants \# RO1-AI51463 and \#P20 RR015587 (COBRE program) to EAF. The authors would like to thank John O'Dowd for critical reading of the manuscript.

\section{Author Contributions}

A.K. performed experiments in Figures 1-3 and compiled all the data. E.F. performed the experiments in Figure 4, proposed the model in Figure 5 and wrote the manuscript.

\section{Conflicts of Interest}

The authors declare no conflict of interest.

\section{References and Notes}

1. Boppana, S.B.; Fowler, K.B.; Britt, W.J.; Stagno, S.; Pass, R.F. Symptomatic congenital cytomegalovirus infection in infants born to mothers with preexisting immunity to cytomegalovirus. Pediatrics 1999, 104, 55-60.

2. Cinque, P.; Marenzi, R.; Ceresa, D. Cytomegalovirus infections of the nervous system. Intervirology 1997, 40, 85-97.

3. Zanghellini, F.; Boppana, S.B.; Emery, V.C.; Griffiths, P.D.; Pass, R.F. Asymptomatic primary cytomegalovirus infection: Virologic and immunologic features. J. Infect. Dis. 1999, 180, 702-707.

4. Britt, W.; Alford, C. Cytomegalovirus. In Fields Virology; Lippincott-Raven Publishers: Philadelphia, PA, USA, 1996; pp. 2493-2523.

5. Dziurzynski, K.; Wei, J.; Qiao, W.; Hatiboglu, M.A.; Kong, L.Y.; Wu, A.; Wang, Y.; Cahill, D.; Levine, N.; Prabhu, S.; et al. Glioma-associated cytomegalovirus mediates subversion of the monocyte lineage to a tumor propagating phenotype. Clin. Canc. Res. 2011, 17, 4642-4649.

6. Michaelis, M.; Baumgarten, P.; Mittelbronn, M.; Driever, P.H.; Doerr, H.W.; Cinatl, J., Jr. Oncomodulation by human cytomegalovirus: Novel clinical findings open new roads. Med. Microbiol. Immunol. 2011, 200, 1-5.

7. Fortunato, E.A.; Dell'quila, M.L.; Spector, D.H. Specific chromosome 1 breaks induced by human cytomegalovirus. Proc. Natl. Acad. Sci. USA 2000, 97, 853-858.

8. Nystad, M.; Fagerheim, T.; Brox, V.; Fortunato, E.A.; Nilssen, O. Human cytomegalovirus (HCMV) and hearing impairment: Infection of fibroblast cells with HCMV induces chromosome breaks at 1q23.3, between loci DFNA7 and DFNA49-Both involved in dominantly inherited, sensorineural, hearing impairment. Mutat. Res. 2008, 637, 56-65.

9. Toesca, A. Central and peripheral myelin in the rat cochlear and vestibular nerves. Neurosci. Lett. 1996, 221, 21-24.

10. Chapman, J.R.; Taylor, M.R.; Boulton, S.J. Playing the end game: DNA double-strand break repair pathway choice. Mol. Cell 2012, 47, 497-510.

11. Rothkamm, K.; Kruger, I.; Thompson, L.H.; Lobrich, M. Pathways of DNA double-strand break repair during the mammalian cell cycle. Mol. Cell Biol. 2003, 23, 5706-5715. 
12. Kulkarni, A.S.; Fortunato, E.A. Stimulation of homology-directed repair at I-SceI-induced DNA breaks during the permissive life cycle of human cytomegalovirus. J. Virol. 2011, 85, 6049-6054.

13. Pierce, A.J.; Johnson, R.D.; Thompson, L.H.; Jasin, M. Xrcc3 promotes homology-directed repair of DNA damage in mammalian cells. Genes Dev. 1999, 13, 2633-2638.

14. Wiese, C.; Pierce, A.J.; Gauny, S.S.; Jasin, M.; Kronenberg, A. Gene conversion is strongly induced in human cells by double-strand breaks and is modulated by the expression of bcl-x(1). Canc. Res. 2002, 62, 1279-1283.

15. Bett, A.J.; Haddara, W.; Prevec, L.; Graham, F.L. An efficient and flexible system for construction of adenovirus vectors with insertions or deletions in early regions 1 and 3 . Proc. Natl. Acad. Sci. USA 1994, 91, 8802-8806.

16. Anglana, M.; Bacchetti, S. Construction of a recombinant adenovirus for efficient delivery of the I-SceI yeast endonuclease to human cells and its application in the in vivo cleavage of chromosomes to expose new potential telomeres. Nucleic Acids Res. 1999, 27, 4276-4281.

17. Luo, M.H.; Fortunato, E.A. Long-term infection and shedding of human cytomegalovirus in T98G glioblastoma cells. J. Virol. 2007, 81, 10424-10436.

18. Greaves, R.F.; Mocarski, E.S. Defective growth correlates with reduced accumulation of a viral DNA replication protein after low-multiplicity infection by a human cytomegalovirus IE1 mutant. J. Virol. 1998, 72, 366-379.

19. Castillo, J.P.; Kowalik, T.F. Human cytomegalovirus immediate early proteins and cell growth control. Gene 2002, 290, 19-34.

20. Short, S.C.; Bourne, S.; Martindale, C.; Woodcock, M.; Jackson, S.P. DNA damage responses at low radiation doses. Rad. Res. 2005, 164, 292-302.

21. Bertrand, P.; Saintigny, Y.; Lopez, B.S. P53's double life: Transactivation-independent repression of homologous recombination. Trends Gen. 2004, 20, 235-243.

22. Rosenke, K.; Samuel, M.A.; McDowell, E.T.; Toerne, M.A.; Fortunato, E.A. An intact sequence-specific DNA-binding domain is required for human cytomegalovirus-mediated sequestration of p53 and may promote in vivo binding to the viral genome during infection. Virology 2006, 348, 19-34.

23. Arias-Lopez, C.; Lazaro-Trueba, I.; Kerr, P.; Lord, C.J.; Dexter, T.; Iravani, M.; Ashworth, A.; Silva, A. P53 modulates homologous recombination by transcriptional regulation of the Rad51 gene. EMBO Rep. 2006, 7, 219-224.

24. Buchhop, S.; Gibson, M.K.; Wang, X.W.; Wagner, P.; Sturzbecher, H.W.; Harris, C.C. Interaction of p53 with the human Rad51 protein. Nucleic Acids Res. 1997, 25, 3868-3874.

25. Rieckmann, T.; Kriegs, M.; Nitsch, L.; Hoffer, K.; Rohaly, G.; Kocher, S.; Petersen, C.; Dikomey, E.; Dornreiter, I.; Dahm-Daphi, J. P53 modulates homologous recombination at I-SceI-induced double-strand breaks through cell-cycle regulation. Oncogene 2013, 32, 968-975.

26. Daboussi, F.; Dumay, A.; Delacote, F.; Lopez, B.S. DNA double-strand break repair signalling: The case of Rad51 post-translational regulation. Cell Signal. 2002, 14, 969-975. 
27. Hwang, E.S.; Zhang, Z.; Cai, H.; Huang, D.Y.; Huong, S.M.; Cha, C.Y.; Huang, E.S. Human cytomegalovirus IE1-72 protein interacts with p53 and inhibits p53-dependent transactivation by a mechanism different from that of IE2-86 protein. J. Virol. 2009, 83, 12388-12398.

28. Klucher, K.M.; Sommer, M.; Kadonaga, J.T.; Spector, D.H. In vivo and in vitro analysis of transcriptional activation mediated by the human cytomegalovirus major immediate-early proteins. Mol. Cell Biol. 1993, 13, 1238-1250.

29. Ashcroft, M.; Kubbutat, M.H.; Vousden, K.H. Regulation of p53 function and stability by phosphorylation. Mol. Cell Biol. 1999, 19, 1751-1758.

30. Casavant, N.C.; Luo, M.H.; Rosenke, K.; Winegardner, T.; Zurawska, A.; Fortunato, E.A. Potential role for p53 in the permissive life cycle of human cytomegalovirus. J. Virol. 2006, 80, 8390-8401.

31. Luo, M.H.; Rosenke, K.; Czornak, K.; Fortunato, E.A. Human cytomegalovirus disrupts both ataxia telangiectasia mutated protein (ATM)- and ATM-Rad3-related kinase-mediated DNA damage responses during lytic infection. J. Virol. 2007, 81, 1934-1950.

32. Ayed, A.; Mulder, F.A.; Yi, G.S.; Lu, Y.; Kay, L.E.; Arrowsmith, C.H. Latent and active p53 are identical in conformation. Nat. Struct. Biol. 2001, 8, 756-760.

33. Guan, K.L.; Dixon, J.E. Eukaryotic proteins expressed in Escherichia coli: An improved thrombin cleavage and purification procedure of fusion proteins with glutathione s-transferase. Anal. Biochem. 1991, 192, 262-267.

34. Snowden, T.; Shim, K.S.; Schmutte, C.; Acharya, S.; Fishel, R. hMSH4-hMSH5 adenosine nucleotide processing and interactions with homologous recombination machinery. J. Biol. Chem. 2008, 283, 145-154.

35. Baydoun, H.H.; Pancewicz, J.; Nicot, C. Human T-lymphotropic type 1 virus p30 inhibits homologous recombination and favors unfaithful DNA repair. Blood 2011, 117, 5897-5906.

36. Becker, S.A.; Lee, T.H.; Butel, J.S.; Slagle, B.L. Hepatitis B virus X protein interferes with cellular DNA repair. J. Virol. 1998, 72, 266-272.

37. Chipitsyna, G.; Slonina, D.; Siddiqui, K.; Peruzzi, F.; Skorski, T.; Reiss, K.; Sawaya, B.E.; Khalili, K.; Amini, S. HIV-1 Tat increases cell survival in response to cisplatin by stimulating Rad51 gene expression. Oncogene 2004, 23, 2664-2671.

38. Durkin, S.S.; Guo, X.; Fryrear, K.A.; Mihaylova, V.T.; Gupta, S.K.; Belgnaoui, S.M.; Haoudi, A.; Kupfer, G.M.; Semmes, O.J. HTLV-1 Tax oncoprotein subverts the cellular DNA damage response via binding to DNA-dependent protein kinase. J. Biol. Chem. 2008, 283, 36311-36320.

39. Groisman, I.J.; Koshy, R.; Henkler, F.; Groopman, J.D.; Alaoui-Jamali, M.A. Downregulation of DNA excision repair by the hepatitis B virus-X protein occurs in p53-proficient and p53-deficient cells. Carcinogenesis 1999, 20, 479-483.

40. Gruhne, B.; Sompallae, R.; Masucci, M.G. Three Epstein-Barr virus latency proteins independently promote genomic instability by inducing DNA damage, inhibiting DNA repair and inactivating cell cycle checkpoints. Oncogene 2009, 28, 3997-4008.

41. Jia, L.; Wang, X.W.; Harris, C.C. Hepatitis B virus X protein inhibits nucleotide excision repair. Int. J. Canc. 1999, 80, 875-879. 
42. Liang, X.; Pickering, M.T.; Cho, N.H.; Chang, H.; Volkert, M.R.; Kowalik, T.F.; Jung, J.U. Deregulation of DNA damage signal transduction by herpesvirus latency-associated M2. J. Virol. 2006, 80, 5862-5874.

43. Prost, S.; Ford, J.M.; Taylor, C.; Doig, J.; Harrison, D.J. Hepatitis B X protein inhibits p53-dependent DNA repair in primary mouse hepatocytes. J. Biol. Chem. 1998, 273, $33327-33332$.

44. Sun, Y.; Huang, Y.C.; Xu, Q.Z.; Wang, H.P.; Bai, B.; Sui, J.L.; Zhou, P.K. HIV-1 Tat depresses DNA-PK(cs) expression and DNA repair, and sensitizes cells to ionizing radiation. Int. J. Radiat. Oncol. Biol. Phys. 2006, 65, 842-850.

45. Trojanek, J.; Croul, S.; Ho, T.; Wang, J.Y.; Darbinyan, A.; Nowicki, M.; Valle, L.D.; Skorski, T.; Khalili, K.; Reiss, K. T-antigen of the human polyomavirus JC attenuates faithful DNA repair by forcing nuclear interaction between IRS-1 and Rad51. J. Cell. Physiol. 2006, 206, 35-46.

46. Mathonnet, G.; Lachance, S.; Alaoui-Jamali, M.; Drobetsky, E.A. Expression of hepatitis B virus $\mathrm{X}$ oncoprotein inhibits transcription-coupled nucleotide excision repair in human cells. Mutat. Res. 2004, 554, 305-318.

47. Mohni, K.N.; Mastrocola, A.S.; Bai, P.; Weller, S.K.; Heinen, C.D. DNA mismatch repair proteins are required for efficient herpes simplex virus 1 replication. J. Virol. 2011, 85, 12241-12253.

48. O'Dowd, J.M.; Zavala, A.G.; Brown, C.J.; Mori, T.; Fortunato, E.A. HCMV-infected cells maintain efficient nucleotide excision repair of the viral genome while abrogating repair of the host genome. PLoS Pathog. 2012, 8, e1003038.

49. Schumacher, A.J.; Mohni, K.N.; Kan, Y.; Hendrickson, E.A.; Stark, J.M.; Weller, S.K. The HSV-1 exonuclease, UL12, stimulates recombination by a single strand annealing mechanism. PLoS Pathog. 2012, 8, e1002862.

50. Deng, C.Z.; AbuBakar, S.; Fons, M.P.; Boldogh, I.; Hokanson, J.; Au, W.W.; Albrecht, T. Cytomegalovirus-enhanced induction of chromosome aberrations in human peripheral blood lymphocytes treated with potent genotoxic agents. Environ. Mol. Mutagen. 1992, 19, 304-310.

51. Duong, F.H.; Christen, V.; Lin, S.; Heim, M.H. Hepatitis C virus-induced up-regulation of protein phosphatase 2a inhibits histone modification and DNA damage repair. Hepatology 2010, 51, 741-751.

52. Lilley, C.E.; Chaurushiya, M.S.; Boutell, C.; Landry, S.; Suh, J.; Panier, S.; Everett, R.D.; Stewart, G.S.; Durocher, D.; Weitzman, M.D. A viral E3 ligase targets RNF8 and RNF168 to control histone ubiquitination and DNA damage responses. EMBO J. 2010, 29, 943-955.

53. Pal, S.; Polyak, S.J.; Bano, N.; Qiu, W.C.; Carithers, R.L.; Shuhart, M.; Gretch, D.R.; Das, A. Hepatitis $\mathrm{C}$ virus induces oxidative stress, DNA damage and modulates the DNA repair enzyme NEIL1. J. Gastroenterol. Hepatol. 2010, 25, 627-634.

54. Philpott, S.M.; Buehring, G.C. Defective DNA repair in cells with human T-cell leukemia/bovine leukemia viruses: Role of Tax gene. J. Natl. Canc. Inst. 1999, 91, 933-942.

55. Ranneberg-Nilsen, T.; Bjoras, M.; Luna, L.; Slettebakk, R.; Dale, H.A.; Seeberg, E.; Rollag, H. Human cytomegalovirus infection modulates DNA base excision repair in fibroblast cells. Virology 2006, 348, 389-397. 
56. Bowman, K.K.; Sicard, D.M.; Ford, J.M.; Hanawalt, P.C. Reduced global genomic repair of ultraviolet light-induced cyclobutane pyrimidine dimers in simian virus 40-transformed human cells. Mol. Carcinog. 2000, 29, 17-24.

57. Yoon, D.; Wang, Y.; Stapleford, K.; Wiesmuller, L.; Chen, J. P53 inhibits strand exchange and replication fork regression promoted by human Rad51. J. Mol. Biol. 2004, 336, 639-654.

58. Restle, A.; Janz, C.; Wiesmuller, L. Differences in the association of p53 phosphorylated on serine 15 and key enzymes of homologous recombination. Oncogene 2005, 24, 4380-4387.

59. Mekeel, K.L.; Tang, W.; Kachnic, L.A.; Luo, C.M.; DeFrank, J.S.; Powell, S.N. Inactivation of p53 results in high rates of homologous recombination. Oncogene 1997, 14, 1847-1857.

60. Boichuk, S.; Hu, L.; Hein, J.; Gjoerup, O.V. Multiple DNA damage signaling and repair pathways deregulated by simian virus 40 large T antigen. J. Virol. 2010, 84, 8007-8020.

61. Kudoh, A.; Iwahori, S.; Sato, Y.; Nakayama, S.; Isomura, H.; Murata, T.; Tsurumi, T. Homologous recombinational repair factors are recruited and loaded onto the viral DNA genome in Epstein-Barr virus replication compartments. J. Virol. 2009, 83, 6641-6651.

62. Cosnefroy, O.; Tocco, A.; Lesbats, P.; Thierry, S.; Calmels, C.; Wiktorowicz, T.; Reigadas, S.; Kwon, Y.; De Cian, A.; Desfarges, S.; et al. Stimulation of the human Rad51 nucleofilament restricts HIV-1 integration in vitro and in infected cells. J. Virol. 2012, 86, 513-526.

63. Fortunato, E.A.; Spector, D.H. P53 and RPA are sequestered in viral replication centers in the nuclei of cells infected with human cytomegalovirus. J. Virol. 1998, 72, 2033-2039.

64. Dziurzynski, K.; Chang, S.M.; Heimberger, A.B.; Kalejta, R.F.; McGregor Dallas, S.R.; Smit, M.; Soroceanu, L.; Cobbs, C.S.; HCMV Glioma Symposium. Consensus on the role of human cytomegalovirus in glioblastoma. Neuro Oncol. 2012, 14, 246-255.

65. Ranganathan, P.; Clark, P.A.; Kuo, J.S.; Salamat, M.S.; Kalejta, R.F. Significant association of multiple human cytomegalovirus genomic loci with glioblastoma multiforme samples. J. Virol. 2012, 86, 854-864. 


\title{
The CMV Life Cycle
}

Reprinted from Viruses. Cite as: Graf, L.; Webel, R.; Wagner, S.; Hamilton, S.T.; Rawlinson, W.D.; Sticht, H.; Marschall, M. The Cyclin-Dependent Kinase Ortholog pUL97 of Human Cytomegalovirus Interacts with Cyclins. Viruses 2013, 5, 3213-3230.

Article

\section{The Cyclin-Dependent Kinase Ortholog pUL97 of Human Cytomegalovirus Interacts with Cyclins}

\section{Laura Graf $^{1}$, Rike Webel ${ }^{1}$, Sabrina Wagner ${ }^{1}$, Stuart T. Hamilton ${ }^{2}$, William D. Rawlinson ${ }^{2}$, Heinrich Sticht ${ }^{3}$ and Manfred Marschall ${ }^{1, *}$}

1 Institute for Clinical and Molecular Virology, University of Erlangen-Nuremberg, 91054 Erlangen, Germany; E-Mails: graf.laura@gmx.de (L.G.); rike.webel@viro.med.uni-erlangen.de (R.W.); sabrina.wagner@viro.med.uni-erlangen.de (S.W.)

2 Virology Division, SEALS, Department of Microbiology, Prince of Wales Hospital, Randwick, NSW 2031, Sydney, Australia; E-Mails: z3058477@zmail.unsw.edu.au (S.T.H.); w.rawlinson@unsw.edu.au (W.D.R.)

3 Division of Bioinformatics, Institute of Biochemistry, University of Erlangen-Nuremberg, 91054 Erlangen, Germany; E-Mail: Heinrich.Sticht@med.uni-erlangen.de

* Author to whom correspondence should be addressed;

E-Mail: manfred.marschall@viro.med.uni-erlangen.de; Tel.: +49-9131-852-6089;

Fax: +49-9131-852-2101.

Received: 14 November 2013; in revised form: 8 December 2013 / Accepted: 9 December 2013 / Published: 18 December 2013

\begin{abstract}
The human cytomegalovirus (HCMV)-encoded protein kinase, pUL97, is considered a cyclin-dependent kinase (CDK) ortholog, due to shared structural and functional characteristics. The primary mechanism of CDK activation is binding to corresponding cyclins, including cyclin $\mathrm{T} 1$, which is the usual regulatory cofactor of CDK9. This study provides evidence of direct interaction between pUL97 and cyclin T1 using yeast two-hybrid and co-immunoprecipitation analyses. Confocal immunofluorescence revealed partial colocalization of pUL97 with cyclin T1 in subnuclear compartments, most pronounced in viral replication centres. The distribution patterns of pUL97 and cyclin T1 were independent of HCMV strain and host cell type. The sequence domain of pUL97 responsible for the interaction with cyclin T1 was between amino acids 231-280. Additional co-immunoprecipitation
\end{abstract}


analyses showed cyclin B1 and cyclin A as further pUL97 interaction partners. Investigation of the pUL97-cyclin T1 interaction in an ATP consumption assay strongly suggested phosphorylation of pUL97 by the CDK9/cyclin T1 complex in a substrate concentration-dependent manner. This is the first demonstration of interaction between a herpesviral CDK ortholog and cellular cyclins.

Keywords: human cytomegalovirus; protein kinase pUL97; cyclins T1, B1 and A; protein-protein interaction; substrate phosphorylation; interaction-mediated regulation

\section{Introduction}

Human cytomegalovirus (HCMV), also known as human herpesvirus 5 (HHV-5), is a member of the $\beta$-Herpesvirinae subfamily. It is a ubiquitous human pathogen of increasing seroprevalence in different populations $(60 \%-90 \%)$ that causes severe systemic diseases in immunosuppressed patients and is the leading infectious cause of birth defects in developed countries [1]. Currently approved antiviral agents for systemic treatment (cidofovir, foscarnet, ganciclovir and valganciclovir) inhibit viral DNA synthesis by targeting the HCMV DNA polymerase, pUL54 [2]. However, drug-resistant virus variants emerge after prolonged therapy, and current antivirals cause frequent adverse side effects. Protein kinases are putative targets of novel antiviral drugs, given their important role in the regulation of HCMV replication [3-8]. Pharmacological cyclin-dependent kinase (CDK) inhibitors interfere with the replication of HCMV and other viruses and are currently being investigated in a number of clinical trials. Roscovitine, a purine analogue that preferentially inhibits CDK1, 2, 5, 7 and 9, has been shown to decrease viral DNA synthesis and production of late viral proteins and infectious virus [4]. Recently, we reported that a novel selective CDK9 inhibitor, R22, exerts anti-cytomegaloviral activity in cell culture models [9]. CDKs are heterodimeric serine/threonine kinases phosphorylating a number of substrate proteins. Upon activation through binding to their regulatory cyclin subunits, CDKs regulate cell cycle progression, transcription, neuronal cytoskeleton organization, apoptosis and other cellular functions. These kinases are promising targets for anti-cytomegaloviral therapy, since the efficiency of HCMV replication is closely connected to CDK activity [4,8-11]. Moreover, HCMV is able to stimulate or suppress CDK activity in order to create an environment favourable for efficient viral transcription, genome replication and assembly of viral particles. At least four CDKs (CDK1, 2, 7 and 9) and their corresponding cyclins are required for efficient HCMV replication and are upregulated in HCMV-infected cells [3,12-15].

HCMV not only modulates CDK regulation of the host cell, but also mimics CDK activity through expression of the serine/threonine protein kinase, pUL97. HCMV pUL97 is considered a CDK ortholog, due to structural and functional similarities. Although pUL97 does not appear to be absolutely required for viral replication, deletion of the ORF UL97 from the viral genome or pharmacological inhibition of pUL97 significantly reduces virus replication, showing the importance of pUL97 activity for efficient virus replication [16,17]. pUL97 regulates HCMV at various stages of replication by phosphorylating viral and cellular proteins (Figure 1). Sequence 
analyses and a three-dimensional pUL97 model suggested conservation of functionally important residues in ATP binding sites and the catalytic centre between pUL97 and CDKs $[18,19]$. Recently, it has been reported that pUL97 phosphorylates cellular retinoblastoma protein $(\mathrm{Rb})$ at the same residues as CDKs, a protein which controls progression through the G1 phase of the cell cycle $[20,21]$. There are further shared substrates of CDKs and pUL97, including nuclear lamins A and C, RNA polymerase II and pUL69 (Figure 1). In addition, inhibition of CDKs potentiates the effect of the pUL97 inhibitor, maribavir, indicating that the functions of CDKs and pUL97 overlap to some extent [22]. Moreover, a yeast complementation assay demonstrated a pUL97-mediated rescue of the proliferation of a Saccharomyces cerevisiae mutant lacking CDK activity [20]. It has been suggested that pUL97 is regulated in a cyclin-independent manner, a conclusion based on the finding that cyclins did not copurify with pUL97 during tandem affinity purification, so that binding of pUL97 to cyclins was considered unlikely [20]. However, in the present study, we provide the first evidence for the interaction of pUL97 with cyclins, emphasizing the functional relation between the viral CDK ortholog pUL97 and cellular CDKs. The meaning of these interactions for phosphorylation-dependent regulatory processes during HCMV replication and possible functional consequences are discussed.

Figure 1. Schematic illustration of the structure of pUL97. Kinase domain (amino acids 337-651): subdivided into 11 subdomains (SD) that are conserved within herpesviral protein kinases and are similarly found in cellular protein kinases; mutation of the invariant lysine residue of SD II (amino acid 355) results in a complete loss of kinase activity $[18,23,24]$. Expression of three pUL97 isoforms: formation by the alternative initiation of translation with start codons at amino acids 1, 74 and 157 [25-27]. Nuclear localization signals (NLS1 amino acids 6-35 and NLS2 amino acids 190-213): mediation of the nuclear import of pUL97 isoforms by importin- $\alpha$ binding [26,28]. Self-interaction domain of pUL97 (amino acids 231-280): formation of dimers and oligomers [29]. Regulation of various processes during human cytomegalovirus (HCMV) replication by pUL97 via phosphorylation of viral and cellular substrates: protein synthesis (RNA polymerase II [30], EF-1 $\delta$ [18,31], pUL69 [32]), viral DNA replication (pUL44 [33]), nuclear capsid egress (lamins A and C, p32, a multi-ligand binding protein, also referred to as $\mathrm{gClqR}$ and $\mathrm{HABP} 1$ [34,35], morphogenesis (pp65 [36]), cell cycle modulation ( $\mathrm{Rb}[20]$ ) and antiviral therapy (ganciclovir [6]). 

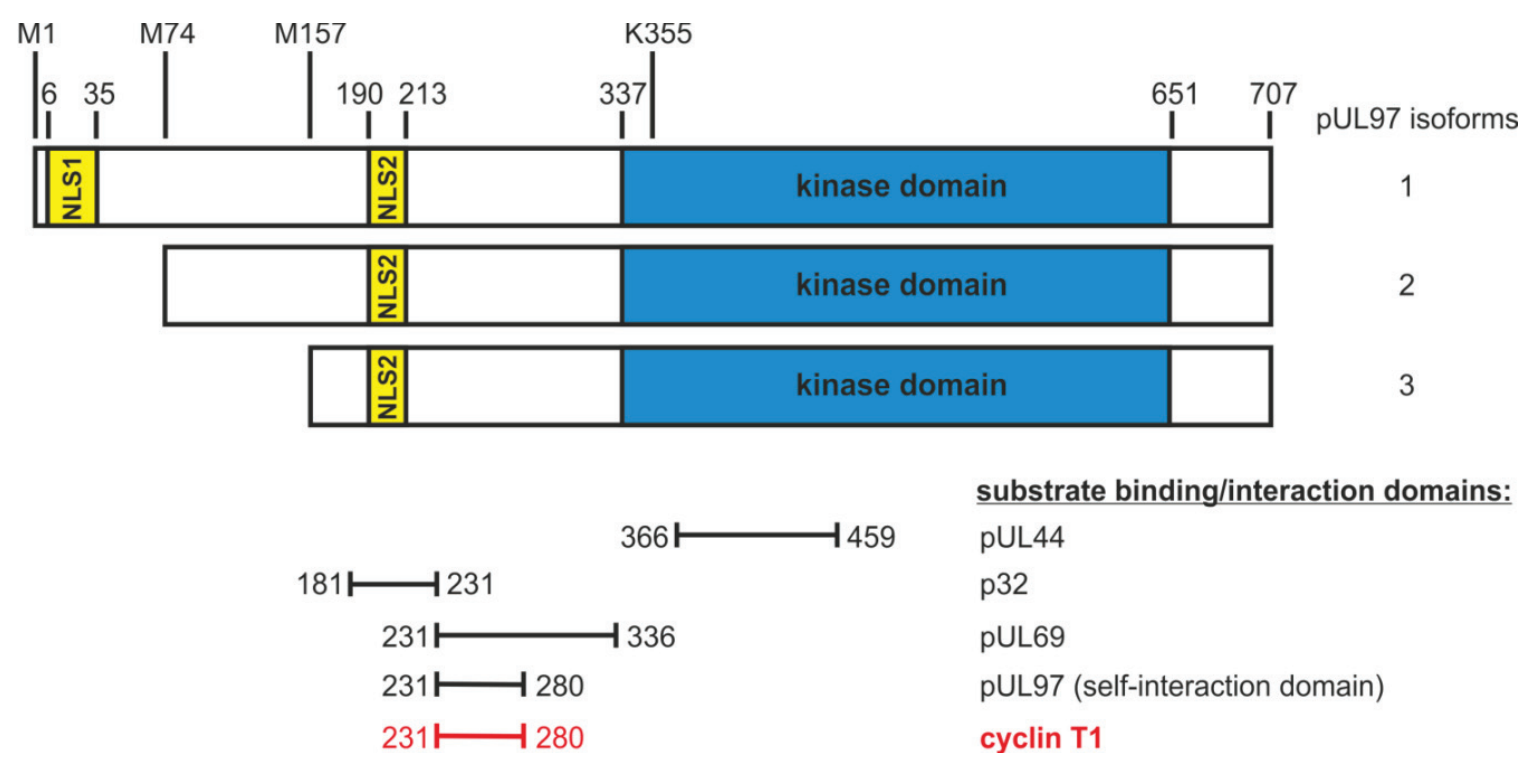

\section{Results}

\subsection{Interaction of the HCMV Protein Kinase pUL97 with Cyclin T1}

Yeast two-hybrid (Y2H) experiments revealed the interaction between pUL97 and cyclin T1 (Figure 2a, row 3). The signal obtained in the filter lift assay was similar to the positive control (interaction between p53 and SV40 T-antigen, Figure 2a, row 1). This was considered specific, since the intrinsic autoactivation effects of the individual fusion proteins were excluded by vector controls (Figure 2a, rows 4 and 5). The interaction between pUL97 and cyclin T1 was confirmed using co-immunoprecipitation (CoIP) analyses with protein lysates from transiently transfected 293 T cells (Figure 2b). Endogenous cyclin T1 was specifically co-immunoprecipitated together with Flag-tagged pUL97 (Figure 2b, lane 1). Positive controls (pUL97 self-interaction, Figure 2b, lane 4) and negative controls (expression of RFP and pUL53, respectively, Figure 2b, lane 2, 3) confirmed the reliability of signals. Further CoIP analyses also demonstrated the pUL97-cyclin T1 interaction in HCMV-infected human foreskin fibroblast (HFF) cells (Figure 2c). pUL97 was co-immunoprecipitated with cyclin T1 (Figure 2c, lanes 2 and 3) and vice versa (Figure 2c, lanes 4 and 5). The intensity of the detected signals was independent of multiplicity of infection, when tested at multiplicity of infection (MOI) 0.5 and 1.0. The known interactions between cyclin T1 and CDK9 [37], as well as between pUL97 and the viral mRNA export factor, pUL69 [32], served as positive controls (Figure 2c, lanes 7-9). The weak signal of co-immunoprecipitated CDK9 in uninfected cells was a result of low expression and subsequent limited immunoprecipitation of cyclin T1. Interestingly, CDK9 did not co-immunoprecipitate with pUL97 (Figure 2c, lane 9), whereas both proteins were detected in the precipitate of cyclin T1. These findings provide the first evidence of the interaction of a herpesviral kinase with a cellular cyclin.

\subsection{Partial Colocalization of pUL97 with Cyclin T1 in Subnuclear Compartments of HCMV-Infected Cells}

In order to study the intracellular localization patterns of pUL97 and cyclin T1, the cell types, HFF, MRC-5, ARPE-19 and TEV-1 were infected with two different HCMV strains, AD169 and Merlin. The results of indirect immunofluorescence analyses are shown in Figure 3. 
Cyclin T1 was found evenly distributed in the nuclei of uninfected cells, regardless of cell type (Figure 3, panels 10, 22, 34, 54). In HCMV-infected cells, however, cyclin T1 was concentrated in subnuclear compartments, partly colocalizing with the viral protein, pUL97 (Figure 3, panels 4, 16, $28,40,48)$. This colocalization was most pronounced in viral replication centres. The kinase pUL97 is a known component of viral replication centres [33], here indicated by co-staining with the viral DNA polymerase processivity factor, pUL44 (Figure 3, panels 6, 18, 30, 42, 50). The pUL97-pUL44 colocalization, previously described in HFFs [33], was confirmed in MRC-5, ARPE-19 and TEV-1 cells. In summary, the subnuclear distribution patterns of pUL97 and cyclin T1 appeared to be rather invariant and did not show detectable differences between cell types and HCMV strains. 
Figure 2. Direct interaction between pUL97 and cyclin T1. (a) Yeast two-hybrid (Y2H) analysis: cyclin T1 fused to the GAL4 activation domain (AD) and pUL97 fused to the GAL4 binding domain (BD) were coexpressed in yeast cells, and colonies were stained in filter lift assays (FLA). (b) co-immunoprecipitation (CoIP) analyses using protein lysates of transfected 293T cells: the self-interaction of pUL97 served as a positive control (lane 4) and pUL53-Flag as a negative control (lane 2). (c) CoIP analyses showing the interaction between pUL97 and cyclin T1 in HCMV-infected cells: human foreskin fibroblasts (HFFs) were infected with HCMV AD169-GFP at a multiplicity of infection (MOI) of 0.5 or 1.0. At six days post-infection, infected and mock-infected (M) cells were lysed and used for CoIP analyses. IP, immunoprecipitation; WB, Western blot analysis.

(a)

\begin{tabular}{|l|l|l|c|c|}
\cline { 2 - 5 } \multicolumn{1}{c|}{} & GAL4-BD & GAL4-AD & $\begin{array}{r}\text { Inter- } \\
\text { action }\end{array}$ & FLA \\
\hline \hline 1 & p53 & SV40-T & ++ & $\bigcirc$ \\
\hline 2 & vector & vector & - & \\
\hline 3 & pUL97 & cyclin T1 & ++ & $\bigcirc$ \\
\hline 4 & vector & cyclin T1 & - & \\
\hline 5 & pUL97 & vector & - & \\
\hline
\end{tabular}

(b)
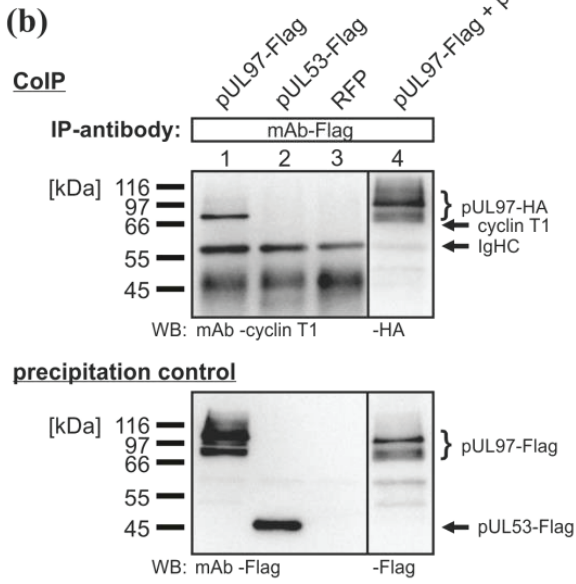

expression control

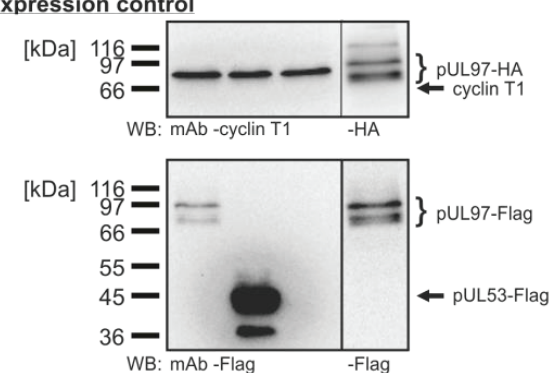

(c)
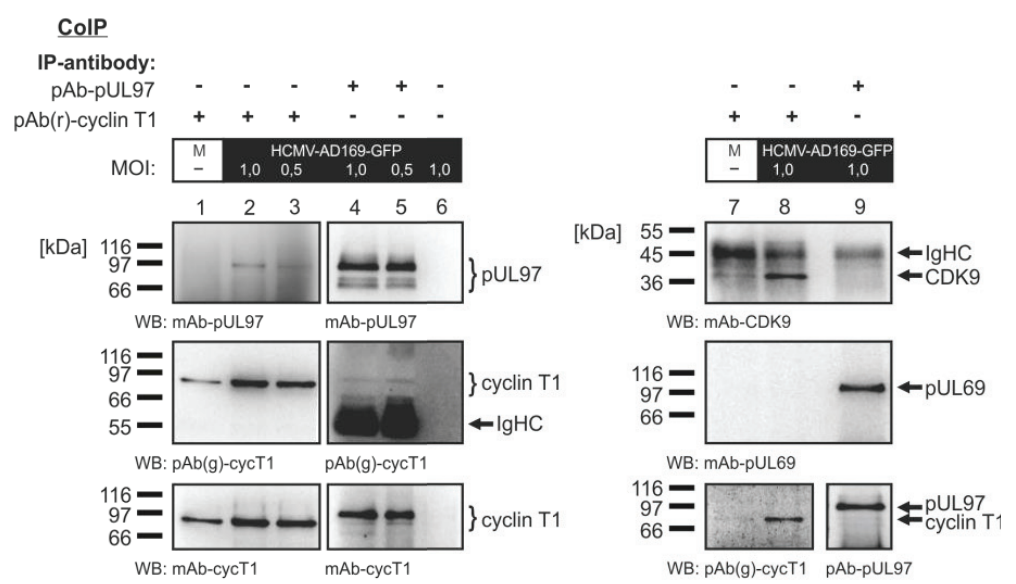

expression control

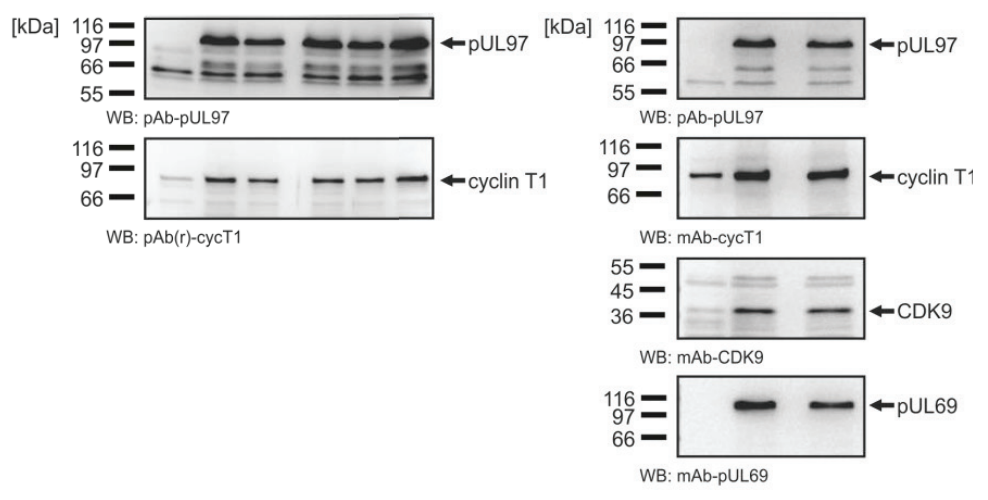


Figure 3. Comparison of the pUL97-cyclin T1 intracellular localization in four human cell types infected with two different HCMV strains using immunofluorescence analyses. HFF, MRC-5, ARPE-19 and TEV-1 cells were infected with HCMV AD169 or HCMV Merlin at various MOIs (1-8: MOI 0.5; 13-20: MOI 0.09; 25-32: MOI 0.03; 37-52: MOI 0.01). Cells were fixed within a range of late time points post-infection (112: 4 dpi, 13-36: 6 dpi, 37-56: 5 dpi) and immunostained with pAb(r)-cyclin (pAb, polyclonal antibody) T1, mAb UL97/pAb-UL97 (mAb, monoclonal antibody) and mAb-UL44. MOCK, uninfected cells.

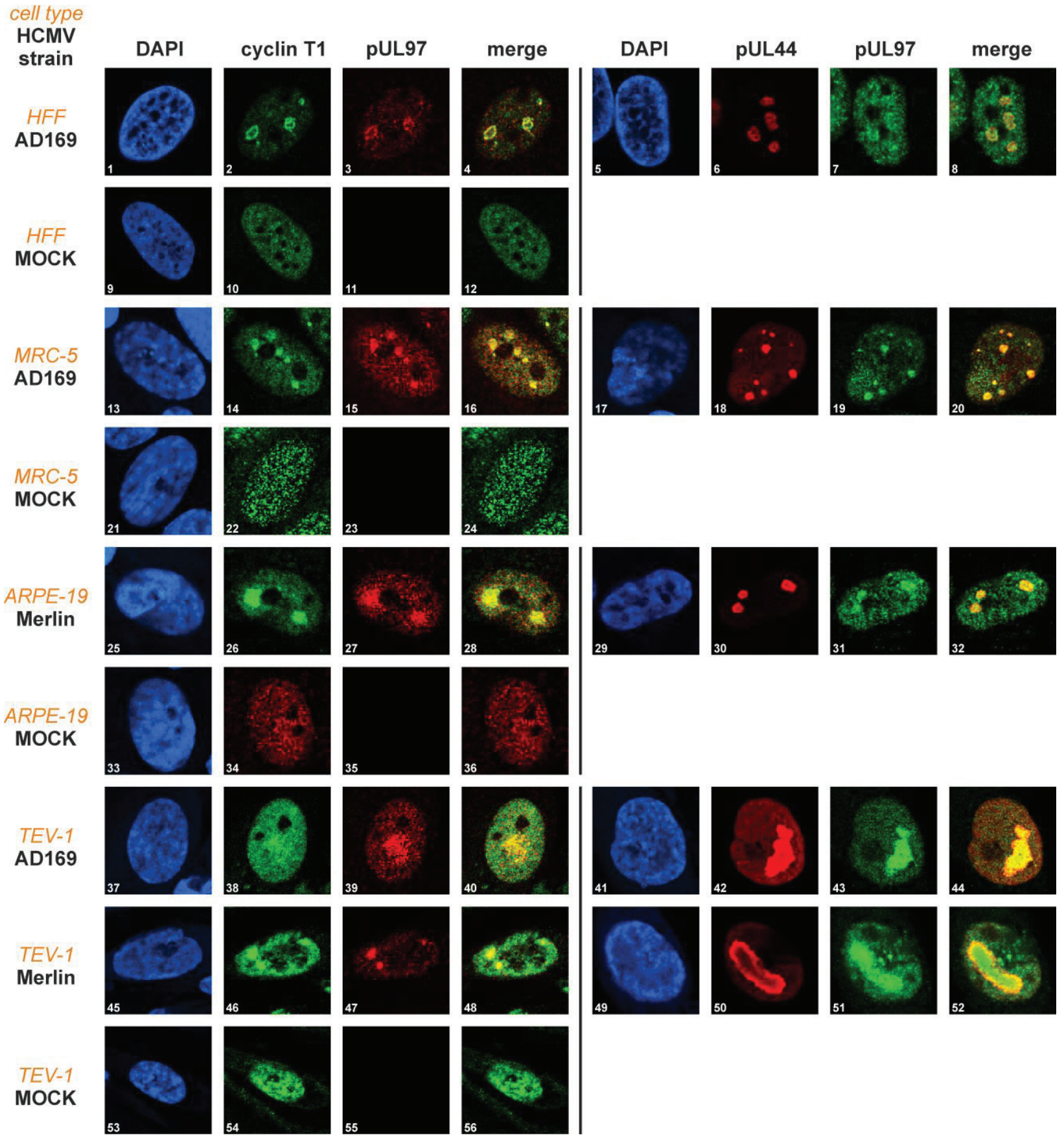




\subsection{Mapping of the Sequence Domain of pUL97 Responsible for the Interaction with Cyclin T1}

CoIP analyses using Flag-tagged N- and C-terminal truncation mutants of pUL97 expressed in 293 T cells were performed to narrow down the sequence domain of pUL97 responsible for the interaction with cyclin T1 (Figure 4a). Initial CoIP analyses revealed that the N-terminus of pUL97 was important for the interaction with cyclin T1 (i.e., amino acids 1-365; data not shown). Further CoIP analyses demonstrated that the first 230 amino acids of pUL97 were dispensable for the interaction with cyclin T1, since pUL97(231-707)-Flag was the smallest N-terminally truncated fragment that was still co-immunoprecipitated, whereas the correlating $\mathrm{C}$-terminal truncation mutant, pUL97(1-230)-Flag, did not interact with cyclin T1 (Figure 4a, lanes 4 and 11). Together with pUL97(1-280)-Flag being the smallest C-terminally truncated pUL97 fragment still interacting with cyclin T (Figure 4a, lane 3), the protein domain of pUL97 responsible for the interaction with cyclin T1 could be mapped to amino acids 231-280. Deletion of the interaction region in construct pUL97( $\Delta 231-280)$-Flag prevented the co-immunoprecipitation of cyclin $\mathrm{T} 1$, confirming the finding of the mapping experiment (Figure 4b, lane 8). All three isoforms of pUL97 contain the cyclin T1 interaction region and interacted with cyclin T1, as shown by CoIP analyses performed with expression constructs encoding individual isoforms (Figure 4b, lane 4 (IF-1, pUL97(MX4)-Flag), lane 5 (IF-2 and IF-3, pUL97(74-707)-Flag) and lane 6 (IF-3, pUL97(157-707)-Flag) [25,26]). It should be mentioned that in CoIP analyses based on HCMV-infected cell lysates, co-immunoprecipitation of isoform 1 with cyclin T1 was mostly detected (Figure 2c, lanes 2 and 3), which, however, might rather refer to a limitation in detection sensitivity than to biochemical differences between pUL97 isoforms expressed in the two systems. Interestingly, the catalytically inactive mutant, pUL97(K355M)-Flag, also interacted with cyclin T1 (Figure 4b, lane 3), suggesting a mode of pUL97-cyclin T1 interaction independent of pUL97 activity.

\subsection{Interaction of the HCMV Protein Kinase pUL97 with Further Cyclins}

Further analysis revealed that pUL97 also interacted with cyclins other than cyclin T1. pUL97 was detected in precipitates of cyclin B1, the regulatory cofactor of CDK1, in a CoIP analysis using protein lysates of transiently transfected 293T cells (Figure 5a, lane 1). Like cyclin T1, cyclin B1 also interacted with the three isoforms of pUL97 (Figure 5a, lanes 4 (IF-1), 5 (IF-2 and IF-3), 6 (IF-3)). Importantly, the catalytically inactive mutant, pUL97(K355M)-Flag, was not co-immunoprecipitated with cyclin B1 (Figure 5a, lane 3). This finding may suggest that the interaction of pUL97 with cyclin B1 is dependent on the kinase activity of pUL97, in contrast to the interaction with cyclin T1. This points to a mechanistic difference between pUL97-cyclin T1 and pUL97-cyclin B1 interactions. Additional CoIP experiments also indicated an interaction between pUL97 and cyclin A (regulatory cofactor of CDK1 and CDK2; Figure 5b, lane 1), albeit with a lower affinity, whereas cyclin $\mathrm{H}$ (a component of the CDK7/cyclin H/MAT1 complex) could not be identified as an interaction partner of pUL97 (Figure 5b, lane 3). These findings strongly suggest that pUL97 does not exclusively interact with cyclin T1, but additionally with a selection of further cyclins. 
Figure 4. Mapping of the sequence domain of pUL97 responsible for the interaction with cyclin T1. Flag-tagged N- and C-terminal amino acid replacement and deletion mutants of pUL97 were transiently expressed in $293 \mathrm{~T}$ cells. Recombinantly expressed RFP was used as the transfection control. At two days post-transfection, CoIP analyses were performed. Either (a) endogenous cyclin T1 was immunoprecipitated using pAb(r)-cyclin T1 or (b) Flag-tagged pUL97 mutants were immunoprecipitated using mAb-Flag. Specificity of precipitation was demonstrated by CoIP using Dynabeads ${ }^{\circledR}$ Protein A without antibody (a, lane 12) and by the negative controls with recombinantly expressed pUL53-Flag (a and b, lane 2). pUL97(MX4)-Flag, multiple amino acid replacement mutant carrying missense mutations in alternative start codons of pUL97 isoform expression: M38A, M74L, M111L and M157A.
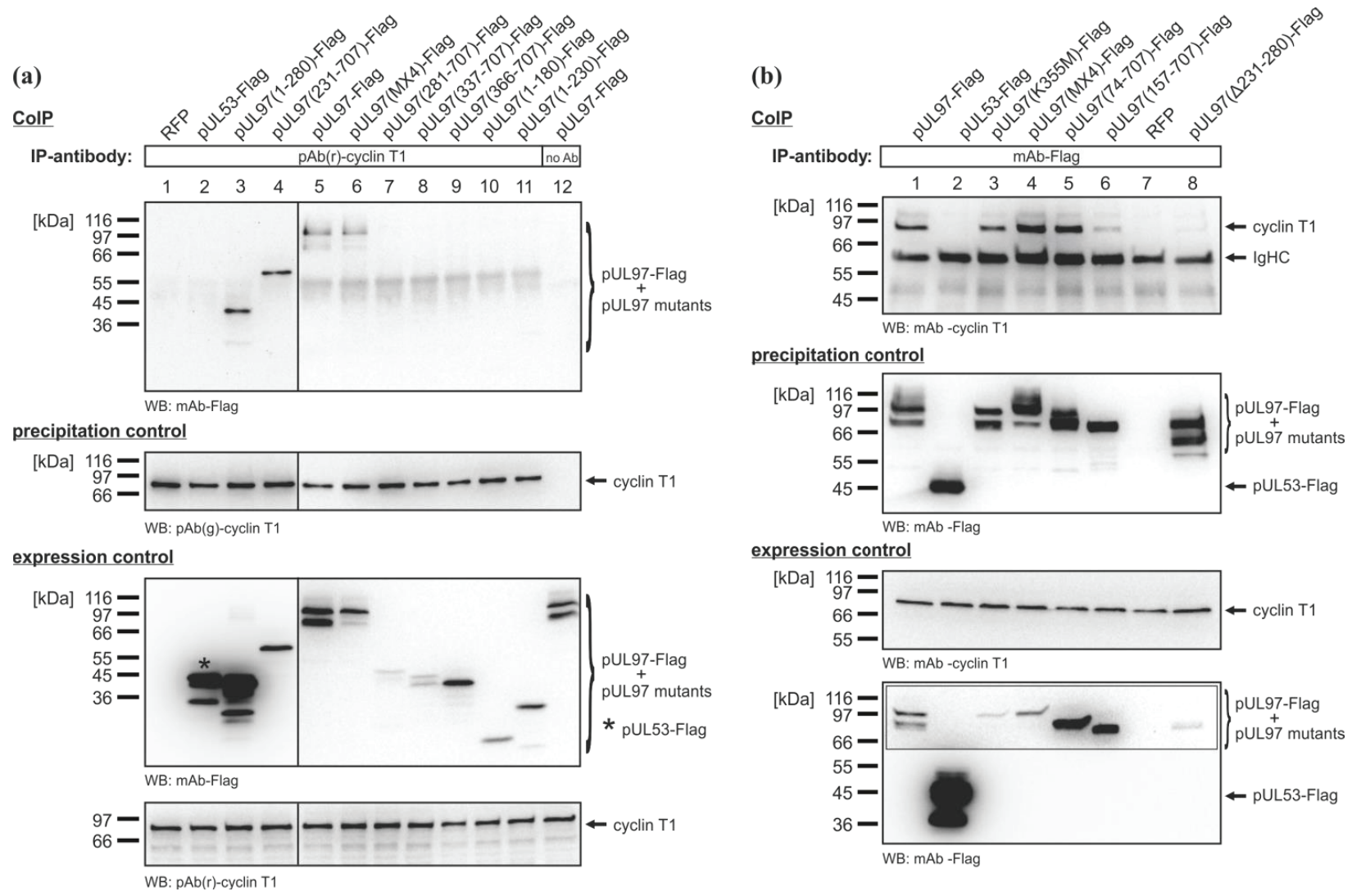

\subsection{Phosphorylation of pUL97 by the CDK9/cyclin T1 Complex in an ATP Consumption Assay}

With pUL97 interacting with cyclin T1, we next examined whether pUL97-cyclin T1 interaction triggered phosphorylation of pUL97 by the CDK9/cyclin T1 complex. Sequence analysis of pUL97 revealed that the mapped cyclin T1 interaction region (amino acids 231-280) did not contain the known, linear cyclin recognition motif, DOC_CYCLIN_1 ([RK].L. $\{0,1\}[$ FYLIVMP]; ELM database [38]), which is found in many CDK substrates. Furthermore, no known CDK phosphorylation site (MOD_CDK_1; ...([ST])P.[KR]; ELM database)) was detected in the amino acid sequence of pUL97. In order to address the question whether pUL97 is phosphorylated by the CDK9/cyclin T1 complex, an ATP consumption assay (ADP-Glo ${ }^{\text {TM }}$ Kinase Assay, Promega, Mannheim, Germany) was performed (ProQinase, Freiburg, Germany). In this nonradioactive in vitro assay, ATP 
consumption of CDK9 was measured as an indirect indicator of CDK9 substrate phosphorylation. Importantly, the results strongly indicate phosphorylation of the catalytically inactive mutant, pUL97(K355M)-Flag, by CDK9/cyclin $\mathrm{T} 1$ in a substrate concentration-dependent manner (Figure $6 \mathrm{c}$ ). The detected signals were clearly increased compared to a background control (mAbFlag (mAb, monoclonal antibody) coated protein A sepharose beads; Figure 6d). Interestingly, an inhibitory, concentration-dependent effect of the control mAb-Flag coated protein A sepharose beads on CDK9 was observed (Figure 6d). If it were the case that CDK9/cyclin T1 was generally inhibited in the presence of protein A sepharose beads, the actual phosphorylation of pUL97(K355M)-Flag might be underestimated under these experimental conditions. RBER-IRStide (artificial fusion protein containing a fragment of human retinoblastoma protein 1, Figure 6a) served as the reference substrate to control the kinase activity of the CDK9/cyclin T1 complex. Bovine histone H1 (a substrate of multiple kinases, excluding CDK9) was used as a negative control to monitor background signals (Figure 6b). CDK9 autophosphorylation signals determined in the absence of substrates were clearly lower than substrate phosphorylation signals (Figure 6a-d, column 1), and substrate background signals measured in the absence of the CDK9/cyclin T1 complex remained at a very low level, close to the detection limit (Figure 6a-d, panel no enzyme). These findings strongly suggest that pUL97 is phosphorylated by the CDK9/cyclin T1 complex. Considering the capability of pUL97 to interact with cyclins other than cyclin T1, the phosphorylation of pUL97 by further CDK/cyclin complexes seems also possible.

\section{Experimental Section}

\subsection{Cell Culture, HCMV Infections and Plasmid Transfection}

Embryonic kidney epithelial cells (293T) and retinal pigment epithelial cells (ARPE-19) were cultivated in Dulbecco's modified Eagle's medium containing 10\% FCS. Primary human foreskin fibroblasts (HFFs) and human foetal lung fibroblasts (MRC-5) were cultivated in MEM containing $7.5 \%$ or $10 \%$ FCS, respectively. Human first-trimester extravillous trophoblast (TEV-1) were cultivated in Ham's F 10 Nutrient Mix supplemented with 10\% FCS. The HCMV infection experiments were performed at a multiplicity of infection (m.o.i.) of 1.0 (or lower as indicated for specific experiments) using HCMV strains AD169, AD169-GFP [39] and Merlin [40]. Transfection of $293 \mathrm{~T}$ cells was performed using polyethylenimine reagent (Sigma-Aldrich, Taufkirchen, Germany), as described previously [29].

\subsection{Antibodies}

The following polyclonal $(\mathrm{pAb})$ and monoclonal $(\mathrm{mAb})$ antibodies were used in the present study: mAb-UL44 (BS 510, kindly provided by Prof. B. Plachter, Univ. Mainz, Mainz, Germany), mAb-UL97 (AL-1, kindly provided by Prof. M. N. Prichard, Univ. Alabama, Birmingham, AL, USA), pAb-UL97 (06/09, kindly provided by Prof. D. M. Coen, Harvard Medical School, Boston, MA, USA), mAb-pUL69 [41], mAb-cyclin A (sc-271645), mAb-cyclin B1 (sc-7393), pAb-cyclin B1 (sc-752), mAb-cyclin H (sc-1662), mAb-cyclin T1 (sc-271348), pAb(r)-cyclin T1 (sc-10750), pAb(g)-cyclin T1 (sc-8127), mAb-CDK9 (sc-13130; all Santa Cruz Biotechnology, Heidelberg, 
Germany), mAb-Flag (Sigma-Aldrich, Heidelberg, Germany) and mAb-HA (12CA5, Roche, Mannheim, Germany). The following fluorescent dye-conjugated secondary antibodies were applied in immunofluorescence analyses: Alexa 488-conjugated goat anti-rabbit $\operatorname{IgG}(\mathrm{H}+\mathrm{L})$ and Alexa 555-conjugated goat anti-mouse IgG $(\mathrm{H}+\mathrm{L})$ (Life Technologies, Darmstadt, Germany).

Figure 5. CoIP analyses used to reveal further cyclin interaction partners of pUL97. Flag-tagged amino acid replacement and deletion mutants of pUL97 were transiently expressed in $293 \mathrm{~T}$ cells. At 2 days post-transfection, cells were lysed and used for co-immunoprecipitation analysis with (a) pAb-cyclin B1 (lanes 1-6) and the Fc fragments of rabbit antibodies as the control (Fc(r), Rabbit IgG Fc-Fragment, Jackson ImmunoResearch; lane 7), respectively; or with (b) mAb-Flag. CoIP analyses with recombinantly expressed pUL53-Flag (a, lane 2; b, lanes 2 and 4) served as a negative control and the self-interaction of pUL97 as the positive control (b, lane 5). * cyclin A-specific band.

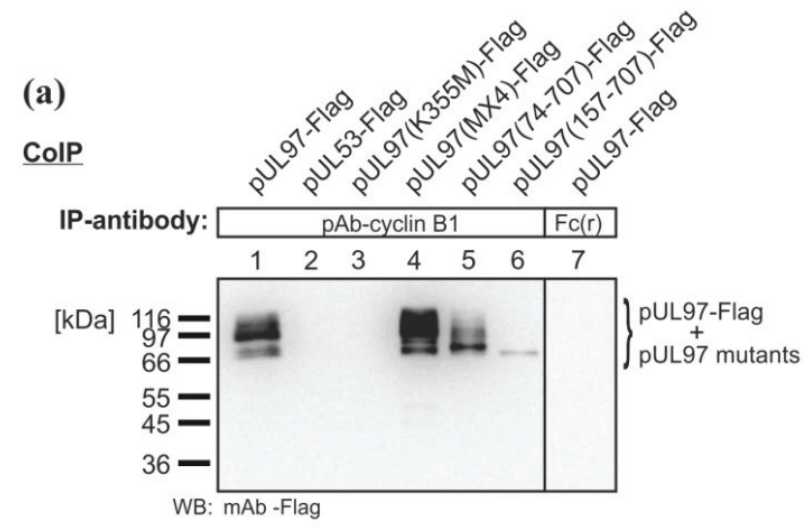

precipitation control

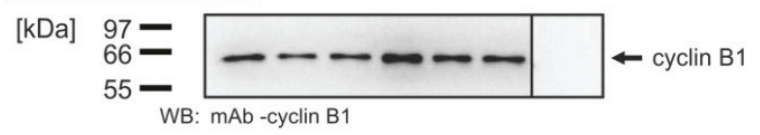

expression control
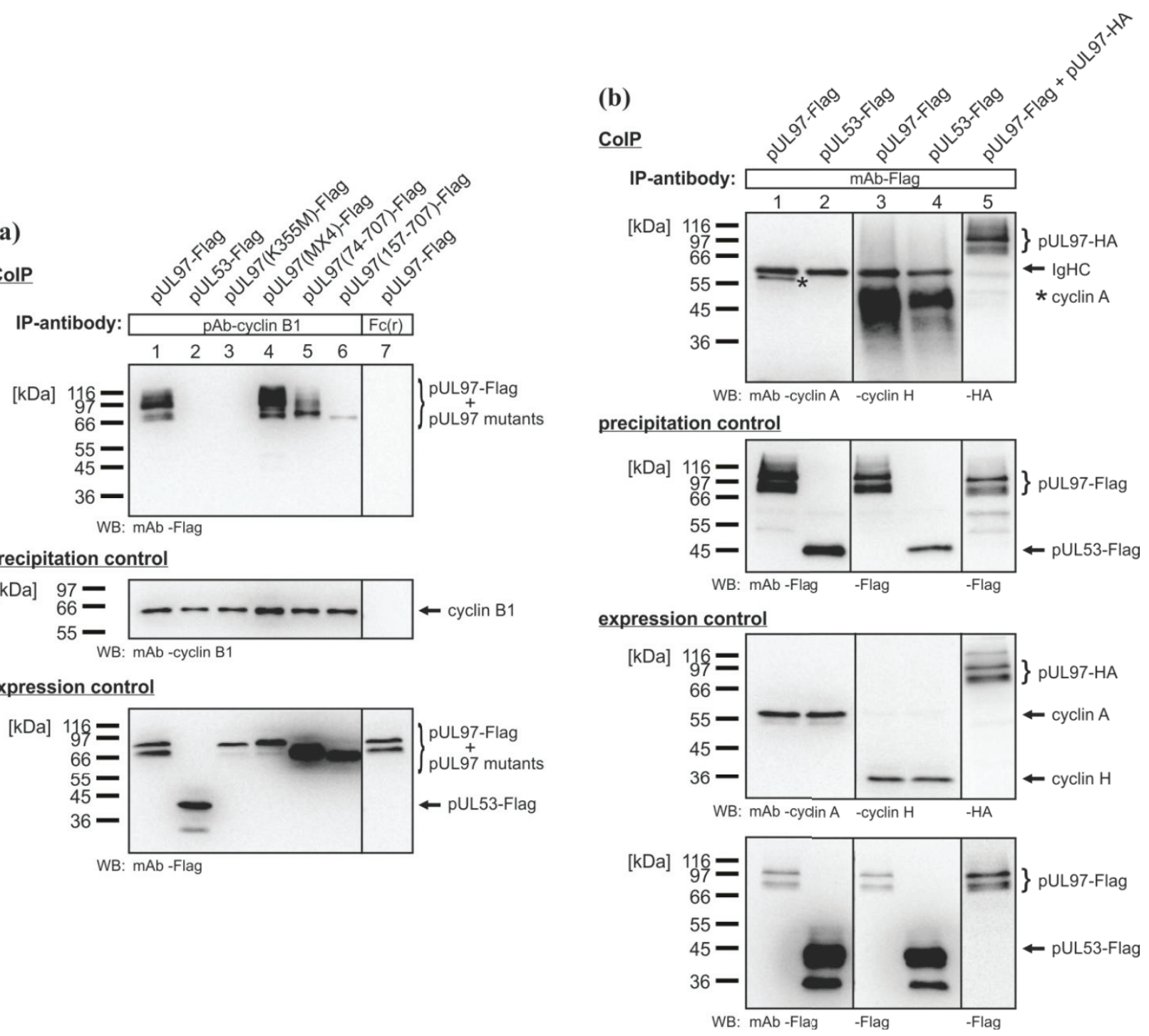
Figure 6. ATP consumption assay used to investigate the putative phosphorylation of pUL97 by the CDK9/cyclin T1 complex. The catalytically inactive mutant pUL97(K355M)-Flag was expressed in 293T cells and 2 days post-transfection, immunoprecipitated from cell lysates using mAb-Flag. Immunoprecipitates and mAb-Flag coated protein A sepharose beads (negative control) were used to determine luminescence signals (counts per second (cps)) as a measure for ADP generation by the active CDK9/cyclin T1 complex; the assay was performed in duplicate $(n=2)$. (a) Specific substrate RBER-IRStide (positive control); (b) substrate bovine histone H1 (negative control); (c) pUL97(K355M)-Flag (substrate in question); (d) mAb-Flag coated protein A sepharose beads (negative control). All substrates were used in increasing amounts.

(a)

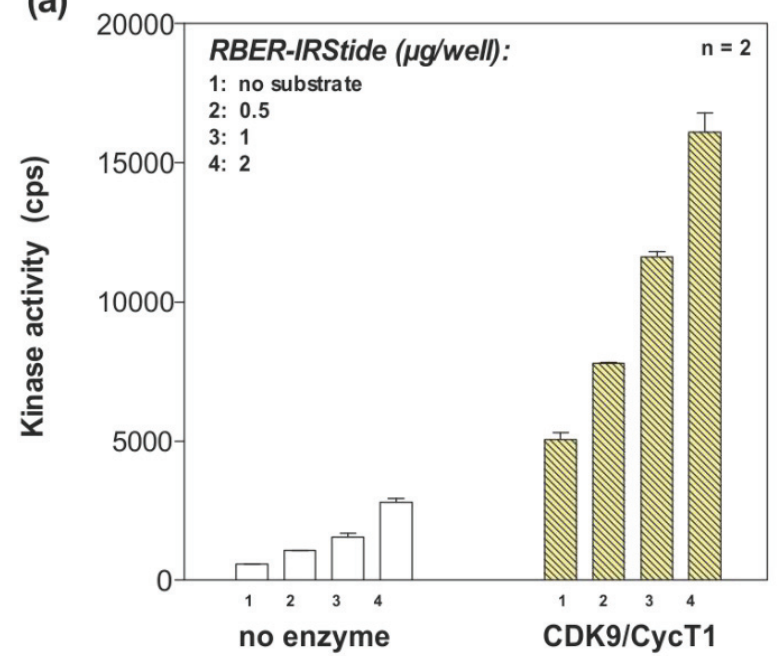

(c)

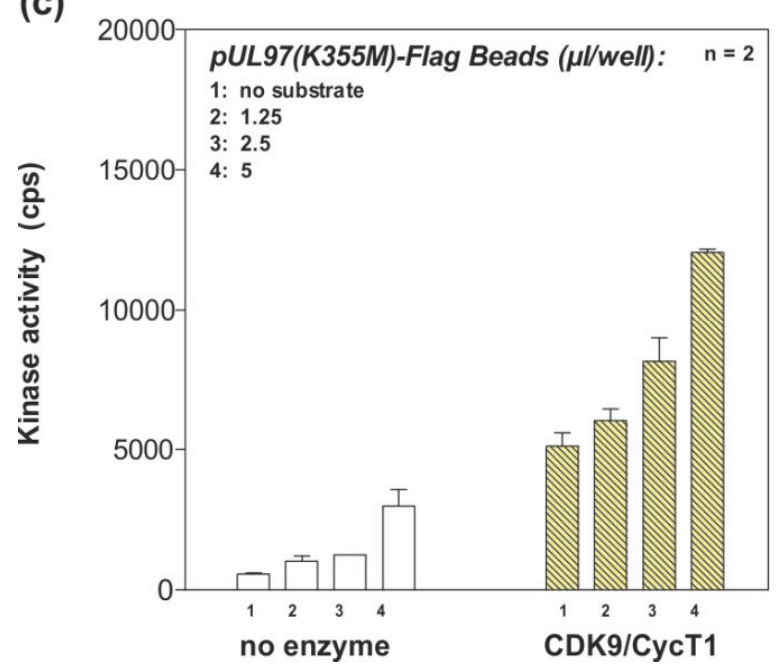

(b)

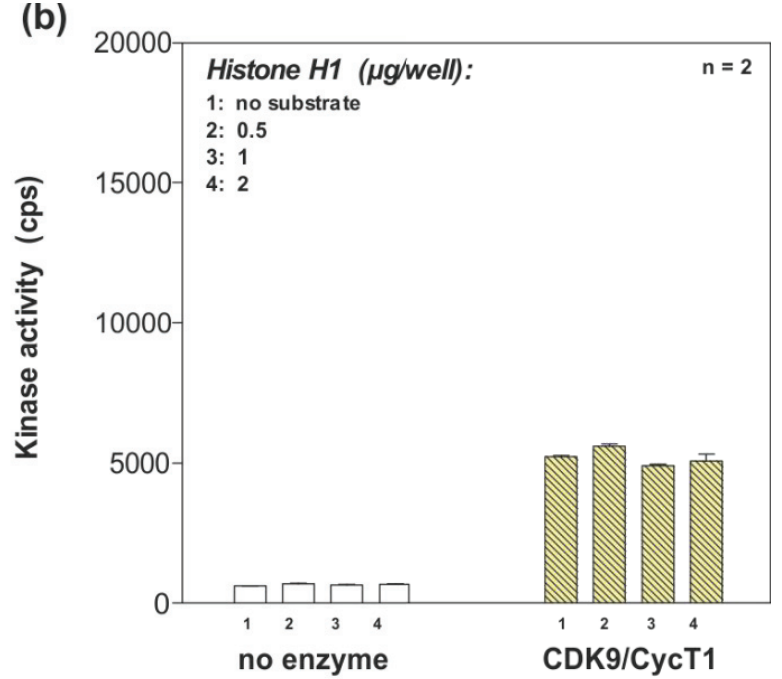

(d)

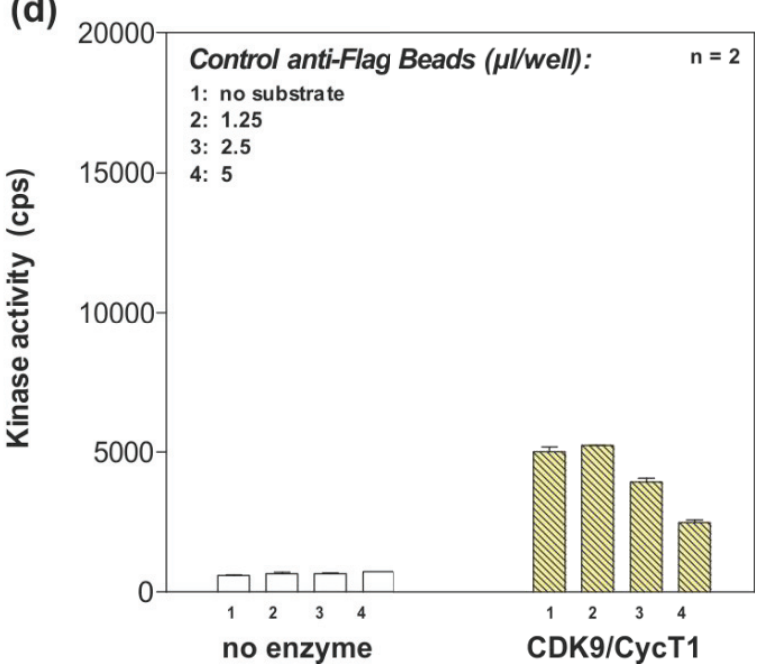

\subsection{Co-Immunoprecipitation}

293 cells were seeded in 10-cm dishes (cell number: $5.1 \times 10^{6}$ ) and transfected with expression plasmids coding for full-length pUL97 or mutants of pUL97 and pUL53, as described previously [23,26,29,42] using the polyethylenimine transfection technique [29]. Red fluorescent protein (RFP; pDsRed1-N1; BD Clontech, Heidelberg, Germany) was used as a transfection 
control. HCMV AD169-GFP-infected HFFs cultivated in cell culture flasks were harvested at 6 days post-infection. Both transfected 293T cells and HCMV infected HFFs were lysed in $500 \mathrm{~mL}$ CoIP buffer (50 mM Tris/HCl (pH 8.0), $150 \mathrm{mM} \mathrm{NaCl,} 5 \mathrm{mM}$ EDTA, 0.5\% NP-40, $1 \mathrm{mM}$ PMSF, $2 \mu \mathrm{g}$ aprotinin $\mathrm{mL}^{-1}, 2 \mu \mathrm{g}$ leupeptin $\mathrm{mL}^{-1}$ and $2 \mu \mathrm{g}$ pepstatin $\mathrm{mL}^{-1}$ ) and incubated with antibody-coated Dynabeads ${ }^{\circledR}$ Protein A $(50 \mu \mathrm{L}$ per sample; Life Technologies, Darmstadt, Germany) for $2 \mathrm{~h}$ at $4{ }^{\circ} \mathrm{C}$ under rotation. The precipitates were washed five times $(1 \mathrm{~mL}$ each) with CoIP buffer before the samples were subjected to standard Western blot analysis using tag- or protein-specific antibodies for the detection of co-immunoprecipitates and protein expression (ECL staining; New England Biolabs, Frankfurt/Main, Germany).

\subsection{Immunofluorescence Analyses}

HFF, MRC-5, ARPE-19 and TEV-1 cells were grown on coverslips and used for infection with various HCMV strains. At indicated time points, cells were fixed with $4 \%$ paraformaldehyde solution (10 min, room temperature) and permeabilized by incubation with $0.2 \%$ Triton $\mathrm{X}-100$ solution $\left(20 \mathrm{~min}, 4{ }^{\circ} \mathrm{C}\right.$ ). Non-specific staining was blocked by incubation with $2 \mathrm{mg} / \mathrm{mL}$ human $\gamma$-globulin (Cohn fraction II; Sigma Aldrich, Taufkirchen, Germany, $30 \mathrm{~min}, 37^{\circ} \mathrm{C}$ ). Proteins were detected by incubation with primary antibodies for $90 \mathrm{~min}$ at $37{ }^{\circ} \mathrm{C}$, followed by incubation with dye-conjugated secondary antibodies for $30 \mathrm{~min}$ at $37{ }^{\circ} \mathrm{C}$. Cell samples were mounted with Vectashield Mounting Medium containing DAPI and analysed using a DMI6000 B microscope and a $63 \times$ HCX PL APO CS oil immersion objective lens (Leica Microsystems, Mannheim, Germany). Confocal laser-scanning microscopy was performed with a TCS SP5 microscope (Leica Microsystems, Mannheim, Germany). Images were processed using the Meta-Imaging series (Molecular Devices, Biberach, Germany), LAS AF software (version 1.8.2 build 1,465; Leica Microsystems) and Adobe Photoshop (version 8.0.1; Adobe Systems Incorporated).

\subsection{ATP Consumption Assay}

An ATP consumption assay (ADP-Glo ${ }^{\mathrm{TM}}$ Kinase Assay, Promega, Mannheim, Germany) was performed in order to measure the CDK9-dependent phosphorylation of pUL97 (ProQinase, Freiburg, Germany). The catalytically inactive mutant, pUL97(K355M)-Flag, was expressed in $293 \mathrm{~T}$ cells. Two days post-transfection, 293T cells were harvested and lysed in $400 \mu \mathrm{L}$ of RIPA lysis buffer (0.1\% SDS, 1\% Na-deoxycholate, 1\% Triton X-100, 0.5\% NP40, 1 mM EDTA, $10 \mathrm{mM}$ Tris/ $\mathrm{HCl}(\mathrm{pH} 7.5), 150 \mathrm{mM} \mathrm{NaCl}$ ) supplemented with protease inhibitors (Complete Mini, Roche; 1 tablet for $10 \mathrm{~mL}$ ). The lysate was incubated with $\mathrm{mAb}$-Flag-coated protein A sepharose beads (200 $\mu \mathrm{L}$ of antibody-coated protein A sepharose beads per sample) for $2 \mathrm{~h}$ under rotation. The immunoprecipitates were washed with RIPA lysis buffer three times. Washed mAb-Flag coated protein A sepharose beads, incubated with protein lysates from 293T cells not expressing pUL97(K355M)-Flag, were prepared as a negative control. The ATP consumption assay was performed as follows. The reaction cocktail, containing $7.5 \mu \mathrm{L}$ of $3.33 \times$ standard ATP consumption assay buffer (167 mM HEPES-NaOH [pH 7.5], $10 \mathrm{mM} \mathrm{MgCl}_{2}, 10 \mu \mathrm{M}$ Na-orthovanadate and $3.33 \mathrm{mM}$ DTT), $7.5 \mu \mathrm{L}$ of a substrate (various amounts of RBER-IRStide (ProQinase, Freiburg, Germany), histone H1 (Sigma-Aldrich, Munich, Germany), pUL97(K355M)-Flag immunoprecipitates 
or mAb-Flag coated protein A sepharose beads), $5 \mu \mathrm{L}$ of the purified kinase complex, CDK9/cyclin T1 (100 ng diluted in $1 \times$ kinase dilution buffer (50 mM HEPES-NaOH (pH 7.5), $0.25 \mathrm{mg} / \mathrm{mL}$ PEG20000, 1 mM DTT), ProQinase, Freiburg, Germany) and $5 \mu \mathrm{L}$ of ATP (UltraPure), was mixed in 96-well white flat bottom half-area plates and incubated for $30 \mathrm{~min}$ at $30{ }^{\circ} \mathrm{C}$ (measured in duplicate). Subsequently, $25 \mu \mathrm{L}$ of $\mathrm{ADP}-\mathrm{Glo}^{\mathrm{TM}}$ reagent were added, and the samples were incubated for $40 \mathrm{~min}$ at room temperature. After the addition of $50 \mu \mathrm{L}$ of ADP-Glo ${ }^{\mathrm{TM}}$ Kinase Detection reagent, the samples were incubated for another $60 \mathrm{~min}$ at room temperature in the dark. Resulting luminescence (counts per second (cps)) as a measure for ADP-generation by the active kinases was determined using a VICTOR reader (Perkin-Elmer, Hamburg, Germany).

\subsection{Yeast-Two-Hybrid System}

Protein interactions were analysed using GAL4 fusion proteins (GAL4-BD, DNA binding domain; GAL4-AD, activation domain) in the yeast two-hybrid system, as described previously [9,34]. pUL97 fused to the GAL4-BD $[33,34]$ and cyclin T1 fused to the GAL4-AD (kindly provided by Dr. Giuliana Napolitano, University of Monte S. Angelo, Italy [43]) were expressed in the Saccharomyces cerevisiae strain, Y153. pGAD424 and pGBT9 (Clontech) served as vector controls and pVA3 (p53) and pTD1 (SV40 large T antigen) as an interaction control. Selection for the presence of bait and interactor plasmids was achieved by cultivation on medium restricting growth to histidine/tryptophan/leucine prototrophy. Selected colonies were analysed for $\beta$-galactosidase activity by filter lift tests.

\section{Conclusions}

Interactions between herpesviral kinases and cellular cyclins have not been described so far. This study provides the first evidence that the HCMV protein kinase, pUL97, interacts with cyclins, as demonstrated by well-established methods of yeast two-hybrid assay, co-immunoprecipitation and confocal microscopy-based colocalization studies. In particular, the sequence domain of pUL97, responsible for the interaction with cyclin T1, was between amino acids 231-280. This biochemically defined interaction region is located within the non-globular N-terminus of pUL97 and is distinct from the pUL97 protein kinase domain, which is determined by subdomains I-XI comprising amino acids 337-651 [18].

Although this cyclin T1 interaction region of pUL97 does not contain a known cyclin recognition motif and the entire pUL97 sequence does not comprise a known CDK phosphorylation motif, the results of an ATP consumption assay strongly suggest that pUL97 is recognized and phosphorylated by the CDK9/cyclin T1 complex. Due to the fact that kinase activity of CDK9/cyclin T1 was determined in an indirect approach by the quantitative determination of ATP consumption, additional in vitro kinase assays using radioactive ATP should be performed to demonstrate the phosphorylation of pUL97 directly and to confirm our conclusion. The detection of further cyclins (cyclin B1 and cyclin A) as interaction partners of pUL97 points towards a more complex mode of pUL97-CDK/cyclin inter-regulation. The question about whether CDK-mediated phosphorylation of pUL97 influences its kinase activity or multifunctionality has not been addressed and requires further investigation. Presently, we are working on the identification of 
motifs in pUL97 mediating cyclin interaction, such as a putative zinc finger motif (see the interaction between cyclin $\mathrm{T} 1$ and that Tat protein of the human immunodeficiency virus [44]) or potential linear interaction motifs [45].

Importantly, the structural and functional similarities between pUL97 and CDKs, the phosphorylation of identical viral and cellular substrates [3] and the interaction between pUL97 and cyclins described here suggest that multimeric complexes may be formed. Such complexes might consist of CDKs, cyclins, pUL97 and further viral proteins or substrates. pUL97 could only transiently be included, so that varying kinase activities might be involved in the regulation of phosphorylation-dependent processes during HCMV replication. They also may only form under certain conditions, as we showed that pUL97 did not co-immunoprecipitate with CDK9. This finding might indicate the lack of interaction or, more likely, a competitive mode of binding of CDK9 and pUL97 to cyclin T1 or the existence of a dynamic complex (trimeric or multimeric) that may only transiently include CDK9, pUL97 and cyclin T1. In the latter scenario, cyclin T1 may play the role of a bridging factor between pUL97 and CDK9, triggering phosphorylation events (see Section 2.5). Interactive regulatory steps of mutual phosphorylation between $\mathrm{CDK} /$ cyclin complexes and pUL97 seem probable in this scenario. The regulation of the activity of the viral RNA transporter, pUL69, is an example of such interplay between CDKs and pUL97. In this case, CDK9 and pUL97 contribute to an activating phosphorylation of pUL69 $[9,32,46]$. The protein region of pUL97 required for the interaction with pUL69 (amino acids 231-336 [32]) overlaps with the cyclin T1 interaction domain of pUL97 (amino acids 231-280), most likely indicating a putative role of cyclin T1 in pUL97-pUL69 interaction. This example of activating pUL69 phosphorylation emphasizes an interplay between CDK and pUL97 activities in phosphorylation-dependent processes during HCMV replication. Interestingly, the mapped region of pUL97 interacting with cyclin T1 is also responsible for the self-interaction of pUL97 (amino acids 231-280 [29]), so that the potential of pUL97 to oligomerize might also be linked to the formation of multimeric complexes. Future experimentation should substantiate this scenario, possibly also including further aspects of pUL97-cyclin interaction, such as a putative regulatory impact on viral DNA synthesis and late gene expression. Taken together, our present and previous findings support the hypothesis that pUL97 not only mimics CDK function, but actively associates with cyclins or $\mathrm{CDK} /$ cyclin heteromers to form functional complexes. Thus, the formation of such cyclin-based complexes may provide a platform for pUL97 and CDKs to undergo mutual trans-phosphorylation and fine-regulation.

\section{Acknowledgments}

We gratefully acknowledge Mark Prichard (UAB, Birmingham, AL, USA) and Donald Coen (Harvard Medical School, Boston, MA, USA), for providing pUL97-specific antibodies, Giuliana Napolitano (University of Monte S. Angelo, Italy) for cyclin T1 clones, Gillian Scott, Jenna Iwasenko and Zin Naing (Virology Div., POW Hospital, Randwick, Australia) for cooperation within an international exchange program, Eileen Socher (Div. Bioinformatics, University of Erlangen-Nuremberg, Germany) for input on bioinformatics, Thomas Stamminger and co-workers for long-term cooperation and Sabine Feichtinger, Corina Hutterer and members of the M.M. research lab for very valuable scientific support. We would like to thank ProQinase GmbH 
(Freiburg, Germany) for performing the ATP consumption assay. This study was supported by the Deutsche Forschungsgemeinschaft SFB796 (C3 and A2), DFG MA 1289/6-1, Wilhelm Sander-Stiftung (grant 2011.085.1) and DAAD/Go8 (grant 54390135).

\section{Conflicts of Interest}

The authors declare no conflict of interest.

\section{References and Notes}

1. Mocarski, E.S.; Shenk, T.; Pass, R.F. Cytomegaloviruses. In Fields Virology, 5th ed.; Knipe, D.M., Howley, P.M., Eds.; Lippincott Williams \& Wilkins: Philadelphia, PA, USA, 2007; Volume 2, pp. 2701-2772.

2. Schreiber, A.; Härter, G.; Schubert, A.; Bunjes, D.; Mertens, T.; Michel, D. Antiviral treatment of cytomegalovirus infection and resistant strains. Expert Opin. Pharmacother. 2009, 10, 191-209.

3. Marschall, M.; Feichtinger, S.; Milbradt, J. Regulatory roles of protein kinases in cytomegalovirus replication. Adv. Virus Res. 2011, 80, 69-101.

4. Marschall, M.; Stamminger, T. Molecular targets for antiviral therapy of cytomegalovirus infections. Future Microbiol. 2009, 4, 731-742.

5. Prichard, M.N. Function of human cytomegalovirus UL97 kinase in viral infection and its inhibition by maribavir. Rev. Med. Virol. 2009, 19, 215-229.

6. Chou, S. Cytomegalovirus UL97 mutations in the era of ganciclovir and maribavir. Rev. Med. Virol. 2008, 18, 233-246.

7. Herget, T.; Marschall, M. Recent developments in anti-herpesviral combination therapy based on protein kinase inhibitors. In New Concepts of Antiviral Therapy; Bogner, E., Holzenburg, A., Eds.; Springer: London, UK, 2006; pp. 351-371.

8. Schang, L.M.; St. Vincent, M.R.; Lacasse, J.J. Five years of progress on cyclindependent kinases and other cellular proteins as potential targets for antiviral drugs. Antivir. Chem. Chemother. 2006, 17, 293-320.

9. Feichtinger, S.; Stamminger, T.; Müller, R.; Graf, L.; Klebl, B.; Eickhoff, J.; Marschall, M. Recruitment of cyclin-dependent kinase 9 to nuclear compartments during cytomegalovirus late replication: Importance of an interaction between viral pUL69 and cyclin T1. J. Gen. Virol. 2011, 92, 1519-1531.

10. Schang, L.M. First demonstration of the effectiveness of inhibitors of cellular protein kinases in antiviral therapy. Expert Rev. Anti Infect. Ther. 2006, 4, 953-956.

11. Sanchez, V.; McElroy, A.K.; Yen, J.; Tamrakar, S.; Clark, C.L.; Schwartz, R.A.; Spector, D.H. Cyclin-dependent kinase activity is required at early times for accurate processing and accumulation of the human cytomegalovirus UL122-123 and UL37 immediate-early transcripts and at later times for virus production. J. Virol. 2004, 78, 11219-11232. 
12. Hutterer, C.; Wandinger, S.K.; Wagner, S.; Müller, R.; Stamminger, T.; Zeitträger, I.; Godl, K.; Baumgartner, R.; Strobl, S.; Marschall, M. Profiling of the kinome of cytomegalovirus-infected cells reveals the functional importance of host kinases Aurora A, ABL and AMPK. Antivir. Res. 2013, 99, 139-148.

13. Kapasi, A.J.; Spector, D.H. Inhibition of the cyclin-dependent kinases at the beginning of human cytomegalovirus infection specifically alters the levels and localization of the RNA polymerase II carboxyl-terminal domain kinases $c \mathrm{kk} 9$ and $\operatorname{cdk} 7$ at the viral transcriptosome. J. Virol. 2008, 82, 394-407.

14. Sanchez, V.; Spector, D.H. Cyclin-dependent kinase activity is required for efficient expression and posttranslational modification of human cytomegalovirus proteins and for production of extracellular particles. J. Virol. 2006, 80, 5886-5896.

15. Tamrakar, S.; Kapasi, A.J.; Spector, D.H. Human cytomegalovirus infection induces specific hyperphosphorylation of the carboxyl-terminal domain of the large subunit of RNA polymerase II that is associated with changes in the abundance, activity, and localization of cdk9 and cdk7. J. Virol. 2005, 79, 15477-15493.

16. Marschall, M.; Stein-Gerlach, M.; Freitag, M.; Kupfer, R.; van den Bogaard, M.; Stamminger, T. Direct targeting of human cytomegalovirus protein kinase pUL97 by kinase inhibitors is a novel principle of antiviral therapy. J. Gen. Virol. 2002, 83, 1013-1023.

17. Prichard, M.N.; Gao, N.; Jairath, S.; Mulamba, G.; Krosky, P.; Coen, D.M.; Parker, B.O.; Pari, G.S. A recombinant human cytomegalovirus with a large deletion in UL97 has a severe replication deficiency. J. Virol. 1999, 73, 5663-5670.

18. Romaker, D.; Schregel, V.; Maurer, K.; Auerochs, S.; Marzi, A.; Sticht, H.; Marschall, M. Analysis of the structure-activity relationship of four herpesviral UL97 subfamily protein kinases reveals partial but not full functional conservation. J. Med. Chem. 2006, 49, 7044-7053.

19. Kuny, C.V.; Chinchilla, K.; Culbertson, M.R.; Kalejta, R.F. Cyclin-dependent kinase-like function is shared by the beta- and gamma-subset of the conserved herpesvirus protein kinases. PLoS Pathog. 2010, 6, 1001092:1-1001092:17.

20. Hume, A.J.; Finkel, J.S.; Kamil, J.P.; Coen, D.M.; Culbertson, M.R.; Kalejta, R.F. Phosphorylation of retinoblastoma protein by viral protein with cyclin-dependent kinase function. Science 2008, 320, 797-799.

21. Prichard, M.N.; Sztul, E.; Daily, S.L.; Perry, A.L.; Frederick, S.L.; Gill, R.B.; Hartline, C.B.; Streblow, D.N.; Varnum, S.M.; Smith, R.D.; Kern, E.R. Human cytomegalovirus UL97 kinase activity is required for the hyperphosphorylation of retinoblastoma protein and inhibits the formation of nuclear aggresomes. J. Virol. 2008, 82, 5054-5067.

22. Hertel, L.; Chou, S.; Mocarski, E.S. Viral and cell cycle-regulated kinases in cytomegalovirus-induced pseudomitosis and replication. PLoS Pathog. 2007, 3, 6:0014-6:0022.

23. Marschall, M.; Stein-Gerlach, M.; Freitag, M.; Kupfer, R.; van den Bogaard, M.; Stamminger, T. Inhibitors of human cytomegalovirus replication drastically reduce the activity of the viral protein kinase pUL97. J. Gen. Virol. 2001, 82, 1439-1450.

24. Hanks, S.K.; Quinn, A.M.; Hunter, T. The protein kinase family: Conserved features and deduced phylogeny of the catalytic domains. Science 1988, 241, 42-52. 
25. Webel, R.; Solbak, S.M.Ø.; Fossen, T.; Auerochs, S.; Sticht, H.; Chou, S.; Marschall, M. Specification of the HCMV pUL97 isoforms: Differences in subcellular localization and functionality. In Proceedings of the 37th Annual International Herpesvirus Workshop, Calgary, Canada, 4-9 August 2012; No. 3.22.

26. Webel, R.; Milbradt, J.; Auerochs, S.; Schregel, V.; Held, C.; Nöbauer, K.; Razzazi-Fazeli, E.; Jardin, C.; Wittenberg, T.; Sticht, H.; Marschall, M. Two isoforms of the protein kinase pUL97 of human cytomegalovirus are differentially regulated in their nuclear translocation. J. Gen. Virol. 2011, 92, 638-649.

27. Webel, R.; Hakki, M.; Prichard, M.; Rawlinson, D.W.; Marschall, M.; Chou, S. Differential properties of three isoforms of cytomegalovirus protein kinase pUL97 affect viral replication and maribavir susceptibility. J. Virol. 2013, to be submitted for publication.

28. Webel, R.; Solbak, S.M.Ø.; Held, C.; Milbradt, J.; Groß, A.; Eichler, J.; Wittenberg, T.; Jardin, C.; Sticht, H.; Fossen, T.; Marschall, M. The nuclear import of isoforms of the cytomegalovirus kinase pUL97 is mediated by differential activity of NLS1 and NLS2 both acting through classical importin- $\alpha$ binding. J. Gen. Virol. 2012, 93, 1756-1768.

29. Schregel, V.; Auerochs, S.; Jochmann, R.; Maurer, K.; Stamminger, T.; Marschall, M. Mapping of a self-interaction domain of the cytomegalovirus protein kinase pUL97. J. Virol. 2007, 88, 395-404.

30. Baek, M.C.; Krosky, P.M.; Pearson, A.; Coen, D.M. Phosphorylation of the RNA polymerase II carboxyl-terminal domain in human cytomegalovirus-infected cells and in vitro by the viral UL97 protein kinase. Virology 2004, 324, 184-193.

31. Kawaguchi, Y.; Matsumura, T.; Roizman, B.; Hirai, K. Cellular elongation factor $1 \delta$ is modified in cells infected with representative alpha-, beta-, or gammaherpesviruses. J. Virol. 1999, 73, 4456-4460.

32. Thomas, M.; Rechter, S.; Milbradt, J.; Auerochs, S.; Müller, R.; Stamminger, T.; Marschall, M. The cytomegaloviral protein kinase pUL97 interacts with the nuclear mRNA export factor pUL69 to modulate its intranuclear localization and activity. J. Gen. Virol. 2009, 90, 567-578.

33. Marschall, M.; Freitag, M.; Suchy, P.; Romaker, D.; Kupfer, R.; Hanke, M.; Stamminger, T. The protein kinase pUL97 of human cytomegalovirus interacts with and phosphorylates the DNA polymerase processivity factor pUL44. Virology 2003, 311, 60-71.

34. Marschall, M.; Marzi, A.; aus dem Siepen, P.; Jochmann, R.; Kalmer, M.; Auerochs, S.; Lischka, P.; Leis, M.; Stamminger, T. Cellular p32 recruits cytomegalovirus kinase pUL97 to redistribute the nuclear lamina. J. Biol. Chem. 2005, 280, 33357-33367.

35. Marschall, M. Institute for Clinical and Molecular Virology, University of Erlangen-Nuremberg, Erlangen, Germany. p32/gC1qR, a multi-ligand binding protein. Unpublished work, 2013.

36. Becke, S.; Fabre-Mersseman, V.; Aue, S.; Auerochs, S.; Sedmak, T.; Wolfrum, U.; Strand, D.; Marschall, M.; Plachter, B.; Reyda, S. Modification of the major tegument protein pp 65 of 1 human cytomegalovirus inhibits viral growth and leads to the enhancement of a protein complex with pUL69 and pUL97 in infected cells. J. Gen. Virol. 2010, 91, 2531-2541.

37. Peng, J.; Zhu, Y.; Milton, J.T.; Price, D.H. Identification of multiple cyclin subunits of human P-TEFb. Genes Dev. 1998, 12, 755-762. 
38. Dinkel, H.; Michael, S.; Weatheritt, R.J.; Davey, N.E.; van Roey, K., Altenberg, B.; Toedt, G.; Uyar, B.; Seiler, M.; Budd, A.; et al. ELM-The database of eukaryotic linear motifs. Nucleic Acids Res. 2012, 40 (Database issue), D242-D251.

39. Marschall, M.; Freitag, M.; Weiler, S.; Sorg, G.; Stamminger, T. Recombinant green fluorescent protein-expressing human cytomegalovirus as a tool for screening antiviral agents. Antimicrob. Agents Chemother. 2000, 44, 1588-1597.

40. Stanton, R.J.; Baluchova, K.; Dargan, D.J.; Cunningham, C.; Sheehy, O.; Seirafian, S.; McSharry, B.P.; Neale, M.L.; Davies, J.A.; Tomasec, P.; et al. Reconstruction of the complete human cytomegalovirus genome in a BAC reveals RL13 to be a potent inhibitor of replication. J. Clin. Investig. 2010, 120, 3191-3208.

41. Winkler, M.; Rice, S.A.; Stamminger, T. UL69 of human cytomegalovirus, an open reading frame with homology to ICP27 of herpes simplex virus, encodes a transactivator of gene expression. J. Virol. 1994, 68, 3943-3954.

42. Milbradt, J.; Auerochs, S.; Marschall, M. Cytomegaloviral proteins pUL50 and pUL53 are associated with the nuclear lamina and interact with cellular protein kinase C. J. Gen. Virol. 2007, 88, 2642-2650.

43. Fraldi, A.; Licciardo, P.; Majello, B.; Giordano, A.; Lania, L. Distinct regions of cyclinT1 are required for binding to CDK9 and for recruitment to the HIV-1 Tat/TAR complex. J. Cell. Biochem. Suppl. 2001, 36, 247-253.

44. Tahirov, T.H.; Babayeva, N.D.; Varzavand, K.; Cooper, J.J.; Sedore, S.C.; Price, D.H. Crystal structure of HIV-1 Tat complexed with human P-TEFb. Nature 2010, 465, 747-751.

45. Marschall, M.; Sticht, H. Institute for Clinical and Molecular Virology and Institute of Biochemistry, University of Erlangen-Nuremberg, Erlangen, Germany. Identification of motifs in pUL97 mediating cyclin interaction. Unpublished work, 2013.

46. Rechter, S.; Scott, G.M.; Eickhoff, J.; Zielke, K.; Auerochs, S.; Müller, R.; Stamminger, T.; Rawlinson, W.D.; Marschall, M. Cyclin-dependent kinases phosphorylate the cytomegalovirus RNA export protein pUL69 to modulate its nuclear localization and activity. J. Biol. Chem. 2009, 284, 8605-8613. 

Reprinted from Viruses. Cite as: Rana, R.; Biegalke, B.J. Human Cytomegalovirus UL34 Early and Late Proteins Are Essential for Viral Replication. Viruses 2014, 6, 476-488.

Article

\title{
Human Cytomegalovirus UL34 Early and Late Proteins Are Essential for Viral Replication
}

\author{
Rico Rana ${ }^{1}$ and Bonita J. Biegalke ${ }^{2, *}$
}

1 Department of Biomedical Sciences, Heritage College of Osteopathic Medicine, Ohio University, 228 Irvine Hall, Athens, OH 45701, USA; E-Mail: rico.rana@yahoo.com

2 Department of Biomedical Sciences and Program in Molecular and Cellular Biology, Heritage College of Osteopathic Medicine, Ohio University, 228 Irvine Hall, Athens, OH 45701, USA

* Author to whom correspondence should be addressed; E-Mail: biegalke@ohio.edu; Tel.: +1-740-593-2377; Fax: +1-740-593-2778.

Received: 10 December 2013; in revised form: 17 January 2014 / Accepted: 21 January 2014 / Published: 28 January 2014

\begin{abstract}
UL34 is one of the $\sim 50$ genes of human cytomegalovirus (HCMV) required for replication in cell culture in human fibroblasts. UL34 encodes highly related early (UL34a) and late (UL34b) proteins that are virtually identical, with the early protein containing an additional 21 amino terminal amino acids. The UL34 proteins are sequence-specific DNA-binding proteins that localize to the nucleus. The HCMV genome contains 14 to 15 UL34 binding sites; two of the UL34 binding sites contribute to transcriptional regulation of two other viral genes, US3 and US9. The roles of the remaining binding sites and the requirement for both UL34 proteins during viral infection remain unknown. We examined the contributions of the early and late UL34 proteins to viral replication by generating HCMV-containing bacterial artificial chromosomes with the initiation codon for the early or the late protein mutated. Neither virus was able to replicate, demonstrating that UL34 expression is required throughout the viral replication cycle. A marked decrease in viral gene expression for each of the mutants suggests that UL34 proteins may contribute generally to transcriptional regulation. Intracellular localization studies demonstrated that UL34 colocalizes with the major immediate early protein, IE2, and the viral DNA polymerase processivity factor, UL44, to viral DNA replication centers. In conclusion, sustained UL34 protein expression is required for viral replication. The sequence-specific DNA binding ability of UL34 proteins, their localization to viral DNA replication centers and their general effects on viral gene expressions suggests that UL34 proteins contribute to the establishment of a nuclear environment necessary for viral gene expression and DNA replication.
\end{abstract}


Keywords: human cytomegalovirus; UL34; sequence-specific DNA-binding protein; nuclear localization

\section{Introduction}

Human cytomegalovirus (HCMV) is predominantly an opportunistic pathogen, causing clinically significant disease in people who have inadequate immune responses, including neonates, transplant recipients, and people with uncontrolled HIV infections [1]. Viral replication initiates with the expression of the immediate early genes, the best characterized of which are IE1 and IE2. IE1 and IE2 initiate the cascade of viral gene expression, regulating the expression of the remainder of the viral genome, resulting in the expression of early and then late genes, and ultimately the production of new virions.

HCMV has a very large genome of $\sim 235 \mathrm{~kb}$ and encodes an estimated 176 genes [2]. Despite the large genome size, only $\sim 50$ genes are required for HCMV replication in cell culture, suggesting that the remaining $70 \%$ of the genes contribute to replication and latency in the human host $[3,4]$. The essential genes of HCMV can be grouped according to function: attachment of the virus to the target cells, transcriptional regulation, viral DNA replication, virion formation, and virion egress. Although there are a relatively small number of essential HCMV genes, to date the functions of several of the essential genes, including UL34, have been minimally characterized.

The UL34 gene is essential for viral replication as determined by Dunn et al. [3] and Yu et al. [4] in their global analyses of the HCMV genome. The UL34 gene is transcribed throughout the viral replication cycle, resulting in the expression of early and late transcripts [5]. The early transcript becomes abundant by 3 hours post-infection (hpi) while the late transcript predominates from 48 hpi throughout the remainder of the viral replication cycle. Two highly related proteins are encoded by the early and late transcripts, with the late protein (UL34b) identical to the early protein (UL34a) except for the absence of 21 amino terminal amino acids. Both UL34 proteins localize to the nucleus and are sequence-specific DNA-binding proteins that act as transcriptional repressors; the interaction of UL34 proteins with the UL34 binding sites in the US3 and US9 genes down-regulates their expression [6,7].

In addition to the UL34 binding sites within the US3 and US9 genes, there are 12 to 13 additional binding sites located in the viral genome. Six of the UL34 binding sites are located within protein coding regions, 3 or 4 binding sites (the number is strain dependent) are in a region flanking the lytic origin of replication, and the remaining 3 binding sites are located 5 ' of protein coding regions [6]. The positions of the UL34 binding sites relative to coding regions suggests that UL34 proteins may be multifunctional, contributing not only to transcriptional repression but also contributing to viral replication in as yet unidentified ways. The experiments described here were undertaken to identify the contributions of each of the UL34 proteins to viral replication and to examine the intracellular localization pattern of UL34 proteins during infection. 


\section{Results and Discussion}

\subsection{Both UL34 Proteins Are Essential for Viral Replication}

Yu et al. [4] and Dunn et al. [3] identified UL34 as essential for viral replication in their global analyses of the HCMV genome. We extended their results by constructing and studying recombinant viruses using the bacterial artificial chromosome (BAC) that contains the HCMV AD169 genome, pHB5 [8]. HCMV-BACs that either entirely lacked UL34 (AUL34), contained UL34 with a mutation in the ATG initiating translation of the early protein [ATG mutated to ATC (methionine to isoleucine), $\Delta \mathrm{E}$ mutant], contained UL34 with a mutation in the ATG initiating the late protein [ATG mutated to GTG (methionine to valine), $\Delta \mathrm{L}$ mutant], or had the UL34 open reading frame restored (UL34 rescue, RUL34). The ability of each of the recombinant viruses to replicate was assayed following electroporation of the HCMV-BACs into primary human fibroblasts, along with a plasmid expressing the tegument protein, pp71. Following electroporation, cells were observed for plaque formation for 4 weeks. The parental BAC, pHB5, and the UL34 rescue BAC (RUL34) gave rise to plaques by 8 days post-transfection. No plaques developed in the cells receiving the $\Delta \mathrm{UL} 34$ mutant, the $\Delta \mathrm{E}$ UL34 mutant or the $\Delta \mathrm{L}$ UL34 mutant BAC during a 4 week observation period. From these results, we concluded that the expression of both UL34 proteins is essential for viral replication.

\subsection{Reduced Viral Gene Expression in the Absence of UL34 Proteins}

To examine the defect in viral replication associated with the absence of UL34 proteins, semi-quantitative RT-PCR reactions were performed on RNA samples extracted following the electroporation of the UL34-HCMV BACs into human fibroblasts. Levels of expression for the essential genes UL32, UL37, UL44, UL46, UL84 and UL123 (IE2) were assayed as were levels of expression of the non-essential UL36 and UL69 genes. IE2, UL36, and UL37 are immediate early genes; UL44 and UL84 are early genes; UL69 is an early/late gene and UL32 and UL46 are late or presumed late genes (Figure 1A). Levels of expression were analyzed at 6 and 8 days post-transfection; time points that correspond approximately to early and late times of infection, based on the time when plaques are visible. Viral transcript levels were normalized to the transcript levels of the cellular gene, glyceraldehyde phosphate dehydrogenase (GAPDH). At 6 and 8 days post-transfection, transcript levels for all genes asssayed were decreased in the UL34 mutant viruses when compared to the UL34 rescued virus (RUL34, Figure 1B,C).

Six days post-transfection, expression of the major immediate early (mIE) gene, IE2, was decreased in the absence of the early, late or both UL34 proteins. Similar to the reduction in the level of IE2 transcripts, levels of UL44, UL84, UL32, and UL46 transcripts were detected at a reduced level for all of the UL34 mutant viruses at 6 days post-transfection (Figure 1B). In contrast, no UL69 or UL37 expression was detected for the UL34 mutant viruses; and UL36 expression was detected only in the UL34 mutant virus expressing the late UL34 protein (UL34b). At 8 days post-transfection, only UL44 transcripts were detectable for the UL34 deleted-BACs, albeit at a much reduced level compared to the UL34 rescued virus (Figure 1C). 
Figure 1. (A) List of genes assayed for expression in cells receiving the recombinant UL34 human cytomegalovirus (HCMV) bacterial artificial chromosome (BACs). (B) and $(\mathbf{C})$ Relative transcript levels for the indicated genes at 6 and 8 days post-transfection. RT-PCR was used to amplify the transcripts for each of the listed genes along with the cellular gene, glyceraldehyde phosphate dehydrogenase (GAPDH). The amplification products were quantified; viral gene levels were normalized to the level of GAPDH amplimers obtained for each of the samples. RUL34 is the UL34 rescued HCMV BAC, $\triangle \mathrm{L}$ UL34 has the initiation codon for the late protein mutated, $\triangle \mathrm{E}$ UL34 has the initiation codon for the early protein mutated and $\Delta$ UL34 has the entire UL34 open reading frame deleted.

A.

\begin{tabular}{|l|l|c|l|}
\hline Gene & Temporal Expression & Essential/non-essential & Function \\
\hline IE2 & immediate early & essential & transcription regulator \\
UL36 & immediate early & non-essential & unknown \\
UL37 & immediate early & essential & anti-apoptotic \\
UL44 & early & essential & viral DNA replication \\
UL84 & early & essential & viral DNA replication \\
UL69 & early/late & non-essential & RNA export \\
UL46 & late & essential & capsid \\
UL32 & late & essential & tegument \\
\hline
\end{tabular}

B.

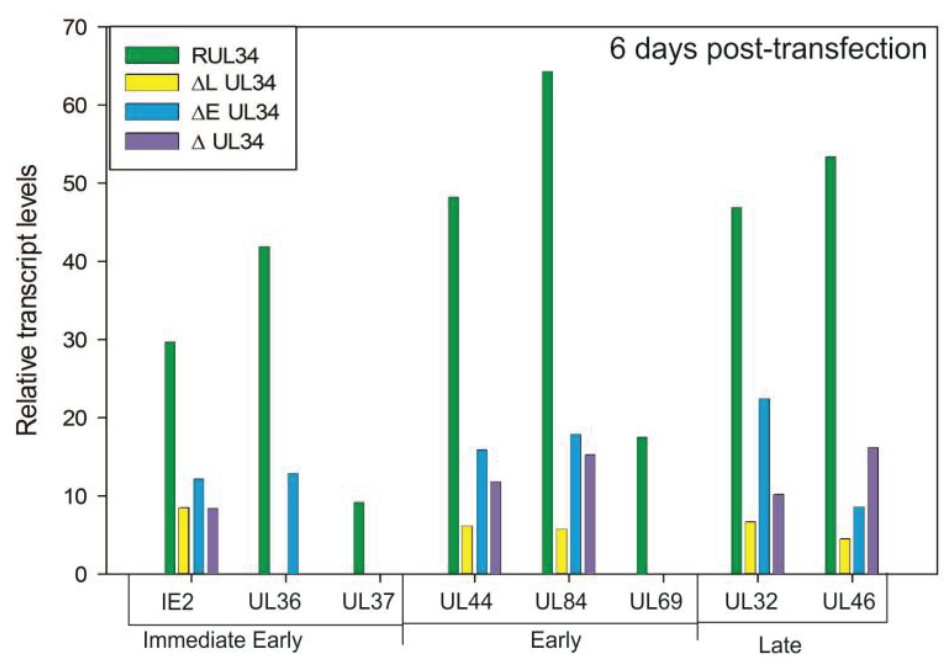

C.

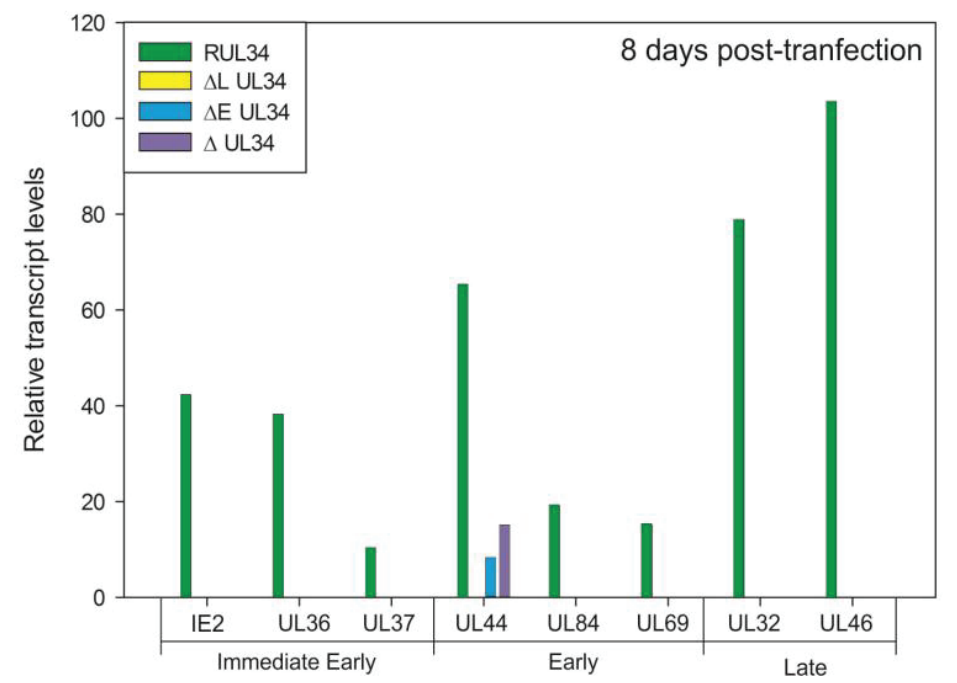


There is no UL34 binding site within the major immediate early gene, and in studies utilizing transient expression assays, UL34 has no activating or repressing effect on the mIE promoter [9]. Consequently, the reduction in IE2 transcript levels seen in the absence of UL34 proteins suggests that UL34 proteins have a general effect on the level of IE2 transcripts. This is supported by the reduction in transcript levels for all viral genes assayed (Figure 1B,C). UL32 and UL37 contain UL34 binding sites within their open reading frames; however, the diminution in transcript levels is consistent for all genes tested, regardless of the presence of a UL34 binding site. Intriguingly, the inability to detect UL69 and UL37 transcripts in the absence of either UL34 protein suggests that both proteins are required for their expression, contrasting with UL36 expression, where transcripts are not detectable in the absence of the late UL34 protein, suggesting that the late UL34 protein (UL34b) is required for UL36 expression.

At 8 days post-transfection, of the genes assayed, only UL44 transcripts were detected for the UL34 mutant viruses, in contrast to the transcripts detected for the UL34 rescued virus (Figure 1C). The lack of either UL34 protein results in a decrease in expression of IE2 and an absence of UL37 expression; proteins critical for later gene expression. These data suggested that the effects of the UL34 mutations were cumulative, that is, a reduction in the expression of essential genes earlier in the infection cycle (day 6) results in very little viral gene expression by 8 days post-transfection, corresponding to the defect in viral replication.

Some variations in transcript levels were seen when comparing the UL34 mutant HCMV BACs. Expression of only the late UL34 protein resulted in detectable levels of UL36 and an increase in the level of UL32 expression, relative to the other UL34 mutant viruses, suggesting that the late protein plays a significant role in the expression of these two genes. There are UL34 binding sites located within the coding regions of UL37 and UL32 that may directly influence gene expression. UL34 proteins have a known transcriptional effect, repressing expression of the US3 and US9 genes [6,7]. However, the data presented in Figure 1 suggests that UL34 has a more general effect on viral gene expression given the lack of UL34 binding sites within the other assayed genes, coupled with the decrease in gene expression. Both forms of the UL34 protein are required for viral replication, with the early and late proteins differentially affecting the expression of other viral genes.

\subsection{UL34 Localizes to Viral DNA Replication Centers}

The UL34 proteins localize to the nucleus when visualized using a tag of enhanced green fluorescent protein (EGFP) or using indirect immunofluorescence [5]. Here, we examined the intracellular location of UL34 proteins during the viral replication cycle, using polyclonal rabbit anti-sera to UL34 and indirect immunofluorescence. Monoclonal antibodies to other viral proteins were used simultaneously to co-label the same infected cell population. Primary human fibroblasts were infected with HCMV strain Towne and fixed and stained at 3, 24, 48, 72 and 96 hours post-infection (hpi).

As UL34 proteins are detectable by 4 hpi using either immunoprecipitation or western blot analyses [5], we initially compared the intracellular pattern of UL34 expression to that of the immediate early protein, IE2. At 3 hpi, UL34 was detected in a punctate staining pattern (Figure 2). Co-labeling with an antibody to IE2 demonstrated that UL34 colocalized with IE2 in the punctate dots at 3 hpi. UL34 continued to colocalize with IE2 throughout the remainder of the viral 
replication cycle, with the staining of UL34 and IE2 increasing as viral replication progressed (Figure 2). At 3 hpi, UL34 was not detectable in all cells positive for IE2, as expected, with IE2 expression activating the expression of other viral genes.

Figure 2. Indirect immunofluorescence was performed on primary human fibroblasts infected with HCMV strain Towne at 3, 24, 48, 72, and 96 hpi using antibodies to UL34 and to IE2. UL34 staining is red, IE2 staining is green; the merged column is the combination of the green and red images. Colocalization is indicated by the yellow color in the merged images. The numbers indicate the time post-infection at which the cells were examined. Control slides, infected cells incubated only with secondary antibodies, are not shown.

UL34

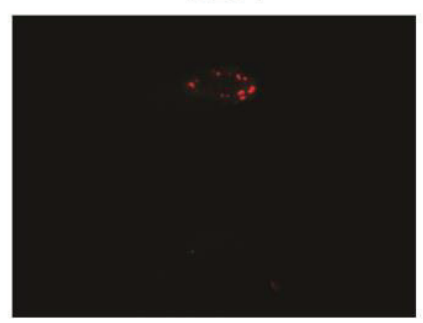

24

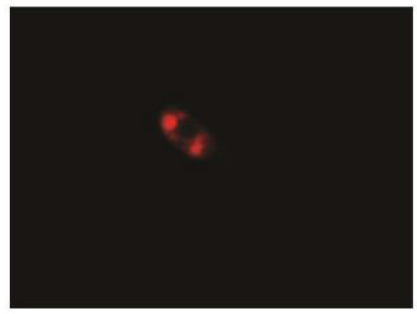

48

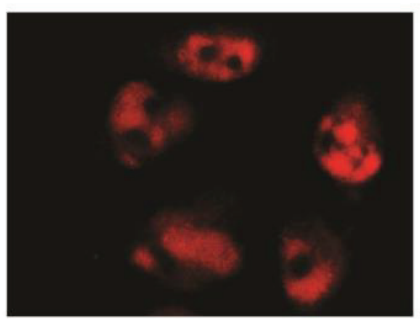

72

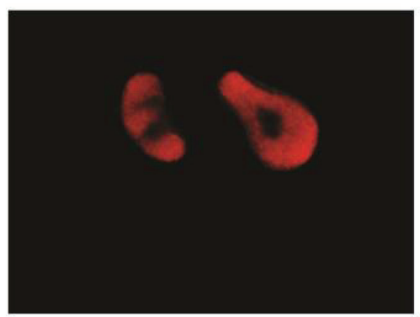

96

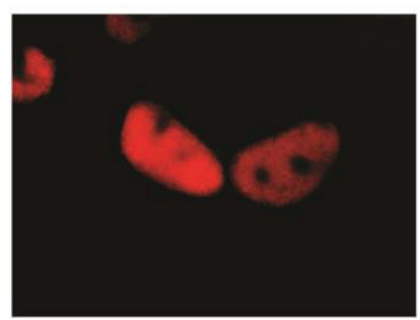

IE2
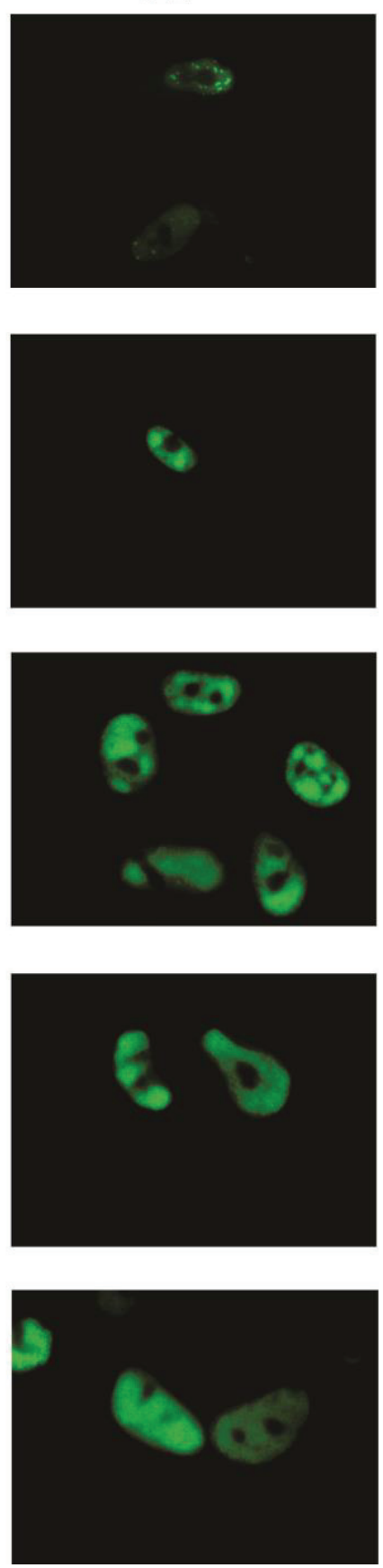

Merged
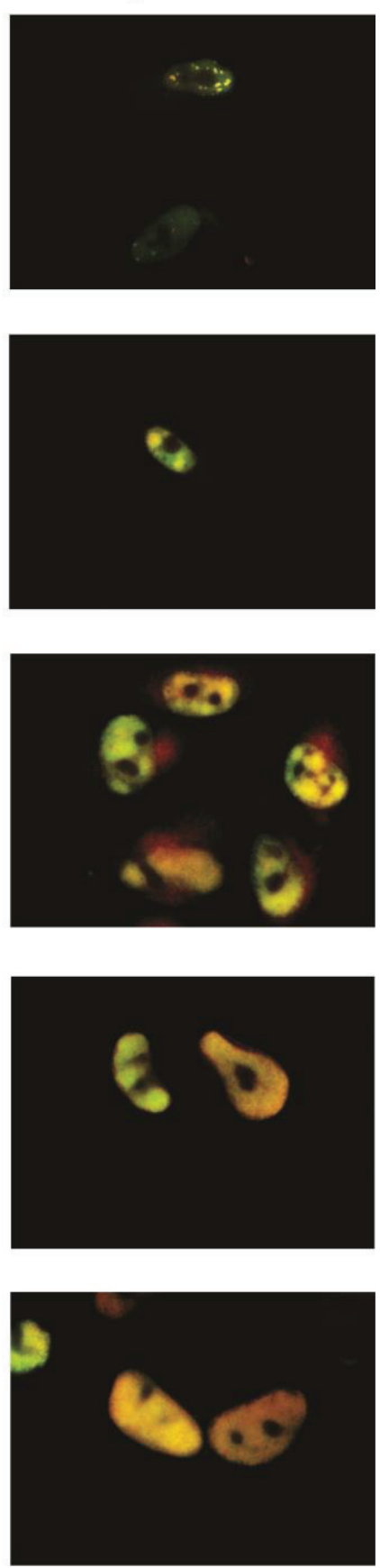
As IE2 is found in viral DNA replication structures [10], the colocalization of UL34 with IE2 suggested that UL34 localizes to viral DNA replication centers. To further examine the intracellular location of UL34, additional immunofluorescence experiments were performed, comparing the intracellular location of UL34 to that of UL44. UL44 encodes a viral DNA polymerase processivity factor; antibody labeling of UL44 is commonly used to identify viral DNA replication centers [11,12]. UL44 was detectable at 24 hours post-infection, localizing as bright nuclear foci with a low level of diffuse nuclear staining (Figure 3). UL34 colocalized with UL44 in the bright nuclear foci. The pattern of colocalization continued throughout the viral replication cycle, with UL34 colocalizing extensively with UL44. Although UL34 colocalizes with UL44 in viral DNA replication centers, UL34 was not identified in the proteins complexing with UL44 [11], suggesting that UL34 does not interact directly with UL44.

Figure 3. Indirect immunofluorescence was performed on primary human fibroblasts infected with HCMV strain Towne at 24, 48, 72, and 96 hpi using antibodies to UL34 and to UL44. UL34 staining is red, UL44 staining is green; the green and red images were merged with the DAPI-stained images (blue) at $24 \mathrm{hpi}$; for the other timepoints, only the red and green images were merged. An enlargement of one of the stained cells is shown in the insert at 24 hpi in the merged column. The extracellular bright green dots seen in the 48 hpi samples were an artifact associated with the secondary antibody.
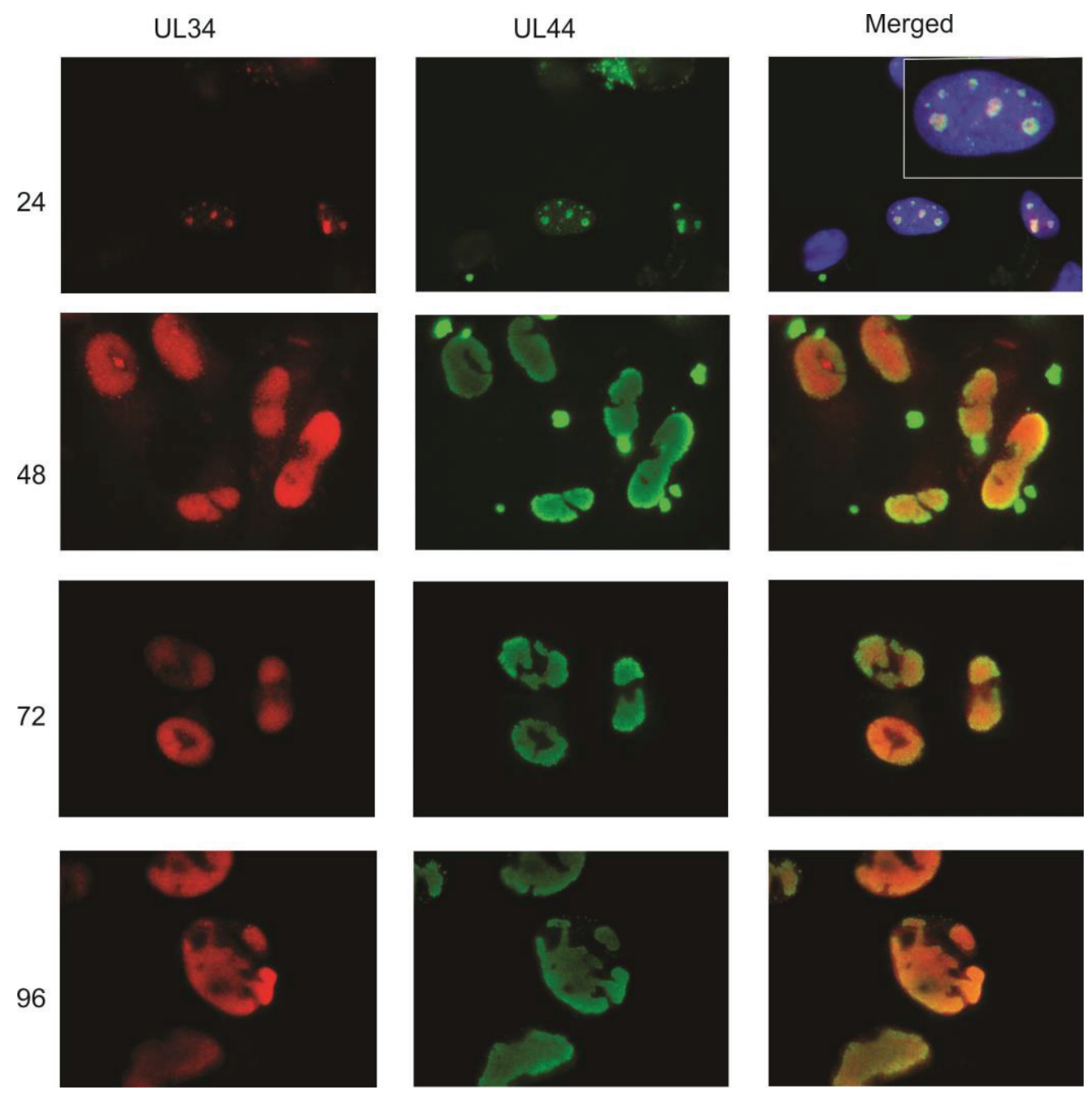
The similar staining patterns of UL34, IE2 and UL44 again suggested that UL34 localizes predominantly to viral replication centers. To compare the intracellular location of UL34 with that of another viral protein found in the nucleus but not in viral DNA replication centers, infected cells were labeled with antisera to UL34 and with a monoclonal antibody to ICP22. The US22 gene encodes ICP22, a tegument protein that is found in the nucleus early in infection and in both the nucleus and cytoplasm at later stages of viral replication [13]. As shown in Figure 4, little colocalization of UL34 with ICP22 is seen, suggesting that UL34 specifically colocalizes with IE2 and UL44 in viral DNA replication centers.

Figure 4. Indirect immunofluorescence was performed on primary human fibroblasts infected with HCMV strain Towne at 24, 48, and 72 hpi using antibodies to UL34 and to ICP22. UL34 staining is red, ICP22 staining is green; the merged column is a combination of the red and green images.

UL34

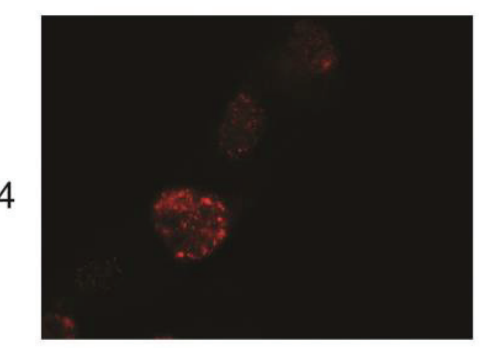

48

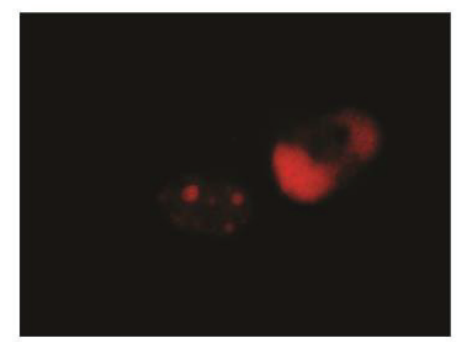

72

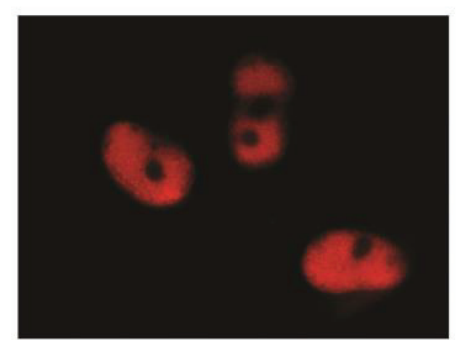

ICP22
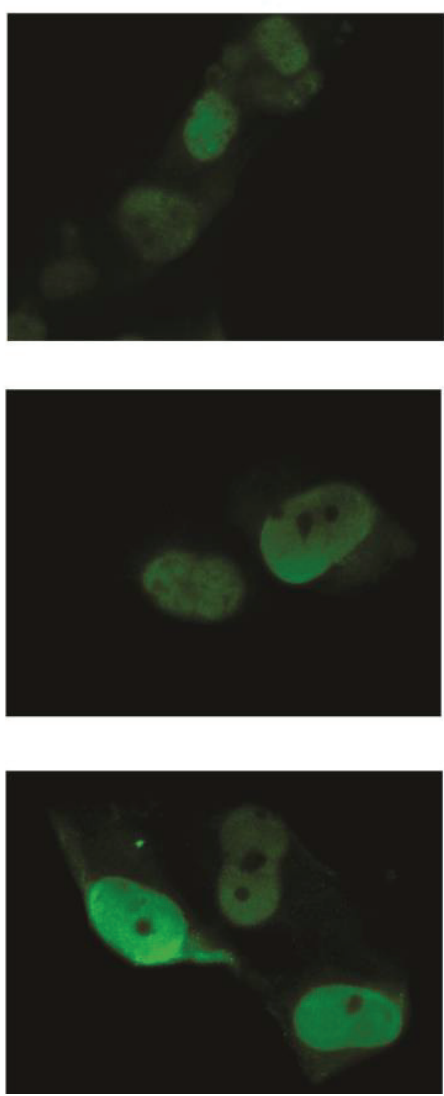

Merged
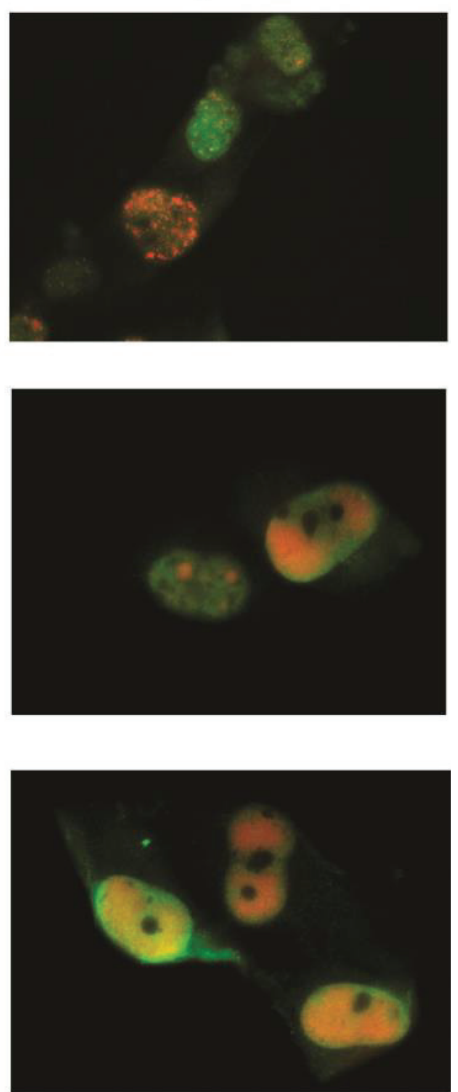

IE2 accumulates at the periphery of promyelocytic leukemia protein-associated nuclear bodies (PODs) [10], becoming incorporated into viral DNA replication centers, along with the core proteins including UL44, as the viral replication cycle continues. Our data demonstrate that UL34, along with IE2 and UL44, is also found in viral DNA replication centers. The contribution of UL34 to viral replication is potentially mediated through the UL34 binding sites located in the viral genome near the origin of lytic replication. The interaction of pUL34 with the ori-lyt region and the localization of UL34 to viral DNA replication center along with the two essential proteins, IE2 and UL44, suggests that UL34 may contribute to the efficiency of viral DNA replication. 


\subsection{UL34 Colocalizes with Nucleolin}

At late stages of infection (96 hpi), UL34 was found widely distributed in the nucleus. The circular areas within the nucleus that did not contain UL34 (See Figure 2, 72 hpi as an example) were identified as nucleoli using an antibody to label the nucleolar protein, fibrillarin (data not shown). However, UL44 colocalizes partially with the cellular protein nucleolin, and the interaction of nucleolin with UL44 is essential for viral replication [11]. The colocalization of UL34 and UL44 suggested that UL34 would colocalize with nucleolin. To examine the interaction of UL34 and nucleolin, infected cells were costained with antibodies to UL34 and to nucleolin. Early in infection (24 hpi), UL34 accumulated in bright nuclear foci adjacent to the nucleolin staining (Figure 5). However, at late times of infection, UL34 partially colocalized with the ring of nucleolin detected at the perimeter of the nucleoli (Figure 5, see inset). These data suggested that nucleolin and nucleoli contribute to organization of UL34 in the nucleus and to the formation and organization of viral DNA replication compartments. Strang et al. [11,12] found nucleolin predominantly dispersed in the nucleus following viral infection (using HCMV strain AD169), and further found that UL44 partially colocalized with nucleolin at the perimeter of viral replication centers. In contrast, using a monoclonal antibody to nucleolin rather than polyclonal antisera, we detected no dispersal of nucleolin. The difference seen in nucleolin distribution following infection may be a result of utilizing different antisera, or alternatively, strain specific differences, with the localization data presented here obtained following infection with HCMV strain Towne. In summary, UL34 proteins are localized in close proximity to nucleolin early in infection; late in infection, UL34 proteins partially colocalize with nucleolin.

Figure 5. Indirect immunofluorescence was performed on primary human fibroblasts infected with HCMV strain Towne at 24 and 96 hpi using antibodies to UL34 and to nucleolin. UL34 staining is red, nucleolin staining is green; the green and red images were merged with the DAPI-stained images (blue) in the merged column at 24 hpi as described for Figure 1; the DAPI-stained image was not included in the merged image for the 96 hpi timepoint. The arrowhead indicates the association of UL34 with nucleolin early in infection. The inset shows an enlargement of the colocalization of UL34 and nucleolin at $96 \mathrm{hpi}$.
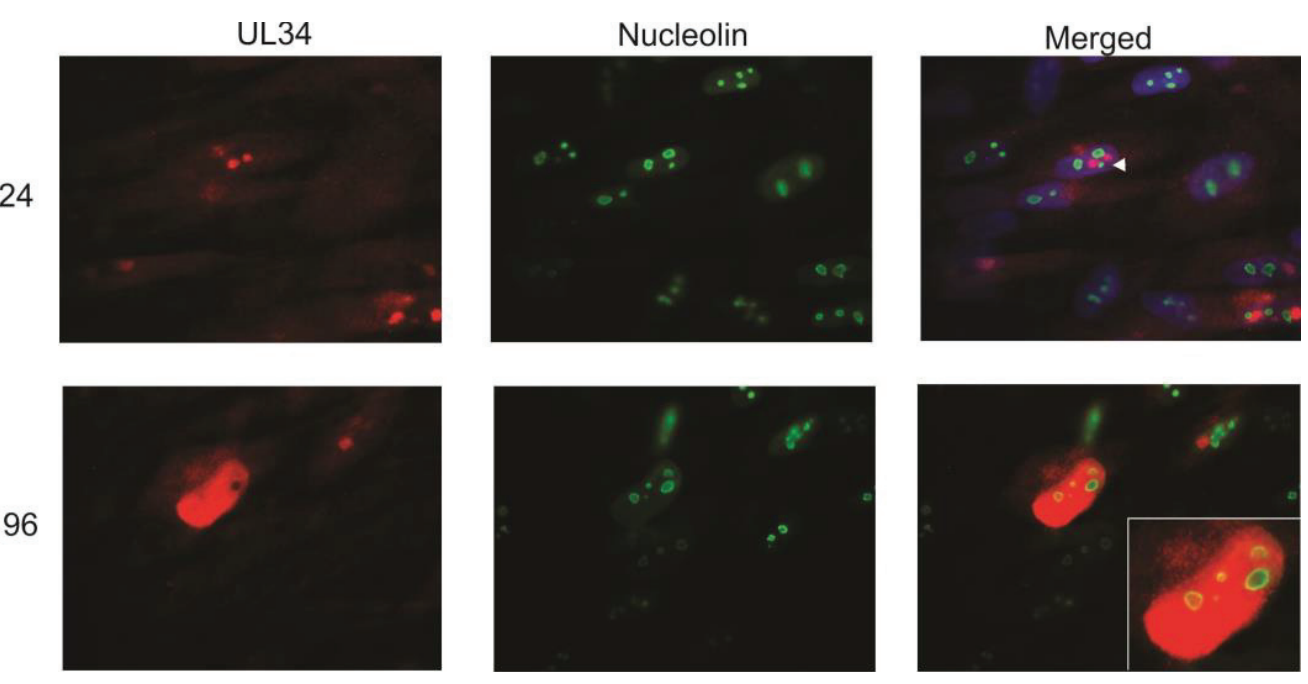


\section{Experimental Section}

\subsection{Virus, Cells and Immunofluorescence}

HCMV strain Towne was propagated in primary human fibroblasts in Dulbecco's minimal essential medium supplemented with glutamine, penicillin, streptomycin and 10\% Nuserum. For immunofluorescence studies, primary human fibroblasts were plated on glass coverslips and infected with HCMV strain Towne at a moi of 1-2 pfu/cell. Prior to antibody staining, cells were washed with phosphate buffered saline (PBS), fixed with 4\% paraformaldehyde and permeabilized using $0.2 \%$ Triton X-100 in PBS. Following four washes with PBS, cells were incubated with $10 \%$ normal human serum at $37{ }^{\circ} \mathrm{C}$ for 1 hour. Primary antibodies were diluted to the recommended concentrations in PBS containing 3\% bovine serum albumin; cells were incubated with the primary antibodies for 1 hour at room temperature. Secondary fluorescent dye-tagged antibodies to mouse or rabbit antibodies were incubated with the cells for 30 minutes at room temperature. Nuclei were stained with 4',6-diamidino-2-phenylindole (DAPI); cells were visualized using a Nikon fluorescence microscope and photographed using a SPOT camera. The following primary antibodies were used: monoclonal antibodies to UL44 (ICP36) and to US22 (ICP22) were obtained from Virusys Corporation, Taneytown, MD, USA; the monoclonal antibody to IE2 was obtained from Millipore, Billerica, MA, USA; the monoclonal antibody to nucleolin was obtained from Abcam, Cambridge, MA, USA, and the fluorescent dye-tagged secondary antibodies were obtained from Life Technologies, Grand Island, NY, USA.

\subsection{Recombinant Virus}

The BAC plasmid, pHB5, which contains the HCMV AD169 genome was kindly provided by U. Koszinowski [8]. A mutated BAC lacking UL34 was constructed as described by Datsenko and Wanner [14] using primers (285, 5'CAGAACCCGTCGCCATTTCCCCTCATATACGGTACACGTCCCC CTGATCTGTGtAGGCTGGAGCTGCTTC $\quad 3^{\prime}$ and 286, 5' AAACCAGAGCGGAACTTGAGAAATCAACGCTTTATTGTTCTCCAGTGACGCATATGAATATCCTCCTTAG 3') which contain sequences complementary to the ends of UL34 and to the kanamycin gene [14]. Briefly, the primers were used to amplify the kanamycin and frt sequences from pKD13; the PCR product was then introduced into pHB5 using pKD46, which expresses the lambda Red genes. Following insertion of the FRT-kanamycin cassette and deletion of the UL34 open reading frame, the kanamycin gene was removed from the BAC using pCP20 which expresses the Flp recombinase.

Recombinant BACs were then constructed by replacing the deleted UL34 gene with either the wild type sequence or with a UL34 gene containing mutations of either the early or late translation initiation codons for the early and the late proteins. pBJ545, which contains the HindIII H fragment of HCMV strain Towne in pST76K_SR, was mutated using oligo pairs 328 and 329 (328, 5' CCTGCGAGCCGCCGAGGTGCGTGACAACGTGGC 3'; 329, 5' GCCACGTTGTCACGC ACCTCGGCGGCTCGCAGG $\left.\quad 3^{\prime}\right)$ or 330 and 331 (330, $5^{\prime}$ ACCGCCCCACCGCCGTCGTCGTCAT CAACTTCATCATCACCACC $\quad$ 3'; 331, 5'

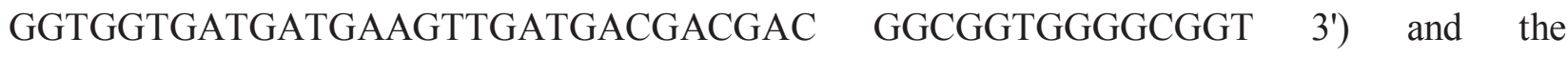


Quickchange mutagenesis kit (Stratagene, Santa Clara, CA, USA) giving rise to pBJ598 (early ATG mutated to ATC) and pBJ599 (late ATG mutated to GTG). pBJ598 and 599 were electroporated into bacteria containing the $\triangle \mathrm{UL} 34-\mathrm{BAC}$ and $\mathrm{pKD} 46$. Resulting colonies were screened using PCR to identify BACs containing the replaced UL34 sequences.

\subsection{Quantitative Reverse Transcriptase Polymerase Chain Reactions}

The following pairs of oligonucleotides were used to amplify a portion of the indicated gene transcript: UL37 (368, 5' TCAGACGATCCGATGAACGT $3^{\prime}$ and 369, 5' TCTCCTCCGAGCCAAAAGTC 3'), UL44 (370, 5' CTAGCCGCACTTTTGCTTCT 3'and 371, 5' ACGGTCTTTCCTCCAAGGAA 3'), UL69 (372, TTAGTCATCCATATCATCGC 3' and 373, GAGCTTAACTTGATGACGCC 3'), UL122 (374, GAGACTTGTTCCTCAGGTCC 3', and 375, 5' CAACATGATCATCCACGCTG 3'), UL36 (376, 5' TCAGTTGTTCATGTAAACGT 3', and 377, ACCACTTTGAACTCTCCTAC 3'), glyceraldehyde phosphate dehydrogenase (GAPDH; 39, 5' AGAGACATCATCCCTGCCTCT 3', and 391, 5' TTTTTCTAGACGGCAGGTCA 3'), UL84 (398; 5' GCAGACCATGGCTAAAGTGA 3' and 399, 5' TTAACCGTACTGGTGAGCGA 3') and Ul32 (20, 5' TGCAGTTTATCGGTCTACAGCG $33^{\prime} ; \quad$ and 21, 5'CGRATCCTTGAGGTGCACAAAG 3'). RNA was extracted from human fibroblasts that had been electroporated with the indicated HCMV-BAC 6 and 8 days post-transfection. RNA was treated with RQ1 DNAse followed by DNAse stop solution. PCR reactions were performed following reverse transcription reactions or in the absence of reverse transcription to confirm the absence of contaminating DNA. Amplimers were quantified following gel electrophoresis.

\section{Conclusions}

The experimental results presented here demonstrate that both UL34 proteins play an essential role in viral replication. Viruses containing in frame point mutations substituted for the initiation codons for the early and the late proteins are replication defective. The absence of UL34 protein expression resulted in a diminution of viral gene expression, and most notably, the absence of UL37 and UL69 transcripts. With the exception of UL34 repression of US3 and US9 expression [6,7], we have been unable to identify any direct effects of UL34 expression on viral gene transcription. This suggests that UL34 proteins are multi-functional, not only specifically repressing the expression of some viral genes, but also having a general effect on viral gene transcription. We postulate that the effects of the UL34 proteins may be to facilitate the establishment of an environment in the nucleus favorable for viral gene expression and viral DNA replication. This notion is supported by the localization of UL34 proteins to viral DNA replication centers. Furthermore, the proximal localization of UL34 to nucleolin suggests that UL34-nucleolin interactions may facilitate the intranuclear positioning of the replication centers. In summary, this work demonstrates the significance of both UL34 proteins, and raises questions about the viral and cellular proteins that UL34 proteins interact with. 


\section{Acknowledgments}

We thank Yingguang Liu for constructing the initial UL34 deletion. We thank the American Heart Association for funding.

\section{Author Contributions}

$\mathrm{RR}$ and BJB designed and conducted the experiments; BJB prepared the manuscript. All authors read and approved the manuscript.

\section{Conflicts of Interest}

The authors declare no conflict of interest.

\section{References and Notes}

1. Mustafa, M.M. Cytomegalovirus infection and disease in the immunocompromised host. Pediatr. Infect. Dis. J. 1994, 13, 249-259.

2. Davison, A.J.; Dolan, A.; Akter, P.; Addison, C.; Dargan, D.J.; Alcendor, D.J.; McGeoch, D.J.; Hayward, G.S. The human cytomegalovirus genome revisited: Comparison with the chimpanzee cytomegalovirus genome. J. Gen. Virol. 2003, 84, 17-28.

3. Dunn, W.; Chou C.; Li H.; Hai R.; Patterson D.; Stolc V.; Zhu H.; Liu F. Functional profiling of a human cytomegalovirus genome. Proc. Nat. Acad. Sci. USA 2003, 100, 14223-14228.

4. Yu, D.; Silva M.C.; Shenk T. Functional map of human cytomegalovirus AD169 defined by global mutational analysis. Proc. Nat. Acad. Sci. USA 2003, 100, 12396-12401.

5. Biegalke, B.J.; Lester E.; Rana R. Characterization of the human cytomegalovirus UL34 gene. J. Virol. 2004, 78, 9579-9583.

6. Liu, Z.; Biegalke B.J. Human cytomegalovirus UL34 binds to multiple sites within the viral genome. J. Virol. 2013, 87, 3587-3591.

7. LaPierre, L.; Biegalke B.J. Identification of a novel transcriptional repressor encoded by human cytomegalovirus. J. Virol. 2001, 75, 6062-6069.

8. Borst, E.-M.; Hahn, G.; Koszinowski, U.H.; Messerle, M. Cloning of the human cytomegalovirus (HCMV) genome as an infectious bacterial artificial chromosome in Escherichia coli: A new approach for construction of HCMV mutants. J. Virol. 2000, 73, 8320-8329.

9. Biegalke, B.J. Ohio University Heritage College of Osteopathic Medicine, Athens, OH, USA. Unpublished work, 2014.

10. Ahn, J.H.; Jang, W.J.; Hayward, G.S. The human cytomegalovirus IE2 and UL112-113 proteins accumulate in viral DNA replication compartments that initiate from the periphery of promyelocytic leukemia protein-associated nuclear bodies (PODs or ND10). J. Virol. 1999, 73, 10458-10471.

11. Strang, B.L.; Boulant S.; Coen D.M. Nucleolin associates with the human cytomegalovirus DNA polymerase accessory subunit UL44 and is necessary for efficient viral replication. J. Virol. 2010, 84, 1771-1784. 
12. Strang, B.L.; Bouland, S.; Chang, L.; Knipe, D.M.; Kirchhausen, T.; Coen, D.M. Human cytomegalovirus UL44 concentrates at the periphery of replication compartments, the site of viral DNA synthesis. J. Virol. 2012, 86, 2089-2095.

13. Mocarski, E.S.; Pereira, L.; McCormick, A.L. Human cytomegalovirus ICP22, the product of the HWLF1 reading frame, is an early nuclear protein that is released from cells. J. Gen. Virol. 1988, 69, 2613-2621.

14. Datsenko, K.A.; Wanner, B.L. One-step inactivation of chromosomal genes in Escherichia coli K-12 using PCR products. Proc. Nat. Acad. Sci. USA 2000, 97, 6640-6645. 

Reprinted from Viruses. Cite as: Niemann, I.; Reichel, A.; Stamminger, T. Intracellular Trafficking of the Human Cytomegalovirus-Encoded 7-trans-Membrane Protein Homologs pUS27 and pUL78 during Viral Infection: A Comparative Analysis. Viruses 2014, 6, 661-682.

Article

\section{Intracellular Trafficking of the Human Cytomegalovirus-Encoded 7-trans-Membrane Protein Homologs pUS27 and pUL78 during Viral Infection: A Comparative Analysis}

\section{Ina Niemann, Anna Reichel and Thomas Stamminger *}

Institute for Clinical and Molecular Virology, University of Erlangen-Nuremberg, Schlossgarten 4, Erlangen 91054, Germany; E-Mails: ina.niemann@viro.med.uni-erlangen.de (I.N.); anna.reichel@viro.med.uni-erlangen.de (A.R.)

* Author to whom correspondence should be addressed;

E-Mail: thomas.stamminger@viro.med.uni-erlangen; Tel.: +49-9131-852-6783;

Fax: +49-9131-852-2101.

Received: 27 November 2013; in revised form: 9 January 2014 / Accepted: 13 January 2014 / Published: 10 February 2014

Abstract: Human cytomegalovirus (HCMV) encodes four G protein-coupled receptor (GPCR) homologs, termed pUS27, pUS28, pUL33, and pUL78. In contrast to the extensively characterized vGPCRs pUS28 and pUL33, knowledge concerning pUS27 and pUL78 is limited. Previous studies already demonstrated constitutive internalization of pUS27 and pUL78, as well as an association with the endosomal machinery, however, these results were mainly obtained using transiently transfected cells. To explore the subcellular localization of both receptors during viral infection, we constructed recombinant HCMVs expressing tagged vGPCRs. Colocalization analyses revealed a predominant association of pUS27 or pUL78 with the trans-Golgi network or the endoplasmic reticulum, respectively. Intriguingly, our data emphasize that protein sorting is highly regulated by viral functions as we detected dramatic changes in the colocalization of pUS27 and pUL78 with endosomal markers during progression of HCMV replication. Furthermore, we observed cell type-dependent differences in trafficking of both vGPCRs between fibroblasts and epithelial cells. Most importantly, infection experiments with a recombinant HCMV carrying tagged versions of pUS27 and pUL78 simultaneously, revealed that these two proteins do not colocalize during viral infection. This contrasts to results of transient expression experiments. In conclusion, our results highlight the importance to investigate vGPCR trafficking in a viral context. 
Keywords: HCMV; cytomegalovirus; 7-transmembrane protein; G protein-coupled receptor; GPCR; US27; UL78; virus infection; localization

\section{Introduction}

G protein-coupled receptors (GPCRs) act as key regulators of numerous cellular processes via transmitting the response of various signal molecules like chemokines, hormones, or neurotransmitters. Thus, it is not surprising that viruses have hijacked mammalian GPCRs during coevolution to ensure efficient viral propagation [1,2]. Thereby, virally encoded GPCRs (vGPCRs) illustrate an effective means to bypass the immune system, modulate cellular functions, and redirect cellular signaling networks [3]. Human cytomegalovirus (HCMV) encodes four GPCR homologs, termed pUS27, pUS28, pUL33, and pUL78 [4,5]. In contrast to pUS27 and pUS28, which are restricted to primate CMVs, pUL33, and pUL78 are highly conserved among all betaherpesviruses. While their expression is not crucial for virus replication in cell culture [6,7], a deletion leads to a significantly diminished pathogenesis in animal experiments [8,9].

The most comprehensively analyzed vGPCR of HCMV is pUS28. As a homolog of CCR1, CCR2, and CX3CR1, it can bind a broad spectrum of chemokines, such as CCL5/RANTES, monocyte chemoattractant protein-1 (MCP-1), and CX3CL1/fractalkine [10-12]. As pUS28 is rapidly internalized in a constitutive manner [13-16], it is believed to function as a chemokine sink to impair leukocyte recruitment thereby dampening the immune response at sites of infection [7,17-19]. Furthermore, similar to other vGPCRs, pUS28 constitutively activates several

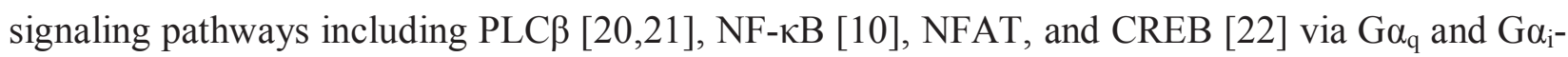
dependent signaling. Due to the strict host specification of all CMVs, it is difficult to determine the role of pUS28 in a physiologically relevant situation in vivo. However, recent reports demonstrated a partial functional complementation between pUS28 and its mouse cytomegalovirus vGPCR homolog pM33 [23,24]. Nevertheless, it is still unknown whether pUS28 plays an essential role during HCMV pathogenesis [7,19].

In contrast to pUS28, the vGPCRs pUS27, pUL78, and pUL33 are still orphan receptors. Interestingly, for pUL33 and its homologs in mouse (pM33) [11] and rat CMV (pR33) [25] a ligand-independent activation of several signaling cascades could be shown [26]. Whereas a functional analysis of pUL33 is difficult in vivo, rodent homologs have been reported to be critical for infection $[8,27,28]$. By means of gene-knockout viruses it was shown that deletion of M33 or R33 resulted in less virulent CMV variants, which neither disseminated nor replicated within the salivary gland. Intriguingly, both pUL33 and pUS28 were able to partially restore the replication defect of a M33-deficient virus indicating shared biological functions between the respective vGPCRs [23].

While pUS28 and pUL33 constitutively activate multiple signaling pathways, the functions of pUS27 and pUL78 are not yet fully understood. As pUS27 and pUS28 are directly adjacent and share 31\% sequence identity, a gene duplication event may have occurred. Similar to pUS28, pUS27 is constitutively internalized and localized to cellular vesicles with endosomal markers (EEA1), to lysosomes (LAMP-1), as well as to Golgi compartments (GM130) [29,30]. A potential 
di-leucine sorting motif in the cytoplasmic domain of pUS27 was reported to be necessary and sufficient for its intracellular localization [29]. The fact that pUS27 is heavily glycosylated [31] and possesses two conserved cysteine residues in the second and third extracellular loop indicates a possible involvement of pUS27 in chemokine binding and signaling in host cells. Notably, O'Connor et al. were able to demonstrate that pUS27 supports viral spread through the extracellular route late during infection in fibroblasts and endothelial cells, but not in epithelial cells [32]. Thus, this study suggested a cell type-dependent function of pUS27, which appears to involve interactions with one or more virus-encoded proteins at the site of virus assembly. Moreover, pUL78 was recently shown to support viral infection affecting a step after plasma membrane binding but before virus entry in epithelial cells [33]. This observation was also cell type-specific as pUL78 seemed to be dispensable for virus replication in fibroblasts and endothelial cells. Furthermore, the rodent variants (pR78, pM78) of pUL78 were used to demonstrate the importance of this vGPCR for viral pathogenesis in vivo [9,34,35]. In line with the three other vGPCRs of HCMV, pUL78 is constitutively internalized [36]. Wagner et al. could show that the process of internalization is dependent on dynamin. In addition, they could identify an association of pUL78 with the endoplasmic reticulum (ER), and its localization in the trans-Golgi network as well as early endosomes. Although pUS27 and pUL78 seem to lack constitutive signaling activity [11], they were demonstrated to colocalize and heteromize with the constitutively active receptor pUS28 in vitro. Thereby, pUL78, in contrast to pUS27, is able to silence pUS28-mediated activation of NF- $\mathrm{\kappa B}$ dependent gene expression [37].

As there is a lack of antibodies against HCMV-encoded GPCRs, most reports assessing the subcellular localization of these proteins were based on transient transfection analyses using tagged receptors. In this study, we describe the construction of recombinant HCMVs expressing either singly tagged pUS27 and pUL78, or a virus, where both proteins were tagged simultaneously, thus, enabling live cell imaging. These viruses were used for a comparative analysis of the subcellular localization of pUS27 and pUL78 after infection of both fibroblasts and epithelial cells. These experiments revealed cell type-dependent differences in trafficking of pUS27 and pUL78. In addition, colocalization analyses with cellular markers showed a substantially different accumulation of both receptors in infected cells compared to transiently transfected cells. Thus, this study emphasizes the necessity to analyze vGPCRs in a viral context in order to obtain physiologically meaningful results concerning their functions during viral replication.

\section{Results}

\subsection{Colocalization of pUS27 and pUL78 in Transiently Transfected Cells}

The viral GPCR pUS28 has previously been shown to colocalize and heteromize with pUL78 or pUS27 in vitro [37]. In order to investigate a possible colocalization of pUS27 with pUL78 in transiently transfected cells, we used tagged versions of both receptors due to a lack of specific antibodies for pUS27 or pUL78. Thus, a FLAG-tag was fused to the $N$-terminal extracellular domain of pUS27. The $C$-terminal intracellular domain of pUL78 was labeled with GFP. To analyze the intracellular vGPCR localization, receptors were transiently expressed in HeLa cells, either alone or in combination. After 24 h, cells were fixed, permeabilized, stained, and analyzed by confocal 
microscopy. Both proteins were detected in a predominantly perinuclear distribution pattern. While pUS27 was only found in dot-like structures (Figure 1b, red), pUL78 showed an additional weaker signal distributed throughout the cytoplasm (Figure 1c, green) as already described in previous reports $[30,36]$. In co-transfection experiments, most of the pUS27 signal overlapped with the dot-like pUL78 signal (Figure 1d). Therefore, both receptors not only colocalize with pUS28 [37], but also show a distinct colocalization among each other presumably in endosomal structures.

Figure 1. Colocalization analysis of FLAG-US27 and UL78-GFP in transiently transfected HeLa cells. HeLa cells were transfected with $1 \mu \mathrm{g}$ of an expression plasmid encoding $N$-terminally tagged wild-type TB40/E FLAG-US27 (b), $C$-terminally tagged wild-type TB40/E UL78-GFP (c), or both plasmids together (d). pcDNA3 was used as a negative control (a). Cells were fixed $24 \mathrm{~h}$ post transfection and permeabilized with Triton $\mathrm{X}-100$. The primary antibody mAb-FLAG in combination with a secondary anti-mouse antibody coupled to Alexa-555 were used to detect pUS27. Cell nuclei were stained with 4',6-diamidino-2-phenylindole (DAPI).

(a) pcDNA3
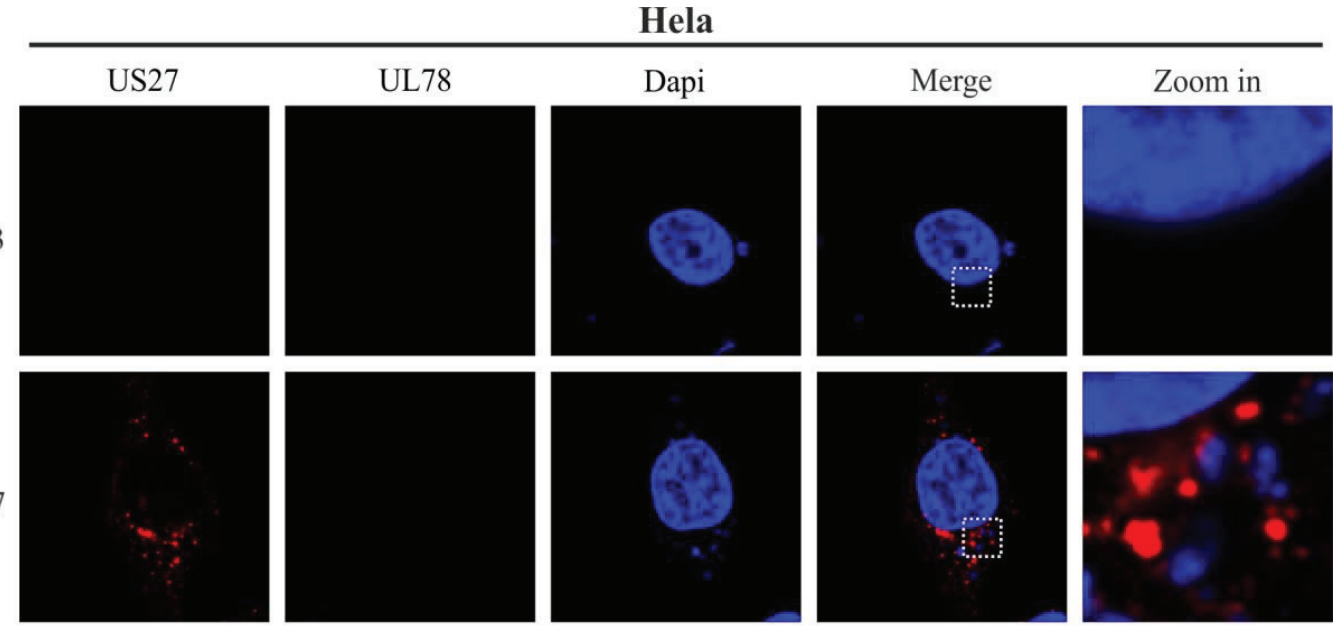

(b) FlagUS27

(c) UL78GFP
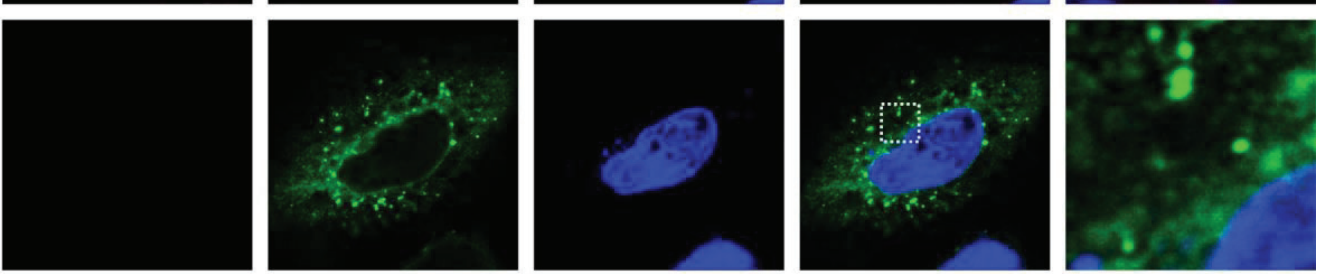

(d) FlagUS27
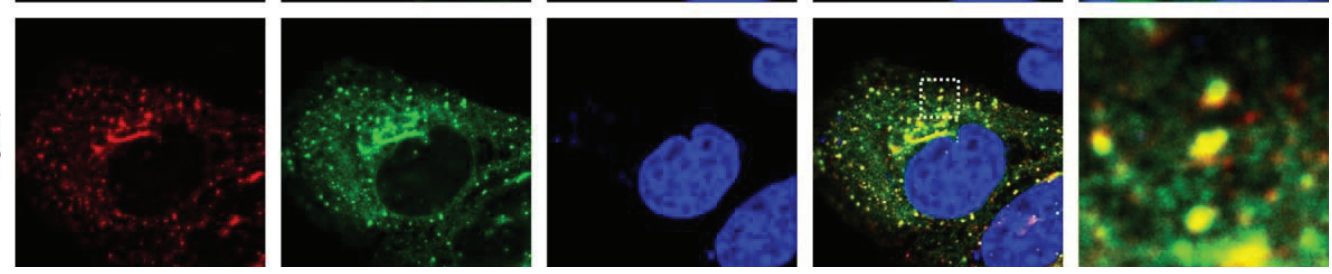

\subsection{Recombinant Viruses Harboring Tagged Versions of pUS27 or pUL78 Show Similar Growth} Kinetics Compared to Wildtype HCMV Strain TB40/E

To analyze whether the intracellular localization patterns of pUS27 and pUL78 as observed in transiently transfected cells $[29,30,36]$ can also be detected during infection, recombinant cytomegaloviruses were engineered via homologous recombination. The two-step, red-mediated 
recombination for markerless DNA manipulation, as described by Tischer et al., was used for the generation of recombinant CMVs [38]. The wild-type TB40/E-based HCMV bacterial artificial chromosome (BAC) TB40-Bac4 [39] was manipulated in order to fuse EYFP to the $C$-terminus of UL78 or US27, resulting in recombinant viruses termed TB40/E-US27-EYFP (US27-EYFP) or TB40/E-UL78-EYFP (UL78-EYFP), respectively (Figure 2a, upper panel). To enable colocalization studies with both vGPCRs in one cell, we additionally generated a recombinant virus carrying tagged versions of US27 and UL78 (Flag and EYFP, respectively), referred to as UL78-EYFP/Flag-US27 (Figure 2a, lower panel). In order to confirm the correct in-frame integration of all tags, BACs were analyzed using various independent methods. Distinct PCR reactions and subsequent nucleotide sequence analyses of all BACs, as well as restriction fragment length polymorphism analysis (RFLP) revealed a correct BAC recombination (data not shown). After reconstitution of infectious viruses, viral growth was analyzed by performing multistep growth curve analyses of wild-type and recombinant TB40/E viruses (Figure 2b). For this purpose, HFFs were infected at an MOI (multiplicity of infection) of 0.1 with either wild-type or recombinant viruses. The supernatants were harvested at $0,3,6,9,12$, and 15 dpi (days post infection). After cell lysis and DNA extraction, real-time PCR was performed to quantify HCMV genome copy numbers. As depicted in Figure 2b, all recombinant viruses replicated with similar growth kinetics compared to the wild-type strain TB40/E. Therefore, the epitope tags do not alter HCMV replication capacities in HFFs. Thus, recombinant viruses can be used to analyze intracellular localization routes of pUS27 and pUL78 during the entire replication cycle, even by live cell imaging.

\subsection{Expression and Localization Patterns of pUS27 and pUL78 Differ upon HCMV Infection of Fibroblasts and Epithelial Cells}

While, up to now, pUS27 localization was only determined in detail in transiently transfected cells $[29,30]$, the intracellular distribution of pUL78 was additionally analyzed at 48 hpi in HFF cells [36]. It has been demonstrated in previous studies that HCMV infection changes the entire morphology of infected cells [40,41]. One of these alterations is the development of a so-called cytoplasmic virion assembly compartment (cVAC). Usually, one cVAC per cell is formed by rearrangement of several cellular compartments including Golgi bodies, the trans-Golgi network and endosomal structures [41-43]. Thus, the dynamic nature of endocytic compartments during infection compels the use of multiple time points in analyses. Furthermore, it is important to analyze vGPCR localization in different cell types, as HCMV can infect a remarkably broad spectrum of different cell types with variable outcomes for the host in vivo [44].

In a first set of experiments we aimed to determine the subcellular localization of pUS27 and pUL78 in human retinal pigment epithelial cells (ARPE-19) and HFFs over the entire replication cycle. For this, HFFs and ARPE-19 were seeded, infected at an MOI of 0.5 or 1 , respectively, and fixed at 6, 24, 48, 72, and $96 \mathrm{~h}$ post infection (hpi). After fixation, infected cells were permeabilized, stained for IE1 detection, and analyzed using confocal microscopy. The progression of virus infection in HFF and ARPE-19 cells is shown in Figure 3. To verify that all detected vGPCR signals were specific for infected cells, IE1 staining was used as a control (Figure 3, red). At $6 \mathrm{~h}$ after HCMV infection, the IE1 protein was already present in the cell nucleus of HFF and ARPE-19 cells. As 
described previously [36], pUL78 (Figure 3, green, middle and right panel) was produced between 6 and 24 hpi. In contrast to that, pUS27 (Figure 3, green, left panel) expression was firstly visible $48 \mathrm{~h}$ after infection. Thus, it appears to be expressed later during HCMV replication. During the entire replication cycle, pUS27 was present in the perinuclear region of the cell. Late in infection, however, pUS27 was not only detected in association with the cVAC, but was additionally found in dot-like structures all over the cytoplasm, which was both observed in HFF and ARPE-19 cells (data not shown). In contrast to the perinuclear distribution of pUS27 at 48 hpi, the pUL78 signal was spread over the entire cytoplasm including defined dot-like structures. As infection progressed (72-96 hpi), pUL78 was increasingly displaced from the cVAC formation site, both in HFF and in ARPE-19 cells. Nevertheless, in epithelial cells dot-like pUL78-positive structures remained in the perinuclear region over the entire replication cycle. This observation suggests that pUL78 may exhibit different functions during infection of epithelial cells versus fibroblasts.

Figure 2. Construction and characterization of recombinant HCMVs expressing tagged US27 and/or UL78. (a) Schematic illustration of the US27 and UL78 genomic loci of HCMV strain TB40/E. Recombinant viruses were generated with $C$-terminal EYFP (US27 or UL78, upper panel) or $N$-terminal FLAG-tag (US27, lower panel); (b) Growth kinetics of recombinant TB40/E-derived viruses. HFF cells were seeded and infected three days later with wild-type and recombinant viruses at an MOI of 0.1. Virus supernatant samples were harvested at indicated times (dpi, days post infection) and digested with proteinase K. Viral DNA was quantitated via Taqman PCR to determine the release of viral particles from infected cells. Each infection was performed in triplicate, and the standard deviations are shown.

(a)

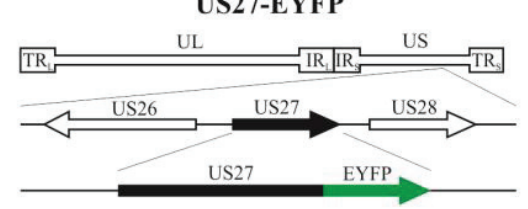

UL78-EYFP

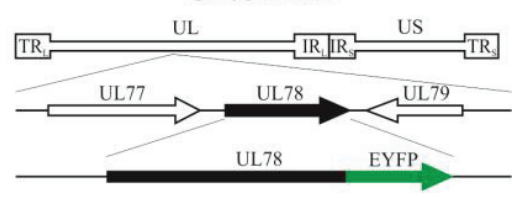

UL78-EYFP/Flag-US27

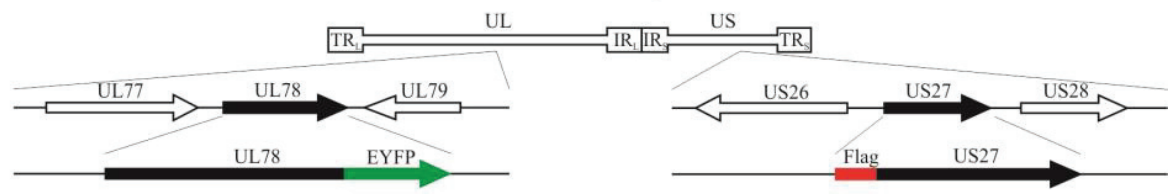

(b)

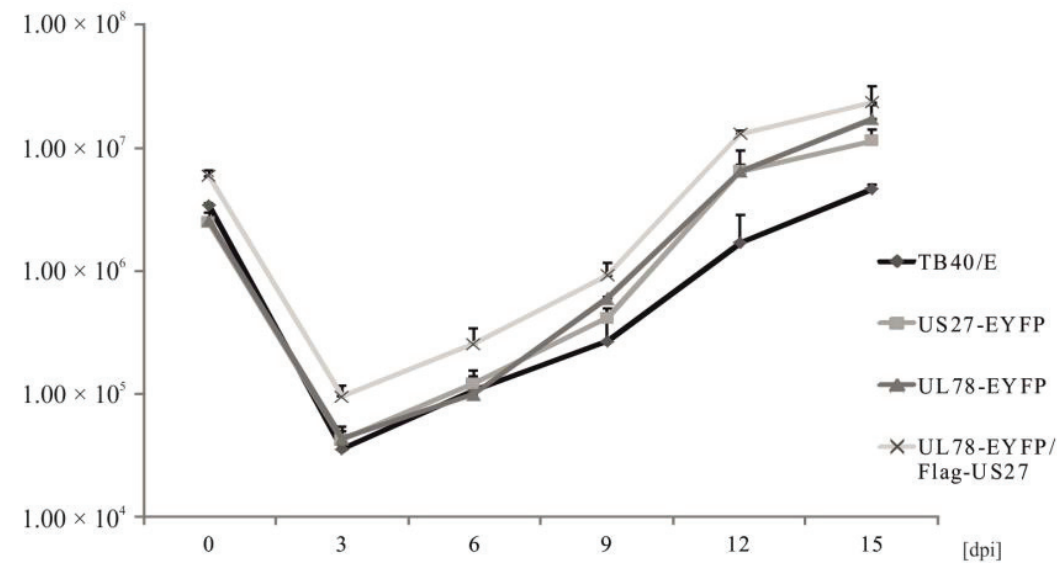

[dpi] 
Figure 3. Subcellular localization of US27-EYFP and UL78-EYFP in infected HFFs and ARPE-19 cells. HFF (left and middle panel) or ARPE-19 cells (right panel) were infected with recombinant TB40/E viruses (MOI: 0.5 or 1) expressing fusion proteins of pUS27 (left panel) or pUL78 (middle and right panel) with EYFP and fixed at different time points during HCMV infection (6-96 hpi). Cells were stained with primary antibody mAb-IE1 (p63-27), and a secondary anti-mouse antibody coupled to Alexa-555. Cell nuclei were stained with DAPI.
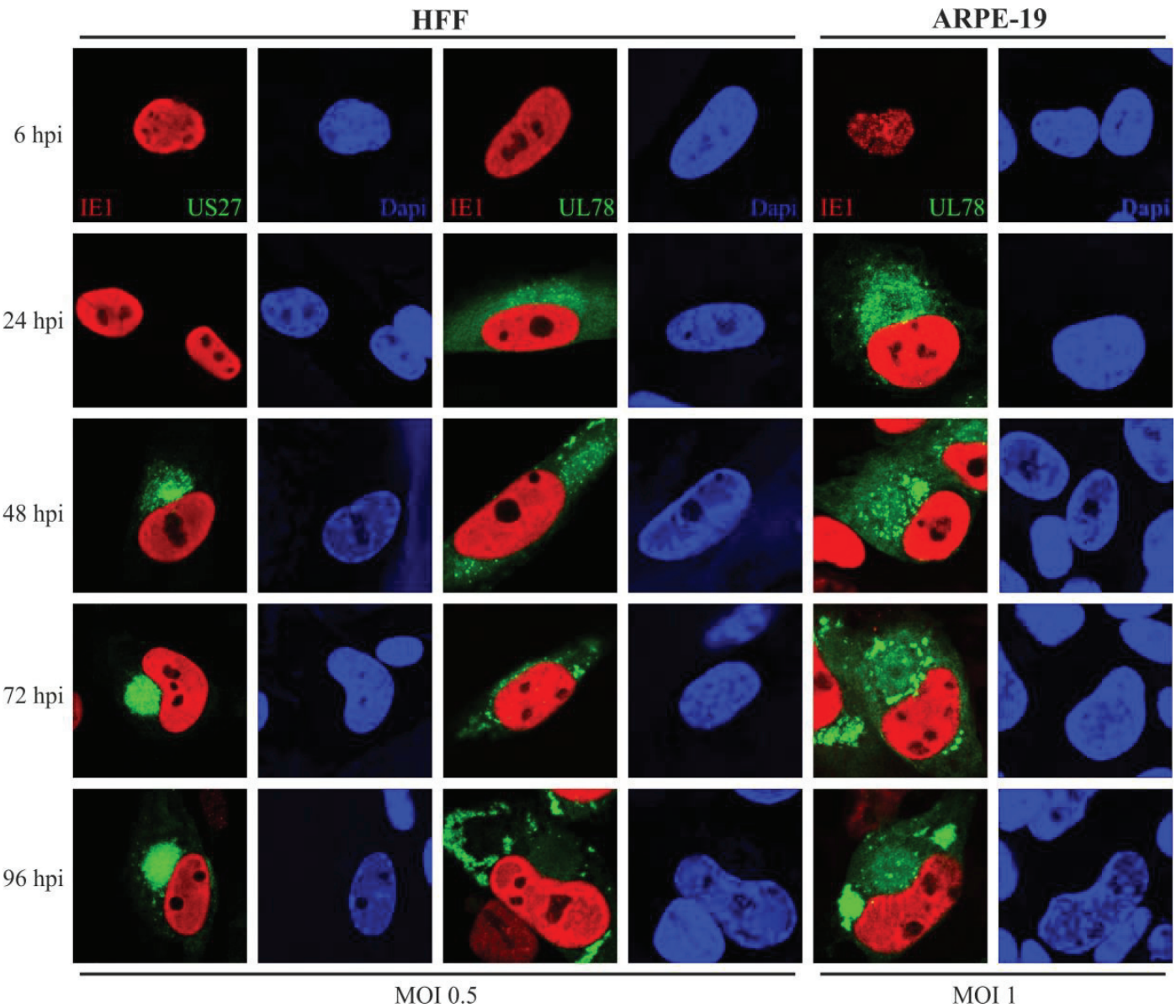

2.4. While Both Receptors Localize to the trans-Golgi Network in Virus Infected Cells, only pUL78 Colocalizes with Calreticulin

To better define the intracellular localization patterns of pUS27 and pUL78 during HCMV infection, we performed indirect immunofluorescence analysis using different cellular markers. In a first experiment, HFF and ARPE-19 cells were infected with either US27-EYFP or UL78-EYFP to identify a potential colocalization with proteins of the Golgi network or the endoplasmic reticulum. Since many vGPCRs, including pUS27, are predicted to be highly glycosylated [31], we expected a colocalization with the cis-Golgi marker GM130 in HFF as well as in ARPE-19 cells. In contrast to previous reports $[29,45]$, we were not able to visualize any colocalization with GM130 in infected cells over the entire replication cycle (data not shown). However, in accordance with transiently transfected receptors [30,36], partial colocalization of pUS27 and pUL78 was detected with the membrane protein TGN46, a marker for the trans-Golgi network, during the progression of HCMV 
infection (Figure 4a). Similar to GM130, the TGN46 signal showed perinuclear localization early in infection (Figure 4a, $24 \mathrm{hpi}$ ). After $48 \mathrm{~h}$, the signal shifted to the cVAC formation site and started forming concentric circles around this virus-induced, cellular structure (Figure 4a, 72 hpi). Upon pUS27 expression, the vGPCR localizes to the trans-Golgi network (TGN) (Figure 4a, left panel), suggesting that the TGN serves as site of glycosylation. This expression pattern lasted until late stages of HCMV infection. Upon comparison of these findings to pUL78, we observed a slightly different pattern. As pUL78 was detected throughout the cytoplasm at early stages (Figure 4a, $6 \mathrm{hpi}$, right panel) and was almost excluded from the virion assembly site late in infection (Figure 4a, 72 hpi, right panel), we detected a partial colocalization of TGN46 with pUL78 but not as striking as with pUS27. Next, we investigated a potential colocalization of both vGPCRs during infection with calreticulin, a marker for the endoplasmic reticulum (ER) (Figure 4b). At early stages of virus infection, calreticulin showed a typical lamellae structure formed around the cell nucleus. After approximately 48 to $72 \mathrm{~h}$, the structure changed and was completely displaced from the site of cVAC formation. As pUS27 is associated with the cVAC, we did not observe any colocalization of this receptor with calreticulin. In contrast to pUS27 (Figure 4b, left panel), pUL78 appeared to be associated with the ER over the entire replication cycle of HCMV (Figure 4b, right panel). The same results were obtained in ARPE-19 cells (data not shown). These results are in accordance with previous studies using transiently transfected cells [36].

Figure 4. Colocalization of US27-EYFP and UL78-EYFP with TGN46 and calreticulin in infected HFFs. HFFs were infected with recombinant TB40/E viruses (MOI: 0.5) expressing fusion proteins of pUS27 (left panel) or pUL78 (right panel) with EYFP and fixed at different time points during HCMV infection (24-96 hpi). Cells were stained with primary antibody (a) pAb-TGN46, diluted 1:300, or (b) pAb-Calreticulin (PA3-900), diluted 1:100, and the respective secondary anti-sheep antibody or anti-rabbit antibody coupled to Alexa-555 (1:400). Cell nuclei were stained with DAPI.

(a)

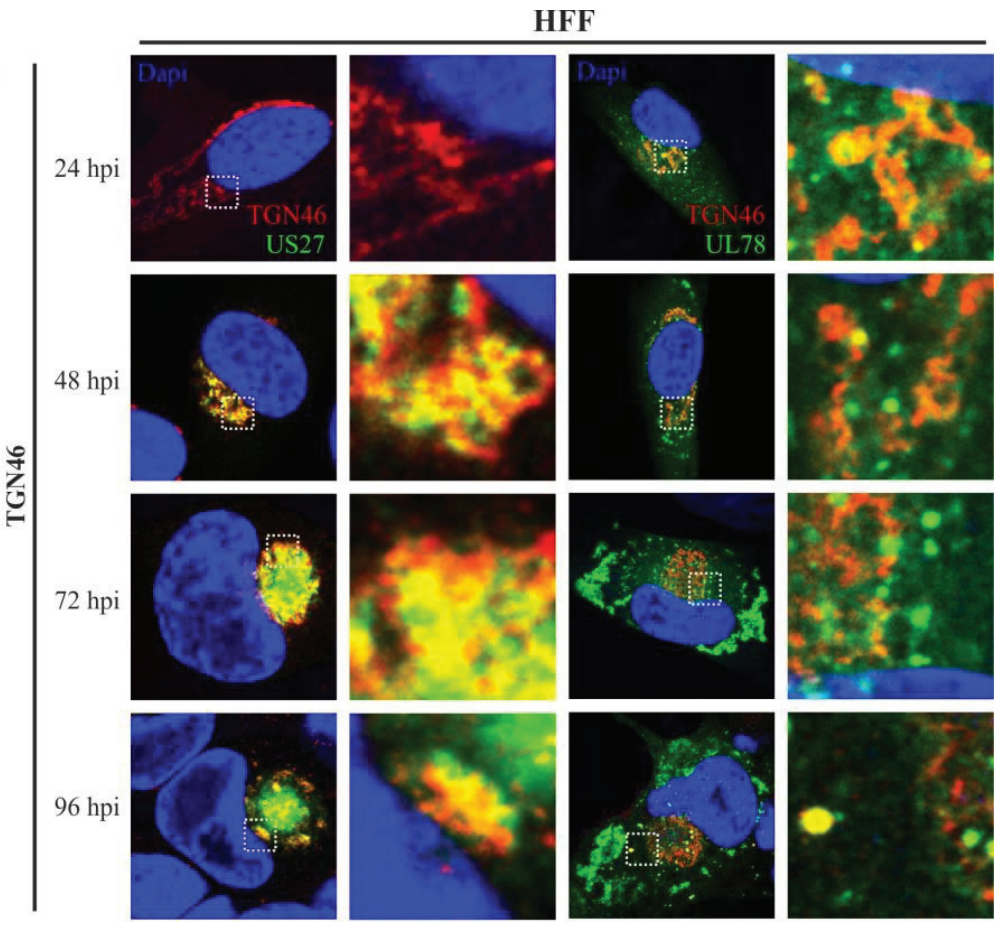


Figure 4. Cont.

(b)

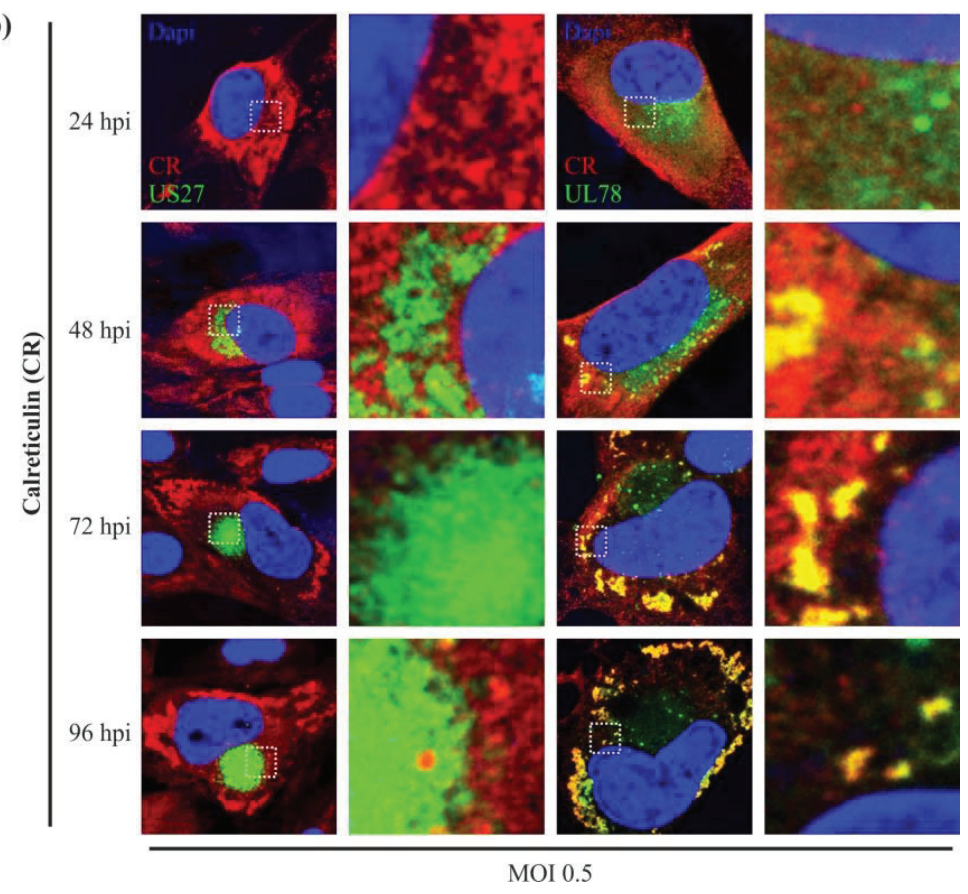

2.5. Endosomal Localization Patterns of pUS27 and pUL78 Diverge in Fibroblasts as Well as in Epithelial Cells during HCMV Replication

Next, we determined whether there is any colocalization of pUS27 or pUL78 with endosomal markers during the time course of HCMV infection. In order to describe endosomal structures in detail, colocalization studies with different endosomal and lysosomal markers were performed. The main components of the endosomal system are early endosomes (EE), recycling endosomes (RE), maturing endosomes (ME), late endosomes (LE), and lysosomes. During cVAC formation, components of the host cell secretory apparatus are rearranged in concentric circles [41]. Thereby, early (EEA1) and recycling (CD71) endosomes were localized in the cVAC center and were enclosed with markers of the trans-Golgi and cis-Golgi network in HFFs (Figures 4 and 5). Markers of the late endocytic pathway (CD63, LAMP-1), however, were found in the cVAC periphery (Figure 6). For pUL78, a partial colocalization with EEA1 was already shown at $48 \mathrm{~h}$ post infection in a in previous report [36]. Here, we demonstrate that co-localization of pUL78 signal with that of EEA1 is not restricted to the $48 \mathrm{~h}$ post infection time point, but in fact occurs throughout the HCMV replication cycle in HFFs (Figure 5, right panel) as well as in ARPE-19 cells (data not shown). In contrast, the pUS27 signal colocalized with EEA1 in HFFs only at late times of infection (Figure 5, left panel). Intriguingly, no colocalization between pUS27 and EEA1 could be detected in ARPE-19 cells, infected with the same recombinant virus (Figure 5, middle panel). Thus, not only pUL78 but also pUS27 may exhibit differential functions in HFFs versus ARPE-19 cells. 
Figure 5. EEA1 staining of US27-EYFP and UL78-EYFP infected HFFs and ARPE-19 cells. HFF (left and right panel) or ARPE-19 cells (middle panel) were infected with recombinant TB40/E viruses (MOI: 0.5 or 1) expressing fusion proteins of pUS27 (left and middle panel) or pUL78 (right panel) with EYFP and fixed at different time points during HCMV infection (24-96 hpi). Cells were stained with primary antibody pAb-EEA1 (H-300), diluted 1:200, and the secondary anti-rabbit antibody coupled to Alexa-555 (1:400). Cell nuclei were stained with DAPI.

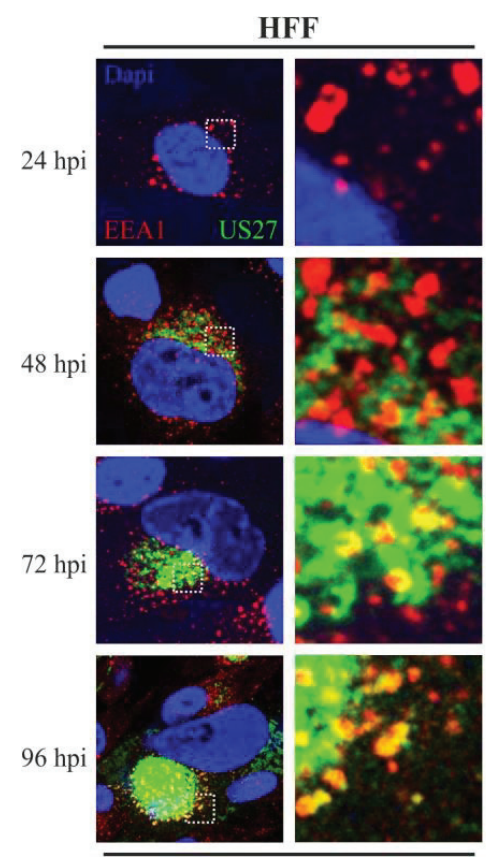

MOI 0.5

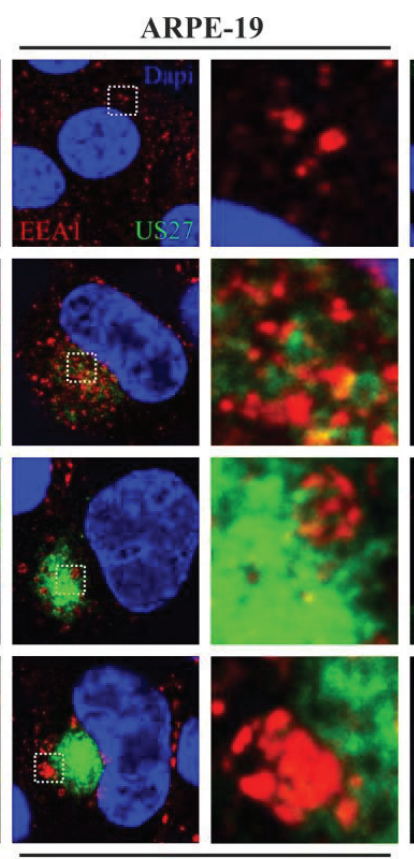

MOI 1

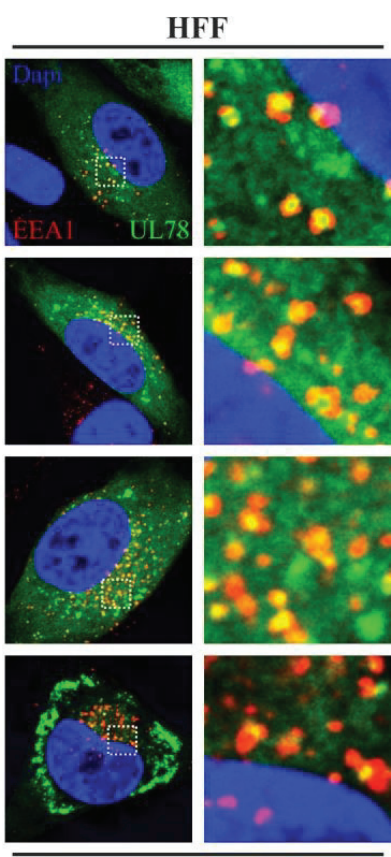

MOI 0.5

The endosomal machinery is a highly complex system. After internalization and bypass of early endosomes, cargo can enter a recycling circuit or a degradative system, in which endosomes mature to MEs, and later to LEs [46]. To further elucidate the fate of HCMV-encoded vGPCRs after internalization, we used markers for REs (CD71), MEs/LEs (CD63), and lysosomes (LAMP1). We expected a colocalization with recycling endosomes, as both receptors were shown to localize to the cell surface $[29,30,36]$. Unfortunately, no overlap of vGPCR signals with CD71 could be detected in any cell type (data not shown). This may suggest that pUS27 and pUL78 recycling is independent of CD71-positive endosomes occurring directly via EEs. Alternatively, both receptors might enter the cellular degradative system immediately after internalization. To address this hypothesis, we next focused on maturing endosomes, late endosomes and lysosomes. CD63, a marker for multivesicular bodies, appeared as dot-like structures (Figure 6a), which were widely spread over the cytoplasm early in HCMV infection (24 hpi). After $48 \mathrm{~h}$, the signal began to concentrate in the perinuclear region. Interestingly, CD63 signal seemed to be downregulated during cVAC formation in HFFs (Figure 6a, left and middle panel), as described previously [43]. In ARPE-19 cells, however, we could not observe any changes in CD63 expression (Figure 6a, right panel). Similar to EEA1, pUL78 began to colocalize with CD63 at 24 hpi (Figure 6a, middle and left panel). This colocalization could be observed throughout the replication cycle in HFFs and ARPE-19 cells and was decreased with the reduction of the CD63 signal in HFFs late in infection (Figure 6a, middle panel). In contrast, pUS27 did not display any colocalization with the cellular 
marker CD63 (Figure 6a, left panel), indicating that this receptor is not present in mature and late endosomes of infected HFFs and ARPE-19 cells (data not shown). Corresponding to CD63, LAMP1, a marker for lysosomes, could be recognized in dot-like structures widely spread throughout the cytoplasm at $24 \mathrm{hpi}$; at $48 \mathrm{hpi}$, the signal started to concentrate in the perinuclear region of the cell (Figure 6b). Furthermore, pUS27 was not observed in lysosomes in HFFs (Figure 6b, left panel) or ARPE-19 cells (data not shown). Nevertheless, pUL78 signal overlapped with LAMP1 (Figure 6b, middle and right panel). This pattern, however, differed strongly dependent on the cell type. While we could demonstrate that pUL78 was localized to lysosomes early in infection in HFFs (Figure 6b, middle panel), the dot-like signals of pUL78, located close to the nucleus, showed clear colocalization with LAMP-1 in ARPE-19 cells starting at $72 \mathrm{~h}$ post infection (Figure 6b, right panel).

Figure 6. CD63 and LAMP1 staining of US27-EYFP and UL78-EYFP infected HFFs and ARPE-19. HFF (left and middle panel) or ARPE-19 cells (right panel) were infected with recombinant TB40/E viruses (MOI: 0.5 or 1) expressing fusion proteins of pUS27 (left panel) or pUL78 (middle and right panel) with EYFP and fixed at different time points during HCMV infection (24-96 hpi). Cells were stained with primary antibody (a) mAb-CD63 (MX-49.129.5) or (b) mAb-LAMP-1 (H5G11), each diluted 1:50, and the secondary anti-mouse antibody coupled to Alexa-555 (1:400). Cell nuclei were stained with DAPI.
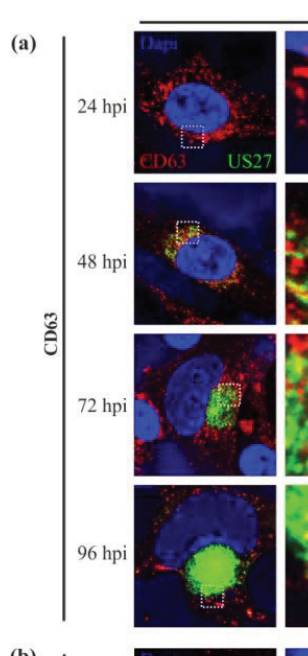

HFF
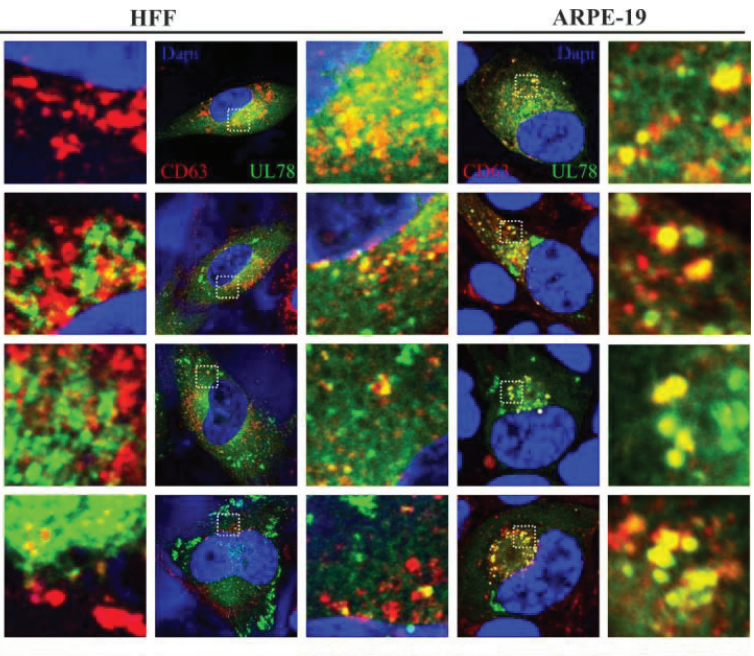

(b)
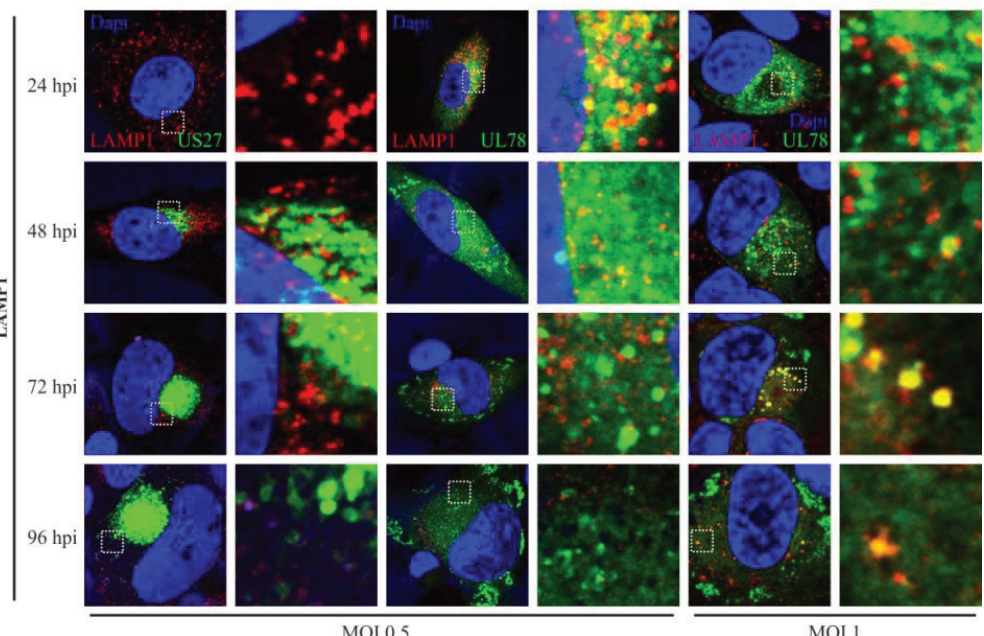
2.6. pUS27 and pUL78 Do Not Colocalize in Infected Fibroblasts or Epithelial Cells throughout the Replication Cycle

Finally, we set out to clarify whether there is any colocalization of both vGPCRs in one cell during HCMV infection. To that end, a recombinant virus carrying tagged versions of the US27, as well as the UL78 gene (Flag/EYFP), was engineered by means of BAC technology (Figure 2) and investigated by immunofluorescence analysis. Colocalization studies with variable cellular markers already revealed that localization patterns of pUS27 and pUL78 differ substantially. Here, we demonstrate for the first time that pUS27 and pUL78 do not display any colocalization throughout the HCMV replicative cycle. This observation was independent of the cell type, as both vGPCR signals did not overlap in HFFs or ARPE-19 cells (Figure 7). In conclusion, this strongly suggests that the localization patterns of virally-encoded GPCRs as determined in transiently transfected cells do not reflect the physiological patterns during infection. Our data highlight the importance to analyze vGPCR functions in a viral context.

Figure 7. Colocalization analysis of Flag-US27 and UL78-EYFP in infected HFFs and ARPE-19 cells. HFF (left panel) or ARPE-19 cells (right panel) were infected with a recombinant TB40/E virus (MOI: 0.5 or 1) expressing fusion proteins of both pUS27 (red) and pUL78 (green) with Flag or EYFP, respectively, and fixed at different time points during HCMV infection (24-96 hpi). Cells were stained with primary antibody $\mathrm{mAb}$-Flag, diluted 1:400, in combination with the secondary anti-mouse antibody coupled to Alexa-555 (1:400) in order to detect pUS27. Cell nuclei were stained with DAPI.

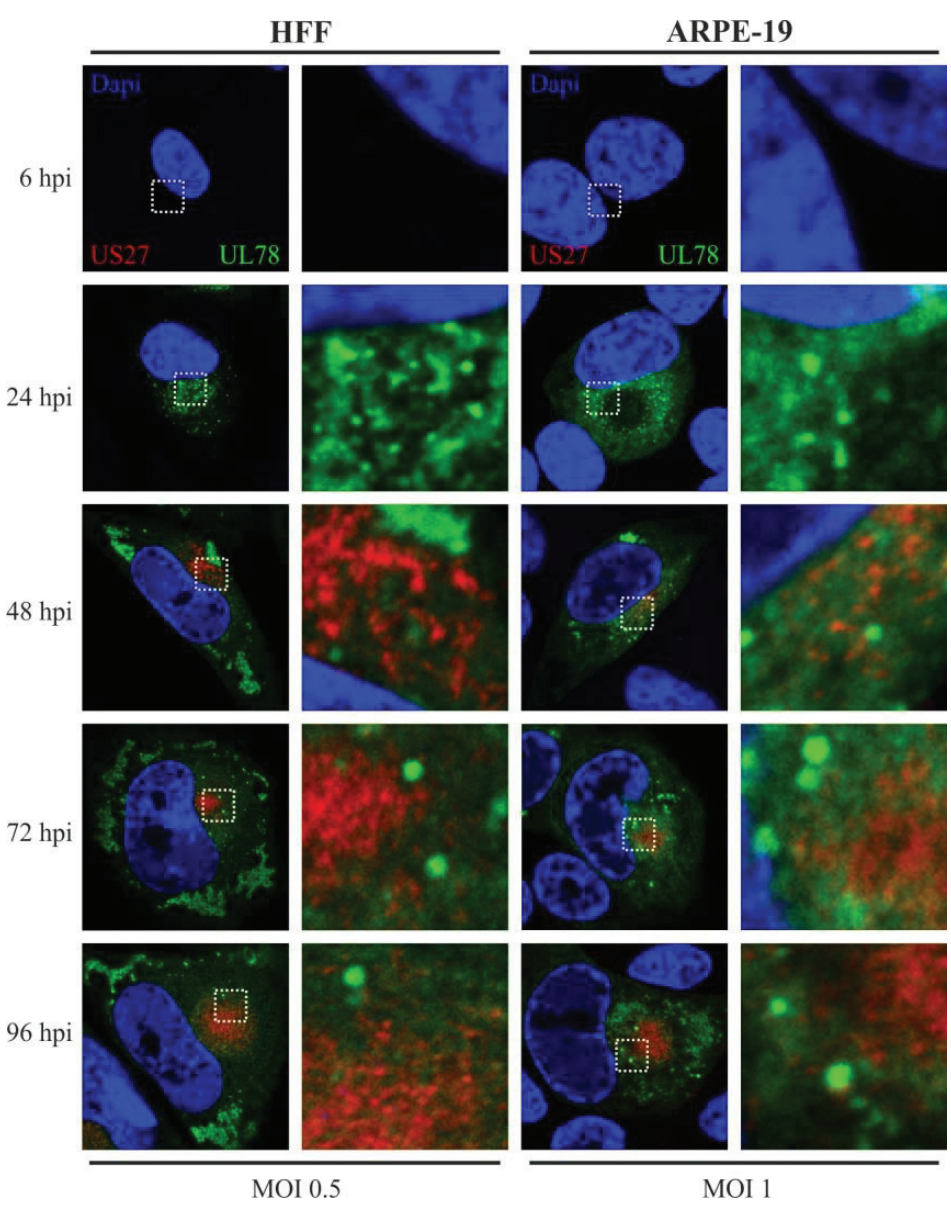




\section{Discussion}

Human cytomegalovirus (HCMV), as all herpesviruses, establishes a lifelong infection in the host. In order to maintain its persistent state, HCMV developed various mechanisms during coevolution with its host to modify the cellular signaling network and to evade the immune system. It is likely that the expression of four homologs of cellular seven-transmembrane receptors termed pUS27, pUS28, pUL33, and pUL78 contributes to the dysregulation of cellular signaling and to immune evasion $[4,5]$. While cellular G-protein coupled receptors (GPCRs) typically localize to the cell surface and induce intracellular signaling cascades upon ligand binding [47], several reports of viral GPCRs (vGPCR) showed a different receptor localization. For instance, in the absence of ligands pUS28 was predominantly found in an intracellular pattern in endosomal compartments [15]. Similar observations were made for the orphan receptors pUS27 and pUL33, where the majority of the protein was detected in the perinuclear region of cells [30]. Moreover, the vGPCR pUL78 seemed to associate mainly with the endoplasmic reticulum (ER) [36]. Unfortunately, most of these results were obtained with transiently expressed receptors. To gain more insight into the subcellular localization of HCMV-encoded vGPCRs during viral infection we performed immunofluorescence analyses of recombinant HCMVs, based on the endotheliotropic strain TB40/E, expressing either EYFP- or Flag-tagged pUS27 and/or pUL78.

In accordance with previous studies, we could confirm that pUL78 has an early/late expression pattern [48], whereas pUS27 is expressed later during infection. While pUS27 exhibits an intracellular perinuclear distribution and is recruited to the cytoplasmic virion assembly complex (cVAC), the early protein pUL78 is observed throughout the cytoplasm. Due to stable colocalization with the $\mathrm{ER}$, which is known to be excluded from the cVAC formation site [41,42], pUL78 is completely displaced from the virus assembly zone in HFFs. Importantly, our results indicate pronounced variations in localization patterns of virally-encoded GPCRs compared to transiently expressed pUS27 or pUL78. Contrary to transiently expressed vGPCRs $[29,45]$, we were not able to visualize any colocalization with the cis-Golgi marker GM130 in HFFs or ARPE-19 cells (data not shown). Similar to recent reports [30,36,45], a colocalization of pUS27 and pUL78, with TGN46, a marker for the trans-Golgi network (TGN), was obvious and stable over the whole replication cycle. Thus, in contrast to Stapleton et al. [29], we suggest the TGN as the site of receptor glycosylation for at least pUS27.

Furthermore, we detected a clear colocalization of pUL78 with EEA1 immediately upon expression, whereas pUS27 started to colocalize with the marker for early endosomes (EEs) at late time points after infection (96 hpi) only in HFFs. This observation differed significantly from the pattern determined for transiently expressed receptors [29,36]. Our data provide evidence that protein sorting is a dynamic process leading to dramatic changes of receptor localization during the entire HCMV replication cycle. Thus, it is of obvious importance to investigate protein localization patterns in a viral context. A surprising finding was that neither pUS27 nor pUL78 signal overlapped with CD71, a marker for recycling endosomes (REs). Both receptors were reported to constitutively internalize [29,30,36]. Hence, we expected a colocalization with REs, which was previously shown for the pUL78 homolog pM78 [45]. However, this result does not necessarily exclude pUS27 and pUL78 recycling in infected cells, since receptor recycling may also occur via 
a direct route from EEs, the so called fast recycling pathway [49], or, as shown for cellular GPCRs, via the TGN [50]. On the other hand, both vGPCRs could enter a cellular degradative system directly after internalization [46]. In contrast to transiently expressed pUS27 [30], we were not able to visualize any colocalization with the cellular markers CD63 and LAMP1. Only pUL78 extensively overlapped with these late endosomal (LE) and lysosomal markers, contrary to a transiently transfected version [36]. Since Das et al. suggested that lysosomes do no longer act as degradation system in infected cells [42], we hypothesized that pUL78 is not degraded but may fulfill a so far unknown function within these structures. However, the colocalization pattern of pUL78 with LEs and lysosomes varied extensively when analyzing different cell types or time points post infection. Consequently, these data again emphasize the highly dynamic protein localization pattern during HCMV replication.

Intriguingly, the most pronounced divergence was detected with our recombinant virus carrying tagged versions of pUS27 and pUL78 simultaneously. In general, it is important to keep in mind that heteromerization of vGPCRs can alter their functions [51]. For example, Tschische et al. showed that heterodimerization of pUL78 with pUS28 led to an almost complete block of pUS28-mediated constitutive NF- $\kappa$ B activation [37]. Besides, pUL78 as well as pUL33 were shown to heteromize with the human CCR5 and CXCR4 receptors thereby modifying GPCR functions and cell surface localization [52]. Thus, we hypothesized that a closer investigation of pUS27/pUL78 heterodimerization might unravel novel roles during virus infection and pathogenesis. Unexpectedly, however, we were not able to visualize any overlap of pUS27 and pUL78 signals in infected cells whereas this was clearly detectable in transient transfection experiments (compare Figures 1 and 7). Consequently, recent heteromerization studies of HCMV-encoded vGPCRs have to be interpreted with caution since the subcellular localization of virally-encoded GPCRs as determined in transiently transfected cells may not reflect the physiological patterns during infection.

In accordance with previous studies [32,33], comparative analyses of fibroblasts and epithelial cells revealed a cell type-dependent trafficking of both pUS27 and pUL78. Moreover, we demonstrate that the subcellular morphology changes not only during infection, but also varies between infected HFFs and ARPE-19 cells. Whereas, as described previously [43], CD63 was downregulated during cVAC formation in HFFs, we did not observe any changes in CD63 expression in ARPE-19 cells. This observation was in agreement with a HFF-specific, decreased colocalization of CD63 with pUL78 late during infection. Similar results were obtained for LAMP1. In addition, not only the colocalization of pUL78 varied between cell types, but also its cytoplasmic distribution: at the beginning of HCMV replication, pUL78 signal was spread throughout the cytoplasm in both analyzed cell types; however, a displacement from the cVAC formation site was only observed in HFFs. In ARPE-19 cells additional dot-like pUL78-positive structures remained in the perinuclear region for the entire replication cycle.

Surprisingly, no colocalization of pUS27 with any endosomal marker was detected in ARPE-19 cells. This raises the question whether pUS27 is localized at the cytoplasmic membrane in epithelial cells. A possible explanation would be that pUS27 internalization occurs via EEA1-negative endosomes, for example APPL-positive ones [53], or in a retrograde manner via the TGN [50]. 
Nonetheless, further investigations will be required to characterize the sorting abilities of pUS27 in more detail.

In conclusion, the results of this study emphasize the importance of analyzing vGPCR sorting in a viral context. Furthermore, we observed an unexpected, cell type-dependent variation in vGPCR receptor localization and sorting suggesting major functional differences of these molecules in terms of their signaling capabilities in various cell types.

\section{Experimental Section}

\subsection{Cells}

HeLa cells were cultivated in Eagle's minimal essential medium (MEM) (GIBCO/BRL) containing $7 \%$ fetal calf serum at $37{ }^{\circ} \mathrm{C}$ and $5 \% \mathrm{CO}_{2}$. Primary human foreskin fibroblasts (HFFs) and the human retinal pigment epithelial cell line ARPE-19 were maintained in MEM, supplemented with $10 \%$ fetal calf serum at $37^{\circ} \mathrm{C}$ and $5 \% \mathrm{CO}_{2}$.

\subsection{Construction of Vectors for Subcellular Localization Analyses}

For the FLAG-US27 construct, a FLAG-epitope was fused to the 5 ' region of the US27 gene (based on TB40/E) after amplification of the US27 coding sequence with primers N-US27-BamHI-for (5'-CTA GGG ATC CAC CAC CTC TAC AAA CCA AAC CTT AAC-3') and N-US27-Xhol-rev (5'-CTA GCT CGA GTT ACA ATA GAA ATT CCT CCT CCC CG-3') followed by insertion into the pcDNA3-derived vector pHM971. For the UL78GFP construct, the GFP coding sequence was fused to the 3 ' region of the UL78 gene (based on TB40/E): after amplification of the UL78 coding sequence with primers UL78-EcoRI-for (5'-CTG AAT TCA TGT CCC CTT CTG TGG AGG AGA C-3') and 2.UL78-BamHI-2nt-rev (5'-TCG GAT CCT GTA ATG CCG TCA CCG TTG CGT CG-3'), the amplicon was inserted into pEGFP-C1 (Clontech, Heidelberg, Germany), resulting in the expression of a $C$-terminally GFP-tagged pUL78.

\subsection{Transient Expression Analyses and Protein Localization}

For immunofluorescence analyses, $4 \times 10^{5} \mathrm{HeLa}$ cells/well were seeded in MEM medium without antibiotics on coverslips until they were grown to approximately $80 \%$ confluence. Transfection of DNA plasmids (2 $\mu \mathrm{g}$ total, with or without pcDNA3/well) was performed by utilizing LipofectamineTM 2000 (Invitrogen, Karlsruhe, Germany) according to the manufacturer's instructions. At $24 \mathrm{~h}$ post-transfection, cells were washed three times with PBSo and fixed by incubation with 4\% PFA for $10 \mathrm{~min}$ at room temperature. After fixation, cells were washed again three times with PBSo and permeabilized with $0.2 \%$ Triton X-100 in PBSo at $4{ }^{\circ} \mathrm{C}$ for 15 min. After three additional washing steps with PBSo, the MAb-Flag (M2 F1804, Sigma-Aldrich, Deisenhofen, Germany), to stain FLAG-US27, diluted 1:400 in PBSo, was applied and cells were incubated for $1 \mathrm{~h}$ at $37^{\circ} \mathrm{C}$. Excessive antibodies were removed by washing three times with PBSo, followed by incubation with anti-mouse Alexa555-conjugated secondary antibody, which was diluted 1:400 in PBSo, and incubated with the cells for a further $45 \mathrm{~min}$ at $37{ }^{\circ} \mathrm{C}$. Cells were mounted using the DAPI Hard-set (4,6-Diamino-2-phenylindol)-containing Vectashield mounting 
medium (Vector Laboratories, Burlingame, CA, USA) and then analyzed using the Leica TCS SP5 confocal laser scanning microscope (Leica, Wetzlar, Germany). Adobe Photoshop package [54] was used for processing of images.

\subsection{Viruses, Generation of Recombinant HCMVs, and Growth Curve Analysis}

All HCMV strains were reconstituted and propagated on human foreskin fibroblasts (HFF) as described previously $[38,55]$ and were based on the HCMV bacterial artificial chromosome (BAC) TB40-Bac4, which was derived from the HCMV strain TB40/E, isolated on endothelial cells [39]. For $C$-terminal and/or $N$-terminal fusion of EYFP- or FLAG-tag to the coding region of US27 and/or UL78 (for details see Figure 2a) a markerless BAC mutagenesis according to Tischer et al. [55] was performed in the E. coli strain GS1783 (kindly provided by Tischer, B.K., Free University of Berlin, Berlin, Germany). For that purpose, linear recombination fragments were generated by amplification of an I-SceI-aphAI cassette from plasmid pEP-S/aphAI (for FLAG-fusion, kindly provided by Tischer, B.K., Free University Berlin, Berlin, Germany) or plasmid pHM3366 (based on pEP-S/aphAI, for EYFP-fusion) via Hot Start PCR with the polymerase Phusion Hot Start (Thermo Fisher Scientific, Waltham, MA, USA) and specific primer pairs (Table 1). For $N$-terminal fusion of the Flag-epitope, two consecutive PCRs had to be performed. Afterwards, the kanamycin cassette was removed by induction of I-SceI followed by a second Red recombination. Resulting BAC clones, termed TB40/E-US27-EYFP (US27-EYFP), TB40/E-Flag-US27 (Flag-US27), or TB40/E-UL78-EYFP (UL78-EYFP), were verified by distinct PCR reactions and subsequent nucleotide sequence analyses, as well as restriction fragment length polymorphism analysis (RFLP, using restriction endonuclease EcoRV-HF, NEB). To generate double-tagged TB40/E-UL78-EYFP/Flag-US27 (UL78-EYFP/Flag-US27), the parental BAC TB40/E-Flag-US27 (Flag-US27) was used to transform E.coli GS1783. Recombination occurred as above with a linear recombination fragment of UL78-EYFP. For virus reconstitution, BAC DNA was isolated from bacteria using the BAC preparation protocol of the PureLink HiPure Plasmid Maxiprep kit (Invitrogen, Karlsruhe, Germany) and transfected into HFFs using X-tremeGENE transfection reagent (Roche, Mannheim, Germany) according to the manufacturer's instructions. Transfected cells were incubated at $37{ }^{\circ} \mathrm{C}$ until plaques appeared, while medium was changed once a week. The supernatant was used for infection of fresh HFF cultures and preparation of virus stocks.

In order to determine the replication capacities of all reconstituted recombinant viruses, multistep growth curve analyses were performed. Therefore, 3 days after seeding, $3.0 \times 10^{5}$ resting HFFs were infected with equal IE1 units $(\mathrm{MOI}=0.1)$ of wild-type TB40/E or recombinant viruses. The viral supernatants were harvested at $0,3,6,9,12$, and 15 days post infection (Figure 2b, dpi, days post infection). Aliquots of the supernatants were treated with proteinase K. Afterwards, to quantify the HCMV genome copy numbers, all samples were subjected to quantitative Real-Time PCR using fluorescence-labeled Taqman probes and the ABI Prism 7500 sequence detector (Applied Biosystems, Foster City, CA, USA) as well as the corresponding software SDS [56]. The viral load was determined by amplification of a region within the exon 4 of the IE1 gene locus (ORF UL123) using the primers CMV5' (5'-AAG CGG CCT CTG ATA ACC AAG-3') and CMV3' (5'-GAG CAG ACT CTC TCA GAG GAT CGG-3') together with the fluorescence labeled 
probe CMV MIE FAM /TAMRA (5'-CAT GCA GAT CTC CTC AAT GCG GCG-3'). Each infection was performed in triplicate.

Table 1. Oligonucleotide primers for generation of recombinant human cytomegalovirus (HCMV) strains.

\begin{tabular}{|c|c|c|}
\hline Name & Construct & Sequence in $5^{\prime}-3^{\prime}$ \\
\hline Flag-kTischer-fw & \multirow{4}{*}{$\begin{array}{l}\text { Flag-US27 } \\
\text { PCR1 }\end{array}$} & GAT TAC AAG GAT GAC GAC GAT AAG \\
\hline \multirow{3}{*}{ Flag-US27-rev } & & CAT ATC AGA ATT GGT TAA TTG GTT GGT AAC ACT ACC TGT AAG \\
\hline & & GTG ATG GAT TAC AAG GAT GAC GAC GAT AAG ATG ACC ACC TCT \\
\hline & & ACA AAC CAA ACC TTA ACA CAG GTG AGC AAC ATG ACA AA \\
\hline Flag-kUS27-rev & \multirow{3}{*}{$\begin{array}{l}\text { Flag-US27 } \\
\text { PCR2 }\end{array}$} & TTT GTC ATG TTG CTC ACC TGT GTT \\
\hline \multirow{2}{*}{ Flag-US27-fw } & & TGT TAT GCT TTT TAC AGG ACC GTT CAG CAG GTA ACA CTA \\
\hline & & CCT GTA AGG TGA TGG ATT ACA AGG ATG ACG ACG ATA AG \\
\hline JS & \multirow{4}{*}{ US27-EYFP } & ATG ACA GAA AAA ATG CAC CTA TGG AGT CCG GGG AGG AGG \\
\hline 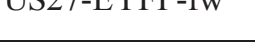 & & AAT TTC TAT TGA TGG TGA GCA AGG GCG AGG AGC TG \\
\hline \multirow{2}{*}{ US27-EYFP-rev } & & GTG CAA TGA GCA AAA ATA GAT GTG CGG CGG ACG CGT GAA \\
\hline & & AGA GGA TCG AAT TAC TTG TAC AGC TCG TCC ATG CCG AG \\
\hline \multirow{2}{*}{ UL78-EYFP-fw } & \multirow{4}{*}{ UL78-EYFP } & GCA CCG ACG GCG AAA ACA CCG TCG CGT CCG ACG CAA CGG \\
\hline & & TGA CGG CAT TAA TGG TGA GCA AGG GCG AGG AGC TG \\
\hline \multirow{2}{*}{ UL78-EYFP-rev } & & GAC GTG ATT TAT CTG CCA CTT TTC TCC CCG CTG CCG TAC \\
\hline & & AGC GCC GCC GCT TAC TTG TAC AGC TCG TCC ATG CCG AG \\
\hline
\end{tabular}

\subsection{HCMV Infection and Protein Localization}

HFF $\left(2 \times 10^{5}\right.$ cells/well $)$ or ARPE-19 cells $\left(4.5 \times 10^{5}\right.$ cells/well $)$ were seeded on coverslips for infection with recombinant viruses. Indirect immunofluorescence analysis was performed as described before for transiently transfected cells. However, after permeabilization, an additional blocking step was performed. Therefore, cells were blocked with human or goat serum diluted 1:10 in PBSo for polyclonal or monoclonal antibodies, respectively, and incubated for 15 min at $37{ }^{\circ} \mathrm{C}$. Afterwards, cells were stained with specific primary antibodies (Table 2) for $1 \mathrm{~h}$ at $37{ }^{\circ} \mathrm{C}$ and appropriate secondary antibodies Alexa-555 anti-sheep, Alexa-555 F(ab)'2 anti-mouse, or Alexa-555 F(ab)'2 anti-rabbit (Invitrogen, Karlsruhe, Germany) for $45 \mathrm{~min}$ at $37^{\circ} \mathrm{C}$ and mounted using the DAPI Hard-set-containing Vectashield mounting medium (Vector Laboratories, Burlingame, CA, USA). Images were acquired as before, using a Leica TCS SP5 confocal laser scanning microscope and finally analyzed using Adobe Photoshop package [54].

Table 2. Antibodies used in this study.

\begin{tabular}{cccc}
\hline Target protein & Localization & Dilution & Nature of antibody (source) \\
\hline IE1 & nucleus & $1: 4$ & MAb, clone p63-27 [57] \\
\hline TGN46 & trans-Golgi network & $1: 300$ & $\begin{array}{c}\text { Sheep polyclonal antibody } \\
\text { (AbD Serotec catalog No. AHP500G) }\end{array}$ \\
\hline calreticulin & endoplasmic reticulum & $1: 100$ & $\begin{array}{c}\text { Rabbit polyclonal antibody } \\
\text { (Thermo Fisher catalog No. PA3-900) }\end{array}$ \\
\hline EEA1 & early endosomes & $1: 200$ & $\begin{array}{c}\text { Rabbit polyclonal antibody, clone H-300 } \\
\text { (SCBT catalog No. 33585) }\end{array}$ \\
\hline CD63 & $\begin{array}{c}\text { multivesicular bodies, } \\
\text { late endosomes }\end{array}$ & $1: 50$ & $\begin{array}{c}\text { MAb, clone 4X-49.129.5 } \\
\text { (SCBT catalog No. 5275) }\end{array}$ \\
\hline LAMP1 & lysosomes & $1: 50$ & MAb, clone H5G11 (SCBT catalog No. 18821) \\
\hline
\end{tabular}




\section{Conclusions}

In conclusion, our data demonstrate that trafficking of the HCMV-encoded GPCRs pUS27 and pUL78 occurs in a cell type-dependent manner suggesting that both receptors may exhibit functional differences in various cell types. Furthermore, we could clearly show that pUS27 as well as pUL78 trafficking are affected by additional viral proteins. Thus, analysis of pUS27 and pUL78 in a viral context is mandatory in order to further decipher the functions of these two vGPCRs during HCMV replication.

\section{Acknowledgments}

We thank C. Sinzger (Ulm, Germany), K. Tischer (Berlin, Germany) and M. Marschall (Erlangen, Germany) for providing reagents; Regina Müller for excellent technical assistance and Myriam Scherer, as well as Nina Reuter, for fruitful discussions. This work was supported by the Interdisciplinary Center for Clinical Research, Project A51, and the DFG (GRK1071, subproject B2). Furthermore, we acknowledge support by the Deutsche Forschungsgemeinschaft and Friedrich-Alexander-Universität Erlangen-Nürnberg within the funding programme Open Access Publishing.

\section{Author Contributions}

I.N. and A.R. performed the experiments. I.N. and T.S. analyzed the data and wrote the manuscript.

\section{Conflicts of Interest}

The authors declare no conflict of interest.

\section{References and Notes}

1. Montaner, S.; Kufareva, I.; Abagyan, R.; Gutkind, J.S. Molecular mechanisms deployed by virally encoded $\mathrm{G}$ protein-coupled receptors in human diseases. Annu. Rev. Pharmacol. Toxicol. 2013, 53, 331-354.

2. Couty, J.P.; Gershengorn, M.C. G-protein-coupled receptors encoded by human herpesviruses. Trends Pharmacol. Sci. 2005, 26, 405-411.

3. Sodhi, A.; Montaner, S.; Gutkind, J.S. Viral hijacking of G-protein-coupled-receptor signalling networks. Nat. Rev. Mol. Cell Biol. 2004, 5, 998-1012.

4. Chee, M.S.; Satchwell, S.C.; Preddie, E.; Weston, K.M.; Barrell, B.G. Human cytomegalovirus encodes three G protein-coupled receptor homologues. Nature 1990, 344, 774-777.

5. Attwood, T.K.; Findlay, J.B. Fingerprinting G-protein-coupled receptors. Protein Eng. 1994, 7, 195-203.

6. Margulies, B.J.; Browne, H.; Gibson, W. Identification of the human cytomegalovirus G protein-coupled receptor homologue encoded by UL33 in infected cells and enveloped virus particles. Virology 1996, 225, 111-125. 
7. Bodaghi, B.; Jones, T.R.; Zipeto, D.; Vita, C.; Sun, L.; Laurent, L.; renzana-Seisdedos, F.; Virelizier, J.L.; Michelson, S. Chemokine sequestration by viral chemoreceptors as a novel viral escape strategy: Withdrawal of chemokines from the environment of cytomegalovirus-infected cells. J. Exp. Med. 1998, 188, 855-866.

8. Cardin, R.D.; Schaefer, G.C.; Allen, J.R.; vis-Poynter, N.J.; Farrell, H.E. The M33 chemokine receptor homolog of murine cytomegalovirus exhibits a differential tissue-specific role during in vivo replication and latency. J. Virol. 2009, 83, 7590-7601.

9. Beisser, P.S.; Grauls, G.; Bruggeman, C.A.; Vink, C. Deletion of the R78 G protein-coupled receptor gene from rat cytomegalovirus results in an attenuated, syncytium-inducing mutant strain. J. Virol. 1999, 73, 7218-7230.

10. Casarosa, P.; Bakker, R.A.; Verzijl, D.; Navis, M.; Timmerman, H.; Leurs, R.; Smit, M.J. Constitutive signaling of the human cytomegalovirus-encoded chemokine receptor US28. J. Biol. Chem. 2001, 276, 1133-1137.

11. Waldhoer, M.; Kledal, T.N.; Farrell, H.; Schwartz, T.W. Murine cytomegalovirus (CMV) M33 and human CMV US28 receptors exhibit similar constitutive signaling activities. J. Virol. 2002, 76, 8161-8168.

12. Gao, J.L.; Murphy, P.M. Human cytomegalovirus open reading frame US28 encodes a functional beta chemokine receptor. J. Biol. Chem. 1994, 269, 28539-28542.

13. Waldhoer, M.; Casarosa, P.; Rosenkilde, M.M.; Smit, M.J.; Leurs, R.; Whistler, J.L.; Schwartz, T.W. The carboxyl terminus of human cytomegalovirus-encoded 7 transmembrane receptor US28 camouflages agonism by mediating constitutive endocytosis. J. Biol. Chem. 2003, 278, 19473-19482.

14. Miller, W.E.; Houtz, D.A.; Nelson, C.D.; Kolattukudy, P.E.; Lefkowitz, R.J. G-protein-coupled receptor (GPCR) kinase phosphorylation and beta-arrestin recruitment regulate the constitutive signaling activity of the human cytomegalovirus US28 GPCR. J. Biol. Chem. 2003, 278, 21663-21671.

15. Fraile-Ramos, A.; Kledal, T.N.; Pelchen-Matthews, A.; Bowers, K.; Schwartz, T.W.; Marsh, M. The human cytomegalovirus US28 protein is located in endocytic vesicles and undergoes constitutive endocytosis and recycling. Mol. Biol. Cell 2001, 12, 1737-1749.

16. Mokros, T.; Rehm, A.; Droese, J.; Oppermann, M.; Lipp, M.; Hopken, U.E. Surface expression and endocytosis of the human cytomegalovirus-encoded chemokine receptor US28 is regulated by agonist-independent phosphorylation. J. Biol. Chem. 2002, 277, 45122-45128.

17. Billstrom, M.A.; Lehman, L.A.; Scott, W.G. Depletion of extracellular RANTES during human cytomegalovirus infection of endothelial cells. Am. J. Respir. Cell Mol. Biol. 1999, 21, 163-167.

18. Randolph-Habecker, J.R.; Rahill, B.; Torok-Storb, B.; Vieira, J.; Kolattukudy, P.E.; Rovin, B.H.; Sedmak, D.D. The expression of the cytomegalovirus chemokine receptor homolog US28 sequesters biologically active CC chemokines and alters IL-8 production. Cytokine 2002, 19, $37-46$.

19. Vieira, J.; Schall, T.J.; Corey, L.; Geballe, A.P. Functional analysis of the human cytomegalovirus US28 gene by insertion mutagenesis with the green fluorescent protein gene. J. Virol. 1998, 72, 8158-8165. 
20. Minisini, R.; Tulone, C.; Luske, A.; Michel, D.; Mertens, T.; Gierschik, P.; Moepps, B. Constitutive inositol phosphate formation in cytomegalovirus-infected human fibroblasts is due to expression of the chemokine receptor homologue pUS28. J. Virol. 2003, 77, 4489-4501.

21. Vischer, H.F.; Leurs, R.; Smit, M.J. HCMV-encoded G-protein-coupled receptors as constitutively active modulators of cellular signaling networks. Trends Pharmacol. Sci. 2006, $27,56-63$.

22. McLean, K.A.; Holst, P.J.; Martini, L.; Schwartz, T.W.; Rosenkilde, M.M. Similar activation of signal transduction pathways by the herpesvirus-encoded chemokine receptors US28 and ORF74. Virology 2004, 325, 241-251.

23. Farrell, H.E.; Abraham, A.M.; Cardin, R.D.; Sparre-Ulrich, A.H.; Rosenkilde, M.M.; Spiess, K.; Jensen, T.H.; Kledal, T.N.; vis-Poynter, N. Partial functional complementation between human and mouse cytomegalovirus chemokine receptor homologues. J. Virol. 2011, 85, 6091-6095.

24. Farrell, H.E.; Abraham, A.M.; Cardin, R.D.; Molleskov-Jensen, A.S.; Rosenkilde, M.M.; vis-Poynter, N. Identification of common mechanisms by which human and mouse cytomegalovirus seven-transmembrane receptor homologues contribute to in vivo phenotypes in a mouse model. J. Virol. 2013, 87, 4112-4117.

25. Gruijthuijsen, Y.K.; Casarosa, P.; Kaptein, S.J.; Broers, J.L.; Leurs, R.; Bruggeman, C.A.; Smit, M.J.; Vink, C. The rat cytomegalovirus R33-encoded G protein-coupled receptor signals in a constitutive fashion. J. Virol. 2002, 76, 1328-1338.

26. Casarosa, P.; Gruijthuijsen, Y.K.; Michel, D.; Beisser, P.S.; Holl, J.; Fitzsimons, C.P.; Verzijl, D.; Bruggeman, C.A.; Mertens, T.; Leurs, R.; et al. Constitutive signaling of the human cytomegalovirus- encoded receptor UL33 differs from that of its rat cytomegalovirus homolog $\mathrm{R} 33$ by promiscuous activation of $\mathrm{G}$ proteins of the $\mathrm{Gq}, \mathrm{Gi}$, and Gs classes. J. Biol. Chem. 2003, 278, 50010-50023.

27. Beisser, P.S.; Vink, C.; van Dam, J.G.; Grauls, G.; Vanherle, S.J.; Bruggeman, C.A. The R33 $\mathrm{G}$ protein-coupled receptor gene of rat cytomegalovirus plays an essential role in the pathogenesis of viral infection. J. Virol. 1998, 72, 2352-2363.

28. Case, R.; Sharp, E.; ned-Jensen, T.; Rosenkilde, M.M.; vis-Poynter, N.; Farrell, H.E. Functional analysis of the murine cytomegalovirus chemokine receptor homologue M33: Ablation of constitutive signaling is associated with an attenuated phenotype in vivo. J. Virol. 2008, 82, 1884-1898.

29. Stapleton, L.K.; Arnolds, K.L.; Lares, A.P.; Devito, T.M.; Spencer, J.V. Receptor chimeras demonstrate that the $C$-terminal domain of the human cytomegalovirus US27 gene product is necessary and sufficient for intracellular receptor localization. Virol. J. 2012, 9, doi:10.1186/1743-422X-9-42.

30. Fraile-Ramos, A.; Pelchen-Matthews, A.; Kledal, T.N.; Browne, H.; Schwartz, T.W.; Marsh, M. Localization of HCMV UL33 and US27 in endocytic compartments and viral membranes. Traffic 2002, 3, 218-232.

31. Margulies, B.J.; Gibson, W. The chemokine receptor homologue encoded by US27 of human cytomegalovirus is heavily glycosylated and is present in infected human foreskin fibroblasts and enveloped virus particles. Virus Res. 2007, 123, 57-71. 
32. O'Connor, C.M.; Shenk, T. Human cytomegalovirus pUS27 G protein-coupled receptor homologue is required for efficient spread by the extracellular route but not for direct cell-to-cell spread. J. Virol. 2011, 85, 3700-3707.

33. O'Connor, C.M.; Shenk, T. Human cytomegalovirus pUL78 G protein-coupled receptor homologue is required for timely cell entry in epithelial cells but not fibroblasts. J. Virol. 2012, $86,11425-11433$.

34. Kaptein, S.J.; Beisser, P.S.; Gruijthuijsen, Y.K.; Savelkouls, K.G.; van Cleef, K.W.; Beuken, E.; Grauls, G.E.; Bruggeman, C.A.; Vink, C. The rat cytomegalovirus R78 G protein-coupled receptor gene is required for production of infectious virus in the spleen. J. Gen. Virol. 2003, $84,2517-2530$.

35. Oliveira, S.A.; Shenk, T.E. Murine cytomegalovirus M78 protein, a G protein-coupled receptor homologue, is a constituent of the virion and facilitates accumulation of immediate-early viral mRNA. Proc. Natl. Acad. Sci. USA 2001, 98, 3237-3242.

36. Wagner, S.; Arnold, F.; Wu, Z.; Schubert, A.; Walliser, C.; Tadagaki, K.; Jockers, R.; Mertens, T.; Michel, D. The 7-transmembrane protein homologue UL78 of the human cytomegalovirus forms oligomers and traffics between the plasma membrane and different intracellular compartments. Arch. Virol. 2012, 157, 935-949.

37. Tschische, P.; Tadagaki, K.; Kamal, M.; Jockers, R.; Waldhoer, M. Heteromerization of human cytomegalovirus encoded chemokine receptors. Biochem. Pharmacol. 2011, 82, 610-619.

38. Tischer, B.K.; von, E.J.; Kaufer, B.; Osterrieder, N. Two-step red-mediated recombination for versatile high-efficiency markerless DNA manipulation in Escherichia coli. Biotechniques 2006, 40, 191-197.

39. Sinzger, C.; Hahn, G.; Digel, M.; Katona, R.; Sampaio, K.L.; Messerle, M.; Hengel, H.; Koszinowski, U.; Brune, W.; Adler, B. Cloning and sequencing of a highly productive, endotheliotropic virus strain derived from human cytomegalovirus TB40/E. J. Gen. Virol. 2008, 89, 359-368.

40. Resnik, K.S.; DiLeonardo, M.; Maillet, M. Histopathologic findings in cutaneous cytomegalovirus infection. Am. J. Dermatopathol. 2000, 22, 397-407.

41. Das, S.; Vasanji, A.; Pellett, P.E. Three-dimensional structure of the human cytomegalovirus cytoplasmic virion assembly complex includes a reoriented secretory apparatus. J. Virol. 2007, 81, 11861-11869.

42. Das, S.; Pellett, P.E. Spatial relationships between markers for secretory and endosomal machinery in human cytomegalovirus-infected cells versus those in uninfected cells. J. Virol. 2011, 85, 5864-5879.

43. Cepeda, V.; Esteban, M.; Fraile-Ramos, A. Human cytomegalovirus final envelopment on membranes containing both trans-Golgi network and endosomal markers. Cell Microbiol. 2010, 12, 386-404.

44. Sinzger, C.; Digel, M.; Jahn, G. Cytomegalovirus cell tropism. Curr. Top. Microbiol. Immunol. 2008, 325, 63-83.

45. Sharp, E.L.; vis-Poynter, N.J.; Farrell, H.E. Analysis of the subcellular trafficking properties of murine cytomegalovirus M78, a 7 transmembrane receptor homologue. J. Gen. Virol. 2009, $90,59-68$. 
46. Huotari, J.; Helenius, A. Endosome maturation. EMBO J. 2011, 30, 3481-3500.

47. Hanyaloglu, A.C.; Von, Z.M. Regulation of GPCRs by endocytic membrane trafficking and its potential implications. Annu. Rev. Pharmacol. Toxicol. 2008, 48, 537-568.

48. Michel, D.; Milotic, I.; Wagner, M.; Vaida, B.; Holl, J.; Ansorge, R.; Mertens, T. The human cytomegalovirus UL78 gene is highly conserved among clinical isolates, but is dispensable for replication in fibroblasts and a renal artery organ-culture system. J. Gen. Virol. 2005, 86, 297-306.

49. Taguchi, T. Emerging roles of recycling endosomes. J. Biochem. 2013, 153, 505-510.

50. Cheng, S.B.; Filardo, E.J. trans-Golgi Network (TGN) as a regulatory node for beta1-adrenergic receptor (beta1AR) down-modulation and recycling. J. Biol. Chem. 2012, 287, 14178-14191.

51. Terrillon, S.; Durroux, T.; Mouillac, B.; Breit, A.; Ayoub, M.A.; Taulan, M.; Jockers, C.; Barberis, R.; Bouvier, M. Oxytocin and vasopressin V1a and V2 receptors form constitutive homo- and heterodimers during biosynthesis. Mol. Endocrinol. 2003, 17, 677-691.

52. Tadagaki, K.; Tudor, D.; Gbahou, F.; Tschische, P.; Waldhoer, M.; Bomsel, M.; Jockers, R.; Kamal, M. Human cytomegalovirus-encoded UL33 and UL78 heteromerize with host CCR5 and CXCR4 impairing their HIV coreceptor activity. Blood 2012, 119, 4908-4918.

53. Zoncu, R.; Perera, R.M.; Balkin, D.M.; Pirruccello, M.; Toomre, D.; De, C.P. A phosphoinositide switch controls the maturation and signaling properties of APPL endosomes. Cell 2009, 136, 1110-1121.

54. Adobe Photoshop CS2; Adobe Systems Incorporated: San Jose, CA, USA, 2003.

55. Tischer, B.K.; Smith, G.A.; Osterrieder, N. En passant mutagenesis: A two step markerless red recombination system Methods. Mol. Biol. 2010, 634, 421-430.

56. ABI PRISM ${ }^{\circledR} 7500$ Sequence Detection System, SDS 1.4 Software Package; Applied Biosystems: Foster City, CA, USA, 2001.

57. Andreoni, M.; Faircloth, M.; Vugler, L.; Britt, W.J. A rapid microneutralization assay for the measurement of neutralizing antibody reactive with human cytomegalovirus. J. Virol. Methods 1989, 23, 157-167. 
Reprinted from Viruses. Cite as: Smith, R.M.; Kosuri, S.; Kerry, J.A. Role of Human Cytomegalovirus Tegument Proteins in Virion Assembly. Viruses 2014, 6, 582-605.

Review

\title{
Role of Human Cytomegalovirus Tegument Proteins in Virion Assembly
}

\author{
Rebecca Marie Smith, Srivenkat Kosuri and Julie Anne Kerry *
}

Department of Microbiology and Molecular Cell Biology, Eastern Virginia Medical School, Norfolk, VA 23501, USA; E-Mails: beckstermw@yahoo.com (R.S.); kosurisr@evms.edu (S.K.)

* Author to whom correspondence should be addressed; E-Mail: kerryja@evms.edu; Tel.: +1-757-446-5663; Fax: +1-757-624-2255.

Received: 2 January 2014; in revised form: 4 February 2014 / Accepted: 4 February 2014 / Published: 6 February 2014

\begin{abstract}
Like other herpesviruses, human cytomegalovirus (HCMV) contains a unique proteinaceous layer between the virion envelope and capsid, termed the tegument. Upon infection, the contents of the tegument layer are delivered to the host cell, along with the capsid and the viral genome, where they facilitate the initial stages of virus replication. The tegument proteins also play important roles in virion assembly and this dual nature makes them attractive potential targets for antiviral therapies. While our knowledge regarding tegument protein function during the initiation of infection has been the subject of intense study, their roles in assembly are much less well understood. In this review, we will focus on recent studies that highlight the functions of HCMV tegument proteins during assembly, and pose key questions for further investigation.
\end{abstract}

Keywords: human cytomegalovirus; tegument; assembly

\section{Introduction}

Despite being near ubiquitous in the population [1], overt human cytomegalovirus (HCMV) disease in adults is typically restricted to the immunocompromised [2,3]. Even in the era of HAART, HCMV is associated with increased risk of progression to AIDS and immune reconstitution inflammatory syndrome in HIV-infected individuals [4]. HCMV also causes significant disease and mortality in transplant recipients [3]. Such patients frequently undergo prophylactic therapy to reduce HCMV-associated complications, leading to viral resistance and 
development of late-onset HCMV disease [5,6]. HCMV is also the most common intrauterine infection in the U.S., with outcomes ranging from death to neurological impairment, sensorineural hearing loss, seizure disorder, cerebral palsy and chorioretinitis [7,8]. Significantly, permanent disability due to congenital HCMV infection is more common than other well-known conditions such as spina bifida and Down's syndrome [9]. Currently available treatments for HCMV infection target the viral DNA replication machinery, and can be quite effective at diminishing the impact of HCMV disease [10]. However, strains resistant to these drugs are emerging at a rate of 5\%-10\% depending on the underlying condition and type of treatment [10], prompting the need to develop new antiviral strategies. The tegument proteins of HCMV have been proposed as potential therapeutic targets due to their key functions in the initiation of infection, virion assembly and particle stability $[11,12]$. While we know a considerable amount regarding the functions of the tegument proteins during the initial phases of virus replication $[11,13]$, our understanding of the specific role of the HCMV tegument proteins in virus assembly lags behind. In this review, we will focus on the functions of tegument proteins in the assembly, egress and stability of virus particles.

\section{Overview of Replication and Assembly}

Replication of HCMV begins with virus binding to cell surface receptors and fusion of the viral envelope with the plasma membrane [14]. At that time, the viral capsid is released into the infected cell along with the components of the virion tegument, a layer of proteins located between the viral envelope and the capsid. The tegument consists of approximately 38 different viral proteins that are released into the host cell upon infection where they play critical roles during the initiation of virus replication [11,12]. The HCMV tegument also contains a number of cellular proteins [15] and mRNA's [16], although it is unclear at this point if these play any role in virus infection, or if they are simply bystanders that are nonspecifically incorporated during the assembly process. During lytic replication, the tegument proteins function to enhance the efficiency of viral immediate early gene expression $[11,12]$. The immediate early proteins are essential for progression through the early phase of gene expression [17,18], which is followed by DNA replication, the late phase of viral gene expression [19] and virion assembly [20].

HCMV assembly occurs in two phases; first, the capsid is formed within the nucleus and the DNA genome is encapsidated [20]. It is likely that at this stage, the initial components of the tegument are added to form the inner tegument, an organized net-like layer that encloses the capsid shell [21]. The capsids then bud through the nuclear envelope and final assembly, including the addition of the bulk of the tegument, occurs in a unique structure consisting of redistributed components of the cellular secretory apparatus known as the assembly complex (AC) [20,22-24]. The majority of the tegument lacks a defined structure [21], and as mentioned above, incorporates a number of cellular proteins and mRNA's $[15,16]$, suggesting that final tegumentation is a relatively nonspecific process. However, there is evidence pointing to a degree of specificity for at least some of the viral tegument components. For example, while three isoforms of the pUL69 protein can be detected in infected cells, only one specific isoform of pUL69 is incorporated into the tegument [25]. Likewise, it has been reported that a hypophosphorylated form of the pp28 tegument protein (product of the UL99 gene) is specifically incorporated into virions [26]. Another possibility to account for the difference in protein isoforms located within the virion versus those found in 
infected cells is modification within the tegument. In that regard, viral and cellular kinases are known tegument components [27,28], and the cellular phosphatases PP1 and PP2A can also be detected in the virion [29]. This raises the possibility that fine tuning of tegument protein post-translational modifications can occur within the virion and have the potential to modulate virus infection. It should be mentioned here that the majority of the tegument proteins can be phosphorylated and the abbreviation "pp" denotes this fact.

Additional studies support the notion that the HCMV tegument is added in an ordered step-wise fashion. For example, there is an abundance of evidence that addition of the pp150 tegument protein, encoded by the UL32 gene, initiates in the nucleus [30,31], and is the primary component of the structured tegument network surrounding the capsid [21]. While the pp150 network clearly plays a role in capsid stability [32,33], it may also provide a structural framework for the addition of subsequent tegument components. Consistent with this, pp150 has been shown by mass spectrometry analysis to interact with a number of capsid and tegument proteins, including the pp71 protein encoded by the UL82 gene [34]. This is of particular note in that pp71, along with the pp65 tegument protein encoded by the $U L 83$ gene, has a relatively tight association with the capsid, suggesting that it may form a secondary, albeit less organized, layer of tegument [35]. Recent studies utilizing yeast two-hybrid analysis have revealed interaction networks centered on a number of hub proteins that may contribute to an ordered tegumentation process [36,37]. For example, the pUL45 tegument protein forms a network hub via interaction with itself, as well as the pUL25, pp150, pUL48, and ppUL69 tegument proteins [36]. Although the precise function of pUL45 is unknown, deletion of this gene causes a defect in plaque formation [38]. A more extensive yeast two-hybrid analysis revealed interactions amongst a number of tegument, capsid and envelop proteins, and also identified the pUL24, pUL25 and pUL89 proteins as network hubs [37]. Intriguingly, both studies showed that a number of the tegument proteins were capable of self-association [36,37]. Such extensive networks of cross-interactions and self-interactions are likely to be critical for efficient tegumentation during the assembly process. Perhaps surprisingly, other than pUL45, most of these hub proteins are not essential for efficient virus replication [39]. A generally accepted concept that accounts for this apparent discrepancy is that multiple overlapping interactions and functions ensures that efficient tegumentation and subsequent infectivity is less dependent on any one tegument protein [20,40].

While it is clear that the majority of the tegument proteins become associated with the virion particle in cytoplasmic assembly complexes [20,40], a number of these proteins contain nuclear localization signals and transit to the nucleus at the initial stages of virus infection $[11,12,41-43]$. Thus, the tegument proteins can be considered as belonging to three major classes; the inner tegument consisting of pp150, which likely initiates tegument incorporation in the nucleus [30,31] and pUL48 [21]; nuclear tegument proteins that initially transit to the nucleus of permissive cells and are later relocalized to the cytoplasmic AC, including pp71, pp65, pTRS1, pUL26, pUL35 and the ppUL97 viral kinase $[11,12,44]$; and the cytoplasmic tegument proteins that retain this localization throughout the course of virus infection, including pp28, pUL71 and pUL25 [45-47]. One of the key questions regarding tegumentation that remains unanswered is whether the nuclear tegument proteins associate with the capsid within the nucleus. Direct evidence for the association of the nuclear tegument proteins with capsids within the nucleus is lacking, although this possibility 
cannot be definitively ruled out [20,40]. If the nuclear tegument proteins do not associate with capsids in the nucleus, how are they redirected to the cytoplasmic AC at the late stages of infection? For some of these proteins, including pp65, pUL94 and ppUL69, there is evidence for shuttling activity between the nucleus and the cytoplasm [48-50]. Thus, the relocalization of these proteins to the cytoplasm could result from modulation of the rate of nuclear import and/or export. For the pp65 protein, phosphorylation clearly plays a role in controlling this process, as inhibition of cellular cyclin dependent kinase (CDK) activity prevents the accumulation of pp65 in the cytoplasm at the late stages of virus infection [51]. In addition, the viral ppUL97 kinase affects the distribution of pp65, with inhibition of ppUL97 resulting in large pp65-containing aggregates [52,53]. However, the formation of these aggregates is more consistent with a role for ppUL97 is regulating the self-association of pp65, rather than a direct effect on trafficking, per se. With other nuclear tegument proteins, domains have been identified that can directly target the proteins to the cytoplasmic AC $[43,54]$. For example, in transfected cells, the pp71 tegument protein colocalizes with markers of the late-trans Golgi Network and early endosomal compartment if the nuclear localization signal is mutated [43]. However, the precise mechanism of this switch in trafficking during the process of virus infection has yet to be identified. Thus, significant gaps in our understanding of HCMV tegumentation remain. Despite this, continued research into the specific functions of the tegument proteins promises new insights into this process, and may yet reveal novel antiviral targets amongst this key class of viral proteins. In the remainder of this review, we will highlight the specific functions of components of the HCMV tegument during virion assembly (Table 1), with a focus on the most recent data.

Table 1. Tegument proteins discussed in detail in this review.

\begin{tabular}{|c|c|c|}
\hline $\begin{array}{l}\text { Gene } \\
\text { Designation }\end{array}$ & $\begin{array}{l}\text { Relative } \\
\text { Abundance a }\end{array}$ & Phenotype ${ }^{b}$ \\
\hline \multicolumn{3}{|c|}{ Inner Tegument } \\
\hline$U L 32(\mathrm{pp} 150)$ & $9.1 \%$ & Essential $[39,55]$ \\
\hline$U L 48$ & $12.6 \%$ & Essential [39]; Severe growth defect ( $>10^{4}$ fold drop in titer) [55] \\
\hline \multicolumn{3}{|c|}{ Nuclear Tegument } \\
\hline$U L 26$ & $0.1 \%$ & Non-essential, Small plaque phenotype with severe growth defect $[39,55]$ \\
\hline UL35 & $0.5 \%$ & $\begin{array}{l}\text { Moderate growth defect }(1,000 \text {-fold decrease in titer }) \text {, Essential at low } \\
\text { multiplicity [56] }\end{array}$ \\
\hline$U L 82(\mathrm{pp} 71)$ & $8.9 \%$ & Non-essential, Severe growth defect at low multiplicity $[57,58]$ \\
\hline$U L 83(\mathrm{pp} 65)$ & $15.4 \%$ & $\begin{array}{l}\text { Non-essential [59], Important for replication in macrophages [60], Slight } \\
\text { growth defect (10-fold) at low multiplicity in fibroblasts [61] }\end{array}$ \\
\hline UL94 & $1.2 \%$ & Essential $[39,62]$ \\
\hline UL97 & $0.1 \%$ & Non-essential, Severe growth defect [63] \\
\hline TRS1 & $0.6 \%$ & Non-essential, Moderate growth defect ( $\sim 200$-fold decrease in titer) [64] \\
\hline \multicolumn{3}{|c|}{ Cytoplasmic Tegument } \\
\hline$U L 71$ & $0.1 \%$ & Essential [39] \\
\hline$U L 99(\mathrm{pp} 28)$ & Unknown & Essential $[39,55,65]$ \\
\hline
\end{tabular}




\section{The Inner Tegument Proteins}

\section{1. pp150 (ppUL32)}

The product of the HCMV UL32 gene, pp150, is the primary component of the inner tegument and forms a filamentous net-like structure that surrounds the capsid [21]. The inner tegument can be distinguished from the outer tegument proteins by virtue of this relatively organized structure, as well as its tight association with the capsid [66]. Recent studies have revealed that pp150 can be co-purified from cells at the late stage of infection in association with capsid components including the major capsid protein (MCP, product of the UL86 gene), pUL46, pUL85, and pUL80.5 [34]. However, co-purification of proteins at this time point probably represents a more general capsid association as opposed to a direct interaction with any one specific component. Additional studies using cryo electron microscopy (cryo-EM) reveal that the smallest capsid protein, pUL48.5, likely makes direct contact with the inner tegument [67]. These studies also determined that the inner tegument is composed of an upper and lower helical bundle joined by a long central helix, with features of the helices consistent with the predicted structure of pp150. Moreover, reduced expression of pUL48.5 mediated by ribozyme inhibition significantly reduced viral yield and resulted in the accumulation of non-infectious enveloped particles (NIEP's) that lack viral DNA [67]. However, this latter finding is distinct from what has been observed by either deletion or knockdown of pp150 directly [32,33]. In these studies, the number of A- (empty), B- (contain scaffold proteins) and C- (DNA filled) capsids within the nuclei of infected cells are comparable in the presence or absence of pp150 [32,33]. In contrast, very few DNA containing capsids or enveloped viral particles could be identified in the cytoplasm in the absence of pp150, suggesting that this protein stabilizes DNA-containing capsids as they progress from the nucleus to the cytoplasmic AC. The discrepancy between the effects of the pUL48.5 and pp150 viral mutants may result from the approaches employed e.g., cryo-EM of extracellular particles [67] versus transmission EM of particles within infected cells [32,33]. However, it is also possible that pUL48.5 has additional functions in virion assembly independent of the interaction with the pp 150 inner tegument protein [67]. What is clear is that the pp150 protein is critical for the efficient formation of enveloped capsids within infected cells and that the mechanism is likely related to effects on capsid stability during post-nuclear events.

Another key question is the cellular site of inner tegument addition. The preponderance of current evidence supports an initial nuclear localization of pp150 derived from the tegument [30,31], perhaps due to the tight association with the capsid [66]. Indeed, this tight association was recently shown to facilitate a novel cis-mode of regulation during HCMV infection [68]. In this study, tegument-derived pp150 was found to be phosphorylated by a cyclin A2-dependent mechanism resulting in restriction of HCMV replication to the G0/G1 phase of the cell cycle. Mutation of the cyclin A2-dependent phosphorylation site blocked the cell cycle restriction effects, but in a genome-specific manner, suggesting that the tight association of pp150 with at least some capsid components is retained during the initial stages of lytic replication. Newly synthesized pp 150 protein appears to be both nuclear and cytoplasmic [34,69], but evidence also suggests that tegument addition begins in the nucleus, as pp150 can be found associated with nuclear B-capsids [31]. This finding would be consistent with formation of an initial inner tegument framework in the nucleus. 
However, it is also clear that as infection progresses, the distribution of pp150 alters to become predominantly cytoplasmic and associated with the AC [30,69,70]. Whether this reflects the accumulation of capsids within the $\mathrm{AC}$ during envelopment, or an independent redistribution of pp150 to facilitate completion of the inner tegument remains an unanswered question.

Another possible function for pp150 is the targeting of capsids to the site of virus assembly. Of note is that pp150 interacts with Bicaudal D1 [71], a protein involved in microtubule dependent cargo transport [72], consistent with an association with the AC [22-24]. Knockdown of Bicaudal D1 or inhibition of Rab6, a Bicaudal D1-interacting protein [73], resulted in decreased localization of pp150 to the $\mathrm{AC}$, and reduced virus replication, although a direct effect on capsid recruitment to the AC was not examined [71,74]. Mass spectrometry analysis also identified clathrin components as pp150 interacting proteins, although a direct role for this interaction in virion assembly or localization to the AC could not be demonstrated [34]. Thus the possibility that pp 150 association with the capsid could participate in the recruitment of capsids to the AC still needs to be addressed experimentally. Finally, a role for the inner tegument as a scaffold that nucleates addition of the remaining tegument makes an attractive, but unproven, model. It is known that pp150 can interact with a number of tegument proteins including pUL48, pTRS1, ppUL69, ppUL97, and pp71 [34], although this may represent association with overlapping tegument interaction networks. Yeast two-hybrid analysis demonstrates direct evidence for pp150 interactions with pUL35, pUL45 and pp71 [36,37], and as already noted pp71 is thought to have a relatively tight association with capsids [35], consistent with pp150 nucleating additional tegument components. Further evidence for a role for pp150 as a tegument scaffold comes from a study examining the pUL96 tegument protein [31]. In this analysis, pUL96 was found to be added to capsids exclusively within the cytoplasm. However, mutation of pUL96 resulted in a similar defect in virus replication to that observed in the pp150 mutant viruses, with an apparent effect on the stability of capsids after or during transition from the nucleus. This data is consistent with the initial association of pp 150 with capsids in the nucleus, and perhaps stabilization of the inner tegument network upon association with pUL96 in the cytoplasm, resulting in nucleocapsids capable of association with additional tegument and finally envelopment in the AC before virus egress from the cell.

\section{2. $p U L 48$}

The protein product of the UL48 gene, pUL48, is a large tegument protein that is tightly associated with the capsid [75], is a component of the inner tegument [21], and localizes to both the nucleus and cytoplasm of infected cells [76]. The best characterized function of the pUL48 is in viral entry where it interacts with pUL47, pUL69 and the pUL86 major capsid protein and is thought to assist in release of the viral genome from the capsid during the initial stages of infection [77]. Like its homologs in other herpesviruses, the HCMV pUL48 has deubiquitinase activity, and mutation of the active site results in a 10 -fold reduction in virus yield [78,79]. However, a specific role for the deubiquitinase activity in virus assembly has not yet been shown. In a more recent study, the nuclear localization signal of pUL48 was mapped, and disruption of nuclear trafficking resulted in a small plaque phenotype [76]. While the nuclear localization could clearly be involved in the established role for pUL48 in virus entry, it also raises an intriguing possibility that this localization could contribute to the initial stages of tegumentation along with pp150. The pUL48 
protein has also been shown to interact with the cellular p180 ER membrane-associated protein [80], and thus could play a role in directing capsids to the cytoplasmic AC. Consistent with this, deletion of the herpes simplex virus (HSV) homolog (pUL36 or VP1/2) results in the accumulation of cytoplasmic unenveloped capsids [81]. Detailed analysis of the HSV pUL36 protein during assembly shows that the protein is added to cytosolic capsids and functions to link the inner and outer tegument $[82,83]$. It remains to be seen whether the HCMV pUL48 protein functions in a similar manner.

\section{The Nuclear Tegument Proteins}

It has been well characterized that a number of HCMV tegument proteins, including pp71 and pp65 (products of the UL82 and UL83 genes, respectively), initially localize to the nucleus of permissively infected cells $[11,12,84,85]$. Of note, both pp71 and pp65 contain nuclear localization signals that can independently target the proteins to the nucleus $[41,43]$. The nuclear trafficking of these proteins during the initial stages of virus infection is associated with their role in enhancing the efficiency of replication and viral gene expression [11]. For example, the pp71 protein has been shown to enhance the transcription of the major immediate early gene region via degradation or relocalization of transcriptional repressors such as Daxx and ATRX [13]. In a similar vein, the pp65 protein was recently shown to enhance activation of the major immediate early promoter via its interaction with the IFI16 protein [61]. It has also been observed that these initially nuclear tegument proteins relocalized to the $\mathrm{AC}$ at the late stages of virus replication. While the precise mechanisms of redistribution remain unclear, as noted above phosphorylation does appear to play a role in some cases $[43,51,53]$. What is perhaps surprising is that a number of these tegument proteins, including pp65 and pp71, are not essential for virus replication [12], emphasizing the apparent redundancy in tegument protein function [20,40]. Despite this, some of the nuclear tegument proteins have been shown to play important roles in virion assembly and stability.

\section{1. $p U L 26$}

The pUL26 protein follows the standard pattern of nuclear tegument protein localization, with an initial nuclear distribution, followed by a perinuclear localization consistent with the AC [44]. When infections with a virus lacking pUL26 were carried out at low multiplicity, expression of the viral immediate early protein levels were reduced relative to the wild type virus, leading to a corresponding decrease in the efficiency of viral DNA replication. Of particular note with respect to assembly, this study showed that the levels of the pp28 and pp65 tegument proteins were diminished within the newly infected cells, although virion levels of both proteins were similar to wild type virus [44]. This finding suggested that the proteins in the mutant virus were somehow less stable, and may correspond to an altered hypophosphorylated form of pp28 being preferentially incorporated into the particles. In a separate study of a pUL26 deletion virus, the authors noted a decrease in particle stability [86]. Specifically, the wild type and revertant viruses were relatively stable ( $\sim 50 \%$ infectivity) for at least two days at 20 degrees, whereas the pUL26 deletion virus retained only $5 \%$ infectivity over the same time period. Electron microscopy revealed nonenveloped extracellular particles derived from cells infected with the pUL26 deletion virus, 
suggesting that the decrease in stability resulted from a loss of the viral envelope due to defective tegumentation. Such an interpretation would be consistent with a role for the tegument analogous to the matrix proteins of RNA viruses in which the tegument forms a critical link between the capsid and the viral envelope [87].

The absence of pUL26 also resulted in the accumulation of immature particles in the cytoplasm associated with electron dense areas, possibly representing aggregates of tegument proteins [86]. This finding is consistent with other data suggesting that disruption of tegumentation leads to nonspecific aggregation of the tegument proteins $[53,56]$, and implies that the interactions amongst the tegument proteins must be tightly regulated. The deletion of the UL26 gene also resulted in increased levels of pp71 in the virion particles [86]. As there is no direct evidence for interaction between pUL26 and pp71, the authors speculate that pUL26 and pp71 may compete for binding to the same capsid protein, and without pUL26, more pp71 becomes incorporated into the virion, perhaps as a compensatory mechanism. This may be one mechanism that accounts for the apparent redundancy of a number of the tegument proteins $[20,40]$.

\section{2. $p U L 35$}

The UL35 gene codes for two isoforms that differ in size; the full-length protein that is expressed at the early stages of infection, and pUL35a consisting of the carboxy-terminal region that is expressed with late kinetics [88]. Both isoforms can interact with the pp71 tegument protein, although there are conflicting reports as to the effect of this interaction on viral gene expression $[88,89]$. Recent studies suggest a role for both pUL35 and pUL35a in controlling ND10 reorganization [90], known to be important for viral replication and gene expression [91]. In addition, pUL35 can interact with and regulate the cellular DNA repair machinery [92]. Deletion of the UL35 gene from HCMV results in decreased numbers of both enveloped viral particles and dense bodies (subviral particles consisting primarily of tegument surrounded by the viral envelope [93]) in the cytoplasm under conditions where viral gene expression is unaffected, suggesting an additional defect in virus assembly [56]. The mechanism of the defect is likely related to the persistence of the pp65 and pp71 proteins in the nucleus, where they form large electron dense accumulations [56], suggesting that the lack of $U L 35$ proteins disrupts the relocalization of these proteins to the AC. Further analysis showed that the smaller isoform, pUL35a, was largely responsible for this effect, as co-transfection of pUL35a with pp71 results in relocalization of both proteins from the nucleus to the cytoplasm [90]. Interestingly, the two proteins colocalized in the cytoplasm in distinct punctate perinuclear structures, similar to those observed when pp71 nuclear localization is abrogated by a specific mutation within the pp71 nuclear localization signal [43]. Together, these findings suggest that pUL35a disrupts recognition of the pp71 non-classical NLS, either through a conformational change or altered phosphorylation, resulting in the nascent pp71 AC localization predominating [43]. It is less clear how deletion of the UL35 gene alters pp65 redistribution, as there was no evidence of a direct interaction between these two proteins in the yeast two-hybrid assays [36,37]. However, recent studies in our laboratory show that affinity purification of the pp71 protein from cells at the late stages of infection results in the co-purification of a number of viral proteins, including pp65 (Table 2). These findings suggest that pp71 and pp65 may exist as a complex, and pUL35a could redistribute both proteins to the 
cytoplasm at the later stages of virus infection [88]. Further, the phenotype of the UL35 gene deletion virus is consistent with sustained retention of the nuclear tegument proteins within the nucleus decreasing the efficiency of virion morphogenesis in the cytoplasm.

Table 2. Viral proteins that co-purify with pp71 ${ }^{\text {a }}$.

\begin{tabular}{lllll}
\hline $\begin{array}{l}\text { Protein } \\
\text { Name }\end{array}$ & Protein Function & $\begin{array}{l}\text { \# of Peptides } \\
\text { Matched }\end{array}$ & $\begin{array}{l}\text { Ion Score }^{\text {b }} \\
(-\mathbf{1 0 L o g}(\mathbf{P}))\end{array}$ & $\begin{array}{l}\text { Sequence } \\
\text { Coverage }\end{array}$ \\
\hline pTRS1 & Transcriptional Regulation/Immune Evasion [12] & 78 & 2439 & $44 \%$ \\
pIRS1 & Transcriptional Regulation/Immune Evasion [12] & 60 & 1809 & $41 \%$ \\
pUL44 & DNA Processivity Factor [94] & 66 & 2413 & $82 \%$ \\
pUL86 & Major Capsid Protein [95] & 5 & 339 & $8 \%$ \\
ppUL97 & Viral Kinase [96] & 5 & 272 & $8 \%$ \\
pp65 & Tegument protein [12] & 236 & 2580 & $71 \%$ \\
pUL50 & Nuclear Egress [97] & 9 & 276 & $26 \%$ \\
pUL52 & Genome Cleavage and Packaging [98] & 7 & 256 & $14 \%$ \\
pUL56 & Terminase Subunit [99,100] & 11 & 385 & $26 \%$ \\
pUL88 & Tegument Protein [100] & 7 & 135 & $10 \%$ \\
pUL35 & Tegument Protein [12] & 6 & 289 & $29 \%$ \\
\hline
\end{tabular}

${ }^{a}$ Proteins were purified using S-protein agarose (Novagen) from cells infected at 72 hpi with a virus expressing a S-tagged version of pp71 [101]. The resultant proteins were subjected to SDS-PAGE using a $4 \%-20 \%$ gel (Jule Biotechnologies, Inc., Milford, CT, USA). Protein bands were visualized by Coomassie Blue staining, excised and subjected to trypsin digestion prior to analysis on an $\mathrm{LTQ}^{\mathrm{TM}}$ Linear Ion Trap tandem Mass Spectrometer (ThermoFinnigan, San Jose, CA, USA). Protein searches were performed using MASCOT ${ }^{\mathrm{TM}}$. Only proteins identified in multiple experiments are included. ${ }^{\mathrm{b}}$ The ion score is based on the calculated probability, $\mathrm{P}$, and provides a measure of the likelihood that the predicted peptide matches the indicated protein (Matrix Science, Boston, MA, USA). For the study shown, a score of $>36$ indicated identity or extensive homology $(p<0.05)$.

\section{3. $p p 71$ ( $p p U L 82)$}

The pp71 tegument protein, encoded by the HCMV UL82 gene, plays a number of roles during the initiation of virus replication, including the degradation and/or inactivation of cellular proteins that would repress viral gene expression [13]. The majority of research on pp71 has focused on its functions during the establishment of infection, and it is unclear what role if any it may play during virus assembly. However, some studies are suggestive of a contribution. For example, as noted above delayed relocalization of the pp71 protein, along with pp65, to the cytoplasm due to the deletion of the UL35 gene results in a decrease in the efficiency of secondary envelopment [56]. A similar effect was observed upon deletion of the TRS1 gene [64]. Other evidence that points to a role in virion assembly is the possibility that pp71 interacts with a number of key virion components including pp150, pUL94, pUL35, pIRS1/TRS1 and the major capsid protein (Table 2) [36,37]. Despite these findings, a definitive role for pp71 in assembly remains to be demonstrated.

However, analysis of pp71 trafficking and post-translational modifications have revealed insight into the mechanisms involved in nuclear tegument protein trafficking and redistribution [43]. In this study, a large region from amino acids 94-300 termed the mid-region (MR) of pp71 was found to be necessary and sufficient for nuclear localization, suggesting the presence of a large 
non-classical nuclear localization signal. Phospho-mapping of pp71 from transfected cells revealed a single phosphorylation site within the MR, at threonine 223 (T223). Mutation of T223 to a phosphomimetic resulted in a block in nuclear localization, and redistribution of pp71 in a punctate perinuclear region. Confocal analysis using intracellular markers demonstrated that cytoplasmic pp71 colocalized with markers of the late trans-Golgi Network and late endosomal compartments. Notably, these cellular components are reorganized along with other structural elements of the secretory pathway into the cytoplasmic assembly complex in infected cells [22-24]. Together, these findings are consistent with pp71 containing an integral signal that denotes trafficking to the $\mathrm{AC}$ that is regulated by phosphorylation [43]. Ongoing studies in our laboratory are directed towards determining the significance of these findings during virus infection.

\section{4. $p p 65$ (ppUL83)}

The pp65 protein is the most abundant component of the tegument, and like the other nuclear tegument proteins initially traffics to the nucleus of permissively infected cells, and then relocalizes to the cytoplasm at the later stages of infection $[42,70]$. While initial reports showed that pp65 played no role in virus infection in vitro [59], more recent studies show that pp65 is important for efficient growth in both monocyte-derived macrophages and fibroblasts $[60,61,102]$. The underlying cause of this growth defect likely involves pp65 functions during the initiation of infection as well as during assembly. For example, pp65 can enhance the activation of the major immediate early promoter through its interaction with the cellular IFI16 transcriptional regulator [61]. Disruption of pp65 expression can also influence the incorporation of other proteins into the virion tegument, such as pUL25, ppUL97 and ppUL69, suggesting that pp65 forms part of a protein interaction network that may be important for assembly [60]. This finding is consistent with yeast two-hybrid analysis that identified pUL25 as a hub protein for an interaction network that included pp65 [37], and studies demonstrating a direct interaction between ppUL97 and pp65 [103]. In spite of these changes to the tegument composition, the virion particles appeared indistinguishable from those resulting from the wild type virus, except for the absence of dense bodies. In this study, a defect in replication of the pp65 mutant virus in monocyte-derived macrophages was observed, although whether this is due to the absence of pp65 per se, or the lack of important viral proteins such as ppUL97 [63] and ppUL69 [104] during the initial stages of replication remains to be definitively established.

A virus containing a 30 amino acid insertion within the pp65 open reading frame, termed RV-VM1, also displayed a defective growth phenotype [102]. In this case, the defect was most likely caused by the retention of pp65 in the nucleus at the late stages of infection, resulting in accumulation of the MCP in the nucleus, reduced numbers of C-capsids in the cytoplasm and a lack of dense body formation. The lack of pp65 relocalization resulted in the formation of large globular structures within the nucleus that contained the pp65 and ppUL69 proteins. Surprisingly, the redistribution of pp150 and pp71 to the $\mathrm{AC}$ were unaffected by the mutation, suggesting that these proteins trafficked independently of pp65 in this case. One possible explanation for this effect is that the insertion within pp65 disrupts the interaction with the pp71 protein. The movement of pp150 into the AC despite the retention of immature capsids in the nucleus is harder to explain, but may be consistent with an independent redistribution of pp150 to the AC to facilitate completion of the inner tegument. The lack of C-capsids in the cytoplasm of cells infected with RV-VM1 implies a 
direct role for pp65 and/or ppUL69 in capsid egress from the nucleus. However, the possibility that nonspecific effects due to the formation of the large globular structures within the nucleus also play a role in this phenotype cannot be excluded. Interestingly, inhibition of ppUL97 kinase activity also results in the accumulation of pp65 in the nucleus of infected cells where it forms large nuclear inclusions, presumably due to self-interaction [53].

Cumulative evidence points to the regulation of pp65 localization and/or self-interaction as important for efficient virion assembly and egress. In that regard, pp65 can shuttle in and out of nucleus with export occurring in a CRM1 dependent manner [48,51]. As mentioned above, control of the rates of nuclear import and export would provide a useful mechanism for the regulation of pp65 localization. Interestingly, the pp65 interacting protein, ppUL69 also functions as a shuttling protein, although it is thought that this protein functions primarily to transport mRNA's from the nucleus to the cytoplasm [105]. Other tegument proteins also regulate the relocalization of pp65, including pUL35, pTRS1 and pUL96 [31,56,64]. It is likely that phosphorylation of pp65 also plays a role in regulating the localization of this protein during infection. For example, the ppUL97 kinase is clearly involved in the regulation of pp65 redistribution to the $\mathrm{AC}$ at the later stages of infection [53] and pp65 is a direct target of the ppUL97 kinase [102]. Infection in the presence of CDK inhibitors also affects both the phosphorylation and localization of pp65 [51]. In order to examine the effect of phosphorylation on pp65 localization and function, we recently used mass spectrometry to determine the sites on which pp65 was phosphorylated at the late stages of infection (Figure 1). This analysis revealed clusters of phosphorylation sites flanking the pp65 nuclear localization signals [41,42], suggesting that phosphorylation may influence the recognition of these signals and alter the distribution of pp65. Interestingly, the 30 amino acid insertion in the RV-VM1 virus occurs at amino acid 387 [102], also in relatively close proximity to the pp65 nuclear localization signals. Indeed, this mutation reduces the susceptibility of the mutant virus to ppUL97 kinase inhibitors. One model to explain this data is that phosphorylation of pp65 by ppUL97 blocks recognition of the nuclear localization signals, resulting in a change in distribution to the cytoplasm. The insertion in the RV-VM1 virus could result in a different pattern of phosphorylation that results in an active nuclear localization signal and subsequent accumulation and aggregation of the protein in the nucleus. Interestingly, two phosphorylation sites were also clustered in the amino terminus in proximity to the self-interaction domain, suggesting that phosphorylation also regulates this property of pp65. This data is certainly consistent with the large aggregates of pp65 that form in the absence of ppUL97 activity [53].

Figure 1. Schematic of pp65 phosphorylation sites at the late stages of virus infection. The pp65 protein was purified from cells infected at 72 hpi by virtue of its association with the pp71 protein (See Table 2). The band corresponding to pp65 was excised from an SDS-PAGE and subjected to mass spectrometric analysis as previously described [43]. Also shown are the pp65 hydrophilic regions (green), sequences required for nuclear localization (red) and the self-interaction domain (blue) $[41,59,106]$.

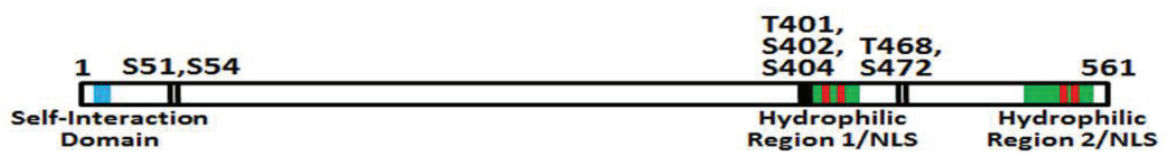




\section{5. $p U L 94$}

The product of the UL94 gene is nominally within the group of nuclear tegument proteins as it has been shown to be located in the nucleus of both transfected and infected cells $[62,107]$. Indeed, similar to the pp65 protein, pUL94 has nuclear:cytoplasmic shuttling activity [50]. However, the major functions of pUL94 during infection that have been reported are associated with its ability to interact with pp28, a cytoplasmic tegument protein [62]. Deletion of the UL94 gene results in defective replication, although viral gene expression and DNA replication are unaffected. The defect in replication is attributed to the accumulation of non-enveloped capsids in the cytoplasm, a phenotype similar to that observed upon deletion of the UL99 gene that encodes pp28 [65]. Thus, it has been concluded that pUL94 works with pp28 to facilitate secondary envelopment of tegumented capsids in the cytoplasm. It has been proposed that pUL94 may regulate the trafficking of the pp28 protein to the cytoplasmic AC $[62,108]$. This finding is in contrast to earlier analysis of the pp28 protein in the absence of virus infection, where it was found to localize to the endoplasmic reticulum-Golgi intermediate compartment, suggesting that trafficking to membranes associated with the AC may be an integral function of the pp28 protein [109]. Despite this discrepancy, it is clear that pUL94 and pp28 work in concert to facilitate secondary envelopment of HCMV virions.

\section{6. $p p U L 97$}

The ppUL97 viral kinase was initially identified by virtue of its ability to phosphorylate the antiviral compound ganciclovir [110]. The kinase activity of the ppUL97 protein is critical for efficient virus replication and it has therefore been the subject of intense investigation as an antiviral target [111]. In regards to virion assembly, a notable function of the ppUL97 viral kinase is in the redistribution of nuclear tegument proteins to the $\mathrm{AC}$, as previously mentioned $[53,102]$. In the absence of this relocalization, the tegument proteins form large nuclear aggregates, suggesting that ppUL97 may regulate the self-association of these tegument proteins [111]. In addition, as the ppUL97 protein can be incorporated into virion particles [28], it is possible that this kinase may modulate phosphorylation of virion components in situ, or immediately after infection. Recent studies also show that ppUL97 can interact with pUL50, and together with pUL53 disrupts the nuclear lamina, enabling efficient capsid egress from the nucleus [112,113]. The ppUL97 protein has also been proposed to play a direct role in the formation of the cytoplasmic AC $[52,114]$. Specifically, deletion of the HCMV UL97 gene or the use of ppUL97 kinase inhibitors results in a modified AC which is more diffuse than that observed in a typical infection, together with the formation of large perinuclear vacuoles [114]. Immuno-electron microscopy revealed that these cytoplasmic vacuoles were surrounded by membranes and contained accumulations of tegument proteins, including pp28 and pp65, as well as the gB envelope protein. The similarity of these vesicles to the nuclear inclusions observed upon inhibition of ppUL97 [111] add further weight to the contention that self-association of tegument proteins is tightly regulated within infected cells. Interestingly, a more severe growth defect was observed when the UL97 gene was deleted versus a kinase dead mutant, or in the presence of a ppUL97 kinase inhibitor [52]. Of note, the presence of dense bodies, tegumented cytoplasmic capsids and viral particle release were detected in the presence of the kinase dead mutant or when the kinase inhibitor NGIC-I was added to the infected 
cells. However, when the UL97 gene was deleted, dense bodies were absent, as was evidence of particle release, and only a few C-capsids were observed in the cytoplasm. This finding suggests that the ppUL97 protein has non-kinase related functions in virion assembly and formation of the AC. However, the possibility that kinase inhibition was not $100 \%$ efficient in the ppUL97 mutant or in the presence of the inhibitor cannot be ruled out. Regardless, further identification of cellular and viral targets of ppUL97 will likely yield more insights into the precise role of this enzyme in virion assembly and egress.

\section{7. $p$ TRS1}

The IRSI and TRS1 genes located in the viral repeat sequences code for highly related, although not identical proteins [115]. The products of both genes are involved in immune evasion through inactivation of the interferon induced kinase, PKR [12]. Uniquely, the pTRS1 protein appears to also be involved in virion assembly. Deletion of the TRS1 gene results in the formation of abnormal viral particles containing decreased levels of the pp65 and pp150 tegument proteins [64]. Extracellular virus particles generated in the absence of pTRS1 also displayed abnormal sedimentation in glycerol-tartrate gradients, indicative of defective particles, with an increase in the formation of dense bodies. A delay in the movement of tegument proteins such as pp65 and pp71 into the cytoplasm at the late stages of infection was also observed, although the large nuclear aggregates observed when ppUL97 is inhibited were not noted. Further analysis of the TRS1-deletion virus revealed decreased capsid formation in the nucleus, suggesting that pTRS1 influences events relatively early in the assembly process [116]. However, the precise function of pTRS1 in capsid assembly remains to be determined. One possibility is that the regulation of the interferon induced kinase, protein kinase R or PKR, by pTRS1 may be involved [117]. This could result in altered phosphorylation of the tegument proteins, either through the direct inhibition of PKR activity, or altered access to substrates by virtue of the relocalization of PKR to the nucleus of infected cells [118]. Some intriguing hints have come from recent studies examining a multiprotein complex that includes pUL84, the ppUL44 DNA processivity factor and the pIRS1/pTRS1 proteins $[119,120]$. Deletion of the amino-terminus of pUL84 diminishes the formation of this complex, and results in an unusual distribution of nuclear capsids in the nucleolar region, as well as a lack of cytoplasmic capsids. This finding suggests that pTRS1 may participate in a complex that regulates the maturation and egress of HCMV capsids.

\section{The Cytoplasmic Tegument Proteins}

\section{1. $p U L 71$}

Two independent studies have implicated a role for the pUL71 protein in secondary envelopment [46,121]. Specifically, deletion of the UL71 gene results in an accumulation of non-enveloped capsids in the AC region of infected cells. In addition, enlarged vesicular structures reminiscent of multi-vesicular bodies were noted, along with distinct changes in the AC including the redistribution of cellular AC markers. At this point, it is unclear whether these observations represent the result of direct effects of pUL71 on the formation of the AC, or whether these 
changes are a secondary consequence of the defect in secondary envelopment. While the precise role of pUL71 in envelopment has yet to be determined, recent data shows that the oligomerization of pUL71 into dimers, trimers and higher-ordered multimers are important for this process [122]. Surprisingly, the pUL71 protein was also found to interact with pUL51 and pUL89 proteins using yeast two-hybrid analysis [37]. The pUL51 and pUL89 proteins are two components of the three subunit terminase subunits required for the cleavage and packaging of DNA within nuclear replication compartments [123]. This finding is inconsistent with the phenotype of the UL71 deletion viruses $[46,121]$, and thus may represent an aberrant result due to the artificial nature of the yeast two-hybrid assays.

\section{2. $p p 28$}

The product of the UL99 gene, pp28, localizes to the cytoplasmic AC in association with cellular membranes as a result of myristoylation in the amino terminus of the protein $[45,70]$. Deletion of the UL99 gene revealed that it is essential for virus replication, and results in the accumulation of tegumented capsids in the cytoplasm, suggesting that the pp28 protein plays a critical role in secondary envelopment $[65,124]$. Detailed mutational analyses of the pp28 open reading frame have identified distinct domains required for both $\mathrm{AC}$ localization and virion assembly with an acidic cluster within the amino terminus being critical for the assembly functions $[26,109]$. Surprisingly, the first 50 of the 190 amino acids of pp28 was capable of conferring both AC localization and at least partially restored the defect in virion assembly [109,125]. Importantly, the incorporation of other tegument proteins into the virion particles was unaffected, suggesting that pp28 functions after the addition of the bulk of the virion tegument [125]. Such a role is consistent with the membrane association of the pp28 protein, and indeed multimerization of the pp28 protein has been proposed to promote membrane deformation and virion budding, akin to the matrix proteins of other viruses [126]. The multimerization function also maps to the first 50 amino acids of pp28, as does its ability to interact with the pUL94 protein that functions with pp28 to facilitate secondary envelopment [108].

It is somewhat surprising that these key functions of the pp28 protein are localized to such a small region with the amino terminus of the protein. One possibility is that additional non-essential functions reside within the remaining portion of the protein. In that regard, recent analysis using mass spectrometry have revealed potential interactions between pp28 and additional viral proteins, including pp65, the major capsid protein and the pUL44 DNA processivity factor [34]. These interactions may represent a component of the functional redundancy of some of the tegument proteins as previously noted $[20,40]$. However, it remains to be seen whether the carboxy-terminal region of pp28 participates in these interactions, or has additional hitherto unknown functions. Another unanswered question in relation to pp28 is the role of phosphorylation in any or all of its functions. It was originally reported that only a hypophosphorylated form of pp28 was packaged into virions [26]. In contrast, deletion of the UL26 gene causes a unique hypophosphorylated form of pp28 to be incorporated into virions and this corresponded to decreased stability of the pp28 protein upon infection [44]. The reason for this apparent discrepancy is unclear, but could be related to the differences in methodologies used for the analysis. The original observation was made using mobility in standard SDS-PAGE analysis [26] whereas the second study utilized 2-D 
gel electrophoresis but did not compare the results to the proteins found within the infected cell [44]. Regardless, it is clear that phosphorylation of pp28 could have a major impact on its function during assembly.

\section{Conclusions}

Insights into the roles of HCMV tegument proteins during virion assembly have advanced in recent years due to enhancements in technology, most notably the ability to rapidly generate viral mutants, and more widespread use of sophisticated microscopy techniques. We now have a more complete understanding of the inner tegument components and how they interact with and stabilize the viral capsid. In addition, we are beginning to decipher the networks that link the nuclear tegument proteins and presumably facilitate their incorporation into the virion particle. We also have important clues regarding the role of the cytoplasmic tegument proteins in secondary envelopment. However, there is still much to be learned and basic questions regarding the mechanism of tegumentation remain unanswered. Specific questions include the potential role of viral and host enzymes in the regulation of virion components in situ; how the addition of the nuclear tegument proteins is coordinated, including the mechanism of redistribution during infection and the role of the capsid in this process; the role of tegument in targeting capsids to the assembly complex; how self-association of the tegument proteins is regulated; identification of specific cellular and viral targets of the ppUL97 viral kinase; and the nature of tegument protein redundancy. Answering these questions are not only vital to our overall understanding of HCMV assembly and egress, but are critical to realize the potential of the tegument proteins as therapeutic targets for intervention.

\section{Acknowledgments}

The authors would like to acknowledge support from the National Institutes of Health (grant AI074800) and the Hazel T. Carmen Charitable Trust to J.A.K. In addition, the authors are grateful to the members of the George L. Wright Jr. Center for Biomedical Proteomics, particularly Julius Nyalwidhe, for assistance with the mass spectrometry experiments.

\section{Author Contributions}

Smith performed the protein purifications and mass spectrometry analysis for the data included in Table 2 and Figure 1 in partial fulfillment of her thesis research for a Master's Degree in Biomedical Sciences. Kosuri assisted with data analysis and provided useful discussion and feedback during the preparation of the manuscript. Kerry researched and wrote the manuscript.

\section{Conflicts of Interest}

The authors declare no conflict of interest. 


\section{References and Notes}

1. Cannon, M.J.; Schmid, D.S.; Hyde, T.B. Review of cytomegalovirus seroprevalence and demographic characteristics associated with infection. Rev. Med. Virol. 2010, 20, 202-213.

2. Drew, W.L. Cytomegalovirus infection in patients with AIDS. Clin. Infect. Dis. 1992, 14, 608-615.

3. Griffiths, P.D.; Clark, D.A.; Emery, V.C. Betaherpesviruses in transplant recipients. J. Antimicrob. Chemother. 2000, 45, 29-34.

4. Steininger, C.; Puchhammer-Stockl, E.; Popow-Kraupp, T. Cytomegalovirus disease in the era of highly active antiretroviral therapy (HAART). J. Clin. Virol. 2006, 37, 1-9.

5. Husain, S.; Pietrangeli, C.E.; Zeevi, A. Delayed onset CMV disease in solid organ transplant recipients. Transpl. Immunol. 2009, 21, 1-9.

6. Limaye, A.P. Antiviral resistance in cytomegalovirus: An emerging problem in organ transplant recipients. Semin. Respir. Infect. 2002, 17, 265-273.

7. Karltorp, E.; Hellstrom, S.; Lewensohn-Fuchs, I.; Carlsson-Hansen, E.; Carlsson, P.I.; Engman, M.L. Congenital cytomegalovirus infection-A common cause of hearing loss of unknown aetiology. Acta Paediatr. 2012, 101, e357-e362.

8. Leung, A.K.; Sauve, R.S.; Davies, H.D. Congenital cytomegalovirus infection. J. Natl. Med. Assoc. 2003, 95, 213-218.

9. Cannon, M.J.; Davis, K.F. Washing our hands of the congenital cytomegalovirus disease epidemic. BMC Publ. Health 2005, 5, 70.

10. Lurain, N.S.; Chou, S. Antiviral drug resistance of human cytomegalovirus. Clin. Microbiol. Rev. 2010, 23, 689-712.

11. Kalejta, R.F. Functions of human cytomegalovirus tegument proteins prior to immediate early gene expression. Curr. Top. Microbiol. Immunol. 2008, 325, 101-115.

12. Kalejta, R.F. Tegument proteins of human cytomegalovirus. Microbiol. Mol. Biol. Rev. 2008, 72, 249-265.

13. Penkert, R.R.; Kalejta, R.F. Tale of a tegument transactivator: The past, present and future of human CMV pp71. Future Virol. 2012, 7, 855-869.

14. Compton, T.; Feire, A. Early events in human cytomegalovirus infection. In Human Herpesviruses: Biology, Therapy, and Immunoprophylaxis; Arvin, A., Campadelli-Fiume, G., Mocarski, E., Moore, P.S., Roizman, B., Whitley, R., Yamanishi, K., Eds.; Cambridge University Press: Cambridge, UK, 2007.

15. Varnum, S.M.; Streblow, D.N.; Monroe, M.E.; Smith, P.; Auberry, K.J.; Pasa-Tolic, L.; Wang, D.; Camp, D.G., 2nd; Rodland, K.; Wiley, S.; et al. Identification of proteins in human cytomegalovirus (HCMV) particles: The HCMV proteome. J. Virol. 2004, 78, 10960-10966.

16. Terhune, S.S.; Schroer, J.; Shenk, T. RNAs are packaged into human cytomegalovirus virions in proportion to their intracellular concentration. J. Virol. 2004, 78, 10390-10398.

17. Stinski, M.F.; Meier, J.L. Immediate-early viral gene regulation and function. In Human Herpesviruses: Biology, Therapy, and Immunoprophylaxis; Arvin, A., Campadelli-Fiume, G., Mocarski, E., Moore, P.S., Roizman, B., Whitley, R., Yamanishi, K., Eds.; Cambridge University Press: Cambridge, UK, 2007. 
18. White, E.A.; Spector, D.H. Early viral gene expression and function. In Human Herpesviruses: Biology, Therapy, and Immunoprophylaxis; Arvin, A., Campadelli-Fiume, G., Mocarski, E., Moore, P.S., Roizman, B., Whitley, R., Yamanishi, K., Eds.; Cambridge University Press: Cambridge, UK, 2007.

19. Anders, D.G.; Kerry, J.A.; Pari, G.S. DNA synthesis and late viral gene expression. In Human Herpesviruses: Biology, Therapy, and Immunoprophylaxis; Arvin, A., Campadelli-Fiume, G., Mocarski, E., Moore, P.S., Roizman, B., Whitley, R., Yamanishi, K., Eds.; Cambridge University Press: Cambridge, UK, 2007.

20. Tandon, R.; Mocarski, E.S. Viral and host control of cytomegalovirus maturation. Trends Microbiol. 2012, 20, 392-401.

21. Yu, X.; Shah, S.; Lee, M.; Dai, W.; Lo, P.; Britt, W.; Zhu, H.; Liu, F.; Zhou, Z.H. Biochemical and structural characterization of the capsid-bound tegument proteins of human cytomegalovirus. J. Struct. Biol. 2011, 174, 451-460.

22. Das, S.; Pellett, P.E. Spatial relationships between markers for secretory and endosomal machinery in human cytomegalovirus-infected cells versus those in uninfected cells. J. Virol. 2011, 85, 5864-5879.

23. Das, S.; Vasanji, A.; Pellett, P.E. Three-dimensional structure of the human cytomegalovirus cytoplasmic virion assembly complex includes a reoriented secretory apparatus. J. Virol. 2007, 81, 11861-11869.

24. Alwine, J.C. The human cytomegalovirus assembly compartment: A masterpiece of viral manipulation of cellular processes that facilitates assembly and egress. PLoS Pathog. 2012, 8, e1002878.

25. Winkler, M.; Stamminger, T. A specific subform of the human cytomegalovirus transactivator protein pUL69 is contained within the tegument of virus particles. J. Virol. 1996, 70, 8984-8987.

26. Jones, T.R.; Lee, S.W. An acidic cluster of human cytomegalovirus UL99 tegument protein is required for trafficking and function. J. Virol. 2004, 78, 1488-1502.

27. Gallina, A.; Simoncini, L.; Garbelli, S.; Percivalle, E.; Pedrali-Noy, G.; Lee, K.S.; Erikson, R.L.; Plachter, B.; Gerna, G.; Milanesi, G. Polo-like kinase 1 as a target for human cytomegalovirus pp65 lower matrix protein. J. Virol. 1999, 73, 1468-1478.

28. Van Zeijl, M.; Fairhurst, J.; Baum, E.Z.; Sun, L.; Jones, T.R. The human cytomegalovirus UL97 protein is phosphorylated and a component of virions. Virology 1997, 231, 72-80.

29. Michelson, S.; Turowski, P.; Picard, L.; Goris, J.; Landini, M.P.; Topilko, A.; Hemmings, B.; Bessia, C.; Garcia, A.; Virelizier, J.L. Human cytomegalovirus carries serine/threonine protein phosphatases PP1 and a host-cell derived PP2a. J. Virol. 1996, 70, 1415-1423.

30. Sampaio, K.L.; Cavignac, Y.; Stierhof, Y.D.; Sinzger, C. Human cytomegalovirus labeled with green fluorescent protein for live analysis of intracellular particle movements. J. Virol. 2005, 79, 2754-2767.

31. Tandon, R.; Mocarski, E.S. Cytomegalovirus pUL96 is critical for the stability of pp150-associated nucleocapsids. J. Virol. 2011, 85, 7129-7141. 
32. Meyer, H.H.; Ripalti, A.; Landini, M.P.; Radsak, K.; Kern, H.F.; Hensel, G.M. Human cytomegalovirus late-phase maturation is blocked by stably expressed UL32 antisense mrna in astrocytoma cells. J. Gen. Virol. 1997, 78, 2621-2631.

33. Tandon, R.; Mocarski, E.S. Control of cytoplasmic maturation events by cytomegalovirus tegument protein pp150. J. Virol. 2008, 82, 9433-9444.

34. Moorman, N.J.; Sharon-Friling, R.; Shenk, T.; Cristea, I.M. A targeted spatial-temporal proteomics approach implicates multiple cellular trafficking pathways in human cytomegalovirus virion maturation. Mol. Cell. Proteomics 2010, 9, 851-860.

35. Trus, B.L.; Gibson, W.; Cheng, N.; Steven, A.C. Capsid structure of simian cytomegalovirus from cryoelectron microscopy: Evidence for tegument attachment sites. J. Virol. 1999, 73, 2181-2192.

36. Phillips, S.L.; Bresnahan, W.A. Identification of binary interactions between human cytomegalovirus virion proteins. J. Virol. 2011, 85, 440-447.

37. To, A.; Bai, Y.; Shen, A.; Gong, H.; Umamoto, S.; Lu, S.; Liu, F. Yeast two hybrid analyses reveal novel binary interactions between human cytomegalovirus-encoded virion proteins. PLoS One 2011, 6, e17796.

38. Patrone, M.; Percivalle, E.; Secchi, M.; Fiorina, L.; Pedrali-Noy, G.; Zoppe, M.; Baldanti, F.; Hahn, G.; Koszinowski, U.H.; Milanesi, G.; et al. The human cytomegalovirus UL45 gene product is a late, virion-associated protein and influences virus growth at low multiplicities of infection. J. Gen. Virol. 2003, 84, 3359-3370.

39. Dunn, W.; Chou, C.; Li, H.; Hai, R.; Patterson, D.; Stolc, V.; Zhu, H.; Liu, F. Functional profiling of a human cytomegalovirus genome. Proc. Natl. Acad. Sci. USA 2003, 100, 14223-14228.

40. Mettenleiter, T.C.; Klupp, B.G.; Granzow, H. Herpesvirus assembly: An update. Virus Res. 2009, 143, 222-234.

41. Gallina, A.; Percivalle, E.; Simoncini, L.; Revello, M.G.; Gerna, G.; Milanesi, G. Human cytomegalovirus pp65 lower matrix phosphoprotein harbours two transplantable nuclear localization signals. J. Gen. Virol. 1996, 77, 1151-1157.

42. Schmolke, S.; Drescher, P.; Jahn, G.; Plachter, B. Nuclear targeting of the tegument protein pp65 (UL83) of human cytomegalovirus: An unusual bipartite nuclear localization signal functions with other portions of the protein to mediate its efficient nuclear transport. J. Virol. 1995, 69, 1071-1078.

43. Shen, W.; Westgard, E.; Huang, L.; Ward, M.D.; Osborn, J.L.; Chau, N.H.; Collins, L.; Marcum, B.; Koach, M.A.; Bibbs, J.; et al. Nuclear trafficking of the human cytomegalovirus pp71 (ppUL82) tegument protein. Virology 2008, 376, 42-52.

44. Munger, J.; Yu, D.; Shenk, T. UL26-deficient human cytomegalovirus produces virions with hypophosphorylated pp28 tegument protein that is unstable within newly infected cells. J. Virol. 2006, 80, 3541-3548.

45. Sanchez, V.; Sztul, E.; Britt, W.J. Human cytomegalovirus pp28 (UL99) localizes to a cytoplasmic compartment which overlaps the endoplasmic reticulum-golgi-intermediate compartment. J. Virol. 2000, 74, 3842-3851. 
46. Womack, A.; Shenk, T. Human cytomegalovirus tegument protein pUL71 is required for efficient virion egress. mBio 2010, 1, e00282-10.

47. Zini, N.; Santi, S.; Riccio, M.; Landini, M.P.; Battista, M.C.; Maraldi, N.M. pUL25 immunolocalization in human cytomegalovirus-infected and gene-transfected cells. Arch. Virol. 2000, 145, 795-803.

48. Frankenberg, N.; Lischka, P.; Pepperl-Klindworth, S.; Stamminger, T.; Plachter, B. Nucleocytoplasmic shuttling and CRM1-dependent MHC class I peptide presentation of human cytomegalovirus pp65. Med. Microbiol. Immunol. 2012, 201, 567-579.

49. Lischka, P.; Rosorius, O.; Trommer, E.; Stamminger, T. A novel transferable nuclear export signal mediates CRM1-independent nucleocytoplasmic shuttling of the human cytomegalovirus transactivator protein pUL69. EMBO J. 2001, 20, 7271-7283.

50. Liu, Y.; Zhang, Z.; Zhao, X.; Wei, H.; Deng, J.; Cui, Z.; Zhang, X.E. Human cytomegalovirus UL94 is a nucleocytoplasmic shuttling protein containing two NLSs and one NES. Virus Res. 2012, 166, 31-42.

51. Sanchez, V.; Mahr, J.A.; Orazio, N.I.; Spector, D.H. Nuclear export of the human cytomegalovirus tegument protein pp65 requires cyclin-dependent kinase activity and the CRM1 exporter. J. Virol. 2007, 81, 11730-11736.

52. Goldberg, M.D.; Honigman, A.; Weinstein, J.; Chou, S.; Taraboulos, A.; Rouvinski, A.; Shinder, V.; Wolf, D.G. Human cytomegalovirus UL97 kinase and nonkinase functions mediate viral cytoplasmic secondary envelopment. J. Virol. 2011, 85, 3375-3384.

53. Prichard, M.N.; Britt, W.J.; Daily, S.L.; Hartline, C.B.; Kern, E.R. Human cytomegalovirus UL97 kinase is required for the normal intranuclear distribution of pp65 and virion morphogenesis. J. Virol. 2005, 79, 15494-15502.

54. Tandon, R.; AuCoin, D.P.; Mocarski, E.S. Human cytomegalovirus exploits ESCRT machinery in the process of virion maturation. J. Virol. 2009, 83, 10797-10807.

55. Yu, D.; Silva, M.C.; Shenk, T. Functional map of human cytomegalovirus AD169 defined by global mutational analysis. Proc. Natl. Acad. Sci. USA 2003, 100, 12396-12401.

56. Schierling, K.; Buser, C.; Mertens, T.; Winkler, M. Human cytomegalovirus tegument protein ppUL35 is important for viral replication and particle formation. J. Virol. 2005, 79, 3084-3096.

57. Bresnahan, W.A.; Hultman, G.E.; Shenk, T. Replication of wild-type and mutant human cytomegalovirus in life-extended human diploid fibroblasts. J. Virol. 2000, 74, 10816-10818.

58. Bresnahan, W.A.; Shenk, T.E. UL82 virion protein activates expression of immediate early viral genes in human cytomegalovirus-infected cells. Proc. Natl. Acad. Sci. USA 2000, 97, 14506-14511.

59. Schmolke, S.; Kern, H.F.; Drescher, P.; Jahn, G.; Plachter, B. The dominant phosphoprotein pp65 (UL83) of human cytomegalovirus is dispensable for growth in cell culture. J. Virol. 1995, 69, 5959-5968.

60. Chevillotte, M.; Landwehr, S.; Linta, L.; Frascaroli, G.; Luske, A.; Buser, C.; Mertens, T.; von Einem, J. Major tegument protein pp65 of human cytomegalovirus is required for the incorporation of pUL69 and pUL97 into the virus particle and for viral growth in macrophages. J. Virol. 2009, 83, 2480-2490. 
61. Cristea, I.M.; Moorman, N.J.; Terhune, S.S.; Cuevas, C.D.; O'Keefe, E.S.; Rout, M.P.; Chait, B.T.; Shenk, T. Human cytomegalovirus pUL83 stimulates activity of the viral immediate-early promoter through its interaction with the cellular IFI16 protein. J. Virol. 2010, 84, 7803-7814.

62. Phillips, S.L.; Bresnahan, W.A. The human cytomegalovirus (HCMV) tegument protein UL94 is essential for secondary envelopment of HCMV virions. J. Virol. 2012, 86, 2523-2532.

63. Prichard, M.N.; Gao, N.; Jairath, S.; Mulamba, G.; Krosky, P.; Coen, D.M.; Parker, B.O.; Pari, G.S. A recombinant human cytomegalovirus with a large deletion in UL97 has a severe replication deficiency. J. Virol. 1999, 73, 5663-5670.

64. Blankenship, C.A.; Shenk, T. Mutant human cytomegalovirus lacking the immediate-early TRS1 coding region exhibits a late defect. J. Virol. 2002, 76, 12290-12299.

65. Silva, M.C.; Yu, Q.C.; Enquist, L.; Shenk, T. Human cytomegalovirus UL99-encoded pp28 is required for the cytoplasmic envelopment of tegument-associated capsids. J. Virol. 2003, 77, 10594-10605.

66. Baxter, M.K.; Gibson, W. Cytomegalovirus basic phosphoprotein (pUL32) binds to capsids in vitro through its amino one-third. J. Virol. 2001, 75, 6865-6873.

67. Dai, X.; Yu, X.; Gong, H.; Jiang, X.; Abenes, G.; Liu, H.; Shivakoti, S.; Britt, W.J.; Zhu, H.; Liu, F.; et al. The smallest capsid protein mediates binding of the essential tegument protein pp150 to stabilize DNA-containing capsids in human cytomegalovirus. PLoS Pathog. 2013, 9, e1003525.

68. Bogdanow, B.; Weisbach, H.; von Einem, J.; Straschewski, S.; Voigt, S.; Winkler, M.; Hagemeier, C.; Wiebusch, L. Human cytomegalovirus tegument protein pp150 acts as a cyclin A2-CDK-dependent sensor of the host cell cycle and differentiation state. Proc. Natl. Acad. Sci. USA 2013, 110, 17510-17515.

69. Hensel, G.; Meyer, H.; Gartner, S.; Brand, G.; Kern, H.F. Nuclear localization of the human cytomegalovirus tegument protein pp150 (ppUL32). J. Gen. Virol. 1995, 76, 1591-1601.

70. Sanchez, V.; Greis, K.D.; Sztul, E.; Britt, W.J. Accumulation of virion tegument and envelope proteins in a stable cytoplasmic compartment during human cytomegalovirus replication: Characterization of a potential site of virus assembly. J. Virol. 2000, 74, 975-986.

71. Indran, S.V.; Ballestas, M.E.; Britt, W.J. Bicaudal D1-dependent trafficking of human cytomegalovirus tegument protein pp1 50 in virus-infected cells. J. Virol. 2010, 84, 3162-3177.

72. Dienstbier, M.; Li, X. Bicaudal-D and its role in cargo sorting by microtubule-based motors. Biochem. Soc. Trans. 2009, 37, 1066-1071.

73. Short, B.; Haas, A.; Barr, F.A. Golgins and GTPases, giving identity and structure to the golgi apparatus. Biochim. Biophys. Acta 2005, 1744, 383-395.

74. Indran, S.V.; Britt, W.J. A role for the small GTPase Rab6 in assembly of human cytomegalovirus. J. Virol. 2011, 85, 5213-5219.

75. Gibson, W. Structure and assembly of the virion. Intervirology 1996, 39, 389-400.

76. Brock, I.; Kruger, M.; Mertens, T.; von Einem, J. Nuclear targeting of human cytomegalovirus large tegument protein pUL48 is essential for viral growth. J. Virol. 2013, $87,6005-6019$. 
77. Bechtel, J.T.; Shenk, T. Human cytomegalovirus UL47 tegument protein functions after entry and before immediate-early gene expression. J. Virol. 2002, 76, 1043-1050.

78. Kim, E.T.; Oh, S.E.; Lee, Y.O.; Gibson, W.; Ahn, J.H. Cleavage specificity of the UL48 deubiquitinating protease activity of human cytomegalovirus and the growth of an active-site mutant virus in cultured cells. J. Virol. 2009, 83, 12046-12056.

79. Wang, J.; Loveland, A.N.; Kattenhorn, L.M.; Ploegh, H.L.; Gibson, W. High-molecular-weight protein (pUL48) of human cytomegalovirus is a competent deubiquitinating protease: Mutant viruses altered in its active-site cysteine or histidine are viable. J. Virol. 2006, 80, 6003-6012.

80. Ogawa-Goto, K.; Irie, S.; Omori, A.; Miura, Y.; Katano, H.; Hasegawa, H.; Kurata, T.; Sata, T.; Arao, Y. An endoplasmic reticulum protein, p180, is highly expressed in human cytomegalovirus-permissive cells and interacts with the tegument protein encoded by UL48. J. Virol. 2002, 76, 2350-2362.

81. Desai, P.J. A null mutation in the UL36 gene of herpes simplex virus type 1 results in accumulation of unenveloped DNA-filled capsids in the cytoplasm of infected cells. J. Virol. 2000, 74, 11608-11618.

82. Sandbaumhuter, M.; Dohner, K.; Schipke, J.; Binz, A.; Pohlmann, A.; Sodeik, B.; Bauerfeind, R. Cytosolic herpes simplex virus capsids not only require binding inner tegument protein pUL36 but also pUL37 for active transport prior to secondary envelopment. Cell. Microbiol. 2013, 15, 248-269.

83. Schipke, J.; Pohlmann, A.; Diestel, R.; Binz, A.; Rudolph, K.; Nagel, C.H.; Bauerfeind, R.; Sodeik, B. The $\mathrm{C}$ terminus of the large tegument protein pUL36 contains multiple capsid binding sites that function differently during assembly and cell entry of herpes simplex virus. J. Virol. 2012, 86, 3682-3700.

84. Hensel, G.M.; Meyer, H.H.; Buchmann, I.; Pommerehne, D.; Schmolke, S.; Plachter, B.; Radsak, K.; Kern, H.F. Intracellular localization and expression of the human cytomegalovirus matrix phosphoprotein pp71 (ppUL82): Evidence for its translocation into the nucleus. J. Gen. Virol. 1996, 77, 3087-3097.

85. Revello, M.G.; Percivalle, E.; Di Matteo, A.; Morini, F.; Gerna, G. Nuclear expression of the lower matrix protein of human cytomegalovirus in peripheral blood leukocytes of immunocompromised viraemic patients. J. Gen. Virol. 1992, 73, 437-442.

86. Lorz, K.; Hofmann, H.; Berndt, A.; Tavalai, N.; Mueller, R.; Schlotzer-Schrehardt, U.; Stamminger, T. Deletion of open reading frame UL26 from the human cytomegalovirus genome results in reduced viral growth, which involves impaired stability of viral particles. J. Virol. 2006, 80, 5423-5434.

87. Liljeroos, L.; Butcher, S.J. Matrix proteins as centralized organizers of negative-sense RNA virions. Front. Biosci. 2013, 18, 696-715.

88. Liu, Y.; Biegalke, B.J. The human cytomegalovirus UL35 gene encodes two proteins with different functions. J. Virol. 2002, 76, 2460-2468.

89. Schierling, K.; Stamminger, T.; Mertens, T.; Winkler, M. Human cytomegalovirus tegument proteins ppUL82 (pp71) and ppUL35 interact and cooperatively activate the major immediate-early enhancer. J. Virol. 2004, 78, 9512-9523. 
90. Salsman, J.; Wang, X.; Frappier, L. Nuclear body formation and PML body remodeling by the human cytomegalovirus protein UL35. Virology 2011, 414, 119-129.

91. Tavalai, N.; Stamminger, T. Interplay between herpesvirus infection and host defense by PML nuclear bodies. Viruses 2009, 1, 1240-1264.

92. Salsman, J.; Jagannathan, M.; Paladino, P.; Chan, P.K.; Dellaire, G.; Raught, B.; Frappier, L. Proteomic profiling of the human cytomegalovirus UL35 gene products reveals a role for UL35 in the DNA repair response. J. Virol. 2012, 86, 806-820.

93. Craighead, J.E.; Kanich, R.E.; Almeida, J.D. Nonviral microbodies with viral antigenicity produced in cytomegalovirus-infected cells. J. Virol. 1972, 10, 766-775.

94. Weiland, K.L.; Oien, N.L.; Homa, F.; Wathen, M.W. Functional analysis of human cytomegalovirus polymerase accessory protein. Virus Res. 1994, 34, 191-206.

95. Chee, M.; Rudolph, S.A.; Plachter, B.; Barrell, B.; Jahn, G. Identification of the major capsid protein gene of human cytomegalovirus. J. Virol. 1989, 63, 1345-1353.

96. He, Z.; He, Y.S.; Kim, Y.; Chu, L.; Ohmstede, C.; Biron, K.K.; Coen, D.M. The human cytomegalovirus UL97 protein is a protein kinase that autophosphorylates on serines and threonines. J. Virol. 1997, 71, 405-411.

97. Milbradt, J.; Auerochs, S.; Marschall, M. Cytomegaloviral proteins pUL50 and pUL53 are associated with the nuclear lamina and interact with cellular protein kinase C. J. Gen. Virol. 2007, 88, 2642-2650.

98. Borst, E.M.; Wagner, K.; Binz, A.; Sodeik, B.; Messerle, M. The essential human cytomegalovirus gene UL52 is required for cleavage-packaging of the viral genome. J. Virol. 2008, 82, 2065-2078.

99. Bogner, E.; Radsak, K.; Stinski, M.F. The gene product of human cytomegalovirus open reading frame UL56 binds the pac motif and has specific nuclease activity. J. Virol. 1998, 72, 2259-2264.

100. Baldick, C.J., Jr.; Shenk, T. Proteins associated with purified human cytomegalovirus particles. J. Virol. 1996, 70, 6097-6105.

101. Lee, S.H.; Kalejta, R.F.; Kerry, J.; Semmes, O.J.; O’Connor, C.M.; Khan, Z.; Garcia, B.A.; Shenk, T.; Murphy, E. BclAF1 restriction factor is neutralized by proteasomal degradation and microRNA repression during human cytomegalovirus infection. Proc. Natl. Acad. Sci. USA 2012, 109, 9575-9580.

102. Becke, S.; Fabre-Mersseman, V.; Aue, S.; Auerochs, S.; Sedmak, T.; Wolfrum, U.; Strand, D.; Marschall, M.; Plachter, B.; Reyda, S. Modification of the major tegument protein pp65 of human cytomegalovirus inhibits virus growth and leads to the enhancement of a protein complex with pUL69 and pUL97 in infected cells. J. Gen. Virol. 2010, 91, 2531-2541.

103. Kamil, J.P.; Coen, D.M. Human cytomegalovirus protein kinase UL97 forms a complex with the tegument phosphoprotein pp65. J. Virol. 2007, 81, 10659-10668.

104. Hayashi, M.L.; Blankenship, C.; Shenk, T. Human cytomegalovirus UL69 protein is required for efficient accumulation of infected cells in the G1 phase of the cell cycle. Proc. Natl. Acad. Sci. USA 2000, 97, 2692-2696.

105. Toth, Z.; Stamminger, T. The human cytomegalovirus regulatory protein UL69 and its effect on mrna export. Front. Biosci. 2008, 13, 2939-2949. 
106. Cui, Z.; Zhang, K.; Zhang, Z.; Liu, Y.; Zhou, Y.; Wei, H.; Zhang, X.E. Visualization of the dynamic multimerization of human cytomegalovirus pp65 in punctuate nuclear foci. Virology 2009, 392, 169-177.

107. Wing, B.A.; Lee, G.C.; Huang, E.S. The human cytomegalovirus UL94 open reading frame encodes a conserved herpesvirus capsid/tegument-associated virion protein that is expressed with true late kinetics. J. Virol. 1996, 70, 3339-3345.

108. Phillips, S.L.; Cygnar, D.; Thomas, A.; Bresnahan, W.A. Interaction between the human cytomegalovirus tegument proteins UL94 and UL99 is essential for virus replication. J. Virol. 2012, 86, 9995-10005.

109. Seo, J.Y.; Britt, W.J. Sequence requirements for localization of human cytomegalovirus tegument protein pp28 to the virus assembly compartment and for assembly of infectious virus. J. Virol. 2006, 80, 5611-5626.

110. Littler, E.; Stuart, A.D.; Chee, M.S. Human cytomegalovirus UL97 open reading frame encodes a protein that phosphorylates the antiviral nucleoside analogue ganciclovir. Nature 1992, 358, 160-162.

111. Prichard, M.N. Function of human cytomegalovirus UL97 kinase in viral infection and its inhibition by maribavir. Rev. Med. Virol. 2009, 19, 215-229.

112. Sharma, M.; Kamil, J.P.; Coughlin, M.; Reim, N.I.; Coen, D.M. Human cytomegalovirus UL50 and UL53 recruit viral protein kinase UL97, not protein kinase C, for disruption of nuclear lamina and nuclear egress in infected cells. J. Virol. 2014, 88, 249-262.

113. Marschall, M.; Marzi, A.; aus dem Siepen, P.; Jochmann, R.; Kalmer, M.; Auerochs, S.; Lischka, P.; Leis, M.; Stamminger, T. Cellular p32 recruits cytomegalovirus kinase pUL97 to redistribute the nuclear lamina. J. Biol. Chem. 2005, 280, 33357-33367.

114. Azzeh, M.; Honigman, A.; Taraboulos, A.; Rouvinski, A.; Wolf, D.G. Structural changes in human cytomegalovirus cytoplasmic assembly sites in the absence of UL97 kinase activity. Virology 2006, 354, 69-79.

115. Chee, M.S.; Bankier, A.T.; Beck, S.; Bohni, R.; Brown, C.M.; Cerny, R.; Horsnell, T.; Hutchison, C.A., 3rd; Kouzarides, T.; Martignetti, J.A.; et al. Analysis of the protein-coding content of the sequence of human cytomegalovirus strain AD169. Curr. Top. Microbiol. Immunol. 1990, 154, 125-169.

116. Adamo, J.E.; Schroer, J.; Shenk, T. Human cytomegalovirus TRS1 protein is required for efficient assembly of DNA-containing capsids. J. Virol. 2004, 78, 10221-10229.

117. Marshall, E.E.; Geballe, A.P. Multifaceted evasion of the interferon response by cytomegalovirus. J. Interferon Cytokine Res. 2009, 29, 609-619.

118. Hakki, M.; Marshall, E.E.; de Niro, K.L.; Geballe, A.P. Binding and nuclear relocalization of protein kinase R by human cytomegalovirus TRS1. J. Virol. 2006, 80, 11817-11826.

119. Strang, B.L.; Geballe, A.P.; Coen, D.M. Association of human cytomegalovirus proteins IRS1 and TRS1 with the viral DNA polymerase accessory subunit UL44. J. Gen. Virol. 2010, 91, 2167-2175.

120. Strang, B.L.; Bender, B.J.; Sharma, M.; Pesola, J.M.; Sanders, R.L.; Spector, D.H.; Coen, D.M. A mutation deleting sequences encoding the amino terminus of human cytomegalovirus UL84 impairs interaction with UL44 and capsid localization. J. Virol. 2012, 86, 11066-11077. 
121. Schauflinger, M.; Fischer, D.; Schreiber, A.; Chevillotte, M.; Walther, P.; Mertens, T.; von Einem, J. The tegument protein UL71 of human cytomegalovirus is involved in late envelopment and affects multivesicular bodies. J. Virol. 2011, 85, 3821-3832.

122. Meissner, C.S.; Suffner, S.; Schauflinger, M.; von Einem, J.; Bogner, E. A leucine zipper motif of a tegument protein triggers final envelopment of human cytomegalovirus. J. Virol. 2012, 86, 3370-3382.

123. Borst, E.M.; Kleine-Albers, J.; Gabaev, I.; Babic, M.; Wagner, K.; Binz, A.; Degenhardt, I.; Kalesse, M.; Jonjic, S.; Bauerfeind, R.; et al. The human cytomegalovirus UL51 protein is essential for viral genome cleavage-packaging and interacts with the terminase subunits pUL56 and pul89. J. Virol. 2013, 87, 1720-1732.

124. Britt, W.J.; Jarvis, M.; Seo, J.Y.; Drummond, D.; Nelson, J. Rapid genetic engineering of human cytomegalovirus by using a lambda phage linear recombination system: Demonstration that pp28 (UL99) is essential for production of infectious virus. J. Virol. 2004, 78, 539-543.

125. Seo, J.Y.; Britt, W.J. Cytoplasmic envelopment of human cytomegalovirus requires the postlocalization function of tegument protein pp28 within the assembly compartment. J. Virol. 2007, 81, 6536-6547.

126. Seo, J.Y.; Britt, W.J. Multimerization of tegument protein pp28 within the assembly compartment is required for cytoplasmic envelopment of human cytomegalovirus. J. Virol. 2008, 82, 6272-6287. 
Reprinted from Viruses. Cite as: Noriega, V.M.; Gardner, T.J.; Redmann, V.; Bongers, G.; Lira, S.A.; Tortorella, D. Human Cytomegalovirus US28 Facilitates Cell-to-Cell Viral Dissemination. Viruses 2014, 6, 1202-1218.

Article

\title{
Human Cytomegalovirus US28 Facilitates Cell-to-Cell Viral Dissemination
}

\author{
Vanessa M. Noriega ${ }^{1}$, Thomas J. Gardner ${ }^{1}$, Veronika Redmann ${ }^{2}$, Gerold Bongers ${ }^{3}$, \\ Sergio A. Lira ${ }^{3}$ and Domenico Tortorella ${ }^{1, *}$
}

1 Department of Microbiology, Icahn School of Medicine at Mount Sinai, One Gustave L. Levy Place, New York, NY 10029, USA; E-Mails: Vanessa.Noriega@mssm.edu (V.M.N.); Thomas.Gardner@mssm.edu (T.J.G.)

2 Department of Pathology and Immunology, Washington University School of Medicine, 660 South Euclid Avenue, St. Louis, MO 63110, USA; E-Mail: vredmann@pathology.wustl.edu

3 Immunology Institute, Icahn School of Medicine at Mount Sinai, One Gustave L. Levy Place, New York, NY 10029, USA; E-Mails: Gerold.Bongers@mssm.edu (G.B.);

Sergio.Lira@mssm.edu (S.A.L.)

* Author to whom correspondence should be addressed;

E-Mail: Domenico.Tortorella@mssm.edu; Tel.: +1-212-241-5447; Fax: +1-212-241-7335.

Received: 20 January 2014; in revised form: 1 March 2014 / Accepted: 4 March 2014 /

Published: 12 March 2014

\begin{abstract}
Human cytomegalovirus (HCMV) encodes a number of viral proteins with homology to cellular G protein-coupled receptors (GPCRs). These viral GPCRs, including US27, US28, UL33, and UL78, have been ascribed numerous functions during infection, including activating diverse cellular pathways, binding to immunomodulatory chemokines, and impacting virus dissemination. To investigate the role of US28 during virus infection, two variants of the clinical isolate TB40/E were generated: TB40/E-US28 ${ }^{\mathrm{YFP}}$ expressing a C-terminal yellow fluorescent protein tag, and $\mathrm{TB} 40 / \mathrm{E}-\mathrm{FLAG}^{\mathrm{YFP}}$ in which a FLAG-YFP cassette replaces the US28 coding region. The TB40/E-US28 ${ }^{\mathrm{YFP}}$ protein localized as large perinuclear fluorescent structures at late times post-infection in fibroblasts, endothelial, and epithelial cells. Interestingly, $\mathrm{US} 28^{\mathrm{YFP}}$ is a non-glycosylated membrane protein throughout the course of infection. US28 appears to impact cell-to-cell spread of virus, as the $\Delta$ US28 virus (TB40/E-FLAG ${ }^{\mathrm{YFP}}$ ) generated a log-greater yield of extracellular progeny whose spread could be significantly neutralized in fibroblasts. Most strikingly, in epithelial cells, where dissemination of virus occurs exclusively by the cell-to-cell route, TB40/E-FLAG ${ }^{\mathrm{YFP}}(\Delta \mathrm{US} 28)$ displayed a
\end{abstract}


significant growth defect. The data demonstrates that HCMV US28 may contribute at a late stage of the viral life cycle to cell-to-cell dissemination of virus.

Keywords: human cytomegalovirus; BAC recombineering; viral GPCR US28; virus dissemination; virus growth; membrane protein biology

\section{Introduction}

Human cytomegalovirus (HCMV) is a widespread pathogen that infects a vast majority of the world's population [1]. HCMV is the prototypic $\beta$-herpesvirus, characterized by its extended replication cycle, restricted host range, and cytopathic effect of pronounced cell swelling [2]. Infection of the healthy, immunocompetent host is typically asymptomatic, with pressure from the immune system leading to establishment of lifelong latent infection within cells of the myeloid lineage [3]. Infection of the immunologically immature or reactivation of latent infection during times of immunosuppression can result in significant disease [4]. In fact, HCMV infection during solid organ or hematopoietic stem cell transplant can have severe implications for the host and can ultimately prove fatal [5].

The exceptionally large HCMV genome encodes for over 200 genes [6], including four putative homologs of cellular G protein-coupled receptors (GPCRs): the HCMV-specific US27 and US28, and the $\beta$-herpesviruses-conserved UL33 and UL78 [7]. GPCRs, also known as seven-transmembrane domain proteins, are integral membrane receptors that sense extracellular ligands to trigger signal transduction networks and coordinate cellular responses [8]. Once activated, these receptors undergo a conformational change, causing activation of an associated heterotrimeric $\mathrm{G}$ protein and leading to production of intracellular secondary messenger molecules to induce downstream signaling pathways. HCMV infection is known to modulate a number of host cellular responses, including intracellular calcium levels, cyclic AMP (cAMP) production, inositol phosphate hydrolysis, and activation of phosphatidylinositol-3-kinase (PI3K) [9]. As constituents of the virion [10-12], several of the HCMV-encoded GPCRs regulate a number of these pathways immediately following infection. Both US28 and UL33 signal constitutively and can alter inositol phosphate production and activation of NF-kB and cAMP response elements (CRE) [13,14]. Furthermore, US28 can bind CC chemokines to induce increases in intracellular calcium levels and migration of infected cells $[15,16]$. Although it shows no constitutive activity [17], US27 was recently found to enhance signaling mediated by endogenous CXCR4, resulting in enhanced calcium mobilization and chemotaxis [18]. To date no activating ligands or signaling properties have been attributed to UL78.

Another intriguing characteristic accorded to HCMV-encoded GPCRs is their contribution to dissemination of virus in vitro. UL78 appears to impact virus growth in both endothelial and epithelial cells [12]. Additionally, UL78 supports infection by coordinating the timely delivery of viral DNA into the nuclei of infected cells [12]. US27 is required for efficient spread by the extracellular route and influences virus growth in fibroblasts and endothelial cells [19]. Expression of the murine cytomegalovirus (MCMV) ortholog M33 protein was shown to be necessary for virus dissemination in vivo but not in tissue culture [20]. An MCMV mutant lacking the GPCR M78 
exhibited a growth defect in culture and reduced pathogenicity in mice [21]. The implication of HCMV-encoded GPCRs as virulence factors to enhance infection is quite intriguing, as their presence within infected cell membranes [22,23] could allow cell-cell communication and modulation of signaling networks within neighboring cells to facilitate propagation.

To determine the role of US28 in HCMV dissemination, mutational analysis of the TB40/E clinical isolate was performed. A YFP derivative of US28 (TB40/E-US28 ${ }^{\mathrm{YFP}}$ ) localized as large perinuclear structures at late times of infection in fibroblasts, endothelial, and epithelial cells. At these late times, US28 ${ }^{\mathrm{YFP}}$ was integrated into cellular membranes, further validating its presence at the interface of infected cells. A $\Delta$ US28 mutant (TB40/E-FLAG ${ }^{\mathrm{YFP}}$ ) produced increased levels of extracellular virus as assayed by both multi-step and single-step growth kinetics. Extracellular virus produced by the $\triangle \mathrm{US} 28$ mutant could be neutralized by the addition of HCMV glycoprotein-specific antibodies and spread of TB40/E-FLAG ${ }^{\mathrm{YFP}}$ by the cell-to-cell route was abrogated in fibroblasts and epithelial cells. These findings implicate the viral GPCR US28 as a factor contributing to cellular dissemination of HCMV.

\section{Results}

\subsection{Generation of HCMV TB40/E US28 Variants}

To extend on studies of viral GPCRs as virulence factors, derivatives of the HCMV clinical isolate TB40/E were generated (Figure 1a). The wild type TB40/E bacterial artificial chromosome (BAC) (herein termed TB40/E wt) was altered to express a chimeric protein in which the carboxy terminus of the US28 coding region was amended with a yellow fluorescent protein tag (TB40/E-US28 ${ }^{\mathrm{YFP}}$ ) (Figure 1a). A second variant was generated in which the US28 coding region was replaced with a DNA cassette encoding a FLAG-tagged YFP chimera (TB40/E-FLAG ${ }^{\text {YFP }}$ ) (Figure 1a). To confirm abrogation of US28 message in the $\triangle \mathrm{US} 28$ (FLAG ${ }^{\mathrm{YFP}}$ ) virus, MRC5 lung fibroblasts were mock-infected or infected with TB40/E wt, TB40/E-US28 ${ }^{\text {YFP }}$ or TB40/E-FLAG ${ }^{\text {YFP }}$ and RNA harvested at 48 hours post-infection, a time when US28 should be abundantly transcribed [24]. RT-PCR analysis with primers specific to a region within US28 demonstrated that US28 messenger RNA continued to be generated during infection with TB40/E wt and TB40/E-US28 ${ }^{\text {YFP }}$, but not with the $\Delta \mathrm{US} 28$ virus (Figure $1 \mathrm{~b}$, lanes $1-4$ ). To further confirm expression of our TB40/E YFP chimeras, fibroblasts were either mock-infected or infected with TB40/E-US28 ${ }^{\mathrm{YFP}}$ or TB40/E-FLAG ${ }^{\mathrm{YFP}}$, harvested at various times post-infection, and analyzed by immunoblot for expression of YFP (Figure 1c). Kinetic analysis confirmed US28 ${ }^{\text {YFP }}$ expression throughout the time course, with maximal expression at 72 hours post-infection (Figure 1c, lanes 1-6). US $28^{\mathrm{YFP}}$ migrated as a broad polypeptide species of approximately $65 \mathrm{kD}$ (Figure 1c, lanes

1-6). FLAG ${ }^{\mathrm{YFP}}$ followed a similar time course of expression, peaking at 72 hours post-infection (Figure 1c, lanes 7-11). When visualized by fluorescence microscopy, the majority of US28 YFP localized intracellularly to vesicular structures concentrated around the nucleus (Figure 1d, center), confirming earlier data for US28 localization in transiently transfected cells [22]. A small portion of US28 ${ }^{\mathrm{YFP}}$ appeared to localize to the cell surface, as US28 undergoes constitutive endocytosis and recycling [22]. TB40/E-FLAG ${ }^{\mathrm{YFP}}$-infected cells expressed fluorescence throughout the cell (Figure 1d, right) while the TB40/E wt parental virus did not express YFP (Figure 1d, left). Taken 
together, the data demonstrates that TB40/E variants of the US28 coding region had been generated to ascertain its role in HCMV virulence.

Figure 1. Generation of TB40/E-US28 variants. (a) Using a bacterial artificial chromosome (BAC) recombineering approach Human cytomegalovirus (HCMV) TB40/E variants were generated that express either chimeric US28 containing a carboxy-terminal YFP tag (US28 ${ }^{\text {YFP }}$ ) or a US28 deletion mutant where the US28 ORF has been replaced with an engineered FLAG-YFP cassette (FLAG ${ }^{\text {YFP }}$ ). YFP sequences are denoted by the diagonally hatched box; FLAG sequences are denoted by the horizontally striped box. $\mathrm{TR}$, terminal repeat; U, unique sequences; IR, inverted repeat; L, long; S, short. (b) Fibroblasts mock-infected or infected $(\mathrm{MOI}=5)$ with TB40/E wt or TB40/E-US28 variants were harvested 48 hours post-infection and subjected to RT-PCR with primers specific to US28 (lanes 1-5) or $\beta$-actin (lanes 6-10). A sample lacking RNA ((-)RNA) was included as a negative control. HCMV US28, $\beta$-actin, and relative DNA standards are indicated. (c) Fibroblasts mock-infected or infected $(\mathrm{MOI}=5)$ with TB40/E-US28 ${ }^{\text {YFP }}$ or TB40/E-FLAG ${ }^{\mathrm{YFP}}$ were harvested at the indicated times and subjected to SDS-PAGE and immunoblot analysis. US28 ${ }^{\mathrm{YFP}}, \mathrm{FLAG}^{\mathrm{YFP}}$, GAPDH, and molecular weight standards are indicated. (d) Fibroblasts infected $(\mathrm{MOI}=5)$ with TB40/E wt, TB40/E-US28 ${ }^{\mathrm{YFP}}$ or TB40/E-FLAG ${ }^{\text {YFP }}$ were harvested 48 hours post-infection and visualized using the EVOS Cell Imaging Systems at $60 \times$ magnification.

(a)

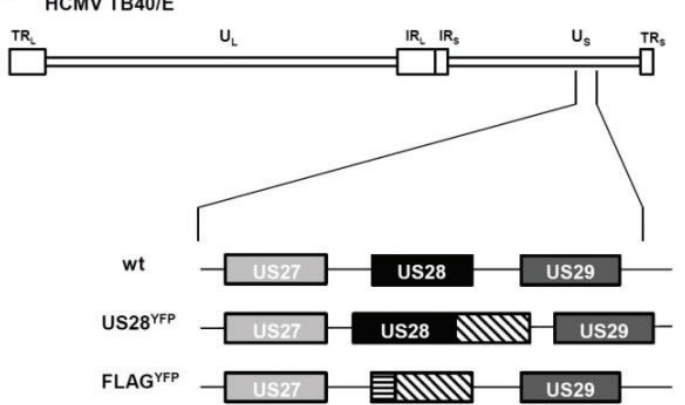

(b)

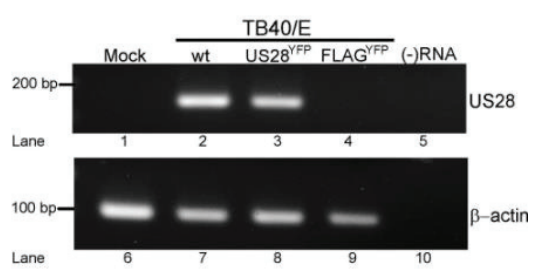

(c)

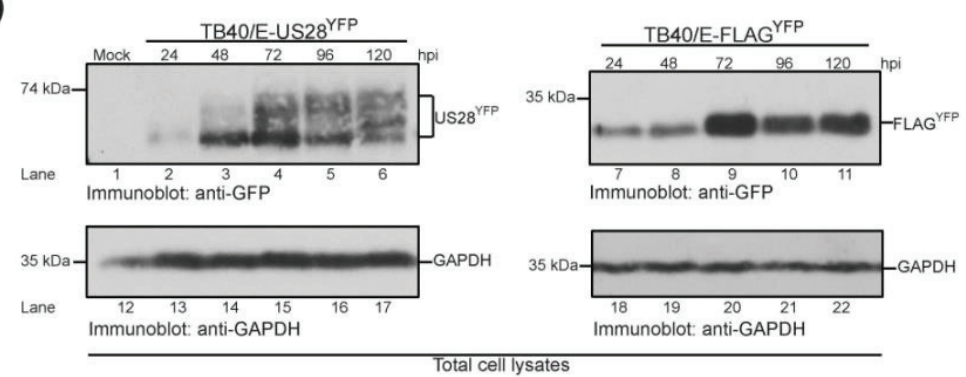

(d)
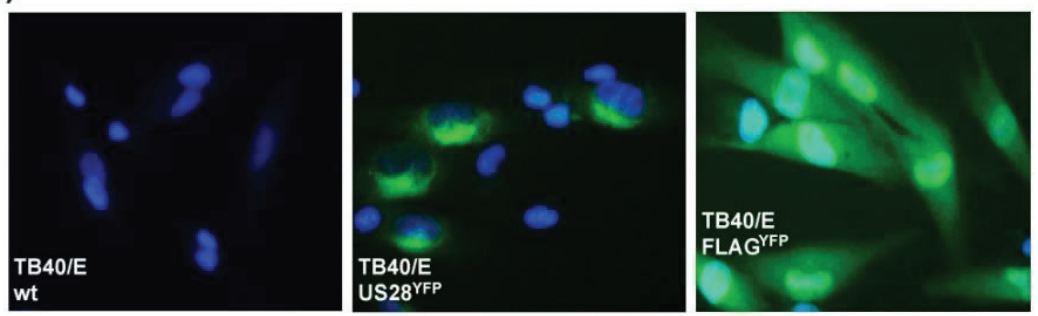


\subsection{HCMV US2 ${ }^{Y F P}$ Localizes as Large Vesicular Structures at Late Times of Infection}

To visualize a time course of US28 ${ }^{\mathrm{YFP}}$ expression, confocal microscopy was performed on fibroblasts infected with TB40/E-US28 ${ }^{\text {YFP }}$ (Figure 2a). At early times post-infection, US28 ${ }^{\text {YFP }}$ localized diffusely throughout the cell (Figure $2 \mathrm{a}$, left). As infection progressed, US28 ${ }^{\mathrm{YFP}}$ coalesced into intense fluorescent perinuclear structures focused on one side of the nucleus (Figure 2a, 48, 72 hpi, arrows). By 72 hours post-infection, these large structures seemed to encroach on the nuclear space (Figure 2a, arrows). These organelles most likely represent viral assembly zones, as US28 has been proposed to be incorporated into assembling virions.

To determine if US28 $8^{\mathrm{YFP}}$ localization into large perinuclear structures late during infection was cell-type specific, infections of human umbilical vein endothelial cells (HUVECs), human microvascular endothelial cells (HMVECs), and ARPE-19 epithelial cells were performed (Figure 2b). Infection with TB40/E-US28 ${ }^{\text {YFP }}$ caused the formation of intense fluorescent granular structures in all cell types assayed (Figure $2 b$, left column). In comparison infection with $\mathrm{TB} 40 / \mathrm{E}_{-F L A G}{ }^{\mathrm{YFP}}$ resulted in diffuse fluorescence throughout the cell for each cell type (Figure $2 \mathrm{~b}$, right column). US28 ${ }^{\text {YFP }}$ expression in endothelial cells was similar to cell surface staining on smooth muscles cells expressing US28, in which the viral GPCR accumulated toward the leading edge of migrating cells [16]. Strikingly, in epithelial cells, US28 $8^{\text {YFP }}$ also appeared to converge at the junction of neighboring infected cells (Figure 2b, bottom left, arrow). Taken together, the results demonstrate that US28 localizes to large perinuclear structures that may represent areas of infectious virus production.

\subsection{Characterization of US2 $8^{Y F P}$ in HCMV-Infected Cells}

\subsubsection{HCMV US28 ${ }^{\mathrm{YFP}}$ Is Integrated into Dense Vesicular Bodies}

The viral GPCRs US27 and UL33 localize to virus-wrapping membranes on HCMV-infected cells [23]. To determine if US28, at late time points post-infection, also traffics to dense vesicles consisting of large complexes, subcellular fractionation was performed on TB40/E-US28 ${ }^{\mathrm{YFP}}$ - and TB40/E-FLAG ${ }^{\mathrm{YFP}}$-infected fibroblasts (Figure 3a). At 72 hours post-infection, cells were lysed using a ball-bearing homogenizer and subjected to two centrifugation steps: nuclei and heavy/dense organelles were spun down at $15,000 \times \mathrm{g}$, followed by separation of cellular membrane and cytoplasm by high-speed centrifugation at $120,000 \times$ g. A substantial amount of US28 $8^{\mathrm{YFP}}$ and FLAG $^{\text {YFP }}$ localized to the $15,000 \times \mathrm{g}$ fraction containing heavy organelles (Figure 3a, lanes 1-3). As US28 ${ }^{\text {YFP }}$ and FLAG ${ }^{\text {YFP }}$ polypeptides are being abundantly synthesized at this late time point of infection (Figure 1c), their localization to this fraction may represent ER membranes contiguous with the nucleus, large protein complexes, and large dense membrane vesicles. Considering the levels of US28 ${ }^{\mathrm{YFP}}$ in the $15,000 \times \mathrm{g}$ fraction, only a small amount of two distinct US28 ${ }^{\mathrm{YFP}}$ species were localized to cellular membranes after high-speed centrifugation (Figure 3a, lane 5), suggesting that US28 ${ }^{\mathrm{YFP}}$ traffics with large protein complexes in dense membrane fractions. Interestingly, FLAG $^{\text {YFP }}$ was also found in this membranous fraction (Figure 3a, lane 6). This may simply represent contamination from the cytoplasmic fraction, as the majority of FLAG ${ }^{\text {YFP }}$, and not US2 $8^{\text {YFP }}$, localizes to the cytoplasm (Figure 3a, lane 8-9). As a control, immunoblot analysis of 
viral glycoproteins was also performed (Figure 3a, lanes 10-18). The glycoprotein gB also localized to the dense cellular membrane fraction (Figure 3a, lanes 10-15) and not the cytoplasmic fraction (Figure 3a, lanes 16-18). A similar result was found for the viral glycoprotein gH (data not shown). Remarkably, US28 ${ }^{\text {YFP }}$ trafficked within dense cellular membranes as early as 24 hours post-infection (Figure 3b, lane 2) probably due to active translation on large ER membranes. Alternatively, the localization of US28 ${ }^{\mathrm{YFP}}$ to the $15,000 \times \mathrm{g}$ fraction may be due to integration of US28 from the HCMV virion into the plasma membrane following infection, but additional experiments are needed to confirm this. Taken together, the data demonstrates that US $28^{\text {YFP }}$ is found in mostly large membrane complexes and localizes to dense vesicles, likely virus assembly zones late during infection.

Figure 2. Formation of US28-expressing structures late during HCMV infection. (a) Confocal microscopy was performed on fibroblasts either mock-infected or infected with TB40/E-US28 ${ }^{\mathrm{YFP}}(\mathrm{MOI}=5)$. At various times post-infection cells were fixed and analyzed using an ImageXpress Ultra plate-scanning confocal microscope. (b) Endothelial and epithelial cells were infected with either TB40/E-US28 ${ }^{\text {YFP }}$ (left) or -FLAG ${ }^{\text {YFP }}$ (right) $(\mathrm{MOI}=25)$ and visualized at 4 days post-infection using the EVOS Cell Imaging Systems at $60 \times$ magnification.

(a)

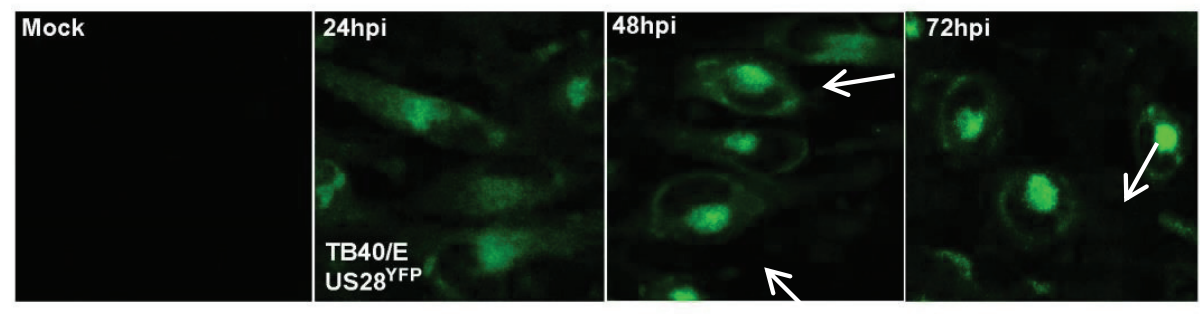

(b)
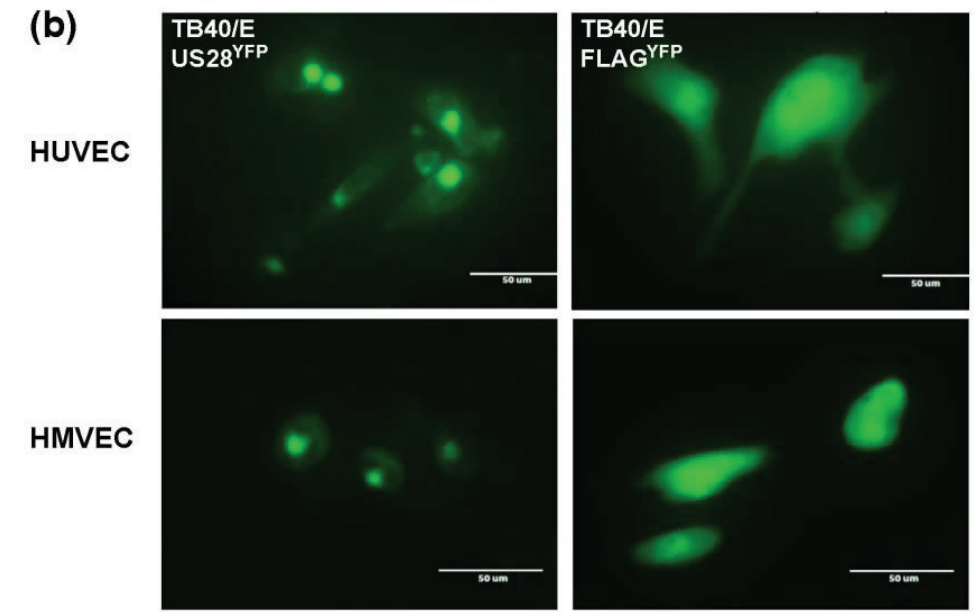

HUVEC

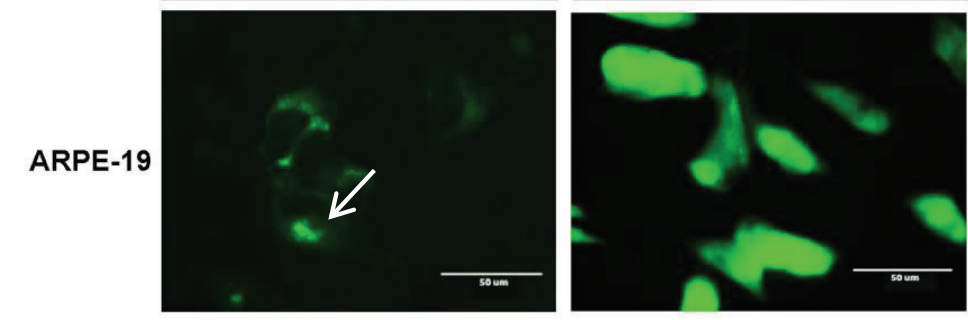


Figure 3. HCMV US28 is a non-glycosylated membrane protein incorporated into infected cells. Fibroblasts mock-infected or infected $(\mathrm{MOI}=5)$ with either TB40/E-US28 ${ }^{\text {YFP }}$ or TB40/E-FLAG ${ }^{\text {YFP }}$ were subjected to subcellular fractionation at 72 (a) or 24 (b) hours post-infection. Cell pellets from the 15,000 and 120,000 $\times \mathrm{g}$ centrifugations $(15 \mathrm{k} \times \mathrm{g}$ pellet, $120 \mathrm{k} \times \mathrm{g}$ pellet $)$ and the $120,000 \times \mathrm{g}$ supernatant $(120 \mathrm{k} \times \mathrm{g}$ supe) were resolved by SDS-PAGE and subjected to immunoblot analysis. $\mathrm{US} 28^{\mathrm{YFP}}, \mathrm{FLAG}^{\mathrm{YFP}}, \mathrm{gB}$, and molecular weight standards are indicated. $\mathrm{gB} *$ indicates the mature form of glycoprotein B. (c) Fibroblasts infected with TB40/E-US28 YFP $(\mathrm{MOI}=5)$ were harvested at the indicated time points and left non-treated $(\mathrm{NT})$ or treated with EndoH $(\mathrm{H})$ or PNGaseF $(\mathrm{F})$. US28 ${ }^{\mathrm{YFP}}$, glycosylated MHC class I heavy chains $(\mathrm{HC}(+) \mathrm{CHO})$, deglycosylated $\mathrm{MHC}$ class I heavy chains $(\mathrm{HC}(-) \mathrm{CHO})$, GAPDH, and molecular weight standards are indicated.

(a)
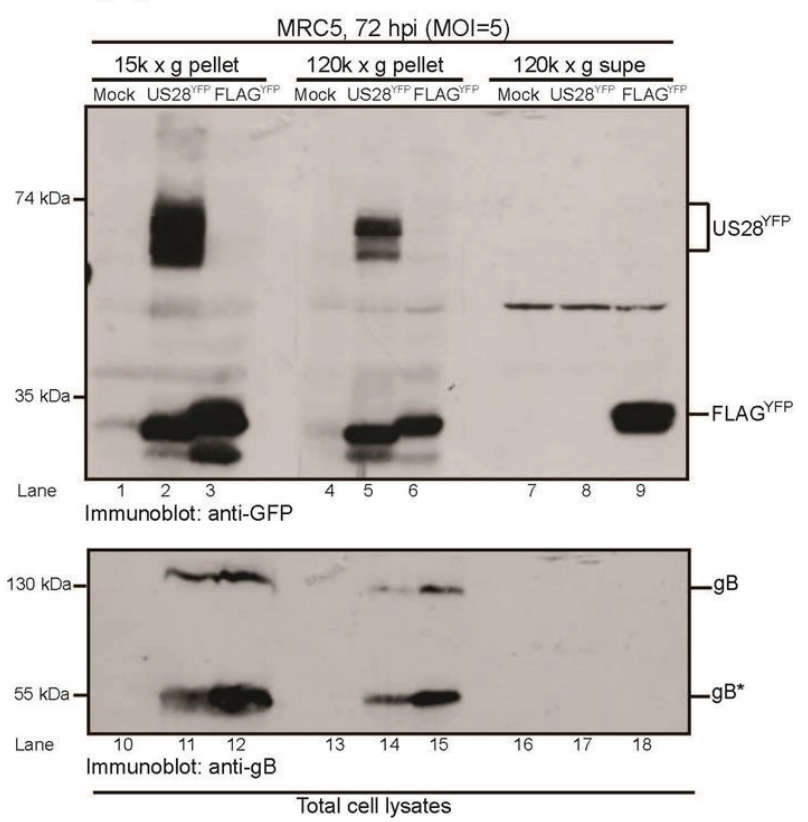

(b)

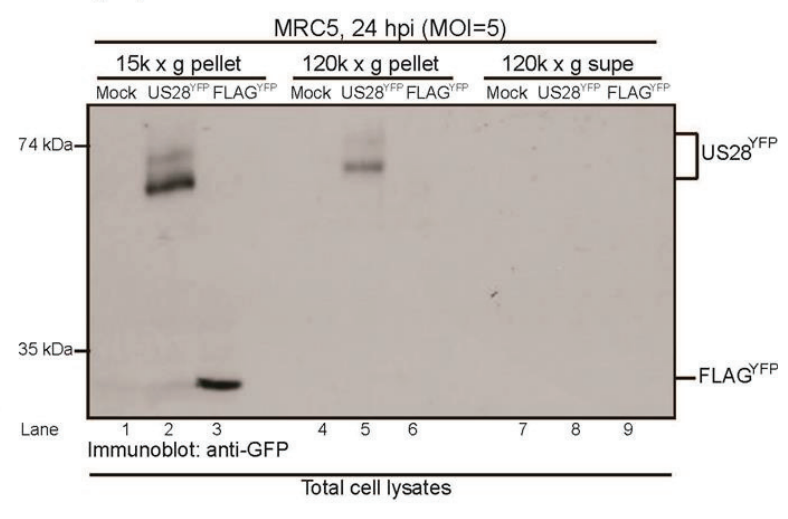

\section{(c)}
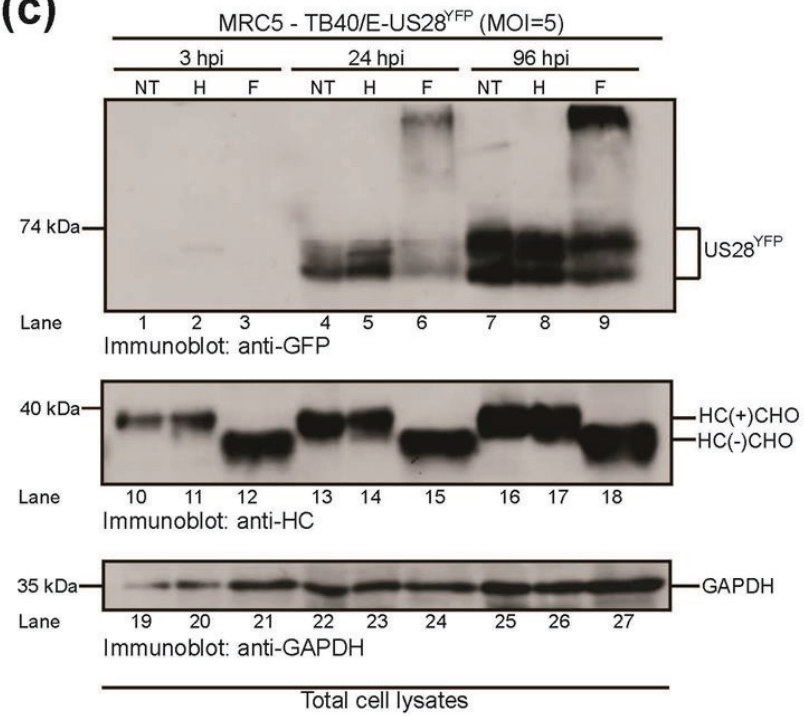


\subsubsection{HMCV US28 Is a Non-Glycosylated Membrane Protein}

As integral membrane proteins, the extracellular portions of GPCRs have the potential to be glycosylated. Both US27 and UL33 are heavily glycosylated while present in infected cell membranes $[10,11]$. The US28 protein annotated from the HCMV genome contains a possible N-linked glycosylation site (amino acids 30-32, NQS). Therefore, to determine if US28 is a glycosylated membrane protein, lysates prepared from TB40/E-US28 ${ }^{\mathrm{YFP}}$-infected fibroblasts at various times post-infection were subjected to digestion by either endoglycosidase $\mathrm{H}(\mathrm{EndoH})$ or peptide: N-glycosidase F (PNGaseF) (Figure 3c). EndoH cleaves high mannose glycans from the core of N-linked glycoproteins while PNGaseF hydrolyzes nearly all types of N-linked glycans from proteins [25]. Unexpectedly, US28 ${ }^{\mathrm{YFP}}$ was insensitive to cleavage by either EndoH or PNGaseF throughout the time course (Figure 3c, lanes 1-9), suggesting that US28 is not glycosylated despite being a membrane protein and trafficking through the secretory compartment [22]. As a control, the sensitivity of the known glycoprotein major histocompatibility complex (MHC) class I heavy chain to cleavage by EndoH and PNGaseF was determined (Figure 3c, lanes 10-18). Class I heavy chains traffic rapidly through the ER, where high-mannose glycans are acquired and cleaved, and is therefore resistant to EndoH digestion [26] (Figure 3c, lanes 11, 14, 17). Class I heavy chains were completely sensitive to cleavage by PNGaseF, resulting in loss of its single glycan (Figure 3c, lanes $12,15,18)$. The findings reveal that HCMV US28 is a unique non-glycosylated membrane protein.

\subsection{Functional Analysis of TB40/E US28 Variants}

\subsubsection{TB40/E $\Delta$ US28 Accumulates Increased Amounts of Extracellular Virus in Fibroblasts}

To determine the growth properties of the TB40/E US28 variants, fibroblasts were infected at both high multiplicity, to study single step growth, and low multiplicity, to determine multi-step growth, and production of infectious extracellular progeny was measured (Figure 4a,b). At high multiplicity of infection (MOI), TB40/E wt and TB40/E-US28 ${ }^{\mathrm{YFP}}$ grew to comparable titers (Figure $4 \mathrm{a}$, solid line $v s$. dotted line). In comparison, TB40/E-FLAG ${ }^{\mathrm{YFP}}(\Delta \mathrm{US} 28)$ displayed a 10 -fold increase in the accumulation of extracellular virus (Figure $4 \mathrm{a}$, dashed line). This effect was amplified at low multiplicity of infection, where TB40/E-FLAG ${ }^{\mathrm{YFP}}$ displayed a 100 -fold increase in viral titers over TB40/E-US28 ${ }^{\mathrm{YFP}}$ (Figure 4b, solid line $v s$. dashed line). The data demonstrates that the loss of US28 results in increased production of extracellular virus during HCMV infection.

\subsubsection{US28 Modulates HCMV Cell-to-Cell Dissemination}

An HCMV mutant virus lacking the tegument phosphoprotein pp28 fails to accumulate extracellular progeny yet mediates cell-to-cell spread of tegument-coated capsids [27]. The accumulation of extracellular virus seen during $\Delta \mathrm{US} 28$ infection could result from a blockade at the level of cell-to-cell spread, thus leading to re-routing of infectious virions into the extracellular milieu. To determine if the $\Delta \mathrm{US} 28$ virus has a defect in cell-to-cell spread of virus, infections were performed in epithelial cells, where spread of TB40/E is exclusively cell-associated [19]. ARPE-19 cells were infected at low multiplicity with either TB40/E-US28 ${ }^{\mathrm{YFP}}$ or TB40/E-FLAG ${ }^{\mathrm{YFP}}$ and infectious progeny measured by determining the titer of cell-associated virus (Figure 4c). In 
comparison to fibroblasts, where TB40/E-FLAG ${ }^{\text {YFP }}$ displayed increased growth properties, the $\Delta \mathrm{US} 28$ virus exhibited a growth defect in epithelial cells (Figure 4c, dashed line). In fact, while titers of cell-associated TB40/E-US28 ${ }^{\text {YFP }}$ increased over the time course, accumulation of intracellular virus became stagnant during TB40/E-FLAG ${ }^{\mathrm{YFP}}$ infection (Figure 4c, solid line vs. dashed line). Taken together, it appears that US28 may contribute to cell-to-cell dissemination during HCMV infection.

Figure 4. HCMV US28 modulates cell-to-cell spread of virus. Infectious extracellular progeny from fibroblasts infected at $5 \mathrm{PFU} /$ cell (a) or $0.1 \mathrm{PFU} /$ cell (b) with TB40/E wt, TB40/E-US28 ${ }^{\mathrm{YFP}}$, or TB40/E-FLAG ${ }^{\mathrm{YFP}}$ were measured by $\mathrm{TCID}_{50}$ assay. Viral titers were assayed in duplicate. Error bars represent standard deviation of the mean. (c) ARPE-19 cells were infected at $0.1 \mathrm{PFU} /$ cell with TB40/E-US28 ${ }^{\mathrm{YFP}}$ or TB40/E-FLAG ${ }^{\mathrm{YFP}}$ and at the indicated times post-infection cell-associated virus was determined by $\mathrm{TCID}_{50}$ assay. Viral titers were assayed in duplicate. Error bars represent standard deviation of the mean.

(a)

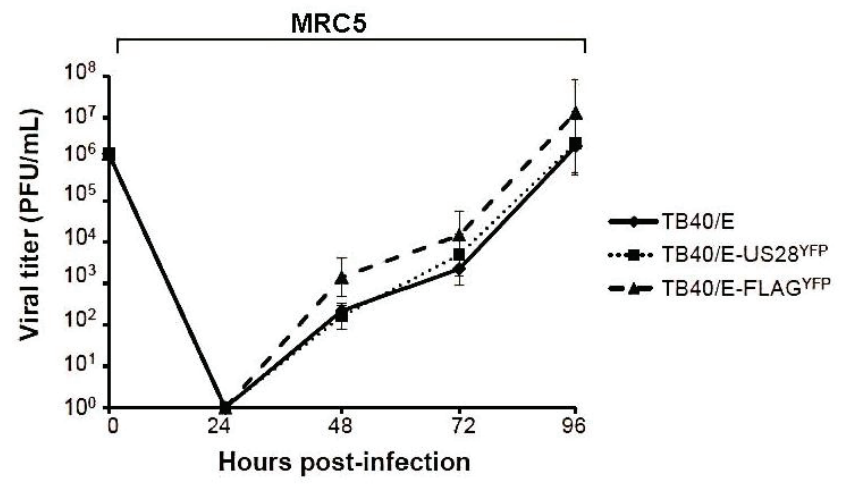

(b)

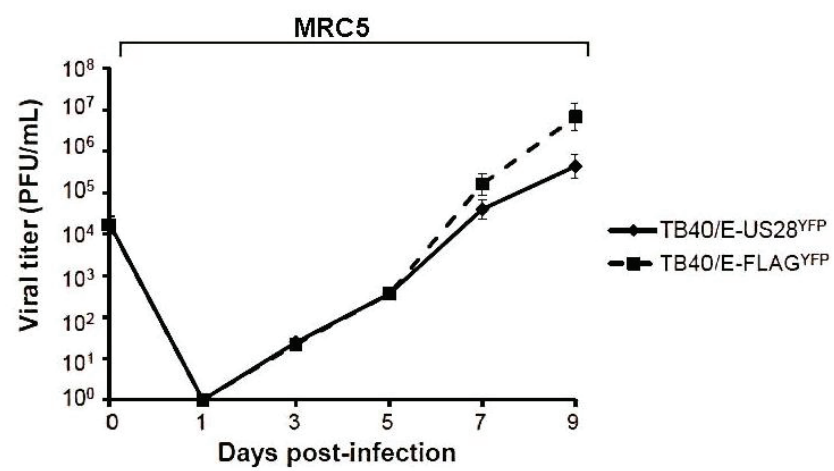

(c)

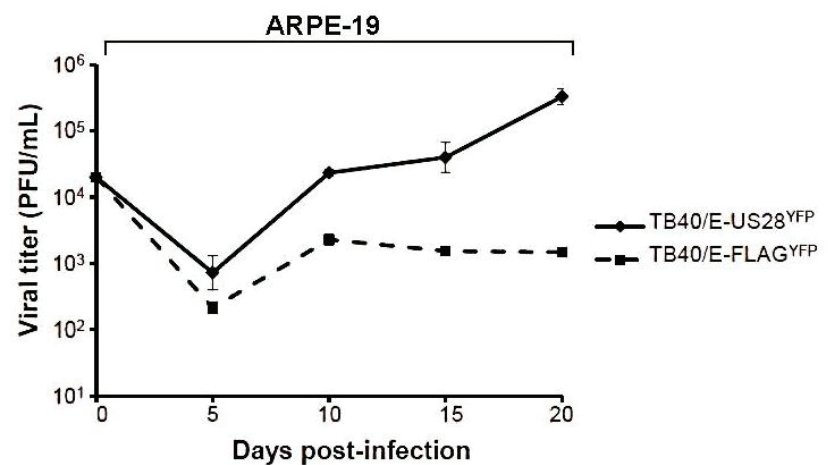




\subsection{Inhibition of TB40/E $\triangle U S 28$ Dissemination by Anti-HCMV Neutralizing Antibody}

When grown in fibroblasts TB40/E-FLAG ${ }^{\mathrm{YFP}}$ produced substantial extracellular virus, suggesting that, in the absence of US28, the virus uses the extracellular route as the main route of dissemination. Therefore, an inhibition of infectious virus in the supernatant of $\Delta U S 28$-infected cells would result in a significant hindrance to dissemination. To determine if TB40/E-FLAG ${ }^{\mathrm{YFP}}$ spread could be ablated in fibroblasts, infected cells were cultured in the presence of the HCMV neutralizing antibody $14-4 \mathrm{~b}$ that recognizes glycoprotein $\mathrm{H}(\mathrm{gH})$ [28]. At approximately two weeks post-infection cells were analyzed by a fluorescence microplate cytometer (Figure 5a) and by fluorescence microscopy (Figure 5b). When grown in the presence of $14-4 \mathrm{~b}$, TB40/E-US28 ${ }^{\mathrm{YFP}}$ total fluorescence was slightly decreased (Figure 5a, left). In comparison, TB40/E-FLAG ${ }^{\text {YFP }}$ fluorescence was significantly reduced in the presence of $14-4 \mathrm{~b}$ (Figure $5 \mathrm{a}$, right). Fluorescence microscopy revealed that although TB40/E-US28 ${ }^{\text {YFP }}$ grown in the presence of $14-4 \mathrm{~b}$ created smaller plaques, cellular syncytia continued to be formed (Figure 5b). In contrast, TB40/E-FLAG ${ }^{\text {YFP }}$ growth was restricted to small pockets of infection in the presence of $14-4 b$, with the absence of plaque formation (Figure $5 b$ ). These findings demonstrate that US28 impacts cell-to-cell spread of virus as TB40/E- $\Delta$ US28 is hindered in its dissemination in culture upon addition of HCMV neutralizing antibodies.

Figure 5. TB40/E $\Delta \mathrm{US} 28$ displays a growth defect when required to use the cell-to-cell route of dissemination. Fibroblasts infected $(\mathrm{MOI}=0.01)$ with either TB40/E-US28YFP or TB40/E-FLAGYFP were cultured in the presence of the HCMV neutralizing antibody 14-4b. Two weeks post-infection cells were analyzed by fluorescent microplate reader (a) or by fluorescence microscopy (b). For (a) YFP fluorescence was assayed in triplicate. Error bars represent standard deviation of the mean; ${ }^{*} p<0.05$, Student's one tailed T-test.

(a)

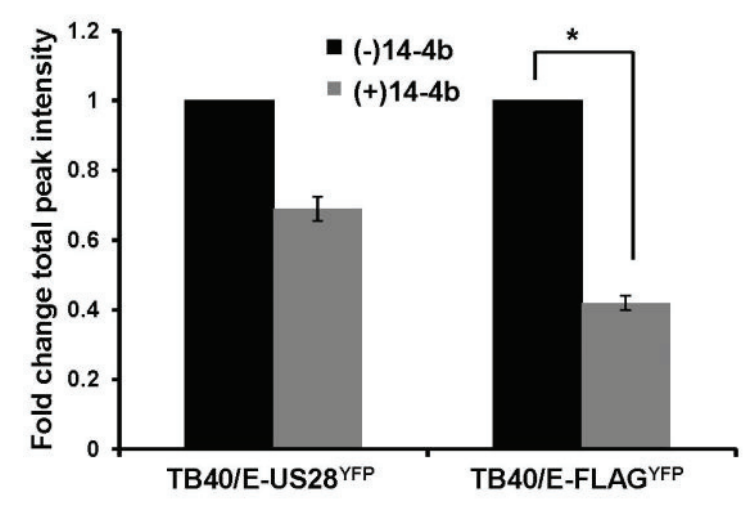

(b)
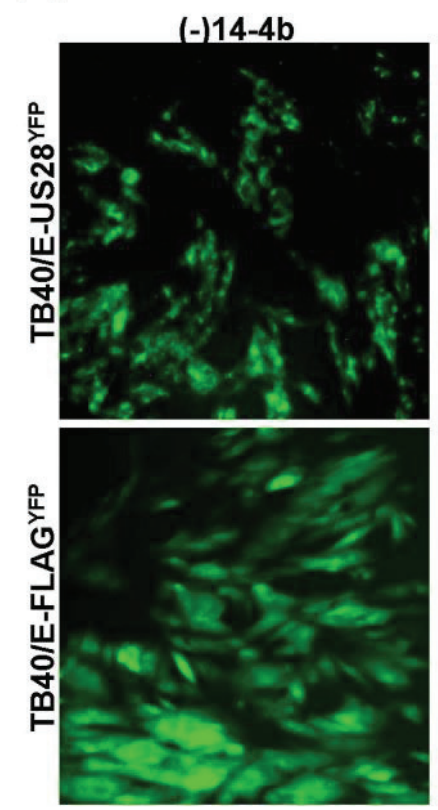


\section{Experimental Section}

\subsection{Cells and Viruses}

Human lung fibroblasts (MRC5) were maintained in Dulbecco's modified Eagle's medium (DMEM) containing 8\% fetal bovine serum (FBS), $1 \mathrm{mM}$ HEPES, $100 \mathrm{U} / \mathrm{mL}$ penicillin, and $100 \mu \mathrm{g} / \mathrm{mL}$ streptomycin. Human retinal pigmented epithelial cells (ARPE-19) were maintained in a 1:1 mixture of complete DMEM (FBS, HEPES, penicillin, streptomycin) and Ham's F-12 medium containing 8\% FBS, $1 \mathrm{mM}$ HEPES, $100 \mathrm{U} / \mathrm{mL}$ penicillin, and $100 \mu \mathrm{g} / \mathrm{mL}$ streptomycin. Human umbilical vein endothelial cells (HUVEC) were cultured in EBM medium supplemented with bovine brain extract (BBE), recombinant human epidermal growth factor (rhEGF), hydrocortisone, ascorbic acid, gentamicin sulfate/amphotericin-B (GA-1000) and 2\% FBS (Lonza Clonetics). $\mathrm{CD} 34^{+} \mathrm{CD} 31^{+}$human microvascular endothelial cells (HMVEC) were cultured in EBM-2 basal medium supplemented with rhEGF, hydrocortisone, recombinant human fibroblast growth factor-beta (rhFGF-B), vascular endothelial growth factor (VEGF), insulin-like growth factor $\left(\mathrm{R}^{3}-\mathrm{IGF}-1\right)$, ascorbic acid, GA-1000, and 5\% FBS (Lonza Clonetics). Cells were maintained at $37{ }^{\circ} \mathrm{C}$ in a humidified atmosphere $\left(95 \% \mathrm{air} / 5 \% \mathrm{CO}_{2}\right)$.

The HCMV bacterial artificial chromosome (BAC) clone of the clinical isolate TB40/E (TB40-BAC4) was a kind gift of Dr. Christian Sinzger (Institute of Virology, University Medical Center Ulm, Ulm, Germany) and Dr. Felicia Goodrum (University of Arizona, Tucson, Arizona, AZ, USA). TB40-BAC4 was used to generate variants expressing a chimeric US28 with a yellow fluorescent protein (YFP) tag (TB40/E-US28 ${ }^{\mathrm{YFP}}$ ) and a US28 deletion virus containing a FLAG-YFP cassette within the US28 open reading frame (TB40/E-FLAG ${ }^{\text {YFP }}(\Delta \mathrm{US} 28)$ ). To generate these viruses TB40-BAC4 was modified using the galK recombineering system as previously described [29]. In brief, the galK gene was amplified by PCR using the following primers: Forward 5'GTGCGTGGACCAGGCGGTGTCCATGCACCGAGGGCAGAACTGGTGCTACC CCTGTTGACA ATTAATCATCGGCA-3'; Reverse 5'-GAGGGGCGGACACGGGGTTTGTATGAAAAGGCCGAGGT AGCGATTTTTTATCAGCACTGTCCTGCTCCTT-3', where the underlined sequences correspond to galK. The PCR product was transformed into recombination-competent E. coli SW105 cells containing TB40-BAC4. GalK-expressing clones were subsequently selected and electroporated with a PCR cassette specific to either US28 ${ }^{\text {YFP }}$ or FLAG ${ }^{\text {YFP }}$ amplified from pcDEF-US28 ${ }^{\text {YFP }}$. Primers used for reversion were as follows: US28 ${ }^{\text {YFP }}$ Forward 5'-GTGCGTGGACCAGG CGGTGTCCATGCACCGAGGGCAGAACTGGTGCTACCATGACACCGACGACGACGACCG-3'; $\mathrm{US} 28^{\mathrm{YFP}} / \mathrm{FLAG}^{\mathrm{YFP}}$ Reverse 5'-GAGGGCGGACACGGGGTTTGTATGAAAAGGCCGAGGTAGCG CTTTTTTATTACTTGTACAGCTCGTCCATGC-3'; FLAG ${ }^{\mathrm{YFP}}$ Forward 5'-GTGCGTGGACCAGGC GGTGTCCATGCACCGAGGGCAGAACTGGTGCTACCATGGACTACAAGGACGACGACGACAC TAGTGCGGCCGCCATGGTGAGC-3'. The resultant clones were chosen following counter-selection against galK and subsequently sequenced to ensure incorporation of YFP and FLAG. Virus stocks were generated by electroporating low passage MRC5s with purified BAC DNA from the respective variants. Cultures were allowed to progress to full cytopathic effect (CPE) and virus was then harvested and purified by centrifugation through a $20 \%$ sorbitol cushion. Virus stocks were stored at $-80{ }^{\circ} \mathrm{C}$ in DMEM containing $8 \%$ FBS plus $1.5 \%$ bovine serum albumin (BSA). Virus stock titers were determined by tissue culture infectious dose $50\left(\mathrm{TCID}_{50}\right)$ assay. 


\subsection{Fluorescence Microscopy}

For fluorescence microscopy, fibroblasts infected at a multiplicity of infection (MOI) of 5 plaque forming units $(\mathrm{PFU}) / \mathrm{mL}$ were visualized 2 days post-infection using the EVOS Cell Imaging Systems (Life Technologies, Grand Island, NY, USA). Images were analyzed using Adobe Photoshop CS5.1 software [30]. ARPE-19 and endothelial cells were infected at an MOI of $25 \mathrm{PFU} / \mathrm{mL}$ and visualized 4 days post-infection. For confocal microscopy, fibroblasts infected at an MOI of $5 \mathrm{PFU} / \mathrm{mL}$ were harvested at various times post-infection and fixed in Cytofix/Cytoperm solution (BD Biosciences, Franklin Lakes, NJ, USA) for 45 minutes at $4{ }^{\circ} \mathrm{C}$. YFP fluorescence was visualized using a Molecular Devices ImageXpress Ultra (IXU) plate-scanning confocal microscope (Integrated Screening Core, Icahn School of Medicine at Mount Sinai, New York, NY, USA). Images were analyzed using MetaExpress software [31].

\subsection{Cell Fractionation and Immunoblot Analysis}

Subcellular fractionation was performed as previously described [32]. In brief, mock-infected and TB40/E-infected fibroblasts were resuspended in $1 \times$ homogenization buffer $(100 \mathrm{mM}$ Tris, $150 \mathrm{mM} \mathrm{NaCl}, 250 \mathrm{mM}$ sucrose, $1.5 \mathrm{mg} / \mathrm{mL}$ aprotinin, and $1 \mu \mathrm{M}$ leupeptin) and mechanically homogenized using a $12-\mu \mathrm{m}$ ball bearing homogenizer (Isobiotec, Hiedelberg, Germany). Samples were centrifuged at $15,000 \times \mathrm{g}$ for 10 minutes at $4{ }^{\circ} \mathrm{C}$ and heavy organelles found in the pellet were lysed directly in $1 \times$ SDS sample buffer $(50 \mathrm{mM}$ Tris, $\mathrm{pH} 6.8,2 \% \mathrm{SDS}, 10 \%$ glycerol, $0.02 \%$ bromphenol blue, $50 \mathrm{mM}$ dithiothreitol). Supernatants were further centrifuged at $120,000 \times \mathrm{g}$ for 1 hour at $4{ }^{\circ} \mathrm{C}$. Pellets containing cellular membranes and supernatants containing cytoplasm were lysed in SDS sample buffer and resolved using SDS-PAGE. Green fluorescent protein (GFP) polyclonal antibody was a kind gift of Dr. Hidde Ploegh (Whitehead Institute, MIT, Cambridge, MA, USA). Polyclonal major histocompatiblity class I heavy chain (MHC class I HC) antibody has been previously described [33]. Anti-glyceraldehyde-3-phosphate dehydrogenase (GAPDH) was purchased from Upstate/Millipore. Monoclonal glycoprotein B $(\mathrm{gB})$ antibody was a kind gift of Dr. William Britt (UAB, Birmingham, AL, USA).

\subsection{N-Linked Protein Glycosylation Analysis}

Endoglycosidase $\mathrm{H}$ (EndoH) and peptide: $N$-glycosidase F (PNGaseF) sensitivity was determined as per the manufacturer's protocol (New England Biolabs, Ipswitch, MA, USA). In brief, polypeptide samples lysed in $1 \%$ SDS were incubated in $1 \times$ denaturing buffer $(0.5 \%$ SDS, $0.04 \mathrm{M}$ dithiothreitol) followed by the addition of $10 \times \mathrm{G} 5$ buffer ( $0.5 \mathrm{M}$ sodium citrate, $\mathrm{pH} 5.5$, for EndoH) or $10 \times \mathrm{G} 7$ buffer $(0.5 \mathrm{M}$ sodium citrate, $\mathrm{pH} 7.5,10 \%$ Nonidet $\mathrm{P}-40$, for PNGaseF) and 1000 units of EndoH or 500 units of PNGaseF. Enzymatic reactions were carried out at $37^{\circ} \mathrm{C}$ for 2 hours.

\subsection{Analysis of Virus Growth and Spread}

Single step growth kinetics were determined by infecting fibroblasts at an MOI of $5 \mathrm{PFU} / \mathrm{mL}$. At the indicated time points post-infection media was collected and virus titers in the supernatant were determined by TCID $_{50}$ assay. Multistep growth kinetic analysis was performed at an MOI of 
0.1 PFU $/ \mathrm{mL}$. For analysis of cell-associated virus yield, ARPE-19 cells were infected at an MOI of $0.1 \mathrm{PFU} / \mathrm{mL}$ and samples harvested by scraping cells into media. Cells were lysed by subjecting them to a single freeze-thaw cycle and sonication. Cellular debris was pelleted by centrifugation and the amount of infectious virus in the resulting supernatant was determined by $\mathrm{TCID}_{50}$ assay.

For neutralization of extracellular virus, fibroblasts were mock-infected or TB40/E-infected at an MOI of $0.01 \mathrm{PFU} / \mathrm{mL}$ for 1 hour at $37^{\circ} \mathrm{C}$ and then placed into media containing $10 \mu \mathrm{g} / \mathrm{mL}$ of the monoclonal anti-gH antibody 14-4b (a kind gift of Dr. William Britt (UAB)). 2 weeks post-infection YFP fluorescence was analyzed using an Acumen ${ }^{\mathrm{e}} \mathrm{X} 3$ laser scanning fluorescence microplate cytometer (TTP LabTech, Cambridge, MA, USA) as previously described [34].

\section{Conclusions}

Many functions have been ascribed to the G protein-coupled receptors encoded by HCMV. In these studies we demonstrate that US28 impacts dissemination of virus by promoting cell-to-cell spread of infectious progeny. The generation of two fluorescent variants of the clinical isolate TB40/E, US28 ${ }^{\mathrm{YFP}}$ and FLAG ${ }^{\mathrm{YFP}}$ ( $\triangle \mathrm{US} 28$ ), allowed us to investigate the role of US28 as a virulence factor during HCMV infection (Figure 1). Infection with TB40/E-US28 ${ }^{\text {YFP }}$ resulted in the formation of intense perinuclear granular structures at late times in fibroblasts, endothelial, and epithelial cells (Figure 2). Interestingly, in epithelial cells, where TB40/E disseminates via the cell-to-cell route, US28 ${ }^{\mathrm{YFP}}$ localized to areas between infected cells (Figure 2b). During infection, US28 ${ }^{\mathrm{YFP}}$ traffics with mostly large dense complexes in the membranes of infected cells (Figure $3 \mathrm{a}, \mathrm{b}$ ). Surprisingly, US28 ${ }^{\mathrm{YFP}}$ did not acquire an N-linked glycan during infection (Figure 3c), ascribing a novel characteristic to this viral membrane protein. Infections with TB40/E-US28 ${ }^{\text {YFP }}$ resulted in growth properties comparable to TB40/E wt (Figure 4a). Strikingly, infection with TB40/E-FLAG ${ }^{\text {YFP }}$, a virus lacking US28, resulted in increased production of extracellular infectious progeny (Figure 4a,b). However, when assayed in epithelial cells, where dissemination occurs via cell-to-cell spread, TB40/E-FLAG ${ }^{\text {YFP }}$ demonstrated a growth defect (Figure 4c) suggesting that US28 plays a role in inter-cellular dissemination of HCMV. Accordingly, when the virus lacking US28 was forced to utilize the cell-to-cell route in fibroblasts by culture in the presence of neutralizing antibodies, a substantial defect in dissemination was observed (Figure 5). Taken together, we can conclude that US28, an integral membrane protein present at the border of adjacent cells, plays a role in dissemination of infectious viral progeny from cell-to-cell.

The scenario may occur during HCMV infection where, at late time points, US28 converges at the interface of viral assembly zones and adjoining cells in order to enhance virus spread to uninfected neighboring cells. The dissemination enhancing activity of US28 likely requires the interaction of this GPCR with a membrane component on adjacent cells. Due to US28's high affinity binding to the chemokine fractalkine [35], this interaction might either promote cell-cell contact or membrane fusion in clinically relevant cell types expressing this $\mathrm{CX}_{3} \mathrm{C}$ membrane-bound chemokine (i.e., endothelial cells). In fact, fusion-enhancing activity has been reported for US28 when expressed together with the HIV Env protein and the G protein of vesicular stomatit is virus (VSV-G) [36]. Heteromerization of US28 and other HCMV GPCRs [37] may alter the physical properties of membranes in a way favorable to fusion with neighboring infected cells. Interestingly, HCMV US27 and UL33 or MCMV M33 did not seem to enhance cell fusion in a way comparable 
to US28 [36], suggesting a novel role for this GPCR in dissemination of HCMV in the host. The pattern of US28 ${ }^{\mathrm{YFP}}$ trafficking and its membrane association would be well-suited to incorporation of this protein into the viral lipid envelope. As a component of the virion, US28 could have a direct role in HCMV entry through its ability to bind target cells and mediate dissemination. This would be most advantageous during latent infection of HCMV when US28, shown to be expressed during latency [38], could mediate adhesion of latently-infected monocytes to activated endothelial cells, where high levels of fractalkine are expressed [39], to potentiate dissemination in the host. Conceivably, virion-bound GPCR US28 could also bind to cells and induce signaling networks to facilitate infection. However, further experiments are necessary to define whether US28 is present in virions and whether it can play a role in virus-cell interaction.

HCMV dissemination can occur via two routes: through production of extracellular virus progeny that subsequently bind and enter new cells to propagate infection or directly from cell to cell with limited exposure of virus to the extracellular environment [27]. Here we define US28 as a factor contributing to cell-to-cell dissemination of HCMV. Our data strongly supports the role of viral-encoded GPCRs as virulence factors in vitro and further adds to our understanding of the multifunctional US28 protein. We also identify US28 as a novel target for anti-HCMV therapeutics to inhibit viral dissemination in the host.

\section{Acknowledgments}

This work was supported in part by the NIH Grant AI101820, the Irma T. Hirschl Trust, and the American Heart Association. V.M.N. is a postdoctoral trainee supported in part by the USPHS Institutional Research Training Award T32-AI07647. T.J.G. is a predoctoral trainee supported by an American Heart Association fellowship.

\section{Author Contributions}

V.M.N. and D.T. conceived of the hypothesis, analyzed all data, and performed all experiments except confocal microscopy performed by V.R. T.J.G. aided in execution of neutralization assay, experimental design, and interpretation of data. G.B. and S.A.L. provided reagents for virus recombineering. V.M.N. and D.T. prepared the manuscript.

\section{Conflicts of Interest}

The authors declare no conflict of interest.

\section{References and Notes}

1. Britt, W. Manifestations of human cytomegalovirus infection: Proposed mechanisms of acute and chronic disease. Curr. Top. Microbiol. Immunol. 2008, 325, 417-470.

2. Arvin, A.M. Human herpesviruses: Biology, therapy, and immunoprophylaxis; Cambridge University Press: Cambridge, New York, NY, USA, 2007; pp. xx, 1388.

3. Soderberg-Naucler, C. Does cytomegalovirus play a causative role in the development of various inflammatory diseases and cancer? J. Intern. Med. 2006, 259, 219-246. 
4. Sissons, J.G.; Carmichael, A.J.; McKinney, N.; Sinclair, J.H.; Wills, M.R. Human cytomegalovirus and immunopathology. Springer Semin. Immunopathol. 2002, 24, 169-185.

5. Razonable, R.R. Epidemiology of cytomegalovirus disease in solid organ and hematopoietic stem cell transplant recipients. Am. J. Health Syst. Pharm. 2005, 62, S7-S13.

6. Murphy, E.; Yu, D.; Grimwood, J.; Schmutz, J.; Dickson, M.; Jarvis, M.A.; Hahn, G.; Nelson, J.A.; Myers, R.M.; Shenk, T.E. Coding potential of laboratory and clinical strains of human cytomegalovirus. Proc. Natl. Acad. Sci. USA 2003, 100, 14976-14981.

7. Vischer, H.F.; Leurs, R.; Smit, M.J. Hcmv-encoded g-protein-coupled receptors as constitutively active modulators of cellular signaling networks. Trends Pharmacol. Sci. 2006, 27, 56-63.

8. Sodhi, A.; Montaner, S.; Gutkind, J.S. Viral hijacking of g-protein-coupled-receptor signalling networks. Nat. Rev. Mol. Cell Biol. 2004, 5, 998-1012.

9. Evers, D.L.; Wang, X.; Huang, E.S. Cellular stress and signal transduction responses to human cytomegalovirus infection. Microbes Infect. 2004, 6, 1084-1093.

10. Margulies, B.J.; Gibson, W. The chemokine receptor homologue encoded by us 27 of human cytomegalovirus is heavily glycosylated and is present in infected human foreskin fibroblasts and enveloped virus particles. Virus Res. 2007, 123, 57-71.

11. Margulies, B.J.; Browne, H.; Gibson, W. Identification of the human cytomegalovirus $g$ protein-coupled receptor homologue encoded by ul33 in infected cells and enveloped virus particles. Virology 1996, 225, 111-125.

12. O’Connor, C.M.; Shenk, T. Human cytomegalovirus pul78 g protein-coupled receptor homologue is required for timely cell entry in epithelial cells but not fibroblasts. J. Virol. 2012, 86, 11425-11433.

13. Casarosa, P.; Gruijthuijsen, Y.K.; Michel, D.; Beisser, P.S.; Holl, J.; Fitzsimons, C.P.; Verzijl, D.; Bruggeman, C.A.; Mertens, T.; Leurs, R.; et al. Constitutive signaling of the human cytomegalovirus-encoded receptor ul33 differs from that of its rat cytomegalovirus homolog r33 by promiscuous activation of g proteins of the gq, gi, and gs classes. J. Biol. Chem. 2003, 278, 50010-50023.

14. Minisini, R.; Tulone, C.; Luske, A.; Michel, D.; Mertens, T.; Gierschik, P.; Moepps, B. Constitutive inositol phosphate formation in cytomegalovirus-infected human fibroblasts is due to expression of the chemokine receptor homologue pus28. J. Virol. 2003, 77, 4489-4501.

15. Billstrom, M.A.; Johnson, G.L.; Avdi, N.J.; Worthen, G.S. Intracellular signaling by the chemokine receptor us28 during human cytomegalovirus infection. J. Virol. 1998, 72, $5535-5544$.

16. Streblow, D.N.; Soderberg-Naucler, C.; Vieira, J.; Smith, P.; Wakabayashi, E.; Ruchti, F.; Mattison, K.; Altschuler, Y.; Nelson, J.A. The human cytomegalovirus chemokine receptor us28 mediates vascular smooth muscle cell migration. Cell 1999, 99, 511-520.

17. Waldhoer, M.; Kledal, T.N.; Farrell, H.; Schwartz, T.W. Murine cytomegalovirus (cmv) m33 and human cmv us 28 receptors exhibit similar constitutive signaling activities. J. Virol. 2002, 76, 8161-8168.

18. Arnolds, K.L.; Lares, A.P.; Spencer, J.V. The us27 gene product of human cytomegalovirus enhances signaling of host chemokine receptor cxcr4. Virology 2013, 439, 122-131. 
19. O’Connor, C.M.; Shenk, T. Human cytomegalovirus pus 27 g protein-coupled receptor homologue is required for efficient spread by the extracellular route but not for direct cell-to-cell spread. J. Virol. 2011, 85, 3700-3707.

20. Davis-Poynter, N.J.; Lynch, D.M.; Vally, H.; Shellam, G.R.; Rawlinson, W.D.; Barrell, B.G.; Farrell, H.E. Identification and characterization of a $\mathrm{g}$ protein-coupled receptor homolog encoded by murine cytomegalovirus. J. Virol. 1997, 71, 1521-1529.

21. Oliveira, S.A.; Shenk, T.E. Murine cytomegalovirus $\mathrm{m} 78$ protein, a g protein-coupled receptor homologue, is a constituent of the virion and facilitates accumulation of immediate-early viral mrna. Proc. Natl. Acad. Sci. USA 2001, 98, 3237-3242.

22. Fraile-Ramos, A.; Kledal, T.N.; Pelchen-Matthews, A.; Bowers, K.; Schwartz, T.W.; Marsh, M. The human cytomegalovirus us2 8 protein is located in endocytic vesicles and undergoes constitutive endocytosis and recycling. Mol. Biol. Cell 2001, 12, 1737-1749.

23. Fraile-Ramos, A.; Pelchen-Matthews, A.; Kledal, T.N.; Browne, H.; Schwartz, T.W.; Marsh, M. Localization of hemv ul33 and us 27 in endocytic compartments and viral membranes. Traffic 2002, 3, 218-232.

24. Stropes, M.P.; Miller, W.E. Functional analysis of human cytomegalovirus pus28 mutants in infected cells. J. Gen. Virol. 2008, 89, 97-105.

25. Maley, F.; Trimble, R.B.; Tarentino, A.L.; Plummer, T.H., Jr. Characterization of glycoproteins and their associated oligosaccharides through the use of endoglycosidases. Anal. Biochem. 1989, 180, 195-204.

26. Noriega, V.M.; Tortorella, D. Human cytomegalovirus-encoded immune modulators partner to downregulate major histocompatibility complex class i molecules. J. Virol. 2009, 83, 1359-1367.

27. Silva, M.C.; Schroer, J.; Shenk, T. Human cytomegalovirus cell-to-cell spread in the absence of an essential assembly protein. Proc. Natl. Acad. Sci. USA 2005, 102, 2081-2086.

28. Li, L.; Coelingh, K.L.; Britt, W.J. Human cytomegalovirus neutralizing antibody-resistant phenotype is associated with reduced expression of glycoprotein h. J. Virol. 1995, 69, 6047-6053.

29. Warming, S.; Costantino, N.; Court, D.L.; Jenkins, N.A.; Copeland, N.G. Simple and highly efficient bac recombineering using galk selection. Nucleic Acids Res. 2005, 33, e36.

30. Adobe Photoshop, CS5.1; Adobe Systems: New York, NY, USA, 2011.

31. MetaXpress, version 5.3; Molecular Devices: Sunnyvale, CA, USA, 2013.

32. Baker, B.M.; Tortorella, D. Dislocation of an endoplasmic reticulum membrane glycoprotein involves the formation of partially dislocated ubiquitinated polypeptides. J. Biol. Chem. 2007, 282, 26845-26856.

33. Noriega, V.M.; Tortorella, D. A bipartite trigger for dislocation directs the proteasomal degradation of an endoplasmic reticulum membrane glycoprotein. J. Biol. Chem. 2008, 283, 4031-4043.

34. Gardner, T.J.; Bolovan-Fritts, C.; Teng, M.W.; Redmann, V.; Kraus, T.A.; Sperling, R.; Moran, T.; Britt, W.; Weinberger, L.S.; Tortorella, D. Development of a high-throughput assay to measure the neutralization capability of anti-cytomegalovirus antibodies. Clin. Vaccine Immunol. 2013, 20, 540-550. 
35. Kledal, T.N.; Rosenkilde, M.M.; Schwartz, T.W. Selective recognition of the membrane-bound cx3c chemokine, fractalkine, by the human cytomegalovirus-encoded broad-spectrum receptor us28. FEBS Lett. 1998, 441, 209-214.

36. Pleskoff, O.; Treboute, C.; Alizon, M. The cytomegalovirus-encoded chemokine receptor us 28 can enhance cell-cell fusion mediated by different viral proteins. J. Virol. 1998, 72, 6389-6397.

37. Tschische, P.; Tadagaki, K.; Kamal, M.; Jockers, R.; Waldhoer, M. Heteromerization of human cytomegalovirus encoded chemokine receptors. Biochem. Pharmacol. 2011, 82, 610-619.

38. Beisser, P.S.; Laurent, L.; Virelizier, J.L.; Michelson, S. Human cytomegalovirus chemokine receptor gene us28 is transcribed in latently infected thp-1 monocytes. J. Virol. 2001, 75, 5949-5957.

39. Goda, S.; Imai, T.; Yoshie, O.; Yoneda, O.; Inoue, H.; Nagano, Y.; Okazaki, T.; Imai, H.; Bloom, E.T.; Domae, N.; Umehara, H. Cx3c-chemokine, fractalkine-enhanced adhesion of thp-1 cells to endothelial cells through integrin-dependent and -independent mechanisms. J. Immunol. 2000, 164, 4313-4320. 



\title{
The Use of Novel Technologies
}

Reprinted from Viruses. Cite as: Reyda, S.; Büscher, N.; Tenzer, S.; Plachter, B. Proteomic Analyses of Human Cytomegalovirus Strain AD169 Derivatives Reveal Highly Conserved Patterns of Viral and Cellular Proteins in Infected Fibroblasts. Viruses 2014, 6, 172-188.

Article

\section{Proteomic Analyses of Human Cytomegalovirus Strain AD169 Derivatives Reveal Highly Conserved Patterns of Viral and Cellular Proteins in Infected Fibroblasts}

\author{
Sabine Reyda ${ }^{1}$, Nicole Büscher ${ }^{1}$, Stefan Tenzer ${ }^{2,3}$ and Bodo Plachter ${ }^{1,3, *}$ \\ 1 Institute for Virology, University Medical Center of the Johannes Gutenberg-University Mainz, \\ Obere Zahlbacher Str. 67, D-55131 Mainz, Germany; E-Mails: reyda@uni-mainz.de (S.R.); \\ bueschni@uni-manz.de (N.B.) \\ 2 Institute for Immunology, University Medical Center of the Johannes Gutenberg-University \\ Mainz, Langenbeckstr. 1, D-55131 Mainz, Germany; E-Mail: tenzer@uni-mainz.de \\ 3 Research Center Immunology, University Medical Center of the Johannes Gutenberg-University \\ Mainz, Langenbeckstr. 1, D-55131 Mainz, Germany \\ * Author to whom correspondence should be addressed; E-Mail: plachter@uni-mainz.de; \\ Tel.: +49-6131-179232; Fax: +49-6131-179038.
}

Received: 15 November 2013; in revised form: 29 December 2013 / Accepted: 30 December 2013 / Published: 7 January 2014

\begin{abstract}
Human cytomegalovirus (HCMV) particle morphogenesis in infected cells is an orchestrated process that eventually results in the release of enveloped virions. Proteomic analysis has been employed to reveal the complexity in the protein composition of these extracellular particles. Only limited information is however available regarding the proteome of infected cells preceding the release of HCMV virions. We used quantitative mass spectrometry to address the pattern of viral and cellular proteins in cells, infected with derivatives of the AD169 laboratory strain. Our analyses revealed a remarkable conservation in the patterns of viral and of abundant cellular proteins in cells, infected for 2 hours, 2 days, or 4 days. Most viral proteins increased in abundance as the infection progressed over time. Of the proteins that were reliably detectable by mass spectrometry, only IE1 (pUL123), pTRS1, and pIRS1 were downregulated at 4 days after infection. In addition, little variation of viral proteins in the virions of the different viruses was detectable, independent of the expression of the major tegument protein
\end{abstract}


pp65. Taken together these data suggest that there is little variation in the expression program of viral and cellular proteins in cells infected with related HCMVs, resulting in a conserved pattern of viral proteins ultimately associated with extracellular virions.

Keywords: human cytomegalovirus; proteomics; mass spectrometry; virions; expression pattern

\section{Introduction}

The human cytomegalovirus (HCMV) is a pathogen of substantial clinical relevance that may lead to severe disease and sequelae after prenatal infection and life threating conditions in immunosuppressed individuals. Considerable interest thus focuses on the development of therapeutic strategies against HCMV infection and disease. One target area for the development of antiviral compounds is particle formation and particle release.

The virions of HCMV are made up by an inner capsid structure, containing the linear double-stranded DNA genome of roughly $235 \mathrm{kbp}$, an attached matrix of viral and cellular proteins, assembled as a tegument layer, and an outer envelope [1]. The $100 \mathrm{~nm}$ icosahedral capsid is composed of the major capsid protein (MCP, pUL86), the minor capsid protein (mCP, pUL85), the mCP-binding protein (mCP-BP, pUL46), the smallest capsid protein (SCP; pUL48.5), and the portal protein (pUL104) [2]. Assembly of the capsids proceeds in the nucleus of infected cells, originating from scaffold-containing procapsids. The viral DNA is encapsidated and selected tegument proteins may become associated with the capsids already at this stage [1,2]. Capsids exit the nucleus by subsequent envelopment and de-envelopment at the nuclear membrane. A key determinant of that process is the nuclear egress complex (NEC) at the inner nuclear membrane that consists of the viral proteins pUL50 and pUL53 [3-6]. Nuclear capsid egress appears to be regulated by both cellular and viral kinases $[3,7,8]$. Following their release into the cytoplasm, the capsids are directed to perinuclear inclusions termed assembly compartments (AC) [9]. The AC consists of cylindrical structures that are assembled from the trans-Golgi network, the endoplasmic reticulum (ER), the ER-Golgi intermediate compartment (ERGIC) and from endosomal compartments, and contains proteins characteristic for endosomal sorting and transport (ESCRT) as well as Rab GTPases [1,10-12]. Tegument attachment to the capsids occurs predominantly at the AC and precedes envelopment. Both virions and subviral dense bodies are enveloped at AC and are subsequently found in cytoplasmic vesicles that are then transported to the cell surface by an exocytotic pathway $[6,13]$. This transport is influenced by the product of the viral UL103 open reading frame (ORF) [14]. Infectious virions and dense bodies are ultimately released at the cell surface.

Early analyses used color staining or radioactive labeling in combination with SDS-PAGE to display the protein pattern of purified HCMV virions [15-18]. Subsequent microsequencing analyses identified a subset of viral and cellular protein components of HCMV virions [19]. Further reports also identified cell derived proteins to be included in HCMV virions, although some of these results have later been challenged as being a result of contamination rather than of packaging [20-24]. A previous study, using mass spectrometry, revealed the high level of complexity of the HCMV virion $[25,26]$. Seventy-one viral proteins were identified to be integral 
constituents of the particle. In addition, over 70 cell-derived proteins were found to be associated with purified particles. Proteomic analyses of the murine CMV virion displayed a similarly complex picture [27].

Mass spectrometry has provided a detailed insight into the protein composition of HCMV virions. There is, however, only limited information available about the proteomic composition of permissively infected fibroblasts preceding the release of particles. Furthermore, analyses of the protein composition of HCMV virions by mass spectrometry were focused on one single AD169-derivative. Up to this point, it remained unclear how culturing of related HCMVs in different laboratories would influence the proteomes of infected cells and virions. To begin to address this issue, we focused on five HCMVs that were all descendants, as bacterial artificial chromosome (BAC) clones, of the AD169 laboratory strain of HCMV. The viruses used for analysis were RV-HB5 [28], RV-HB15 (AD169-RV) [29]) and RV-BADwt [30], and the pp65 deletion mutants RV-Hd65 [31], RV-KB14 [32]. The RV-HB5 is the first BAC-derived HCMV strain, originally cloned by Borst and colleagues by inserting a BAC-vector into the US2-US6 gene region of the AD169 strain [28]. The RV-HB15 is a modified version of RV-HB5, where the US2-US6 deletion was repaired and the BAC vector was removed by cre-lox recombination at virus reconstitution, leaving one single loxP site behind in the genome. [29]. The RV-BADwt is a full length AD169 strain that was cloned by inserting a self-excisable BAC-vector between the ORFs US28 and US29, again leaving one single loxP site behind, following the US28 ORF [30]. The pp65neg strain RV-Hd65 was derived by replacing the UL83 (pp65) ORF from AD169-BAC by a neomycin resistance cassette, leaving behind 151 5'-terminal base pairs of the pp65 ORF [31]. The AD169-BAC is the clone used to reconstitute RV-HB15. The neomycin resistance cassette was removed using FLP-recombinase mediated excision in E. coli. The BAC vector was removed by cre-lox recombination at virus reconstitution. The pp65neg strain RV-KB14 was generated by inserting a tetracycline resistance cassette into the UL83 (pp65) ORF of pAD/cre (the BAC clone used for reconstitution of RV-BADwt), thereby deleting all of the pp65-coding region except for $1525^{\prime}$ base pairs of the ORF [32]. All viruses were characterized before with respect to their genomic structure and biologic properties.

The data analyses presented here show a remarkable conservation of the overall levels of viral and cellular proteins in fibroblasts, infected with RV-HB15 or RV-BADwt. Most viral proteins increased in their steady-state levels in infected cells up to 4 days after infection (dpi). Only IE1 (pUL123), IRS1 and TRS1 appeared to be downregulated at 4 dpi. No gross alteration of the viral protein content of the virions of pp65pos- or pp65neg-viruses were seen.

\section{Results and Discussion}

\subsection{Conserved Pattern of Viral Protein Expression in Infected Cells}

To address the steady-state levels of viral proteins in HFF, cells were infected at an m.o.i. of 1 with RV-HB15 and RV-BAD, respectively. Cells were collected and processed after 2 hpi, 2 dpi, or 4 dpi, respectively, and analyzed by mass spectrometry.

The patterns of viral proteins that were detectable at 2 hpi were divergent between RV-HB15 and RV-BADwt (Figure 1A). This, however, was not surprising, considering the low amounts of 
viral proteins relative to the prevalence of cellular proteins at this early time after infection (data not shown). The precision of the measurement of viral proteins against this high background was limited, thus compromising a direct comparison in this case. It was yet remarkable that viral proteins could be detected at this early time after infection, underscoring the sensitivity of the proteomic approach. In contrast to these results, the patterns of viral proteins at 2 dpi were comparable between RV-HB15 and RV-BADwt (Figure 1B). This indicated that the protein expression of these two viruses was highly conserved over the passaging on human fibroblasts. This was corroborated by the patterns observed at 4 dpi (Figure 1C). As expected, the pattern changed from 2 to 4 dpi for both viruses. At 2 hpi and 2 dpi, regulatory proteins, like IRS1, or proteins for DNA replication, like UL44 were most prominent (Figure 2 and Table 1). Note that, at this time point, pUL44 was the most abundant protein in infected cells. The levels of the regulatory proteins IE1 (UL123), TRS1 and IRS1 already peaked at 2 dpi. These three were the only viral proteins that appeared to be downregulated, resulting in a decreased abundance at 4 dpi (Figure 2). At the later time point, structural proteins more prominently shaped the pattern of expression in HCMV infected cells, pp65 being by far the most abundant representative. Surprisingly, however, UL44 was also highly expressed at 4 dpi, although other proteins, involved in DNA replication did not reach that level. The reason for this abundance of UL44 in HCMV infected fibroblasts is unclear at this point.

The proteomic approach shown here provided an impressive reflection of the long known burst of viral protein expression in infected fibroblasts. Whereas viral proteins constituted below $1 \%$ of the total protein mass in infected cells at $2 \mathrm{hpi}$, this increased to over $20 \%$ at 4 dpi (data not shown). Given the long half-life of many abundant cellular proteins, these results underscore the intensity with which HCMV diverts the cellular biosynthesis to its own use.

\subsection{Comparable Impact of RV-HB15-and RV-BADwt-Infection on the Cellular Proteome}

Proteomic analyses of the expression patterns of viral proteins in infected HFF displayed relatively conserved patterns between two different AD169 derived viruses. To test the influence of the two viruses on the levels of cell proteins in infected HFF, the mass spectrometry data of infected fibroblasts were analyzed with regard to cellular proteins (Figure 3 and Supplementary Table 1). Uninfected cells (mock) that were collected at different times after passage showed a proteomic pattern that displayed little change over time. This result compellingly documented the accuracy and the reproducibility of the method. The protein pattern of cells that were infected for 2 hours was identical to the pattern of mock infected HFF for both tested viruses. This was not surprising, as no gross changes were expected to occur at this early time. The pattern started to change at $2 \mathrm{dpi}$, and these changes were even more pronounced at $4 \mathrm{dpi}$. The alterations were consistent with the results of many reports detailing the dramatic impact of HCMV infection on cellular gene expression [1,33,34]. Little differences were, however, seen between RV-HB15- and RV-BADwt-infected cells at any of the tested time points. This indicates that HCMV infection with $\mathrm{AD} 169$-derived viruses results in a rather uniform pattern of changes in the infected cell proteome (details of the data set can be found in Supplementary Table 1). Further analyses are, however, required to investigate if the pattern of cell protein expression varies, when viruses other than AD169-derivatives are analyzed. In addition the experimental setup chosen here can only provide a 
pattern-analysis of the infected cell proteome. A more detailed insight into the HCMV-induced changes of particular host cell proteins will await further analyses.

Figure 1. Proteomic analysis of viral proteins expressed in infected HFF. Fibroblasts were infected with RV-HB15 or RV-BADwt. At 2 hpi, 2 dpi, and 4 dpi, the cells were collected and analyzed, using nanoUPLC mass spectrometry. The relative abundance of individual proteins is shown in the pie charts. TOP3-Intensity was calculated as the average intensity of the three best ionizing peptides and is proportional to the molar amount of the respective protein in the sample. Proteins were sorted in decreasing abundance according to the values determined for four-day RV-HB15 infected fibroblasts and numbered accordingly, as indicated in the box. (A) samples obtained for analysis at $2 \mathrm{hpi}$; (B) samples obtained for analysis at $2 \mathrm{dpi}$; (C) samples obtained for analysis at 4 dpi. 

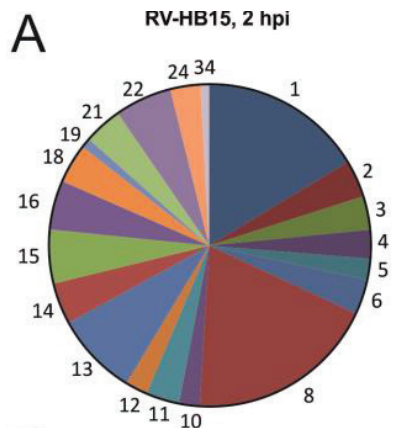

B

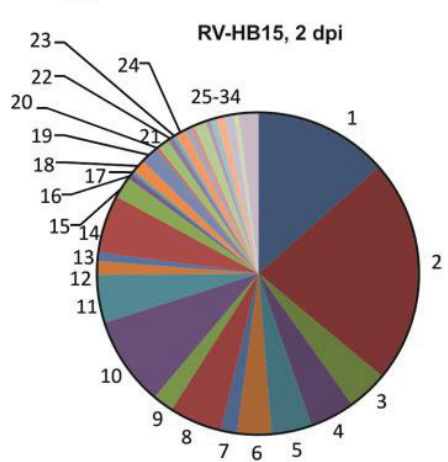

C

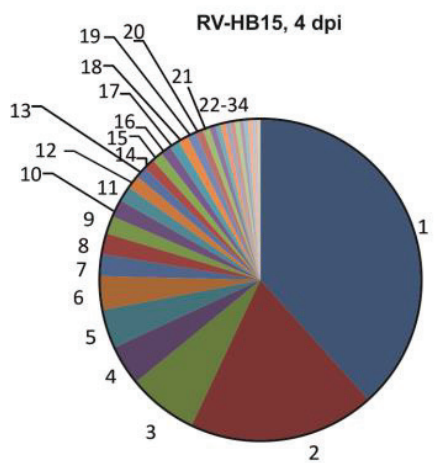

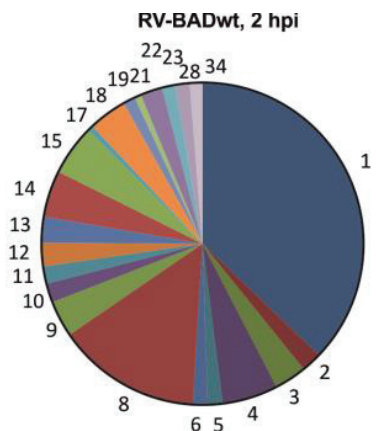
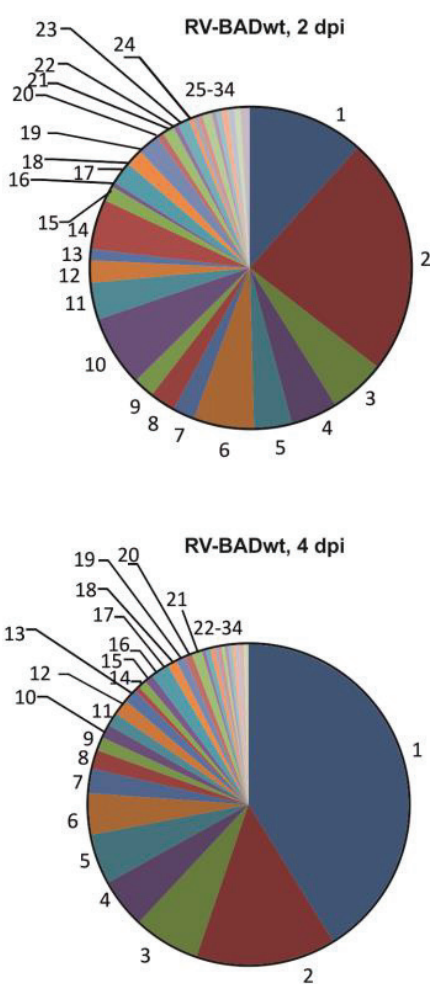

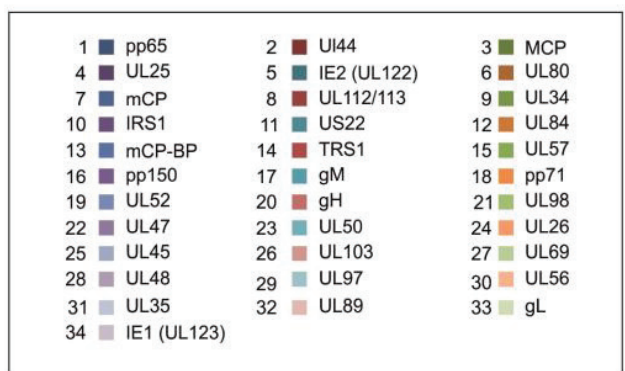

Figure 2. Time course of viral protein levels in infected HFF. The data shown in the pie charts in Figure 1 are displayed in bar chart format to show the course for each individual protein. (A) and (C), viral proteins in RV-HB15 infected cells. (B) and (D), viral proteins in RV-BADwt infected cells. Note that the scales are different in $(\mathbf{A})$ and (B) versus (C) and (D). TOP3-intensity was calculated as the average intensity of three best ionizing peptides and is proportional to the molar amount of the respective protein in the sample. 
A

HB15 2hpi

 $3.0 \times 10^{5}$
$2.5 \times 10^{5}$
$2.0 \times 10^{5}$
$1.5 \times 10^{5}$
$1.0 \times 10^{5}$
$0.5 \times 10^{5}$

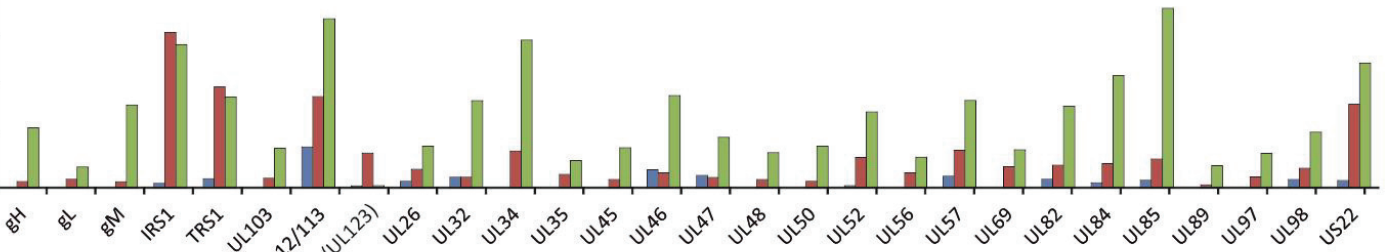

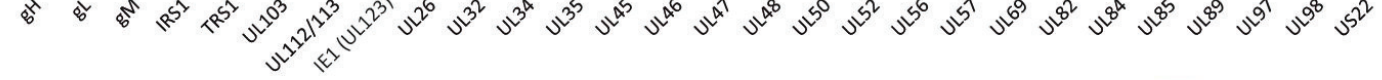

BADwt 2hpi

B

BADwt 2dpi

$3.0 \times 10^{5}$

$2.5 \times 10^{5}$

$2.0 \times 10^{5}$

$1.5 \times 10^{5}$

$1.0 \times 10^{5}$

$0.5 \times 10^{5}$

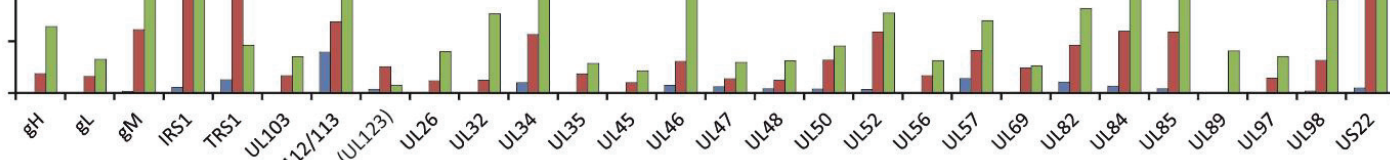

C

D
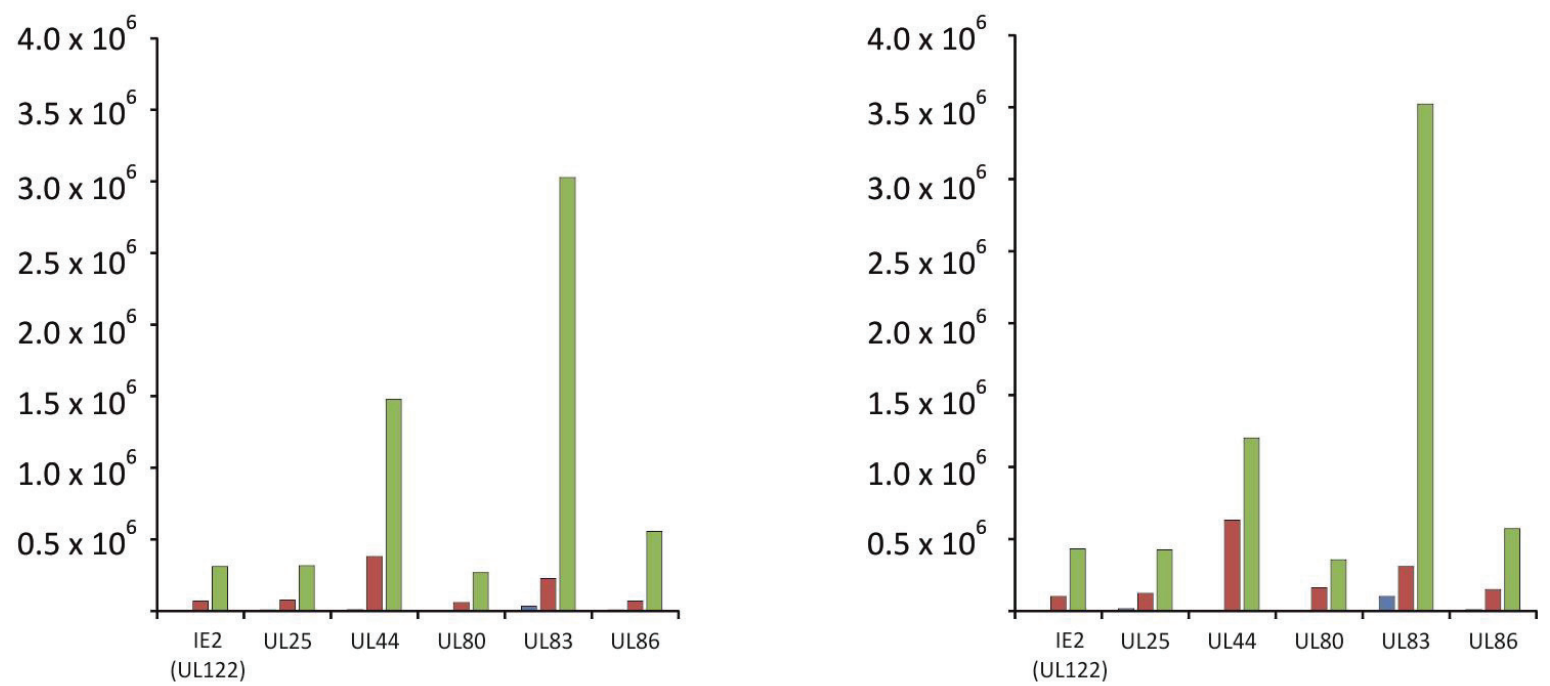

HB15 2hpi HB15 2dpi HB15 4dpi

BADwt 2hpi $\square$ BADwt 2dpi $\square$ BADwt 4dpi 


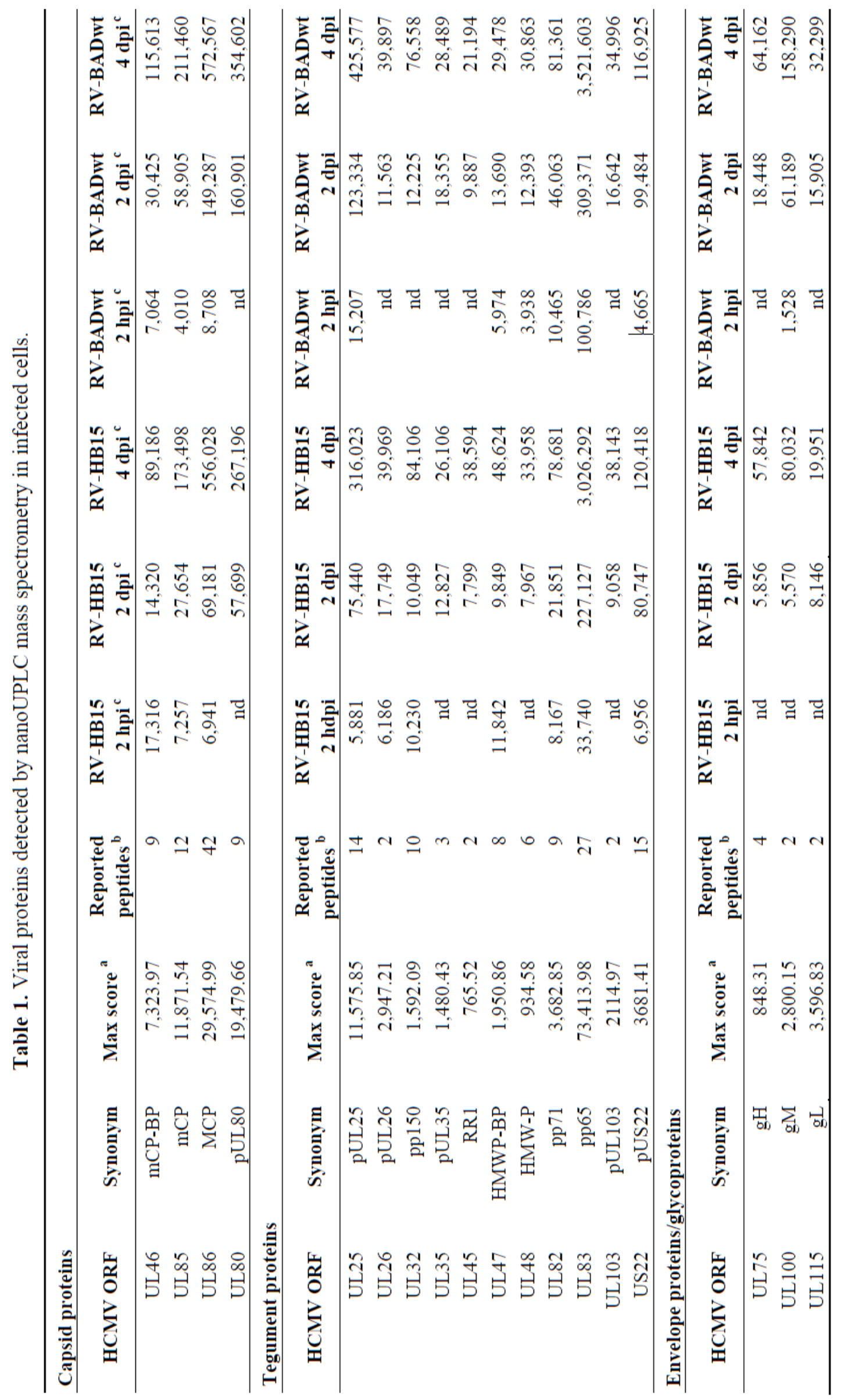




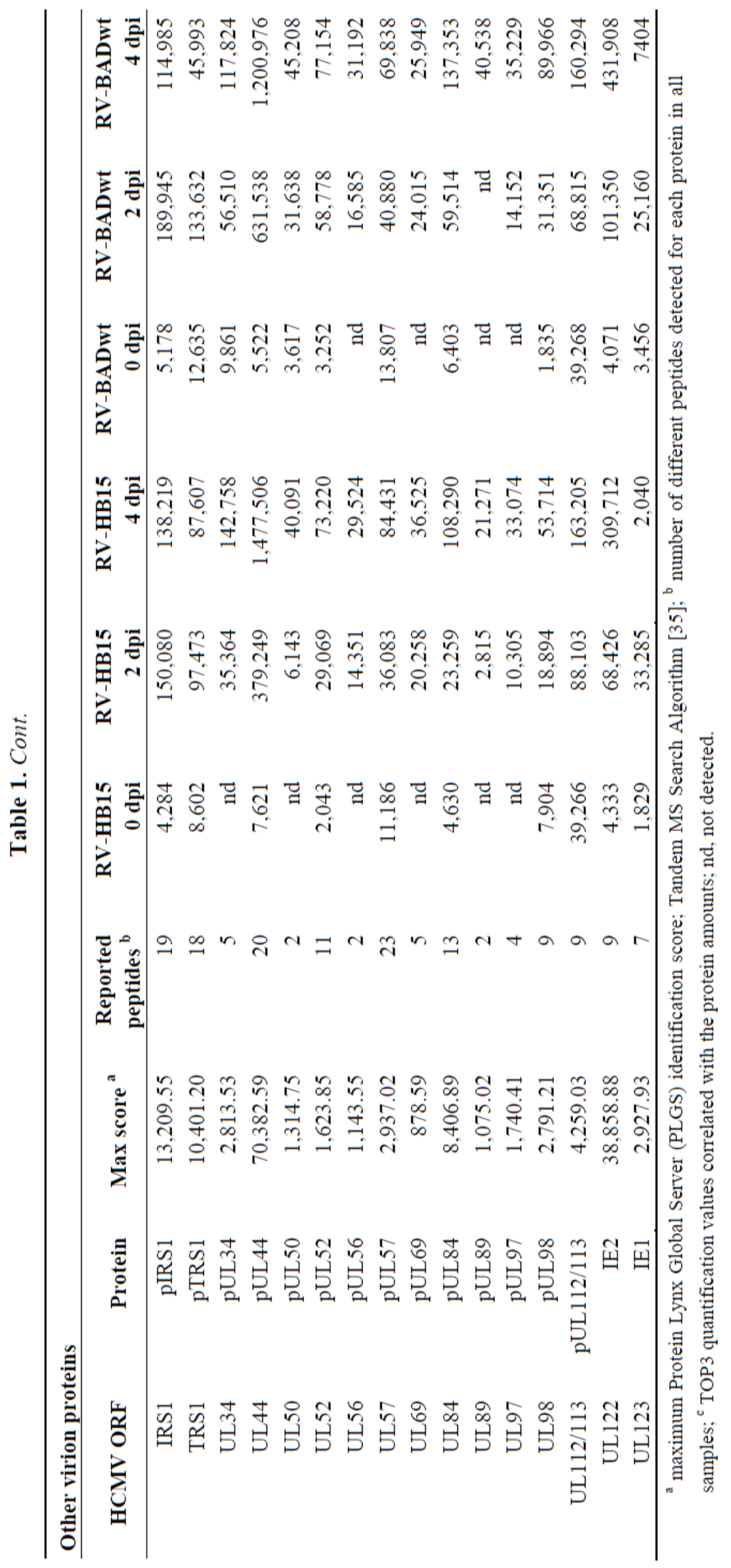


Figure 3. Proteomic analysis of cellular proteins in infected HFF. Fibroblasts were infected with RV-HB15 or RV-BADwt. After 2 hpi, 2 dpi or 4 dpi, the cells were collected and analyzed using nanoUPLC mass spectrometry. The relative frequency of the viral proteins in the samples is shown in the pie charts. Proteins were sorted according to frequencies found in four day RV-HB15 infected fibroblasts, displayed from left to right in the caption. The 100 most frequent proteins in RV-HB15 infected HFF are shown. A detailed representation of the data is provided in the Supplementary Table 1.
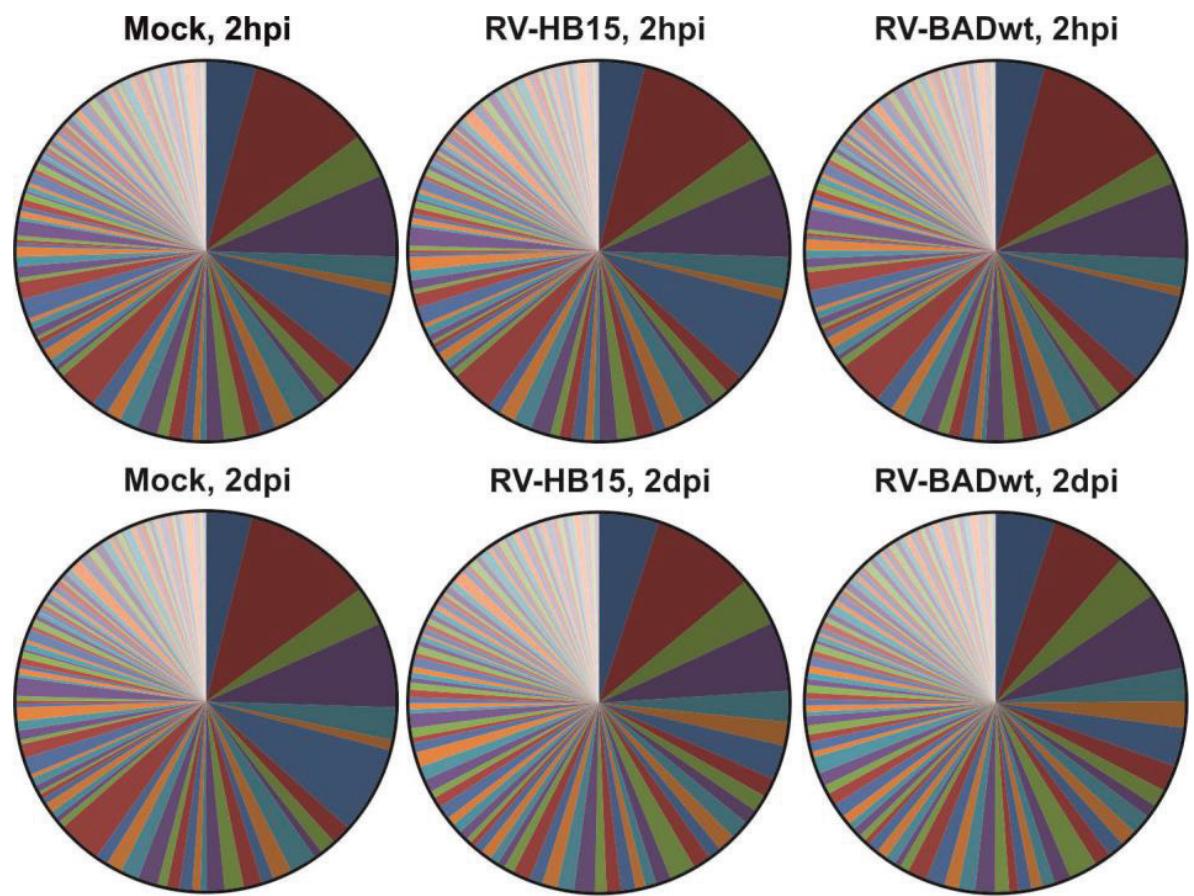

RV-BADwt, 2dpi
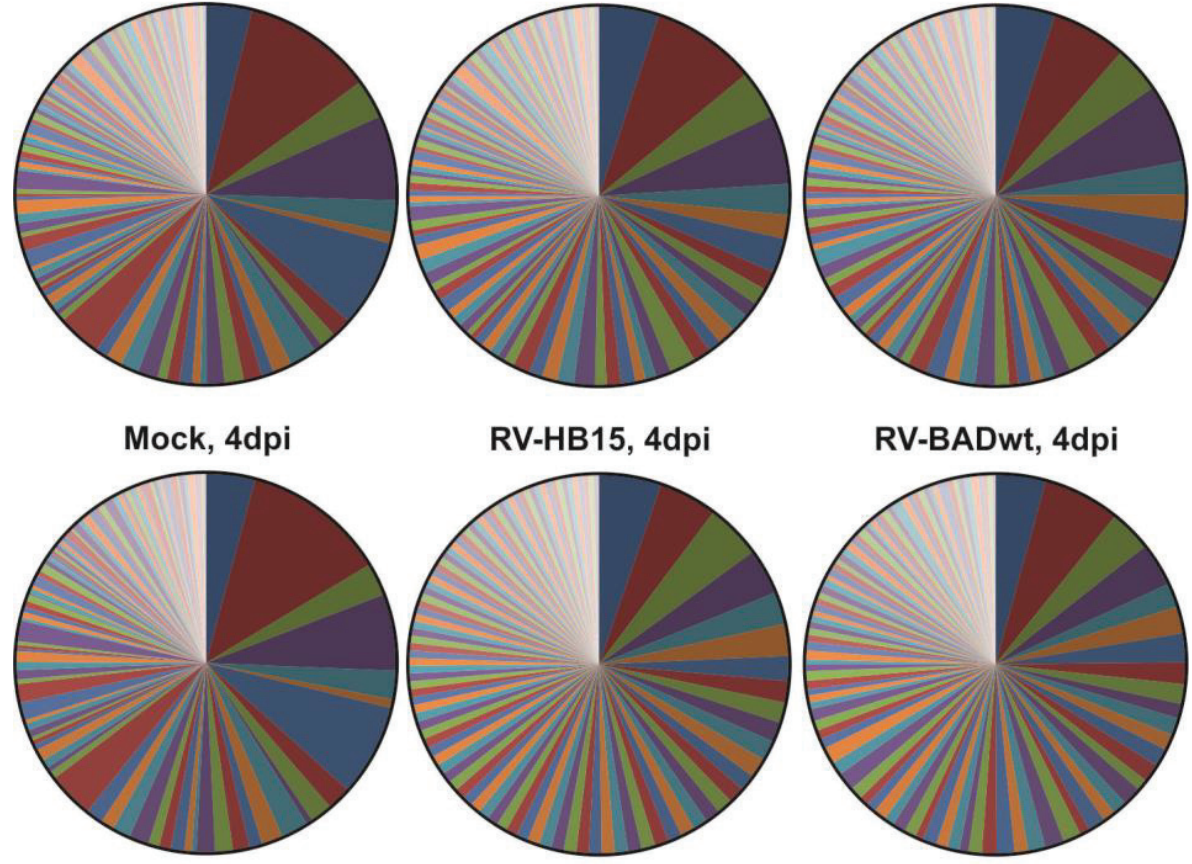

\begin{tabular}{|c|c|c|c|c|c|}
\hline E G3P & АCTB & EF1A1 & 口 VIME & - $\mathrm{H}_{4}$ & HSP7C \\
\hline aCTC & - H2B1D & ENOA & EF2 & ANXA2 & KPYM \\
\hline 口 TPIS & TBA1B & H2A2B & COF1 & на90в & 口33 \\
\hline CALR & LDHA & GRP78 & 는1 & TBB5 & LMNA \\
\hline DROF1 & ACTS & PDIA3 & CLH1 & DPIB & ALDOA \\
\hline PRDX1 & TKT & MDHM & GSTP1 & TBB2C & CH60 \\
\hline DPYL2 & — SERPH & ATPB & — PPIA & NPM & $\mathrm{H} 2 \mathrm{~A} 2 \mathrm{C}$ \\
\hline HSP71 & ATPA & HS90A & SYAC & G6PI & ELLNC \\
\hline RS3 & PGAM1 & ENPL & YBOX1 & RTN4 & aRP75 \\
\hline L LRC59 & EL23A & H2B1J & 口 PGK1 & RAN & EF1A2 \\
\hline LDHB & RL18 & EF1G & NDKB & RS27A & PTBP1 \\
\hline HNRPK & TBA1A & IQGA1 & [ PDIA1 & NUCL & H31 \\
\hline PRDX6 & CALX & ANXA5 & MYH9 & H2AV & GELS \\
\hline GDIB & MDHC & ROA2 & ADT3 & RLA1 & RLAO \\
\hline RS15 & AHNK & IF4A1 & RL7 & TCPD & RL4 \\
\hline $\mathrm{S} 10 \mathrm{AB}$ & HSPB1 & RS17L & PDIA6 & TERA & S10A6 \\
\hline RS8 & MPCP & TCPQ & XRCC5 & & \\
\hline
\end{tabular}

RV-BADwt, 4dpi

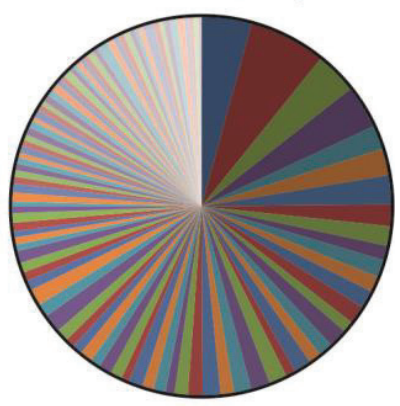




\subsection{Conserved Stoichiometry of Viral Proteins in Virions of AD169-Derived Viruses}

Viral protein levels in HFF were comparable between the two AD169-derived viruses. We next asked the question, if this conservation was also reflected in the proteomes of virions from the different viruses. For this, also two AD169 variants were used that did not express the most abundant tegument protein pp65, in addition to three pp65positive (pp65-pos) viruses. Proteomic analyses showed a striking level of similarity in the protein pattern of the three pp65-pos viruses (RV-HB5; RV-HB15; RV-BADwt) and the two pp65-negative (pp65-neg) viruses (RV-Hd65; RV-KB14), respectively (Figure 4). As expected, the most prominent constituent of the pp65pos viruses was pp65, followed by the major capsid protein, pp71, pp150 and pUL94. These results were comparable to what has been published before for the proteome of AD169 virions [25]. There was some variation in the relative molarity of pp65, which is a non-essential tegument protein [18]. The number of pp65 molecules that are included in the tegument may thus vary. Accordingly, the relative copy numbers of some of the tegument proteins, known to interact with pp65 [36-38] showed subtle alterations which may be related to variations in pp65 content. There were also some differences in the ranking of abundance of some other virion proteins, compared to previously published data [25]. We do not know the reasons for this at this point. Different infection and particle purification strategies, e.g., with regard to the time point of collection of infectious supernatant or with regard to ultracentrifugation may account for these variations. In addition, different mass spectrometry protocols were used. In our study, exogenously added enolase was employed as an internal standard, providing a high level of confidence with respect to relative quantification. However, more detailed analyses of the virion composition, including different strains, will have to be performed to more accurately display relative abundances of individual proteins in HCMV virions.

The compositions of the two pp65neg viruses and the three pp65pos viruses were virtually identical, except for the lack of pp65 in the former (Figure 4). Note that RV-Hd65 is a derivative of AD169-BAC [29], the BAC, used for reconstitution of RV-HB15 (AD169-RV), and RV-KB14 is a derivative of $\mathrm{pAD} / \mathrm{cre}$ [30], the $\mathrm{BAC}$ used for reconstitution of RV-BADwt. The slight differences seen in the protein pattern of the two parental viruses were not detectable in the pp65neg variants.

Taken together these results showed that the viral protein composition of extracellular virions of AD169 descendants is subject to only subtle variations. This argues in favor of a conserved process of viral protein packaging within a given strain of HCMV. Remarkably, however, the pattern did not change dramatically by removing pp65 which, in our analyses, comprised up to one third of the total mass of viral proteins in the particle. Despite the absence of pp65, tegument assembly appeared to follow along a controlled process that resulted in protein patterns that were comparable between pp65pos and pp65neg viruses. It is likely that the network of tegument protein interactions, as reported by others [37,39] will be instrumental to this process; yet this hypothesis still awaits confirmation. 
Figure 4. Proteomic analysis of HCMV proteins from purified virions. Virions were purified by glycerol-tartrate-gradient-centrifugation from supernatants of 6-7 day infected foreskin fibroblasts. The pp65pos strains (RV-HB5, RV-HB15, and RV-BADwt) and the pp65neg strains (RV-Hd65 and RV-KB14) were used for analysis. Purified virions were analyzed using nanoUPLC mass spectrometry. The stoichiometry of individual proteins, normalized to exogenously added enolase is shown in the pie charts. Proteins were sorted in decreasing abundance according to the values obtained for RV-HB15 virions and numbered accordingly, as indicated in the figure caption.
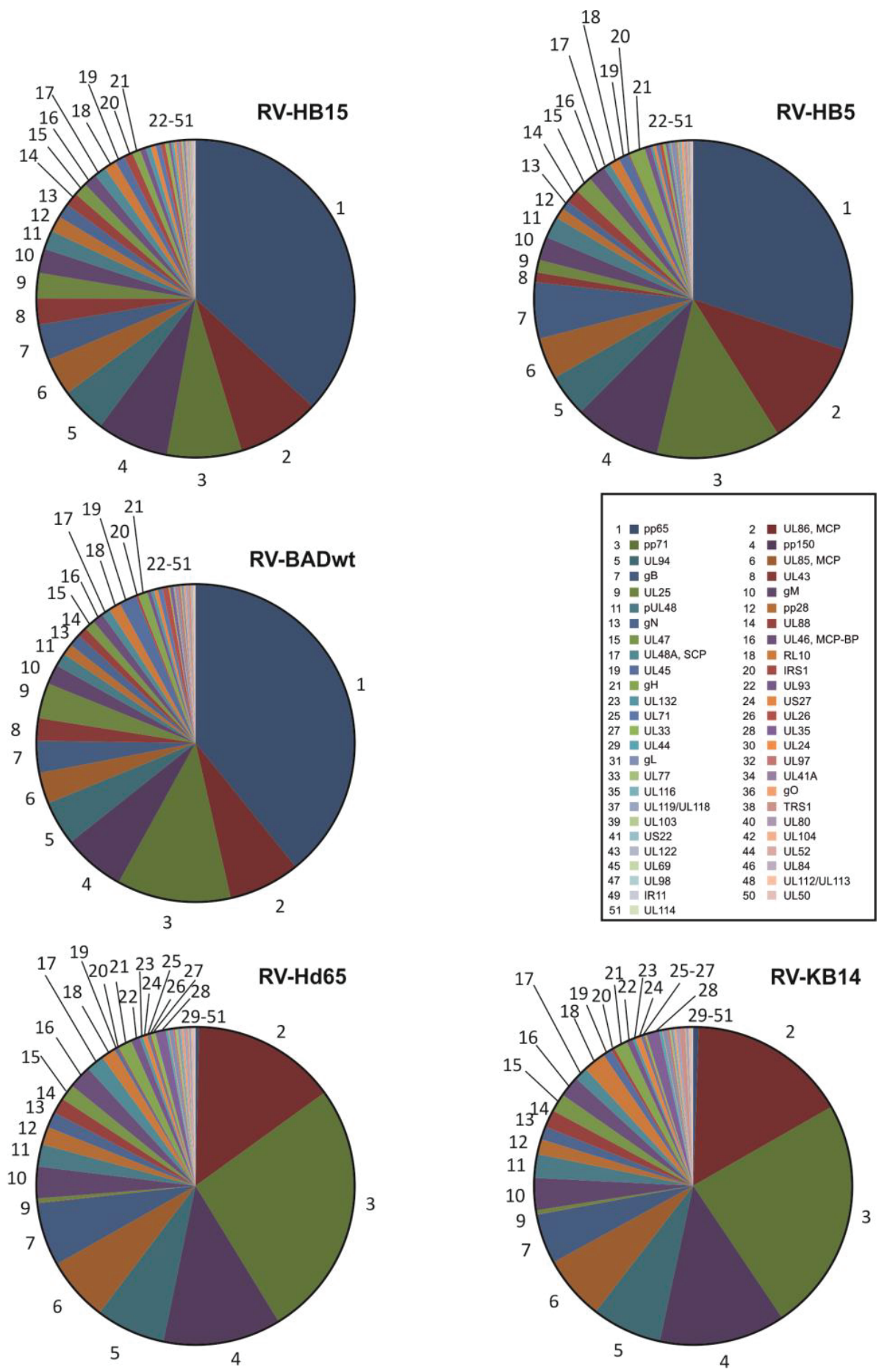


\section{Experimental Section}

For proteomic analyses of infected cells, HFF were infected at a multiplicity of infection (MOI) of 1 in each case. For this, culture supernatants from 6-7 day infected HFF had been collected and stored at $-80^{\circ} \mathrm{C}$. One sample was thawed and analyzed for infectivity, using IE1 (UL123) specific antibody staining. An MOI, based on serial dilution of the supernatants was calculated. For proteomic analyses of virions, cells were infected in a way that all cells showed a cytopathic effect at day one of infection.

Mass spectrometry was performed as described elsewhere [32]. Briefly, sample preparation and protein digestion of virions were performed as described in [32]. Nanoscale LC separation of tryptic peptides was performed with a nanoAcquity system (Waters Corporation, Manchester, UK) equipped with a BEH C18 $1.7 \mu \mathrm{m}, 75 \mu \mathrm{m} \times 150 \mathrm{~mm}$ analytical reversed-phase column (Waters Corporation) in direct injection mode as described before [40]. Mobile phases, gradients and flow rates were chosen as described in [32] and $0.2 \mu \mathrm{L}$ of sample (50 $\mathrm{ng}$ of total protein) was injected per technical replicate. Running conditions were as described in [32].

Mass spectrometric analysis of tryptic peptides from infected cells and virions was performed in quintuplicate using a QTOF-Premier mass spectrometer (Waters Corporation) with a typical resolution of at least 10,000 FWHM (full width half maximum) as described before [32]. All analyses were performed in positive mode ESI using instrument settings and nanoLockspray calibration as described before [32].

Continuum LC-MS data were processed and searched using ProteinLynx GlobalSERVER version 2.5.2 (Waters Corporation) [41]. Protein identifications were obtained by searching a custom compiled database containing sequences of human and HCMV proteins from the Uniprot database. Sequence information of enolase 1 (S. cerevisiae) and bovine trypsin were added to the databases to normalize the data sets or to conduct absolute quantification as described before [42]. Database search was performed allowing a maximal mass deviation of $15 \mathrm{ppm}$ for precursor ions and $30 \mathrm{ppm}$ for fragment ions with one missed cleavage allowed and fixed carbamidomethyl-cysteine and variable methionine oxidation set as the modifications. For valid protein identification, the following criteria had to be met: at least two peptides detected with together at least seven fragments. The false positive rate for protein identification was set to $1 \%$ based on search of a triple randomized database. Guideline identification criteria were applied for all searches.

For the absolute quantification of proteins, we employed a well established label-free quantitative proteomics workflow that we have previously used both for the quantification of nanoparticle protein coronas [40] and also higher complexity samples such as myelin [42]. Label-free quantification using the TOP3-approach allows both the relative and absolute quantification of proteins. TOP3-Intensity was calculated as the average intensity of three best ionizing peptides and is proportional to the molar amount of the respective protein in the sample [43]. Values were normalized across technical replicates and samples using ISOQuant [44]. For the proteomic analysis of HFF, cells were infected at an MOI of 1 for $2 \mathrm{~h}, 2 \mathrm{~d}$, or $4 \mathrm{~d}$, respectively. After that, cells were collected by removing the culture supernatant and subsequently adding $5 \mathrm{~mL}$ of $50 \mathrm{mM}$ EDTA solution in $1 \times$ PBS. They were detached from the support after an incubation time of 5-15 minutes at $37{ }^{\circ} \mathrm{C}$ and were subsequently centrifuged at $470 \mathrm{~g}$ for five minutes. The pellet was 
resuspended in $10 \mathrm{~mL}$ of PBS and the cells were centrifuged again as described above. This was repeated once. The final pellet was resuspended in 2-3 mL PBS and the cells were counted. 0.5 millions of cells each were transferred to $1.8 \mathrm{~mL}$ Eppendorf tubes and the cells were again centrifuged at $1,300 \mathrm{~g}$ for three minutes. The supernatant was carefully removed and the tubes were stored at $-80{ }^{\circ} \mathrm{C}$ until further mass spectrometry. The samples were subsequently analyzed by mass spectrometry as detailed above.

Virions were purified from the culture supernatants of HCMV infected human foreskin fibroblasts (HFF). For this, cells were infected in a way that all cells showed a typical cytopathic effect at day 1 after infection. Culture supernatants were collected at 6-7 days of infection and were purified by gradient ultracentrifugation as originally described by Irmiere and Gibson [15]. For this, the culture supernatants were collected and centrifuged for $10 \mathrm{~min}$ at $1,900 \mathrm{~g}$ to remove cells and debris. After that, the supernatant was collected and centrifuged at $95,000 \times \mathrm{g}\left(70 \mathrm{~min}, 10^{\circ} \mathrm{C}\right)$ in a SW32Ti rotor in a Beckman Optima L-90K ultracentrifuge. Pellets were resuspended with $2 \mathrm{~mL} 1 \times$ PBS. Na-tartrate gradients were prepared directly before use. For this, $4 \mathrm{~mL}$ of $35 \% \mathrm{Na}$-tartrate solution in $0.04 \mathrm{M}$ Na-phosphate buffer, $\mathrm{pH} 7.4$ were applied in one, and $5 \mathrm{~mL}$ of $15 \% \mathrm{Na}$-tartrate/30\% glycerol solution in $0.04 \mathrm{M}$ Na-phosphate buffer, $\mathrm{pH} 7.4$ were applied in the second column of a gradient mixer. The gradients were prepared by slowly dropping the solutions into Beckman Ultraclear $^{\mathrm{TM}}$ centrifuge tubes $(14 \times 89 \mathrm{~mm})$, positioned at an angle of $45^{\circ} .1 \mathrm{~mL}$ of the viral particles was then carefully layered on top of the gradient. Ultracentrifugation was performed in slow deceleration mode in a Beckman SW41 swing out rotor for $60 \mathrm{~min}$ at 90,000 g and $10^{\circ} \mathrm{C}$. Particles were collected from the gradient, illuminated by light scattering. For this, the centrifuge tube was penetrated with a hollow needle below the band and samples were carefully drawn from the tube with a syringe. The particles were then washed once with $1 \times$ PBS and centrifuged in a SW41 swing out rotor for $90 \mathrm{~min}$ at $100,000 \mathrm{~g}$ and $10^{\circ} \mathrm{C}$. Following that centrifugation step, the pellets were resuspended in 120-150 $\mu \mathrm{L} 1 \times$ PBS. The protein concentration of the purified virions was determined with the Pierce BCA Protein Assay Kit (Thermo Scientific, Bonn, Germany). Twenty $\mu \mathrm{g}$ aliquots of the virions were then pelleted by ultracentrifugation for $60 \mathrm{~min}, 100,000 \mathrm{~g}$ at $10^{\circ} \mathrm{C}$ and stored at $-80^{\circ} \mathrm{C}$ for analysis by mass spectrometry.

\section{Conclusions}

We are only at the advent in our understanding of the complexity of HCMV particle morphogenesis. Mass spectrometry has provided a technical leap in our attempts to understand the molecular events that direct the formation and release of infectious progeny. The results indicate that there are only limited variations in both the intracellular and the virion proteome with regard to viral proteins in derivatives of one particular laboratory strain, AD169. This argues in favor of a highly ordered process of viral protein expression and interaction to ultimately result in packaging and virion release. In line with this, also the proteome of cellular proteins appeared to be conserved following infection with different viruses. However, the data also immediately foster the request for analogous proteomic analyses of other HCMV strains, clinical isolates being of particular interest in this respect. 


\section{Acknowledgments}

This work was supported by a grant from the Deutsche Forschungsgemeinschaft, (DFG), Clinical Research Unit 183 (KFO 183) and by the Research Center for Immunology of the University Medical Center of the University of Mainz, Mainz, Germany. We gratefully acknowledge the donation of BAC-derived viruses from Thomas Shenk, Ulrich Koszinowski, Martin Messerle, Eva Borst, and Gabriele Hahn.

\section{Conflicts of Interest}

The authors declare no conflict of interest.

\section{References and Notes}

1. Mocarski, E.S.; Shenk, T.; Griffiths, P.D.; Pass, R.F. Cytomegaloviruses. In Fields Virology, 6th ed.; Knipe, D.M., Howley, P.M., Eds.; Wolters Kluwer Lippincott Williams \& Wilkins: Philadelphia, PA, USA, 2013; pp. 1960-2014.

2. Gibson, W.; Bogner, E. Morphogenesis of the cytomegalovirus virion and subviral particles. In Cytomegaloviruses: From Molecular Pathogenesis to Intervention, 2th ed.; Reddehase, M.J., Ed.; Caister Academic Press: Norfolk, UK, 2013; pp. 230-246.

3. Milbradt, J.; Auerochs, S.; Marschall, M. Cytomegaloviral proteins pUL50 and pUL53 are associated with the nuclear lamina and interact with cellular protein kinase C. J. Gen. Virol. 2007, 88, 2642-2650.

4. Milbradt, J.; Auerochs, S.; Sticht, H.; Marschall, M. Cytomegaloviral proteins that associate with the nuclear lamina: Components of a postulated nuclear egress complex. J. Gen. Virol. 2009, 90, 579-590.

5. Camozzi, D.; Pignatelli, S.; Valvo, C.; Lattanzi, G.; Capanni, C.; Dal, M.P.; Landini, M.P. Remodelling of the nuclear lamina during human cytomegalovirus infection: Role of the viral proteins pUL50 and pUL53. J. Gen. Virol. 2008, 89, 731-740.

6. Colberg Poley, A.M.; Williamson, C.A. Intracellular sorting and trafficking of cytomegalovirus proteins during permissive infection. In Cytomegaloviruses From Molecular Pathogenesis to Intervention, 2th ed.; Reddehase, M.J., Ed.; Caister Academic Press: Norfolk, UK, 2013; pp. 196-229.

7. Marschall, M.; Feichtinger, S.; Milbradt, J. Regulatory roles of protein kinases in cytomegalovirus replication. Adv. Virus Res. 2011, 80, 69-101.

8. Krosky, P.M.; Baek, M.C.; Coen, D.M. The human cytomegalovirus UL97 protein kinase, an antiviral drug target, is required at the stage of nuclear egress. J. Virol. 2003, 77, 905-914.

9. Sanchez, V.; Greis, K.D.; Sztul, E.; Britt, W.J. Accumulation of virion tegument and envelope proteins in a stable cytoplasmic compartment during human cytomegalovirus replication: Characterization of a potential site of virus assembly. J. Virol. 2000, 74, 975-986.

10. Das, S.; Vasanji, A.; Pellett, P.E. Three-dimensional structure of the human cytomegalovirus cytoplasmic virion assembly complex includes a reoriented secretory apparatus. J. Virol. 2007, 81, 11861-11869. 
11. Das, S.; Pellett, P.E. Spatial relationships between markers for secretory and endosomal machinery in human cytomegalovirus-infected cells versus those in uninfected cells. J. Virol. 2011, 85, 5864-5879.

12. Indran, S.V.; Britt, W.J. A role for the small GTPase Rab6 in assembly of human cytomegalovirus. J. Virol. 2011, 85, 5213-5219.

13. Tandon, R.; AuCoin, D.P.; Mocarski, E.S. Human cytomegalovirus exploits ESCRT machinery in the process of virion maturation. J. Virol. 2009, 83, 10797-10807.

14. Ahlqvist, J.; Mocarski, E. Cytomegalovirus UL103 controls virion and dense body egress. J. Virol. 2011, 85, 5125-5135.

15. Irmiere, A.; Gibson, W. Isolation and characterization of a noninfectious virion-like particle released from cells infected with human strains of cytomegalovirus. Virology 1983, 130, $118-133$.

16. Roby, C.; Gibson, W. Characterization of phosphoproteins and protein kinase activity of virions, noninfectious enveloped particles, and dense bodies of human cytomegalovirus. J. Virol. 1986, 59, 714-727.

17. Jahn, G.; Scholl, B.C.; Traupe, B.; Fleckenstein, B. The two major structural phosphoproteins (pp65 and pp150) of human cytomegalovirus and their antigenic properties. J. Gen. Virol. 1987, 68, 1327-1337.

18. Schmolke, S.; Kern, H.F.; Drescher, P.; Jahn, G.; Plachter, B. The dominant phosphoprotein pp65 (UL83) of human cytomegalovirus is dispensable for growth in cell culture. J. Virol. 1995, 69, 5959-5968.

19. Baldick, C.J., Jr.; Shenk, T. Proteins associated with purified human cytomegalovirus particles. J. Virol. 1996, 70, 6097-6105.

20. Grundy, J.E.; McKeating, J.A.; Griffiths, P.D. Cytomegalovirus strain AD169 binds beta 2 microglobulin in vitro after release from cells. J. Gen. Virol. 1987, 68, 777-784.

21. Stannard, L.M. Beta 2 microglobulin binds to the tegument of cytomegalovirus: An immunogold study. J. Gen. Virol. 1989, 70, 2179-2184.

22. Wright, J.F.; Kurosky, A.; Pryzdial, E.L.; Wasi, S. Host cellular annexin II is associated with cytomegalovirus particles isolated from cultured human fibroblasts. J. Virol. 1995, 69, 4784-4791.

23. Giugni, T.D.; Soderberg, C.; Ham, D.J.; Bautista, R.M.; Hedlund, K.O.; Moller, E.; Zaia, J.A. Neutralization of human cytomegalovirus by human CD13-specific antibodies. J. Infect. Dis. 1996, 173, 1062-1071.

24. Michelson, S.; Turowski, P.; Picard, L.; Goris, J.; Landini, M.P.; Topilko, A.; Hemmings, B.; Bessia, C.; Garcia, A.; Virelizier, J.L. Human cytomegalovirus carries serine/threonine protein phosphatases PP1 and a host-cell derived PP2A. J. Virol. 1996, 70, 1415-1423.

25. Varnum, S.M.; Streblow, D.N.; Monroe, M.E.; Smith, P.; Auberry, K.J.; Pasa-Tolic, L.; Wang, D.; Camp, D.G.; Rodland, K.; Wiley, S.; et al. Identification of proteins in human cytomegalovirus (HCMV) particles: The HCMV proteome. J. Virol. 2004, 78, 10960-10966.

26. Caposio, P.; Streblow, D.N.; Nelson, J.A. Cytomegalovirus proteomics. In Cytomegaloviruses From Molecular Pathogenesis to Intervention, 2th ed.; Reddehase, M.J., Ed.; Caister Academic Press: Norfolk, UK, 2013; pp. 86-108. 
27. Kattenhorn, L.M.; Mills, R.; Wagner, M.; Lomsadze, A.; Makeev, V.; Borodovsky, M.; Ploegh, H.L.; Kessler, B.M. Identification of proteins associated with murine cytomegalovirus virions. J. Virol. 2004, 78, 11187-11197.

28. Borst, E.M.; Hahn, G.; Koszinowski, U.H.; Messerle, M. Cloning of the human cytomegalovirus (HCMV) genome as an infectious bacterial artificial chromosome in Escherichia coli: A new approach for construction of HCMV mutants. J. Virol. 1999, 73, 8320-8329.

29. Hobom, U.; Brune, W.; Messerle, M.; Hahn, G.; Koszinowski, U.H. Fast screening procedures for random transposon libraries of cloned herpesvirus genomes: Mutational analysis of human cytomegalovirus envelope glycoprotein genes. J. Virol. 2000, 74, 7720-7729.

30. Yu, D.; Smith, G.A.; Enquist, L.W.; Shenk, T. Construction of a self-excisable bacterial artificial chromosome containing the human cytomegalovirus genome and mutagenesis of the diploid TRL/IRL13 gene. J. Virol. 2002, 76, 2316-2328.

31. Besold, K.; Frankenberg, N.; Pepperl-Klindworth, S.; Kuball, J.; Theobald, M.; Hahn, G.; Plachter, B. Processing and MHC class I presentation of human cytomegalovirus pp65-derived peptides persist despite gpUS2-11-mediated immune evasion. J. Gen. Virol. 2007, 88, 1429-1439.

32. Hesse, J.; Reyda, S.; Tenzer, S.; Besold, K.; Reuter, N.; Krauter, S.; Büscher, N.; Stamminger, T.; Plachter, B. Human cytomegalovirus pp71 stimulates major histocompatibility complex class i presentation of IE1-derived peptides at immediate early times of infection. J. Virol. 2013, 87, 5229-5238.

33. Zhu, H.; Cong, J.P.; Mamtora, G.; Gingeras, T.; Shenk, T. Cellular gene expression altered by human cytomegalovirus: Global monitoring with oligonucleotide arrays. Proc. Natl. Acad. Sci. USA 1998, 95, 14470-14475.

34. Rabinowitz, J.D.; Shenk, T. Human cytomegalovirus metabolomics. In Cytomegalovirus from Molecular Pathogenesis to Intervention, 2th ed.; Reddehase, M.J., Ed.; Caister Academic Press: Norfolk, UK, 2013; pp. 59-67.

35. Protein Lynx Global Server (PLGS) Ion Accounting Search Algorithm. PLGS is available from Waters, Manchester, UK. Available online: http://www.waters.com/ (accessed on 21 October 2013).

36. Chevillotte, M.; Landwehr, S.; Linta, L.; Frascaroli, G.; Luske, A.; Buser, C.; Mertens, T.; von Einem, J. Major tegument protein pp65 of human cytomegalovirus is required for the incorporation of pUL69 and pUL97 into the virus particle and for viral growth in macrophages. J. Virol. 2009, 83, 2480-2490.

37. To, A.; Bai, Y.; Shen, A.; Gong, H.; Umamoto, S.; Lu, S.; Liu, F. Yeast two hybrid analyses reveal novel binary interactions between human cytomegalovirus-encoded virion proteins. PLoS One 2011, 6, e17796.

38. Becke, S.; Fabre-Mersseman, V.; Aue, S.; Auerochs, S.; Sedmak, T.; Wolfrum, U.; Strand, D.; Marschall, M.; Plachter, B.; Reyda, S. Modification of the major tegument protein pp65 of human cytomegalovirus inhibits virus growth and leads to the enhancement of a protein complex with pUL69 and pUL97 in infected cells. J. Gen. Virol. 2010, 91, 2531-2541. 
39. Phillips, S.L.; Bresnahan, W.A. Identification of binary interactions between human cytomegalovirus virion proteins. J. Virol. 2011, 85, 440-447.

40. Tenzer, S.; Docter, D.; Rosfa, S.; Wlodarski, A.; Kuharev, J.; Rekik, A.; Knauer, S.K.; Bantz, C.; Nawroth, T.; Bier, C.; et al. Nanoparticle size is a critical physicochemical determinant of the human blood plasma corona: a comprehensive quantitative proteomic analysis. ACS Nano. 2011, 5, 7155-7167.

41. ProteinLynx GlobalSERVER, version 2.5.2; Waters Corporation: Manchester, UK, 2011.

42. Patzig, J.; Jahn, O.; Tenzer, S.; Wichert, S.P.; de Monasterio-Schrader, P.; Rosfa, S.; Kuharev, J.; Yan, K.; Bormuth, I.; Bremer, J.; et al. Quantitative and integrative proteome analysis of peripheral nerve myelin identifies novel myelin proteins and candidate neuropathy loci. J. Neurosci. 2011, 31, 16369-16386.

43. Silva, J.C.; Gorenstein, M.V.; Li, G.Z.; Vissers, J.P.; Geromanos, S.J. Absolute quantification of proteins by LCMSE: A virtue of parallel MS acquisition. Mol. Cell Proteomics. 2006, 5, $144-156$.

44. Distler, U.; Kuharev, J.; Navarro, P.; Levin, Y.; Schild, H.J.; Tenzer, S. Drift time-specific collision energies enable deep-coverage data-independent acquisition proteomics. Nat. Methods 2013, doi:10.1038/nmeth.2767. 
Reprinted from Viruses. Cite as: Schleiss, M.R.; McAllister, S.; Armién, A.G.; Hernandez-Alvarado, N.; Fernández-Alarcón, C.; Zabeli, J.C.; Ramaraj, T.; Crow, J.A.; McVoy, M.A. Molecular and Biological Characterization of a New Isolate of Guinea Pig Cytomegalovirus. Viruses 2014, 6, 448-475.

Article

\title{
Molecular and Biological Characterization of a New Isolate of Guinea Pig Cytomegalovirus
}

\author{
Mark R. Schleiss ${ }^{1}{ }^{1} *$, Shane McAllister ${ }^{1}$, Anibal G. Armién ${ }^{2}$, Nelmary Hernandez-Alvarado ${ }^{1}$, \\ Claudia Fernández-Alarcón ${ }^{1}$, Jason C. Zabeli ${ }^{1}$, Thiruvarangan Ramaraj ${ }^{3}$, John A. Crow ${ }^{3}$ \\ and Michael A. McVoy ${ }^{4}$
}

1 Division of Pediatric Infectious Diseases, Department of Pediatrics, University of Minnesota Medical School, Minneapolis, MN 55455, USA;

E-Mails: smcallis@umn.edu (S.M.); hernande@umn.edu (N.H.-A.);

ferna128@umn.edu (C.F.-A.); zabe043@umn.edu (J.C.Z.)

2 Department of Veterinary Population Medicine and Veterinary Diagnostic Laboratory, College of Veterinary Medicine, Saint Paul, MN 55108, USA; E-Mail: armie001@umn.edu

3 National Center for Genome Resources (NCGR), Santa Fe, NM 87505, USA;

E-Mails: tr@ncgr.org (T.R.); jac@ncgr.org (J.A.C.)

4 Division of Pediatric Infectious Diseases, Department of Pediatrics, Virginia Commonwealth University School of Medicine, Richmond, VA 23298, USA; E-Mail: mmcvoy@vcu.edu

* Author to whom correspondence should be addressed; E-Mail: schleiss@umn.edu;

Tel.: +1-612-624-1112; Fax: +1-612-626-9924.

Received: 18 November 2013; in revised form: 9 January 2014 / Accepted: 9 January 2014 /

Published: 27 January 2014

Abstract: Development of a vaccine against congenital infection with human cytomegalovirus is complicated by the issue of re-infection, with subsequent vertical transmission, in women with pre-conception immunity to the virus. The study of experimental therapeutic prevention of re-infection would ideally be undertaken in a small animal model, such as the guinea pig cytomegalovirus (GPCMV) model, prior to human clinical trials. However, the ability to model re-infection in the GPCMV model has been limited by availability of only one strain of virus, the 22122 strain, isolated in 1957. In this report, we describe the isolation of a new GPCMV strain, the CIDMTR strain. This strain demonstrated morphological characteristics of a typical Herpesvirinae by electron microscopy. Illumina and PacBio sequencing demonstrated a genome of 232,778 nt. Novel open reading frames ORFs not found in reference strain 22122 
included an additional MHC Class I homolog near the right genome terminus. The CIDMTR strain was capable of dissemination in immune compromised guinea pigs, and was found to be capable of congenital transmission in GPCMV-immune dams previously infected with salivary gland-adapted strain 22122 virus. The availability of a new GPCMV strain should facilitate study of re-infection in this small animal model.

Keywords: guinea pig cytomegalovirus; cytomegalovirus strain variation; CMV immune evasion; congenital cytomegalovirus infection; congenital CMV vaccines

\section{Introduction}

Development of a vaccine against human cytomegalovirus (HCMV) is a major public health priority [1]. The suggestion that passively transferred antibody protects the fetus against infection and injury [2] has driven efforts to develop recombinant subunit vaccines targeting major envelope glycoproteins, such as glycoprotein $\mathrm{B}(\mathrm{gB})[3,4]$. Although clinical trials of recombinant $\mathrm{gB}$ vaccines have shown some degree of effectiveness in preventing HCMV infection and disease in high risk populations [5,6], vaccine mediated protection with vaccines targeting this single envelope glycoprotein appears to be incomplete. Moreover, the effectiveness of natural immunity in preventing congenital HCMV infection and its attendant sequelae is itself a matter of some controversy. A number of recent studies have described fetal HCMV transmission in women with preconception immunity, due to re-infection with new strains of HCMV [7-12]. Such infections can produce sequelae identical to those observed in congenitally infected infants born to women with primary HCMV infection in pregnancy $[13,14]$. These observations certainly complicate HCMV vaccine design, and suggest that: (1) for full protection, an HCMV vaccine may need to elicit responses superior to those conferred by natural immunity; (2) there may be a strong rationale for vaccinating women of childbearing age who are already HCMV seropositive, in addition to targeting and immunizing seronegative women, toward the goal of preventing re-infection with subsequent transmission of the "new" strain.

Several clinical studies have documented the phenomena of re-infection in women of childbearing age. In one prospective study performed at the University of Alabama-Birmingham, serum specimens from 46 women with preconceptional immunity against HCMV obtained during a previous pregnancy and a new pregnancy were analyzed for antibodies against the strain-specific epitopes of HCMV glycoprotein $\mathrm{H}(\mathrm{gH})$, and the nucleotide sequences of the $\mathrm{gH}$ gene from seven HCMV isolates were determined. Ten of the 16 mothers with infected children (62\%) acquired new antibody specificities against $\mathrm{gH}$, as compared with only 4 of the 30 mothers of uninfected infants (13\%), suggesting that acquisition of an infection with a virus expressing a novel strain-specific $\mathrm{gH}$ genotype during pregnancy was associated with congenital transmission [9]. In another study in Brazil that followed 7,848 women prospectively, sera from 40 mothers of congenitally infected infants and 109 mothers of uninfected control newborns were analyzed for strain-specific anti-HCMV antibodies, based not only on polymorphisms within $\mathrm{gH}$ binding sites, but also a second antibody reactivity site on $\mathrm{gB}[14,15]$. Seven of $40(17.5 \%)$ study women, but only 5 of $109(4.6 \%)$ controls 
acquired antibodies reactive with new HCMV strains during pregnancy $(p=0.002)$, suggesting that maternal reinfection by new strains of HCMV is a major source of congenital infection in this population. In a study of re-infection (based on acquisition of new $\mathrm{gB}$ and/or $\mathrm{gH}$ antibody specificities) in 205 seropositive women performed by Ross and colleagues at UAB, approximately one-third of the study participants (59 of 205) were noted to have reinfection, using this definition, during follow-up [10]. The molecular and immunological correlates of re-infection are unclear. There is some evidence that $\mathrm{gB}$ polymorphisms in clinical isolates may be less important for re-infection than polymorphisms in $\mathrm{gH}$ and other envelope glycoproteins. In a study in Brazil, infections in immunocompetent women with strains corresponding to more than one gB genotype were not common [16]. Additionally, in a study of the HCMV strains acquired longitudinally in women who developed infection in spite of being enrolled in the recombinant $\mathrm{gB}$ vaccine trial at $\mathrm{UAB}[5]$, there was no selection for or against any non-vaccine $\mathrm{gB}$ subtype, in spite of women being immunized only with gB protein corresponding to the Towne (gB1 subtype) strain [17]. Other evidence suggests that the response (or lack thereof) to the envelope glycoprotein $\mathrm{N}$ ( $\mathrm{gN}$ ) may play a role in predisposing to re-infection with new HCMV strains expressing heterologous gB and/or gH genotypes [18-20].

Irrespective of the mechanism(s) involved, the issue of re-infection is a major challenge in vaccine design. There is increasing evidence that congenital HCMV infections after nonprimary maternal infections can lead to symptomatic disease and substantial long-term sequelae. Notably, recent evidence from a study at UAB indicated that the incidence of hearing loss in infants infected after nonprimary maternal infection was similar to that in infected infants born to women with primary infection [13], although in this study, infants in the primary infection group were more likely to demonstrate progressive and severe or profound hearing loss, compared to infants in the non-primary group.

Since the consequences of re-infection and congenital transmission with a newly acquired strain in pregnant seropositive women can be similar to those that occur after primary infection and transmission in CMV-seronegative women $[10,11,13,14]$, the study of re-infection in a small animal model of congenital transmission would be very useful for the modeling of vaccine strategies to prevent maternal re-infection [21,22]. Ideally, HCMV reinfection would be studied in an animal model prior to clinical vaccine trials. Unfortunately, the strict species-specificity of cytomegaloviruses precludes preclinical testing of HCMV vaccines in animals. However, a number of rodent and primate cytomegaloviruses are useful in modeling HCMV vaccines and therapies, given the conservation of many immunogenic structural proteins amongst the various viruses [23-25].

Among the small animal models, the guinea pig cytomegalovirus (GPCMV) is uniquely useful, since, in contrast to other rodent models, transplacental infection of the fetus occurs following viral challenge during pregnancy $[24,26]$. Hence, the GPCMV model is particularly well-suited to the study of vaccines against congenital infection. However, until now this model has relied exclusively on a single strain of GPCMV, 22122, isolated by Hartley in 1957 [27]. While it has been feasible to study re-infection by experimentally inoculating strain 22122 virus into naturally seropositive guinea pigs [28], the lack of defined genetic information on other GPCMV strains (e.g., those presumably latent in the seropositive animals used in the above mentioned studies) has 
made it impossible to study of the role of strain variation in fetal infection in the setting of preconception immunity.

In this report, we describe the isolation of a novel strain of GPCMV, the CIDMTR strain. This communication represents the first report of detailed characterization of a GPCMV isolate since the original publication of isolation of the 22122 strain [27]. Although sequence and ORF structure were generally well conserved with the 22122 strain of GPCMV, the CIDMTR strain demonstrated some differences in genome structure, particularly in the right-hand end of the viral genome. There are also substantive differences in some protein coding sequences between the two strains, including sequences in envelope glycoproteins, suggesting that these proteins may have been the targets of immune selection during the evolution of GPCMV in the guinea pig host. We describe in this report the morphology and DNA sequence of this newly isolated strain, and report preliminary experiments regarding its pathogenesis in vivo. The availability of a second strain of GPCMV should enable the study of re-infection and, potentially, the development of vaccine strategies designed to protect against maternal re-infection in the guinea pig model of congenital cytomegalovirus infection.

\section{Results and Discussion}

\subsection{Isolation of the CIDMTR Strain}

In the course of ongoing vaccine and pathogenesis studies, guinea pigs were screened at the time of purchase for GPCMV antibodies, using an ELISA based on GPCMV strain 22122 [29]. Within a group of 24 guinea pigs purchased from a commercial source, $5(21 \%)$ were found to be GPCMV-seropositive by ELISA. Western blot analysis was performed using sera from commercially purchased, ELISA-positive animals using purified strain 22122 virions as the source of target antigens (Figure 1). These studies confirmed that sera from these animals were broadly cross-reactive with GPCMV strain 22122 virion-associated polypeptides, suggesting that these animals were infected with GPCMV strains that were closely related antigenically to strain 22122.

One of the seropositive animals identified in these preliminary experiments was immunosuppressed with cyclophosphamide, $100 \mathrm{mg} / \mathrm{kg}$ [32], and seven days later the animal was sacrificed and salivary gland (SG) homogenates harvested. Presumptive virus in the SG homogenate was passaged in vivo by inoculation of two GPCMV seronegative inbred strain 2 guinea pigs with $1 \mathrm{~mL}$ SG homogenate by subcutaneous route in the dorsal neck. Both of these animals demonstrated DNAemia (3.2 and $6.9 \times 10^{3}$ genomes $/ \mathrm{mL}$ of blood, respectively) at 14 days post-inoculation. Three weeks following inoculation, these animals were also immunosuppressed with $100 \mathrm{mg} / \mathrm{kg}$ of cyclophosphamide. One week later, these animals were sacrificed and SG homogenates were cultured on guinea pig lung fibroblast cells (GPLs). Eleven days after inoculation plaques with characteristic cytopathic effect (CPE) were observed in one of the SG homogenate cultures (designated $\mathrm{P}_{0}$ ). Supernatant from this flask was used to inoculate GPLs to generate a $\mathrm{P}_{1}$ stock of virus. When CPE was extensive (approximately 2 weeks later), an aliquot was removed for electron microscopy (EM) studies (described below, Section 2.2) and the remainder of this flask was expanded for large-scale virus propagation (20 flasks). These flasks were then incubated for an additional week prior to harvest of $\mathrm{P}_{2}$ viral stock (Figure 1b). Some of these infected flasks were 
used for DNA purification for Illumina MiSeq and Pacific Biosciences PacBio RS sequencing, as described below. The remainder of this stock was used for in vivo challenge experiments in guinea pigs, as described in Section 2.4.

Figure 1. Western blot analysis of sera from three "naturally seropositive" commercially purchased outbred Hartley guinea pigs. (a) Western analysis using a pooled, high-titer polyclonal anti-GPCMV antisera from guinea pigs immunized with adjuvanted viral particles [30] or sera from three "natural seropositive" guinea pigs (GP1, GP2, GP3) obtained from a commercial supplier, using sucrose gradient-purified virions from 22122 strain as target antigen. Two independently derived, monospecific anti-GPCMV gB antibodies (moab 29-29 [31] (gB1) and moab IE321 (gB2)) are included as controls. (b) Photomicrographs comparing CPE of low-passage $\left(\mathrm{P}_{2}\right)$ CIDMTR virus (right panel) to GPCMV strain 22122 (left panel) following infection of GPL cells. CIDMTR plaques are more rounded, with elliptical appearance, compared to the more spindle-like morphology of 22122-infected fibroblasts. Plaques photographed at $60 \times$ magnification.

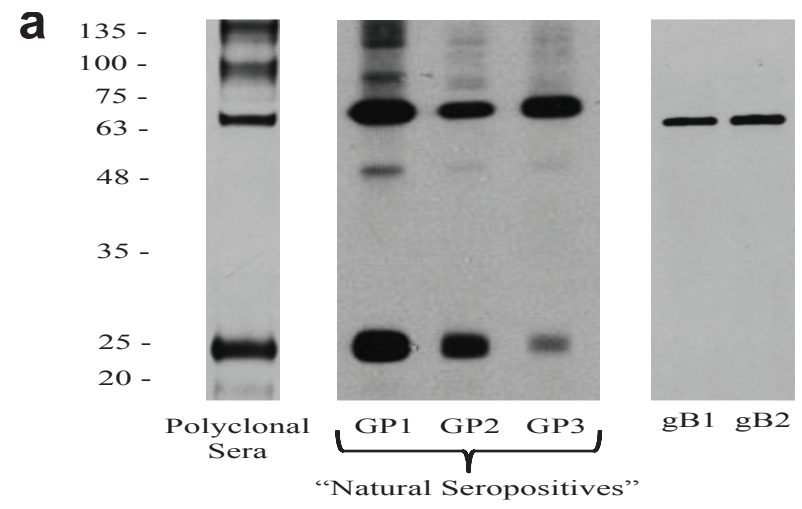

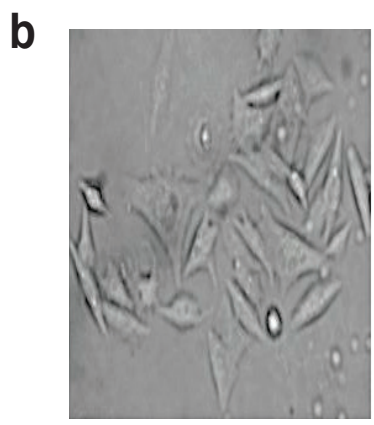

$\operatorname{ATCC}(22122)$

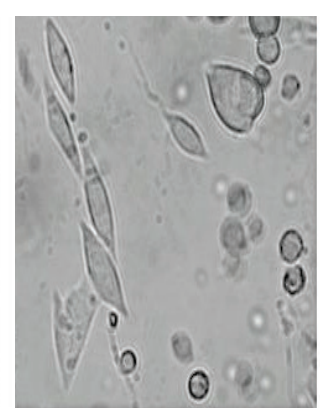

CIDMTR

\subsection{Morphological Analyses by EM}

To confirm that the isolated virus had morphological characteristics of a cytomegalovirus, EM was performed. Analysis of $\mathrm{P}_{1}$ virus stock by conventional microscopy (Section 2.1) revealed 20\% of the cells showed cytopathic effect. Cells were enlarged with large intranuclear and intracytoplasmic basophilic inclusions visible upon examination by light microscopy on thick section of plastic preparation stained with Toluidine blue (data not shown). Ultrastructurally, both intranuclear and intracytoplasmic inclusions were noted, interpreted as representative of nuclear and cytoplasmic viral factories (Figure 2A,B). Nuclear inclusions were typical for the center of replication and assembly characteristic of Herpesvirinae (Figure 2C). These nuclear factories showed a large amount of fibrillary electron dense material admixed with moderate to large numbers of empty A-capsids, scaffold-containing B-capsids, and DNA-containing C-capsids that are characteristic of herpesvirus capsid formation and genome packaging (Figure 2C; most capsids in this image are B-capsids). By comparison, a virtually identical appearance was noted in EMs from strain 22122-infected fibroblasts (Figure 2H). First envelopment was acquired at the inner nuclear membrane. The EM morphology supports a model whereby de-envelopment of nucleocapsids takes place at the outer nuclear membrane during egress, with the second envelopment and tegumentation occurring in the cytoplasm factory (Figure 2D). Rearrangement of 
Golgi cisterns, Golgi vesicles, multivesicular body and endoplasmic reticulum in conjunction with formation of nucleocapsids produced large aggregations that were often mixed with electron dense material. Numerous dense bodies were present in the intercellular space (Figure 2A,F). On negative contrast preparation, a large number of non-infectious enveloped particles $(220.24 \pm 48.15 \mathrm{~nm})$ and dense bodies $(357.83 \pm 97.85 \mathrm{~nm}$ ) were observed (Figure $2 \mathrm{G}$ ). Infectious mature virions consisted of an envelope containing a $116.5 \pm 2.68 \mathrm{~nm}$ in diameter icosahedral capsid with capsomeres of approximately $11.66 \pm 1.87 \mathrm{~nm}$. The CIDMTR strain of GPCMV presented similar cytopathic and pathogenic effects as those demonstrated by ATCC strain 22122 [33-35]. Nevertheless, some differences were noted between these two strains. The ATCC strain in contrast to the CIDMTR strain, demonstrated more efficient infection of fibroblasts, even at the same multiplicity of infection (MOI; MOI of 1), with over $80 \%$ of fibroblastic cells exhibiting cytopathic effect. The nuclear and cytoplasmic factories were less pronounced in the CIDMTR-infected cells, with generation of reduced quantities of infectious and non-infectious enveloped viral particles and dense bodies (data not shown).

\subsection{Sequencing and Sequence Analysis}

Viral DNA $\left(\mathrm{P}_{2}\right)$ was purified from the CIDMTR strain after two passages in GLFs and subjected to sequence analysis using the MiSeq and PacBio platforms as described in Section 3. The CIDMTR genome was 232,778 nt in length. Overall sequence homology to strain 22122 (accession \#KC503762.1) was 98\%, consistent with the hypothesis suggested by immunoblotting that CIDMTR is a GPCMV and not a novel and distinct betaherpesvirus of guinea pigs. As discussed further in Section 4, a possibility existed that genetic diversity of GPCMV isolates could be restricted due to bottlenecks in the breeding history of Hartley guinea pigs, from which both 22122 and CIDMTR are derived. Historically, HCMV strains were differentiated on the basis of restriction pattern polymorphisms. Epidemiologically unrelated isolates, considered unique HCMV strains, exhibit predominantly identical restriction patterns with occasional unique fragments. To compare CIDMTR to 22122 in these terms, genomic sequences for each virus were used to generate in silico-predicted restriction patterns for three enzymes (EcoR I, Hind III, and Xba I). As shown in Figure 3a, the majority of fragments matched between the two virus genomes in all three predicted restriction enzyme patterns, while for each restriction pattern a few unique (unmatched) fragments were evident. These results are consistent with CIDMTR and 22122 being distinct GPCMV strains in a manner similar to that of HCMV strains.

Sequence comparison revealed three regions of striking discrepancies between the two genome sequences (Figure 3b). The first was located between nucleotides 199227 and 203071 of the 22122 sequence. Within this 3,845-bp region, 1,437 bps were entirely missing from the CIDMTR genome, while the remainder corresponded with a poorly conserved $(50 \%$ average nucleotide conservation) 2,366-bp region in CIDMTR. As a result, one of the three gp138 family ORFs previously annotated in 22122 was missing from CIDMTR (Figure 3c). Curiously, this region corresponds to a hot-spot for spontaneous deletions that occur in response to over length genomes in GPCMV that also roughly corresponds to regions of instability/rearrangements in HCMV and rhesus cytomegalovirus [36]. 
The second region of discrepancy was located between nucleotides 221608 and 224743 of the CIDMTR sequence. Within this 3,136-bp region, 1,424 bps were entirely missing from the 22122 genome, and the remainder correspond with a poorly conserved (68\% average nucleotide conservation) 1,684-bp region in 22122. Surprisingly, the additional sequences in CIDMTR encoded a fourth putative MHC class I homolog, annotated gp147.1, that lies adjacent to three MHC class I homologs ( $g$ p147, gp148, and gp149) that were previously identified in 22122 (Figure 3c).

Figure 2. Transmission electron microscopy (EM) microphotographs of fibroblasts infected with the CIDMTR Strain of GPCMV. (A) Plastic embedded preparation contrasted with uranyl acetate/lead citrate of two enlarged fibroblasts showing cell intranuclear and cytoplasmic viral inclusions and numerous dense bodies (white arrowheads) in the intercellular space (bar $=10 \mu \mathrm{m})$. (B) Magnification of another CIDMTR-infected cell revealed nuclear $(\mathrm{N})$ viral replication and nucleocapsid assembly sites (small square), as well as a large maturation site (asterisk) and dense body formation in the cytoplasm (large square, bar $=2 \mu \mathrm{m}$ ). (C) Additional magnification in which replication and capsid assembly in the nucleus (small square) is appreciated; note empty capsids (arrow) and DNA containing nucleocapsids (arrowhead; bar $=0.5 \mu \mathrm{m}$ ). (D) Magnification of virus maturation sites within the cytoplasm (large square in panel (B)) reveals electron dense material formed by aggregation of nucleocapsids and cell organelles such as Golgi systems, endoplasmic reticulum and vesicles (bar $=0.5 \mu \mathrm{m}$ ). (E) Capsid is demonstrated becoming coated with tegument proteins and then acquiring its final envelope by budding into vesicles (arrows; bar $=0.5 \mu \mathrm{m}$ ). (F) Several dense bodies are present in the intercellular space (bar $=0.5 \mu \mathrm{m})$. (G) Negative contrast preparation of enveloped B-capsid (arrow) and dense body (asterisk, bar $=0.5 \mu \mathrm{m}$ ) is demonstrated. (H) EM of strain 22122-infected cells demonstrating virtually identical morphology; A, B and C capsids are identified as described in text.

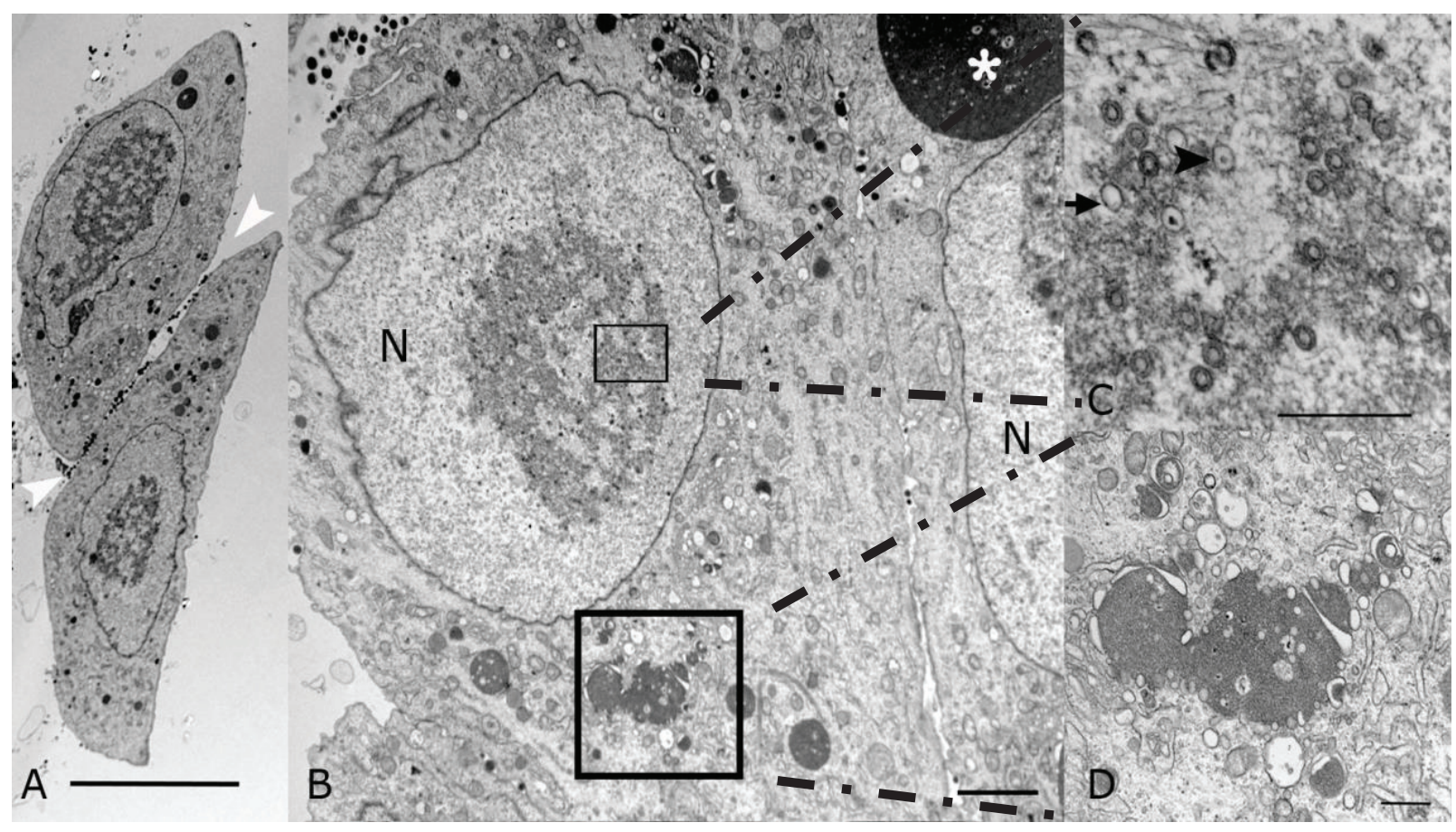


Figure 2. Cont.

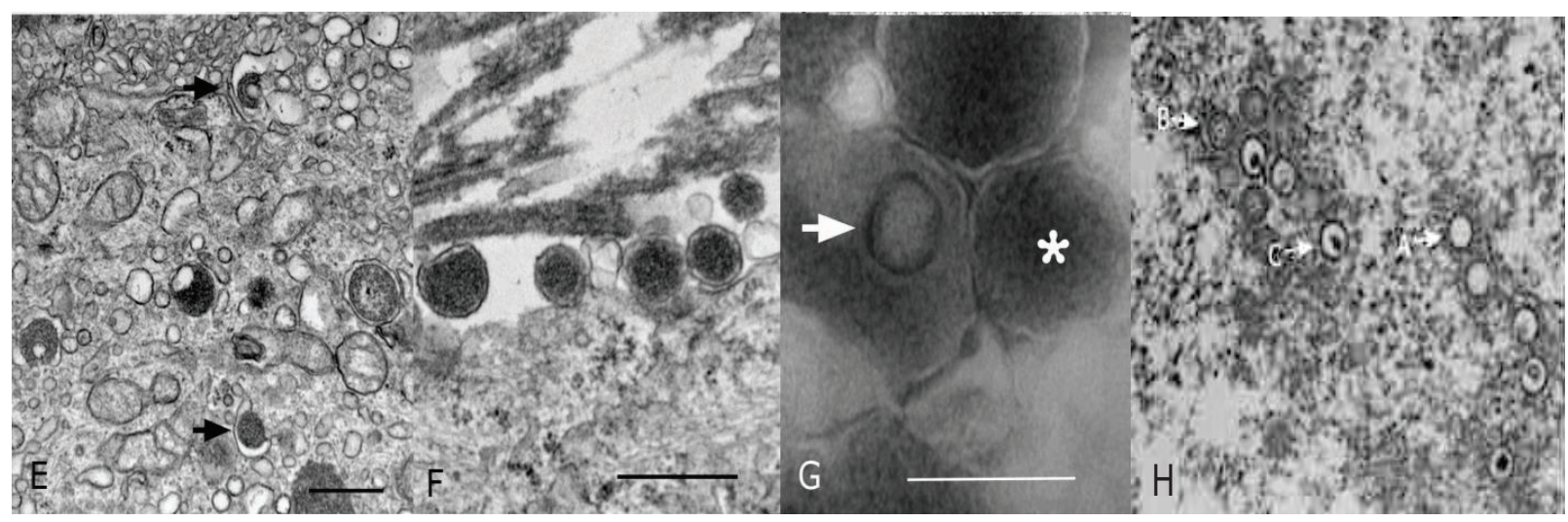

The third region of discrepancy was located between nucleotides 230176 and 231697 of the 22122 sequence. Within this 1,522-bp region, 107 bps were missing from the CIDMTR genome, and the remainder correspond with a poorly conserved (51\% average nucleotide conservation) 1,383-bp region in CIDMTR. This discrepant region partially overlaps gp149 and results in CIDMTR- and 22129-encoded gp149 proteins that lack amino acid homology in residues 1-134 (22122) or 1-164 (CIDMTR) but are highly conserved within the remaining C-terminal 494 residues.

Finally, like 22122, the CIDMTR genome was found to have direct terminal repeat sequences, although the CIDMTR repeats were slightly shorter (841 nt) than those in 22122 (953 nt).

Figure $3 \mathrm{~b}$ highlights the regions of DNA sequence dissimilarity when the CIDMTR strain is compared to strain 22122. Figure 3c illustrates in map format the regions of genomic discontinuity. To confirm that these large-scale rearrangements did not arise during passage of virus in cell culture, PCR and sequence analyses were performed directly on both DNA purified from the original salivary gland homogenate (no tissue culture passage), and on DNA from the tissue culture-derived isolate $\left(\mathrm{P}_{0}\right)$, with identical results (see Section 2.5 and Figure 4). The sequences of the PCR-generated fragments were identical to the Illumina/PacBio genome sequence, confirming that discontinuities with strain 22122 did not arise as an artifact of tissue culture passage. It therefore appears that, as it appears in its natural host, the CIDMTR strain lacks the gp138.1 gene found in 22122. Similarly, 22122 lacks the fourth MHC class I homolog gene (gp147.1) found in CIDMTR. As the original SG extract from which 22122 was cultured is no longer available, it is not possible to conduct similar studies to determine whether gp147.1 was absent from 22122 prior to its initial isolation in cell culture or was lost during subsequent in vitro/in vivo passage. Nevertheless, these results suggest that naturally occurring strains of GPCMV may be polymorphic with respect to the presence or absence of entire genes. Similar findings have been observed when comparing the Smith strain of murine cytomegalovirus to strains isolated from wild mice [37]. Further analyses of other primary virus isolates from "naturally infected" guinea pigs will be necessary to confirm this hypothesis and to determine if such polymorphisms are limited to specific genes, such as gp138.1 and perhaps gp147.1, or include others.

Table 1 summarizes the predicted ORFs identified in the CIDMTR strain. ORFs that are highly conserved in cytomegaloviruses are noted in the table in upper case/bold font (e.g., GP55). In contrast, ORFs appearing unique to GPCMV are noted in lower case (e.g., gp138). The "C" designation in Table 1 refers to the complimentary strand. Splicing sites are predicted based on 
previously published reports and these need empiric confirmation in future studies unless otherwise indicated (Figures 4 and 5); exons are described as coding exons.

Figure 3. Strain-specific genome structure differences between CIDMTR and 22122. (a) In silico analysis of predicted restriction endonuclease profiles for CIDMTR strain and 22122 (ATCC) strain with enzymes EcoRI, HindIII, and XbaI. Predictions and profile generated using program CLC Main Workbench 6 [40]. (b) Dot-matrix comparison of CIDMTR vs. 22122 genomic sequences. Two genome regions demonstrating sequence variability resulting in ORFs unique to each strain are indicated by shaded boxes. (c) Linear map comparison of the major areas of genome discontinuity. Linear maps prepared using XPlasMap [41]. Colored boxes in blue represent predicted conserved ORFs. Light red and green colored ORFs demonstrate discordance between the two strains. In the CIDMTR strain, an additional ORF not annotated in 22122, 149.1, is also noted (dark red). Dashed lines indicate the positions where the sequences differ. See Table 1 for a full list of sequenced gene annotations.

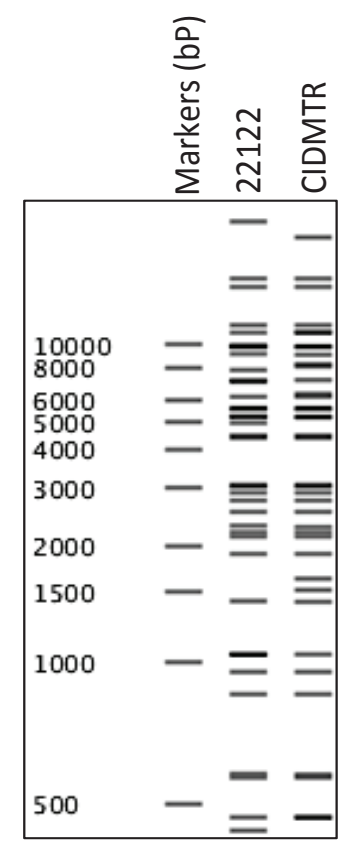

EcoR I

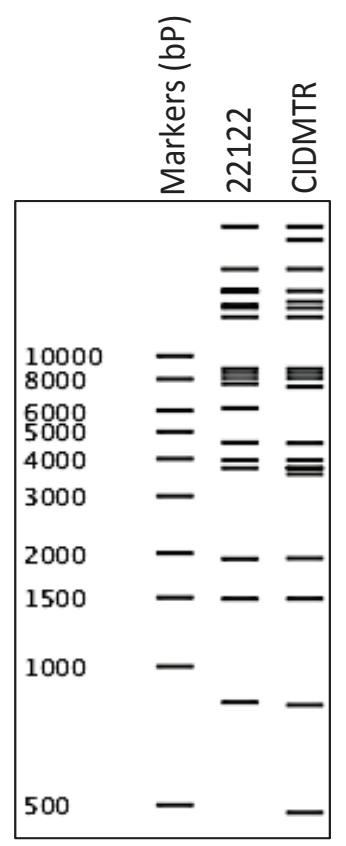

Hind III

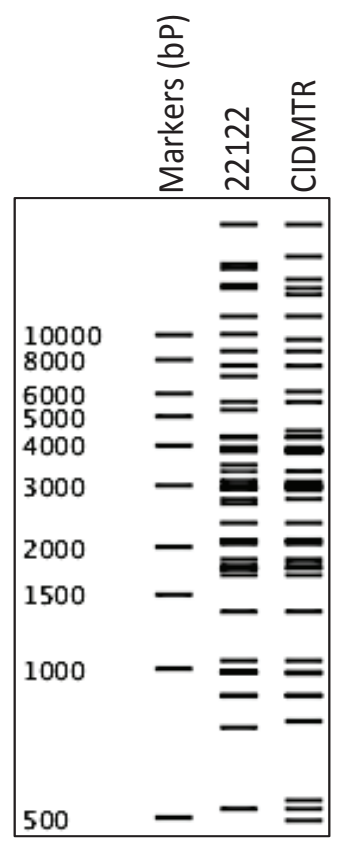

Xba I

(a) 
Figure 3. Cont.

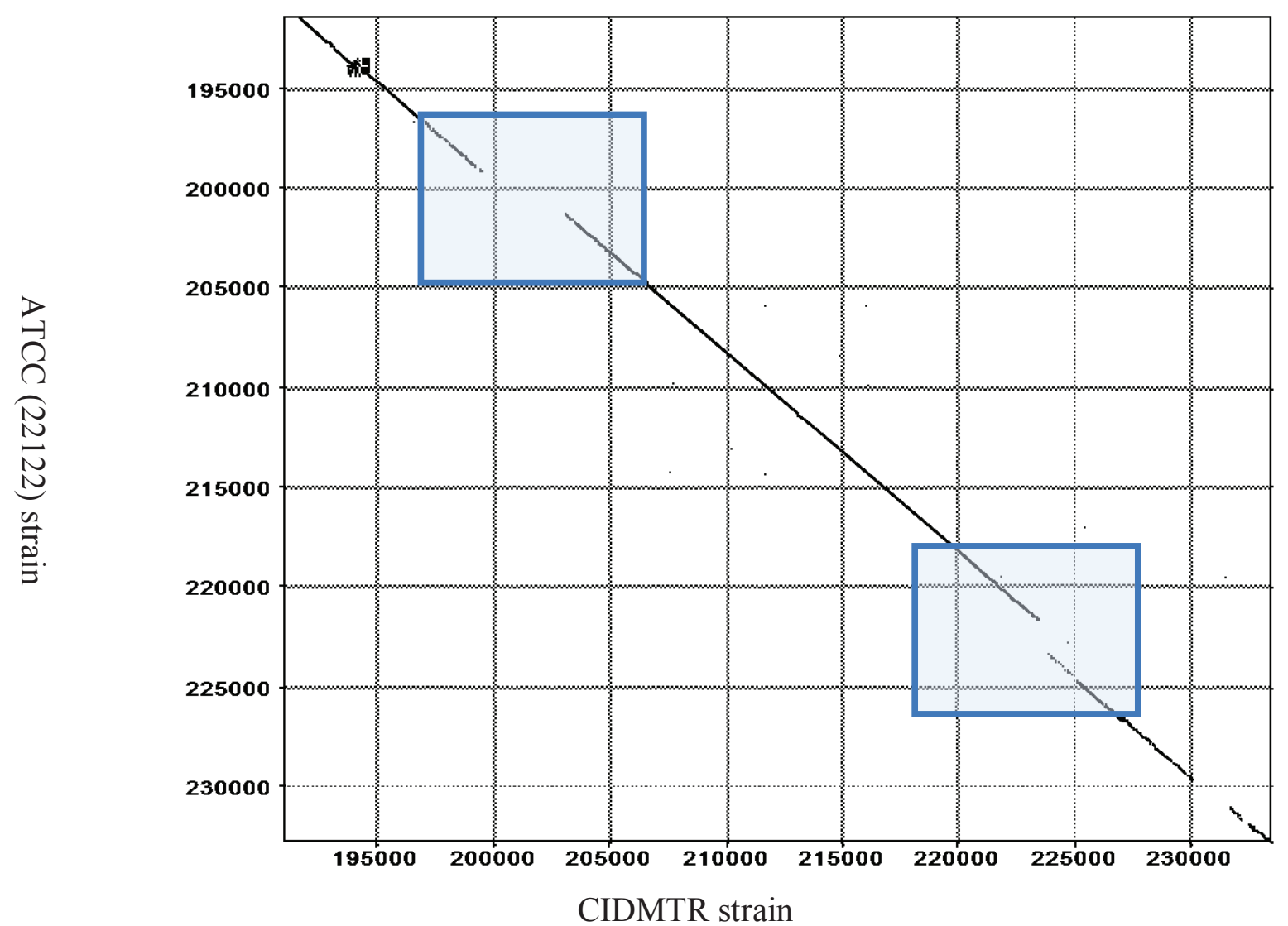

(b)
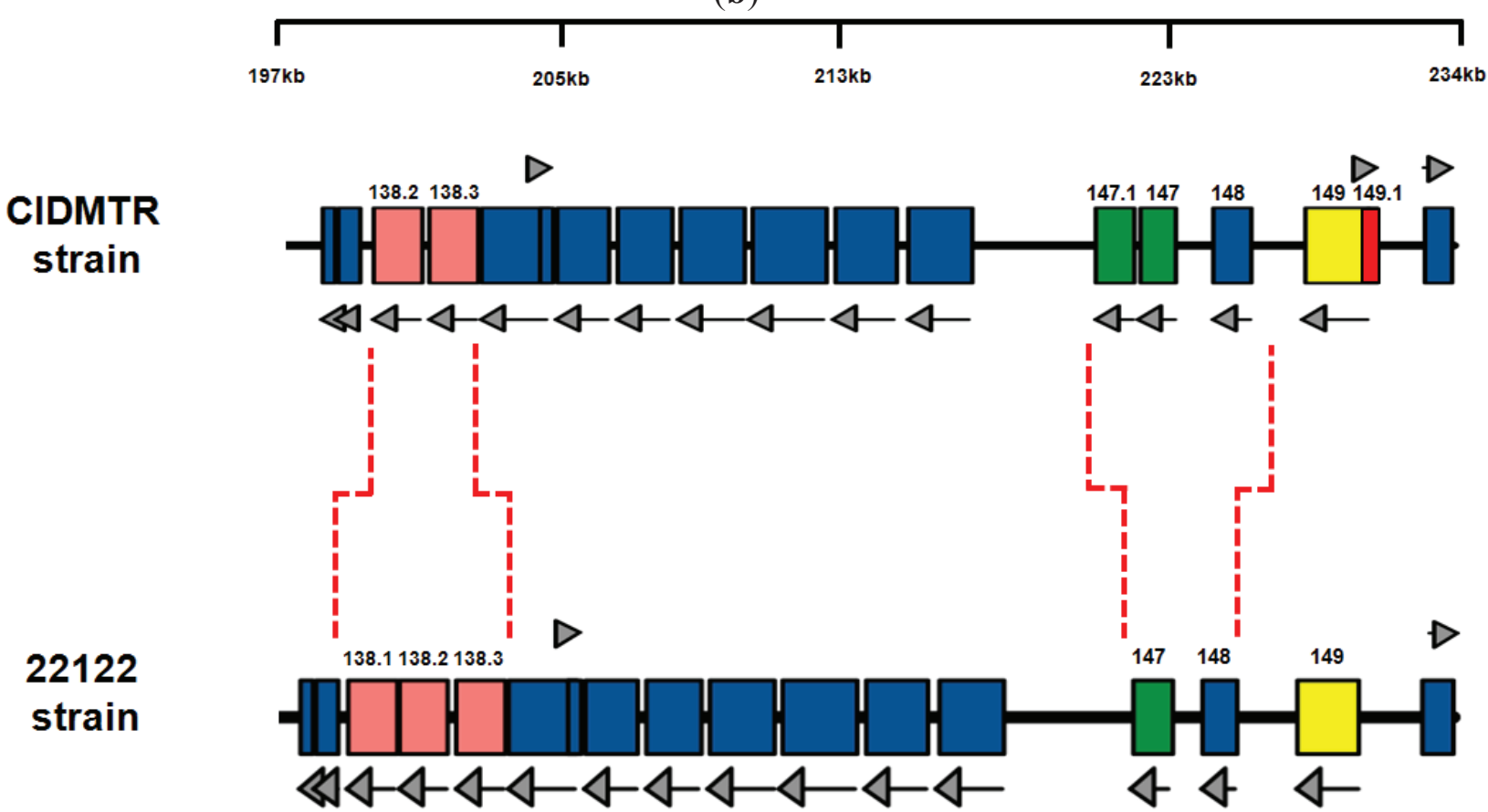

(c) 
Table 1. GPCMV Strain CIDMTR Predicted ORFs.

\begin{tabular}{|c|c|c|c|c|c|}
\hline \multicolumn{6}{|c|}{ GPCMV Open Reading Frames (ORFs): CIDMTR Strain } \\
\hline$\underline{\text { ORF }}$ & Strand & Begin & End & Codons & Notes \\
\hline gp1 & $\mathrm{C}$ & 12,464 & 12,769 & 101 & GPCMV MIP $1 \square$; CC chemokine homolog \\
\hline $\mathrm{gp} 2$ & & 14,840 & 15,685 & 281 & Homology to MCMV M69 $^{\text {a }}$ \\
\hline gp3 & $\mathrm{C}$ & 17,197 & 19,563 & 788 & Homology to THV T5 $5^{\mathrm{b}}$; US22 superfamily \\
\hline gp4 & $\mathrm{C}$ & 20,829 & 21,152 & 107 & Homology to RCMV r136 ${ }^{\mathrm{d}}$ \\
\hline gp5 & $\mathrm{C}$ & 26,721 & 27,818 & 365 & Homology to MCMV m32a \\
\hline GP23 & $\mathrm{C}$ & 33,299 & 34,501 & 400 & UL23 homolog; US22 gene superfamily \\
\hline GP24 & $\mathrm{C}$ & 34,739 & 35,956 & 405 & UL24 homolog; US22 superfamily \\
\hline GP25 & & 36,542 & 38,194 & 550 & UL25 homolog; tegument protein \\
\hline GP26 & $\mathrm{C}$ & 38,360 & 39,043 & 227 & UL26 homolog \\
\hline GP27 & $\mathrm{C}$ & 39,166 & 41,211 & 681 & UL27 homolog \\
\hline GP28 & $\mathrm{C}$ & 41,311 & 42,378 & 355 & UL28 homolog; US22 superfamily \\
\hline GP28.1 & $\mathrm{C}$ & 42,866 & 44,287 & 473 & UL28 homolog; US22 superfamily \\
\hline GP29 & $\mathrm{C}$ & 44,653 & 46,623 & 656 & UL29 homolog; US22 superfamily \\
\hline gp29.1 & $\mathrm{C}$ & 47,247 & 47,861 & 204 & US22 superfamily \\
\hline GP30 & $\mathrm{C}$ & 49,082 & 50,800 & 572 & UL30 homolog \\
\hline GP31 & & 51,094 & 52,572 & 492 & UL31 homolog \\
\hline GP32 & $\mathrm{C}$ & 52,665 & 54,365 & 566 & UL32 homolog \\
\hline GP33 & & 54,585 & 55,868 & 427 & UL33 homolog; 7-TMR GPCR superfamily \\
\hline GP34 & & 56,221 & 57,804 & 527 & UL34 homolog \\
\hline GP35 & & 58,008 & 59,666 & 552 & UL35 homolog \\
\hline GP37 & $\mathrm{C}$ & 59,788 & 60,711 & 307 & UL37 homolog \\
\hline GP38 & $\mathrm{C}$ & 61,068 & 61,988 & 306 & UL38 homolog \\
\hline gp38.1 & $\mathrm{C}$ & 62,705 & 63,262 & 185 & Positional homolog of HCMV UL40 \\
\hline gp38.2 & $\mathrm{C}$ & 63,620 & 64,933 & 437 & Positional homolog of HCMV UL41a \\
\hline gp38.3 & $\mathrm{C}$ & 65,624 & 66,478 & 284 & Positional homolog of HCMV UL42 \\
\hline gp38.4 & $\mathrm{C}$ & 66,997 & 67,362 & 121 & Homology to RCMV r42 $^{\mathrm{d}}$ \\
\hline GP43 & $\mathrm{C}$ & 67,951 & 68,964 & 337 & UL43 homolog \\
\hline GP44 & $\mathrm{C}$ & 68,952 & 70,175 & 407 & UL44 homolog \\
\hline GP45 & $\mathrm{C}$ & 70,887 & 73,673 & 928 & UL45 homolog \\
\hline GP46 & $\mathrm{C}$ & 73,779 & 74,867 & 362 & UL46 homolog \\
\hline GP47 & & 74,674 & 77,787 & 1037 & UL47 homolog \\
\hline GP48 & & 77,784 & 84,155 & 2123 & UL48 homolog \\
\hline GP48.2 & $\mathrm{C}$ & 84,238 & 84,468 & 76 & UL48a homolog \\
\hline GP49 & $\mathrm{C}$ & 84,479 & 86,119 & 546 & UL49 homolog \\
\hline GP50 & $\mathrm{C}$ & 86,088 & 87,158 & 356 & UL50 homolog \\
\hline GP51 & $\mathrm{C}$ & 87,281 & 87,580 & 99 & UL51 homolog; terminase subunit \\
\hline GP52 & & 87,900 & 89,480 & 526 & UL52 homolog \\
\hline GP53 & & 89,473 & 90,459 & 328 & UL53 homolog \\
\hline gp53.1 & $\mathrm{C}$ & 90,196 & 90,537 & 113 & Homology to RhCMV rh86; YP 068179.1 \\
\hline GP54 & $\mathrm{C}$ & 90,551 & 93,904 & 1117 & UL54 homolog; DNA polymerase \\
\hline GP55 & $\mathrm{C}$ & 93,947 & 96,652 & 901 & UL55 homolog; glycoprotein B \\
\hline GP56 & $\mathrm{C}$ & 96,549 & 98,816 & 755 & UL56 homolog; terminase subunit \\
\hline GP57 & $\mathrm{C}$ & 98,967 & 102,650 & 1227 & UL57 homolog \\
\hline gp57.1 & $\mathrm{C}$ & 104,411 & 104,926 & 171 & Homolog to RCMV r23.1 ${ }^{\text {d }}$ \\
\hline GP69 & $\mathrm{C}$ & 108,260 & 111,430 & 1056 & UL69 homolog \\
\hline GP70 & $\mathrm{C}$ & 112,136 & 115,339 & 1067 & UL70 homolog; helicase-primase \\
\hline GP71 & & 115,338 & 116,114 & 258 & UL71 homolog \\
\hline GP72 & $\mathrm{C}$ & 116,277 & 117,350 & 357 & UL72 homolog; dUTPase \\
\hline GP73 & & 117,432 & 117,833 & 133 & UL73 homolog; glycoprotein N \\
\hline
\end{tabular}


Table 1. Cont.

\begin{tabular}{|c|c|c|c|c|c|}
\hline \multicolumn{6}{|c|}{ GPCMV Open Reading Frames (ORFs): CIDMTR Strain } \\
\hline$\underline{\text { ORF }}$ & $\underline{\text { Strand }}$ & Begin & End & $\underline{\text { Codons }}$ & Notes \\
\hline GP73.5ex1 & & 117,860 & 117,869 & 3 & \\
\hline GP73.5ex2 & & 119,005 & 119,198 & 64 & \\
\hline GP74 & $\mathrm{C}$ & 117,780 & 118,904 & 374 & UL74 homolog; glycoprotein O \\
\hline GP75 & $\mathrm{C}$ & 119,349 & 121,529 & 726 & UL75 homolog; glycoprotein H \\
\hline GP76 & & 121,694 & 122,533 & 279 & UL76 homolog \\
\hline GP77 & & 122,247 & 124,091 & 614 & UL77 homolog \\
\hline GP78 & & 124,473 & 125,717 & 414 & UL78 homolog; 7-TMR GPCR superfamily \\
\hline GP79 & $\mathrm{C}$ & 125,914 & 126,861 & 315 & UL79 homolog \\
\hline GP80 & & 126,722 & 129,031 & 769 & UL80 homolog; CMV protease \\
\hline GP80.5 & & 127,610 & 129,031 & 473 & UL80.5 \\
\hline GP82 & $\mathrm{C}$ & 129,324 & 130,889 & 521 & UL82 homolog; pp71 \\
\hline GP83 & $\mathrm{C}$ & 131,109 & 132,809 & 566 & UL83 homolog; pp65 \\
\hline GP84 & $\mathrm{C}$ & 133,051 & 134,487 & 478 & UL84 homolog \\
\hline GP85 & $\mathrm{C}$ & 134,785 & 135,696 & 303 & UL85 homolog \\
\hline GP86 & $\mathrm{C}$ & 135,977 & 140,026 & 1349 & UL86 homolog \\
\hline GP87 & & 140,407 & 143,328 & 973 & UL87 homolog \\
\hline GP88 & & 143,231 & 144,499 & 422 & UL88 homolog \\
\hline GP89ex2 & $\mathrm{C}$ & 144,545 & 145,675 & 376 & UL89 homolog; terminase subunit, exon 2 \\
\hline GP91 & & 146,102 & 146,365 & 87 & UL91 homolog \\
\hline GP92 & & 146,362 & 146,991 & 209 & UL92 homolog \\
\hline GP93 & & 146,957 & 148,732 & 591 & UL93 homolog \\
\hline GP94 & & 148,644 & 149,681 & 345 & UL94 homolog \\
\hline GP89ex1 & $\mathrm{C}$ & 150,032 & 150,913 & 291 & UL89 homolog; terminase subunit, exon 1 \\
\hline GP95 & & 150,967 & 152,235 & 422 & UL95 homolog \\
\hline GP96 & & 152,468 & 152,830 & 120 & UL96 homolog \\
\hline GP97 & & 152,910 & 154,727 & 605 & UL97 homolog; protein kinase \\
\hline GP98 & & 154,747 & 156,531 & 594 & UL98 homolog; alkaline nuclease \\
\hline GP99 & & 156,444 & 156,965 & 173 & UL99 homolog; pp28 \\
\hline gp99.1 & & 157,155 & 157,769 & 204 & Homology to RCMV r4 ${ }^{\text {d }}$ \\
\hline GP100 & $\mathrm{C}$ & 157,278 & 158,327 & 349 & UL100 homolog; glycoprotein M \\
\hline GP102 & & 158,657 & 160,942 & 761 & UL102 homolog \\
\hline GP103 & $\mathrm{C}$ & 161,127 & 161,852 & 241 & UL103 homolog \\
\hline GP104 & $\mathrm{C}$ & 161,815 & 163,908 & 697 & UL104 homolog; portal \\
\hline GP105 & & 163,748 & 166,531 & 927 & UL105 homolog; helicase-primase \\
\hline GP112ex1 & & 176,745 & 177,498 & 315 & UL112 homolog; replication accessory, ex 1 \\
\hline GP112ex2 & & 177,606 & 177,782 & & UL112 homolog; replication accessory, ex 2 \\
\hline GP112ex3 & & 178,115 & 178,131 & & UL122 homolog; replication accessory, ex 3 \\
\hline GP114 & $\mathrm{C}$ & 179,126 & 179,920 & 264 & UL114 homolog; uracil glycosylase \\
\hline GP115 & $\mathrm{C}$ & 179,986 & 180,762 & 258 & UL115 homolog; glycoprotein L \\
\hline GP116 & $\mathrm{C}$ & 180,755 & 181,654 & 299 & Homology to THV t1 $16^{\text {b }} ; \mathrm{Fc}$ receptor//Ig \\
\hline GP117 & $\mathrm{C}$ & 181,877 & 183,262 & 461 & UL117 homolog \\
\hline gp119.1 & $\mathrm{C}$ & 184,418 & 185,167 & 249 & Similar to MCMV in ACE95619.1 \\
\hline GP121.2 & $\mathrm{C}$ & 185,160 & 185,834 & 224 & Betaherpesvirus B7D8, accession AFK83957 \\
\hline GP121.4 & $\mathrm{C}$ & 186,299 & 187,174 & 291 & UL121 homolog; Tupaia t121.4, NP 116476 \\
\hline GP122ex3 & $\mathrm{C}$ & 187,993 & 189,677 & & \\
\hline GP122ex2 & $\mathrm{C}$ & 191,079 & 191,311 & 677 & UL122 homolog; HCMV IE2 \\
\hline${ }^{8} \mathrm{GP122ex1}$ & $\mathrm{C}$ & 191,403 & 191,518 & & \\
\hline GP123ex3 & $\mathrm{C}$ & 189,907 & 190,985 & & \\
\hline GP123ex2 & $\mathrm{C}$ & 191,079 & 191,311 & 475 & UL123 homolog; HCMV IE1 \\
\hline${ }^{8}$ GP123ex1 & $\mathrm{C}$ & 191,403 & 191,518 & & \\
\hline
\end{tabular}


Table 1. Cont.

\begin{tabular}{|c|c|c|c|c|c|}
\hline \multicolumn{6}{|c|}{ GPCMV Open Reading Frames (ORFs): CIDMTR Strain } \\
\hline$\underline{\text { ORF }}$ & Strand & Begin & End & Codons & Notes \\
\hline GP128 & & 195,400 & 196,455 & 351 & Similar to Bat HSV B126; US22 Family \\
\hline gp130 & & 196,655 & 196,999 & 114 & \\
\hline GP129ex3 & $\mathrm{C}$ & 196,432 & 196,690 & \multirow{3}{*}{178} & \multirow{3}{*}{ Homolog of HCMV UL128 } \\
\hline GP129ex2 & & 196,768 & 196,890 & & \\
\hline GP129ex1 & $\mathrm{C}$ & 196,974 & 197,128 & & \\
\hline GP131ex2 & $\mathrm{C}$ & 197,133 & 197,469 & \multirow{2}{*}{191} & \multirow{2}{*}{ Homolog of HCMV UL130 } \\
\hline GP131ex1 & $\mathrm{C}$ & 197,550 & 197,788 & & \\
\hline GP133 & $\mathrm{C}$ & 197,788 & 198,174 & 128 & Homolog of HCMV UL131 \\
\hline GP134 & $\mathrm{C}$ & 198,268 & 198,951 & 227 & \\
\hline gp138.2 & $\mathrm{C}$ & 199,367 & 200,875 & 502 & \\
\hline gp138.3 & $\mathrm{C}$ & 201,090 & 202,607 & 505 & \\
\hline gp139 & $\mathrm{C}$ & 202,706 & 204,793 & 695 & THV T5; US22 superfamily \\
\hline gp140 & & 204,522 & 204,929 & 135 & Homology to CCMV UL132 \\
\hline gp141 & $\mathrm{C}$ & 205,053 & 206,660 & 535 & HCMV US23; US22 superfamily \\
\hline gp142 & $\mathrm{C}$ & 206,928 & 208,622 & 564 & HCMV US24; US22 superfamily \\
\hline gp143 & $\mathrm{C}$ & 208,875 & 210,866 & 663 & THV T5; US22 superfamily \\
\hline gp144 & $\mathrm{C}$ & 211,109 & 213,403 & 764 & US26; US22 gene superfamily \\
\hline gp145 & $\mathrm{C}$ & 213,677 & 215,575 & 632 & HCMV IRS1/TRS1; US22 superfamily \\
\hline gp146 & $\mathrm{C}$ & 215,932 & 217,914 & 660 & HCMV IRS1/TRS1; US22 superfamily \\
\hline gp147.1 & $\mathrm{C}$ & 221,739 & 222,935 & 398 & MHC class I homolog \\
\hline gp147 & $\mathrm{C}$ & 223,124 & 224,218 & 364 & MHC class I homolog \\
\hline gp148 & $\mathrm{C}$ & 225,379 & 226,560 & 393 & MHC class I homolog \\
\hline gp149 & $\mathrm{C}$ & 228,236 & 230,209 & 657 & MHC class I homolog \\
\hline gp149.1 & & 230,163 & 230,465 & 100 & Unique ORF sequence in CIDMTR \\
\hline
\end{tabular}

$\S$ An observation of note is the codon, ATA, spanning nucleotides 191516-191518 (minus strand). This codon is ATG in strain 22122, which was annotated as the start codon for both IE2 (GP122; exons 3, 4 and 5) and IE1 (GP123; exons 3, 4 and 6) [42-44]. Whether alternative splicing is occurring in the CIDMTR strain, or the start codon for the IE1/IE2 proteins is different between the two strains of GPCMV (i.e., in exon 4, not exon 3), requires further evaluation; this is described in greater detail in Section 2.4.

Amino acid sequences predicted from CIDMTR genes encoding conserved envelope glycoproteins were compared to those from strain 22122. There was striking sequence conservation of the $\mathrm{gB}$ homolog; although 13 SNPs were noted, only 2 coding changes were observed (overall identity of 99\%). In contrast, sequence analyses of GP74 ( $\mathrm{gO})$ demonstrated the greatest degree of sequence divergence, with 303/374 (81\% identity) noted, followed by GP75 (gH), with 613/726 (84\%) identity compared to the 22122 strain. GP73, the homolog of HCMV glycoprotein N, a protein known to exhibit substantial sequence divergence across clinical isolates [19,38,39], demonstrated 92\% identity between the CIDMTR and 22122 strains. These results confirm that CIDMTR represents a GPCMV strain distinct from 22122 as the amino acid sequences of $\mathrm{gH}$, gO, and $\mathrm{gN}$ clearly diverged through gradual evolutionary processes and cannot be attributed to abrupt, limited events such as insertions, deletions, or duplications. This further implies that despite potential bottlenecks imposed by selective breeding, GPCMV, as it exists within commercial breeding colonies, exhibits significant strain diversity of a nature similar to that of HCMV. These findings 
suggest that GPCMV may provide a useful model to study the impact of naturally occurring strain variation on viral pathogenesis, particularly in the area of nonprimary infection and disease.

\subsection{Sequence Characterization of DNA from Original SG Homogenate in Region of IE $1 / 2$ Start Codon}

A surprising finding from the DNA sequence analysis was the finding of an ATA codon as the putative start codon of the IE1/IE2 protein product [42]. To examine this issue, sequence analyses of PCR-amplified DNA from the original salivary gland homogenates, as well as salivary gland homogenates from further in vivo passages, were undertaken. DNA from the original salivary gland homogenate that was the source of the CIDMTR isolate was compared to DNA from GPL cells infected with CIDMTR following cell culture passage. The PCR was done with the primer pair designated IE splice 3' P1 (5'-TGCGAAGCGATCTCTCTCAAC-3') and IE splice 5' P1 (5'-GTGGTTGTACGTGTCGTCGTCA-3'), which was predicted to produce an 864-bp product from CIDMTR DNA. The purified DNA was cloned and three clones from each reaction were sequenced. This analysis indicated that the original salivary gland source of the CIDMTR strain contained viral DNA that encoded an ATG codon (as does 22122), the putative start codon for the IE1/IE2 protein product, but that the tissue cultured-derived CIDMTR strain, following two passages in fibroblasts, had a DNA sequence corresponding to an ATA codon.

To further examine splicing of the IE region from the CIDMTR strain (tissue culture-derived; CIDMTR-TC) virus, reverse-transcriptase PCR was performed on RNA harvested at immediate early times post-infection. RNA was extracted from GPL cells infected with 22122 or CIDTMR at 4 hours post-inoculation cDNA was synthesized from $1 \mu \mathrm{g}$ of total RNA. Conventional PCR was carried out using cDNA as template. PCR used primer pair IE exon4 F1 (5'-CCGCATTTTCTGAGGGTGTT-3') and IE exon1 R1 (5'-CATGCCAGTTCCCTGTGCTG-3'), and primer pair IE exon5 F1 (5'- GGAAGATGTCCACTTGGAAG-3') and IE exon2 R1 (5'- ACGTAGCCGAGAAGTAAAGT-3'). The expected product sizes for primer pair IE exon4 F1/IE exon1 R1 and IE exon5 F1/IE exon2 R1 were 473 bp and 508 bp, respectively.

A product of the expected size was purified, cloned into pCR2.1, and multiple clones with inserts from each reaction were sequenced. Notably, all CIDMTR-derived clones contained ATA at the putative (collinear) IE1/IE2 gene product start codon, confirming the DNA sequencing analysis. In contrast, all the 22122 clones contained an ATG at this position. The observed exon junctions were as described in the 22122 strain. The ATG codon in exon 3 was originally annotated as the putative IE1/2 start codon in 22122 [41] and the DNA sequence analysis of DNA from the CIDMTR strain purified directly from the salivary gland homogenate also demonstrated an ATG codon in exon 3. Thus, the finding of this ATA by DNA and RT-PCR sequencing in tissue culture-passaged CIDMTR virus was surprising. Since IE1/2 is abundantly expressed and ATA is known as an inefficient initiator of protein translation, we looked for ATGs that might serve as alternative start sites. Tissue culture-passaged CIDMTR strain may employ a start codon in exon four for the IE1 and IE2 gene products (Figure 4), although the Kozak consensus sequence of this putative start codon ( $\mathrm{T}$ at $-3, \mathrm{~A}$ at +4$)$ is relatively weak [45]. 
Figure 4. RT-PCR mapping of CIDMTR strain and 22122 strain splice sites. (a) Cartoon representation of the IE1/2 gene locus illustrating positions of primer pairs used for RT-PCR. Introns are indicated by straight lines and exons are blue boxes, although exon 1 and 2 are non-coding. (b) Results of RT-PCR reactions e4-1, e5-2, and GAPDH (control) using RNA from uninfected cells or cells infected with 22122 or CIDMTR. (c) RT-PCR consensus sequence of CIDMTR strain. Exon junctions are highlighted in blue. The first gray highlighted sequence is an ATA codon; this is an ATG codon and the putative start codon for the 22122 IE1/2 proteins, but is not conserved in the CIDMTR sequence in tissue culture passaged virus. The second gray highlighted sequence may therefore represent the putative start codon for CIDMTR TC IE1/2.

(a)

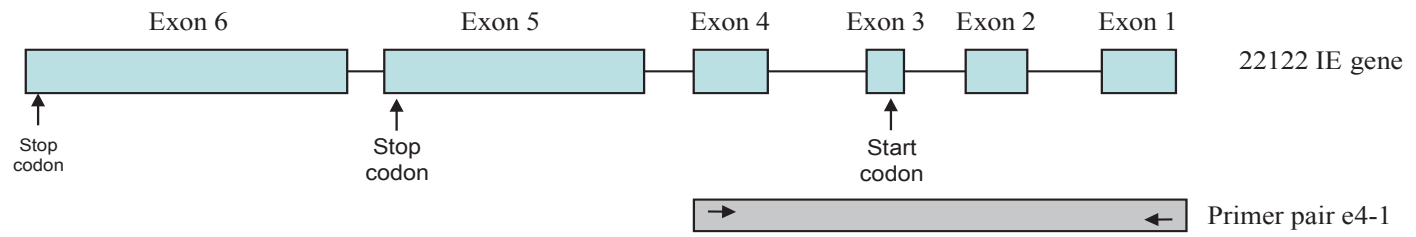

(b)

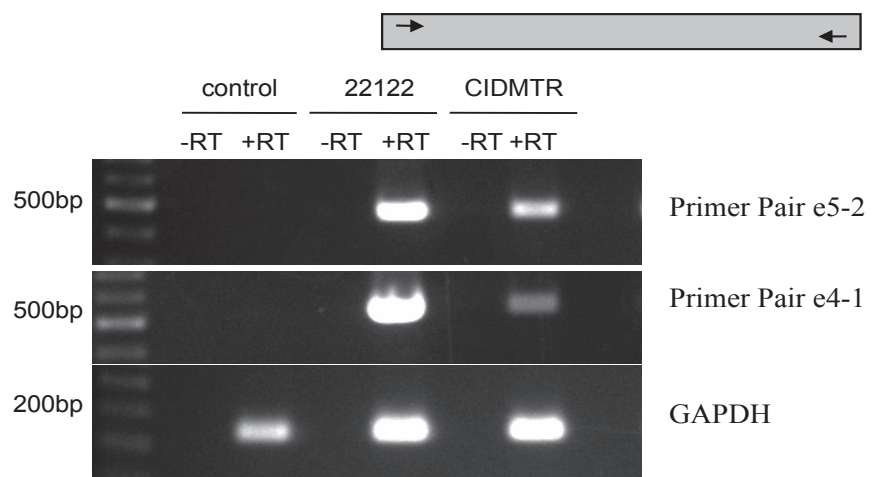

(c)

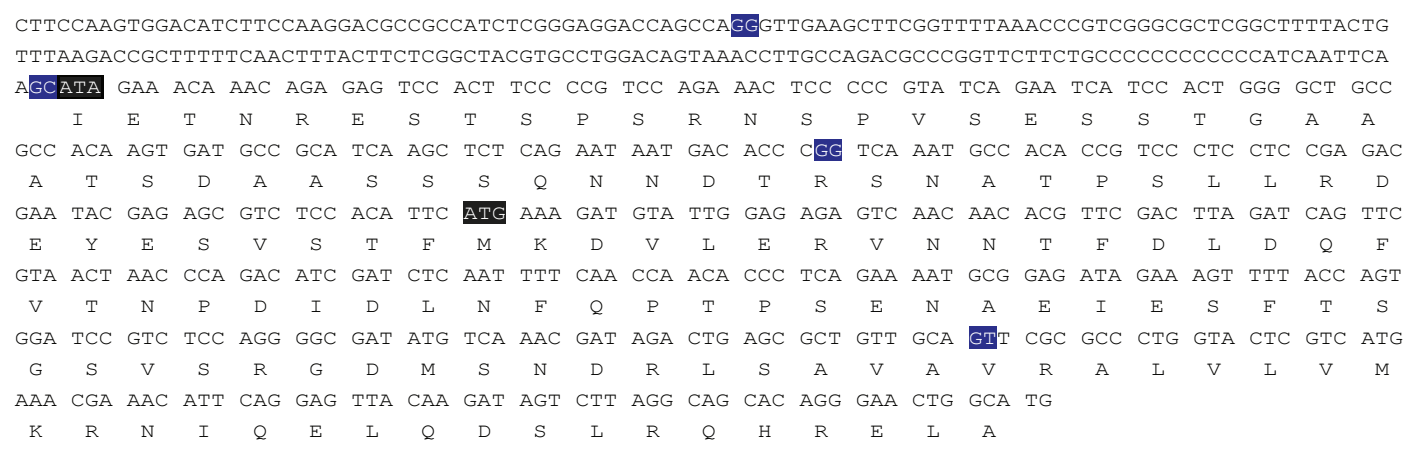

To further define IE transcription from the CIDMTR-TC virus, 5' RACE was performed. RNA was extracted and cDNA was synthesized with a specific sequence attached to the $5^{\prime}$ UTR for capped mRNAs. After ligation of a specific RNA oligo to the 5'UTR of mRNAs, cDNA was synthesized using oligo-dT and random hexamers. The cDNA was used as template for a first round of PCR using GeneRacer 5' Primer and primer IE2 P5 (5'-GGCGTCAATGGGCTCGGGTTTGAT-3'). Two nested PCR reactions were then performed with the GeneRacer 5' Nested Primer, per the manufacturer's specifications, and IE2 P5 or IE exon3 P1 (5'-GGCAGCCCCAGTGGATGATTCTGATA-3') (Figure 5b). Purified DNA was cloned and sequenced. Results matched the previously described splice sites for strain 22122 [42]. 
Using this primer combination, the RT-PCR product was $734 \mathrm{nt}$ in size (5 nt longer than the homologous ATCC/22122 cDNA). The substitution of ATA for the putative ATCC/22122 IE2/3 start codon was once again noted. We did not observe other in-frame start codons for the CIDMTR strain IE1/2 other than the one identified in exon 4 . Further experiments will be required to identify the start codon for IE1/2; possibly, both 22122 and CIDMTR strains use the start codon in exon 4, in spite of its suboptimal Kozak consensus. Alternatively, growth of the CIDMTR strain in fibroblasts may select for mutation of the exon 3 start codon, even upon minimal passage. Development of guinea pig epithelial/endothelial cell for isolation and passage of CIDMTR virus is an important future priority, to determine whether in vivo and in vitro passage result in different selective pressure on viral sequences.

Figure 5. 5' RACE analysis of CIDMTR IE region. (a) Schematic of IE gene indicating primers pair used in RACE. (b) nPCR gels for 22122 and CIDMTR using RNA purified under IE conditions. The CIDMTR RACE product is $734 \mathrm{nt}$ compared to 729 nt for the 22122 strain, explaining its slightly higher migration by gel electrophoresis. (c) RACE consensus sequence of CIDMTR IE. Exon junctions highlighted in blue. First gray highlighted sequence is co-linear to the start codon for 22122; second highlighted sequence represents putative start codon for CIDMTR IE1/2.

(a)

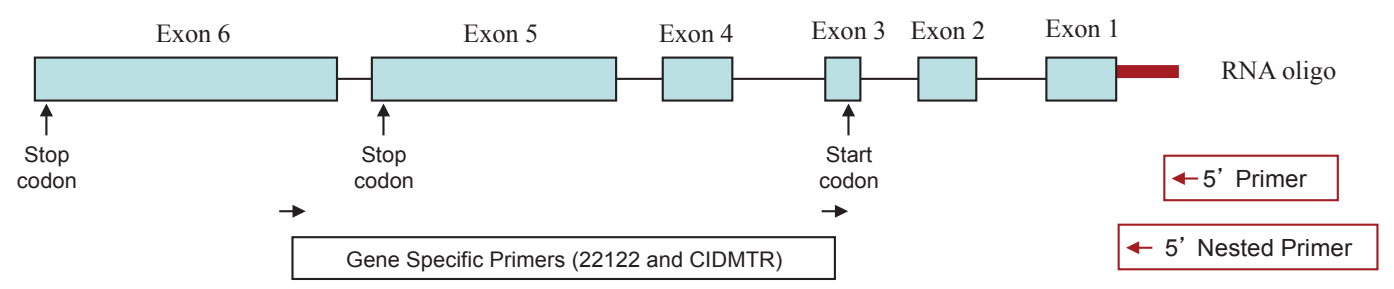

(b)

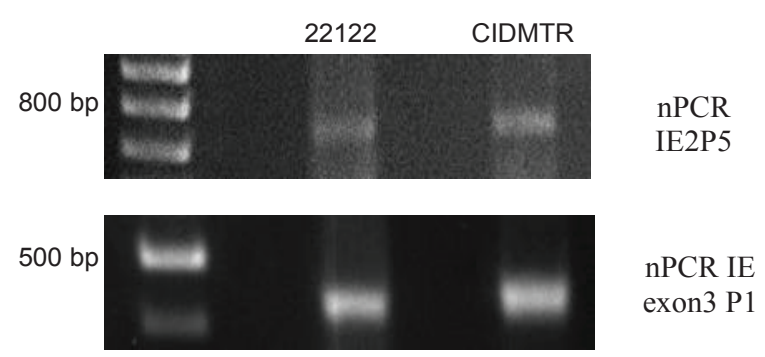

(c)

* AGAGCGAGGAGGCTCTGCAAGAGAGCACATCGTCCAGGAGCGACACCCCGCGGCTGCCCAGAGGACTGCACCTTGGACCACGCCTGCTTCGGAGCGCC GCCATTTTCTTCACCAGGTGGCCACGCTTCCAAGTGGACATCTTCCAAGTGGACATCTTCCAAGGACGCCGCCATCTCGGGAGGACCAGCCAGGGTTG AAGCTTCGGTTTTAAACCCGTCGGGCGCTCGGCTTTTACTGTTTAAGACCGCTTTTTCAACTTTACTTCTCGGCTACGTGCCTGGACAGTAAACCTTG CCAGACGCCCGGTTCTTCTGCCCCCCCCCCCCATCAATTCAAGCATA GAA ACA AAC AGA GAG TCC ACT TCC CCG TCC AGA AAC TCC $\begin{array}{llllllllllllllllllll}I & E & T & N & R & E & S & T & S & P & S & R & N & S\end{array}$ CCC GTA TCA GAA TCA TCC ACT GGG GCT GCC GCC ACA AGT GAT GCC GCA TCA AGC TCT CAG AAT AAT GAC ACC CGG $\begin{array}{llllllllllllllllllllllllllll}P & V & S & \text { E } & S & S & T & G & A & A & A & T & S & D & A & A & S & S & S & Q & N & N & D & T & R\end{array}$ TCA AAT GCC ACA CCG TCC CTC CTC CGA GAC GAA TAC GAG AGC GTC TCC ACA TTC ATG AAA GAT GTA TTG GAG AGA $\begin{array}{lllllllllllllllllllllllllll}S & N & A & T & P & S & L & L & R & D & E & Y & E & S & V & S & T & F & M & K & D & V & L & E & R\end{array}$ GTC AAC AAC ACG TTC GAC TTA GAT CAG TTC GTA ACT AAC CCA GAC ATC GAT CTC AAT TTT CAA CCA ACA CCC TCA $\begin{array}{llllllllllllllllllllllllll}V & N & N & T & F & D & L & D & Q & F & V & T & N & P & D & I & D & L & N & F & Q & P & T & P & S\end{array}$ GAA AAT GCG GAG ATA GAA AGT TTT ACC AGT GGA TCC GTC TCC AGG GGC GAT ATG TCA AAC GAT AGA CTG AGC GCT $\begin{array}{lllllllllllllllllllllllllll} & \text { E } & N & A & E & I & E & S & F & T & S & G & S & V & S & R & G & D & M & S & N & D & R & L & S & A\end{array}$ GTT GCA GAC ACG CCC GAG GGA TGG CTG ACA CCT TCC ATC TCA CAG ATC AAA CCC GAG CCC ATT GAC GCC

$\begin{array}{llllllllllllllllllllllll}\mathrm{V} & \mathrm{A} & \mathrm{D} & \mathrm{T} & \mathrm{P} & \mathrm{E} & \mathrm{G} & \mathrm{W} & \mathrm{L} & \mathrm{T} & \mathrm{P} & \mathrm{S} & \mathrm{I} & \mathrm{S} & \mathrm{Q} & \mathrm{I} & \mathrm{K} & \mathrm{P} & \mathrm{E} & \mathrm{P} & \mathrm{I} & \mathrm{D} & \mathrm{A}\end{array}$ 


\subsection{PCR Confirmation of GPCMV-CIDMTR Genome Structure}

To confirm the structure of the CIDMTR strain compared to the 22122 strain, PCR was performed on viral DNA from both strains, using primers spanning the mismatched regions observed in the sequence analysis comparisons (Figure 3b, boxed/shaded regions). Two primers pairs were used for each region of mismatch (mismatch region 1 and mismatch region 2). PCR was performed using primer pairs mismatch-region $1 \mathrm{~F} 1 / \mathrm{R} 1$ and mismatch-region 1 F2/R2, that amplify $\mathrm{a} \sim 4-\mathrm{kb}$ region for the 22122 strain, but a $\sim 2.5-\mathrm{kb}$ region for the CIDMTR strain. The amplification region using primer pairs mismatch-region 2 F1/R1 or mismatch-region 2 F2/R2 was predicted to be $\sim 2.2 \mathrm{~kb}$ for the 22122 strain and $\sim 3.7 \mathrm{~kb}$ for the CIDMTR strain. Primer sequences are indicated in Table 2. The results of these experiments confirmed that the genome configuration was precisely as predicted from the deep sequence analysis. Moreover, the PCR was also performed on DNA purified directly from the original salivary gland homogenate from which the CIDMTR isolate was obtained (data not shown). These results confirmed that the insertions and deletions identified by sequencing (Figure 3c) did not arise as an artifact of limited passage of virus in cell culture. The PCR results are shown in Figure 6.

Table 2. Primer Sequences.

\begin{tabular}{cc}
\hline Primer Name & Primer Sequence \\
\hline Mismatch Region 1 F1 & 5'-GTGAGACGTAAGAATAGCTTGC-3' \\
Mismatch Region 1 F2 & 5'-GATCCTTAGACTCTATCACGG-3' \\
Mismatch Region 1 R1 & 5'-GTGTTGTCACAATTGGCACATG-3' \\
Mismatch Region 1 R2 & 5'-ACATGGTCACGACAGAATC-3' \\
Mismatch Region 2 F1 & 5'-GTGGACAGGATCCCCAAATT-3' \\
Mismatch Region 2 F2 & 5'-CCAAATTTCTGTCGTCGGCG-3' \\
Mismatch Region 2 R1 & 5'-TGTTTCCGTGTCTGTCTCCGT-3' \\
Mismatch Region 2 R2 & 5'-GTCTTAGCCCGAGACCTTC-3' \\
\hline
\end{tabular}

\subsection{Infection In Vivo and Development of a Real-Time PCR Assay for Detection of CIDMTR DNA}

To evaluate DNAemia and end-organ infection in the course of in vivo studies, a strain-specific real-time PCR assay was developed in order to differentiate the GPCMV-CIDMTR strain from the 22122 strain. This assay focused on the amplification of sequences corresponding to the CIDMTR strain GP147.1 ORF (Table 1), since this sequence is absent in the 22122 strain. A GPCMV 147.1 specific PCR primer pair, consisting of CIDMTR147.1_464F (5'-ATGCAACATAGCGTGCTGAC-3') and CIDMTR147.1_583R (5'-GGGACAAAAGCACGATGAAC-3') was designed and utilized for the real-time PCR assay (described in detail in 3.5). These primers amplified a 120 bp region of the 147.1 gene specific for the CIDMTR strain. DNA was extracted from either $100 \mu \mathrm{L}$ citrated blood or from fresh frozen tissues samples, as described in the Methods section. For quantitative PCR, both previously validated primers for the GP83 gene [sequences shared by both strains] and novel primers for the GPCMV 147.1 gene [sequences only found in the CIDMTR strain] were used for real-time PCR assay. There was strong concordance between the viral load estimates identified by the gp147.1 and 
GP83 real-time primers (data not shown). As a negative control, GPCMV 147.1 primers were used in several PCR assays of 22122 DNA, with consistently negative results (data not shown).

Figure 6. Strain-specific PCR assays differentiate ATCC 22122 strain of GPCMV from CIDMTR strain. (a) Primers were designed to amplify across discontinuous/unique regions as demonstrated in Figure 3b. Lane 1, kb ladder; lane 2, mismatch region 1 F1/R1, ATCC; lane 3, mismatch region 1, F1/R1, CIDMTR; lane 4, mismatch region 1, no template control; lane 5, mismatch region $1 \mathrm{~F} 2 / \mathrm{R} 2$, ATCC; lane 6 , mismatch region 1 F2/R2, CIDMTR; lane 7, mismatch region 1 F2/R2, no template control; lane 8, mismatch region 2 F1/R1, ATCC; lane 9, mismatch region 2 F1/R2, CIDMTR; lane 10, mismatch region $2 \mathrm{~F} 1 / \mathrm{R} 1$, no template control; lane 11, mismatch region $2 \mathrm{~F} 2 / \mathrm{R} 2$, ATCC; lane 12, mismatch region 2 F2/R2, CIDMTR; lane 13, mismatch region 2 F2/R2, no template control. (b) Restriction polymorphisms were as predicted by DNA sequence. PCR amplification products were digested with enzymes as indicated. Lane 1, kb ladder; lane 2, mismatch region $1 \mathrm{~F} 1 / \mathrm{R} 1 \mathrm{ATCC}, \mathrm{Bgl} 2$; lane 3, mismatch region 1 F1/R1 CIDMTR, $B g l$ 2; lane 4, mismatch region 1 F2/R2 ATCC, $B g l$ 2; lane 5, mismatch region $1 \mathrm{~F} 2 / \mathrm{R} 2 \mathrm{CIDMTR}, B g l$ 2; lane 6, mismatch region $1 \mathrm{~F} 1 / \mathrm{R} 1 \mathrm{ATCC}$, BamH I; lane 7, mismatch region 1 F1/R1 CIDMTR, BamH I; lane 8, mismatch region 1 F2/R2 ATCC, BamH I; lane 9, mismatch region 1 F2/R2, BamH I; lane 10, mismatch region 1 F1R1 ATCC, EcoR V; lane 11, mismatch region 1 F1/R1 CIDMTR, EcoR V; lane 12, mismatch region $1 \mathrm{~F} 2 / \mathrm{R} 2 \mathrm{ATCC}, E c o R \mathrm{~V}$; lane 13, mismatch region $1 \mathrm{~F} 2 / \mathrm{R} 2$, EcoR V; lane 14, mismatch region 2 F1R1 ATCC, EcoR I; lane 15, mismatch region 2 F1R1 CIDMTR, EcoR I; lane 16, mismatch region 2 F2/R2 ATCC, EcoR I; lane 17, mismatch region 2 F2/R2, EcoR I.

a

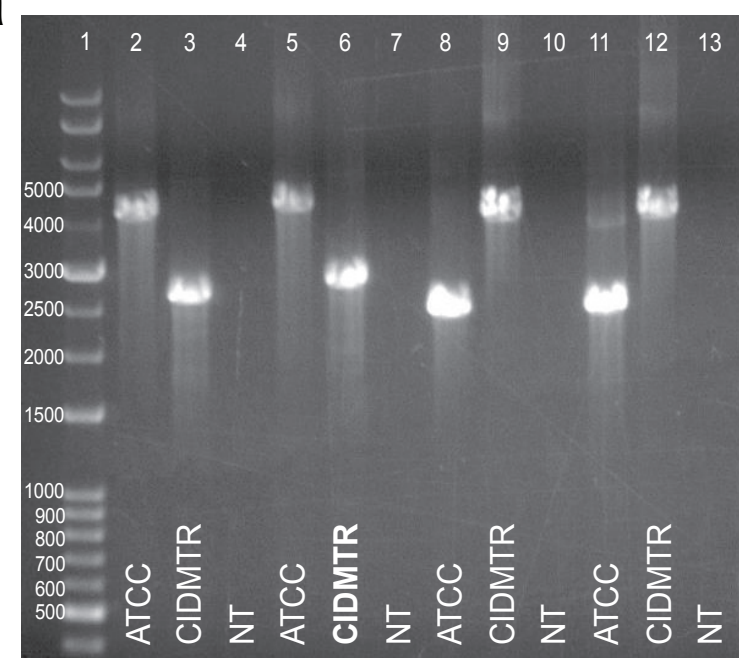

b

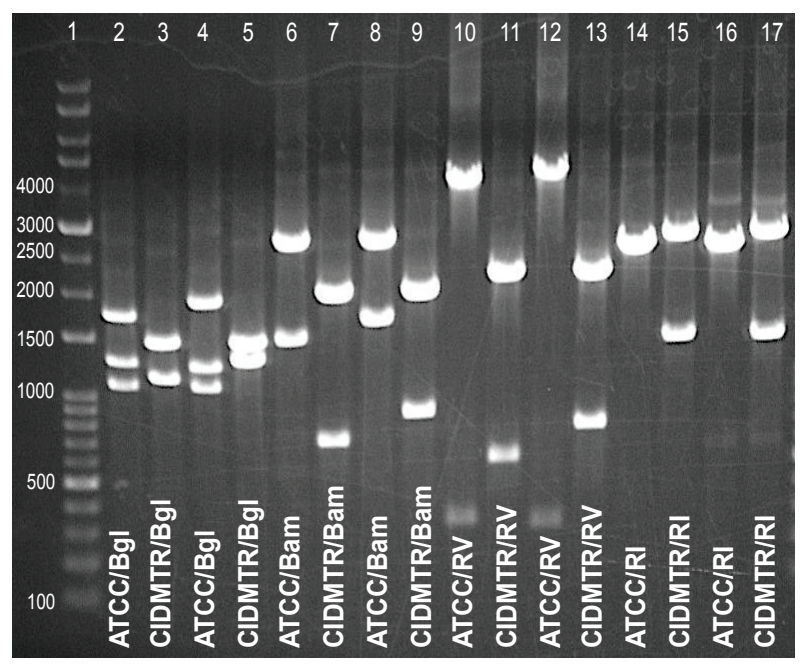

Next, twelve young, GPCMV-seronegative outbred Hartley guinea pigs were divided into two groups of six/group. Each group was inoculated with CIDMTR strain virus (p1) at a dose of $1 \times 10^{5} \mathrm{pfu}$, administered subcutaneously, as described in Section 3. Group $1(\mathrm{n}=6)$ was treated with $200 \mathrm{mg} / \mathrm{kg}$ cyclophosphamide on day -1 and $50 \mathrm{mg} / \mathrm{kg}$ on day +6 following viral inoculation as described previously [32]; group $2(n=6)$ was sham-treated (PBS only). Whole blood and sera 
samples were collected on day $0,3,7$, and 21. Animals were sacrificed on day 21 and tissue, including lung, liver, spleen, and brain, were collected for PCR analysis. In group 1, 5/6 animals were DNAemic, peaking at day 7 (mean, $3.2+/-0.3 \log _{10}$ genomes $/ \mathrm{mL}$ ) while in group 2, the prevalence of DNAemia was lower (3/6 animals; $2.5+/-0.35 \log _{10}$ genomes $/ \mathrm{mL}, p=0.06$ compared to group 1). Viral DNA was most readily recovered from spleen upon dissection at day 21 post-infection. All 6 animals from each group had recoverable CIDMTR strain DNA in the spleen. Total spleen viral load in group 1 was $2.4+/-0.07 \log _{10}$ copies/mg and was $2.4+/-0.1$ $\log _{10}$ copies/mg in group 2 ( $\mathrm{p}=$ NS compared to group 1$)$.

The ability of the CIDMTR strain to infect pregnant animals previously inoculated with the 22122 strain was next assessed. A total of 6 female seronegative Hartley guinea pigs received a primary infection with SG homogenate GPCMV (22122) at a dose of $1 \times 10^{4} \mathrm{pfu}$, administered subcutaneously. A parallel group of animals were sham-treated (PBS only). Then, 5 weeks after inoculation, the animals in both groups were mated with GPCMV-seronegative breeders. Dams originally inoculated with the SG virus (22122 strain) were documented to undergo seroconversion; in addition, all were demonstrated to have low-level DNAemia after inoculation with 22122 virus (data not shown). Approximately 5 weeks after the initiation of mating, group 1 was challenged subcutaneously with CIDMTR strain at a titer of $6 \times 10^{5}$ pfu. A total of 19 pups were born in the experimental group; all pups were liveborn. When pup tissues were analyzed, 4/19 (21\%) of these animals had recoverable CIDMTR strain-specific DNA isolated from the salivary gland. One of these animals also had detectable DNA in the brain.

\section{Experimental Section}

\subsection{Cells, Virus and DNA Preparation}

GPCMV (strain 22122, ATCC VR682), and CPCMV/CIDMTR were propagated on guinea pig lung fibroblast cells (GPL; ATCC CCL 158) in F-12 medium supplemented with $10 \%$ fetal calf serum (FCS; Gibco-BRl), 10,000 IU of penicillin/liter, $10 \mathrm{mg}$ of streptomycin/liter (Gibco-BRL), and $7.5 \% \mathrm{NaHCO} 3$ (Gibco-BRL). When the $\mathrm{P}_{1}$ passaged CIDMTR virus exhibited extensive CPE in GPL cell culture, cells and virions were pelleted. Aliquots were fixed in phosphotungstic acid for EM studies (see below) and the remainder washed in $10 \mathrm{mM}$ Tris (pH 8.0)/1 mM EDTA (TE), and lysis buffer $(200 \mathrm{mM} \mathrm{NaCl}, 2 \% \mathrm{SDS}, 200 \mu \mathrm{g} / \mathrm{mL}$ proteinase $\mathrm{K}$, in TE), was added. Cells and virions were gently suspended, inverted, and incubated at $68{ }^{\circ} \mathrm{C}$ overnight. Following transfer to a $37{ }^{\circ} \mathrm{C}$ water bath, three successive phenol-chloroform extractions were performed, followed by ethanol precipitation.

\subsection{Transmission Electron Microscopy}

Cells were fixed in $1 \mathrm{~mL}$ of $2.5 \%$ glutaraldehyde in $0.1 \mathrm{M}$ sodium cacodylate buffer and post fixed with $1 \%$ osmium tetroxide in $0.1 \mathrm{M}$ sodium cacodylate buffer (all reagents from Electron Microscopy Sciences, Hatfield, PA, USA). After three washes in distilled water, samples were dehydrated using a 25\%-100\% ethyl alcohol gradient. Samples were then infiltrated with 2:1 ethanol: Embed 812 resin (Electron Microscopy Sciences, Hatfield, PA, USA) for 1 hour and 
subsequently transferred to a 1:2 ethanol: Embed 812 resin mixture for 1 hour. Cells were further infiltrated with $100 \%$ resin and were embedded and incubated at $58{ }^{\circ} \mathrm{C}$ for 24 hours to polymerize the resin. Embedded samples were trimmed and sectioned on a Leica UC6 Ultramicrotome (Leica Microsystems, Vienna, Austria). Thin sections $(60-70 \mathrm{~nm})$ were obtained and collected on a 200 mesh copper grid (Electron Microscopy Sciences, Hatfield, PA, USA) using a perfect loop. Grids were contrasted with 5\% uranyl acetate for 20 minutes and Santos' lead citrate for 6 minutes. For negative contrast, virions and dense bodies were collected from $\mathrm{P}_{1}$ supernatants of infected fibroblasts, transferred to airfuge tubes (Beckman-Coulter, Brea, CA, USA), and centrifuged at 30 PSI using an airfuge (Beckman-Coulter, Brea, CA, USA) for 20 minutes on parafilm and formvar coated copper grids (Electron Microscopy Sciences, Hatfield, PA, USA). Excess liquid was wicked and the grids were stained with $1 \%$ phosphotungstic acid for one minute. All sections were observed under JEOL 1200 EX II transmission electron microscope (JEOL LTD, Tokyo, Japan). Images were obtained using a Veleta $2 \mathrm{~K} \times 2 \mathrm{~K}$ camera with iTEM software (Olympus SIS, Munster, Germany) [46].

\subsection{Deep Sequencing and Sequence Analyses}

For sequence analysis of CIDMTR viral DNA, virions were purified as described previously [44], and lysis buffer $(200 \mathrm{mM} \mathrm{NaCl}, 2 \% \mathrm{SDS}$, and $200 \mu \mathrm{g} / \mathrm{mL}$ proteinase $\mathrm{K}$ in Tris-EDTA [TE]) was added. Following incubation at $68{ }^{\circ} \mathrm{C}$ overnight, three phenol-chloroform extractions were performed, followed by ethanol precipitation of viral DNA. Genomic sequencing was performed using Illumina MiSeq and Pacific Biosciences PacBio RS platforms. Approximately 5.2 million 151-bp paired-end MiSeq reads were generated at the University of Minnesota's Biomedical Genomics Center with a nominal insert size of $400 \mathrm{bp}$. Removal of low quality reads and PhiX sequence resulted in a set of 4.0 million cleaned reads, approximately $11,000 \times$ coverage. Initial scaffolds were generated from the cleaned Illumina reads using the ABySS assembler (version 1.3.4) [47]. Scaffold quality was assessed manually by comparison with the reference strain, $22122[44,48,49]$, and by remapping the reads using Bowtie 2 and scrutinizing local coverage and consensus using Tablet [50] and SAMtools [51]. Special attention was paid to correct alignment and orientation of the paired ends. Regions of weak coverage and scaffold gaps were identified and closed either by manual local assembly [52] or by Sanger sequencing. Independent validation of the pseudomolecule was also performed using the longer PacBio RS reads, validating the Sanger sequencing and manual assembly. SMRT Analysis software [53] produced 998 high quality ("corrected") reads ranging between $509-15,898 \mathrm{bp}$, median $6,257 \mathrm{bp}$ (approximately $27 \times$ coverage), as well as another set of scaffolds. These data were used to evaluate the Illumina assembly, specifically its structural correctness, and to correct misassembled repeat regions. The resulting complete genome was deposited with the EMBL Nucleotide Sequence Database (accession number HG531783).

\subsection{RT-PCR and RACE Analyses}

Reverse-transcriptase PCR was performed on RNA harvested at immediate early times post-infection. RNA was extracted from GPL cells infected with 22122 or CIDTMR, at 4 hours post-inoculation, using the RNeasy mini kit (Qiagen, Hilden, North Rhine-Westphalia, Germany) 
according to the manufacturer's instructions. RNA was treated with RNase-free DNase Set (Qiagen) while in the column according to manufacturer's instructions. cDNA was synthesized from $1 \mu \mathrm{g}$ of total RNA using Quantitect Reverse Transcription kit (Qiagen). Conventional PCR was carried out using cDNA as template and AmpliTaq Gold Fast PCR Master Mix (Invitrogen, Carlsbad, CA, USA). Primers are as described in Section 2.4. The PCR products were run in a $0.7 \%$ agarose gel. A band of the expected size was cut form the gel and purified using QIAquick Gel Extraction Kit (Qiagen). The purified DNA was cloned into pCR2.1 (Invitrogen) using the TA Cloning Kit (Invitrogen). Three of clones with inserts from each reaction were sequenced with T3 promoter, M13 Reverse and the PCR primers.

For 5' RACE analysis, RNA was used as template for cDNA synthesis using the GeneRacer kit (Invitrogen) in order to generate a cDNA with a specific sequence attached to the 5' UTR for only mRNAs that were capped. After ligation of a specific RNA oligo to the 5'UTR of mRNAs, cDNA was synthesized using a combination of oligo-dT and random hexamers. The cDNA was used as template for a first round of PCR using GeneRacer 5' Primer and primer IE2 P5 (5'-GGCGTCAATGGGCTCGGGTTTGAT-3'). In order to increase the specificity and quantity of the PCR product, nested PCR (nPCR) was performed in a dilution $(1: 20)$ of the PCR product. Two nPCR reactions were performed with the GeneRacer 5' Nested Primer, per the manufacturer's specifications, and IE2 P5 or IE exon3 P1 (5'- GGCAGCCCCAGTGGATGATTCTGATA-3'). A band of the expected size was cut form the gel and purified using QIAquick Gel Extraction Kit (Qiagen). The purified DNA was cloned into pCR4-TOPO (Invitrogen) using the TOPO TA Cloning. Clones were analyzed for the present of the insert by digestion with EcoR I. Three of the clones with insert from each reaction were sequenced with T3 promoter, M13 forward $(-20)$ and the PCR primers.

\subsection{GPCMV-CIDMTR Strain PCR Assay}

For confirmation of viral genome structural differences noted by deep sequencing, PCR was performed for each virus using primers indicated in Table 2. The PCR reaction was performed in a $50 \mu \mathrm{L}$ of total volume using GoTaq long PCR Master Mix from Promega and $1.0 \mu \mathrm{M}$ primers. The DNA template was total genomic and viral DNA extracted from GPL cells infected with 22122 or CIDMTR tissue culture strain. The conditions for the PCR were: initial denaturation at $95{ }^{\circ} \mathrm{C}$ for $2 \mathrm{~min}$, followed by $95^{\circ} \mathrm{C}$ for $30 \mathrm{~s}, 53{ }^{\circ} \mathrm{C}$ for $30 \mathrm{~s}, 72{ }^{\circ} \mathrm{C}$ for $4 \mathrm{~min}$ for a total of 35 cycles, and elongation at $72{ }^{\circ} \mathrm{C}$ for $10 \mathrm{~min}$. The PCR product $(4 \mu \mathrm{L})$ was subjected to electrophoresis in a $0.7 \%$ agarose gel. The PCR product from mismatch $1 \mathrm{~F} 2 / \mathrm{R} 2$ and mismatch 2 F2/R2 were purified from the gel using the Geneclean ${ }^{\circledR}$ II kit (MP Biomedicals, Santa Ana, CA, USA) following the manufacturer's instructions. The cleaned PCR product was then sequenced by Sanger sequencing (Functional Biosciences, Madison, WI, USA). Purified PCR products were also subjected to restriction endonuclease comparisons as indicated in Figure 5b.

The real-time PCR assay focused on the amplification of sequences corresponding to the CIDMTR strain gp147.1 ORF (Table 1), since this sequence is absent in the 22122 strain sequence. A GPCMV gp147.1 specific real-time PCR primer pair, consisting of CIDMTR147.1_464F (5'-ATGCAACATAGCGTGCTGAC-3') and CIDMTR147.1_583R (5'-GGGACAAAAGCACGATGAAC-3') was designed and utilized for the real-time PCR assay. 
These primers amplified a $120 \mathrm{bp}$ region of the gp147.1 gene specific for the CIDMTR strain. The specific hydrolysis probe used for detection was CIDMTR147.1_494P (FAM-GTGTTCGTGTCCTTGATCGTACGCA-BHQ1). A second GPCMV 147.1 specific primer pair, CIDMTR147.1_225F (5'-AATGGTTCGCTACGGACATC-3') and CIDMTR147.1_368R (5'-CGGACAACGGAACATACTTG-3') was also utilized in real-time PCR assays. These primers amplified a 144 bp region of 147.1 specific for the CIDMTR strain. The specific hydrolysis probe used for detection with this primer pair was CIDMTR147.1_262P (FAM-TTCCTCGACGAAGCTCGCGGTATAAT-BHQ1). In each instance, the PCR reactions were performed in a $25 \mu \mathrm{L}$ reaction, using LightCycler 480 Probes Master from Roche (Penzberg, Bavaria, Germany); as well as $0.4 \mu \mathrm{M}$ primers, $0.1 \mu \mathrm{M}$ probe and $0.4 \mathrm{u} / \mu \mathrm{L}$ of UNG. PCR was performed using the LightCycler 480 Real-Time PCR System (Roche) under the following conditions: initial denaturation at $95{ }^{\circ} \mathrm{C}$ for $10 \mathrm{~min}$, followed by $95{ }^{\circ} \mathrm{C}$ for $10 \mathrm{~s}, 56{ }^{\circ} \mathrm{C}$ for $15 \mathrm{~s}$, $72{ }^{\circ} \mathrm{C}$ for $10 \mathrm{~s}$ for a total of 45 cycles, then a final hold step at $40{ }^{\circ} \mathrm{C}$. The first primer pair ( $464 \mathrm{~F}$ and 583R) was chosen for detection of viral genome for in vivo studies. Data were analyzed with the LightCycler Data Analysis Software (version 1.5; Roche) [54] using standard curves generated using serial dilutions of plasmid pCR2.1 with gp147.1 at known concentrations. Negative results were arbitrarily assigned a level of 50 for the purpose of statistical comparisons, based upon limit-of-detection analyses observed in other real-time PCR experiments [55].

\subsection{Animal Challenge Studies}

All animal studies were performed with the approval of the University of Minnesota Institutional Animal Care and Use Committee (IACUC). Some animals were immune suppressed at day $-1(200 \mathrm{mg} / \mathrm{kg})$ and day $+6(50 \mathrm{mg} / \mathrm{kg})$ with cyclophosphamide delivered by intraperitoneal injection. For experiments described in Section 2.6, both cyclophosphamide-treated and untreated animals ( $\mathrm{n}=6$ /group) were challenged with CIDMTR strain virus $\left(\mathrm{P}_{1}\right.$ workpool) at a dose of $1 \times 10^{5}$ pfu by subcutaneous injection. Blood samples were collected at day $0,3,7$ and 21 post-inoculation and animals humanely sacrificed at day 21 for collection of tissue samples for PCR analyses. Pregnancy/challenge studies were conducted as described in Section 2.6. Liveborn pups were sacrificed within 72 hours of delivery for DNA extraction and subsequent PCR.

\section{Conclusions}

The 22122 strain was originally isolated by Hartley in 1957 [27]. As the only characterized isolate of GPCMV it was used in virtually all subsequent GPCMV research. However, GPCMV infection is common among animals in commercial breeding colonies. In a longitudinal study conducted by Hsiung and colleagues from 1974 to 1979, GPCMV-neutralizing antibody was observed in $25 \%$ of Hartley strain guinea pigs obtained from commercial sources while virus was isolated from only 6 of 204 animals [56]. The percentages of antibody-positive animals obtained from different sources varied from shipment to shipment, ranging from $8 \%-50 \%$. It does not appear that any of these viral isolates were retained. Thus, given only a single characterized isolate, the extent to which diverse GPCMV strains have been maintained within these domesticated populations was not previously known. Given that Hartley guinea pigs are descended from animals 
imported from South America to Europe in the 16th century, and that these animals subsequently underwent centuries of selective breeding, first as pets and later as research animals, the possibility existed that bottlenecks in the breeding history of Hartley guinea pigs could have limited the genetic diversity of GPCMV strains currently endemic in commercial breeding facilities.

In the current study, a second virus was independently isolated and characterized, again from a Hartley strain guinea pig obtained from a commercial supplier in the United States. Based on a high degree of genetic relatedness to 22122, predominantly similar restriction endonuclease patterns, and an conserved overall genomic structure, we conclude that CIDMTR is a GPCMV and not a novel and distinct betaherpesvirus. However, based on restriction pattern polymorphisms and significant divergence of amino acid sequences for several envelope glycoproteins (particularly gH and gO), we conclude that CIDMTR and 22122 represent two distinct strains of GPCMV and are not minor variants of the same strain. This further suggests that GPCMV strain diversity has been sustained within commercial breeding colonies and that other strains exhibiting similar levels of divergence may exist and could potentially be exploited to further extend this important animal model.

The passage history of 22122 is uncertain. In the original report by Hartley, two parallel isolations of GPCMV were reported: one, from salivary gland homogenate obtained from guinea pigs purchased by a commercial supplier in Yonkers, NY, and a second isolate obtained from a second supplier in New York state. Reactivation of virus may have been driven by allogeneic responses engendered from injecting SG extract into an animal with a different allotype, as has been suggested may drive HCMV and MCMV reactivation during transplantation [57,58]. Allogeneic responses may similarly have played a role, along with cyclophosphamide immunosuppression, in reactivation of the CIDMTR strain, since repeated tissue culture of salivary gland explants directly obtained from seropositive animals failed to result in growth of virus (data not shown). With respect to the original isolation of 22122, it is worth noting that serial in vivo passages may have occurred in the context, for some animals, of "mixed infection", since Hartley noted that $7.7 \%(3 / 39)$ of "control NIH" strain guinea pigs aged 5 months or older were naturally infected, as evidenced by infrequent salivary gland inclusions [27]. This strain underwent 22 additional passages in cell culture of fibroblasts, and appears to be the strain that eventually was deposited with ATCC. The precise date of deposit is unclear, but reports of GPCMV research from the 1960s [59] and early 1970s describe obtaining this strain directly from the NIH, while studies since the late 1970s describe obtaining the virus from ATCC [60]. Between its original isolation by Hartley and its submission to ATCC, it appears to have undergone 54 additional passages in guinea pigs, and six additional passages in cell culture ( 3 passages in guinea pig embryo fibroblasts, and 3 passages in CCL 158 cells [61]. The 22122 strain derives from multiple rounds of both in vivo passage, some possibly occurring in the context of mixed infection, and 25-30 passages in cell culture. Thus, it is possible that 22122 underwent changes after isolation from the initial animal, either in cell culture or during in vivo passage.

In contrast, CIDMTR was subjected to minimal passage (one passage in vivo and two passages in cell culture) prior to genomic characterization. Thus, the genomic structure and sequence of the CIDMTR strain may more likely represent a bona fide "wild-type" GPCMV sequence than does the 22122 strain. 
Sequence comparison with the 22122 strain revealed generally good conservation of protein coding sequences, although three areas of substantial discontinuity were noted. Thus, each strain contains unique sequences that can be used as markers to distinguish the strains during in vivo coinfection experiments. One of the regions unique to CIDMTR contains an ORF encoding a fourth putative MHC class I homolog not found in the 22122 strain. Since the three putative MHC class I homologs found in 22122 appear to be important for the in vivo pathogenesis of infection [55], further functional comparisons of the two strains will be of interest. In spite of minimal cell culture passage, at least one mutation, in the IE1/IE2 start codon, was observed in the tissue culture-adapted CIDMTR virus, compared to sequences amplified from salivary gland homogenate. This observation is similar to reported nucleotide sequence comparisons between several open reading frames in the DNA of different laboratory-adapted strains and clinical isolates of HCMV that has revealed amino-terminal sequence extensions of ORFs with alternate start codon usage [62]. Sequence differences with respect to start codon usage have also been noted upon comparison of laboratory-passaged and "wild" isolates of MCMV [37]. Future sequence analysis of CIDMTR DNA propagated solely in vivo is therefore warranted. Studies in immune competent and immune compromised guinea pigs confirm the ability of the CIDMTR virus to disseminate and produce viremia (DNAemia). Variation in glycoprotein protein coding sequences were noted, particularly for the $\mathrm{gH}$ and $\mathrm{gO}$ proteins, suggesting that this virus may be useful for the study of re-infection of immune guinea pigs in the guinea pig model of congenital cytomegalovirus infection. Cross-neutralization studies examining strain-specific antibody responses to envelope glycoproteins would enhance the usefulness of this new strain for the modeling of vaccine-mediated protection against re-infection and congenital transmission in this uniquely valuable model.

\section{Acknowledgments}

Support from NIH HD038416, HD044864 and HD068322 is acknowledged.

\section{Author Contributions}

M.R.S. conceived of the experiments and approach. A.A. performed electron microscopy studies. S.M., N.H.-A., C.F.-A., J.C.Z., and M.R.S. conducted experiments, and performed data analysis. T.A.R. and J.C. performed sequence analysis. M.M. analyzed data and assisted in writing the manuscript. All other authors also assisted in writing the manuscript.

\section{Conflicts of Interest}

The authors declare no conflict of interest relevant to this work.

\section{EMBL Accession Number}

The genome sequence for this strain has been deposited with the EMBL Nucleotide Sequence Database under the accession number HG531783. The CIDMTR strain has been deposited with ATCC (Rockville, MD, USA; accession number PTA-120714), or is available upon request from the corresponding author. 


\section{References and Notes}

1. Sung, H.; Schleiss, M.R. Update on the current status of cytomegalovirus vaccines. Expert Rev. Vaccines 2010, 9, 1303-1314.

2. Nigro, G.; Adler, S.P.; La Torre, R.; Best, A.M. Passive immunization during pregnancy for congenital cytomegalovirus infection. N. Engl. J. Med. 2005, 353, 1350-1362.

3. Griffiths, P.; Plotkin, S.; Mocarski, E.; Pass, R.; Schleiss, M.; Krause, P.; Bialek, S. Desirability and feasibility of a vaccine against cytomegalovirus. Vaccine 2013, 31, B197-B203.

4. Krause, P.R.; Bialek, S.R.; Boppana, S.B.; Griffiths, P.D.; Laughlin, C.A.; Ljungman, P.; Mocarski, E.S.; Pass, R.F.; Read, J.S.; Schleiss, M.R.; et al. Priorities for cmv vaccine development. Vaccine 2013, 32, 4-10.

5. Pass, R.F.; Zhang, C.; Evans, A.; Simpson, T.; Andrews, W.; Huang, M.L.; Corey, L.; Hill, J.; Davis, E.; Flanigan, C.; et al. Vaccine prevention of maternal cytomegalovirus infection. N. Engl. J. Med. 2009, 360, 1191-1199.

6. Griffiths, P.D.; Stanton, A.; McCarrell, E.; Smith, C.; Osman, M.; Harber, M.; Davenport, A.; Jones, G.; Wheeler, D.C.; O'Beirne, J.; et al. Cytomegalovirus glycoprotein-b vaccine with mf59 adjuvant in transplant recipients: A phase 2 randomised placebo-controlled trial. Lancet 2011, 377, 1256-1263.

7. Ahlfors, K.; Ivarsson, S.A.; Harris, S. Report on a long-term study of maternal and congenital cytomegalovirus infection in sweden. Review of prospective studies available in the literature. Scand. J. Infect. Dis. 1999, 31, 443-457.

8. Ahlfors, K.; Harris, S.; Ivarsson, S.; Svanberg, L. Secondary maternal cytomegalovirus infection causing symptomatic congenital infection. N. Engl. J. Med. 1981, 305, 284.

9. Boppana, S.B.; Rivera, L.B.; Fowler, K.B.; Mach, M.; Britt, W.J. Intrauterine transmission of cytomegalovirus to infants of women with preconceptional immunity. N. Engl. J. Med. 2001, 344, 1366-1371.

10. Ross, S.A.; Arora, N.; Novak, Z.; Fowler, K.B.; Britt, W.J.; Boppana, S.B. Cytomegalovirus reinfections in healthy seroimmune women. J. Infect. Dis. 2010, 201, 386-389.

11. Yamamoto, A.Y.; Mussi-Pinhata, M.M.; Boppana, S.B.; Novak, Z.; Wagatsuma, V.M.; Oliveira Pde, F.; Duarte, G.; Britt, W.J. Human cytomegalovirus reinfection is associated with intrauterine transmission in a highly cytomegalovirus-immune maternal population. Am. J. Obstet. Gynecol. 2010, 202, 297 e291-298.

12. Mussi-Pinhata, M.M.; Yamamoto, A.Y.; Moura Brito, R.M.; de Lima Isaac, M.; de Carvalho e Oliveira, P.F.; Boppana, S.; Britt, W.J. Birth prevalence and natural history of congenital cytomegalovirus infection in a highly seroimmune population. Clin. Infect. Dis. 2009, 49, 522-528.

13. Ross, S.A.; Fowler, K.B.; Ashrith, G.; Stagno, S.; Britt, W.J.; Pass, R.F.; Boppana, S.B. Hearing loss in children with congenital cytomegalovirus infection born to mothers with preexisting immunity. J. Pediatr. 2006, 148, 332-336. 
14. Yamamoto, A.Y.; Mussi-Pinhata, M.M.; Isaac, M.D.; Amaral, F.R.; Carvalheiro, C.G.; Aragon, D.C.; Manfredi, A.K.; Boppana, S.B.; Britt, W.J. Congenital cytomegalovirus infection as a cause of sensorineural hearing loss in a highly immune population. J. Pediatr. Infect. Dis. 2011, 30, 1043-1046.

15. Novak, Z.; Ross, S.A.; Patro, R.K.; Pati, S.K.; Reddy, M.K.; Purser, M.; Britt, W.J.; Boppana, S.B. Enzyme-linked immunosorbent assay method for detection of cytomegalovirus strain-specific antibody responses. Clin. Vaccine Immunol. 2009, 16, 288-290.

16. Yamamoto, A.Y.; Mussi-Pinhata, M.M.; de Deus Wagatsuma, V.M.; Marin, L.J.; Duarte, G.; Figueiredo, L.T. Human cytomegalovirus glycoprotein b genotypes in brazilian mothers and their congenitally infected infants. J. Med. Virol. 2007, 79, 1164-1168.

17. Murthy, S.; Hayward, G.S.; Wheelan, S.; Forman, M.S.; Ahn, J.H.; Pass, R.F.; Arav-Boger, R. Detection of a single identical cytomegalovirus (cmv) strain in recently seroconverted young women. PLoS One 2011, 6, e15949.

18. Kropff, B.; Burkhardt, C.; Schott, J.; Nentwich, J.; Fisch, T.; Britt, W.; Mach, M. Glycoprotein $\mathrm{n}$ of human cytomegalovirus protects the virus from neutralizing antibodies. PLoS Pathog. 2012, 8, e1002999.

19. Pati, S.K.; Novak, Z.; Purser, M.; Arora, N.; Mach, M.; Britt, W.J.; Boppana, S.B. Strain-specific neutralizing antibody responses against human cytomegalovirus envelope glycoprotein n. Clin. Vaccine Immunol. 2012, 19, 909-913.

20. Burkhardt, C.; Himmelein, S.; Britt, W.; Winkler, T.; Mach, M. Glycoprotein n subtypes of human cytomegalovirus induce a strain-specific antibody response during natural infection. J. Gen. Virol. 2009, 90, 1951-1961.

21. Sabbaj, S.; Pass, R.F.; Goepfert, P.A.; Pichon, S. Glycoprotein b vaccine is capable of boosting both antibody and cd4 t-cell responses to cytomegalovirus in chronically infected women. J. Infect. Dis. 2011, 203, 1534-1541.

22. Schleiss, M.R. Could therapeutic vaccination of cytomegalovirus-seropositive persons prevent reinfection and congenital virus transmission? J. Infect. Dis. 2011, 203, 1513-1516.

23. Kern, E.R. Pivotal role of animal models in the development of new therapies for cytomegalovirus infections. Antivir. Res. 2006, 71, 164-171.

24. Schleiss, M.R. Nonprimate models of congenital cytomegalovirus (cmv) infection: Gaining insight into pathogenesis and prevention of disease in newborns. ILAR J. 2006, 47, 65-72.

25. Yue, Y.; Wang, Z.; Abel, K.; Li, J.; Strelow, L.; Mandarino, A.; Eberhardt, M.K.; Schmidt, K.A.; Diamond, D.J.; Barry, P.A. Evaluation of recombinant modified vaccinia ankara virus-based rhesus cytomegalovirus vaccines in rhesus macaques. Med. Microbiol. Immunol. 2008, 197, 117-123.

26. Bia, F.J.; Miller, S.A.; Davidson, K.H. The guinea pig cytomegalovirus model of congenital human cytomegalovirus infection. Birth. Defects Orig. Artic. Ser. 1984, 20, 233-241.

27. Hartley, J.W.; Rowe, W.P.; Huebner, R.J. Serial propagation of the guinea pig salivary gland virus in tissue culture. Proc. Soc. Exp. Biol. Med. 1957, 96, 281-285.

28. Johnson, K.P.; Connor, W.S. Guinea pig cytomegalovirus: Transplacental transmission. Brief report. Arch. Virol. 1979, 59, 263-267. 
29. Schleiss, M.R.; Bourne, N.; Stroup, G.; Bravo, F.J.; Jensen, N.J.; Bernstein, D.I. Protection against congenital cytomegalovirus infection and disease in guinea pigs, conferred by a purified recombinant glycoprotein b vaccine. J. Infect. Dis. 2004, 189, 1374-1381.

30. Bratcher, D.F.; Bourne, N.; Bravo, F.J.; Schleiss, M.R.; Slaoui, M.; Myers, M.G.; Bernstein, D.I. Effect of passive antibody on congenital cytomegalovirus infection in guinea pigs. J. Infect. Dis. 1995, 172, 944-950.

31. Britt, W.J.; Harrison, C. Identification of an abundant disulfide-linked complex of glycoproteins in the envelope of guinea pig cytomegalovirus. Virology 1994, 201, 294-302.

32. Schleiss, M.R.; Bernstein, D.I.; McVoy, M.A.; Stroup, G.; Bravo, F.; Creasy, B.; McGregor, A.; Henninger, K.; Hallenberger, S. The non-nucleoside antiviral, bay 38-4766, protects against cytomegalovirus (cmv) disease and mortality in immunocompromised guinea pigs. Antivir. Res. 2005, 65, 35-43.

33. Fong, C.K.; Bia, F.; Hsiung, G.D. Ultrastructural development and persistence of guinea pig cytomegalovirus in duet cells of guinea pig submaxillary gland. Arch. Virol. 1980, 64, 97-108.

34. Fong, C.K.; Bia, F.; Hsiung, G.D.; Madore, P.; Chang, P.W. Ultrastructural development of guinea pig cytomegalovirus in cultured guinea pig embryo cells. J. Gen. Virol. 1979, 42, $127-140$.

35. Fong, C.K.; Brigati, D. Ultrastructural localization of viral antigen in nuclear inclusions of cytomegalovirus infected guinea pig cells. Arch. Virol. 1982, 74, 125-133.

36. Cui, X.; McGregor, A.; Schleiss, M.R.; McVoy, M.A. The impact of genome length on replication and genome stability of the herpesvirus guinea pig cytomegalovirus. Virology 2009, 386, 132-138.

37. Smith, L.M.; McWhorter, A.R.; Masters, L.L.; Shellam, G.R.; Redwood, A.J. Laboratory strains of murine cytomegalovirus are genetically similar to but phenotypically distinct from wild strains of virus. J. Virol. 2008, 82, 6689-6696.

38. Pignatelli, S.; Dal Monte, P.; Rossini, G.; Chou, S.; Gojobori, T.; Hanada, K.; Guo, J.J.; Rawlinson, W.; Britt, W.; Mach, M. et al. Human cytomegalovirus glycoprotein n (gpul73-gn) genomic variants: Identification of a novel subgroup, geographical distribution and evidence of positive selective pressure. J. Gen. Virol. 2003, 84, 647-655.

39. Pignatelli, S.; Dal Monte, P.; Rossini, G.; Lazzarotto, T.; Gatto, M.R.; Landini, M.P. Intrauterine cytomegalovirus infection and glycoprotein n (gn) genotypes. J. Clin. Virol. 2003, $28,38-43$.

40. CLC Workbench software, version 6.0; CLC: Cambridge, MA, USA. Available online: http://www.clcbio.com/products/clc-main-workbench/ (accessed on 23 January 2014).

41. XPlasMap, version 0.99; Available online: http://www.iayork.com/XPlasMap/ (accessed on 16 January 2014).

42. Yamada, S.; Nozawa, N.; Katano, H.; Fukui, Y.; Tsuda, M.; Tsutsui, Y.; Kurane, I.; Inoue, N. Characterization of the guinea pig cytomegalovirus genome locus that encodes homologs of human cytomegalovirus major immediate-early genes, ul128, and ul130. Virology 2009, 391, 99-106. 
43. Nozawa, N.; Yamamoto, Y.; Fukui, Y.; Katano, H.; Tsutsui, Y.; Sato, Y.; Yamada, S.; Inami, Y.; Nakamura, K.; Yokoi, M.; et al. Identification of a 1.6 kb genome locus of guinea pig cytomegalovirus required for efficient viral growth in animals but not in cell culture. Virology 2008, 379, 45-54.

44. Yang, D.; Tamburro, K.; Dittmer, D.; Cui, X.; McVoy, M.A.; Hernandez-Alvarado, N.; Schleiss, M.R. Complete genome sequence of pathogenic guinea pig cytomegalovirus from salivary gland homogenates of infected animals. Genome Announc. 2013, 1, e0005413.

45. Kozak, M. An analysis of 5'-noncoding sequences from 699 vertebrate messenger rnas. Nucleic Acids Res. 1987, 15, 8125-8148.

46. iTEM software, Version 9.0; Olympus SIS: Münster, Germany, 2014.

47. Simpson, J.T.; Wong, K.; Jackman, S.D.; Schein, J.E.; Jones, S.J.; Birol, I. Abyss: A parallel assembler for short read sequence data. Genome Res. 2009, 19, 1117-1123.

48. Kanai, K.; Yamada, S.; Yamamoto, Y.; Fukui, Y.; Kurane, I.; Inoue, N. Re-evaluation of the genome sequence of guinea pig cytomegalovirus. J. Gen. Virol. 2011, 92, 1005-1020.

49. Schleiss, M.R.; McGregor, A.; Choi, K.Y.; Date, S.V.; Cui, X.; McVoy, M.A. Analysis of the nucleotide sequence of the guinea pig cytomegalovirus (gpcmv) genome. Virol. J. 2008, 5, 139.

50. Milne, I.; Bayer, M.; Cardle, L.; Shaw, P.; Stephen, G.; Wright, F.; Marshall, D. Tablet-Next generation sequence assembly visualization. Bioinformatics 2010, 26, 401-402.

51. Li, H.; Handsaker, B.; Wysoker, A.; Fennell, T.; Ruan, J.; Homer, N.; Marth, G.; Abecasis, G.; Durbin, R. The sequence alignment/map format and samtools. Bioinformatics 2009, 25, 2078-2079.

52. Waterhouse, A.M.; Procter, J.B.; Martin, D.M.; Clamp, M.; Barton, G.J. Jalview version 2-A multiple sequence alignment editor and analysis workbench. Bioinformatics 2009, 25, 1189-1191.

53. SMRT Analysis software, Version 1.4.0; Pacific Biosciences: Menlo Park, CA, USA, 2014. Available online: http:/www.pacificbiosciences.com/products/software/secondary-analysis/ (accessed on 16 January 2014).

54. LightCycler Data Analysis Software, version 1.5; Roche: Indianapolis, IN, USA, 2014.

55. Crumpler, M.M.; Choi, K.Y.; McVoy, M.A.; Schleiss, M.R. A live guinea pig cytomegalovirus vaccine deleted of three putative immune evasion genes is highly attenuated but remains immunogenic in a vaccine/challenge model of congenital cytomegalovirus infection. Vaccine 2009, 27, 4209-4218.

56. Hsiung, G.D.; Bia, F.J.; Fong, C.K. Viruses of guinea pigs: Considerations for biomedical research. Microbiol. Rev. 1980, 44, 468-490.

57. Hummel, M.; Zhang, Z.; Yan, S.; DePlaen, I.; Golia, P.; Varghese, T.; Thomas, G.; Abecassis, M.I. Allogeneic transplantation induces expression of cytomegalovirus immediate-early genes in vivo: A model for reactivation from latency. J. Virol. 2001, 75, 4814-4822.

58. Soderberg-Naucler, C.; Fish, K.N.; Nelson, J.A. Reactivation of latent human cytomegalovirus by allogeneic stimulation of blood cells from healthy donors. Cell 1997, 91, 119-126.

59. Middelkamp, J.N.; Patrizi, G.; Reed, C.A. Light and electron microscopic studies of the guinea pig cytomegalovirus. J. Ultrastruct. Res. 1967, 18, 85-101. 
60. Choi, Y.C.; Hsiung, G.D. Cytomegalovirus infection in guinea pigs. II. Transplacental and horizontal transmission. J. Infect. Dis. 1978, 138, 197-202.

61. ATCC History of GPCMV. Available online: http://www.atcc.org/products/all/VR682.aspx\#history/ (accessed on 23 January 2014).

62. Brondke, H.; Schmitz, B.; Doerfler, W. Nucleotide sequence comparisons between several strains and isolates of human cytomegalovirus reveal alternate start codon usage. Arch. Virol. 2007, 152, 2035-2046. 

Reprinted from Viruses. Cite as: Gnanandarajah, J.S.; Gillis, P.A.; Hernandez-Alvarado, N.; Higgins, L.; Markowski, T.W.; Sung, H.; Lumley, S.; Schleiss, M.R. Identification by Mass Spectrometry and Immune Response Analysis of Guinea Pig Cytomegalovirus (GPCMV) Pentameric Complex Proteins GP129, 131 and 133. Viruses 2014, 6, 727-751.

Article

\title{
Identification by Mass Spectrometry and Immune Response Analysis of Guinea Pig Cytomegalovirus (GPCMV) Pentameric Complex Proteins GP129, 131 and 133
}

\author{
Josephine S. Gnanandarajah ${ }^{1}$, Peter A. Gillis ${ }^{1}$, Nelmary Hernandez-Alvarado ${ }^{1}$, \\ LeeAnn Higgins ${ }^{2}$, Todd W. Markowski ${ }^{2}$, Heungsup Sung ${ }^{1}$, Sheila Lumley ${ }^{1}$ and \\ Mark R. Schleiss ${ }^{1, *}$
}

1 Departments of Pediatrics, University of Minnesota Medical School, 2001 6th Street SE, Minneapolis, MN 55455, USA; E-Mails: gnan0007@umn.edu (J.S.G.); gill0221@umn.edu (P.A.G.); hernande@umn.edu (N.H.-A.); sung@amc.seoul.kr (H.S.); sheilalumley@gmail.com (S.L.)

2 Department of Biochemistry, Molecular Biology and Biophysics, University of Minnesota, 321 Church Street SE, Minneapolis, MN 55455, USA; E-Mails: higgi022@umn.edu (L.H.); marko025@umn.edu (T.W.M.)

* Author to whom correspondence should be addressed; E-Mail: schleiss@umn.edu; Tel.: +1-612-624-1112; Fax: +1-612-626-9924.

Received: 18 November 2013; in revised form: 3 January 2014 / Accepted: 14 January 2014 / Published: 13 February 2014

Abstract: Development of a vaccine against congenital infection with human
cytomegalovirus (HCMV) is a major public health priority. A potential vaccine target
receiving considerable recent attention is the pentameric complex (PC) of HCMV
proteins consisting of gL, gH, UL128, UL130, and UL131, since some antibodies
against these target proteins are capable of potently neutralizing virus at epithelial and
endothelial cell surfaces. Recently, homologous proteins have been described for
guinea pig cytomegalovirus (GPCMV), consisting of gH, gL, and the GPCMV proteins
GP129, GP131, and GP133. To investigate these proteins as potential vaccine targets,
expression of GP129-GP133 transcripts was confirmed by reverse-transcriptase PCR.
Mass spectrometry combined with western blot assays demonstrated the presence of
GP129, GP131, and GP133 proteins in virus particles. Recombinant proteins
corresponding to these PC proteins were generated in baculovirus, and as GST fusion
proteins. Recombinant proteins were noted to be immunoreactive with convalescent 
sera from infected animals, suggesting that these proteins are recognized in the humoral immune response to GPCMV infection. These analyses support the study of PC-based recombinant vaccines in the GPCMV congenital infection model.

Keywords: guinea pig cytomegalovirus; cytomegalovirus vaccine; pentameric complex; congenital cytomegalovirus infection; baculovirus

\section{Introduction}

Development of a vaccine against human cytomegalovirus (HCMV) is a major public health priority [1]. Although a vaccine could be useful in several patient populations at risk for HCMV-associated disease, a vaccine that could prevent congenital infection and its attendant sequelae would be of particular value. HCMV infection elicits both cellular and humoral immune responses. The suggestion that passively transferred anti-HCMV antibody during pregnancy protects the fetus against infection and HCMV-induced injury [2] has driven efforts to develop recombinant vaccines targeting major envelope glycoproteins. Development of an optimal glycoprotein vaccine is complicated by the complexity of virus entry and the variety of mechanisms employed. HCMV uses two different entry mechanisms to infect various cell types (fibroblasts, epithelial cells, endothelial cells, and macrophages). Fibroblast entry is mediated by glycoprotein complexes $\mathrm{gB}, \mathrm{gH} / \mathrm{gL} / \mathrm{gO}$, and $\mathrm{gM} / \mathrm{gN}$, but entry into epithelial cells, endothelial cells, and macrophages requires the gH/gL/UL128/UL130/UL131 complex [3-5]. Recently, it was demonstrated that the majority of virus-neutralizing antibodies in hyperimmune globulin target the gH/gL/UL128/UL130/UL131 complex [6]. Moreover, it was recently shown that fetal CMV transmission was more likely in the setting of a delayed or diminished maternal antibody response to the $\mathrm{gH} / \mathrm{gL} / \mathrm{pUL128} / 130 / 131$ complex during primary infection [7], further underscoring the potential usefulness of targeting these proteins for vaccine development [8]. Therefore, although clinical trials of recombinant $\mathrm{gB}$ vaccines (which have shown some degree of effectiveness in preventing CMV infection and disease in high risk populations) are ongoing [9,10], there is also considerable interest in developing vaccines targeting the HCMV pentameric complex (PC).

Ideally, a HCMV vaccine strategy would be tested in an animal model prior to clinical trials. Unfortunately, the strict species-specificity of CMVs precludes preclinical testing of HCMV vaccines in animals. However, a number of rodent and primate CMVs are useful in modeling HCMV vaccines and therapies, given the conservation of many immunogenic structural proteins amongst the various viruses [11-13]. The rhesus CMV (RhCMV) encodes highly conserved homologs of the UL128/UL130/UL131 members of the PC. Recently, a modified vaccinia virus Ankara virus (MVA) was described that stably coexpresses all five RhCMV proteins homologous to the HCMV PC [14]. RhCMV-naïve rhesus macaques vaccinated with the MVA construct developed antibodies that blocked infection of monkey kidney epithelial cells and rhesus fibroblasts. In addition, following subcutaneous RhCMV challenge at eight weeks post-vaccination, vaccinated animals demonstrated reduced plasma viral loads. 
Although the RhCMV model is a valuable system that recapitulates many of the pathologies of HCMV congenital infection, the expense of rhesus macaques and the difficulty in establishing RhCMV seronegative animal colonies makes it difficult to conduct large-scale studies comparing vaccines against congenital infection. Among the small animal models, the guinea pig CMV (GPCMV) is uniquely useful, since, in contrast to rodent models, transplacental infection of the fetus occurs following viral challenge during pregnancy $[12,15]$. Hence, the GPCMV model is well-suited to the study of vaccines against congenital infection. Inoue described a region of the GPCMV genome that appeared to be genetically unstable, in the setting of serial virus passage in fibroblasts that contained homologs of the HCMV UL128/UL130/UL131 genes [16,17]. More recently, Feierbach [18] further defined the proteins encoded by this region and determined that the GPCMV genes GP129, GP131, and GP133 represented the ancillary protein members (along with GP75 $[\mathrm{gH}]$ and gp115 [gL]) of the PC (Table 1). These studies were undertaken to characterize these genes and express the encoded proteins in recombinant systems, toward the goal of examining antibody responses of naturally and experimentally infected guinea pigs to these proteins. Knowledge of the patterns of immune responses to these GPCMV proteins should prove useful in the evaluation of potential subunit vaccines targeting the PC in the GPCMV model.

Table 1. Guinea pig cytomegalovirus (GPCMV) 129-133 Locus.

\begin{tabular}{ccccc}
\hline $\begin{array}{c}\text { GPCMV } \\
\text { Protein }\end{array}$ & $\begin{array}{c}\text { HCMV } \\
\text { Homolog }\end{array}$ & Codons & Predicted M & $\begin{array}{c}\text { Amino Acid Similarity to } \\
\text { HCMV (\%) }\end{array}$ \\
\hline GP129 & UL128 & 179 & $20.6 \mathrm{kDa}$ & $48 \%$ \\
GP131 & UL130 & 192 & $21.8 \mathrm{kDa}$ & $36 \%$ \\
GP133 & UL131 & 127 & $14.7 \mathrm{kDa}$ & $29 \%$ \\
\hline
\end{tabular}

\section{Results and Discussion}

\subsection{Transcriptional Analyses of GP129, 131 and 133}

Initially, transcription from the GPCMV GP129, 131, and 133 locus was examined by reverse-transcriptase PCR (RT-PCR). Primer design was based upon published primer sequences reported by Inoue [17]. Primers used for these studies are summarized in Table 2. Previous reports indicated that these genes are encoded by single mRNAs, and the GP129 and GP131 RNAs, but not the GP133 RNA, are spliced (Figure 1A). To confirm and extend these findings, RNA was purified from GPCMV-infected GPL cells at 8, 12, 24, and 48 hours post-inoculation. A 48-hour time point was also collected in the presence of phosphonoacetic acid (PAA), $300 \mu \mathrm{g} / \mathrm{mL}$. The viral stock used was P1-passaged GPCMV Strain 22122 purchased directly from the American Type Culture Collection (ATCC; Manassas, VA, USA). As an additional control, cells were inoculated with vAM403, an eGFP-expressing recombinant GPCMV [19] known to lack the $1.6 \mathrm{~kb}$ region containing the GP129-133 locus [16]. The RT-PCR products predicted for each gene were: 540 base pairs (bp) for GP129 transcript; 515 bp for GP131; and 384 bp for GP133. Transcripts specific for GP131 and GP133 were apparent as early as 12 hours post-infection (Figure 1B,C). A GP129-specific transcript could be faintly detected as soon as 12 hours post-infection, and was abundantly present at 24 hours post-infection (Figure 1A). These data differ from those reported by Inoue and colleagues [17], who 
did not note any GP129-specific transcript until $\sim 48$ hours post-infection, although this analysis was performed by northern blot, which may have been less sensitive than RT-PCR. Similar to Inoue's findings, we noted that the spliced GP129 transcript was not identified in the presence of PAA, consistent with its classification as a late gene. However, we noted transcripts corresponding to the spliced GP131 mRNA as soon as 12 hours post-infection and continuing at high levels throughout 48 hours post-infection even in the presence of PAA (Figure 1B), suggesting that GP131 is an early gene, in contrast to the previous report by Inoue that suggested (based on northern blot analysis) that both GP129 and GP131 were late genes [17].

Table 2. Primers used in GP129, 131 and 133 Cloning and Protein Expression Studies.

\begin{tabular}{|c|c|}
\hline ORF & Primer Sequences \\
\hline GP129 (Baculovirus and & 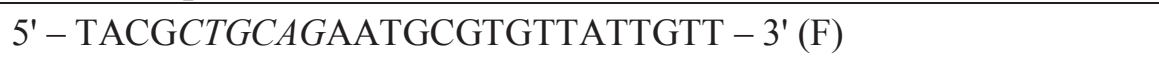 \\
\hline Transcript Analysis) & $5^{\prime}$ - TACGAGATCTTTACTTCCCGTTACC - 3' (R) \\
\hline \multirow{2}{*}{ GP129 GST (R1) } & 5' - TACGGGATCCTATACCCGTCCCGGTATCTTTG - 3' (F) \\
\hline & $5^{\prime}$ - TACGCTCGAGTTAAGTATTCCCACATCGTACTAATC - 3' (R) \\
\hline \multirow{2}{*}{ GP129 GST (R2) } & 5' - TACGGGATCCTCGCGGCAAGAACTCCAT - 3' (F) \\
\hline & 5' - TACGCTCGAGTTAACGGTAGGTCACCCCCAAG - 3' (R) \\
\hline \multirow{2}{*}{ GP129 GST (R3) } & $5^{\prime}$ - TACGGGATCCAACGGTTTATTATGCACCTTTC - 3' (F) \\
\hline & 5' - TACGCTCGAGTTACTTCCCGTTACCATCGAC - 3' (R) \\
\hline \multirow{2}{*}{ GP131 (Baculovirus) } & 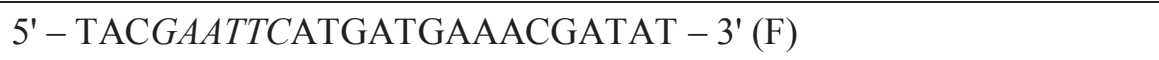 \\
\hline & $5^{\prime}$ - TACGCTGCAGTTATCACGTCCAGTT - 3' (R) \\
\hline \multirow{2}{*}{$\begin{array}{l}\text { GP131 (Transcript } \\
\text { Analysis) }\end{array}$} & $5^{\prime}$ - ATAATGATGAAACGATAT - 3' (F) \\
\hline & 5' - TTATCACGTCCAGTTCCA - 3' (R) \\
\hline \multirow{2}{*}{ GP131 GST (R1) } & $5^{\prime}$ - GATAGGATCCTTTTACGCCTCGTTCGGA - 3' (F) \\
\hline & 5' - AATACTCGAGGTTCGTCAGGGTCAGGAC - 3' (R) \\
\hline \multirow{2}{*}{ GP131 GST (R2) } & 5' - GATAGGATCCCGCCGAATAGATTACGGA - 3' (F) \\
\hline & 5' - AATACTCGAGCCACAAGAAGGACGAATC - 3' (R) \\
\hline \multirow{2}{*}{ GP131 GST (R3) } & $5^{\prime}$ - CGTGGGATCCTGGCATTATACGATACGG - 3' (F) \\
\hline & 5' - AATACTCGAGCAGGCAAGCGATAGAATC - 3' (R) \\
\hline \multirow{2}{*}{ GP133 (Baculovirus) } & 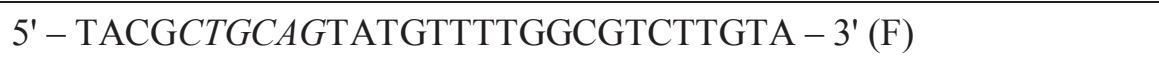 \\
\hline & 5' - TACGAGATCTTTATGCTCTGTCTATGC - 3' (R) \\
\hline \multirow{2}{*}{$\begin{array}{l}\text { GP133 (Transcript } \\
\text { Analysis) }\end{array}$} & 5' - TATGTTTTGGCGTCTTGTA - 3' (F) \\
\hline & $5^{\prime}$ - TTATGCTCTGTCTATGC - 3' (R) \\
\hline \multirow{2}{*}{ GP133 GST (R1) } & 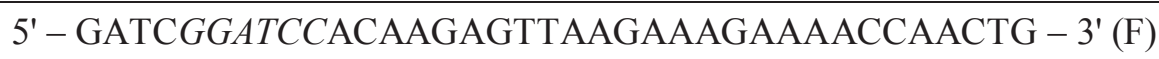 \\
\hline & 5' - GATCCTCGAGTTAGGAGTCCGCTAACGTATG - 3' (R) \\
\hline \multirow{2}{*}{ GP133 GST (R2) } & 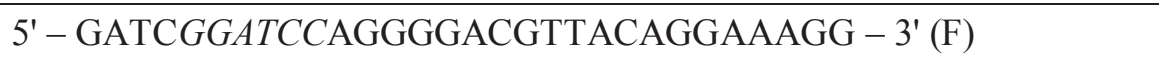 \\
\hline & 5' - GATCCTCGAGTTAAGTGCTTTGTTGAATAGAAATACG - 3' (R) \\
\hline \multirow{2}{*}{ GAPDH } & 5' - ATCTCATCGTATTTGGCCGGT - 3' (F) \\
\hline & 5' - AATGGGAAGCTCACAGGTATGG - 3' (R) \\
\hline
\end{tabular}


Figure 1. Transcription of GP129, 131 and 133 as assessed by reverse-transcriptase PCR (RT-PCR). RNA from ATCC GPCMV-infected cells (Lanes 2, 4, 6, 8, 10) and vAM403-infected cells (Lanes 3, 5, 7, 9 and 11) was purified at 8 hours (Lanes 2, 3), 12 hours (Lanes 4, 5), 24 hours (Lanes 6, 7) and 48 hours (Lanes 8, 9) post-infection. A 48-hour time point was also collected in the presence of phosphonoacetic acid (PAA) (Lanes 10, 11). Lane 1 represents RT-PCR of RNA from uninfected cells. Electrophoresis was performed on a $1.5 \%$ agarose gel. Molecular weights markers are the $1 \mathrm{~Kb}$-Opti-DNA marker from ABM (Applied Biological Materials Inc., Richmond, BC, Canada). (a) GP129-specific transcripts. Predicted splice product of $540 \mathrm{nt}$ is noted (arrow), but is not present in infected cells incubated with PAA. (b) GP131-specific transcripts. Predicted splice product of $515 \mathrm{nt}$ is noted (arrow). (c) GP133-specific transcripts demonstrating no evidence of splicing. Predicted $384 \mathrm{nt}$ product is noted (arrow). (d) GAPDH control.

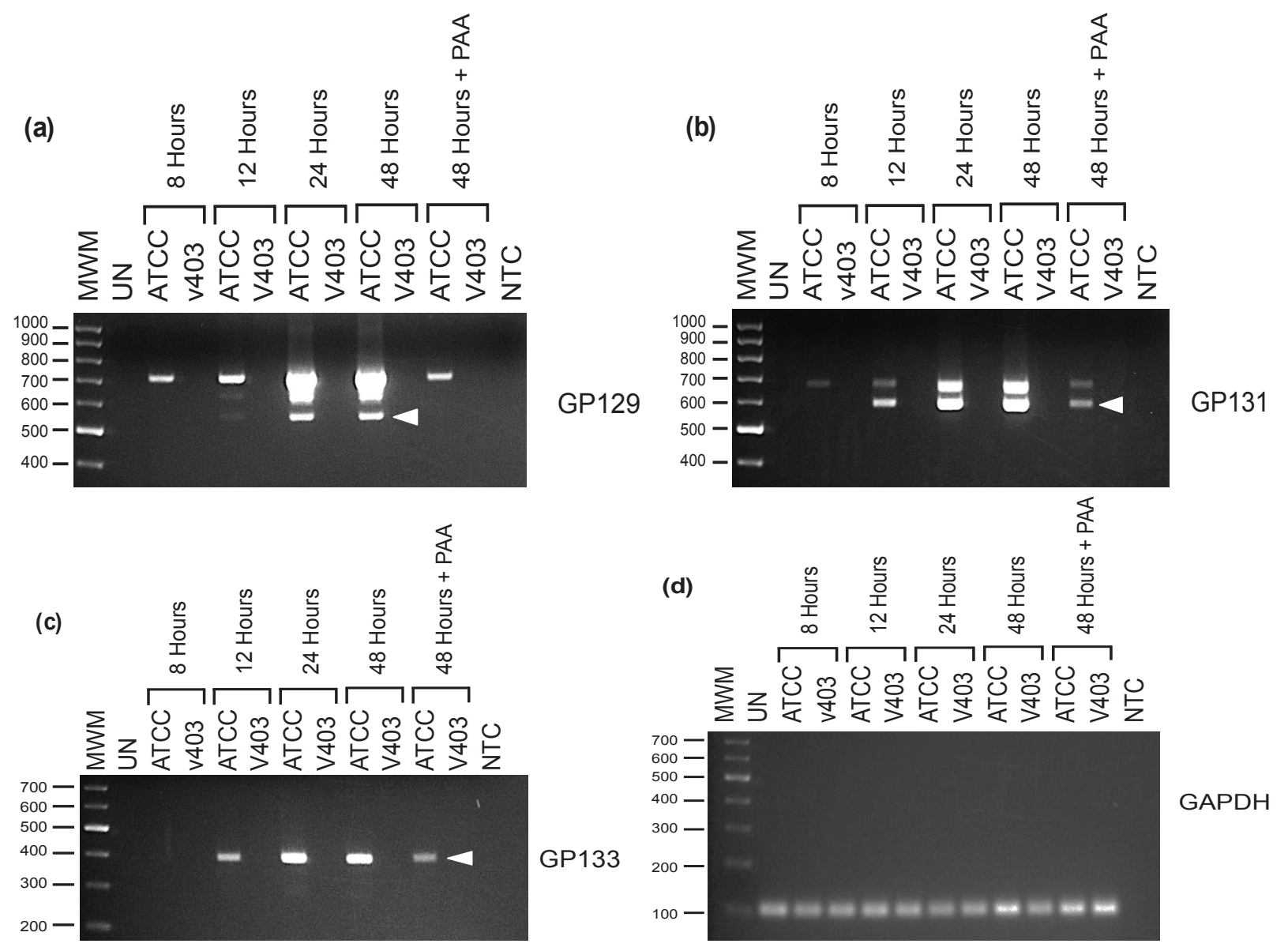

We saw no evidence of splicing of the GP133 transcript, and this RNA continued to be expressed to high levels through 48 hours post-infection. This transcript was also expressed in the presence of PAA. RNA species larger than we predicted were noted for the GP129 and GP131 transcripts. Absence of any signal in the absence of reverse transcriptase makes the possibility of DNA contamination unlikely. These were presumed to represent larger, unspliced RNAs driven by promoters upstream of the GP129-133 promoter region. RNA purified from vAM403-infected cells was negative for GP129, 131 and 133 RT-PCR products, as expected, since this virus lacks the 
region of the GPCMV genome encoding these ORFs. Guinea pig GAPDH cDNA was amplified to control for RNA recovery (1D). No bands were noted in the no-RT control (data not shown).

\subsection{Mass Spectrometry Analysis}

GPCMV virus particles were purified by sequential sucrose [20] and glycerol-tartrate density gradient centrifugation [21] as described in Section 3.2. Particles were examined by coomassie blue stain (Figure 2a) and transmission electron microscopy (EM; Figure 2b) to confirm homogeneity. Particles were probed with a monoclonal antibody (moab) to glycoprotein B, IE321, generated in our laboratory (Figure 2c), to further confirm their integrity. Particles were resolved by SDS PAGE followed by coomassie staining, and bands were excised from the gel for mass spectrometry. Three peptides corresponding to two unique sequences for GP129 were identified from a region of the gel with an approximate molecular weight of $25 \mathrm{kD}$. Additionally, six peptides corresponding to four unique sequences corresponding to GP131 were identified from a protein band excised from a region with a molecular weight spanning approximately 20-22 kD. Finally, seven peptides from four unique sequences of GP133 were identified from a protein band with an approximate molecular weight of $18 \mathrm{kD}$. Results are summarized in Table 3. Mass spectra data are provided in Appendix Figures A1-A3.

Figure 2. Characterization of GPCMV viral particles. (a) Coomassie blue stain (5 $\mu \mathrm{g})$ of purified virus particles by $12 \%$ gel SDS-PAGE. (b) Transmission electron microscope microphotograph of purified particles. Negative contrast preparation of purified particles demonstrates enveloped virions (arrows). Dense bodies are also noted. Magnification, $150,000 \times$. (c) Western blot analysis of viral particles probed with IE3-21 monoclonal antibody recognizing GPCMV gB (1:2,000 dilution; arrow).

(a)

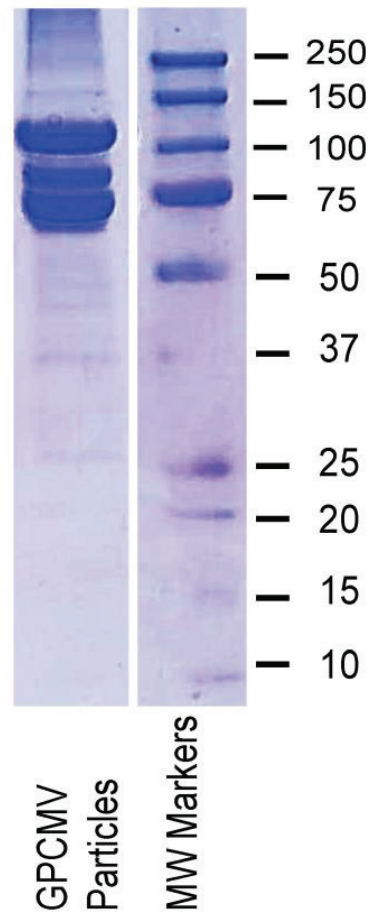

(b)

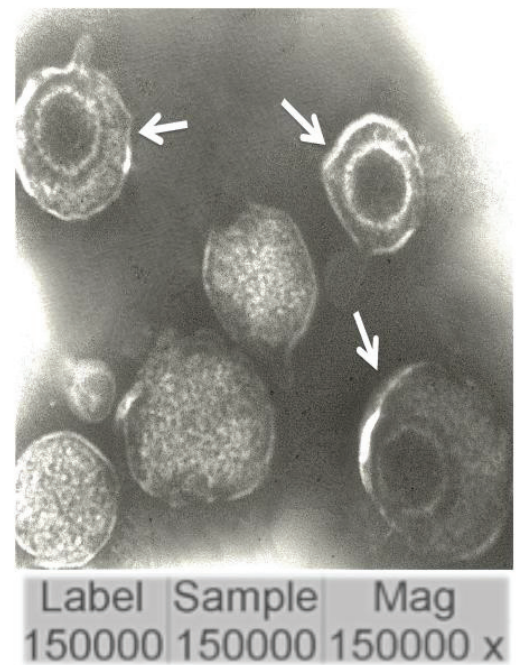

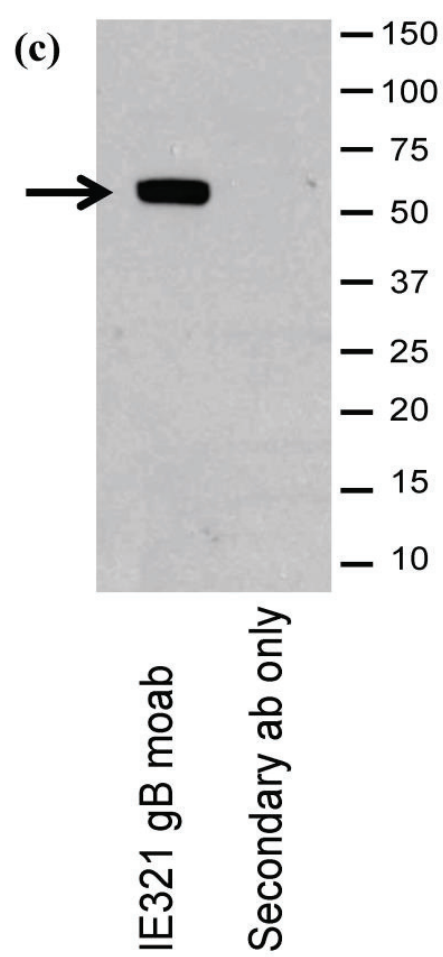


Table 3. Mass spectrometry identification of peptides corresponding to GP129, 131 and 133. Other major virion structural proteins included as controls for mass spectrometry analyses (protein probability calculations performed as described in materials and methods).

\begin{tabular}{cccccc}
\hline ORF & $\begin{array}{c}\text { Unique } \\
\text { Spectra }\end{array}$ & Unique Peptide Sequences & $\begin{array}{c}\text { \% } \\
\text { Coverage }\end{array}$ & Max $\mathbf{X}_{\text {corr }}$ & Probability \\
\hline GP 129 & 2 & $\begin{array}{c}\text { MPSVQSKPEKPSILGVTYR } \\
\text { VDYTVMIPTPHFPR }\end{array}$ & 18 & 3.37 & $99 \%$ \\
\hline GP131 & $6 \quad \begin{array}{c}\text { RIDYGSTGTAASTLPSLTSLR } \\
\text { TYFGDRDSSFLWHYTIRT } \\
\text { DCDVYVTSR HPADSIACLL }\end{array}$ & 29 & 5.01 & $99.9 \%$ \\
\hline GP133 & 7 & $\begin{array}{c}\text { EDEGIDTWWLGGVTDNTR } \\
\text { VKKENQLAHYILK } \\
\text { KGLVIDGIR TIVLTHHR }\end{array}$ & 39 & 4.51 & $99.9 \%$ \\
\hline GP83 & 74 & 61 & 59 & 62 & $100 \%$ \\
\hline GP25 & 78 & 55 & 77 & 5.9 & $100 \%$ \\
\hline gB & 51 & 37 & 31 & 5.1 & $100 \%$ \\
\hline
\end{tabular}

\subsection{Characterization of GP129 and 131 Proteins}

Initially, an anti-peptide rabbit antiserum was generated targeting a GP131-specific peptide sequence, $\mathrm{NH}_{2}-\mathrm{CYYPSTPIPKSFVKHVDTTRSLPE-COOH}$, conjugated with keyhole limpet hemocyanin (see Section 3 for details on generation of antibody). This antibody was used in western blot assay to examine purified virions (purified from passage 1 ATCC Strain 22122 GPCMV), as well as infected cell lysates, for expression of the GP131 protein. Immune sera consistently demonstrated the appearance of a broadly migrating band at approximately $22 \mathrm{kDa}$. In some experiments, the band appeared to separate into two discrete bands at $\sim 20$ and $\sim 22 \mathrm{kDa}$ (Figure 3A). To confirm the specificity of this response for GP131, westerns were also performed using a recombinant, eGFP-expressing GPCMV, vAM403, known to lack the $1.6 \mathrm{~kb}$ region spanning the GP129-133 locus. Western assay targeting vAM403 proteins using the GP131 rabbit polyclonal antibody did not demonstrate presence of this band in purified lysates from cells infected with this virus, as expected (Figure 3A, Lane 2).

The GP131-specific antibody was next examined in an immunofluorescence assay using cells transfected with a GP131 expression plasmid, pKTS 789. Immune, (but not pre-immune) sera identified a protein in GP131-transfected cells, but only under conditions of permeabilization (Figure 3B). This result is compatible with the known requirement of GP131 to be co-expressed with $\mathrm{gH}$ in order for cell surface localization.

To identify GP129 in virions, a GP129-specific antibody was next generated following vaccination of naïve guinea pigs with a GST fusion protein, designated GST_R3_GP129 (described in Section 3). Three guinea pigs were immunized with fusion protein. All animals generated ELISA responses to GST_R3_129 (data not shown). The GST_R3_GP129 immune, but not pre-immune, sera identified a broadly migrating band in purified GPCMV virions (Figure 4). This result suggested that the GP129 protein is expressed in virions, and migrates broadly in the $\sim 25 \mathrm{kDa}$ range by SDS-PAGE. These data confirmed the identification of GP129-specific peptides present in the mass spectrometry analyses of virus particles. 
Figure 3. Characterization of GP131 protein. (a) Western blot analysis using anti-peptide GP131 antibody. Virion particles were probed with either pre-immune sera (left panel) or immune sera (right panel) following purification of virus particles from cells infected either with ATCC GPCMV (Lane 1 in each panel) or vAM403 (Lane 2 in each panel). A band of approximately $20-22 \mathrm{kDa}$ was noted with immune, but not pre-immune sera. On shorter exposure, this band appeared as a doublet. (b) Effect of cell permeabilization on detection of GP131. GPL cells were transfected with a GP131 expression plasmid and immunofluorescence performed with anti-GP131 antibody. Little to no protein expression was detected in non-permeabilized cells (middle panel), but protein expression was readily detected following permeabilization (right panel). Left panel demonstrates pre-immune antibody control.
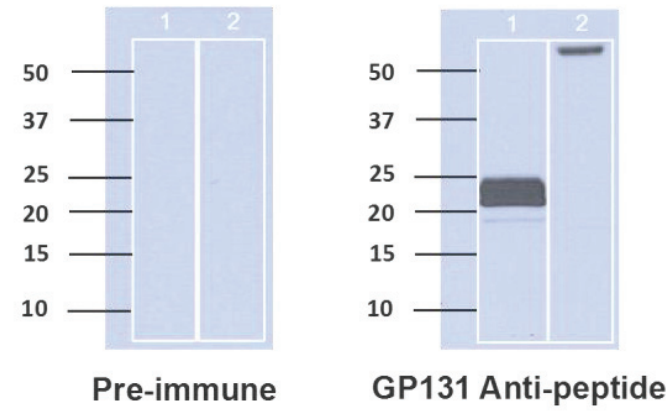

(a)

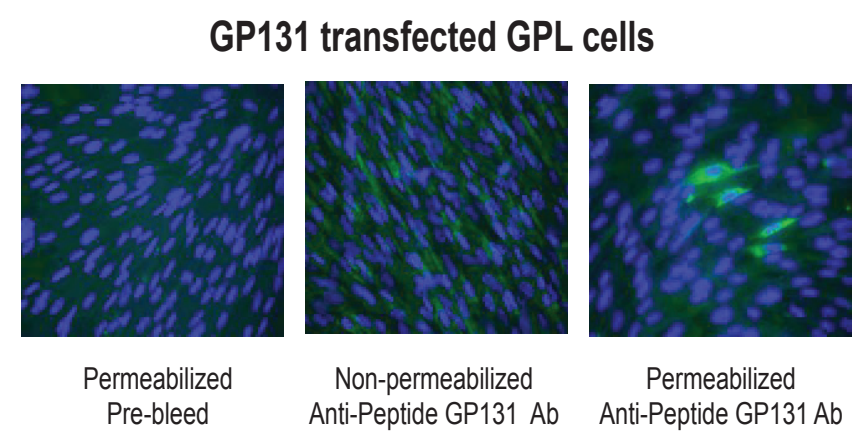

(b)

Figure 4. Identification of GP129 in virus particles. Western blot analysis using an anti-GST/GP129 fusion protein derived antibody. Following electrophoresis with $12 \%$ Bis-Tris gel, western transfer was performed and virion particles were probed (1:200 dilution) with either pre-immune sera (left panel) or immune sera (right panel). GST_R3_129 immune (but not pre-immune) antisera identified a broadly migrating band with MW of the predominate species at $\sim 25 \mathrm{kDa}$ (arrow). Band was not identified in blots of vAM403 (lacking GP129-133 locus) virus particles probed with identical antibodies (data not shown).

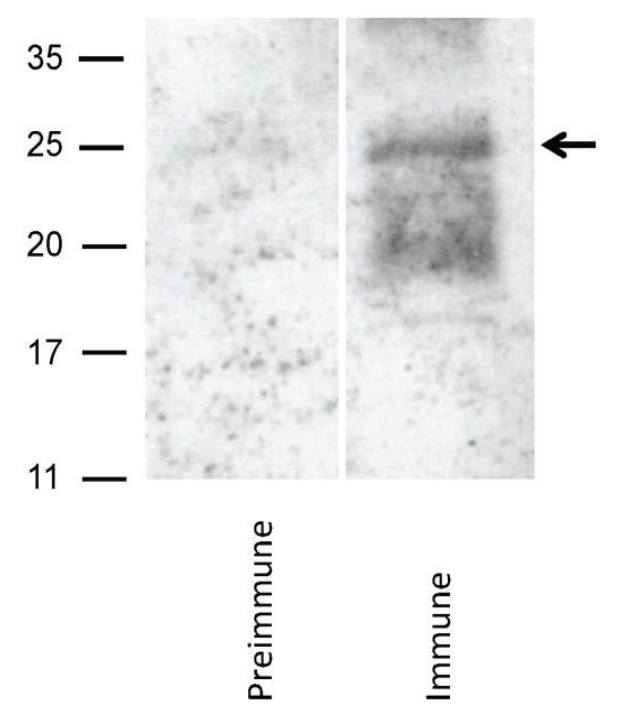


2.4. Characterization of Guinea Pig Antibody Reactivity to Recombinant-Expressed GP129, 131, and 133

To examine the guinea pig immune response to GP129, 131, and 133, a series of GST fusion proteins were generated, corresponding to predicted immunogenic domains in the respective proteins. Primers used in the generation of these fusion proteins are indicated in Table 2. Immune responses in guinea pig convalescent sera from infected animals were readily demonstrable to GP129 fusion proteins. Three domains of GP129 (GP129 R1, R2, and R3; Figure 5) were cloned as GST fusion proteins and western blots probed with anti-GPCMV antisera obtained from experimentally infected animals. The R3 domain (Figure 6A) was the domain most highly immunoreactive with convalescent anti-GPCMV sera from infected animals. This region of the GP129 contains a cysteine residue highly conserved with HCMV and RhCMV UL128 (the homolog of GP129). Although interactions between UL128 and the other constituents of the pentameric complex are not believe to involve disulfide bond formation, this region of the UL128 protein in HCMV is known to be the target of neutralizing monoclonal antibodies [22,23]. When GP129 was expressed in recombinant baculovirus, purified recombinant protein was also immunoreactive with immune sera from animals challenged with salivary gland-adapted GPCMV (Figure 6B). Pre-immune sera failed to recognize the recombinant protein. These analyses strongly suggest that the GP129 protein is a target of the guinea pig antibody response following GPCMV infection.

Figure 5. Subcloning of GP129, 131, 133 proteins. (a) Schematic map of the GPCMV GP129, 131 and 133 ORFs, patterned after [18]. These ORFs map to the rightward end of the GPCMV genome. Genome coordinates of this region are indicated (based on sequence Genbank KC503762 [24]). In contrast to HCMV, the GP131 (UL130) homolog is spliced, but the GP133 (UL131 homolog) is not. (b) GPCMV GP129, GP131, and GP133 ORFs. Predicted van Heinje signal sequence cleavage site is indicated by arrow. Cysteine residues are outlined in dark blue. Potential glycosylation sites (N-X-T and N-X-S) are shown in black outline. Regions of ORFs subcloned as GST fusion proteins are indicated. For GP129 and GP131, pink region represents R1 region; blue, R2 region; yellow, R3. For GP133, pink region is R1 and yellow region R2 (see Table 2 and text for details).

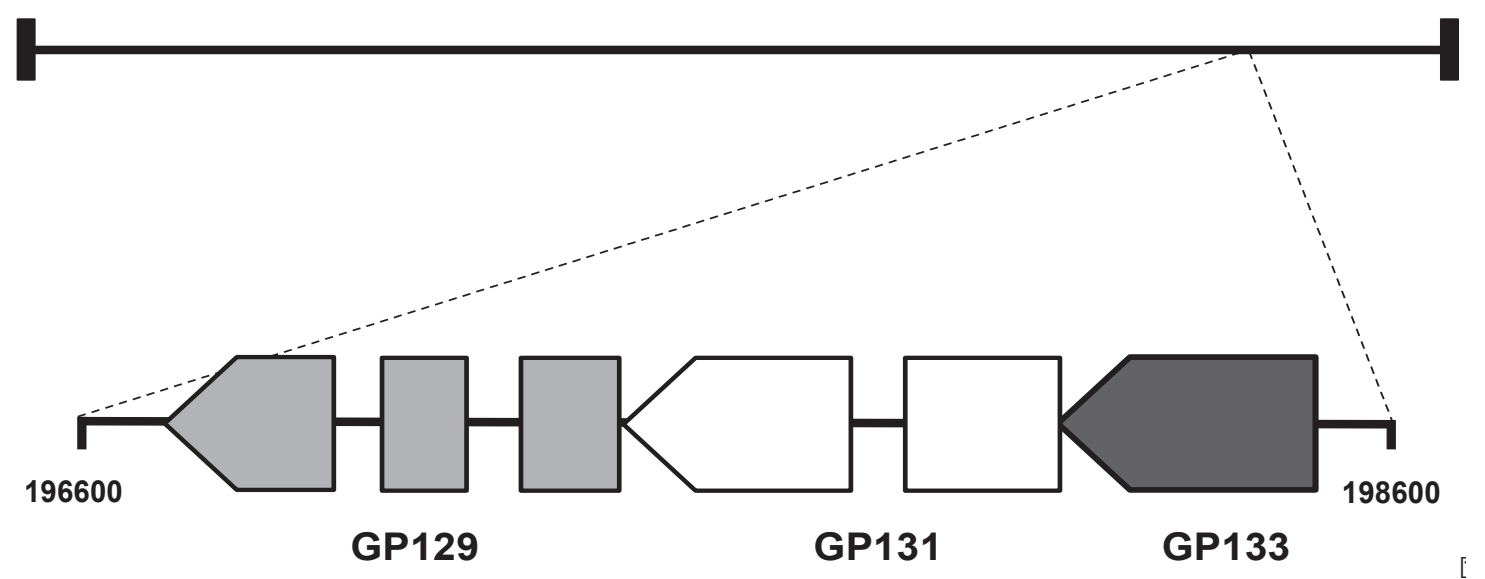

(a) 
Figure 5. Cont.

$$
\begin{aligned}
& \text { MRVIVLLVMFYYTRP GIFDDPCCIYSSKDRRVQHSTTSNDTWRLVRCGNTLMVAKRYTDSFCEF } \\
& \text { SLEENLFESLALNVSRQELHVLAPECKFGPVEVGINKQVRCIRYPRMPSVQSKPEKPSILGVTYRV } \\
& \text { DYTVMIPTPHFPRDF NGLLCTFLEKNDTFYNTTVDVCGSEFYSVDGNGK** }
\end{aligned}
$$

$$
\text { MMKRYLVLLPWIMFYASFGRA GRCYYPSTPIPKSFVKHVDTTRSLPECENDTVAVLTLTNGAK }
$$$$
\text { LYVNMLNTWIDGYITTLQYAIPPTLSDIFAFIK RRIDYGSTGTAASTLPSLTSLRTYFGDRDSSFLW }
$$$$
\text { HYTIRMKDGAKTLDCDVYVTSRVHFVLNSYEAVQTVLFEGGVVISRHPADSIACLLINWNWT* }
$$

MFWRLVYVYLVSLLLSIGA EDEGIDTWWLGGVTDNTRVKKENQLAHYILKTIVLTHHRRLRT GDECTEQLSNDLDIHSVHTLADSIRRI RGRYRKGLVSIDGIRISIQQSTRTQQKGLWISARIDRA*

(b)

Figure 6. Antibodies from GPCMV-seropositive guinea pigs react with recombinant forms of GP129. (a) Analysis of GP129/GST fusion proteins. Three GP129 domains (Figure 5b) were cloned in-frame with GST using vector pGEX 6-P as described in materials and methods. Constructs evaluated by western blot included GST only; GST-GP129R1 fusion; GST-GP129R2 fusion; and GST-GP129R3 fusion, without IPTG induction (uninduced) and following IPTG induction (induced). Anti-GPCMV antisera from immune, experimentally infected guinea pigs demonstrated strongest immunoreactivity with GST-GP129R3 fusion protein. In some experiments, some signal could be observed with GP129R2 fusion protein. Data shown is a representative result from an experimentally infected animal. All fusion proteins expressed to high levels following IPTG induction as demonstrated by western analysis with anti-GST monoclonal antibody (data not shown). No background reactivity was noted with serum from GPCMV-seronegative animals or with pre-immune sera from experimentally infected guinea pigs (data not shown). (b) Western blot analysis of recombinant baculovirus-expressed GP129. The GP129 was cloned in-frame with GFP and a 6-His tag. This resulted in a fusion protein of $\sim 55 \mathrm{kDa}$, made up of $\sim 6 \mathrm{kDa}$ from the 6 -His tag and vector sequences; $28 \mathrm{kDa}$ from the GFP sequence; and $\sim 21 \mathrm{kDa}$ from the GP129 sequences. Purified recombinant protein was subjected to SDS-PAGE and western assays performed as described in materials and methods section. For some western blots, a control baculovirus expressing a recombinant form of the GPCMV GP73.5 glycoprotein was also evaluated. Top panel, both the anti-GFP and anti-6 His antibodies were immunoreactive with GP73.5 ( $\sim 37 \mathrm{kDa})$ and GP129 $(\sim 55 \mathrm{kDa})$ fusion proteins, as expected. A higher MW band was noted at $\sim 65-70 \mathrm{kDa}$, possibly 
representing a glycosylated variant of the baculovirus-expressed GP129 fusion protein. Sera, raised against the GST-GP129-R3 fusion protein (Figure 5a), was also used to probe baculovirus-expressed GP129 as a control. Immune, but not preimmune, sera (1:200 dilution) from a guinea pig immunized with the GST fusion protein were reactive with the baculovirus expressed protein, but not proteins in uninfected SF9 cells. Again, both the $55 \mathrm{kDa}$ fusion protein as well as a larger species of $65-70 \mathrm{kDa}$, were identified. (c) Sera from experimentally infected animals were immunoreactive with the GP129-baulovirus fusion protein. Results shown are representative pre- and post-infection sera (1:200 dilution) from two GPCMV-infected guinea pigs (GP1; GP2) demonstrating immunoreactivity with bacolovirus-GP129 fusion protein (arrow), but not a pre-immune guinea pig serum (NEG).
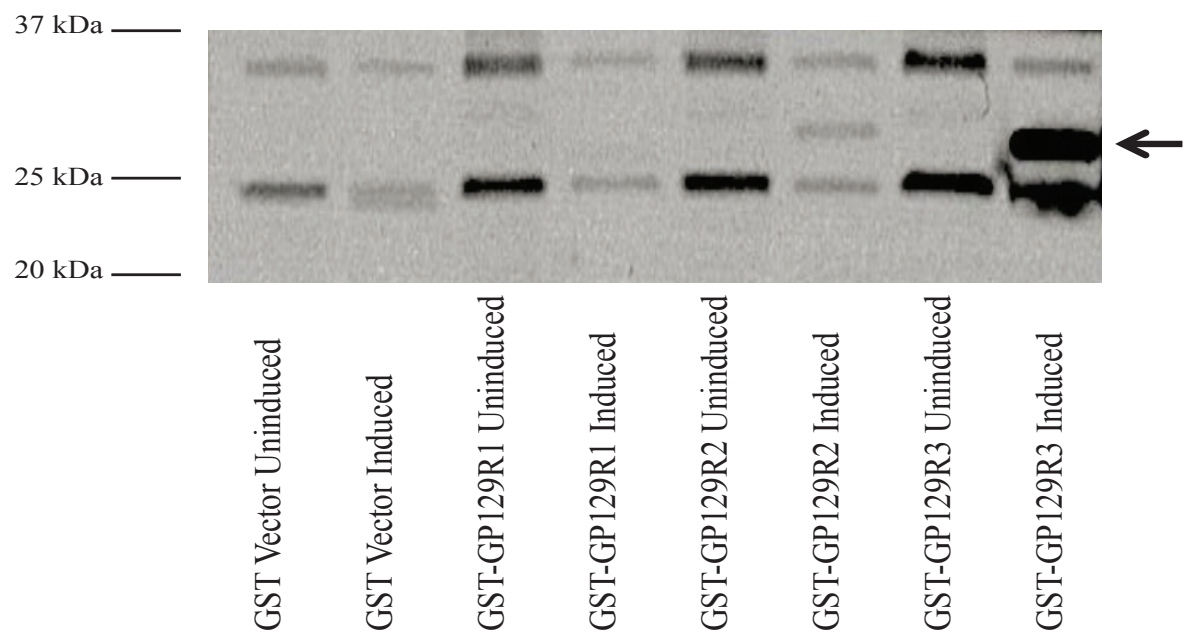

(a)
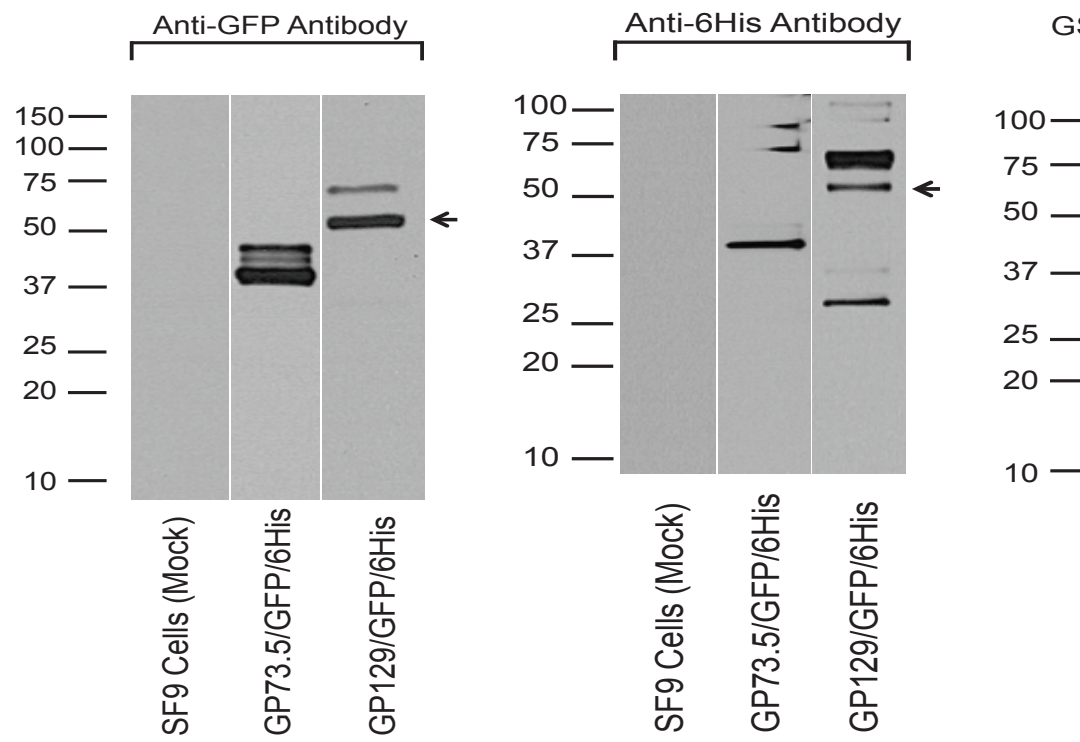

GST/GP129-R3 Polyclonal Antibody

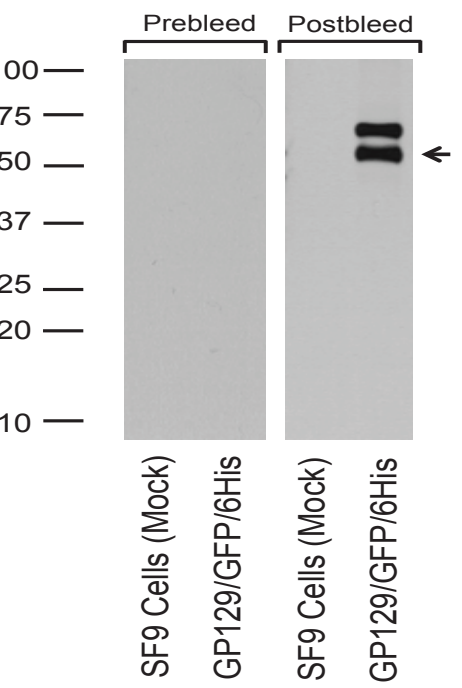

(b) 
Figure 6. Cont.

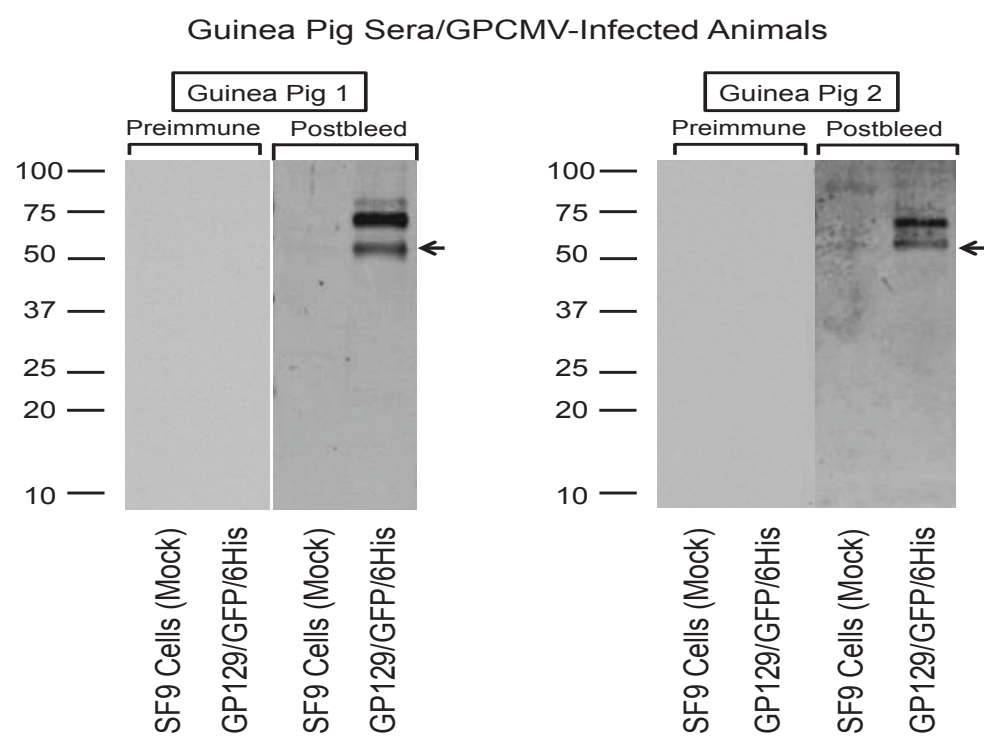

(c)

The guinea pig antibody response to GP131 was next evaluated, using recombinant GP131 in western blot assay along with sera from GPCMV-seropositive guinea pigs. As was the approach for GP129, the GP131 coding sequence was first subcloned as three GST fusion proteins, spanning potentially immunogenic domains of the GP131 protein (GP131 R1, R2 and R3). The GST fusion proteins were purified and, following western transfer, blots were probed with sera from experimentally infected GPCMV-seropositive animals (1:200 dilution; details as noted in Figure 6). A representative result from a GPCMV-infected animal is shown. These analyses demonstrated (Figure 7) that convalescent, but not pre-immune, sera from GPCMV-infected guinea pigs recognized the GST fusion protein corresponding to domains R1, R2 and R3 of the GP131 ORF.

Figure 7. Characterization of antibody responses of GPCMV-infected guinea pigs to recombinant GP131 proteins. Western blots examining reactivity of anti-GPCMV antisera with uninduced and IPTG-induced GST-GP131R1, R2, and R3 fusion proteins. GP133R1 and GP131R3 were most consistently reactive with post-infection antisera (1:200 dilution), but not preimmune sera (data not shown). Representative result from an infected animal is shown. 37 and $25 \mathrm{kDa}$ markers are indicated in right side of figure.
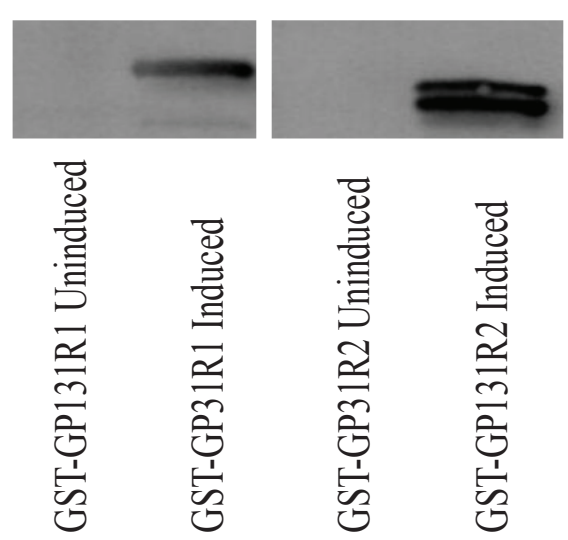

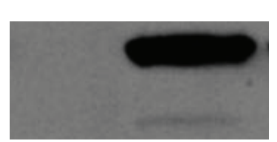

$-37 \mathrm{kDa}$

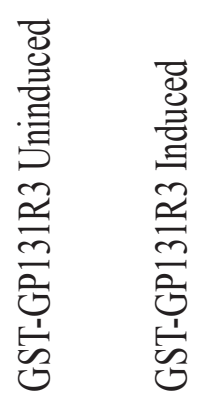


Finally, antibody responses to recombinant GP133 proteins were assessed. GP133 coding sequences were subcloned as depicted in Figure 5b. GST fusion constructs corresponding to Region 1 (predicted MW of $31.8 \mathrm{kDa}$ ) and Region 2 (predicted MW of $28.5 \mathrm{kDa}$ ) were evaluated, as well as a full-length GST fusion construct spanning both domains (R1/R2 construct; predicted MW of 34.8). These fusion protein clones were generated using primers as depicted in Table 2. The GST fusion proteins were purified from insoluble inclusion bodies and, following western transfer, blots were probed with convalescent sera from GPCMV seropositive animals that had been infected subcutaneously with SG virus. Results were confirmed with three independently GPCMV-infected guinea pigs; Figure 8 demonstrates representative results from one of these animals. These analyses demonstrated that convalescent, but not pre-immune (data not shown), sera from GPCMV-infected guinea pigs recognized the proteins corresponding to both the R1 and the R1/R2 regions of GP133 expressed as GST fusions, but not the R2 region of the GP133 protein. Reactivity of sera from infected animals, in contrast, was not noted with recombinant GP133 expressed as a GFP/6-His fusion protein in recombinant baculovirus (data not shown). This may have been due to loss of reactive epitopes engendered by fusion of the GFP and 6-His tags on the amino-terminal coding sequences of the recombinant GP133 protein. Further experiments will be necessary to resolve this question.

Figure 8. Characterization of antibody responses from GPCMV-immune guinea pigs to recombinant GP133 protein. (a) Antisera from a GPCMV-infected animal [GPCMV $(+)$ ] demonstrates immunoreactivity with the GST-GP133_R1_fusion protein (arrow) but not with purified GST protein. Sera is not reactive with GST-R2 fusion protein (right panel). Anti-GST moab (1:5,000 dilution) demonstrated reactivity both with the GST-GP133R1, $\mathrm{R} 2$, and R1/2 fusion proteins, as well as the purified GST protein. (b) Western analysis of GST-GP133R1/2 fusion protein. Both GST (GST vector) and GST-GP133R1/2 fusion protein are reactive with anti-GST moab; however, only sera from GPCMV-infected animal [GPCMV(+), 1:200 dilution] was reactive with the GST-R1/2 fusion protein. For both experiments, pre-immune sera from GPCMV-infected guinea pig was unreactive with all GP133/GST fusion proteins and with the GST parent protein itself (data not shown).
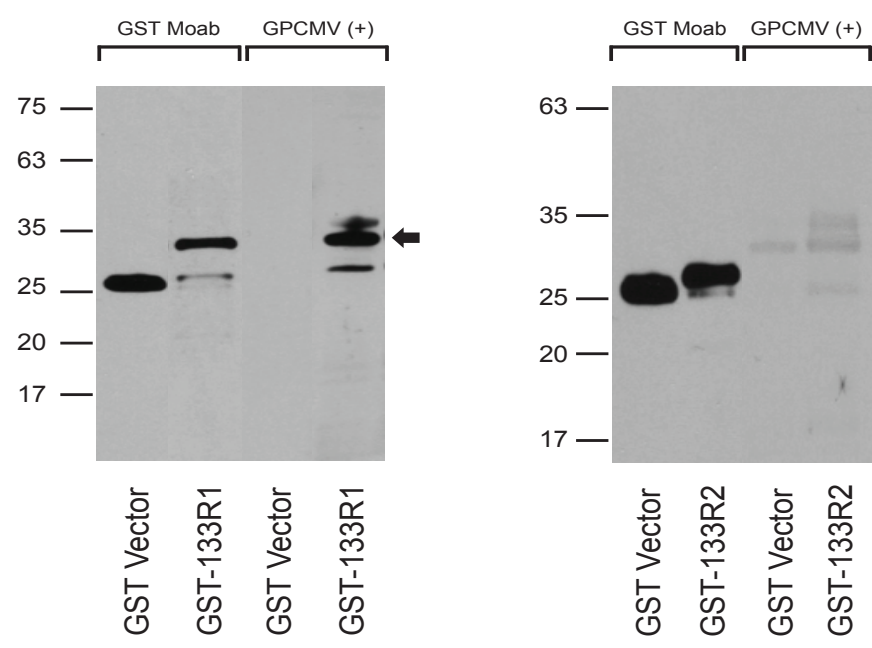

(a)

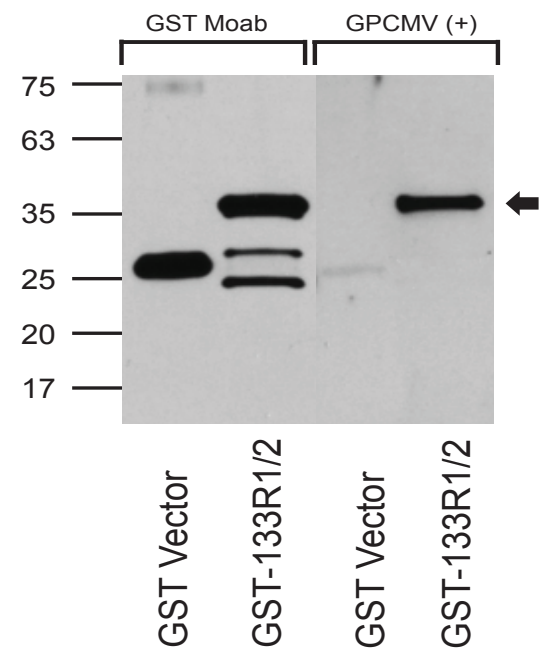

(b) 
Table 4 summarizes the immunoreactivity of the various GST fusion constructs (depicted in Figure 5b) with immune sera from infected guinea pigs post-infection with SG virus. Designations: $(-)$, no immunoreactivity; $(+)$, minimal immunoreactivity; $(++)$, moderate immunoreactivity; $(+++)$ consistent, high-level immunoreactivity.

Table 4. Summary of reactivity of sera from infected guinea pigs with GST-GP129, 131 and 133 fusion proteins.

\begin{tabular}{cc}
\hline $\begin{array}{c}\text { GST Fusion Protein } \\
\text { Domain }\end{array}$ & $\begin{array}{c}\text { Immunoreactivity with Guinea Pig Sera Post-Experimental } \\
\text { Infection with GPCMV }\end{array}$ \\
\hline GP129-R1 & - \\
GP129-R2 & + \\
GP129-R3 & +++ \\
GP131-R1 & ++ \\
GP131-R2 & + \\
GP131-R3 & +++ \\
GP133-R1 & ++ \\
GP133-R2 & - \\
\hline
\end{tabular}

\section{Experimental Section}

\subsection{Cells and Virus}

GPCMV (Strain 22122/VR682 from American Type Culture Collection [ATCC], Manassas, VA, USA), and vAM403, an enhanced green fluorescent protein (eGFP)-tagged GPCMV [19] were propagated on guinea pig fibroblast lung cells (GPL; ATCC CCL 158) in F-12 medium supplemented with 10\% fetal calf serum (FCS; Gibco-BRL), 10,000 IU of penicillin/liter, $10 \mathrm{mg}$ of streptomycin/liter, and 7.5\% NaHCO3 (Gibco-BRL).

\subsection{EM and Mass Spectrometry Analyses}

For mass spectrometry analyses, GPCMV (Strain 22122; ATCC VR-682) was propagated on GPL cells as described previously, and virus particles were purified from culture supernatants by density gradient centrifugation. Virus was purchased from ATCC and subjected to only one passage in GPL culture to minimize selection for genome variants with deletion in the GP129-133 locus [16]. Briefly, cellular debris was removed from the culture supernatant by repeated centrifugation at $4,000 \times \mathrm{g}$ for $15 \mathrm{~min}$ and supernatant was collected. GPCMV particles were separated by centrifugation at $11,000 \times \mathrm{g}$ for $30 \mathrm{~min}$ and pellet was washed twice in phosphate-buffered saline (PBS). Virion particles were resuspended in PBS and loaded onto a $20 \%-70 \%$ sucrose gradient $(27 \mathrm{~mL})$ in SW 32 centrifuge tubes and subjected to ultracentrifugation at $22,000 \mathrm{RPM} / 25^{\circ} \mathrm{C}$ for $60 \mathrm{~min}$. The visualized band was then subjected to glycerol-tartrate gradient centrifugation as described elsewhere [21]. Particles were washed and protein concentration was quantified by BCA assay (Thermo Fisher Scientific, Waltham, MA, USA). Particles were also subjected to electron microscopy. First, particles were centrifuged at 30 PSI using an airfuge (Belkman-Coulter, Brea, CA, USA) for 20 minutes on parafilm and formvar coated copper grids (Electron Microscopy Sciences, 
Hatfield, PA, USA). Excess liquid was wicked and the grids were stained with 1\% phosphotungstic acid for one minute. All sections were observed under JEOL 1200 EX II transmission electron microscope (JEOL LTD, Tokyo, Japan). Images were obtained using a Veleta $2 \mathrm{~K} \times 2 \mathrm{~K}$ camera with iTEM software (Olympus SIS, Munster, Germany) [25]. GPCMV particles were then resolved by SDS-PAGE and resolved protein bands were visualized by Coomassie stain. Visible bands were excised and subjected to in-gel trypsin digestion [26]. The digested peptide mixtures were analyzed using LTQ LC-MS/MS system or Velos Orbitrap LC-MS/MS system (ThermoFisher, San Jose, CA, USA) as previously described [27,28]. Tandem mass spectra were searched with Sequest version 2.7 (ThermoFisher, San Jose, CA, USA) and peptide and probability scores were applied in Scaffold 3.6.5 (Proteome Software, Inc., Portland, OR, USA) [29] as specified by Protein Prophet [30] and Peptide Prophet [31]. Mass spectra were searched against a combined protein FASTA database with sequence entries from NCBI Reference Sequence Cavia porcellus [32] NCBI nr Caviid herpesvirus 2 [33] and common lab contaminants from [34]. Manual inspection of all spectra validated the peptide assignments made by Scaffold.

\subsection{Transcriptional Analyses}

Guinea pig lung fibroblasts (GPL) were infected with ATCC GPCMV P1 virus at MOI of 0.1. After one hour of infection at $37{ }^{\circ} \mathrm{C}$, cells were washed with PBS and cultured in F12 medium supplemented with $10 \%$ fetal calf serum, 10,000 IU/L penicillin, $10 \mathrm{mg} / \mathrm{L}$ streptomycin, and 7.5\% NaHCO3. RNA was extracted from infected GPL cells at 8, 12, 24 and 48 hours post-infection using RNeasy mini kit (Qiagen, Venlo, Limburg, NL) according to the manufacturer's instructions. RNA was treated with RNase-free DNase Set (Qiagen) while in the column according to manufacturer's instructions. cDNA was synthesized from $1 \mu \mathrm{g}$ of total RNA using either Quantitect Reverse Transcription kit (Qiagen). Conventional PCR was carried out using cDNA as template and goTaq (Promega, Madison, WI, USA). The PCR for GAPDH were done using primer pair 5'-ATCTCATCGTATTTGGCCGGT-3' and 5'-AATGGGAAGCTCACAGGTATGG-3'. The conditions for GAPDH PCR were: initial denaturation at $95^{\circ} \mathrm{C}$ for $2 \mathrm{~min}$, followed by $95{ }^{\circ} \mathrm{C}$ for 30 s, $58{ }^{\circ} \mathrm{C}$ for $30 \mathrm{~s}, 72{ }^{\circ} \mathrm{C}$ for $35 \mathrm{~s}$ for a total of 25 cycles, and elongation at $72{ }^{\circ} \mathrm{C}$ for $7 \mathrm{~min}$. GP129 PCR primers are listed in Table 2. The conditions of the GP129 PCR were: initial denaturation at $95{ }^{\circ} \mathrm{C}$ for $2 \mathrm{~min}$, followed by $95{ }^{\circ} \mathrm{C}$ for $30 \mathrm{~s}, 56{ }^{\circ} \mathrm{C}$ for $30 \mathrm{~s}, 72{ }^{\circ} \mathrm{C}$ for $35 \mathrm{~s}$ for a total of 35 cycles, and elongation at $72{ }^{\circ} \mathrm{C}$ for $7 \mathrm{~min}$. The primers for GP131 PCR were 5'-ATAATGATGAAACGATAT-3' and 5'-TTATCACGTCCAGTTCCA-3'; the conditions were the same as GP131 but the annealing temperature was $48^{\circ} \mathrm{C}$. GP133 PCR was done using primer pair 5'-TATGTTTTGGCGTCTTGTA-3' and 5'-TTATGCTCTGTCTATGC-3', and the same conditions as GAPDH. Amplicons from the PCR were resolved by agarose gel electrophoresis and visualized by ethidium bromide staining.

\subsection{Recombinant Baculovirus Expression}

To analyze the protein products encoded by GP133, GP131 and GP129 regions of the GPCMV genome, proteins were expressed in recombinant baculovirus. Baculovirus shuttle plasmids were generated. Briefly, ORF coding sequences GP129, GP131 and GP133 were amplified from cDNA 
by PCR using the primers listed in Table 2. Amplicons were resolved by agarose gel electrophoresis and visualized by ethidium bromide staining. The purified amplicons were ligated into digested pAc-HLT-A-GFP vector (BD Biosciences, San Jose, CA, USA) at the C-terminus of the GFP-His tag following conventional cloning methods. The resultant clones encoding GP133, GP131 and GP129 were designated pKTS 824, pKTS 791 and pKTS 814, respectively. Recombinant baculoviruses were generated by co-transfecting Sf9 cells with aforementioned plasmids and BaculoGold ${ }^{\circledR}$ DNA (BD Biosciences) according to manufacturer's instructions.

\subsection{Recombinant Antigenic GP129, GP131 and GP133 Fragment Expression}

Antigenic domains for GP129, 131 and 133 were predicted using MacVector software (version 12.0) [35] by calculating antigenic index based on the results of Kyte-Doolittle hydrophilicity, surface probability, protein flexibility, and Chou-Fasman and Robson-Garnier secondary structure analyses. To screen for potential antigenic regions of GP129 that are recognized by the GPCMV guinea pig sera, three predicted antigenic fragments $\mathrm{R} 1\left(\mathrm{TYR}_{12}\right.$ through $\left.\mathrm{THR} \mathrm{R}_{50}\right), \mathrm{R} 2\left(\mathrm{SER}_{79}\right.$ through $\left.\mathrm{ARG}_{129}\right)$ and $\mathrm{R} 3\left(\mathrm{ASN}_{146}\right.$ through $\left.\mathrm{LYS}_{179}\right)$ were expressed as GST fusion proteins. Briefly, R1, R2 and R3 coding sequences of GP129 were amplified from cDNA by PCR using the primers listed in Table 2. The purified amplicons were ligated into linearized pGEX-6P vector (GE Healthcare Biosciences, Pittsburgh, PA, USA) at the C-terminus of the GST following conventional cloning methods. The resultant clones encoding R1-GP129, R2-GP129, R3-GP129 were designated pKTS 828, 829 and 830, respectively. For GP131, the clones were designated as pKTS 818, 819, and 820 (domains R1, R2, and R3, respectively). For GP133, clones encoding R1-GP133 and R2-GP133 were designated as pKTS 831 and pKTS 832 respectively.

The aforementioned plasmids corresponded to these fusion proteins were sequenced, and then were transformed into MAX EFFICIENCY ${ }^{\circledR}$ DH5 $\alpha$ chemically competent cells (Invitrogen, Carlsbad, CA, USA) and screened on LB plates containing ampicillin $(100 \mu \mathrm{g} / \mathrm{mL})$. DH5 $\alpha$ cells harboring the above plasmids were selected to express GST fusion proteins under IPTG induction $(0.5 \mathrm{mM})$. GST-fusion proteins were extracted and purified using Glutathione agarose beads (Pierce, Rockford, IL, USA) according to the manufacturer's instructions.

\subsection{Western Blot}

Recombinant GP129, GP131 and GP133 proteins were characterized by western blot. Briefly, $80 \%$ confluent monolayer of Sf9 cells were infected with recombinant baculovirus and after 120 hours of infection, the cells were harvested and washed twice with PBS. Cellular proteins were extracted and recombinant GP129, GP131 and GP133 fusions proteins were purified using Ni-NTA agarose beads according to the manufacturer's instruction (Qiagen). Either purified recombinant GP129, GP131 or GP133-expressing Sf9 cell lysates were resolved by SDS PAGE (Novex-10\%-20\% Tris-HCl, Life Technologies, Carlsbad, CA, USA) and electro blotted to PVDF membrane. Blots were blocked with 5\% skim milk solution in PBS containing $0.1 \%$ Tween-20 for 2 hours. Blots were probed with anti-GFP monoclonal (1:3,000 dilution), anti-6-His monoclonal (1:2,000 dilution), anti-GST monoclonal (1:5,000 dilution), preimmune and post-infection sera from guinea pigs (1:200 dilution for most experiments as indicated in individual figure legends), pre-immune 
rabbit sera (both at 1:2,000 dilution), anti R3-GP129 guinea pig sera (1:2,000 dilution) or rabbit affinity-purified, GP131 anti-peptide antibody (concentration $1 \mu \mathrm{g} / \mathrm{mL}$ ) for one hour, followed by incubation with either horseradish peroxidase (HRP)-conjugated anti-guinea pig (1:10,000 dilution, Sigma, St. Louis, MO, USA), HRP-conjugated anti-rabbit (1:2,000 dilution; Cell Signaling Technology, Danvers, MA, USA) or HRP-conjugated anti mouse (1:10,000; GE Healthcare Biosciences) secondary antibody. Anti-GPCMV gB moab IE321 was used at a 1:2,000 dilution. Binding of antibody was detected by adding enhanced chemiluminescent substrate (GE Healthcare Biosciences) for HRP and bands were visualized by exposing the blot to an X-ray film.

\subsection{Generation of Antibodies}

GP131-specific anti-peptide antibodies were raised in rabbits (US Biological, Salem, MA, USA). A synthetic peptide, $\mathrm{NH}_{2}$-CYYPSTPIPKSFVKHVDTTRSLPE-COOH, was generated and conjugated with keyhole limpet hemocyanin (KLH). Rabbits were immunized with $200 \mu \mathrm{g}$ of the conjugate and complete Freunds adjuvant (CFA), followed by three subsequent doses of the conjugate $(100 \mu \mathrm{g})$ and incomplete Freunds adjuvant (IFA) at 21, 42 and 72 days following initial immunization. Serum was collected seven days after final immunization. GP131-specific anti-peptide antibodies were purified from the aforementioned rabbit serum by affinity column chromatography using cyanogen bromide-activated sepharose 4B gel coupled to $\mathrm{NH}_{2}$-CYYPSTPIPKSFVKHVDTTRSLPE-COOH.

R3-GP129 antibodies were raised in Strain 2 guinea pigs (University of Minnesota RAR facility, Minneapolis, MN, USA). GPCMV-seronegative guinea pigs were immunized with $50 \mu \mathrm{g}$ of the purified GST_R3_GP129 and incomplete Freunds adjuvant (IFA), followed by three subsequent doses of the GST_R3_GP129 (30 $\mu \mathrm{g})$ and incomplete Freunds adjuvant (IFA) at 21, 42 and 72 days following initial immunization. Sera were collected seven days after final immunization.

GPCMV sera were generated by infecting seronegative Hartley strain guinea pigs (Elm Hill laboratories, Chelmsford, MA, USA) with salivary gland-adapted GPCMV. Infection was by subcutaneous route with a dose of $5 \times 10^{5} \mathrm{pfu}$. Animals were monitored for seroconversion by ELISA assay and western blot, and preimmune and immune (post-infection) sera were used to probe GP129, 131 and 133 recombinant proteins at dilutions of 1:200 as described in individual figure legends.

\section{Conclusions}

These results confirm and extend the observations of Feierbach by identifying the GP129, 131 and 133 proteins in virus particles purified from GPCMV-infected cells [18]. There are potential limitations to our analyses. We performed these studies using strain 22122 of GPCMV from the ATCC. This strain is known to harbor two genomic variants of GPCMV, with the second variant containing a $1.6 \mathrm{~kb}$ genome deletion that lacks the GP129-133 locus. It is possible that our studies underestimated the presence of GP129-133 proteins by selecting for the deletion variant in cell culture in fibroblasts [16]. However, we used virus passaged only one time in GPL cells, and by PCR analysis we were able to confirm that the majority of the genome variants in cell culture were full-length, compatible with our finding of the GP129-133 proteins by mass spectrometry. Another 
potential limitation of these studies was the lack of full characterization of whether there were dense bodies co-purifying with virions in our purified virus. A more detailed characterization of GPCMV virions and dense bodies will be required in future studies to elucidate what differences exist in the proteome of these respective particles.

Using anti-peptide antisera (GP131) and an anti-GST fusion protein antibody (GP129), we were able to identify both the GP131 and GP129 proteins in purified virus particles by western blot. The GP131 was found to migrate as a doublet at $\sim 20$ and $22 \mathrm{kDa}$. The virion-associated GP129 migrated at $\sim 25 \mathrm{kDa}$, compatible with its predicted potential to undergo more extensive glycosylation (Figure $5 b$ ). The GP129 protein also appeared to migrate as a double (at $\sim 55$ and $\sim 65 \mathrm{kDa}$ ) in Sf9 cells infected with a recombinant baculovirus expressing GP129 as a GFP/6-His fusion, also compatible with differential glycosylated isoforms of the protein in insect cells. Experiments with glycosylation inhibitors will be required to examine this question in further detail. We were unable to identify GP133 in virions using an anti-GST antibody raised against a GP133-GST fusion protein (data not shown). However, the finding of GP133 by mass spectrometry analysis of viral particles provides assurance that this protein is expressed in the mature virion.

Using GST fusions and, for GP129, a recombinant baculovirus-expressed protein, we were able to identify antibody responses in serum from guinea pigs experimentally infected with GPCMV. We were unable to identify immunoreactivity of sera from GPCMV-infected animals with recombinant insect cell-expressed forms of GP131 and 133. Both the GP131 and GP133 baculovirus-expressed proteins were reactive with anti-6-His and anti-GFP antibodies at the predicted MWs of $\sim 56$ and $\sim 49 \mathrm{kDa}$, respectively (data not shown), indicating that the recombinant baculovirus fusion protein was being expressed in insect cells. It may be that fusion of GFP and/or 6-His at the amino-terminus of these fusion proteins rendered relevant epitopes inaccessible. Our study was also limited by examination of a relatively small number of sera from infected animals.

In summary, GPCMV encodes homologs of HCMV PC complex proteins, GP129, 131, and 133, and these proteins are expressed and can be identified in virions by mass spectrometry. Furthermore, using recombinant GST and baculovirus expressed proteins, we observe that the GP129/131/133 constituents of the GPCMV PC appear to elicit an antibody response in the context of viral infection in animals. These observations reinforce interest in studying the GPCMV PC as a potential target of protective subunit vaccination in the GPCMV model of congenital infection. The precise protein-protein interactions in this complex remain to be elucidated. In a model of the protein-protein interactions that make up the gH/gL/UL128/UL130/UL131 complex, the HCMV $\mathrm{gH}$ protein is predicted to be inserted in the viral envelope, and interacts with gL through disulfide bond formation. Johnson et al. characterized the HCMV PC using a system of HCMV mutants lacking UL128-131, differentially reconstituted with non-replicating adenovirus vectors expressing $\mathrm{gH}, \mathrm{gL}, \mathrm{UL} 128$, UL130, and UL131 [4]. The assembly of a pentameric structure was further suggested by the observation of increased export of $\mathrm{gH} / \mathrm{gL}$ complexes from the endoplasmic reticulum (ER) to the Golgi apparatus and cell surface when UL128, UL130, and UL131 were expressed with $\mathrm{gH} / \mathrm{gL}$ [4]. Direct covalent disulfide bonds between HCMV gH/gL and UL130/UL131 were suggested by differential immunoprecipitation of heterodimers in reducing and non-reducing environments; direct non-covalent interactions between gH/UL130, gL/UL128, and UL128/UL130 were also observed. These complex interrelationships remain to be elucidated for 
the GPCMV PC. Evaluation of GPCMV neutralization following immunization with GPCMV pentameric complex constituents should help clarify which of these proteins are most important in elucidation of a protective immune response, and subunit vaccine studies in guinea pigs should help provide a useful proof-of-concept for the potential usefulness of a PC-based HCMV vaccine.

\section{Acknowledgments}

Support from NIH HD038416 and HD044864 is acknowledged.

\section{Author Contributions}

The experiments were designed by M.R.S. with assistance from J.S.G., P.A.G., N.H.-A., S.L. and H.S., J.S.G., P.A.G., N.H.-A., and H.S. conducted cloning experiments, analysis of gene expression at RNA and protein level, and immune assays. L.H. and T.W.M. performed mass spectrometry experiments. J.S.G. wrote the first draft of the manuscript assisted by M.R.S. All authors participated in data analysis and contributed to the writing of the manuscript.

\section{Conflict of Interest}

The authors declare no conflict of interest relevant to this work.

\section{References and Notes}

1. Sung, H.; Schleiss, M.R. Update on the current status of cytomegalovirus vaccines. Expert Rev. Vaccines 2010, 9, 1303-1314.

2. Nigro, G.; Adler, S.P.; La Torre, R.; Best, A.M. Passive immunization during pregnancy for congenital cytomegalovirus infection. N. Engl. J. Med. 2005, 353, 1350-1362.

3. Ryckman, B.J.; Jarvis, M.A.; Drummond, D.D.; Nelson, J.A.; Johnson, D.C. Human cytomegalovirus entry into epithelial and endothelial cells depends on genes ul128 to ul150 and occurs by endocytosis and low-ph fusion. J. Virol. 2006, 80, 710-722.

4. Ryckman, B.J.; Rainish, B.L.; Chase, M.C.; Borton, J.A.; Nelson, J.A.; Jarvis, M.A.; Johnson, D.C. Characterization of the human cytomegalovirus $\mathrm{gh} / \mathrm{gl} / \mathrm{ul} 128-131$ complex that mediates entry into epithelial and endothelial cells. J. Virol. 2008, 82, 60-70.

5. Revello, M.G.; Gerna, G. Human cytomegalovirus tropism for endothelial/epithelial cells: Scientific background and clinical implications. Rev. Med. Virol. 2010, 20, 136-155.

6. Fouts, A.E.; Chan, P.; Stephan, J.P.; Vandlen, R.; Feierbach, B. Antibodies against the $\mathrm{gh} / \mathrm{gl} / \mathrm{ul128} / \mathrm{ul130/ \textrm {ul131 }}$ complex comprise the majority of the anti-cytomegalovirus (anti-cmv) neutralizing antibody response in cmv hyperimmune globulin. J. Virol. 2012, 86, 7444-7447.

7. Lilleri, D.; Kabanova, A.; Revello, M.G.; Percivalle, E.; Sarasini, A.; Genini, E.; Sallusto, F.; Lanzavecchia, A.; Corti, D.; Gerna, G. Fetal human cytomegalovirus transmission correlates with delayed maternal antibodies to $\mathrm{gh} / \mathrm{gl} / \mathrm{pul128-130-131} \mathrm{complex} \mathrm{during} \mathrm{primary} \mathrm{infection.}$ PLoS One 2013, 8, e59863. 
8. Fu, T.M.; Wang, D.; Freed, D.C.; Tang, A.; Li, F.; He, X.; Cole, S.; Dubey, S.; Finnefrock, A.C.; ter Meulen, J.; et al. Restoration of viral epithelial tropism improves immunogenicity in rabbits and rhesus macaques for a whole virion vaccine of human cytomegalovirus. Vaccine 2012, 30, 7469-7474.

9. Pass, R.F.; Zhang, C.; Evans, A.; Simpson, T.; Andrews, W.; Huang, M.L.; Corey, L.; Hill, J.; Davis, E.; Flanigan, C.; et al. Vaccine prevention of maternal cytomegalovirus infection. N. Engl. J. Med. 2009, 360, 1191-1199.

10. Griffiths, P.D.; Stanton, A.; McCarrell, E.; Smith, C.; Osman, M.; Harber, M.; Davenport, A.; Jones, G.; Wheeler, D.C.; O'Beirne, J.; et al. Cytomegalovirus glycoprotein-b vaccine with mf59 adjuvant in transplant recipients: A phase 2 randomised placebo-controlled trial. Lancet 2011, 377, 1256-1263.

11. Kern, E.R. Pivotal role of animal models in the development of new therapies for cytomegalovirus infections. Antivir. Res. 2006, 71, 164-171.

12. Schleiss, M.R. Nonprimate models of congenital cytomegalovirus (cmv) infection: Gaining insight into pathogenesis and prevention of disease in newborns. ILAR J. 2006, 47, 65-72.

13. Yue, Y.; Wang, Z.; Abel, K.; Li, J.; Strelow, L.; Mandarino, A.; Eberhardt, M.K.; Schmidt, K.A.; Diamond, D.J.; Barry, P.A. Evaluation of recombinant modified vaccinia ankara virus-based rhesus cytomegalovirus vaccines in rhesus macaques. Med. Microbiol. Immunol. 2008, 197, 117-123.

14. Wussow, F.; Yue, Y.; Martinez, J.; Deere, J.D.; Longmate, J.; Herrmann, A.; Barry, P.A.; Diamond, D.J. A vaccine based on the rhesus cytomegalovirus ul128 complex induces broadly neutralizing antibodies in rhesus macaques. J. Virol. 2013, 87, 1322-1332.

15. Bia, F.J.; Miller, S.A.; Davidson, K.H. The guinea pig cytomegalovirus model of congenital human cytomegalovirus infection. Birth Defects Orig. Artic. Ser. 1984, 20, 233-241.

16. Nozawa, N.; Yamamoto, Y.; Fukui, Y.; Katano, H.; Tsutsui, Y.; Sato, Y.; Yamada, S.; Inami, Y.; Nakamura, K.; Yokoi, M.; et al. Identification of a $1.6 \mathrm{~kb}$ genome locus of guinea pig cytomegalovirus required for efficient viral growth in animals but not in cell culture. Virology 2008, 379, 45-54.

17. Yamada, S.; Nozawa, N.; Katano, H.; Fukui, Y.; Tsuda, M.; Tsutsui, Y.; Kurane, I.; Inoue, N. Characterization of the guinea pig cytomegalovirus genome locus that encodes homologs of human cytomegalovirus major immediate-early genes, ul128, and ul130. Virology 2009, 391, 99-106.

18. Auerbach, M.; Yan, D.; Fouts, A.; Xu, M.; Estevez, A.; Austin, C.D.; Bazan, F.; Feierbach, B. Characterization of the guinea pig $\mathrm{cmv} \mathrm{gh} / \mathrm{gl} / \mathrm{gp} 129 / \mathrm{gp} 131 / \mathrm{gp} 133$ complex in infection and spread. Virology 2013, 441, 75-84.

19. McGregor, A.; Schleiss, M.R. Molecular cloning of the guinea pig cytomegalovirus (gpcmv) genome as an infectious bacterial artificial chromosome (bac) in Escherichia coli. Mol. Genet. Metab. 2001, 72, 15-26.

20. Paglino, J.C.; Brady, R.C.; Schleiss, M.R. Molecular characterization of the guinea-pig cytomegalovirus glycoprotein 1 gene. Arch. Virol. 1999, 144, 447-462.

21. Talbot, P.; Almeida, J.D. Human cytomegalovirus: Purification of enveloped virions and dense bodies. J. Gen. Virol. 1977, 36, 345-349. 
22. Macagno, A.; Bernasconi, N.L.; Vanzetta, F.; Dander, E.; Sarasini, A.; Revello, M.G.; Gerna, G.; Sallusto, F.; Lanzavecchia, A. Isolation of human monoclonal antibodies that potently neutralize human cytomegalovirus infection by targeting different epitopes on the gh/g1/ul128-131a complex. J. Virol. 2010, 84, 1005-1013.

23. Lilleri, D.; Kabanova, A.; Lanzavecchia, A.; Gerna, G. Antibodies against neutralization epitopes of human cytomegalovirus gh/gl/pul128-130-131 complex and virus spreading may correlate with virus control in vivo. J. Clin. Immunol. 2012, 32, 1324-1331.

24. Yang, D.; Tamburro, K.; Dittmer, D.; Cui, X.; McVoy, M.A.; Hernandez-Alvarado, N.; Schleiss, M.R. Complete genome sequence of pathogenic guinea pig cytomegalovirus from salivary gland homogenates of infected animals. Genome Announc. 2013, 1, e0005413.

25. iTEM, Version 9.0; Olympus Soft Imaging Solutions GmbH: Münster, Germany, 2014.

26. Shevchenko, A.; Wilm, M.; Vorm, O.; Mann, M. Mass spectrometric sequencing of proteins silver-stained polyacrylamide gels. Anal. Chem. 1996, 68, 850-858.

27. Lin-Moshier, Y.; Sebastian, P.J.; Higgins, L.; Sampson, N.D.; Hewitt, J.E.; Marchant, J.S. Re-evaluation of the role of calcium homeostasis endoplasmic reticulum protein (cherp) in cellular calcium signaling. J. Biol. Chem. 2013, 288, 355-367.

28. Fallon, A.M.; Witthuhn, B.A. Proteasome activity in a naive mosquito cell line infected with wolbachia pipientis walbb. In Vitro Cell Dev. Biol. Anim. 2009, 45, 460-466.

29. Scaffold, Version 3.6.5; Proteome Software, Inc.: Portland, OR, USA, 2014.

30. Nesvizhskii, A.I.; Keller, A.; Kolker, E.; Aebersold, R. A statistical model for identifying proteins by tandem mass spectrometry. Anal. Chem. 2003, 75, 4646-4658.

31. Keller, A.; Nesvizhskii, A.I.; Kolker, E.; Aebersold, R. Empirical statistical model to estimate the accuracy of peptide identifications made by $\mathrm{ms} / \mathrm{ms}$ and database search. Anal. Chem. 2002, 74, 5383-5392.

32. National Center for Biotechnology Information (NCBI) Taxonomy Browser Site. Available online: http:/www.ncbi.nlm.nih.gov/Taxonomy/Browser/wwwtax.cgi?id=10141/ (accessed on 15 January 2014).

33. NCBI Cavia Porcellus (Domestic Guinea Pig) Genome Project Report. Available online: http://www.ncbi.nlm.nih.gov/genome/?term=cavia/ (accessed on 15 January 2014).

34. Global Proteome Machine Web Site: Common Repository of Adventitious Proteins Page. Available online: http://www.thegpm.org/crap/_(accessed on 15 January 2014).

35. MacVector, Version 12.0; MacVector software: Cary, NC, USA, 2014. 


\section{Appendix}

Figure A1. Mass spectrometry data for GP129. Representative mass spectra for each of the unique peptides identified using Velos Orbitrap LC-MS/MS system for GP129. $\mathrm{B}$ peaks are labeled in red. Y peaks are labeled in blue. Additional charge variants are marked in green. (a) Spectrum for MPSVQSKPEKPSILGVTYR. (b) Spectrum for VDYTVMIPTPHFPR.

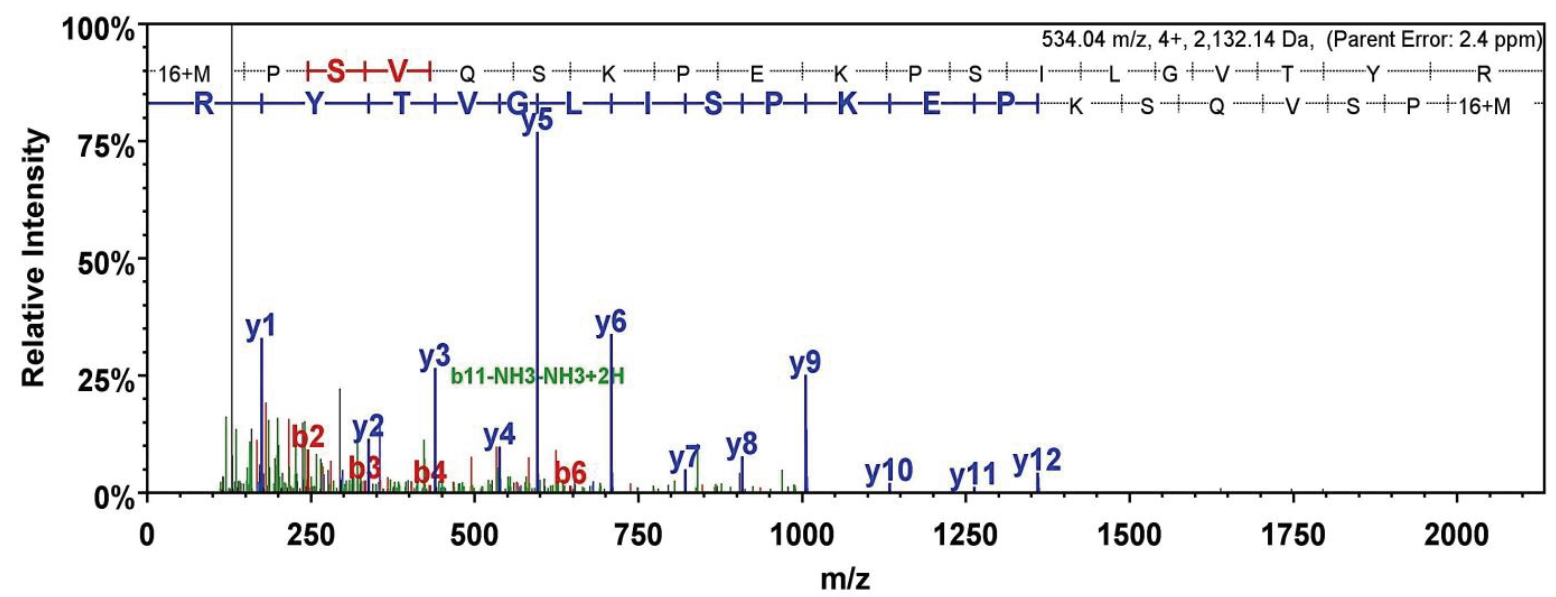

(a)

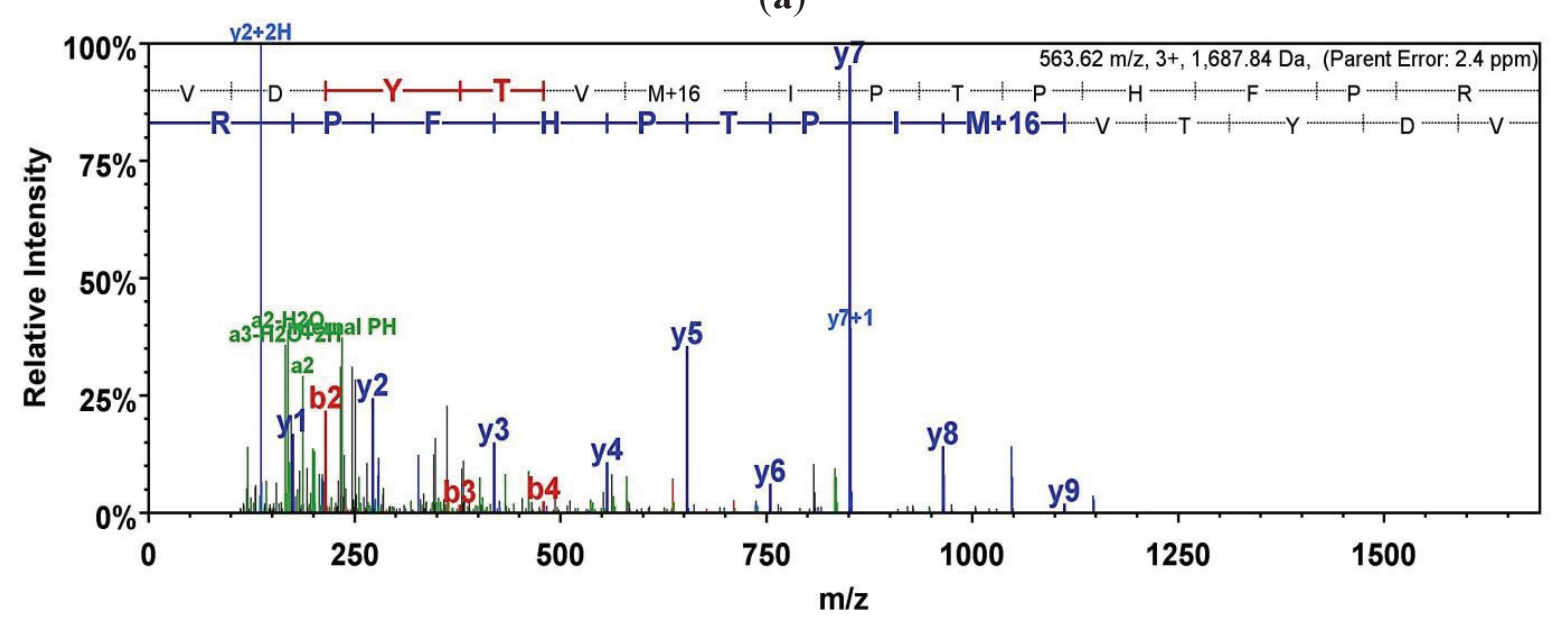

(b) 
Figure A2. Mass spectrometry data for GP131. Representative mass spectra for each of the unique peptides identified using Velos Orbitrap LC-MS/MS system for GP131. B peaks are labeled in red. Y peaks are labeled in blue. Additional charge variants are marked in green. (a) Spectrum for RIDYGSTGTAASTLPSLTSLR. (b) Spectrum for TYFGDRDSSFLWHYTIR. (c) Spectrum for TLDCDVYVTSR. (d) Spectrum for HPADSIACLL.

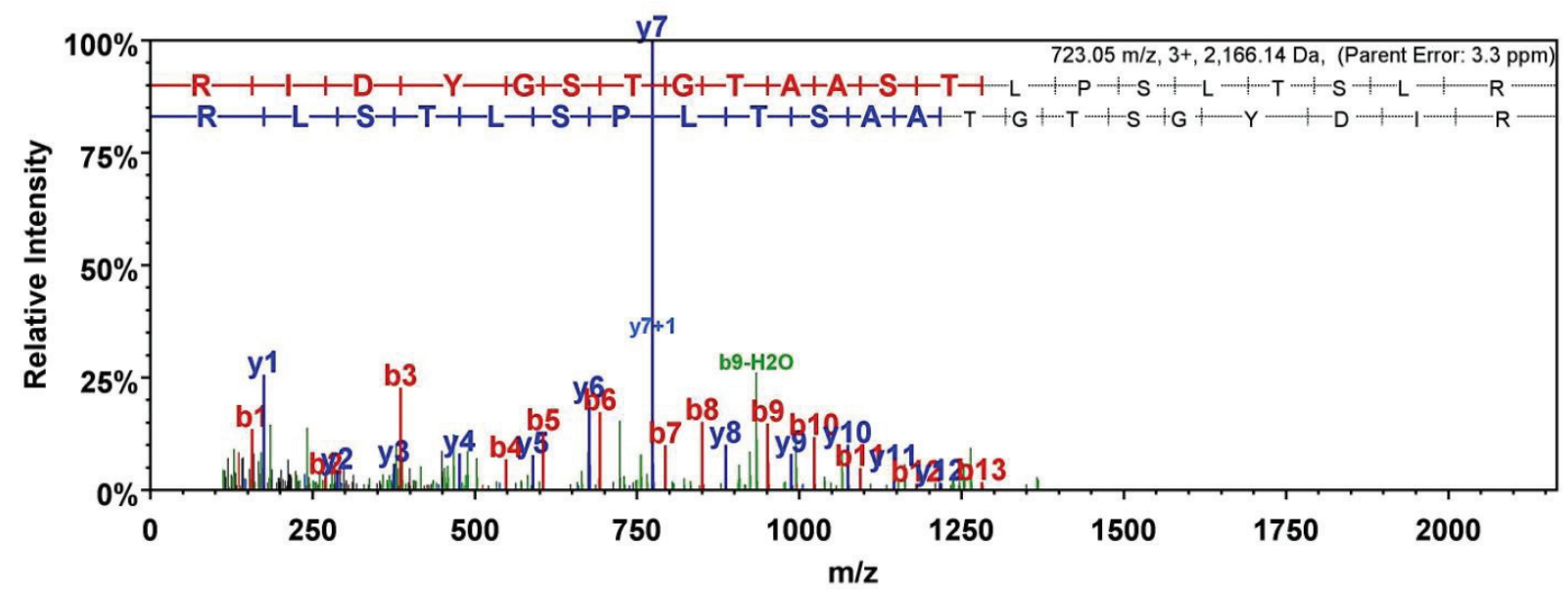

(a)

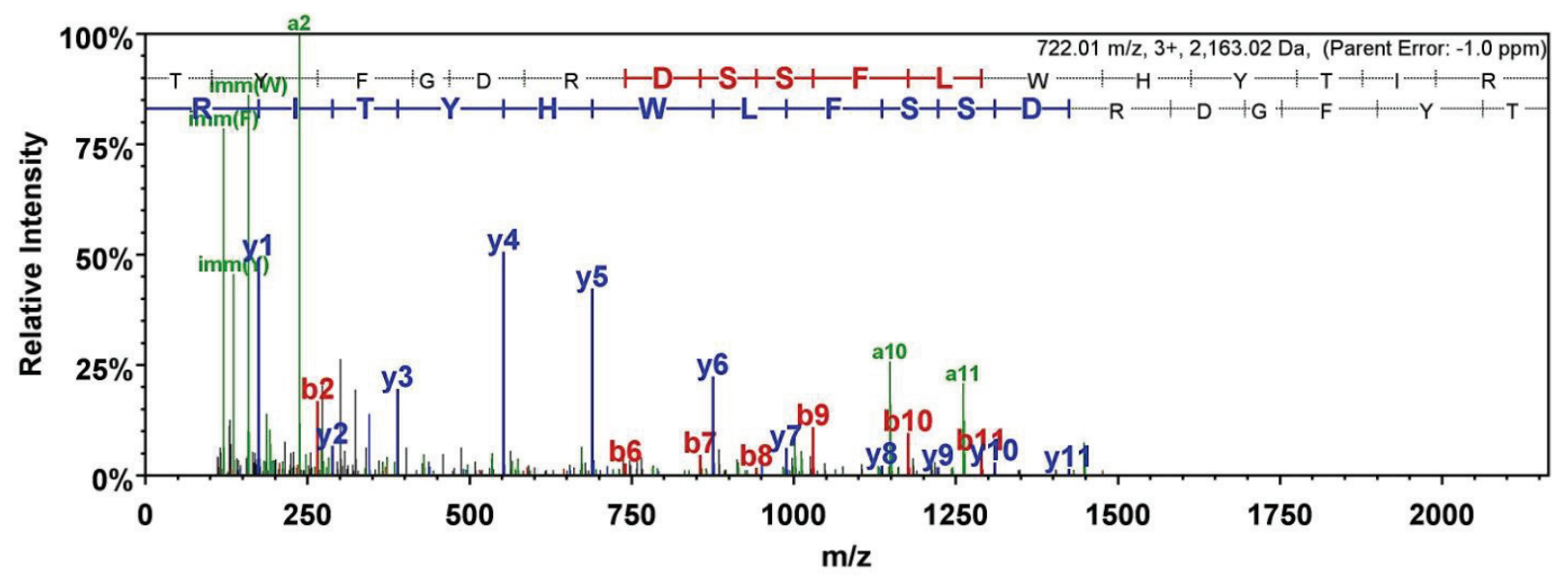

(b)

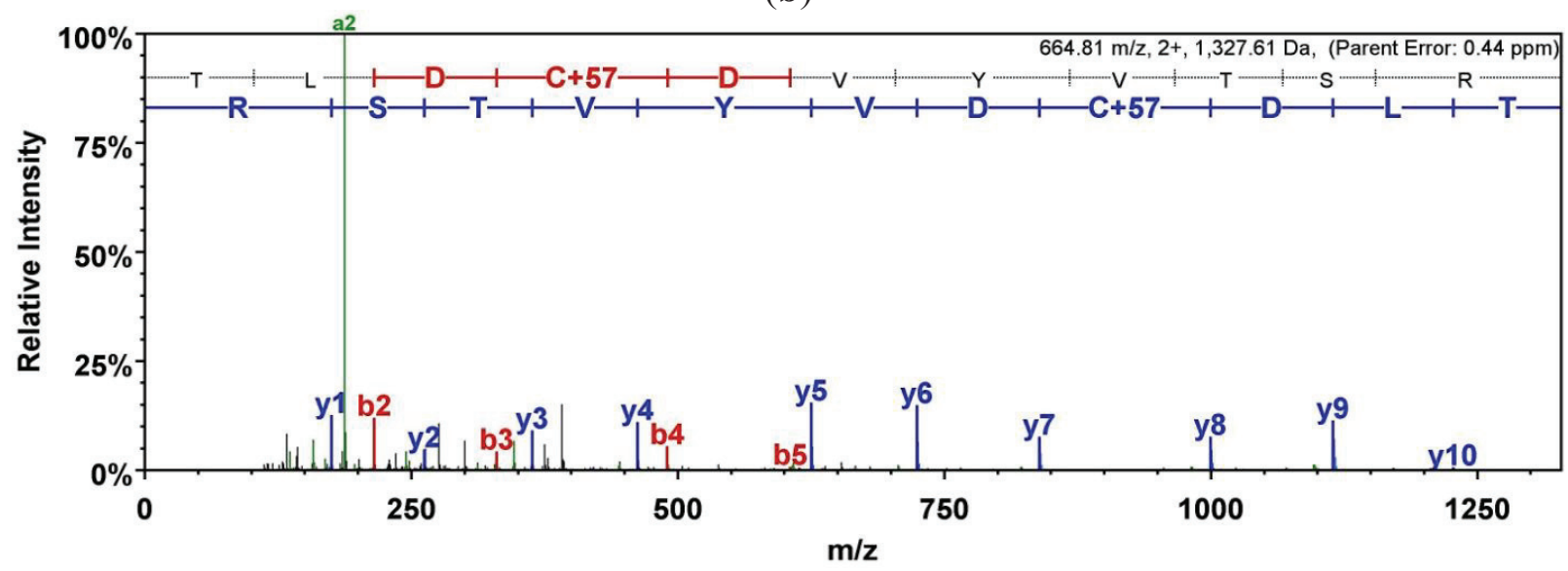

(c) 
Figure A2. Cont.

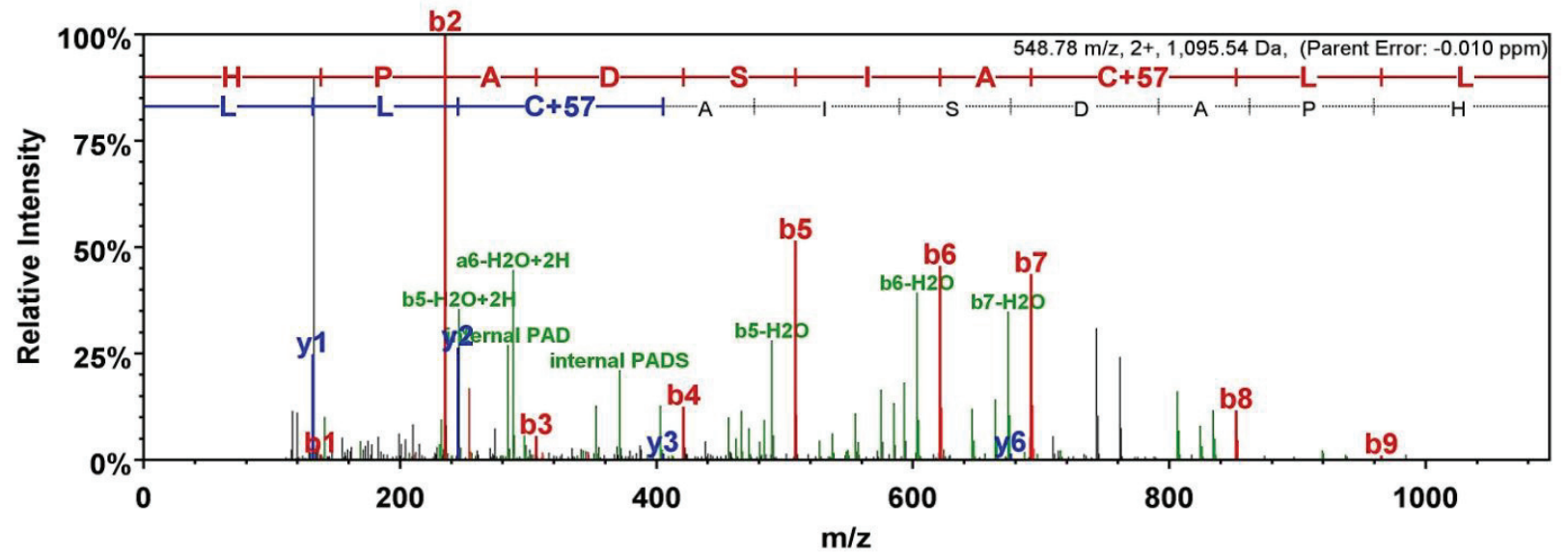

(d)

Figure A3. Mass spectrometry data for GP133. Representative mass spectra for each of the unique peptides identified using Velos Orbitrap LC-MS/MS system for GP133. B peaks are labeled in red. Y peaks are labeled in blue. Additional charge variants are marked in green. (a) Spectrum for EDEGIDTWWLGGVTDNTR. (b) Spectrum for KENQLAHYILK. (c) Spectrum for TIVLTHHR. (d) Spectrum for KGLVSIDGIR.

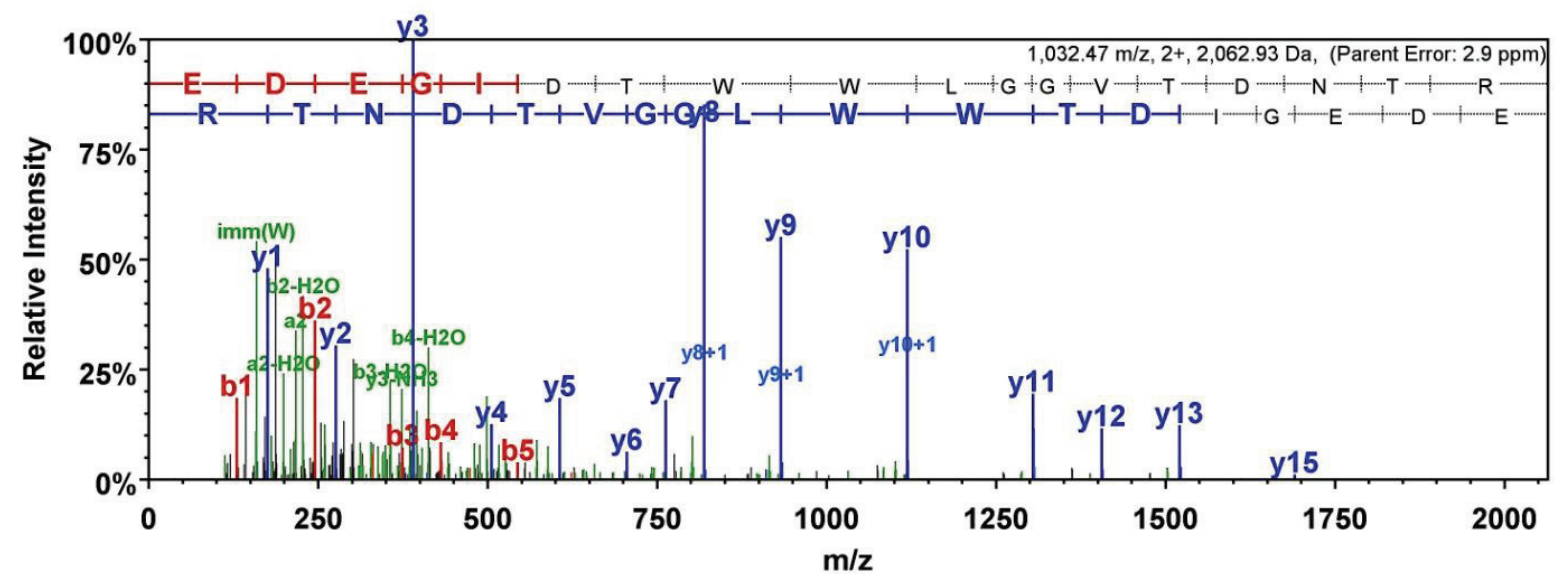

(a)

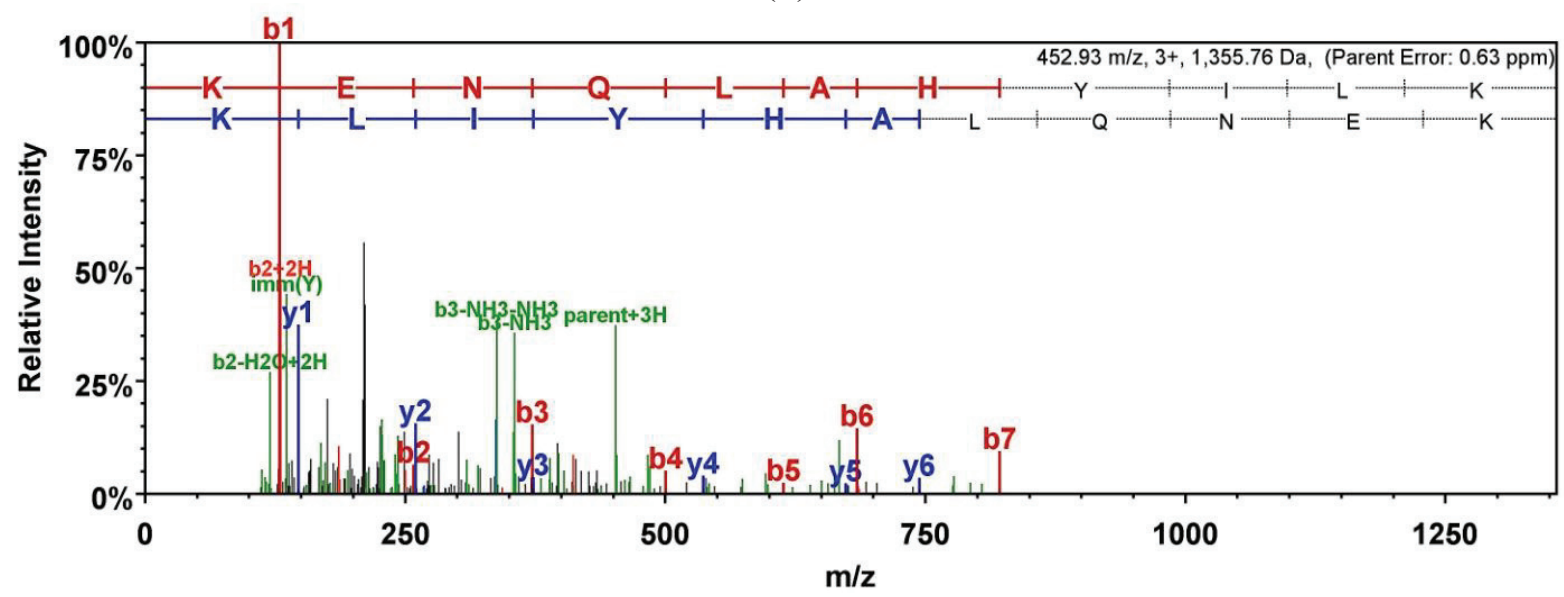

(b) 
Figure A3. Cont.

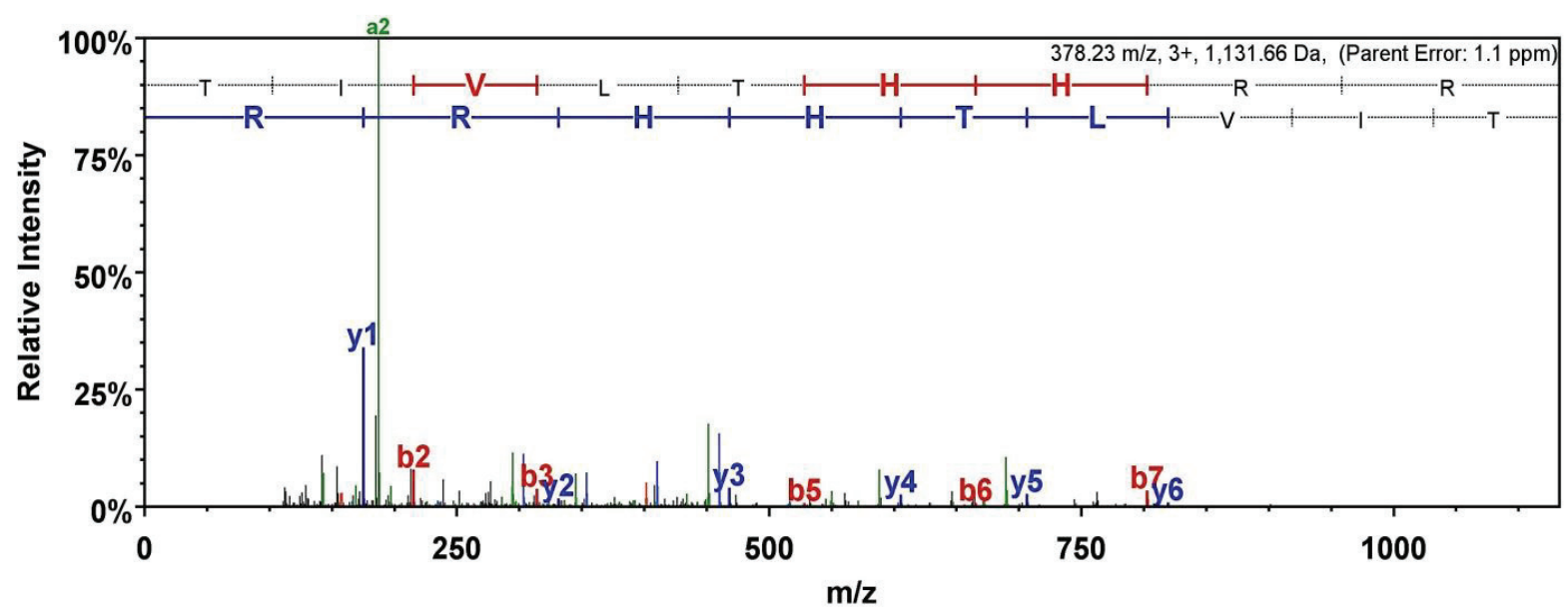

(c)

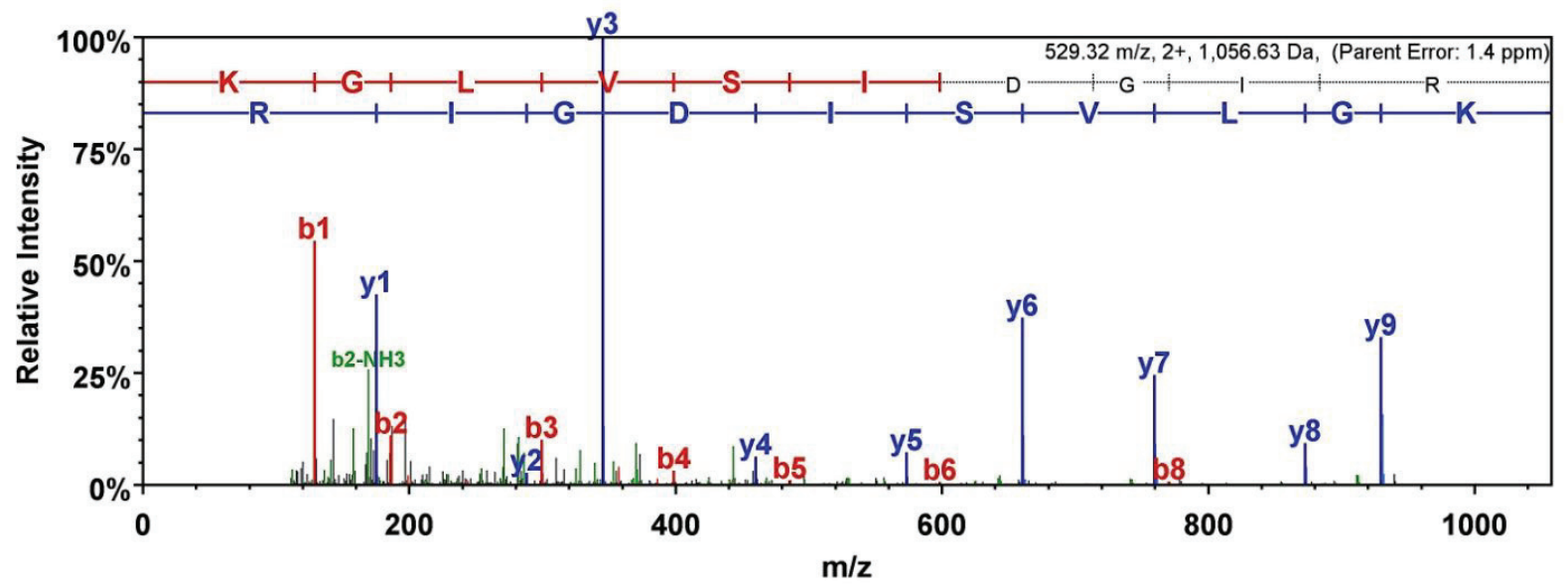

(d) 

Reprinted from Viruses. Cite as: Martínez, F.P.; Tang, Q. Identification of Cellular Proteins that Interact with Human Cytomegalovirus Immediate-Early Protein 1 by Protein Array Assay. Viruses 2014, 6, 89-105.

Article

\title{
Identification of Cellular Proteins that Interact with Human Cytomegalovirus Immediate-Early Protein 1 by Protein Array Assay
}

\section{Francisco Puerta Martínez and Qiyi Tang *}

Department of Microbiology/RCMI Program, Ponce School of Medicine and Health Sciences, Ponce, PR 00716, USA

* Author to whom correspondence should be addressed; E-Mail: qtang@psm.edu; Tel.: +1-787-840-2575 (ext. 2215); Fax: +1-787-841-5159.

Received: 7 November 2013; in revised form: 10 December 2013 / Accepted: 20 December 2013/ Published: 31 December 2013

\begin{abstract}
Human cytomegalovirus (HCMV) gene expression during infection is characterized as a sequential process including immediate-early (IE), early (E), and late (L)-stage gene expression. The most abundantly expressed gene at the IE stage of infection is the major IE (MIE) gene that produces IE1 and IE2. IE1 has been the focus of study because it is an important protein, not only for viral gene expression but also for viral replication. It is believed that IE1 plays important roles in viral gene regulation by interacting with cellular proteins. In the current study, we performed protein array assays and identified 83 cellular proteins that interact with IE1. Among them, seven are RNA-binding proteins that are important in RNA processing; more than half are nuclear proteins that are involved in gene regulations. Tumorigenesis-related proteins are also found to interact with IE1, implying that the role of IE1 in tumorigenesis might need to be reevaluated. Unexpectedly, cytoplasmic proteins, such as Golgi autoantigen and GGA1 (both related to the Golgi trafficking protein), are also found to be associated with IE1. We also employed a coimmunoprecipitation assay to test the interactions of IE1 and some of the proteins identified in the protein array assays and confirmed that the results from the protein array assays are reliable. Many of the proteins identified by the protein array assay have not been previously reported. Therefore, the functions of the IE1-protein interactions need to be further explored in the future.
\end{abstract}


Keywords: human cytomegalovirus (HCMV); major immediate-early (MIE); IE1; protein-protein interaction; protein array

\section{Introduction}

Human cytomegalovirus (HCMV) has been defined as one of the major infectious agents related to AIDS. HCMV infects large populations in general and causes serious diseases in immunocompromised individuals [1-3]. A sequential process of viral events is characterized for CMV infection in permissive host cells [3], including viral entry, immediate-early (IE) and early gene expression, DNA replication, late (L) gene expression, viral packaging and release. Major immediate-early (MIE) gene expression is one of the earliest events during CMV infection. MIE genes are the most abundantly expressed viral genes at the IE stage and give rise to several nuclear phosphoproteins that are critical for the next stage of gene expression and viral replication [4-14]. Hence, the HCMV MIE gene has been the focus of study. IE1 is believed to be the first de novo viral protein produced after HCMV infection and is essential for HCMV replication when HCMV is infected at a low multiplicity of infection (MOI).

In general, IE1 has been considered as a gene activator. How IE1 activates gene expression is not fully understood. Several mechanisms that are involved in this activation (by IE1) have been described by different groups. First, IE1 interacts with nuclear corepressors such as PML, Daxx, and Sp100, thereby reducing their repressive effects on gene expression $[15,16]$. It was found by different groups independently that IE1 interacts with HDAC and inhibits HDAC's activity [15,17]. Second, IE1 disperses ND10, a nuclear domain that contains many different nuclear suppressive proteins and responds to interferon stimulation [18-21]. In addition, innate cellular defense directed by interferons was disrupted by IE1 via inhibiting JAK-STAT signaling and by interacting with STAT1 [22-24]. The IE1 protein counteracts virus- or type I IFN-induced ISG activation via complex formation with STAT1 and STAT2 resulting in the reduced binding of ISGF3 to ISREs [22-25]. Last, IE1 is important for HCMV to arrest the cell cycle in the G1 phase that favors the infected HCMV for the cellular microenvironment. IE1 binds the Rb-related p107 protein and relieves its repression of E2F-responsive promoters [26]; IE1 also induces p53 accumulation through activating the p53 pathway by increasing the levels of p19 Arf and by inducing the phosphorylation of p53 at Ser15 [27], which might also relate to the HCMV-caused "G1 arrest" of infected cells. The mechanisms used by IE1 to activate viral gene expression perhaps all depend on IE1-protein interactions. Therefore, it is important to identify the cellular proteins interacting with IE1 at a global level.

\section{Results}

One of the major challenges in the post-genomic era is to explore the functional elements in the human genome. It also applies to the virus-host interaction. Identifying the cellular proteins that interact with the important virus proteins will certainly contribute to the understanding of the mechanisms that the virus uses for its gene expression and replication. Protein arrays constitute a 
powerful tool for high throughput and multiplexed protein analysis, including protein detection, the investigation of protein interactions with various types of molecules, and the determination of protein functions [28]. Protein array technology is highly sensitive and generates large amounts of data in a single experiment with comparatively low sample consumption; therefore, it is highly economical [28]. In current studies, we used protein array assays to screen cellular proteins that interact with HCMV IE1. Here we report our experimental results.

First, we isolated the IE1 from an IE1-producing cell line (previously called U373-IE1, now called U-251 MG-IE1) [29] using a specific anti-IE1 antibody that was later incubated with beads-conjugated secondary antibody after which the beads were washed in binding/wash buffer (20 mM Na $2 \mathrm{HPO}_{4}, 0.15 \mathrm{M} \mathrm{NaCl}, \mathrm{pH}$ 7.0). The pulled-down IE1 was washed off from the secondary antibody-bound beads with elution buffer (0.1 M glycine, $\mathrm{pH} 2-3)$, and the eluted solution was immediately neutralized with neutralization buffer $(1 \mathrm{M}$ Tris, $\mathrm{pH} 8.5)$. The isolated IE1 was confirmed by Western blot assay as shown in the top of Figure 1. Then the isolated IE1 was incubated at room temperature for 1 hour with the $22 \mathrm{~cm} \times 22 \mathrm{~cm}$ PVDF membranes presenting up to 7390 in situ expressed human proteins (Cat\# Unipex_1,_2, library \# 9027, \#9028, imaGenes $\mathrm{GmbH}$, Berlin, Germany). After $24 \mathrm{~h}$ of incubation with primary anti-IE1 antibody at $4{ }^{\circ} \mathrm{C}$, the membrane was washed with the buffer 2 times followed by incubation with a horseradish peroxidase-coupled secondary antibody (GE Healthcare Bio-Sciences, Pittsburgh, PA, USA) and detection with enhanced chemiluminescence (Pierce, Rockford, IL, USA), according to standard methods. As can be seen in Figure 1A,B, 83 cellular proteins were found to bind to IE1. In the PVDF membrane, each in situ expressed cellular protein is presented in doublets, which is why we can see 2 dots together for each protein. We set up cutoffs according to the background index. Figure 1A,B represents 2 PVDF membranes that were processed at the same time with the same samples. The 83 proteins are listed in the Table 1 with their Gene bank \#, name, function, and references.

Further analysis of the 83 proteins revealed some important information: First, some of them have already been reported to interact with IE1. These are ATRX (\#13) [15], splicing factor, arginine/serine-rich 2 (\#40) [18], and Daxx (\#65) [30]. Second, some of them are reported to be important for CMV infection but they have not been shown to interact with IE1, these are CDC25A (\#10) [31], ATF4 (\#41) [32], BCL-2 (\#46) [33], and Ataxin (\#39) [34]. In addition, 7 of the proteins that interact with IE1 are RNA binding proteins: RNA binding motif protein 10 (RBM10) (\#5), RNA binding motif protein 5 (\#20), Staufen, RNA binding protein (\#26), splicing factor, arginine/serine-rich 2 (\#40), heterogeneous nuclear ribonucleoprotein F (\#52), RD RNA binding protein (\#59), and PRP40 pre-mRNA processing factor $40 \mathrm{~B}$ (\#60). All the RNA-bonding proteins are important for pre-mRNA processing. To our surprise, neither HDAC1, 2, or 3 nor TRIM19 (PML) is on the list; however, HDAC10 (\#15) and TRIM33 (\#57) are both detected as IE1-binding proteins with high signals (Figure 1).

Since most of the IE1-interacting proteins in the Table 1 have not been reported, we need to validate the reliability of the results from the protein arrays. For that purpose, we performed a coimmunoprecipitation (coIP) assay using antibodies on hand to examine whether the proteins can be pulled down by the anti-IE1 antibody. The IE1-producing cell line (U-251 MG-IE1) [29] was cultured at $37{ }^{\circ} \mathrm{C}$ until it reached $95 \%$ confluence. The nuclear extract was prepared according to the protocol as described in Material and Method section. The nuclear extracts were incubated with 
anti-IE1 antibody or with normal IgG and then secondary antibody-conjugated beads. After several washings, the eluted complexes from the beads were examined by Western blot assay using antibodies against the proteins as indicated in Figure 2. All the proteins from the list that are examined by Western blot assay were positive. We also examined the tubulin as control and it was negative. Therefore, the results from the protein arrays are reliable.

Figure 1. Protein Array. The isolated IE1 was incubated with the two PVDF membranes, which was followed by incubation with a horseradish peroxidase-coupled secondary antibody (GE Healthcare Bio-Sciences, Pittsburgh, PA, USA) and detection with enhanced chemiluminescence (GE Healthcare Bio-Sciences, Pittsburgh, PA, USA), according to standard methods. Some genes overlap between the two PVDF membranes.
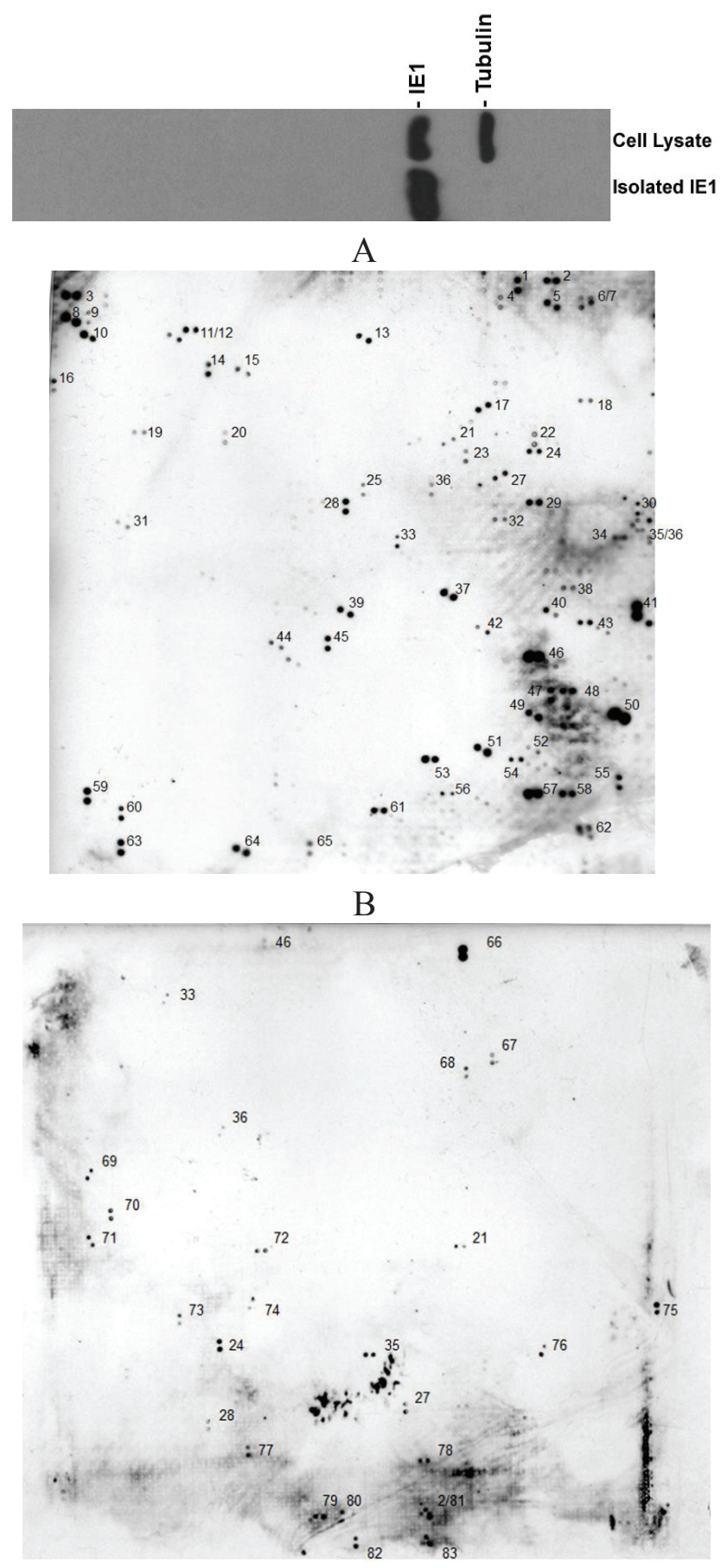
Table 1. List of the Human Cytomegalovirus (HCMV) IE1 Protein-Protein Interactions (PPIs) by Protein Array Assay. The positive spots in Figure 1A,B were listed in this table according to the manufacturer's annotation table. The dots are all in doublets. Some genes overlap between the two PVDF membranes. Cellular Proteins Interacting with Human Cytomegalovirus IE1 (Identified by Protein Array Assays).

\begin{tabular}{|c|c|c|c|}
\hline GenBank entry\# & Name of the protein & Function & Ref. \\
\hline AF217982 & CDK5 regulatory subunit associated protein & Tumorigenesis and metastasis & {$[35]$} \\
\hline D84294 & TPRDI (tetratricopeptide repeat) & Down syndrome & [36] \\
\hline ВC069268 & Golgi autoantigen, golgin subfamily a & Tentacular matrix & [37] \\
\hline NM_007144 & Polycomb group ring Finger 2 (PCGF2) & Embryogenesis and tumorigenesis & [38] \\
\hline NM_005676 & RNA binding motif protein 10 (RBM10) & RNA-related apoptosis & [39] \\
\hline Z11584 & NuMA protein & Spindle orientation & [40] \\
\hline $\mathrm{BC} 022880$ & Breast carcinoma amplified sequence 2 & Negative regulator of p53 & [41] \\
\hline BC057387 & LSM14B, SCD6 homolog B & Regulation of translation & [42] \\
\hline BG354577 & CDCA4 Cell division cycle associated gene 4 & Transcription regulation & [43] \\
\hline NM_001789 & Cell division cycle 25 homolog A (CDC25A) & Cell cycle regulation & [44] \\
\hline NM_003026 & SH3-domain GRB2-like 2 (SH3GL2) & Tumorigenesis & [45] \\
\hline NM_203505 & GTPase activating protein binding protein 2 & Stress granule formation & [46] \\
\hline XM_001128623 & Transcriptional regulator ATRX & Transcriptional repression & [47] \\
\hline NM_153273 & Inositol hexaphosphate kinase 1 (IHPK1) & Type 2 diabetes & [48] \\
\hline NM_032019 & Histone deacetylase 10 (HDAC10) & Cancer metastasis & [49] \\
\hline NM_001040653 & ZXD family zinc finger C (ZXDC) & MHC gene transcription & [50] \\
\hline NM_138383 & $\begin{array}{l}\text { Actin-bundling protein with BAIAP2 homology } \\
\text { (ABBA-1) }\end{array}$ & unknown & [51] \\
\hline NM_001082486 & $\begin{array}{l}\text { Adrenocortical dysplasia homolog (mouse) } \\
\text { (ACD), transcript variant } 1\end{array}$ & Tumorigenesis & [52] \\
\hline AK122898 & ADP-ribosylation factor binding protein GGA1 & Trans-olgi network & [53] \\
\hline AB208813 & RNA binding motif protein 5 & Gene splicing factor & [39] \\
\hline NM_170677 & Meis homeobox 2 (MEIS2) & Unknown & [54] \\
\hline NM_000038 & Adenomatosis polyposis coli (APC) & Tumor supresor & [55] \\
\hline NM_003660 & $\begin{array}{c}\text { Protein tyrosine phosphatase, receptor type } \mathrm{f} \\
\text { polypeptide (PTPRF), interacting protein (liprin), } \\
\text { alpha } 3 \text { (PPFIA3) }\end{array}$ & Unknown & {$[56]$} \\
\hline NM_012398 & $\begin{array}{c}\text { Phosphatidylinositol-4-phosphate 5-kinase, type } \\
\text { I, gamma (PIP5K1C) }\end{array}$ & Lethal contractural syndrome & [57] \\
\hline NM_003861 & WD repeat domain 22 (WDR22) & Unknown & N/A \\
\hline NM_017453 & Staufen, RNA binding protein & mRNA traffic & [58] \\
\hline BC017222 & Sequestosome 1 & Signal transduction & [59] \\
\hline NM_152586 & Ubiquitin specific peptidase 54 (USP54) & Unknown & {$[60]$} \\
\hline NM_014868 & Ring finger protein 10 (RNF10) & Type 2 diabetes & [61] \\
\hline BC041897 & $\begin{array}{l}\text { SplA/ryanodine receptor domain and SOCS box } \\
\text { containing } 3\end{array}$ & Inflammation & [62] \\
\hline AB209534 & Tumor rejection antigen (gp96) 1 & Unknown & N/A \\
\hline NM_001080424 & Jumonji domain containing 3 (JMJD3) & Histone demethylation & [63] \\
\hline NM_006312 & Nuclear receptor co-repressor 2 (NCOR2) & Gene suppressor & [64] \\
\hline AK124656 & Gamma enolase (EC 4.2.1.11) & Neural tissue development & [65] \\
\hline
\end{tabular}


Table 1. Cont.

\begin{tabular}{|c|c|c|c|}
\hline GenBank entry\# & Name of the protein & Function & Ref. \\
\hline AK123065 & Sperm acrosomal protein & Motility of the spermatozoon & [66] \\
\hline NM_001098800 & Melanoma antigen family D 4 (MAGED4) & Renal cell carcinoma & [67] \\
\hline NM_021098 & $\begin{array}{l}\text { Calcium channel, voltage-dependent, } \mathrm{T} \text { type, alpha } \\
\qquad 1 \mathrm{H} \text { subunit }(\mathrm{CACNA} 1 \mathrm{H})\end{array}$ & T-type $\mathrm{Ca}(2+)$ channel activity & [68] \\
\hline NM_001417 & Eukaryotic translation initiation factor 4B (EIF4B) & Translation control & [69] \\
\hline NM_002973 & Ataxin 2 (ATXN2) & Spinocerebellar ataxia type 2 & [70] \\
\hline ВC070086 & Splicing factor, arginine/serine-rich 2 & Gene splicing & [18] \\
\hline NM_001675 & Activating transcription factor 4 (ATF4) & Gene transcription & [71] \\
\hline AB209149 & Phenol-sulfating phenol sulfotransferase 1 & Transfer of a sulfonate moiety & [72] \\
\hline AY509035 & Roundabout-like protein 3 (ROBO3) & Horizontal gaze palsy & [73] \\
\hline NM_014751 & Metastasis suppressor 1 (MTSS1) & Metastasis & [74] \\
\hline AY335491 & GON4L isoform C (GON4L) & Hematopoiesis & [75] \\
\hline NM_014739 & BCL2-associated transcription factor 1 & Transcriptional repression & [76] \\
\hline AB067518 & KIAA1931 protein & Unknown & N/A \\
\hline NM_003200 & $\begin{array}{l}\text { Transcription factor } 3 \text { (E2A immunoglobulin } \\
\text { enhancer binding factors E12/E47) (TCF3) }\end{array}$ & Transcription regulation & [77] \\
\hline AB209197 & Protein phosphatase $1(\mathrm{PP} 1)$ & Multiple functions & [78] \\
\hline NM_020226 & PR domain containing 8 (PRDM8) & Gene repressor & [79] \\
\hline XM_166659 & OTU domain containing 1 (OTUD1) & Unknown & N/A \\
\hline NM_001098208 & Heterogeneous nuclear ribonucleoprotein F (HNRPF) & Gene splicing & [80] \\
\hline AB209441 & Fibroblast growth factor receptor 3 isoform 1 precursor & Development & [81] \\
\hline NM_152643 & $\begin{array}{l}\text { Kinase non-catalytic C-lobe domain (KIND) } \\
\text { containing } 1 \text { (KNDC1) }\end{array}$ & Unknown & N/A \\
\hline NM_001982 & $\begin{array}{l}\text { V-erb-b2 erythroblastic leukemia viral oncogene } \\
\text { homolog } 3 \text { (ERBB3) }\end{array}$ & $\begin{array}{l}\text { Cell proliferation or } \\
\text { differentiation }\end{array}$ & [82] \\
\hline NM_015695 & Bromodomain and PHD finger containing, 3 (BRPF3) & Fetal liver erythropoiesis & [83] \\
\hline NM_015906 & Tripartite motif-containing 33 (TRIM33) & Tumor suppressors & [84] \\
\hline NM_032127 & Chromosome 11 open reading frame 56 (C11orf56) & $\begin{array}{l}\text { FTS and Hook-interacting } \\
\text { protein }\end{array}$ & [85] \\
\hline NM_002904 & RD RNA binding protein (RDBP) & Repress RNA polymerase II & $\mathrm{N} / \mathrm{A}$ \\
\hline NM_012272 & PRP40 pre-mRNA processing factor $40 \mathrm{~B}$ (PRPF40B) & Gene splicing & [86] \\
\hline NM_020967 & Nuclear receptor coactivator 5 (NCOA5) & Gene regulator & [87] \\
\hline NM_001080495 & KIAA1856 protein (KIAA1856) & Unknown & $\mathrm{N} / \mathrm{A}$ \\
\hline NM_022748 & Tensin 3 (TNS3) & Signal transduction & [88] \\
\hline ВC063642 & $\begin{array}{l}\text { Phosphodiesterase 4D interacting protein } \\
\text { (myomegalin) }\end{array}$ & Control microtubules & [89] \\
\hline AB209493 & Death-associated protein 6 (DAXX) & Development and Cancer & [90] \\
\hline NM_003482 & $\begin{array}{l}\text { Myeloid/lymphoid or mixed-lineage leukemia } 2 \\
\text { (MLL2) }\end{array}$ & Lymphomagenesis & [91] \\
\hline NM_033396 & Tankyrase 1 binding protein 1 , (TNKS1BP1) & Unknown & [92] \\
\hline AY729650 & Intersex-like protein & Unknown & [93] \\
\hline BC110647 & Immediate-early response 2 & Unknown & N/A \\
\hline $\mathrm{AB} 014581$ & KIAA0681 protein & Unknown & N/A \\
\hline
\end{tabular}


Table 1. Cont.

\begin{tabular}{cccc}
\hline GenBank entry\# & Name of the protein & Function & Ref. \\
\hline NM_004235 & Kruppel-like factor 4 (gut) (KLF4) & Transactivation and growth & [94] \\
suppression & Unknown & N/A \\
AB051455 & KIAA1668 protein & Autophagy activation & {$[95]$} \\
AF045458 & Serine/threonine kinase ULK1 (ULK1) & Unknown \\
NM_001003694 & Bromodomain and PHD finger containing, 1 (BRPF1) & No \\
NM_003626 & protein tyrosine phosphatase, receptor type, f polypeptide & Unknown & N/A \\
& (PTPRF), interacting protein (liprin), alpha 1 (PPFIA1) & & \\
NM_006291 & tumor necrosis factor, alpha-induced protein 2 & Unknown & N/A \\
AB209643 & (TNFAIP2) & Unknown & N/A \\
NM_002857 & smoothelin isoform b & Peroxisomal assembly & {$[96]$} \\
AB061669 & peroxisomal biogenesis factor 19 (PEX19) & Signaling and inflammation & [97] \\
NM_014678 & receptor for advanced glycation end-products & Nnknown \\
NM_006887 & ZAPS domain family, member 2 (SAPS2) & Unknown & N/A \\
AB208876 & zinger protein 36, C3H type-like 2 (ZFP36L2) & Wnt signaling pathway & {$[98]$} \\
NM_004530 & axin 1 isoform & Metastasis and inflammation & [99] \\
\hline
\end{tabular}

Figure 2. Validation of some IE1 PPIs by coIP. The nuclear extract from the IE1-producing cell line was prepared and incubated with anti-IE1 antibody followed by incubation with secondary bound beads. The pulled-down complexes were examined by Western blot using antibodies against the protein, as indicated on the right hand side.

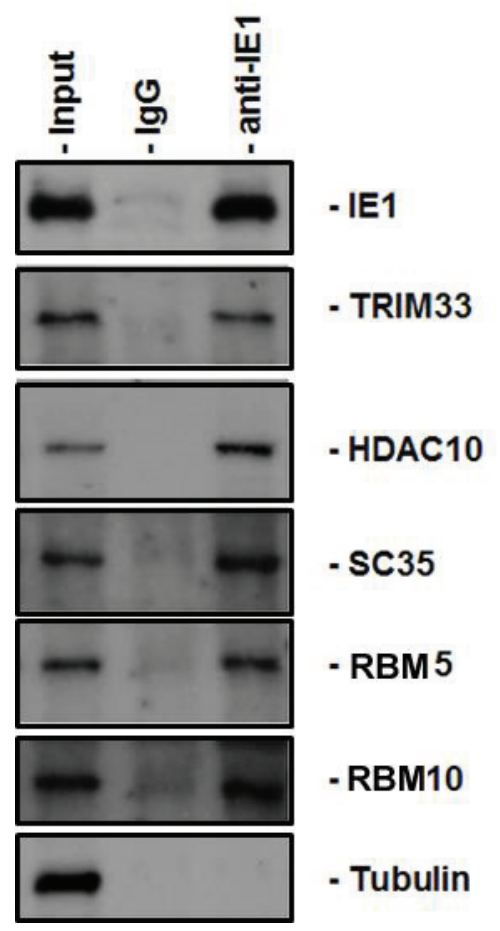

\section{Discussion}

HCMV infects a large part of the population and has serious consequences for immunocompromised persons with AIDS or who have received organ transplants and for newborns after congenital 
infection. In fact, HCMV is the leading viral cause of congenital birth defects [1]. More than 30\% of primary HCMV infections in pregnant women result in placental transmission and clinical syndromes. Congenital infection is not uncommon [100-102]. Of symptomatic newborns, about $12 \%$ die and half of the survivors develop mental retardation, vision loss, and/or sensorineural deafness [103]. Interference strategies commonly target the early events of the replication cycle by using approved nucleoside analogs such as ganciclovir, the nucleotide analog cidofovir, and foscarnet. However, these can lead to resistance [104]. In vitro anti-sense oligonucleotides against the HCMV immediate-early protein 2 (IE2) have proven effective [105], as has targeting the UL36 and UL37 sites [105,106]. These attempts show that targeting the HCMV IE part of the propagation cycle may be effective; however, none of these treatments are permissible or feasible for use on a potentially infected fetus. The lack of suitable treatment modalities has especially serious consequences for the congenitally infected fetus and for patients with impaired immune systems. The challenge is to find treatment modalities that do not depend on the inhibition of the DNA replication process. This has attracted our investigation to the immediate-early (IE) stage of HCMV infection. Understanding IE1's function is our first step in the development of a practical strategy against HCMV replication via interfering with IE1 function.

In many cases, proteins play their biological role through interactions with other proteins. Especially for viral infection, viruses need to manipulate cellular function via interacting with cellular proteins. Therefore, the systematic identification and characterization of protein-protein interactions (PPIs) is considered a key strategy to understand protein function. In the case of HCMV viral immediate-early products, IE1 might be the most important protein that initiates the early interactions between the virus and cells. The number of possible contacts between protein surfaces is astronomical. In this context, protein array technology opens up new avenues for the characterization of viral proteins and the identification of molecular partners involved in virus-mediated regulatory networks in cells. The array technology used in the present study started with incubation of IE1 with the PVDF membranes that carry GST-tagged human fusion proteins. Those fusion proteins were expressed in E. coli, and after native lysis with lysozyme, crude protein extracts were prepared under non-denaturing conditions in a 384-well plate format. The crude bacterial cell extracts were used for incubation overnight with the high-density spotted PVDF membranes, which were used to detect IE1-interacting proteins.

Using the protein array, eighty-three cellular proteins in total were identified to be PPIs of HCMV IE1. Most of them were gene regulators. Only 3 IE1 PPIs from the list have been reported. However, many HCMV-mediated cell biological activities have been linked to IE1 (which link is also shown in these array assays); these activities include, among others, the interaction of IE1 with BCL-2, CDC25A, which interaction might be important for HCMV-induced "G1 arrest". Some cellular proteins reported to interact with IE1 are not on the list, they might interact with IE1 only indirectly or the printed proteins are in different isoforms. For example, some isoforms of PML was found to interact with IE1 [15] but is not on the list because the other isoforms of PML are printed on the PVDF membranes. As shown on the Table 1, 7 RNA binding proteins are found to interact with IE1, implying that IE1 has a novel function in viral mRNA processing.

In conclusion, our current effort in characterizing IE1's PPIs resulted in the identification of 83 cellular proteins that directly interact with HCMV IE1. The results have been partially validated by 
coIP assay. With the data listed in the Table 1, we will further investigate the function of the PPIs of IE1.

\section{Experimental}

\subsection{Tissue Culture and Viruses}

The IE1-producing cell lines (U-251 MG-IE1) [29] were maintained in Dulbecco's modified Eagle's medium (DMEM) supplemented with 10\% fetal calf serum (FCS) and 1\% penicillin-streptomycin.

\subsection{Antibodies}

The monoclonal antibody against IE1 (MAB810) and the rabbit antibodies against cellular proteins were purchased from Santa Cruz Biotechnology, Inc. (Santa Cruz, CA, USA). The rabbit antibodies included anti-SC35 (sc-28720), -TRIM33 (sc-68424), -HDAC10 (sc-130775), and -tubulin (sc-5546). Rabbit antibodies against RBM5 (ab69970) and RBM 10 (ab126112) were bought from Abcam (Cambridge, MA, USA)

\subsection{Preparation of Nuclear Extracts}

Nuclear extracts were obtained essentially as described previously [15]. Briefly, monolayer cells were washed with PBS and scraped into fresh Eppendorf tubes. Cell pellets were resuspended in cold buffer A (10 mM HEPES-KOH, pH 7.9, at $4{ }^{\circ} \mathrm{C}, 1.5 \mathrm{mM} \mathrm{MgCl}_{2}, 10 \mathrm{mM} \mathrm{KCl}, 0.5 \mathrm{mM}$ dithiothreitol, $0.2 \mathrm{mM}$ phenylmethylsulfonyl fluoride) and incubated for $10 \mathrm{~min}$ at $4{ }^{\circ} \mathrm{C}$. Then the cells were dounced with 10-20 plunges in a Kontes-B (Wheaton, DC, USA) Dounce Homogenizer (Pestle B) and poured into new bottles after centrifugation. The pellets were resuspended in cold buffer C (20 mM HEPES-KOH, pH 7.9, 25\% glycerol, $420 \mathrm{mM} \mathrm{NaCl}, 1.5 \mathrm{mM} \mathrm{MgCl} 2,0.2 \mathrm{mM}$ EDTA, $0.5 \mathrm{mM}$ dithiothreitol, $0.2 \mathrm{mM}$ phenylmethylsulfonyl fluoride) by vortexing and incubated for 30 min at $4{ }^{\circ} \mathrm{C}$. The pellets were dounced again with 10-20 plunges in Kontes-B (Wheaton) Dounce Homogenizer (Pestle B), and clarified extracts were transferred to fresh tubes and stored at $-70{ }^{\circ} \mathrm{C}$ until use.

\subsection{Coimmunoprecipitation}

Antibodies were coupled to protein G-Sepharose beads (GE Healthcare Bio-Sciences, Pittsburgh, PA, USA), according to the manufacturer's instructions. After a wash with PBS- $0.1 \%$ bovine serum albumin, the beads were incubated overnight at $4{ }^{\circ} \mathrm{C}$ with clarified extracts, washed again in PBS- $0.1 \%$ bovine serum albumin, and resuspended in a mixture of PBS and $2 \times$ Laemmli buffer (20 $\mu \mathrm{L}$ of each). After being heated for $5 \mathrm{~min}$ at $95{ }^{\circ} \mathrm{C}$, the beads were removed by centrifugation and supernatants were analyzed by SDS-PAGE and immunoblotting.

\subsection{Immunoblot Analysis}

Proteins were separated by sodium dodecyl sulfate- $7.5 \%$ polyacrylamide gel electrophoresis [107] (10 to $20 \mu \mathrm{g}$ loaded in each lane), transferred to nitrocellulose membranes (Amersham Inc., 
Piscataway, NJ, USA), and blocked with 5\% nonfat milk for $60 \mathrm{~min}$ at room temperature. Membranes were incubated overnight at $4{ }^{\circ} \mathrm{C}$ with primary antibody followed by incubation with a horseradish peroxidase-coupled secondary antibody (Amersham Inc.) and detection with enhanced chemiluminescence (Pierce, Rockford, IL, USA), according to standard methods. Membranes were stripped with stripping buffer (100 mM $\beta$-mercaptoethanol, 2\% SDS, $62.5 \mathrm{mM}$ Tris-HCl, pH 6.8), washed with PBS- $0.1 \%$ Tween 20, and used to detect additional proteins.

\section{Conclusions}

We currently used a protein array assay to identify HCMV IE1 interacting proteins. There are 83 cellular proteins that are PPIs of IE1 and most of the PPIs have not been reported previously. The interactions have been partially validated by coIP method which confirmed that the protein array assay is reliable.

\section{Acknowledgements}

This study was supported by a pilot grant from the Research Center for Minority Institutes (RCMI) program (2G12RR003050-24/8G12MD007579-27) (Q.T.), an American Cancer Society grant (RSG-090289-01-MPC) (Q.T), and NIH/NCRR U54RR022762 (Q.T.). We acknowledge the instrument support of the PSMHS Molecular Biology Core Laboratory. Finally, we are grateful to Bob Ritchie of the Ponce School of Medicine and Health Sciences/RCMI Publications Office (G12 RR003050/8G12MD007579-27) for his help with manuscript preparation.

\section{Conflicts of Interest}

The authors declare no conflict of interest.

\section{References and Notes}

1. Sweet, C. The pathogenicity of cytomegalovirus. FEMS Microbiol. Rev. 1999, 23, 457-482.

2. Landolfo, S.; Gariglio, M.; Gribaudo, G.; Lembo, D. The human cytomegalovirus. Pharmacol. Ther. 2003, 98, 269-297.

3. Mocarski, E.S., Jr.; Shenk, T.; Pass, R.F. Cytomegaloviruses, 5th ed.; Lippincott Williams \& Wilkins: Philadelphia, PA, USA, 2006.

4. Tang, Q.; Li, L.; Maul, G.G. Mouse cytomegalovirus early M112/113 proteins control the repressive effect of IE3 on the major immediate-early promoter. J. Virol. 2005, 79, 257-263.

5. Tang, Q.; Maul, G. Immediate early interactions and epigenetic defense mechanisms. In In Cytomegaloviruses: Molecular Biology and Immunology; Reddehase, M.J., Ed.; Hethersett, Horizon Scientific Press: Norwich, UK, 2005.

6. Hagemeier, C.; Walker, S.M.; Sissons, P.J.; Sinclair, J.H. The 72K IE1 and 80K IE2 proteins of human cytomegalovirus independently trans-activate the c-Fos, c-Myc and hsp70 promoters via basal promoter elements. J. Gen. Virol. 1992, 73, 2385-2393. 
7. Liu, B.; Hermiston, T.W.; Stinski, M.F. A cis-acting element in the major immediate-early (IE) promoter of human cytomegalovirus is required for negative regulation by IE2. J. Virol. 1991, 65, 897-903.

8. Scully, A.L.; Sommer, M.H.; Schwartz, R.; Spector, D.H. The human cytomegalovirus IE2 86-kilodalton protein interacts with an early gene promoter via site-specific DNA binding and protein-protein associations. J. Virol. 1995, 69, 6533-6540.

9. Awasthi, S.; Isler, J.A.; Alwine, J.C. Analysis of splice variants of the immediate-early 1 region of human cytomegalovirus. J. Virol. 2004, 78, 8191-8200.

10. Sadanari, H.; Yamada, R.; Yamagoshi, T.; Ohnishi, K.; Matsubara, K.; Fukuda, S.; Tanaka, J. The major immediate-early genes of human cytomegalovirus induce two novel proteins with molecular weights of 91 and 102 kilodaltons. Arch. Virol. 2000, 145, 1257-1266.

11. Ahn, J.H.; Hayward, G.S. The major immediate-early proteins IE1 and IE2 of human cytomegalovirus colocalize with and disrupt PML-associated nuclear bodies at very early times in infected permissive cells. J. Virol. 1997, 71, 4599-4613.

12. Meier, J.L.; Stinski, M.F. Effect of a modulator deletion on transcription of the human cytomegalovirus major immediate-early genes in infected undifferentiated and differentiated cells. J. Virol. 1997, 71, 1246-1255.

13. Stenberg, R.M. The human cytomegalovirus major immediate-early gene. Intervirology 1996, 39, 343-349.

14. Stenberg, R.M.; Thomsen, D.R.; Stinski, M.F. Structural analysis of the major immediate early gene of human cytomegalovirus. J. Virol. 1984, 49, 190-199.

15. Tang, Q.; Maul, G.G. Mouse cytomegalovirus immediate-early protein 1 binds with host cell repressors to relieve suppressive effects on viral transcription and replication during lytic infection. J. Virol. 2003, 77, 1357-1367.

16. Lee, H.R.; Kim, D.J.; Lee, J.M.; Choi, C.Y.; Ahn, B.Y.; Hayward, G.S.; Ahn, J.H. Ability of the human cytomegalovirus IE1 protein to modulate sumoylation of PML correlates with its functional activities in transcriptional regulation and infectivity in cultured fibroblast cells. J. Virol. 2004, 78, 6527-6542.

17. Nevels, M.; Paulus, C.; Shenk, T. Human cytomegalovirus immediate-early 1 protein facilitates viral replication by antagonizing histone deacetylation. Proc. Natl. Acad. Sci. USA 2004, 101, 17234-17239.

18. Ahn, J.H.; Hayward, G.S. Disruption of PML-associated nuclear bodies by IE1 correlates with efficient early stages of viral gene expression and DNA replication in human cytomegalovirus infection. Virology 2000, 274, 39-55.

19. Ishov, A.M.; Stenberg, R.M.; Maul, G.G. Human cytomegalovirus immediate early interaction with host nuclear structures: Definition of an immediate transcript environment. J. Cell Biol. 1997, 138, 5-16.

20. Lee, H.R.; Huh, Y.H.; Kim, Y.E.; Lee, K.; Kim, S.; Ahn, J.H. N-Terminal determinants of human cytomegalovirus IE1 protein in nuclear targeting and disrupting PML-associated subnuclear structures. Biochem. Biophys. Res. Commun. 2007, 356, 499-504.

21. Maul, G.G.; Negorev, D.; Bell, P.; Ishov, A.M. Review: Properties and assembly mechanisms of ND10, PML bodies, or PODs. J. Struct. Biol. 2000, 129, 278-287. 
22. Paulus, C.; Krauss, S.; Nevels, M. A human cytomegalovirus antagonist of type I IFN-dependent signal transducer and activator of transcription signaling. Proc. Natl. Acad. Sci. USA 2006, 103, 3840-3845.

23. Huh, Y.H.; Kim, Y.E.; Kim, E.T.; Park, J.J.; Song, M.J.; Zhu, H.; Hayward, G.S.; Ahn, J.H. Binding STAT2 by the acidic domain of human cytomegalovirus IE1 promotes viral growth and is negatively regulated by SUMO. J. Virol. 2008, 82, 10444-10454.

24. Krauss, S.; Kaps, J.; Czech, N.; Paulus, C.; Nevels, M. Physical requirements and functional consequences of complex formation between the cytomegalovirus IE1 protein and human STAT2. J. Virol. 2009, 83, 12854-12870.

25. Knoblach, T.; Grandel, B.; Seiler, J.; Nevels, M.; Paulus, C. Human cytomegalovirus IE1 protein elicits a type II interferon-like host cell response that depends on activated STAT1 but not interferon-gamma. PLoS Pathog. 2011, 7, e1002016.

26. Zhang, Z.; Huong, S.M.; Wang, X.; Huang, D.Y.; Huang, E.S. Interactions between human cytomegalovirus IE1-72 and cellular p107: Functional domains and mechanisms of up-regulation of cyclin E/cdk2 kinase activity. J. Virol. 2003, 77, 12660-12670.

27. Castillo, J.P.; Frame, F.M.; Rogoff, H.A.; Pickering, M.T.; Yurochko, A.D.; Kowalik, T.F. Human cytomegalovirus IE1-72 activates ataxia telangiectasia mutated kinase and a p53/p21-mediated growth arrest response. J. Virol. 2005, 79, 11467-11475.

28. Hu, S.; Xie, Z.; Onishi, A.; Yu, X.; Jiang, L.; Lin, J.; Rho, H.S.; Woodard, C.; Wang, H.; Jeong, J.S.; et al. Profiling the human protein-DNA interactome reveals ERK2 as a transcriptional repressor of interferon signaling. Cell 2009, 139, 610-622.

29. Tang, Q.; Maul, G.G. Mouse cytomegalovirus crosses the species barrier with help from a few human cytomegalovirus proteins. J. Virol. 2006, 80, 7510-7521.

30. Reeves, M.; Woodhall, D.; Compton, T.; Sinclair, J. Human cytomegalovirus IE72 protein interacts with the transcriptional repressor hDaxx to regulate LUNA gene expression during lytic infection. J. Virol. 2010, 84, 7185-7194.

31. Gaspar, M.; Shenk, T. Human cytomegalovirus inhibits a DNA damage response by mislocalizing checkpoint proteins. Proc. Natl. Acad. Sci. USA 2006, 103, 2821-2826.

32. Xuan, B.; Qian, Z.; Torigoi, E.; Yu, D. Human cytomegalovirus protein pUL38 induces ATF4 expression, inhibits persistent JNK phosphorylation, and suppresses endoplasmic reticulum stress-induced cell death. J. Virol. 2009, 83, 3463-3474.

33. Andoniou, C.E.; Andrews, D.M.; Manzur, M.; Ricciardi-Castagnoli, P.; Degli-Esposti, M.A. A novel checkpoint in the Bcl-2-regulated apoptotic pathway revealed by murine cytomegalovirus infection of dendritic cells. J. Cell Biol. 2004, 166, 827-837.

34. Tower, C.; Fu, L.; Gill, R.; Prichard, M.; Lesort, M.; Sztul, E. Human cytomegalovirus UL97 kinase prevents the deposition of mutant protein aggregates in cellular models of Huntington's disease and ataxia. Neurobiol. Dis. 2011, 41, 11-22.

35. Mak, G.W.; Lai, W.L.; Zhou, Y.; Li, M.; Ng, I.O.; Ching, Y.P. CDK5RAP3 is a novel repressor of p14ARF in hepatocellular carcinoma cells. PLoS One 2012, 7, e42210.

36. Tsukahara, F.; Hattori, M.; Muraki, T.; Sakaki, Y. Identification and cloning of a novel cDNA belonging to tetratricopeptide repeat gene family from Down syndrome-critical region 21q22.2. J. Biochem. 1996, 120, 820-827. 
37. Munro, S. The golgin coiled-coil proteins of the Golgi apparatus. Cold Spring Harb. Perspect. Biol. 2011, 3, doi:10.1101/cshperspect.a005256.

38. Hennig, L.; Derkacheva, M. Diversity of Polycomb group complexes in plants: Same rules, different players? Trends Genet. 2009, 25, 414-423.

39. Sutherland, L.C.; Rintala-Maki, N.D.; White, R.D.; Morin, C.D. RNA binding motif (RBM) proteins: A novel family of apoptosis modulators? J. Cell Biochem. 2005, 94, 5-24.

40. Seldin, L.; Poulson, N.D.; Foote, H.P.; Lechler, T. NuMA localization, stability and function in spindle orientation involves 4.1 and Cdk1 interactions. Mol. Biol. Cell 2013, 24, 3651-3662.

41. Kuo, P.C.; Tsao, Y.P.; Chang, H.W.; Chen, P.H.; Huang, C.W.; Lin, S.T.; Weng, Y.T.; Tsai, T.C.; Shieh, S.Y.; Chen, S.L. Breast cancer amplified sequence 2, a novel negative regulator of the p53 tumor suppressor. Cancer Res. 2009, 69, 8877-8885.

42. Huang, Y.C.; Schmitt, M.; Yang, Z.; Que, L.G.; Stewart, J.C.; Frampton, M.W.; Devlin, R.B. Gene expression profile in circulating mononuclear cells after exposure to ultrafine carbon particles. Inhal. Toxicol. 2010, 22, 835-846.

43. Tategu, M.; Nakagawa, H.; Hayashi, R.; Yoshida, K. Transcriptional co-factor CDCA4 participates in the regulation of JUN oncogene expression. Biochimie 2008, 90, 1515-1522.

44. Shen, T.; Huang, S. The role of Cdc25A in the regulation of cell proliferation and apoptosis. Anticancer Agents Med. Chem. 2012, 12, 631-639.

45. Trub, T.; Frantz, J.D.; Miyazaki, M.; Band, H.; Shoelson, S.E. The role of a lymphoid-restricted, Grb2-like SH3-SH2-SH3 protein in T cell receptor signaling. J. Biol. Chem. 1997, 272, 894-902.

46. Matsuki, H.; Takahashi, M.; Higuchi, M.; Makokha, G.N.; Oie, M.; Fujii, M. Both G3BP1 and G3BP2 contribute to stress granule formation. Genes Cells 2013, 18, 135-146.

47. Salomoni, P.; Khelifi, A.F. DAXX: Death or survival protein? Trends Cell Biol. 2006, 16, 97-104.

48. Kamimura, J.; Wakui, K.; Kadowaki, H.; Watanabe, Y.; Miyake, K.; Harada, N.; Sakamoto, M.; Kinoshita, A.; Yoshiura, K.; Ohta, T.; et al. The IHPK1 gene is disrupted at the 3p21.31 breakpoint of $\mathrm{t}(3 ; 9)$ in a family with type 2 diabetes mellitus. J. Hum. Genet. 2004, 49, 360-365.

49. Song, C.; Zhu, S.; Wu, C.; Kang, J. Histone Deacetylase (HDAC) 10 suppresses cervical cancer metastasis through inhibition of matrix metalloproteinase (MMP) 2 and 9 Expression. J. Biol. Chem. 2013, 288, 28021-28033.

50. Al-Kandari, W.; Jambunathan, S.; Navalgund, V.; Koneni, R.; Freer, M.; Parimi, N.; Mudhasani, R.; Fontes, J.D. ZXDC, a novel zinc finger protein that binds CIITA and activates MHC gene transcription. Mol. Immunol. 2007, 44, 311-321.

51. Machesky, L.M.; Johnston, S.A. MIM: A multifunctional scaffold protein. J. Mol. Med. 2007, 85, 569-576.

52. Else, T.; Trovato, A.; Kim, A.C.; Wu, Y.; Ferguson, D.O.; Kuick, R.D.; Lucas, P.C.; Hammer, G.D. Genetic p53 deficiency partially rescues the adrenocortical dysplasia phenotype at the expense of increased tumorigenesis. Cancer Cell 2009, 15, 465-476.

53. Boman, A.L.; Zhang, C.; Zhu, X.; Kahn, R.A. A family of ADP-ribosylation factor effectors that can alter membrane transport through the trans-Golgi. Mol. Biol. Cell 2000, 11, 1241-1255. 
54. Nakamura, T.; Jenkins, N.A.; Copeland, N.G. Identification of a new family of Pbx-related homeobox genes. Oncogene 1996, 13, 2235-2242.

55. Wong, M.H.; Hermiston, M.L.; Syder, A.J.; Gordon, J.I. Forced expression of the tumor suppressor adenomatosis polyposis coli protein induces disordered cell migration in the intestinal epithelium. Proc. Natl. Acad. Sci. USA 1996, 93, 9588-9593.

56. Katoh, M. Identification and characterization of mouse Ppfial gene in silico. Int. J. Mol. Med. 2003, 12, 263-267.

57. Narkis, G.; Ofir, R.; Landau, D.; Manor, E.; Volokita, M.; Hershkowitz, R.; Elbedour, K.; Birk, O.S. Lethal contractural syndrome type 3 (LCCS3) is caused by a mutation in PIP5K1C, which encodes PIPKI gamma of the phophatidylinsitol pathway. Am. J. Hum. Genet. 2007, 81, 530-539.

58. Miki, T.; Takano, K.; Yoneda, Y. The role of mammalian Staufen on mRNA traffic: A view from its nucleocytoplasmic shuttling function. Cell Struct. Funct. 2005, 30, 51-56.

59. Geetha, T.; Vishwaprakash, N.; Sycheva, M.; Babu, J.R. Sequestosome 1/p62: Across diseases. Biomarkers 2012, 17, 99-103.

60. Rigden, D.J.; Liu, H.; Hayes, S.D.; Urbe, S.; Clague, M.J. Ab initio protein modelling reveals novel human MIT domains. FEBS Lett. 2009, 583, 872-878.

61. Huang, K.; Nair, A.K.; Muller, Y.L.; Piaggi, P.; Bian, L.; del Rosario, M.; Knowler, W.C.; Kobes, S.; Hanson, R.L.; Bogardus, C.; et al. Whole exome sequencing identifies variation in CYB5A and RNF10 associated with adiposity and type 2 diabetes. Obesity 2013, doi:10.1002/oby.20647.

62. Kuang, Z.; Yao, S.; Xu, Y.; Lewis, R.S.; Low, A.; Masters, S.L.; Willson, T.A.; Kolesnik, T.B.; Nicholson, S.E.; Garrett, T.J.; et al. SPRY domain-containing SOCS box protein 2: Crystal structure and residues critical for protein binding. J. Mol. Biol. 2009, 386, 662-674.

63. Svotelis, A.; Bianco, S.; Madore, J.; Huppe, G.; Nordell-Markovits, A.; Mes-Masson, A.M.; Gevry, N. H3K27 demethylation by JMJD3 at a poised enhancer of anti-apoptotic gene BCL2 determines ERalpha ligand dependency. EMBO J. 2011, 30, 3947-3961.

64. Privalsky, M.L. Regulation of SMRT and N-CoR corepressor function. Curr. Top. Microbiol. Immunol. 2001, 254, 117-136.

65. Marangos, P.J.; Schmechel, D. The neurobiology of the brain enolases. Essays Neurochem. Neuropharmacol. 1980, 4, 211-247.

66. Topfer-Petersen, E.; Cechova, D.; Henschen, A.; Steinberger, M.; Friess, A.E.; Zucker, A. Cell biology of acrosomal proteins. Andrologia 1990, 22, 110-121.

67. Kramer, B.F.; Schoor, O.; Kruger, T.; Reichle, C.; Muller, M.; Weinschenk, T.; Hennenlotter, J.; Stenzl, A.; Rammensee, H.G.; Stevanovic, S. MAGED4-expression in renal cell carcinoma and identification of an HLA-A*25-restricted MHC class I ligand from solid tumor tissue. Cancer Biol. Ther. 2005, 4, 943-948.

68. Kuwahara, K.; Takano, M.; Nakao, K. Pathophysiological significance of T-type $\mathrm{Ca}^{2+}$ channels: Transcriptional regulation of T-type $\mathrm{Ca}^{2+}$ channel-Regulation of CACNA1H by neuron-restrictive silencer factor. J. Pharmacol. Sci. 2005, 99, 211-213. 
69. Shahbazian, D.; Parsyan, A.; Petroulakis, E.; Hershey, J.; Sonenberg, N. eIF4B controls survival and proliferation and is regulated by proto-oncogenic signaling pathways. Cell Cycle 2010, 9, 4106-4109.

70. Bonini, N.M.; Gitler, A.D. Model organisms reveal insight into human neurodegenerative disease: Ataxin-2 intermediate-length polyglutamine expansions are a risk factor for ALS. J. Mol. Neurosci. 2011, 45, 676-683.

71. Rutkowski, D.T.; Kaufman, R.J. All roads lead to ATF4. Dev. Cell 2003, 4, 442-444.

72. Bernier, F.; Soucy, P.; Luu-The, V. Human phenol sulfotransferase gene contains two alternative promoters: Structure and expression of the gene. DNA Cell Biol. 1996, 15, 367-375.

73. Bosley, T.M.; Salih, M.A.; Jen, J.C.; Lin, D.D.; Oystreck, D.; Abu-Amero, K.K.; MacDonald, D.B.; al Zayed, Z.; al Dhalaan, H.; Kansu, T.; et al. Neurologic features of horizontal gaze palsy and progressive scoliosis with mutations in ROBO3. Neurology 2005, 64, 1196-1203.

74. Du, P.; Ye, L.; Li, H.; Yang, Y.; Jiang, W.G. The tumour suppressive role of metastasis suppressor-1, MTSS1, in human kidney cancer, a possible connection with the SHH pathway. J. Exp. Ther. Oncol. 2012, 10, 91-99.

75. Lu, P.; Hankel, I.L.; Hostager, B.S.; Swartzendruber, J.A.; Friedman, A.D.; Brenton, J.L.; Rothman, P.B.; Colgan, J.D. The developmental regulator protein Gon4l associates with protein YY1, co-repressor Sin3a, and histone deacetylase 1 and mediates transcriptional repression. J. Biol. Chem. 2011, 286, 18311-18319.

76. McPherson, J.P.; Sarras, H.; Lemmers, B.; Tamblyn, L.; Migon, E.; Matysiak-Zablocki, E.; Hakem, A.; Azami, S.A.; Cardoso, R.; Fish, J.; et al. Essential role for Bclaf1 in lung development and immune system function. Cell Death Differ. 2009, 16, 331-339.

77. Yi, F.; Pereira, L.; Merrill, B.J. Tcf3 functions as a steady-state limiter of transcriptional programs of mouse embryonic stem cell self-renewal. Stem Cells 2008, 26, 1951-1960.

78. Fong, N.M.; Jensen, T.C.; Shah, A.S.; Parekh, N.N.; Saltiel, A.R.; Brady, M.J. Identification of binding sites on protein targeting to glycogen for enzymes of glycogen metabolism. J. Biol. Chem. 2000, 275, 35034-35039.

79. Ross, S.E.; McCord, A.E.; Jung, C.; Atan, D.; Mok, S.I.; Hemberg, M.; Kim, T.K.; Salogiannis, J.; Hu, L.; Cohen, S.; et al. Bhlhb5 and Prdm8 form a repressor complex involved in neuronal circuit assembly. Neuron 2012, 73, 292-303.

80. Honore, B.; Rasmussen, H.H.; Vorum, H.; Dejgaard, K.; Liu, X.; Gromov, P.; Madsen, P.; Gesser, B.; Tommerup, N.; Celis, J.E. Heterogeneous nuclear ribonucleoproteins H, H', and F are members of a ubiquitously expressed subfamily of related but distinct proteins encoded by genes mapping to different chromosomes. J. Biol. Chem. 1995, 270, 28780-28789.

81. Keegan, K.; Johnson, D.E.; Williams, L.T.; Hayman, M.J. Isolation of an additional member of the fibroblast growth factor receptor family, FGFR-3. Proc. Natl. Acad. Sci. USA 1991, 88, 1095-1099.

82. Jaiswal, B.S.; Kljavin, N.M.; Stawiski, E.W.; Chan, E.; Parikh, C.; Durinck, S.; Chaudhuri, S.; Pujara, K.; Guillory, J.; Edgar, K.A.; et al. Oncogenic ERBB3 mutations in human cancers. Cancer Cell 2013, 23, 603-617. 
83. Mishima, Y.; Miyagi, S.; Saraya, A.; Negishi, M.; Endoh, M.; Endo, T.A.; Toyoda, T.; Shinga, J.; Katsumoto, T.; Chiba, T.; et al. The Hbo1-Brd1/Brpf2 complex is responsible for global acetylation of H3K14 and required for fetal liver erythropoiesis. Blood 2011, 118, 2443-2453.

84. Hatakeyama, S. TRIM proteins and cancer. Nat. Rev. Cancer 2011, 11, 792-804.

85. Wiemann, S.; Weil, B.; Wellenreuther, R.; Gassenhuber, J.; Glassl, S.; Ansorge, W.; Bocher, M.; Blocker, H.; Bauersachs, S.; Blum, H.; et al. Toward a catalog of human genes and proteins: Sequencing and analysis of 500 novel complete protein coding human cDNAs. Genome Res. 2001, 11, 422-435.

86. Maciejewski, J.P.; Padgett, R.A. Defects in spliceosomal machinery: A new pathway of leukaemogenesis. Br. J. Haematol. 2012, 158, 165-173.

87. Sauve, F.; McBroom, L.D.; Gallant, J.; Moraitis, A.N.; Labrie, F.; Giguere, V. CIA, a novel estrogen receptor coactivator with a bifunctional nuclear receptor interacting determinant. Mol. Cell Biol. 2001, 21, 343-353.

88. Lo, S.H.; Weisberg, E.; Chen, L.B. Tensin: A potential link between the cytoskeleton and signal transduction. Bioessays 1994, 16, 817-823.

89. Roubin, R.; Acquaviva, C.; Chevrier, V.; Sedjai, F.; Zyss, D.; Birnbaum, D.; Rosnet, O. Myomegalin is necessary for the formation of centrosomal and Golgi-derived microtubules. Biol. Open 2013, 2, 238-250.

90. Ishov, A.M.; Vladimirova, O.V.; Maul, G.G. Daxx-mediated accumulation of human cytomegalovirus tegument protein pp71 at ND10 facilitates initiation of viral infection at these nuclear domains. J. Virol. 2002, 76, 7705-7712.

91. Morin, R.D.; Mendez-Lago, M.; Mungall, A.J.; Goya, R.; Mungall, K.L.; Corbett, R.D.; Johnson, N.A.; Severson, T.M.; Chiu, R.; Field, M.; et al. Frequent mutation of histone-modifying genes in non-Hodgkin lymphoma. Nature 2011, 476, 298-303.

92. Seimiya, H.; Smith, S. The telomeric poly(ADP-ribose) polymerase, tankyrase 1, contains multiple binding sites for telomeric repeat binding factor 1 (TRF1) and a novel acceptor, 182-kDa tankyrase-binding protein (TAB182). J. Biol. Chem. 2002, 277, 14116-14126.

93. Kuuselo, R.; Savinainen, K.; Azorsa, D.O.; Basu, G.D.; Karhu, R.; Tuzmen, S.; Mousses, S.; Kallioniemi, A. Intersex-like (IXL) is a cell survival regulator in pancreatic cancer with 19q13 amplification. Cancer Res. 2007, 67, 1943-1949.

94. Geiman, D.E.; Ton-That, H.; Johnson, J.M.; Yang, V.W. Transactivation and growth suppression by the gut-enriched Kruppel-like factor (Kruppel-like factor 4) are dependent on acidic amino acid residues and protein-protein interaction. Nucleic Acids Res. 2000, 28, 1106-1113.

95. Wong, P.M.; Puente, C.; Ganley, I.G.; Jiang, X. The ULK1 complex: Sensing nutrient signals for autophagy activation. Autophagy 2013, 9, 124-137.

96. Mayerhofer, P.U.; Kattenfeld, T.; Roscher, A.A.; Muntau, A.C. Two splice variants of human PEX19 exhibit distinct functions in peroxisomal assembly. Biochem. Biophys. Res. Commun. 2002, 291, 1180-1186.

97. Kierdorf, K.; Fritz, G. RAGE regulation and signaling in inflammation and beyond. J. Leukoc. Biol. 2013, 94, 55-68. 
98. Kikuchi, A. Roles of Axin in the Wnt signalling pathway. Cell. Signal. 1999, 11, 777-788.

99. Martignetti, J.A.; Aqeel, A.A.; Sewairi, W.A.; Boumah, C.E.; Kambouris, M.; Mayouf, S.A.; Sheth, K.V.; Eid, W.A.; Dowling, O.; Harris, J.; et al. Mutation of the matrix metalloproteinase 2 gene (MMP2) causes a multicentric osteolysis and arthritis syndrome. Nat. Genet. 2001, 28, 261-265.

100. Stagno, S.; Pass, R.F.; Cloud, G.; Britt, W.J.; Henderson, R.E.; Walton, P.D.; Veren, D.A.; Page, F.; Alford, C.A. Primary cytomegalovirus infection in pregnancy. Incidence, transmission to fetus, and clinical outcome. JAMA 1986, 256, 1904-1908.

101. Revello, M.G.; Gerna, G. Diagnosis and management of human cytomegalovirus infection in the mother, fetus, and newborn infant. Clin. Microbiol. Rev. 2002, 15, 80-715.

102. Revello, M.G.; Zavattoni, M.; Furione, M.; Lilleri, D.; Gorini, G.; Gerna, G. Diagnosis and outcome of preconceptional and periconceptional primary human cytomegalovirus infections. J. Infect. Dis. 2002, 186, 553-557.

103. Ramsay, M.E.; Miller, E.; Peckham, C.S. Outcome of confirmed symptomatic congenital cytomegalovirus infection. Arch. Dis. Child. 1991, 66, 1068-1069.

104. Chou, S.; Marousek, G.; Guentzel, S.; Follansbee, S.E.; Poscher, M.E.; Lalezari, J.P.; Miner, R.C.; Drew, W.L. Evolution of mutations conferring multidrug resistance during prophylaxis and therapy for cytomegalovirus disease. J. Infect. Dis. 1997, 176, 786-789.

105. Anderson, K.P.; Fox, M.C.; Brown-Driver, V.; Martin, M.J.; Azad, R.F. Inhibition of human cytomegalovirus immediate-early gene expression by an antisense oligonucleotide complementary to immediate-early RNA. Antimicrob. Agents Chemother. 1996, 40, 2004-2011.

106. Smith, J.A.; Pari, G.S. Expression of human cytomegalovirus UL36 and UL37 genes is required for viral DNA replication. J. Virol. 1995, 69, 1925-1931.

107. Munch, K.; Messerle, M.; Plachter, B.; Koszinowski, U.H. An acidic region of the 89K murine cytomegalovirus immediate early protein interacts with DNA. J. Gen. Virol. 1992, 73, 499-506. 

Reprinted from Viruses. Cite as: Elbasani, E.; Gabaev, I.; Steinbrück, L.; Messerle, M.; Borst, E.M. Analysis of Essential Viral Gene Functions after Highly Efficient Adenofection of Cells with Cloned Human Cytomegalovirus Genomes. Viruses 2014, 6, 354-370.

Article

\title{
Analysis of Essential Viral Gene Functions after Highly Efficient Adenofection of Cells with Cloned Human Cytomegalovirus Genomes
}

\author{
Endrit Elbasani, Ildar Gabaev, Lars Steinbrück, Martin Messerle and Eva Maria Borst * \\ Department of Virology, Hannover Medical School, Carl-Neuberg-Strasse 1, 30625 Hannover, \\ Germany; E-Mails: elbasani.endrit@mh-hannover.de (E.E.); gabaev.ildar@mh-hannover.de (I.G.); \\ steinbrueck.lars@mh-hannover.de (L.S.); messerle.martin@mh-hannover.de (M.M.) \\ * Author to whom correspondence should be addressed; E-Mail: borst.eva@mh-hannover.de; \\ Tel.: +49-511-5324320; Fax: +49-511-5328736.
}

Received: 14 December 2013; in revised form: 10 January 2014 / Accepted: 13 January 2014 / Published: 23 January 2014

\begin{abstract}
Human cytomegalovirus (HCMV) has a large $240 \mathrm{~kb}$ genome that may encode more than 700 gene products with many of them remaining uncharacterized. Mutagenesis of bacterial artificial chromosome (BAC)-cloned CMV genomes has greatly facilitated the analysis of viral gene functions. However, the roles of essential proteins often remain particularly elusive because their investigation requires the cumbersome establishment of suitable complementation systems. Here, we show that HCMV genomes can be introduced into cells with unprecedented efficiency by applying a transfection protocol based on replication-defective, inactivated adenovirus particles (adenofection). Upon adenofection of several permissive cell types with HCMV genomes carrying mutations in essential genes, transfection rates of up to $60 \%$ were observed and viral proteins of all kinetic classes were found expressed. This enabled further analyses of the transfected cells by standard biochemical techniques. Remarkably, HCMV genomes lacking elements essential for viral DNA replication, such as the lytic origin of replication, still expressed several late proteins. In conclusion, adenofection allows the study of essential HCMV genes directly in BACtransfected cells without the need for sophisticated complementation strategies.
\end{abstract}

Keywords: human cytomegalovirus; bacterial artificial chromosomes; essential viral genes; transfection; adenovirus 


\section{Introduction}

Human cytomegalovirus (HCMV) is still a serious threat for immunocompromised individuals such as AIDS patients and transplant recipients [1,2]. HCMV is also the leading viral cause of birth defects, resulting in severe long-term sequelae, e.g., hearing loss or mental retardation [3]. Currently, there is no effective CMV vaccine in use and the available medication has severe side effects (myelosuppression, nephrotoxicity) and can lead to the emergence of drug-resistant virus strains $[4,5]$. Understanding HCMV gene functions is necessary to identify new antiviral drug targets and for the rational design of a CMV vaccine. The 240-kbp HCMV genome is the largest among all mammalian DNA viruses, and according to a recent study it has the potential to encode more than 700 gene products [6]. The cloning of cytomegalovirus genomes as infectious bacterial artificial chromosomes (BAC) in E. coli and the establishment of suitable mutagenesis techniques has tremendously advanced the investigation into HCMV gene products [7]. Still, especially the roles of essential viral proteins remain largely elusive, which is mainly due to the lack of appropriate complementation systems. Random mutagenesis of HCMV genomes indicated that approximately 40 of the proposed 165 canonical open reading frames (ORF) [8,9] are essential for viral growth in vitro $[10,11]$. Yet, several features of $\mathrm{HCMV}$, e.g., its slow replication cycle and the limited range of cell types supporting efficient growth, have impeded attempts to complement mutants with mutations in essential viral genes. Successful examples include a few cell lines expressing the essential protein of interest [12-16], as well as inducible systems based on tetracycline-regulated transcription [17,18], or the fusion of essential HCMV proteins to a destabilizing domain [19-23]. However, each of these procedures has limitations, such as low virus productivity on the complementing cells, occurrence of escape mutants (rescuants), insufficient tightness of conditional gene regulation, or impairment of viral protein function upon fusion to regulatory domains.

We therefore asked whether it is possible to analyze the phenotypic consequences of the disruption of an essential viral ORF directly in cells transfected with the respective mutant HCMV BACs. Transfection of permissive primary cells with the 240-kbp HCMV BACs is very inefficient, resulting in few transfected cells only, and immortalized cell lines widely employed for complementation of $\alpha$-herpesvirus mutants do not support the full replication cycle of HCMV. Utilizing commercially available transfection reagents, infectious viral progeny can be reconstituted from HCMV BACs, because few successfully transfected cells will give rise to infectious progeny that finally spreads throughout the cultures. However, the transfection efficiencies achieved so far did not allow the analysis of the transfected cells by common virological and biochemical techniques. In this study, we evaluated an alternative method to introduce HCMV BACs into permissive cells, namely an adenovirus-based gene delivery protocol that was pioneered by Matthew Cotten and coworkers [24-27]. Adenovirus particles derived from a replication-deficient mutant are chemically and physically inactivated and serve as carriers for the HCMV BACs, which results in the uptake of the BACs via the natural infection route of the adenovirus. Firstly, the BAC DNA is condensed with cationic low molecular weight 
polyethyleneimine (PEI), yielding a BAC-PEI complex with an overall positive charge. Secondly, this complex is bound to the adenovirus (Ad) particles, presumably through interaction with the negatively charged hexon protein of the viral capsid. By receptor-mediated endocytosis, the BAC-PEI-Ad complexes are then taken up by the cells, and the ability of PEI to act as a proton sponge together with the endosomolytic activity of adenovirus capsid proteins enable the escape of the transfected DNA into the cytosol.

We applied this method, which we termed adenofection, to different HCMV-permissive cell types and used various HCMV BACs in which essential genomic regions had been deleted. The excellent delivery rates of the mutated HCMV genomes, particularly into an epithelial cell line, made it possible to examine the transfected cells for expression, localization, and interactions of viral proteins, as well as for testing of viral DNA replication. In summary, by using the adenofection technique, essential viral gene functions can be assessed immediately after transfection of the HCMV BAC mutants, which obviates the requirement to develop individual complementation strategies.

\section{Results and Discussion}

\subsection{Adenovirus-Mediated Delivery of HCMV BACs into Different Cell Types and Optimization of the Procedure}

Previously, we had already set up the adenofection method (Figure 1A) to reconstitute virus from HCMV BACs in primary human foreskin fibroblasts (HFF) [28]. HFF is the prototype cell type for propagating HCMV, however, these primary cells are particularly difficult to transfect. In these previous experiments, adenofection gave better results than all commercially available transfection reagents we had tried before, yielding approximately 100-200 transfected cells per $3.5 \mathrm{~cm}$ dish. Here, we examined the transfection efficiency of other human cell types after adenofection with an EGFP-expressing HCMV BAC (pHG-13) that does not give rise to infectious progeny due to a deletion in the essential ORF UL104 (see Table 1). Since primary HFF originate from various sources and may therefore vary in their properties, we instead used commercially available primary fetal lung fibroblasts (MRC-5), which in addition are also easier to transfect than HFF [29]. Besides MRC-5, we tested two epithelial cell lines, retinal pigmented epithelial cells having an extended life span due to expression of human telomerase reverse transcriptase (hTERT-RPE-1 cells, further referred to as RPE-1), and A549 cells (a lung adenocarcinoma epithelial cell line) as well as the human glioblastoma cell line U373. All of these cells can be infected with HCMV and support replication of the virus, although with different efficacy. Because the adenofection protocol is sensitive to bacterial lipopolysaccharide (LPS) that is potentially present in plasmid preparations and interferes with DNA-PEI complex formation and can also provoke cytotoxicity in the presence of adenovirus [30], we first compared BAC DNA purified by standard protocols to that obtained by a method removing most of the LPS. Indeed, when using endotoxin-free BAC DNA, transfection rates increased twofold over those obtained with LPS-containing DNA (data not shown), even though the LPS-chelating substance polymyxin B was added during adenofection, which alleviates LPS-mediated toxicity [31]. Therefore, all of the following experiments were done with endotoxin-free BAC DNA. The different cell types were 
adenofected with $1 \mu \mathrm{g}$ of pHG-13 each, and EGFP expression was analyzed the following day by flow cytometry. As can be seen in Figure 1B, transfection of RPE-1 cells was most efficient, with $64 \%$ of cells scoring positive for EGFP, followed by MRC-5 (13\%), A549 (6.5\%) and U373 cells (2\%). To our knowledge, such high delivery rates of HCMV BACs into permissive cells have not been achieved previously by applying any other transfection technique. It is conceivable that by optimizing parameters even higher transfection efficiencies can be achieved. It has for instance been reported that the success of the method greatly depends on the formation of a DNA-PEI complex which is of optimal size and has a positive net charge [27]. The size of these complexes can be tuned by varying the sodium chloride concentration of the HBS solution employed to dilute both DNA and PEI (see Experimental Section), and the charge of the complex is given by the molar ratio of the anionic DNA to polyethylenimine (a polycation). We performed all adenofection experiments in the presence of $150 \mathrm{mM}$ sodium chloride as initially proposed [24,26], which results in larger complexes compared to that prepared in the absence of sodium chloride [27]. If smaller complexes are beneficial to transfection of HCMV BACs remains to be tested. Related to that, we noticed that the transfection of HCMV BACs is enhanced when increasing the amount of PEI 2000. For smaller DNA molecules, maximum delivery was reported when the molar ratio of PEI nitrogen to DNA phosphate $(\mathrm{N}: \mathrm{P})$ was around 10, and was not further improved using higher N:P ratios [27]. However, there is some evidence that larger DNA molecules may require a higher N:P ratio than smaller plasmids [26]. In our HCMV BAC transfection protocol the N:P ratio is $50(1 \mu \mathrm{g}$ of DNA equals $3 \mathrm{nmol}$ of phosphate, and $15 \mu \mathrm{L}$ of $10 \mathrm{mM}$ PEI 2000 solution correspond to $150 \mathrm{nmol}$ of nitrogen). We also tried N:P ratios of 33 and 67 (see below) and did not see a major effect on transfection efficiency, yet lowering the N:P ratio to 20 resulted in a markedly decreased delivery rate (not shown). Therefore, by determining the best relation between sodium chloride, HCMV BAC DNA, PEI 2000, and also the amount of adenovirus particles (i.e., the size and charge of the final PEI-DNA-adenovirus complex that is taken up by the cells) the procedure may be further improved.

Since RPE-1 cells were most efficiently transfected by adenofection, all subsequent experiments were performed with this cell type. Next we tested whether the amount of BAC DNA and the cell confluence have an impact on transfection efficiency. Six different HCMV BACs harboring deletions in essential genomic regions (pHG-11 to pHG-14, pHG- $\Delta 3.7$, and pHG- $\Delta 1.5$; see Table 1) and the parental BAC pHG were used to transfect either $80 \%$ or $100 \%$ confluent RPE-1 cells with $0.75 \mu \mathrm{g}(\mathrm{N}: \mathrm{P}=67)$ or $1.5 \mu \mathrm{g}(\mathrm{N}: \mathrm{P}=33)$ of each BAC DNA, respectively (Figure 2A). One day post transfection, the number of EGFP-expressing cells was determined by flow cytometry. The DNA amounts used had little influence on the number of EGFP-positive cells, whereas (as also true for other transfection protocols) there was a slight improvement in transfection efficiency when using subconfluent cell cultures. Occasionally we observed variable transfection efficiencies (e.g., compare pHG-14 to pHG, white bars in Figure 2A, right part), which is perhaps due to the fact that preparations of high molecular weight DNA such as of the $240 \mathrm{~kb}$ HCMV BACs tend to be inhomogeneous, resulting in differing DNA contents during handling of the samples. Still, in general adenofection allowed the uptake of the HCMV BACs into 40\%-60\% of the RPE-1 cells, which represents an outstanding delivery rate not obtained with any other transfection reagent tested so far [32]. 
Figure 1. (A) Principle of the adenofection method. BAC DNA is condensed with low molecular weight polyethylenimine (PEI 2000). Addition of replication-incompetent, chemically and physically inactivated adenovirus particles leads to binding of the BAC-PEI complexes to the virions. The DNA-loaded adenovirus particles are taken up by the cells, and the endosomolytic activity of virion protein helps to release the DNA into the cytosol; (B) Adenofection of different human cell types with the EGFP-expressing HCMV BAC pHG-13. RPE-1: retinal pigmented epithelial cell line, A549: lung adenocarcinoma epithelial cell line, MRC-5: primary embryonic lung fibroblasts, U373: human astrocytoma cell line. Transfection efficiency is given as percentage of EGFP-positive cells on day 1 post transfection. Transfections were done in quadruplicates, and standard deviations are indicated. Inlay: example of FACS analysis of adenofected RPE-1 cells.
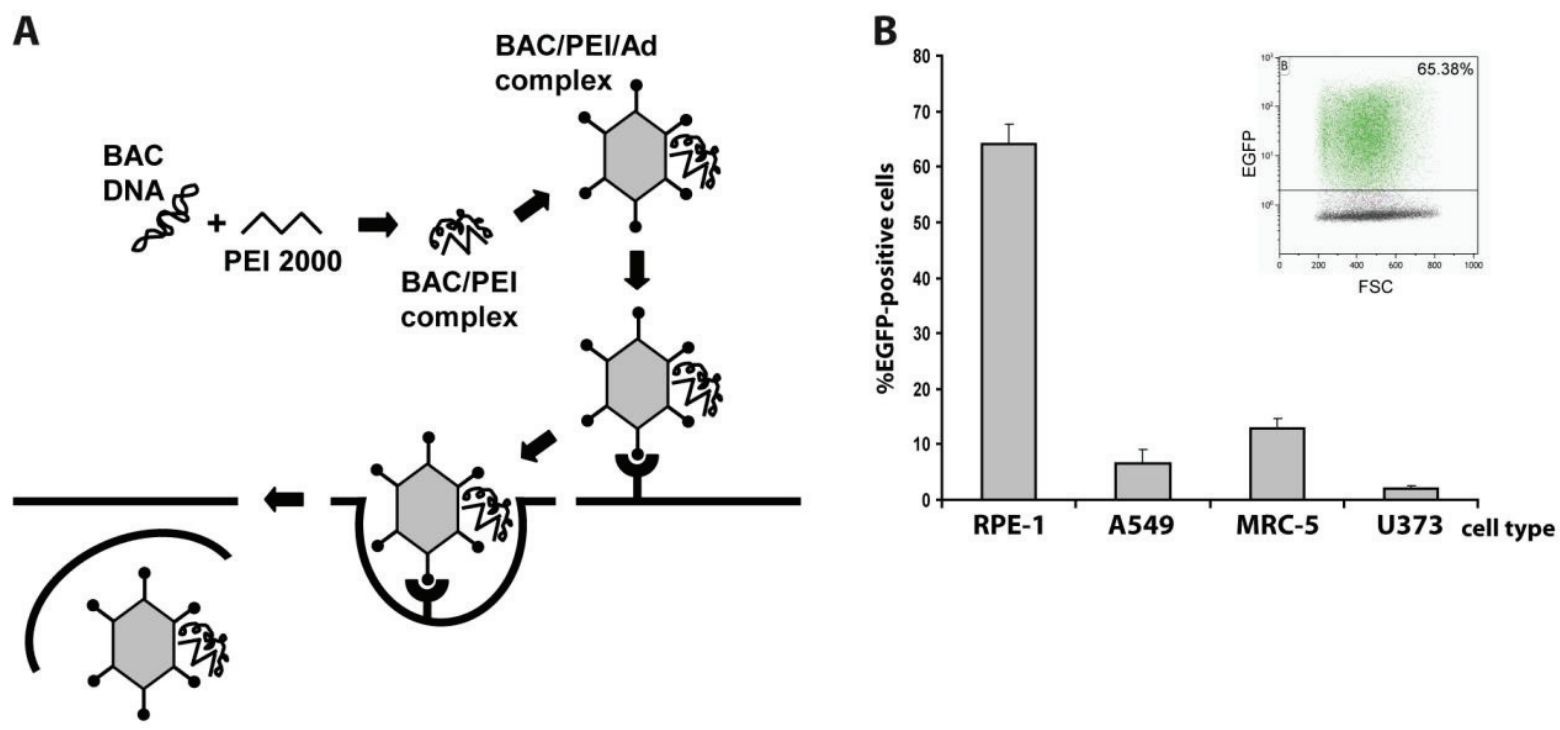

Table 1. HCMV BACs used in this study.

\begin{tabular}{cc}
\hline Name of HCMV BAC & Mutation \\
\hline pHG & EGFP ORF under control of HCMV MIEP \\
(parental BAC) & deletion of UL1-10 [28] \\
pHG-11 & deletion in UL77 \\
pHG-12 & deletion in UL93 \\
pHG-13 & deletion in UL104 \\
pHG-14 & deletion in UL44 \\
pHG- $\Delta 1.5$ & 1.5 kb deletion in oriLyt [28] \\
pHG- $\Delta 3.7$ & 3.7 kb deletion in oriLyt [28] \\
pHG- $\Delta 52$ & deletion in UL52 [14]
\end{tabular}


Figure 2. Evaluation of adenofection conditions and late protein expression in adenofected cells. (A) Subconfluent or confluent RPE-1 cells were transfected with 750 or 1,500 ng of the HCMV BACs indicated. On day 1 post transfection the percentage of EGFP-expressing cells was determined by flow cytometry. Each bar represents the mean value of two transfections; (B) RPE-1 cells transfected with the HCMV BACs indicated were stained on day 4 post transfection with DAPI and an antibody against the major capsid protein (MCP). Ten random images were taken per setting, and the percentages of EGFP-positive cells (white bars) and MCP-expressing cells (grey bars) were determined. The index numbers following the names of the BACs (/1 and /2) indicate that 750 and $1,500 \mathrm{ng}$ of BAC DNA were used for adenofection, respectively.
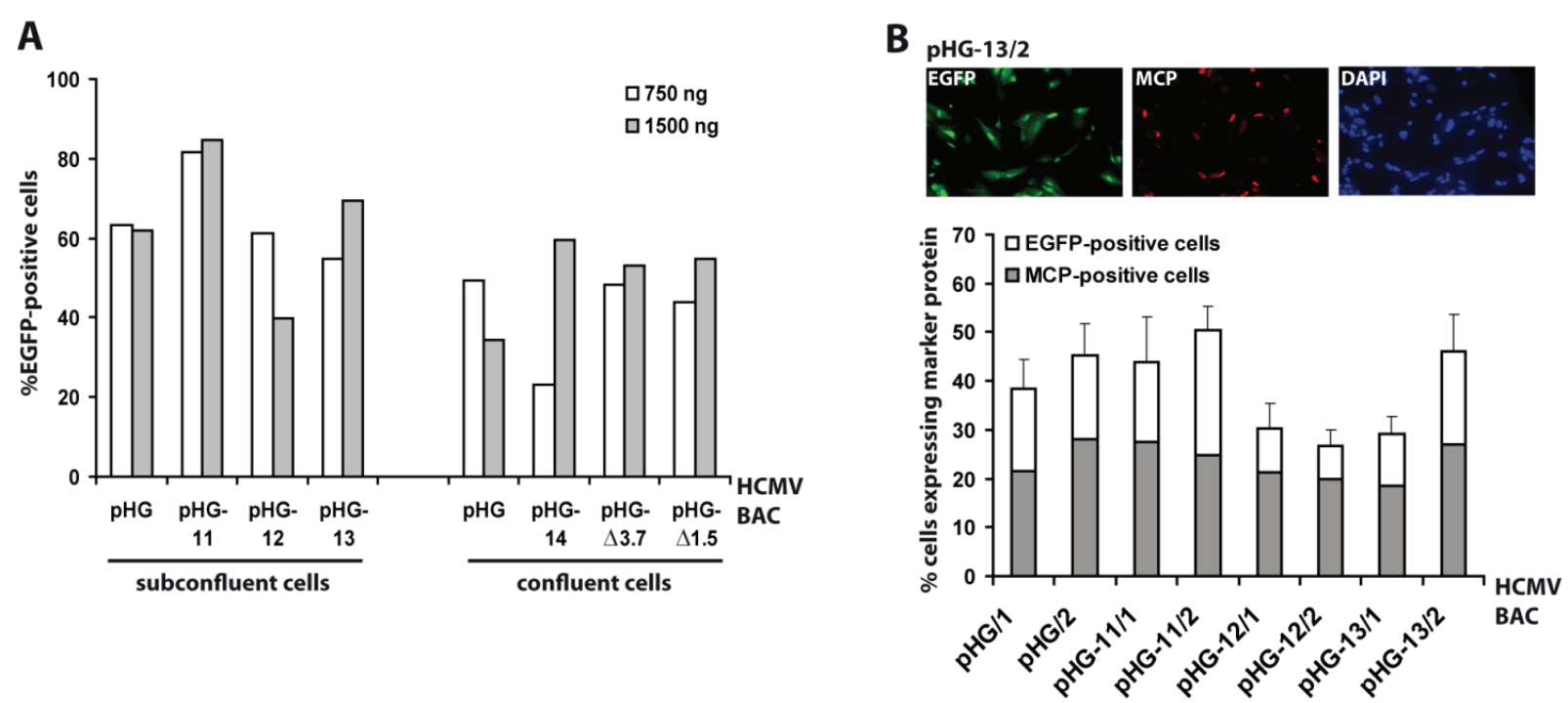

\subsection{Viral Gene Expression in Adenofected Cells}

So far, we measured the outcome of adenofection by detection of EGFP, which is under control of the HCMV major immediate-early promoter (MIEP) [33]. EGFP-expression thus directly represents transfection efficiency, because gene expression driven by the HCMV MIEP occurs very early after transfection (and infection) and is independent of the synthesis of other viral proteins. However, most experiments to be performed with HCMV BAC-transfected cells will not only require the initiation of viral gene expression, but also progression to the early and late phases of the HCMV life cycle. Therefore, we analyzed how many of the EGFP-positive cells proceed to the late phase of the infection cycle. A portion of the cells transfected with $\mathrm{pHG}$ or $\mathrm{pHG}-11$ to $\mathrm{pHG}-13$ (cf. Figure 2A, left part) was seeded onto cover slips and examined on day four post transfection for the presence of EGFP and the major capsid protein (MCP; a late viral protein) by immunofluorescence (Figure 2B). Compared to the data obtained by FACS analysis, transfection efficiency evaluated by fluorescence microscopy gave slightly lower values, probably because the latter technique is the less sensitive one. We found that in the majority of the EGFP-expressing cells MCP was detected as well, and also displayed the expected nuclear localization, with the percentage of MCP-positive cells (related to the EGFP-expressing cells) ranging from 50\% (pHG-11/2) to $75 \%$ (pHG-12/2). This result is similar to what is observed after infection with HCMV, where it is known that not all cells expressing immediate-early proteins also make it into 
the late phase and lead to plaque formation, which can be explained by defective virus particles and/or an unfavorable cellular environment (e.g., failure of viral genomes to overcome intrinsic cellular defense mechanisms such as sequestration at PML bodies). Likewise, it is conceivable that not all of the $240 \mathrm{~kb}$ HCMV BAC molecules remain intact during the DNA preparation and transfection procedure. Moreover, progression through the productive infection cycle may be impaired in the absence of tegument proteins that usually counteract the function of cellular restriction factors early in infection $[34,35]$. This may be overcome by simultaneously transfecting the cells with plasmids encoding the respective tegument proteins, such as pp71 which is known to enhance gene expression from HCMV DNA [36].

Next, we aimed at detecting expression of other viral proteins following adenofection and also included early and additional late proteins. Furthermore, we wanted to investigate late gene expression in the absence of HCMV genome replication. The kinetics of herpesviral protein expression is typically analyzed by keeping infected cells in the presence of inhibitors that interfere with protein synthesis (e.g., cycloheximide, CHX) or DNA replication (phosphonoacetic acid, PAA). Viral genes transcribed in the presence of CHX are classified as immediate-early, and those expressed despite the presence of PAA are considered to be early genes. In contrast, it is believed that late proteins are synthesized only in the absence of any inhibitor, i.e., following viral genome replication. Cells were adenofected with HCMV BACs pHG-14, pHG- $\Delta 1.5$ or pHG- $\Delta 3.7$ to investigate whether late protein synthesis strictly relies on viral replication. $\mathrm{pHG}-14$ has a deletion in the UL44 open reading frame (ORF), whose gene product pUL44 is the accessory factor of the viral DNA polymerase and is required for HCMV lytic origin (oriLyt)-dependent genome replication [37,38]. In pHG- $\Delta 1.5$ and $\mathrm{pHG}-\Delta 3.7$, a $1.5 \mathrm{~kb}$ or $3.7 \mathrm{~kb}$ region of oriLyt was deleted, and upon transfection these BACs do not produce infectious progeny [28]. We first ensured that these BACs have indeed a defect in viral genome replication. Adenofected RPE-1 cells were harvested on days 4 and 5 post transfection, and total DNA was extracted from the cells. The DNA samples were examined by dot blot as well as by restriction analysis, followed by Southern blotting. As is obvious from Figure 3A, viral DNA was only detected in cells transfected with the parental BAC $\mathrm{pHG}$, but not after transfection of $\mathrm{pHG}-14$, $\mathrm{pHG}-\Delta 1.5$, or $\mathrm{pHG}-\Delta 3.7$. We conclude from this data that these HCMV genomes are deficient for viral DNA replication.

We then checked viral protein expression in the adenofected cells by immunoblotting (Figure 3B). The $72 \mathrm{kDa}$ IE1 protein (Figure 3B, panel IE1, lower band) was detected in comparable amounts with all of the mutants as well as with the parental BAC. Interestingly, the higher molecular mass form of IE1, presumably representing sumoylated IE1, was markedly reduced when viral replication was impaired, in line with observations of others which showed that this version of IE1 is rather made during the late phase of the HCMV life cycle [39]. UL44, an early gene product, was expressed by all BACs except pHG-14, in which the UL44 ORF was disrupted. Remarkably, compromising viral replication had different effects on the levels of the other proteins tested, ranging from almost complete absence (UL50, UL53, UL104, SCP, pp150: all late proteins) to more or less reduced, but still clearly detectable amounts (UL52, UL99, MCP, pp65: late proteins, and UL56, UL89: early-late proteins). It is also of note that UL52, which was previously designated as a true late protein due to its complete lack of expression in the presence of PAA $[14,40]$ was found in considerable amounts after transfection of the replication-defective genomes. These data 
may be explained by recent results of Nitzsche and colleagues, who found that viral DNA replication is needed to mark the HCMV genome with methylated histones that define transcriptionally active chromatin, namely H3K4me2 [23]. Most interestingly, the tested HCMV genomic regions were differentially influenced by impairing viral replication, for instance occupancy of UL32 sequences (coding for pp150) with H3K4me2 was much more dependent on HCMV genome replication than that of the UL99 gene, which was still labeled with this activating histone mark quite efficiently in both the presence of ganciclovir and after knock-down of the viral polymerase. This is consistent with the expression of the UL99 protein and the absence of pp 150 (UL32) in cells transfected with HCMV BACs defective for genome replication. A similar mechanism may apply to the other HCMV proteins tested by us in this study.

Figure 3. Analysis of viral genome replication and protein expression in adenofected cells. (A) Viral DNA levels in RPE-1 cells adenofected with the indicated HCMV BACs. Total DNA was isolated on days four and five post transfection and analyzed by dot blot hybridization using a UL93-specific probe (left part). Right part: the DNA samples obtained on day five were treated with HindIII and DpnI, followed by Southern blotting. The ${ }^{32} \mathrm{P}$-labelled probe specific for the UL93 ORF detects a $7.5 \mathrm{~kb}$ HindIII fragment; (B) Viral protein expression after adenofection with the depicted HCMV BACs. Cell lysates were prepared on day four post transfection and analyzed for the given proteins by immunoblotting.
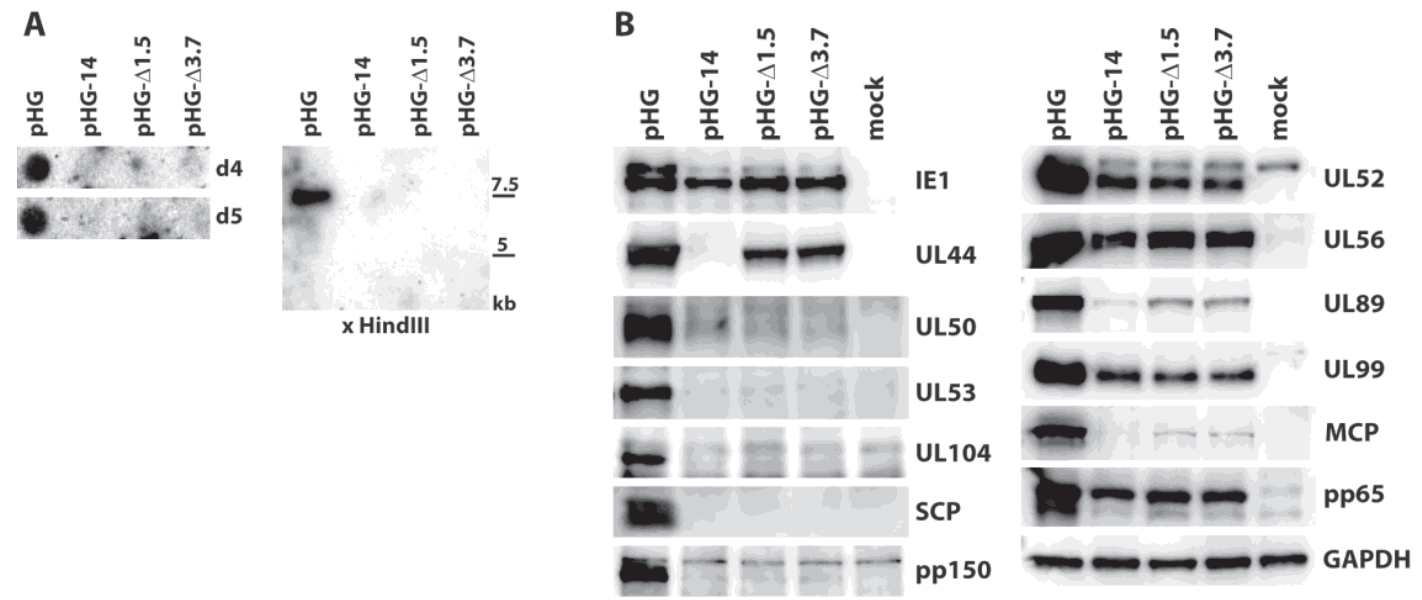

\subsection{Impact of UL52 on HCMV Terminase Proteins}

Besides genome replication, the nuclear phase of the HCMV life cycle comprises the assembly of capsid shells, as well as packaging of viral genomes into these preformed capsids. HCMV proteins mediating genome cleavage and encapsidation are the terminase subunits UL56 and UL89. We have recently demonstrated that UL51 is also essential for cleavage of viral concatemeric DNA and the generation of DNA-filled capsids. Moreover, UL51 forms a complex with UL56 and UL89 and seems to be required for the correct localization of UL56 and UL89 to nuclear replication compartments [40,41]. Likewise, we have identified an essential role of UL52 in genome cleavage-packaging, but there was no evidence for UL52 being also part of the terminase complex $[14,40]$. The precise function of UL52 thus remains elusive. 
Figure 4. Investigation into RPE-1 cells adenofected with the UL52-deleted genome pHG- $\Delta 52$. (A) Transfected cells were harvested on days zero to six post transfection (dpt) and expression of the indicated proteins was analyzed by immunoblotting. Please note that the pp65 antibody exhibits some cross-reactivity with cellular proteins as observed previously [42]; (B) Interaction of the terminase subunits UL56 and UL89 in the absence of UL52. RPE-1 cells adenofected with pHG- $\Delta 52$ were subjected to immunoprecipitation with $\alpha$-UL56 and $\alpha$-UL89 monoclonal antibodies on day 4 post transfection, followed by immunoblotting using the same antibodies. Input: whole cell lysate before IP, hc: IgG heavy chain; (C) Subcellular localization of HCMV terminase subunits in the presence or absence of UL52. RPE-1 cells were adenofected with pHG or pHG- $\triangle 52$ and analyzed on day 4 post transfection by confocal laser scanning microscopy using monoclonal antibodies against UL51, UL56, and UL89.
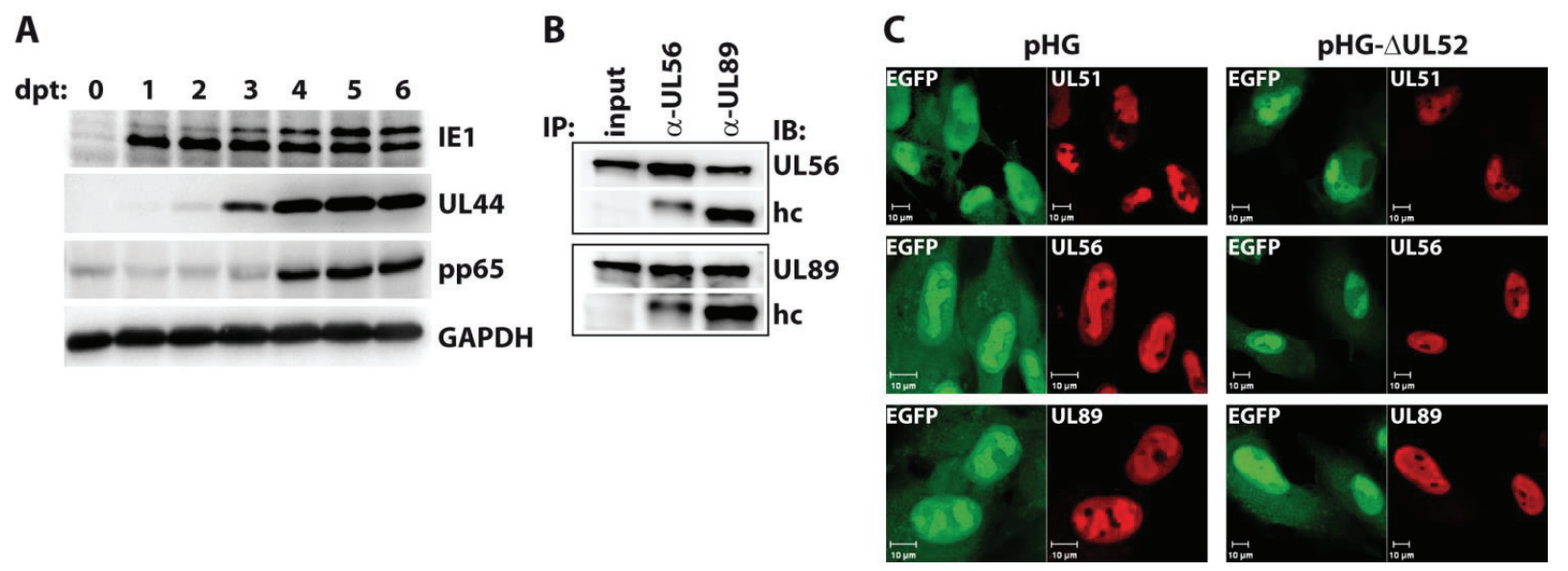

We now asked whether the absence of UL52 may influence the interactions among the terminase subunits or their subnuclear localization. To this end, RPE-1 cells were adenofected with the BAC genome pHG- $\triangle$ UL52, which carries a disrupted UL52 ORF. First, we checked the expression kinetics of representative immediate-early (IE1), early (UL44) and late proteins (pp65) in the transfected cells. Cells were harvested on days 0 to 6 post transfection, and viral proteins were analyzed by immunoblotting. As is obvious from Figure 4A, IE1, UL44 and pp65 were expressed with the expected kinetics, with IE1 already being detectable on day 1, UL44 coming up on day 2, and pp65 being clearly visible on day 4 and later. The higher molecular mass form of IE1 was detected in distinct amounts on day 3 and later only, which is in agreement with our finding that the abundance of this version of IE1 seems to be increased following viral replication (cf. Figure 3B). To investigate the interaction of the terminase subunits, pHG- $\Delta$ UL52-transfected cells were lysed on day four and subjected to immunoprecipitation using monoclonal antibodies (mAb) directed against UL56 and UL89 [40]. Figure 4B shows that the binding of UL56 to UL89 was still seen when UL52 was missing, since immunoprecipitation of UL56 also brought down UL89 (Figure 4B, second lane), and vice versa (Figure 4B, third lane). We could not test for binding to UL51 in this setting, because the UL51 mAb exhibits no distinct reactivity in immunoblotting. Since the UL51 antibody performs well in immunofluorescence experiments, we examined the subcellular distribution of UL51, UL56 and UL89 in the absence of UL52 by confocal laser scanning microscopy in RPE-1 cells transfected with pHG- $\Delta$ UL52 or the parental 
BAC pHG. As can be seen in Figure 4C, the terminase subunits were all found in the cell nucleus and were enriched within the replication compartments, despite the lack of UL52 (Figure 4C, right part), as is the case in cells transfected with the parental BAC genome pHG (Figure 4C, left part). Thus, UL52 does not seem to play a role in the interactions among the HCMV terminase proteins or their correct intracellular localization.

\section{Experimental Section}

\subsection{Cells}

hTERT-RPE-1 cells were obtained from Clontech and were cultivated in Dulbecco's modified Eagle's medium (DMEM)/Ham's F-12 (Sigma, Saint Louis, MO, USA, D6421), supplemented with $5 \%$ fetal calf serum (FCS), $100 \mathrm{U} / \mathrm{mL}$ of penicillin, $100 \mu \mathrm{g} / \mathrm{mL}$ of streptomycin sulfate, $2 \mathrm{mM}$ glutamine, and $0.348 \%$ sodium bicarbonate. A549 and MRC-5 cells (ATCC) were kept in DMEM (Sigma, D5796), containing 10\% FCS and the mentioned antibiotics. The U-373 MG (Uppsala) cell line was purchased from Sigma (Cat. No. 08061901) and corresponds to a new deposit of the cells from the laboratory in Uppsala, Sweden, where they were originally isolated. U-373 MG cells were cultivated according to the instructions of the supplier.

\subsection{HCMV Bacterial Artificial Chromosomes}

The parental HCMV BAC pHG, carrying the enhanced green fluorescent protein (EGFP) ORF under control of the major immediate-early promoter (MIEP) and containing a deletion of the non-essential genes UL1 to UL10 was described before [28,33]. The construction of pHG- $\Delta 52$ lacking most of the UL52 open reading frame (ORF) was reported elsewhere [14], and pHG- $\Delta 1.5$ and pHG- $\Delta 3.7$ harboring deletions in the HCMV lytic origin region have also been described [28]. pHG-11, pHG-13 and pHG-14, having deletions in essential viral genes UL77, UL104 and UL44, respectively, were generated by red $\alpha \beta \gamma$-mediated recombination in E. coli as described [43]. Oligonucleotides used for mutagenesis are given in Table 2, and comprised sequences homologous to the desired integration site in the BAC, as well as priming regions to the kanamycin resistance (KnR) gene flanked by mutant FRT sites [28]. The KnR sequence was subsequently removed from all BACs by Flp-mediated recombination as reported [43]. pHG-12, in which UL93 is disrupted, was constructed by en passant mutagenesis using the primers indicated in Table 2, with a KnR cassette carrying an I-SceI site as a template [44]. Further details on the construction of these BACs will be available from the authors upon request. Large-scale purification of HCMV BACs was done either with the Nucleobond PC 100 columns, or by using the Nucleobond PC 500 EF kit (both Macherey \& Nagel, Düren, Germany), which yields mostly endotoxin-free DNA. BAC DNA was resuspended in TE buffer (10 $\mathrm{mM}$ Tris- $\mathrm{HCl} \mathrm{pH}$ 8.0/1 mM EDTA) and DNA concentrations were determined by measuring each preparation three times using a Nanodrop device. 
Table 2. Oligonucleotides used for HCMV BAC mutagenesis.

\begin{tabular}{|c|c|}
\hline Primer name & Nucleotide sequence \\
\hline UL77-ko.for & 5'-acgatgccatcacgggaccegccgecgecccgtctgacgtggaaaagtgccacctgcagat-3' \\
\hline UL77-ko.rev & 5'-ccgaggacgttcgccetttatgcagcgagcgacacgtggtgcaggaacacttaacggctga-3' \\
\hline UL104-ko.for & 5'-gctgtcgtcgtaggcggcggccacgatctcgccgaaggagagaaaagtgccacctgcagat-3' \\
\hline UL104-ko.rev & 5'-gtgctgggcggectccgcgacattttatatcagtacgccgcaggaacacttaacggctga-3' \\
\hline UL44-ko.for & 5'-gggaccgcgecgtgcgegegttcccaggcacgcggcccgcgaaaagtgccacetgcagat-3' \\
\hline UL44-ko.rev & 5'-tcgctcgctcgcgcccgctccttagtcgagacttgcacgctcaggaacacttaacggctga-3' \\
\hline UL93-ko.for & $\begin{array}{l}5 \text { '-agtcgaactataccgggcgttggacgcttatcgggegtagtgactgattcgegccgtgcgcaa- } \\
\text { ggatgacgacgataag-3' }\end{array}$ \\
\hline UL93-ko.rev & $\begin{array}{l}\text { 5'-agtacagcgtagatctcgtcgegcacggegegaatcagtcactacgecegataagcgtccagcc-agtgttacaa- } \\
\text { ccaatt-3' }\end{array}$ \\
\hline
\end{tabular}

\subsection{Preparation of Adenovirus Stocks and Transfection by Adenofection}

The adenovirus d11014 mutant [45], having a deletion in the E4 region, and the complementing cell line W162 [46] were kindly provided by Gary Ketner, Johns Hopkins University School of Medicine, MD, USA. To produce the adenovirus stock, ten T175 flasks of W162 cells were infected at an MOI of 0.01-0.1. At approximately day three post infection, when all cells were rounded and detached, cells were collected by centrifugation and the pellet was transferred to $-80{ }^{\circ} \mathrm{C}$. Virus was released by adding $5 \mathrm{~mL}$ of serum-free DMEM, followed by two freeze-thaw cycles. Cellular debris was removed by centrifugation at 3,800 $\times \mathrm{g}$ for $20 \mathrm{~min}$, and virus in the supernatant was purified by two rounds of isopycnic cesium chloride gradient centrifugation. For the first centrifugation step, $3 \mathrm{~mL}$ of a $\mathrm{CsCl}$ solution with a density of $1.2 \mathrm{~g} / \mathrm{mL}$ was carefully added to a tube containing $3 \mathrm{~mL}$ of a $\mathrm{CsCl}$ solution with a density of $1.4 \mathrm{~g} / \mathrm{mL}$. Virus-containing supernatant was layered on top, and centrifugation was done for $90 \mathrm{~min}$ at $100,000 \times \mathrm{g}, 20{ }^{\circ} \mathrm{C}$ in an SW41 Ti rotor, without deceleration. The virus-containing band was identified by light scattering and removed by puncturing the tube with a hollow needle, and was then diluted to a total volume of $2 \mathrm{~mL}$ with $10 \mathrm{mM}$ Tris-HCl/1 mM EDTA pH 7.5. The second purification step was done via a continuous $\mathrm{CsCl}$ gradient prepared by mixing $5.4 \mathrm{~mL}$ of $\mathrm{CsCl}$ solution density 1.2 with $4.6 \mathrm{~mL}$ of $\mathrm{CsCl}$ solution density $1.4 \mathrm{using}$ a gradient maker, and centrifugation took place for $24 \mathrm{~h}$ at $100,000 \times \mathrm{g}$, $20{ }^{\circ} \mathrm{C}$ without deceleration. After harvesting of the virus band as described above, the samples were desalted using PD-10 columns (GE Healthcare, Uppsala, Sweden), and fractions of $0.5 \mathrm{~mL}$ each were collected by elution in $\mathrm{HBS} / 40 \%$ glycerol (HBS is $20 \mathrm{mM}$ HEPES, $150 \mathrm{mM} \mathrm{NaCl}$ $\mathrm{pH}$ 7.4). Virus-containing fractions were identified by measuring protein concentrations with the Pierce BCA protein assay kit (Thermo Scientific, Rockford, IL, USA), and protein-positive fractions were pooled. To inactivate the virus, 8-methoxy-psoralen (Sigma, M-3501) was added to a final concentration of $0.33 \mathrm{mg} / \mathrm{mL}$, the sample was transferred on ice and exposed to UV light $(366 \mathrm{~nm})$ for $30 \mathrm{~min}$, with rotation of the dish every $10 \mathrm{~min}$. The inactivated virus particles were again purified by PD-10 columns, and fractions scoring positive in the BCA protein assay were pooled and stored at $-80{ }^{\circ} \mathrm{C}$ in $\mathrm{HBS} / 40 \%$ glycerol. Concentration of adenovirus particles was determined by calculating $0.3 \mathrm{mg} / \mathrm{mL}$ of (capsid) protein being equivalent to $1 \times 10^{12}$ particles $/ \mathrm{mL}$. 
For adenofection of HCMV BACs, the DNA amounts indicated in the figures were diluted in $250 \mu \mathrm{L}$ of HBS. $15 \mu \mathrm{L}$ of $10 \mathrm{mM}$ PEI 2000 stock solution (polyethylenimine MW 2000, Sigma, Cat. No. 40870-0, $0.9 \mathrm{mg} / \mathrm{mL} \mathrm{H}_{2} \mathrm{O}$ ), mixed before usage, was diluted in $250 \mu \mathrm{L}$ of HBS and mixed vigorously again. The diluted PEI 2000 solution was slowly added to the diluted DNA, with constant gentle flicking of the tube, and the sample was incubated for $20 \mathrm{~min}$ at room temperature (RT). $3 \times 10^{9}$ particles of inactivated adenovirus were added, and the sample was incubated at RT for another $20 \mathrm{~min}$. Cells cultured in six-well plates were washed with PBS, and $1.5 \mathrm{~mL} / \mathrm{well}$ of serum-free DMEM containing polymyxin B $(30 \mu \mathrm{g} / \mathrm{mL})$ was added. The transfection complexes were added to the cells, which were then incubated for $5 \mathrm{~h}$ at $37{ }^{\circ} \mathrm{C}, 5 \% \mathrm{CO}_{2}$. After that, the cells were washed twice with PBS and further cultivated in complete growth medium with polymyxin B $(30 \mu \mathrm{g} / \mathrm{mL})$.

\subsection{Flow Cytometry}

Cells were adenofected with the HCMV BAC genomes indicated in the figure legends. One day later, cells were trypsinized, washed with PBS containing $2 \mathrm{mM}$ EDTA, and fixed with 3\% paraformaldehyde $(w / v)$ in PBS/2mM EDTA. One-color flow cytometry was applied to detect GFP-positive cells. A total of 30,000 events were collected by a Beckman Coulter FC500 flow cytometer and analyzed using the Kaluza software (Beckman Coulter, Brea, CA, USA) [47].

\subsection{Viral DNA Replication Assay}

RPE-1 cells adenofected with the respective HCMV BACs were trypsinized on days four or five post transfection, and total DNA was isolated using the DNeasy Blood and Tissue kit (Qiagen, Hilden, Germany). For dot blot, $5 \mu \mathrm{L}$ of each sample (corresponding to approximately $30 \mathrm{ng}$ of total DNA each) was applied to a nylon membrane. Denaturing of the DNAs was achieved by exposing the membrane to filter paper soaked with $0.5 \mathrm{M} \mathrm{NaOH} / 1.5 \mathrm{M} \mathrm{NaCl}$ twice for three min each, followed by neutralization of the membrane four times for three min each using $0.5 \mathrm{M}$ Tris- $\mathrm{HCl} / 1.5 \mathrm{M} \mathrm{NaCl} \mathrm{pH}$ 7.2. For restriction analysis, $40 \mu \mathrm{L}$ of each DNA sample obtained on day five (corresponding to approximately $240 \mathrm{ng}$ of total DNA each) was treated with DpnI to cut residual transfected BAC DNA, followed by treatment with HindIII. After gel electrophoresis, the DNAs were transferred to a nylon membrane by Southern blotting, and hybridized to a ${ }^{32} \mathrm{P}$-labelled probe generated by PCR amplification of HCMV UL93 sequences using primers UL93-N.for (5'-cgcggatccttctatgccgtcttcactacg-3') and UL93-N.rev (5'-cgcaagcttgcgactgcgccaaaaggaatt-3') with HCMV BAC pHG as a template.

\subsection{Protein Biochemistry}

Immunofluorescence microscopy of adenofected cells was done as described previously [14], and immunoprecipitation as well as immunoblotting was performed as reported recently [40]. Additionally, the SuperSignal Western Blot Enhancer reagents (Thermo Scientific, Cat. No. 46640) and the SuperSignal West Femto Maximum Sensitivity Substrate (Thermo Scientific, Cat. No. 34096) were applied. Antibodies commercially available were directed against HCMV IE1 (NEA-9221, PerkinElmer, Boston, MA, USA), UL99 (Fitzgerald, MA, USA, Cat. No. 10-C50), 
and pp65 (Novus Biologicals, Littleton, CO, USA, Cat. No. NB110-57244). The antibody recognizing the major capsid protein was kindly provided by Klaus Radsak, UL44 and pp150 monoclonal antibodies were a gift of Bodo Plachter, University of Mainz, Germany, UL50 and UL53 antibodies were obtained from Manfred Marschall, University of Erlangen, Germany, and the monoclonal antibody directed against the HCMV small capsid protein was provided by William Britt, Birmingham, Alabama. The monoclonal antibodies specific for UL51, UL52, UL56, and UL89 are described elsewhere [40]. The UL104-specific antibody was generated by immunization of mice as described [40], using a recombinant protein comprising the N-terminal part of pUL104. Primers for amplification of UL104 sequences were UL104-N.for (5'-cgcaagcttggcgtactgatataaaatgt-3') and UL104-N.rev (5'-cgcggatccgagcgaaaccactggaacga-3'). The resulting PCR product was cut with HindIII and BamHI and cloned into the bacterial expression vector pQE-30 via the same restriction sites.

\section{Conclusions}

In this work, we present a novel approach to analyze essential HCMV gene functions by transfecting cells with BACs that are deleted for the gene of interest. Transfection is based on applying replication-deficient, inactivated adenovirus particles as carriers, a method that was initially developed by Cotten and coworkers for BACs of up to $170 \mathrm{~kb}$, and that proved to be superior to commercially available transfection reagents $[26,27]$. We now show that this approach works also perfectly well with HCMV BACs, which are of larger size (240 kbp). We used particles of an adenovirus mutant that has a deletion in the essential E4 region and is propagated on a complementing cell line. However, other adenovirus mutants may be employed as well, such as d1312 lacking E1a sequences and which can be grown on 293 cells [24], although under certain conditions cellular factors can substitute for E1a functions, resulting in toxicity due to low level adenoviral gene expression [25]. Due to the natural tropism of human adenovirus the adenofection method is currently restricted to human cells. However, it is conceivable that the protocol can be extended to BAC-cloned herpesviruses of other species, for instance mouse adenovirus mutants could be generated and tested for adenofection of murine cells. Transfection can also be performed through double condensation of BAC DNA with low and high molecular weight PEI only [48]. In our hands, this approach also gave good transfection efficiencies (data not shown), although the high transfection rates described here $(40 \%-60 \%)$ were only reached upon including adenovirus particles.

The excellent transfection efficiencies with HCMV BACs found after adenofection have not been possible before with other transfection methods applied so far. RPE-1 cells performed best in these experiments, which is of note because this cell type is also useful to propagate clinical HCMV isolates [49]. This enables faster reconstitution of viral progeny from HCMV BAC genomes, which could be of advantage for high throughput analysis of a library of HCMV mutants. Furthermore, the new delivery approach allows the study of the phenotypic consequences of the lack of given essential proteins by various techniques such as immunofluorescence, immunoblotting, or immunoprecipitation. Other assays, for instance pulsed field gel electrophoresis of viral DNA or electron microscopy, are also feasible, especially when the adenofected cells are sorted for EGFP-positive cells before applying the respective techniques. We think that virtually any 
investigation can be done with BAC-transfected cells, at least as steps of the infection cycle subsequent to delivery of the viral genomes into the nucleus are concerned. Although RNAs and virion proteins delivered by the HCMV particle, which can enhance viral gene expression are absent following adenofection, all viral proteins tested were produced in sufficient amounts to perform downstream analyses. We consider the adenofection procedure as safe since infectious adenovirus was not observed in the transfection experiments. Moreover, recombination between adenoviral and HCMV DNA appears highly unlikely, because (i) the genomes do not share significant homologous regions; (ii) the adenovirus DNA is cross-linked by treatment with psoralen and UV light and is thus inert; and (iii) there is no selection pressure on the occurrence of recombination.

In summary, adenofection of HCMV BACs enables the analysis of essential viral genes directly after transfection, without the need to establish complementing systems for the HCMV mutants of interest.

\section{Acknowledgments}

We thank Matthew Cotten for sharing protocols and reagents as well as Gary Ketner (Johns Hopkins University School of Medicine) for providing the adenovirus mutant d11014 and complementing W162 cells. Confocal microscopy was done in the Research Core Unit for Laser Microscopy at the Hannover Medical School.

This work was supported by individual grants ME1102/3-1 and BO4196/1-2 (to M.M. and E.M.B.) and a grant of the International Research Training Group 1273 (to E.E. and M.M.) from the Deutsche Forschungsgemeinschaft. I.G. was supported by the Impuls- und Vernetzungsfond of the Helmholtz Association, VISTRIE grant VH-VI-424-4 (to M.M.).

\section{Author Contributions}

EMB, IG and MM designed the experiments; EE, LS and EMB performed the experiments and analyzed data. EMB and MM prepared the manuscript. All authors read and approved the final manuscript.

\section{Conflicts of Interest}

The authors declare no conflict of interest.

\section{References and Notes}

1. Griffiths, P.D. Burden of disease associated with human cytomegalovirus and prospects for elimination by universal immunisation. Lancet Infect. Dis. 2012, 12, 790-798.

2. Ljungman, P.; Hakki, M.; Boeckh, M. Cytomegalovirus in hematopoietic stem cell transplant recipients. Infect. Dis. Clin. N. Am. 2010, 24, 319-337.

3. Nigro, G.; Adler, S.P. Cytomegalovirus infections during pregnancy. Curr. Opin. Obstet. Gynecol. 2011, 23, 123-128.

4. Biron, K.K. Antiviral drugs for cytomegalovirus diseases. Antivir. Res. 2006, 71, 154-163. 
5. Le Page, A.K.; Jager, M.M.; Iwasenko, J.M.; Scott, G.M.; Alain, S.; Rawlinson, W.D. Clinical aspects of cytomegalovirus antiviral resistance in solid organ transplant recipients. Clin. Infect. Dis. 2013, 56, 1018-1029.

6. Stern-Ginossar, N.; Weisburd, B.; Michalski, A.; Le, V.T.; Hein, M.Y.; Huang, S.X.; Ma, M.; Shen, B.; Qian, S.B.; Hengel, H.; et al. Decoding human cytomegalovirus. Science 2012, 338, $1088-1093$.

7. Ruzsics, Z.; Borst, E.M.; Bosse, J.B.; Brune, W.; Messerle, M. Manipulating cytomegalovirus genomes by BAC mutagenesis: Strategies and applications. In Cytomegaloviruses: From Molecular Pathogenesis to Intervention; Reddehase, M.J., Ed.; Caister Academic Press: Hethersett, Norwich, UK, 2013; pp. 37-57.

8. Davison, A.J.; Dolan, A.; Akter, P.; Addison, C.; Dargan, D.J.; Alcendor, D.J.; McGeoch, D.J.; Hayward, G.S. The human cytomegalovirus genome revisited: Comparison with the chimpanzee cytomegalovirus genome. J. Gen. Virol. 2003, 84, 17-28.

9. Dolan, A.; Cunningham, C.; Hector, R.D.; Hassan-Walker, A.F.; Lee, L.; Addison, C.; Dargan, D.J.; McGeoch, D.J.; Gatherer, D.; Emery, V.C.; et al. Genetic content of wild-type human cytomegalovirus. J. Gen. Virol. 2004, 85, 1301-1312.

10. Yu, D.; Silva, M.C.; Shenk, T. Functional map of human cytomegalovirus AD169 defined by global mutational analysis. Proc. Natl. Acad. Sci. USA 2003, 100, 12396-12401.

11. Dunn, W.; Chou, C.; Li, H.; Hai, R.; Patterson, D.; Stolc, V.; Zhu, H.; Liu, F. Functional profiling of a human cytomegalovirus genome. Proc. Natl. Acad. Sci. USA 2003, 100, $14223-14228$.

12. Mocarski, E.S.; Kemble, G.W.; Lyle, J.M.; Greaves, R.F. A deletion mutant in the human cytomegalovirus gene encoding IE1(491aa) is replication defective due to a failure in autoregulation. Proc. Natl. Acad. Sci. USA 1996, 93, 11321-11326.

13. Silva, M.C.; Yu, Q.C.; Enquist, L.; Shenk, T. Human cytomegalovirus UL99-encoded pp28 is required for the cytoplasmic envelopment of tegument-associated capsids. J. Virol. 2003, 77, 10594-10605.

14. Borst, E.M.; Wagner, K.; Binz, A.; Sodeik, B.; Messerle, M. The essential human cytomegalovirus gene UL52 is required for cleavage-packaging of the viral genome. J. Virol. 2008, 82, 2065-2078.

15. Sanders, R.L.; Clark, C.L.; Morello, C.S.; Spector, D.H. Development of cell lines that provide tightly controlled temporal translation of the human cytomegalovirus IE2 proteins for complementation and functional analyses of growth-impaired and nonviable IE2 mutant viruses. J. Virol. 2008, 82, 7059-7077.

16. Mohr, H.; Mohr, C.A.; Schneider, M.R.; Scrivano, L.; Adler, B.; Kraner-Schreiber, S.; Schnieke, A.; Dahlhoff, M.; Wolf, E.; Koszinowski, U.H.; et al. Cytomegalovirus replicon-based regulation of gene expression in vitro and in vivo. PLoS Pathog. 2012, 8, e1002728.

17. McVoy, M.A.; Mocarski, E.S. Tetracycline-mediated regulation of gene expression within the human cytomegalovirus genome. Virology 1999, 258, 295-303.

18. Rupp, B.; Ruzsics, Z.; Sacher, T.; Koszinowski, U.H. Conditional cytomegalovirus replication in vitro and in vivo. J. Virol. 2005, 79, 486-494. 
19. Glass, M.; Busche, A.; Wagner, K.; Messerle, M.; Borst, E.M. Conditional and reversible disruption of essential herpesvirus proteins. Nat. Methods 2009, 6, 577-579.

20. Qian, Z.; Leung-Pineda, V.; Xuan, B.; Piwnica-Worms, H.; Yu, D. Human cytomegalovirus protein pUL117 targets the mini-chromosome maintenance complex and suppresses cellular DNA synthesis. PLoS Pathog. 2010, 6, e1000814.

21. Perng, Y.C.; Qian, Z.; Fehr, A.R.; Xuan, B.; Yu, D. The human cytomegalovirus gene UL79 is required for the accumulation of late viral transcripts. J. Virol. 2011, 85, 4841-4852.

22. Tandon, R.; Mocarski, E.S. Cytomegalovirus pUL96 is critical for the stability of pp150-associated nucleocapsids. J. Virol. 2011, 85, 7129-7141.

23. Nitzsche, A.; Steinhausser, C.; Mucke, K.; Paulus, C.; Nevels, M. Histone H3 lysine 4 methylation marks postreplicative human cytomegalovirus chromatin. J. Virol. 2012, 86, 9817-9827.

24. Cotten, M.; Wagner, E.; Zatloukal, K.; Phillips, S.; Curiel, D.T.; Birnstiel, M.L. High-efficiency receptor-mediated delivery of small and large (48 kilobase gene constructs using the endosome-disruption activity of defective or chemically inactivated adenovirus particles. Proc. Natl. Acad. Sci. USA 1992, 89, 6094-6098.

25. Cotten, M. Adenovirus-augmented, receptor-mediated gene delivery and some solutions to the common toxicity problems. Curr. Top. Microbiol. Immunol. 1995, 199, 283-295.

26. Baker, A.; Cotten, M. Delivery of bacterial artificial chromosomes into mammalian cells with psoralen-inactivated adenovirus carrier. Nucleic Acids Res. 1997, 25, 1950-1956.

27. Baker, A.; Saltik, M.; Lehrmann, H.; Killisch, I.; Mautner, V.; Lamm, G.; Christofori, G.; Cotten, M. Polyethylenimine (PEI) is a simple, inexpensive and effective reagent for condensing and linking plasmid DNA to adenovirus for gene delivery. Gene Ther. 1997, 4, 773-782.

28. Borst, E.M.; Messerle, M. Analysis of human cytomegalovirus oriLyt sequence requirements in the context of the viral genome. J. Virol. 2005, 79, 3615-3626.

29. Borst, E. Department of Virology, Hannover Medical School, Hannover, Germany. Unpublished observation, 2013.

30. Cotten, M.; Baker, A.; Saltik, M.; Wagner, E.; Buschle, M. Lipopolysaccharide is a frequent contaminant of plasmid DNA preparations and can be toxic to primary human cells in the presence of adenovirus. Gene Ther. 1994, 1, 239-246.

31. Cotten, M.; Saltik, M. Intracellular delivery of lipopolysaccharide during DNA transfection activates a lipid A-dependent cell death response that can be prevented by polymyxin B. Hum. Gene Ther. 1997, 8, 555-561.

32. Borst, E.; Elbasani, E. Department of Virology, Hannover Medical School, Hannover, Germany. Unpublished data, 2013.

33. Borst, E.M.; Messerle, M. Development of a cytomegalovirus vector for somatic gene therapy. Bone Marrow Transplant. 2000, 25, S80-S82.

34. Kalejta, R.F. Functions of human cytomegalovirus tegument proteins prior to immediate early gene expression. Curr. Top. Microbiol. Immunol. 2008, 325, 101-115. 
35. Bishop, C.L.; Ramalho, M.; Nadkarni, N.; May, K.W.; Higgins, C.F.; Krauzewicz, N. Role for centromeric heterochromatin and PML nuclear bodies in the cellular response to foreign DNA. Mol. Cell Biol. 2006, 26, 2583-2594.

36. Baldick, C.J., Jr.; Marchini, A.; Patterson, C.E.; Shenk, T. Human cytomegalovirus tegument protein pp71 (ppUL82) enhances the infectivity of viral DNA and accelerates the infectious cycle. J. Virol. 1997, 71, 4400-4408.

37. Pari, G.S.; Kacica, M.A.; Anders, D.G. Open reading frames UL44, IRS1/TRS1, and UL36-38 are required for transient complementation of human cytomegalovirus oriLyt-dependent DNA synthesis. J. Virol. 1993, 67, 2575-2582.

38. Silva, L.A.; Loregian, A.; Pari, G.S.; Strang, B.L.; Coen, D.M. The carboxy-terminal segment of the human cytomegalovirus DNA polymerase accessory subunit UL44 is crucial for viral replication. J. Virol. 2010, 84, 11563-11568.

39. Nevels, M.; Brune, W.; Shenk, T. SUMOylation of the human cytomegalovirus 72-kilodalton IE1 protein facilitates expression of the 86-kilodalton IE2 protein and promotes viral replication. J. Virol. 2004, 78, 7803-7812.

40. Borst, E.M.; Kleine-Albers, J.; Gabaev, I.; Babic, M.; Wagner, K.; Binz, A.; Degenhardt, I.; Kalesse, M.; Jonjic, S.; Bauerfeind, R.; et al. The human cytomegalovirus UL51 protein is essential for viral genome cleavage-packaging and interacts with the terminase subunits pUL56 and pUL89. J. Virol. 2013, 87, 1720-1732.

41. Wang, J.B.; Zhu, Y.; McVoy, M.A.; Parris, D.S. Changes in subcellular localization reveal interactions between human cytomegalovirus terminase subunits. Virol. J. 2012, 9, 315.

42. Borst, E.M.; Ständker, L.; Wagner, K.; Schulz, T.F.; Forssmann, W.G.; Messerle, M. A peptide inhibitor of cytomegalovirus infection from human hemofiltrate. Antimicrob. Agents Chemother. 2013, 57, 4751-4760.

43. Borst, E.M.; Benkartek, C.; Messerle, M. Use of bacterial artificial chromosomes in generating targeted mutations in human and mouse cytomegaloviruses. In Current Protocols in Immunology; John Wiley \& Sons: New York, NY, USA, 2007; Chapter 10, Unit 10.32, pp. 10.32.1-10.32.30, doi:10.1002/0471142735.im1032s77.

44. Tischer, B.K.; Smith, G.A.; Osterrieder, N. En passant mutagenesis: A two step markerless red recombination system. Methods Mol. Biol. 2010, 634, 421-430.

45. Bridge, E.; Ketner, G. Redundant control of adenovirus late gene expression by early region 4. J. Virol. 1989, 63, 631-638.

46. Weinberg, D.H.; Ketner, G. A cell line that supports the growth of a defective early region 4 deletion mutant of human adenovirus type 2. Proc. Natl. Acad. Sci. USA 1983, 80, 5383-5386.

47. Kaluza Cytometry Software, version 1.2; Beckman Coulter: Brea, CA, USA, 2012.

48. Cotten, M. Axxima Pharmaceuticals AG, Munich, Germany. Personal communication, 2001.

49. Murrell, I.; Tomasec, P.; Wilkie, G.S.; Dargan, D.J.; Davison, A.J.; Stanton, R.J. Impact of sequence variation in the UL128 locus on production of human cytomegalovirus in fibroblast and epithelial cells. J. Virol. 2013, 87, 10489-10500. 

Reprinted from Viruses. Cite as: Bhuvanendran, S.; Salka, K.; Rainey, K.; Sreetama, S.C.; Williams, E.; Leeker, M.; Prasad, V.; Boyd, J.; Patterson, G.H.; Jaiswal, J.K.; Colberg-Poley, A.M. Superresolution Imaging of Human Cytomegalovirus vMIA Localization in Sub-Mitochondrial Compartments. Viruses 2014, 6, 1612-1636.

Article

\title{
Superresolution Imaging of Human Cytomegalovirus vMIA Localization in Sub-Mitochondrial Compartments
}

\author{
Shivaprasad Bhuvanendran ${ }^{1}$, Kyle Salka ${ }^{1}$, Kristin Rainey ${ }^{2}$, Sen Chandra Sreetama ${ }^{1}$, \\ Elizabeth Williams ${ }^{1}$, Margretha Leeker ${ }^{1}$, Vidhya Prasad ${ }^{1}$, Jonathan Boyd ${ }^{3}$, \\ George H. Patterson ${ }^{2, *}$, Jyoti K. Jaiswal ${ }^{1,4, *}$ and Anamaris M. Colberg-Poley ${ }^{1,4,5, *}$
}

1 Research Center for Genetic Medicine, Children's Research Institute, Children's National Health System, 111 Michigan Avenue, NW, Washington, DC 20010, USA; E-Mails: SBhuvanendran@childrensnational.org (S.B.); kyle.salka@gmail.com (K.S.); Sreetama.SenChandra@childrensnational.org (S.C.S.); elizabeth.anne.williams.umd@gmail.com (E.W.); maggie.leeker@gmail.com (M.L.); VPrasad@childrensnational.org (V.P.)

2 Section on Biophotonics, National Institute of Biomedical Imaging and Bioengineering, National Institutes of Health, Bethesda, MD 20892, USA; E-Mail: kristin.rainey@nih.gov

3 Life Science Division, Leica Microsystems, Inc., 1700 Leider Lane, Buffalo Grove, IL 60089, USA; E-Mail: Jonathan.Boyd@Leica-Microsystems.com

4 Department of Integrative Systems Biology, George Washington University School of Medicine and Health Sciences, Washington, DC 20037, USA

5 Department of Biochemistry and Molecular Medicine, George Washington University School of Medicine and Health Sciences, Washington, DC 20037, USA

* Authors to whom correspondence should be addressed;

E-Mails: pattersg@mail.nih.gov (G.H.P.); jkjaiswal@childrensnational.org (J.K.J.); acolberg-poley@childrensnational.org (A.M.C.-P.); Tel.: +1-202-476-3984 (A.M.C.-P.); Fax: +1-202-476-6014 (A.M.C.-P.).

Received: 17 January 2014; in revised form: 16 March 2014 / Accepted: 27 March 2014 / Published: 9 April 2014

Abstract: The human cytomegalovirus (HCMV) viral mitochondria-localized inhibitor
of apoptosis (vMIA) protein, traffics to mitochondria-associated membranes (MAM),
where the endoplasmic reticulum (ER) contacts the outer mitochondrial membrane
(OMM). vMIA association with the MAM has not been visualized by imaging. Here,
we have visualized this by using a combination of confocal and superresolution 
imaging. Deconvolution of confocal microscopy images shows vMIA localizes away from mitochondrial matrix at the Mitochondria-ER interface. By gated stimulated emission depletion (GSTED) imaging, we show that along this interface vMIA is distributed in clusters. Through multicolor, multifocal structured illumination microscopy (MSIM), we find vMIA clusters localize away from MitoTracker Red, indicating its OMM localization. GSTED and MSIM imaging show vMIA exists in clusters of $\sim 100-150 \mathrm{~nm}$, which is consistent with the cluster size determined by Photoactivated Localization Microscopy (PALM). With these diverse superresolution approaches, we have imaged the clustered distribution of vMIA at the OMM adjacent to the ER. Our findings directly compare the relative advantages of each of these superresolution imaging modalities for imaging components of the MAM and sub-mitochondrial compartments. These studies establish the ability of superresolution imaging to provide valuable insight into viral protein location, particularly in the sub-mitochondrial compartments, and into their clustered organization.

Keywords: HCMV vMIA; MAM; mitochondria; OMM; matrix; confocal microscopy; superresolution microscopy; GSTED; MSIM; PALM

\section{Introduction}

The mitochondria-associated membrane (MAM) sub-compartment of the endoplasmic reticulum (ER) plays critical roles in ER-mitochondrial cross-talk by allowing efficient transfer of calcium $\left(\mathrm{Ca}^{2+}\right)$ from the ER to mitochondria without elevating cytosolic $\mathrm{Ca}^{2+}$ levels [1-3]. A macromolecular complex, composed of MAM inositol 1,4,5 trisphosphate receptors (IP3R), cytosolic glucose response protein 75 (GRP75) and outer mitochondrial membrane (OMM)-localized voltage dependent anion channel (VDAC) generates the high $\mathrm{Ca}^{2+}$ microdomains needed for $\mathrm{Ca}^{2+}$ transfer from ER to mitochondria (Figure 1) [4]. Constitutive low level IP3R-mediated $\mathrm{Ca}^{2+}$ transfer, needed for $\mathrm{Ca}^{2+}$ dependent mitochondrial enzymes, maintains normal cellular metabolism [5]. Nonetheless, continued mitochondrial $\mathrm{Ca}^{2+}$ influx drives the adaptive metabolic phase of early ER stress and can result in mitochondrial-mediated apoptosis [6,7]. Lipids, including phospholipids, cholesterol, and ceramide, are synthesized in the MAM and transferred to the OMM $[1,8,9]$. In addition, the MAM is enriched in internal lipid rafts [10], which can serve to connect extrinsic and intrinsic apoptotic pathways [11]. Finally, mitochondrial antiviral responses have also been recently linked to the MAM $[7,12,13]$.

The viral mitochondria-localized inhibitor of apoptosis (vMIA), encoded by the human cytomegalovirus (HCMV) UL37 exon 1 (UL37x1) immediate early gene, inhibits mitochondrialmediated programmed cell death and increases viral progeny production during permissive infection [14-19]. vMIA traffics sequentially from the ER to mitochondria and is present at ER-mitochondria contact sites known as MAM [20-24]. At the ER, vMIA causes ER $\mathrm{Ca}^{2+}$ efflux [15]. It associates with MAM lipid rafts in close proximity to sigma 1 receptor (Sig-1R) [25], which is a chaperone affecting $\mathrm{Ca}^{2+}$ efflux from the ER. Additionally, vMIA recruits Bax to MAM 
lipid rafts and induces Bax proteasome-mediated degradation, thereby augmenting vMIA's antiapoptotic activity [26,27]. Because of its sequential trafficking [20,23], vMIA can relocalize a cellular defense protein, viperin, from the ER to mitochondria where viperin assumes a new role of a major effector to induce lipogenesis metabolism during HCMV infection [28,29]. At mitochondria, vMIA blocks Bax-mediated permeabilization of the OMM [17,18,30,31], reduces ATP synthesis [28,29,32], causes mitochondrial fragmentation [12,30,33,34], and controls HtrA2/Omi-induced cell death through very late times of HCMV infection [35].

Figure 1. Endoplasmic reticulum (ER), mitochondria-associated membranes (MAM), and mitochondrial sub-compartments visualized. Viral mitochondria-localized inhibitor of apoptosis (vMIA) localization in the MAM and mitochondrion sub-compartments was imaged using the following markers: preprolactin signal sequence (ss) fused to the $\mathrm{N}$-terminus of the red fluorescent protein (RFP) with a KDEL ER retention signal at its C-terminus (ss-RFP-KDEL, for ER) [36] and mitochondrial Cox 4 leader fused to Tag blue fluorescent protein (Mito-BFP)/mitochondrial targeting sequence from human cytochrome c oxidase subunit VIII to the N-terminus of Discosoma RFP (DsRed-Mito, for mitochondrial matrix) [37]. We use MitoTracker Red as an intermembrane space (IMS) marker based upon the superresolution imaging of MitoTracker by others [38,39] and our own results herein. Contacts between the ER and mitochondria are shown, with the MAM $\mathrm{Ca}^{2+}$ signaling complex components on the ER (IP3R3), cytosol (GRP75) and outer mitochondrial membrane (OMM) (voltage dependent anion channel (VDAC)). MAM Ca ${ }^{2+}$ efflux from the ER is regulated by chaperones (BiP, Sig-1R) as well as vMIA. Lipid rafts (blue) containing the $\mathrm{Ca}^{2+}$ signaling complex and vMIA are shown. These components are shown in the figure.

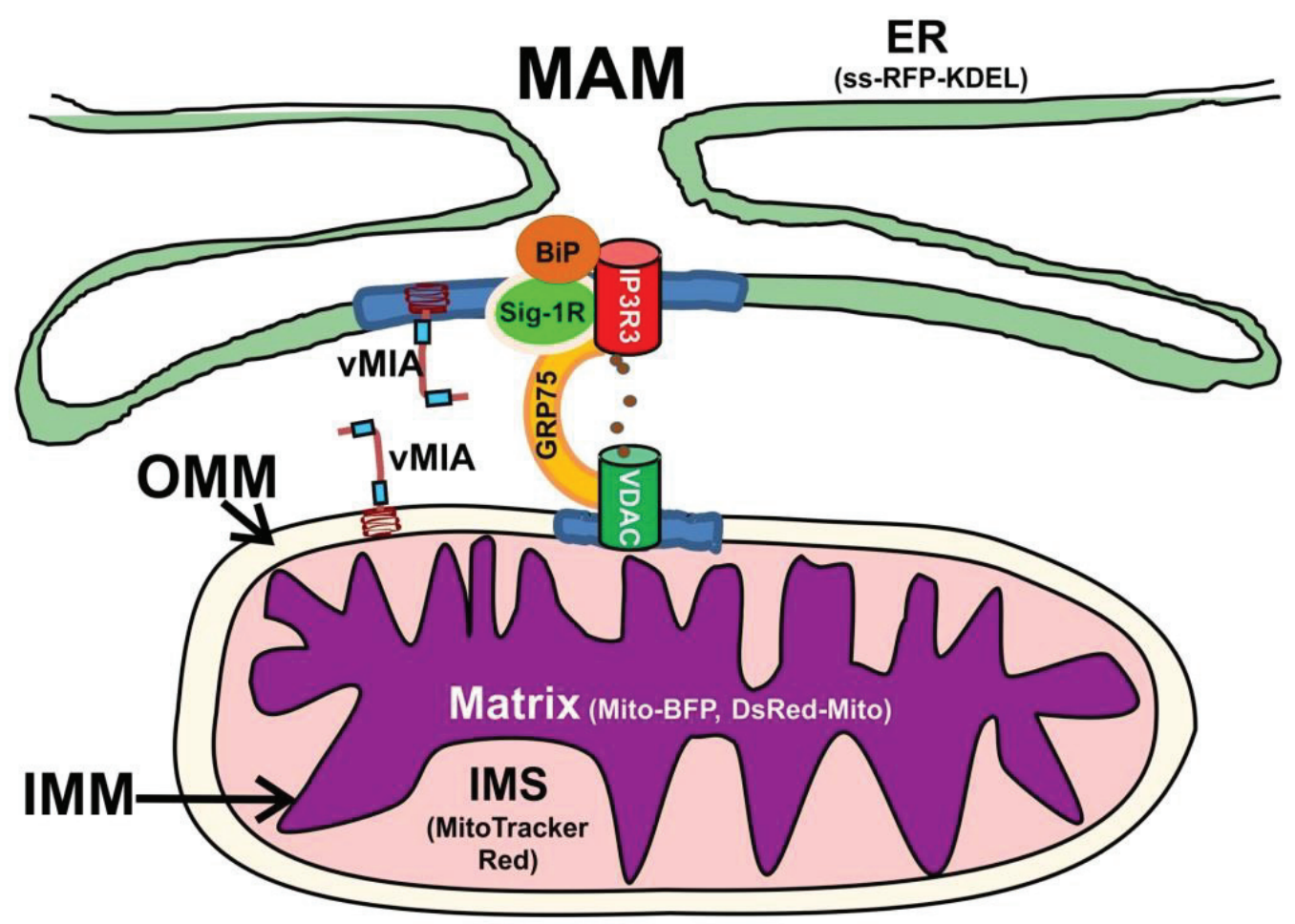

Mitochondrion 
vMIA is N-terminally anchored to ER and mitochondrial membranes by an uncleaved hydrophobic leader and its downstream C-terminal sequences are cytosolic [20]. This topology was confirmed by vMIA's sensitivity to protease digestion in ER and mitochondrial fractions [22]. Immune electron microscopy (EM) of stably transfected HeLa cells has localized vMIA-myc using anti-myc antibody primarily at the OMM [16]. However, fixation, embedding and staining of specimens for EM severely compromise membrane morphology. Moreover, immune EM limits identifying the distribution of the protein populations such that only a fraction of the molecules in a given cellular organelle can be detected, thereby offering limited information about spatial distribution of the targeted molecule. Thus, the exact pattern of vMIA distribution and functional organization along the mitochondria was not detected by immune EM analysis. This EM analysis aside, vMIA imaging has primarily utilized conventional confocal microscopy [15-18,20,23,24,30-32,35,40]. Using multicolor confocal microscopy, we have previously found that enhanced green fluorescent protein (EGFP) tagged vMIA partially co-localizes with MAM, lipid raft, and mitochondrial markers [20,21,23]. Further, vMIA has been co-localized with mitochondrial markers from the OMM and matrix [17,18,20,21,23-25,32].

A major challenge in precisely defining vMIA's localization in the ER, MAM and sub-mitochondrial compartments by confocal microscopy results from the close proximities of the ER and OMM membranes (10-25 nm) and of the OMM and inner mitochondrial membrane (IMM) at the MAM, which are below its diffraction limit. While confocal microscopy can theoretically produce a resolution down to $\sim 200 \mathrm{~nm}$, this resolution of visible light is seldom achieved in practice due to numerous optical aberrations associated with biological specimens as well as noise associated with the detected fluorescence. Some of this can be corrected by deconvolution of confocal images [41]. Mitochondria typically have a diameter of 200-500 nm [38]. Thus, it is not possible to determine vMIA distribution within sub-mitochondrial compartments using conventional confocal microscopy. For this, we turned to superresolution microscopy, which allows imaging beyond the limitations imposed by diffraction, to improve insight into vMIA's distribution in sub-mitochondrial compartments.

Superresolution microscopy overcomes the physical limit imposed by diffraction. Multiple approaches have been developed to resolve fluorescent signals below diffraction limit and these include structured illumination (e.g., structured illumination microscopy, SIM; multifocal SIM, MSIM), reduction of point spread function by grounding emissions outside of the excitation center (e.g., stimulated emission depletion, STED; gated STED, GSTED) or activation of single fluorophores (e.g., photoactivated localization microscopy, PALM; stochastic optical reconstruction microscopy, STORM). Each of these approaches has its own strengths and weaknesses. In this study, we examined the localization of vMIA by deconvolved confocal microscopy and three superresolution microscopy techniques namely MSIM, single-color GSTED, and PALM.

MSIM uses sparse 2D excitation patterns [42] moved in sequential steps to fully illuminate the specimen. Images are collected at each step and used in post processing to derive a superresolution image. Superresolution is achieved by first defining the precise location of the illumination spots. Once the focal spots in each of the images are defined, these are digitally pinholed followed by scaling the spots by a factor of 0.5 and then summing these images over all positions. The pinholed, scaled, and summed images are then subjected to Richardson-Lucy deconvolution to gain $\sim 2$-fold 
improvement in resolution. Although MSIM sacrifices speed compared to confocal microscopy, it maintains the optical sectioning and was shown to provide resolution-doubling characteristics of SIM to $\sim 140 \mathrm{~nm}[42]$.

Further improvement in resolution over MSIM is obtained using STED microscopy, where a $592 \mathrm{~nm}$ wavelength doughnut shaped beam is used to drive the fluorochromes in the doughnut to ground state by stimulated emission resulting in $<50 \mathrm{~nm}$ resolution $[43,44]$. Time gating of the short fluorescent lifetimes caused by stimulated emission results in the GSTED approach further improves the spatial resolution. STED microscopy has previously shown that the mitochondrial inner membrane organizing system (MINOS) forms clusters within mitochondria of primary human fibroblasts [45] while two color STED found VDAC type 3 and hexokinase I clusters on the OMM of human osteosarcoma cells [46]. Similarly, cytochrome c oxidase subunit 2 and VDAC1 were found in clusters in purified mitochondria from murine heart [47].

Pointillistic imaging based superresolution microscopy approach, PALM offers the highest resolution microscopy $(\sim 25 \mathrm{~nm})$ used in these studies. PALM uses photoactivatable fluorescent proteins and precise localization of single molecules to overcome diffraction limitations. PALM is based on high density, single molecule localization in which single molecule signals are fitted with 2D Gaussian functions to provide a more precise estimate of the molecule's location. PALM and several related techniques use photoactivatable, photoswitchable, or photoconvertible fluorescent proteins [48], which initially have little fluorescence or their fluorescence can be turned "off' in the spectral region under detection before they are actively turned "on" during imaging. Conservatively setting the precision cutoff at $\sim 50 \mathrm{~nm}$ can often produce images resolved at that value which is $\sim 3$-fold improvement over the MSIM images and comparable to the GSTED images. Similar to MSIM, PALM provides the ability to image multiple colors and better resolution than confocal, but slower imaging speed.

\section{Results and Discussion}

\subsection{Conventional Confocal Imaging of vMIA Localization with Mitochondrial Markers}

We first used confocal microscopy to monitor mitochondrial distribution of vMIA in HCMV permissive cells, human foreskin fibroblasts (HFFs) expressing vMIA-EGFP, which traffics indistinguishably from untagged vMIA $[15,21,23,26]$. We labeled the mitochondrial matrix by using $S$. cerevisiae mitochondrial Cox 4 leader fused to Tag blue fluorescent protein (Mito-BFP) [37] (Figure 2). As we previously found [20,21,23,24], vMIA-EGFP fluorescence substantially co-localized with a matrix marker, Mito-BFP [37] (Figure 2A). The zoom of one of the larger mitochondrion (Figure 2C) shows an example of the level of detail available when imaging these markers with confocal microscopy. With the spatial resolution we obtained for this image (FWHM for vMIA-EGFP $=298 \mathrm{~nm}$ ), vMIA-EGFP distribution was only marginally distinguishable from Mito-BFP. With the image acquired 3-times below the Nyquist limit, deconvolution of the confocal Z-stack images improved the spatial resolution (FWHM for vMIA-EGFP $=182 \mathrm{~nm}$ ) (Figure 2B). This improved resolution allowed us to distinguish the presence of vMIA at the rim of the mitochondria, away from the matrix marker Mito-BFP (Figure 2D). This is quantified by the intensity profile for the dotted line marked along the deconvolved image of the mitochondrion 
(Figure 2E). This imaging also demonstrated that vMIA is not uniformly distributed along the mitochondrial periphery, hinting at the possibility of clustering of vMIA on the mitochondrial surface (Figure 2D); however, the clusters of molecules were not clearly resolvable even after deconvolution of the confocal microscopy images. In summary, confocal microscopy showed the presence of vMIA at the periphery of mitochondria and partially resolved it from the matrix marker.

Figure 2. Monitoring mitochondrial localization of vMIA by confocal microscopy. Primary human foreskin fibroblasts (HFFs) lipofected with vectors expressing vMIA-EGFP and Mito-BFP were fixed with 4\% paraformaldehyde (PFA) at 22 hours after transfection as described in the methods. (A) Images show a single optical plane for a cell expressing vMIA-EGFP (green) and Mito-BFP (pseudocolored red) imaged using confocal microscopy and (B) the same image plane following deconvolution of the entire Z-stack. (C,D) The boxed region of interest is enlarged on the right. (E) Intensity profile of vMIA-EGFP (green) and Mito-BFP (red) emissions along the pixels marked by the dotted line on the deconvolved image are shown by the plot. For higher resolution images, see Supplemental Figure S1.

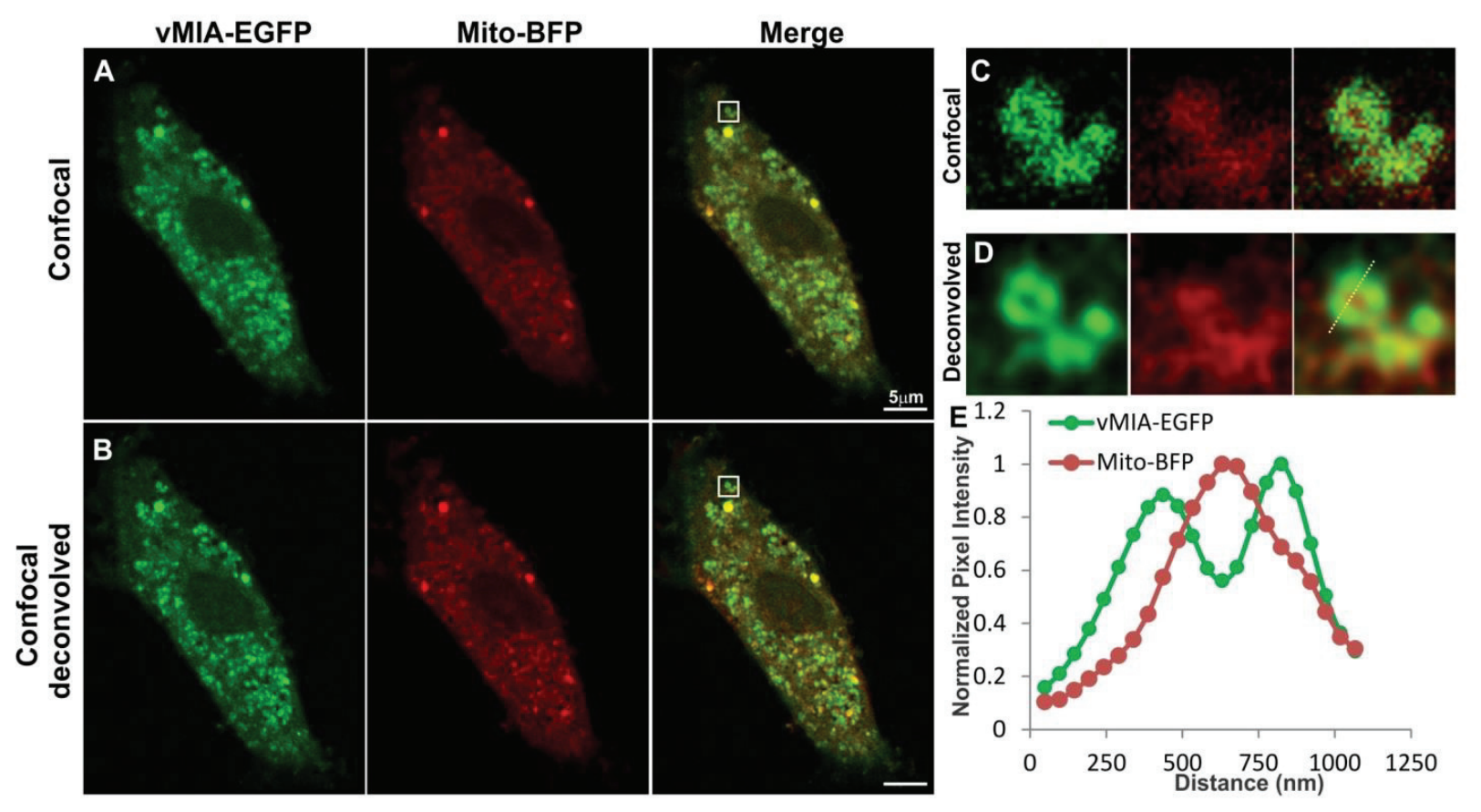

In these studies, we detected altered mitochondrial morphology in HFFs expressing vMIA consistent with previous literature [30,33,34,49]. To determine if the altered mitochondrial morphology correlated with vMIA levels, we examined mitochondria morphology in cells expressing vMIA (Figure 3). We found that mitochondria (red) not expressing vMIA or expressing low levels of vMIA (R1) maintained tubular morphology (Figure 3A). Conversely, mitochondria expressing higher levels of vMIA (R2) showed fragmented, vesicular morphology. Multiple tubular mitochondria (blue arrows) not expressing or expressing low levels of vMIA were also observed in another cell (Figure 3B). These results suggest that threshold levels of vMIA are required for mitochondrial fragmentation and vesiculation. 
With the ability of the deconvolved confocal image to resolve vMIA distribution from the mitochondrial matrix marker (Mito-BFP), we next examined the distribution of these markers with respect to that of the ER (ss-RFP-KDEL), which has the bovine preprolactin signal sequence (ss) fused to monomeric red fluorescent protein (RFP) with the KDEL ER retention [36] sequence (graciously provided by Dr. J. Lippincott-Schwartz) (Figure 4A). HFFs expressing the three fluorophore-tagged proteins showed that vMIA partially colocalized with ER and mitochondrial markers as we previously found [20,21,23,24,26,50]. Moreover, the intensity profile (Figure 4B) of the pixels along the line shown in the zoomed boxed region of interest (Figure 4C) showed that vMIA-EGFP is located at the interface of Mito-BFP and ss-RFP-KDEL. Although there is detection of the interfaces between the ER and mitochondria, there is substantial overlap of the mitochondrial OMM (green) and matrix (blue) sub-compartments, which limits the ability of confocal microscopy to compellingly resolve the sub-mitochondrial distribution of vMIA.

Figure 3. Mitochondrial fragmentation by vMIA exhibits a threshold effect. Primary HFFs transfected with vectors to express vMIA-EGFP and Mito-BFP were fixed with 4\% PFA at 22 hours after transfection as described in the methods and visualized by confocal deconvolution microscopy. (A) Maximal intensity projection of a 3-D image showing a pair of cells expressing differing levels of vMIA-EGFP (green), but comparable level of Mito-BFP (pseudocolored red). Insets showing the zoom of regions R1 and R2 highlights the change in mitochondrial morphology (as shown by Mito-BFP) in cells expressing low level (R1) or high level (R2) of vMIA-GFP (B) Maximal intensity 3-D projection of another cell expressing Mito-BFP (pseudocolored red in the merge) and showing varying levels of vMIA-EGFP (green in the merge) on the individual mitochondria. While most of the mitochondria in this cell have lost their tubular appearance, red arrows point to the individual mitochondrion that show low or no vMIA-GFP expression have remained tubular. Scale bars represent $5 \mu \mathrm{m}$. For higher resolution images, see Supplemental Figure S2.

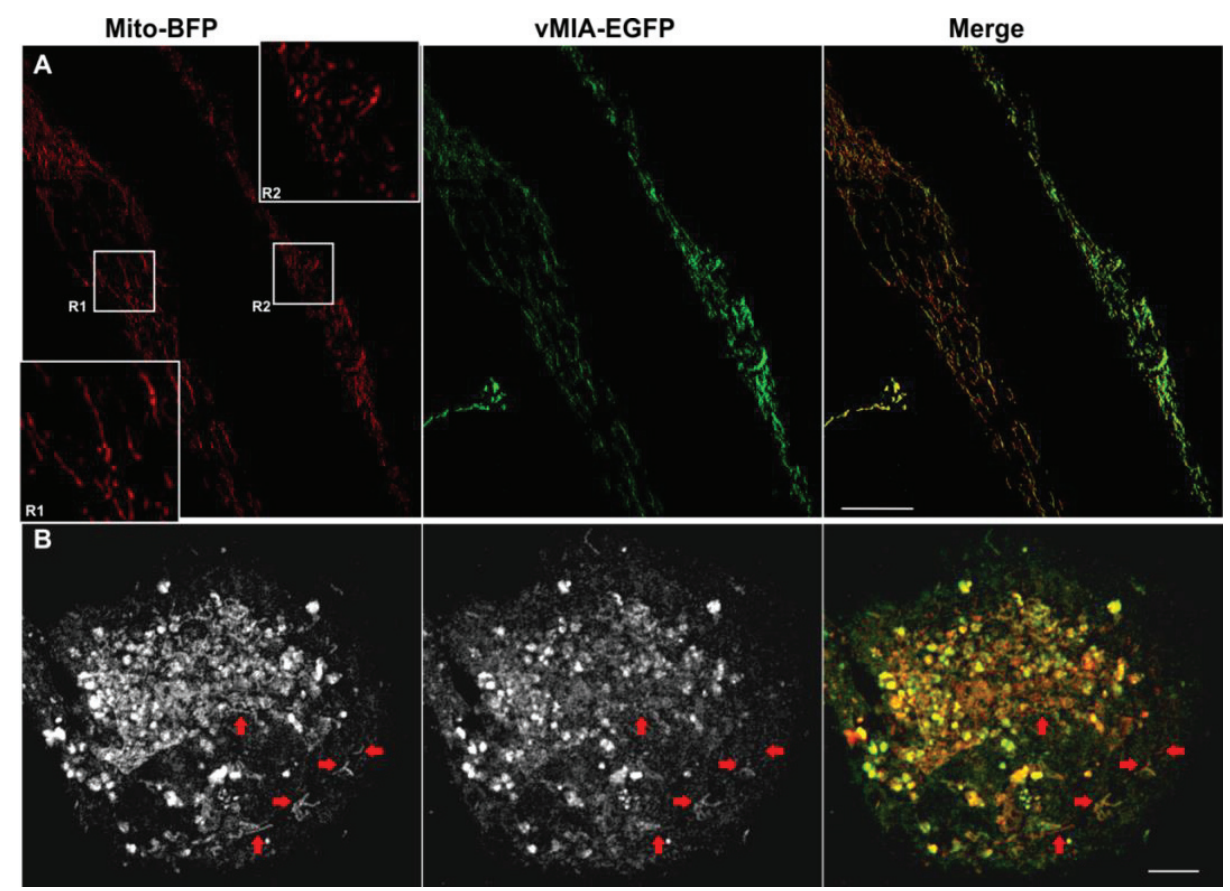


Figure 4. Confocal microscopy imaging of ER-mitochondria interface. HFFs were lipofected with vectors expressing vMIA-EGFP (OMM), ss-RFP-KDEL (ER) and Mito-BFP (matrix) and fixed with 4\% PFA at 25 hours after transfection as described below and previously published [23]. (A) Cells expressing vMIA-EGFP (green), ss-RFP-KDEL (red) and Mito-BFP (blue) were imaged using confocal microscopy. (C) The boxed region of interest from the merged image is enlarged. (B) Line scans of vMIA-EGFP (green), ss-RFP-KDEL (red) and Mito-BFP (blue) emissions are shown. For higher resolution images, see Supplemental Figure S3.
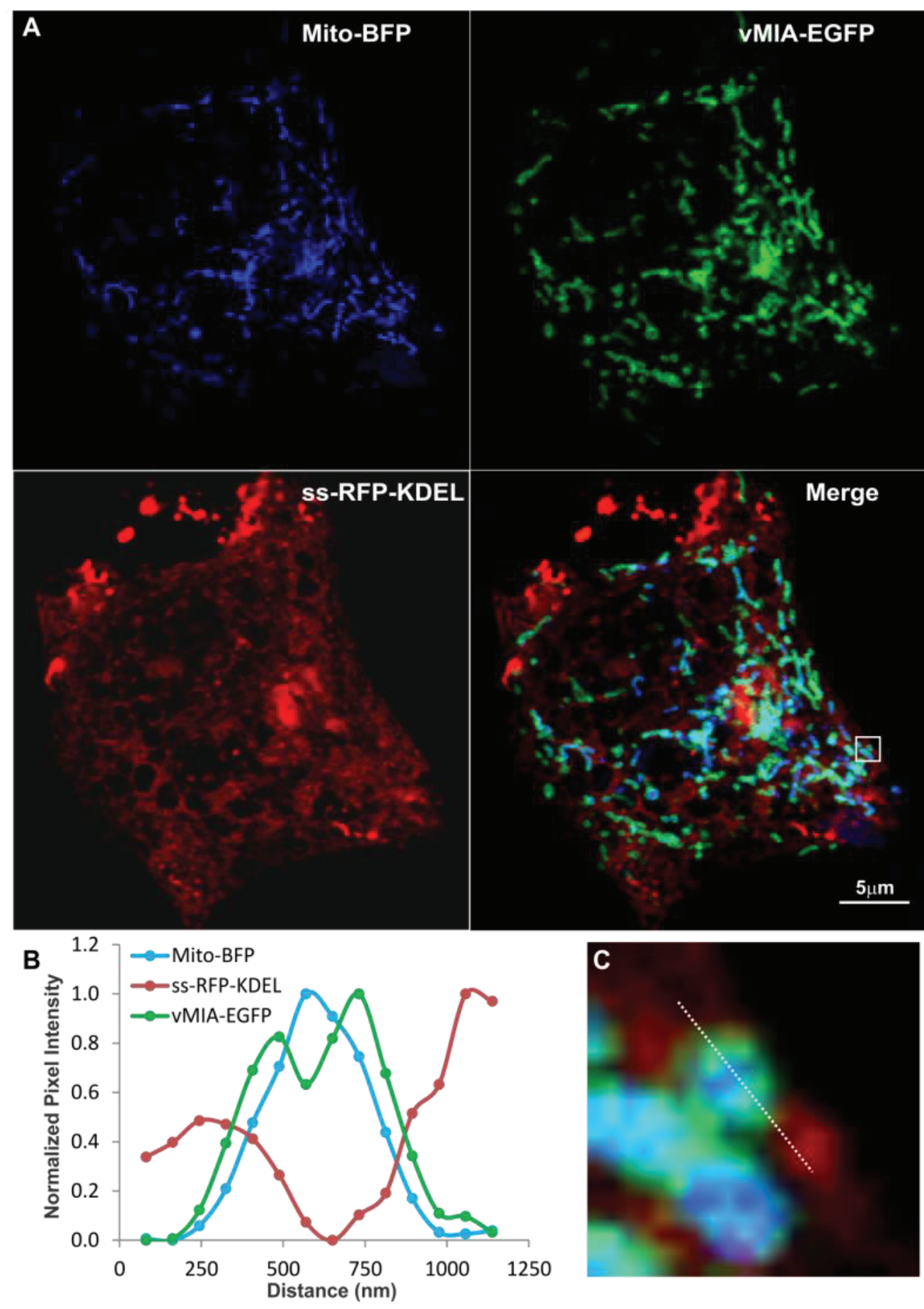


\subsection{Single Color GSTED Imaging of vMIA Localization}

To image vMIA beyond the diffraction limit imposed by the visible light, we used GSTED, an earlier variant, STED, has been used to study clustered distribution of several mitochondrial proteins at the OMM and IMM by immune localization of intact or isolated mitochondria [45-47]. As use of antibody affects precision of localization due to increased distance added on by the presence of primary and fluorescently tagged secondary antibodies and imaging isolated mitochondria provides localization outside the biologically relevant subcellular context, we undertook in situ imaging of vMIA-EGFP in permissive HFFs (Figure 5A). Following deconvolution of the GSTED image, we detected vMIA-EGFP (green) in the periphery of a tubular mitochondrion (Figure 5B), which is consistent with the above suggestion of OMM localization by the confocal microscopy and of biochemical literature [16,20,22]. Intensity profile of pixels marked by the line shown in the zoomed GSTED image of the region R2 confirmed that vMIA is localized at the periphery of mitochondria, distinguishable from DsRed-Mito, used as a matrix marker and imaged by confocal microscopy (Figure 5D,E). GSTED showed improved resolution of vMIA (FWHM = $75 \mathrm{~nm}$ ) OMM location compared to confocal imaging of vMIA (Figure 5B). This increase in spatial resolution also offered conclusive evidence to support clustered distribution of vMIA along the OMM (Figure 5C). Thus, superresolution GSTED resolved the presence of vMIA at the OMM and its clustered distribution in mitochondria in permissive HFFs. It also showed that vMIA exists as $<100 \mathrm{~nm}$ clusters at the OMM irrespective of if the mitochondrion is tubular (Figure 5B) or fragmented (Figure 5D).

As clustering of vMIA along the OMM is an unprecedented phenotype for a viral protein, we performed GSTED imaging of VMIA-EGFP in another permissive (human astrocytoma) cell line, U373-Tet-ON cells [51] (Figure 6A). GSTED imaging detected vMIA-EGFP (green) in U373-Tet-ON cells also showed that similar to HFFs, vMIA is present at the rim of the mitochondria (Figure 6B). This rim-like distribution of vMIA-EGFP was confirmed by the intensity profile of pixels marked by the line shown in the zoomed region (R2, Figure 6B). GSTED showed improved resolution of vMIA location $(<50 \mathrm{~nm})$ compared to confocal imaging $(200 \mathrm{~nm})$ (Figure 6C). Similar to our findings in HFFs (Figure 5), vMIA was present in clusters on the mitochondria in transfected U373-Tet-ON cells (Figure 6D,E). However, mitochondria of the U373-Tet-ON cells expressing vMIA are considerably less tubular (Figure 6) than mitochondria from transfected HFFs expressing vMIA (Figure 5). These differences in mitochondrial morphology likely represent cell-type specific differences in the effects of vMIA, which is known to alter mitochondrial morphology [30,32,33,52]. 
Figure 5. Gated stimulated emission depletion (GSTED) microscopy of vMIA-EGFP in human fibroblasts. (A) HFFs were lipofected with vectors expressing vMIA-EGFP and DsRed-Mito. At 24 hours post transfection, cells were methanol fixed as described as below and elsewhere [23] and imaged using GSTED (vMIA-EGFP) and confocal microscopy (DsRed-Mito, vMIA-EGFP) followed by deconvolution of both the images. (B) Zoomed, merged image of a tubular mitochondrion in the boxed region (R1) is shown. This includes DsRed-Mito confocal (red), vMIA-EGFP confocal (blue) and vMIA-EGFP GSTED (green). Each channel is also presented individually. (C) Intensity profile of the pixels marked by the dotted line on the GSTED panel demonstrates the clustered distribution of vMIA along the entire length of the OMM of this mitochondrion. (D) The zoomed, merged image of a mitochondrion in the boxed region (R2) is shown. (E) The normalized intensity profile along the line shown on the R2 image, which demonstrates the significant improvement in visualizing the vMIA distribution along OMM by GSTED as compared to confocal imaging and its improved resolution of its localization away from the matrix and at the OMM. For higher resolution images, see Supplemental Figure S4.

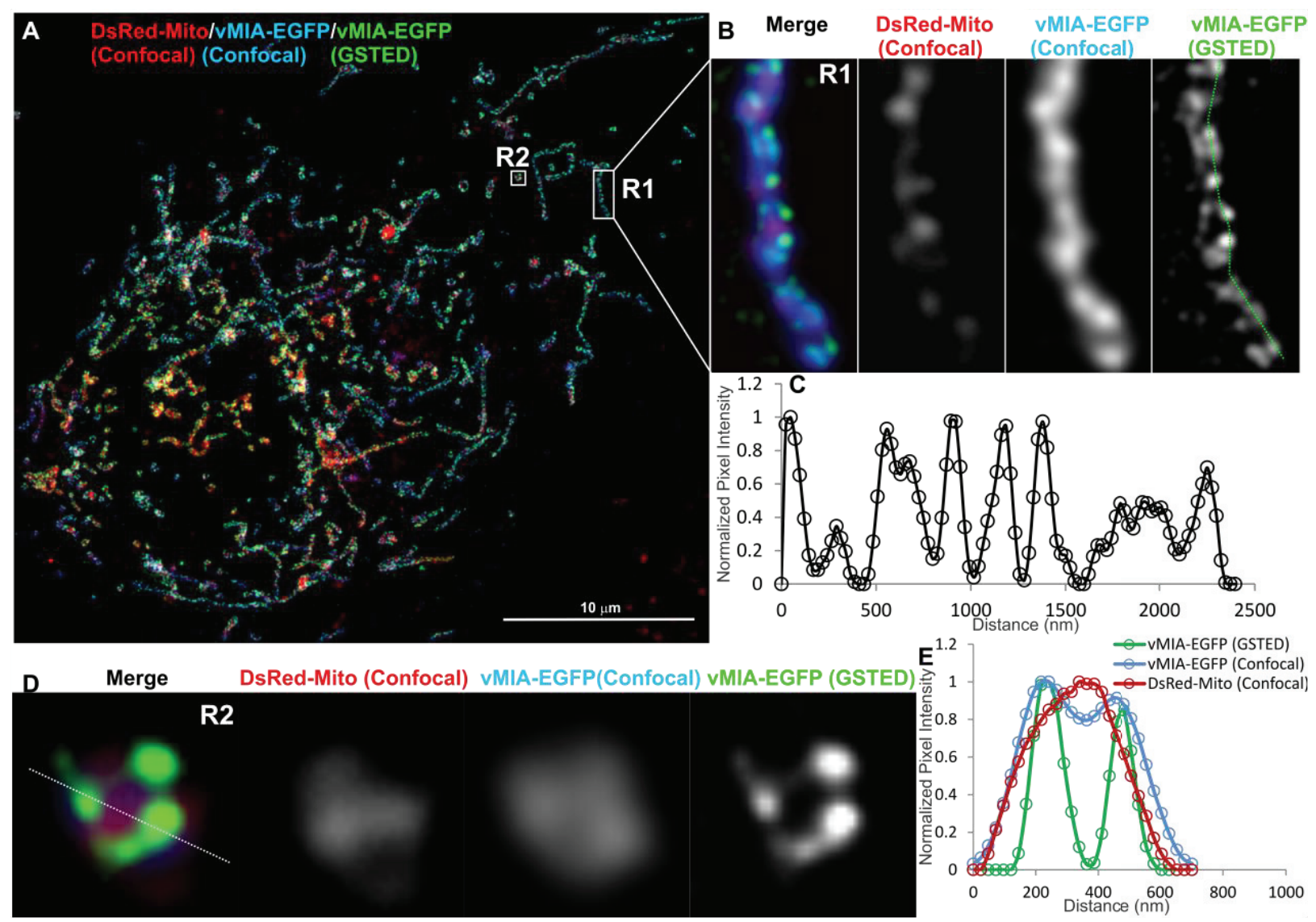


Figure 6. GSTED microscopy of vMIA-EGFP in human astrocytoma cells. U373-Tet-ON cells expressing the tetracycline controlled transactivator (tTA) [51] were lipofected with the tetracycline responsive element (TRE-Tight) promoter driving expression of vMIA-EGFP. At 24 hours post transfection, cells were treated with doxycycline (Dox) for 60 minutes, labeled with MitoTracker Red $(0.5 \mu \mathrm{M})$ and imaged live using Confocal (vMIA-GFP and MitoTracker Red) and GSTED (vMIA-GFP). (A) An optical slice showing deconvolved confocal image of a cell expressing vMIA-EGFP and a zoom of the region corresponding to the boxed region (R1) are shown in the left panel. Right panel presents the zoom of the R1 region showing the deconvolved GSTED (bottom) and deconvolved confocal MitoTracker (top) channels. (B) Zoomed confocal images of the mitochondrion in the region R2 showing the various channels acquired-Confocal images of MitoTracker Red and vMIA-EGFP as well as GSTED image of vMIA-EGFP. (C) Normalized intensity profile along the dotted line shown on the confocal and GSTED images of R2 demonstrate the improved resolution of vMIA localization by GSTED imaging as compared to confocal imaging. (D) Zoom of region R3 showing GSTED image of vMIA (E) Intensity profile of the pixels marked by the red line in panel (D). For higher resolution images, see Supplemental Figure S5.

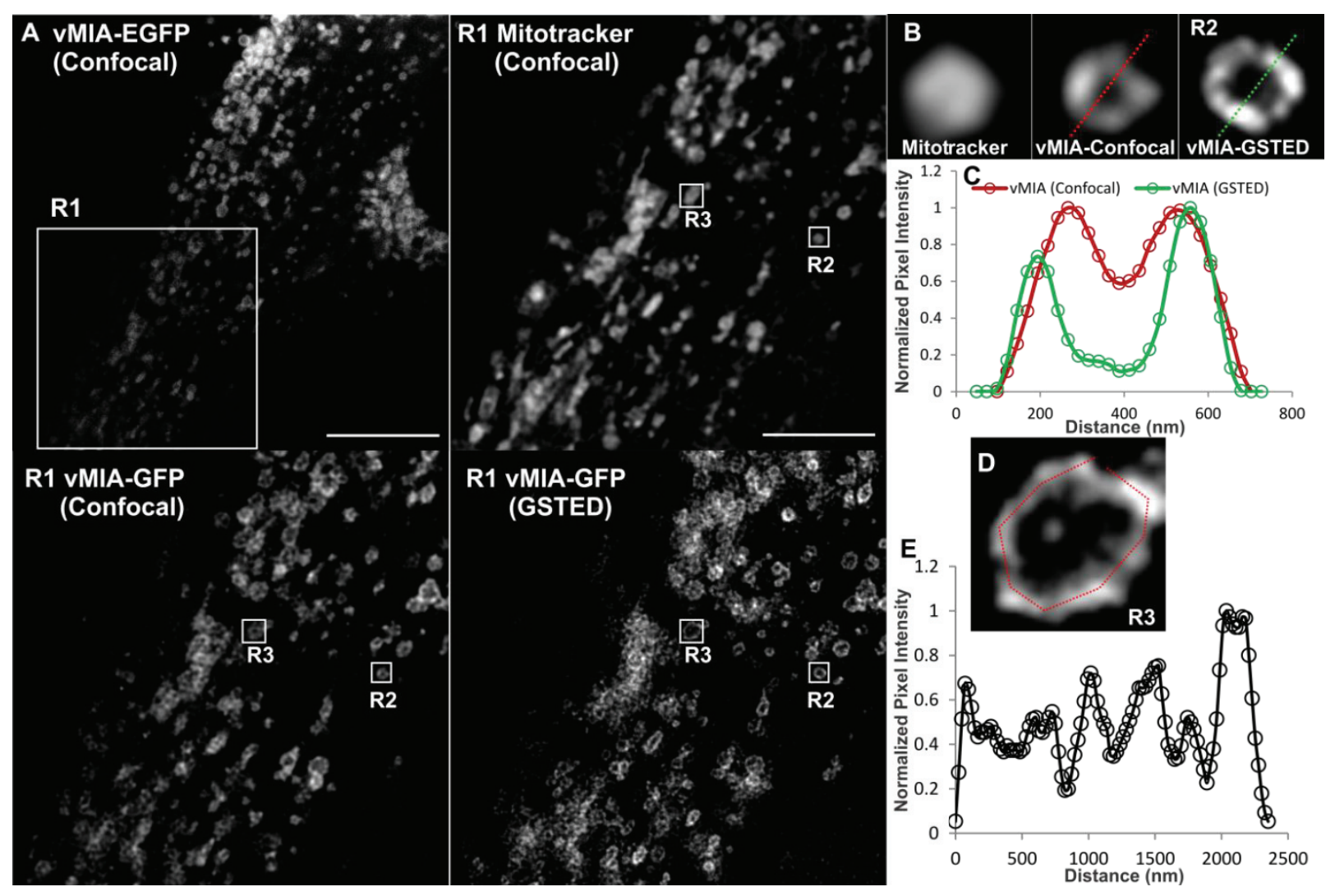

\subsection{MSIM Imaging of vMIA and Mitochondrial Marker}

With a restricted set of compatible fluorophores that can be used for multicolor superresolution imaging by GSTED, for simultaneous superresolution imaging of vMIA-EGFP in context of organelle markers we made use of an alternative superresolution imaging approach-MSIM. We performed MSIM imaging of doxycycline (Dox) treated HFFs dually transfected with the 
tetracycline transactivator [53] and the TRE-Tight promoter driving vMIA-EGFP (Figure 7). We observed that vMIA localized distinctly from the mitochondrial marker we used here-MitoTracker Red, which appears to localize to the IMS [38]. As expected, widefield imaging of vMIA-EGFP and MitoTracker Red showed predominant colocalization of the two fluorophores (Figure 7A). With the observed spatial resolution of $302 \mathrm{~nm}$ for this image there was little resolution of the vMIA and MitoTracker labeling (Figure 7B). The overlap of the signals was documented by the intensity profile of each fluorophore along the pixels marked by the dotted line shown in the zoom of the region R3 (Figure 7B). MSIM images of the two fluorophores for this cell showed improved resolution of vMIA-EGFP and MitoTracker Red (Figure 7C). Intensity profile of MSIM image of each fluorophore along the pixels marked by the dotted line shown in the zoom of the region R3 showed partial separation of the vMIA-EGFP fluorescence peaks from those of the MitoTracker fluorescence peaks such that the bimodal vMIA peaks are farther away to the outside of the bimodal MitoTracker peaks (FWHM $=120 \mathrm{~nm}$ ) (Figure 7D). This distribution of the two markers is in agreement with the vMIA localization to the OMM, surrounding the IMS localized MitoTracker Red. Furthermore, MSIM imaging in individual mitochondrion (R1 and R2 in Figure 7E) confirmed our previous observations of vMIA localization in clusters at the OMM. The presence of vMIA clusters was verified by intensity profile of MSIM image of each fluorophore along the pixels marked by the dotted line drawn along the rim of a mitochondrion in R2 (Figure 7F). These vMIA clusters were obscured by diffraction in widefield imaging (Figure 7B) and confocal microscopy (Figures 2-4) but detected by superresolution GSTED imaging (Figures 5 and 6). Together, these results show that MSIM partially resolves vMIA location at mitochondria periphery, away from IMS, and in clusters of diffraction limited size. The ER and OMM make contacts at the MAM. In addition, using superresolution microscopy multiple mitochondrial proteins including the OMM VDAC1, VDAC3 and Tom 20 proteins have been shown to exist in clusters as functional mitochondrial complexes [46,47,54]. vMIA clustering could represent the contact sites between the ER and OMM or could indicate the vMIA association with functional complexes at the OMM. Use of above approaches allowed us to narrow down the size of vMIA clusters to be in the range of $100 \mathrm{~nm}$, but this is close to the resolution of imaging modalities used.

\subsection{PALM Imaging of PA-mCherry-vMIA}

To visualize vMIA clusters by yet another independent superresolution approach and obtain a better estimate of the size of vMIA clusters, we made use of the pointillistic imaging superresolution imaging approach PALM. Here, we expressed vMIA tagged with PAmCherry, a photoactivatable red fluorescent protein [55], in HFFs and visualized them using 2D PALM imaging (Figure 8). Most of the molecules in this image localized with precision better than $25 \mathrm{~nm}$, but here we have conservatively rendered all molecules which have been localized to $<25 \mathrm{~nm}$ precision as 2D Gaussian distributions with $25 \mathrm{~nm}$ sigmas. Similar to our MSIM and GSTED results above, qualitative observations suggest a non-uniform distribution of vMIA-PAmCherry on the periphery of the mitochondria when rendered in this manner. PALM imaging also affords a second, rather straightforward analysis of molecule distributions, which is relevant to this work, cluster analysis. Several methods are developed for this sort of analysis, but we opted for pair 
correlation analysis in quantitatively determining which regions of each mitochondrion displayed increased vMIA protein densities.

Figure 7. Imaging vMIA localization using widefield microscopy and MSIM. HFFs were transfected with vectors expressing TRE-tight promoter-vMIA-EGFP (green) and its tetracycline controlled transactivator (tTA) [53]. Transfected cells were Dox-treated $(0.2 \mu \mathrm{g} / \mathrm{mL})$ for one hour, MitoTracker Red treated $(0.5 \mu \mathrm{M}$ for $5 \mathrm{~min}$, red) and methanol fixed as described [23]. 25 slices $(0.2 \mu \mathrm{m}$ step size) were collected using $488 \mathrm{~nm}$ and $561 \mathrm{~nm}$ excitation lasers. For each channel, 256 multifocal excited images were collected as the excitation array was stepped $\sim 1$ pixel in a $16 \times 16$ grid pattern. (A) Summation of the images produces the widefield fluorescence image, which shows high degree of co-localization between vMIA-EGFP and MitoTracker Red. (B) Zoomed images of mitochondria in the region marked R3 are shown and the plot below shows the intensity profile through the region marked by dotted line. (C) The MSIM image is shown. (D) The zoomed images of region R3 and the line scan for the region marked by dotted line show the improved ability to resolve the vMIA-EGFP and MitoTracker Red staining through the use of MSIM. (E) MSIM images of two mitochondria (R1 and R2) are shown. (F) Intensity profile through the perimeter of mitochondrion in R2 marked by dotted line is shown in the plot. This indicates non-uniform distribution of vMIA-GFP along the mitochondrial membrane. For higher resolution images, see Supplemental Figure S6.

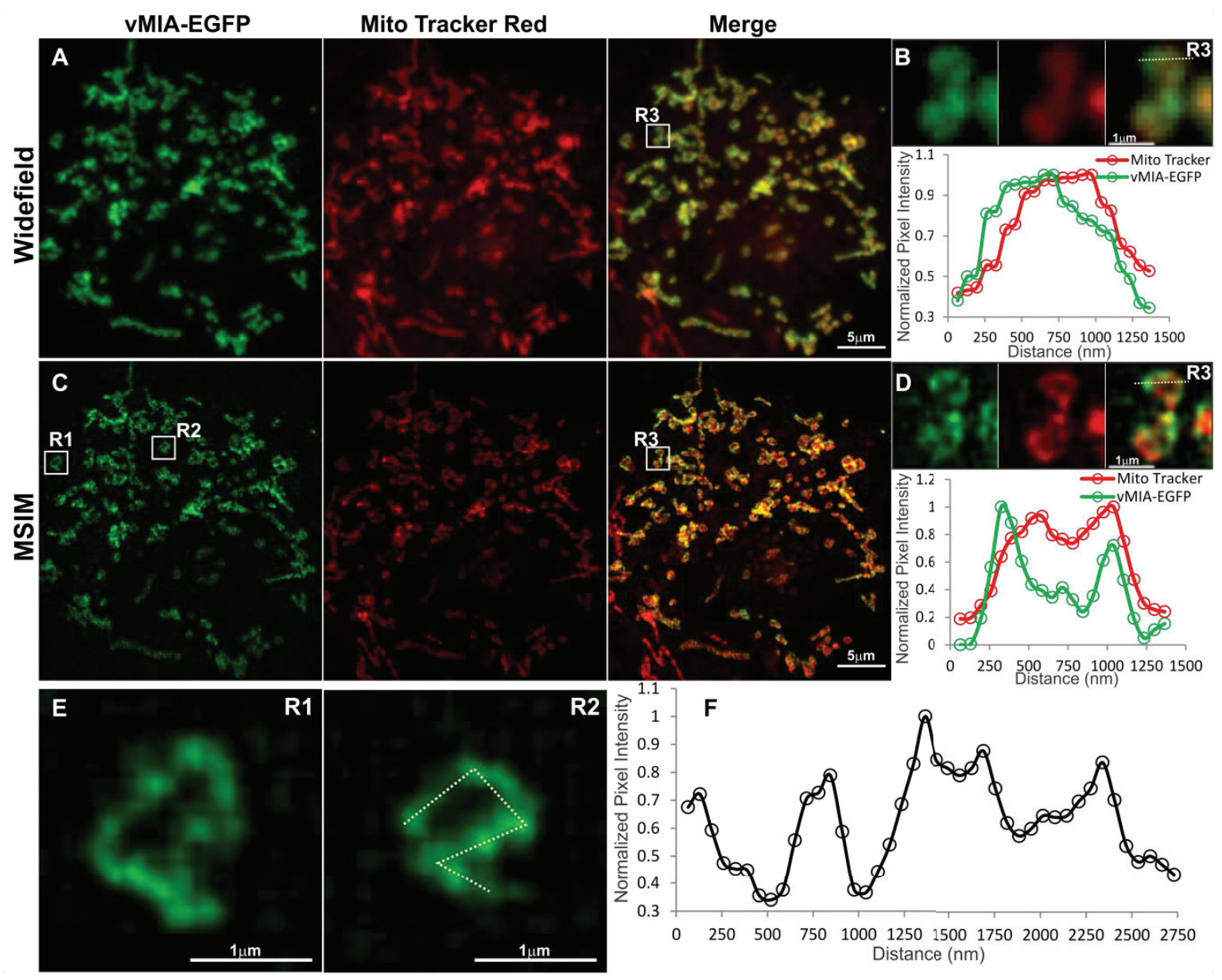


Figure 8. PALM imaging of vMIA-PAmCherryl. HFFs were transfected with vMIA-PAmCherry and imaged by PALM. (A) The image shows 301666 molecules that were localized to $\leq 25 \mathrm{~nm}$ precision out of 515207. Molecules are plotted as two-dimensional Gaussian distributions with $25 \mathrm{~nm}$ standard deviations. (B) The image shows a small region in (A) indicated by the red square. The molecule positions in a binary image were used in pair correlation analysis and the maximum $\mathrm{g}(\mathrm{r})$ derived from using each molecule as a reference point was plotted as the pixel values. (C) The image shows the same region in the red square color-coded to display the varying degrees of molecule densities. The Look Up Table (LUT) and calibration bar are included in the upper right corner. The distributions of cluster sizes (D) and the number of particles per cluster (E) were determined from the pair correlation analysis results. The maximum cluster radius was limited to $500 \mathrm{~nm}$ and the minimum number of particles per cluster was limited to 20. Please note that the $\mathrm{Y}$ axis in (E) is a log scale to better indicate the lower percentages at the higher particle numbers. The scale bar in (A) is $5 \mu \mathrm{m}$ and in $(B, C)$ is $1 \mu \mathrm{m}$. High resolution images of the entire field of view are available in the SOM (see Supplemental Figures S7, S10 and S11).

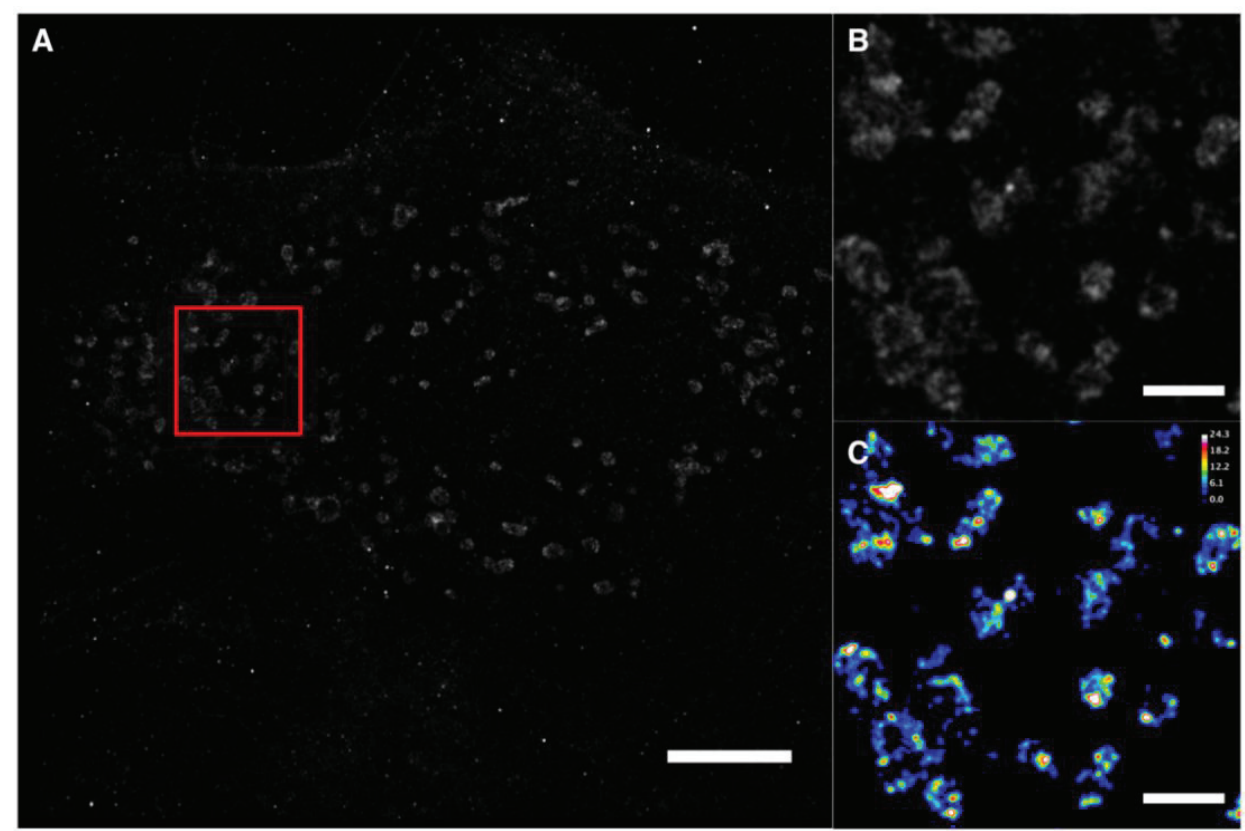

D

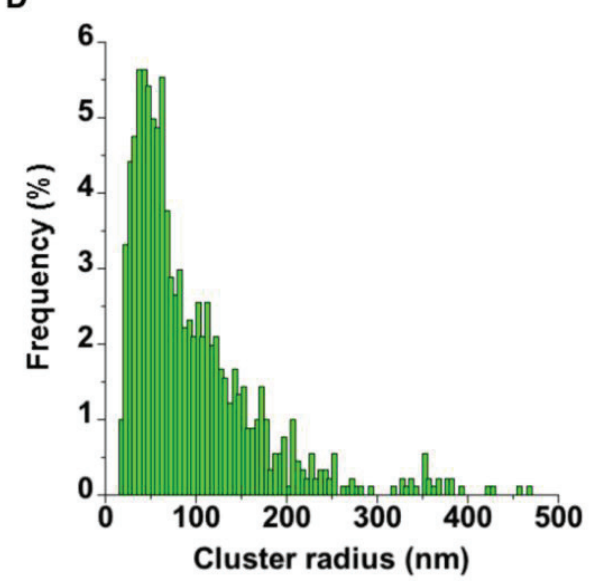

E

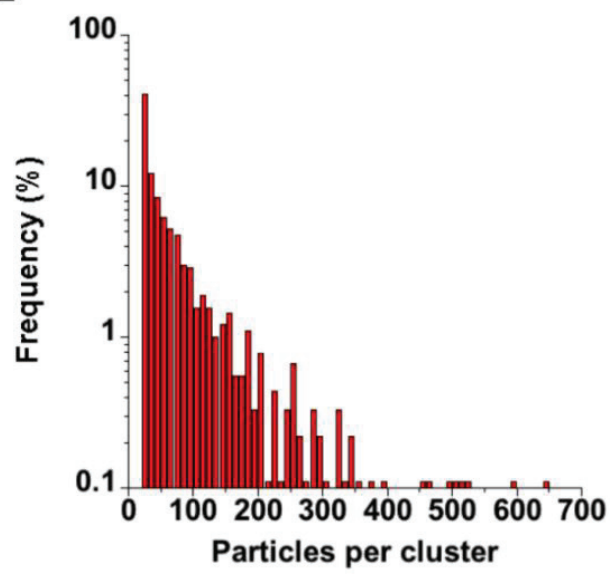


In these analyses, the function $\mathrm{g}(\mathrm{r})$ is determined by comparing the protein density in a local "shell" region around each position with the average density of all molecules of interest (see Supplemental Figure S8). Simulated images containing various levels of clustering are shown in Supplemental Figure S9A-D. For these, the factor $g$ is plotted as a function of the radius and for images containing no clusters, this remains close to 1 as the radius decreases toward zero (Supplemental Figure S9E). However, in images with clusters, the $\mathrm{g}(\mathrm{r})$ deviates from 1 at approximately the diameter of the clusters (Supplemental Figure S9E). If the cluster radii increase, the $g(r)$ deviates at a higher value of $r$. If the molecule density in the cluster increases, the maximum $g(r)$ increases. Thus, the maximum $g(r)$ can reflect the relative amplitude of the molecule density surrounding each reference particle. This is important for our simple analysis in which we compare the local protein density with the whole image density. With these criteria, the entire mitochondrion could be considered a cluster. In our analyses, we used the average density of all molecules in the image localized to $<25 \mathrm{~nm}$ precision. We plotted the $\mathrm{g}(\mathrm{r})$ maximum value obtained for each reference molecule in a new image at the appropriate location (Supplemental Figure S10). Indeed, we find that most of the molecules localized to the mitochondria show $\mathrm{g}(\mathrm{r})$ values greater than 1 , but we also note that regions resembling the clusters observed with MSIM and GSTED have much higher $\mathrm{g}(\mathrm{r})$ values than surrounding peripheral regions (Figure 8 ). Using the results from pair correlation analysis, the cluster sizes (Figure 8D) and the number of particles per cluster (Figure 8E) were determined. Cluster radii show a broad distribution with a mean radius of approximately $95 \mathrm{~nm}$ and suggest that a majority are $<100 \mathrm{~nm}$. The number of particles per cluster is also broadly distributed and indicates that a majority of the clusters (approximately 60\%) contain 50 particles or less.

\section{Experimental Section}

\subsection{Cell Culture and Lipofection}

HFFs were cultured in Dulbecco's Modified Eagle's medium containing 10\% fetal calf serum (FCS, Hyclone, Logan, UT), $100 \mathrm{U} / \mathrm{mL}$ of penicillin, $100 \mu \mathrm{g} / \mathrm{mL}$ of streptomycin, $2 \mathrm{mM}$ L-Glutamine (Life Technologies, Grand Island, NY) as previously described [56]. U373-Tet-ON cells were also cultured in the $10 \% \mathrm{FCS}, 100 \mathrm{U} / \mathrm{mL}$ of penicillin, $100 \mu \mathrm{g} / \mathrm{mL}$ of streptomycin, $2 \mathrm{mM}$ L-Glutamine but maintained under selective pressure using $0.6 \mathrm{mg} / \mathrm{mL}$ Geneticin (Life Technologies).

Cells were seeded at 20\%-50\% confluency on sterile $18 \mathrm{~mm}$ (for confocal or GSTED) or $25 \mathrm{~mm}$ (for PALM and MSIM) cover slips in six-well plates $\left(9.72 \mathrm{~cm}^{2}\right.$ per well). Twenty-four hours later cells were transiently transfected using Lipofectamine 2000 (Life Technologies) suspended in Opti-MEM (Life Technologies), according to the manufacturer's protocols. DNA ( $\mu \mathrm{g})$ :lipid ( $\mu \mathrm{L})$ ratios for transfection were at 1:1.77, with approximately $0.5 \mu \mathrm{g}$ total DNA used per $\mathrm{cm}^{2}$ of available plating surface area. Cells were harvested 22-25 hours after transfection by fixation with methanol or 4\% PFA in PBS and mounted with Prolong Gold Antifade. GSTED imaging was performed on live U373-Tet-ON cells treated with Dox $(0.5 \mu \mathrm{M})$. 


\subsection{Construction of pTRE-Tight Promoter-vMIA-EGFP}

The vMIA/UL37x1 protein-EGFP open reading was isolated from p1242 [15] by restriction enzyme digestion with EcoRI and NotI. The fragment was ligated into EcoRI/NotI digested TRE-Tight vector (Clontech, Mountain View, CA, USA) and the ligation product was transformed into competent E. coli (strain DH5 alpha).

\subsection{Confocal Microscopy}

The confocal images were acquired using the Olympus FV1000 confocal microscope. An UPlanSApo 100x/1.40NA oil objective was used to obtain an oversampled $1024 \times 1024$ image with $49 \mathrm{~nm}$ pixel and a z-stack with a step size of $120 \mathrm{~nm}$. The Mito-BFP and the vMIA-EGFP were sequentially excited using a $405 \mathrm{~nm}$ diode laser and a $488 \mathrm{~nm}$ Argon laser, respectively, and collected between $425 \mathrm{~nm}-475 \mathrm{~nm}$ and $500 \mathrm{~nm}-545 \mathrm{~nm}$ in the spectral detectors. In the triple labeled cells, ss-RFP-KDEL was also sequentially collected using $575 \mathrm{~nm}-675 \mathrm{~nm}$ filter after excitation with a $559 \mathrm{~nm}$ diode laser.

\subsection{GSTED Microscopy}

The GSTED images were acquired using a Leica TCS SP5 gated STED (GSTED) microscope that was equipped with a super continuum white light laser (WLL) and hybrid detectors adapted for time gated imaging which allow elimination of low spatial frequency from the final super resolved image [57]. The $592 \mathrm{~nm}$ depletion laser delivers $0.3 \mathrm{~W}$ at the focal plane. A HCX PL APO $100 \times 1.40$ NA oil objective was used to obtain a $1600 \times 1600$ image with a $24.2 \mathrm{~nm}$ pixel. The DsRed-Mito confocal image was acquired using the $560 \mathrm{~nm}$ excitation and collected between $569 \mathrm{~nm}-665 \mathrm{~nm}$. A sequential GSTED image of UL37-EGFP was obtained using a $488 \mathrm{~nm}$ excitation, $592 \mathrm{~nm}$ depletion, time gating from 1-8 ns, and collected between $500 \mathrm{~nm}-544 \mathrm{~nm}$. Using the tunability of the white light laser and prism based spectral emission. "Lambda lambda" scans were acquired for an excitation range of $470 \mathrm{~nm}-590 \mathrm{~nm}$ and an emission range of $485 \mathrm{~nm}-605 \mathrm{~nm}$. Both excitation and emission scans were taken with $10 \mathrm{~nm}$ wavelength steps between measurements and was used to confirm the excitation and emission properties of the EGFP fluorophore imaged on this microscope in the GSTED or confocal mode.

\subsection{Deconvolution Analysis}

Blurring due to out of focus signal and Poisson noise in the confocal and GSTED images was removed by carrying out deconvolution using the Huygens Essential software supplied by Scientific Volume Imaging B.V. (Hilversum, The Netherlands). For confocal images, deconvolution was done using 3D images acquired as described above. For GSTED images, 2D deconvolution was done on the using images acquired as discussed below. For generating pixel intensity plots images were imported and analyzed in the Metamorph Premier (7.7.0) software supplied by Molecular Devices, LLC (Sunnyvale, CA, USA). 
The MSIM microscope used for these experiments is a homebuilt machine using an Olympus IX-71 widefield microscope as previously described [58] and with modifications detailed below. A $100 \mathrm{~mW} 405 \mathrm{~nm}$ Cube laser (Coherent, Inc., Santa Clara, CA, USA), $50 \mathrm{~mW} 488$ laser (Oxxius, Lannion, France, LBX-488-50-CIR-PP), $50 \mathrm{~mW} 561$ Sapphire laser (Coherent, Inc.), and $100 \mathrm{~mW}$ $640 \mathrm{~nm}$ Cube laser (Coherent, Inc.) served as illumination sources for blue, green, red, and far-red fluorophores, respectively. The light from each laser was filtered by placing a 405/10 nm BrightLine single-band bandpass filter (Semrock Inc., Rochester, NY, USA, FF01-405-10/25) at the aperture of the 405 laser, a 488/6 nm BrightLine single-band bandpass filter (Semrock Inc., FF01-488-6/25) at the aperture of the 488 laser, a 561/4 nm BrightLine single-band bandpass filter (Semrock Inc., FF01-561-6/25) at the aperture of the 561 laser, and a 640/8 nm MaxDiode laser clean-up filter (Semrock Inc., LL01-640-8/12.5) at the aperture of the 640 laser. The laser beams were collimated or expanded and collimated with insertion of pairs of lenses after the clean-up filters. The 405 laser has a $2 \times$ telescope consisting of a $50 \mathrm{~mm}$ focal length lens (Thorlabs Inc., Newton, NJ, USA, LA1131-A) and a $100 \mathrm{~mm}$ focal length lens (Thorlabs Inc., LA1509-A). The 488 laser has a $1 \times$ telescope consisting of a pair of $100 \mathrm{~mm}$ focal length lenses (Thorlabs Inc., LA1509-A). The 561 laser has a $3 \times$ telescope consisting of a $50 \mathrm{~mm}$ focal length lens (Thorlabs Inc., LA1131-A) and a $150 \mathrm{~mm}$ focal length lens (Thorlabs Inc., LA1433-A). The 640 laser has a $2 \times$ telescope consisting of a $50 \mathrm{~mm}$ focal length lens (Thorlabs Inc., LA1131-A) and a $100 \mathrm{~mm}$ focal length lens (Thorlabs Inc., AC254-100-A). Mechanical shutters along with acousto-optic tunable filter (AA Opto-electronic Inc., Orsay, France, AOTFnC-400.650) controlled by Micro-Manager [59] allowed for laser wavelength selection, laser power tuning and laser shuttering. The laser beams were expanded $5 \times$ using a $40 \mathrm{~mm}$ focal length lens (Thorlabs Inc., AC254-40-A) and a $200 \mathrm{~mm}$ focal length lens (Thorlabs Inc., AC254-200-A). Collected fluorescence was filtered through a 480/40 bandpass filter (Chroma Technology Corp, Bellows Falls, VT, USA D480/40m) for $405 \mathrm{~nm}$ excitation, a 525/45 bandpass filter (Semrock, FF01-525/45-25) for $488 \mathrm{~nm}$ excitation, a 600/37 bandpass filter (Semrock, FF01-600/37-25) for $561 \mathrm{~nm}$ excitation, and a EdgeBasic 635 longpass filter (Semrock, BLP01-635R-25) for $640 \mathrm{~nm}$ excitation. All other components in the excitation and emission paths, such as optical components, 2D galvanometer, z-stage and camera, are the same as previously published [58].

\subsection{MSIM Data Collection and Analysis}

MSIM data were collected for each slice as previously published [58] except the galvanometer was stepped in a $16 \times 16$ grid to collect 256 frames for each $512 \times 512$ pixel wide field of view. Post-processing was performed as previously published [58] on freely available software [60]. Deconvolution was performed on the MPSS images using a program written in Python (freely available at [60] implementing Richardson-Lucy deconvolution [58,61-63]. 


\subsection{PALM Data Analysis}

Single molecule images were analyzed as previously described [64] using PeakSelector (a program written in IDL by Harald Hess and Gleb Shtengel, Howard Hughes Medical Institute, Janelia Farms, Ashburn, VA, USA). The molecule localization data were output as an Ascii file which was used by an ImageJ macro (available upon request) [65] to plot the molecule positions and precisions on images with $5 \mathrm{~nm}$ pixels.

Pair correlation analysis was performed on binary images displaying peak positions rendered as single $5 \mathrm{~nm}$ pixels. The $\mathrm{g}(\mathrm{r})$ function was determined for each particle by using the equation

$$
g(r)=\rho(\delta r) / \rho\left(A_{N}\right)
$$

where each particle is treated as a reference particle. The particle density in a "shell" region of $\delta \mathrm{r}$ is determined and then divided by the particle density in a region containing all of the particles of interest, $A_{N}$. In these analyses, the entire image was used as $A_{N}$ (Supplemental Figure S8). The analysis was performed using an ImageJ macro (available upon request) run in FIJI [66]. Briefly, the particle density $\rho$ in region $A_{N}, \rho(\delta r)$, was determined by dividing the total number of rendered molecules by the area of the image. The particle density $\rho$ in region $\delta r, \rho(\delta r)$, was determined by first counting the number of particles in areas $r 1$ and $r 2$ (Supplemental Figure S8). The number of particles in region $\delta \mathrm{r}$ was determined by subtracting the $\mathrm{r} 1$ count from the $\mathrm{r} 2$ count. The area of $\delta \mathrm{r}$ was determined using $\pi \mathrm{r}^{2}$ by subtracting the area the $\mathrm{r} 1$ region from the area of the $\mathrm{r} 2$ region. The $\rho(\delta r)$ was calculated by dividing the $\delta r$ count by the area of $\delta r$.

The $\mathrm{g}(\mathrm{r})$ as a function of the radius $\mathrm{r} 1$ for each peak was fit with an exponential equation

$$
\mathrm{g}(\mathrm{r})=\mathrm{A} \times \exp (-(1 / \mathrm{B}) \times \mathrm{r})+\mathrm{C}
$$

where $\mathrm{A}$ is the amplitude, $\mathrm{B}$ is the radius of the cluster, and $\mathrm{C}$ is the offset. Only peaks with $\mathrm{g}(\mathrm{r})$ fitted with a correlation coefficient $\mathrm{R}^{2}>0.9$ were rendered in the $\mathrm{g}(\mathrm{r})$ image. To display these data, the molecule peak position image was rendered by setting the pixel values for each particle to the maximum $g(r)$ determined for that peak when using it as a reference in the analysis (Supplemental Figure S10). A color coded LUT with calibration is included for comparison of different regions of the cell. Additional processing on this image included a Gaussian blur with a $25 \mathrm{~nm}$ sigma to represent the uncertainty in molecule localization (Supplemental Figure S11).

\section{Conclusions}

Because superresolution microscopy is an emerging technology, it has had very limited use in the study of viruses. To date, superresolution imaging has primarily focused on RNA viruses including imaging of rotavirus virions [67], influenza hemagglutinin clusters [68], HIV assembly [64,69,70] and HIV transfer [71]. More recently, single copy viral genomes including those of DNA viruses have been imaged using superresolution microscopy [72]. To our knowledge, our studies are the first superresolution imaging of any human herpesvirus protein, in particular of a HCMV vMIA.

HCMV vMIA imaging by confocal/deconvolution microscopy indicated its OMM localization, which was confirmed by GSTED, MSIM, and PALM imaging. Additionally these imaging modalities all demonstrate that at the OMM vMIA is present in clusters, indicating nanoscale localization of HCMV vMIA at the mitochondrial periphery, away from IMS and matrix. While 
this clustering is also suggested by deconvolved images obtained by the confocal microscopy, diffraction limits the ability to visualize the distribution of MAM clusters. This indicates that the vMIA domains are generally spaced closer than the diffraction limit. This inference has been supported by the ability to resolve them by each of the superresolution imaging approaches used here. Use of PALM imaging allowed us to estimate the sizes of these clusters at approximately 95 $\mathrm{nm}$, which is in agreement with the cluster sizes indicated by the GSTED and MSIM approaches.

Intriguingly, clusters of cellular mitochondrial proteins have been observed using superresolution microscopy. STED microscopy found that MINOS, which maintains IMM morphology, forms clusters often in an ordered inner mitochondrial distribution [45]. Tom 20 and Tom22, components of the translocase of the outer mitochondrial membrane, are clustered at the OMM with densities that correlate with mitochondrial membrane potential [73]. VDAC1 and VDAC3 have also been found by superresolution imaging to be distributed in clusters in the OMM. Cytosolic hexokinase I which associates with VDAC3 is partly also localized in clusters with VDAC3 [46,47]. Our superresolution imaging suggests that vMIA may target and associate with clustered OMM proteins. Alternatively, vMIA traffics efficiently to the MAM [20-23,26,74]. vMIA clustering may represent sites of contacts between the ER and OMM, where vMIA could be transferred between the two organelles. The use of vMIA mutants and known cellular markers of MAM should allow us to distinguish these possibilities.

Superresolution imaging has the clear potential to provide valuable insight into the nanoscale organization of viral machineries, which provide essential replicative processes. Case in point is the detection of non-uniform distribution of vMIA at the OMM. This distribution was not detectable by conventional microscopy because of its diffraction limited resolution. Further, this technology allows virologists to study how viruses alter cellular organelles to establish replication compartments and virus assembly. However, the superresolution approach to be used should be carefully selected. Our approach here involved three techniques with complementary advantages in nanoscale studies of vMIA localization. GSTED relies primarily on imaging of one fluorophore impacting on the ability to place the viral proteins in the in situ biological context of cellular organelles or structures. Secondary markers can be imaged by GSTED using the appropriately labeled secondary antibodies (e.g., Alexa 532). GSTED provides greater resolution ( $<50 \mathrm{~nm}$ ) than MSIM. However, use of primary and secondary antibodies can adversely affect precision of protein localization at the nanoscale resolution. Nonetheless, because of its resolution, GSTED provided compelling evidence for vMIA clustering in mitochondria and localization at the OMM. Conversely, MSIM is lower resolution than GSTED microscopy. However, MSIM makes use of the same fluorophores used for conventional confocal microscopy. This enables imaging of viral proteins in the context of multiple fluorophore tagged proteins from different sub-cellular compartments. For our experiments, MSIM provided sufficient resolution to confirm the location and clustering of vMIA at the OMM.

PALM imaging provides the highest resolution used in these studies but imaging sufficient molecules to obtain meaningful information requires hours of acquisition and post-acquisition processing. This limits most PALM imaging to fixed specimens. Given the increasing palette of photoactivatable fluorophores and increasing availability of these powerful microscopy techniques much can be learned by their application to multiple viruses and their processes. 
Here, we have used three different superresolution techniques with multiple markers to image the localization and clustering of vMIA. These all confirm the presence of the clusters on the outer membranes of mitochondria and are helping to define the cluster physical characteristics. In general, the clusters are found closer together than the limits of resolution by widefield and confocal microscopy. Examples of these include the plot profiles in Figures 5-7 compared with the widefield or confocal counterparts. These results show that given time, the imaging techniques used here will help discern on a sub-mitochondria scale where are the components of MAMs located in relation to each other as well as other cellular players.

A second important finding here is that we may put a conservative upper limit on the diffraction-limited vMIA cluster size of approximately $150 \mathrm{~nm}$. Since this comes from the data collected with three independent imaging techniques, it provides a more reasonable estimate than simply relying on a single technique.

Why is the size of importance? At these scales, small cluster sizes dictate the maximum number of molecules which can fit inside. Given the number of processes in which MAMs are known to play a role, this imposes limits on the number of molecules which can participate in signaling events. Thus, every MAM may not have all of the components to initiate all processes and this brings up another nagging question in the MAM field. Are the MAMs homogeneous or have differing compositions which imply different functions? Now that these structures can be readily imaged with optical techniques using multiple color markers, answers to this question will be far more straightforward to derive than with previous efforts. In summary, our studies show that superresolution imaging provides valuable insight into sub-diffraction resolution of viral protein location, particularly in the sub-mitochondrial compartments, and into their clustered arrangement.

\section{Acknowledgments}

This work was partially funded by the NSF grant (MCB 1244509) to ACP and JKJ. The confocal microscopy imaging was supported by a core grant (1P30HD40677) to the Children's Intellectual and Developmental Disabilities Research Center. This work was also supported by the Intramural Research Program of the National Institutes of Health including the National Institute of Biomedical Imaging and Bioengineering.

The authors thank Yan Su for construction of pTRE-Tight promoter-vMIA-EGFP plasmid, Jennifer Lippincott-Schwartz for providing ss-RFP-KDEL and Gary Thomas for the gift of U373-Tet-ON cells. We thank Maria Ingaramo for technical assistance with the MSIM image collection and analysis. We thank Andrew York and Hari Shroff for sharing MSIM analysis and deconvolution software. We thank Harald Hess for the use of the PALM analysis software, PeakSelector.

\section{Author Contributions}

S.B. and S.C.S. performed confocal microscopy, K.S., M.L., and V.P. performed transfections, E.W. and K.R. collected and analyzed the MSIM data, J.B. performed GSTED imaging, S.B., S.C.S. and J.K.J. performed the deconvolution and analysis of the confocal and GSTED images, G.H.P. collected and analyzed the PALM images, G.H.P., J.K.J. and A.C.P. designed experiments, analyzed results and wrote the manuscript. All edited the manuscript. 


\section{Conflicts of Interest}

Jonathan Boyd is an employee of Leica Microsystems. The other authors declare no conflict of interest.

\section{References and Notes}

1. Hayashi, T.; Rizzuto, R.; Hajnoczky, G.; Su, T.P. MAM: More than just a housekeeper. Trends Cell Biol. 2009, 19, 81-88.

2. Bononi, A.; Missiroli, S.; Poletti, F.; Suski, J.M.; Agnoletto, C.; Bonora, M.; de Marchi, E.; Giorgi, C.; Marchi, S.; Patergnani, S.; et al. Mitochondria-associated membranes (MAMs) as hotspot $\mathrm{Ca}(2+)$ signaling units. Adv. Exp. Med. Biol. 2012, 740, 411-437.

3. Marchi, S.; Patergnani, S.; Pinton, P. The endoplasmic reticulum-mitochondria connection: One touch, multiple functions. Biochim. Biophys. Acta 2014, 1837, 461-469.

4. Szabadkai, G.; Bianchi, K.; Varnai, P.; de Stefani, D.; Wieckowski, M.R.; Cavagna, D.; Nagy, A.I.; Balla, T.; Rizzuto, R. Chaperone-mediated coupling of endoplasmic reticulum and mitochondrial Ca2+ channels. J. Cell Biol. 2006, 175, 901-911.

5. Cardenas, C.; Miller, R.A.; Smith, I.; Bui, T.; Molgo, J.; Muller, M.; Vais, H.; Cheung, K.H.; Yang, J.; Parker, I.; et al. Essential regulation of cell bioenergetics by constitutive InsP3 receptor Ca2+ transfer to mitochondria. Cell 2010, 142, 270-283.

6. Bravo, R.; Vicencio, J.M.; Parra, V.; Troncoso, R.; Munoz, J.P.; Bui, M.; Quiroga, C.; Rodriguez, A.E.; Verdejo, H.E.; Ferreira, J.; et al. Increased ER-mitochondrial coupling promotes mitochondrial respiration and bioenergetics during early phases of ER stress. J. Cell Sci. 2011, 124, 2143-2152.

7. Castanier, C.; Arnoult, D. Mitochondrial localization of viral proteins as a means to subvert host defense. Biochim. Biophys. Acta 2010, 1813, 575-583.

8. Stiban, J.; Caputo, L.; Colombini, M. Ceramide synthesis in the endoplasmic reticulum can permeabilize mitochondria to proapoptotic proteins. J. Lipid Res. 2008, 49, 625-634.

9. Stone, S.J.; Vance, J.E. Phosphatidylserine synthase-1 and -2 are localized to mitochondria-associated membranes. J. Biol. Chem. 2000, 275, 34534-34540.

10. Hayashi, T.; Fujimoto, M. Detergent-resistant microdomains determine the localization of sigma-1 receptors to the endoplasmic reticulum-mitochondria junction. Mol. Pharmacol. 2010, 77, 517-528.

11. Gajate, C.; Gonzalez-Camacho, F.; Mollinedo, F. Lipid raft connection between extrinsic and intrinsic apoptotic pathways. Biochem. Biophys. Res. Commun. 2009, 380, 780-784.

12. Castanier, C.; Garcin, D.; Vazquez, A.; Arnoult, D. Mitochondrial dynamics regulate the RIG-I-like receptor antiviral pathway. EMBO Rep. 2010, 11, 133-138.

13. Horner, S.M.; Liu, H.M.; Park, H.S.; Briley, J.; Gale, M., Jr. Mitochondrial-associated endoplasmic reticulum membranes (MAM) form innate immune synapses and are targeted by hepatitis C virus. Proc. Natl. Acad. Sci. USA 2011, 108, 14590-14595.

14. Reboredo, M.; Greaves, R.F.; Hahn, G. Human cytomegalovirus proteins encoded by UL37 exon 1 protect infected fibroblasts against virus-induced apoptosis and are required for efficient virus replication. J. Gen. Virol. 2004, 85, 3555-3567. 
15. Sharon-Friling, R.; Goodhouse, J.; Colberg-Poley, A.M.; Shenk, T. Human cytomegalovirus pUL37x1 induces the release of endoplasmic reticulum calcium stores. Proc. Natl. Acad. Sci. USA 2006, 103, 19117-19122.

16. Goldmacher, V.S.; Bartle, L.M.; Skaletskaya, A.; Dionne, C.A.; Kedersha, N.L.; Vater, C.A.; Han, J.W.; Lutz, R.J.; Watanabe, S.; Cahir McFarland, E.D.; et al. A cytomegalovirus-encoded mitochondria-localized inhibitor of apoptosis structurally unrelated to Bcl-2. Proc. Natl. Acad. Sci. USA 1999, 96, 12536-12541.

17. Arnoult, D.; Bartle, L.M.; Skaletskaya, A.; Poncet, D.; Zamzami, N.; Park, P.U.; Sharpe, J.; Youle, R.J.; Goldmacher, V.S. Cytomegalovirus cell death suppressor vMIA blocks Bax- but not Bak-mediated apoptosis by binding and sequestering Bax at mitochondria. Proc. Natl. Acad. Sci. USA 2004, 101, 7988-7993.

18. Poncet, D.; Larochette, N.; Pauleau, A.L.; Boya, P.; Jalil, A.A.; Cartron, P.F.; Vallette, F.; Schnebelen, C.; Bartle, L.M.; Skaletskaya, A.; et al. An anti-apoptotic viral protein that recruits Bax to mitochondria. J. Biol. Chem. 2004, 279, 22605-22614.

19. Hayajneh, W.A.; Colberg-Poley, A.M.; Skaletskaya, A.; Bartle, L.M.; Lesperance, M.M.; Contopoulos-Ioannidis, D.G.; Kedersha, N.L.; Goldmacher, V.S. The sequence and antiapoptotic functional domains of the human cytomegalovirus UL37 exon 1 immediate early protein are conserved in multiple primary strains. Virology 2001, 279, 233-240.

20. Mavinakere, M.S.; Williamson, C.D.; Goldmacher, V.S.; Colberg-Poley, A.M. Processing of human cytomegalovirus UL37 mutant glycoproteins in the endoplasmic reticulum lumen prior to mitochondrial importation. J. Virol. 2006, 80, 6771-6783.

21. Bozidis, P.; Williamson, C.D.; Colberg-Poley, A.M. Mitochondrial and secretory human cytomegalovirus UL37 proteins traffic into mitochondrion-associated membranes of human cells. J. Virol. 2008, 82, 2715-2726.

22. Bozidis, P.; Williamson, C.D.; Wong, D.S.; Colberg-Poley, A.M. Trafficking of UL37 proteins into mitochondrion-associated membranes during permissive human cytomegalovirus infection. J. Virol. 2010, 84, 7898-7903.

23. Williamson, C.D.; Colberg-Poley, A.M. Intracellular Sorting Signals for Sequential Trafficking of Human Cytomegalovirus UL37 Proteins to the Endoplasmic Reticulum and Mitochondria. J. Virol. 2010, 84, 6400-6409.

24. Colberg-Poley, A.M.; Patel, M.B.; Erezo, D.P.; Slater, J.E. Human cytomegalovirus UL37 immediate-early regulatory proteins traffic through the secretory apparatus and to mitochondria. J. Gen. Virol. 2000, 81, 1779-1789.

25. Williamson, C.D.; Colberg-Poley, A.M. Access of viral proteins to mitochondria via mitochondria-associated membranes. Rev. Med. Virol. 2009, 19, 147-164.

26. Williamson, C.D.; Zhang, A.; Colberg-Poley, A.M. The human cytomegalovirus protein UL37 exon 1 associates with internal lipid rafts. J. Virol. 2011, 85, 2100-2111.

27. Zhang, A.; Hildreth, R.L.; Colberg-Poley, A.M. Human cytomegalovirus inhibits apoptosis by proteasome-mediated degradation of Bax at endoplasmic reticulum-mitochondrion contacts. J. Virol. 2013, 87, 5657-5668.

28. Seo, J.Y.; Yaneva, R.; Hinson, E.R.; Cresswell, P. Human cytomegalovirus directly induces the antiviral protein viperin to enhance infectivity. Science 2011, 332, 1093-1097. 
29. Seo, J.Y.; Cresswell, P. Viperin regulates cellular lipid metabolism during human cytomegalovirus infection. PLoS Pathog. 2013, 9, e1003497.

30. Norris, K.L.; Youle, R.J. Cytomegalovirus proteins vMIA and m38.5 link mitochondrial morphogenesis to Bcl-2 family proteins. J. Virol. 2008, 82, 6232-6243.

31. Ma, J.; Edlich, F.; Bermejo, G.A.; Norris, K.L.; Youle, R.J.; Tjandra, N. Structural mechanism of Bax inhibition by cytomegalovirus protein vMIA. Proc. Natl. Acad. Sci. USA 2012, 109, 20901-20906.

32. Poncet, D.; Pauleau, A.L.; Szabadkai, G.; Vozza, A.; Scholz, S.R.; Le Bras, M.; Briere, J.J.; Jalil, A.; Le Moigne, R.; Brenner, C.; et al. Cytopathic effects of the cytomegalovirus-encoded apoptosis inhibitory protein vMIA. J. Cell Biol. 2006, 174, 985-996.

33. McCormick, A.L.; Smith, V.L.; Chow, D.; Mocarski, E.S. Disruption of mitochondrial networks by the human cytomegalovirus UL37 gene product viral mitochondrion-localized inhibitor of apoptosis. J. Virol. 2003, 77, 631-641.

34. Roumier, T.; Szabadkai, G.; Simoni, A.M.; Perfettini, J.L.; Paulau, A.L.; Castedo, M.; Metivier, D.; Badley, A.; Rizzuto, R.; Kroemer, G. HIV-1 protease inhibitors and cytomegalovirus vMIA induce mitochondrial fragmentation without triggering apoptosis. Cell Death Differ. 2006, 13, 348-351.

35. McCormick, A.L.; Roback, L.; Mocarski, E.S. HtrA2/Omi terminates cytomegalovirus infection and is controlled by the viral mitochondrial inhibitor of apoptosis (vMIA). PLoS Pathog. 2008, 4, e1000063.

36. Altan-Bonnet, N.; Sougrat, R.; Liu, W.; Snapp, E.L.; Ward, T.; Lippincott-Schwartz, J. Golgi inheritance in mammalian cells is mediated through endoplasmic reticulum export activities. Mol. Biol. Cell 2006, 17, 990-1005.

37. Friedman, J.R.; Lackner, L.L.; West, M.; DiBenedetto, J.R.; Nunnari, J.; Voeltz, G.K. ER tubules mark sites of mitochondrial division. Science 2011, 334, 358-362.

38. Shim, S.H.; Xia, C.; Zhong, G.; Babcock, H.P.; Vaughan, J.C.; Huang, B.; Wang, X.; Xu, C.; Bi, G.Q.; Zhuang, X. Super-resolution fluorescence imaging of organelles in live cells with photoswitchable membrane probes. Proc. Natl. Acad. Sci. USA 2012, 109, 13978-13983.

39. Shao, L.; Kner, P.; Rego, E.H.; Gustafsson, M.G. Super-resolution 3D microscopy of live whole cells using structured illumination. Nat. Methods 2011, 8, 1044-1046.

40. Arnoult, D.; Skaletskaya, A.; Estaquier, J.; Dufour, C.; Goldmacher, V.S. The murine cytomegalovirus cell death suppressor m38.5 binds Bax and blocks Bax-mediated mitochondrial outer membrane permeabilization. Apoptosis 2008, 13, 1100-1110.

41. Biggs, D.S. 3D deconvolution microscopy. Curr. Protoc. Cytom. 2010, doi:10.1002/0471142956.cy1219s52.

42. York, A.G.; Parekh, S.H.; Dalle Nogare, D.; Fischer, R.S.; Temprine, K.; Mione, M.; Chitnis, A.B.; Combs, C.A.; Shroff, H. Resolution doubling in live, multicellular organisms via multifocal structured illumination microscopy. Nat. Methods 2012, 9, 749-754.

43. Hell, S.W. Far-field optical nanoscopy. Science 2007, 316, 1153-1158.

44. Hell, S.W.; Wichmann, J. Breaking the diffraction resolution limit by stimulated emission: stimulated-emission-depletion fluorescence microscopy. Opt. Lett. 1994, 19, 780-782. 
45. Jans, D.C.; Wurm, C.A.; Riedel, D.; Wenzel, D.; Stagge, F.; Deckers, M.; Rehling, P.; Jakobs, S. STED super-resolution microscopy reveals an array of MINOS clusters along human mitochondria. Proc. Natl. Acad. Sci. USA 2013, 110, 8936-8941.

46. Neumann, D.; Buckers, J.; Kastrup, L.; Hell, S.W.; Jakobs, S. Two-color STED microscopy reveals different degrees of colocalization between hexokinase-I and the three human VDAC isoforms. PMC Biophys. 2010, 3, 4.

47. Singh, H.; Lu, R.; Rodriguez, P.F.; Wu, Y.; Bopassa, J.C.; Stefani, E.; Toro, L. Visualization and quantification of cardiac mitochondrial protein clusters with STED microscopy. Mitochondrion 2012, 12, 230-236.

48. Lippincott-Schwartz, J.; Patterson, G.H. Photoactivatable fluorescent proteins for diffraction-limited and super-resolution imaging. Trends Cell Biol. 2009, 19, 555-565.

49. Pauleau, A.L.; Larochette, N.; Giordanetto, F.; Scholz, S.R.; Poncet, D.; Zamzami, N.; Goldmacher, V.S.; Kroemer, G. Structure-function analysis of the interaction between Bax and the cytomegalovirus-encoded protein vMIA. Oncogene 2007, 26, 7067-7080.

50. Hildreth, R.L.; Bullough, M.D.; Zhang, A.; Chen, H.L.; Schwartz, P.H.; Panchision, D.M.; Colberg-Poley, A.M. Viral mitochondria-localized inhibitor of apoptosis (UL37 exon 1 protein) does not protect human neural precursor cells from human cytomegalovirus-induced cell death. J. Gen. Virol. 2012, 93, 2436-2446.

51. Crump, C.M.; Hung, C.H.; Thomas, L.; Wan, L.; Thomas, G. Role of PACS-1 in trafficking of human cytomegalovirus glycoprotein B and virus production. J. Virol. 2003, 77, 11105-11113.

52. Colberg-Poley, A.M.; Williamson, C.D. Intracellular sorting and trafficking of cytomegalovirus proteins during permissive infection. In Cytomegaloviruses: From Molecular Pathogenesis to Intervention, 2nd ed.; Reddehase, M.J., Ed.; Caister Academic Press/Horizon: Norwich, UK, 2013; Volume I, pp. 196-229.

53. Gossen, M.; Bujard, H. Tight control of gene expression in mammalian cells by tetracycline-responsive promoters. Proc. Natl. Acad. Sci. USA 1992, 89, 5547-5551.

54. Watanabe, S.; Punge, A.; Hollopeter, G.; Willig, K.I.; Hobson, R.J.; Davis, M.W.; Hell, S.W.; Jorgensen, E.M. Protein localization in electron micrographs using fluorescence nanoscopy. Nat. Methods 2011, 8, 80-84.

55. Subach, F.V.; Patterson, G.H.; Manley, S.; Gillette, J.M.; Lippincott-Schwartz, J.; Verkhusha, V.V. Photoactivatable mCherry for high-resolution two-color fluorescence microscopy. Nat. Methods 2009, 6, 153-159.

56. Adair, R.; Liebisch, G.W.; Su, Y.; Colberg-Poley, A.M. Alteration of cellular RNA splicing and polyadenylation machineries during productive human cytomegalovirus infection. J. Gen. Virol. 2004, 85, 3541-3553.

57. Vicidomini, G.; Moneron, G.; Han, K.Y.; Westphal, V.; Ta, H.; Reuss, M.; Engelhardt, J.; Eggeling, C.; Hell, S.W. Sharper low-power STED nanoscopy by time gating. Nat. Methods 2011, 8, 571-573.

58. Ingaramo, M.; York, A.G.; Wawrzusin, P.; Milberg, O.; Hong, A.; Weigert, R.; Shroff, H.; Patterson, G.H. Two-photon excitation improves multifocal structured illumination in thick scattering tissue. Proc. Natl. Acad. Sci. USA 2014, in press. 
59. Edelstein, A.; Amodaj, N.; Hoover, K.; Vale, R.; Stuurman, N. Computer control of microscopes using microManager. Curr. Protoc. Mol. Biol. 2010, doi:10.1002/0471142727.mb1420s92.

60. York, A.G. MSIM Superresolution Fluorescence Microscopy of Multicellular Organisms. Available online: http://code.google.com/p/msim/ (accessed on 3 May 2012).

61. Richardson, W.H. Bayesian-based iterative method of image restoration. JOSA 1972, 62, 55-59.

62. Lucy, L. An iterative technique for the rectification of observed distributions. Astron. J. 1974, 79, 745-754.

63. York, A.G.; Chandris, P.; Nogare, D.D.; Head, J.; Wawrzusin, P.; Fischer, R.S.; Chitnis, A.; Shroff, H. Instant super-resolution imaging in live cells and embryos via analog image processing. Nat. Methods 2013, 10, 1122-1126.

64. Betzig, E.; Patterson, G.H.; Sougrat, R.; Lindwasser, O.W.; Olenych, S.; Bonifacino, J.S.; Davidson, M.W.; Lippincott-Schwartz, J.; Hess, H.F. Imaging intracellular fluorescent proteins at nanometer resolution. Science 2006, 313, 1642-1645.

65. Schneider, C.A.; Rasband, W.S.; Eliceiri, K.W. NIH Image to ImageJ: 25 years of image analysis. Nat. Methods 2012, 9, 671-675.

66. Schindelin, J.; Arganda-Carreras, I.; Frise, E.; Kaynig, V.; Longair, M.; Pietzsch, T.; Preibisch, S.; Rueden, C.; Saalfeld, S.; Schmid, B.; et al. Fiji: An open-source platform for biological-image analysis. Nat. Methods 2012, 9, 676-682.

67. Willig, K.I.; Kellner, R.R.; Medda, R.; Hein, B.; Jakobs, S.; Hell, S.W. Nanoscale resolution in GFP-based microscopy. Nat. Methods 2006, 3, 721-723.

68. Gudheti, M.V.; Curthoys, N.M.; Gould, T.J.; Kim, D.; Gunewardene, M.S.; Gabor, K.A.; Gosse, J.A.; Kim, C.H.; Zimmerberg, J.; Hess, S.T. Actin mediates the nanoscale membrane organization of the clustered membrane protein influenza hemagglutinin. Biophys. J. 2013, 104, 2182-2192.

69. Roy, N.H.; Chan, J.; Lambele, M.; Thali, M. Clustering and mobility of HIV-1 Env at viral assembly sites predict its propensity to induce cell-cell fusion. J. Virol. 2013, 87, 7516-7525.

70. Grover, J.R.; Llewellyn, G.N.; Soheilian, F.; Nagashima, K.; Veatch, S.L.; Ono, A. Roles played by capsid-dependent induction of membrane curvature and Gag-ESCRT interactions in tetherin recruitment to HIV-1 assembly sites. J. Virol. 2013, 87, 4650-4664.

71. Felts, R.L.; Narayan, K.; Estes, J.D.; Shi, D.; Trubey, C.M.; Fu, J.; Hartnell, L.M.; Ruthel, G.T.; Schneider, D.K.; Nagashima, K.; et al. 3D visualization of HIV transfer at the virological synapse between dendritic cells and T cells. Proc. Natl. Acad. Sci. USA 2010, 107, 13336-13341.

72. Wang, I.H.; Suomalainen, M.; Andriasyan, V.; Kilcher, S.; Mercer, J.; Neef, A.; Luedtke, N.W.; Greber, U.F. Tracking viral genomes in host cells at single-molecule resolution. Cell Host Microbe 2013, 14, 468-480.

73. Wurm, C.A.; Neumann, D.; Lauterbach, M.A.; Harke, B.; Egner, A.; Hell, S.W.; Jakobs, S. Nanoscale distribution of mitochondrial import receptor Tom20 is adjusted to cellular conditions and exhibits an inner-cellular gradient. Proc. Natl. Acad. Sci. USA 2011, 108, 13546-13551.

74. Williamson, C.D.; DeBiasi, R.L.; Colberg-Poley, A.M. Viral product trafficking to mitochondria, mechanisms and roles in pathogenesis. Infect. Disord. Drug Targets 2012, 12, 18-37. 

Reprinted from Viruses. Cite as: Sijmons, S.; Van Ranst, M.; Maes, P. Genomic and Functional Characteristics of Human Cytomegalovirus Revealed by Next-Generation Sequencing. Viruses 2014, 6, 1049-1072.

Review

\title{
Genomic and Functional Characteristics of Human Cytomegalovirus Revealed by Next-Generation Sequencing
}

\author{
Steven Sijmons *, Marc Van Ranst and Piet Maes \\ Laboratory of Clinical Virology, Rega Institute for Medical Research, K.U.Leuven, \\ Minderbroedersstraat 10, Leuven BE-3000, Belgium; \\ E-Mails: marc.vanranst@uzleuven.be (M.V.R.); piet.maes@rega.kuleuven.be (P.M.) \\ * Author to whom correspondence should be addressed; E-Mail: steven.sijmons@uzleuven.be; \\ Tel.: +32-16-332-166; Fax: +32-16-332-131.
}

Received: 20 January 2014; in revised form: 11 February 2014/ Accepted: 11 February 2014 / Published: 5 March 2014

\begin{abstract}
The complete genome of human cytomegalovirus (HCMV) was elucidated almost 25 years ago using a traditional cloning and Sanger sequencing approach. Analysis of the genetic content of additional laboratory and clinical isolates has lead to a better, albeit still incomplete, definition of the coding potential and diversity of wild-type HCMV strains. The introduction of a new generation of massively parallel sequencing technologies, collectively called next-generation sequencing, has profoundly increased the throughput and resolution of the genomics field. These increased possibilities are already leading to a better understanding of the circulating diversity of HCMV clinical isolates. The higher resolution of next-generation sequencing provides new opportunities in the study of intrahost viral population structures. Furthermore, deep sequencing enables novel diagnostic applications for sensitive drug resistance mutation detection. RNA-seq applications have changed the picture of the HCMV transcriptome, which resulted in proof of a vast amount of splicing events and alternative transcripts. This review discusses the application of next-generation sequencing technologies, which has provided a clearer picture of the intricate nature of the HCMV genome. The continuing development and application of novel sequencing technologies will further augment our understanding of this ubiquitous, but elusive, herpesvirus.
\end{abstract}

Keywords: cytomegalovirus; genomics; transcriptomics; next-generation sequencing; drug resistance; deep sequencing 


\section{Introduction}

Human cytomegalovirus (HCMV), the prototype member of the herpesvirus subfamily Betaherpesvirinae, has a worldwide distribution and infections with this virus are extremely common. Seroprevalences in the adult population vary from $45 \%$ to $100 \%$, increasing with age and varying with geographic location and socio-economic background [1]. HCMV causes only mild to no symptoms in immunocompetent individuals, but the virus is never cleared and establishes a latent infection for the lifetime of its host [2]. Primary infection, reactivation, or reinfection of immunocompromised individuals, such as transplant recipients and AIDS patients, results in considerable morbidity and mortality [3]. Furthermore, it is the most important congenital infection in both developed and developing countries, causing sensorineural hearing loss and neurodevelopmental delays [4]. The double-stranded DNA (dsDNA) genome of wild-type HCMV strains has a size of around $235 \mathrm{~kb}$, which is longer than all other human herpesviruses and one of the longest genomes of all human viruses in general. It has the characteristic herpesvirus class $\mathrm{E}$ genome architecture, consisting of two unique regions (unique long UL and unique short US), both flanked by a pair of inverted repeats (terminal/internal repeat long TRL/IRL and internal/terminal repeat short IRS/TRS). Both sets of repeats share a region of a few hundred bps, the so-called " $a$ sequence"; the other regions of the repeats are sometimes referred to as " $b$ sequence" and "c sequence" (Figure 1). The genome exists as an equimolar mixture of four genomic isomers by inversion of UL and US regions [5].

Figure 1. Class E genome of HCMV. The unique long and unique short regions are indicated as UL and US. Repeat regions are indicated as $a, b$ and $c$ sequences, where primes designate inverted orientations. Sequences $a b$ and $b^{\prime} a$ ' correspond to the terminal/internal repeat long (TRL/IRL); sequences $a{ }^{\prime} c^{\prime}$ and $c a$ correspond to the internal/terminal repeat short (IRS/TRS). Top: typical genome arrangement of wild-type strains; bottom: genome arrangement of strain AD169 is given as an example of a laboratory-adapted strain. Genome rearrangements (deletion of UL 3' end and replacement by an inverted copy of UL 5' end) that have occurred during extensive passaging are indicated in red between the wild-type and laboratory-adapted configurations.

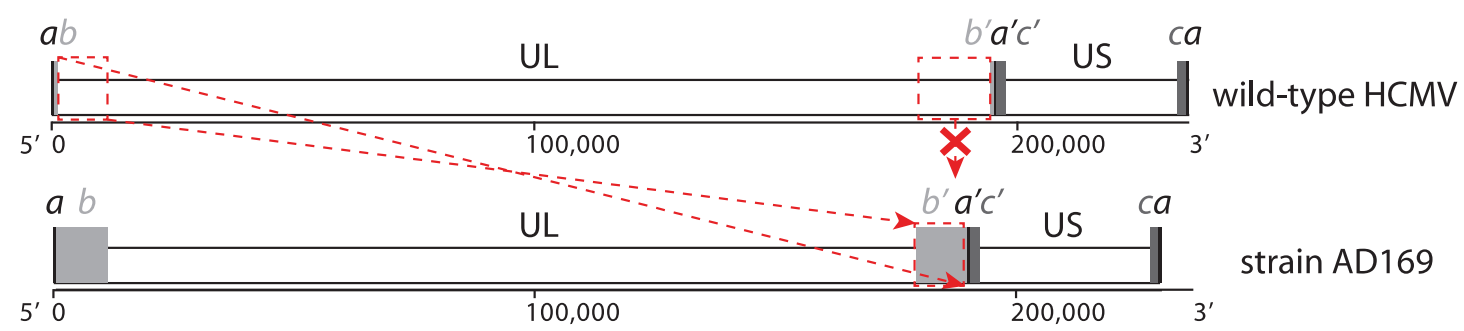

The commercial introduction of new DNA sequencing technologies, referred to as next-generation sequencing (NGS), has immensely impacted the field of genomics. These novel technologies generate a massive throughput at a considerably lower per base cost than traditional Sanger sequencing, and obviate the need for laborious cloning procedures [6-8]. NGS has already shown its promise in virological research with applications in virus discovery and metagenomics, whole 
genome analysis, deep sequencing of viral populations, novel diagnostic assays, and studies of virus-host interactions through transcriptome and epigenome studies [9-11]. In this review, we will highlight the recent progress that has contributed to the understanding of HCMV genomics through the application of NGS technologies.

\section{HCMV Genomics before the Introduction of NGS}

\subsection{Genome Alterations during Cell Culture Adaptation}

When the first complete genome sequence of HCMV was published in 1990, it was the largest contiguous sequence generated at the time $[12,13]$. The authors estimated that the effort to sequence the genome of the laboratory-adapted strain AD169 with M13 shotgun cloning and Sanger sequencing was equivalent to a 12-year workload for one person. The laboratory-adapted strains AD169 and Towne had been passaged extensively in human fibroblast cell lines and were found to cause no or very low virulence in seronegative individuals during vaccine studies [14-16]. In contrast, the Toledo strain had been passaged significantly less and produced mononucleosis syndromes when administered to seropositive individuals [17]. When genome regions of AD169, Towne, and Toledo were compared through restriction enzyme profiles, hybridization, and sequencing techniques, it was observed that AD169 and Towne had lost genome segments of $15 \mathrm{~kb}$ and $13 \mathrm{~kb}$, respectively. These segments were situated at the 3' end of the UL region (sometimes referred to as the UL/b' region) and were replaced by an inverted copy of the 5 ' end of the UL region, leading to an enlargement of the $b$ repeats (Figure 1). This missing UL/b' segment was also found to be present in an additional set of five low-passage clinical isolates like Toledo, confirming that it is a universal hallmark of wild-type HCMV strains and clearly contains factors that are dispensable for fibroblast replication, but essential for virulence in vivo [18,19].

In addition to these genome rearrangements, strains AD169 and Towne display more subtle alterations of their coding capacity in genes inside - variants of AD169 and Towne with a more or less intact $\mathrm{UL} / b$ ' region exist [20] — and outside the UL/b' region. AD169 has open reading frame (ORF) disrupting mutations in genes RL5A, RL13, UL36, and UL131A; Towne is affected in genes RL13, UL1, UL40, UL130, US1, and US9 [20-24]. Even the low-passage strain Toledo is mutated in genes RL13, UL9 and UL128 [25]. Almost all strains passaged in fibroblast cell cultures display one or more mutations in the UL128 locus (UL128L) and in the genes of the RL11 family, indicating a role in cell tropism for these gene products. UL128L consists of genes UL128, UL130, and UL131A (Figure 2). and its products form a complex with the viral glycoproteins gH and gL. While this complex is dispensable for growth in fibroblasts, it is essential for endothelial and epithelial cell tropism [26-28]. The RL11 gene family contains 14 genes at the 5' end of the UL region (RL5A, RL6, RL11-UL1, UL4-UL11, Figure 2) that are dispensable for growth in

fibroblasts and are functionally poorly characterized [23,29,30]. Several of these genes show a remarkable genetic variability between different clinical isolates [25,31]. The majority of the RL11 genes have a characteristic domain (RL11D) that shares homology with the CR1 domain of the adenovirus E3 genes [23]. These proteins could function as modulators of a set of variable host proteins, and similarities to the immunoglobulin IgD family have been proposed [12]. Recently, some RL11 genes have indeed been implicated in immune evasive functions [32-34]. 
Figure 2. Genome annotation of the low-passage HCMV strain Merlin (GenBank accession NC_006273). The dsDNA genome is visualized as a single line; nucleotide positions are given in bps. Terminal and internal repeat regions (TRL, IRL/IRS and TRS) are indicated with white boxes. Arrows represent genes; different gene families are designated with different color codes, as illustrated below the genome. The four large non-coding RNAs and the origin of lytic replication are also represented.

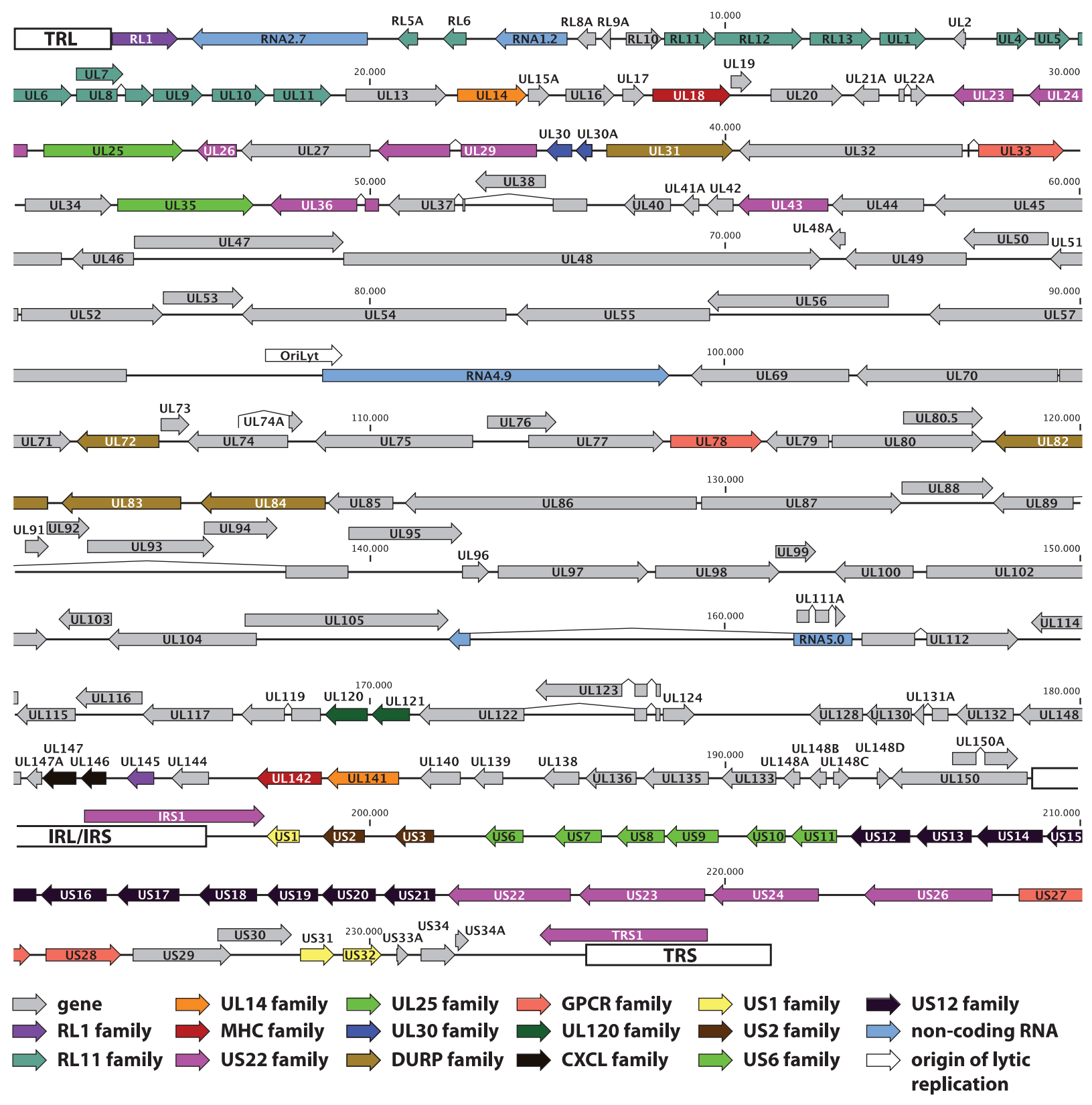

While the consensus sequence of the low-passage strain Merlin only has a mutation in gene UL128, cloning of the strain into a bacterial artificial chromosome (BAC) vector showed that it was also defective in gene RL13 [25,35]. Since RL13 mutations were present at different sites in different clones, this was not noticeable in the consensus sequence. Repair of UL128 in the Merlin BAC repressed replication in fibroblast cells, but not in epithelial cells and, consequently, novel mutations only emerged in fibroblasts. On the contrary, repair of RL13 impaired replication in 
fibroblast cells as well as in epithelial cells, and mutants appeared rapidly in both cell types. Mutational dynamics of clinical HCMV isolates were further analyzed in fibroblasts, epithelial, and endothelial cells by recording all mutations in four isolates up to passage 50 or more [36]. Several additional genes were mutated in some strains, but only UL128L and RL13 invariably mutated in fibroblast cells (RL13 in all cell types). Taken together, these studies stress the inherent instability of HCMV isolates when passaged in cell culture. Thus, in vitro studies with HCMV strains should allow for these limitations, and researchers should be mindful of the genetic changes that have altered the coding capacity and functionality of the strain under study.

\subsection{Genome Annotation}

The publication of the first complete genome sequence of HCMV was accompanied by a first genome annotation, predicting 208 ORFs that were potentially protein encoding [12]. Only ORFs encoding for proteins with a minimum length of 100 amino acids were considered, with a maximal overlap of $60 \%$ between ORFs. As the authors expected, this preliminary annotation wrongly predicted some ORFs and at the same time excluded genuine ORFs that were either too small or highly spliced. Since the results were based on the laboratory-adapted strain AD169, the ORFs encoded by the UL/b' region (Figure 1 ) were also missed $[18,19]$. The annotation was further refined by comparison with chimpanzee, rhesus and murine cytomegalovirus genomes [37,38] and by sequence analysis of additional clinical isolates of HCMV [25,39]. The low-passage strain Merlin has become the reference strain for wild-type HCMV, and its sequence entry (NCBI GenBank accession NC_006273, Table 1) currently contains a set of 170 genes. Several sets of genes share some sequence similarity and are thought to have originated from duplication events and subsequent divergence of structure and function [12,13]. These 15 gene families are indicated on the genome map in Figure 2.

Table 1. HCMV complete genome sequences available on NCBI GenBank, listed in order of submission date.

\begin{tabular}{|c|c|c|c|c|c|}
\hline $\begin{array}{l}\text { GenBank } \\
\text { accession }\end{array}$ & Strain name & Clinical source & Passage history & Ref. & $\begin{array}{c}\text { Submission } \\
\text { date }\end{array}$ \\
\hline X17403 & AD169 & Adenoids of a 7-year old girl & $\begin{array}{l}\text { Passaged extensively in } \\
\text { human fibroblasts }\end{array}$ & {$[12]$} & $\begin{array}{c}\text { December 6, } \\
1989\end{array}$ \\
\hline BK00039' & $\begin{array}{l}\text { AD169 } \\
\text { varUK }\end{array}$ & Adenoids of a 7-year old girl & $\begin{array}{l}\text { Passaged extensively in } \\
\text { human fibroblasts }\end{array}$ & {$[40]$} & May 1, 2002 \\
\hline $\begin{array}{l}\text { NC_006273* } \\
\text { GU179001 }\end{array}$ & Merlin & $\begin{array}{l}\text { Urine from a congenitally } \\
\text { infected infant }\end{array}$ & $\begin{array}{c}\text { Passaged } 3 \text { times in human } \\
\text { fibroblasts }\end{array}$ & {$[25]$} & $\begin{array}{l}\text { September } \\
27,2002\end{array}$ \\
\hline AY315197 & Towne varS & $\begin{array}{c}\text { Urine of a 2-month-old } \\
\text { infant with microcephaly and } \\
\text { hepatosplenomegaly }\end{array}$ & $\begin{array}{l}\text { Passaged extensively in } \\
\text { human fibroblasts }\end{array}$ & {$[30]$} & June 6, 2003 \\
\hline AC146851 & Towne-BAC & $\begin{array}{c}\text { Urine of a 2-month-old } \\
\text { infant with microcephaly and } \\
\text { hepatosplenomegaly }\end{array}$ & $\begin{array}{l}\text { BAC clone from a plaque } \\
\text { purified Towne derivative } \\
\text { (varS) }\end{array}$ & {$[39]$} & $\begin{array}{l}\text { October 14, } \\
2003\end{array}$ \\
\hline AC146904 & PH-BAC & $\begin{array}{c}\text { Transplant patient with } \\
\text { HCMV disease }\end{array}$ & $\begin{array}{l}\text { BAC clone from isolate } \mathrm{PH} \\
\text { (passaged less than } 12 \text { times) }\end{array}$ & {$[39]$} & $\begin{array}{c}\text { October 21, } \\
2003\end{array}$ \\
\hline
\end{tabular}


Table 1. Cont.

\begin{tabular}{|c|c|c|c|c|c|}
\hline $\begin{array}{l}\text { GenBank } \\
\text { accession }\end{array}$ & Strain name & Clinical source & Passage history & Ref. & $\begin{array}{c}\text { Submission } \\
\text { date }\end{array}$ \\
\hline AC146905 & Toledo-BAC & $\begin{array}{l}\text { Urine from a congenitally } \\
\text { infected infant }\end{array}$ & $\begin{array}{l}\text { BAC clone from a plaque } \\
\text { purified Toledo derivative }\end{array}$ & {$[39]$} & $\begin{array}{l}\text { October 21, } \\
2013\end{array}$ \\
\hline AC146906 & TR-BAC & $\begin{array}{l}\text { AIDS patient with CMV } \\
\text { retinitis }\end{array}$ & BAC clone from isolate TR & [39] & $\begin{array}{l}\text { October 21, } \\
2013\end{array}$ \\
\hline AC146907 & FIX-BAC & $\begin{array}{l}\text { Cervical secretions of a } \\
\text { pregnant woman with a } \\
\text { primary HCMV infection }\end{array}$ & $\begin{array}{c}\text { BAC clone from isolate } \\
\text { VR1814 }\end{array}$ & [39] & $\begin{array}{l}\text { October 21, } \\
2013\end{array}$ \\
\hline AC146999 & AD169-BAC & Adenoids of a 7-year old girl & $\begin{array}{l}\text { BAC clone from a plaque } \\
\text { purified AD169 derivative } \\
\text { (varATCC) }\end{array}$ & [39] & $\begin{array}{l}\text { October 31, } \\
2013\end{array}$ \\
\hline EF999921 & $\begin{array}{l}\text { TB40/E clone } \\
\text { TB40-BAC4 }\end{array}$ & $\begin{array}{l}\text { Throat wash of a bone } \\
\text { marrow transplant recipient }\end{array}$ & $\begin{array}{l}\text { BAC clone from TB40/E } \\
\text { passaged } 5 \text { times in human } \\
\text { fibroblasts and } 22 \text { times in } \\
\text { human endothelial cells }\end{array}$ & {$[41]$} & $\begin{array}{l}\text { June } 25, \\
2007\end{array}$ \\
\hline FJ527563 & $\begin{array}{l}\text { AD169 } \\
\text { varUC }\end{array}$ & Adenoids of a 7-year old girl & $\begin{array}{l}\text { Passaged extensively in } \\
\text { human fibroblasts }\end{array}$ & {$[20]$} & $\begin{array}{l}\text { December 1, } \\
2008\end{array}$ \\
\hline FJ616285 & Towne varL & $\begin{array}{c}\text { Urine of a 2-month-old } \\
\text { infant with microcephaly and } \\
\text { hepatosplenomegaly }\end{array}$ & $\begin{array}{l}\text { Passaged extensively in } \\
\text { human fibroblasts }\end{array}$ & {$[20]$} & $\begin{array}{c}\text { January } 9 \\
2009\end{array}$ \\
\hline GQ221973 & HAN13 & Bronchoalveolar lavage & $\begin{array}{c}\text { Passaged } 3 \text { times in human } \\
\text { fibroblasts }\end{array}$ & {$[42]$} & $\begin{array}{l}\text { May } 28, \\
2009\end{array}$ \\
\hline GQ221974 & 3157 & $\begin{array}{l}\text { Urine from a congenitally } \\
\text { infected infant }\end{array}$ & $\begin{array}{c}\text { Passaged } 3 \text { times in human } \\
\text { fibroblasts }\end{array}$ & {$[42]$} & $\begin{array}{l}\text { May } 28, \\
2009\end{array}$ \\
\hline GQ221975 & JP & $\begin{array}{l}\text { Post mortem prostate tissue } \\
\text { from an AIDS patient }\end{array}$ & Unpassaged & {$[42]$} & $\begin{array}{l}\text { May } 28 \\
2009\end{array}$ \\
\hline GQ396662 & HAN38 & Bronchoalveolar lavage & $\begin{array}{c}\text { Passaged } 2 \text { times in human } \\
\text { fibroblasts }\end{array}$ & {$[42]$} & $\begin{array}{l}\text { July } 17 \text {, } \\
2009\end{array}$ \\
\hline GQ396663 & HAN20 & Bronchoalveolar lavage & $\begin{array}{l}\text { Passaged } 2 \text { times in human } \\
\text { fibroblasts }\end{array}$ & {$[42]$} & $\begin{array}{l}\text { July } 17, \\
2009\end{array}$ \\
\hline GQ466044 & 3301 & $\begin{array}{l}\text { Urine from a congenitally } \\
\text { infected infant }\end{array}$ & Unpassaged & {$[42]$} & $\begin{array}{l}\text { August 7, } \\
2009\end{array}$ \\
\hline GU179288 & U8 & $\begin{array}{l}\text { Urine from a congenitally } \\
\text { infected infant }\end{array}$ & Unpassaged & {$[42]$} & $\begin{array}{l}\text { November } 5 \text {, } \\
2009\end{array}$ \\
\hline GU179289 & VR1814 & $\begin{array}{l}\text { Cervical secretions of a } \\
\text { pregnant woman with a } \\
\text { primary HCMV infection }\end{array}$ & Unpassaged & {$[42]$} & $\begin{array}{l}\text { November } 5, \\
2009\end{array}$ \\
\hline GU179290 & U11 & $\begin{array}{l}\text { Urine from a congenitally } \\
\text { infected infant }\end{array}$ & Unpassaged & {$[42]$} & $\begin{array}{l}\text { November } 5 \text {, } \\
2009\end{array}$ \\
\hline GU179291 & AF1 & Amniotic fluid & Unpassaged & {$[42]$} & $\begin{array}{c}\text { November 5, } \\
2009\end{array}$ \\
\hline GU937742 & Toledo & $\begin{array}{l}\text { Urine from a congenitally } \\
\text { infected infant }\end{array}$ & $\begin{array}{c}\text { Passaged several times in } \\
\text { human fibroblasts }\end{array}$ & {$[25]$} & $\begin{array}{c}\text { February 26, } \\
2010\end{array}$ \\
\hline HQ380895 & $\mathrm{JHC}$ & $\begin{array}{l}\text { Blood from a bone marrow } \\
\text { transplant patient }\end{array}$ & $\begin{array}{c}\text { Plaque purified and } \\
\text { passaged } 3 \text { times in human } \\
\text { fibroblasts }\end{array}$ & {$[43]$} & $\begin{array}{c}\text { October } 7 \\
2010\end{array}$ \\
\hline
\end{tabular}


Table 1. Cont.

\begin{tabular}{|c|c|c|c|c|c|}
\hline $\begin{array}{l}\text { GenBank } \\
\text { accession }\end{array}$ & Strain name & Clinical source & Passage history & Ref. & $\begin{array}{c}\text { Submission } \\
\text { date }\end{array}$ \\
\hline JX512197 & 6397 & $\begin{array}{c}\text { Urine from a congenitally } \\
\text { infected infant }\end{array}$ & $\begin{array}{c}\text { Passaged } 3 \text { times in human } \\
\text { fibroblasts }\end{array}$ & - & $\begin{array}{l}\text { August 21, } \\
2012\end{array}$ \\
\hline JX512198 & Davis & $\begin{array}{l}\text { Liver biopsy from a } \\
\text { congenitally infected infant }\end{array}$ & $\begin{array}{l}\text { Passaged many times in } \\
\text { human fibroblasts }\end{array}$ & - & $\begin{array}{l}\text { August 21, } \\
2012\end{array}$ \\
\hline JX512199 & HAN1 & Bronchoalveolar lavage & No information & - & $\begin{array}{l}\text { August 21, } \\
2012\end{array}$ \\
\hline JX512200 & HAN2 & Bronchoalveolar lavage & $\begin{array}{c}\text { Passaged } 3 \text { times in human } \\
\text { fibroblasts }\end{array}$ & - & $\begin{array}{l}\text { August 21, } \\
2012\end{array}$ \\
\hline JX512201 & HAN3 & Bronchoalveolar lavage & $\begin{array}{c}\text { Passaged } 3 \text { times in human } \\
\text { fibroblasts }\end{array}$ & - & $\begin{array}{l}\text { August 21, } \\
2012\end{array}$ \\
\hline JX512202 & HAN8 & Bronchoalveolar lavage & $\begin{array}{c}\text { Passaged } 3 \text { times in human } \\
\text { fibroblasts }\end{array}$ & - & $\begin{array}{l}\text { August 21, } \\
2012\end{array}$ \\
\hline JX512203 & HAN12 & Bronchoalveolar lavage & $\begin{array}{c}\text { Passaged } 3 \text { times in human } \\
\text { fibroblasts }\end{array}$ & - & $\begin{array}{l}\text { August 21, } \\
2012\end{array}$ \\
\hline JX512204 & HAN16 & Urine from an infant & $\begin{array}{c}\text { Passaged } 2 \text { times in human } \\
\text { fibroblasts }\end{array}$ & - & $\begin{array}{l}\text { August 21, } \\
2012\end{array}$ \\
\hline JX512205 & HAN19 & Bronchoalveolar lavage & $\begin{array}{c}\text { Passaged } 2 \text { times in human } \\
\text { fibroblasts }\end{array}$ & - & $\begin{array}{l}\text { August 21, } \\
2012\end{array}$ \\
\hline JX512206 & HAN22 & Bronchoalveolar lavage & $\begin{array}{c}\text { Passaged } 2 \text { times in human } \\
\text { fibroblasts }\end{array}$ & - & $\begin{array}{l}\text { August 21, } \\
2012\end{array}$ \\
\hline JX512207 & HAN28 & Bronchoalveolar lavage & $\begin{array}{c}\text { Passaged } 3 \text { times in human } \\
\text { fibroblasts }\end{array}$ & - & $\begin{array}{l}\text { August 21, } \\
2012\end{array}$ \\
\hline JX512208 & HAN31 & Bronchoalveolar lavage & $\begin{array}{c}\text { Passaged } 2 \text { times in human } \\
\text { fibroblasts }\end{array}$ & - & $\begin{array}{l}\text { August 21, } \\
2012\end{array}$ \\
\hline KC519319 & $\mathrm{BE} / 9 / 2010$ & Urine from an infant & $\begin{array}{c}\text { Passaged } 2 \text { times in human } \\
\text { fibroblasts }\end{array}$ & - & $\begin{array}{c}\text { January } 23, \\
2013\end{array}$ \\
\hline KC519320 & $\mathrm{BE} / 10 / 2010$ & $\begin{array}{c}\text { Urine from a congenitally } \\
\text { infected infant }\end{array}$ & $\begin{array}{c}\text { Passaged } 2 \text { times in human } \\
\text { fibroblasts }\end{array}$ & - & $\begin{array}{c}\text { January 23, } \\
2013\end{array}$ \\
\hline KC519321 & $\mathrm{BE} / 11 / 2010$ & Urine from an infant & $\begin{array}{c}\text { Passaged } 2 \text { times in human } \\
\text { fibroblasts }\end{array}$ & - & $\begin{array}{c}\text { January } 23 \text {, } \\
2013\end{array}$ \\
\hline KC519322 & $\mathrm{BE} / 21 / 2010$ & $\begin{array}{l}\text { Urine from a pulmonary } \\
\text { transplant recipient }\end{array}$ & Unpassaged & - & $\begin{array}{c}\text { January } 23 \text {, } \\
2013\end{array}$ \\
\hline KC519323 & $\mathrm{BE} / 27 / 2010$ & $\begin{array}{l}\text { Urine from a renal transplant } \\
\text { recipient }\end{array}$ & $\begin{array}{c}\text { Passaged } 4 \text { times in human } \\
\text { fibroblasts }\end{array}$ & - & $\begin{array}{c}\text { January } 23, \\
2013\end{array}$ \\
\hline KF021605 & TR & $\begin{array}{l}\text { Vitreous humor from eye of } \\
\text { HIV-positive male }\end{array}$ & $\begin{array}{l}\text { Passaged several times in } \\
\text { human fibroblasts }\end{array}$ & {$[44]$} & May 9, 2013 \\
\hline KF297339 & $\begin{array}{l}\text { TB40/E clone } \\
\text { Lisa }\end{array}$ & $\begin{array}{l}\text { Throat wash of a bone } \\
\text { marrow transplant recipient }\end{array}$ & $\begin{array}{c}\text { Generated on human } \\
\text { fibroblasts by passaging } \\
\text { strain TB40/E once, plaque } \\
\text { purifying } 3 \text { times and } \\
\text { passaging once more }\end{array}$ & {$[45]$} & $\begin{array}{l}\text { June } 26 \text {, } \\
2013\end{array}$ \\
\hline
\end{tabular}

${ }^{\circ}$ NCBI GenBank release file 199.0; search performed on 15 January 2014; patent sequences, transgenic strains, and incomplete sequences were not included; *NCBI Reference Sequence (RefSeq); "Update from AD169 entry X17403 by the addition of the 929 bp missing region, encompassing UL42 and UL43, and the correction of sequencing errors. 


\subsection{Genetic Diversity}

Complete sequence analysis of several clinical isolates not only assisted the refinement of the genome annotation, but also led to the understanding that several regions of the HCMV genome are variable between different isolates [25,39]. Studies of individual gene sequences from viral glycoprotein genes [46-48], virulence-determining genes from the UL/b' region [49-51], and RL11 genes $[31,52,53]$ have been conducted to establish the existence of separate clusters of polymorphisms or genotypes (reviewed in [54-56]). Despite their variability, individual genotypes display remarkable sequence stability both within the host as in the population, and most genotypes seem to have a worldwide distribution [50,57-60]. Based on these data, it is hypothesized that the selective forces that have shaped the currently circulating genotypes were active during the evolution of early humans or even earlier and were modulated by founder and bottleneck events. In more recent times, migrations of human populations have redistributed and mixed these genotypes on a worldwide scale [56,59,61]. Furthermore, the very low incidence of gene linkage in the HCMV genome probably illustrates the predominant role of recombination in the generation of the existing genetic diversity [31,50,62-64].

The existence of distinct genotypes of several genes has attracted interest because of the potential differences in pathogenicity. If such correlations would be observed, this could provide novel diagnostic tools to tailor medical interventions. While some studies investigating genes UL55 (glycoprotein B) [65,66], UL73 (glycoprotein N) [67,68], and UL144 (TNF- $\alpha$-like receptor) [69-71] have presented data involving specific genotypes with different disease outcomes, there is no overall consensus on these correlations yet (reviewed in [54-56]). To establish the feasibility of using viral genotype data as prognostic markers in patient follow-up, more comprehensive studies that include larger sets of variable genes, if not complete genome sequences, will be necessary. In this regard, a study making use of gene sequences from only four genes (UL144, UL146, UL147 and US28) could train an artificial neural network to correctly predict congenitally-infected infants to be symptomatic or asymptomatic at birth in $90 \%$ of cases [72]. Considering the progress in sequencing technology and its implementation in HCMV genomics, this type of investigation should now become more feasible on a full genome scale.

\section{Characterization of Complete HCMV Genomes Using NGS}

Table 1 gives an overview of all complete HCMV genome sequences that are currently publicly available. If each individual strain is only counted once-some strains have multiple isolate sequences published-, this amounts to a total of 35 strains; 31 of these strains can be considered low-passage (or unpassaged) clinical isolates. While a considerable proportion of these sequences were still deduced using traditional Sanger sequencing, the labor intensity of these approaches precludes routine and high-throughput application of complete genome sequencing. Over the past 5 years, NGS technologies have begun to show their promise in becoming a novel, scalable, cost-effective, and time-efficient way of characterizing HCMV genome diversity.

The first application of NGS to HCMV genomics was published in 2009 and investigated the genome architecture of laboratory-adapted strains AD169 and Towne in detail (Table 2) [20]. While the published sequences of AD169 (varUK and AD169-BAC/varATCC) and Towne 
(varS/varRIT3) missed the entire $\mathrm{UL} / b$ ' region (Table 1), it had already been noted that some variants did seem to contain this region $[73,74]$. For AD169, this variant constituted a separate stock (varUC), but it was unclear whether this was an AD169 variant with an intact UL/b' or a different strain altogether. For Towne, the original stock was a mixture of the varS/varRIT3 variant, that was cloned into a BAC and sequenced [30,39], and an apparently intact varL variant, for which the UL/b' region had been characterized [25]. The exact nature of the AD169 varUC stock and the mixed Towne stock containing both varS and varL was determined by generating sequencing reads with the Genome Analyzer (Illumina) and mapping these onto appropriate reference sequences with or without the UL/b' region. The results showed that AD169 varUC was indeed an AD169 variant with a nearly intact $\mathrm{UL} / b$ ' region, only missing a $3.2 \mathrm{~kb}$ region affecting genes UL144, UL142, UL141, and UL140. Furthermore, the presence of both varS and varL variants in the Towne stock was experimentally confirmed. In a similar fashion, other studies have sequenced specific transgenic BAC clones of strains Merlin and Towne using NGS to characterize genetic changes that have occurred during passaging and cloning of these BACs (Table 2) $[35,75,76]$.

Table 2. Overview of studies on the HCMV genome making use of NGS technology, ranked in chronological order.

\begin{tabular}{|c|c|c|c|c|c|}
\hline First author & Title & Journal & $\begin{array}{c}\text { NGS } \\
\text { technology }\end{array}$ & Ref. & $\begin{array}{c}\text { Publication } \\
\text { date }\end{array}$ \\
\hline Bradley et al. & $\begin{array}{l}\text { High-throughput sequence analysis of variants of } \\
\text { human cytomegalovirus strains Towne and AD169. }\end{array}$ & J. Gen. Virol. & $\mathrm{IGA}^{\circ}$ & {$[20]$} & $\begin{array}{c}\text { June } 24, \\
2009\end{array}$ \\
\hline $\begin{array}{l}\text { Cunningham } \\
\text { et al. }\end{array}$ & $\begin{array}{l}\text { Sequences of complete human cytomegalovirus } \\
\text { genomes from infected cell cultures and clinical } \\
\text { specimens. }\end{array}$ & J. Gen. Virol. & $\mathrm{IGA}^{\circ}$ & {$[42]$} & $\begin{array}{c}\text { November } \\
11,2009\end{array}$ \\
\hline Görzer et al. & $\begin{array}{l}\text { Deep sequencing reveals highly complex dynamics } \\
\text { of human cytomegalovirus genotypes in transplant } \\
\text { patients over time. }\end{array}$ & J. Virol. & GSF* & {$[77]$} & $\begin{array}{c}\text { May 12, } \\
2010\end{array}$ \\
\hline Stanton et al. & $\begin{array}{l}\text { Reconstruction of the complete human } \\
\text { cytomegalovirus genome in a BAC reveals RL13 to } \\
\text { be a potent inhibitor of replication. }\end{array}$ & J. Clin. Investig. & $\mathrm{IGA}^{\circ}$ & {$[35]$} & $\begin{array}{l}\text { August 2, } \\
2010\end{array}$ \\
\hline Görzer et al. & $\begin{array}{l}\text { The impact of PCR-generated recombination on } \\
\text { diversity estimation of mixed viral populations by } \\
\text { deep sequencing. }\end{array}$ & J. Virol. Methods & GSF* & {$[78]$} & $\begin{array}{l}\text { August 4, } \\
2010\end{array}$ \\
\hline Jung et al. & $\begin{array}{l}\text { Full genome sequencing and analysis of human } \\
\text { cytomegalovirus strain JHC isolated from } \\
\text { a Korean patient. }\end{array}$ & Virus Res. & GSF* & {$[43]$} & $\begin{array}{c}\text { January 19, } \\
2011\end{array}$ \\
\hline Renzette et al. & $\begin{array}{l}\text { Extensive genome-wide variability of human } \\
\text { cytomegalovirus in congenitally infected infants. }\end{array}$ & PLoS Pathog. & $\mathrm{IGA}^{\circ}$ & [79] & $\begin{array}{c}\text { May 19, } \\
2011\end{array}$ \\
\hline James et al. & $\begin{array}{l}\text { Cyclopropavir inhibits the normal function of the } \\
\text { human cytomegalovirus UL97 kinase. }\end{array}$ & $\begin{array}{l}\text { Antimicrob. } \\
\text { Agents } \\
\text { Chemother. }\end{array}$ & $\mathrm{IGA}^{\circ}$ & {$[80]$} & $\begin{array}{l}\text { July } 25 \text {, } \\
2011\end{array}$ \\
\hline Stark et al. & $\begin{array}{l}\text { High-resolution profiling and analysis of viral and } \\
\text { host small RNAs during human } \\
\text { cytomegalovirus infection. }\end{array}$ & J. Virol. & $\mathrm{IGA}^{\circ}$ & {$[81]$} & $\begin{array}{l}\text { October 19, } \\
2011\end{array}$ \\
\hline
\end{tabular}


Table 2.Cont.

\begin{tabular}{|c|c|c|c|c|c|}
\hline First author & Title & Journal & $\begin{array}{l}\text { NGS } \\
\text { technology }\end{array}$ & Ref. & $\begin{array}{c}\text { Publication } \\
\text { date }\end{array}$ \\
\hline Gatherer et al. & $\begin{array}{l}\text { High-resolution human cytomegalovirus } \\
\text { transcriptome. }\end{array}$ & $\begin{array}{l}\text { Proc. Natl. Acad. } \\
\text { Sci. U. S. A. }\end{array}$ & $\mathrm{IGA}^{\circ}$ & {$[82]$} & $\begin{array}{l}\text { November } \\
22,2011\end{array}$ \\
\hline $\begin{array}{l}\text { Bhattacharjee } \\
\text { et al. }\end{array}$ & $\begin{array}{l}\text { Genetic analysis of cytomegalovirus in } \\
\text { malignant gliomas. }\end{array}$ & J. Virol. & $\mathrm{IGA}^{\circ}$ & {$[83]$} & $\begin{array}{l}\text { April 11, } \\
2012\end{array}$ \\
\hline Meshesha et al. & $\begin{array}{l}\text { The microRNA Transcriptome of Human } \\
\text { Cytomegalovirus (HCMV). }\end{array}$ & Open Virol. J. & $\mathrm{IGA}^{\circ}$ & {$[84]$} & $\begin{array}{l}\text { April 11, } \\
2012\end{array}$ \\
\hline $\begin{array}{c}\text { Stern- } \\
\text { Ginossar et al. }\end{array}$ & Decoding human cytomegalovirus. & Science & $\begin{array}{l}\mathrm{IGA}^{\circ} \\
\mathrm{HiSeq}^{\wedge}\end{array}$ & {$[85]$} & $\begin{array}{l}\text { November } \\
23,2012\end{array}$ \\
\hline Rossetto et al. & $\begin{array}{l}\text { Cis and trans acting factors involved in human } \\
\text { cytomegalovirus experimental and natural } \\
\text { latent infection of CD14 }(+) \text { monocytes and } \\
\text { CD34 }(+) \text { cells. }\end{array}$ & PLoS Pathog. & MiSeq' & {$[86]$} & $\begin{array}{c}\text { May 23, } \\
2013\end{array}$ \\
\hline Sahoo et al. & $\begin{array}{l}\text { Detection of cytomegalovirus drug resistance } \\
\text { mutations by next-generation sequencing. }\end{array}$ & $\begin{array}{l}\text { J. Clin. } \\
\text { Microbiol. }\end{array}$ & GSJ" & {$[87]$} & $\begin{array}{l}\text { August 28, } \\
2013\end{array}$ \\
\hline Renzette et al. & $\begin{array}{l}\text { Rapid intrahost evolution of human } \\
\text { cytomegalovirus is shaped by demography and } \\
\text { positive selection. }\end{array}$ & PLoS Genet. & $\mathrm{IGA}^{\circ}$ & {$[88]$} & $\begin{array}{l}\text { September } \\
26,2013\end{array}$ \\
\hline Brechtel et al. & $\begin{array}{l}\text { Complete Genome Sequence of a Cytomegalovirus } \\
\text { Towne-BAC (Bacterial Artificial Chromosome) } \\
\text { Isolate Maintained in Escherichia coli for } 10 \text { Years } \\
\text { and Then Serially Passaged in Human Fibroblasts. }\end{array}$ & $\begin{array}{l}\text { Genome } \\
\text { Announc. }\end{array}$ & MiSeq' & {$[75]$} & $\begin{array}{l}\text { September } \\
26,2013\end{array}$ \\
\hline Brechtel et al. & $\begin{array}{l}\text { Complete Genome Sequence of a UL96 Mutant } \\
\text { Cytomegalovirus Towne-BAC (Bacterial Artificial } \\
\text { Chromosome) Isolate Passaged in Fibroblasts To } \\
\text { Allow Accumulation of Compensatory Mutations. }\end{array}$ & $\begin{array}{l}\text { Genome } \\
\text { Announc. }\end{array}$ & MiSeq' & {$[76]$} & $\begin{array}{l}\text { October 24, } \\
2013\end{array}$ \\
\hline
\end{tabular}

${ }^{\circ}$ Genome Analyzer (Illumina); * 454 GS FLX (Roche); ^ HiSeq (Illumina); ‘ MiSeq (Illumina); “ 454 GS Junior (Roche).

The previous studies made proper use of NGS technology to elucidate the stock composition of laboratory-adapted strains, but, then again, they could employ the existing sequence information to direct the assembly of the millions of sequencing reads that are generated during a typical Illumina run. In order to apply NGS to the genome characterization of novel clinical isolates, this assembly approach needed some adjustment. Because of the sequence variability in substantial regions of the HCMV genome, direct mapping of NGS reads from unknown isolates to existing reference sequences leads to a lack of coverage in these areas, simply because the novel sequences are too divergent from the chosen reference strain. To assemble the sequence information from novel clinical isolates, alternative approaches were devised that start with a de novo or reference-independent assembly of sequence reads (Table 2) [42]. The longer sequences formed by de novo assembly, the so-called contigs, are scaffolded against a reference sequence to produce a strain-specific reference that can be used for a mapping or reference-dependent assembly like before. The final strain sequence is optimized through manual inspection of the read alignment and correction of misassemblies by iterative mapping and/or PCR sequencing. 
A comparative analysis was made of the effectiveness of sequencing complete HCMV genomes from clinical isolates through both Sanger sequencing of overlapping PCR products and NGS analysis of infected cell cultures and unamplified clinical material with the Genome Analyzer [42]. Both approaches were successful, but the PCR and Sanger sequencing method proved to be much more labor-intensive and, by consequence, less amenable to high-throughput application. However, the NGS approach is not specifically directed towards viral DNA and analyzes the total DNA present in an isolate. Whole cell culture extracts are heavily contaminated with cellular DNA and the viral loads in unamplified clinical material can be very low. This was illustrated for strain 3301 (Table 1); only 3\% of sequence reads that were collected directly from the sample were of viral origin. While it was possible to reconstruct the complete genome using these $3 \%$ of reads, such an approach also precludes any high-throughput prospect. This limitation was recently confirmed when strain BE/21/2010 (Table 1) was amplified using undirected whole genome amplification and only yielded 12\% HCMV-specific NGS reads (Sijmons et al, unpublished results). This study did realize higher levels of viral DNA (mostly $>90 \%$ ) by combining limited cell culture amplification, nuclease digestion of unencapsidated (cellular) DNA, purification of viral DNA, and whole genome amplification. A series of validation experiments showed that the generated genome sequences did not undergo major alterations during these procedures and were still representative for the strain in the original clinical isolate.

As discussed previously, cell culture passaging leads to disruptive mutations in genes that are inhibitory or non-essential for growth in that cell type. Genes RL13 and UL128L seem to be the first that are affected when passaging a strain in fibroblast cells [35,36]. Interestingly, RL13 and UL128L genes do not show obvious disruptive mutations in most of the clinical isolates analyzed by NGS after limited culturing $[42,43]$. This suggests that these strains are still in a very early phase of genetic adaptation to fibroblast replication; although it cannot be ruled out that these genes are mutated at different sites in different clones of the population, like in the case of RL13 in strain Merlin [35]. Several observations imply that some ORF-disrupting mutations may be present in the original clinical isolate and are not an artifact of culturing [42]. Most importantly, the sequence characterization of strains JP and BE/21/2010 directly from clinical material (Table 1) has shown disruptive mutations in genes RL5A, UL9, UL111A, and UL150 that are definitely culture-independent. Furthermore, identical indels and point mutations were shared between unrelated isolates, which suggests that these could be derived from a common ancestor circulating in the human population. Finally, the presence of individual mutations in RL5A, UL1, UL9, and UL111A in passaged strains was confirmed by PCR sequencing of the original sample (Sijmons et al, unpublished results). Analysis of a larger number of clinical isolates will reveal the complete set of genes that can be disabled in clinical isolates, their occurrence in different patient populations, and potential implications for strain pathogenicity.

\section{Deep Sequencing of Intrahost HCMV Populations}

Accumulating data shows that infections with multiple HCMV strains are no exceptions, neither in immunocompromised nor in immunocompetent hosts (reviewed in [56]). This could probably be the product of both simultaneous and consecutive virus transmission events $[89,90]$. Multiple infections could result in a higher pathogenic potential because of trans-complementation between 
strains [91]. This prediction is confirmed by data about the effect of strain multiplicity in transplant patients [92-95]. Because of the large amounts of sequence reads that are generated by NGS technologies, these are ideally suited to characterize the dynamics of mixed viral populations in greater depth (reviewed in $[96,97]$ ). The first study to apply this approach to HCMV populations analyzed PCR amplicons of the hypervariable genes UL73, UL74, and UL139 in lung transplant recipients using 454 GS FLX (Roche) technology (Table 2) [77]. Viral populations consisted of mixtures of up to six genotypes, with one or two types accounting for the majority of the population and the other genotypes present at frequencies of $0.1 \%-10 \%$. When serial samples of patients were compared, the genotype frequencies fluctuated in a seemingly stochastic fashion. The authors speculated these fluctuations could be caused by sporadic and stochastic events that lead to differential reactivation of latent genomes. While the abundance of the individual genotypes changed, their sequences did not, confirming the stability of hypervariable HCMV genes [44,51,53]. In a follow-up study, the authors warned against the formation of artificial recombinants during PCR amplification when interpreting results from amplicon deep sequencing experiments [78].

The previous studies characterized intrahost population diversity by analyzing a set of genes that are highly variable between hosts. However, applying such a deep sequencing approach to a complete genome does not suffer from a selection bias towards certain genome regions and can provide a more comprehensive picture of the diversity and dynamics of viral populations inside the host. Sequencing complete genomes from unamplified clinical material, results in a low proportion of viral NGS reads, which would impair any deep sequencing effort [42]. Cell culture amplification on the other hand would almost certainly alter the composition of viral populations. Therefore, a workflow was devised that characterized complete HCMV genomes using overlapping PCR amplicons $[79,83,88]$. As an internal control, BAC clones of AD169 and Toledo strains were resequenced to establish a set of quality filtering thresholds that helped distinguishing genuine intrahost variants from PCR and sequencing errors. In a first study, the viral populations of three congenitally infected infants were characterized [79]. Surprisingly, for a dsDNA virus encoding a polymerase with proofreading capacity, estimates of the genetic diversity of these populations were comparable to quasispecies RNA viruses like HIV and dengue virus. Population variants were clustered in two groups. Variants present at high frequencies $(\geq 90 \%)$ accounted for $20 \%$ of reads, while low-frequency variants $(\leq 10 \%)$ represented $73 \%$ of reads. This population structure is comparable to the study of UL73, UL74 and UL139 amplicons in lung transplant recipients, which also found one or two variants present at higher frequencies and a set of low-frequency variants $(0.1 \%-10 \%)$ [77]. Interestingly, when ORF-specific intrahost diversities were estimated, ORFs encoding glycoproteins or immune-evasive functions showed the lowest intrahost diversity estimates. While they are considered to have the highest interhost diversity, studies focusing on these ORFs may underestimate intrahost diversity. Compared to the results in congenitally infected infants, intrahost diversity estimates were lower in malignant gliomas [83]. A potential explanation for this discrepancy could be the higher levels of replication during congenital infection, which would lead to a higher accumulation of de novo mutations. More data from different patient groups is warranted to provide a broader view of the range of intrahost diversity estimates, the mechanisms that shape them, and potential implications for patient health. 
To better understand the dynamics of these genome populations, serial urine and plasma isolates were sampled from five infants with a symptomatic HCMV infection at birth [88]. When serial isolates of the same compartment were compared, the majority of SNPs had a similar frequency, and consensus sequences differed only by $0.2 \%$ at the nucleotide level, demonstrating the overall stability of the populations. This result is in clear accordance with previous results that have illustrated the stability of HCMV genotypes in vivo [50,57,59]. However, comparison of isolates of different compartments (urine and plasma) at the same time point showed that consensus sequences differed by approx. 1\%; comparable to the divergence of HCMV strains from different hosts. Subsequently, the observed dynamics were modeled using both demographic variables (population size and structure) and selective pressures. Intercompartment differentiation was shaped by strong bottleneck events and the calculation of bottleneck timing enabled estimation of the timing of infection and compartment colonization. While the effects of positive selection in the same compartment were small, strong evidence of positive selection was found when comparing different compartments. The large differences between viral populations in plasma and urine pose important questions about how representative the secreted virus (urine) is for the virus that circulates (plasma). It is conceivable that other compartments might show other diversification dynamics. However, the total number of patients analyzed in this study is relatively low and the data about intercompartment diversification are only based on one patient. From the presented data, it is unclear whether this patient was infected by a single strain or multiple strains. Rephrasing the issue: are we looking at the differentiation of one single quasispecies cloud or the segregation of multiple quasispecies clouds that could have had inherent differences in their respective cell tropisms? Analysis of the intercompartment diversity in additional patients will be needed to improve the understanding of the dynamics of these virus populations, which obviously could have very important implications for diagnostics, treatment, and vaccine development.

The deep sequencing capabilities of NGS technology show great promise for the sensitive detection of drug resistance mutations. This approach has already proven its use in drug resistance testing for HIV, HCV, and HBV (reviewed in [10]). While Sanger sequencing of UL54 and UL97 PCR amplicons is still the gold standard for detection of drug resistance mutations in HCMV isolates, this method suffers from a lack of sensitivity, often failing when plasma viral loads drop below 1,000 copies/mL and/or mutant frequencies are lower than $10 \%-20 \%$. Recently, the use of NGS technology in resistance mutation detection was evaluated for the first time for HCMV [87]. Using the benchtop 454 GS Junior system (Roche), the study showed the ability to reproducibly detect resistance mutations at frequencies lower than $20 \%$ and at viral loads lower than 1000 copies $/ \mathrm{mL}$. This improvement in sensitivity will help studying the abundance, dynamics, and importance of low-frequency drug-resistant variants. The high throughput of NGS could result in a decrease of the time and cost of resistance detection. In addition, full genome characterization of drug-resistant variants with NGS can potentially lead to the discovery of new resistance mutations in other genome regions.

\section{NGS in HCMV Transcriptome Studies}

The high throughput of NGS provided new opportunities for the field of transcriptomics; the study of the total coding and non-coding RNA that is transcribed in a given cell type [98]. NGS, 
often referred to as RNA-Seq in this context, has now surpassed microarrays as the method of choice for transcriptomic research, since it is much better at detecting rare variants and does not depend on a priori sequence knowledge. Transcriptomic analysis of HCMV in an infected cell can contribute to the genome annotation by revealing complex transcriptional processes that often cannot be predicted based on the genome sequence alone. HCMV transcription is characterized by the presence of multiple transcripts sharing common 5' or 3' ends, complex and adaptable splicing patterns, antisense transcription, and transcription of non-coding and miRNAs (reviewed in [99]). Several NGS-based studies have added important insights into these processes.

The first study to use RNA-Seq in HCMV transcriptome research characterized the polyA RNA content of human fetal foreskin fibroblast cells, $72 \mathrm{~h}$ after infection with the Merlin strain, when virion production is underway [82]. The four large non-coding RNAs that are encoded in the HCMV genome (RNA2.7, RNA1.2, RNA4.9, and RNA5.0; Figure 2) accounted for a staggering $65.1 \%$ of viral transcription. These RNAs probably do not function via translation, since they do not overlap significantly with potential ORFs. Large non-coding RNAs are implicated in gene regulation in eukaryotes [100]. Especially RNA2.7 was transcribed massively, making up almost half of the viral transcripts. RNA2.7 inhibits apoptosis by regulation of mitochondria-induced cell death [101]. Furthermore, antisense transcripts were found throughout the genome, but are generally present at a lower level than their sense counterparts. Antisense transcription is increasingly being recognized as being involved in gene regulation, both in pro- and eukaryotes (reviewed in [102]). It can be hypothesized that the antisense transcripts of HCMV provide the virus with an additional mechanism of regulating its expression. Because of the high levels of transcription from non-coding and antisense regions, coding regions only made up one third of transcription. Within these coding regions, splicing patterns were found to be more numerous and complex than previously appreciated. While some of these splicing events are essential for proper expression, others probably have more subtle regulatory roles or could be non-functional byproducts of normal transcription. Four new HCMV genes were identified based on this transcriptome study (RL8A, RL9A, UL150A, and US33A; Figure 2), indicating the added value of this kind of study for genome annotation.

The added complexity that is caused by transcription and translation was further illustrated by a study that made use of a novel technique, i.e., ribosome profiling [85]. This technique characterizes the "translatome" by generating libraries of ribosome-protected mRNA fragments. Examining these ribosome footprints, the authors identified 751 ORFs, only 147 of which were previously recognized. ORFs were positioned within existing ORFs (both in-frame and out of frame), upstream of existing ORFs, antisense of existing ORFs, and within presumably non-coding regions. Multiple translation products were also identified on the long non-coding RNAs RNA2.7, RNA1.2, and RNA4.9. Tandem mass spectrometry and protein tagging experiments confirmed the translation of a large proportion of these novel ORFs. Furthermore, this study highlighted the use of alternative 5' ends, enabling the virus to express different proteins from overlapping coding regions in a temporally regulated fashion.

The discovery that microRNAs (miRNAs), small RNAs that target and silence complementary mRNAs, are not restricted to eukaryotes, but also present in several viruses, including HCMV, added yet another weapon to the viral armory [103-107]. While miRNAs are non-immunogenic 
and only require minimal space in the genome, they offer the virus an efficient way of regulating both viral and host gene expression. Two studies have used NGS technology to characterize the profile of small RNAs that are expressed in HCMV-infected cells $[81,84]$. These studies used different HCMV strains (Towne vs. AD169), harvested RNA at different time points post-infection (72 h vs. $96 \mathrm{~h}$ ), and assigned and verified novel miRNAs in different ways. The fraction of small RNAs encoding viral miRNAs was $20 \%$ and $5 \%$, respectively. The seven miRNAs with the highest expression levels were identical in both, although in a different order. While the second study identified more novel miRNAs from NGS data, the functionality of the only two novel miRNAs reported in the first study was confirmed in transfection assays. The first study also reported that all 22 characterized miRNAs were incorporated into the endogenous host silencing machinery, further highlighting their functionality [81]. Additionally, the authors identified novel small viral RNAs, distinct from miRNAs that were especially observed across the long non-coding RNAs, such as RNA2.7. These could be related to the ribosome footprints that were found on these RNAs [85].

Recently, the HCMV transcriptome of CD14+ and CD34+ cells in experimental and natural latency was characterized through deep sequencing, identifying transcripts that had not previously been related to latency [86]. These include UL44 and UL84 transcripts, normally involved in lytic DNA replication, and the large non-coding RNAs RNA2.7 and RNA4.9.

In addition to providing insights into the viral expression profile during productive and latent infection, NGS-based transcriptome studies can also study the virus-host interface by simultaneously monitoring the changes in cellular transcription. Two studies have already illustrated this for murine cytomegalovirus, but similar studies on HCMV have not yet been published $[108,109]$.

It has to be noted that the complex set of transcripts that were identified through deep sequencing still await further characterization to identify their potential functions. As the authors admit, some of the predicted RNAs and proteins could be aberrant byproducts of normal transcription/translation without further function and/or could be rapidly degraded [82,85]. These studies do offer the first glance at a complex regulatory network that the virus can use to finely balance its replication, including alternative splicing, antisense transcription, large and small non-coding RNAs, and miRNAs.

\section{Conclusions}

A quarter of a century has now passed since the publication of the first complete HCMV genome sequence ushered in the age of HCMV genomics. In the meantime, insights have accumulated regarding the genetic alterations of laboratory-adapted strains, the genome annotation has been progressively fine-tuned, and regions of high nucleotide diversity have been identified. The advent of NGS technology has changed the scope and pace of genomic research and is beginning to show its promise in the HCMV field. However, NGS is still a relatively recent technology and the interpretation of the massive amounts of sequence data requires considerable bioinformatics expertise. Error identification and correction protocols are not completely standardized yet and this precludes the routine application in clinical settings.

Sequencing the complete genome of a clinical HCMV isolate is now possible in a considerably higher throughput and speed than a few years ago. Analysis of a large set of genomes from a 
diverse group of patients will improve our understanding of the circulating genetic diversity and variability in coding capacity of wild-type HCMV. This could lead to novel insights into the association of genetic diversity and viral pathogenicity, which has eluded the field for years. Furthermore, routine sequencing of transgenic strains to identify unintended genetic alterations should now be possible.

Deep sequencing has shown that intrahost populations of HCMV are remarkably diverse. A better understanding of the dynamics of these populations could have important implications for treatment and vaccine development. The sensitivity of NGS will also improve the standard of drug resistance mutation detection.

RNA-Seq applications have already altered our understanding of the transcriptional complexity during HCMV-infection. Further characterization of these transcripts is warranted and could provide novel insights into mechanisms of viral pathogenicity and potential treatment options. Additionally, simultaneous characterization of the changes in the host transcriptome during infection will reveal currently unknown virus-host interactions.

Meanwhile, a new generation of single-molecule sequencing technologies are being developed [110] or have already found their way to the market [111] (reviewed in [112]). These technologies require much less input material than NGS, making them more attractive for sequencing directly from clinical material. They do not need a library amplification step, ruling out possible artifacts caused by this step. Furthermore, they provide considerable longer read lengths, which facilitate genome assembly and could provide insights into the mutual relations of single variants in intrahost populations. Finally, these technologies can characterize DNA modifications, enabling the direct analysis of epigenetic changes in the genome [113,114]. Undoubtedly, application of single-molecule sequencing to HCMV genomics will aid in deciphering this complex herpesvirus.

\section{Acknowledgments}

S.S. and P.M. are supported by the Research Foundation Flanders (FWO- "Fonds voor Wetenschappelijk Onderzoek, Vlaanderen").

\section{Author Contributions}

S.S., M.V.R. and P.M. wrote the manuscript.

\section{Conflicts of Interest}

The authors declare no conflict of interest.

\section{References and Notes}

1. Cannon, M.J.; Schmid, D.S.; Hyde, T.B. Review of cytomegalovirus seroprevalence and demographic characteristics associated with infection. Rev. Med. Virol. 2010, 20, 202-213.

2. Reeves, M.; Sinclair, J. Aspects of human cytomegalovirus latency and reactivation. Curr. Top. Microbiol. Immunol. 2008, 325, 297-313. 
3. Boeckh, M.; Geballe, A.P. Cytomegalovirus: Pathogen, paradigm, and puzzle. J. Clin. Investig. 2011, 121, 1673-1680.

4. Manicklal, S.; Emery, V.C.; Lazzarotto, T.; Boppana, S.B.; Gupta, R.K. The "silent" global burden of congenital cytomegalovirus. Clin. Microbiol. Rev. 2013, 26, 86-102.

5. Murphy, E.; Shenk, T. Human cytomegalovirus genome. Curr. Top. Microbiol. Immunol. 2008, 325, 1-19.

6. Metzker, M.L. Sequencing technologies-The next generation. Nat. Rev. Genet. 2010, 11, $31-46$.

7. Zhang, J.; Chiodini, R.; Badr, A.; Zhang, G. The impact of next-generation sequencing on genomics. J. Genet. Genomics 2011, 38, 95-109.

8. Koboldt, D.C.; Steinberg, K.M.; Larson, D.E.; Wilson, R.K.; Mardis, E.R. The next-generation sequencing revolution and its impact on genomics. Cell 2013, 155, 27-38.

9. Radford, A.D.; Chapman, D.; Dixon, L.; Chantrey, J.; Darby, A.C.; Hall, N. Application of next-generation sequencing technologies in virology. J. Gen. Virol. 2012, 93, 1853-1868.

10. Capobianchi, M.R.; Giombini, E.; Rozera, G. Next-generation sequencing technology in clinical virology. Clin. Microbiol. Infect. 2013, 19, 15-22.

11. Barzon, L.; Lavezzo, E.; Costanzi, G.; Franchin, E.; Toppo, S.; Palu, G. Next-generation sequencing technologies in diagnostic virology. J. Clin. Virol. 2013, 58, 346-350.

12. Chee, M.S.; Bankier, A.T.; Beck, S.; Bohni, R.; Brown, C.M.; Cerny, R.; Horsnell, T.; Hutchison, C.A., 3rd.; Kouzarides, T.; Martignetti, J.A.; et al. Analysis of the protein-coding content of the sequence of human cytomegalovirus strain AD169. Curr. Top. Microbiol. Immunol. 1990, 154, 125-169.

13. Bankier, A.T.; Beck, S.; Bohni, R.; Brown, C.M.; Cerny, R.; Chee, M.S.; Hutchison, C.A., 3rd.; Kouzarides, T.; Martignetti, J.A.; Preddie, E.; et al. The DNA sequence of the human cytomegalovirus genome. DNA Seq. 1991, 2, 1-12.

14. Just, M.; Buergin-Wolff, A.; Emoedi, G.; Hernandez, R. Immunisation trials with live attenuated cytomegalovirus TOWNE 125. Infection 1975, 3, 111-114.

15. Plotkin, S.A.; Farquhar, J.; Horberger, E. Clinical trials of immunization with the Towne 125 strain of human cytomegalovirus. J. Infect. Dis. 1976, 134, 470-475.

16. Neff, B.J.; Weibel, R.E.; Buynak, E.B.; McLean, A.A.; Hilleman, M.R. Clinical and laboratory studies of live cytomegalovirus vaccine Ad-169. Proc. Soc. Exp. Biol. Med. 1979, $160,32-37$.

17. Quinnan, G.V., Jr.; Delery, M.; Rook, A.H.; Frederick, W.R.; Epstein, J.S.; Manischewitz, J.F.; Jackson, L.; Ramsey, K.M.; Mittal, K.; Plotkin, S.A.; et al. Comparative virulence and immunogenicity of the Towne strain and a nonattenuated strain of cytomegalovirus. Ann. Intern. Med. 1984, 101, 478-483.

18. Cha, T.A.; Tom, E.; Kemble, G.W.; Duke, G.M.; Mocarski, E.S.; Spaete, R.R. Human cytomegalovirus clinical isolates carry at least 19 genes not found in laboratory strains. J. Virol. 1996, 70, 78-83.

19. Prichard, M.N.; Penfold, M.E.; Duke, G.M.; Spaete, R.R.; Kemble, G.W. A review of genetic differences between limited and extensively passaged human cytomegalovirus strains. Rev. Med. Virol. 2001, 11, 191-200. 
20. Bradley, A.J.; Lurain, N.S.; Ghazal, P.; Trivedi, U.; Cunningham, C.; Baluchova, K.; Gatherer, D.; Wilkinson, G.W.; Dargan, D.J.; Davison, A.J. High-throughput sequence analysis of variants of human cytomegalovirus strains Towne and AD169. J. Gen. Virol. 2009, 90, 2375-2380.

21. Skaletskaya, A.; Bartle, L.M.; Chittenden, T.; McCormick, A.L.; Mocarski, E.S.; Goldmacher, V.S. A cytomegalovirus-encoded inhibitor of apoptosis that suppresses caspase-8 activation. Proc. Natl. Acad. Sci. USA 2001, 98, 7829-7834.

22. Yu, D.; Smith, G.A.; Enquist, L.W.; Shenk, T. Construction of a self-excisable bacterial artificial chromosome containing the human cytomegalovirus genome and mutagenesis of the diploid TRL/IRL13 gene. J. Virol. 2002, 76, 2316-2328.

23. Davison, A.J.; Akter, P.; Cunningham, C.; Dolan, A.; Addison, C.; Dargan, D.J.; Hassan-Walker, A.F.; Emery, V.C.; Griffiths, P.D.; Wilkinson, G.W. Homology between the human cytomegalovirus RL11 gene family and human adenovirus E3 genes. J. Gen. Virol. 2003, 84, 657-663.

24. Akter, P.; Cunningham, C.; McSharry, B.P.; Dolan, A.; Addison, C.; Dargan, D.J.; Hassan-Walker, A.F.; Emery, V.C.; Griffiths, P.D.; Wilkinson, G.W.; et al. Two novel spliced genes in human cytomegalovirus. J. Gen. Virol. 2003, 84, 1117-1122.

25. Dolan, A.; Cunningham, C.; Hector, R.D.; Hassan-Walker, A.F.; Lee, L.; Addison, C.; Dargan, D.J.; McGeoch, D.J.; Gatherer, D.; Emery, V.C.; et al. Genetic content of wild-type human cytomegalovirus. J. Gen. Virol. 2004, 85, 1301-1312.

26. Hahn, G.; Revello, M.G.; Patrone, M.; Percivalle, E.; Campanini, G.; Sarasini, A.; Wagner, M.; Gallina, A.; Milanesi, G.; Koszinowski, U.; et al. Human cytomegalovirus UL131-128 genes are indispensable for virus growth in endothelial cells and virus transfer to leukocytes. J. Virol. 2004, 78, 10023-10033.

27. Wang, D.; Shenk, T. Human cytomegalovirus virion protein complex required for epithelial and endothelial cell tropism. Proc. Natl. Acad. Sci. USA 2005, 102, 18153-18158.

28. Revello, M.G.; Gerna, G. Human cytomegalovirus tropism for endothelial/epithelial cells: Scientific background and clinical implications. Rev. Med. Virol. 2010, 20, 136-155.

29. Yu, D.; Silva, M.C.; Shenk, T. Functional map of human cytomegalovirus AD169 defined by global mutational analysis. Proc. Natl. Acad. Sci. USA 2003, 100, 12396-12401.

30. Dunn, W.; Chou, C.; Li, H.; Hai, R.; Patterson, D.; Stolc, V.; Zhu, H.; Liu, F. Functional profiling of a human cytomegalovirus genome. Proc. Natl. Acad. Sci. USA 2003, 100, $14223-14228$.

31. Sekulin, K.; Gorzer, I.; Heiss-Czedik, D.; Puchhammer-Stockl, E. Analysis of the variability of CMV strains in the RL11D domain of the RL11 multigene family. Virus Genes 2007, 35, 577-583.

32. Engel, P.; Perez-Carmona, N.; Alba, M.M.; Robertson, K.; Ghazal, P.; Angulo, A. Human cytomegalovirus UL7, a homologue of the SLAM-family receptor CD229, impairs cytokine production. Immunol. Cell Biol. 2011, 89, 753-766. 
33. Gabaev, I.; Steinbruck, L.; Pokoyski, C.; Pich, A.; Stanton, R.J.; Schwinzer, R.; Schulz, T.F.; Jacobs, R.; Messerle, M.; Kay-Fedorov, P.C. The human cytomegalovirus UL11 protein interacts with the receptor tyrosine phosphatase CD45, resulting in functional paralysis of $\mathrm{T}$ cells. PLoS Pathog. 2011, 7, e1002432.

34. Cortese, M.; Calo, S.; D'Aurizio, R.; Lilja, A.; Pacchiani, N.; Merola, M. Recombinant Human Cytomegalovirus (HCMV) RL13 Binds Human Immunoglobulin G Fc. PloS One 2012, 7, e50166.

35. Stanton, R.J.; Baluchova, K.; Dargan, D.J.; Cunningham, C.; Sheehy, O.; Seirafian, S.; McSharry, B.P.; Neale, M.L.; Davies, J.A.; Tomasec, P.; et al. Reconstruction of the complete human cytomegalovirus genome in a BAC reveals RL13 to be a potent inhibitor of replication. J. Clin. Investig. 2010, 120, 3191-3208.

36. Dargan, D.J.; Douglas, E.; Cunningham, C.; Jamieson, F.; Stanton, R.J.; Baluchova, K.; McSharry, B.P.; Tomasec, P.; Emery, V.C.; Percivalle, E.; et al. Sequential mutations associated with adaptation of human cytomegalovirus to growth in cell culture. J. Gen. Virol. 2010, 91, 1535-1546.

37. Davison, A.J.; Dolan, A.; Akter, P.; Addison, C.; Dargan, D.J.; Alcendor, D.J.; McGeoch, D.J.; Hayward, G.S. The human cytomegalovirus genome revisited: comparison with the chimpanzee cytomegalovirus genome. J. Gen. Virol. 2003, 84, 17-28.

38. Murphy, E.; Rigoutsos, I.; Shibuya, T.; Shenk, T.E. Reevaluation of human cytomegalovirus coding potential. Proc. Natl. Acad. Sci. USA 2003, 100, 13585-13590.

39. Murphy, E.; Yu, D.; Grimwood, J.; Schmutz, J.; Dickson, M.; Jarvis, M.A.; Hahn, G.; Nelson, J.A.; Myers, R.M.; Shenk, T.E. Coding potential of laboratory and clinical strains of human cytomegalovirus. Proc. Natl. Acad. Sci. USA 2003, 100, 14976-14981.

40. Dargan, D.J.; Jamieson, F.E.; MacLean, J.; Dolan, A.; Addison, C.; McGeoch, D.J. The published DNA sequence of human cytomegalovirus strain AD169 lacks 929 base pairs affecting genes UL42 and UL43. J. Virol. 1997, 71, 9833-9836.

41. Sinzger, C.; Hahn, G.; Digel, M.; Katona, R.; Sampaio, K.L.; Messerle, M.; Hengel, H.; Koszinowski, U.; Brune, W.; Adler, B. Cloning and sequencing of a highly productive, endotheliotropic virus strain derived from human cytomegalovirus TB40/E. J. Gen. Virol. 2008, 89, 359-368.

42. Cunningham, C.; Gatherer, D.; Hilfrich, B.; Baluchova, K.; Dargan, D.J.; Thomson, M.; Griffiths, P.D.; Wilkinson, G.W.; Schulz, T.F.; Davison, A.J. Sequences of complete human cytomegalovirus genomes from infected cell cultures and clinical specimens. J. Gen. Virol. 2010, 91, 605-615.

43. Jung, G.S.; Kim, Y.Y.; Kim, J.I.; Ji, G.Y.; Jeon, J.S.; Yoon, H.W.; Lee, G.C.; Ahn, J.H.; Lee, K.M.; Lee, C.H. Full genome sequencing and analysis of human cytomegalovirus strain JHC isolated from a Korean patient. Virus Res. 2011, 156, 113-120.

44. Murrell, I.; Tomasec, P.; Wilkie, G.S.; Dargan, D.J.; Davison, A.J.; Stanton, R.J. Impact of sequence variation in the UL128 locus on production of human cytomegalovirus in fibroblast and epithelial cells. J. Virol. 2013, 87, 10489-10500. 
45. Tomasec, P.; Wang, E.C.; Davison, A.J.; Vojtesek, B.; Armstrong, M.; Griffin, C.; McSharry, B.P.; Morris, R.J.; Llewellyn-Lacey, S.; Rickards, C.; et al. Downregulation of natural killer cell-activating ligand CD155 by human cytomegalovirus UL141. Nat. Immunol. 2005, 6, 181-188.

46. Chou, S.W.; Dennison, K.M. Analysis of interstrain variation in cytomegalovirus glycoprotein B sequences encoding neutralization-related epitopes. J. Infect. Dis. 1991, 163, 1229-1234.

47. Pignatelli, S.; Dal Monte, P.; Landini, M.P. gpUL73 (gN) genomic variants of human cytomegalovirus isolates are clustered into four distinct genotypes. J. Gen. Virol. 2001, 82, 2777-2784.

48. Rasmussen, L.; Geissler, A.; Cowan, C.; Chase, A.; Winters, M. The genes encoding the gCIII complex of human cytomegalovirus exist in highly diverse combinations in clinical isolates. J. Virol. 2002, 76, 10841-10848.

49. Lurain, N.S.; Kapell, K.S.; Huang, D.D.; Short, J.A.; Paintsil, J.; Winkfield, E.; Benedict, C.A.; Ware, C.F.; Bremer, J.W. Human cytomegalovirus UL144 open reading frame: Sequence hypervariability in low-passage clinical isolates. J. Virol. 1999, 73, 10040-10050.

50. Lurain, N.S.; Fox, A.M.; Lichy, H.M.; Bhorade, S.M.; Ware, C.F.; Huang, D.D.; Kwan, S.P.; Garrity, E.R.; Chou, S. Analysis of the human cytomegalovirus genomic region from UL146 through UL147A reveals sequence hypervariability, genotypic stability, and overlapping transcripts.Virol. J. 2006, 3, 4.

51. Qi, Y.; Mao, Z.Q.; Ruan, Q.; He, R.; Ma, Y.P.; Sun, Z.R.; Ji, Y.H.; Huang, Y. Human cytomegalovirus (HCMV) UL139 open reading frame: Sequence variants are clustered into three major genotypes. J. Med. Virol. 2006, 78, 517-522.

52. Hitomi, S.; Kozuka-Hata, H.; Chen, Z.; Sugano, S.; Yamaguchi, N.; Watanabe, S. Human cytomegalovirus open reading frame UL11 encodes a highly polymorphic protein expressed on the infected cell surface. Arch. Virol. 1997, 142, 1407-1427.

53. Bar, M.; Shannon-Lowe, C.; Geballe, A.P. Differentiation of human cytomegalovirus genotypes in immunocompromised patients on the basis of UL4 gene polymorphisms. J. Infect. Dis. 2001, 183, 218-225.

54. Pignatelli, S.; Dal Monte, P.; Rossini, G.; Landini, M.P. Genetic polymorphisms among human cytomegalovirus (HCMV) wild-type strains. Rev. Med. Virol. 2004, 14, 383-410.

55. Puchhammer-Stockl, E.; Gorzer, I. Cytomegalovirus and Epstein-Barr virus subtypes-The search for clinical significance. J. Clin. Virol. 2006, 36, 239-248.

56. Puchhammer-Stockl, E.; Gorzer, I. Human cytomegalovirus: An enormous variety of strains and their possible clinical significance in the human host. Future Virol. 2011, 6, 259-271.

57. Stanton, R.; Westmoreland, D.; Fox, J.D.; Davison, A.J.; Wilkinson, G.W. Stability of human cytomegalovirus genotypes in persistently infected renal transplant recipients. J. Med. Virol. 2005, 75, 42-46.

58. Pignatelli, S.; Dal Monte, P.; Rossini, G.; Chou, S.; Gojobori, T.; Hanada, K.; Guo, J.J.; Rawlinson, W.; Britt, W.; Mach, M.; et al. Human cytomegalovirus glycoprotein N (gpUL73-gN) genomic variants: Identification of a novel subgroup, geographical distribution and evidence of positive selective pressure. J. Gen. Virol. 2003, 84, 647-655. 
59. Bradley, A.J.; Kovacs, I.J.; Gatherer, D.; Dargan, D.J.; Alkharsah, K.R.; Chan, P.K.; Carman, W.F.; Dedicoat, M.; Emery, V.C.; Geddes, C.C.; et al. Genotypic analysis of two hypervariable human cytomegalovirus genes. J. Med. Virol. 2008, 80, 1615-1623.

60. Bates, M.; Monze, M.; Bima, H.; Kapambwe, M.; Kasolo, F.C.; Gompels, U.A. High human cytomegalovirus loads and diverse linked variable genotypes in both HIV-1 infected and exposed, but uninfected, children in Africa. Virology 2008, 382, 28-36.

61. McGeoch, D.J.; Rixon, F.J.; Davison, A.J. Topics in herpes virus genomics and evolution. Virus Res. 2006, 117, 90-104.

62. Chou, S.W. Reactivation and recombination of multiple cytomegalovirus strains from individual organ donors. J. Infect. Dis. 1989, 160, 11-15.

63. Rasmussen, L.; Geissler, A.; Winters, M. Inter- and intragenic variations complicate the molecular epidemiology of human cytomegalovirus. J. Infect. Dis. 2003, 187, 809-819.

64. Faure-Della Corte, M.; Samot, J.; Garrigue, I.; Magnin, N.; Reigadas, S.; Couzi, L.; Dromer, C.; Velly, J.F.; Dechanet-Merville, J.; Fleury, H.J.; et al.Variability and recombination of clinical human cytomegalovirus strains from transplantation recipients. J. Clin. Virol. 2010, 47, 161-169.

65. Shepp, D.H.; Match, M.E.; Ashraf, A.B.; Lipson, S.M.; Millan, C.; Pergolizzi, R. Cytomegalovirus glycoprotein B groups associated with retinitis in AIDS. J. Infect. Dis. 1996, 174, 184-187.

66. Torok-Storb, B.; Boeckh, M.; Hoy, C.; Leisenring, W.; Myerson, D.; Gooley, T. Association of specific cytomegalovirus genotypes with death from myelosuppression after marrow transplantation. Blood 1997, 90, 2097-2102.

67. Rossini, G.; Pignatelli, S.; Dal Monte, P.; Camozzi, D.; Lazzarotto, T.; Gabrielli, L.; Gatto, M.R.; Landini, M.P. Monitoring for human cytomegalovirus infection in solid organ transplant recipients through antigenemia and glycoprotein $\mathrm{N}(\mathrm{gN})$ variants: Evidence of correlation and potential prognostic value of gN genotypes. Microbes Infect. 2005, 7, 890-896.

68. Pignatelli, S.; Lazzarotto, T.; Gatto, M.R.; Dal Monte, P.; Landini, M.P.; Faldella, G.; Lanari, M. Cytomegalovirus gN genotypes distribution among congenitally infected newborns and their relationship with symptoms at birth and sequelae. Clin. Infect. Dis. 2010, 51, 33-41.

69. Arav-Boger, R.; Willoughby, R.E.; Pass, R.F.; Zong, J.C.; Jang, W.J.; Alcendor, D.; Hayward, G.S. Polymorphisms of the cytomegalovirus (CMV)-encoded tumor necrosis factor-alpha and beta-chemokine receptors in congenital CMV disease. J. Infect. Dis. 2002, 186, 1057-1064.

70. Arav-Boger, R.; Battaglia, C.A.; Lazzarotto, T.; Gabrielli, L.; Zong, J.C.; Hayward, G.S.; Diener-West, M.; Landini, M.P. Cytomegalovirus (CMV)-encoded UL144 (truncated tumor necrosis factor receptor) and outcome of congenital CMV infection. J. Infect. Dis. 2006, 194, 464-473.

71. Waters, A.; Hassan, J.; de Gascun, C.; Kissoon, G.; Knowles, S.; Molloy, E.; Connell, J.; Hall, W.W. Human cytomegalovirus UL144 is associated with viremia and infant development sequelae in congenital infection. J. Clin. Microbiol. 2010, 48, 3956-3962. 
72. Arav-Boger, R.; Boger, Y.S.; Foster, C.B.; Boger, Z. The use of artificial neural networks in prediction of congenital CMV outcome from sequence data. Bioinform. Biol. Insights 2008, 2, 281-289.

73. Kemble, G.; Duke, G.; Winter, R.; Spaete, R. Defined large-scale alterations of the human cytomegalovirus genome constructed by cotransfection of overlapping cosmids. J. Virol. 1996, 70, 2044-2048.

74. Hahn, G.; Rose, D.; Wagner, M.; Rhiel, S.; McVoy, M.A. Cloning of the genomes of human cytomegalovirus strains Toledo, TownevarRIT3, and Towne long as BACs and site-directed mutagenesis using a PCR-based technique. Virology 2003, 307, 164-177.

75. Brechtel, T.; Tyner, M.; Tandon, R. Complete Genome Sequence of a Cytomegalovirus Towne-BAC (Bacterial Artificial Chromosome) Isolate Maintained in Escherichia coli for 10 Years and Then Serially Passaged in Human Fibroblasts. Genome Announc. 2013, 1, e00693-13.

76. Brechtel, T.M.; Tyner, M.; Tandon, R. Complete Genome Sequence of a UL96 Mutant Cytomegalovirus Towne-BAC (Bacterial Artificial Chromosome) Isolate Passaged in Fibroblasts To Allow Accumulation of Compensatory Mutations. Genome Announc. 2013, 1, e00901-13.

77. Gorzer, I.; Guelly, C.; Trajanoski, S.; Puchhammer-Stockl, E. Deep sequencing reveals highly complex dynamics of human cytomegalovirus genotypes in transplant patients over time. J. Virol. 2010, 84, 7195-7203.

78. Gorzer, I.; Guelly, C.; Trajanoski, S.; Puchhammer-Stockl, E. The impact of PCR-generated recombination on diversity estimation of mixed viral populations by deep sequencing. J. Virol. Methods2010, 169, 248-252.

79. Renzette, N.; Bhattacharjee, B.; Jensen, J.D.; Gibson, L.; Kowalik, T.F. Extensive genome-wide variability of human cytomegalovirus in congenitally infected infants. PLoS Pathog. 2011,7, e1001344.

80. James, S.H.; Hartline, C.B.; Harden, E.A.; Driebe, E.M.; Schupp, J.M.; Engelthaler, D.M.; Keim, P.S.; Bowlin, T.L.; Kern, E.R.; Prichard, M.N. Cyclopropavir inhibits the normal function of the human cytomegalovirus UL97 kinase. Antimicrob. Agents Chemother. 2011, $55,4682-4691$.

81. Stark, T.J.; Arnold, J.D.; Spector, D.H.; Yeo, G.W. High-resolution profiling and analysis of viral and host small RNAs during human cytomegalovirus infection. J. Virol. 2012, 86, 226-235.

82. Gatherer, D.; Seirafian, S.; Cunningham, C.; Holton, M.; Dargan, D.J.; Baluchova, K.; Hector, R.D.; Galbraith, J.; Herzyk, P.; Wilkinson, G.W.; et al. High-resolution human cytomegalovirus transcriptome. Proc. Natl. Acad. Sci. USA 2011, 108, 19755-19760.

83. Bhattacharjee, B.; Renzette, N.; Kowalik, T.F. Genetic analysis of cytomegalovirus in malignant gliomas. J. Virol. 2012, 86, 6815-6824.

84. Meshesha, M.K.; Veksler-Lublinsky, I.; Isakov, O.; Reichenstein, I.; Shomron, N.; Kedem, K.; Ziv-Ukelson, M.; Bentwich, Z.; Avni, Y.S. The microRNA Transcriptome of Human Cytomegalovirus (HCMV). Open Virol. J. 2012, 6, 38-48. 
85. Stern-Ginossar, N.; Weisburd, B.; Michalski, A.; Le, V.T.; Hein, M.Y.; Huang, S.X.; Ma, M.; Shen, B.; Qian, S.B.; Hengel, H.; et al. Decoding human cytomegalovirus. Science 2012, 338, 1088-1093.

86. Rossetto, C.C.; Tarrant-Elorza, M.; Pari, G.S. Cis and trans acting factors involved in human cytomegalovirus experimental and natural latent infection of CD14 (+) monocytes and CD34 (+) cells. PLoS Pathog. 2013, 9, e1003366.

87. Sahoo, M.K.; Lefterova, M.I.; Yamamoto, F.; Waggoner, J.J.; Chou, S.; Holmes, S.P.; Anderson, M.W.; Pinsky, B.A. Detection of cytomegalovirus drug resistance mutations by next-generation sequencing. J. Clin. Microbiol. 2013, 51, 3700-3710.

88. Renzette, N.; Gibson, L.; Bhattacharjee, B.; Fisher, D.; Schleiss, M.R.; Jensen, J.D.; Kowalik, T.F. Rapid intrahost evolution of human cytomegalovirus is shaped by demography and positive selection. PLoS Genet. 2013, 9, e1003735.

89. Gorzer, I.; Kerschner, H.; Redlberger-Fritz, M.; Puchhammer-Stockl, E. Human cytomegalovirus (HCMV) genotype populations in immunocompetent individuals during primary HCMV infection. J. Clin. Virol. 2010, 48, 100-103.

90. Ross, S.A.; Novak, Z.; Pati, S.; Patro, R.K.; Blumenthal, J.; Danthuluri, V.R.; Ahmed, A.; Michaels, M.G.; Sanchez, P.J.; Bernstein, D.I.; et al. Mixed infection and strain diversity in congenital cytomegalovirus infection. J. Infect. Dis. 2011, 204, 1003-1007.

91. Cicin-Sain, L.; Podlech, J.; Messerle, M.; Reddehase, M.J.; Koszinowski, U.H. Frequent coinfection of cells explains functional in vivo complementation between cytomegalovirus variants in the multiply infected host. $J$. Virol. 2005, 79, 9492-9502.

92. Humar, A.; Kumar, D.; Gilbert, C.; Boivin, G. Cytomegalovirus (CMV) glycoprotein B genotypes and response to antiviral therapy, in solid-organ-transplant recipients with CMV disease. J. Infect. Dis. 2003, 188, 581-584.

93. Coaquette, A.; Bourgeois, A.; Dirand, C.; Varin, A.; Chen, W.; Herbein, G. Mixed cytomegalovirus glycoprotein B genotypes in immunocompromised patients. Clin. Infect. Dis. 2004, 39, 155-161.

94. Puchhammer-Stockl, E.; Gorzer, I.; Zoufaly, A.; Jaksch, P.; Bauer, C.C.; Klepetko, W.; Popow-Kraupp, T. Emergence of multiple cytomegalovirus strains in blood and lung of lung transplant recipients. Transplantation 2006, 81, 187-194.

95. Manuel, O.; Asberg, A.; Pang, X.; Rollag, H.; Emery, V.C.; Preiksaitis, J.K.; Kumar, D.; Pescovitz, M.D.; Bignamini, A.A.; Hartmann, A.; et al. Impact of genetic polymorphisms in cytomegalovirus glycoprotein B on outcomes in solid-organ transplant recipients with cytomegalovirus disease. Clin. Infect. Dis. 2009, 49, 1160-1166.

96. Beerenwinkel, N.; Zagordi, O. Ultra-deep sequencing for the analysis of viral populations. Curr. Opin. Virol. 2011, 1, 413-418.

97. Beerenwinkel, N.; Gunthard, H.F.; Roth, V.; Metzner, K.J. Challenges and opportunities in estimating viral genetic diversity from next-generation sequencing data. Front. Microbiol. 2012, 3, 329.

98. McGettigan, P.A. Transcriptomics in the RNA-seq era. Curr. Opin. Chem. Biol. 2013, 17, $4-11$. 
99. Ma, Y.; Wang, N.; Li, M.; Gao, S.; Wang, L.; Zheng, B.; Qi, Y.; Ruan, Q. Human CMV transcripts: An overview. Future Microbiol. 2012, 7, 577-593.

100. Chinen, M.; Tani, T. Diverse functions of nuclear non-coding RNAs in eukaryotic gene expression. Front. Biosci. 2012, 17, 1402-1417.

101. Reeves, M.B.; Davies, A.A.; McSharry, B.P.; Wilkinson, G.W.; Sinclair, J.H. Complex I binding by a virally encoded RNA regulates mitochondria-induced cell death. Science 2007, 316, 1345-1348.

102. Su, W.Y.; Xiong, H.; Fang, J.Y. Natural antisense transcripts regulate gene expression in an epigenetic manner. Biochem. Biophys. Res. Commun. 2010, 396, 177-181.

103. Bartel, D.P. MicroRNAs: target recognition and regulatory functions. Cell 2009, 136, 215-233.

104. Pfeffer, S.; Zavolan, M.; Grasser, F.A.; Chien, M.; Russo, J.J.; Ju, J.; John, B.; Enright, A.J.; Marks, D.; Sander, C.; et al. Identification of virus-encoded microRNAs. Science 2004, 304, 734-736.

105. Dunn, W.; Trang, P.; Zhong, Q.; Yang, E.; van Belle, C.; Liu, F. Human cytomegalovirus expresses novel microRNAs during productive viral infection. Cell. Microbiol. 2005, 7, 1684-1695.

106. Grey, F.; Antoniewicz, A.; Allen, E.; Saugstad, J.; McShea, A.; Carrington, J.C.; Nelson, J. Identification and characterization of human cytomegalovirus-encoded microRNAs. J. Virol. 2005, 79, 12095-12099.

107. Tuddenham, L.; Pfeffer, S. Roles and regulation of microRNAs in cytomegalovirus infection. Biochim. Biophys. Acta 2011, 1809, 613-622.

108. Marcinowski, L.; Lidschreiber, M.; Windhager, L.; Rieder, M.; Bosse, J.B.; Radle, B.; Bonfert, T.; Gyory, I.; de Graaf, M.; Prazeres da Costa, O.; et al. Real-time transcriptional profiling of cellular and viral gene expression during lytic cytomegalovirus infection. PLoS Pathog. 2012, 8, e1002908.

109. Juranic Lisnic, V.; Babic Cac, M.; Lisnic, B.; Trsan, T.; Mefferd, A.; das Mukhopadhyay, C.; Cook, C.H.; Jonjic, S.; Trgovcich, J. Dual analysis of the murine cytomegalovirus and host cell transcriptomes reveal new aspects of the virus-host cell interface. PLoS Pathog. 2013, 9, e1003611.

110. Yang, Y.; Liu, R.; Xie, H.; Hui, Y.; Jiao, R.; Gong, Y.; Zhang, Y. Advances in nanopore sequencing technology. J. Nanosci. Nanotechnol. 2013, 13, 4521-4538.

111. Coupland, P.; Chandra, T.; Quail, M.; Reik, W.; Swerdlow, H. Direct sequencing of small genomes on the Pacific Biosciences RS without library preparation. BioTechniques 2012, 53, 365-372.

112. Thompson, J.F.; Milos, P.M. The properties and applications of single-molecule DNA sequencing. Genome Biol. 2011, 12, 217.

113. Korlach, J.; Turner, S.W. Going beyond five bases in DNA sequencing. Curr. Opin. Struct. Biol. 2012, 22, 251-261.

114. Davis, B.M.; Chao, M.C.; Waldor, M.K. Entering the era of bacterial epigenomics with single molecule real time DNA sequencing. Curr. Opin. Microbiol. 2013, 16, 192-198. 
Reprinted from Viruses. Cite as: Yang, Z.; Vu, G.-P.; Qian, H.; Chen, Y.-C.; Wang, Y.; Reeves, M.; Zen, K.; Liu, F. Engineered RNase P Ribozymes Effectively Inhibit Human Cytomegalovirus Gene Expression and Replication. Viruses 2014, 6, 2376-2391.

Article

\title{
Engineered RNase P Ribozymes Effectively Inhibit Human Cytomegalovirus Gene Expression and Replication
}

\author{
Zhu Yang ${ }^{1,2,3, \dagger}$, Gia-Phong Vu ${ }^{4, \dagger}$, Hua Qian ${ }^{5, \dagger}$, Yuan-Chuan Chen ${ }^{4}$, Yu Wang ${ }^{2,3}$, \\ Michael Reeves ${ }^{6}$, Ke Zen ${ }^{1}$ and Fenyong Liu ${ }^{4,6, *}$
}

1 Institute of Virology, School of Life Sciences, Nanjing University, Nanjing, Jiangsu 210093, China; E-Mails: nooney1986@163.com (Z.Y.); kzen@nju.edu.cn (K.Z.)

2 Taizhou Institute of Virology, Taizhou, Jiangsu 225300, China; E-Mail: sin_angel@foxmail.com

3 Jiangsu Affynigen Biotechnologies, Inc., Taizhou, Jiangsu 225300, China

4 Program in Comparative Biochemistry, University of California, Berkeley, CA 94720, USA; E-Mails: giaphongvu@berkeley.edu (G.-P.V.); yuchuan1022@berkeley.edu (Y.-C.C.)

5 Department of Gynecology, People's Hospital of Taizhou, Taizhou, Jiangsu 225300, China; E-Mail: huaqian1965@hotmail.com

6 School of Public Health, University of California, Berkeley, CA 94720, USA; E-Mail: mreeves@berkeley.edu

$\dagger$ These authors contribute equally to this study.

* Author to whom correspondence should be addressed; E-Mail: liu_fy@berkeley.edu; Tel.: +1-510-643-2436; Fax: +1-510-643-9955.

Received: 8 April 2014; in revised form: 19 May 2014 / Accepted: 23 May 2014 /

Published: 13 June 2014

\begin{abstract}
RNase P ribozyme can be engineered to be a sequence-specific gene-targeting agent with promising application in both basic research and clinical settings. By using an in vitro selection system, we have previously generated RNase P ribozyme variants that have better catalytic activity in cleaving an mRNA sequence than the wild type ribozyme. In this study, one of the variants was used to target the mRNA encoding human cytomegalovirus (HCMV) essential transcription factor immediate-early protein 2 (IE2). The variant was able to cleave IE2 mRNA in vitro 50 -fold better than the wild type ribozyme. A reduction of about $98 \%$ in IE2 expression and a reduction of 3500 -fold in viral production was observed in HCMV-infected cells expressing the variant compared to a $75 \%$ reduction in IE2 expression and a 100 -fold reduction in viral production in cells expressing the ribozyme derived from the wild type sequence. These
\end{abstract}


results suggest that ribozyme variants that are selected to be highly active in vitro are also more effective in inhibiting the expression of their targets in cultured cells. Our study demonstrates that RNase P ribozyme variants are efficient in reducing HCMV gene expression and growth and are potentially useful for anti-viral therapeutic application.

Keywords: ribozyme; RNase P; gene targeting; cytomegalovirus

\section{Introduction}

Human cytomegalovirus (HCMV) is a common herpesvirus and usually affects individuals with compromised immune system [1]. This virus causes birth defects including mental problems [2]. Furthermore, HCMV can cause debilitating symptoms in AIDS patients, such as blindness and gastrointestinal diseases [3,4]. New therapeutic approaches are needed to combat and control this important opportunistic pathogen.

Gene targeting approaches using nucleic acid-based molecules to target specific mRNA sequences of choice represent promising therapeutic strategies [5,6]. One example is small interfering RNAs (siRNAs), which were used to target several human viruses effectively $[5,7,8]$. Ribozymes can also inactivate viral mRNA sequences and diminish viral infection in human cells [9-12].

RNase P functions in cells to process tRNA precursors (ptRNA) into mature tRNAs [13-15]. In Escherichia coli, RNase P forms a holoenzyme containing a protein (C5 protein) and a catalytically active RNA (M1 RNA) [16]. Previous studies have shown that the enzyme recognizes its substrate through the tertiary structure and not the primary sequence. (Figure 1A) [13,14,17]. Any mRNA substrate can be targeted and hydrolyzed by M1 ribozyme through the binding of a complementary external guide sequence (EGS) to the substrate to resemble the structure of the tRNA module that includes the 5' leader sequence, the acceptor stem, the T-stem, and the 3' CCA sequence (Figure 1) [18,19]. A ribozyme targeting any mRNA sequence of choice, M1GS RNA, can be designed by linking a guide sequence (GS) to the 3' terminus of M1 RNA (Figure 1C) [20,21]. The guide sequence can basepair with an mRNA target and thus, allows M1GS to bind to the mRNA. Previous studies had designed M1GS ribozymes to target cellular genes and the essential genes of herpes simplex virus 1 (HSV-1) and HCMV [22-24]. The ribozymes were effective in reducing HSV-1 and HCMV growth 1000-fold and 150-fold, respectively [22,23].

Compared to other gene-targeting approaches such as RNAi, the M1GS ribozyme has several advantages. Ribozymes have not been shown to saturate the cellular machinery required for their processing while high levels of siRNAs can overwhelm the RNAi machinery that contains various cellular factors and may affect the normal cellular functions of these factors [5,25-28]. Furthermore, by interacting with specific cellular proteins including the RNase P protein subunits [14,29], RNase $\mathrm{P}$ ribozymes may improve their intracellular stability and catalytic activities in cells. RNase $\mathrm{P}$ ribozyme represents a unique and promising class of gene-targeting agents for therapeutic application [30]. 
Figure 1. Schematic presentation of RNase P/M1GS ribozyme's substrates. (A-C) pre-tRNA (ptRNA), a natural substrate (A); EGS:mRNA complex (B); and M1GS binding to its substrate $(\mathbf{C})$. Filled arrows denote the cleavage sites. (D) Substrate ie2-39 with boxed region representing the complementary sequence to the guide sequence of M1GS.

A

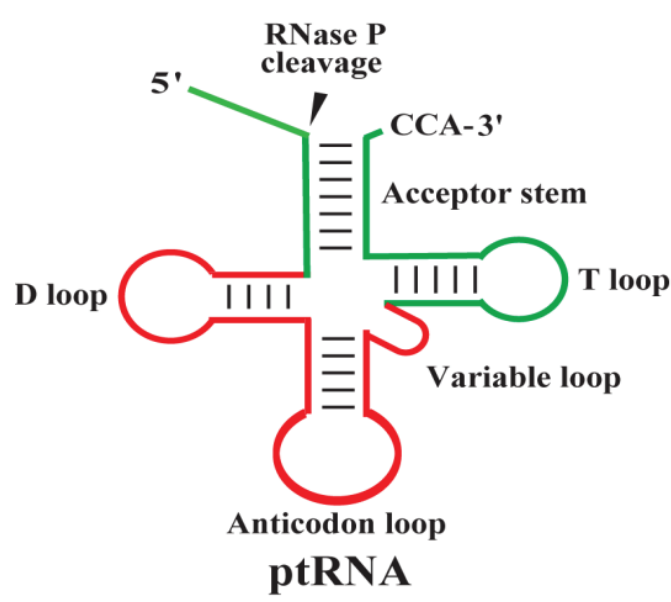

B

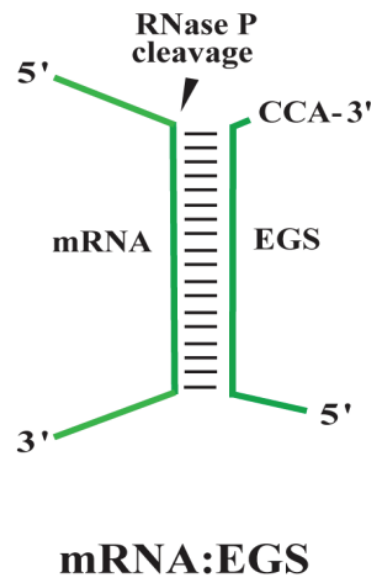

$\mathrm{C}$

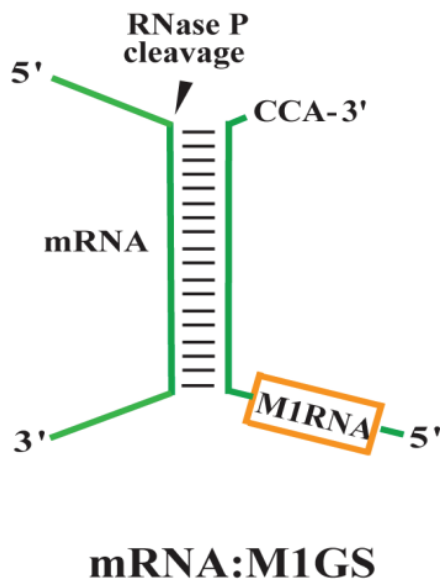

$\mathrm{D}$ \begin{tabular}{crrr} 
ie2-39 & \multicolumn{1}{c}{ GGTGTCAATCAT } & GCCGGTATCGATTCCAGTAGGATCCCG \\
1 & 12 & 39
\end{tabular}

Increasing the catalytic efficiency of the RNase P ribozyme is essential for M1GS technology to be used as an effective gene-targeting approach. Previously, we have generated RNase P ribozyme variants through an in vitro selection system that have better catalytic activity in cleaving an mRNA sequence than the wild type M1 RNA ribozyme [31]. For the study, a highly active ribozyme variant was designed to hydrolyze the exon 5 region of the mRNA encoding HCMV immediate-early 2 (IE2) protein. IE2 encodes a HCMV major transcription regulatory factor and its expression is needed for the expression of viral early $(\beta)$ and late $(\gamma)$ genes $[1,32-34]$. Thus, IE2 protein expression is essential for HCMV replication in cultured cells. Inhibition of IE2 expression would result in shutting down gene expression and replication of HCMV. We investigated the activity of the constructed ribozyme in generating cleavage of IE2 mRNA sequence in vitro and in shutting down HCMV infection in cells. Our data showed that the ribozyme variant cleaved the target IE2 mRNA sequence at least 50-fold more efficiently than the wild type ribozyme. More importantly, greater reduction in IE2 expression and HCMV growth was observed in cells expressing the variant. Our results suggest that RNase $\mathrm{P}$ ribozyme variants can be used as promising gene-targeting agents for anti-CMV applications. 


\section{Results}

\subsection{Targeting and Cleavage Activities of the MIGS RNAs in Vitro}

Since most mRNA species inside cells form complex secondary structures and are associated with proteins, a targeting region must be accessible for ribozyme binding and catalytic cleavage of the target mRNA. We used dimethyl sulphate (DMS) [21,35] to map the accessible regions of IE2 mRNA in vivo [36]. To map these regions, we infected human U373MG cells with HCMV and then incubated these cells with culture media that contained DMS [36]. It is expected that DMS would enter the cells and modify the nucleotides of the accessible mRNA regions. We then isolated the total mRNAs. Primer extension assays using reverse transcriptase were used to map the IE2 mRNA regions that were modified by DMS [36]. A position, 30 nucleotides downstream from the 5' terminus of exon 5, was selected as the targeting site. The chosen targeting region (designated as IE2 RNA) was extensively modified by DMS and was predicted to be accessible for M1GS binding and cleavage (data not shown).

We had previously carried out an in vitro selection procedure and isolated M1GS RNA variants that have higher catalytic activity in cleaving HSV-1 thymidine kinase (TK) mRNA than the wild type M1 RNA [31]. However, little research has been done to examine whether these variants are also effective in targeting HCMV mRNAs to shut down viral infection. To investigate these issues, we chose variant 661 (designated as V661) for the study because this selected variant is highly active in cleaving target RNAs in vitro (e.g., IE2 and TK and mRNA) (Table 1) [31]. This variant contains two point mutations (i.e., $\mathrm{A}_{94} \rightarrow \mathrm{G}_{94}$ and $\mathrm{G}_{194} \rightarrow \mathrm{C}_{194}$ ) [31]. Little is known about the contribution of these nucleotides to the activity of M1GS RNAs in cleaving an mRNA substrate. The effect of the point mutations on the activity of RNase P ribozymes has not been studied.

Table 1. Overall cleavage rate $\left[\left(\mathrm{k}_{\mathrm{cat}} / \mathrm{K}_{\mathrm{m}}\right)^{\mathrm{s}}\right]$ and binding affinity $\left(\mathrm{K}_{\mathrm{d}}\right)$ of RNase $P$ ribozymes with substrate ie2-39.

\begin{tabular}{|c|c|c|}
\hline Enzyme & $\begin{array}{c}\left(\mathbf{k}_{\mathrm{cat}} / \mathbf{K}_{\mathrm{m}}\right)^{\mathrm{s}} \\
\left(\mu \mathbf{M}^{-1} \cdot \mathbf{m i n}^{-1}\right) \\
\end{array}$ & $\begin{array}{c}\mathbf{K}_{\mathbf{d}} \\
(\mathbf{n M})\end{array}$ \\
\hline M1-IE2 & $0.20 \pm 0.05$ & $0.34 \pm 0.06$ \\
\hline V661-IE2 & $10.5 \pm 0.5$ & $0.30 \pm 0.05$ \\
\hline M1-IE2-C & $<5 \times 10^{-6}$ & $0.33 \pm 0.07$ \\
\hline V661-IE2-C & $<5 \times 10^{-6}$ & $0.32 \pm 0.07$ \\
\hline M1-TK & $<5 \times 10^{-6}$ & ND \\
\hline
\end{tabular}

The values shown are the average derived from triplicate experiments. $p<0.01$. "ND", not determined.

To construct IE2 mRNA-cleaving ribozymes from the variant and wild type M1 sequence, V661-IE2 and M1-IE2, the 3' termini of V661 and M1 RNA were covalently linked with an 18 nucleotides guide sequence that binds to the IE2 mRNA sequence, respectively. We included two control RNase P ribozymes, M1-IE2-C and V661-IE2-C. M1-IE2-C was generated from C102 RNA, an M1 mutant with mutations $\left(\mathrm{A}_{347} \mathrm{C}_{348} \rightarrow \mathrm{C}_{347} \mathrm{U}_{348}, \mathrm{C}_{353} \mathrm{C}_{354} \mathrm{C}_{355} \mathrm{G}_{356} \rightarrow \mathrm{G}_{353} \mathrm{G}_{354} \mathrm{~A}_{355} \mathrm{U}_{356}\right)$ at the catalytic $\mathrm{P} 4$ domain that rendered it inactive in cleaving a pre-tRNA [37]. V661-IE2-C was generated from V661-IE2 and contained the mutations found in C102. While sharing identical 
guide sequence with M1-IE2 and V661-IE2, both M1-IE2-C and V661-IE2-C were not expected to be functional because of the $\mathrm{P} 4$ mutations.

We measured the catalytic activity $\left(\mathrm{k}_{\mathrm{cat}} / \mathrm{K}_{\mathrm{m}}\right)^{\mathrm{s}}$ with kinetic analyses for these ribozymes in cleaving substrate ie2-39 of the 39 nucleotide long IE2 mRNA target sequence (Figure 1D). These results, shown in Table 1, indicate that V661-IE2 is about 50-fold more active than M1-IE2 in cleaving substrate ie2-39 $(p<0.01)$. As expected, V661-IE2-C and M1-IE2-C were at least $10^{4}$-fold less active than M1-IE2 RNA in cleaving substrate ie2-39, possibly because the P4 mutations abolished the catalytic activity of the ribozymes $(p<0.01)$.

Detailed gel-shift assays indicate that the binding affinities of V661-IE2-C and M1-IE2-C to substrate ie2-39, as measured by the dissociation constant $\left(\mathrm{K}_{\mathrm{d}}\right)$, are similar to those of V661-IE2 and M1-IE2 (Table 1). Since V661-IE2-C and M1-IE2-C are non-functional but contain identical guide sequence complementary to ie2-39 and exhibit similar affinity to ie2-39 as V661-IE2 and M1-IE2, these ribozymes serve as controls for the antisense effect in our experiments.

\subsection{Expression of Ribozymes in Human Cell Culture}

The DNA sequences coding for V661-IE2, M1-IE2, V661-IE2-C, and M1-IE2-C were subcloned into LXSN retroviral vector and placed under the constitutively expressed U6 RNA promoter $[19,21,38]$. To construct cell lines that express M1GS RNAs, we generated retroviral vectors that contained the genes for M1GS RNAs by transfecting LXSN-M1GS DNAs into the amphotropic packaging cells (PA317). We subsequently infected human U373MG cells with the retroviruses and generated stable cell lines expressing the M1GSs. A cell line expressing ribozyme M1-TK, which targeted HSV-1 TK mRNA, was constructed to control for M1GS RNA with a mismatched guide sequence [22]. No cleavage of substrate ie2-39 by M1-TK was observed in vitro (data not shown, Table 1).

Northern blot analysis was performed to assay M1GS RNA expression in individual cell clones (Figure 2, lanes 5-8). Human H1 RNA was used as the loading and internal control (Figure 2, lanes $1-4)[14,36]$. No difference in cell growth and viability was observed for up to two months between cell lines expressing M1GS and a control line with empty vector LXSN DNA, suggesting that ribozymes did not result in significant cytotoxicity (data not shown). For subsequent experiments, only cell lines exhibiting similar ribozyme expression levels were used.

Figure 2. M1GS ribozymes expression with Northern blot analysis in parental U373MG cells (-, lanes 1 and 5) and cells with M1-IE2-C (lanes 2 and 6), M1-IE2 (lanes 3 and 7), and V661-IE2 (lanes 4 and 8). H1 RNA control (lanes 1-4).

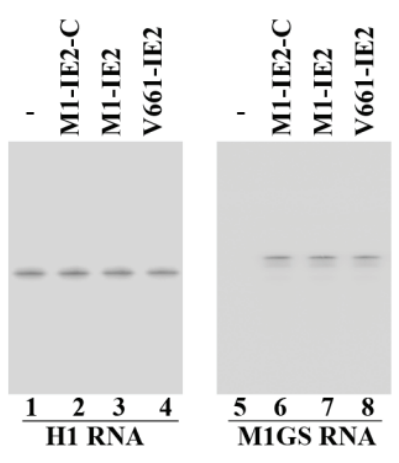


Cells were infected with HCMV at a multiplicity of infection (MOI) of 1. Total RNAs were then isolated from these cells. HCMV immediate early $5 \mathrm{~kb}$ RNA, which is expressed from the region spanning viral UL106 and UL111 with its 5' end at nucleotide position 159627 and its putative 3' end at position 154829 of the HCMV (AD169) genome [1,39], was used as an internal loading control for IE2 mRNA expression.

Figure 3 shows northern analysis of IE2 mRNA levels, which are quantitated in Table 2. A reduction of about $98 \%$ and $75 \%(p<0.04)$ in IE2 mRNA expression was detected in cells that expressed V661-IE2 and M1-IE2, respectively (Figure 3, lanes 5 and 8).

Figure 3. Northern analysis of the levels of human cytomegalovirus (HCMV) mRNAs. Cells $\left(n=1 \times 10^{6}\right)$ were infected with HCMV $(\mathrm{MOI}=1)$ and were harvested at either 8 (A-B) or $24 \mathrm{~h}(\mathbf{C})$ post-infection. We used RNAs isolated from parental U373MG cells (-, lanes 3, 7, and 11) and cell lines that expressed V661-IE2 (lanes 1, 5, and 9), M1-IE2-C (lanes 2, 6, and 10), and M1-IE2 (lanes 4, 8, and 12). RNA samples were hybridized to a DNA probe coding for HCMV $5 \mathrm{~kb}$ RNA (lanes 1-4), IE2 mRNA (lanes 5-8), and US2 mRNA (lanes 9-12).

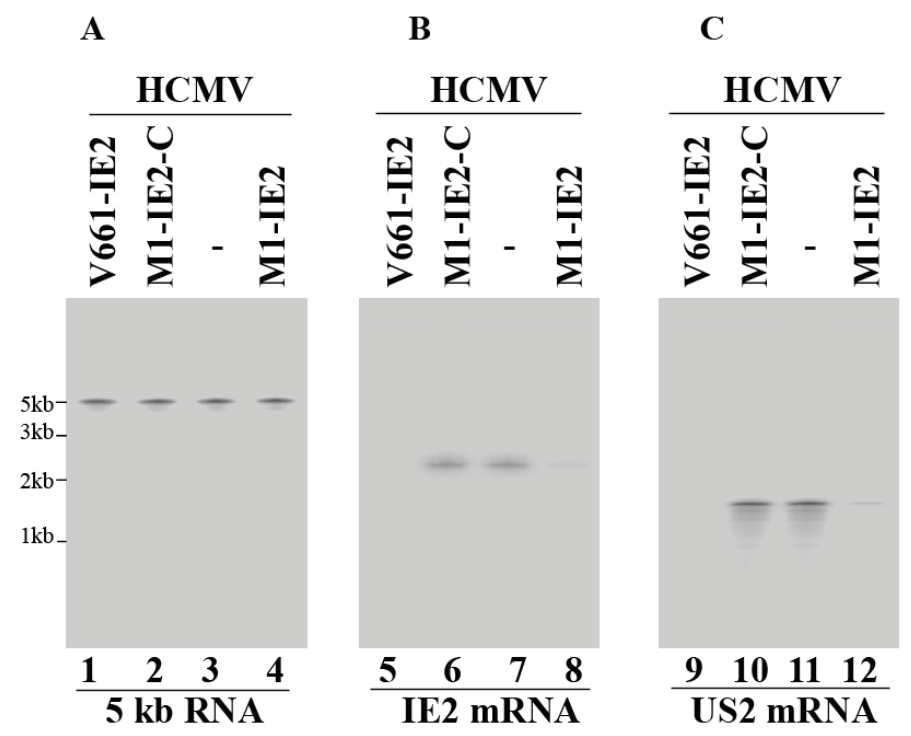

In comparison, cells that expressed M1-IE2-C and V661-IE2-C showed little reduction $(<10 \%)$ (Figure 3, lane 6, data not shown) (Table 2). An antisense effect could explain the low level of inhibition found in cells that expressed M1-IE2-C and V661-IE2-C. This is because these control ribozymes contained the same guide sequence as M1-IE2 and V661-IE2 but did not exhibit catalytic activity. These observations suggest that the significant reduction of IE2 mRNA expression in cells that expressed V661-IE2 and M1-IE2 was due to the catalytic cleavage of the target mRNA by these ribozymes. IE2 mRNA cleavage products were not detected in our northern analyses possibly due to degradation by intracellular RNases. 
Table 2. HCMV mRNA and protein expression in cells expressing M1-TK, M1-IE2-C, V661-IE2-C, M1-IE2, or V661-IE2, and cells not expressing any ribozyme (U373MG). The values represent the levels of inhibition of gene expression as compared to the levels of inhibition in U373MG cells.

\begin{tabular}{|c|c|c|c|c|c|c|c|}
\hline & \multirow{2}{*}{$\begin{array}{c}\text { Viral Gene } \\
\text { Class } \\
\end{array}$} & \multicolumn{6}{|c|}{ Ribozymes } \\
\hline & & U373MG & M1-IE2-C & V661-IE2-C & M1-IE2 & V661-IE2 & M1-TK \\
\hline IE2 mRNA & $\alpha$ & $0 \%$ & $6 \%$ & $5 \%$ & $75 \% \pm 7 \%$ & $98 \% \pm 7 \%$ & $1 \%$ \\
\hline US2 mRNA & $\beta$ & $0 \%$ & $1 \%$ & $1 \%$ & $70 \% \pm 6 \%$ & $96 \% \pm 8 \%$ & $0 \%$ \\
\hline IE2 protein & $\alpha$ & $0 \%$ & $4 \%$ & $5 \%$ & $75 \% \pm 8 \%$ & $98 \% \pm 8 \%$ & $0 \%$ \\
\hline UL44 protein & $\beta, \gamma$ & $0 \%$ & $1 \%$ & $0 \%$ & $71 \% \pm 8 \%$ & $95 \% \pm 7 \%$ & $1 \%$ \\
\hline UL99 protein & $\gamma$ & $0 \%$ & $0 \%$ & $1 \%$ & $72 \% \pm 7 \%$ & $94 \% \pm 6 \%$ & $0 \%$ \\
\hline $\mathrm{gH}$ protein & $\gamma$ & $0 \%$ & $0 \%$ & $0 \%$ & $71 \% \pm 8 \%$ & $98 \% \pm 7 \%$ & $1 \%$ \\
\hline
\end{tabular}

The values represent arithmetic means of three independent experiments performed in triplicate. $p<0.04$.

The values of standard deviation that were less than $5 \%$ are not shown.

IE2 protein levels in M1GS-expressing cells are expected to decrease due to the corresponding reduction in the level of IE2 mRNA. Proteins were isolated from cells, separated in gels, and transferred to membranes. The membranes were stained with an anti-IE2 antibody (anti-IE2) (Figure 4, lanes 5-8), and the expression levels of HCMV IE2 protein were determined. Actin was used as an internal and loading control (Figure 4, lanes 1-4). The results are summarized in Table 2 from three independent experiments: $98 \%$ and $75 \%$ reduction in IE2 protein expression $(p<0.04)$ was found in cells with V661-IE2 and M1-IE2 RNA, respectively. In comparison, little reduction $(<10 \%)$ was detected in cells with V661-IE2-C, M1-IE2-C, and M1-TK RNAs (Table 2).

\subsection{Increased Reduction of HCMV Infection by the Ribozymes}

The expressions of HCMV $\beta$ (early) and $\gamma$ (late) genes are expected to be decreased due to the inhibition of IE2 expression [1,33]. To explore this, US2 mRNA level (a $\beta$ mRNA) (Figure 3, lanes 9-12) as well as UL99 protein level (a $\gamma$ protein) (Figure 4, lanes 9-12) were determined. We used HCMV $5 \mathrm{~kb}$ RNA and human actin as the internal loading controls. Our results demonstrated $95 \%-96 \%$ and $71 \%-73 \%$ reduction $(p<0.04)$ in the expression levels of these genes in cells with V661-IE2 and M1-IE2, respectively. Limited inhibition was found in cells with M1-IE2-C, V661-IE2-C, and M1-TK (Figures 3 and 4, Table 2). Similar results were also observed in the expression of HCMV polymerase processivity factor UL44 (a $\beta$ protein) and viral glycoprotein $\mathrm{H}$ (gH) (a $\gamma$ protein) (Table 2). Thus, V661-IE2 and M1-IE2 expressing cells appeared to exhibit overall viral $\beta$ and $\gamma$ gene expression inhibition.

The ribozymes also appeared to inhibit viral growth. After 5 days post-infection, we observed a 3500 - and 100-fold reduction $(p<0.04)$ in viral titers in cells that expressed V661-IE2 and M1-IE2, respectively (Figure 5). In comparison, we observed no reduction in cells with control M1-IE2-C, V661-IE2-C, or M1-TK ribozymes (Figure 5, data not shown). 
Figure 4. Levels of HCMV proteins observed by Western Blot. Actin control (lanes 1-4), IE2 (lanes 5-8), and UL99 (lanes 9-12). Cells $\left(n=1 \times 10^{6}\right)$ were infected with HCMV (MOI = 1) and were harvested at either 24 (lanes 1-8) or $72 \mathrm{~h}$ (lanes 9-12) post-infection.

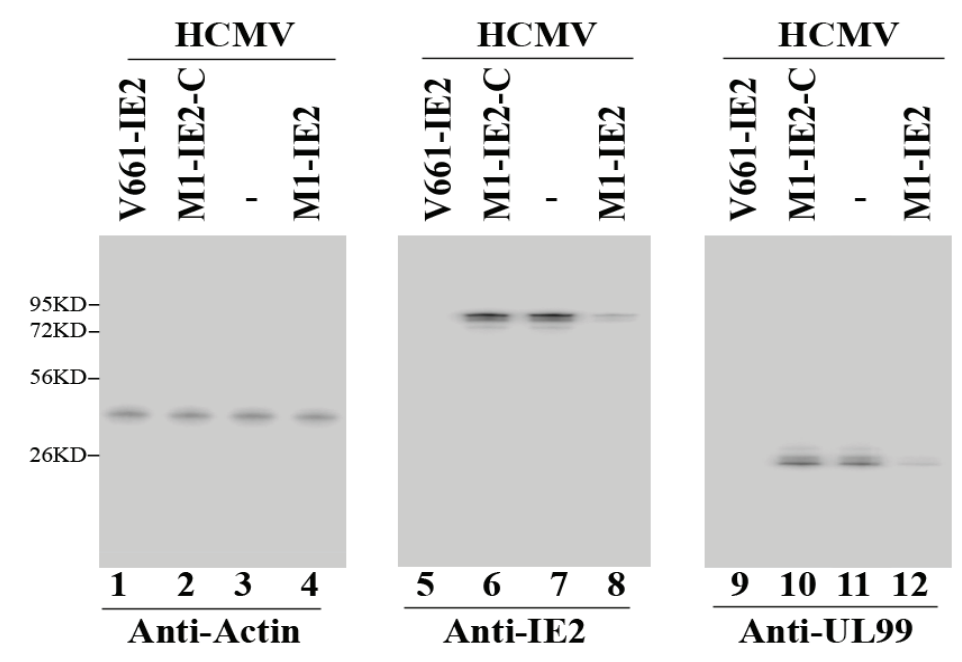

Figure 5. HCMV growth in various cell lines expressing M1GS and the parental U373MG cells. These values are the average from three experiments with error bars showing the standard deviation. $p<0.04$.

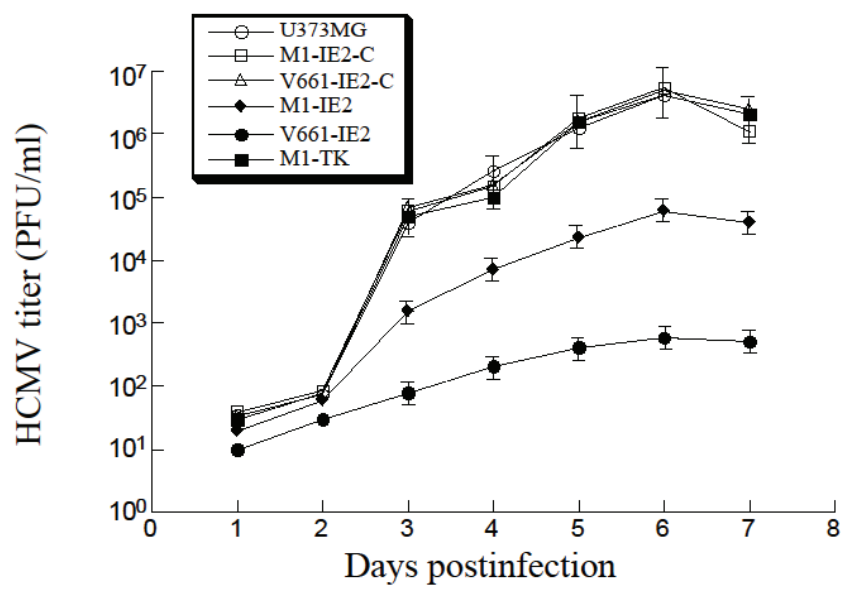

\section{Discussion}

Ribozyme-based gene targeting represents a promising therapeutic approach [5]. For ribozyme-mediated gene targeting to work efficiently, M1GS needs to be highly specific, catalytically efficient, and easily delivered to the target of choice. We have constructed M1GS RNAs that target the exon 5 region of HCMV IE2 mRNA and have shown that the ribozymes target the substrate efficiently in vitro. Moreover, 98\% reduction in the expression level of IE2 and 3,500-fold reduction in viral growth were observed in cells with a ribozyme variant (i.e., V661-IE2). In comparison, we detected little reduction $(<10 \%)$ in IE2 expression and HCMV growth in cells with V661-IE2-C and M1-IE2-C. While non-functional, V661-IE2-C and M1-IE2-C contained identical guide 
sequence and showed similar binding affinity to the targeting mRNA as V661-IE2 and M1-IE2. Thus, our results imply that the overall observed inhibition of HCMV infection with M1-IE2 and V661-IE RNA was primarily due to specific ribozyme-mediated cleavage of the target mRNA.

Limited information is available about what limits M1GS-mediated gene-targeting efficacy in cultured cells [30]. Not much is known about how to improve the efficacy of M1GS RNA in blocking HCMV infection in vivo. In this report, M1GS was designed to target a DMS-accessible sequence of IE2 mRNA and was constitutively expressed by the U6 promoter. Our design should enhance the chance for M1GS to find the mRNA target. With the design, we postulated that the efficacy of RNase P ribozyme cleavage in cultured cells is dictated by its catalytic efficiency $\left[\left(\mathrm{k}_{\mathrm{cat}} / \mathrm{K}_{\mathrm{m}}\right)^{\mathrm{s}}\right]$. It is conceivable that enhancing catalytic activity of M1GS may reduce target mRNA expression more effectively in vivo. Indeed, V661-IE2, which was more efficient in cleaving ie2-39 in vitro, blocked IE2 expression and viral growth in cultured cells more effectively than M1-IE2, the wild type M1GS (Tables 1 and 2). The difference between the in vivo efficacies of V661-IE2 and M1-IE2 (e.g., 98\% vs. 75\%) is less than the difference of in vitro cleavage efficiencies (more than 50 -fold difference). A possible explanation is that about $1 \%-2 \%$ of the target mRNA substrates are unavailable for ribozyme cleavage because they may be bound by ribosome for translation prior to being bound by the ribozymes. Our work implies that increasing catalytic efficiencies $\left[\left(\mathrm{k}_{\mathrm{cat}} / \mathrm{K}_{\mathrm{m}}\right)^{\mathrm{s}}\right]$ correlates with better ribozyme-mediated effect in reducing HCMV growth, and further suggests that improving the in vitro catalytic efficiencies of the ribozymes should lead to increased anti-HCMV efficacies in tissue culture. Thus, our study provides a guideline for the generation of highly effective RNase P ribozyme variants.

The antiviral effect of RNase P ribozymes appears to be specifically induced by the cleavage of the IE2 mRNA. First, cells expressing the ribozymes appeared normal with no toxicity for up to two months (data not shown). Second, the antiviral effect of the ribozyme (inhibition of viral growth) appears to be due to the decrease of IE2 expression. We detected overall reduction of viral $\beta$ and $\gamma$ gene expression (e.g., US2, UL44, UL99, and gH) in cells expressing functional M1-IE2 and V661-IE2 but not control M1-IE2-C or V661-IE2-C. The levels of reduction of IE2 expression correlate well with the levels of reduction in viral $\beta$ and $\gamma$ gene expression. In comparison, we detected no reduction in other viral immediate-early RNA (e.g., 5 kb RNA and UL36 mRNA) expression in these cells (Figure 3A, Table 2, data not shown) $[22,40]$. Thus, the antiviral effect of M1GS ribozyme may be induced by the cleavage of the IE2 mRNA.

Our study showed that variant V661-IE2 is more active in cleaving IE2 mRNA sequence than M1-IE2 in vitro (Table 1). Furthermore, a ribozyme targeting the HSV-1 TK mRNA sequence, V661-TK, was derived from variant V661. V661-TK also cleaved the TK mRNA sequence in vitro more efficiently than M1-TK, which was derived from the wild type ribozyme [31]. Thus, V661 may be used to construct highly efficient ribozymes to target any specific mRNAs. This variant has two point mutations: $\mathrm{A}_{94} \rightarrow \mathrm{G}_{94}$ and $\mathrm{G}_{194} \rightarrow \mathrm{C}_{194}$. Little is known about the roles of these two nucleotides in the M1GS-mediated cleavage of an mRNA substrate. Our study indicated that the point mutations at these two positions increased the overall cleavage rate $\left(\mathrm{k}_{\mathrm{cat}} / \mathrm{K}_{\mathrm{m}}\right)$ without affecting the binding affinity $\left(\mathrm{K}_{\mathrm{d}}\right)$ to the mRNA substrate (Table 1). These results imply that the mutations may have no effect on the interactions of the ribozyme to the target mRNA. Perhaps these mutations increase the overall cleavage rate by stabilizing the active site and facilitating the 
folding of the overall structure of the ribozyme. Further biochemical characterization of V661 and other ribozyme variants will elucidate the mechanism by which mutations found in the variants increase the gene-targeting activity of the ribozymes and provide insight into the construction of highly effective ribozymes for gene targeting applications.

HCMV is a member of the human herpesvirus family [41,42]. Like other herpesviruses, HCMV can actively replicate or enter into latency [1]. When HCMV reactivates from latent infection and initiates lytic infection, IE2 is among the first proteins expressed and is essential for viral replication [1]. To study the functionality of M1GS ribozyme in HCMV latent infection, M1GS can be delivered into latently infected cells including $\mathrm{CD}^{+} 4^{+}$bone marrow progenitor cells. These studies will determine if RNase P ribozymes diminish IE2 expression and block HCMV reactivation. One potential challenge is to develop appropriate vectors for effective delivery and efficient expression of the ribozymes in $\mathrm{CD} 34^{+}$bone marrow progenitor cells. These and further studies should provide insight into the development of M1GSs as potentially effective anti-HCMV therapeutics.

\section{Experimental Section}

\subsection{Viruses, Cells and Antibodies}

HCMV (strain AD169) was grown in human astrocytoma U373MG cells and foreskin fibroblasts in Dulbecco's modified Eagle medium (DMEM) [22,32]. The anti-UL44 and UL99 monoclonal antibodies were obtained from Virusys (Taneytown, MD, USA). Other antibodies were described previously $[22,32]$.

\subsection{Mapping of the Accessible Regions of HCMV IE2 mRNA in Cells}

Detailed protocol for using dimethyl sulfate (DMS) to map the accessible regions of mRNA was described previously [36]. Briefly, DMS was incubated with HCMV-infected cells for 5-10 min. The cells were then lysed and the supernatant containing the cellular lysate was transferred to another tube for total RNA isolation with phenol-chloroform extraction and ethanol precipitation. Primer extension assays were performed with radiolabeled oligonucleotides in order to map the DMS modification sites [21,35], following the procedure described previously [36]. Phenol chloroform was used to extract the primer extension products followed by ethanol precipitation and separation in $8 \%$ denaturing gels. Sites that blocked primer extension, which represent potential locations modified by DMS, were identified in denaturing gels and analyzed using a STORM840 Phosphorimager [36].

\subsection{Ribozyme Studies in Vitro}

The ie2-39 DNA template was obtained by annealing oligonucleotide AF25 (5'GGAATTCTAATACGACTCACTATAG-3') that contains a T7 promoter with oligonucleotide sie2-39 (5'-CGGGATCCTACTGGAATCGATACCGGCATGATTGACACCTATAGTGAGTCGTATTA3'). Plasmid pFL117, pV661, and pC102, which encode M1 RNA, variant V661, and mutant $\mathrm{C} 102$, respectively, have been described previously [31,37]. C102 contains point mutations $\left(\mathrm{A}_{347} \mathrm{C}_{348} \rightarrow \mathrm{C}_{347} \mathrm{U}_{348}, \mathrm{C}_{353} \mathrm{C}_{354} \mathrm{C}_{355} \mathrm{G}_{356} \rightarrow \mathrm{G}_{353} \mathrm{G}_{354} \mathrm{~A}_{355} \mathrm{U}_{356}\right)$, which render it inactive [37]. 
Plasmids pFL117, pV661, and pC102 were used as templates to construct ribozymes M1-IE2, V661-IE2, and M1-IE2-C, respectively. Plasmid pV661-C, which was derived from pV661 and contained the mutations found in $\mathrm{C} 102$, was used as the template to construct ribozyme V661-IE2-C. The 5' PCR primer was AF25 while the 3' primer was M1IE23 (5'CCCGCTCGAGAAAAAATGGTGCCGGTATCGATTCCAGTTGTGGAATTGTG-3'). Ribozymes and RNA substrate ie2-39 were synthesized with T7 RNA polymerase [12]. Kinetic analyses and gel shift assays were performed as described previously $[31,43,44]$.

\subsection{Construction of the M1GS-Expressing Cell Lines}

The DNA sequences coding for the M1GSs were subcloned into retroviral vector LXSN and placed under the control of the U6 RNA promoter [12,21,45]. The retroviral vector DNAs containing the M1GS sequence were transfected into amphotropic PA317 cells. Culture supernatants containing retroviruses were used to infect human U373MG cells, and neomycin-resistant cells were selected in the presence of neomycin $(600 \mu \mathrm{g} / \mathrm{mL})$ (GE Healthcare, Piscataway, NJ) and were eventually cloned $[12,21,45]$.

\subsection{Assaying of Gene Expression and Viral Infection}

Cells $\left(n=1 \times 10^{6}\right)$ were either mock-infected or infected with HCMV at a multiplicity of infection (MOI) of 1. Viral mRNA or protein samples were isolated from the infected cells at various time points as described previously $[22,44]$. Specifically, the samples used for the detection of the IE2 mRNA and protein expression were harvested at 8 and $24 \mathrm{~h}$ post-infection, respectively. Similarly, the samples used for the detection of the HCMV immediate-early UL36 mRNA were harvested at $8 \mathrm{~h}$ post-infection. The samples used for the detection of the US2 mRNA expression were harvested at $24 \mathrm{~h}$ post-infection while those used for the detection of the protein expression of UL44, UL99, and gH were harvested at $72 \mathrm{~h}$ post-infection. For controls, the samples for the detection of the $5 \mathrm{~kb}$ RNA expression were harvested at 8 and $24 \mathrm{~h}$ post-infection while the samples for the detection of the actin protein expression were harvested at 24 and $72 \mathrm{~h}$ post-infection.

Western and northern blot analyses for the expression levels of HCMV proteins and mRNAs and ribozymes were performed as described previously [12,22,40]. In the northern blot analysis experiments, the RNA samples were separated in 1\% agarose gels that contained formaldehyde, transferred to membranes, hybridized with the $\left[{ }^{32} \mathrm{P}\right]$-radiolabeled DNA probes that contained the HCMV DNA sequences or the DNA sequences coding for M1 RNA and H1 RNA, and analyzed with a STORM840 Phosphorimager [12,22,40]. The radiolabeled DNA probes used in the northern blot analysis experiments were synthesized from plasmids using a random primed labeling kit (Roche Applied Science, Indianapolis, IN).

In the western blot analysis experiments, the protein samples were separated on $9 \%(\mathrm{v} / \mathrm{v})$ SDS-polyacrylamide gels cross-linked with N,N-methylenebisacrylamide. The separated proteins were transferred electrically to nitrocellulose membranes and reacted with the antibodies against human actin and HCMV proteins. The membranes were subsequently stained with a chemiluminescent substrate with the aid of a Western chemiluminescent substrate kit (GE Healthcare) and quantitated with a STORM 840 Phosphorimager [12,22,40]. 
In order to accurately assay the viral $\mathrm{mRNA}$ /protein expression and increase the sensitivities of our detection assays, a series of diluted RNA and protein samples (1-100-fold dilution) were used and quantitation was performed in the linear range of RNA and protein detection. For example, two-fold changes in RNA and protein samples resulted in a two-fold change in signal bracketing the range of experimental values in our experiments. Furthermore, to obtain an accurate measurement of the expression level of a specific mRNA or protein, we carried out three separate and independent experiments. In each of these three experiments, cells were grown in triplicate and samples were collected from these cultures and analyzed. The results were the arithmetic average of the three experiments $[12,22,40]$.

To study the M1GS-mediated inhibition of viral replication, U373MG cells $\left(n=5 \times 10^{5}\right)$ expressing M1GS were infected with HCMV at an MOI of 1. Viral stocks were prepared from cells harvested at 1 day interval for 7 days post-infection, and viral titers in the stocks were assayed by infecting human foreskin fibroblasts in triplicate, following previously described procedures [12,22].

\subsection{Statistical Analysis}

Statistical significance was determined using Student's $t$ test for paired samples [46]. All the experiments were carried out in triplicate, and repeated three times. We considered differences statistically significant at a value of $p \leq 0.05$.

\section{Conclusions}

HCMV represents a significant global public health concern and developing novel anti-HCMV strategies is critical for the treatment and prevention of HCMV infection and associated diseases. RNase P ribozyme can act as a sequence-specific gene-targeting agent with promising applications in clinical settings. Here, we report the anti-HCMV activity of a ribozyme derived from a novel RNase P variant in cultured cells. The ribozyme variant cleaved the target IE2 mRNA in vitro and shut down IE2 expression in human cells more efficiently than the wild type ribozyme. Furthermore, cells expressing the variant exhibited more than a 3500-fold reduction in HCMV growth. Our study demonstrates that RNase P ribozyme variants are efficient in reducing HCMV infection and are potentially useful for anti-viral therapeutic applications.

\section{Acknowledgments}

We thank Phong Trang, Hao Gong and Xiaohong Jiang for excellent editorial assistance and technical help. G. V. and Y. C. were partially supported by a Block Grant Predoctoral Fellowship (UC-Berkeley). G. V. is a recipient of a University of California Dissertation Fellowship award. This research has been supported by grants from National Basic Research Program of China (973 Program, No. 2011CB504800), National Natural Science Foundation of China (No. 31100128 and 81030031), National Mega Project on Major Drug Development of China, National Small Business Innovation and Research (SBIR) Program of China, the Technology R \& D Program of Jiangsu Province, China (BG20077035 and BG2008662), and NIH (RO1-AI041927 and RO1-DE014842). 


\section{Author Contributions}

Z.Y., G.-P.V., H.Q., Y.W., Y.-C.C., K.Z. and F.L. designed the study, analyzed the results, and wrote the paper. Z.Y., G.-P.V., H.Q., Y.-C.C. and M.R. conducted experiments. All authors have read and approved the final manuscript.

\section{Conflicts of Interest}

The authors declare no conflict of interest.

\section{References and Notes}

1. Mocarski, E.S.; Shenk, T.; Pass, R.F. Cytomegalovirus. In Fields Virology; Knipe, D.M., Howley, P.M., Griffin, D.E., Martin, M.A., Lamb, R.A., Roizman, B., Straus, S.E., Eds.; Lippincott-William \& Wilkins: Philadelphia, PA, USA, 2007; pp. 2701-2772.

2. Boppana, S.B.; Ross, S.A.; Fowler, K.B. Congenital cytomegalovirus infection: Clinical outcome. Clin. Infect. Dis. 2013, 57, S178-S181.

3. Palella, F.J., Jr.; Delaney, K.M.; Moorman, A.C.; Loveless, M.O.; Fuhrer, J.; Satten, G.A.; Aschman, D.J.; Holmberg, S.D. Declining morbidity and mortality among patients with advanced human immunodeficiency virus infection. HIV Outpatient Study Investigators. $N$. Engl. J. Med. 1998, 338, 853-860.

4. Gallant, J.E.; Moore, R.D.; Richman, D.D.; Keruly, J.; Chaisson, R.E. Incidence and natural history of cytomegalovirus disease in patients with advanced human immunodeficiency virus disease treated with zidovudine. The Zidovudine Epidemiology Study Group. J. Infect. Dis. 1992, 166, 1223-1227.

5. Scherer, L.J.; Rossi, J.J. Approaches for the sequence-specific knockdown of mRNA. Nat. Biotechnol. 2003, 21, 1457-1465.

6. Stein, C.A.; Cheng, Y.C. Antisense oligonucleotides as therapeutic agents - Is the bullet really magical? Science 1993, 261, 1004-1012.

7. Wiebusch, L.; Truss, M.; Hagemeier, C. Inhibition of human cytomegalovirus replication by small interfering RNAs. J. Gen. Virol. 2004, 85, 179-184.

8. Jacque, J.M.; Triques, K.; Stevenson, M. Modulation of HIV-1 replication by RNA interference. Nature 2002, 418, 435-438.

9. Zu Putlitz, J.; Yu, Q.; Burke, J.M.; Wands, J.R. Combinatorial screening and intracellular antiviral activity of hairpin ribozymes directed against hepatitis B virus. J. Virol. 1999, 73, 5381-5387.

10. Yu, M.; Ojwang, J.; Yamada, O.; Hampel, A.; Rapapport, J.; Looney, D.; Wong-Staal, F. A hairpin ribozyme inhibits expression of diverse strains of human immunodeficiency virus type 1. Proc. Natl. Acad. Sci. USA 1993, 90, 6340-6344.

11. Sarver, N.; Cantin, E.M.; Chang, P.S.; Zaia, J.A.; Ladne, P.A.; Stephens, D.A.; Rossi, J.J. Ribozymes as potential anti-HIV-1 therapeutic agents. Science 1990, 247, 1222-1225. 
12. Yang, Y.H.; Li, H.; Zhou, T.; Kim, K.; Liu, F. Engineered external guide sequences are highly effective in inducing RNase $\mathrm{P}$ for inhibition of gene expression and replication of human cytomegalovirus. Nucleic Acids Res. 2006, 34, 575-583.

13. Altman, S.; Kirsebom, L.A. Ribonuclease P. In The RNA World; Gesteland, R.F., Cech, T.R., Atkins, J.F., Eds.; Cold Spring Harbor Press: Cold Spring Harbor, NY, USA, 1999; pp. 351-380.

14. Gopalan, V.; Altman, S., RNase P:structure and catalysis. In The RNA World; Gesteland, R., Cech, T., Atkins, J., Eds.; Cold Spring Harbor Laboratory Press: Cold Spring Harbor, NY, USA, 2006; Volume 277, Chapter 6.1. Available online: http://rna.cshl.edu/ (accessed on 18 May 2014).

15. Kazantsev, A.V.; Pace, N.R. Bacterial RNase P: A new view of an ancient enzyme. Nat. Rev. Microbiol. 2006, 4, 729-740.

16. Guerrier-Takada, C.; Gardiner, K.; Marsh, T.; Pace, N.; Altman, S. The RNA moiety of ribonuclease $\mathrm{P}$ is the catalytic subunit of the enzyme. Cell 1983, 35, 849-857.

17. Marvin, M.C.; Engelke, D.R. Broadening the mission of an RNA enzyme. J. Cell Biochem. 2009, 108, 1244-1251.

18. Forster, A.C.; Altman, S. External guide sequences for an RNA enzyme. Science 1990, 249, 783-786.

19. Yuan, Y.; Hwang, E.S.; Altman, S. Targeted cleavage of mRNA by human RNase P. Proc. Natl. Acad. Sci. USA 1992, 89, 8006-8010.

20. Frank, D.N.; Harris, M.; Pace, N.R. Rational design of self-cleaving pre-tRNA-ribonuclease P RNA conjugates. Biochemistry 1994, 33, 10800-10808.

21. Liu, F.; Altman, S. Inhibition of viral gene expression by the catalytic RNA subunit of RNase P from Escherichia coli. Genes Dev. 1995, 9, 471-480.

22. Trang, P.; Lee, M.; Nepomuceno, E.; Kim, J.; Zhu, H.; Liu, F. Effective inhibition of human cytomegalovirus gene expression and replication by a ribozyme derived from the catalytic RNA subunit of RNase P from Escherichia coli. Proc. Natl. Acad. Sci. USA 2000, 97, 5812-5817.

23. Trang, P.; Kilani, A.F.; Kim, J.; Liu, F. A ribozyme derived from the catalytic subunit of RNase P from Escherichia coli is highly effective in inhibiting replication of herpes simplex virus 1. J. Mol. Biol. 2000, 301, 817-816.

24. Cobaleda, C.; Sanchez-Garcia, I. In vitro inhibition by a site-specific catalytic RNA subunit of RNase P designed against the BCR-ABL oncogenic products: A novel approach for cancer treatment. Blood 2000, 95, 731-737.

25. Zhou, J.; Rossi, J.J. Current progress in the development of RNAi-based therapeutics for HIV-1. Gene Ther. 2011, 18, 1134-1138.

26. Ditzler, M.A.; Bose, D.; Shkriabai, N.; Marchand, B.; Sarafianos, S.G.; Kvaratskhelia, M.; Burke, D.H. Broad-spectrum aptamer inhibitors of HIV reverse transcriptase closely mimic natural substrates. Nucleic Acids Res. 2011, 39, 8237-8247.

27. Held, D.M.; Kissel, J.D.; Patterson, J.T.; Nickens, D.G.; Burke, D.H. HIV-1 inactivation by nucleic acid aptamers. Front. Biosci. 2006, 11, 89-112. 
28. Zeng, W.; Chen, Y.C.; Bai, Y.; Trang, P.; Vu, G.P.; Lu, S.; Wu, J.; Liu, F. Effective inhibition of human immunodeficiency virus 1 replication by engineered RNase P ribozyme. PLoS One 2012, 7, e51855.

29. Mann, H.; Ben-Asouli, Y.; Schein, A.; Moussa, S.; Jarrous, N. Eukaryotic RNase P: Role of RNA and protein subunits of a primordial catalytic ribonucleoprotein in RNA-based catalysis. Mol. Cell 2003, 12, 925-935.

30. Liu, F. Ribonuclease P as a Tool; Springer: New York, NY, USA, 2010; pp. 257-276.

31. Kilani, A.F.; Trang, P.; Jo, S.; Hsu, A.; Kim, J.; Nepomuceno, E.; Liou, K.; Liu, F. RNase $\mathrm{P}$ ribozymes selected in vitro to cleave a viral mRNA effectively inhibit its expressionin cell culture. J. Biol. Chem. 2000, 275, 10611-10622.

32. Dunn, W.; Chou, C.; Li, H.; Hai, R.; Patterson, D.; Stolc, V.; Zhu, H.; Liu, F. Functional profiling of human cytomegalovirus genome. Proc. Natl. Acad. Sci. USA 2003, 100, 14223-14228.

33. Marchini, A.; Liu, H.; Zhu, H. Human cytomegalovirus with IE-2 (UL122) deleted fails to express early lytic genes. J. Virol. 2001, 75, 1870-1878.

34. Yu, D.; Silva, M.C.; Shenk, T. Functional map of human cytomegalovirus AD169 defined by global mutational analysis. Proc. Natl. Acad. Sci. USA 2003, 100, 12396-12401.

35. Zaug, A.J.; Cech, T.R. Analysis of the structure of Tetrahymena nuclear RNAs in vivo: Telomerase RNA, the self-splicing rRNA intron, and U2 snRNA. RNA 1995, 1, 363-374.

36. Jiang, X.; Chen, Y.C.; Gong, H.; Trang, P.; Lu, S.; Liu, F. Ribonuclease P-mediated inhibition of human cytomegalovirus gene expression and replication induced by engineered external guide sequences. RNA Biol. 2012, 9, 1186-1195.

37. Kim, J.J.; Kilani, A.F.; Zhan, X.; Altman, S.; Liu, F. The protein cofactor allows the sequence of an RNase P ribozyme to diversify by maintaining the catalytically active structure of the enzyme. RNA 1997, 3, 613-623.

38. Bertrand, E.; Castanotto, D.; Zhou, C.; Carbonnelle, C.; Lee, N.S.; Good, P.; Chatterjee, S.; Grange, T.; Pictet, R.; Kohn, D.; et al. The expression cassette determines the functional activity of ribozymes in mammalian cells by controlling their intracellular localization. $R N A$ 1997, 3, 75-88.

39. Zhu, H.; Cong, J.P.; Mamtora, G.; Gingeras, T.; Shenk, T. Cellular gene expression altered by human cytomegalovirus: Global monitoring with oligonucleotide arrays. Proc. Natl. Acad. Sci. USA 1998, 95, 14470-14475.

40. Zhou, T.; Kim, J.; Kilani, A.F.; Kim, K.; Dunn, W.; Jo, S.; Nepomuceno, E.; Liu, F. In vitro selection of external guide sequences for directing RNase P-mediated inhibition of viral gene expression. J. Biol. Chem. 2002, 277, 30112-30120.

41. Kieff, E.D.; Rickinson, A.B. Epstein-Barr virus and its replication. In Fields Virology; Knipe, D.M., Howley, P.M., Griffin, D.E., Martin, M.A., Lamb, R.A., Roizman, B., Straus, S.E., Eds.; Lippincott-William \& Wilkins: Philadelphia, PA, USA, 2007; pp. 2604-2654.

42. Roizman, B.; Knipe, D.M.; Whitley, R.J. Herpes simplex viruses. In Fields Virology; Knipe, D.M., Howley, P.M., Griffin, D.E., Martin, M.A., Lamb, R.A., Roizman, B., Straus, S.E., Eds.; Lippincott-William \& Wilkins: Philadelphia, PA, USA, 2007; pp. 2503-2601. 
43. Fedor, M.J.; Uhlenbeck, O.C. Kinetics of intermolecular cleavage by hammerhead ribozymes. Biochemistry 1992, 31, 12042-12054.

44. Trang, P.; Lee, J.; Kilani, A.F.; Kim, J.; Liu, F. Effective inhibition of herpes simplex virus 1 gene expression and growth by engineered RNase P ribozyme. Nucleic Acids Res. 2001, 29, 5071-5078.

45. Miller, A.D.; Rosman, G.J. Improved retroviral vectors for gene transfer and expression. Biotechniques 1989, 7, 980-990.

46. Chen, Y.; Shen, A.; Rider, P.J.; Yu, Y.; Wu, K.; Mu, Y.; Hao, Q.; Liu, Y.; Gong, H.; Zhu, Y.; et al. A liver-specific microRNA binds to a highly conserved RNA sequence of hepatitis B virus and negatively regulates viral gene expression and replication. FASEB J. 2011, 25, $4511-4521$. 


\author{
MDPI AG \\ Klybeckstrasse 64 \\ 4057 Basel, Switzerland \\ Tel. +41616837734 \\ Fax +41613028918 \\ http://www.mdpi.com/ \\ Viruses Editorial Office \\ E-mail: viruses@mdpi.com \\ http://www.mdpi.com/journal/viruses
}





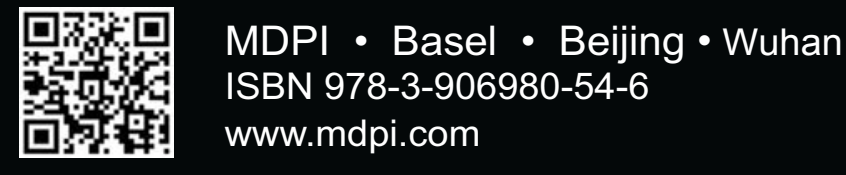

